\title{
Hanford Grout Technology Program \\ Leach Test of Cladding Removal Waste Grout Using Hanford Groundwater
}

R. J. Serne

W. J. Martin

V. L. Legore

September 1995

Prepared for the U.S. Department of Energy under Contract DE-AC06-76RLO 1830

Pacific Northwest Laboratory

Richland, Washington 99352 


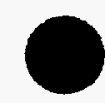

○

0 


\section{DISCLAIMER}

Portions of this document may be illegible in electronic image products. Images are produced from the best available original document. 


\section{Summary}

This report describes laboratory experiments performed during 1986-1990 designed to produce empirical leach rate data for cladding removal waste (CRW) grout. At the completion of the laboratory work funding was not available for report completion, and only now during final grout closeout activities, is the report published. The leach rates serve as inputs to computer codes used in assessing the potential risk from the migration of waste species from disposed grout. This report discusses chemical analyses conducted on samples of CRW grout, and the results of geochemical computer code calculations that help identify mechanisms involved in the leaching process.

The semi-infinite solid diffusion model was selected as the most representative model for describing leaching of grouts. The use of this model with empirically derived leach constants yields conservative predictions of waste release rates, provided no significant changes occur in the grout leach processes over long time periods.

The test methods included three types of leach tests--the American Nuclear Society (ANS) 16.1 intermittent solution exchange test, a static leach test, and a once-through flow column test. The synthetic CRW used in the tests was prepared in five batches using simulated liquid waste spiked with several radionuclides: iodine $\left({ }^{125} \mathrm{I}\right)$, carbon $\left({ }^{14} \mathrm{C}\right)$, technetium $\left({ }^{99} \mathrm{Tc}\right)$, cesium $\left({ }^{137} \mathrm{Cs}\right)$, strontium $\left({ }^{85} \mathrm{Sr}\right)$, americium $\left({ }^{21} \mathrm{Am}\right)$, and plutonium $\left({ }^{38} \mathrm{Pu}\right)$. The grout was formed by mixing the simulated liquid waste with dry blend containing Type I and Type II Portland cement, class F fly ash, Indian Red Pottery clay, and calcium hydroxide. The mixture was allowed to set and cure at room temperature in closed containers for at least 46 days before it was tested.

When a typical Hanford groundwater was used as the CRW grout leachant, only seven stable constituents ( $\mathrm{Na}, \mathrm{K}, \mathrm{F}, \mathrm{B}, \mathrm{Al}, \mathrm{SO}_{4}$ and $\left.\mathrm{NO}_{3}\right)$ and the three radionuclides $\left({ }^{125} \mathrm{I},{ }^{99} \mathrm{Tc}\right.$, and $\left.{ }^{85} \mathrm{Sr}\right)$ leached in measurable concentrations. The rate of leaching and the chemical composition of the leachates were dependent upon how often the groundwater leachant was exchanged. The leach rates (expressed in terms of cumulative effective diffusion coefficients, $\mathrm{D}_{\mathrm{e}}$ ) showed that $\mathrm{Na}, \mathrm{SO}_{4}$, and ${ }^{125} \mathrm{I}$ leached faster than $\mathrm{K}_{\text {and }} \mathrm{NO}_{3}$, all with effective diffusivities ranging from $10^{-9}$ to $10^{-10} \mathrm{~cm}^{2} / \mathrm{s}$. Species that leached considerably slower include ${ }^{85} \mathrm{Sr}, \mathrm{B}, \mathrm{F}, \mathrm{Al}$, and ${ }^{99} \mathrm{Tc}$, with effective diffusivities of approximately $10^{-12} \mathrm{~cm}^{2} / \mathrm{s}$. The other radionuclides $\left({ }^{14} \mathrm{C},{ }^{137} \mathrm{Cs},{ }^{241} \mathrm{Am}\right.$, and ${ }^{238} \mathrm{Pu}$ ), which showed little to no leaching, had effective diffusivities of $10^{-13} \mathrm{~cm}^{2} / \mathrm{s}$ or less.

The general trend of leaching behavior of CRW grout is similar to that experienced with another previously studied grouted Hanford liquid waste, phosphate/sulfate waste (PSW), despite the fact that CRW grout generated more alkalinity. Both PSW and CRW waste grouts showed a continued decrease in release rate for most species with time, even after extended periods of contact. Both PSW and CRW grouts exhibited calcite precipitation on the surface, resulting in an "armoring" phenomenon that may reduce the effective diffusivity of some species. An overall comparison of mass balance data from all leach test methods showed that in tests during which large amounts of groundwater contacted the grouts, relatively large amounts of $\mathrm{Ca}$ and $\mathrm{Mg}$ were precipitated.

The diffusion-based leach theory appears to adequately describe upper limit (bounding) release values in the short laboratory experiments conducted. However, accurate and detailed predictions 
should consider chemical reactions that lower leach rates below that predicted by diffusion alone. Trace metals, such as $\mathrm{Cd}, \mathrm{Cr}, \mathrm{Cu}, \mathrm{Pb}$, and $\mathrm{Zn}$, were present in the simulated waste in low concentrations, while the actual CRW liquid waste may contain higher concentrations of these metals. We did not find any measurable releases of $\mathrm{Ba}, \mathrm{Cd}, \mathrm{Cr}, \mathrm{Cu}, \mathrm{Fe}, \mathrm{Mn}, \mathrm{Pb}$, or $\mathrm{Zn}$ in the leachates. We hypothesize that solubility will control trace metal leach rates at insignificant levels because of the high $\mathrm{pH}$ aqueous environment. One element of particular abundance in the $\mathrm{CRW}$ grout is $\mathrm{Zr}$. No appreciable levels of $\mathrm{Zr}$ were observed in the leachate. Zirconium is readily hydrolyzed and is fairly insoluble at high $\mathrm{pH}$ environments.

In the flow-through column leach test, the initial contact between the groundwater and grout produced a white fibrous precipitate (believed to be calcium carbonate) in the solution surrounding the grout sample. The effluent samples showed a release of $\mathrm{F}^{-}, \mathrm{NO}_{3}^{-}, \mathrm{SO}_{4}^{2-}$, and $\mathrm{B}(\mathrm{OH})_{4}^{-}$. The general release trend for these elements was an initially high concentration that dropped off to near or ambient concentrations. The trace metal behavior (no appreciable leaching) was similar to that observed in both the static and ANS 16.1 leach tests.

Several combined leach-adsorption tests were also run where grout was packed along with Hanford soil into a column through which groundwater was percolated. The pH of leachate in combined grout/sediment leach tests and the once-through column test (no sediment present) gradually decreased with time. The CRW grout generates more alkalinity than the PSW grouts; therefore, the pH of CRW grout systems remains more alkaline for longer periods of time. The calcium and magnesium concentrations in the effluent from all leach tests were lower than those in the influent, possibly indicating that calcite and brucite precipitation reactions are controlling these elements.

The combined sediment/grout leach tests performed with crushed grout in several configurations showed a number of similarities with the leach test using intact grout embedded in sediment: 1) the lowered $\mathrm{Ca}, \mathrm{Mg}$, and $\mathrm{Sr}$ values suggested that precipitation of these elements occurred; 2) the $\mathrm{NO}_{3}^{-}$and $\mathrm{SO}_{4}^{2-}$ values returned to ambient groundwater concentrations over time; 3) the $\mathrm{F}^{-}$and borate effluent concentrations reached steady-state; and 4) the $\mathrm{Cl}^{-}$concentrations remained fairly constant. The disaggregated grout allowed for the more rapid flushing of alkalinity, $\mathrm{Na}, \mathrm{K}$, and anions, suggesting that the release of these elements is diffusion controlled in intact grout specimens. Past experiments using crushed PSW grout gave similar results. These similarities help enforce our assumptions about the controlling leach mechanisms for certain species, and provide defensible data for use in performance assessment predictions.

Comparison of results with grouts leached in sediment to those without sediment demonstrated that an ion exchange process where $\mathrm{K}$ replaces $\mathrm{Na}$ and $\mathrm{Ca}$ on soil sites occurs during early leaching time.

The data presented in this report were compared to results from similar tests previously performed on PSW grouts. Many of the trends observed in the two sets of tests are similar, suggesting that differences in starting liquid waste composition may be muted after solidification occurs in optimal grout blends. 


\section{Acknowledgments}

This study was funded during 1986-90 by Westinghouse Hanford Company (Westinghouse) as part of the Hanford Grout Technology Program. We wish to acknowledge the program direction, support, and peer review efforts of J. J. Zimmer, L. C. Brown, R. S. Shirley, M. T. Jansky, J. W. Shade and J. A. Voogd from WHC and M. S. Hanson and J. H. Westsik, Jr. from PNL.

We wish to thank R. O. Lokken, P. F. C. Martin, and L. A. Bray of PNL for making the radioactive grout samples used in this study.

We thank the following PNL staff members for analytical support: F. T. Hara and D. E. Rinehart (inductively coupled plasma and ion chromatography), R. W. Sanders (x-ray fluorescence), and K. M. McFadden (total organic carbon). We thank M. G. Stinson for typing the original manuscript. Finally, we thank J. H. Westsik, Jr., J. M. Creer, and C. T. Kincaid (PNL) for allocating the funds to publish this report in 1995. 
0

○

-

L

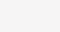

.

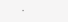

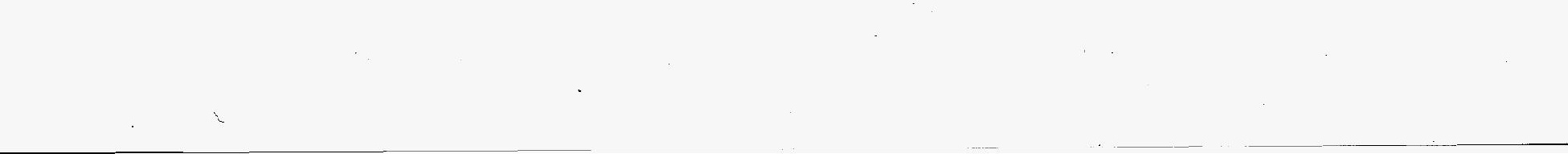




\section{Contents}

Summary $\ldots \ldots \ldots \ldots \ldots \ldots \ldots \ldots \ldots \ldots \ldots \ldots \ldots \ldots \ldots \ldots$ iii

Acknowledgments $\ldots \ldots \ldots \ldots \ldots \ldots \ldots \ldots \ldots \ldots \ldots \ldots \ldots \ldots \ldots$

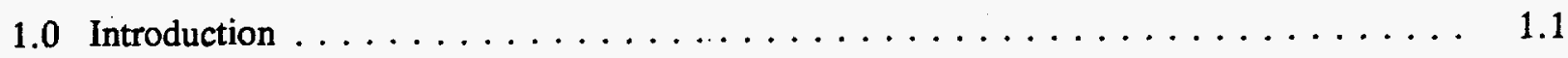

Hydrologic and Chemical Processes Controlling Leachate Generation and
Subsequent Migration $\ldots \ldots \ldots \ldots \ldots \ldots \ldots \ldots \ldots \ldots \ldots \ldots \ldots \ldots \ldots$

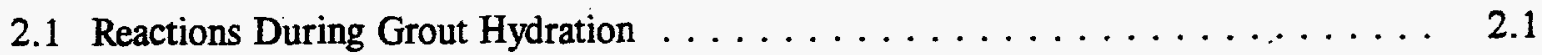

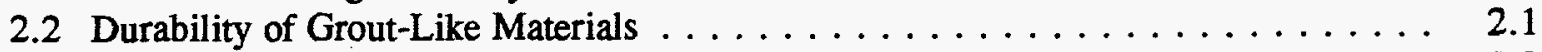

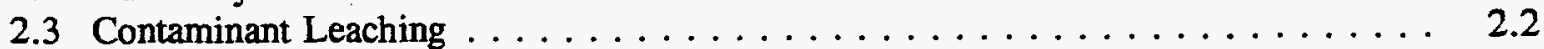

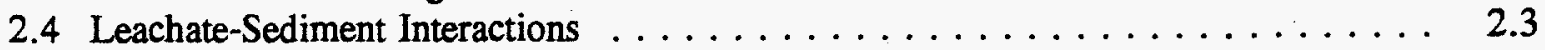

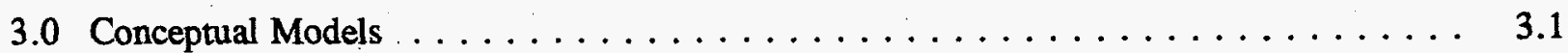

3.1 Semi-Infinite Solid Diffusion Leach Model . . . . . . . . . . . . . . . 3.1

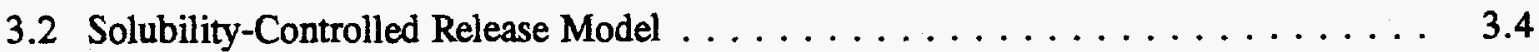

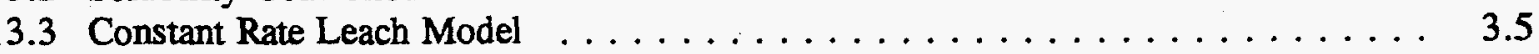

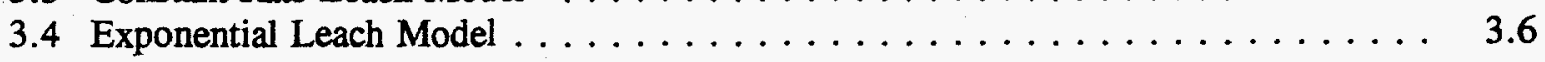

4.0 Materials $\ldots \ldots \ldots \ldots \ldots \ldots \ldots \ldots \ldots \ldots \ldots \ldots \ldots \ldots \ldots \ldots \ldots$

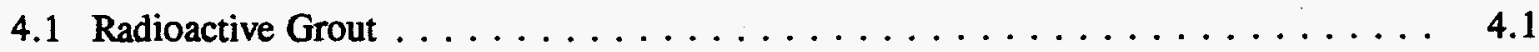

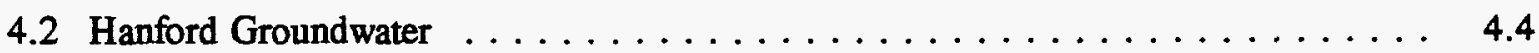

4.3 Hanford Sediment . . . . . . . . . . . . . . . . . . . 4.9

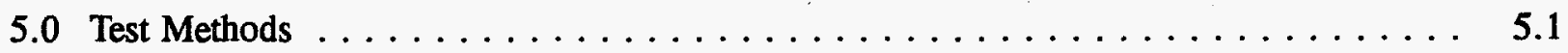

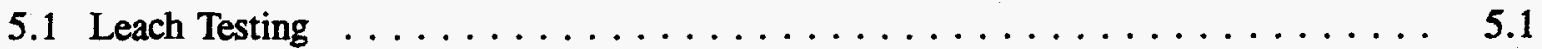

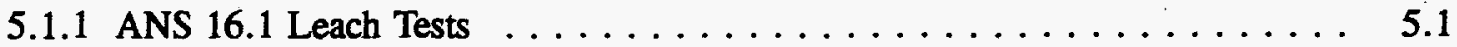

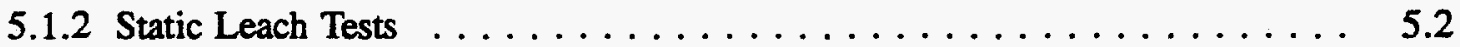

5.1 .3 Flow-through Column Leach Test $\ldots \ldots \ldots \ldots \ldots \ldots$

5.2 Combined Leach Test $\ldots \ldots \ldots \ldots \ldots \ldots \ldots \ldots \ldots \ldots \ldots \ldots$

5.3 Solids Analysis $\ldots \ldots \ldots \ldots \ldots \ldots \ldots \ldots \ldots \ldots \ldots \ldots \ldots$

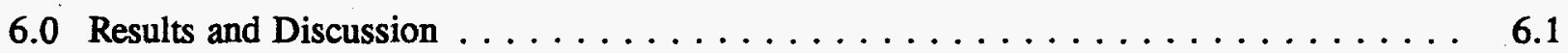

6.1 Chemical Analysis of Leachates From ANS 16.1 Leach Tests on Intact CRW Grouts $\ldots \ldots \ldots \ldots \ldots \ldots \ldots \ldots \ldots \ldots \ldots$ 
6.2 Chemical Analysis of Leachates from Static Leach Tests on Intact CRW Grouts

6.3 Chemical Analysis of Leachates From Flow-Through Leach Tests

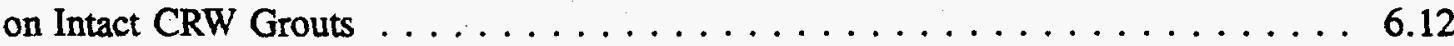

6.4 Chemical Analysis of Leachates from Combined Leach/Adsorption Tests on Intact CRW Grouts

6.5 Chemical Analysis of Effluents from Grout Leach Tests and Combined Leach/Adsorption Tests Using Crushed Grout . . . . . . . . . . . . 6.21

6.6 Cumulative Effective Diffusion Coefficients for Samples of Intact Grout . . . . . . . . . . . . . . . . . . . . . 6.23

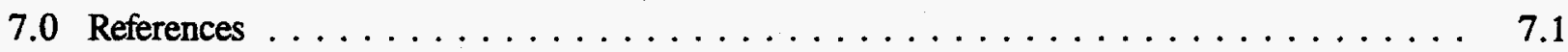

Appendix A - Chemical Compositions of Leachates and Column Effluents $\ldots \ldots \ldots \ldots$

Appendix B - Cumulative Fraction Leached and Effective Diffusion Coefficient Data . . . . . B.1 Appendix C - Mass Balance of Leach Solutions $\ldots \ldots \ldots \ldots \ldots \ldots \ldots$ 


\section{Figures}

4.1 Particle-Size Distribution of Hanford Sediment $\ldots \ldots \ldots \ldots \ldots \ldots$

5.1 Various Configurations of Combined Tests $\ldots \ldots \ldots \ldots \ldots \ldots$

6.1 Comparison of ANS 16.1 Test Leachates for PSW and CRW Grouts . . . . . . . 6.1

6.2 Calcium Values for PSW and CRW Grouts, ANS 16.1 Test $\ldots \ldots \ldots \ldots . \ldots \ldots$

6.3 Magnesium Values for PSW and CRW Grouts, ANS 16.1 Test $\ldots \ldots \ldots . \ldots$

6.4 Sodium Concentrations for PSW and CRW Grouts, ANS 16.1 Test $\ldots \ldots \ldots \ldots .4$

6.5 Potassium Concentrations for PSW and CRW Grouts, ANS 16.1 Test $\ldots \ldots \ldots .4$

6.6 Strontium Concentration vs. Time for ANS 16.1 Test Using CRW Grout $\ldots \ldots \ldots .5$

6.7 Sodium Concentration vs. Time for CRW Grout $\ldots \ldots \ldots \ldots \ldots$

6.8 Potassium Concentration vs. Time for CRW Grout $\ldots \ldots \ldots \ldots \ldots \ldots$

6.9 Fluoride Concentration vs. Time for CRW Grout $\ldots \ldots \ldots \ldots \ldots$

6.10 Nitrate Concentration vs. Time for CRW Grout $\ldots \ldots \ldots \ldots \ldots \ldots$

$6.11 \mathrm{pH}$ Values for Grout Leachates in Hanford Groundwater . . . . . . . . . . . 6.9

6.12 Potassium Concentration vs. Time for the CRW Static Leach Test . . . . . . . . . 6.10

6.13 Sodium Concentration vs. Time for the CRW Static Leach Test $\ldots \ldots \ldots . \ldots$

6.14 Nitrate Concentration vs. Time for CRW Grout $\ldots \ldots \ldots \ldots \ldots \ldots \ldots \ldots$

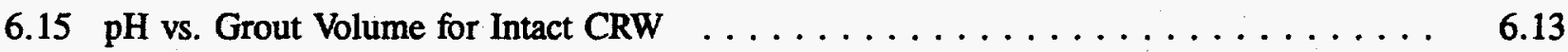

6.16 Sodium Concentration vs. Leachant/Grout Volume for Intact CRW Grouts in
Hanford Groundwater $\ldots \ldots \ldots \ldots \ldots \ldots \ldots \ldots \ldots \ldots \ldots \ldots \ldots \ldots \ldots \ldots$

6.17 Potassium Concentration vs. Leachant/Grout Volume for Intact CRW Grouts in Hanford Groundwater $\ldots \ldots \ldots \ldots \ldots \ldots \ldots \ldots \ldots \ldots \ldots$

$6.18 \mathrm{pH}$ Values for Leach Test vs. Combined Leach Test $\ldots \ldots \ldots \ldots \ldots$

6.19 Calcium Concentration for Leach Test vs. Combined Leach Test $\ldots \ldots \ldots . \ldots$ 
6.20 Sodium Concentration for Leach Test vs. Combined Leach Test . . . . . . . . . . 6.17

6.21 Potassium Concentration vs. Pore Volume for Intact CRW Grouts Packed

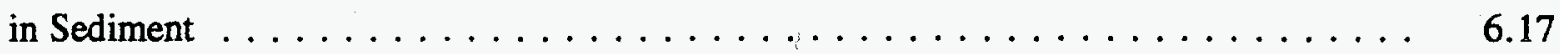

6.22 Fluoride Concentration for Leach Test vs. Combined Leach Test . . . . . . . . . . 6.18

6.23 Nitrate Concentration for Leach Test vs. Combined Leach Test . . . . . . . . . . 6.19

6.24 Sulfate Concentration for Leach Test vs. Combined Leach Test . . . . . . . . . 6.19 


\section{Tables}

4.1 Chemical Composition of CRW Waste Solution $\ldots \ldots \ldots \ldots \ldots \ldots$

4.2 Waste Batches Spiked with Radionuclides $\ldots \ldots \ldots \ldots \ldots \ldots \ldots$

4.3 Dry Blend for CRW Grout $\ldots \ldots \ldots \ldots \ldots \ldots \ldots \ldots \ldots \ldots \ldots$

4.4 Chemical Composition of the CRW Grout $\ldots \ldots \ldots \ldots \ldots \ldots$

4.5 Initial Inventory of Elemental Components for Each Sample Tested, $\mathrm{mg} \ldots \ldots .5$

4.6 Initial Inventory of the Radioactive Components in the Samples Tested, $\mu \mathrm{Ci} \ldots \ldots .6$

4.7 Chemical Composition of Hanford Well Water Over Time $\ldots \ldots \ldots \ldots \ldots$

5.1 Sampling Schedule for ANS 16.1 Leach Tests $\ldots \ldots \ldots \ldots \ldots \ldots \ldots$

5.2 Sampling Schedule for Static Leach Tests $\ldots \ldots \ldots \ldots \ldots \ldots \ldots$

6.1 Sodium and Potassium Mass Leached (mg) from CRW and PSW Grouts Using Two Test Methods $\ldots \ldots \ldots \ldots \ldots \ldots \ldots \ldots \ldots \ldots \ldots \ldots \ldots \ldots$

6.2 Mass Balance Relationships for Various Test Methodologies $\ldots \ldots \ldots \ldots .20$

6.3 Comparison of Mass Balances for the Combined Leach/Adsorption Test of

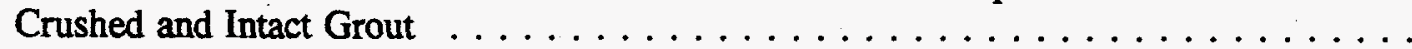

6.4 Effective Diffusion Coefficient Values Obtained From Standard Leach Tests $\ldots \ldots . . . \quad 6.24$

6.5 Flow-Through Column Data for Intact Grouts $\mathrm{D}_{\mathrm{c}} \ldots \ldots \ldots \ldots \ldots \ldots$ 


\subsection{Introduction}

A Grout Treatment Facility (GTF) was constructed on the Hanford Site near Richland, Washington, and was to be used to immobilize and dispose of the low-level fraction of liquid, radioactive wastes as a solidified grout. The grout disposal program was funded through the U.S. Department of Energy (DOE) and managed by Westinghouse Hanford Company (Westinghouse), with technical support from Oak Ridge National Laboratory (ORNL) and Pacific Northwest Laboratory (PNL). ${ }^{\text {(a) }}$ In 1993 a decision was made to halt grouting activities until other solidification methods, such as glass vitrification, could be further developed.

In the GTF, low-level fractions of liquid wastes are mixed with blends of grout-forming solids (Portland cement, fly ash, clays, and other components) to produce a slurry. This slurry is pumped to near-surface disposal sites (engineered vaults) where the grout hardens, thus immobilizing the waste. As part of the program to demonstrate the acceptability of grout as a final disposal method, performance assessment studies are being conducted (e.g. Sewart et al. 1987 and Kincaid et al. 1995). These studies generated computer-model predictions that are used to assess the impact of the grout disposal method on long-term public health and safety.

An assessment of the long-term risks posed by grout requires data on the ability of grout to resist leaching and/or diffusion of waste species contained in the grout via contact with water that would percolate into the waste after the engineered vault degrades. Leaching data are also needed for determining the ability of the soil (sediment) that surrounds the grout vaults to retard the mobility of any wastes released from the grout. Any changes that may occur with time (up to 10,000 years) in the ability of grout to contain wastes and the ability of sediment to retard waste migration must also be factored into a credible performance assessment.

This report describes specific laboratory experiments performed during 1986-1990 that produced empirical leach rate data for a particular waste stream, cladding removal waste (CRW). At the completion of the laboratory work funding was not available for report completion, and only now during final grout closeout activities, is the report published. The report provides empirical leach rates for several radionuclides and stable chemicals. The leach rate values are direct inputs to the performance assessment models used to forecast environmental impacts. This report also discusses other chemical analyses and geochemical computer code simulations that were used to help identify mechanisms that control the release of species from the solid grout waste form during contact with groundwater. Knowledge of the controlling chemical and physical processes is necessary to justify the extrapolation of the empirical laboratory data to long time periods and the very large scale of the grout disposal system.

The report is divided into sections that discuss the hydrologic and chemical processes controlling grout leaching, models used to understand such processes, materials and methods used in the leaching tests, and data generated from the experiments. Appendices $A$ through $\mathrm{C}$ contain the data used to support the analyses.

(a) Pacific Northwest Laboratory is operated by Battelle Memorial Institute for the U.S. Department of Energy under Contract DE-AC06-76RLO 1830. 


\subsection{Hydrologic and Chemical Processes Controlling Leachate Generation and Subsequent Migration}

Hanford grouts are formed by mixing liquid wastes with dry solids, including Portland cement, fly ash, and other additives. The cement acts as a binder and the fly ash is added to react with excess calcium hydroxide from the cement, thereby reducing permeability and leach rates. Fly ash also improves the ability of the slurry to be pumped. Additives such as clay may be used as suspending agents and to adsorb or sequester specific contaminants. The wastes and solids used in the formulation may affect reactions that control the rate and composition of leachate generation, and the subsequent migration of leached species. For each specific type of liquid waste, a grout recipe was developed by other project participants to optimize the processing characteristics of the grout and the stability of the waste form, and to mitigate long-term leaching. A specific description of the CRW grout recipe used in these leach tests is given in Section 5.0.

\subsection{Reactions During Grout Hydration}

The Portland cement used in grout formulation is a mixture of tricalcium silicate $\left(3 \mathrm{CaO} \cdot \mathrm{SiO}_{2}\right)$, dicalcium silicate $\left(2 \mathrm{CaO} \cdot \mathrm{SiO}_{2}\right)$, tricalcium aluminate $\left(3 \mathrm{CaO} \cdot \mathrm{Al}_{2} \mathrm{O}_{3}\right)$, tetracalcium aluminoferrite $\left(4 \mathrm{CaO} \cdot \mathrm{Al}_{2} \mathrm{O}_{3} \cdot \mathrm{Fe}_{2} \mathrm{O}_{3}\right.$ ), and other constituents. Upon wetting, these minerals hydrate, forming polymeric chains of mostly amorphous calcium silicates, crystalline aluminoferrites and aluminates, and portlandite $\left[\mathrm{Ca}(\mathrm{OH})_{2}\right]$. When other chemicals are present, such as sulfate in wastes or bicarbonates in the groundwater leachant, other reactions occur, forming compounds such as ettringite $\left(3 \mathrm{CaO} \cdot \mathrm{Al}_{2} \mathrm{O}_{3} \cdot 3 \mathrm{CaSO}_{4} \cdot 31 \mathrm{H}_{2} \mathrm{O}\right)$, gypsum $\left(\mathrm{CaSO}_{4} \cdot 2 \mathrm{H}_{2} \mathrm{O}\right)$, calcite $\left(\mathrm{CaCO}_{3}\right)$, and carbonated calcium aluminates and ferrites.

During hydration and curing, the permeability of the grout decreases significantly. This decrease in permeability retards the mobility of waste components inside the grout. Furthermore, some of the waste constituents chemically react with the grout minerals or additives and become more strongly bound in the grout matrix. Cations that hydrolyze at high $\mathrm{pH}$ values, such as many metals and the actinides (e.g., ${ }^{241} \mathrm{Am}$ and ${ }^{238} \mathrm{Pu}$ ), could be expected to form highly insoluble hydroxides in the high $\mathrm{pH}$ environment inside the grout. Certain clay additives adsorb cations such as cesium and Sr. Matsuzuru and Ito (1978a) show that the addition of $10 \%$ to $20 \%$ by weight of zeolite (a form of clay) to the dry grout ingredients can decrease the effective leach rate of cesium by two to three orders of magnitude. In the CRW grout formulation, Indian Red Pottery clay is added to adsorb cesium, plutonium, and americium. Thus, some waste constituents are sequestered in the grout by strong chemical interactions through adsorption and precipitation. However, other waste constituents are physically isolated in the small pores of the grout and migration is controlled by diffusion processes.

\subsection{Durability of Grout-Like Materials}

Roy and Langton (1982) studied ancient human artifacts from Cyprus and Greece (water containers, aqueducts, and other findings) that were made using materials similar to Portland cement, and 
found those artifacts to be relatively intact. Nearly 40 samples from Greece and Cyprus dating from 400 B.C. to 200 A.D. were extensively studied by these investigators. Mineralogic, surface, and bulk chemical analyses confirmed that the cementitious materials in the artifacts had remained relatively intact for up to 3000 years in the presence of surface and subsurface waters. Many of the artifacts, such as aqueducts, bath basins, and drinking mugs, were used to contain water or were in contact with water nearly continuously over this length of time. Reaction products of the kind previously discussed were identified (e.g., hydrated calcium aluminate and hydrated calcium ferrite crystals as well as calcite and carbonated calcium aluminates and ferrites). The presence of these reaction products attests to the durability of the artifacts, and suggests that modern cementitious materials will be at least as durable as the artifacts.

\subsection{Contaminant Leaching}

Water percolating through Hanford sediments may contact the solid waste forms and leach waste constituents from the grout once the integrity of the engineered vaults is breached. Currently, the groundwater recharge rate at Hanford is not known with certainty, but for bounding calculations, a value between 0.5 and $5.0 \mathrm{~cm} / \mathrm{yr}$ was adopted for the environmental impact statement for the disposal of Hanford defense waste (HDW-EIS) (U.S. DOE 1987).

Qualitative permeability tests conducted at PNL have shown that more than 7.6 bars [110 lb/in. $\left.{ }^{2}\right]$ over-pressure is required to force water to flow through grout. Thus, it seems reasonable that any recharge water that is not diverted around the grout by the various proposed interbarriers in the engineered vault will encounter the low permeability grout monolith, provided that the waste forms are not fractured. Because the surrounding sediments are rather coarse and permeable, any significant water flow should be diverted through the sediments as opposed to directly flowing through the grout. Thus, a waste release model based on diffusion and chemical reaction processes seems appropriate in order to project the long-term impacts of grout disposal.

For grouts containing radioactive and chemically hazardous wastes, the release of wastes is most often described as an effective diffusion process--dissolved wastes diffuse through interconnected pores out of the grout waste form and the engineered vault that contains it into the surrounding sediments. In reality, the actual processes that control grout leaching are complex, but most of the available literature on grout and cement leaching argue fairly conclusively that an effective (or lumped) diffusion model satisfactorily explains the observed short-term laboratory data. Using the effective diffusion model, most radionuclides, and, by inference, inorganic hazardous chemicals leaching from cementitious waste forms exhibit a wide range of diffusion coefficients ranging from $10^{-6}$ to less than $10^{-13} \mathrm{~cm}^{2} / \mathrm{s}$ (see Tables in Appendices A and B). For comparison, most cations and anions exhibit a diffusion coefficient of approximately $10^{-5} \mathrm{~cm}^{2} / \mathrm{s}$ in water (Dayal, Arora and Morcos 1983). The large range in the effective diffusion coefficients observed for species in grout is a reflection of the other processes, such as adsorption, precipitation, and mineral substitution, that impede the release of some constituents.

An effective diffusion model is used for understanding contaminant release because it is simple and yet satisfactorily describes most laboratory leach data collected from tests that were conducted over periods ranging from several months to several years. More complicated models usually require a complete analysis of the chemical and mineralogical nature of the grout both before and after contact 
with leachant, as well as a complete chemical characterization of the leachate solution versus time. Furthermore, the grout and its interstitial water probably vary, both chemically and physically, with position relative to the grout surface such that bulk analyses of the grout solid and bulk leachate are only approximate estimates of the reacting system. The use of microanalytical techniques that allow analyses at the surface of the grout and at various depths may be necessary to accurately describe all of the interactions and to predict long-term changes in the waste retentive properties of the grout. Very few researchers experimenting with leaching from low-level radioactive waste forms have attempted detailed analyses of the bulk solids and leachates, let alone the potentially numerous microenvironments that can exist in a grout matrix.

Should long-term performance assessment analyses indicate that release and transport of contaminants from grout must be reduced, a moisture barrier could be placed around and/or over the disposed grout and engineered vault to further reduce advection of water and therefore reduce contaminant transport rates. Though a moisture barrier may reduce the advective transport of waste constituents from grout waste forms, diffusive transport of contaminants through the pore water may still be significant. It follows that the diffusive transport pathway could control the release and transport of wastes buried beneath an intact protective barrier. The diffusion-dominated water pathway would conduct contaminants in all directions to advection-dominated water transport pathways outside the barrier's influence.

A coupled advection-diffusion and chemical reaction transport model may be required to describe release and transport because both of these processes may provide significant contributions to the overall transport rate. Such a combined advection-diffusion with chemical reaction transport model will require detailed hydrologic knowledge of the neighboring barrier and unsaturated sediments, and an understanding of the mechanisms of release of contaminants from the grout surface.

\subsection{Leachate-Sediment Interactions}

It is assumed that after contaminants from within the grout matrix diffuse to the surface of the grout waste form (and any additional vault barriers), they will contact the partially saturated sediments that surround the vault. Upon contacting the sediments, the leached contaminants can be advected away by percolating water or diffused away through the pore water contained in the partially saturated vadose sediments. By either pathway, the leached contaminant species will travel through two types of sediments before reaching the biosphere: the vadose zone and the saturated aquifer. In both the vadose and saturated aquifer sediments, chemical reactions, such as adsorption and precipitation, and physical processes, such as diffusion and dispersion, will change the composition and concentration of the leachate. In the semiarid climate at Hanford, it is likely that the concentrations of many chemical constituents in solution at the vault-sediment interface will be at their maximum values (i.e., limited by their solubilities). Also, after an initial stabilization period, it is unlikely that redox and $\mathrm{pH}$ conditions will change dramatically as the leachate is transported away from the sediments surrounding the grout. From these two assumptions, it is inferred that any dissolution/precipitation reactions that might occur would happen near and/or within the surface of the grout, the vault engineered barriers, and the sediment directly contacting the vault. 
Beyond the barrier-sediment interface the contaminants will likely be present at such low concentrations that adsorption reactions onto the sediment will be the only chemical process to further reduce contaminant transport. 


\subsection{Conceptual Models}

Laboratory leaching and adsorption tests that were outlined in the literature were reviewed to help identify the proper conceptual models. The conceptual models or the constructs used to analyze the empirical data are especially important because they dictate the types of experiments that should be run and what parameters should be measured or controlled.

The categories of leach models discussed here include semi-infinite solid diffusion, solubilitycontrolled release, constant release rate, and exponential release rate. These models were developed to explain empirical leaching data. Each of these models is described in the following section.

\subsection{Semi-Infinite Solid Diffusion Leach Model}

This type of diffusion model is the most common release model used to describe the leaching of grouts and other cement waste forms. This model is used by researchers at Brookhaven National Laboratory (Kalb and Columbo 1984; Arora and Dayal 1984; Dayal, Schweitzer and Davis 1984; Dayal, Arora and Morcos 1983); ORNL (Moore, Godbee and Kibbey 1977; Godbee, Anders and Nielson 1980; Clark 1977); the Japan Atomic Energy Research Institute (Matsuzuru and Moriyama 1982; Moriyama, Dojiri and Matsuzuru 1977); and the United Kingdom Atomic Energy Authority, Harwell (Sambell, Smitton and Elsden 1982). The model is used to analyze laboratory leach data on cylindrical specimens of cement or grout waste forms in which nuclear wastes are incorporated. This model is endorsed by the American Nuclear Society (ANS) and is the basis for their recommended leach test ANS-16.1, "Measurement of the Leachability of Solidified Low-Level Radioactive Wastes by a Short-Term Test Procedure" (ANS 1986). The theoretical equations for diffusion-controlled leaching are based on the following assumptions:

1. Once leaching commences, the concentration of the species being leached is zero in the solution at the waste form surface. In other words, dissolved waste is swept away from the surface as soon as it diffuses out of the waste form.

2. The leachant solution is continually moving past the waste form and its composition never changes significantly.

3. The solid waste form is homogeneous and remains essentially unchanged chemically and physically during the leach process. In other words, no significant chemical alterations occur and the mass of constituents removed from the waste form is negligible in comparison to the total mass of each constituent in the waste form. This is known as the semi-infinite requirement.

4. The surface is smooth and does not deteriorate with time or acquire a protective layer (i.e., the surface area is constant and equal to the geometric surface area).

5. There are no time-dependent interactions among the leachable species, the leachant, and the solid matrix. 
6. Each radionuclide and hazardous chemical of interest is present as only one chemical species.

7. The leachable species are mobilized by the leachant such that bulk diffusion is the limiting process.

Although the semi-infinite solid diffusion leach model is based on these seven simplifying assumptions, it has been accepted by many researchers. It is used to interpret laboratory leach tests on cement and grout waste forms in which the leachant is changed periodically. This diffusion model appears to successfully describe most empirical data collected to date.

The mathematical description of a diffusion model that meets the above assumptions yields a diffusion coefficient for each measured species. The effective diffusion coefficient combines all of the chemical and physical interactions that may actually be occurring into a single value that defines release rate.

Two mathematical descriptions of this model exist that are commonly used to analyze laboratory data based on the leaching of semi-infinite cylindrical solids, as follows:

$$
D_{\theta}=\frac{\pi}{4}\left[\frac{\sum_{n=1}^{N} a_{n}}{A_{0}}\right]^{2}\left[\frac{V}{S}\right]^{2} \frac{1}{t}
$$

Equation (3.1) is used to calculate the diffusion coefficient over all cumulative time, whereas the following equation (3.2) is used to calculate diffusion coefficient during each incremental time interval.

$$
D_{e}^{\prime}=\pi\left(\frac{a_{n}}{A_{0}}\right)^{2}\left(\frac{V}{S}\right)^{2} \frac{T}{(\Delta t)_{n}^{2}}
$$

where $D_{e}=$ effective cumulative diffusion coefficient $\left(\mathrm{cm}^{2} / \mathrm{s}\right), \mathrm{D}_{e}^{\prime}=$ instantaneous diffusion coefficient $\left(\mathrm{cm}^{2} / \mathrm{s}\right)$

$\mathrm{a}_{\mathrm{n}}=$ amount of species of interest leached during each leach interval

(e.g., g or $\mu \mathrm{Ci}$ )

$\mathrm{A}_{\mathrm{o}}=$ total amount of species in solid waste form (e.g., $\mathrm{g}$ or $\mu \mathrm{Ci}$ )

$\mathrm{V}=$ volume of solid waste form $\left(\mathrm{cm}^{3}\right)$

$S=$ geometric surface area of waste form $\left(\mathrm{cm}^{2}\right)$

$t=$ total elapsed time since start of leaching $(s)=\Sigma(\Delta t)_{n}=t_{n}$

$T=\left[1 / 2\left(\sqrt{t_{n}}+\sqrt{t_{n-1}}\right)\right]^{2}$

$(\Delta t)_{n}=$ time duration of interval $n(s)$

$\mathrm{n}=$ number of time intervals

$\mathbf{N}=$ total number of time intervals 
If the leach experiments satisfy the seven conditions previously mentioned, both equations (3.1) and (3.2) will give the same effective diffusion coefficient at all times (i.e., $\mathrm{D}_{e}=\mathrm{D}_{\mathrm{e}}^{\prime}$ ).

At Hanford an asphalt/aggregate diffusion barrier was planned to be placed around the CRW grout waste forms to further impede the release of contaminants into the surrounding soil. According to the proposed disposal system design, the water flow rate in the sediments around the engineered vault/ diffusion barrier was expected to be fast relative to the diffusion rate of contaminants through the various engineered barriers. However, it is doubtful that the flow rate of water around grout within the engineered vault will be fast enough to meet the first of the several assumptions, namely, that dissolved waste diffusing out of the grout will be swept away from the surface and satisfy the boundary condition, concentration $=0$ at $t>0$. If diffusion is in fact the controlling process, the semi-infinite diffusion model should overpredict the release of species from CRW grout, because any buildup of leached material near the grout surface will decrease the diffusive driving force. Furthermore, if protective barriers remain intact, the water within the various barriers will not flow but instead will transmit leached species via diffusion. Some of the barriers may have lower effective diffusion coefficients than the CRW grout and thus these barriers would further slow down contaminant transport. Diffusion in the partially saturated sediments surrounding the engineered vault is assumed to occur more rapidly than diffusion from the grout and engineered barriers. Again, the assumption that all leached species are rapidly swept away from the waste form surface would lead to an overprediction of the actual mass released from grout placed within an engineered barrier.

Over a 10,000-year time frame (which is assumed in the HDW-EIS to be the length of time that the grout disposal system must provide protection of public health and safety), the mass removed from the grout could begin to deplete the original waste inventory such that the third assumption, that for a semiinfinite solid, would lead to higher calculated releases than would calculations for a finite solid. Thus, the semi-infinite approach is conservative. The ANS 16.1 procedure (ANS 1986) gives correction tables for various geometries to correct predicted release rates if the semi-infinite criterion is violated. Furthermore, Moriyama et al. (1975) and Nestor (1980) give exact mathematical expressions for calculating $D_{e}$ values for certain finite geometric shapes. To ascertain whether more accurate results could be obtained using finite solid corrections, a useful rule of thumb is that corrections become important only if more than $20 \%$ of the total mass of a constituent of interest has leached from the waste form. A second check is to test whether the product, $D_{e} \cdot \sqrt{t}$, is much less than $r$, the characteristic length parameter for a solid shape. If so, to use the semi-infinite model equations over the time frame of interest is technically justifiable.

The $\mathrm{D}_{\mathrm{c}}$ (cumulative values) listed in Appendix B are corrected when $\Sigma \mathrm{a}_{\mathrm{a}} / \mathrm{A}_{\mathrm{o}}$ exceeds $20 \%$. The corrections are based on the characteristic lengths (diameter and height) of the sample cylinders per the protocol given by the ANS 16.1 procedure (ANS 1986).

It seems quite likely that CRW grout and concrete disposed in Hanford sediments will react with the carbon dioxide and carbonate-rich groundwaters to form calcite and carbonate-rich solids similar to those found in the artifacts from Cyprus and Greece (Roy and Langton 1982). From a few exploratory results on CRW grout using scanning electron microscopy, the calcite produced in these interactions forms a protective layer on the grout that would impede the diffusion process of most species. The 
actual extent of the reduction in diffusion has not yet been explored and the impact over long periods of time is less certain.

Three other diffusion-based models for release by a semi-infinite medium include the effects of 1) concentration-dependent dissolution; 2) surface dissolution represented by a moving boundary; and 3) time-dependent, first-order dissolution. Mathematical expressions for the cumulative fraction leached, $\Sigma \mathrm{a}_{\mathrm{n}} / \mathrm{A}_{\mathrm{o}}$, by these three models can be found in Moore, Godbee and Kibbey (1977). These three models have rarely been used by experimenters, probably because each requires identification of one or two additional parameters for which no a priori values are known.

If Equation (3.1) is rearranged, we can get a relationship that, when plotted as a function of the square root of time, should yield a straight line:

$$
\frac{\sum_{n=1}^{N} a_{n}}{A_{0}} \frac{V}{S}=2\left(\frac{D_{\theta}}{\pi}\right)^{1 / 2} t^{1 / 2}
$$

It is often found that actual leach data indicate a much faster release in the early time periods of the leach test and later satisfy the linear dependence with the square root of time. This situation occurred in the leaching experiments performed on all Hanford grouts (see Serne et al. 1987, 1989a,b and 1992). Matsuzuru and Moriyama (1982); Godbee, Anders and Nielson (1980); Matsuzuru and Ito (1978b), and Dayal, Arora and Morcos (1983) discuss leach experiments that show a similar initially rapid release. Data can be analyzed using linear regression on Equation (3.4) to provide a best-fit $D_{e}$ value (from the slope), and an empirical "instantaneous wash-off fraction, " $\alpha$ ", from the intercept at $t$ $=0$ :

$$
\frac{\Sigma a_{n}}{A_{0}} \frac{V}{S}=2\left(\frac{D_{e}}{\pi}\right)^{1 / 2} t^{1 / 2}+\alpha
$$

The actual mechanism or cause of the "instantaneous wash-off" has not been conclusively identified and perhaps could reflect that the experiments themselves do not satisfy all seven model assumptions discussed earlier. At any rate, after a relatively short time period, the data do start obeying Equations (3.1) and (3.3)-i.e., leach rate being proportional to the square root of time. If we assume that the leach data would continue to follow Equation (3.4) for very long time periods, we would predict an initial pulse release (the magnitude equal to $\alpha$ ) followed by a release rate proportional to the square root of time until the inventory of contaminant in the grout is exhausted.

\subsection{Solubility-Controlled Release Model}

If the laboratory data show that a constant or steady-state concentration is rapidly established in the leachate for a particular chemical or radionuclide, it can be speculated that a solid phase within the grout or a reaction product at the grout surface is limiting the solution concentration. Predictions of 
release over the long term can then be determined assuming that the concentration in the leachate remains constant until the controlling solid phase is exhausted. This constant concentration model is the simplest of the solubility-controlled release models. The solubility of any solid is defined by the product of the thermodynamic activity of each of the species formed upon dissolution. For example, if the release of ${ }^{90} \mathrm{Sr}$ and ${ }^{60} \mathrm{Co}$ from the grouted waste happened to be controlled by the solubility of $\mathrm{SrCO}_{3}$ and $\mathrm{Co}(\mathrm{OH})_{2}$ solids, then the products $\left[\mathrm{Sr}^{+2}\right]\left[\mathrm{CO}_{3}^{-2}\right]$ and $\left[\mathrm{Co}^{+2}\right]\left[\mathrm{OH}^{-}\right]^{2}$ would remain constant during dissolution. The symbol [ ] denotes thermodynamic activity of aqueous species that can be related to solution concentration. If the $\mathrm{pH}$ of the leachate changes, both $\left[\mathrm{OH}^{-}\right]$and $\left[\mathrm{CO}_{3}^{-2}\right]$ would change, and thus the $\left[\mathrm{Sr}^{+2}\right]$ and $\left[\mathrm{Co}^{+2}\right]$ would also change to maintain a constant solubility product. Thus, additional information, particularly the effects of $\mathrm{pH}$ changes, must be considered when applying solubility-controlled release models. Therefore, concentrations of dissolved species can vary even though solubility is being controlled by a single solid phase.

To manage all of the ancillary information and calculate the resulting solubility-controlled concentrations (which, as mentioned, can vary), a thermodynamic equilibrium code is often used. Examples of how to use such codes are found in Deutsch, Jenne and Krupka (1982); Strachan, Krupka and Grambow (1984); and Peterson et al. (1984). These codes may be used to evaluate whether certain solids could be controlling the concentrations of species in various leachates. Peterson et al. (1984) and Peterson, Martin and Serne (1986) show how such codes can be used to estimate limiting solution concentrations that should result when a liquid waste equilibrates with a sediment. The approach requires specifying precipitation and dissolution reactions. The leaching of Hanford grout can be evaluated using the same principles. An analysis of CRW grout leach data using these principles is discussed in Chapter 6.0 of this report.

\subsection{Constant Rate Leach Model}

One of the simplest release models is based on the assumption that a constituent leaches at a constant rate from the solid waste under all conditions and over all times until the waste form is depleted. The mathematical description of such a release is

$$
\frac{d A}{d t}=\lambda_{L} A_{0} \text { or } A=\lambda_{L} A_{0} \Delta t
$$

where $\mathrm{A}=$ mass released from waste solid $(\mathrm{g})$

$A_{0}=$ initial mass of constituent in the waste solid (g)

$\lambda_{L}=$ constant leach rate $\left(t^{-1}\right)$

$\Delta t=$ elapsed time for leaching $(t)$.

This type of model is often used for bounding or scoping calculations when actual data on solid waste leaching are not available. Laboratory leach data on cement and grout low-level nuclear waste forms do not show constant leach rate behavior. In general, the cement waste forms exhibit a release rate that decreases with advancing time from an initially high release rate. The constant rate leach model is not considered to be technically realistic for analyses of Hanford grouts, although it may be adequate for bounding calculations. 


\subsection{Exponential Leach Model}

A second simple release model is based on the assumption that the solid leaches such that the constituents are removed at an exponentially decreasing rate until the total waste form completely dissolves. The mathematical description of such a release model is

$$
\frac{d A}{d t}=\lambda_{I} A_{0} e^{-\lambda L t} \text { or } A=A_{0}\left(1-e^{-\lambda L \Delta t}\right)
$$

where the symbols have the same meaning and units as Equation 3.5. This model allows the mass released from the waste solid (A) to increase rapidly at first and change more slowly at longer times. This type of behavior better describes the leaching of cement waste forms, but is not as accurate as the diffusion-based models previously described. There is no physical process that relates exponential decay to the rate of leaching; thus, this model is typically not used. 


\subsection{Materials}

The materials used in these experiments (radioactive grout, groundwater, and sediment) are described in this section. The fabrication of the CRW grouts, their chemical and radiologic contents are discussed. The chemical composition of the groundwater and particle size of the sediment are also provided.

\subsection{Radioactive Grout}

The grout waste form used in this experiment was made from CRW, a low-level radioactive, liquid waste. For purposes of this study, CRW was prepared according to a neutralization process that involves neutralizing the acidic waste solution to $\mathrm{pH} \geq 12$ using concentrated sodium hydroxide $(\mathrm{NaOH})$. The slurry was allowed to settle, and approximately $60 \%$ of the supernatant was decanted. The solution was analyzed using the solution analysis techniques outlined in Chapter 5.0. Results from the analyses are listed in Table 4.1. The waste solution used was a chemically simulated liquid waste spiked with known quantities of radionuclides. Five batches of spiked liquid waste were prepared from a large nonradioactive stock solution. Predetermined amounts of radionuclides were added to each batch (Table 4.2). This waste slurry (the settled solids and liquid [40\%] not decanted and discarded) was mixed with the dry blend described in Table 4.3.

The composition of the CRW grout is listed in Table 4.4. A ratio of $7 \mathrm{lb}$ of grout-forming solids per gallon of liquid waste $(840 \mathrm{~g} / \mathrm{L})$ was used to produce the grout. The grout slurry was poured into plastic containers of various sizes that were capped to prevent moisture loss. The grout samples were cured at room temperature in the sealed plastic containers. A 28-day curing period is required prior to beginning the leach tests; the actual curing times reflect additional time prior to the initiation of experiments.

Just before starting each experiment, the individual grout samples were removed from their containers, weighed, and measured. Obvious cracks or defects were noted, and severely cracked samples were discarded. Cracking was usually a result of removing the sample from its hard plastic container. On all the samples, a few specks of loose grout clung to the main piece of grout. The specks were wiped off before weighing the sample.

Several small samples of grout were crushed and dried overnight at $105^{\circ} \mathrm{C}$. These samples on average contained $50 \%$ water as determined by simple weight loss measurement. Subsamples of the dried grout were submitted for total chemical analyses by two methods: 1) total fusion with lithium metaborate and dissolution followed by measurements with inductively coupled plasma (ICP), and 2) direct analyses of solid samples by $x$-ray fiuorescence (XRF). The dried grout also contained water of hydration or bound water (about $3.5 \%$ by weight), as determined by further heating to $300^{\circ} \mathrm{C}$. Upon igniting at $1000^{\circ} \mathrm{C}$ for 24 hours, the grout lost an additional $4.5 \%$ to $5.0 \%$ of its weight.

Because neither ICP nor XRF analyses alone provide complete information on all the chemical constituents, a composite of the results from both analyses was used (Table 4.4). Therefore, an 
Table 4.1. Chemical Composition of CRW Waste Solution

Constituents

Al

As

B

$\mathrm{Ca}$

$\mathrm{Cd}$

Co

$\mathrm{Cr}$

$\mathrm{Fe}$

Gd

$\mathrm{K}$

La

$\mathrm{Li}$

$\mathrm{Mg}$

$\mathrm{Mn}$

Mo

$\mathrm{Na}$

$\mathrm{Ni}$

$P$

$\mathrm{Pb}$

Si

$\mathrm{Zn}$

$\mathrm{Zr}$

$\mathrm{NH}_{4}$

$\mathrm{NO}_{2}$

$\mathrm{NO}_{3}$

F

$\mathrm{Cl}$

\section{Acidic Solution}
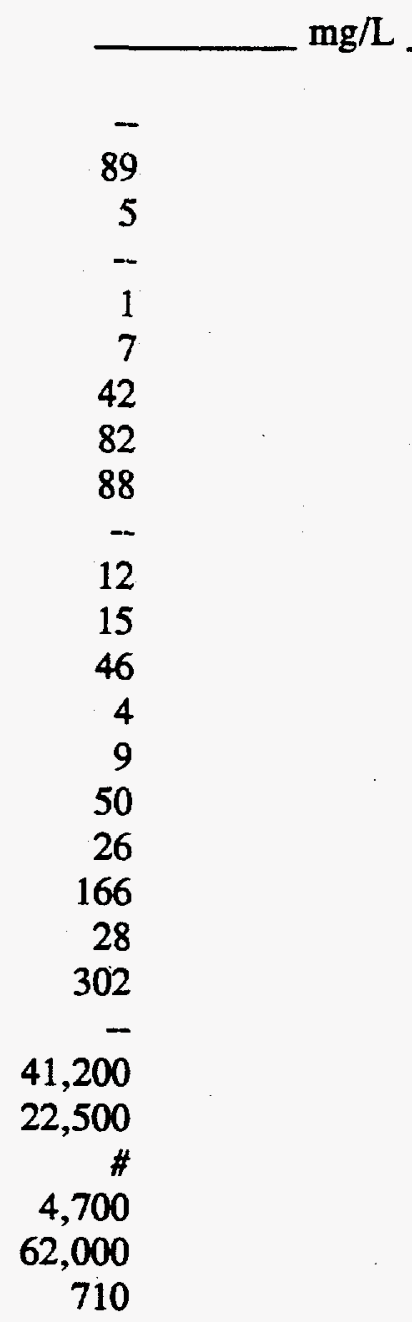

35

-

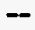

0.3

-

$-$

0.15

--

$-$

25

$-$

$-$

$-$

$-$

0.33

19,900

-

$-$

31

0.068

1.2

12,582

400

2,540

10,617

507

(-) Below detection limits.

(\#) Not determined. 
Table 4.2. Waste Batches Spiked with Radionuclides

\begin{tabular}{ccc} 
Batch Number & Radionuclide & Ouantity Added, mCi/L \\
\hline 1 & ${ }^{241} \mathrm{Am}$ & 0.5 \\
& ${ }^{238} \mathrm{Pu}$ & 0.538 \\
2 & ${ }^{14} \mathrm{C}$ & 0.5 \\
3 & ${ }^{99} \mathrm{Tc}$ & $0.02-0.05$ \\
4 & ${ }^{125} \mathrm{I}$ & 0.5 \\
5 & ${ }^{137} \mathrm{Cs}$ & 0.1 \\
& ${ }^{85} \mathrm{Sr}$ & 0.1
\end{tabular}

Table 4.3. Dry Blend for CRW Grout

Material

Portland cement LA (Type III)

Class F fly ash (Centralia, WA)

Calcium hydroxide

Indian Red Pottery Clay
Fraction, wt\%

42

40

10

Table 4.4. Chemical Composition of the CRW Grout

\section{Constituent}

$\mathrm{Al}_{2} \mathrm{O}_{3}$

$\mathrm{BaO}$

$\mathrm{CaO}$

$\mathrm{SiO}_{2}$

$\mathrm{Fe}_{2} \mathrm{O}_{3}$

$\mathrm{Na}_{2} \mathrm{O}$

$\mathrm{MgO}$

$\mathrm{TiO}_{2}$

$\mathrm{MnO}_{2}$

$\mathrm{SrO}$

$\mathrm{ZrO}_{2}$

$\mathrm{SO}_{4}$

$\mathrm{K}_{2} \mathrm{O}$

$\mathrm{NO}_{3}$

$\mathrm{NO}_{2}$

$\mathrm{Cl}$

F

$\mathrm{NH}_{4}$
Percent Weight

in Grout

10.2

0.1

36.6

28.8

3.82

9.47

1.26

1.5

0.04

0.13

5.56

0.65

0.49

0.19

0.02

0.02

4.08

0.5 
accurate mass balance is not possible. However, the values reported in Table 4.4 were assumed to be correct and were used to establish initial inventories, $A_{0}$, in the leach equations presented previously [i.e., Equations (3.1) through (3.4)]. Tables 4.5 and 4.6 give the $A_{o}$ values for nonradioactive and radioactive components, respectively, of the grout samples used in each leach test performed.

\subsection{Hanford Groundwater}

Using data from the Hanford Groundwater Monitoring Program, a PNL-managed program, a well (\#6-S3-25) was identified on the Hanford Site that is free of contamination and provides water representative of natural waters in the unconfined aquifer underlying Hanford. Periodically, large volumes of water were pumped from the well into pre-rinsed plastic containers. The well water was filtered through a $0.45-\mu \mathrm{m}$ membrane and stored for use in various laboratory experiments. A complete chemical analysis of the water was conducted on each new batch. No significant changes in the water composition from batch to batch and during storage in the laboratory were observed. Cation/anion balances were reasonable, suggesting that all significant dissolved constituents were measured and accounted.

The chemical composition of seven batches of this groundwater is listed in Table 4.7. The computer code MINTEQ (Felmy, Girvin and Jenne 1984) was used to determine whether or not this groundwater is in equilibrium with carbon dioxide and any common minerals found in Hanford sediments. Using average analyses based on values from Table 4.7, MINTEQ calculations showed the Hanford groundwater to be slightly enriched in dissolved $\mathrm{CO}_{2}\left(\mathrm{CO}_{2}=-2.9 \mathrm{~atm}\right.$ versus $-3.5 \mathrm{~atm}$ in equilibrium with air). MINTEQ also indicated that the groundwater might be in equilibrium with several minerals, including barite $\left(\mathrm{BaSO}_{4}\right)$, boehmite $\left[(\mathrm{AlO}(\mathrm{OH})]\right.$, dolomite $\left[\mathrm{Ca}, \mathrm{Mg}\left(\mathrm{CO}_{3}\right)\right]$, calcite $\left(\mathrm{CaCO}_{3}\right)$, magnesite $\left(\mathrm{MgCO}_{3}\right)$, sepiolite $\left[\mathrm{Mg}_{4}\left(\mathrm{Si}_{6} \mathrm{O}_{15}\right)(\mathrm{OH})_{2} \cdot 6 \mathrm{H}_{2} \mathrm{O}\right.$ ], gibbsite $\left[\mathrm{Al}(\mathrm{OH})_{3}\right]$, amorphous silica $\mathrm{SiO}_{2}, \mathrm{MnHPO}_{4}$, willemite $\left(\mathrm{Zn}_{2} \mathrm{SiO}_{4}\right)$, otavite $\left(\mathrm{CdCO}_{3}\right)$, cerrusite $\left(\mathrm{PbCO}_{3}\right)$ and analcime $\left(\mathrm{NaAlSi}_{2} \mathrm{O}_{6} \cdot \mathrm{H}_{2} \mathrm{O}\right)$. The sediments are known to contain trace quantities of calcite and amorphous silica.

Thus, the concentrations of calcium and magnesium in the groundwater appear to be controlled by either the pure carbonates or the mixed carbonate dolomite. Either gibbsite or boehmite might be controlling the aluminum concentration. Amorphous $\mathrm{SiO}_{2}$ appears to be controlling silicon concentrations. The carbonate system might also be controlling cadmium and lead concentrations, and a zinc silicate and barium sulfate may be controlling the zinc and barium concentrations, respectively. The concentrations of most trace metals appear to be below the solubility limits of common minerals and, thus, may be controlled by adsorption reactions.

We are assuming that the groundwater used in the leach tests is chemically similar to pore waters formed by meteoric water percolating through the vadose sediments near the land surface. This assumption is reasonable because in previous experiments, pore water from extracted vadose zone core samples that were contacted with deionized water for up to several days was similar to the groundwater (Serne et al. 1993). 
Table 4.5. Initial Inventory of Elemental Components for Each Sample Tested, mg

A. AHS 16.1 Leach Test

\begin{tabular}{|c|c|c|c|c|}
\hline \multirow[b]{2}{*}{ Element } & \multicolumn{4}{|c|}{ Sample } \\
\hline & ANS2-4 & ANS3-4 & ANS4-8 & ANS5-5 \\
\hline Al & 1669.2 & 1705.51 & 1538.62 & 1656.08 \\
\hline $\mathrm{Ca}$ & 8086.36 & 8262.24 & 7453.75 & 8022.8 \\
\hline$K$ & 125.81 & 128.24 & 115.97 & 124.82 \\
\hline $\mathrm{Mg}$ & 234.92 & 240.03 & 216.55 & 233.08 \\
\hline $\mathrm{Na}$ & 2166.87 & 2214 & 1997.35 & 2149.84 \\
\hline$S i$ & 4160.64 & 4251.14 & 3835.15 & 4127.94 \\
\hline $\mathrm{NO}_{3}$ & 58.73 & 60.01 & 54.14 & 58.27 \\
\hline $\mathrm{SO}_{4}^{3}$ & 200.92 & 205.29 & 185.2 & 199.34 \\
\hline & 1261.17 & 1288.61 & 1162.51 & 1251.26 \\
\hline $\mathrm{H}_{3} \mathrm{BO}_{3}$. & 191.03 & 195.19 & 176.09 & 189.53 \\
\hline
\end{tabular}

B. Static Leach Test

\begin{tabular}{|c|c|c|c|c|}
\hline \multirow[b]{2}{*}{ Element } & \multicolumn{4}{|c|}{ Sample } \\
\hline & $5 T 2-17$ & ST3-18 & ST4-13 & ST5-17 \\
\hline A) & 1727.78 & 1652.11 & 1736.63 & 1743.95 \\
\hline $\mathrm{Ca}$ & 8370.14 & 8003.59 & 8413 & 8448.48 \\
\hline K & 130.22 & 124.52 & 130.89 & 131.44 \\
\hline $\mathrm{Mg}$ & 243.17 & 232.52 & 244.41 & 245.44 \\
\hline $\mathrm{Na}$ & 2242.92 & 2144.69 & 2254.4 & 2263.91 \\
\hline Si & 4306.65 & 4118.05 & 4328.71 & 4346.96 \\
\hline $\mathrm{NO}_{3}$ & 60.79 & 58.13 & 61.1 & 61.36 \\
\hline $\mathrm{SO}_{4}^{3}$ & 207.97 & 198.86 & 209.04 & 209.92 \\
\hline & 1305.43 & 1248.26 & 1312.12 & 1317.65 \\
\hline $\mathrm{H}_{3} \mathrm{BO}_{3}$ & 197.73 & 189.07 & 198.75 & 199.58 \\
\hline
\end{tabular}

C. Column Experiment

\begin{tabular}{|c|c|c|c|}
\hline \multirow[b]{2}{*}{ Element } & \multicolumn{3}{|c|}{ Sample $e^{(a)}$} \\
\hline & COLCI & COLTI & COLII \\
\hline A1 & 2096.65 & 2111.9 & 2060.34 \\
\hline $\mathrm{Ca}$ & 10157.09 & 10230.99 & 9981.2 \\
\hline K & 158.02 & 159.17 & 155.29 \\
\hline Mg & 295.08 & 297.23 & 289.97 \\
\hline $\mathrm{Na}$ & 2721.76 & 2741.56 & 2674.63 \\
\hline$S i$ & 522.09 & 5264.11 & 5135.59 \\
\hline $\mathrm{NO}_{3}$ & 73.77 & 74.31 & 72.49 \\
\hline $\mathrm{SO}_{4}^{\mathrm{J}}$ & 252.37 & 254.21 & 248 \\
\hline & 1584.13 & 1595.66 & 1556.7 \\
\hline $\mathrm{H}_{3} \mathrm{BO}_{3}$ & 239.95 & 241.69 & 235.79 \\
\hline
\end{tabular}

\begin{tabular}{|c|c|c|c|}
\hline \multirow[b]{2}{*}{ Element } & \multicolumn{3}{|c|}{ Sample $e^{(a)}$} \\
\hline & COLLTI & COLLT2 & $\overline{C O L L T} 3$ \\
\hline AI & 2060.34 & 2120.44 & 2066.75 \\
\hline $\mathrm{Ca}$ & 9981.2 & 10272.38 & 10012 \\
\hline$k$ & 155.29 & 159.82 & 155.77 \\
\hline $\mathrm{Mg}$ & 289.97 & 298.43 & 290.87 \\
\hline $\mathrm{Na}$ & 2674.63 & 2752.65 & 2682.94 \\
\hline $\mathrm{Si}$ & 5135.59 & 5285.4 & 5151.56 \\
\hline $\mathrm{NO}_{3}$ & 72.49 & 74.61 & 72.72 \\
\hline $\mathrm{SO}_{4}^{3}$ & 248 & 255.24 & 248.77 \\
\hline$F^{\triangleleft}$ & 1566.7 & 1602.11 & 1561.54 \\
\hline $\mathrm{H}_{3} \mathrm{BO}_{3}$ & 235.79 & 242.67 & 236.53 \\
\hline
\end{tabular}

(a) Column experiment, no sediment.

(b) Column experiment, sediment present.

D. Crushed Grout Tests

\begin{tabular}{cc}
\multicolumn{2}{c}{$\begin{array}{c}\text { Samples CGT, CGC, SCGI } \\
\text { (in sediments) }\end{array}$} \\
\hline $\mathrm{Al}$ & 1547.15 \\
$\mathrm{Ca}$ & 7495.1 \\
$\mathrm{~K}$ & 116.61 \\
$\mathrm{Mg}$ & 217.75 \\
$\mathrm{Na}$ & 2008.43 \\
$\mathrm{Si}$ & 3856.42 \\
$\mathrm{NO}$ & 54.55 \\
$\mathrm{SO}_{4}^{3}$ & 186.23 \\
$\mathrm{~F}^{4}$ & 1168.96 \\
$\mathrm{H}_{380^{3}}$ & 177.06
\end{tabular}

\begin{tabular}{cc}
\multicolumn{2}{c}{$\begin{array}{c}\text { Samples } \\
\text { (no sediments }\end{array}$} \\
\hline $\mathrm{A}$ present) \\
$\mathrm{Ca}$ & 270 \\
$\mathrm{~K}$ & 1308 \\
$\mathrm{Mg}$ & 20.35 \\
$\mathrm{Na}$ & 38 \\
$\mathrm{Si}$ & 350.5 \\
$\mathrm{NO}_{3}$ & 673 \\
$\mathrm{SO}_{4}^{3}$ & 9.5 \\
$\mathrm{~F}^{4}$ & 32.5 \\
$\mathrm{H}_{3} \mathrm{BO}_{3}$ & 204 \\
& 30.9
\end{tabular}


Table 4.6. Initial Inventory of the Radioactive Components in the Samples Tested, $\mu \mathrm{Ci}$

A. Column Experiments

\begin{tabular}{|c|c|c|}
\hline Sample Name & Isotope & Quantity \\
\hline COL T1 & TC & $1.834 E+00$ \\
\hline $\mathrm{COL} \mathrm{T2}$ & ${ }^{99} \mathrm{TC}$ & $1.827 E+00$ \\
\hline $\operatorname{COL} 3 A$ & ${ }^{99}$ TC & $1.795 E+00$ \\
\hline $\mathrm{COL} 3 \mathrm{~B}$ & ${ }^{99} T \mathrm{C}$ & $1.870 E+00$ \\
\hline CRUSHED SCGT & ${ }^{99}$ Tc & $1.351 E+00$ \\
\hline CRUSHED CGT & ${ }^{99} \mathrm{Tc}$ & $1.351 E+00$ \\
\hline CRUSHED BCGT & ${ }^{99} \mathrm{Tc}$ & $2.650 \mathrm{E}-01$ \\
\hline $\operatorname{COL} 11$ & ${ }^{125} \mathrm{I}$ & $9.723 E-01$ \\
\hline COL 12 & $125 \mathrm{I}$ & $1.007 E+00$ \\
\hline $\operatorname{COL} 2 A$ & $125_{I}$ & $9.320 E-01$ \\
\hline $\operatorname{COL} 2 B$ & $125 \mathrm{I}$ & $9.320 E-01$ \\
\hline CRUSHED SCGI & 1251 & $3.800 E-01$ \\
\hline CRUSHED CGI & 125 & $3.800 E-01$ \\
\hline CRUSHED BCGI & & $1.759 \mathrm{E}-02$ \\
\hline $\mathrm{COL} \mathrm{Cl}$ & ${ }^{137} \mathrm{Cs}$ & $4.103 E+00$ \\
\hline $\mathrm{COLCl}$ & & $7.768 \mathrm{E}-02$ \\
\hline $\mathrm{COLC} C 2$ & ${ }^{137} \mathrm{Cs}$ & 4. $196 E+00$ \\
\hline $\mathrm{COL} \mathrm{C2}$ & ${ }^{85} \mathrm{Sr}$ & $7.945 \mathrm{E}-02$ \\
\hline COL $1 A$ & ${ }^{137} \mathrm{Cs}$ & $4.124 E+00$ \\
\hline COL $1 A$ & ${ }^{85} \mathrm{Sr}$ & $7.318 \mathrm{E}-02$ \\
\hline $\mathrm{COL} 1 \mathrm{~B}$ & $137 \mathrm{Cs}$ & $4.130 E+00$ \\
\hline $\mathrm{COL} 1 \mathrm{~B}$ & & $7.328 \mathrm{E}-02$ \\
\hline CRUSHED SCGC & ${ }^{137} \mathrm{Cs}$ & $3.045 \mathrm{E}+00$ \\
\hline CRUSHED SCGC & & $3.165 \mathrm{E}-02$ \\
\hline CRUSHED CGC & ${ }^{137} \mathrm{Cs}$ & $3.045 E+00$ \\
\hline CRUSHED CGC & ${ }^{85} \mathrm{Sr}$ & $3.165 \mathrm{E}-02$ \\
\hline CRUSHED BCGC & ${ }^{137}$ Cs & $5.970 E-01$ \\
\hline CRUSHED BCGC & ${ }^{85} \mathrm{Sr}$ & $1.605 E-03$ \\
\hline
\end{tabular}

B. ANS 16.1 Leach Test

\begin{tabular}{|c|c|c|}
\hline Sample Name & Isotope & Quantity \\
\hline ANS $1-B$ & & $1.344 E+01$ \\
\hline ANS $1-B$ & ${ }^{9} \mathrm{Pu}$ & $1.440 E+01$ \\
\hline ANS $1-13$ & ${ }^{41} \mathrm{Am}$ & $1.289 E+01$ \\
\hline ANS $1-13$ & ${ }^{239} \mathrm{Pu}$ & $1.382 E+01$ \\
\hline ANS $2-4$ & ${ }^{14} \mathrm{C}$ & $1.646 \mathrm{E}+00$ \\
\hline ANS 2-5 & ${ }^{14} \mathrm{C}$ & $1.676 \mathrm{E}+00$ \\
\hline ANS $3-4$ & ${ }^{99} \mathrm{Tc}$ & $1.481 E+00$ \\
\hline ANS $3-5$ & ${ }^{99} \mathrm{~T}$ & $1.473 E+00$ \\
\hline ANS $3-19$ & ${ }^{99} \mathrm{Tc}$ & $1.484 E+00$ \\
\hline ANS 4-8 & $125_{1}$ & $9.214 E+00$ \\
\hline
\end{tabular}


Table 4.6. (contd)

B. ANS 16.1 Leach Test (cont'd)

\begin{tabular}{l} 
Sample Name \\
\hline ANS $4-9$ \\
ANS $5-5$ \\
ANS $5-5$ \\
ANS $5-14$ \\
ANS $5-14$
\end{tabular}

$\begin{array}{lll}\frac{\text { Isotope }}{125} & & \text { Quantity } \\ & & 9.410 \mathrm{E}+00 \\ { }^{85} \mathrm{Cs} & & 3.241 \mathrm{E}+00 \\ { }_{\mathrm{Sr}}^{137} \mathrm{Cs} & & 6.438 \mathrm{E}-01 \\ { }^{85_{\mathrm{Sr}}} & & 3.418 \mathrm{E}+00 \\ & 6.791 \mathrm{E}-01\end{array}$

C. Static Leach Test

\begin{tabular}{l} 
Sample Name \\
\hline STA $1-17$ \\
STA $1-17$ \\
STA $1-18$ \\
STA $1-18$ \\
STA $2-17$ \\
STA $2-18$ \\
STA $3-18$ \\
STA $4-13$ \\
STA $4-19$ \\
STA $5-17$ \\
STA 5-17 \\
STA 5-19 \\
STA $5-19$
\end{tabular}

\begin{tabular}{lll}
\multicolumn{1}{c}{ Isotope } & & Quantity \\
\cline { 1 - 1 }${ }^{241} \mathrm{Am}$ & & $1.373 \mathrm{E}+01$ \\
${ }^{239} \mathrm{Pu}$ & & $1.472 \mathrm{E}+01$ \\
${ }^{241} \mathrm{Am}$ & & $1.273 \mathrm{E}+01$ \\
${ }^{239} \mathrm{Pu}$ & & $1.364 \mathrm{E}+01$ \\
${ }^{14} \mathrm{C}$ & & $1.704 \mathrm{E}+00$ \\
${ }^{14} \mathrm{C}$ & & $1.659 \mathrm{E}+00$ \\
${ }^{99} \mathrm{TC}$ & & $1.435 \mathrm{E}+00$ \\
${ }^{125} \mathrm{I}$ & & $1.040 \mathrm{E}+01$ \\
${ }_{125} \mathrm{I}$ & & $9.487 \mathrm{E}+00$ \\
${ }_{137} \mathrm{Cs}$ & & $3.412 \mathrm{E}+00$ \\
${ }^{85} \mathrm{Sr}$ & & $6.779 \mathrm{E}-01$ \\
${ }^{137} \mathrm{Cs}$ & & $3.387 \mathrm{E}+00$ \\
${ }^{85} \mathrm{Sr}$ & & $6.729 \mathrm{~S}-01$
\end{tabular}


Table 4.7. Chemical Composition of Hanford Well Water Over Time

\begin{tabular}{|c|c|c|c|c|c|c|c|c|}
\hline \multirow[b]{2}{*}{ Item } & \multirow[b]{2}{*}{ Quantity } & \multicolumn{7}{|c|}{ Date Sampled } \\
\hline & & $\begin{array}{c}\text { Date } \\
\text { Unknown } \\
\end{array}$ & $1-30-86$ & $5-7-86$ & $8-8-86$ & $9-3-86$ & $11-10-86$ & $5-11-87$ \\
\hline $\mathrm{pH}$ & 9.1 & 8.1 & 8.5 & 7.82 & 7.99 & 8.05 & 8.36 & \\
\hline Eh & $\mathrm{mv}$ & 295 & 380 & 297 & 325 & 382 & 315 & 351 \\
\hline A7 & $\mathrm{mg} / \mathrm{L}$ & $<0.100$ & $<0.03$ & $<0.03$ & $<0.03$ & $<0.03$ & 0.029 & $<0.03$ \\
\hline$B$ & $\mathrm{mg} / \mathrm{L}$ & 0.28 & 0.08 & 0.074 & 0.076 & 0.08 & 0.077 & 0.08 \\
\hline $\mathrm{H}_{3} \mathrm{BO}_{3}$ & $\mathrm{mg} / \mathrm{L}$ & 1.6 & 0.46 & 0.4231 & 0.043 & 0.46 & 0.44 & 0.46 \\
\hline $\mathrm{Ba}^{3}$ & $\mathrm{mg} / \mathrm{L}$ & 0.06 & 0.052 & 0.054 & 0.27 & 0.057 & 0.053 & 0.056 \\
\hline $\mathrm{Ca}$ & $\mathrm{mg} / \mathrm{L}$ & 56 & 53.7 & 55 & 55 & 54.9 & 51.7 & 50.6 \\
\hline $\mathrm{Cd}$ & $\mathrm{mg} / \mathrm{L}$ & $<0.005$ & $<0.004$ & $<0.004$ & $<0.004$ & $<0.004$ & $<0.004$ & $<0.004$ \\
\hline $\mathrm{Cr}$ & $\mathrm{mg} / \mathrm{L}$ & $<0.015$ & $<0.02$ & $<0.02$ & $<0.02$ & $<0.02$ & $<0.02$ & $<0.02$ \\
\hline $\mathrm{Cu}$ & $\mathrm{mg} / \mathrm{L}$ & $<0.005$ & $<0.004$ & $<0.004$ & $<0.004$ & $<0.004$ & 0.005 & $<0.004$ \\
\hline $\mathrm{Fe}$ & $\mathrm{mg} / \mathrm{L}$ & $<0.010$ & $<0.005$ & $<0.005$ & $<0.005$ & $<0.005$ & 0.008 & $<0.005$ \\
\hline K & $\mathrm{mg} / \mathrm{L}$ & 7.8 & 7.1 & 4.9 & 6.1 & 6 & 7.3 & 8 \\
\hline $\mathrm{Mg}$ & $\mathrm{mg} / \mathrm{L}$ & 14.4 & 14.4 & 14.1 & 14 & 14.3 & 13.2 & 13.4 \\
\hline Mn & $\mathrm{mg} / \mathrm{L}$ & $<0.010$ & 0.026 & 0.068 & $<0.002$ & 0.13 & 0.117 & 0.087 \\
\hline $\mathrm{Na}$ & $\mathrm{mg} / \mathrm{L}$ & 25 & 25 & 25.6 & 24 & 26 & 23.8 & 24.9 \\
\hline$P$ & $\mathrm{mg} / \mathrm{L}$ & $<0.100$ & $<0.100$ & $<0.100$ & $<0.1$ & $<0.1$ & $<0.1$ & $<0.1$ \\
\hline as $\mathrm{PO}_{4}$ & $\mathrm{mg} / \mathrm{L}$ & $<0.3$ & $<0.3$ & $<0.3$ & $<0.3$ & $<0.3$ & $<0.3$ & $<0.3$ \\
\hline & $\mathrm{mg} / \mathrm{L}$ & $<0.050$ & $<0.06$ & $<0.050$ & $<0.06$ & $<0.06$ & $<0.06$ & $<0.06$ \\
\hline S & $\mathrm{mg} / \mathrm{L}$ & 28 & -- & -- & -- & - & -- & -- \\
\hline as $\mathrm{SO}_{4}$ & $\mathrm{mg} / \mathrm{L}$ & 85 & -- & -- & -- & -- & -- & -- \\
\hline & $\mathrm{mg} / \mathrm{L}$ & & 15.1 & 15.5 & 14.5 & 15.6 & 14.6 & 14.9 \\
\hline Sr & $\mathrm{mg} / \mathrm{L}$ & 0.25 & 0.242 & 0.238 & 0.234 & 0.25 & 0.229 & 0.236 \\
\hline Zn & $\mathrm{mg} / \mathrm{L}$ & $<0.010$ & $<0.02$ & $<0.02$ & $<0.02$ & $<0.02$ & $<0.02$ & $<0.02$ \\
\hline $\mathrm{F}^{-}$ & $\mathrm{mg} / \mathrm{L}$ & $<0.05$ & $<0.1$ & 0.7 & 0.6 & 0.7 & 0.68 & $<0.7$ \\
\hline $\mathrm{Cl}^{-}$ & $\mathrm{mg} / \mathrm{L}$ & 22 & 21 & 22 & 25 & 27 & 21 & 21 \\
\hline $\mathrm{NO}_{2}^{-}$ & $\mathrm{mg} / \mathrm{L}$ & $<0.03$ & $<0.1$ & $<0.2$ & $<0.1$ & $<0.1$ & $<0.1$ & $<0.1$ \\
\hline $\mathrm{NO}_{3}^{-}$ & $\mathrm{mg} / \mathrm{L}$ & 0.1 & $<1.0$ & 0.2 & $<0.3$ & $<0.5$ & $<0.5$ & 19 \\
\hline $\mathrm{SO}_{4}^{2-}$ & $\mathrm{mg} / \mathrm{L}$ & 86 & 69 & 78 & 78 & 73 & 63 & 67 \\
\hline $\mathrm{PO}_{4}^{3-}$ & $\mathrm{mg} / \mathrm{L}$ & $<1.2$ & $<3.0$ & $<1.0$ & $<0.1$ & $<0.1$ & $<0.1$ & $<0.1$ \\
\hline $\mathrm{HCO}_{3}^{-}$ & $\mathrm{mg} / \mathrm{L}$ & 171 & 150 & 176.9 & 183.6 & 183.6 & 251.7 & -- \\
\hline $\mathrm{CO}_{3}^{2-}$ & $\mathrm{mg} / \mathrm{L}$ & $2: 1$ & 6 & 0 & 0.0 & 0.0 & 0.0 & -- \\
\hline TOT. ALK. & $\mathrm{mg} / \mathrm{L}$ & 92.16 & 81.0 & 87 & 90.3 & 90.3 & 123.8 & 92.4 \\
\hline $\mathrm{OH}^{-}$ & $\mathrm{mg} / \mathrm{L}$ & 0 & 0 & 0 & 0 & 0 & 0 & -- \\
\hline TOC & $\mathrm{mg} / \mathrm{L}$ & 1 & 1.3 & 1.7 & 0.45 & 1.05 & 0.27 & 0.45 \\
\hline IC & $\mathrm{mg} / \mathrm{L}$ & 34.1 & 32.77 & 34.8 & 33.94 & 26.91 & 35.2 & 34.48 \\
\hline Cations & meq $/ L$ & 5.28 & 5.14 & 5.15 & 5.11 & 5.21 & 4.90 & - \\
\hline Anions & meq/L & 5.55 & 4.85 & 5.22 & 5.38 & 5.33 & 6.07 & -- \\
\hline
\end{tabular}




\subsection{Hanford Sediment}

The sediment used for this study was obtained from the AP Tank Farm excavation in the 200 East Area of the Hanford Site and represents the sediments expected to exist in the nearby grout disposal site. The sediment sample was air dried, mixed, and sieved through $2-\mathrm{mm}$ screens prior to its use in the laboratory experiments.

The particle size analysis was performed by a combination of wet sieving and the hydrometer method, ASTM D-422. The results from the analysis, which are portrayed in Figure 4.1, show that the sediment is $93 \%$ sand, $5 \%$ silt, and $2 \%$ clay. Chemical characterization data on the sediment have not been collected to date.

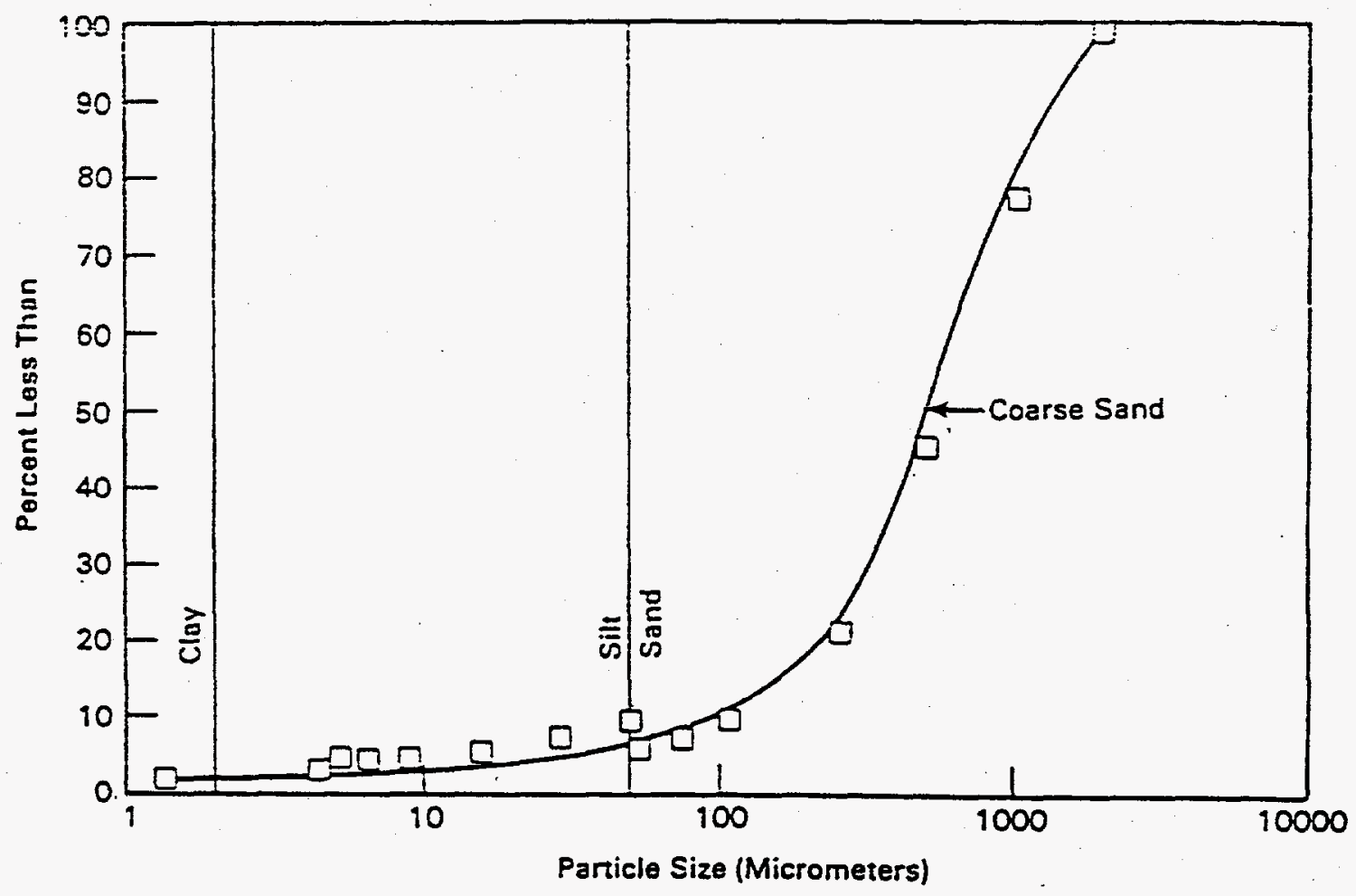

Figure 4.1. Particle-Size Distribution of Hanford Sediment 


\subsection{Test Methods}

In this section we outline the details of the procedures used in determining leaching characteristics of CRW grout.

\subsection{Leach Testing}

Three types of leach tests were conducted on the CRW grout: 1) an intermittent solution exchange test (ANS 16.1 leach test), 2) a static leach test, and 3) a flow-through column test.

\subsubsection{ANS 16.1 Leach Tests}

The ANS 16.1 leach test consists of a procedure whereby the leachate is sampled and replaced at specific intervals (ANS 1986). All the ANS 16.1 leach tests on CRW grout were conducted using intact right cylinders of $3.2 \mathrm{~cm}$ in diameter and 4.5 to $4.6 \mathrm{~cm}$ in length. The leachant was Hanford groundwater from well \#6-S3-25. The groundwater was filtered through a $0.45-\mu \mathrm{m}$ filter prior to use. Duplicate ANS 16.1 experiments were conducted at room temperature. Table 5.1 lists the sampling schedule.

To initiate the ANS 16.1 leach tests, $600 \mathrm{~mL}$ of leachant was added to 1000 -mL wide-mouth polyethylene jars. Using unwaxed dental floss, the grout specimens were anchored securely to the lids of each jar and then suspended in the leachant. Two containers were used for each specimen in an alternating sequence that allowed the amount of radionuclide adsorbed on the inside surface of the containers to be determined while the waste specimen was leaching in the other container.

Table 5.1. Sampling Schedule for ANS 16.1 Leach Tests

\begin{tabular}{ccccc} 
Sample \# & Cumulative Time & & Sample \# & Cumulative Time \\
\cline { 2 - 3 } 1 & 30 seconds & 9 & 27 days \\
2 & 2 hours & 10 & 50 days \\
3 & 7 hours & 11 & 63 days \\
4 & 24 hours & 12 & 92 days \\
5 & 48 hours & 13 & 122 days \\
6 & 72 hours & 14 & 134 days \\
7 & 13 days & 15 & 182 days \\
8 & 20 days & 16 & 302 days
\end{tabular}


At each sampling interval the leachate was removed, filtered through a $0.45-\mu \mathrm{m}$ Millex Millipore MF membrane filter, and analyzed. Measurements of $\mathrm{pH}$, Eh, and alkalinity were taken as quickly as possible before any of these parameters changed following exposure to ambient atmosphere. The $\mathrm{pH}$ was measured using a Corning Model $130 \mathrm{pH}$ meter and a combination electrode. The Eh was measured using a Corning Model $130 \mathrm{pH}$ meter and a Bradley James combination reference-platinum electrode. The alkalinity, reported as $\mathrm{mg} / \mathrm{L}$ carbonate, was measured by standard methods using a Mettler DL-40 memotitrator and a micro-combination pH electrode [Method \#403 from Greenberg et al. (1981)]. The remaining samples were preserved in $25-\mathrm{mL}$ plastic scintillation vials for additional analyses, as follows:

1. Approximately $20 \mathrm{~mL}$ was provided for anion analyses by ion chromatography (IC).

2. Approximately $20 \mathrm{~mL}$ was provided for cation and other dissolved consituent analysis by ICP emission spectrometry. This sample was acidified with ultra-pure $\mathrm{HCl}$ to a $\mathrm{pH}$ of $<2$.

3. Approximately $15 \mathrm{~mL}$ was provided for total organic carbon (TOC) analysis.

4. Exactly $15 \mathrm{~mL}$ acidified to $\mathrm{pH}<2$ was provided for radionuclide counting.

5. To monitor radionuclide and chemical plateout on containers at each sampling interval, the containers were rinsed with a solution consisting of $10 \mathrm{~mL}$ of deionized water and $10 \mathrm{~mL}$ of a pH 2 nitric acid solution.

The cation and other dissolved constituents in solution were measured using an ICP Jarrell Ash model \#975 plasma autocomp spectrophotometer. We report data for the following elements: Al, boron, $\mathrm{Ba}, \mathrm{Ca}, \mathrm{Cd}, \mathrm{Cr}, \mathrm{Cu}, \mathrm{Fe}, \mathrm{K}, \mathrm{Mg}, \mathrm{Mn}, \mathrm{Na}$, phosphorous, $\mathrm{Pb}$, sulfur, silicon, $\mathrm{Sr}$, and $\mathrm{Zn}$. The anions in solution, $\mathrm{F}^{-}, \mathrm{Cl}^{-}, \mathrm{NO}_{2}^{-}, \mathrm{NO}_{3}^{-}, \mathrm{SO}_{4}^{2-}$, and $\mathrm{PO}_{4}^{3-}$, were measured using a Dionex Model \#12 ion chromatograph. The TOC and inorganic carbon were measured using a Dohrmann DC80 carbon analyzer.

The radionuclide analyses were performed for gamma emitters using a 5- in. x 5- in. sodium iodide detector and an intrinsic germanium detector connected to a Nuclear Data 6700 analyzer. The alpha and beta emitters were analyzed using a Beckman LS 9800 liquid scintillator.

The counting geometries were $15 \mathrm{~mL}$ of solution in a $25-\mathrm{mL}$ scintillation vial for the gamma emitters, and 1 to $2 \mathrm{~mL}$ of sample in $15 \mathrm{~mL}$ of scintillation cocktail placed in a $25-\mathrm{mL}$ scintillation vial for alpha and beta emitters.

The excess leachate was archived in 500-mL polyethylene bottles in case further analyses would be necessary. All samples were stored at room temperature.

\subsubsection{Static Leach Tests}

Ten right cylinders of CRW grout, two from each batch of waste, were suspended in separate 1-L, wide-mouthed polyethylene jars filled with $600 \mathrm{~mL}$ of Hanford groundwater. At the prescribed time, $50 \mathrm{~mL}$ of leachate was removed from the container and filtered through a $0.45-\mu \mathrm{m}$ membrane. 
The effluents were analyzed for the same constituents and treated exactly as described for the ANS 16.1 test leachates. To replenish the leachate removed for analysis, $50 \mathrm{~mL}$ of fresh Hanford groundwater was added and the experiment was continued. It is believed that the impact of replenishing the leachate with $50 \mathrm{~mL}$ of groundwater should have little effect on the solution chemistry-i.e., by the time a given leachate is sampled again, the solution is assumed to have re-equilibrated as if there never was a disturbance.

The sampling schedule for the static leach tests is shown in Table 5.2.

\subsubsection{Flow-through Column Leach Test}

Flow-through column leach tests were conducted to determine the impact of flowing leachant on leach rates. We performed this test on two types of grout: 1) a right cylinder of CRW grout and 2) $5 \mathrm{~g}$ of crushed grout suspended in a nylon mesh bag in the lower section of a column. The dimensions of the column used for this experiment were length, 11.2 to $13.6 \mathrm{~cm}$, and inner diameter, $5.2 \mathrm{~cm}$. Inlet and outlet tubes in the end caps of the column were used to add leachant (Hanford groundwater) and to collect leachate, respectively. The leachant flowed into the bottom of the column, around the grout sample, and out the top of the column. The flow rate, which was controlled by a syringe pump, was approximately $0.20 \mathrm{~mL} / \mathrm{h}$. The respective linear pore velocities ranged from $1.7 \times 10^{-3} \mathrm{~cm} / \mathrm{h}(15 \mathrm{~cm} / \mathrm{yr})$ to $1.98 \times 10^{-1} \mathrm{~cm} / \mathrm{h}(17.5 \mathrm{~m} / \mathrm{yr})$. For comparison, the actual recharge rate expected in Hanford sediments is estimated to be between 0.5 to $5 \mathrm{~cm} / \mathrm{yr}$.

The effluent from the flow-through test was collected in a sealed container to minimize evaporation and loss or gain of $\mathrm{CO}_{2}$. Samples were collected daily. When the solution chemistry began to indicate signs of stabilizing, samples were collected at progressively longer time intervals. Each aliquot was split into four samples that were analyzed for 1) Eh, pH, and alkalinity; 2) major cations and selected trace metals; 3) major anions, inorganic carbon, and organic carbon; and 4) radionuclide content using methods described earlier. The aliquots were filtered to remove any suspended particulates. Thus, only dissolved and colloidal species $(<0.45 \mu \mathrm{m})$ were assumed to be present in the solution analyses.

Table 5.2. Sampling Schedule for Static Leach Tests

\begin{tabular}{ccccc}
$\begin{array}{c}\text { Sample } \\
\text { Number }\end{array}$ & Cumulative Time & $\begin{array}{c}\text { Sample } \\
\text { Number }\end{array}$ & Cumulative Time \\
\cline { 2 - 3 } & & & \\
1 & 7 days & 9 & 70 days \\
2 & 13 days & 10 & 76 days \\
3 & 20 days & 11 & 104 days \\
4 & 27 days & 12 & 134 days \\
5 & 41 days & 13 & 190 days \\
6 & 48 days & 14 & 315 days \\
7 & 55 days & 15 & 424 days \\
8 & 62 days & &
\end{tabular}




\subsection{Combined Leach Test}

Cladding removal waste (CRW) grouts were packed within Hanford sediment in a flow-through column test to study the impacts of this more realistic case. In these column experiments, CRW grout samples of the same composition (except for the radionuclide content) were placed in the lower part of a column with the same dimensions as described in Section 5.1.3. The grout sample was supported on approximately $50 \mathrm{~g}$ of Hanford sediment, which filled $1.0 \mathrm{~cm}$ of the column. Additional sediment was packed around the grout sample and added to fill the remaining void space to the top of the column.

The crushed grout samples were arranged in two configurations, as illustrated in Figure 5.1. Configuration $C$ was a layer of grout that filled the inner diameter of the column. This configuration ensured total contact with the solution as the groundwater passed through the column. Configuration B had the same shape as the intact grout sample and was located in the middle of the column, surrounded by sediment. Both configurations used $35 \mathrm{~g}$ of grout samples, which is approximately that of the intact samples. Hanford groundwater was pumped into the bottom of the column where it flowed through the bottom layer of sediment, around and/or through the grout sample, and then through the upper layer of sediment. The same syringe pump and flow rates as described in Section 5.1.3 were used. Effluent
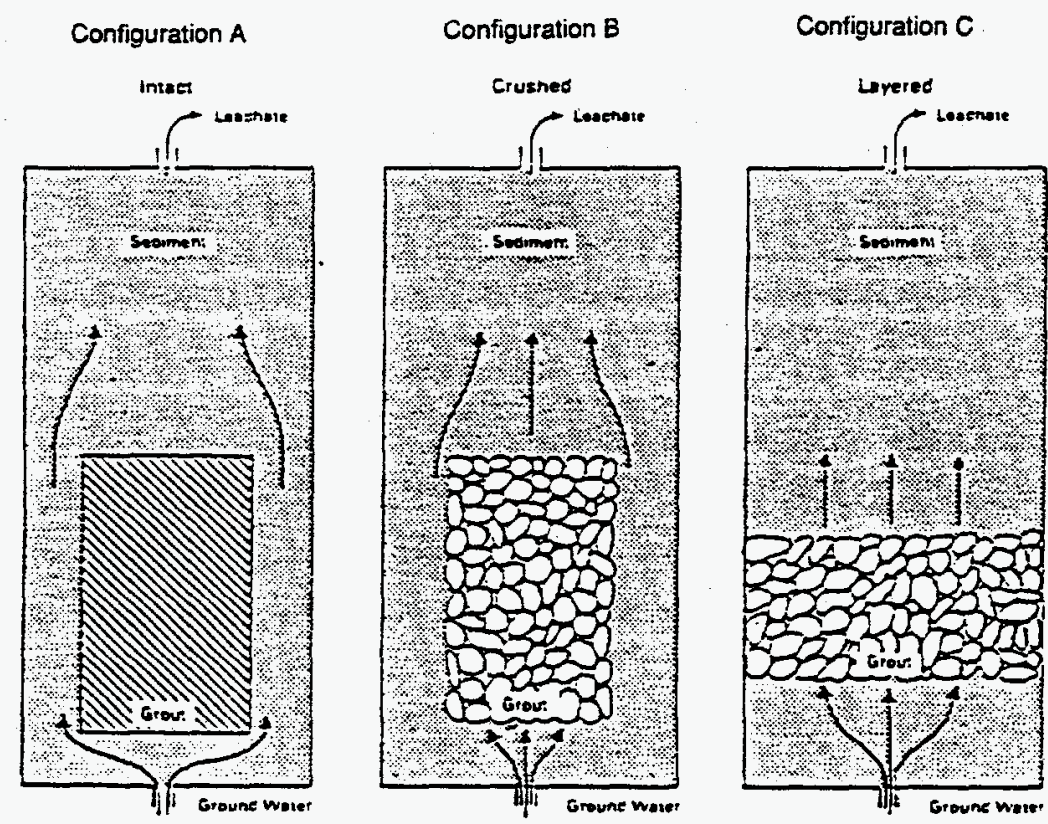

Figure 5.1. Various Configurations of Combined Tests 
from this experiment was collected in covered containers to minimize evaporation and incorporation of $\mathrm{CO}_{2}$ gas. The effluent had been sampled periodically and total chemical characterization performed as previously described.

\subsection{Solids Analysis}

The solid samples for radionuclide counting were prepared by thoroughly mixing $1 \mathrm{~g}$ of sample with $4 \mathrm{~g}$ of cellulose and $14 \mathrm{~g}$ of clean sand in a scintillation vial. The contents of the vial were then pressed into a wafer using a Carver Model $M$ laboratory press. The wafers were pressed at 50,000 psi $(345 \mathrm{MPa})$ for 2 minutes, yielding a wafer $2.25 \mathrm{in} .(5.7 \mathrm{~cm})$ in diameter and $0.125 \mathrm{in} .(0.32 \mathrm{~cm})$ thick.

The solid samples were counted using an intrinsic germanium detector or a coaxial germanium detector connected to a Nuclear Data 6700 analyzer. Samples showing high activity were counted for 10 minutes, and those exhibiting a low level of activity were counted for 1 hour.

Trace metal contents in the sediments are determined either directly by energy-dispersive XRF or by lithium metaborate total fusion-acid dissolution followed by ICP analysis, depending on the sensitivity of the method for each particular element.

The results of these analyses were used for characterization and determination of the initial inventories presented previously (Table 4.1). 


\subsection{Results and Discussion}

This chapter summarizes and discusses results from the leach tests on CRW grout.

\subsection{Chemical Analysis of Leachates From ANS 16.1 Leach Tests on Intact CRW Grouts}

Four of the eight leachates from synthetic CRW grout specimens that were tested using ANS 16.1 methodology were analyzed. The results are provided in Tables A.1 through A.4 of Appendix A. The leachant solution was Hanford groundwater.

Cladding removal waste grout is very reactive and quickly forces the solution $\mathrm{pH}$ to values above 10. The $\mathrm{pH}$ values rise above 11 when contact times reach at least 20 days between solution exchanges. The maximum $\mathrm{pH}$ observed in leachates was 12.15 for the sample with a 10-day contact time and 13-day total elapsed time. The long-term, steady-state $\mathrm{pH}$ after 410 days of contact (18 solution exchanges) was 11.7 . In contrast, previous experiments with $60 \%$ phosphate $140 \%$ sulfate (PSW) grout samples produced leachates that never exceeded a pH of 10 and reached a steady-state $\mathrm{pH}$ of 8.3 (see Serne et al. 1987, 1989b). The comparison of the $\mathrm{pH}$ values for PSW and CRW grout leachate is shown in Figure 6.1. The difference in $\mathrm{pH}$ values is likely caused by the dissolution of excess calcium hydroxide present in the CRW solid blend or the initial high $\mathrm{NaOH}$ content of the CRW liquid. PSW was the first Hanford waste to be grouted. It originates from spent ion exchange resin and various $\mathrm{N}$ reactor cleanup activities. PSW wastes are considered less dangerous than CRW wastes.

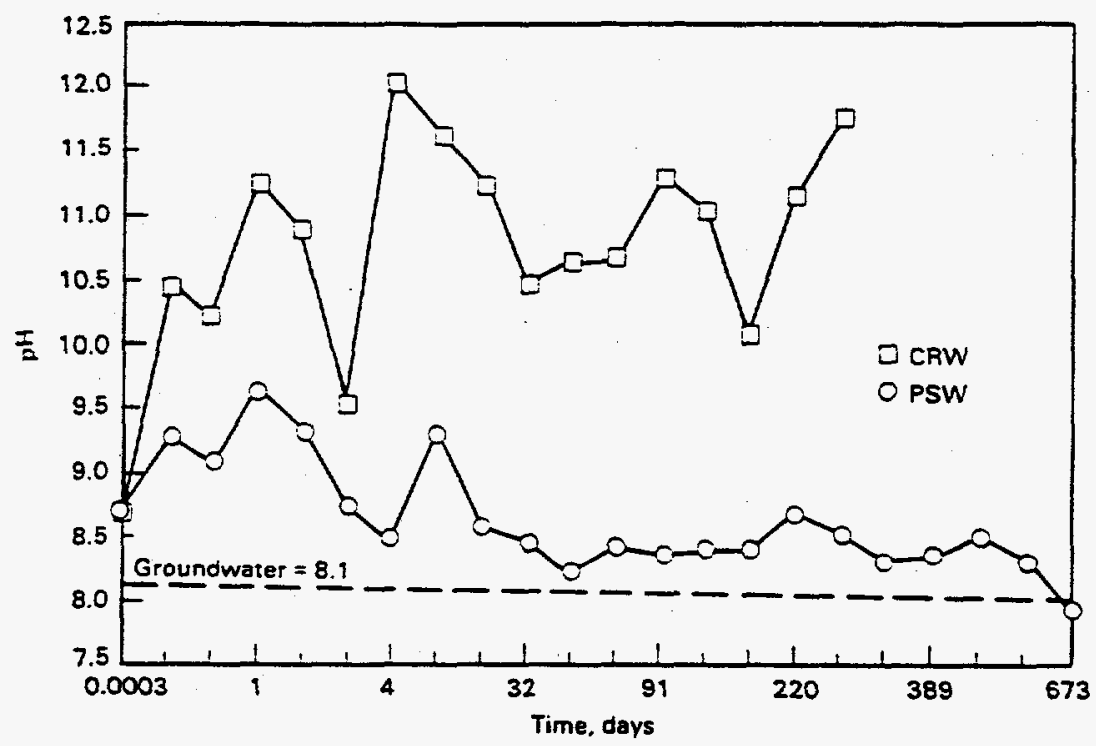

Axis not drawn to scale. Points represent samples taken at discrete time periods that increase with time.

Figure 6.1. Comparison of ANS 16.1 Test Leachates for PSW and CRW Grouts 
Excluding the 1-day and 13-day leachate, none of the CRW leach solutions contained significant concentrations of aluminum. Generally, the aluminum concentrations in the leachates were less than $0.03 \mathrm{mg} / \mathrm{L}$. It appears that the $\mathrm{pH}$ must exceed 12 before appreciable amounts of aluminum dissolve from CRW grouts. Calcium concentrations in the CRW leachates were always less than concentrations in the ambient groundwater (Figure 6.2). For those samples with residence times greater than 20 days, the calcium concentrations were around $1 \mathrm{mg} / \mathrm{L}$. In general, the results also show systematic losses of inorganic carbon $\left(\mathrm{HCO}_{3}\right.$ and $\left.\mathrm{CO}_{3}\right)$, suggesting significant precipitation of calcium carbonate on the CRW grout specimen surfaces. After similar test periods, CRW and PSW grouts removed $500 \mathrm{mg}$ and $300 \mathrm{mg}$ of calcium, respectively, from the leachant. Figure 6.2 shows that CRW grout removes substantially more calcium from the groundwater. Therefore one might expect a thicker reaction layer of $\mathrm{CaCO}_{3}$ to form on CRW grout specimens than PSW specimens.

Magnesium concentrations in the CRW leachates were always less than those in the ambient groundwater and, when $\mathrm{pH}$ values were greater than 11 , the magnesium concentrations were typically less than $1 \mathrm{mg} / \mathrm{L}$ (Figure 6.3). In contrast, the previously tested PSW grouts did not show any significant precipitation of magnesium. Mass balance calculations showed that CRW grouts removed $120 \mathrm{mg}$ of magnesium, while PSW grouts removed only $8 \mathrm{mg}$. In the CRW grout tests, precipitation of brucite $\left[\mathrm{Mg}(\mathrm{OH})_{2}\right]$ or coprecipitation of $\mathrm{Mg}$ with calcite is suspected.

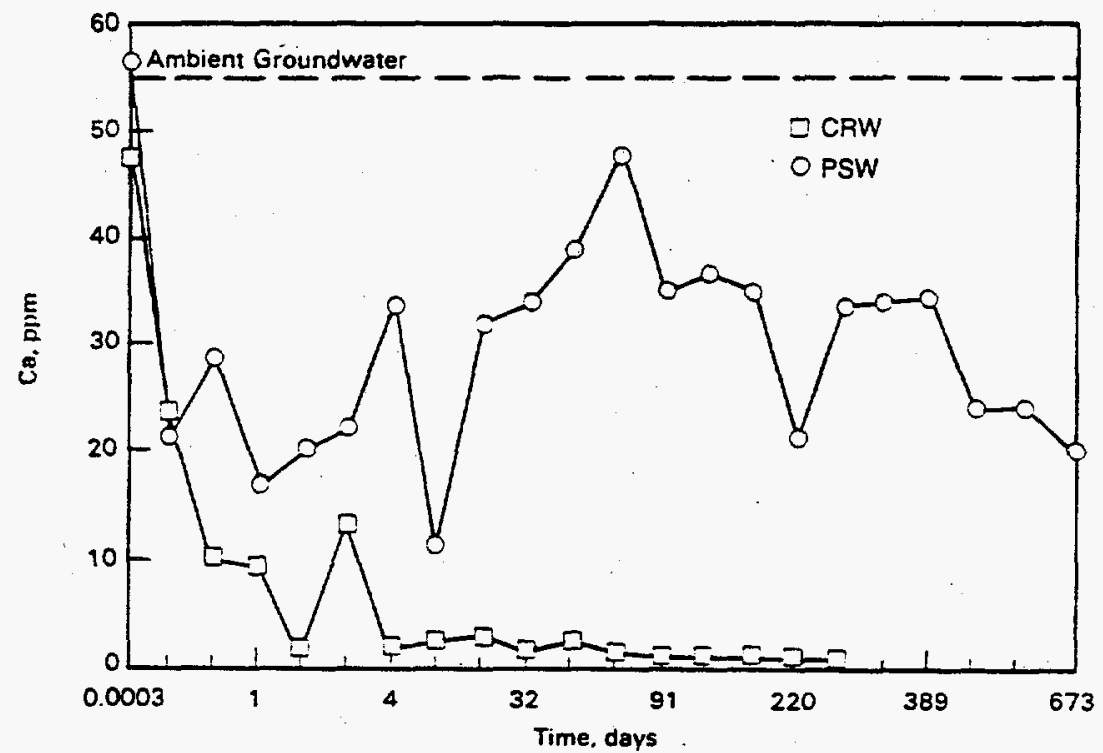

Axis not drawn to scale. Points represent samples taken at discrete time periods that increase with time.

Figure 6.2. Calcium Values for PSW and CRW Grouts, ANS 16.1 Test (Grout Leachates in Hanford Groundwater) 


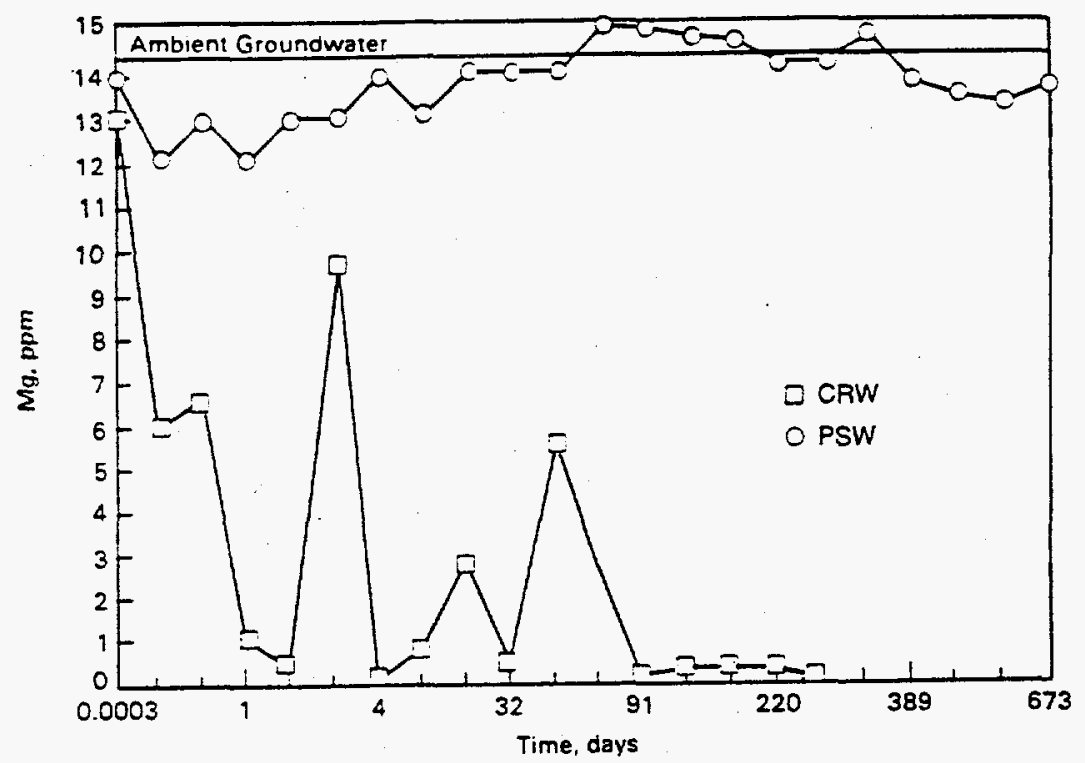

Axis not drawn to scale. Points represent samples taken at discrete time periods that increase with time.

Figure 6.3. Magnesium Values for PSW and CRW Grouts, ANS 16.1 Test (Grout Leachates in Hanford Groundwater)

The CRW leachates showed continual leaching of potassium and sodium (Figure 6.4 and 6.5 ). After 302 days, the cumulative mass leached ranged from 39 to $47 \mathrm{mg}$ for potassium and from 1300 to $1500 \mathrm{mg}$ for sodium. Continual leaching of these alkali metals is similar to that observed for the PSW grout samples, except the magnitude of release is higher for CRW leachates. The PSW tests released only 13 and $150 \mathrm{mg}$ of potassium and sodium, respectively. The higher mass leached from CRW grout was caused by the much higher concentrations of salts in the CRW liquid waste stream.

Silicon concentrations in the CRW leachates were lower than in the ambient Hanford groundwater, suggesting that precipitation occurred. Similarly, strontium concentrations were lower in the CRW leachates than in the groundwater used to leach the grout (Figure 6.6). Perhaps strontium is incorporated into the calcium, magnesium, and carbonate precipitates that coat the grout surfaces. The net strontium precipitation should minimize strontium-90 leaching from the actual CRW grout. The sampling numbering system consisted of two numbers. The first signifies which batch the sample came from (see Table 4.2) while the second number signifies which hardened specimen (replicate) was used.

Although the CRW grouts contained significant concentrations of zirconium ( $4.12 \%$ by dry weight), no appreciable concentrations of zirconium were observed in the leachates. This is not surprising, because zirconium readily hydrolyzes and is rather insoluble in all aqueous solutions that are not strong acids. 


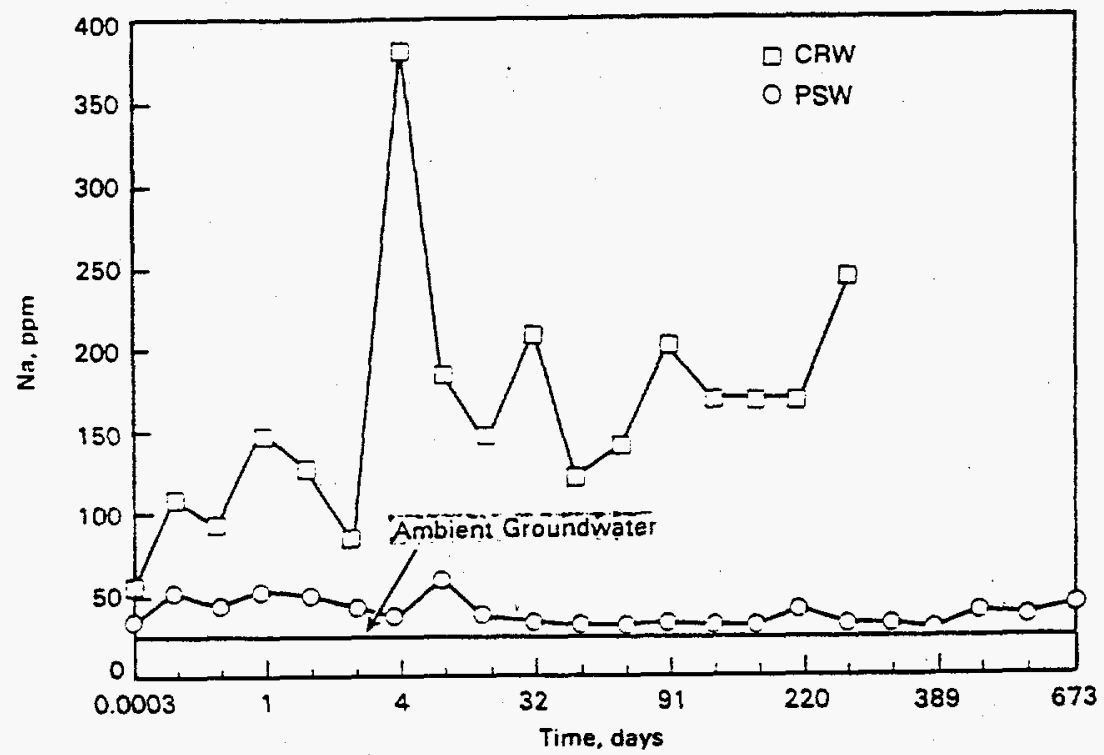

Axis not drawn to scale. Points represent samples taken at discrete time periods that increase with time.

Figure 6.4. Sodium Concentrations for PSW and CRW Grouts, ANS 16.1 Test (Grout Leachates in Hanford Groundwater)

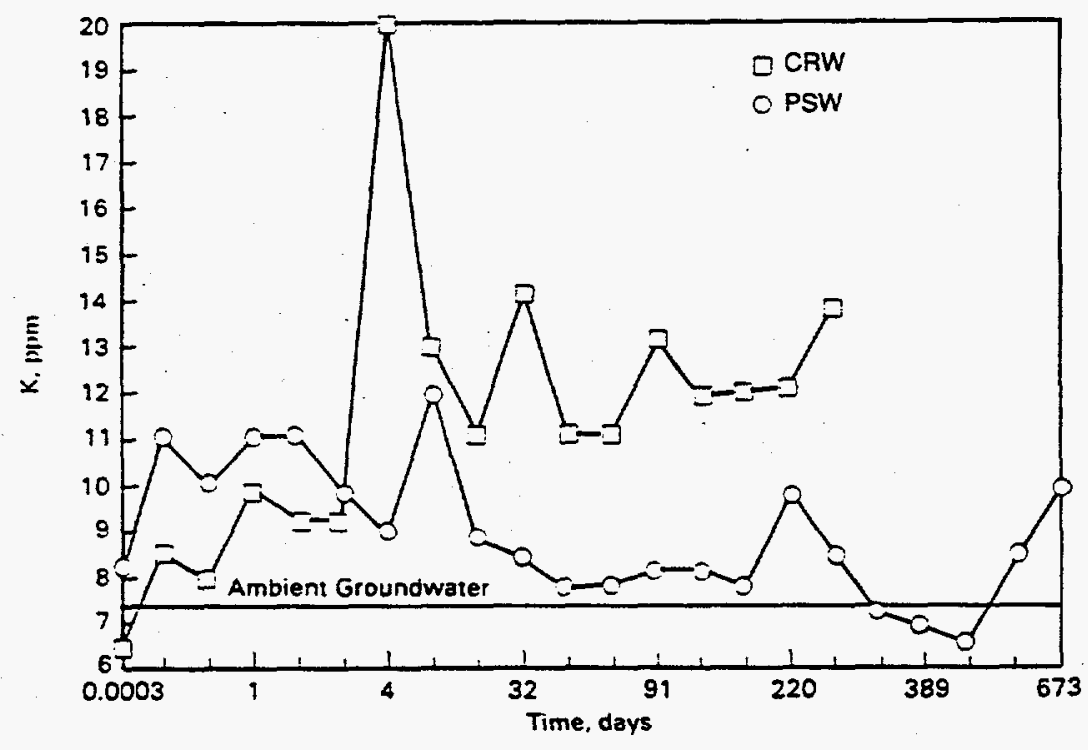

Axis not drawn to scale. Points represent samples taken at discrete time periods that increase with ime.

Figure 6.5. Potassium Concentrations for PSW and CRW Grouts, ANS 16.1 Test (Grout Leachates in Hanford Groundwater) 


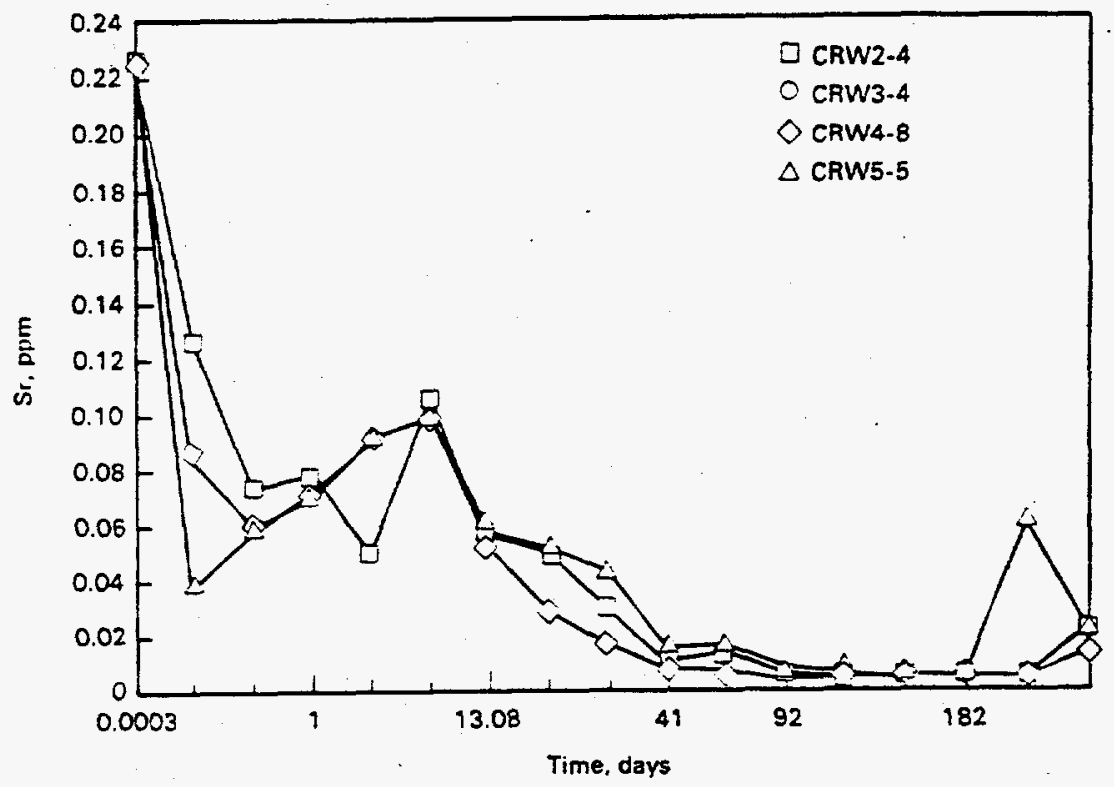

Axis not drawn to scale. Points represent samples taken at discrete time periods that increase with time.

Figure 6.6. Strontium Concentration vs. Time for ANS 16.1 Test Using CRW Grout

The CRW grout also contained about $4 \%$ (by dry weight) fluoride, which slowly leaches from the grout. The fluorine behavior suggests that a low-solubility compound is slowly releasing fluoride, but geochemical code calculations have not identified the controlling compound. Fluorite $\left(\mathrm{CaF}_{2}\right)$ is apparently not the controlling phase, because the leachates were significantly undersaturated with respect to fluorite. If fluorite was the controlling phase, the leachates would have had much higher fluoride concentrations. Less soluble, mixed $\mathrm{PO}_{4}-\mathrm{F}$ minerals from the apatite family are possibly controlling fluoride concentrations. Phosphate present at $0.22 \%$ (by dry weight) did not appear to leach from the CRW grout, probably because of the insolubility of apatite (alkaline-earth phosphates) minerals.

The grout contained a very small amount of chloride $(0.02 \%$ by dry weight) that may have been sporadically released in the ANS 16.1 test. In general, the chloride concentrations in the CRW leachates were similar to that in the ambient groundwater, but frequently the leachate appeared to contain a measurable increase of chloride. No patterns appeared among the four replicates.

The CRW grout contained $0.19 \%$ (by dry weight) nitrate, which leaches from the grout. (Additional discussion on nitrate appears in Section 6.6.) Sulfate leached from the grouts for the first 100 days and then perhaps actually started precipitating out of solution at longer times. Because various batches of groundwater were used over the years of the leach experiment and the various batches of groundwater showed some fluctuation in ambient sulfate concentration, this variation may 
have caused the apparent change from leaching to removal of sulfate. Groundwater samples were obtained periodically throughout the year as demand warranted. The variation in ambient groundwater chemistry was shown in Table 4.7.

The grout contained $0.11 \%$ boron (by dry weight). Leachates showed a fairly rapid leaching of boron followed by a slow release, suggesting diffusion-controlled release of this constituent.

The synthetic CRW grouts did not leach appreciable amounts of organic carbon; however, actual $\mathrm{CRW}$ waste streams probably contain larger amounts of more complex organics. Therefore, no conclusions should be drawn on the fate of organic carbon in CRW grouts based on these data for synthetic grouts.

In general, the leach behavior of major CRW grout constituents was similar to that for PSW grout. The exception was magnesium, which appeared to precipitate in the higher $\mathrm{pH}$ environment caused by the excess lime or high $\mathrm{NaOH}$ content in the $\mathrm{CRW}$ grout. The agreement in leachate chemical composition among the four replicates was good, as evidenced in Figures 6.7 through 6.10 for sodium, potassium, fluoride, nitrate, and borate. Thus it could appear that each CRW grout specimen behaves in a similar fashion.

The synthetic $\mathrm{CRW}$ grout did not leach appreciable amounts of $\mathrm{Ba}, \mathrm{Cd}, \mathrm{Cr}, \mathrm{Cu}, \mathrm{Fe}, \mathrm{Mn}, \mathrm{Pb}$, or $\mathrm{Zn}$, although actual CRW wastes may contain higher concentrations of these metals. Thus, we cannot make conclusive statements regarding the fates of these metals in actual CRW grout. However, we speculate that, barring strong organic chelation, these metals will not leach at significant concentrations in the highly alkaline aqueous environment. The ANS 16.1 tests suggest that, among nonradioactive species, fluoride leaching may be the most significant potential problem in terms of assessing. performance of grouted CRW over time despite the apparent solubility limit on fluoride release.

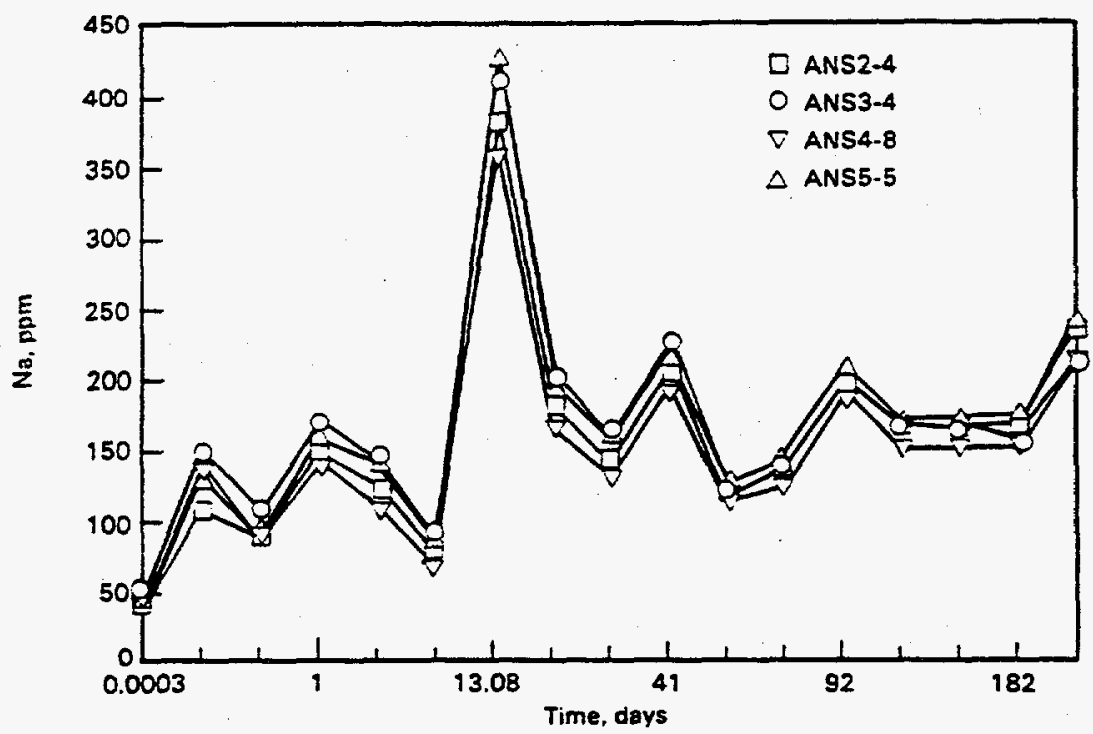

Axis not drawn to scale. Points represent samples taken at discrete time periods that increase with time.

Figure 6.7. Sodium Concentration vs. Time for CRW Grout (ANS 16.1 Test) 


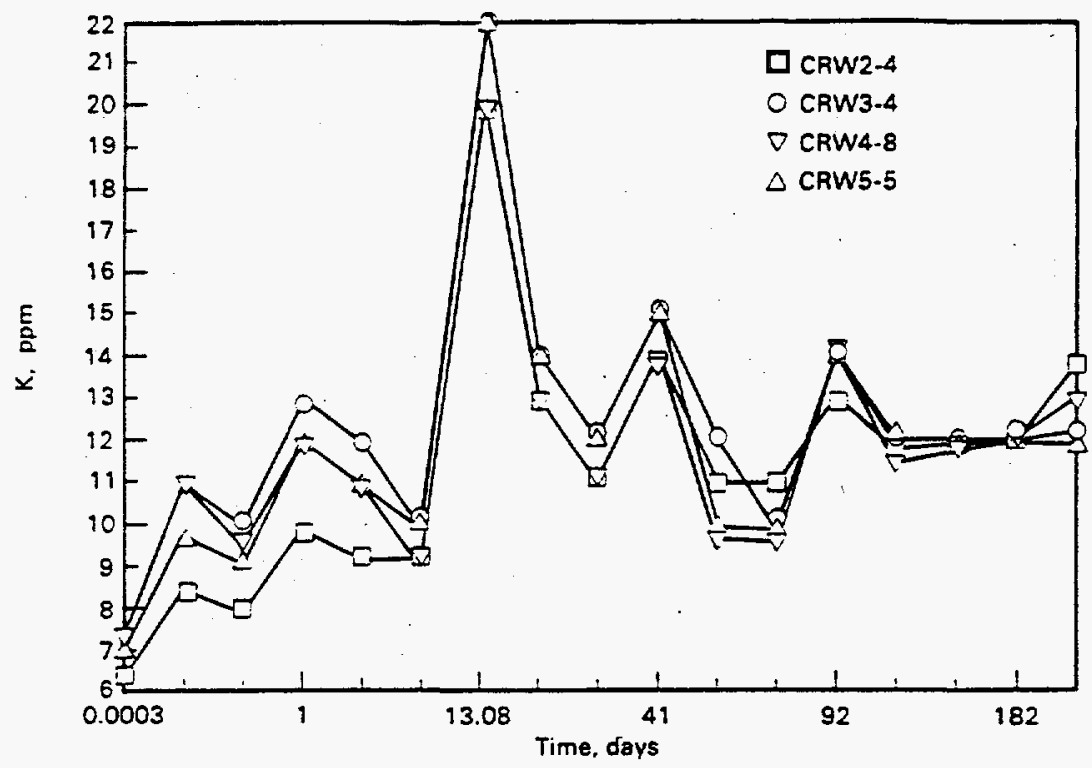

Axis not drawn to scale. Points represent samples taken at discrete time periods that increase with time.

Figure 6.8. Potassium Concentration vs. Time for CRW Grout (ANS 16.1 Test)

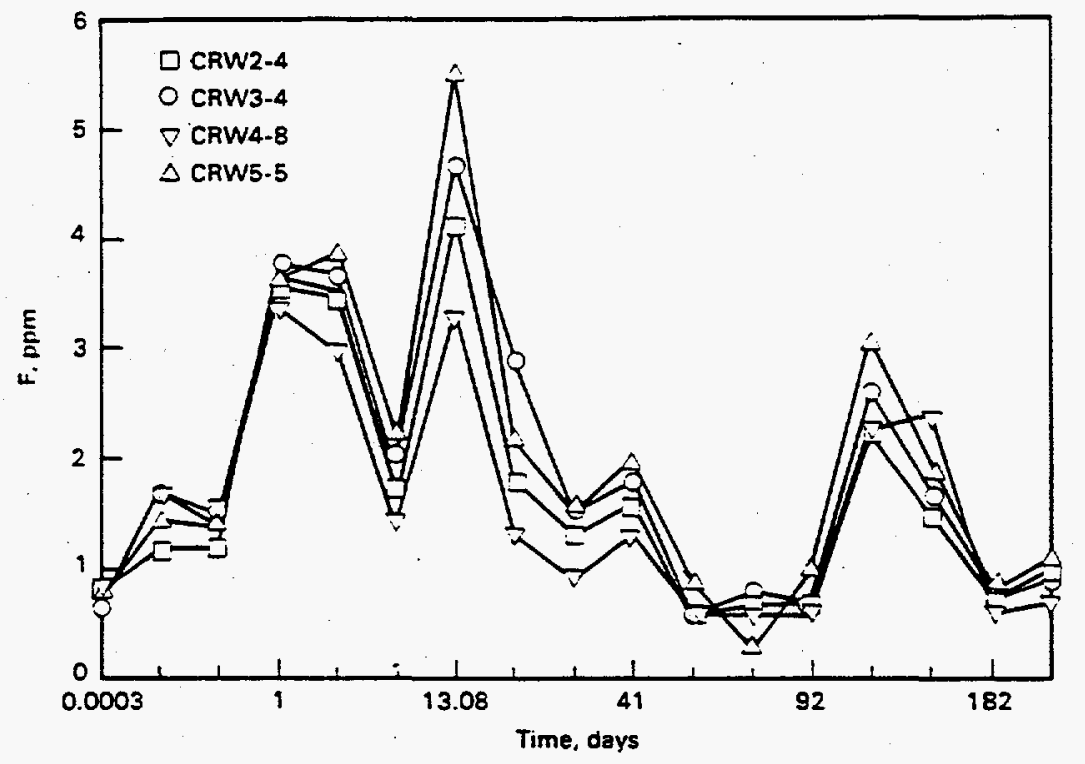

Axis not drawn to scale. Points represent samples taken at discrete time periods that increase with time.

Figure 6.9. Fluoride Concentration vs. Time for CRW Grout (ANS 16.1 Test) 


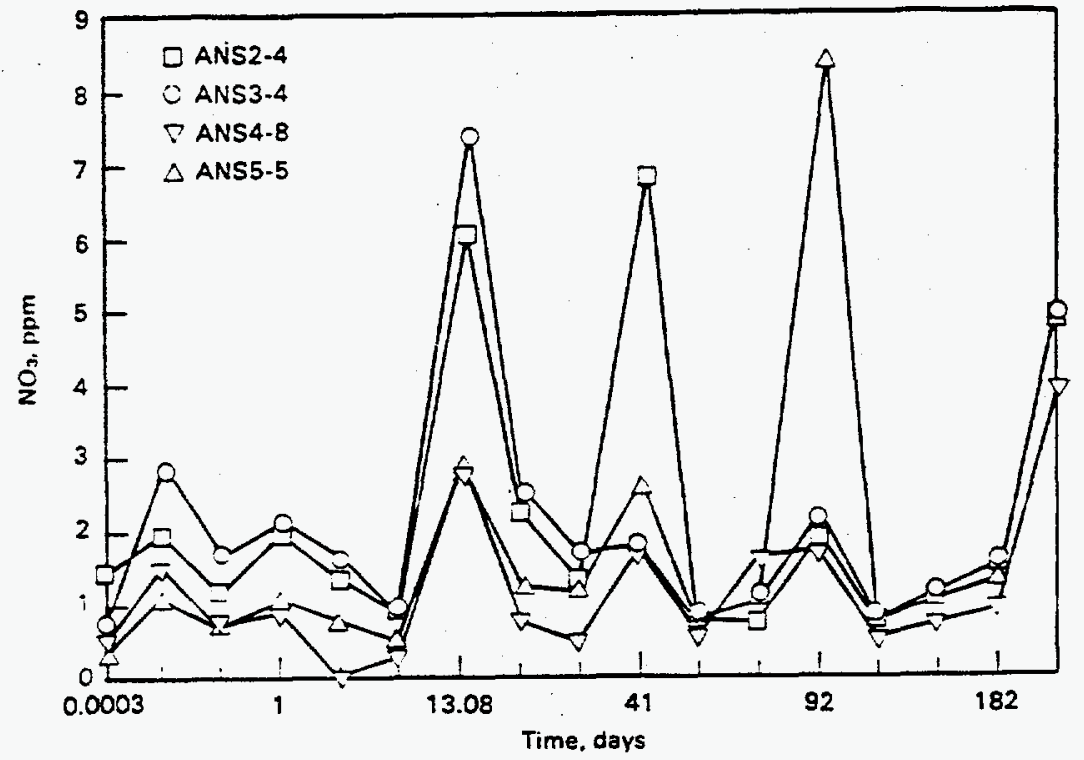

Axis not drawn to scale. Points represent samples taken at discrete time periods that increase with time.

Figure 6.10. Nitrate Concentration vs. Time for CRW Grout (ANS 16.1 Test)

\subsection{Chemical Analysis of Leachates from Static Leach Tests on Intact CRW Grouts}

Leachates from synthetic CRW grout specimens that were subjected to static leach tests were analyzed. The results are provided in Tables A.5 through A.8 of Appendix A. For the first 55 days of contact in the leachant solution, all eight samples were analyzed; after that time, only one leachate from each set (total of 4) was analyzed. The $\mathrm{pH}$ of the static leachates rose to above 12 within the first sampling period and appeared to reach steady state in the range of $12.5 \pm 0.3$. This value is about 0.7 to $1.0 \mathrm{pH}$ units higher than that for the CRW leachates from the ANS 16.1 test, where complete solution exchange occurs numerous times. The $\mathrm{pH}$ value is in the same range as that for the static leach tests previously performed on PSW grout, which suggests that all grouts will tend to force the pH to a value near 12.5 when the volume of water is limited. The $\mathrm{pH}$ values of the static leachates from the two grout leach tests are compared in Figure 6.11.

The leachate calcium concentrations for the static leach tests reached a steady-state value of $4 \pm 1 \mathrm{mg} / \mathrm{L}$ after 7 days. The magnesium concentrations dropped below detection limits throughout the tests. Thus, alkaline earth metals, including strontium, appeared to be precipitating from the groundwater leachant. The likely reaction products are pure carbonates, mixed calcium-magnesiumstrontium carbonates, and brucite $\left[\mathrm{Mg}(\mathrm{OH})_{2}\right]$. We found that these reactions may also occur in the ANS 16.1 test on CRW grout and the static leach test on PSW grout. Less precipitation, especially of 


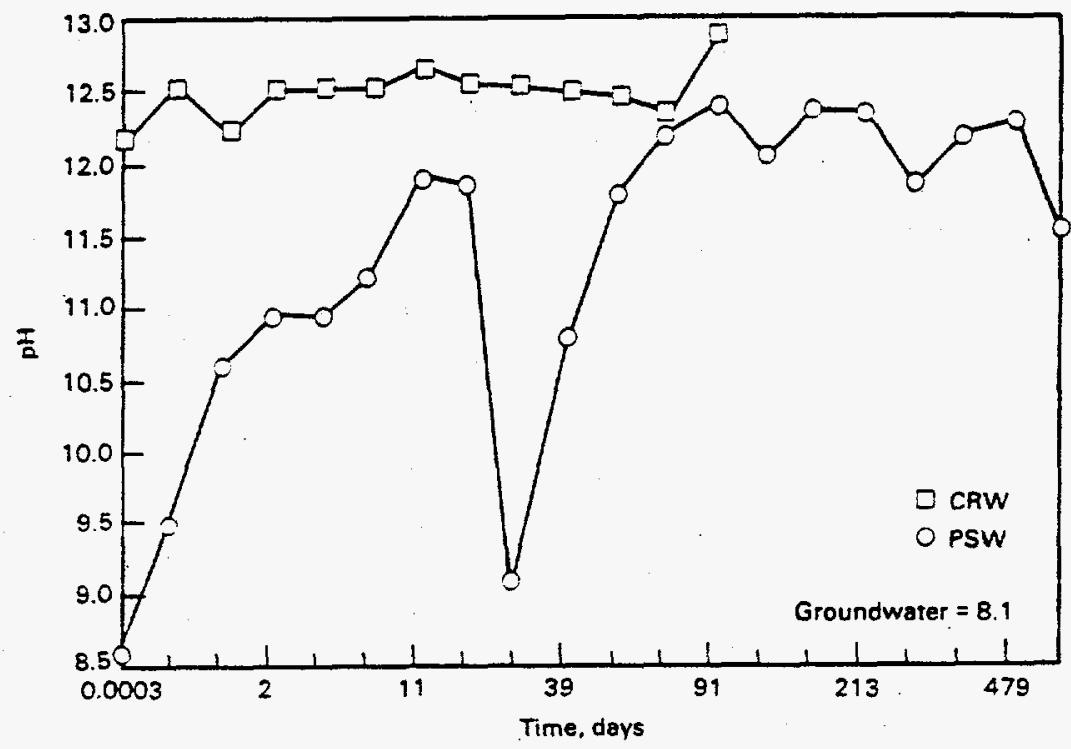

Axis not drawn to scale. Points represent samples taken at discrete time periods that increase with time.

Figure 6.11. pH Values for Grout Leachates in Hanford Groundwater (Static Leach Test)

$\mathrm{Mg}$, was found in the PSW grout tests using the ANS 16.1 protocol. The masses of calcium, magnesium, and inorganic carbon removed in the static CRW leach tests $(67 \mathrm{mg}$ of calcium, $18 \mathrm{mg}$ of magnesium, and $11 \mathrm{mg}$ of inorganic carbon) were about $15 \%$ of the values measured in the ANS 16.1 tests. Therefore, we speculated that the surface coatings for the static leach tests will be less copious because of the smaller amounts of these ions that are available for precipitation.

The high $\mathrm{pH}$ in the static test permitted some aluminum dissolution from the CRW grout, in contrast to the ANS 16.1 tests. There may have been a small amount of silicon precipitation (as evidenced by low silicon concentrations in leachates) in the early portion of the CRW static tests, but by the 200-day contact time, there did not appear to be significant losses of silicon. In contrast, the CRW ANS 16.1 test produced measurable amounts of silicon precipitation. (See mass balance tables in Appendix C.)

The potassium in the CRW static test leachates rapidly reached a steady-state value of $45 \pm 5 \mathrm{mg} / \mathrm{L}$ (within 20 days); the sodium rapidly reached a concentration of $1050 \pm 150 \mathrm{mg} / \mathrm{L}$ (Figures 6.12 and 6.13). Thus, it appears that leaching of these alkali metals from the CRW grout occurs rapidly and then stops. Alternatively, these concentrations may represent solubility limits of some compounds yet to be identified. In contrast, the ANS 16.1 CRW leachates continually removed small amounts of potassium and sodium with each solution exchange. On a mass balance basis, the ANS $16.1 \mathrm{CRW}$ tests leached $-1500 \mathrm{mg}$ of sodium and $42 \pm 3 \mathrm{mg}$ of potassium, while the static CRW leach test leached $1400 \mathrm{mg}$ of sodium and $51 \mathrm{mg}$ of potassium. A comparison to mass leached from PSW grout 


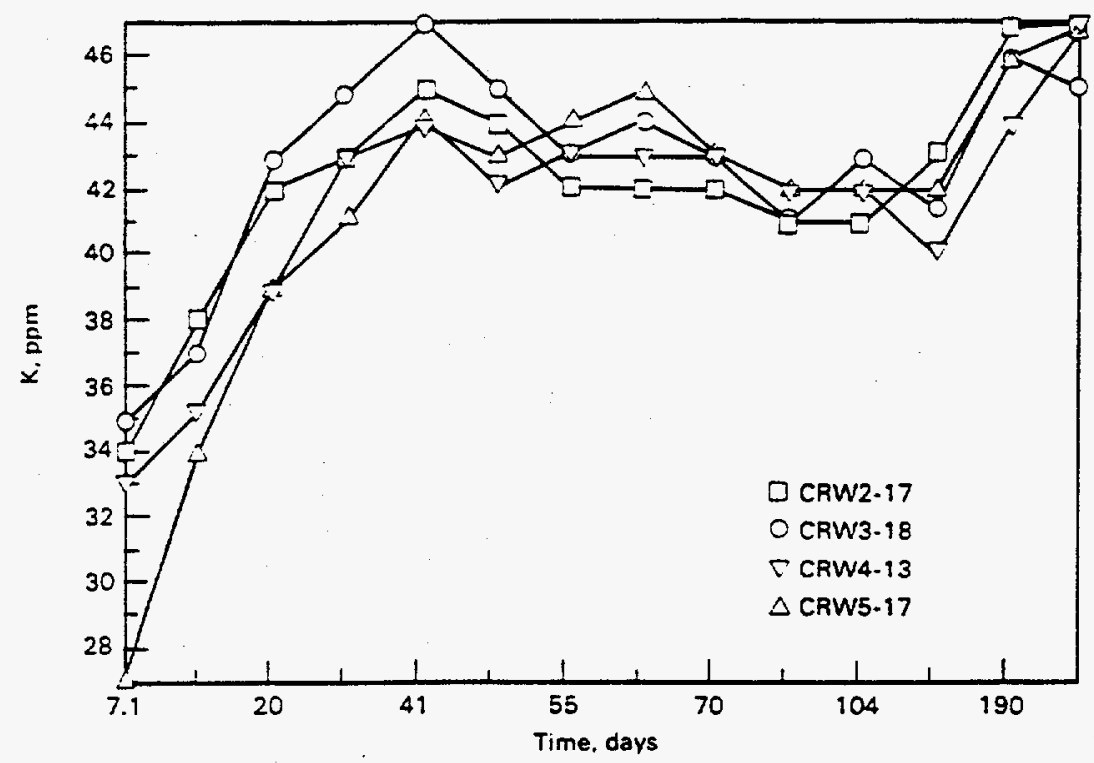

Axis not drawn to scale. Points represent samples taken at discrete time periods that increase with time.

Figure 6.12. Potassium Concentration vs. Time for the CRW Static Leach Test

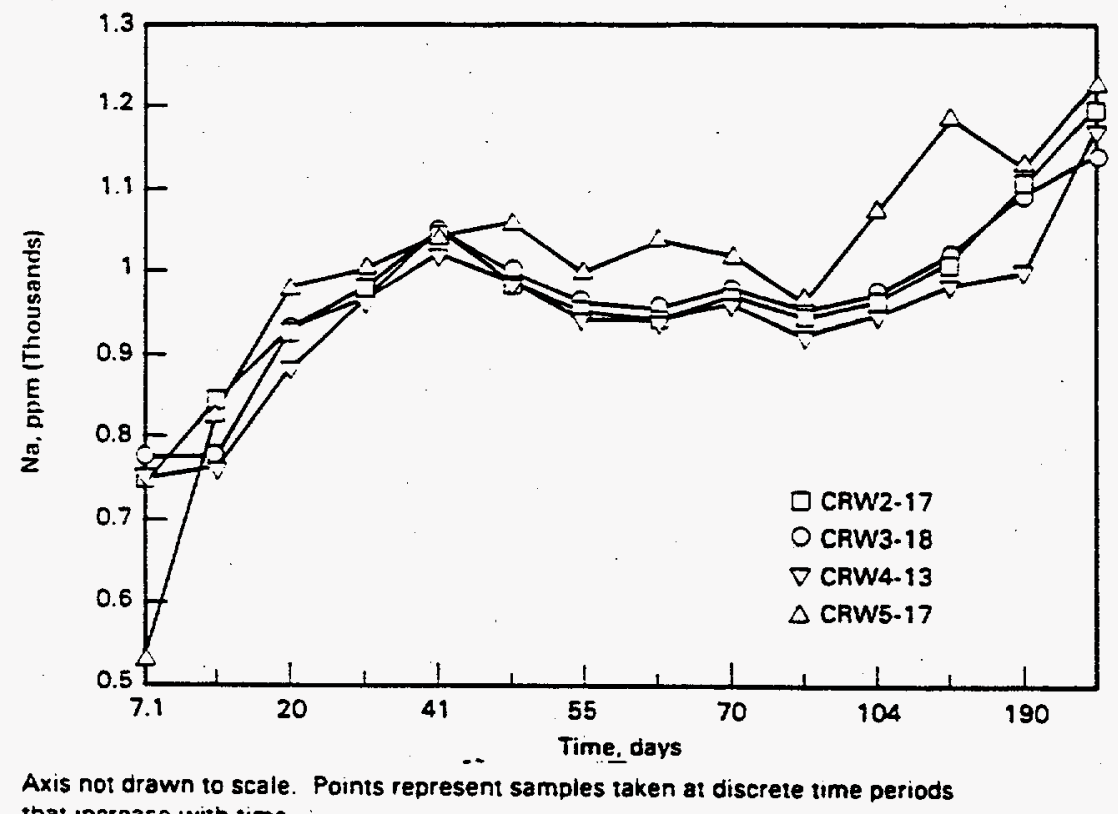

Figure 6.13. Sodium Concentration vs. Time for the CRW Static Leach Test 
is shown in Table 6.1. The mass of sodium and potassium leached from CRW grouts did not differ very much between the two test methods. On the other hand, results for the PSW grout showed significantly larger masses of the two elements leached by the static.leach test. We have speculated that the PSW static leach tests did not allow much carbonate precipitation onto the grout surfaces. For CRW grouts, carbonate precipitation occurred under both test conditions; however, we still suggest that much more carbonate armoring occurs under ANS 16.1 test conditions. It is not clear why there is no difference in the CRW grout leach data for the two types of $\mathrm{Na}$ and $\mathrm{K}$ tests, if carbonate armoring is in fact different. The large difference in mass leached for sodium, and to a lesser extent potassium, between the grout types (CRW vs. PSW) reflects the much larger inventories of those elements in CRW grout.

The fluoride concentrations in static test leachates rapidly reached $10 \pm 1 \mathrm{mg} / \mathrm{L}$ (within the first 7 days) and slowly increased to $15 \pm 5 \mathrm{mg} / \mathrm{L}$ after 100 days. (This leaching trend will be discussed in Section 6.6.) There was little difference in the mass of fluoride leached between the ANS 16.1 and static CRW leach tests: $12.9 \pm 2.3$ and $15.6 \pm 1.2 \mathrm{mg}$, respectively.

The nitrate concentrations in static leach test leachates reached $6 \mathrm{mg} / \mathrm{L}$ within the first sampling period ( 7 days), and then seemed to level out through 27 days; between 27 and 41 days, all the samples showed an abrupt jump to $15 \mathrm{mg} / \mathrm{L}$. Nitrate concentrations increased very slightly through 190 days, and then increased to $23 \pm 2 \mathrm{mg} / \mathrm{L}$ beyond 300 days. These trends are shown in Figure 6.14. The total mass of nitrate leached was similar for the two tests.

Sulfate concentrations rapidly rose to values around $170 \pm 10 \mathrm{mg} / \mathrm{L}$ within the first sample period. Between 7 and 190 days, the sulfate concentrations remained in the range of $160 \pm 20$, while an increase to about $200 \mathrm{mg} / \mathrm{L}$ occurred between 190 and 300 days.

Boron, reported as boric acid $\left(\mathrm{H}_{3} \mathrm{BO}_{3}\right)$, quickly reached a concentration of $4 \pm 1 \mathrm{mg} / \mathrm{L}$, and, after 190 days, reached values of $6 \pm 1 \mathrm{mg} / \mathrm{L}$. Barium, cadmium, chromium, copper, iron, manganese, phosphate, lead, zinc, and zirconium remained near or below detection limits in all the static leach test leachates. These results are similar to leachates from the ANS 16.1 test.

Table 6.1. Sodium and Potassium Mass Leached (mg) from CRW and PSW Grouts Using Two Test Methods

\begin{tabular}{|c|c|c|c|c|}
\hline \multirow[b]{2}{*}{ Element } & \multicolumn{2}{|c|}{ CRW Grout } & \multicolumn{2}{|c|}{ PSW Grout } \\
\hline & ANS 16.1 & Static & ANS 16.1 & Static \\
\hline $\mathbf{N a}$ & 1500 & 1400 & 145 & 281 \\
\hline $\mathbf{K}$ & 42 & 51 & 12 & 37 \\
\hline
\end{tabular}




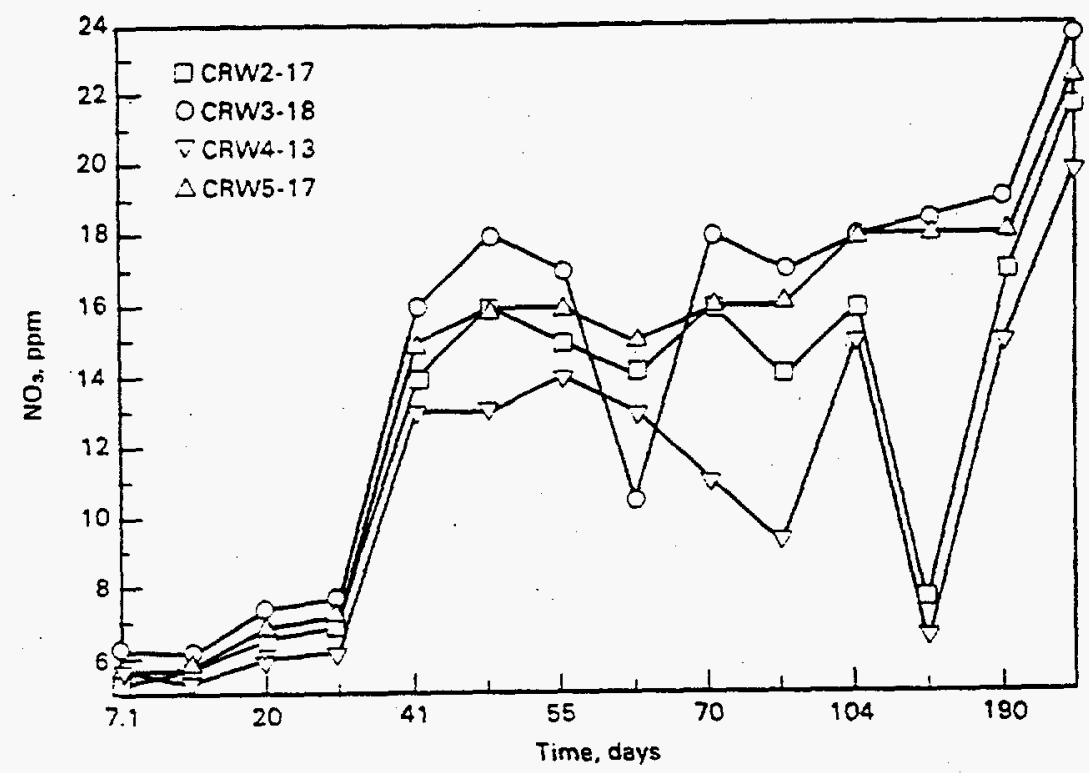

Axis not drawn to scale. Points represent samples taken at discrete time periods that increase with time.

Figure 6.14. Nitrate Concentration vs. Time for CRW Grout (Static Leach Test)

From a regulatory standpoint, the CRW static leachates exceeded maximum permissible concentrations in drinking water for fluoride, while nitrate reached about $50 \%$ of the allowable maximum.

\subsection{Chemical Analysis of Leachates From Flow-Through Leach Tests on Intact CRW Grouts}

For the flow-through leach tests, an intact grout cylinder was suspended in the middle of a Plexiglas column and groundwater flowed past the specimen at a fairly constant rate of 0.4 to 0.6 grout volumes per day. The leachates were collected in small aliquots (a few pore volumes per sample). Complete chemical and radionuclide characterization was performed on almost every sample. Details of the chemical analyses are presented in Tables A.12 through A.14 (Appendix A); radionuclide data are in Appendix B.

The initial effluent was quite alkaline, with a pH range of 12.5 to 12.9. Later effluents showed erratic but generally decreasing trends in $\mathrm{pH}$ to values as low as 8.5 (Figure 6.15). The legends on Figures $6.15-6.17$ designate which radionuclide was present in the grout. For these plots we can ignore this distinction and consider the separate specimens as replicates. The calcium and magnesium concentrations of the initial effiuent were reduced to a range of 1 to $4 \mathrm{mg} / \mathrm{L}$ and less than $0.06 \mathrm{mg} / \mathrm{L}$, respectively. Strontium concentrations also decreased from the ambient groundwater value. For the CRW grout ANS 16.1 and static leach tests, it appeared that calcium carbonate, mixed carbonates, and brucite may have formed on the grout surface. A visible white "fibrous" material was seen growing on the surface soon after the groundwater contacted the grout in all three types of leach tests. 


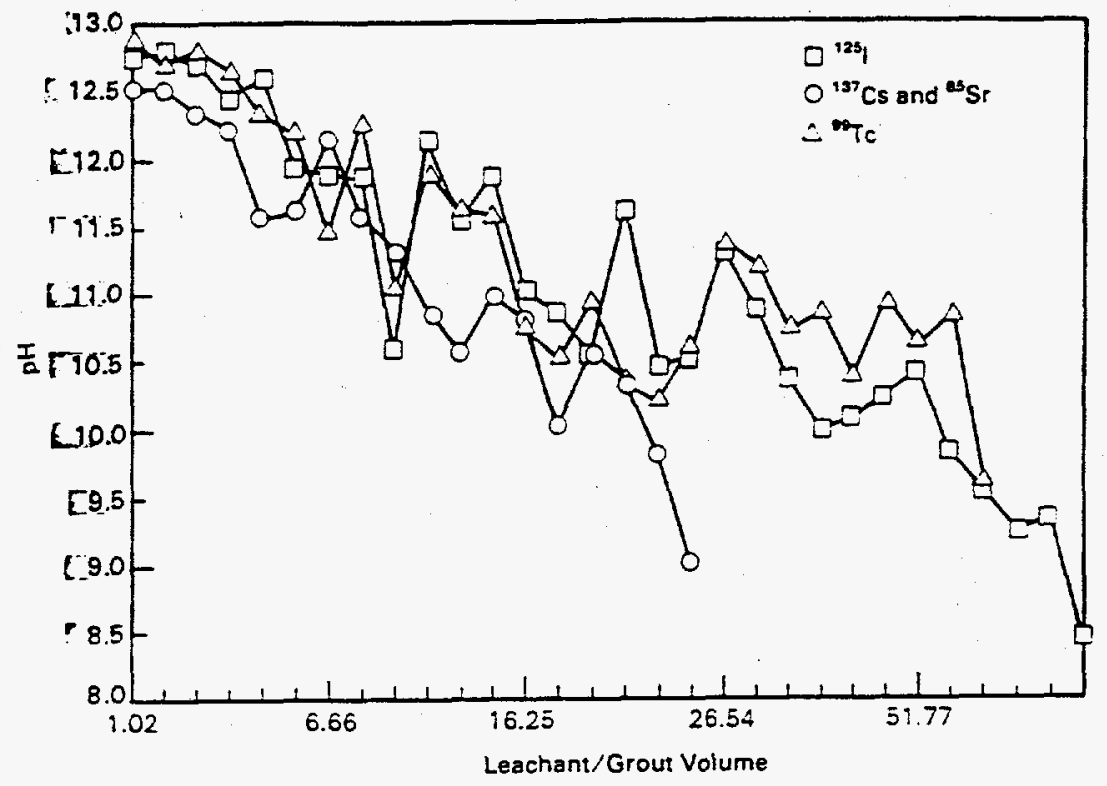

Axis not drawn to scale. Points represent samples taken at discrete time periods that increase with time.

Figure 6.15. $\mathrm{pH}$ vs. Grout Volume for Intact CRW (Grouts in Hanford Groundwater). (Three separate tests; each specimen contains different radionuclides.)

Large concentrations of potassium and sodium were present in the initial effluents from the flowthrough CRW leach tests. Throughout the 80- to 288-day test period, the effluents contained higherthan-ambient concentrations of potassium and sodium. Figures 6.16 and 6.17 show the $\mathrm{Na}$ and $\mathrm{K}$ leachate data plotted versus number of grout volumes of effluent collected.

The initial aluminum concentrations in the flow-through column effluent reached values as large as $33 \mathrm{mg} / \mathrm{L}$, then the concentrations dropped to below detection limits $(<0.03 \mathrm{mg} / \mathrm{L})$. The aluminum concentrations appeared to be controlled by the $\mathrm{pH}$. At $\mathrm{pH}$ values of greater than 11.5 , aluminum dissolution occurred. Silicon concentrations in the flow-through column effluent were consistently somewhat less than the ambient groundwater concentrations.

The effluents from the flow-through leach test showed that CRW grouts released fluoride, nitrate, sulfate, and borate. The initial effluents showed the highest concentrations, then concentrations dropped to near-ambient groundwater values for sulfate. However, fluoride, nitrate, and borate concentrations remained above the ambient groundwater levels for the duration of the test. Fluoride concentrations leveled off at about $6 \pm 1 \mathrm{mg} / \mathrm{L}$, a value approximately 4 times larger than the maximum permissible concentration (MPC) in drinking water. This steady-state concentration suggests a solubility control. Nitrate effiuent concentrations leveled off at $1 \pm 1 \mathrm{mg} / \mathrm{L}$, a value much below the drinking water MPC ( $44 \mathrm{mg} / \mathrm{L})$. Boric acid effluent concentrations leveled off at a value of $1 \pm 0.5 \mathrm{mg} / \mathrm{L}$. Phosphate concentrations in the effluents were consistently below the detection limit $(<0.3 \mathrm{mg} / \mathrm{L})$. 


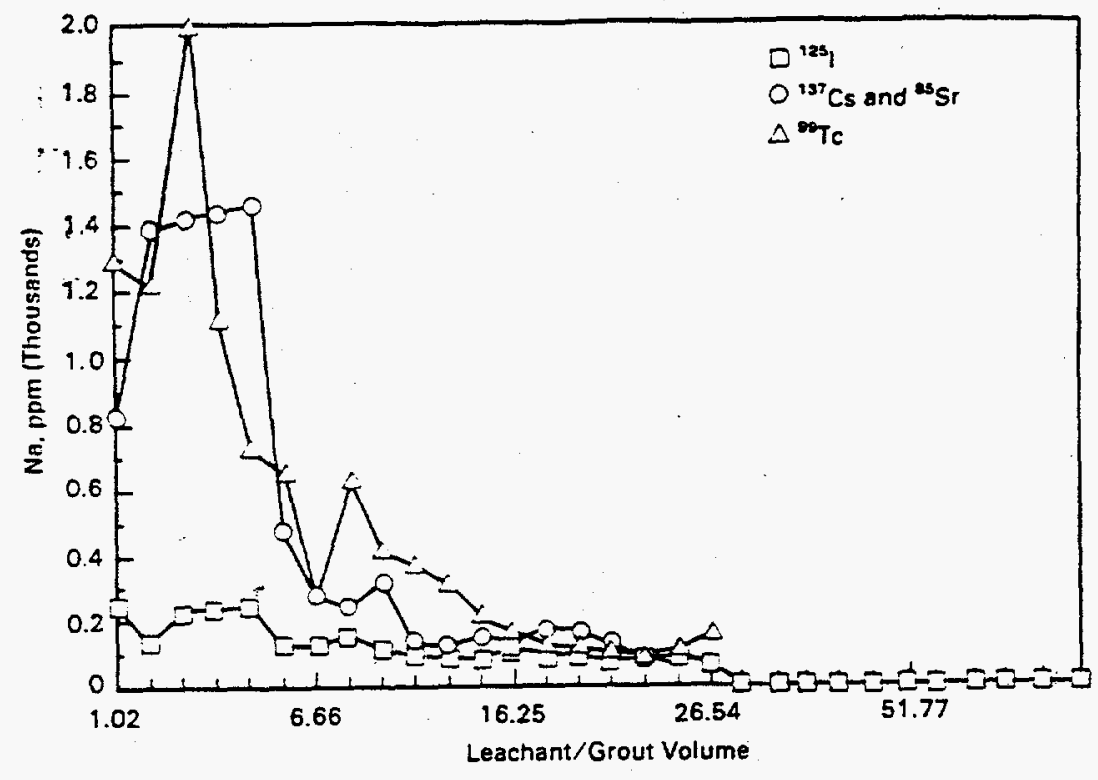

Axis not drawn to scale. Points represent samples taken at discrete time periods that increase with time.

Figure 6.16. Sodium Concentration vs. Leachant/Grout Volume for Intact CRW Grouts in Hanford Groundwater (three separate tests; each specimen contains different radionuclides).

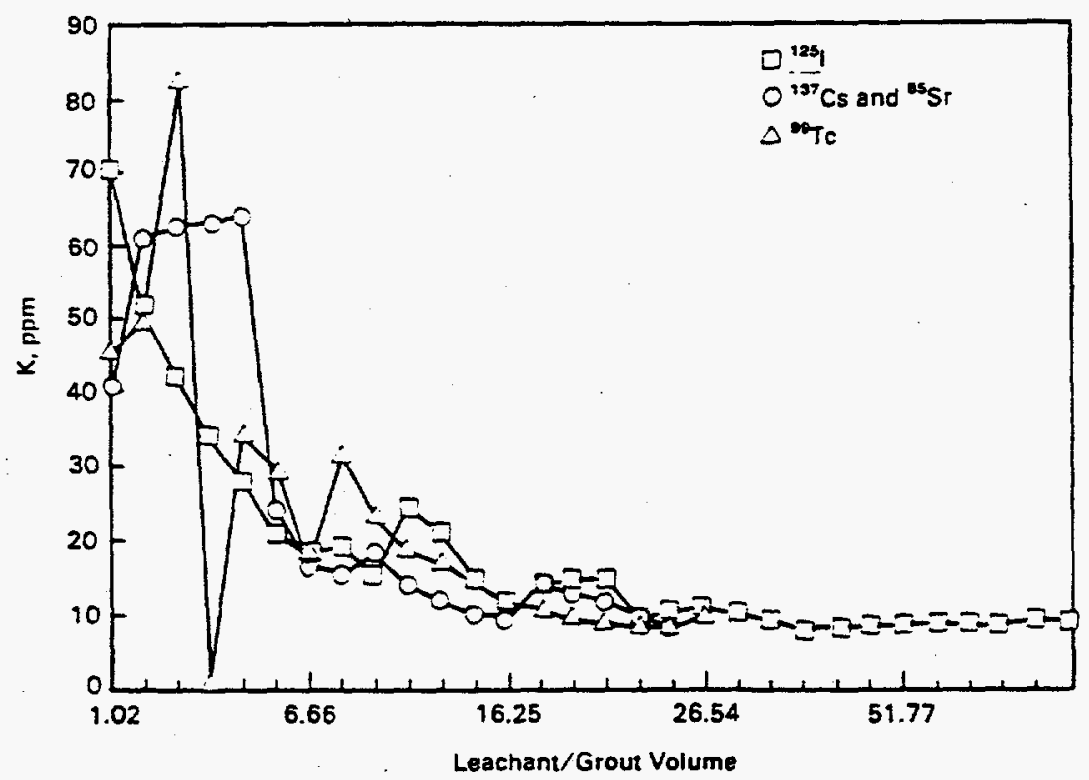

Axis not drawn to scale. Points represent samples taken at discrete time periods that increase with time.

Figure 6.17. Potassium Concentration vs. Leachant/Grout Volume for Intact CRW Grouts in Hanford Groundwater (three separate tests; each specimen contains different radionuclides). 
The total organic carbon content of the leachate was 10 to $20 \mathrm{mg} / \mathrm{L}$ in initial effluents, decreasing to near-detection limits at later times. The source of the organic carbon in the synthetic grout was not identified. As for other components, there was no release of barium, cadmium, chromium, copper, iron, manganese, lead, or zinc. These results agree with CRW grout leachate results for the ANS 16.1 and static leach tests. The zinc concentrations in the flow-through column effluent were quite sporadic, but they do suggest that some zinc was leached in the first three grout volumes.

\subsection{Chemical Analysis of Leachates from Combined Leach/Adsorption Tests on Intact CRW Grouts}

For the combined leach/adsorption tests, intact grout specimens were packed in Hanford sediment and groundwater was flowed through the column. The effluent $\mathrm{pH}$ values for the combined test were similar to the results for the leach tests without sediment, as shown in Figure 6.18. Initial effluent $\mathrm{pH}$ values were 12.2 to 12.4 , suggesting that the sediment-buffering capacity was quickly depleted. With continual flushing, the effluent $\mathrm{pH}$ dropped below 10 (after 65 to 75 pore volumes) and finally reached about 8.1 , the ambient groundwater $\mathrm{pH}$ (after 120 pore volumes). The CRW grout generated much more alkalinity than PSW grout and forced the effluent $\mathrm{pH}$ to remain highly alkaline for long periods of time.

The effluent calcium, magnesium, and strontium concentrations remained below ambient groundwater concentrations throughout the combined leach/adsorption test periods. Figure 6.19 shows the calcium concentrations in the effluents for the flow-through leach and combined leach/adsorption test. As with the other three test methodologies (ANS 16.1, static, and flow-through leach test), we suspect that carbonate and brucite precipitation reactions control the leachate concentrations of these alkaline earths.

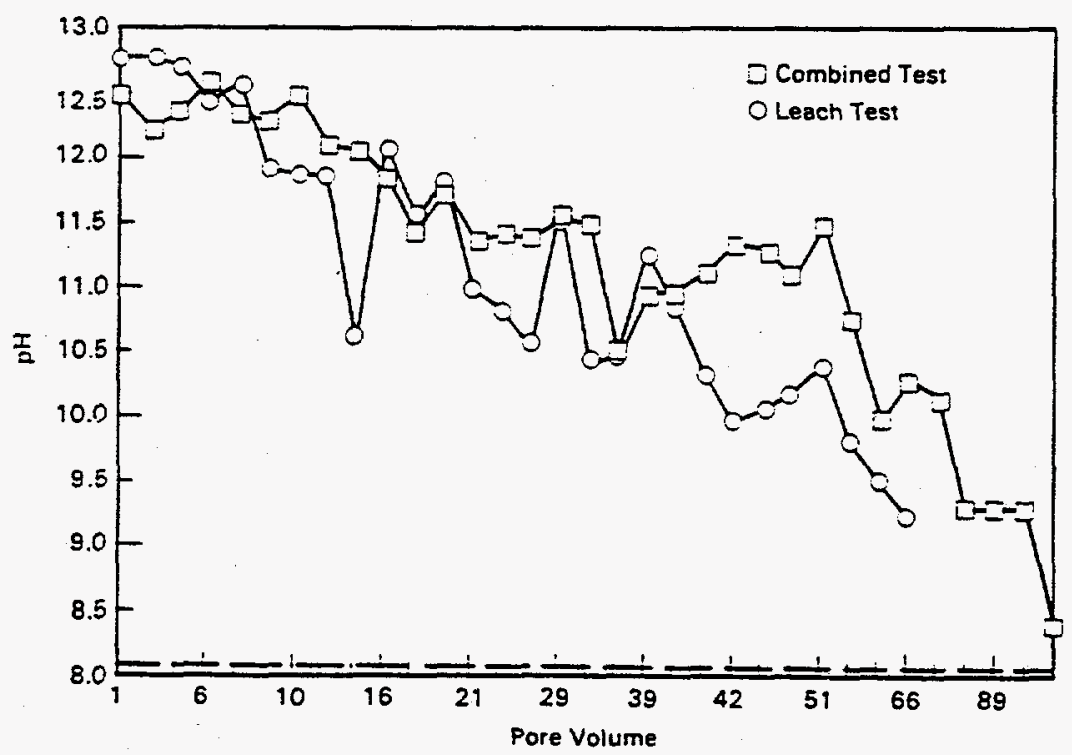

Axis not drawn to scale. Points represent samples taken at discrete time periods that increase with time.

Figure 6.18. pH Values for Leach Test vs. Combined Leach Test 


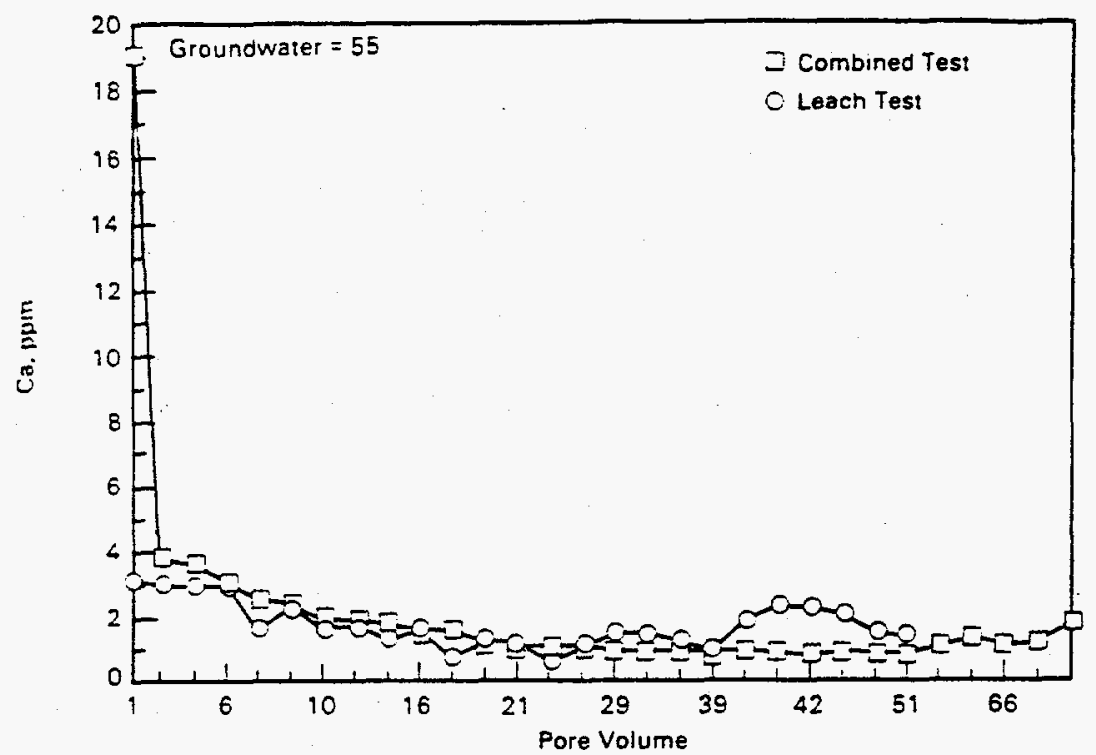

Axis not drawn to scale. Points represent samples taken at discrete time periods that increase with time.

Figure 6.19. Calcium Concentration for Leach Test vs. Combined Leach Test

The effluents from the combined leachate/adsorption tests initially contained large concentrations of sodium ( 800 to $1100 \mathrm{mg} / \mathrm{L}$ ). Later effluents still contained higher than-ambient sodium concentrations, as did effuents from all the test methodologies studied (Figure 6.20). In contrast, initial effluents from the combined grout/sediment test contained lower-than-ambient concentrations of potassium, whereas all other leach tests without sediment showed significant amounts of potassium release. In these combined tests, a pulse of potassium was released after 80 pore volumes (see Figure 6.21). Again the legend on Figure 6.21 reflects which radionuclide was present in the grout specimens packed into sediment. For this discussion ignore the nuclides and consider the data as coming from replicate tests. The delay in potassium release was likely caused by potassium exchanging onto the sediment cation exchange sites and releasing other cations such as sodium and calcium. At later times, the exchange dynamics forced potassium from the sediment and a pulse of potassium occurred in the effluent.

Aluminum concentrations in the effuents from the combined leach test were larger than those for the ambient groundwater. The initial large release observed in the flow test without sediment was dampened in tests with sediment; however, by the end of the tests, the tests with sediment released more aluminum mass. There was a much larger release of silicon from the combined tests than from the leach-only tests. The sediment contained large amounts of relatively soluble silicon under alkaline conditions. 


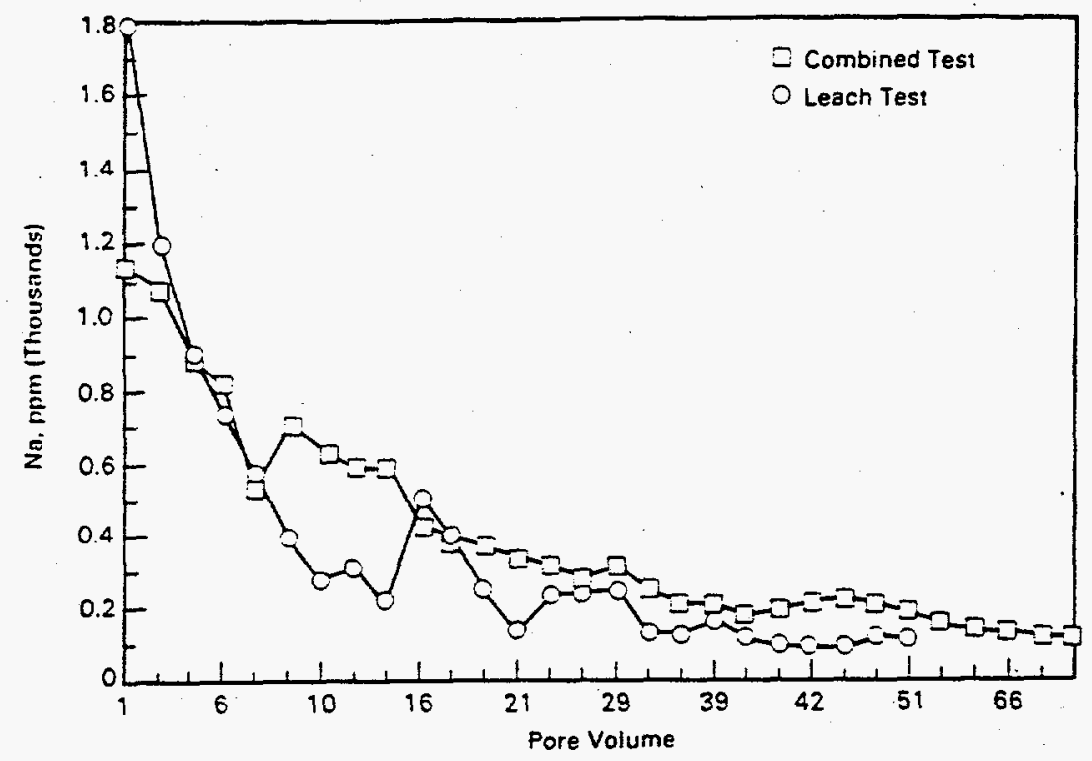

Axis not drawn to scale. Points represent samples taken at discrete time periods that increase with time.

Figure 6.20. Sodium Concentration for Leach Test vs. Combined Leach Test

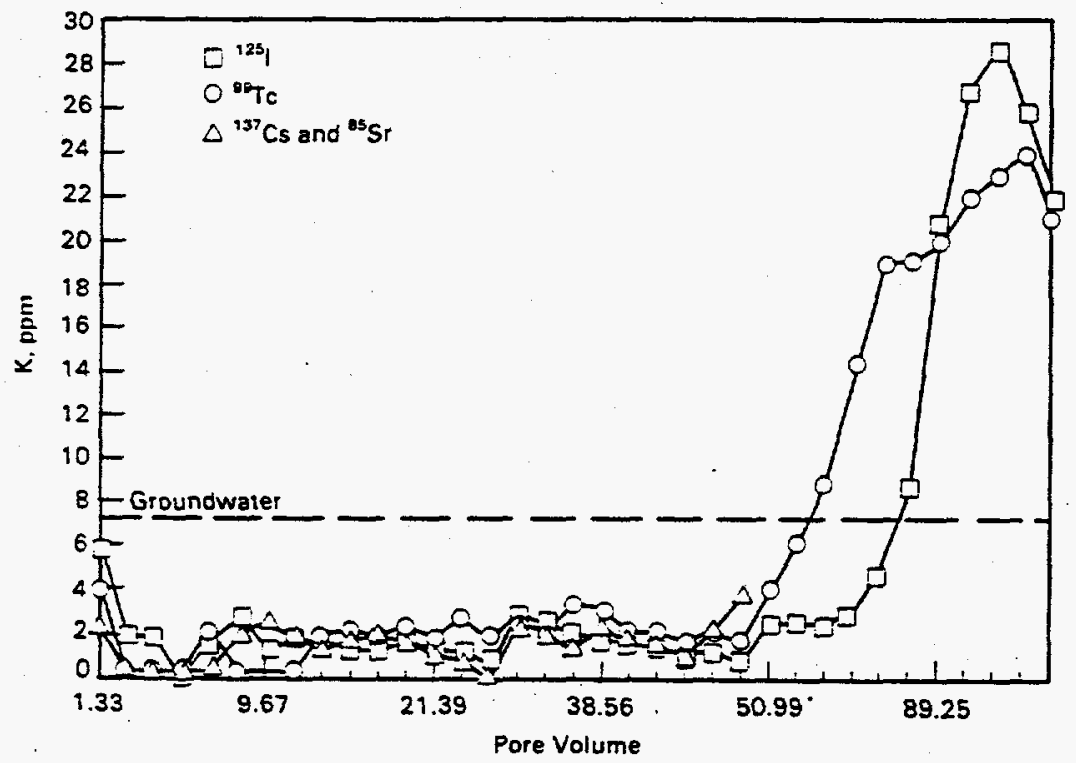

Axis not drawn to scale. Points represent samples taken at discrete time periods that increase with time.

Figure 6.21. Potassium Concentration vs. Pore Volume for Intact CRW Grouts Packed in Sediment 
The anion concentrations in effluents from the flow-through tests with sediment were very high initially, eventually dropping to values similar to those observed in the flow-through tests without sediment. The first pore volume of effluent from the combined tests contained 13 to $22 \mathrm{mg} / \mathrm{L}$ fluoride, 40 to $90 \mathrm{mg} / \mathrm{L}$ nitrate, up to $880 \mathrm{mg} / \mathrm{L}$ sulfate, and 10 to $30 \mathrm{mg} / \mathrm{L}$ boric acid.

The effluent concentrations of fluoride, nitrate, and sulfate from flow-through tests with and without sediments are shown in Figures $6.22,6.23$, and 6.24 , respectively. The first two species exceeded allowable drinking water limits in the initial effluent.

It is not clear at this time why the presence of sediment seems to enhance the initial concentrations of anions found in effluents. Dissolution of soluble salts within the sediment may contribute to this effect. After this initial flux, the fluoride concentrations from the combined test reached steady state at about $4 \mathrm{mg} / \mathrm{L}$, slightly less than the $6 \mathrm{mg} / \mathrm{L}$ for tests without sediment. The nitrate in the combined test effluent suggests that a second pulse of nitrate exited the sediment columns near the end of the tests. The steady-state concentrations of sulfate and boric acid in the effluent were similar to the values from the tests without sediment.

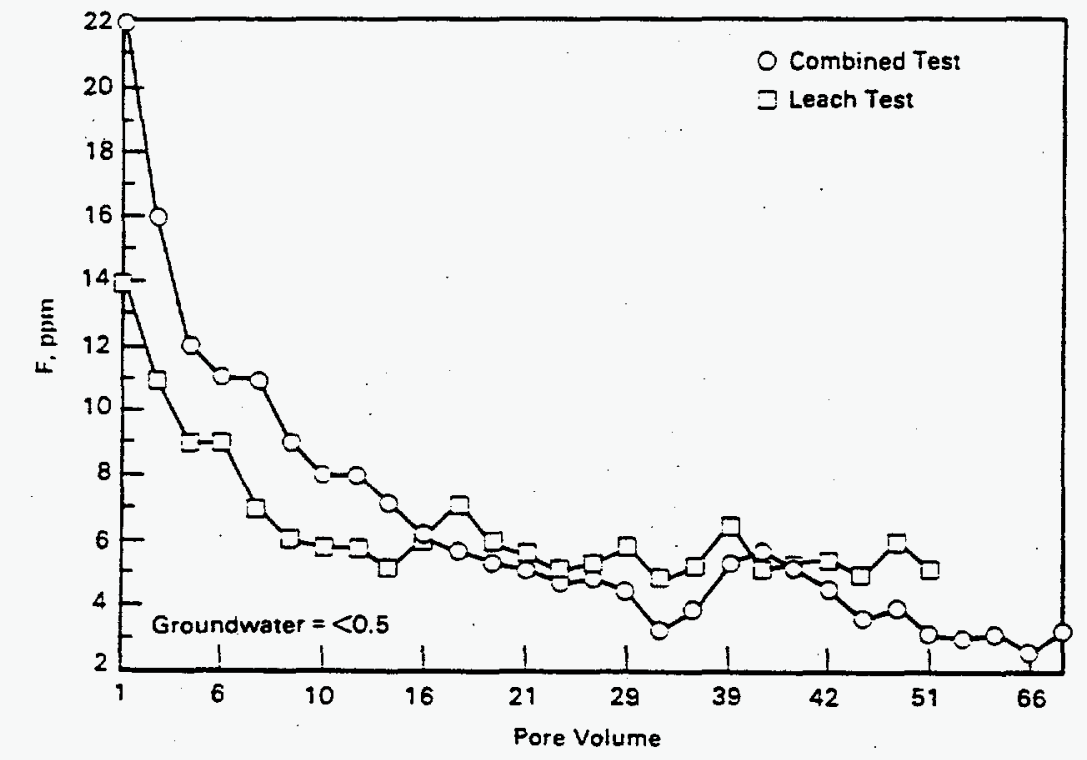

Axis not drawn to scale. Points represent samples taken at discrete time periods that increase with time.

Figure 6.22. Fluoride Concentration for Leach Test vs. Combined Leach Test 


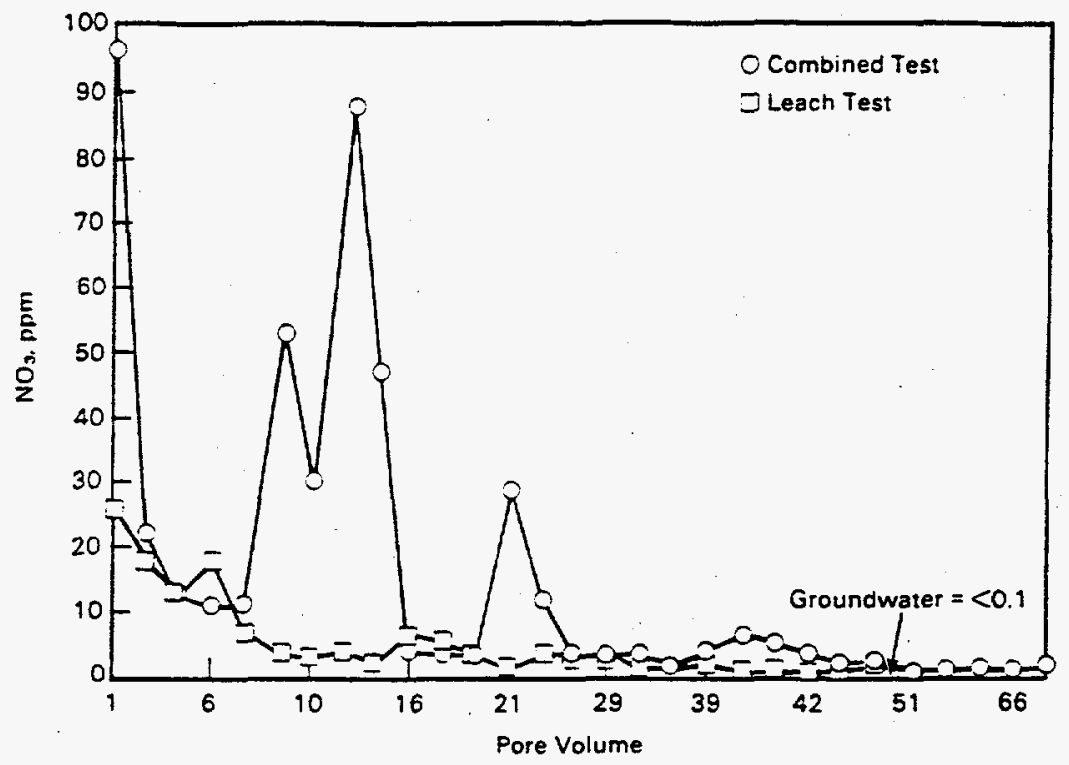

Axis not drawn to scale. Points represent samples taken at discrete time periods that increase with time.

Figure 6.23. Nitrate Concentration for Leach Test vs. Combined Leach Test

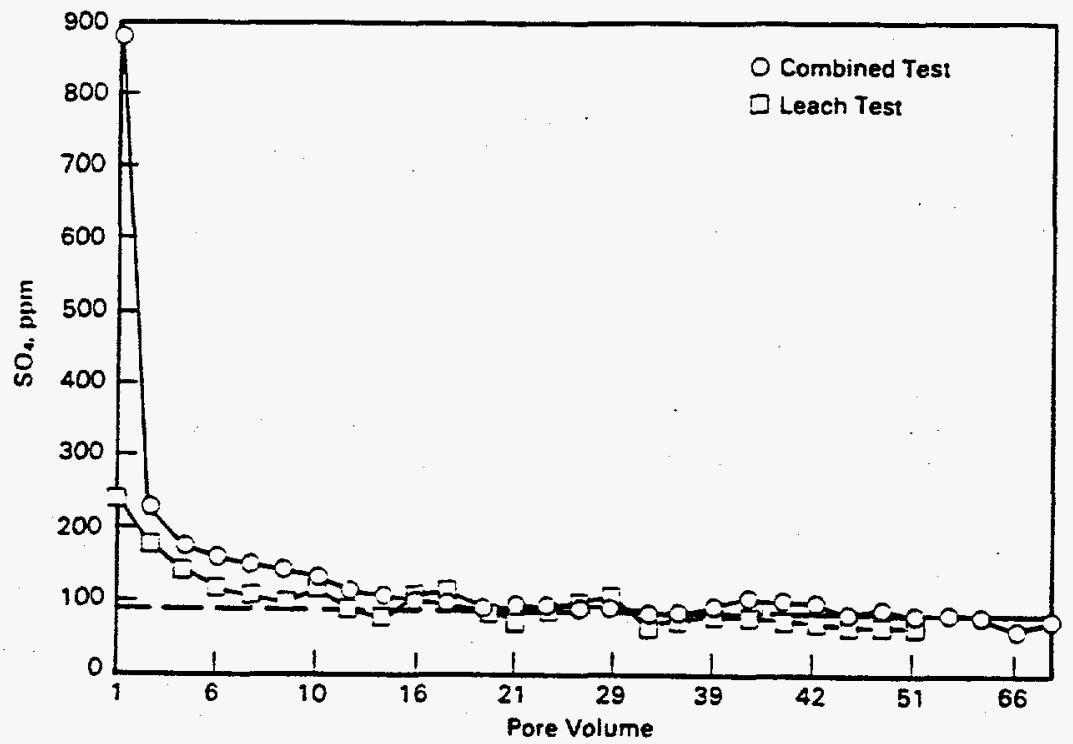

Axis not drawn to scale. Points represent samples taken at discrete time periods that increase with time.

Figure 6.24. Sulfate Concentration for Leach Test vs. Combined Leach Test 
Table 6.2 shows a comparison of the mass balance calculations for constituents in the effiuents for the four test methods. The mass balance data for the flow-through tests are those for the tests that were run the longest, and that had total effluent volumes more similar to the total volume of solution exchanges in the ANS 16.1 tests.

For those tests in which greater volumes of groundwater contacted the grout (ANS 16.1 and both column flow tests), relatively large amounts of calcium and magnesium were precipitated out of the groundwater. All of the tests showed that comparable masses of sodium and potassium were leached from the grout, but recall that the potassium shows up in the combined leach test effluent at the very end, while the bulk of the potassium leached in the other three tests shows up immediately.

All tests showed a net leaching of fluoride, nitrate, and boron. Both flow tests suggested that significantly greater amounts of fluoride were leached compared to the static and ANS 16.1 leach tests. The combined leach/adsorption test appeared to release significantly larger amounts of nitrate, although there was high variability between replicate tests.

Table 6.2. Mass Balance Relationships for Various Test Methodologies [(-) mg precipitated from groundwater and $(+) \mathrm{mg}$ leached from grout]

Type of Test

\begin{tabular}{|c|c|c|c|c|}
\hline Constituent & ANS 16.1 & Static & $\begin{array}{c}\text { Flow-Through } \\
\text { Leach }\end{array}$ & $\begin{array}{l}\text { Combined } \\
\text { Leach/ } \\
\text { Adsorption }\end{array}$ \\
\hline $\mathrm{Ca}$ & $-504.0 \pm 18.0$ & $-67.4 \pm 3.9$ & -576.0 & -571.0 \\
\hline $\mathrm{Na}$ & $+1434.0 \pm 107.0$ & $+1328.0 \pm 68$ & +1366.0 & +1683.0 \\
\hline $\mathbf{K}$ & $+42.0 \pm 3.0$ & $+46.4 \pm 2.8$ & +41.0 & +40.0 \\
\hline $\mathbf{M g}$ & $-118.0 \pm 11.0$ & $-18.3 \pm 0.7$ & -143.0 & -150.0 \\
\hline $\mathrm{Al}$ & $+0.4 \pm 0.4$ & $+21.4 \pm 2.7$ & +8.7 & +29.6 \\
\hline $\mathrm{Si}$ & $-68.0 \pm 4.0$ & $-0.4 \pm 1.8$ & -62.0 & +91.0 \\
\hline $\mathbf{F}$ & $+12.9 \pm 2.9$ & $+15.6 \pm 1.2$ & +63.0 & +41.0 \\
\hline $\mathrm{NO}_{3}$ & $+28.0 \pm 20.0$ & $+21.9 \pm 1.2$ & +24.0 & +60.0 \\
\hline $\mathrm{B}(\mathrm{OH})_{4}^{-}$ & $+3.6 \pm 1.9$ & $+6.7 \pm 0.8$ & +9.0 & +14.3 \\
\hline $\mathrm{SO}_{4}$ & $-35.0 \pm 27.0$ & $+127.0 \pm 17$ & -18.0 & +88.0 \\
\hline
\end{tabular}


The combined adsorption test released silicon, probably from dissolution of sediment material rather than grout. The ANS 16.1 and flow-through leach tests showed net silicon precipitation. The static test (with the highest average $\mathrm{pH}$ conditions) and combined grout/sediment leach test showed net aluminum leaching. In the combined leach/adsorption test the sediment may be the source. In the static test, silicon release must have come from the grout itself. Sulfate shows variable behavior, with apparent precipitation in two leach tests and apparent leaching in the static and combined tests.

\subsection{Chemical Analysis of Effluents from Grout Leach Tests and Combined Leach/Adsorption Tests Using Crushed Grout}

For conservative-bounding purposes, several grout specimens were disaggregated and used in two test methodologies. Five grams of crushed grout were placed in fine-mesh nylon bags, suspended in Plexiglas columns, and contacted with groundwater in a once-through flow mode. In the combined tests, a weight equivalent to that of the intact samples (i.e., $35 \mathrm{~g}$ ) of disaggregated grout was packed into Hanford sediment columns in either of the two configurations previously shown (see Figure 4.1). The two configurations are called the "standing" and "layer" configurations (" $\mathrm{B}$ " and " $\mathrm{C}$," respectively).

The leach experiment on the bag of disaggregated grout showed leachates that instantly rose to $\mathrm{pH}$ values of around 12 . However, after two pore volumes the $\mathrm{pH}$ of the effluents started to drop; by eight pore volumes the $\mathrm{pH}$ was similar to that of ambient groundwater.

As with the other leach tests, the calcium, magnesium, and strontium concentrations were initially quite low compared to those in the groundwater, suggesting precipitation. After about 12 pore volumes, the calcium, magnesium, and strontium effluent concentrations had risen to about 30,10 , and $0.2 \mathrm{mg} / \mathrm{L}$, respectively, which represents $54 \%, 69 \%$, and $80 \%$, respectively, of the ambient groundwater values. The initial potassium and sodium effluent concentrations were high, but after 9 to 15 pore volumes, the effluents were similar to those in ambient groundwater.

The initial leachates from the bagged, crushed grout showed a net leaching of aluminum and silicon. However, after the first three pore volumes the aluminum concentrations dropped to values near the ambient groundwater, and silicon values dropped below the ambient groundwater value. As in the other leach tests there was an initial flush of anions, but the peak concentrations were higher than those for tests with intact grouts, and the pulse duration was shorter. Nitrate and sulfate effluent concentrations dropped to ambient conditions within 3 to 4 pore volumes. Fluoride and boric acid rapidly reached steady-state effluent concentrations of 9 and $0.8 \mathrm{mg} / \mathrm{L}$, respectively. The chloride concentrations remained constant at the ambient groundwater value; phosphate concentrations remained near the detection limit throughout the leach test. The rapid flushing of alkalinity, sodium, potassium, and anions suggests that disaggregated grout allows the leaching to rapidly reach completion for readily soluble species. The chemical reactions remain the same, but the effects of the slow diffusion of leachable species out of the intact grout are removed by disaggregation. Further, it may be possible that more components are released before the carbonate shell (coating) can form over all the pieces. The detailed chemical analysis data for flow-through leach tests on bagged, crushed grout are presented in Tables A.15 through A.17. 
The chemical analysis of effluents from disaggregated grout packed in Hanford sediment is presented in Tables A.18 through A.20. The effluent $\mathrm{pH}$ values were very basic at first (12.0 to 13.2) and remained basic for the duration of the tests (30 to 185 pore volumes). In contrast to the effluents from intact grouts packed in sediment, the effluents from the crushed grout tests did not consistently drop below $\mathrm{pH} 10$ after 65 to 75 pore volumes. One test of crushed grout continued beyond 30 pore volumes (see Table A.19), and the pH finally dropped below 10 after 110 pore volumes. This release of extra alkalinity from the crushed grout tests is not surprising. The more the monolith is compromised (crushed), the faster the excess $\mathrm{Ca}(\mathrm{OH})_{2}$ can dissolve and interact with the soil. On the other hand, the initial $\mathrm{pH}$ values for the crushed grout combined leach/adsorption tests were not significantly higher than the $\mathrm{pH}$ values for the tests of intact grout. Apparently, chemical reactions controlled the effluent $\mathrm{pH}$ at values near $12.5 \pm 0.2$ no matter how much alkalinity was available. $\mathrm{Ca}(\mathrm{OH})_{2}$ in equilibrium with water fixes the $\mathrm{pH}$ at 12.5 , thus we believe that the excess $\mathrm{Ca}(\mathrm{OH})_{2}$ in the CRW grout controls system $\mathrm{pH}$ until it is completely dissolved.

In general, the alkaline earth cations (calcium, magnesium, and strontium) in crushed grout/sediment combined tests showed the same trends (net precipitation) as did the other leach and combined leach/adsorption tests. The calcium concentrations in the effluent from the crushed grout/sediment column test were initially low, but rose to about $40 \mathrm{mg} / \mathrm{L}$ after 60 pore volumes. In contrast, combined tests with intact grout had calcium effluents that remained low throughout the test. The data for inorganic carbon also suggest that the crushed test removed more carbonate ions from the inflowing groundwater and, to maintain saturation, allowed more calcium to be present in solution. The intact grouts removed less carbonate and thus, to maintain a fixed activity product $\left(\left[\mathrm{Ca}^{2+}\right]\left[\mathrm{CO}_{3}{ }^{2-}\right]\right)$, the solutions contacting intact grout specimens have less dissolved calcium. As with the crushed grout leach test (no sediment), later effluents showed higher concentrations of strontium. In fact, between 60 pore volumes and the end of the experiment (185 pore volumes), effluent strontium concentrations exceeded the groundwater ambient value. This suggests that strontium initially precipitates or is bound by adsorption reactions and later redissolves or desorbs. In no other test configuration did effluent concentrations for strontium rise above ambient groundwater values.

As occurred in the crushed grout leach test and the intact grout/sediment fiow-through test, sodium effluent concentrations in the crushed grout/sediment tests were initially high, suggesting rapid leaching. The sodium effluent concentrations of tests with crushed grouts dropped to ambient groundwater values within 15 pore volumes in the leach test (no sediment) and after 50 pore volumes in the combined grout/sediment test. This suggests that crushing allows rapid leaching, which exhausts the leachable sodium source from the grout. For intact grout, the tests continue to slowly remove sodium.

The potassium concentrations in effiuents that have contacted soil, whether from intact or crushed grout, are low initially in contrast to the potassium concentrations for the leach-only tests. We speculate that the potassium is leaching from the grout but exchanges onto the sediments. After 40 pore volumes for crushed grout and 60 to 70 pore volumes for intact grout, the potassium values in the effluent rose above ambient groundwater values, suggesting desorption. A similar trend was observed in tests of PSW grout (see Serne et al. 1987).

The most striking chemical data from the tests with crushed grout packed in sediment resulted from the very rapid flushing of nitrate and sulfate (within 10 pore volumes). Later effiuents contained essentially ambient groundwater concentrations of these two constituents. For intact grouts packed in 
sediment, nitrate effluent concentrations remained above ambient for as long as tests were run. This again suggests that crushing grout allows rapid depletion of readily soluble constituents. There was also a very large release of fluoride and boric acid from the crushed grouts packed in sediment, but instead of dropping off to groundwater concentrations the effluent remained above ambient. This suggests either two distinct sources with differing leach kinetics or solubility control. In the crushed grout tests packed in sediments, initial concentrations of fluoride, nitrate, sulfate, and sodium exceeded primary and secondary drinking water standards.

Table 6.3 compares the total mass of selected elements that precipitated or leached in combined leach/adsorption tests of equal weights of crushed and intact grout. For each method, the tests that ran for the longest time are presented.

There are no clear trends in the data for the totals leached or precipitated. Thus, although crushing the grout caused material to be rapidly released, by the end of the tests the total mass released or precipitated was not distinctly different than those tests using intact specimens. In fact, it appeared that the intact grout precipitated more calcium and actually leached more sodium, potassium, and nitrate. The crushed samples released more fluoride and boron. Silicon behavior seemed to vary between the two tests.

\subsection{Cumulative Effective Diffusion Coefficients for Samples of Intact Grout}

Appendix B contains all of the diffusion coefficient data. Both instantaneous and cumulative diffusion coefficient values are reported for each test (except the static leach tests) for selected species that leached from the grout. Leach rates for the flow-through column tests were also calculated using

Table 6.3. Comparison of Mass Balances for the Combined Leach/Adsorption Test of Crushed and Intact Grout (mg) (-precipitated, + leached)

\begin{tabular}{|c|c|c|}
\hline Component & Crushed Grout & Intact Grout \\
\hline $\mathrm{Ca}$ & -232.0 & -571.0 \\
\hline $\mathrm{Na}$ & +1240.0 & +1683.0 \\
\hline K & +22.4 & +40.0 \\
\hline Mg & -137.0 & -150.0 \\
\hline $\mathrm{Al}$ & +52.8 & +29.6 \\
\hline $\mathrm{Si}$ & -9.0 & +91.0 \\
\hline $\mathbf{F}$ & +89.8 & +41.0 \\
\hline $\mathrm{NO}_{3}$ & +23.8 & +59.5 \\
\hline $\mathrm{B}(\mathrm{OH})_{4}^{-}$ & +28.2 & +14.3 \\
\hline $\mathrm{SO}_{4}$ & +62.9 & +87.8 \\
\hline
\end{tabular}


Materials Characterization Center (MCC)-4 protocol. However, only the cumulative effective diffusion coefficients are discussed here; until large-scale leaching tests are performed and the "surface wash-off" phenomenon is explored further, we suggest that cumulative effective diffusion coefficients are the best representation of the leach data from a performance assessment standpoint. The cumulative effective diffusion coefficient includes all the mass leached and is calculated at the longest time period of study. The values are smaller (by up to two orders of magnitude) than some of the initial instantaneous values, and generally larger (by a factor of two) than instantaneous diffusion coefficients at the later times.

Table 6.4 lists effective cumulative diffusion coefficients for the intact $\mathrm{CRW}$ grouts leached in Hanford groundwater. For the species potassium, sodium, fluoride, nitrate, ${ }^{14} \mathrm{C},{ }^{125} \mathrm{I},{ }^{137} \mathrm{Cs},{ }^{241} \mathrm{Am}$, and ${ }^{238} \mathrm{Pu}$, there were no significant differences in the diffusion coefficients between ANS 16.1 and static leach tests. Boron, aluminum, technetium, and perhaps ${ }^{85} \mathrm{Sr}$ leached more rapidly in the static leach test. Furthermore, sulfate leached from grout in the static leach tests but not in the ANS 16.1 tests. The apparently higher leach rates from the static leach tests for several constituents are counter to expectations based solely on diffusion theory. As with the PSW grout, we suspect that coatings of carbonate minerals on the CRW grout surfaces may be lowering the leachability of ANS 16.1

Table 6.4. Effective Diffusion Coefficient (cumulative $D_{e}$ ) Values Obtained From Standard Leach Tests $\left(\mathrm{cm}^{2} / \mathrm{s}\right)$

\begin{tabular}{lccc} 
Component & ANS 16.1 Test & Static Test \\
\cline { 2 - 3 } $\mathrm{K}$ & $1.3 \times 10^{-9} \pm 0.4 \times 10^{-9}$ & $1.6 \times 10^{-9} \pm 0.2 \times 10^{-9}$ \\
$\mathrm{Na}$ & $6.4 \times 10^{-9} \pm 2.1 \times 10^{-9}$ & $5.2 \times 10^{-9} \pm 0.7 \times 10^{-9}$ \\
$\mathrm{Al}$ & $*$ & $1.6 \times 10^{-12} \pm 0.4 \times 10^{-12}$ \\
$\mathrm{H}_{3} \mathrm{BO}_{3}$ & $2.4 \times 10^{-12} \pm 1.0 \times 10^{-12}$ & $1.6 \times 10^{-10} \pm 2.9 \times 10^{-10}$ \\
$\mathrm{~F}$ & $2.0 \times 10^{-12} \pm 1.8 \times 10^{-12}$ & $1.4 \times 10^{-12} \pm 0.4 \times 10^{-12}$ \\
$\mathrm{NO}_{3}$ & $1.2 \times 10^{-9} \pm 0.5 \times 10^{-9}$ & $1.7 \times 10^{-9} \pm 0.3 \times 10^{-9}$ \\
$\mathrm{SO}_{4}$ & $*$ & $7.0 \times 10^{-9} \pm 3.0 \times 10^{-9}$ \\
${ }^{14} \mathrm{C}$ & $2.0 \times 10^{-13}$ & $1.0 \times 10^{-13}$ \\
${ }^{99} \mathrm{Tc}$ & $2.0 \times 10^{-12}$ & $2.0 \times 10^{-11}$ \\
${ }^{125} \mathrm{I}$ & $3.4 \times 10^{-9}$ & $2.0 \times 10^{-9}$ to $7.0 \times 10^{-8}$ \\
${ }^{137} \mathrm{Cs}$ & $1.0 \times 10^{-15}$ & $4.0 \times 10^{-15}$ \\
${ }^{85} \mathrm{Sr}$ & $5.0 \times 10^{-12}$ & $10.0^{-12}$ to $10.0^{-10}$ \\
${ }^{241} \mathrm{Am}$ & $6.0 \times 10^{-16}$ & $1.0 \times 10^{-17}$ \\
${ }^{238} \mathrm{Pu}$ & $1.0 \times 10^{-16}$ & $2.0 \times 10^{-16}$ \\
$*$ did not leach. & & \\
& &
\end{tabular}


specimens more so than in the static leach test specimens. The loss of calcium and inorganic carbon in ANS 16.1 tests of CRW grout was 8 to 10 times greater than for static leach tests. Thus, we expect a greater degree of coating on specimens from the ANS 16.1 tests.

The data in Table 6.4 suggest the following trends. Sodium and ${ }^{125} \mathrm{I}$ leach slightly faster than $\mathrm{K}$ and $\mathrm{NO}_{3}^{-}$. The species ${ }^{85} \mathrm{Sr}, \mathrm{B}, \mathrm{F}$, and ${ }^{95} \mathrm{Tc}$ leach significantly slower than these four. Even slower leach rates are found for ${ }^{14} \mathrm{C}$; and the slowest leach rates of all are found for the species ${ }^{137} \mathrm{Cs},{ }^{241} \mathrm{Am}$, and ${ }^{208} \mathrm{Pu}$. In the static leach tests, sulfate leached at rates similar to $\mathrm{Na}$ and ${ }^{125} \mathrm{I}$.

In comparison, PSW grout showed an effective diffusion coefficient for $\mathrm{Na}$ that was one-tenth as large, and an effective diffusion coefficient for $\mathrm{K}$ that was one-tenth to one-hundredth as large. The effective diffusion coefficient for nitrite from PSW grout was quite variable, but the largest value found is similar to the nitrate value for CRW grout. The effective diffusion coefficient for ${ }^{85} \mathrm{Sr}$ in PSW grout was similar to the lower values $\left(10^{-12} \mathrm{~cm}^{2} / \mathrm{s}\right)$ found for CRW grout. The effective diffusion coefficient for ${ }^{137} \mathrm{Cs}$ in PSW grout was 100 times higher than that in CRW grout. We speculate that the observed higher diffusion coefficients for $\mathrm{Na}$ and $\mathrm{K}$ from CRW grout versus PSW grout are caused by the larger fraction of these cations found in the liquid waste stream as compared to the total inventory in the grout, which includes contributions from the dry blend. That is, much more of the Na and K in PSW grout is present in the cement, fly ash, and clay and, therefore, may not be as leachable as the $\mathrm{Na}$ and $\mathrm{K}$ originally present in the $\mathrm{CRW}$ liquid waste.

Effective diffusion coefficients were also calculated from the flow-through leach and combined grout/sediment tests. The effective diffusivities (cumulative) at the end of the tests are shown in Table 6.5. For most constituents, the presence or absence of the sediment makes little difference in the calculated diffusion coefficients. The one exception is nitrate, for which the diffusion coefficient was actually larger when sediment was present. A water leach test of the sediment shows that it contains nitrates that contribute to the mass leached and thus bias the diffusion coefficient to a larger value.

The presence of sediment did not change the leaching of ${ }^{99} \mathrm{Tc}$ and ${ }^{125} \mathrm{I}$, although it significantly lowered the leaching of ${ }^{137} \mathrm{Cs}$ and perhaps ${ }^{85} \mathrm{Sr}$. Batch adsorption tests with $\mathrm{CRW}$ leachate from the ANS 16.1 tests showed no adsorption of ${ }^{99} \mathrm{Tc}$ and ${ }^{125} \mathrm{I}$ (unpublished data). Previous adsorption tests with PSW leachates has shown considerable adsorption of ${ }^{85} \mathrm{Sr}$ and ${ }^{137} \mathrm{Cs}$ (see Serne et al. 1987). Thus, it appears that ${ }^{129} \mathrm{I}$ and ${ }^{99} \mathrm{Tc}$ that leach from grout waste forms will travel through sediment with little, if any, additional retardation by adsorption reactions.

A comparison between the flow-through leach test (Table 6.5) and the ANS 16.1 and static leach tests (Table 6.4) shows that most constituents (except ${ }^{137} \mathrm{Cs}$ and ${ }^{85} \mathrm{Sr}$ ) leach more rapidly in the flowthrough test. The effective diffusion coefficients (cumulative) for the flow-through leach test were 2 to 5 times larger than the static and ANS 16.1 test results for $\mathrm{Na}, \mathrm{K}, \mathrm{F}^{-}$, and ${ }^{125} \mathrm{I}$. The flow-through leach test values for boric acid and ${ }^{90} \mathrm{Tc}$ tend to be 10 to 100 times larger than those measured in comparable "jar" tests. Some of the differences may be caused by the shorter time periods used in the flowthrough tests and the fact that there is more inherent scatter in the data from flow-through tests. The data in Table 6.5 represent only those tests carried out for longer time periods, and thus they contain minimal replication. More tests using the flow-through methodology would be needed to better establish whether perceived test differences are real. 
Table 6.5. Flow-Through Column Data for Intact Grouts $D_{e}$ (cumulative) $\left(\mathrm{cm}^{2} / \mathrm{s}\right.$ )

$\begin{array}{lcc}\text { Component } & \text { Leach } & \text { Combined Leach/Adsorption } \\ \mathrm{K} & 1.5 \text { to } 6.5 \times 10^{-9} & 0.0 \text { to } 2.2 \times 10^{-9} \\ \mathrm{Na} & 0.6 \text { to } 2.0 \times 10^{-8} & 0.5 \text { to } 1.0 \times 10^{-8} \\ \mathrm{Al} & 0.9 \text { to } 2.9 \times 10^{-12} & 1.4 \text { to } 3.7 \times 10^{-12} \\ \mathrm{H}_{3} \mathrm{BO}_{3} & 1.5 \text { to } 5.0 \times 10^{-11} & 1.8 \text { to } 4.4 \times 10^{-11} \\ \mathrm{~F} & 2.0 \text { to } 4.6 \times 10^{-11} & 4.6 \text { to } 8.3 \times 10^{-12} \\ \mathrm{NO}_{3} & 1.6 \text { to } 5.5 \times 10^{-9} & 0.3 \text { to } 1.5 \times 10^{-8} \\ { }^{14} \mathrm{C} & \text { no data } & \text { no data } \\ { }^{99} \mathrm{Tc} & 0.3 \text { to } 1.2 \times 10^{-10} & 0.6 \text { to } 3.3 \times 10^{-10} \\ { }^{125} \mathrm{I} & 0.1 \text { to } 3.4 \times 10^{-8} & 0.8 \text { to } 4.0 \times 10^{-8} \\ { }^{137} \mathrm{Cs} & 10^{-26} & 10^{-27} \\ { }^{85} \mathrm{Sr} & 10^{-23} & 10^{-23} \\ { }^{241} \mathrm{Am} & \text { no data } & \text { no data } \\ { }^{238} \mathrm{Pu} & \text { no data } & \text { no data }\end{array}$

Radionuclide leach data from the flow-through tests with crushed grout are reported in Appendix B, Tables B.20-B.23. Aside from Tc for which leachate data suggest more ${ }^{90} \mathrm{Tc}$ was leached than was present in the starting inventory, the cumulative effective diffusion coefficients do not differ greatly from those in Table 6.5 (right-hand column) for intact grout. The ${ }^{99} \mathrm{Tc}$ data show much faster release for the crushed grout packed in sediments but mass balance is bad (more Tc leached than present). The release of other mobile species, $\mathrm{Na},{ }^{125} \mathrm{I}$ and $\mathrm{NO}_{3}$ from crushed grout packed in sediment is a factor of 1 to 3 larger than for intact grout placed in sediment. This small increase is caused by the crushing that exposes much larger surface area of the waste form. 


\subsection{References}

ANS. 1986. Measurement of the Leachibility of Solidified Low-Level Radioactive Wastes by a ShortTerm Procedure. ANS 16.1, American Nuclear Society, Champaign, Illinois.

Arora, H. and R. Dayal. 1984. Solidification and Leaching of Boric Acid and Resin LWR Wastes. NUREG/CR-3909, Nuclear Regulatory Commission, Washington, D.C.

Clark, W. E. 1977. "The Isolation of Radioiodine with Portland Cement Part 1: Scoping Leach Studies." Nucl. Tech. 36:215-221.

Coles, D. G., and L. D. Ramspott. 1982. ${ }^{106}$ Ru Migration in a Deep Tuffaceous Alluvium Aquifer, Nevada Test Site: Discrepancy Between Field Observations and Prediction from Laboratory Measurements." Science 215:1235-1237.

Dayal, R., H. Arora and N. Morcos. 1983. Estimation of Cesium-137 Release from Waste/Cement Composites Using Data from Small-Scale Specimen. NUREG/CR-3382, Nuclear Regulatory Commission, Washington, D.C.

Dayal, R., D. C. Schweitzer and R. E. Davis. 1984. "Wet and Dry Cycle Leaching: Aspects of Research in the Unsaturated Zone." In NRC Nuclear Waste Geochemistry '83, eds. D. H. Alexander and G. F. Birchard, pp. 440-453. NUREG/CP-0052, Nuclear Regulatory Commission, Washington, D.C.

Deutsch, W. J., E. A. Jenne and K. M. Krupka. 1982. "Solubility Equilibria in Basalt Aquifers: The Columbia Plateau, Eastern Washington, U.S.A." Chemical Geology 36:15-34.

Felmy, A. R., D. C. Girvin and E. A. Jenne. 1984. MINTEQ: A Computer Program for Calculating Aqueous Geochemical Equilibria. EPA-600/3-84-032, U.S. Environmental Protection Agency, Athens, Georgia.

Godbee, H. W., O. U. Anders and R. M. Nielson, Jr. 1980. "Application of Mass Transport Theory to the Leaching of Radionuclides from Waste Solids." Nucl. and Chem. Waste Management 1:29-35.

Greenberg, A. E., J. J. Connors, D. Jensen, and M. A. H. Franson, eds. 1981. Standard Methods for the Examination of Wastes and Wastewater. 15th ed., Method \#403. American Public Health Association, Washington, D.C.

Heilman, M. D. et al. 1965. "The Ethylene Glycol Monoethyl Ether (EGME) Technique for Determining Soil Surface Area." Soil Sci. 100(6):409-413.

Kalb, P. D., and P. Columbo. 1984. "Full-Scale Leaching Study of Commercial Reactor Waste Forms." In Waste Management '84, Vol. 2, ed. R. G. Post, pp. 189-194. The University of Arizona Press, Tempe, Arizona. 
Kincaid, C. T., J. W. Shade, G. A. Whyatt, M. G. Piepho, K. Rhoads, J. A. Voogd, J. H. Westsik, Jr., M. D. Freshley, K. A. Blanchard, and B. G. Lauzon. 1995. Performance Assessment of Grouted Double-Shell Tank Waste Disposal at Hanford.

WHC-SD-WM-EE-004, Revision 1, Volumes 1 and 2, Westinghouse Hanford Company, Richland, Washington.

Matsuzuru, H. and A. Ito. 1978a. "Immobilization of Cesium-137 in Cement-Waste Composites by Addition of Mineral Zeolites." Health Phys. 34:643-648.

Matsuzuru, H. and A. Ito. 1978b. "Effects of Dimension of Specimen on Amounts of ${ }^{137} \mathrm{Cs},{ }^{90} \mathrm{Sr}$ and ${ }^{60}$ Co Leached from Matrix of Hardened Cement Grout." J. of Nucl. Sci. and Techn. 15:296-301.

Matsuzuru, H. and N. Moriyama. 1982. "Leaching Radionuclides from a Cement Composite Incorporating Evaporatory Concentrates Generated at a Pressurized Water Reactor Nuclear Power Plant." Nuclear Sci. and Eng. 80:14-25.

Moody, J. B. 1981. Radionuclide Migration/Retardation: Research and Development Technology Status Report. ONWI-321, Office of Nuclear Waste Isolation, Battelle Memorial Institute, Columbus, Ohio.

Moore, J. G., H. W. Godbee and A. H. Kibbey. 1977. "Leach Behavior of Hydrofracture Grout Incorporating Radioactive Wastes." Nuclear Technology 32:39-59.

Moriyama, N., S. Dojiri and H. Matsuzuru. 1977. "Leaching of ${ }^{137} \mathrm{Cs}$ from the Ion Exchange Resin Incorporated in Polyethylene or Cement Composite." Health Phys. 32:549-552.

Moriyama, N., H. Matsuzuru, S. Dojiri and Y. Wadachi. 1975. Analytical Evaluation on the Leaching of Radionuclides from Waste Solids. JAERI-M 6302, Japan Atomic Energy Research Institute, Tokyo, Japan.

Muller, A. B., D. Langmuir and L. E. Duda. 1983. "The Formulation of an Integrated PhysicochemicalHydrologic Model for Predicting Waste Nuclide Retardation in Geologic Media." In Scientific Basis for Waste Management VI, Vol. 15, D. G. Brookins, ed., pp. 547-564. Materials Research Society, Pittsburgh, Pennsylvania.

Nestor, C. W., Jr. 1980. Diffusion from Solid Cylinders. ORNL/CSD/TM-84, Oak Ridge National Laboratory, Oak Ridge, Tennessee.

Peterson, S. R., R. J. Serne, A. R. Felmy, R. L. Erikson, K. M. Krupka and G. W. Gee. 1984. "Interactions of Acidic Solutions with Sediments: A Case Study." In Proceedings of NRC Nuclear Waste Geochemistry ' 83 , eds. E. H. Alexander and G. F. Birchard, pp. 513-535. NUREG/CP-0052, Nuclear Regulatory Commission, Washington, D.C.

Peterson, S. R., W. J. Martin, and R. J. Serne. 1986. Predictive Geochemical Modeling of Contaminant Concentrations in Laboratory Columns and in Plumes Migrating from Uranium Mill Tailings Waste Impoundments. NUREG-CR/4520, U.S. Nuclear Regulatory Commission, Washington, D.C. 
Reardon, R. J. 1981. "Kd's - Can They be Used to Describe Reversible Ion Sorption Reaction in Contaminant Migration?" Ground Water 19:279-286.

Roy, D. M. and C. A. Langton. 1982. Longevity of Borehole and Shaft Sealing Materials: 2. Characterization of Cement-Based Ancient Building Materials. ONWI-202, Office of Nuclear Waste Isolation, Battelle Memorial Institute, Columbus, Ohio.

Sambell, R. A. J., C. Smitton and A. Elsden. 1982. "A Contribution Towards a Standard Leach Test for Immobilized Low and Medium Level Waste." Nuclear and Chemical Waste Management 3:125129.

Serne, R. J. and J. F. Relyea. 1983. "The Status of Radionuclide Sorption-Desorption Studies Performed by the WRTT Program." In The Technology of High-Level Nuclear Waste Disposal, Vol. 1. DOE/TIC-4621, pp. 203-254. Technical Information Center, U. S. Department of Energy, Washington, D.C.

Serne, R. J., R. O. Lokken, and L. J. Criscenti. 1992. "Characterization of Grouted-Low-Level Waste to Support Performance Assessment," Waste Management 12:271-287.

Serne, R. J., W. J. Martin, R. O. Lokken, V. L. LeGore, C. W. Lindenmeier and P. F. C. Martin. 1989a. Leach and EP Toxicity Tests on Grouted Waste from Tank 106-AN. PNL-6960, Pacific Northwest Laboratory, Richland, Washington.

Serne, R. J., W. J. Martin, S. B. McLaurine, S. P. Airhart, V. L. LeGore and R. L. Treat. 1987. Laboratory Leach Tests of HFW Grout and Leachate Adsorption Tests Using Hanford Sediment. PNL-6019, Pacific Northwest Laboratory, Richland, Washington.

Serne, R. J., W. J. Martin, V. L. LeGore, C. L. Lindenmeier, S. B. McLaurine, P. F. C. Martin, and R. O. Lokken. 1989b. Leach Tests on Grouts Made with Actual and Trace Metal-Spiked Synthetic Phosphate/Sulfate Waste. PNL-7121, Pacific Northwest Laboratory, Richland, Washington.

Serne, R. J., C. W. Lindenmeier, J. L. Conca, J. A. Campbell, V. L. LeGore, J. E. Amonette, K. J. Cantrell, and M. I. Wood. 1993. Solid-Waste Leach Characteristics and Contaminant-Sediment Interactions Vol. 1: Batch Leach and Adsorption Tests and Sediment Characterization, PNL-8889 Vol. 1, Pacific Northwest Laboratory, Richland, Washington.

Sewart, G. H., W. T. Farris, D. G. Huizenga, A. H. McMakin, G. P. Streile and R. L. Treat. 1987. Long-term Performance Assessment of Grouted Phosphate/Sulfate Waste from N Reactor Operations. PNL-6152, Pacific Northwest Laboratory, Richland, Washington.

Strachan, D. M., K. M. Krupka and B. E. Grambow. 1984. "Solubility Interpretations of Leach Tests on Nuclear Waste Glass." Nuclear and Chemical Waste Management 5:87-99.

U.S. Department of Energy. 1987. Final Environmental Impact Statement: Disposal of Hanford Defense, High-Level, Transuranic and Tank Wastes. DOE/EIS-0113, U.S. Department of Energy, Washington, D.C. 
Appendix A

Chemical Compositions of Leachates and Column Effluents 


\section{Appendix A}

\section{List of Tables}

Table A.1 - ANS 16.1 Leach Test on Whole Grout with Groundwater (ANS 2-4)

Table A.2 - ANS 16.1 Leach Test on Whole Grout with Groundwater (ANS 3-4)

Table A.3 - ANS 16.1 Leach Test on Whole Grout with Groundwater (ANS 4-8)

Table A.4 - ANS 16.1 Leach Test on Whole Grout with Groundwater (ANS 5-5)

Table A.5 - Static Leach Test on Whole Grout with Groundwater (ST2-17)

Table A.6 - Static Leach Test on Whole Grout with Groundwater (ST3-18)

Table A.7 - Static Leach Test on Whole Grout with Groundwater (ST4-13)

Table A.8 - Static Leach Test on Whole Grout with Groundwater (ST5-17)

Table A.9 - Column Adsorption Test on Whole Grout in Sediment (COLT1)

Table A.10 - Column Adsorption Test on Whole Grout in Sediment (COLI1)

Table A.11 - Column Adsorption Test on Whole Grout in Sediment (COLC1)

Table A.12 - Flow-Through Leach Test on Whole Grout with Groundwater (COLLT3)

Table A.13 - Flow-Through Leach Test on Whole Grout with Groundwater (COLLT2)

Table A.14 - Flow-Through Leach Test on Whole Grout with Groundwater (COLLT1)

Table A.15 - Flow-Through Leach Test on Crushed Grout with Groundwater (BCGT)

Table A.16 - Flow-Through Leach Test on Crushed Grout with Groundwater (BCGI)

Table A.17 - Flow-Through Leach Test on Crushed Grout with Groundwater (BCGC)

Table A.18 - Column Adsorption Test on Crushed Grout in Sediment (CGT)

Table A.19 - Column Adsorption Test on Crushed Grout in Sediment (CGC)

Table A.20 - Column Adsorption Test on Crushed Grout in Sediment (SCGI) 
TABLE A.1. ANS 16.1 Leach Test on whole Grout with Groundwater (ANS2-4)

\begin{tabular}{|c|c|c|c|c|c|c|c|c|c|c|c|}
\hline SAMPLE & ------ & $\begin{array}{l}\text { HELL } \\
\text { HATER }\end{array}$ & 1 & 2 & 3 & 4 & 5 & 6 & 7 & $a$ & 7 \\
\hline TIHE & days & $-\infty-\infty$ & 0.0003 & 0.0833 & 0.292 & 1 & 2 & 3 & 13 & 20 & 27 \\
\hline $\mathrm{pH}$ & & a.1 & 8.72 & 10.44 & 10.19 & 11.30 & 10.88 & 9.51 & 12.08 & 11.65 & 11.25 \\
\hline Eh & ay & 295 & 259 & 180 & 201 & 324 & 325 & 330 & 309 & 228 & 169 \\
\hline Hil & $19 / 1$ & 0.1 & 0.11 & 0.05 & $\{0.03$ & 0.04 & $<0.03$ & 0.05 & 1.08 & $\langle 0.03$ & 60.03 \\
\hline Ba & $\lg / 1$ & 0.06 & 0.041 & 0.016 & 0.005 & 0.000 & 0.003 & 0.017 & 0.006 & 0.006 & 0.004 \\
\hline$\tilde{\complement a}$ & $\log / 1$ & 56 & 49.6 & 23.5 & 9.9 & $9 . j$ & 1.1 & 13.2 & 1.6 & 2.4 & 3.0 \\
\hline Co & $\operatorname{ag} / 1$ & $<0.005$ & 50.004 & 60.004 & 80.004 & 0.005 & $<0.004$ & 60.004 & 60.004 & 80.004 & 50.004 \\
\hline$[r$ & $v g / 1$ & 60,015 & 60.02 & 60.02 & (i). 02 & $<0.02$ & 60.02 & 60.02 & 10.02 & $<0.02$ & $\langle 0,0\rangle$ \\
\hline Cu & moil & 60.0105 & $<0.604$ & 60.604 & 60.004 & 0.004 & 10.004 & 50.004 & $<0.004$ & 10.004 & 10.0014 \\
\hline $\mathrm{Fe}_{\mathrm{E}}$ & mạjl & 0.01 & 0.041 & 0.005 & $<0.005$ & 00.005 & 60.005 & 0.006 & 60.005 & $\angle 0.005$ & (0.0.005 \\
\hline 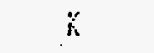 & $\operatorname{dg} / 1$ & 7.0 & 6.3 & 0.5 & $7 . \hat{7}$ & 9.9 & $\$ .2$ & 3.2 & 20.0 & 13.0 & 11.0 \\
\hline$\because g$ & $g / 1$ & 14.4 & 13.10 & 5.34 & 6.63 & 1.00 & $0.3 \hat{2}$ & 9.70 & 60.06 & 6.75 & 2.86 \\
\hline $\mathrm{Mn}$ & ag! & 0.01 & 10.002 & 60.002 & $<0.002$ & $<0.002$ & 60.602 & $<0.002$ & $i \hat{v} .002$ & 60.602 & 60. 602 \\
\hline Ha & $\$ q i !$ & 25 & 46 & 168 & 31 & 149 & 125 & 80 & 383 & 183 & 143 \\
\hline$F$ & ggi! & 0.1 & 0.37 & 0.1 & 0.14 & 0.21 & $<0.1$ & 0.15 & $<0.1$ & $10 . i$ & 0.1 \\
\hline$j \equiv 84$ & $\| \stackrel{̣}{i} !$ & $\{0.3\}$ & 1.13 & 60.31 & 0.43 & 0.64 & $\langle\hat{i} .31$ & 0.46 & 0.31 & 0.31 & $\langle i, 3 i$ \\
\hline Ft & ag̣ & 0.05 & 60.06 & 0.06 & 30.06 & Co.vo & 50.06 & 00.06 & 00.06 & $\{0.06$ & 60.06 \\
\hline $3 i$ & ing $j$ & 15 & 13.5 & 11.7 & 11.4 & 10.6 & 8.6 & 12.4 & 10.3 & 8.7 & 3. 6 \\
\hline Sr & $a g / 1$ & 0.25 & 0.227 & 0.127 & 0.073 & 0.079 & 0.049 & 0.106 & 0.056 & 0.050 & 0.030 \\
\hline in & all & 00.01 & 60.02 & 0.02 & $<0.02$ & $\langle 0 . \hat{v} 2$ & (iv. 02 & 10.02 & 0.02 & 60.02 & 60.02 \\
\hline ir & $8 g / 1$ & $\therefore 0.008$ & 0.0 & 0.0 & 60.008 & 0.0 & $<0.008$ & 0.0 & 0.0 & 40.000 & $(0.000$ \\
\hline$F-$ & $\operatorname{sq} / !$ & 0.5 & 0.8 & 1.2 & 1.2 & 3.6 & 3.5 & 1.7 & 4.2 & 1.8 & 1.3 \\
\hline C:- & nğ!! & 22 & 36 & 37 & 32 & 24 & 26 & 24 & 23 & 23 & 25 \\
\hline $\mathrm{M} \mathrm{Ti}_{2}-$ & agil & 0.03 & 0.4 & 60.3 & 60.3 & $<0.3$ & 0.3 & $\{0.3$ & 60.3 & $<0.3$ & 0.3 \\
\hline 40̄̄3- & $\lg / !$ & $0 . !$ & 1.4 & 2.1 & 1.2 & 2.0 & 1.4 & 0.7 & 6.1 & 2.3 & 1.3 \\
\hline $394--$ & sgil & B6 & 81 & 93 & $B 4$ & 89 & 84 & 75 & 107 & 82 & 82 \\
\hline hiojo- & แบ่ & 171 & 164.7 & 280.4 & 192.4 & 0.0 & 234.2 & 73.2 & 0.0 & 0.0 & 50.4 \\
\hline $\mathrm{CO} 3--$ & aqu! & 2.1 & 3.6 & 10.8 & 121.4 & 96.0 & 14.4 & 52.8 & 100.0 & 84. 0 & 126.0 \\
\hline OH- & $\operatorname{tgn} f$ & 0 & 0.0 & 0.0 & 0.0 & 24.5 & 0.0 & 0.0 & 199.0 & 32.3 & $\dot{y} . \dot{v}$ \\
\hline $\bar{E}$ & $43 / 1$ & 0.06 & 0.101 & 0.122 & 0.127 & 0.111 & 0.390 & 0.173 & 0.076 & 0.070 & 0.072 \\
\hline$\therefore \equiv 4360.3$ & ดQ $/ 1$ & 0.39 & 0.58 & 1.04 & 0.73 & $0.6 j$ & 0.51 & 0.39 & 1).43 & 0.43 & 0.41 \\
\hline Itî̀ & \#प̣ & i & 1.25 & 1.55 & 0.77 & 1.40 & 1.47 & 1.29 & 1.400 & 1.25 & 1.40 \\
\hline FO:-- & mýl & $(1.0)$ & $\langle 5.0$ & (5.0 & (5. & i5. & 15.0 & $<5.0$ & 15.0 & 15.0 & 6.0 \\
\hline Cótions & $x \in Q / 1$ & 5. . 20 & 5.73 & $6.6 j$ & 5.21 & $7.3 !$ & 2.72 & 3.38 & 8.57 & 8.47 & b. \\
\hline miritorls & ieq 11 & 5.54 & 5.75 & $6.3 \dot{0}$ & 10.10 & 7.54 & 6.51 & 5.46 & 17.94 & 8.65 & $i .71$ \\
\hline TOT. HLLL.* & 和/1 & 92.16 & 85 & 154 & $2 ! 6$ & 137 & 130 & B9 & 434 & 186 & 151 \\
\hline II & $\operatorname{mil}$ & 34.1 & 23.98 & 25.62 & 17.33 & 20.51 & 20.27 & 21.97 & $20.10 \overline{0}$ & 17.85 & $1 i .10$ \\
\hline
\end{tabular}

(\#) Data not available.
(\#) Total Alkalinity as c03--. 
TABLE A.1. contd

\begin{tabular}{|c|c|c|c|c|c|c|c|c|c|c|}
\hline SAMPLE & $\cdots$ & 10 & 11 & 12 & 13 & 14 & 15 & 16 & 17 & 18 \\
\hline TIME & days & 41 & 50 & 63 & 92 & 122 & 134 & 182 & 302 & 410 \\
\hline $\mathrm{pH}$ & & 10.50 & 10.65 & 10.66 & 11.29 & 11.05 & 10.03 & 11.12 & 11.74 & 11.6ó \\
\hline Eh & $\Delta V$ & 132 & 205 & 168 & 201 & 214 & 327 & 228 & 235 & 275 \\
\hline Al & $g / 1$ & 0.03 & $<0.03$ & $<0.03$ & $<0.03$ & $<0.03$ & --- & $\langle 0.03$ & 60.03 & $\{0.03$ \\
\hline Ba & $g / 1$ & $<0.002$ & 0.002 & $<0.002$ & $\$ 0.002$ & $<0.002$ & ---- & $<0.002$ & 0.002 & $\{0.002$ \\
\hline $\mathrm{Ca}$ & $9 / 1$ & 1.4 & 2.7 & 1.6 & 1.1 & 1.0 & --.- & 1.0 & 0.a & 0.7 \\
\hline Co & $g y / 1$ & 80.004 & $<0.004$ & $<0.004$ & $\$ 0.004$ & $<0.004$ & -... & $<0.004$ & $<0.004$ & 60.004 \\
\hline or & $9 / 1$ & $<0.02$ & $<0.02$ & $<0.02$ & $<0.02$ & $<0.02$ & --- & $\langle 0.02$ & $<0.02$ & $\therefore(0.02$ \\
\hline $\mathrm{Cu}$ & $g / l$ & 60.004 & $<0.004$ & $\langle 0.004$ & $<0.004$ & $<0.004$ & --. & $<0.004$ & $\{0.004$ & 60.004 \\
\hline $\mathrm{Fe}$ & $1 g / 1$ & $<0.005$ & 0.006 & 0.136 & 0.008 & $<0.005$ & -.- & $<0.005$ & 10.005 & 60.015 \\
\hline$k$ & $a / I$ & 14.0 & 11.0 & i1. 0 & 13.0 & 11.8 & -..- & $: 2.0$ & 13. & 13.6 \\
\hline Mg & $9 g / 1$ & 0.46 & 5.53 & 2.57 & 0.13 & 0.31 & $-\cdots$ & 0.28 & 60.06 & 0.15 \\
\hline Hn & $9 / 1$ & $<0.002$ & 0.002 & $<0.002$ & $<0.002$ & $<0.002$ & --. & 0.002 & $<0.002$ & $<0.062$ \\
\hline $\mathrm{Ha}$ & $9 / 1$ & 210 & 118 & 138 & 200 & 168 & --- & 169 & 241 & 205 \\
\hline $\mathrm{F}$ & $\log / 1$ & 10.1 & $<0.1$ & $<0.1$ & $<0.1$ & $<0.1$ & -..- & 0.1 & (6. 1 & 0.1 \\
\hline d5 $\mathrm{PO}$ & $4 / 1$ & $\{0.3\}$ & $\langle 0.31$ & $<0.31$ & $<0.31$ & $\langle 0.3\rangle$ & $-\cdots$ & 60.31 & $<0.31$ & 4.31 \\
\hline$P b$ & $a g / l$ & $<0.06$ & 80.06 & $<0.06$ & $<0.06$ & $<0.06$ & ---- & 0.06 & $<0.06$ & 0.16 \\
\hline $\mathrm{Si}$ & $a g / 1$ & 7.6 & 8.6 & 6.2 & 5.1 & 4.3 & -.-- & 4.8 & 5.2 & 3.4 \\
\hline $5 r$ & $g / 1$ & 0.011 & 0.014 & 0.006 & 0.006 & 0.005 & ---. & 0.005 & $0.02 !$ & 0.000 \\
\hline in & ogil & $<0.02$ & 0.022 & $<0.02$ & $<0.02$ & $<0.02$ & $-\cdots$ & 80.02 & 0.033 & $\langle i, i j i$ \\
\hline Ir & $g / 1$ & $<0.008$ & $<0.008$ & $\$ 0.008$ & $<0.008$ & 00.008 & ---- & $\{0,008$ & 0.008 & <0.005 \\
\hline F- & $19 / 1$ & 1.6 & 0.6 & 0.7 & 0.7 & 2.3 & $\cdots$ & 0.7 & 1.0 & i. 7 \\
\hline [1- & $g / 1$ & 26 & 55 & 24 & $2 B$ & 52 & -- & 22 & 23 & 23 \\
\hline HC2- & $9 g / 1$ & $<0.3$ & 0.2 & $<0.2$ & 0.5 & $\langle 0.2$ & -- & 60.3 & 10.1 & 0.3 \\
\hline H03- & sg/1 & 6.9 & 0.8 & 0.8 & 2.0 & 0. 8 & -- & 1.0 & $5 . j$ & 2.4 \\
\hline $504--$ & $9 q / 1$ & 72 & 84 & 81 & 83 & 60 & $\cdots$ & 71 & 75 & 70 \\
\hline HCO3- & $\Delta / 1$ & 128.1 & 155.7 & 0.0 & 0.0 & 244.0 & 51.2 & 0.0 & 0.00 & 0.04 \\
\hline COS3-- & $9 \varphi / 1$ & 176.4 & 50.4 & 100.8 & 102.0 & 60.0 & $6 j$ & $12 b$ & 84.00 & 196.40 \\
\hline OH- & og/1 & 0.0 & 0.0 & 24.9 & 74.9 & 0.0 & 0.0 & 66.3 & 76.60 & 46.92 \\
\hline B & $g / 1$ & 0.097 & 0.066 & 0.066 & 0.080 & 0.071 & $\cdots$ & 0.062 & 0.601 & 0.070 \\
\hline as 493803 & sọil & 0.55 & 0.38 & 0.39 & 0.46 & $0.4 i$ & --- & 0.46 & 0.50 & 6.40 \\
\hline$i 0 C$ & gii & $\{.37$ & 1.46 & 2.76 & 1.35 & 0.80 & $\cdots$ & $\dot{v} .9$ & --- & $2 . \hat{v}$ \\
\hline fOH- & $00 / 1$ & $\$ 5.0$ & 11.0 & 22.0 & $<3.0$ & $(2,0)$ & $\cdots$ & $<5$ & $: 5$ & $\{5, j\}$ \\
\hline CATIOHS & apq/1 & 9.60 & 6.00 & 6.57 & 9.10 & 7.68 & $-\cdots$ & 7.73 & 10.87 & 9.29 \\
\hline ANIONS & MEq/1 & 10.56 & 6.39 & 7.31 & 10.46 & 8.92 & $\cdots$ & 10.26 & 10.96 & 5.32 \\
\hline TOT.rilk. & $\$ q / 1$ & 239 & 126 & 145 & 233 & 85 & 88 & 243 & 2550 & 173 \\
\hline If & $\log / 1$ & 19.71 & 19.14 & 49.11 & 26.16 & 19.20 & --- & 21.5 & 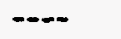 & $75 . \overline{4}$ \\
\hline
\end{tabular}

(--) Data not available.

(\#) Total Alkalinity as C03--. 
TABLE A.2. ANS 16.1 Leach Test on whole Grout with Groundwater (ANS3-4)

\begin{tabular}{|c|c|c|c|c|c|c|c|c|c|c|c|}
\hline & & WELL & & & & & & & & & \\
\hline SAKPLE & ---- & HATER & 1 & 2 & 3 & 4 & 5 & 6 & 7 & 8 & 9 \\
\hline TIME & days & -- & 0.0003 & 0.0833 & 0.292 & $\cdot 1$ & 2 & 3 & 13 & 20 & 27 \\
\hline $\mathrm{pH}$ & & 8.1 & 8.66 & 11.00 & 10.61 & 11.32 & 11.28 & 10.55 & 12.15 & 11.74 & 11.31 \\
\hline Eh & av & 295 & 258 & 162 & 183 & 320 & 306 & 321 & 241 & 277 & 174 \\
\hline Al & $g / 1$ & 10.1 & 0.13 & 0.08 & 0.05 & 0.63 & 0.10 & 0.09 & 1.71 & 0.04 & 0.04 \\
\hline $\mathrm{Ba}$ & $g / 1$ & 0.06 & 0.044 & 0.010 & 0.006 & 0.009 & 0.013 & 0.015 & 0.005 & 0.003 & 0.093 \\
\hline $\mathrm{C}_{\mathrm{d}}$ & $\lg i l$ & 56 & 51.9 & 13.7 & 7.8 & 7.5 & 12.8 & 13.5 & 14.2 & 2.0 & 2.6 \\
\hline Cd & $9 / 1$ & $<0.005$ & 0.005 & $\$ 0.004$ & $<0.004$ & $<0.004$ & $<0.004$ & $<0.004$ & $<0.004$ & $<0.005$ & $<0.005$ \\
\hline$\Sigma r$ & $\lg f 1$ & 0.015 & 0.03 & $<0.02$ & $<0.02$ & $<0.02$ & $<0.02$ & 10.02 & 10.02 & $<0.02$ & 60.02 \\
\hline $\mathrm{Cu}$ & $9 / 1$ & $<0.005$ & 10.004 & 60.004 & 10.004 & $<0.004$ & $<0.004$ & $<0.004$ & 10.004 & $<0.004$ & $<0.004$ \\
\hline $\mathrm{Fe}$ & $\lg h$ & 10.01 & 0.013 & 0.005 & 0.005 & 0.005 & $<0.005$ & 0.010 & $<0.005$ & $<0.005$ & 0.007 \\
\hline$k$ & $\lg i$ & 7.8 & 7.4 & 11.0 & 10.0 & 13.0 & 12.0 & 10.0 & 22.0 & 14.0 & 12.0 \\
\hline $\mathrm{Ng}$ & $90 / 1$ & 14.4 & 13.20 & 2.48 & 4.70 & 0.29 & 1.69 & 8.50 & 30.06 & 0.20 & 1.60 \\
\hline Mn & $\lg / 1$ & $\div 0.01$ & 0.003 & 60.002 & $<0.002$ & $\{0.002$ & $<0.002$ & 0.002 & $<0.002$ & $<0.002$ & $(0.002$ \\
\hline $\mathrm{Na}$ & $\operatorname{ag} / 1$ & 25 & 36 & 152 & 108 & 173 & 144 & . $\quad 86$ & 418 & 206 & 161 \\
\hline$f$ & $\mathrm{ag} / 1$ & 0.1 & 0.31 & 60.1 & 0.13 & 0.12 & 10.1 & 0.11 & $<0.1$ & $\$ 0.1$ & 60.1 \\
\hline$a=804$ & $\operatorname{mg} / 1$ & $\{0.31$ & 0.95 & 60.31 & 0.39 & 0.37 & $\langle 0.3\rangle$ & 0.34 & $\langle 0.31$ & 60.31 & $\langle 0.31$ \\
\hline Fb & $9 / 1$ & 10.05 & $\therefore 0.06$ & 10.06 & $(0.06$ & $<0.06^{\circ}$ & $<0.06$ & 10.06 & 80.06 & 60.06 & $<0.06$ \\
\hline $5 \mathrm{i}$ & $\lg / 1$ & 15 & 13.8 & 11.3 & $11: 0$ & 10.0 & 10.5 & 11.8 & 10.3 & 9.3 & 8.3 \\
\hline$\hat{\mathrm{j}} \mathrm{r}$ & nọfi & 0.25 & 0.224 & 0.083 & 0.060 & 0.070 & 0.092 & 0.099 & 0.051 & 0.029 & 0.017 \\
\hline in & ngil & 10.01 & $\{0.02$ & $\{0.02$ & $<0.02$ & $<0.02$ & $<0.02$ & $<0.02$ & 10.02 & 10.02 & 80.02 \\
\hline$i r$ & $\lg / 1$ & 60.008 & 60.008 & 0.012 & 60.008 & $\$ 0.008$ & $<0.008$ & 0.017 & 10.008 & 10.009 & 10.008 \\
\hline$F-$ & $9 i 1$ & $<0.5$ & 0.6 & 1.7 & 1.5 & 3.8 & 3.7 & 2.0 & 4.7 & 2.9 & 1.5 \\
\hline $\mathrm{Cl}-$ & sqil & 22 & 29 & 34 & 30 & 25 & 26 & 24 & 24 & 36 & 24 \\
\hline ing 2- & $00 / 1$ & 60.03 & 10.3 & 00.3 & $<0.3$ & 0.3 & $<0.3$ & 20.3 & 10.3 & $\langle 0.3$ & 60.3 \\
\hline NO3- & $\log / 1$ & 0.1 & 0.6 & 2.7 & 1.7 & 2.2 & 1.7 & 0.9 & 7.5 & 2.6 & 1.7 \\
\hline S04-- & $\$ 0 /]$ & 86 & 79 & 102 & 91 & 95 & 86 & 82 & 107 & 84 & 82 \\
\hline HCOJ- & $19 / 1$ & 171 & -- & 448.0 & 294.6 & 269.0 & 166.5 & 166.5 & $0 . \hat{0}$ & 320.3 & 25.6 \\
\hline Coj-- & $\lg / 1$ & 2.1 & --- & 76 & 176 & 12.6 & 50 & 25 & 202 & 70 & 104 \\
\hline $\mathrm{OH}-$ & $\operatorname{tg} i]$ & 0 & -- & 0.0 & 0.0 & 0.0 & 0.0 & 0.0 & 671.2 & 0.0 & 0.0 \\
\hline E & $\log i$ & 0.06 & 0.073 & 0.250 & 0.177 & 0.217 & 0.150 & 0.097 & 0.207 & 0.098 & 0.001 \\
\hline as H3803 & agil & 0.34 & 0.53 & 1.43 & 1.01 & 1.24 & 0.86 & 0.55 & 1.18 & 0.56 & 0.96 \\
\hline$T O C$ & $\operatorname{ag} / 1$ & $!$ & $-\infty$ & $\cdots$ & $\cdots$ & $--\infty$ & $-\infty$ & -- & $-\infty$ & $\cdots$ & $\cdots$ \\
\hline F'4-- & $\operatorname{mol} 1$ & 10.0 & $<5.0$ & 65.0 & 65.6 & $<5.0$ & $\langle 5.0$ & $(5.0)$ & $\langle 5.0$ & \$5. 0 & 65.0 \\
\hline CATIOYS & neq il & 5.23 & 5.44 & 7.78 & 5.72 & 8.25 & 7.35 & 5.36 & 19.44 & 9.13 & 7.56 \\
\hline AHIONS & seq/1 & 5.54 & -- & 13.25 & 13.71 & 7.72 & 9.01 & 7.07 & 99.64 & 10.89 & 3.52 \\
\hline TOT. ALLK. * & 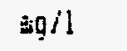 & 72.16 & -- & 296 & 321 & 145 & 183 & 132 & 1386 & 233 & 176 \\
\hline IC & $80 ! i$ & 34.1 & $-\cdots$ & --- & $-\cdots$ & $--\infty$ & -- & $-\infty$ & - & $-\infty$ & \\
\hline
\end{tabular}

\footnotetext{
(--) Data not available.

(\#) Total Alkalinity as C03--.
} 
TABLE A.2. contd

\begin{tabular}{|c|c|c|c|c|c|c|c|c|c|}
\hline SAYTPLE & - & 10 & 11 & 12 & 13 & 14 & 15 & 16 & 17 \\
\hline TIME & days & 41 & 50 & 63 & 92 & 122 & $\$ 34$ & 182 & 302 \\
\hline $\mathrm{pH}$ & & 11.69 & 10.96 & 10.69 & 11.32 & 11.06 & 9.76 & 10.93 & 11.6 \\
\hline E⿸t & ov & 175 & 193 & 165 & 186 & 209 & 214 & 267 & $3 \mathbf{j}$ \\
\hline Al & $\operatorname{ag} / 1$ & $\quad 0.05$ & 0.04 & $<0.03$ & 0.03 & $<0.03$ & --- & $<0.03$ & 0.0 \\
\hline$B a$ & gil & $<0.002$ & 0.002 & $<0.002$ & $<0.002$ & $<0.002$ & $\cdots$ & 80.002 & $<0.002$ \\
\hline$C_{2}$ & $\operatorname{sg} / 1$ & 1.2 & 1.9 & 1.3 & 1.0 & 1.2 & --. & 0.9 & 0.8 \\
\hline Cd & $g / 1$ & $<0.005$ & 0.006 & $<0.004$ & $<0.004$ & 60.004 & --- & $<0.004$ & 00.004 \\
\hline $\mathrm{Cr}$ & $g / 1$ & $<0.02$ & $<0.02$ & $<0.02$ & $<0.02$ & $<0.02$ & $\cdots$ & $<0.02$ & 00.02 \\
\hline $\mathrm{Cu}$ & $\log / 1$ & $<0.004$ & $<0.004$ & $<0.004$ & 0.005 & $<0.004$ & --- & $\langle 0.004$ & $<0.004$ \\
\hline $\mathrm{Fe}$ & $i g / 1$ & $<0.005$ & 0.006 & $<0.005$ & 0.008 & 60.005 & --- & 60.005 & 0.005 \\
\hline K & $\log / 1$ & 15.0 & 12.0 & 10.0 & 14.0 & 12.0 & -.-- & 12.0 & 12.2 \\
\hline $\mathrm{Mg}$ & 11 & 0.1 & $4.07:$ & 1.91 & 0.14 & 0.34 & --- & 0.34 & $<0.06$ \\
\hline $\mathrm{Mn}$ & $g / 1$ & $<0.002$ & 0.002 & $<0.002$ & $<0.002$ & $<0.002$ & --- & 16.002 & $<0.002$ \\
\hline $\mathrm{Ha}$ & $40 / 1$ & 229 & 126 & 140 & 200 & 169 & -- & 161 & 217 \\
\hline$F$ & & 60.1 & $<0.1$ & $<0.1$ & $<0.1$ & $<0.1$ & $\cdots$ & $\therefore 0.1$ & $<0.1$ \\
\hline ds PO4 & & $\langle 0.3\rangle$ & $<0.31$ & 00.31 & 0.31 & $<0.31$ & - & $\langle 0.3 i$ & $<0.31$ \\
\hline $\mathrm{Fb}$ & $g / 1$ & $\langle 0.06$ & 0.06 & $<0.06$ & 10.06 & 10.06 & -..- & 10.06 & 00.06 \\
\hline $3 i$ & & 7.3 & 7.6 & 11.5 & 4.3 & 4.6 & $\cdots$ & 4.7 & 4.7 \\
\hline$S r$ & II & 0.008 & $0.00 B$ & 0.004 & 0.005 & 0.005 & $\ldots$ & 0.004 & 0.014 \\
\hline In & & $<0.02$ & $<0.02$ & $<0.02$ & $<0.02$ & $<0.02$ & $-\cdots$ & $<0.02$ & 0.054 \\
\hline Ir & & $<0.008$ & $<0.008$ & 0.024 & $\angle 0,00 B$ & 10.008 & --- & $<0.008$ & $<0.008$ \\
\hline F- & 11 & 1.8 & 0.6 & 0.8 & 0.7 & 2.6 & -..- & 0.7 & 0.9 \\
\hline $\mathrm{Cl}-$ & & 26 & 36 & 31 & 31 & 51 & $\ldots$ & 35 & 22 \\
\hline $\mathrm{HO} \hat{z}^{-}$ & & $<0.3$ & $<0.2$ & 60.2 & $<0.5$ & $i 0.2$ & $\ldots$ & 60.3 & $<0.3$ \\
\hline KOJ - & & 1.9 & 0.8 & 1.1 & 2.3 & 0.8 & -... & 1.6 & 5.0 \\
\hline 504-- & & 75 & 87 & 78 & 32 & 60 & $-\ldots$ & 68 & 71 \\
\hline $\mathrm{HCOS}-$ & & 0.0 & 2005.0 & 0.0 & 0.0 & 0.0 & 38.43 & 0.0 & 0.00 \\
\hline [03-- & & 265 & 25 & 110 & 113 & 139 & 63.00 & 198 & 92.30 \\
\hline OH- & & 0.0 & 0.0 & 28.6 & 71.4 & 28.6 & 0.00 & 25.5 & 83.61 \\
\hline 5 & & 0.100 & 0.076 & 0.065 & 0.080 & 0.065 & --- & 0.680 & 0.096 \\
\hline as $\mathrm{HBBOJ}$ & & 0.57 & 0.43 & 0.37 & 0.46 & 0.37 & -..- & 0.45 & 0.55 \\
\hline 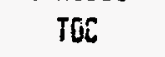 & & -- & $\cdots$ & --- & $-\cdots$ & 0.8 & -- &.-- & -.-- \\
\hline PO4--. & & $<0.5$ & $<1.0$ & $<2.0$ & 2.0 & 22.0 & --- & $<0.5$ & 0.5 \\
\hline CATIOKS & & 10.41 & 6.22 & 6.57 & 9.12 & 7.74 & 0.00 & 7.30 & 9.79 \\
\hline AHIOKS & neq/I & 11.26 & 5.88 & 8.09 & 10.70 & 9.21 & 2.73 & 10.57 & 30.85 \\
\hline TOT.ÄLK.ะ & $\log$ & 265 & 126 & iót & 237 & 189 & 82 & $24 j$ & 253 \\
\hline IC & igil & - & -- & . & -..- & 19.1 & 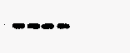 & --- & -..- \\
\hline
\end{tabular}

\footnotetext{
(--) Data not available.

(\#) Total Alkalinity as CO3--.
} 
TABLE A.3. ANS 16.1 Leach Test on whole Grout with Groundwater (ANS4-8)

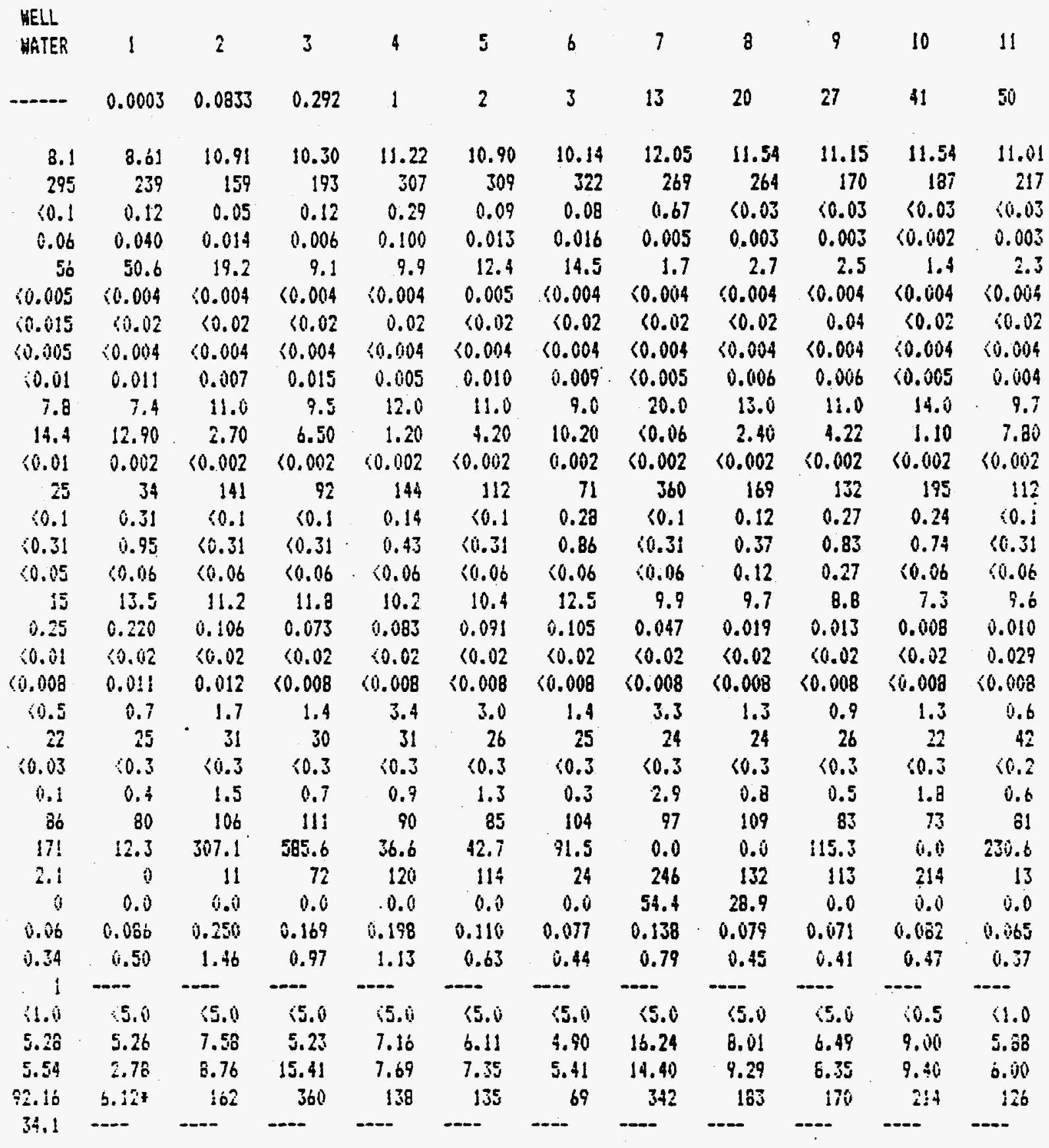

(--) Data not available.

(\#) Total Alkalinity as CO3--. 
TABLE A.3. contd

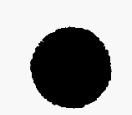

\begin{tabular}{|c|c|c|c|c|c|}
\hline 12 & 13 & 14 & 15 & 16 & 17 \\
\hline 64 & 92 & 122 & 134 & 182 & 302 \\
\hline 10.54 & 10.98 & 11.10 & 9.86 & 10.65 & 11.60 \\
\hline 172 & 216 & 202 & 197 & 266 & 336 \\
\hline 10.03 & 0.03 & $<0.03$ & 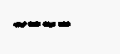 & $<0.03$ & $<0.03$ \\
\hline$<0.002$ & $<0.002$ & $<0.002$ & -- & $<0.002$ & $<0.002$ \\
\hline 1.3 & 1.1 & 0.9 & --.- & 0.9 & 0.9 \\
\hline 0.004 & $<0.004$ & $<0.004$ & $-\cdots$ & $\langle 0.004$ & $<0.004$ \\
\hline$<0.02$ & $<0.02$ & $<0.02$ & $-\cdots$ & 10.02 & 10.02 \\
\hline 40.004 & 0.004 & $<0.004$ & 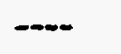 & $<0.004$ & $<0.004$ \\
\hline$<0.005$ & 0.008 & 0.024 & -- & $<0.005$ & $(0.005$ \\
\hline 9.6 & 14.0 & 11.5 & $\cdots$ & 12.0 & 13.0 \\
\hline 3.67 & 0.24 & 0.82 & $\cdots$ & 0.71 & 00.06 \\
\hline$<0.002$ & 10.002 & $\{0.002$ & - & 10.002 & $<0.002$ \\
\hline 124 & 190 & $|5|$ & $\cdots$ & 152 & 218 \\
\hline 0.1 & $<0.1$ & $<0.1$ & $\cdots$ & 0.10 & $<0.1$ \\
\hline 60.31 & $\langle 0.3\rangle$ & 0.31 & $\cdots$ & 0.31 & $<0.3$ \\
\hline$<0.06$ & 60.06 & $<0.06$ & - & $<0.06$ & $<0.06$ \\
\hline 3.4 & 5.2 & 4.4 & -- & 5.0 & 5.0 \\
\hline 0.004 & 0.005 & 0.004 & $-\cdots$ & 0.004 & 0.012 \\
\hline 10.02 & 00.02 & $(0.02$ & $\cdots$ & 50.02 & 0.093 \\
\hline 60.008 & $<0.008$ & $<0.008$ & $\cdots$ & $<0.008$ & $<0.008$ \\
\hline 0.6 & 0.6 & 2.2 & $\cdots$ & 0.6 & $\begin{array}{ll}\cdot & 0.7\end{array}$ \\
\hline 23 & 32 & 32 & $\rightarrow$ & 21 & 23 \\
\hline 60.2 & $<0.5$ & $<0.2$ & -- & 10.3 & $<0.1$ \\
\hline 1.7 & 1.8 & 0.6 & $m$ & 1.0 & 4.0 \\
\hline 85 & 84 & 58 & $-\cdots$ & 69 & 76 \\
\hline 0.0 & 0.0 & 0.0 & 89.7 & 0.0 & 0.00 \\
\hline 134 & 113 & 126 & 38 & 108 & 93.00 \\
\hline 17.8 & 53.6 & 17.9 & 0.0 & 66.3 & 87.66 \\
\hline 0.065 & 0.080 & 0.068 & $\cdots$ & 0.079 & 0.090 \\
\hline$\dot{v} .57$ & 0.46 & 0.39 & $\cdots$ & 0.45 & 0.51 \\
\hline$\cdots$ & - & 0.7 & $\cdots$ & - & $-\cdots$ \\
\hline 2.0 & \$3.0 & 2.0 & $\langle 5.0$ & S. & $\langle 5.0$ \\
\hline 6.00 & B. 70 & 6.97 & 0.00 & 7.02 & 9.86 \\
\hline 7.36 & 9.75 & 8.12 & 2.73 & 9.74 & 10.61 \\
\hline 145 & 208 & 158 & 82 & 225 & 248 \\
\hline$\cdots$ & & 19.1 & --- & $\cdots$ & $-\infty$ \\
\hline
\end{tabular}

(--) Data not available.

(\#) Total Alkalinity as C03--. 
TABLE A.4. ANS 16.1 Leach Test on Whole Grout with Groundwater (ANS5-5)

\begin{tabular}{|c|c|c|c|c|c|c|c|c|c|c|c|}
\hline & & HELL & & & & & & & & & \\
\hline SAKPLE & ---.- & WATER & 1 & 2 & 3 & 4 & 5 & 6 & 7 & 8 & 9 \\
\hline TIME & days & -..-- & 0.0003 & 0.0833 & 0.292 & 1 & 2 & 3 & 13 & 20 & 27 \\
\hline $\mathrm{pH}$ & & 8.1 & 3.58 & 10.98 & 10.47 & 11.05 & 11.21 & 10.59 & 12.13 & 11.73 & 11.35 \\
\hline Eh & av & 295 & 243 & $17 !$ & 188 & 248 & $25 !$ & 256 & 313 & 272 & 185 \\
\hline Al & $1 g / 1$ & 0.1 & 0.09 & $\{0.03$ & $<0.03$ & 0.19 & 0.10 & 0.08 & 2.11 & 0.09 & 0.04 \\
\hline $\mathrm{Bz}$ & ng/1 & 0.06 & 0.040 & 60.002 & 0.004 & 0.006 & 0.014 & 0.013 & 0.005 & 0.005 & 0.004 \\
\hline$\lceil\dot{a}$ & ogil & 56 & 50.6 & 1.6 & 7.8 & 8.0 & 12.6 & 13.1 & 1.5 & 2.3 & 2.7 \\
\hline$C d$ & $\operatorname{ag} / 1$ & 10.005 & $<0.004$ & 0.005 & $<0.004$ & $<0.004$ & $<0.004$ & 0.010 & $<0.004$ & $<0.004$ & 0.005 \\
\hline Cr & igil & 60.015 & 10.02 & 60.02 & $<0.02$ & 0.08 & 50.02 & $<0.02$ & $\langle 0.02$ & $<0.02$ & 80.02 \\
\hline $\mathrm{Cu}$ & $a g / 1$ & $<0.005$ & 0.004 & 80.004 & $\leqslant 0.004$ & 60.004 & $<0.004$ & $<0.004$ & $<0.004$ & $<0.004$ & 60.004 \\
\hline $\mathrm{FE}$ & $\operatorname{sg} / 1$ & $<0.01$ & 10.005 & 0.014 & $<0.005$ & $<0.005$ & 0.010 & 0.009 & 10.005 & 0.010 & 0.088 \\
\hline$K$ & $\operatorname{sg} / 1$ & 7.8 & 6.7 & 9.8 & 9.1 & 12.0 & 11.0 & 10.0 & 22.0 & 14.0 & 12.0 \\
\hline $\mathrm{Hg}_{9}$ & $\mathrm{ag} / \mathrm{l}$ & 14.4 & 13.00 & 2.00 & 5.09 & 0.25 & 2.59 & 8.70 & $\$ 0.06$ & 0.30 & 1.50 \\
\hline Mn & $g ! 1$ & $<0.01$ & 0.004 & 10.002 & $<0.002$ & $<0.002$ & 0.002 & 0.002 & $<0.602$ & 60.002 & 0.002 \\
\hline $\mathrm{Na}$ & $\operatorname{sgil}$ & 25 & 38 & 132 & 94 & 161 & 142 & 90 & 430 & 200 & 157 \\
\hline$F$ & $g / 1$ & 10.1 & 0.11 & 0.1 & 0.21 & $<0.1$ & 0.20 & 0.20 & 0.14 & 0.15 & $\langle\bar{u} .1$ \\
\hline as $\mathrm{POA}$ & agil & 60.31 & 0.34 & $\{0.3\}$ & 0.60 & $<0.31$ & 0.61 & 0.61 & 0.43 & 0.46 & $\{0.31$ \\
\hline $\mathrm{Pb}$ & $0 / 1$ & 10.05 & 00.06 & 10.06 & $<0.06$ & $<0.06$ & 10.06 & 0.10 & $<0.06$ & 0.09 & 0.11 \\
\hline $\mathrm{si}$ & $\$ 0 i l$ & 15 & 13.5 & 10.2 & 10.8 & 10.1 & 10.7 & 12.3 & 11.1 & 3.7 & 3.0 \\
\hline $5 r$ & Ing/1 & 0.25 & 0.222 & 0.038 & 0.058 & 0.072 & 0.091 & 0.100 & 0.058 & 0.052 & 0.043 \\
\hline in & $39 / 1$ & $<0.0 \mathrm{i}$ & 60.02 & $<0.02$ & 60.02 & $<0.02$ & 60.02 & $<0.02$ & $<0.02$ & $<0.02$ & $30.0) 2$ \\
\hline Ir & $\log / 1$ & 60.008 & 10.008 & $\{0.008$ & 60.008 & 80.008 & $<0.008$ & $<0.008$ & $\angle 0.00 \mathrm{~B}$ & $<0.068$ & 60.003 \\
\hline F- & $\operatorname{ag} / 1$ & $\$ 0.5$ & 0.7 & 1.5 & 1.4 & 3.7 & 3.9 & 2.2 & 5.5 & 2.2 & 1.5 \\
\hline $\mathrm{Cl}-$ & $9 q / 1$ & 22 & 27 & 34 & 29 & 31 & 27 & 25 & 26 & 24 & 32 \\
\hline Ho?- & aqi & 10.03 & 0.3 & 60.3 & 00.3 & $: 0.3$ & 10.3 & 10.3 & $<0.3$ & 60.3 & 10.3 \\
\hline HOS- & mọ/l & $\hat{0.1}$ & 0.3 & 1.1 & 0.7 & 1.1 & 0.8 & 0.5 & 3.0 & 1.3 & 1.2 \\
\hline $504--$ & min 1 & 36 & 80 & 102 & 90 & 94 & 90 & $8 \bar{j}$ & 106 & 85 & 85 \\
\hline HCO3- & ag $i$ & 171 & 128.1 & 0.0 & 0.0 & 666.3 & 0.0 & 76.9 & 0.0 & 0.0 & 0.0 \\
\hline COS3-- & $29 i$ & 2.1 & 13 & 294 & 193 & 29 & 126 & 76 & 176 & $\$ 01$ & 176 \\
\hline $\mathrm{OH}-$ & $09 i$ & 0 & 0.0 & 41.7 & 27.4 & 0.0 & 21.4 & 0.0 & 288.4 & 46.4 & 0.0 \\
\hline B & agil & 0.06 & 0.107 & 0.227 & 0.140 & 0.192 & 0.136 & 0.104 & 0.225 & 0.093 & 0.083 \\
\hline as $H 360 j$ & anil & 0.34 & 0.al & 1.30 & 0.80 & 1.09 & 0.78 & 0.59 & 1.29 & 0.53 & $0.4 i$ \\
\hline $\mathrm{iOC}$ & $\$ g i !$ & 1 & ---- & $\cdots$ & --- & --- & --- & --- & $-\cdots$ & $\cdots$ & -- \\
\hline FUt--- & noil & $\therefore 1.0$ & 0.0 & 15.0 & $\langle 5.0$ & ธ. .0 & 5.0 & (5.) & $\langle 5.0$ & (5.0) & (5.o \\
\hline CAATHONS & เeq/1 & 5.28 & 5.43 & 6.24 & 5.94 & 7.73 & 7.29 & 5.53 & 19.34 & 9.19 & 7.37 \\
\hline 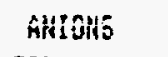 & necil & 5.54 & 5.15 & 10.68 & 7.61 & 10.34 & 8.47 & 6.50 & 22.75 & 8.85 & a.82 \\
\hline TOT. HLL.: & gi! & 92.16 & 76 & 221 & 145 & 214 & 164 & 113 & 580 & 183 & 176 \\
\hline If & $9: 1$ & 34.1 & -- & $\cdots$ & --- & $\cdots$ & -..- & --- & -- & --- & ---- \\
\hline
\end{tabular}

(--) Data not available.

(\#) Total Alkalinity as C03--. 
TABLE A.4. contd

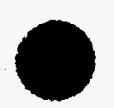

\begin{tabular}{|c|c|c|c|c|c|c|c|c|c|}
\hline SAMPLE & $-\cdots$ & 10 & 11 & 12 & 13 & 14 & 15 & 16 & 17 \\
\hline TIME & days & 41 & 50 & 64 & 92 & 122 & 134 & 182 & 302 \\
\hline pH & & $\cdot 11.57$ & 11.20 & 10.75 & 11.32 & 11.06 & 9.76 & 10.58 & 11.74 \\
\hline Eh & ov & 187 & 199 & 172 & 186 & 209 & 214 & 242 & 356 \\
\hline Al & $\mathrm{og} / 1$ & 0.06 & 0.09 & $<0.03$ & 0.04 & 10.03 & 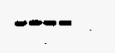 & $<0.03$ & 10.03 \\
\hline $8 \mathrm{a}$ & $g / 1$ & $<0.002$ & 0.002 & $<0.002$ & $<0.002$ & $<0.002$ & $\cdots$ & 0.002 & 0.003 \\
\hline $\mathrm{Ca}$ & $g / 1$ & 1.4 & 2.6 & 1.8 & 1.1 & 1.2 & ---- & 0.9 & $0 . \hat{0}$ \\
\hline Cd & $g / l$ & $<0.004$ & $<0.004$ & $<0.004$ & $<0.004$ & $\{0.004$ & ---- & $<0.004$ & $<0.004$ \\
\hline $\mathrm{Cr}$ & $\log / 1$ & $<0.02$ & $<0.02$ & $<0.02$ & 60.02 & 60.02 & --- & $<0.02$ & $<0.02$ \\
\hline $\mathrm{Cu}$ & $\Delta / 1$ & $<0.004$ & $<0.004$ & $<0.004$ & 0.005 & 0.004 & $\cdots$ & 80.004 & $<0.0014$ \\
\hline $\mathrm{Fe}$ & $g / 1$ & $<0.005$ & $<0.005$ & $<0.005$ & 0.011 & 0.007 & $-\cdots$ & 60.105 & $\{0.005$ \\
\hline$k$ & $g / 1$ & 15.0 & 10.0 & 9.9 & 14.0 & 11.8 & ---- & 12.0 & 13.5 \\
\hline $\mathrm{rg}$ & $g / 1$ & 0.32 & $3.8 B$ & 1.86 & 0.14 & 0.20 & --- & 0.15 & 0.06 \\
\hline $\mathrm{Mn}$ & $g / 1$ & $<0.002$ & $<0.002$ & 80.002 & $<0.002$ & $<0.002$ & --- & 60.002 & 10.002 \\
\hline $\mathrm{Ha}$ & og $/ 1$ & 223 & 128 & 143 & 210 & 170 & -. & 175 & 250 \\
\hline$P$ & $g / 1$ & 0.10 & $<0.1$ & $<0.1$ & $<0.1$ & $<0.1$ & - & $<0.1$ & 0.1 \\
\hline $35 \mathrm{PO4}$ & ag/1 & 0.31 & $\langle 0.31$ & 0.31 & 00.31 & 20.31 & -.-- & 00.31 & 0.31 \\
\hline $\mathrm{Pb}$ & agil & 10.06 & $<0.06$ & 80.06 & 10.06 & $<0.06$ & ---- & $<0.06$ & 00.06 \\
\hline $5 i$ & $\log n$ & 7.4 & 8.0 & 6.9 & 4.9 & 4.8 & -..- & 4.9 & 5.4 \\
\hline $5 r$ & ag $/ 1$ & 0.016 & 0.017 & 0.009 & 0.007 & 0.006 & $-\ldots$ & $0.060^{\circ}$ & 0.022 \\
\hline In & $\log / 1$ & $<0.02$ & 0.037 & $<0.02$ & 0.02 & 60.02 & $\ldots$ & 10.02 & 0.0355 \\
\hline$i r$ & $\lg / 1$ & $<0.008$ & $<0.008$ & $<0.008$ & $<0.008$ & $(0.008$ & - & $\{0.008$ & $<0.008$ \\
\hline F- & agil & 2.0 & 0.9 & 0.3 & 1.0 & 3.1 & --- & 0.8 & 1.1 \\
\hline Cl- & $99 / 1$ & 25 & 26 & 25 & 33 & 52 & -..- & 21 & 24 \\
\hline $\mathrm{HO}^{2-}$ & $g / 1$ & $<0.3$ & $<0.2$ & 60.2 & 0.1 & 50.2 &.-- & $\{0.3$ & <0.1 \\
\hline NDS- & $9 / 1$ & 2.7 & 0.8 & 1.1 & 8.5 & 0.9 & $-\cdots$ & 1.4 & 5.6 \\
\hline 504-- & gil & 89 & 84 & 83 & B4 & 57 & $\cdots$ & 66 & 79 \\
\hline $\mathrm{HCOS}-$ & $19 / 1$ & 371.5 & 230.6 & 0.0 & 0.0 & 0.0 & 51.2 & 0.00 & $\dot{0} .00$ \\
\hline COJ-- & ag/l & 63 & 13 & 151 & $B B$ & 126 & 63 & 108.60 & 113.60 \\
\hline OH- & $\operatorname{sil}$ & 0.0 & 0.0 & 14.3 & 85.7 & 42.8 & 0.0 & 96.90 & 96.45 \\
\hline B & ag/1 & 0.105 & 0.071 & 0.073 & 0.090 & 0.071 & -.- & ن. 680 & $0.10 i$ \\
\hline as & og $/ 1$ & 0.60 & 0.41 & 0.42 & 0.51 & 0.41 & $-\ldots$ & 0.45 & 0.58 \\
\hline TOC & $9 g / l$ & $\ldots$ & $\cdots$ & --- & $-\infty$ & 1.0 & --- & -- & --- \\
\hline POA- - & $\operatorname{ag} / 1$ & 0.5 & $\$ 1.0$ & $<2.0$ & $\langle 3.0$ & 22.0 & $-\cdots$ & $(5 . i)$ & 15.6 \\
\hline CATiJONS & ะzq/1 & 10.17 & 6.27 & 6.71 & 9.56 & 7.77 & 0.00 & 7.98 & 11.26 \\
\hline HAllons & aeq/1 & 8.35 & 5.58 & B. 42 & 10.95 & 9.62 & 2.94 & 11.98 & 11.94 \\
\hline TOT.ALK. * & agil & 246 & 126 & 176 & 239 & 202 & 38 & 279 & 284 \\
\hline IC & 3011 & -..- & --- & -.-- & $\ldots$ & 13.6 & $\cdots$ & $\cdots$ & $-\cdots$ \\
\hline
\end{tabular}

(---) Data not available.

(\#) Total Alkalinity as C03--. 
$\mathrm{OI}^{\circ} \mathrm{V}$

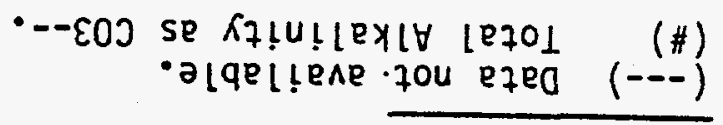

\begin{tabular}{|c|c|c|c|c|c|c|c|c|c|c|c|c|}
\hline$\cdots$ & $\because 4$ & $\cdots$ & $i \operatorname{sit}$ & $\cdots$ & $\angle>81$ & --- & $D^{0} \pi$ & -- & $5 \cdot 0 z$ & $!+n$ & ling & II \\
\hline$\cdots$ & 99:। & $\cdots$ & OISI & rtOI & $+L L$ & $5 O L$ & $590 i$ & tors & not & 9123 & $1.16 n$ & ะทามด \\
\hline-- & $29^{\circ} 0 S$ & -- & $58 \cdot 86$ & 98.68 & 28.08 & $20 \cdot 82$ & 9200 & $z \cdot h z$ & $\angle B \cdot 4 \hat{i}$ & tsis & i/bas & SHOINS \\
\hline EI'LA & 6012 & $00^{\circ} \mathrm{cs}$ & $56^{\circ} \mathrm{St}$ & Lt'ot & $2 L^{\prime}$ it & $60^{\circ} \mathrm{LS}$ & $99^{\circ} L \mathcal{L}$ & $n i \cdot 9 s$ & 129 & 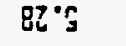 & lobat & EMPI:LY \\
\hline Q5: & $\begin{array}{l}\text { 5) } \\
0.7\end{array}$ & s? & 5) & (1.5) & $\begin{array}{l}0.57 \\
t=z\end{array}$ & 0.5: & $\begin{array}{l}8.5 \\
0.27\end{array}$ & $\begin{array}{l}0.5\rangle \\
---\end{array}$ & $\begin{array}{l}0: 5> \\
105\end{array}$ & 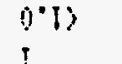 & $1 / 60$ & $--60 j$ \\
\hline $20^{\circ} t$ & $16^{\circ} \mathrm{b}$ & $6 t^{\circ}+$ & $+6 . t$ & $\left.z \Sigma^{\prime}\right\}$ & $21 \%$ & $80 \%$ & $6 t: \Omega$ & $19 \%$ & $Z L \div$ & tron & $1 / 6 x$ & SOSeH se \\
\hline $29 e^{\circ} 0$ & 0280 & $58: \circ 0$ & $9 \angle L O 0$ & $992 \% 0$ & $i 2 l^{\circ} 0$ & HILO & $6 \in 9 \cdot 0$ & 9080 & $059 \%$ & $90 \%$ & $y^{64}$ & 5 \\
\hline--- & e.tst & $\cdots$ & 5.665 & $5 \cdot 965$ & 0.0 & S'08z & $0.8 z z$ & $0 \cdot 142$ & 8.625 & 0 & Vig日 & $-H 0$ \\
\hline$\ldots$ & tos & $\cdots$ & $25 z$ & 891 & 896 & 012 & 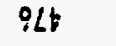 & 821 & $81 \Sigma$ & $i " z$ & $1 / 6 \%$ & --200 \\
\hline ---- & $0^{\prime} 0$ & - & 0.0 & 0.0 & $2 \cdot ? i$ & 0.0 & $0^{\circ} 0$ & $0 \%$ & 0.0 & ILI & $1 /$ ou & HCISH \\
\hline$i \hat{s i}$ & $8+!$ & $0 \angle 3$ & $0 L 1$ & 891 & 891 & ESI & 891 & toI & 191 & 98 & I!sin & $--68 s$ \\
\hline n.6! & 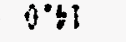 & 6.9 & 6.9 & $0^{\circ} 9$ & 9.9 & 0.9 & 8.5 & 279 & $2 \cdot 5$ & 100 & lion & - \\
\hline 20 & 50 & $5^{\circ} 0$ & +0 & 10 & se & $t 00$ & 20 & 1.0 & $\varepsilon^{\circ} \mathrm{C}$ & $200 \%$ & 1/60 & $-20 \%$ \\
\hline$\tau$ & $z^{2}$ & $8 z$ & 62 & $L$ & $L$ & $i z$ & 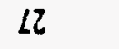 & $8 z$ & 12 & $\pi$ & libe & $-1]$ \\
\hline $0 \cdot 11$ & 0.11 & 0.01 & 0.01 & 1.6 & 0.01 & 56 & 1.5 & $8 \% 6$ & $1 \cdot 6$ & $\mathrm{sel}^{\circ}$ & $v^{6}$ & -5 \\
\hline $\left.800^{\circ} 0\right\rangle$ & $\left.800^{\circ} 0\right\rangle$ & $600^{\circ} 0$ & $110^{\circ} 0$ & $800^{\circ} 0 ;$ & $020^{\circ} 0$ & $010^{\circ} 0$ & $120^{\circ} 0$ & $\left.800^{\circ} 0\right\rangle$ & 120.0 & $800^{\circ} 0 \%$ & $1 / 6$ & $I I$ \\
\hline $\left.20^{\circ} 0\right\rangle$ & $20^{\circ} 03$ & $\left.20^{\circ} 0\right\rangle$ & $\left.20^{\circ} 0\right)$ & $\left.20^{\circ} 0\right\rangle$ & $\left.20^{\circ} 0\right\rangle$ & $\left.20^{\circ} 0\right)$ & $\left.20^{\circ} 0\right\rangle$ & $\left.20^{\circ} 0\right\rangle$ & 20.08 & $10 \%$ & $1 / 60$ & uz \\
\hline $610^{\circ} 0$ & 150.0 & $560^{\circ} 0$ & 2710 & $180^{\circ} 0$ & $801 \%$ & $\varepsilon \angle 0^{\circ} 0$ & $660^{\circ} 0$ & $880^{\circ} 0$ & $\varepsilon B 0^{\circ} 0$ & s:0 & lite & JS \\
\hline$z \cdot s$ & $8: 21$ & $9 \cdot 8$ & 9.11 & E:01 & E.11 & Z"0l & 0.11 & rot & 8.01 & st & $y^{6 *}$ & !S \\
\hline $\left.90^{\circ} 0\right\rangle$ & $\left.90^{\circ} 0\right)$ & 90.05 & 90.05 & $\left.90^{\circ} 0\right)$ & $\left.90^{\circ} 0\right\rangle$ & $\left.90^{\circ} 0\right\rangle$ & $90^{\circ} 0^{\circ}$ & $\left.90^{\circ} 0\right\rangle$ & $90 \%$ & $50.0\rangle$ & $1 / 60$ & Qd \\
\hline$\left[0^{\circ} 0\right)$ & {$\left[C^{\circ} 0\right\rangle$} & $1 \varepsilon^{\circ} 0$ & $\left|c^{\circ} 0\right\rangle$ & $98 \cdot 0$ & $\left\{\left[0^{\circ} 0\right\rangle\right.$ & $\left\{5^{\circ} 0\right\}$ & $15 \cdot 0 ?$ & $2 t^{\circ} 0$ & $\left.10^{\circ} 0\right\rangle$ & $\left\{\Sigma^{\circ} 0\right\}$ & $V^{66}$ & fod $\leqq 9$ \\
\hline$(\cdot 0)$ & $|\cdot 0\rangle$ & $01^{\circ} 0$ & $|\cdot 0\rangle$ & SI.0 & $\lceil\cdot 0\rangle$ & {$[0\rangle$} & $\lceil\cdot 0\rangle$ & 610 & $|\cdot 0\rangle$ & 10 & $1 / 60$ & $d$ \\
\hline r50I & $\mathrm{SSOI}$ & 696 & 086 & 606 & OEb & 058 & 048 & 808 & Obl & $\Omega$ & $1 / 60$ & EH \\
\hline $200^{\circ} 0$ & $\left.200^{\circ} 0\right\rangle$ & $200^{\circ} 03$ & $\left.200^{\circ} 0\right\rangle$ & $\left.200^{\circ} 0\right\rangle$ & $\left.200^{\circ} 0\right\rangle$ & $\left.200^{\circ} 0\right\rangle$ & $\left.200^{\circ} 0\right\rangle$ & $200^{\circ} 0 ?$ & $200^{\circ} 03$ & 10.03 & $1 / 69$ & UH \\
\hline $90^{\circ} 0 ;$ & $90^{\circ} 03$ & $\left.90^{\circ} 0\right\rangle$ & $50^{\circ} 0$ & $\left.90^{\circ} 0\right\rangle$ & $90^{\circ} 03$ & $\left.90^{\circ} 0\right\rangle$ & $90^{\circ} 0 ;$ & $90^{\circ} 0 ?$ & $\left.90^{\circ} 0\right\rangle$ & 501 & $1 / 60$ & $6 y$ \\
\hline 94 & st & $2 b$ & St & $0 t$ & zh & LI & $9 !$ & 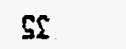 & ts & 8.6 & $1 / 60$ & $x$ \\
\hline $5000 \%$ & $967^{\circ} 0$ & $800^{\circ} 0$ & $010 \%$ & $\left.500)^{\circ} 0\right\rangle$ & 610.0 & $900 \%$ & $010^{\circ} 0$ & $150^{\circ} 0$ & $\$ 10^{\circ} 0$ & $10.0 \%$ & $v^{6}$ & af \\
\hline $\left.500^{\circ} 0\right)^{\circ}$ & $\left.100^{\circ} 0\right\rangle$ & $500^{\circ} 0 y^{j}$ & $400^{\circ} 03$ & 600.03 & $\left.600^{\circ} 0\right\rangle$ & $600^{\circ} 03$ & $\left.600^{\circ} 0\right\rangle$ & $\left.500^{\circ} 0\right\rangle$ & $\left.600^{\circ} 0\right\rangle$ & $500^{\circ} 0 ;$ & $1 / 64$ & ח \\
\hline $20 \% ?$ & $50^{\circ} 0$ & $\left.20^{\circ} 0\right)$ & $20^{\circ} 03$ & $\left.20^{\circ} 0\right\rangle$ & $\left.20^{\circ} 0\right\rangle$ & $\left.20^{\circ} 0\right\rangle$ & $\left.20^{\circ} 0\right\rangle$ & $\left.20^{\circ} 0\right\rangle$ & $20 \cdot 0\rangle$ & sio.0; & $1 / 60$ & 13 \\
\hline to0.0\% & $500^{\circ} 03$ & $100.0\rangle$ & 500.02 & $\left.600^{\circ} 0\right\rangle$ & $100.0\rangle$ & $\left.600^{\circ} 0\right\rangle$ & $\left.\$ 00^{\circ} 0\right\rangle$ & $\$ 00.07$ & $\left.100^{\circ} 0\right\rangle$ & $\left.500^{\circ} 0\right\rangle$ & $1 / 60$ & PJ \\
\hline S's & $\varepsilon^{\prime} 0$ & $L^{\circ} \Omega$ & 9.6 & $L^{\circ} 0$ & $2 \%$ & $L^{\prime} 0$ & $l^{\prime} s$ & $s^{\prime} s$ & $\Sigma^{\circ} \Omega$ & 99 & I/6n & B] \\
\hline $500^{\circ} 0$ & 900.0 & $600 \%$ & $210^{\circ} 0$ & $800^{\circ} 0$ & 110.0 & $800^{\circ} 0$ & 600.0 & 600.0 & 600.0 & $90 \%$ & I/60 & eg \\
\hline $6 \%$ & 0.51 & 8.01 & $9 \cdot 21$ & $|\cdot c|$ & I’ZI & $r \cdot z r$ & $t \cdot 11$ & $\tau \bullet \bullet$ & 8.01 & 100 & $u^{6 \pi}$ & IH \\
\hline SSI & $6 \subseteq !$ & हाI & SZI & $2 I Z$ & 612 & ZIL & $6 L Z$ & 192 & 982 & 562 & A & 4 \\
\hline ו" & $25^{\circ}$ 지 & $s t^{\circ} \bar{\prime}$ & $15^{\circ} 2 !$ & $8 g^{\circ} 21$ & $\Sigma \Sigma \tau^{\circ} \tau$ & $\varepsilon Z^{\prime} Z I$ & SS:ZI & $\left\{b^{\circ} Z\right]$ & LI'Z! & 1.8 & & Ho \\
\hline It & it & $L I$ & $I Z$ & $o z$ & 02 & EI & $\{1$ & $l$ & 6 & - & SKEP & 3411 \\
\hline $9-81$ & $9-41$ & $4-8 !$ & $t-L I$ & $\varepsilon-8[$ & {$[-1]$} & $2-81$ & $z-61$ & $|-8|$ & $|-| \mid$ & $\begin{array}{r}83164 \% \\
773 \mathrm{H}\end{array}$ & $\cdots$ & I 57d \\
\hline
\end{tabular}

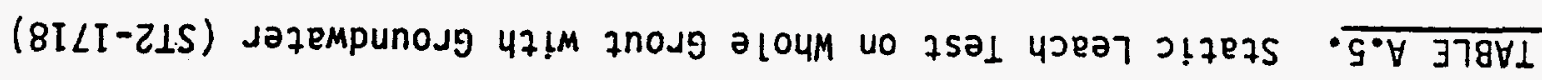


TABLE A.5. contd

\begin{tabular}{|c|c|c|c|c|c|c|c|c|c|c|c|}
\hline SAMFLE I & $\cdots$ & $17-7$ & $18-7$ & $17-8$ & $18-8$ & $17-9$ & $17-10$ & $17-11$ & $17-12$ & $17-13$ & $18-14$ \\
\hline TIME & days & 48 & 48 & 55 & 55 & 62 & 70 & 76 & 104 & 134 & 190 \\
\hline $\mathrm{pH}$ & & 12.51 & 12.52 & 12.63 & 13.14 & 12.55 & 12.53 & 12.48 & 12.44 & $12.3 \mathrm{~J}$ & 12.36 \\
\hline Eh & or & 139 & 143 & 153 & 160 & 114 & 164 & 164 & 132 & 157 & 153 \\
\hline Al & $9 / 1$ & 12.1 & 13.3 & 11.5 & 12.6 & 11.3 & 11.5 & 11.2 & 12.1 & 13.5 & ló.t \\
\hline $\mathrm{Ba}$ & $9 / 1$ & 0.012 & 0.012 & 0.012 & 0.013 & 0.012 & 0.011 & 0.011 & 0.014 & 0.010 & 0.110 \\
\hline $\mathrm{Ca}$ & $29 / 1$ & 4.4 & 4.4 & 4.0 & 4.3 & 4.1 & 4.1 & 4.0 & 4.0 & 4.2 & 4.7 \\
\hline Cd & $a / 1$ & $<0.004$ & $<0.004$ & $<0.004$ & $<0.004$ & $<0.004$ & $<0.004$ & $\because(i .004$ & $<0.004$ & 60.104 & 60.004 \\
\hline$\delta r$ & $2 g / 1$ & 0.03 & $<0.02$ & $<0.02$ & $<0.02$ & $<0.02$ & $<0.02$ & 10.02 & 50.62 & 0.02 & 0.02 \\
\hline $\mathrm{Cu}$ & $g / 1$ & 0.006 & 0.005 & $<0.004$ & $<0.004$ & 10.004 & $<0.004$ & $<0.004$ & 0.005 & 80.1004 & 6. Gota \\
\hline $\mathrm{Fe}$ & $\triangle 9 / 1$ & 0.014 & 0.010 & 0.008 & 0.007 & 0.031 & 0.014 & 0.026 & 0.021 & 6.010 & Q.uig \\
\hline$k$ & $g / 1$ & 14 & 45 & 42 & 43 & 42 & 42 & 41 & 41 & 43 & 47 \\
\hline $\mathrm{Hg}$ & $a g / 1$ & 0.07 & 10.06 & $<0.06$ & $<0.06$ & 0.05 & $\langle 0.06$ & 60.06 & 10.06 & $\angle 0.00$ & 4.12 \\
\hline Mn & $g / 1$ & $<0.002$ & $<0.002$ & $<0.002$ & $<0.002$ & 10.002 & 60.002 & $i 0.002$ & 0.003 & $6 \hat{v}, \mathrm{iv2}$ & 0.0002 \\
\hline $\mathrm{Na}$ & $\lg / 1$ & 982 & 990 & 950 & 964 & 939 & 970 & $\$ 40$ & 960 & 1008 & 1100 \\
\hline$P$ & $g / 1$ & $<0.1$ & $<0.1$ & $<0.1$ & $<0.1$ & 10.1 & 10.1 & 10.1 & $<0.1$ & 0.1 & 0.10 \\
\hline ¿5 PO4 & $q / 1$ & $<0.31$ & $\langle 0.31$ & $<0.31$ & 00.31 & 00.31 & $\{0.3\}$ & 60.31 & $\{0.3\}$ & 60.31 & $0.3 i$ \\
\hline Fb & $9 g / 1$ & $<0.06$ & $<0.06$ & $<0.06$ & $<0.06$ & 10.06 & 80.06 & $<0.06$ & $\angle 0.06$ & 10.06 & 60.06 \\
\hline$j i$ & $\log / 1$ & 12.4 & 11.9 & 12.3 & 11.7 & 12.5 & 14.6 & 13.7 & 13.5 & 14.3 & 14.6 \\
\hline Sr & $\operatorname{og} / 1$ & 0.116 & 0.122 & 0.135 & 0.121 & 0.115 & 0.115 & 0.116 & 0.117 & 0.119 & 0.130 \\
\hline in & $9 / 1$ & $<0.02$ & $<0.02$ & $<0.02$ & 80.02 & 10.02 & $\$ 0.02$ & 10.02 & 0.060 & (0.0ิ2 & 0.076 \\
\hline Ir & $.9 / 1$ & $<0.008$ & 00.008 & 0.102 & 0.035 & 0.047 & $<0.008$ & $<0.008$ & 60.008 & 60.008 & 10.006 \\
\hline$F-$ & $2 g / 1$ & 9.9 & 9.9 & 10.0 & 9.9 & 9.9 & १.5 & 9.4 & 11.0 & 12.0 & 13.0 \\
\hline $\mathrm{Cl}-$ & $g / 1$ & 26 & 27 & 27 & 26 & 23 & 24 & 20 & 22 & 23 & 23 \\
\hline HO2- & $9 q / 1$ & 0.3 & 0.4 & 0.3 & 0.3 & 0.4 & 0.3 & 3.4 & 10.1 & 0.2 & 80.3 \\
\hline H03- & $\operatorname{lng} / 1$ & 16.0 & 15.0 & 15.0 & 16.0 & 14.0 & 16.0 & 14.0 & 16.0 & 7.6 & 17.0 \\
\hline $504--$ & $4 n$ & 165 & 168 & 159 & 161 & 160 & 159 & 150 & 150 & 175 & 160 \\
\hline $\mathrm{HCO3-}$ & $\operatorname{ag} / 1$ & 0.0 & --- & 0.0 & $-\cdots$ & 0.0 & 0.0 & 0.0 & 0.0 & 0.6 & 6.8 \\
\hline$[03--$ & $g / 1$ & 202 & - & 176 & --- & 202 & 178 & 454 & 177 & 199 & 35 \\
\hline JH- & $g / 1$ & 621.1 & -- & 600.1 & --- & 599.5 & 577.4 & 385.6 & 635.3 & b78.j & 785.4 \\
\hline 5 & $a / 1$ & 0.660 & 0.811 & 0.740 & 0.780 & 0.731 & 0.750 & 0.750 & 0.760 & 0.846 & $1 . \hat{0}$ \\
\hline is $\mathrm{H} 3 \mathrm{BOO}$ & $g / 1$ & 3.77 & 4.64 & 4.23 & 4.46 & 4.16 & 4.33 & 4.33 & 4.34 & 4.85 & 5.60 \\
\hline$T x$ & mq $/ \mathrm{l}$ & 2.4 & $m$ & 2.3 & & 2.4 & 5.1 & 4.4 & 2.2 & 2.9 & $\cdots$ \\
\hline Fo4--- & $\operatorname{sol} / 1$ & 11.0 & 11.0 & $(1.0$ & 《1.0 & 62.0 & 6.0 & 35.6 & 6.0 & 1.0 & (0.5 \\
\hline ERTIOHS & meq/1 & 44.10 & 44.42 & 42.6 & 43.2 & 42.1 & 43.46 & 42.12 & 42.59 & 45.19 & 15.27 \\
\hline AHIOHS & agq/I & 45.62 & -- & 46.05 & $\ldots$ & 46.80 & 44.81 & 42.44 & 47.95 & 53.23 & D́.es \\
\hline TOT.ALK.* & $\operatorname{ag} / 1$ & 1298 & $\cdots$ & 1235 & ---- & 1260 & 1197.00 & 1154.00 & 1298.00 & 1386.60 & 1701.00 \\
\hline IC & gil & 18.3 & - & 22.1 & -..- & 17.1 & 41.5 & 30.3 & 14.7 & 31.5 & --- \\
\hline
\end{tabular}

(--) Data not available.

(\#) Total Alkalinity as CO3--. 


\begin{tabular}{|c|c|}
\hline $17-15$ & $18-16$ \\
\hline 315 & 424 \\
\hline 12.85 & 11.7 \\
\hline 233 & 30 \\
\hline 19.4 & 21. \\
\hline 0.009 & 0.002 \\
\hline 3.6 & 1.6 \\
\hline$\{0.004$ & 00.004 \\
\hline$\{0.02$ & 0.02 \\
\hline$\$ 0.604$ & $\{0.004$ \\
\hline 0.024 & 0.005 \\
\hline 47 & 48 \\
\hline 10.06 & $\langle 0.06$ \\
\hline$<0.002$ & $<0.002$ \\
\hline 1200 & 1163 \\
\hline 0.1 & $\{0.1$ \\
\hline 0.3 & 60.3 \\
\hline 10.06 & $<0.06$ \\
\hline 16.8 & 17.4 \\
\hline 0.101 & 0.046 \\
\hline 60.02 & 60.02 \\
\hline 60.008 & 60.008 \\
\hline 15.0 & 12.0 \\
\hline 23 & 27 \\
\hline 60.1 & 0.1 \\
\hline 22.0 & 22.0 \\
\hline 213 & 200 \\
\hline 0.00 & 0.00 \\
\hline 774.00 & 276.06 \\
\hline 365.50 & 749.11 \\
\hline 1.110 & 1.240 \\
\hline 6.35 & 7.09 \\
\hline 4.5 & -.- \\
\hline$\$ 0.5$ & 6.5 \\
\hline 53.50 & 51.87 \\
\hline $53.5 j$ & 59.16 \\
\hline 419.00 & 1595.04 \\
\hline 40.7 & \\
\hline
\end{tabular}

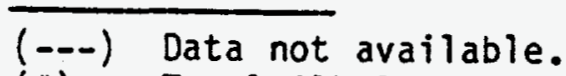

(\#) Total Alkalinity as C03-.. 
TABLE A.6. Static Leach Test on whole Grout with Groundwater (ST3-1819)

MELL

SAMPLE -.-. HATER $18-1 \quad 19-1 \quad 18-2 \quad 19-2 \quad 18-3 \quad 19-3 \quad 18-4 \quad 19-4 \quad 18-6$

\begin{tabular}{|c|c|c|c|c|c|c|c|c|c|c|c|}
\hline TIKE & days & י--חי - & 7 & 7 & 13 & 13 & 20 & 20 & 27 & 27 & 41 \\
\hline $\mathrm{pH}$ & & 8.1 & 12.49 & 12.51 & 12.40 & 12.43 & 12.30 & 12.59 & 12.52 & 12.52 & 12.53 \\
\hline Eh & ov & 295 & 284 & 289 & 304 & $26 j$ & 229 & 202 & 119 & 110 & 116 \\
\hline Al & $2 g / 1$ & $<0.1$ & 14.0 & 15.1 & 11.9. & 15.4 & 15.1 & 15.9 & 15.4 & 15.5 & 16.1 \\
\hline $\mathrm{Ba}$ & $g n$ & 0.06 & 0.008 & 0.009 & 0.009 & 0.011 & 0.009 & 0.008 & 0.011 & 0.100 & 0.005 \\
\hline Ca & $9 / 1$ & 56 & 3.3 & 4.3 & 3.6 & 3.9 & 1.4 & 5.2 & 4.1 & 5.1 & 0.7 \\
\hline $\mathrm{CO}$ & $09 / 1$ & $<0.005$ & 0.005 & 60.004 & $<0.004$ & 80.004 & $<0.004$ & 0.004 & 0.004 & 00.004 & 00,604 \\
\hline Cr & $\mathrm{mg} / 1$ & $<0.015$ & $<0.02$ & $<0.02$ & $<0.02$ & $<0.02$ & $<0.02$ & 10.02 & $\{0.002$ & 0.03 & 30.02 \\
\hline Cu & og/1 & $<0,005$ & $<0.004$ & $<0.004$ & $<0.004$ & 60.004 & 00.004 & 00.004 & 10.004 & $(0.004$ & 0.005 \\
\hline Fe & $9 g / 1$ & $<0.01$ & 0.015 & 0.018 & 0.006 & 0.010 & 0.005 & 0.009 & 0.007 & 0.013 & 0.026 \\
\hline$k$ & $\operatorname{sg} / 1$ & 7.8 & 35 & 37 & 37 & 42 & 43 & 44 & 45 & 46 & 47 \\
\hline$M g$ & $a g / 1$ & 14.4 & $<0.06$ & 80.06 & $<0.06$ & $<0.06$ & 60.06 & 10.06 & $\$ 0.06$ & 0.06 & 60.06 \\
\hline in & $29 / 1$ & 60.01 & 60.002 & 80.002 & $<0.002$ & 80.002 & $<0.002$ & 0.002 & $\langle 0.002$ & 60.002 & 6.002 \\
\hline $\mathrm{Ha}$ & $9 / 1$ & 25 & 776 & 808 & 776 & 900 & 932 & 956 & 969 & 1000 & $104 \hat{f}$ \\
\hline$f$ & $\log / 1$ & $<0.1$ & 0.20 & $<0.1$ & $<0.1$ & 10.1 & 0.17 & 0.10 & $<0.1$ & 0.14 & 0.19 \\
\hline as PQ4 & $\operatorname{\Delta og} / 1$ & $\langle 0.31$ & 0.61 & $\langle 0.31$ & 00.31 & $\{0.31$ & 0.52 & 0.31 & 0.31 & 0.43 & ن.58 \\
\hline$P b$ & 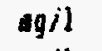 & $<0.05$ & 80.06 & $<0.06$ & $<0.06$ & $<0.06$ & 60.06 & $<0.06$ & 60.06 & $\langle 0.06$ & 00.06 \\
\hline $\mathrm{Gi}$ & ogil & 15 & 9.8 & 9.7 & $\$ .1$ & 9.9 & 9.7 & 9.9 & 10.0 & 9.2 & 11.2 \\
\hline $5 r$ & $a g / l$ & 0.25 & 0.085 & 0.104 & 0.091 & 0.113 & 0.098 & 0.081 & 0.119 & 0.120 & 0.038 \\
\hline$i n$ & $\Delta g / 1$ & 10.01 & 10.02 & 10.02 & 60.02 & 60.02 & $<0.02$ & $<0.02$ & 80.02 & $\langle\hat{i} .02$ & 80.02 \\
\hline Ir & ag $/$ l & 60.008 & 0.0 & $<0.008$ & 0.0 & 80.008 & 0.0 & 10.608 & 0.0 & 80.008 & 0.3 \\
\hline F- & agil & $<0.5$ & 8.4 & 9.4 & 7.7 & S.B & 9.4 & 160.0 & 10.0 & 10.0 & 13.0 \\
\hline $\mathrm{Cl}-$ & $g / 1$ & 22 & 27.0 & 27 & 25 & 25 & 27 & 27 & 27 & 28 & 23 \\
\hline 102- & $g / 1$ & 60.03 & 0.7 & 0.7 & 0.5 & 0.7 & 0.7 & 0.8 & 0.7 & 0.8 & $<0.3$ \\
\hline H03- & igil & 0.1 & 6.2 & 6.2 & 6.1 & $7 . \dot{j}$ & 3.4 & 7.7 & 7.7 & 3.4 & 16.0 \\
\hline $504-\infty$ & agil & Gá & 184.0 & 180 & 156 & 180 & 175 & 187 & 182 & [5! & 664 \\
\hline $\mathrm{HCOJ-}$ & agil & 171 & 0.0 & 0.0 & 0.0 & 0.0 & 0.0 & 6.0 & $0 . \hat{0}$ & --- & 8.8 \\
\hline 20$\}:-$ & $\Delta g / 1$ & 2.1 & 328 & 403 & 378 & 415 & 277 & 743 & 327 & $\cdots$ & 227 \\
\hline $\mathrm{OH}-$ & ogill & 0 & 332.7 & 492.7 & 385.6 & 510.7 & 535.6 & 324.9 & 557.1 & -- & 042.5 \\
\hline B & agil & 0.06 & 0.881 & 0.895 & 0.750 & 0.916 & 0.900 & 0.746 & 0.910 & 01.985 & 1.070 \\
\hline is 43603 & $a g / 1$ & 0.34 & .5 .04 & 5.12 & 4.29 & 5.24 & 5.15 & 5.41 & 5.20 & $5.5 j$ & 0.12 \\
\hline$T D C$ & ag $/ 1$ & 1 & --- & $\cdots$ & --- & $-\infty$ & $-\cdots$ & $\cdots$ & --. & ---- & $\cdots$ \\
\hline Fo4 -.. & $\lg / 1$ & $\{1.0$ & $\langle 5.0$ & 55.0 & $\langle 5.0$ & <.5.0 & $\langle 5.0$ & $(5.0)$ & 25.0 & 45.0 & 0.0 \\
\hline Câtioñs & $\triangle E q / 1$ & 5.28 & 34.80 & 36.28 & .38 .87 & 40.39 & 41.69 & 42.95 & 43.49 & 44.95 & 46.85 \\
\hline ANIONS & aeq/1 & 5.54 & 39.32 & 44.76 & 39.83 & 49.15 & 46.01 & 49.37 & $49,0.14$ & $\cdots$ & 50.27 \\
\hline OT. ATLK,* & $\Delta / 1$ & 92.16 & 1021 & 1184 & 1058 & 1317 & 1222 & 1317 & 1310 & -- & SOaj \\
\hline IC & $g / 1$ & 34.1 & $\cdots$ & $\cdots$ & -- & $\cdots$ & $\cdots$ & ---- & $\cdots$ & --. & $\cdots$ \\
\hline
\end{tabular}

(--) Data not available.

(\#) Total Alkalinity as C03-.. 
TABLE A.6. contd

\begin{tabular}{|c|c|c|c|c|c|c|c|c|c|c|c|}
\hline SAMPLE & - & $19-6$ & $18-7$ & $19-7$ & $18-8$ & $19-8$ & $18-9$ & $18-10$ & $18-11$ & $18-12$ & $18-13$ \\
\hline TIME & days & 41 & 48 & 48 & 55 & 55 & 62 & 70 & 76 & 104 & 134 \\
\hline $\mathrm{pH}$ & & 12.55 & 12.54 & 12.57 & 12.58 & 12.60 & 12.57 & 12.43 & 12.52 & 12.13 & 12.38 \\
\hline Eh & iv & 127 & 135 & 178 & 149 & 151 & 101 & 210 & 181 & 145 & 139 \\
\hline$\hat{H} \mathrm{l}$ & ogil & 16.8 & 15.6 & 15.9 & 14.5 & 15.2 & 14.6 & 14.9 & 14.3 & 15.9 & 17.0 \\
\hline 82 & $g / 1$ & 0.004 & 0.013 & 0.014 & 0.015 & 0.013 & 0.012 & 0.012 & 0.012 & 0.010 & 0.010 \\
\hline $\mathrm{Ca}$ & $10 / 1$ & 1.2 & 4.5 & 5.5 & 3.9 & 5. 2 & 4.0 & 4.2 & 4.1 & 4.0 & 4.2 \\
\hline $\mathrm{Cd}$ & $9 / 1$ & $<0.604$ & 0.008 & 10.004 & $<0.004$ & $<0.604$ & 0.008 & $<0.004$ & $<0.004$ & (0.004 & 00.004 \\
\hline $\mathrm{Cr}$ & $2 g / 1$ & 0.02 & 10.02 & 60.02 & $\$ 0.02$ & 60.02 & $<0.02$ & $<0.02$ & $<0.02$ & $<0.02$ & $<0.02$ \\
\hline $\mathrm{Cu}$ & $\log / 1$ & $<0.004$ & 0.008 & 0.008 & $<0.004$ & 60.004 & $<0.004$ & 0.011 & 0.004 & $<0.004$ & 60.604 \\
\hline $\mathrm{Fe}$ & $a g / 1$ & 0.006 & 1.580 & 0.0117 & 0.006 & 0.010 & 0.012 & 0.015 & 0.009 & 0.017 & 0.014 \\
\hline$k$ & $g / 1$ & 48 & 45 & 47 & 43 & 45 & 44 & 43 & 41 & 43 & 42 \\
\hline $\mathrm{Hg}$ & $\log$ & 10.06 & $<0.06$ & 0.08 & 00.06 & 10.06 & $<0.06$ & 60.06 & $<0.06$ & 00.06 & $\{0.06$ \\
\hline $\mathrm{Mn}$ & $g / 1$ & $<0.002$ & 0.008 & 0.003 & 10.002 & 60.002 & $\$ 0.002$ & $\{0.002$ & 60.002 & 80.002 & $\{0.002$ \\
\hline $\mathrm{Na}$ & $\operatorname{sig} / 1$ & 1075 & 1000 & 1020 & 762 & 998 & 955 & 980 & 950 & 970 & 1015 \\
\hline$f$ & $\lg j 1$ & 0.26 & $<0.1$ & 0.1 & $<0.1$ & 10.1 & $<0.1$ & $<0.1$ & $<0.1$ & 10.1 & 0.1 \\
\hline 35 FO4 & $9 g^{\prime}$ & 0.30 & 10.31 & $(0.31$ & $(0.31$ & $<0.31$ & $(0.31$ & $<0.31$ & $\{0.3\}$ & 0.31 & 10.31 \\
\hline Fb & $\operatorname{ag} / 1$ & 00.06 & 0.06 & 60.06 & $<0.06$ & $<0.06$ & $<0.06$ & 0.06 & 20.06 & $<0.00$ & $<0.06$ \\
\hline$\hat{\mathrm{j} i}$ & $\lg j]$ & 11.0 & 11.2 & 11.2 & 11.3 & 11.2 & 10.1 & 12.7 & 12.7 & 12.8 & 13.6 \\
\hline $5 r$ & $\log / 1$ & 0.049 & 0.129 & 0.140 & 0.128 & 0.140 & 0.126 & 0.126 & 0.125 & 0.128 & 0.124 \\
\hline In & oil & 10.02 & $\{0.02$ & 10.02 & 0.028 & 0.022 & $<0.02$ & 0.270 & $<0.02$ & 50.02 & 60.02 \\
\hline $2 r$ & $g / 1$ & $0 . i$ & $<0.008$ & $<0.008$ & 0.0 & 0.0 & 0.0 & 0.3 & 0.0 & $<0.008$ & $<0.008$ \\
\hline$F-$ & 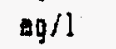 & 12.0 & 10.2 & 10.2 & 9.7 & 10.0 & $9 . \hat{7}$ & 11.0 & 9.7 & 11.0 & --- \\
\hline $\mathrm{Cl}_{-}^{-}$ & $9 / 1$ & 22 & 26 & 26 & 26 & 27 & 19 & 24 & 23 & 22 & -..- \\
\hline HO?- & $-g / 1$ & 60.3 & 0.7 & 0.7 & 0.6 & 0.6 & 0.6 & 0.5 & 0.4 & $<0.1$ & -..-- \\
\hline No3- & $\operatorname{sg} / !$ & 17.0 & 18.0 & 18.0 & 17.0 & 18.0 & 10.5 & 18.0 & 17.0 & 18.0 & ---- \\
\hline 504-- & sgil & 167 & 183 & 183 & 172 & 179 & 173 & 178 & 173 & 150 & -.-- \\
\hline $\mathrm{HCOJ}-$ & al /: & -- & 0.0 & 0.0 & 0.0 & $\cdots$ & 0.0 & 0.0 & 0.0 & 0.0 & 0.0 \\
\hline CO3-- & $g / \mathrm{i}$ & -- & 202 & 378 & 252 & -- & 202 & 226 & 454 & 176 & 378 \\
\hline $\mathrm{OH}-$ & $g / 1$ & --- & 671.2 & 589.1 & 621.2 & -.. & 571.1 & 593.0 & 407.0 & $635 . \dot{J}$ & 571.2 \\
\hline $\bar{B}$ & $\operatorname{uug} i$ & 1.090. & 0.346 & 0.787 & 0.720 & 0.550 & 0.906 & 0.730 & 0.890 & 0.960 & 1.100 \\
\hline as $4530 \mathrm{~J}$ & $g / 1$ & 6.23 & 5.41 & 5.64 & 5.26 & 5.43 & 5.18 & 5.37 & 5.14 & 5.49 & 6.27 \\
\hline TOC & $\theta 9$ & --- & -- & -- & -- & -.- & $\ldots$ & $\ldots$ & --- & --- & --- \\
\hline FO4--- & $5 g /:$ & 45.0 & $<1.0$ & a.0 & 11.0 & $\$ 1.0$ & 22.0 & 10.5 & 10.5 & 0.5 & --- \\
\hline CÁTJONS & aeqil & 48.03 & 44.86 & 45.83 & 43.12 & 44.82 & 41.42 & 43.73 & 42.56 & 43.42 & $\{5.40$ \\
\hline AllioNs & กอบ /] & -- & 48.72 & 49.77 & 50.10 & $\ldots$ & 45.24 & 47.82 & 44.26 & $\$ 7.97$ & $\cdots$ \\
\hline TOT.ALK.* & igil & -- & 1386 & 1410 & 1348 & -- & 1210 & 1272 & 1172 & 1298 & 1336 \\
\hline It & ingil & --.- & ---- & $\ldots$ & $\cdots$ & $\ldots-$ & $-\cdots$ & --- & $\ldots$ & $\ldots$ & $\ldots$ \\
\hline
\end{tabular}

(--) Data not available.

(\#) Total Alkalinity as C03--. 
TABLE A.6: contd

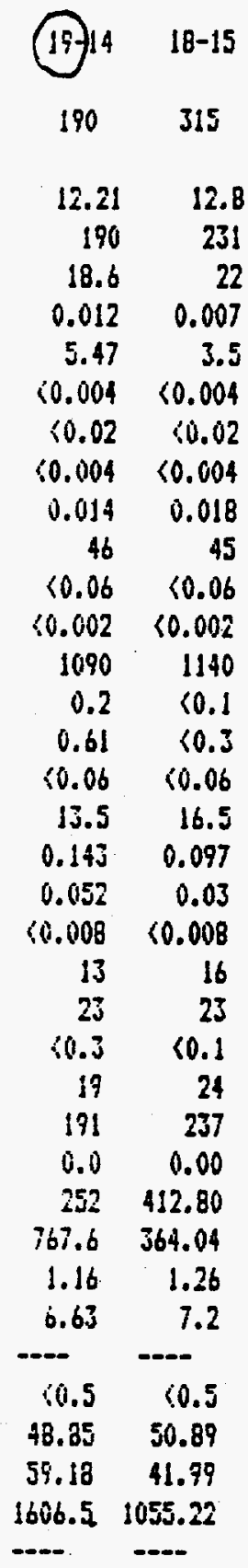

(---) Data not available.

(\#) Total Alkalinity as c03--. 
TABLE A.7. Static Leach Test on whole Grout with Groundwater (ST4-1319)

\begin{tabular}{|c|c|c|c|c|c|c|c|c|c|c|c|}
\hline SAMFLL I & -.-- & $\begin{array}{l}\text { WELL } \\
\text { MATER }\end{array}$ & $13-1$ & $19-1$ & $13-2$ & $19-2$ & $13-3$ & $19-3$ & $13-4$ & $19-4$ & $13-6$ \\
\hline TIME & days & - & 7 & $?$ & 13 & 13 & 20 & 20 & 27 & 27 & 41 \\
\hline $\mathrm{pH}$ & & 8.1 & 12.47 & 12.43 & 12.38 & 12.34 & 12.59 & 12.54 & $\lfloor 2.51$ & 12.48 & 12.50 \\
\hline Eh & av & 295 & 294 & 294 & 278 & 304 & 215 & 215 & 133 & 126 & 123 \\
\hline AI & $g / 1$ & $<0.1$ & 12.3 & 11.8 & 10.7 & 10.3 & 12.2 & 11.2 & 4.8 & 11.8 & 8.0 \\
\hline Ba & $g / 1$ & 0.06 & 0.011 & 0.009 & 0.007 & 0.010 & 0.008 & 0.012 & 0.007 & 0.013 & 0.005 \\
\hline$C_{d}$ & $g / 1$ & 56 & 4.0 & 4.0 & 0.5 & 0.7 & 1.5 & 4.2 & 4.4 & 4.8 & 3.1 \\
\hline $\mathrm{Cd}$ & $\Delta g / 1$ & $\langle 0.005$ & $<0.004$ & $\langle 0.005$ & $\{0.004$ & 10.005 & $<0.004$ & $<0.005$ & $<0.004$ & $<0.005$ & 20.004 \\
\hline $\mathrm{Cr}$ & $19 / 1$ & $\langle 0.015$ & $<0.02$ & 10.02 & $<0.02$ & 60.02 & $<0.02$ & $\leqslant 0.02$ & $<0.02$ & $\langle 0.02$ & $\therefore 0.02$ \\
\hline $\mathrm{Cu}$ & ag $/ 1$ & 50.005 & $<0.004$ & $<0.604$ & 10.004 & $\because 0.004$ & $<0.004$ & 10.004 & $<0.004$ & 0.008 & $<0.004$ \\
\hline $\mathrm{Fe}$ & igh & 60.01 & 0.016 & 0.016 & 0.007 & 0.013 & 0.005 & 0.012 & 10.005 & 0.015 & 60.005 \\
\hline$k$ & $\sin / 1$ & 7.8 & 33 & 30 & 35 & 32 & 39 & 35 & 43 & 37 & 44 \\
\hline$n_{q}$ & $g / 1$ & 14.4 & 10.06 & 10.06 & $\$ 0.06$ & 0.06 & $<0.06$ & 10.06 & 60.06 & 10.06 & 60.06 \\
\hline th & $n g / 1$ & $<0.01$ & $<0.002$ & $<0.002$ & $<0.002$ & 80.602 & 10.002 & 50.002 & $<0.002$ & 00.002 & $<0.002$ \\
\hline$N_{a}$ & $0 g i l$ & 25 & 748.0 & 672.0 & 762.0 & 685.0 & $8 B 3$ & 765 & 964 & 813 & 1020 \\
\hline$P$ & $\log / 1$ & 60.1 & $<0.1$ & $<0.1$ & $<0.1$ & 0.1 & $<0.1$ & $<0.1$ & 60.1 & 0.12 & 60.1 \\
\hline js P04 & ag $/ !$ & $\langle 0.31$ & $(0.31:$ & 00.31 & $<0.3 !$ & $\{0.3\}$ & $(0.31$ & $\langle 0.31$ & 0.31 & 0.37 & 0.31 \\
\hline $\mathrm{Pb}$ & hig/1 & $<0.05$ & $\{0.06$ & 0.06 & 0.06 & 10.06 & 60.06 & $<0.06$ & 00.06 & $<0.06$ & $\therefore 0.06$ \\
\hline $\mathbf{S}$ & $\log / 1$ & 15 & 10.00 & 9.70 & 9.70 & 9.60 & 10.2 & 9.8 & 6.1 & 10.2 & 5.0 \\
\hline $5 r$ & $g / 1$ & 0.25 & 0.099 & 0.098 & 0.068 & 0.082 & 0.091 & 0.117 & 0.104 & 0.122 & 0.059 \\
\hline in & $=g / 1$ & $<0.01$ & $<0.02$ & 0.020 & $<0.02$ & 60.02 & $<0.02$ & $<0.02$ & 10.02 & 60.02 & $\$ 0.02$ \\
\hline $7 r$ & $\operatorname{ag} / 1$ & 00.008 & 0.036 & $<0.008$ & 0.029 & 0.017 & $<0.008$ & $\$ 0.008$ & $<0.008$ & 60.008 & 0.093 \\
\hline F- & qqil & 50.5 & 9.8 & 9.4 & 8.8 & 8.8 & 10.0 & 9.4 & 9.8 & 9.8 & 11.0 \\
\hline $\mathrm{Cl}_{-}^{-}$ & $6 / 1$ & 22 & 27 & 26 & 25 & 27 & 24 & 25 & 26 & 25 & 22 \\
\hline $102-$ & $90 i 1$. & $0.0 j$ & 0.7 & 0.5 & 0.5 & 0.5 & 0.7 & 0.7 & 0.7 & 0.5 & 60.3 \\
\hline NDS- & ag/l & 0.1 & 5.6 & 6.2 & 5.3 & 6.2 & 6.0 & 5.3 & 6.2 & 5.7 & 13.0 \\
\hline $504--$ & $g / 1$ & 86 & 170 & 161 & 149 & 144 & 156. & 158 & $16 i$ & 191 & 137 \\
\hline $\mathrm{HC}_{\mathrm{C} \mathrm{S}^{-}}$ & $g / 1$ & $i i 1$ & 170.8 & 0.6 & 0.0 & 0.0 & 0.0 & 0.0 & 0.0 & $\cdots$ & 0.0 \\
\hline $203--$ & igil & 2.1 & 27 & 192 & 192 & 480 & 216 & 216 & 227 & -- & 328 \\
\hline $\mathrm{OH}-$ & ofil & 0 & 0.0 & 374.0 & 442.0 & 204.0 & 510.0 & $4 i 4.8$ & 721.1 & -- & 599.6 \\
\hline$\hat{\mathbf{D}}$ & 10/1 & 0.06 & 0.709 & 0.681 & 0.655 & $0 .\{23$ & 0.710 & 0.628 & 0.752 & 0.069 & 0.772 \\
\hline ¿5 H3BOJ & ogil & 0.34 & 4.05 & 3.69 & 3.75 & 3.56 & 4.06 & 3.59 & 4.30 & 3.82 & $4.4 i$ \\
\hline TOC & $49 / 1$ & 1 & --- & $\cdots$ & --.- & $-\cdots$ & -- & $-\infty$ & $-\ldots$ & -.- & $-\cdots$ \\
\hline Fu4--- & ogil & :i.0 & $\$ 5.0$ & 85.0 & 65.0 & 65.0 & $\langle 5.0$ & 85.0 & $i \mathbf{5}, \dot{u}$ & <5.0 & 60.5 \\
\hline CATIOKS & sea/l & 5.28 & 33.57 & 30.17 & 34.05 & 30.57 & 39.48 & 34.37 & $\$ 3.23$ & 36.55 & 45.03 \\
\hline AH:ENS & 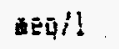 & 5.54 & 35.08 & 33.15 & 36.92 & 32.46 & $\$ 1.90$ & 36.38 & 54.86 & -- & 50.49 \\
\hline TGT. ALLK. & $\operatorname{mog} i 1$ & 92.16 & 900 & 352 & 972 & 240 & 1116 & 948 & 1499 & -- & 1386 \\
\hline IC & -ioj $j$ & 34.1 & ---- & ---- & $\cdots$ & ---- & $\cdots$ & ---- & --- & -.-- & $-\infty$ \\
\hline
\end{tabular}

(---) Data not available.

(\#) Total Alkalinity as CO3--. 
TABLE A.7. contd

\begin{tabular}{|c|c|c|c|c|c|c|c|c|c|c|c|}
\hline 5AMPLE & $\cdots$ & $19-6$ & $13-7$ & $19-7$ & $13-8$ & $19-8$ & $13-9$ & $13-10$ & $13-11$ & $13-12$ & $13-1 j$ \\
\hline TIME & days & 41 & 48 & 48 & 55 & 55 & 62 & 70 & 76 & 104 & 134 \\
\hline pH & & 12.45 & 12.51 & 12.49 & 12.59 & 12.55 & 12.31 & 12.31 & 12.51 & 12.42 & 12.41 \\
\hline Eh & ev & 127 & 127 & 123 & 152 & 155 & 161 & 161 & 199 & 148 & 135 \\
\hline Al & $9 / 1$ & 12.2 & 12.3 & 12.0 & 11.8 & 11.6 & 11.7 & 11.7 & 11.2 & 12.8 & 1 \\
\hline $\mathrm{Ba}$ & g $/ 1$ & 0.004 & 0.012 & 0.017 & 0.016 & 0.014 & 0.012 & 0.015 & 0.013 & 0.011 & 0.012 \\
\hline $\mathrm{Ca}$ & $g / 1$ & 3.3 & 4.9 & 4.9 & 4.6 & 4.9 & 4.6 & 4.6 & 4.1 & 4.6 & 5.18 \\
\hline Cd & $g / 1$ & 80.004 & $<0.004$ & 0.004 & $<0.004$ & 30.004 & 0.004 & $\$ 0.004$ & $<0.004$ & $\$ 0.004$ & $<0.004$ \\
\hline $\mathrm{Cr}$ & $9 / 1$ & $<0.02$ & $<0.02$ & $\leqslant 0.02$ & 0.03 & 10.02 & 10.02 & 60.02 & 30.02 & 60.02 & $\langle 0.02$ \\
\hline $\mathrm{Cu}$ & $g / 1$ & $<0.004$ & $<0.004$ & $\$ 0.004$ & $<0.004$ & 10.004 & 0.004 & 0.007 & 0.005 & 0.004 & 0.004 \\
\hline $\mathrm{Fe}$ & $19 / 1$ & $<0.005$ & 0.010 & 0.017 & 0.012 & 0.011 & 0.017 & 0.020 & 0.009 & 0.026 & 0.06 \\
\hline$k$ & $9 / 1$ & 41 & 42 & 38 & 43 & 41) & 43 & 43 & 212 & 42 & 41) \\
\hline $\mathrm{Mg}$ & $\lg / 1$ & $<0.06$ & $<0.06$ & $<0.06$ & 80.06 & 0.10 & 80.06 & 0.07 & $\langle 0.06$ & $<0.00$ & 60.06 \\
\hline Hn & $\lg / 1$ & 60.002 & $<0.002$ & $<0.002$ & 0.002 & $\langle 0.002$ & 80.002 & 20.002 & $\langle 0.1002$ & 60.002 & 0.002 \\
\hline $\mathrm{Ha}$ & $9 / 1$ & 914 & 989 & 892 & 940 & 857 & 938 & 960 & 920 & 945 & 380. \\
\hline$P$ & $\lg / 1$ & $<0.1$ & $<0.1$ & 00.1 & 00.1 & $<0.1$ & 60.1 & 60.1 & $<0.1$ & 0.1 & 60.1 \\
\hline as $\mathrm{f04}$ & $\Delta g / 1$ & $<0.31$ & $<0.31$ & $\langle 0.31$ & 10.31 & $(0.3)$ & $(0.31$ & 00.31 & 00.31 & 10.31 & 0.31 \\
\hline$P b$ & $2 g / 1$ & $<0.06$ & $<0.06$ & $<0.06$ & 10.06 & 10.06 & 10.016 & $<0.06$ & 10.06 & 40.06 & 60.06 \\
\hline $\mathbf{j i}$ & $\mathrm{Ag} / \mathrm{l}$ & 11.2 & 11.8 & 11.7 & 12.0 & 11.9 & 12.4 & 13.3 & 13.4 & 13.6 & 13.7 \\
\hline Sr & $\Delta g / 1$ & 0.070 & 0.137 & 0.130 & 0.137 & 0.132 & 0.136 & 0.139 & 0.133 & 0.153 & 0.158 \\
\hline In & $9 g / 1$ & $<0.02$ & 0.041 & 0.056 & 0.023 & $<0.02$ & 50.02 & 0.030 & 10.02 & 60.02 & 00.02 \\
\hline $2 r$ & $\operatorname{mg} / !$ & 0.042 & 0.043 & 0.025 & $<0.008$ & 0.021 & 0.011 & 0.030 & 20.000 & 60.006 & 0.008 \\
\hline F- & $g / 1$ & 10.0 & 8.7 & 9.3 & 9.9 & 9.2 & 9.5 & 10.4 & 7.5 & 11.0 & 12 \\
\hline $\mathrm{Cl}-$ & $g / 1$ & 23 & 23 & 24 & 26 & 26 & 17 & 22 & 23 & 22 & 25 \\
\hline $\mathrm{HO}_{2}^{-}$ & $a / 1$ & 60.3 & 0.5 & 0.5 & 0.6 & 0.6 & 0.6 & 1.6 & 0.4 & 6.2 & 34.2 \\
\hline HOS- & $g / 1$ & 12.0 & 13.0 & 12.0 & 14.0 & 12.0 & 13.0 & 71.0 & 9.2 & 25.6 & 0.5 \\
\hline $504--$ & $9 / 1$ & 133 & 144 & 147 & 150 & 143 & 147 & 172 & 143 & 140 & 150 \\
\hline $\mathrm{HCOJ-}$ & $\log / 1$ & $\cdots$ & 0.0 & $\cdots$ & 0.0 & 0.0 & 0.0 & 0.0 & 0.0 & $\dot{y} .0$ & 4.8 \\
\hline $203--$ & $49 / 1$ & -- & 227 & $\cdots$ & 227 & 265 & 176 & .255 & 630 & $20 \%$ & 202 \\
\hline DH- & $n ! / 1$ & $\cdots$ & 556.9 & $\cdots$ & 614.0 & 472.4 & 614.6 & 577.5 & 129.2 & 670.5 & 610.5 \\
\hline 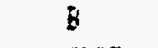 & $4 g / 1$ & 0.710 & 0.713 & 0.651 & 0.688 & 0.634 & 0.691 & 0.310 & 0.670 & 6.730 & i. 785 \\
\hline ¿5 HJBOJ & gill & 4.01 & 9.08 & 3.72 & 3.93 & $3.6 j$ & 3.75 & 4.10 & 3.37 & 4.17 & 4.47 \\
\hline TOC & $g / 1$ & --- & $-\ldots$ & $\cdots$ & $\cdots$ & $\therefore$ & $\cdots$ & $\cdots$ & --- & $\cdots$ & $\cdots$ \\
\hline PO4--- & g $/ 1$ & $<0.5$ & $\langle 1.0$ & 31.0 & 1.0 & 01.0 & 2.0 & $(5.0)$ & 5.0 & 0.0 & $\because 1.0$ \\
\hline CATIOHS & eq/1 & 40.96 & 44.32 & 40.00 & 42.20 & 38.53 & 42.12 & 43.03 & 41.29 & 42.40 & 43.70 \\
\hline AMIDHS & neq/1 & -- & 42.37 & -- & 46.33 & +2.23 & 46.33 & 48.55 & 35.71 & 50.60 & 50.54 \\
\hline TUT.ALK.: & $2 g / 1$ & $\cdots$ & 1210 & $\cdots$ & 1310 & 1134 & 1260 & 1274 & 1058 & j336 & 1579.7 \\
\hline Ii & agn $/ 1$ & $-\infty$ & - & $m$ & -- & -- & - & -- & -- & $\cdots$ & -..- \\
\hline
\end{tabular}

(-) Data not available.

(\#) Total Alkalinity as C03--. 
TABLE A.7. contd

\begin{tabular}{|c|c|}
\hline $13-16$ & $13-15$ \\
\hline 190 & 315 \\
\hline 12.24 & 12.87 \\
\hline 207 & 243 \\
\hline 14.5 & 17 \\
\hline 0.012 & 0.013 \\
\hline 4.84 & 5.7 \\
\hline 80.004 & $(0.004$ \\
\hline$\{0.02$ & $<0.02$ \\
\hline$\lceil 0.004$ & $<0.004$ \\
\hline 0.14 & 0.018 \\
\hline 44 & 47 \\
\hline$<0.06$ & $<0.06$ \\
\hline$<0.002$ & $\{0.002$ \\
\hline 995 & 1170 \\
\hline 50.1 & $<0.1$ \\
\hline$<0.31$ & 60.31 \\
\hline$<0.06$ & 00.06 \\
\hline 14.8 & 15.7 \\
\hline 0.13 & 0.139 \\
\hline 10.05 & $\langle 0.05$ \\
\hline 60.608 & $\angle 0.008$ \\
\hline 12 & 14 \\
\hline 22 & 21 \\
\hline$\langle 0.3$ & $\langle 0.1$ \\
\hline 15 & 20 \\
\hline 15.3 & 193 \\
\hline 0.0 & 0.00 \\
\hline 252 & 774.00 \\
\hline 342.6 & 170.60 \\
\hline 0.77 & 0.95 \\
\hline 4.4 & 5.43 \\
\hline$\cdots$ & $-\infty$ \\
\hline 60.5 & 0.5 \\
\hline 44.64 & 52.36 \\
\hline 50.89 & 42.65 \\
\hline 1386 & 1109.4 \\
\hline & \\
\hline
\end{tabular}

$\begin{array}{ll}(---) & \text { Data not available. } \\ \text { (\#) } & \text { Total Alkalinity as c03--. }\end{array}$ 
TABLE A.8. Static Leach Test on Whole Grout with Groundwater (ST5-1719)

\begin{tabular}{|c|c|c|c|c|c|c|c|c|c|c|c|}
\hline SAMPLE I & --.-- & $\begin{array}{l}\text { HELL } \\
\text { HATER }\end{array}$ & $17-1$ & $19-1$ & $17-2$ & $19-2$ & $17-3$ & $19-3$ & $17-4$ & $19-4$ & $17-6$ \\
\hline IIME & days & ---- & 7 & 7 & 13 & 13 & 20 & 20 & 27 & 27 & 41 \\
\hline pH & & 8.1 & 12.29 & 12.44 & 12.38 & 12.01 & 12.80 & 12.78 & 12.49 & 12.70 & 12.51 \\
\hline Eh & av & 295 & 303 & 302 & 303 & 304 & 214 & 217 & 111 & 112 & 114 \\
\hline Al & $\Delta g / 1$ & $<0.1$ & 5.3 & 13.4 & 11.8 & 11.3 & 14.7 & 14.1 & 14.8 & 13.6 & 15.7 \\
\hline Ba & $9 g / 1$ & 0.06 & 0.007 & 0.006 & 0.008 & 0.008 & 0.007 & 0.007 & 0.009 & 0.007 & $<0.002$ \\
\hline$i$ & $\Delta / 1$ & $.5 b$ & 3.4 & 3.4 & 2.6 & 2.3 & 1.6 & 1.5 & 2.4 & 2.6 & 1.2 \\
\hline Co & $\log / 1$ & 60.005 & 0.007 & $\langle 0.007$ & $<0.004$ & $<0.007$ & 10.004 & 60.607 & $<0.0014$ & $\langle 0.007$ & 60.604 \\
\hline Cr & $g / 1$ & $<0.015$ & $<0.02$ & $<0.02$ & 30.02 & 60.02 & 0.02 & $<0.02$ & $<0.02$ & $<0.02$ & 60.02 \\
\hline $\mathrm{Cu}$ & $\lg / 1$ & $<0.005$ & $<0.004$ & $<0.004$ & 60.004 & $<0.004$ & 60.004 & 60.004 & 10.004 & 60.604 & C6. 0014 \\
\hline $\mathrm{Fe}$ & $g / 1$ & $<0.01$ & 0.011 & 0.008 & $<0.005$ & 10.005 & $<0.005$ & 0.007 & 0.115 & 0.010 & $\angle 0.005$ \\
\hline$k$ & $90 / 1$ & 7.8 & 27 & 33 & 34 & 34 & 39 & 40 & 41 & 40 & 44 \\
\hline Mg & $\lg / 1$ & 14.4 & $<0.06$ & $<0.06$ & $<0.06$ & $<0.06$ & 50.06 & $<0.06$ & $<0.06$ & 30.06 & 60.06 \\
\hline Mn & 11 & 60.01 & 10.002 & $<0.002$ & $<0.002$ & $<0.002$ & $<0.002$ & $\$ 0.002$ & $<0.002$ & (0).602 & 00.0002 \\
\hline $\mathrm{Ha}$ & $g / 1$ & 25 & 523 & 818 & 628 & 819 & 981 & 975 & 1000 & 993 & 1042 \\
\hline$P$ & & $<0.1$ & 0.19 & $\langle 0.1$ & $<0.1$ & 0.20 & 30.1 & 0.1 & 0.12 & 0.30 & 0.17 \\
\hline as P04 & $\ln / 1$ & $\{0.31$ & 0.58 & $\langle 0.3\rangle^{\circ}$ & $\langle 0.31$ & 0.58 & $\langle 0.31$ & $\{\hat{u} .31$ & 0.37 & $0.6 j$ & 3.52 \\
\hline $\mathrm{Pb}$ & $9 i$ & $<0.05$ & 10.06 & $<0.06$ & $<0.06$ & $\angle 0.06$ & $\therefore 0.06$ & 10.06 & 10.160 & Co. 06 & 60.06 \\
\hline $5 i$ & $g / 1$ & 15 & 8.8 & 10.1 & 9.7 & 9.8 & 10.3 & 30.6 & 10.7 & 10.5 & 12.0 \\
\hline $5 f$ & $/ 1$ & 0.25 & 0.063 & 0.085 & 0.087 & 0.084 & 0.090 & 0.086 & 0.096 & 0.0184 & 0.640 \\
\hline$i \pi$ & / & 10.01 & 0.024 & 0.027 & 0.037 & 60.02 & $\langle 0.02$ & 60.02 & 30.62 & 10.62 & 0.02 \\
\hline Ir & & $<0.008$ & $<0.008$ & $<0.008$ & $<0.008$ & 00.008 & (0.008 & 0.008 & $<0.006$ & 1). 100 & 6. 607 \\
\hline F- & & $<0.5$ & 9.4 & 4.5 & 8.1 & 8.8 & 10.0 & 10.0 & 10.0 & $1 i .0$ & 11.0 \\
\hline $\mathrm{Cl}_{1} \mathrm{-}^{\circ}$ & & 22 & 27 & 20 & 25 & 24 & 26 & 25 & 27 & 26 & 23 \\
\hline $\mathrm{inO}^{-}$ & & 00.03 & 0.5 & 0.3 & 0.5 & 0.5 & 0.7 & 0.7 & $0 . \bar{i}$ & 0.7 & 60.3 \\
\hline $\mathrm{NOJ}-$ & & 0.1 & 5.7 & 3.0 & 5.7 & 5.7 & 6.9 & 7.0 & 7.2 & $7 . \mathrm{J}$ & 15.6 \\
\hline $504--$ & & 86 & 170 & 59 & 149 & 153 & 156 & 172 & 161 & 172 & 153 \\
\hline HCOJ- & & 171 & 0.0 & 0.0 & 0.0 & 307.0 & 0.0 & -- & 0.0 & $\dot{0}, \dot{0}$ & $\dot{v}, \dot{0}$ \\
\hline Co3-- & & 2.1 & 428 & 504 & 327 & 950 & 454 & -- & 277 & 346 & 253 \\
\hline $\mathrm{OH}-$ & 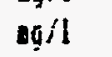 & 0 & 128.5 & 324.7 & 421.0 & 0.0 & 492.7 & -- & $656 . ;$ & 570.3 & S2. \\
\hline i & & 0.06 & 0.414 & 0.814 & 0.739 & 0.674 & 0.329 & 0.829 & 6.244 & $\dot{0} . \Delta \bar{i}$ & 6.900 \\
\hline as 45800 & agil & 0.34 & 2.37 & 4.65 & 4.23 & 3.65 & 4.74 & 4.79 & 4.85 & 4.01 & $\overline{3} .30$ \\
\hline ToC & gil & 1 & $\ldots$ & --- & --- &.-- & --- & ---- & -..- & --- & -..- \\
\hline Fint-.. & & 31.0 & ใ..5 & 0.5 & 60.5 & ४j. & ด.ร & $0 . \bar{J}$ & 0.5 & 61,5 & 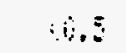 \\
\hline Chitouss & & 5.28 & $23.6 \mathrm{i}$ & 41.90 & 37.06 & i1. 89 & 45.33 & 47.84 & 34.64 & 30.79 & $46.5 i$ \\
\hline HHions & se & 5.54 & 26.67 & 36.84 & 40.05 & 41.39 & 48.74 & -.- & $52.6 \bar{i}$ & 5.96 & $5 . \mathrm{i4}$ \\
\hline 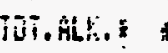 & Agi! & 52.16 & 655 & 1677 & 1071 & 1100 & j323 & $\cdots$ & 1430 & $\mathrm{iski}$ & $i+\div 4$ \\
\hline If & agil & 34.1 & -.. & $-\infty$ & $\ldots$ & $-\cdots$ & --- & $-\cdots$ & $\ldots$ & $\ldots$ & $-\ldots$ \\
\hline
\end{tabular}

(--) Data not available.

(\#) Total Alkalinity as C03--. 
TABLE A.8. contd

\begin{tabular}{|c|c|c|c|c|c|c|c|c|c|c|c|}
\hline MPLE 1 & -..- & $19-6$ & $17-7$ & $19-7$ & $17-8$ & $19-8$ & $17-9$ & $17-10$ & $17-11$ & $17-12$ & $17-13$ \\
\hline TIME & day 5 & 41 & 48 & 48 & 55 & 55 & 62 & 70 & 76 & 104 & 134 \\
\hline $\mathrm{pH}$ & & 12.51 & 12.55 & 12.53 & 12.62 & 12.57 & 12.55 & 12.29 & 12.50 & 12.42 & 12.40 \\
\hline Eh & $\Delta v$ & 117 & 126 & 113 & 152 & 153 & 112 & 143 & 160 & 146 & 142 \\
\hline iil & ag/1 & 12.3 & 16.1 & 15.4 & 15.2 & 14.7 & 15.9 & 15.4 & 14.6 & --- & 17.7 \\
\hline $\mathrm{Ba}$ & o. $/ 1$ & $\{0.002$ & 0.012 & 0.011 & 0.013 & 0.010 & 0.011 & 0.011 & 0.011 & --- & 0.008 \\
\hline Cà & 11 & 1.3 & 3.2 & 3.0 & 2.8 & 2.3 & 3.4 & 3.5 & 2.9 & $\cdots$ & 3.1 \\
\hline Co & i! & $\{0.004$ & $<0.004$ & $<0.004$ & $<0.004$ & 0.008 & $<0.004$ & $<0.004$ & $<0.004$ & $\cdots$ & 00.004 \\
\hline ir & $89 / 1$ & $(0.02$ & $<0.02$ & 10.02 & $<0.02$ & $<0.02$ & 10.02 & $<0.02$ & 0.02 & --- & 60.02 \\
\hline Cu & & $\{0.004$ & $<0.004$ & 50.004 & 0.005 & $<0.004$ & 0.005 & 80.004 & $<0.004$ & --- & 10.004 \\
\hline $\mathrm{Fe}$ & & $\{0.005$ & -0.026 & 0.034 & 0.011 & 0.019 & 0.023 & 0.018 & 0.017 & $\cdots$ & 0.013 \\
\hline$k$ & & 44 & 43 & 42 & 44 & $4 j$ & 45 & 43 & 42 & -- & 42 \\
\hline $\mathrm{Kg}$ & $9 n$ & $<0.06$ & 30.06 & $\$ 0.06$ & $<0.06$ & 00.06 & $\$ 0.06$ & $\{0.06$ & $<0.06$ & $\cdots$ & $\therefore i{ }_{0}$ (1) \\
\hline Mn & lo $i]$ & 60.002 & $<0.002$ & $<0.002$ & $<0.002$ & $<0.002$ & $<0.002$ & $<0.002$ & $(0.002$ & $\cdots$ & 0.0062 \\
\hline $\mathrm{Ha}$ & & 1034 & 1060 & 1027 & 1000 & 980 & 1040 & 1020 & 960 & --- & 1185 \\
\hline$P$ & & 0.1 & $<0.1$ & 0.10 & $<0.1$ & 80.1 & $<0.1$ & 0.10 & 0.10 & --- & $<0.1$ \\
\hline as FO4 & 9 & $<0.31$ & $\langle 0.31$ & $0.3 !$ & $\langle 0.3\}$ & $\langle 0.3 !$ & 10.31 & 0.31 & 0.31 & --- & 40.31 \\
\hline $\mathrm{Pb}$ & of & $<0.06$ & $<0.06$ & 10.06 & 80.06 & 0.06 & $<0.06$ & $<0.06$ & 0.06 & - & 0.06 \\
\hline $5 i$ & $g$ & 8.0 & 12.5 & 13.2 & 12.8 & 13.6 & 13.8 & 14.3 & 14.3 & --- & 16.5 \\
\hline Sr & & 0.037 & 0.103 & 0.068 & 0.103 & 0.084 & 0.107 & 0.099 & 0.094 & --- & 0.050 \\
\hline in & 40̣. & $\{0.02$ & 0.072 & 0.054 & 0.046 & 0.047 & $\$ 0.02$ & 0.030 & 0.020 & $\cdots$ & 0.030 \\
\hline ir & 的 & 0.090 & $\angle 0.008$ & 0.008 & 60.008 & 0.018 & $<0.008$ & 80.008 & $<0.008$ & $\cdots$ & 20.008 \\
\hline$F-$ & & 12.0 & 10.8 & 11.4 & 11.0 & 11.0 & 11.0 & 11.2 & 11.5 & 14.0 & $\cdots$ \\
\hline $\mathrm{Cl}-$ & 10 & 22 & 26 & 25 & 27 & 26 & 19 & 22 & 24 & 23 & $\cdots$ \\
\hline NO:- & al & 0.3 & 0.5 & 0.5 & 0.6 & 0.6 & 0.6 & 0.5 & 0.4 & $<0.1$ & ---- \\
\hline$: 03-$ & $g i$ & 15.0 & 16.0 & 16.0 & 16.0 & 16.0 & 15.0 & 16.0 & 16.0 & 38.0 & $-\cdots$ \\
\hline $504--$ & g. & 150 & 174 & 171 & 168 & 165 & 168 & 167 & 16 & 160 & ---- \\
\hline HeO3- & & 0.0 & 0.0 & 0.0 & 0.0 & 0.0 & 0.0 & 0.0 & 0. & 0.0 & 0.0 \\
\hline 6 inj-- & oull & 302 & 277 & i285 & 253 & 983 & 353 & 227 & 227 & 176 & 315 \\
\hline $\mathrm{OH}-$ & $9 g / 1$ & 690.5 & 655.7 & $\$ 2.6$ & 685.2 & 257.6 & 549.8 & 614.0 & 599.8 & 685.4 & 676.3 \\
\hline 3 & 由all & 0.990 & 0.916 & 0.375 & 0.876 & 0.860 & 0.909 & 0.880 & 0.830 & -- & 0.33 \\
\hline $5 \mathrm{HJBCJ}$ & agi! & 5.is & 5.24 & 5.00 & 5.01 & 4.72 & 5.20 & 5.03 & 4.74 & --- & 9.74 \\
\hline TGC & $a g / 1$ & $-\ldots$ & --- & --- & - & --- . & --- & --- & ---- & $\cdots$ & $-\cdots$ \\
\hline PQ4--- & $\operatorname{ng} / 1$ & 40.5 & (1.0) & 41.0 & $\$ 1.0$ & $\$ 1.0$ & 12.0 & $\langle 5.0$ & 65.0 & 13.0 & $\cdots$ \\
\hline 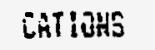 & aeqil & 46.15 & 47.36 & 45.88 & 44.76 & 43.84 & 43.80 & 45.63 & 42.97 & $-\cdots$ & --.- \\
\hline Hillons & aeq $/ 1$ & 50.63 & 50.31 & 47.75 & 53.88 & 52.93 & 49.03 & 46.80 & 48.01 & 51.31 & $\cdots$ \\
\hline TOT. ALSE, & ng̣il & 1380 & 1436 & 1361 & $146 i$ & $143 \dot{b}$ & 1323 & 1310 & 1285 & 1386 & 1512 \\
\hline II & $\lg / 1$ & $\ldots$ & -- & & -.- & $\cdots$ & 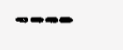 & --- & --- & 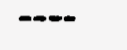 & \\
\hline
\end{tabular}

(--) Data not available.

(\#) Total Alkalinity as C03--. 
TABLE A.8. contd

$$
\begin{aligned}
& 17-14 \cdot 17-15 \\
& 190 \quad 315 \\
& 12.21 \quad 12.83 \\
& 172 \quad 243 \\
& 19.6 \quad 22.6 \\
& 0.007 \quad 0.006 \\
& 1.8 \quad 2.6 \\
& 0.004<0.004 \\
& 60.02 \quad 60.02 \\
& 00.004<0.004 \\
& 0.044 \quad 0.026 \\
& 46 \quad 47 \\
& 0.06<0.06 \\
& \$ 0.002<0.002 \\
& 1125 \quad 1230 \\
& <0.1<0.1 \\
& \text { <0.3! }<0.31 \\
& 60.06 \quad 00.06 \\
& 21.2 \quad 20.0 \\
& \begin{array}{lll}
0.055 & 0.073
\end{array} \\
& 0.065 \quad 0.030 \\
& \because 0.008 \quad<0.008 \\
& 20 \quad 17 \\
& 23 \quad 23 \\
& 10.3<0.1 \\
& 18 \quad 23 \\
& 176 \cdot 229 \\
& 0.0 \quad 0.00 \\
& 504 \quad 774.00 \\
& 571.2 \quad 453.22 \\
& 1.1 \quad 1.21 \\
& 6.29 \quad 6.92 \\
& 10.5 \quad 0.5 \\
& 50.16 \quad 54.81 \\
& 56.08 \quad 59.15 \\
& 1512 \quad 1573.8
\end{aligned}
$$

$---$

(---) Data not available.

(\#) Total Alkalinity as CO3--. 
TABLE A.9. Column Absorption Test an Whole Grout in Sediment (COLTI)

\begin{tabular}{|c|c|c|c|c|c|c|c|c|c|c|c|}
\hline & & & & & & & & & & & \\
\hline SAMPLE & $-\cdots$ & WATER & 1 & 2 & 3 & 4 & 5 & $b$ & 7 & 8 & 3 \\
\hline THME & days & $-\infty$ & 3 & 6 & 9 & 13 & 17 & 20 & 23 & 27 & 30 \\
\hline ph & & B.1 & 12.27 & 12.13. & 12.30 & 12.37 & 12.31 & 12.12 & 12.12 & 11.88 & 12.05 \\
\hline Eh & ay & 295 & 192 & 269 & 165 & 125 & 160 & 185 & 154 & 278 & 155 \\
\hline Al & $9 / 1$ & 80.1 & 1.52 & 3.70 & 4.50 & 6.40 & 6.30 & 5.56 & 6.60 & 7.77 & 7.90 \\
\hline $\mathrm{Ba}$ & gil & 0.06 & 0.008 & $\$ 0.002$ & 10.002 & $<0.002$ & $<0.002$ & $<0.002$ & $<0.002$ & 60.002 & 00.002 \\
\hline $\mathrm{Ca}$ & gil & 56 & 18.1 & 3.6 & 3.3 & 2.9 & 2.2 & 2.0 & 2.2 & 2.1 & 1.8 \\
\hline Co & $g / !$ & $<0.005$ & 50.004 & $<0.004$ & $<0.004$ & 60.004 & 60.004 & $\leqslant 0.004$ & $<0.004$ & $<0.604$ & 10.004 \\
\hline $\mathrm{Cr}$ & $0 / 1$ & 60.015 & $<0.02$ & $<0.02$ & $<0.02$ & $<0.02$ & <0.02 & $<0.02$ & $<0.02$ & $<0.02$ & 0.02 \\
\hline Cu & nig'! & $<0.005$ & 0.060 & 0.008 & 0.020 & $<0.004$ & 0.009 & 0.014 & 0.011 & 0.100 & $<0.004$ \\
\hline$F E$ & gil & 50.01 & $<0.005$ & 0.012 & 0.016 & 0.014 & 0.049 & $<0.005$ & $<0.005$ & 60.005 & 0.030 \\
\hline$k$ & $g / 1$ & 7.8 & 4.2 & 0.3 & 0.3 & 80.3 & 2.3 & $<0.3$ & 60.3 & 50.5 & 2.0 \\
\hline $\mathrm{Hg}$ & $g i l$ & 19.4 & 0.06 & $\leqslant 0.06$ & $(0.06$ & 10.06 & 10.06 & $<0.06$ & $<0.06$ & $<0.06$ & $<0.06$ \\
\hline $\mathrm{Nn}$ & $g / 1$ & $<0.01$ & $<0.002$ & 10.002 & $10.00 \overline{2}$ & $<0.002$ & 10.002 & $<0.002$ & $\{0.002$ & $<0.002$ & $\langle 0.002$ \\
\hline $\mathrm{Na}$ & $g / 1$ & 25 & 794 & 930 & 810 & 760 & 600 & 540 & 570 & 550 & 550 \\
\hline$p$ & $69 / 1$ & \{0.1 & $(0.1$ & 60.1 & $<0.1$ & 0.1 & $<0.1$ & 80.1 & 60.1 & $<0.1$ & 0. 14 \\
\hline $35 \mathrm{POA}_{4}$ & ogil & 10.3 & $\langle 0.31$ & 0.31 & $(0.31$ & 0.31 & $<0.31$ & $(0.31$ & $\{0.31$ & $\{0.3\}$ & 0. 43 \\
\hline $\mathrm{Pb}$ & $\operatorname{ng} / 1$ & $<0.05$ & $<0.06$ & 10.06 & $<0.16$ & $<0.06$ & 80.06 & 10.06 & 10.06 & 10.06 & 10.06 \\
\hline $3 i$ & $\lg i 1$ & 15 & 116.0 & 167.0 & 152.0 & 121.0 & 93.0 & 97.4 & 96.3 & 90.9 & 81.6 \\
\hline$S r$ & $i 1$ & 0.25 & 0.114 & 0.018 & 0.018 & 0.014 & 0.010 & 0.011 & 0.010 & 0.010 & 0.608 \\
\hline $2 n$ & $a g / 1$ & $\{0.01$ & 10.02 & $<0.02$ & 0.050 & 0.060 & 0.080 & $<0.02$ & 60.02 & $<0.02$ & $<0.008$ \\
\hline $2 r$ & & 0.008 & 0.008 & 00.008 & $<0.008$ & $<0.008$ & $<0.008$ & $<0.008$ & $<0.008$ & $10.008^{\circ}$ & $<0.008$ \\
\hline $\mathrm{F}-$ & il & 60.5 & 13.0 & 8.5 & 8.5 & 13.0 & 5.7 & 6.0 & 5.0 & 5.0 & 4.0 \\
\hline [l- & & 22 & 32 & 21 & 23 & 11 & 22 & 23 & 16 & 23 & 21 \\
\hline $\mathrm{H} 02-$ & 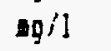 & 0.0 .3 & 30.1 & 33.0 & 13.0 & .3 .0 & 10.1 & 20.1 & 80.1 & 80.1 & $\langle 0.5$ \\
\hline NGS- & agil & 0.1 & 30.0 & 13.0 & 12.0 & 7.7 & 11.0 & 8.0 & 10.0 & 5.0 & 5.0 \\
\hline 304-- & $\$ 0 ! !$ & $\partial \dot{b}$ & 540 & 130 & 140 & 110 & 100 & 117 & 108 & 100 & 110 \\
\hline $\mathrm{HCOSJ}-$ & $9 / 1$ & 171 & 0.0 & 0.0 & 0.0 & 0.0 & 0.0 & 0.0 & 0.0 & 0.0 & 0.0 \\
\hline $\mathrm{COS}_{-1}$ & aqil & 2.1 & 378 & 315 & 252 & 189 & 1235 & 265 & 225 & 412 & 359.7 \\
\hline OH- & $\Delta q ! 1$ & 0 & 321.3 & 464.1 & 374.9 & 339.2 & 14.3 & 235.5 & 283.7 & 139.4 & 0.0 \\
\hline 8 & ogil & 0.06 & 3.300 & 1.100 & 0.940 & 0.610 & 0.420 & 0.470 & 0.392 & 0.320 & 0.299 \\
\hline as HZS:DJ & gil & 0.34 & 18.87 & 6.29 & 5.37 & 3.49 & 2.40 & 2.68 & 2.24 & 1.83 & i. .71 \\
\hline 100 & $\Delta / 1$ & 1 & 32.9 & 11.0 & 15.6 & 6.3 & 5.6 & 7.7 & 5.6 & 4.7 & -... \\
\hline F84--- & $40 / 1$ & 41.0 & $\$ 5.0$ & $\langle 5.0$ & $\langle 5.0$ & 35.0 & $\langle 5.0$ & 85.0 & $\langle 5.0$ & $: 5.0$ & $\{5.0$ \\
\hline EATIOHE & MEQ $/ 1$ & 5.28 & 35.59 & $40.8 j$ & 35.40 & 33.20 & 26.26 & 23.59 & 24.90 & 24.03 & 24.06 \\
\hline AHIONS & Eq/1 & 5.54 & 45.63 & .31 .98 & 34.88 & 29.88 & 45.34 & 26.37 & 27.47 & 25.17 & 22.28 \\
\hline TET.ALK.* & ạil & 32.16 & 945 & 1134 & 914 & 788 & 1260 & 680 & 725 & 658 & 573 \\
\hline IC & agil & 33.1 & 34.3 & 65.1 & 233.7 & 26.1 & 35.9 & 39.2 & 26.5 & 63.2 & $\cdots$ \\
\hline & & & 1.44 & $2.7 !$ & 4.49 & 6.66 & 8.47 & 9.65 & 11.47 & 14.36 & 16 \\
\hline
\end{tabular}

(--) Data not available.

(\#) Total Alkalinity as c03-- 
TABLE A.9. contd

\begin{tabular}{|c|c|c|c|c|c|c|c|c|c|c|c|}
\hline SAMPLE & -.... & 10 & 11 & 12 & 13 & 14 & 15 & 16 & 17 & 18 & 19 \\
\hline TIME & days. & 34 & 37 & 41 & 44 & 49 & 52 & 58 & 62 & 65 & 67 \\
\hline $\mathrm{pH}$ & & 11.50 & 11.86 & 11.74 & 11.54 & 11.46 & 11.75 & 11.66 & 11.32 & 11.32 & 10.76 \\
\hline Eh & iv & 230 & 304 & 196 & 177 & 235 & 253 & 191 & 280 & 220 & 261 \\
\hline Al & $a g / 1$ & 7.17 & 6.91 & 6.68 & 6.46 & 11.00 & 10.80 & 10.00 & 7.34 & 6.01 & 5.65 \\
\hline $8 \mathrm{a}$ & ag/l & $<0.002$ & $<0.002$ & $<0.002$ & $<0.002$ & $<0.002$ & $<0.002$ & $\$ 0.002$ & 0.002 & $(0.602$ & 0.002 \\
\hline Ca & $g / 1$ & 1.6 & 1.6 & 1.4 & 1.4 & 0.7 & 1.0 & 0.8 & 0.8 & 0.3 & 0.7 \\
\hline$\hat{C O U}^{\prime}$ & $\mathrm{ag} / \mathrm{l}$ & $<0.004$ & $<0.004$ & $<0.004$ & $<0.004$ & $<0.004$ & $<0.004$ & $\langle 0,004$ & 10.004 & $(0.00) 9$ & 60.004 \\
\hline $\mathrm{Cr}$ & $g / l$ & $<0.02$ & $<0.02$ & $<0.02$ & $\$ 0.02$ & $<0.02$ & 10.02 & 10.02 & 30.02 & 60.02 & 0.02 \\
\hline Cu & $g / 1$ & $<0.004$ & $<0.004$ & $<0.004$ & $<0.004$ & $<0.004$ & $<0.004$ & 10.004 & 10.004 & 60.604 & 0.004 \\
\hline $\mathrm{Fe}$ & $g / 1$ & 0.033 & 0.038 & 0.032 & 0.024 & 0.038 & 0.041 & 0.031 & 0.032 & 0.641 & 0.032 \\
\hline$k$ & 011 & 2.2 & 1.9 & 2.4 & 1.8 & 2.7 & 1.8 & 2.8 & 2.6 & 3.4 & 3.2 \\
\hline $\mathrm{Mg}$ & $2 g / 1$ & $<0.06$ & 60.06 & $<0.06$ & $<0.06$ & $<0.06$ & 10.06 & 60.06 & $i 0.06$ & $\angle 0.06$ & 40,016 \\
\hline $\mathrm{Mn}$ & $g / 1$ & $<0.002$ & $<0.002$ & $<0.002$ & $<0.002$ & 60.002 & 10.002 & $<0.002$ & $<0.002$ & 10.002 & $\langle 6.002$ \\
\hline $\mathrm{Na}$ & $a / l$ & 414 & 363 & 348 & 346 & 402 & 354 & 347 & 241 & $20 j$ & 195 \\
\hline$P$ & $\log / 1$ & 0.15 & 0.12 & 0.13 & $<0.1$ & $<0.1$ & $<0.1$ & 60.1 & $<0.1$ & 0.12 & 0.11 \\
\hline 25 PO4 & $9 / 1$ & 0.45 & 0.36 & 0.10 & $<0.3$ & $<0.3$ & 60.3 & 0.3 & 10.3 & 0.37 & 0.34 \\
\hline Pb & $g / 1$ & $<0.06$ & 80.06 & $<0.06$ & $<0.06$ & $<0.06$ & 60.06 & $\langle 0.06$ & 0.06 & 60.06 & 10.06 \\
\hline $\mathbf{j i}$ & $a g / 1$ & 67.6 & 58,4 & 55.0 & 52.9 & 50.6 & 42.0 & 41.0 & $24 . ?$ & 21.1 & 22.0 \\
\hline Sr & $g / 1$ & 0.007 & 0.007 & 0.007 & 0.005 & $0.00 \mathrm{j}$ & 0.003 & 0.003 & 0.002 & 0.005 & $\hat{v} .603$ \\
\hline in & $g / 1$ & $<0.008$ & $<0.008$ & $<0.008$ & $\angle 0.00 B$ & $\langle 0.008$ & 0.020 & <ú. (106 & 60.006 & 0.608 & $\{0.006$ \\
\hline Ir & $\lg i l$ & $<0.008$ & $<0.008$ & $<0.008$ & $<0.008$ & 60.008 & $<0.008$ & $\{0.008$ & $<0.006$ & 60.006 & $6 \hat{0.008}$ \\
\hline F- & $\log / 1$ & 4.0 & 3.5 & 3.4 & 5.6 & 3.5 & 4.8 & 4.7 & 3.6 & 4.1 & 4.4 \\
\hline $\mathrm{Cl}-$ & $g / 1$ & 24 & 20 & 21 & 25 & 25 & 23 & 23 & 23 & 10 & 23 \\
\hline 1102- & $\ln / 1$ & 80.1 & $<0.1$ & 10.1 & $<0.1$ & 60.1 & $<0.1$ & 80.1 & 0.1 & 60.1 & 6.1 \\
\hline NOJ- & $\operatorname{sg} i$ & $4 . j$ & 3.6 & 3.5 & 5.0 & 3.8 & 4.1 & 3.9 & 1.7 & 2.1 & 2.3 \\
\hline 504-- & $a g n$ & 96 & 88 & 88 & 100 & 105 & 98 & .88 & 86 & 85 & 98 \\
\hline $\mathrm{HLOJ}-$ & $90 / 1$ & 0.0 & 0.0 & 146.4 & 0.0 & 0.0 & 0.0 & 0.0 & 0.0 & 0.0 & 0.0 \\
\hline co3-- & $\operatorname{mg} / 1$ & 754.4 & 849.9 & 660.8 & 567.3 & 614.1 & 630.3 & 597.8 & 341.6 & 124 & $15 i$ \\
\hline $\mathrm{OH}-$ & $\log / 1$ & 0.0 & 0.0 & 0.0 & 0.0 & 0.0 & 0.0 & 0. & 0.0 & 46.2 & 26.6 \\
\hline$B$ & $2 q / 1$ & 0.243 & 0.211 & 0.202 & 0.193 & 0.397 & 0.340 & 0.330 & 0.186 & 0.195 & $0.2 ! !$ \\
\hline as H3BOJ & $0 q / 1$ & 1.39 & 1.21 & 1.16 & 1.10 & 2.27 & 1.84 & 1.88 & 1.07 & $1.1 i$ & $1.2 !$ \\
\hline TOC & iqil & -- & --- & -- & -- & - & -.- &.-- & -..- & $-\cdots$ & --- \\
\hline PO4--- & $19 / 1$ & 25.0 & $\langle 5.0$ & $\langle 5.0$ & 35.0 & 15.0 & $<5.0$ & 3.0 & 65.0 & 3.6 & $\langle 5.0$ \\
\hline CATIDARS & $\mathrm{q} / 1$ & 18.14 & 15.92 & 15.27 & 15.17 & 17.59 & 15.47 & 15.20 & $10.5 i$ & $8.90^{\circ}$ & 0.60 \\
\hline AHIONS & & 20.26 & 20.44 & 26.73 & 16.57 & 18.04 & 17.11 & 15.71 & 11.26 & 8.50 & 7.66 \\
\hline TOT.ALK.* & $\ln 11$ & 519 & 534 & 722 & 402 & 477 & 423 & 387 & 258 & 209.19 & $20 \mathrm{i}$ \\
\hline II & ig/l & --- & -- & $-\infty$ & -- & --.- & $-\infty$ & --- & $\cdots$ & ---- & --- \\
\hline & & 18.56 & 20.64 & 23.jó & 25.27 & 21.75 & 33.7 & 27.25 & 31.44 & 33.80 & 37.15 \\
\hline
\end{tabular}

(--) Data not available.

(\#) Total Alkalinity as C03--. 
TABLE A.9. contd

\begin{tabular}{|c|c|c|c|c|c|c|c|c|c|c|c|c|}
\hline SAHPLE & ................ & 20 & 21 & 22 & 23 & 24 & 26 & 28 & 30 & 32 & 34 & 36 \\
\hline TIME & days 5 & 72 & 76 & 80 & $B 4$ & 87 & 94 & 100 & 107 & 115 & 121 & 128 \\
\hline $\mathrm{pH}$ & & 11.20 & 10.72 & 10.85 & 10.37 & 10.93 & 10.6 & 10.83 & 9.62 & 9.67 & 9.51 & 9.99 \\
\hline Eh & ev & 204 & 286 & 257 & 151 & 401 & 209 & 259 & 276 & 275 & 235 & 349 \\
\hline Al & $\Delta / 1$ & 5.02 & 4.32 & 4.15 & 3.51 & 3.12 & 2.8 & 2.3 & 1.31 & 1.23 & 1.14 & 1.13 \\
\hline Ba & $89 / 1$ & 60.002 & $<0.002$ & $<0.002$ & $<0.002$ & 10.002 & $<0.002$ & $<0.002$ & $<0.002$ & $<0.002$ & 0.003 & 00.002 \\
\hline Ca & ond & 0.7 & 0.8 & 0.8 & 0.8 & 0.9 & 0.97 & 1.1 & 1.67 & 1.57 & 4.74 & $1 . ?$ \\
\hline Co & sil & $<0.004$ & $<0.004$ & $<0.004$ & $<0.004$ & 80.004 & $<0.004$ & $<0.004$ & $\$ 0.004$ & $<0.004$ & $<0.004$ & $<0.004$ \\
\hline $\mathrm{Cr}$ & 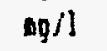 & $\{0.02$ & 30.02 & 30.02 & 50.02 & $<0.02$ & $<0.02$ & $<0.02$ & $<0.02$ & 60.02 & 60.02 & $<0.02$ \\
\hline $\mathrm{Cu}$ & ogil & $<0.004$ & 10.004 & 80.004 & $<0.004$ & $<0.004$ & $<0.004$ & $<0.004$ & $<0.004$ & 10.004 & $<0.004$ & (1.606 \\
\hline $\mathrm{Fe}$ & $a g / 1$ & 0.018 & 0.017 & 0.0009 & 0.007 & 0.009 & 0.019 & 0.01 & 0.014 & 0.009 & 0.009 & 0.000 \\
\hline$k^{\prime}$ & $30 / 1$ & 2.1 & 2.1 & 1.7 & 2.2 & $1: 7$ & 4 & 6.2 & 9 & 14.4 & 19 & 19.1 \\
\hline $\mathrm{Kg}$ & $g / 1$ & $<0.06$ & $<0.06$ & 10.06 & 00.06 & $\{0.06$ & 10.06 & $<0.06$ & $<0.06$ & $<0.06$ & 0.06 & 00.06 \\
\hline $\mathrm{Mn}$ & $0 / 1$ & $<0.002$ & $<0.002$ & $<0.002$ & $<0.002$ & 00.002 & $<0.002$ & $<0.002$ & $<0.002$ & 80.002 & 60.002 & 00.002 \\
\hline $\mathrm{Ha}$ & $a g / 1$ & 171 & 168 & 163 & $15 !$ & 140 & 135 & 119 & 100 & 103 & 104 & 101 \\
\hline$f$ & $g / 1$ & $<0.1$ & $<0.1$ & $<0.1$ & $<0.1$ & $<0.1$ & $<0.1$ & $<0.1$ & 10.1 & $<0.1$ & (i). & 0.1 \\
\hline as P04 & agil & 10.3 & $<0.3$ & 60.3 & 60.3 & $\{0.3$ & $\{0.3$ & 40.3 & $<0.3$ & 60.3 & $10.3^{3}$ & $\{0 . j$ \\
\hline fb & ag $i !$ & $<0.06$ & $<0.06$ & $\{0.06$ & 10.06 & $<0.06$ & $(0.06$ & $\langle 0.06$ & $<0.06$ & 00.06 & $\langle 0.00$ & $(0.06$ \\
\hline $\mathrm{Si}$ & $\operatorname{tg} / 1$ & 19.2 & 20.2 & 18.7 & 15.8 & 16.9 & 15.8 & 11.1 & 8.34 & 10.7 & $1: .8$ & 13 \\
\hline sr & aq $/ 1$ & 0.003 & 0.004 & 0.003 & 0.006 & 0.004 & 0.004 & 0.004 & 0.009 & 0. & 0.022 & 4.614 \\
\hline in & $g i l$ & 0.054 & $<0.008$ & $<0.008$ & 0.066 & 0.078 & 0.060 & $0.0 \dot{0}$ & 0.031 & 0.022 & $60.0 \hat{2}$ & 0.021 \\
\hline $2 r$ & $g / 1$ & 0.008 & $<0.008$ & $<0.008$ & $<0.008$ & 60.008 & 0.068 & $<0.008$ & $<0.008$ & $\langle 0.000$ & 20.208 & $\$ 0.008$ \\
\hline F- & ang 1 & 4.0 & 4.0 & 3.6 & 3.8 & 4.3 & --- & 3 & 3.6 & 3.3 & 3.6 & 3.7 \\
\hline $\mathrm{Cl}-$ & $n g / 1$ & 25 & 28 & 25 & 25 & 26 & $\cdots$ & 23 & 24 & 25 & 22 & 21 \\
\hline $1102-$ & $\operatorname{mg} / 3$ & 10.1 & $<0.1$ & 0.1 & 0.1 & $<0.1$ & $-\cdots$ & $<0.1$ & 0.3 & $\therefore 0.3$ & 4.7 & 3.4 \\
\hline $\mathrm{MES}-$ & $\log / 1$ & 2.2 & 2.0 & 1.8 & 2.1 & 2.8 & ---- & 2 & 4.6 & 4.5 & 7 & 7 \\
\hline SE4-- & onil & 91 & 107 & 91 & 91 & 100 & $-\infty$ & 83 & 87 & 79 & 77 & $3 i$ \\
\hline$H C 0.3-$ & ag/i & 0.0 & 0.0 & 0.0 & 22.4 & $14 i .64$ & 31.48 & 828,67 & 41.37 & 89.18 & 104.92 & 31.46 \\
\hline [153-- & ag $j 1$ & 134 & 154 & 104 & 144 & 51.50 & 118.68 & 123.84 & 41.26 & 30.96 & 25.86 & 61.32 \\
\hline $\mathrm{OH}^{-}$ & -gil & 26.1 & 9.1 & 31.7 & 0.0 & 0.00 & 0.00 & 0.00 & 0.00 & 0.00 & 0.00 & 0.30 \\
\hline 6 & dquil & 0.193 & 0.219 & 0.183 & 0.376 & 4.209 & 0.17 & 0.15 & 0.164 & 0.175 & $0.19 !$ & $0.19 ?$ \\
\hline A5 H3̈ล03 & $\operatorname{cg} i 1$ & 1.10 & 1.25 & 1.04 & 1.01 & 1.17 & 0.97 & 0.85 & 0.93 & 1 & 1.09 & 1.13 \\
\hline TQC & $20 / 1$ & $\cdots$ & $-\cdots$ & $\cdots$ & --- & $\cdots$ & $\cdots$ & $-\cdots$ & 3.13 & 2.58 & 1.6 & 2.31 \\
\hline P04--- & ggil & 15.0 & 65.0 & 6.0 & 15.0 & is.o & & 60.3 & 60.4 & 60.4 & 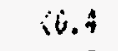 & 60.4 \\
\hline Sitrons & aeqis & $7.5 j$ & 7.40 & 7.18 & 6.67 & 6.53 & 6.03 & 5.35 & 4.67 & 4.33 & 5.25 & 4.95 \\
\hline AH1045 & neqii & 7.01 & 9.09 & 8.31 & 8.16 & 7.13 & ---- & 20.78 & 4.22 & 5.65 & 5.03 & 5.25 \\
\hline TOT. ALK.: & $3 q / 1$ & 180 & 170 & 160 & 155 & $12 !$ & 134.16 & 531.48 & 61.82 & $74 . \overline{8} \bar{i}$ & 37.4 & 77.4 \\
\hline II & $69 / 1$ & --- & --- & $\cdots$ & --- & $\cdots$ & --- & --- & 21.66 & 19.64 & 27.23 & 20.93 \\
\hline f. VQL. & & 39.13 & 41.76 & 46.26 & 47.11 & 51.02 & 56.32 & 64.06 & 67.83 & $72 . j$ & 74.56 & 73.24 \\
\hline
\end{tabular}

(--) Data not available.

(\#) Total Alkalinity as CO3-.. 
TABLE A.9. contd

\begin{tabular}{|c|c|c|c|c|c|c|c|c|}
\hline SAMFLE & & 39 & 41 & 43 & 45 & 47 & 49 & 50 \\
\hline TIME & days & 142 & 153 & 163 & 172 & 181 & 221 & 251 \\
\hline $\mathrm{PH}$ & & 9.89 & 9.49 & 9.4 & 9.5 & 8.45 & 7.99 & \\
\hline Eh & av & 359 & 236 & 371 & 367 & 353 & 332 & $2 B$ \\
\hline $\mathrm{Al}$ & $19 / 1$ & 1.23 & 0.871 & 0.611 & 0.468 & 0.38 & 0.297 & 0.165 \\
\hline 82 & $9 / 1$ & $<0.002$ & $<0.002$ & $<0.002$ & 0.004 & 0.004 & 0.004 & 0.008 \\
\hline$C_{2}$ & $g / 1$ & 1.88 & 2.42 & 3.22 & 4.9 & 5.07 & 6.65 & 14.1 \\
\hline Cd & $g / 1$ & $<0.004$ & 60.004 & $<0.004$ & $<0.004$ & $<0.004$ & $<0.004$ & 10.004 \\
\hline $\mathrm{Cr}$ & $9 g / 1$ & 60.02 & $<0.02$ & $<0.02$ & $<0.02$ & $<0.02$ & 10.02 & 0.02 \\
\hline Cu & $\lg / 1$ & $<0.004$ & $<0.004$ & $<0.004$ & $<0.004$ & $<0.004$ & $<0.604$ & $<0.004$ \\
\hline $\mathrm{Fe}$ & $a q / 1$ & 0.005 & 0.012 & 0.007 & $<0.005$ & 0.01 & 0.01 & 10.005 \\
\hline$K$ & $a g / 1$ & 20 & 22 & 23 & 24 & 21 & 17 & 16 \\
\hline Ng & $9 / 1$ & $<0.06$ & $<0.06$ & $<0.06$ & $<0.06$ & $<0.16$ & $<0.06$ & 0.519 \\
\hline in & $9 / 1$ & $<0.002$ & $<0.002$ & $<0.002$ & $<0.002$ & 0.003 & 60.003 & $<0.002$ \\
\hline $\mathrm{Ha}$ & $g / 1$ & 85.4 & 74 & 67 & 64.8 & 77.2 & 62.3 & 52.7 \\
\hline 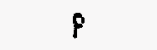 & $\lg / 1$ & $<0.1$ & $<0.1$ & $<0.1$ & 0.1 & $<0.1$ & $<0.1$ & $<0.1$ \\
\hline 25 P04 & $9 / 1$ & 60.3 & $<0.3$ & $<0.3$ & $<0.3$ & $<0.3$ & 60.3 & $<0.3$ \\
\hline $\mathrm{Fb}$ & $\lg / 1$ & $\{0.06$ & 10.06 & 10.06 & 00.06 & 00.06 & $<0.06$ & 0.06 \\
\hline $\mathrm{si}$ & $\Delta g / 1$ & 9.11 & 6.5 & 6.29 & 6.78 & 3.43 & 6.6 & $7 ; 08$ \\
\hline or & $9 / 1$ & 0.008 & 0.012 & 0.015 & 0.022 & 0.025 & 0.032 & 0.084 \\
\hline in & $9 / 1$ & 60.02 & $<0.02$ & 30.02 & $<0.02$ & $\$ 0.02$ & $(0.02$ & 60.02 \\
\hline Ir & $9 / 1$ & $\angle 0.00 B$ & 10.008 & $<0.008$ & 10.008 & $<0.008$ & $<0.008$ & $<0.008$ \\
\hline F- & ag/1 & 3.7 & 4 & 3.6 & 4.3 & 4.2 & 3.6 & 3.02 \\
\hline $\mathrm{Cl}-$ & $/ 1$ & 23.7 & 23.7 & 23.3 & 27 & 25 & 23 & 22.1 \\
\hline 102- & 11 & 1.4 & 1.1 & 1 & 1.4 & 1.4 & 60.3 & 60.3 \\
\hline HOS- & & 6.2 & 7.8 & 6.9 & 6.8 & 19 & 60.5 & 4.92 \\
\hline 504-- & /1 & 82 & $B 1$ & 78 & 32 & 84 & 80 & 78.8 \\
\hline $\mathrm{HCOS-}$ & ogil & 42.05 & 62.95 & 47.21 & 15.74 & 52.46 & 56.12 & 50.12 \\
\hline CO3-- & $90 / 1$ & 46.40 & 20.64 & 20.64 & 30.96 & 15.46 & 0.00 & 0.00 \\
\hline OH- & $a / 1$ & 0.00 & 0.00 & 0.00 & 0.00 & 0.00 & 0.00 & 0.00 \\
\hline B & $\log / 1$ & 0.143 & 0.138 & 0.13 & 0.162 & 0.198 & 0.146 & 0.14 \\
\hline as 43500 & $\lg / 1$ & 0.32 & 0.79 & 0.74 & 0.93 & 1.13 & 0.63 & 0.8 \\
\hline TOC & $g / 1$ & 6.91 & 2.37 & 2.49 & $\cdots$ & 0.9 & $0 . \overline{8} \bar{i}$ & ---- \\
\hline P04-- & $\log / 1$ & $<0.4$ & $<0.4$ & 0.4 & <0.4 & 60.4 & 0.4 & 60.4 \\
\hline CATIOHS & seq/1 & 4.32 & 3.91 & 3.67 & 3.68 & 4.15 & 3.48 & 3.45 \\
\hline AHIOHS & aeq/] & 4.95 & 4.45 & 4.08 & 4.14 & 4.34 & 3.43 & 3.42 \\
\hline TOT.ALK, & $g / 1$ & 67.08 & 51.6 & 13.86 & 38.7 & 41.28 & 27.6 & 27.6 \\
\hline If & $\log / 1$. & 18.28 & 17.18 & 15.58 & --- & 13.74 & 10.5 &..-- \\
\hline P. YOL. & & 30.19 & 95.45 & 101.7 & 107.75 & 111.33 & 135.14 & 151.35 \\
\hline
\end{tabular}

(--) Data not available.

(\#) Total Alkalinity as C03--. 
TABLE A.10. Column Absorption Test on Whole Grout in Sediment (COL1)

\begin{tabular}{|c|c|c|c|c|c|c|c|c|c|c|c|}
\hline SAMPLE & --.-- & $\begin{array}{l}\text { WELL } \\
\text { WATER }\end{array}$ & 1 & 2 & 3 & 4 & 5 & 6 & 7 & 8 & 9 \\
\hline TIME & days & --.-- & 3 & 6 & 9 & 13 & 17 & 20 & 23 & 27 & 30 \\
\hline $\mathrm{pH}$ & & B.1 & 12.45 & 12.21 & 12.35 & 12.59 & 12.35 & 12.29 & 12.48 & 12.10 & 12.08 \\
\hline Eh & av & 295 & 257 & 180 & 310 & 173 & 252 & .220 & 160 & 202 & 294 \\
\hline Al & $g / 1$ & $<0.1$ & 1.52 & 7.50 & 4.80 & 7.50 & 9.30 & 8.90 & 9.94 & 10.00 & 10.30 \\
\hline $8 a$ & $\log / 1$ & 0.06 & 0.008 & $<0.002$ & $<0.002$ & $<0.002$ & $<0.002$ & $<0.002$ & $<0.002$ & $<0.002$ & 10.002 \\
\hline $\mathrm{Ca}$ & $g / 1$ & 56 & 19.1 & 3.8 & 3.6 & 3.0 & 2.5 & 2.4 & 1.9 & 1.9 & 1.7 \\
\hline$c d$ & $0 / 1$ & $<0.005$ & $<0.004$ & $<0.004$ & $<0.004$ & $<0.004$ & $<0.004$ & $<0.004$ & $<0.004$ & $<0.004$ & $<0.004$ \\
\hline Er & $9 / 1$ & $<0.015$ & $<0.02$ & $<0.02$ & $<0.02$ & $<0.02$ & $<0.02$ & $<0.02$ & $<0.02$ & $\leqslant 0.02$ & $\{0.02$ \\
\hline $\mathrm{Cu}$ & $\log / 1$ & 00.005 & 0.076 & 0.038 & 0.022 & 0.016 & 0.008 & 0.018 & 0.005 & $<0.004$ & $<0.004$ \\
\hline $\mathrm{Fe}$ & $\exists g / 1$ & $\{0.01$ & 10.005 & 0.020 & $<0.005$ & 0.024 & $<0.005$ & $\langle 0.005$ & $<0.005$ & $<0.005$ & 0.038 \\
\hline$k$ & $\log / 1$ & 7.8 & $6 . J$ & 1.9 & 3.9 & 60.3 & 1.6 & 2.8 & 1.4 & 1.9 & 1.8 \\
\hline $\mathrm{H}_{9}$ & $g i l$ & 14.4 & $<0.06$ & $<0.06$ & $<0.06$ & $<0.06$ & $<0.06$ & $<0.06$ & $<0.06$ & $<0.06$ & $<0.16$ \\
\hline rin & 90 & $\langle 0.0 !$ & $<0.002$ & $<0.602$ & $<0.002$ & $<0.002$ & $<0.002$ & $<0.002$ & $<0.002$ & $<0.002$ & 60.002 \\
\hline $\mathrm{Na}$ & $\log / 1$ & 25 & 1130 & 1080 & . 890 & 820 & 530 & 710 & 640 & 590 & 590 \\
\hline $\mathbf{P}$ & $g / 1$ & $<0.1$ & 0.20 & 60.1 & $<0.1$ & $<0.1$ & $<0.1$ & 0.17 & 0.13 & 0.15 & $<0.1$ \\
\hline as $\mathrm{POA}$ & $g / 1$ & $\vdots 0.3$ & $\{0.3$ & $\{0.3$ & 10.3 & $<0.3$ & $\langle 0.3$ & 0.52 & 0.39 & 0.46 & $<0.3$ \\
\hline $\mathrm{Pb}$ & $\log / 1$ & $<0.05$ & $<0.06$ : & 10.06 & 00.06 & 10.06 & $<0.06$ & 10.06 & $<0.06$ & 0.06 & $\$ 0.06$ \\
\hline si & $\operatorname{ag} i !$ & 15 & 103.0 & 150.0 & 159.0 & 115.0 & 103.0 & 108.0 & 89.4 & 85.9 & 36.8 \\
\hline $5 r$ & $\lg i$ & 0.25 & 0.126 & 0.020 & 0.018 & 0.014 & 0.012 & 0.012 & 0.009 & 0.010 & 0.008 \\
\hline in & $g i !$ & $<0.01$ & 10.02 & 10.02 & 0.040 & $\{0.02$ & 0.050 & $<0.02$ & $(0.02$ & 10.02 & 60.02 \\
\hline ir & $\lg / 1$ & $\therefore 0.008$ & $<0.008$ & 60.008 & $<0.008$ & 0.1 & 10.008 & 80.008 & $<0.008$ & 40.008 & 10.008 \\
\hline F- & ag̣ /1 & \{0.5 & 22.0 & 16.0 & 12.0 & 11.0 & 11.0 & 9.0 & 8.0 & 8.0 & 3.0 \\
\hline $\mathrm{Cl}-$ & $9 / 1$ & 22 & 34 & 22 & 23 & 21 & 21 & 24 & 24 & 23 & 22 \\
\hline HQ2:- & $9 g / 1$ & 00.03 & $<0.1$ & 33.0 & $\$ 3.0$ & $<3.0$ & $\{0.1$ & $<0.1$ & 10.1 & $\$ 0.1$ & 60.5 \\
\hline $1605-$ & $60 / 1$ & 0.1 & 96.0 & 22.0 & 13.0 & 11.0 & 11.0 & 8.8 & 3.0 & 5.3 & 5.0 \\
\hline $304--$ & gil & 86 & 880 & 230 & 175 & 160 & 150 & $144^{\circ}$ & 137 & 116 & 110 \\
\hline $\mathrm{HLO3}^{-}$ & ay !! & 171 & 0.0 & 0.0 & 0.0 & $0 . \hat{0}$ & $0 . \hat{v}$ & 0.0 & 0.0 & 0.0 & 0.0 \\
\hline $\mathrm{COB}--$ & in $i !$ & 2.1 & 504 & 315 & 378 & 252 & 378 & 376 & 223 & 314 & 266 \\
\hline $3 \mathrm{~K}^{-}$ & agil & 0 & 392.7 & 589.1 & 303.5 & 392.7 & 370.3 & 310.6 & 300.4 & 214.2 & 218.2 \\
\hline E & inil & 0.06 & 5.240 & 2.300 & 1.400 & 1.200 & 1.460 & $0.240^{\circ}$ & 0.570 & 0.804 & 0.523 \\
\hline d5 $H 33063$ & agi 1 & 0.34 & 27.97 & ij.ió & 6.00 & 6.36 & 8.00 & 4.84 & 4.90 & 9.60 & 2.99 \\
\hline TOC & $\pm 0 / 1$ & i & 21.4 & 11.6 & 11.1 & 0.2 & 5.9 & 7.4 & 4.7 & 3.8 & --- \\
\hline PQS4-- & $\operatorname{tg} i$ & $(0.1$ & 6.5 & 6.5 & $<.5$ & 6.5 & $\therefore .5$ & $<.5$ & 6.5 & <.5 & 6.5 \\
\hline Cinlughs & 由ec /! & 5.28 & 50.25 & 47.26 & 38.93 & 35.82 & 23.21 & 31.07 & 27.96 & 25.80 & 25.78 \\
\hline AHIOHS & SEq/1 & 5.54 & 62.66 & 51.76 & 35.81 & 36.40 & 40.54 & 35.16 & 29.11 & 26.64 & 25.06 \\
\hline Tot. AlLA. & $\mathrm{ag} / 1$ & 92.16 & 1197 & 1355 & 914 & 945 & 1077 & 926 & 753 & 692 & $65 !$ \\
\hline $\mathbb{I}$ & $\mathrm{ag} / 1$ & 33.1 & 45.4 & 50.2 & 56.7 & 30.3 & 48.6 & 46.2 & 37.1 & 37.6 & --- \\
\hline F. VOL. & & & 1.33 & 2.72 & 4.15 & 5.89 & 0.72 & 3.01 & 9.67 & 11.94 & 13.74 \\
\hline
\end{tabular}

\footnotetext{
(--) Data not available.

(\#) Total Alkalinity as c03--.
} 


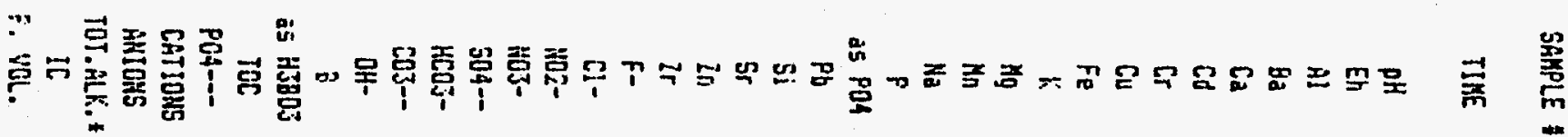

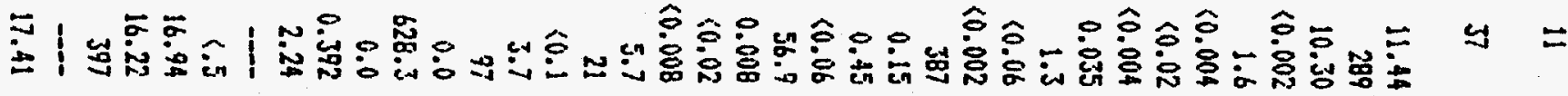
广̊

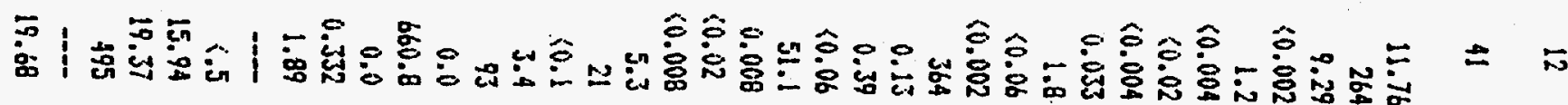
垫|

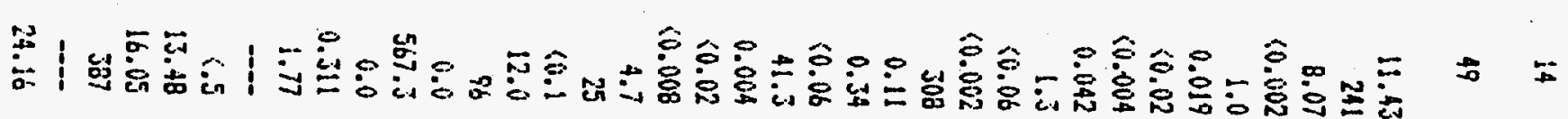

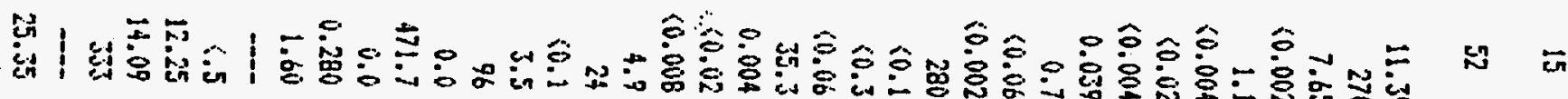
算| 出|

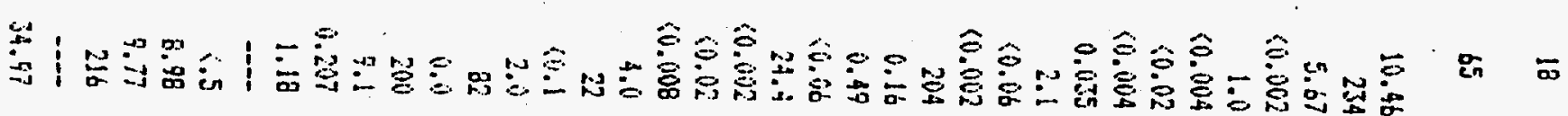

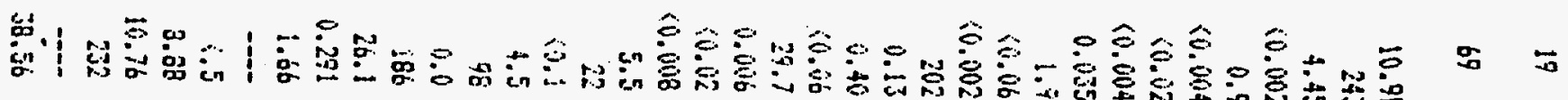


TABLE A.10. contd

\begin{tabular}{|c|c|c|c|c|c|c|c|c|c|c|c|}
\hline SAYPLE & -...- & 20 & 22 & 23 & 24 & 26 & 28 & 30 & 32 & 34 & 36 \\
\hline TIME & days & 72 & 80 & 84 & 87 & 94 & 100 & 107 & 115 & 121 & 128 \\
\hline pH & & 10.98 & 11.38 & 11.33 & 11.08 & 11.52 & 10.8 & 9.99 & 10.31 & 10.17 & 9.3 \\
\hline Eh & av & 210 & 319 & 151 & 395 & 261 & 227 & 258 & 230 & 246 & 341 \\
\hline Al & $a g / 1$ & 4.69 & 5.75 & 5.84 & 5.13 & 5.1 & 3.9 & 2.44 & 1.97 & 1.68 & 1.27 \\
\hline 83 & $n g / 1$ & 0.002 & $<0.002$ & $<0,002$ & $<0.002$ & $<0.002$ & $<0.002$ & $<0.002$ & $10.00_{2}^{n}$ & 10.002 & $<0.002$ \\
\hline$C_{3}$ & $\mathrm{ag} / 1$ & 1.0 & 0.8 & 0.9 & 0.9 & 0.85 & 1.1 & 1.37 & 1.11 & 1.19 & 1.81 \\
\hline Cd & og/l & 60.004 & 10.004 & 60.004 & $<0.004$ & $<0.004$ & $<0.004$ & 80.004 & $<0.004$ & $<0.004$ & $\$ 0.004$ \\
\hline$c r$ & $\Delta / 1$ & $<0.02$ & $<0.02$ & $<0.02$ & 60.02 & $<0.02$ & $\langle 0.02$ & $<0.02$ & 10.02 & 10.02 & 80.02 \\
\hline $\mathrm{Cu}$ & ig & $<0.004$ & $\$ 0.004$ & $<0.004$ & $<0.004$ & $<0.004$ & $<0.004$ & 80.004 & $<0.004$ & $<0.004$ & $<0.004$ \\
\hline Fe & ag $/ 1$ & 0.020 & 0.023 & 0.008 & 0.011 & 0.015 & 0.017 & 0.028 & 0.024 & 60.005 & 0.007 \\
\hline$k$ & $a / l$ & 1.8 & 1.2 & $1 . j$ & 0.9 & 2.6 & 2.6 & 2.5 & 3 & 4.9 & 8.7 \\
\hline $\mathrm{Mg}$ & $g / 1$ & $<0.06$ & $<0.06$ & $<0.06$ & $<0.06$ & 60.06 & 20.06 & $\{0.08$ & $\$ 0.06$ & 10.06 & 0.06 \\
\hline $\mathrm{Mn}$ & nด $/ 1$ & 0.002 & $<0.002$ & $<0.002$ & $(0.002$ & $<0.002$ & $<0.002$ & $<0.002$ & $\langle 0.002$ & 80.002 & $<0.002$ \\
\hline $\mathrm{Ha}$ & $g / 1$ & $17 !$ & 250 & 216 & 202 & 177 & 147 & 129 & 123 & 104 & 101 \\
\hline$f$ & $g / 1$ & 60.1 & $<0.1$ & 60.1 & $<0.1$ & $<0.1$ & $<0.1$ & $<0.1$ & 0.1 & $<0.1$ & 0.1 \\
\hline as 904 & $9 q / 1$ & $\{0.3$ & 10.3 & $<0.3$ & 10.3 & 60.3 & $<0.3$ & 10.3 & 60.3 & $\langle 0.3$ & 10.3 \\
\hline$F b$ & $\pm 9 ! 1$ & $<0.06$ & $<0.06$ & $<0.06$ & $<0.06$ & $\langle 0.06$ & $<0.06$ & $<0.06$ & 10,06 & 0.06 & 0.06 \\
\hline$j i$ & $9 / 1$ & 33.0 & 39.0 & 30.3 & 28.0 & 21.5 & 14.3 & 11.9 & 13.4 & 11.1 & 9.95 \\
\hline sr & eqh & 0.004 & 0.007 & 0.005 & 0.006 & 0.004 & 0.005 & 0.008 & 0.006 & 0.004 & 0.009 \\
\hline in & $\operatorname{mg} / 1$ & 0.092 & 0.019 & 0.205 & 0.062 & 0.04 & 0.02 & 0.025 & 0.021 & $\langle 0.02$ & 0.053 \\
\hline Ir & ogil & 60.008 & $<0.008$ & $<0.008$ & 10.008 & $\angle 0.008$ & $<0.008$ & 0.016 & 0.01 & 0.013 & $<0.008$ \\
\hline$F-$ & agil & 5.8 & 4.6 & 3.7 & 4.1 & 3.2 & 3.1 & 3.3 & 3.1 & 2.6 & 3.3 \\
\hline Cl- & ag 11 & 25 & 24 & 23 & 25 & 23 & 24 & 24 & 23 & 21 & 23 \\
\hline wo2- & ug/l & 80.1 & $<0.1$ & $<0.1$ & 0.1 & 0.1 & $<0.1$ & $<0.3$ & 10.3 & 2.5 & 4.3 \\
\hline HCIS- & $\mathrm{g} / 1$ & 6.8 & 3.7 & 2.3 & 2.8 & 1.6 & 1.5 & 1.9 & 1.6 & i.4 & 2.3 \\
\hline S04-- & agil & 110 & 106 & 91 & 97 & 86 & 88 & $B 6$ & 79 & 72 & al \\
\hline $\mathrm{HCDS}-$ & ag/1 & 0.0 & 0.0 & 22.4 & 299.02 & 0.00 & 365.76 & 141.64 & 99.67. & 26.23 & 57.71 \\
\hline 2033-- & ag $/ 1$ & 184 & 154 & 144 & 30.96 & 129.00 & 10.32 & 20.64 & 41.28 & 77.40 & 36.12 \\
\hline AH- & no $/ 1$ & 32.9 & 67.4 & 0.0 & 0.00 & 33.63 & 0.00 & 0.00 & 0.00 & 0.00 & 0.60 \\
\hline$B$ & aqi & 0.313 & 0.302 & 0.207 & 0.223 & 0.17 & 0.15 & 0.167 & 0.167 & 0.138 & 0.155 \\
\hline as 43603 & มุ̣i! & 1.79 & 1.72 & 1.18 & 1.27 & 0.97 & 0.85 & 0.76 & 0.96 & 0.79 & 0.287 \\
\hline TOC & anil & $\cdots$ & $-\infty$ & -- & $\cdots$ & --- & --- & $\cdots$ & 2.13 & $\cdots$ & --- \\
\hline FD4- - & Kg $/ 1$ & $\therefore .5$ & 6.5 & 8.5 & 6.5 & 8.5 & i.5 & $s 0.4$ & $\langle 0.4$ & 60.4 & 80.4 \\
\hline Cirillons & neq $/ 1$ & 7.53 & 10.95 & 9.48 & 2.86 & $7.8 !$ & 6.52 & 5.75 & 5.49 & $4.7 !$ & 4.71 \\
\hline ANIONS & equ/l & 11.48 & 12.31 & 7.96 & 3.74 & 6.93 & 9.06 & 5.70 & 5.28 & 5.66 & 4.39 \\
\hline TOT. ALLK.: & moil & 243 & 273 & 155 & 178 & 188.34 & 190.2 & 70.3 & 90.3 & 90.3 & 64.5 \\
\hline if & $n$ & ---- & $\cdots$ & $\cdots$ & $-\cdots$ & -- & $-\infty$ & $-\cdots$ & 18.7 & --- & ---- \\
\hline F. VCL. & & 39.63 & 42.27 & 44.7 & 46.18 & 50.99 & 57.84 & 61.36 & 66.1 & 72.36 & 77.15 \\
\hline
\end{tabular}

(--) Data not available.

(\#) Total Alkalinity as C03--. 
TABLE A.10. contd

\begin{tabular}{|c|c|c|c|c|c|c|c|c|}
\hline \multicolumn{2}{|c|}{ SAMPLE $1 \cdots$} & 39 & 41 & 43 & 45 & 47 & 49 & 50 \\
\hline TIME & days & 142 & 149 & 156 & 168 & 177 & 213 & 243 \\
\hline DH & & 9.33 & 9.32 & 9.42 & 9.16 & 8.17 & 8.08 & B.11 \\
\hline Eh & av & 364 & 301 & 383 & 372 & 322 & 326 & \\
\hline Al & $\ln / 1$ & 0.898 & 0.666 & 0.515 & 0.376 & 0.205 & 0.101 & 0.171 \\
\hline $\mathrm{Ba}$ & $\lg / 1$ & 0.003 & 0.004 & 0.006 & 0.007 & 0.011 & 0.009 & 0.01 \\
\hline Ca & $19 / 1$ & 2.69 & 3.58 & 5.1 & 6.48 & 13 & 12.5 & 14.6 \\
\hline Cd & $g / 1$ & $<0.004$ & $<0.004$ & $<0.004$ & $<0.004$ & $\$ 0.004$ & $<0.004$ & 0.004 \\
\hline $\mathrm{Cr}$ & $9 g / 1$ & $<0.02$ & $<0.02$ & $<0.02$ & $<0.02$ & $<0.02$ & $\langle 0.02$ & $<0.02$ \\
\hline Cu & $2 g / 1$ & $<0.004$ & $<0.004$ & $<0.004$ & $<0.004$ & $<0.004$ & $<0.004$ & 30.004 \\
\hline $\mathrm{Fe}$ & $g / 1$ & 0.01 & 0.005 & 0.008 & 0.005 & 0.005 & $<0.005$ & 60.005 \\
\hline$k$ & $\operatorname{sg} / 1$ & 21 & 27 & 29 & 26 & 22 & 13 & 12 \\
\hline $\mathrm{Mg}$ & $\operatorname{sg} / 1$ & $<0.06$ & $<0.06$ & 0.151 & 0.371 & 1.66 & 2.16 & 3.45 \\
\hline Mn & $\log / 1$ & $<0.002$ & $<0.002$ & $<0.002$ & $<0.002$ & $<0.002$ & $<0.002$ & $<0.002$ \\
\hline $\mathrm{Ha}$ & ingil & 84.2 & 69.6 & 64.1 & 64.3 & 51.8 & 56.5 & 52.9 \\
\hline $\mathbf{P}$ & $\operatorname{sg} / 1$ & $<0.1$ & $<0.1$ & $<0.1$ & $<0.1$ & 0.1 & $<0.1$ & 60.1 \\
\hline d5 P04 & $g g / 1$ & $<0 . j$ & $<0.3$ & $<0.3$ & $<0.3$ & 80.3 & 60.3 & 0.3 \\
\hline $\mathrm{Fb}$ & ogil & $<0.06$ & $<0.06$ & $<0.06$ & $<0.06$ & $<0.06$ & $<0.06$ & 10.06 \\
\hline $5 i$ & $\Delta g / 1$ & 8.65 & 7.19 & 7.31 & 8.05 & 8.53 & 8.5 & 7.08 \\
\hline ir & g / / & 0.012 & 0.018 & 0.029 & 0.037 & 0.08 & 0.072 & 0.084 \\
\hline in & egil & $<0.02$ & $<0.02$ & $<0.02$ & 80.02 & $<0.02$ & $\langle 0,02$ & $<0.02$ \\
\hline Ir & $9 / 1$ & $<0.008$ & $<0.008$ & $<0.008$ & $<0.008$ & $<0.008$ & 0.016 & 00.008 \\
\hline$F-$ & $g / 1$ & 3.1 & 3.1 & 2.7 & .3 & 4.2 & & 2.95 \\
\hline $\mathrm{Cl}-$ & $9 / 1$ & 25.8 & 22.6 & 23 & 22.6 & 23 & 27 & 22.6 \\
\hline $102-$ & and & 3.1 & 2.5 & 1.8 & 0.8 & 1.4 & $\{0.3$ & 60.3 \\
\hline HOJ- & $/ 1$ & 3.6 & 5.3 & 5.3 & 4.4 & 5.7 & $\langle 0.5$ & 9.58 \\
\hline $504--$ & $\log / 1$ & 79 & 77 & 76 & 80 & 72 & 80 & 78.8 \\
\hline HCOJ- & $g / 1$ & 47.25 & 2.46 & 57.71 & 57.71 & 69.05 & .49 .27 & 39.26 \\
\hline $\mathrm{COJ}--$ & $n$ & 30.96 & 20.64 & 15.48 & 15.48 & 10.32 & 0.00 & 11.09 \\
\hline BH- & $0 / 1$ & 0.00 & 0.00 & 0.00 & 0.00 & 0.00 & 0.00 & 0.06 \\
\hline H & agil & 0.135 & 0.132 & 0.125 & 0.157 & 0.117 & 0.136 & 6.134 \\
\hline $15 \mathrm{H3B03}$ & $19 / 1$ & 0.772 & 0.75 & 0.71 & 0.76 & 0.67 & 0.78 & 0.77 \\
\hline TOC & $9 / 1$ & $\cdots$ & -- & -.- & $\cdots$ & --- & $\cdots$ & --- \\
\hline PO4--- & & 60.4 & 60.4 & 80.4 & 60.4 & 60.4 & 60.4 & 86.4 \\
\hline CATIOHS & il & 4.34 & 3.30 & 3.80 & 3.82 & 3.60 & 3.59 & 3.62 \\
\hline AMIGNS & Aeq/1 & 4.48 & 4.10 & 3.97 & 4.02 & 3.97 & 3.39 & 3.60 \\
\hline TOT.ALK.A & $g / 1$ & 54.2 & 46.44 & 43.86 & 43.86 & 44.28 & 24.24 & 30.36 \\
\hline$\Omega$ & $a g / 1$ &.- & $\cdots$ & --- & -.- & $-\infty$ & $\cdots$ & --- \\
\hline V VUL. & & 39.25 & 94.68 & 102.67 & 111.94 & 123.04 & 149.07 & $16 \overline{1.17}$ \\
\hline
\end{tabular}

(--) Data not available.

(\#) Total Alkalinity as C03--. 
TABLE A.11. Column Absorption Test on Whole Grout in Sediment (COLC1)

\begin{tabular}{|c|c|c|c|c|c|c|c|c|c|c|c|}
\hline SAMPLE & -...... & $\begin{array}{l}\text { HELL } \\
\text { HATER }\end{array}$ & 1 & 2 & 3 & 4 & 5 & 6 & 7 & $B$ & 9 \\
\hline TIME & days & - & 3 & 6 & 9 & 13 & 17 & 20 & 23 & 27 & 30 \\
\hline pH & & 8.1 & 12.59 & 12.16 & 12.45 & 12.37 & 12.02 & 12. 18 & 12.10 & 11.76 & 11.87 \\
\hline Eh & ov & 295 & 181 & 284 & 136 & 156 & 210 & 231 & 162 & 312 & 183 \\
\hline Al & $19 / 1$ & $<0.1$ & 4.72 & 5.80 & 8.40 & 7.80 & 6.50 & 7.70 & 8.84 & 9.80 & 9.48 \\
\hline $8 a$ & $a g / 1$ & 0.06 & 0.006 & $<0.002$ & $<0.002$ & $<0.002$ & $<0.002$ & $<0.002$ & $<0.002$ & $<0.002$ & $<0.002$ \\
\hline $\mathrm{Cd}_{\mathrm{d}}$ & ind & 56 & 10.2 & 3.0 & 3.1 & 2.4 & 2.0 & 1.8 & 1.7 & 1.8 & 1.6 \\
\hline Cd & og $/ 1$ & $<0.005$ & $<0.004$ & $<0.004$ & $<0.004$ & $<0.004$ & $<0.004$ & $<0.004$ & $<0.004$ & $<0.004$ & $<0.004$ \\
\hline $\mathrm{Cr}$ & $g / 1$ & $\{0,015$ & $<0.02$ & 60.02 & 00.02 & $<0.02$ & 60.02 & $<0.02$ & $<0.02$ & $<0.02$ & 00.02 \\
\hline Cus & og/l & 60.005 & 0.058 & 0.008 & 0.008 & 10.004 & 0.008 & 0.011 & 0.009 & ‘.004 & 5.004 \\
\hline$F_{\bar{E}}$ & ogi! & 60.01 & $<0.005$ & 80.005 & $<0.005$ & $<0.005$ & $<0.005$ & $<0.005$ & 0.009 & 0.031 & 0.016 \\
\hline$k$ & $0 / 1$ & 7.8 & 2.4 & 0.3 & 0.3 & 0.3 & 0.3 & 2.0 & 2.6 & 1.8 & 1.3 \\
\hline $\mathrm{Hg}$ & $g / 1$ & 14.4 & $<0.06$ & $<0.06$ & $<0.06$ & 10.06 & $<0.06$ & $<0.06$ & 60.06 & $\leqslant 0.06$ & $<0.06$ \\
\hline $\mathrm{Mn}$ & 11 & 50.01 & $<0.002$ & $<0.002$ & $\{0.002$ & $<0.002$ & $<0.002$ & $<0.002$ & $<0.002$ & $<0.002$ & $<0.002$ \\
\hline $\mathrm{Na}$ & & 25 & 1120 & 940 & 940 & 610 & 530 & 540 & 524 & 470 & 490 \\
\hline $\mathrm{F}$ & & 00.1 & $<0.1$ & $<0.1$. & $<0.1$ & 60.1 & $<0.1$ & 0.12 & 0.15 & 0.15 & 0.12 \\
\hline is PO4 & $g / l$ & 60.3 & 10.3 & 60.3 & $<0.3$ & $<0.3$ & 60.3 & 0.37 & 0.45 & 0.45 & 0.37 \\
\hline $9 b$ & 11 & $<0.05$ & 0.06 & 0.06 & 10.06 & 10.06 & 80.06 & $\{0.06$ & $<0.06$ & 10.06 & $\langle 0.06$ \\
\hline si & 11 & 15 & 158.0 & 160.0 & 135.0 & 82.0 & 80.0 & 87.2 & 76.2 & 72.0 & 65.5 \\
\hline $5 r$ & 11 & 0.25 & 0.020 & 0.020 & 0.020 & 0.014 & 0.010 & 0.012 & 0.010 & 0.010 & 0.009 \\
\hline in & $49 / 1$ & 10.01 & 0.044 & 0.020 & 0.020 & 0.020 & 0.020 & 0.087 & 0.057 & 0.025 & 0.027 \\
\hline Ir & $99 / 1$ & & $10.00 \mathrm{~B}$ & 0.008 & $<0.008$ & $<0.008$ & $<0.008$ & $<0.008$ & $<0.008$ & $<0.008$ & $<0.008$ \\
\hline F- & 11 & 0.5 & 15.0 & 9.6 & 8.2 & 5.5 & 6.0 & 7.0 & 6.0 & 6.0 & 6.0 \\
\hline Cl- & & 22 & 26 & 21 & 2 & 21 & 20 & 22 & 23 & 23 & 20 \\
\hline HD2- & & 00.03 & $<0.1$ & 63 & 53 & 63 & 60.1 & 60.1 & 10.1 & 6.1 & 00.1 \\
\hline $103-$ & & 0.1 & 42.0 & 15.0 & 10.0 & 5.0 & 7.5 & 8.0 & 9.0 & 4.0 & 6.0 \\
\hline $504--$ & & 86 & 380 & 140 & $12:$ & 100 & 110 & 126 & 109 & 103 & 120 \\
\hline incoj- & & $17 !$ & 0.0 & 0.0 & 0.0 & 0.0 & 0.0 & 0.0 & 0.0 & 0.0 & 0.0 \\
\hline isis-- & & 2.1 & 376 & 126 & 315 & 252 & 252 & 136 & 167 & 222 & 186 \\
\hline $\mathrm{CH}_{-}$ & og & i & 535.5 & 571.2 & 428.4 & 267.8 & 178.5 & 340.5 & 289.7 & 139.4 & 179.6 \\
\hline 5 & & 0.06 & 3.080 & 1.100 & 0.750 & 0.390 & 0.480 & 0.563 & 0.445 & 0.360 & 0.339 \\
\hline $5 \mathrm{HJSOZ}$ & & 0.34 & 9.49 & 6.27 & 4.28 & 2.23 & 2.74 & 3.22 & 2.54 & 2.05 & 1.93 \\
\hline TOC & & 1 & 15.8 & 3.4 & 0.8 & 3.7 & 0.5 & 4.3 & 3.0 & 2.5 & 3.0 \\
\hline PDA - - & & $\$ 1.0$ & $\$ 1.0$ & 81.0 & $<1.0$ & $(1.0$ & 21.0 & $<0.1$ & 10.1 & 30.1 & 0.1 \\
\hline CÁTIONS & $Q / 1$ & 5.28 & 49.27 & 41.03 & 41.04 & 26.65 & 23.16 & 23.63 & 23.44 & 20.58 & 21.42 \\
\hline ANIOHS & Eq $/ !$ & 5.54 & 54.25 & 42.15 & 39.61 & 27.29 & 22.23 & 28.35 & 25.71 & 18.78 & 24.25 \\
\hline TOT.ḦLK. & og/1 & 72.16 & 1323 & 1134 & 1071 & 725 & 567 & 737 & 670 & 468 & 503 \\
\hline lit & ag $/ 1$ & 34.1 & 43.4 & 45.3 & 40.7 & 27.1 & 54.1 & 23.0 & 23.1 & $3 i .4$ & 28.3 \\
\hline OFE 'OUL. & & & 1.78 & 3.74 & 0.52 & 9.8 & 10.78 & 11.99 & 13.53 & 15.73 & 17.31 \\
\hline
\end{tabular}

(--) Data not available.

(\#) Total Alkalinity as C03--. 
TABLE A.11. contd

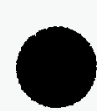

\begin{tabular}{|c|c|c|c|c|c|c|c|c|c|c|c|}
\hline SAMPLE & $\cdots$ & 10 & 11 & 12 & 13 & 14 & 15 & 16 & 17 & 13 & 19 \\
\hline TIKE & days & 34 & 37 & 41 & 44 & 49 & 52 & 58 & 62 & 65 & 69 \\
\hline $\mathrm{pH}$ & & 11.79 & 11.78 & 11.71 & 11.73 & 11.49 & 11.57 & 11.42 & 11.25 & 10.80 & 10.91 \\
\hline Eh & av & 268 & 271 & 215 & 241 & 237 & 252 & 179 & 270 & 217 & 266 \\
\hline Al & $9 / 1$ & 9.19 & 8.73 & 8.23 & 7.70 & 6.78 & 7.16 & 6.87 & 5.45 & 3.91 & 3.56 \\
\hline $\mathrm{Ba}$ & $a g / 1$ & $<0.002$ & $<0.002$ & $<0.002$ & $<0.002$ & $<0.002$ & $<0.002$ & $<0.002$ & $<0.002$ & 60.002 & $\because 0.002$ \\
\hline$C_{z}$ & $g / 1$ & 1.4 & 1.3 & 1.2 & 1.1 & 1.0 & 1.3 & 1.2 & 1.0 & 0.9 & 0.8 \\
\hline Cd & 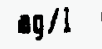 & $<0.004$ & $<0.004$ & $<0.004$ & $<0.004$ & $<0.004$ & $<0.004$ & $\leqslant 0.004$ & $<0.004$ & 60.004 & $<0.004$ \\
\hline $\mathrm{Cr}$ & $g / 1$ & $<0.02$ & $<0.02$ & $<0.02$ & 10.02 & $<0.02$ & 0.04 & $<0.02$ & $<0.02$ & $\langle 0.02$ & 80.02 \\
\hline Cu & $n g / 1$ & <.004 & <.004 & 6.004 & $<.004$ & $<.004$ & C.004 & K.004 & 6.004 & 0.004 & $\therefore .0014$ \\
\hline Fe & $19 / 1$ & 0.016 & 0.028 & 0.027 & 0.016 & $<0.005$ & 0.150 & 0.025 & 0.027 & 0.041 & $0.02 B$ \\
\hline$k$ & $\operatorname{gg} / 1$ & 1.8 & 1.9 & 1.7 & 1.0 & 0.9 & 60.3 & 2.5 & 2.2 & 1.3 & 2.4 \\
\hline $\mathrm{ng}$ & $g / 1$ & $<0.06$ & $<0.06$ & $<0.06$ & 10.06 & $<0.06$ & $<0.06$ & 60.06 & 50.06 & 10.06 & 60.06 \\
\hline $\mathrm{kn}$ & $g / 1$ & $<0.002$ & $\leqslant 0.002$ & $<0.002$ & $<0.002$ & $<0.002$ & 0.022 & 60.002 & 60.002 & 00.002 & 00.002 \\
\hline $\mathrm{Ha}$ & ag/1 & 362 & 327 & . 297 & 300 & 282 & 284 & 308 & 222 & 168 & 167 \\
\hline 8 & $\Delta g / 1$ & 0.15 & 0.15 & 0.15 & 0.12 & $<0.1$ & 10.1 & 0.10 & 0.10 & $\{0.1$ & 60.1 \\
\hline is 904 & $\lg / 1$ & 0.45 & 0.45 & 0.45 & 0.36 & 80.3 & $<0.3$ & 0.31 & 0.31 & 60.3 & 60.3 \\
\hline$P_{b}$ & $\mathrm{gg} / 1$ & $<0.06$ & $<0.06$ & $<0.06$ & 10.06 & $\{0.06$ & $<0.06$ & $\leqslant 0.06$ & 0.06 & 0.06 & 60.06 \\
\hline $\mathrm{ji}$ & $9 / 1$ & 56.2 & 49.8 & 46.6 & 43.6 & 91.8 & 39.1 & 42.7 & 23.8 & 19.1 & 20.2 \\
\hline $\mathrm{Sr}$ & $8 g / 1$ & 0.009 & 0.009 & 0.007 & 0.006 & 0.005 & 0.005 & 0.006 & 0.004 & 0.005 & 0.605 \\
\hline in & $9 q / 1$ & 0.071 & 0.047 & 0.065 & $<0.112$ & $<0.02$ & $<0.02$ & 60.02 & 60.02 & 0.02 & $\therefore 0.02$ \\
\hline ir & $g / 1$ & $<0.008$ & $<0.00 B$ & 10.008 & $<0.008$ & 10.008 & $\therefore 0.008$ & $<0.008$ & $<0.008$ & $<0.008$ & 50.608 \\
\hline F- & $9 / 1$ & 4.8 & 4.7 & 4.7 & 4.8 & 4.8 & 4.7 & 5.2 & 3.0 & 4.1 & 4.5 \\
\hline $\mathrm{Cl}-$ & $\log / 1$ & 21 & 21 & 20 & 25 & 25 & 24 & 22 & 17 & 22 & 23 \\
\hline $\mathrm{HOZ}^{-}$ & $g / 1$ & $<0.1$ & $<0.1$ & 10.1 & $<0.1$ & $<0.1$ & $<0.1$ & 20.1 & $<0.1$ & 0.1 & 60.1 \\
\hline NOJ- & $g / 1$ & 3.6 & 3.4 & 3.2 & 4.3 & 3.7 & 3.4 & 3.3 & 1.2 & 1.5 & 1.3 \\
\hline S04-- & agil & 93 & 91 & 88 & 94 & 97 & 31 & .98 & ij & 78 & 31 \\
\hline $\mathrm{HCOJ}-$ & $a g i l$ & 0.0 & 0.0 & 0.0 & 0.0 & 0.0 & 0.0 & 0.0 & $\hat{0.0}$ & 0.0 & 0.0 \\
\hline $\mathrm{COJ}--$ & $89 / 1$ & 218 & 628.3 & 707.6 & 976.0 & 504.3 & 378.2 & 488.0 & 288.7 & 202 & 1it \\
\hline $\mathrm{OH}-$ & $g / 1$ & 130.9 & 0.0 & 0.0 & 0.0 & 0.0 & 0.0 & 0.0 & 0.0 & 9.6 & 17.6 \\
\hline B & $g / 1$ & 0.282 & 0.257 & $0.2 \mathrm{B2}$ & 0.267 & 0.247 & 0.240 & 0.271 & 0.135 & 0.138 & 0.168 \\
\hline as H360J & $g / 1$ & 1.61 & 1.47 & 1.61 & 1.52 & 1.12 & 1.37 & 1.55 & 0.77 & 0.73 & 6.76 \\
\hline TOC & $\lg i 1$ & 2.4 & 2.3 & 2.5 & 3.0 & 2.4 & 2.5 & $\therefore .7$ & 1.3 & 1.2 & 1.5 \\
\hline P04- - & gil & 10.1 & 60.5 & 10.5 & $<0.5$ & 10.5 & $<0.5$ & 10.5 & 60.5 & $\langle i .5$ & 60.5 \\
\hline CÂTIONG & meqil & 15.75 & 14.34 & 13.02 & 15.13 & 12.34 & 12.43 & 13.52 & 9.76 & 7.37 & 7.37 \\
\hline AHIOHS & eqq/l & 17.31 & 15.83 & 17.73 & 28.25 & i4.41 & 11.91 & 14.59 & 9.27 & 9.77 & 3.77 \\
\hline 10T.ALK.ะ & $\operatorname{mg} / 1$ & 449 & 394 & 451 & 758 & $34 !$ & $27 !$ & 348 & 219 & 217 & 155 \\
\hline IC & gil & 33.6 & 26.4 & 25.6 & 31.7 & 27.0 & 27.7 & 35.1 & 20.1 & 45.9 & $2: .5$ \\
\hline FERE YOL. & & 19.79 & 21.84 & 24.34 & 26.2 & 28.68 & 30.69 & 33.67 & 38 & 41.07 & 45.21 \\
\hline
\end{tabular}

(--) Data not available.

(\#) Total Alkalinity as CO3--. 


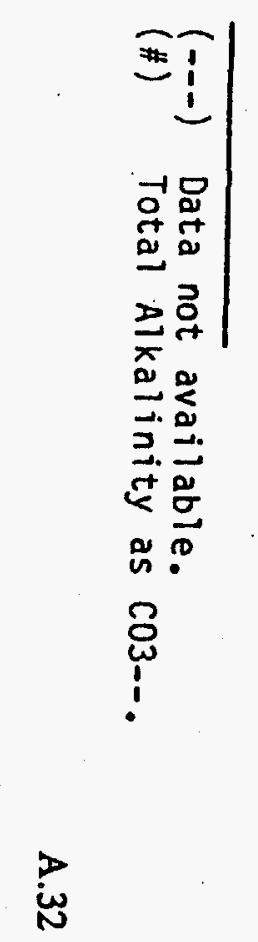

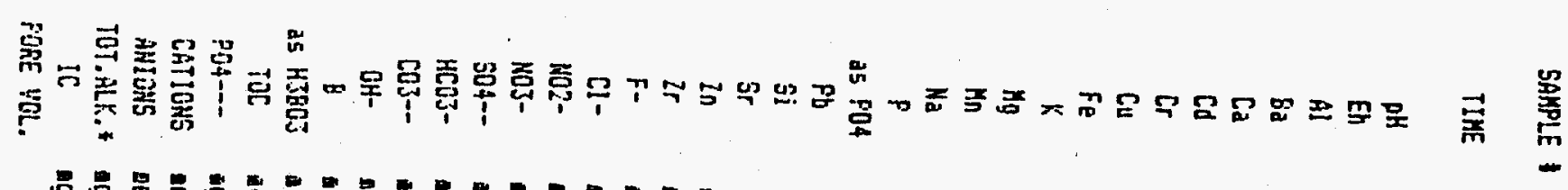

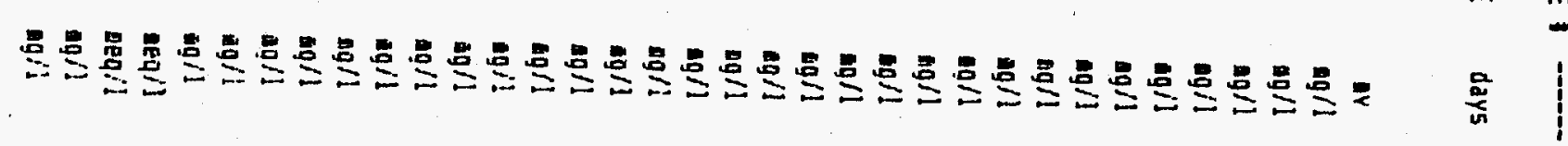

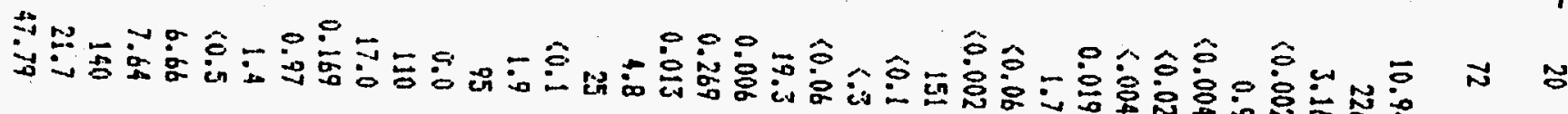

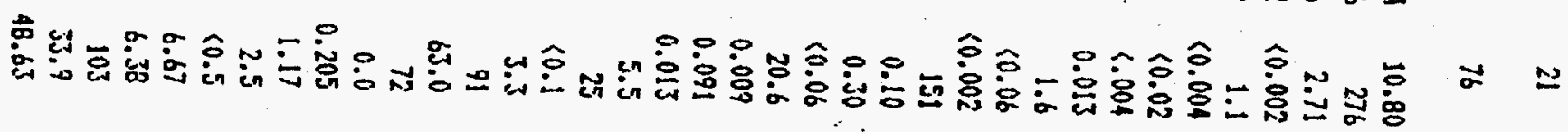

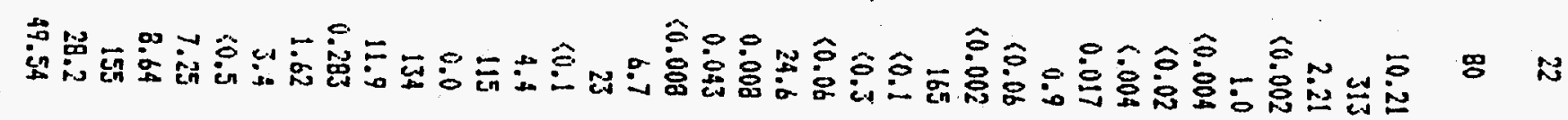

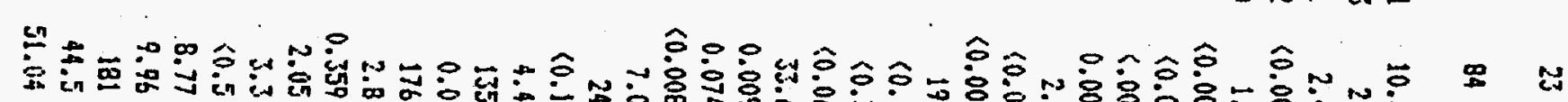

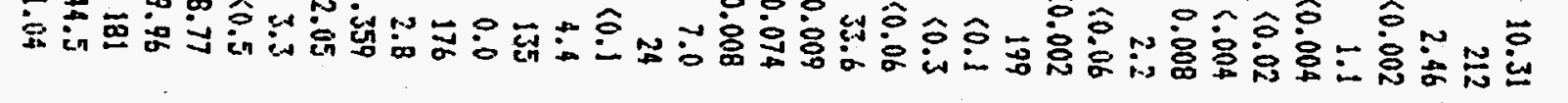

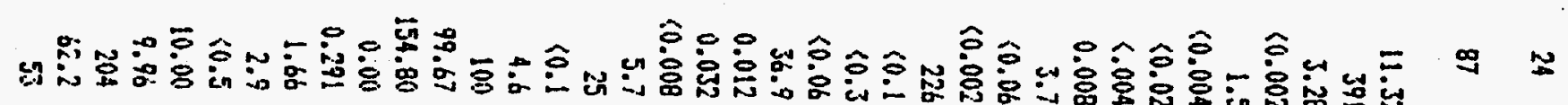


TABLE A.12. Flow Through Leach Test on Whole Grout with Groundwater

(COLLT3)

MELL

SAMPLE WATER

\begin{tabular}{|c|c|}
\hline TIME & days \\
\hline $\begin{array}{l}\text { pH } \\
\text { Eh }\end{array}$ & \\
\hline $\begin{array}{l}\text { Eh } \\
\hat{A l}\end{array}$ & $\begin{array}{c}\text { av } \\
\text { og/l }\end{array}$ \\
\hline $\mathrm{Bd}$ & $9 / 1$ \\
\hline Ca & $9 g / 1$ \\
\hline$C 0$ & $9 / 1$ \\
\hline ir & igil \\
\hline $\mathrm{Cu}$ & $\Delta / 1$ \\
\hline $\mathrm{Fe}$ & $9 / 1$ \\
\hline$k^{\prime}$ & $a g / l$ \\
\hline $\mathrm{Hg}$ & $\mathrm{ng} / \mathrm{l}$ \\
\hline in & $a g / 1$ \\
\hline $\mathrm{Na}$ & ag/l \\
\hline$F$ & $g / 1$ \\
\hline as P04 & 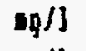 \\
\hline Pt & $\operatorname{ag} / 1$ \\
\hline $5 i$ & $g i l$ \\
\hline $5 r$ & $\Delta / 1$ \\
\hline in & ogil \\
\hline ir & $0 / 1$ \\
\hline$F^{*}$ & ail \\
\hline $\mathrm{Cl}^{-}$ & $\log / 1$ \\
\hline $\mathrm{HOZ}-$ & gil \\
\hline $403-$ & 60/1 \\
\hline 504-- & 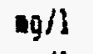 \\
\hline $\mathrm{HCOJ}-$ & $09 / 1$ \\
\hline COJ-- & $9 g / 1$ \\
\hline $\mathrm{OH}-$ & $g / 1$ \\
\hline $\bar{B}$ & gil. \\
\hline ; HJEOJ & $09 / 1$ \\
\hline TOC & $g / 1$ \\
\hline PO4--- & agil \\
\hline CATIOANS & \$eq/! \\
\hline hillons & eeq/I \\
\hline OT.ALK. & $g i 1$ \\
\hline ic & $00 / 1$ \\
\hline
\end{tabular}

FORE YGL,

$$
8.10 \quad 12.51 \quad 12.51
$$

$8.10 \quad 12.51$

12.51

12.34

10.1

12.70

22.00

0.060

0.008

0.008

123

56.0

0.005

1.6

1.3

$\begin{array}{lll}123 & 244 \\ \cdots & - & 317\end{array}$

$=$

4

5

6:

7

911

$<0.015$

$\$ 0.004$

80.004

80.02

0.02

$=$

60.005

$<0.00$

80.005

0.008

7.8

14.40

38.0

0.020

$\begin{array}{rrr}- & - & 0.02 \\ -- & - & 0.004\end{array}$

$<0.01$

10.06

61.0

$\begin{array}{rrr}-\cdots & \cdots & <0.005 \\ -\cdots & -\cdots & 64.0\end{array}$

29

29

32

39

45

$\begin{array}{lll}0.01 & 60.002 & <0.002\end{array}$

$\begin{array}{rr}0.002 & \quad 0.002 \\ 227 & 1380\end{array}$

<0.

$<0.31$

10.05

$<0.1$

0.13

$<0.3$

0.40

15.0

10.06

0.06

0.250

13.2

16.7

0.062

0.071

$<0.008$

0.064

0.065

i0.5

$<0.008$

60.008

12.7

21. 0

22

$<0.03$

20

20

0.1

$<0.1$

$<0.5$

13.0

22.0

171.0

2

102

215

670

0.060

-.-

731.0

$1.440 \quad 1.230$
$0.24 \quad 7.03$

0.34

7.03

-. $\quad \cdots \quad 10.06$

--. -.- $\quad<0.002$

$\begin{array}{lll}--- & -- & 1460 \\ --- & -- & 10.8\end{array}$

0.30

60.06

16.0

0.104

0.068

60.008

15. 0

15.0
20

$<0.1$

24.0

210

0.0

11.61

12.16

11.55

11.32

172
0.09

$3 . j B$

$28 \mathrm{~J}$

278

0.002

0.008

2.88

4.23

$<0.004$

1.4

1.4

2.0

0.02

$<0.02$

0.004

0.004

$\$ 0.004$

0.004

50.02

0.02

0.005

0.013

0.004

(0.004

24.0

16.4

0.105

$\$ 0.005$

10.06

60.002

30.06 .

15.2

18.2

$\{0.002$

469

60.3

6.1

10.3

60.06

60.06

10.4

12.2

60.06

10.06

0.043

0.658

(0.002

10.602

1.0

20.1

(6).

$5.28 \quad 37.01$

0.5

0.000

0.030

$24 i$

$\therefore 0.1$

0.3

324

66.

0.06

00.3

0.06

11.3

12.7

$0.054 \quad 0.062$

0.037

6. 044

6.0

0.009

6.2

60.008

5.6

ใט..

5.7

23
$60 . j$

30.3

107

(1.0)

99

0.0205 .4

218

122

168.86

108.36

85.6

0.60

0.30

0.440

1.7

$\begin{array}{rrr}736.0 & 0.0 & 47.6 \\ -\cdots & -3 & 9.15\end{array}$

2.53

40.5

<0.5

$5.54 \quad---\quad 71.86$

$32 \quad \cdots \quad 1360$

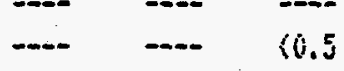

12.45

...- $\quad$-... 16.20

12.42

34.1

$2064 \quad 549$

362

273

9.33

201

23
60.1

3.0

435.42

110.60

$0.60 \quad 23.35$

$0.227 \quad 8.315$

$1.30 \quad 1.00$

(1).5 i. 5

$10.84 \quad 19.56$

$13.34 \quad 7.65$

$330 \quad 134$

$\begin{array}{lllllllll}1.06 & 1.7 & 3.18 & 6.6 & 9.94 & 12.36 & 13.93 & 16.95 & 24.89\end{array}$

$(--)$ Data not available.

(\#) Total ATkalinity as c03--. 
TABLE A.12. contd

\begin{tabular}{|c|c|c|c|c|c|c|c|c|c|c|}
\hline SAKPLE & 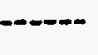 & 13 & 15 & 17 & 19 & 22 & 24 & 26 & 28 & 30 \\
\hline TIME & days & 52 & 60 & 67 & 73 & 87 & 94 & 101 & 113 & 122 \\
\hline pht & & 10.89 & 10.56 & 10,98 & 10.79 & 9.99 & 10.56 & 10.37 & 9.82 & 9.0 \\
\hline Eh & sv & 289 & 298 & 272 & 323 & 347 & 268 & 345 & 327 & 34 \\
\hline Al & $9 / 1$ & 0.32 & 0.06 & 1.01 & 0.86 & 1.35 & 1.22 & 0.52 & $<0.03$ & 10.03 \\
\hline 83 & $90 / 1$ & 0.004 & 0.006 & 0.002 & 0.002 & 0.005 & 0.004 & 0.007 & 0.013 & 0.015 \\
\hline $\mathrm{Ca}$ & $\log / 1$ & 1.1 & 3.8 & 1.1 & 0.9 & 1.7 & 0.8 & 1.7 & 4.7 & 6.6 \\
\hline Cd & $g / 1$ & 10.004 & $<0.004$ & 00.004 & $<0.004$ & 50.004 & $<0.004$ & $<0,004$ & $<0.004$ & $<0.004$ \\
\hline $\mathrm{cr}$ & $g / 1$ & 00.02 & 10.02 & $<0.02$ & $<0.02$ & 10.02 & $<0.02$ & 30.02 & $<0.02$ & 50.02 \\
\hline Cu & $g / l$ & $<0.004^{\circ}$ & $<0.004$ & 0.005 & 0.009 & $<0.004$ & 60.004 & 80.004 & $<0.004$ & $<0.004$ \\
\hline FE & $g / l$ & $<0.005$ & 0.007 & 1.100 & 10.005 & 0.005 & 0.019 & 0.006 & $\{0.005$ & 0.013 \\
\hline$k$ & $\Delta 4 / 1$ & 9.9 & 9.9 & 10.0 & 9.8 & 14.0 & 13.0 & 12.0 & 10.0 & 6.4 \\
\hline $\mathrm{Kg}$ & ogil & 10.06 & 10.06 & 50.06 & $<0.06$ & $\{0.06$ & 60.06 & 10.06 & $<0.06$ & 3.45 \\
\hline $\mathrm{Hn}$ & $19 / 1$ & $\langle 0.002$ & $<0.002$ & $\langle 0.002$ & $<0.002$ & $<0.002$ & $<0.002$ & 80.002 & $<0.002$ & 80.002 \\
\hline $\mathrm{Na}$ & $9 / 1$ & 132 & 121 & 155 & 132 & 175 & 165 & 123 & 79 & 61 \\
\hline$p$ & ag /l & $<0.1$ & $<0.1$ & 0.1 & 80.1 & 80.1 & $<0.1$ & 20.1 & $<0.1$ & $<0.1$ \\
\hline a5 PDG & $9 / 1$ & $\langle 0.3$ & $<0.3$ & $\{0.3$ & $\{0.3$ & 60.3 & 80.3 & $<0.3$ & $\{0.3$ & $\{0.3$ \\
\hline Fb & $\log / 1$ & 10.06 & 10.06 & 10.06 & 10.06 & 10.06 & 50.06 & 80.06 & 40.06 & $<0.06$ \\
\hline $5 i$ & $99 / 1$ & 9.7 & 8.6 & 12.4 & 10.0 & 12.6 & 11.3 & 9.3 & 6.5 & 9.3 \\
\hline $5 r$ & $69 / 1$ & 0.046 & $<0.002$ & 0.034 & 0.032 & 0.051 & 0.046 & 0.070 & 0.114 & 60.002 \\
\hline In & $19 / 1$ & 0.029 & 00.02 & $\{0.02$ & 0.024 & 10.02 & 80.02 & $\{0.02$ & 60.02 & $<0.02$ \\
\hline Ir & هִ & 0.012 & 0.015 & $\zeta 0.008$ & $<0.008$ & 0.025 & 0.014 & 60.0108 & $<0.008$ & 0.018 \\
\hline$F-$ & agil & 5.0 & 5.2 & 6.0 & 5.3 & 0.3 & 6.3 & 5.1 & 5.3 & 5.6 \\
\hline $\mathrm{al}$ & ag $/$ & 22 & 25 & 23 & 24 & 24 & 24 & 24 & 22 & 23 \\
\hline $102-$ & $g / 1$ & $\langle 0.3$ & $<0.3$ & $<0.1$ & $\{0.5$ & 60.3 & 0.3 & $\{0.3$ & $\{0.3$ & $\{0.3$ \\
\hline $\mathrm{HOJ-}$ & 011 & 1.6 & 1.5 & 2.7 & 2.0 & 3.5 & 3.2 & 2.3 & 1.4 & 1.2 \\
\hline S64t-- & aqil & 77 & 82 & 83 & 81 & 89 & 87 & B1 & 76 & 74 \\
\hline $\mathrm{HCOS}-$ & 8qil & 8.86 & 31.48 & $0.9 !$ & 0.00 & 69.10 & 41.97 & 0.00 & 31.48 & 26.23 \\
\hline COS-- & 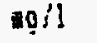 & 108.36 & 72.24 & 195.60 & 2.24 & 112.68 & 108.36 & 1.24 & 103.20 & 5.80 \\
\hline OH- & $9 q / 1$ & 0.00 & 0.00 & 0.00 & 32.16 & 0.00 & & .19 & 0. & 0.00 \\
\hline $\bar{B}$ & agi! & 0.133 & 0.138 & 0.208 & 0.144 & 0.195 & 0.169 & 0.127 & 0.118 & 0.143 \\
\hline as H3803 & $a g / 1$ & 0.76 & 0.79 & 1.17 & 0.82 & 1.11 & 0.97 & 0.73 & 0.67 & 0.82 \\
\hline 100 & $09 / 1$ & $\cdots$ & $\cdots$ & $\cdots$ & $\cdots$ & $\cdots$ & $\cdots$ & $\cdots$ & $-\infty$ & $\cdots$ \\
\hline$F[4-\cdots$ & in & $(0.5$ & 60.5 & 00.5 & 60.5 & $<6.4$ & 0.4 & 10.4 & $<0.1$ & 80.4 \\
\hline CÁTIGHE & ail & 6.05 & 5.61 & 7.05 & 6.04 & 8.05 & 7.55 & 5.74 & 3.91 & 3.49 \\
\hline AKIGHS & qil & 6.15 & 2.73 & 6.75 & 7.00 & 8.34 & 3.17 & 5.65 & 3.46 & 3.79 \\
\hline TOT, ḾLK.: & $\operatorname{mag} i]$ & 201 & 88 & 186 & 127 & 163 & 129 & 95 & 113 & 39 \\
\hline IC & agil & $\cdots$ & $\cdots$ & 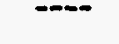 & - & $\cdots$ & $\cdots$ & $\cdots$ & $\cdots$ & $\cdots$ \\
\hline HE VGL. & & 30.42 & 35.89 & 3.91 & 2.39 & 47.29 & 8.65 & 4.56 & 84.77 & 73.33 \\
\hline
\end{tabular}

$(--)$ Data not available.

(\#) Total Alkalinity as CO3--. 
TABLE A.12. contd

\begin{tabular}{|c|c|}
\hline 32 & 33 \\
\hline 158 & 188 \\
\hline 8.31 & 8.48 \\
\hline 323 & 258 \\
\hline 60.03 & 00.03 \\
\hline 0.028 & 0.041 \\
\hline 17.1 & 26.4 \\
\hline 60.004 & $<0.004$ \\
\hline$<0.02$ & $<0.02$ \\
\hline$<0.004$ & 80.004 \\
\hline 0.006 & 80.006 \\
\hline 8.4 & 7 \\
\hline 10.70 & 13.4 \\
\hline 10.002 & 0.002 \\
\hline 46 & 40.1 \\
\hline$<0.1$ & 80.1 \\
\hline$\{0.3$ & 0.3 \\
\hline 80.06 & 10.06 \\
\hline 13.4 & 14.9 \\
\hline 0.209 & 0.221 \\
\hline 00.02 & $<0.02$ \\
\hline$<0.008$ & $\$ 0.008$ \\
\hline 4.0 & 2.57 \\
\hline 23 & 22.1 \\
\hline$<0.3$ & $<0.3$ \\
\hline 1.4 & 0.5 \\
\hline 78 & 74.6 \\
\hline 84.18 & 101.20 \\
\hline 5.52 & 11.04 \\
\hline 0.00 & 0.00 \\
\hline 0.117 & 0.111 \\
\hline 6.67 & 0.634 \\
\hline$\langle 0.4$ & 0.5 \\
\hline 3.37 & 4.35 \\
\hline 4.07 & 4.34 \\
\hline 17 & 60.72 \\
\hline -...- & $\cdots$ \\
\hline
\end{tabular}

$89.62 \quad 104.2$

(\#) Data not available.
(\#) 
TABLE A.13. Flow Through Leach Test on whole Grout with Groundwater (COLLT2)

\begin{tabular}{|c|c|c|c|c|c|c|c|c|c|c|c|}
\hline SAMPLE & -...-. & $\begin{array}{l}\text { WELL } \\
\text { WATER }\end{array}$ & 1 & 2 & 3 & 4 & 5 & 6 & 7 & $B$ & 9 \\
\hline TIME & days & 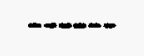 & 3 & 10 & 14 & 17 & 21 & 24 & 28 & 31 & 35 \\
\hline $\mathrm{pH}$ & & 8.1 & 12.78 & 12.79 & 12.70 & 12.43 & 12.59 & 11.93 & 11.89 & 11.87 & 10.60 \\
\hline Eh & $\mathbf{E r}$ & 295 & 135 & 149 & 156 & 144 & 263 & 168 & 221 & 259 & 221 \\
\hline A] & mọil & $<0.1$ & 24.00 & 17.00 & 13.00 & 12.00 & 8.85 & 5.49 & 4.16 & 4.75 & 2.50 \\
\hline $\mathrm{Ba}$ & $\lg / 1$ & 0.06 & $<0.002$ & 0.007 & 0.007 & 0.006 & 0.006 & $<0.002$ & 0.005 & 0.004 & 0.004 \\
\hline $\begin{array}{l}\mathrm{Ca} \\
\mathrm{Cd}\end{array}$ & $10 / 1$ & $\begin{array}{r}56 \\
00.005\end{array}$ & $\begin{array}{r}3.2 \\
<0.004\end{array}$ & $\begin{array}{r}3.0 \\
0.004\end{array}$ & $\begin{array}{r}3.0 \\
00.004\end{array}$ & $\begin{array}{r}3.0 \\
<0.004\end{array}$ & $\begin{array}{r}1.6 \\
<0.004\end{array}$ & $\begin{array}{r}2.3 \\
<0.004\end{array}$ & $\begin{array}{r}1.6 \\
<0.004\end{array}$ & $\begin{array}{r}1.7 \\
50.004\end{array}$ & $\begin{array}{r}1.3 \\
0.004\end{array}$ \\
\hline Cr & $0 / 1$ & $0(0.015$ & 10.02 & 60.02 & $<0.02$ & $<0.02$ & $<0.02$ & 60.02 & 0.02 & $<0.02$ & 30.02 \\
\hline $\mathrm{Cu}$ & $\lg / 1$ & 60.005 & $\{0.004$ & 0.010 & 0.010 & 0.014 & $\leqslant 0.004$ & $<0.004$ & 20.004 & $<0.004$ & $<0.004$ \\
\hline $\mathrm{Fe}$ & $g / 1$ & 10.01 & 0.022 & 0.013 & 0.025 & 0.029 & 0.015 & 0.007 & $<0.005$ & 0.005 & 0.144 \\
\hline$r$ & $90 / 1$ & 7.8 & 70.0 & 51.0 & 42.0 & 34.0 & 28.0 & 21.0 & 18.0 & 19.0 & 15.0 \\
\hline $\mathrm{Hg}$ & $\log / 1$ & 14.4 & 0.06 & 0.06 & 0.08 & 0.06 & 0.06 & $<0.06$ & $<0.06$ & 10.06 & 80.06 \\
\hline $\mathrm{Kn}_{\mathrm{n}}$ & $g / 1$ & 0.01 & $<0.002$ & $\{0.002$ & $<0.002$ & 60.002 & $<0.002$ & $\{0.002$ & 10.002 & $<0.002$ & $<0.002$ \\
\hline $\mathrm{Ha}$ & il & 25 & 1800 & 1200 & 910 & 760 & 570 & 400 & 278 & 317 & 212 \\
\hline$p$ & $\operatorname{hg} / 1$ & 10.1 & $<0.1$ & $<0.1$ & $<0.1$ & $<0.1$ & $<0.1$ & 00.1 & $<0.1$ & 60.1 & $(0)$. \\
\hline as $\mathrm{PO4}$ & $9 / 1$ & $<0.31$ & 60.3 & $<0.3$ & $<0.3$ & 60.3 & $<0.3$ & $<0.3$ & $<0.3$ & 60.3 & $\langle 0.3$ \\
\hline$F b$ & ogil & $<0.05$ & 10.06 & $<0.06$ & $<0.06$ & 30.06 & $<0.06$. & $<0.06$ & $<0.06$ & $<0.06$ & 0.06 \\
\hline $3 i$ & ogil & 15 & 14.5 & 12.0 & 10.8 & 10.0 & 10.5 & 9.7 & 10.3 & 10.4 & 10.2 \\
\hline sr & $9 / 1$ & 0.25 & 0.106 & 0.082 & 0.080 & 0.076 & 0.080 & 0.077 & 0.075 & 0.068 & 0.064 \\
\hline In & $9 / 1$ & $<0.01$ & 0.140 & 0.090 & 0.052 & 0.100 & $<0.02$ & $<0.02$ & 0.102 & 0.021 & 0.022 \\
\hline $7 r$ & $\lg / 1$ & 60.008 & $<0.008$ & $<0,008$ & $<0.008$ & $<0.008$ & $<0.008$ & $<0.008$ & $<0.008$ & $<0.008$ & $<0.008$ \\
\hline F- & $9 / 1$ & $\$ 0.5$ & 14.0 & 11.0 & 9.0 & 9.0 & 7.0 & 0.0 & 5.8 & 5.6 & 5.2 \\
\hline $\mathrm{Cl}-$ & $\lg / 1$ & 22 & 22 & $2 j$ & 21 & 20 & 22 & 23 & 23 & 25 & 22 \\
\hline H02- & ag $/ 1$ & 00.03 & <3.0 & 00.1 & 0.1 & $<0.1$ & 30.1 & $<0.5$ & 60.1 & $<0.1$ & $<0 .:$ \\
\hline $\mathrm{HOJ}^{-}$ & $\operatorname{og} i 1$ & 0.1 & 26.0 & 18.0 & 13.0 & $18 .(6$ & 7.0 & 4.0 & 3.3 & 4.1 & 2.4 \\
\hline SO4-- & il & ố & 240 & 180 & 144 & 120 & 110 & 100 & 120 & 500 & 80 \\
\hline HELIJ- & $g / 1$ & 171 & 0.0 & 0.0 & 0.0 & 0.0 & 0.0 & 0.0 & 0.0 & 0.0 & 0.0 \\
\hline $\mathrm{CO}=-$ & gil & 2.1 & 4060.1 & 2615.1 & 2636.5 & 2150.7 & 1079.1 & 786.9 & 597.8 & 707.6 & 331.6 \\
\hline $\mathrm{BH}-$ & $0 / 1$ & 0 & 83.6 & 101.5 & 58.3 & 39.2 & 61.3 & 0 & 0 & $\hat{0}$ & 0 \\
\hline 8 & $a g i l$ & 0.06 & 1.000 & 1.200 & 0.900 & 0.780 & 0.489 & 0.3 & 0.260 & 0.284 & 0.163 \\
\hline is $\mathrm{HJBOJ}$ & $\operatorname{seg} / 1$ & 0.34 & 5.72 & 6.86 & 5.15 & 4.16 & 2.79 & 1.62 & 1.49 & 1.02 & 0.93 \\
\hline$T D C$ & 8qil & 1 & 13.6 & 9.5 & 0.4 & 6.7 & 4.3 & 3.5 & 3.7 & 3.5 & 2.7 \\
\hline Fo:--- & ingil & 01.0 & 11.0 & 31.0 & $\$ 1.0$ & 01.0 & 11.0 & 31.0 & $(1.0)$ & 91.0 & $\{1,0$ \\
\hline CHTIUKS & Ieq/1 & 5.28 & 80.22 & 53.64 & 40.80 & 34.07 & 25.59 & 18.05 & 12.64 & 14.36 & 4.67 \\
\hline ANEONS & $\pm \in q / 1$ & 5.54 & 86.64 & 58.80 & 52.58 & 44.73 & 29.50 & 18.36 & 15.64 & 17.10 & 19.64 \\
\hline TOT.ALY.* & gii & 92.16 & 2394 & 1806 & 1449 & 1227 & 783 & 457 & 304 & 426 & 271 \\
\hline IC & ngil & 34.1 & 41.1 & 99.9 & 28.7 & 19.3 & 30.1 & 20.7 & 24.2 & 24.3 & 34.9 \\
\hline F. VGL. & & & 1.02 & 1.88 & 2.65 & 3.05 & 4.69 & 5.87 & 0.66 & 7.83 & 9.32 \\
\hline
\end{tabular}

(--) Data not available.

(\#) Total Alkalinity as C03--. 


\section{TABLE A.13. contd}

\begin{tabular}{|c|c|c|c|c|c|c|c|c|c|c|c|}
\hline SAKPLE & - & 10 & 11 & 12 & 13. & 14 & 15 & 16 & 17 & iB & 19 \\
\hline IIME & days & 45 & 52 & 56 & 59 & 63 & bb & 70 & 74 & 76 & $8 i$ \\
\hline pH & & 12.11 & 11.55 & 11.85 & 11.00 & 10.85 & 10.55 & $11.6 \mathrm{j}$ & 10.45 & 10.51 & 11.31 \\
\hline Eh & ar & 168 & 178 & 220 & 209 & 222 & 234 & 219 & 304 & 192 & 331 \\
\hline Al & $\lg / 1$ & 7.29 & 5.97 & 3.17 & 0.35 & 2.42 & -- & 2.43 & 0.06 & 0.17 & 0.73 \\
\hline 82 & ig $/ 1$ & 0.006 & 0.004 & 0.005 & 0.004 & 0.004 & -.. & 0.006 & 0.005 & 0.005 & 0.004 \\
\hline Ca & $a / 1$ & 1.7 & 0.8 & 1.2 & 1.2 & 0.7 & --- & 1.5 & 1.4 & 1.2 & 1.0 \\
\hline $\mathrm{Co}$ & 911 & $<0.004$ & $<0.004$ & $<0.004$ & $<0.004$ & 10,004 & -..- & $<0.004$ & $<0.004$ & 60.004 & $\{0.004$ \\
\hline $\mathrm{Cr}$ & $\Delta / 1$ & $<0.02$ & 10.02 & $<0.02$ & $\langle 0 . \dot{v} 2$ & $<0.02$ & -..-- & $<0.02$ & 60.02 & 60.02 & 0.02 \\
\hline $\mathrm{Cu}$ & ag/l & 60.004 & $<0.004$ & $<0.004$ & 10.004 & $<0.004$ & -...- & $<0.004$. & $<0.004$ & 60.004 & 0.004 \\
\hline $\mathrm{Fe}$ & $8 / 1$ & $<0.005$ & $<0.005$ & 00.005 & 0.010 & 0.000 & -..- & 10.005 & 0.005 & 00.005 & 60.005 \\
\hline$k$ & $\mathrm{gg} / 1$ & 24.4 & 21.0 & 15.0 & 11.0 & 14.0 & ---- & 35.0 & 9.7 & lo.c & 11.4 \\
\hline $\mathrm{Mg}$ & $\operatorname{sg} / 1$ & $<0.06$ & 50.06 & $<0.06$ & $<0.06$ & $<0.06$ & --.- & 10.06 & 0.28 & 0.17 & 10.06 \\
\hline th & $g / 1$ & $<0.002$. & 10.002 & $<0.002$ & $<0.002$ & 10.002 & $-\ldots$ & $<0.002$ & $(0.01) 2$ & 60.002 & 60.002 \\
\hline $\mathrm{Ha}$ & $a g / 1$ & 509 & 391 & 243 & 137 & 235 & -..- & 248 & 120 & 121 & 160 \\
\hline$F$ & $0 g / 1$ & $<0.1$ & $<0.1$ & 0.1 & $<0.1$ & $<0.1$ & ---- & $<0.1$ & 60.1 & 0.1 & 10.1 \\
\hline $35 \mathrm{PO4}$ & and $/ 1$ & $<0.3$ & $<0.3$ & 60.3 & $<0.3$ & $<0.3$ & -... & $<0.3$ & 60.3 & 60.3 & 60.3 \\
\hline Po & $\log / 1$ & $<0.06$ & $<0.06$ & 10.06 & 80.06 & $\therefore 0.06$ & $\cdots$ & $<0.06$ & 80.016 & 60.06 & 10.06 \\
\hline $\mathrm{si}$ & $g / 1$ & 11.4 & 11.0 & 11.0 & 9.5 & 12.8 & $\cdots$ & 11.9 & 9.7 & 20.6 & 13.5 \\
\hline $5 r$ & ag $/ 1$ & 0.084 & 0.057 & 0.074 & 0.064 & 0.056. & -... & 0.069 & 0. vेb7 & 0.064 & 0.050 \\
\hline In & ind & $<0.02$ & 50.02 & 10.02 & 10.02 & $\leqslant 0.02$ & $\cdots$ & 60.02 & $(0.02$ & 0.643 & 6.057 \\
\hline $3 r$ & $\ln n$ & $<0.008$ & $<0.008$ & $\langle 0,008$ & 60.008 & 0.045 & $\cdots$ & 10.008 & 80.000 & ט. 300 & (U. OUE \\
\hline F- & $g i l$ & b.l & 7.2 & 5.9 & 5.6 & 5.1 & $\cdots$ & 5.7 & 4.8 & 5.5 & 3. \\
\hline $\mathrm{Cl}-$ & agn $/ 1$ & 22 & 23 & 22 & 23 & 24 & $\cdots$ & 27 & 23 & 23 & 23 \\
\hline $\mathrm{HO2}-$ & $\log / 1$ & $<0.1$ & $<0.1$ & $<0.1$ & $<0.1$ & $<0.1$ & --- & 80.1 & $\langle 0 .:$ & iu. & iv.: \\
\hline $1103-$ & gil & 6.8 & 5.6 & 3.3 & 1.5 & 3.7 & -- & 4.3 & 1.4 & 1.6 & 2.5 \\
\hline 504-- & $\lg / l$ & 114 & 119 & 92 & 80 & 98 & $-\cdots$ & 112 & 76 & 80 & 84 \\
\hline $\mathrm{HCOJ-}$ & $g / 1$ & 0.0 & 0.0 & 0.0 & 0.0 & 6.0 & $0 . \hat{\hat{v}}$ & 0.0 & 0.0 & 0.0 & 178.32 \\
\hline $\cos -2$ & $9 / 1$ & 1179.3 & 488.0 & 455.5 & 108 & 200 & 312 & $\therefore \quad 124$ & 72 & 82 & 51.60 \\
\hline 84- & $a g / 1$ & 0 & 0 & 0 & 26.6 & 22.7 & 36.5 & 109.4 & 9.1 & 26.6 & 0.00 \\
\hline$B$ & og $/ 1$ & 0.470 & 0.350 & 0.242 & 0.126 & 0.266 & $-\cdots$ & 0.271 & 0.146 & 4.164 & $0.2 ! 8$ \\
\hline as $\mathrm{HJBOJ}$ & $9 / 1$ & 2.68 & 2.00 & 1.38 & 0.72 & 1.52 & -.-- & 1.55 & 0.83 & 0.93 & 1.24 \\
\hline TOC & $g n$ & -- & 4.5 & 19.1 & 19.9 & 46.7 & $20 . \hat{?}$ & -..- & --- & -..- & --- \\
\hline PO4--- & $g / 1$ & $\langle 1.0$ & $(1.0$ & 11.0 & $<1.0$ & 3.0 & $\langle 1.0$ & $(1.0)$ & $(i, 0)$ & $(1.0$ & 01.0 \\
\hline CATIOHS & seqil & 22.85 & 17.58 & 11.02 & 6.30 & $10.6 \bar{i}$ & -..- & 11.25 & 5.56 & 5.61 & 7.30 \\
\hline AMIOKS & Irqia & 25.10 & 15.97 & 12.20 & 7.60 & 11.05 & -.- & 14.04 & 6.14 & 6.75 & 7.46 \\
\hline TOT.ALK.* & agil & 650 & 371 & 279 & 155 & 240 & 380 & 317 & iNi8 & 129 & 137 \\
\hline IC & $a g / 1$ & $-\cdots$ & 63.5 & $16 . j$ & 17.0 & 42.6 & 17.3 & $\cdots$ & --- & $\cdots$ & --- \\
\hline VOL. & & 10.24 & 12.09 & 14.72 & 16.25 & 17.18 & 17.38 & $20 . .16$ & 3.52 & 25.71 & 26.54 \\
\hline
\end{tabular}

(--) Data not available.

(\#) Total Alkalinity as CO3--. 
TABLE A.13. contd

\begin{tabular}{|c|c|c|c|c|c|c|c|c|c|c|c|}
\hline SAMPLE I & $\cdots$ & 21 & 23 & 25 & 27 & 29 & 31 & 34 & 36 & 38 & 40 \\
\hline TIME & days & 88 & 94 & 101 & 109 & 115 & 122 & 136 & 143 & 150 & 162 \\
\hline $\mathrm{PH}$ & & 10.88 & 10.35 & 9.97 & 10.07 & 10.23 & 10.42 & 9.64 & 9.53 & 9.27 & 9.36 \\
\hline Eh & or & $28 B$ & 284 & 288 & 286 & 268 & 307 & 356 & 304 & 345 & 334 \\
\hline Â! & $9 / 1$ & 0.14 & 60.03 & $<0.03$ & $<0.03$ & 0.15 & 0.18 & 0.07 & 0.028 & 0.069 & 0.162 \\
\hline $\mathrm{Bd}$ & $g / 1$ & 0.006 & 0.008 & 0.007 & 0.006 & 0.003 & 0.004 & 0.008 & 0.012 & 0.008 & 0.008 \\
\hline$C_{\mathbf{a}}$ & $g / 1$ & 1.9 & 2.4 & 2.3 & 2.1 & 1.5 & 1.36 & 3.35 & 4.17 & 3.17 & 3.74 \\
\hline $\mathrm{Cd}$ & $9 / !$ & $<0.004$ & $<0.004$ & $<0.004$ & $<0.004$ & $<0.004$ & $<0.004$ & 10.004 & $<0.004$ & $<0.004$ & $(0,004$ \\
\hline $\mathrm{Cr}$ & og $/ 1$ & $<0.02$ & $<0.02$ & $<0.02$ & 80.02 & 10.02 & $\{0.02$ & $<0.02$ & 60.02 & $(0.02$ & $<0.02$ \\
\hline $\mathrm{Cu}$ & $9 q / 1$ & 10.004 & $<0.004$ & $<0.004$ & $<0.004$ & $<0.004$ & 0.007 & 80.004 & $<0.004$ & 10.004 & $<0.004$ \\
\hline $\mathrm{Fe}$ & $g / 1$ & 0.006 & $<0.005$ & $<0.005$ & 0.008 & $<0.005$ & $<0.005$ & $<0.005$ & 60.005 & 10.005 & 0.005 \\
\hline$k$ & ag/l & 10.5 & 9.5 & 8.3 & B.t & .8 .8 & 9 & 9.2 & 8.8 & 0.6 & 7.2 \\
\hline $\mathrm{xg}$ & $\lg / 1$ & 0.41 & 0.70 & 0.54 & 0.39 & 0.06 & 0.215 & 0.649 & 1.22 & 0.283 & 1.24 \\
\hline $\mathrm{m}$ & $g / 1$ & $<0.002$ & $10.00\}$ & $<0.002$ & $<0.002$ & $\$ 0.002$ & 10.002 & $<0.002$ & 10.002 & $<0.002$ & 10.0012 \\
\hline Ha & iqil & 110 & 91 & 88 & 27 & 115 & 102 & 83.3 & 79.2 & 87.a & 93.1 \\
\hline$p$ & agil & 0.1 & $<0.1$ & 0.1 & $<0.1$ & $<0.1$ & $<0.1$ & 60.1 & 0.1 & $<0.1$ & (0.1 \\
\hline d5 F04 & $\circ g / 1$ & 50.3 & 10.3 & $<0.3$ & $<0.3$ & $<0.3$ & $<0.3$ & 60.3 & $<0.3$ & $<0.3$ & 60.3 \\
\hline $\mathrm{Pb}$ & $g / 1$ & $\$ 0.06$ & $<0.06$ & 10.06 & $<0.06$ & $<0.06$ & $<0.06$ & 10.06 & $<0.06$ & $<0.06$ & 80.06 \\
\hline$\hat{\mathrm{s}} \mathrm{i}$ & $m y / 1$ & 9.8 & 8.9 & 9.5 & 9.1 & 12.1 & 10.5 & 9.53 & 8.62 & 10.5 & 10.4 \\
\hline Sr & $\operatorname{mg} / 1$ & 0.061 & 0.076 & 0.075 & 0.066 & $0.04 !$ & 0.05 & 0.085 & 0.12 & 0.169 & 0.086 \\
\hline In & $g / 1$ & 0.040 & 0.030 & 0.047 & 0.033 & 60.02 & 0.024 & 0.02 & $<0.02$ & $\{0.02$ & 30.02 \\
\hline Ir & $2 g / 1$ & $<0.008$ & 0.01 & 0.1 & 0.03 & 0.035 & $<0.008$ & $\$ 0.008$ & $<0.008$ & 00.008 & $i 0 . \dot{v}(i B$ \\
\hline F- & sil & 5.2 & 5.4 & 5.5 & 5.0 & 6.1 & 5.2 & 6.3 & 6.5 & b.ô & 0.8 \\
\hline $\mathrm{Cl}-$ & $9 / 1$ & 23 & 23 & 24 & 23 & 21 & 21 & 21 & 23 & 23 & 22 \\
\hline $\mathrm{HO2}-$ & $69 / 1$ & $<0.1$ & $<0.1$ & 0.3 & 60.3 & <0.1 & 0.1 & 60.3 & $\{0.3$ & $<0.3$ & 10.3 \\
\hline NOJ- & $g / 1$ & 1.6 & 1.2 & 1.2 & 1.3 & 2.0 & 1.6 & 2.2 & 1.5 & 1.7 & 2.4 \\
\hline $584-$ & ag $/ 1$ & 84 & . 82 & 78 & 74 & 75 & 73 & 71 & 78 & 79 & 80 \\
\hline HCOS- & $g / 1$ & -- & 31.52 & $57.7 !$ & 94.43 & 0.00 & 0.00 & 31.48 & 47.21 & 31.46 & 62.75 \\
\hline $603--$ & $4 i 1$ & --- & 46.40 & 30.76 & 20.64 & 103.20 & 25.80 & 51.60 & 20.64 & 41.28 & 41.28 \\
\hline OH- & agil & --- & 0.00 & 0.00 & 0.00 & 5.84 & 42.40 & 0.00 & 0.00 & 0.60 & 0.60 \\
\hline 8 & agil & 0.140 & 0.150 & 0.155 & 0.145 & 0.202 & 0.164 & 0.137 & 0.139 & $0 . \mid 71$ & 0.18 \\
\hline 2543303 & $\log / 1$ & 0.80 & 0.85 & 0.39 & 0.83 & 1.15 & 0.94 & 0.78 & 0.79 & O. 78 & 1.62 \\
\hline io: & 19 & -- & 2.3 & 2.6 & 2.2 & 2.0 & 3.52 & 5.66 & 4.52 & 3.55 & 3.60 \\
\hline FOS--- & $a g / 1$ & 11.0 & $<1.0$ & 10.4 & 6.4 & 0.5 & $<0.5$ & iv. 4 & $<0.4$ & (0.4 & C0.4 \\
\hline Cot JaHS & seqi! & 5.18 & 4.38 & 4.22 & 4.14 & 5.31 & 4.75 & 4.51 & 3.78 & 4.27 & 4.37 \\
\hline ANI DNS & aeqil & ---- & 4.75 & 4.61 & 4.73 & 6.31 & 5.76 & 4.67 & 4.10 & 4.57 & 2,41 \\
\hline TUT.ALK, & $=0 i 1$ & $\ldots$ & 62 & 59 & $\delta 7$ & 114 & 100.62 & 67.08 & 43.86 & 56.76 & 72.24 \\
\hline Ii & $n g / !$ & ---- & 15.0 & 18.8 & 12.0 & 38.5 & 21.9 & 24.74 & 17.47 & 20.7 & 21.26 \\
\hline f. val. & & 31.79 & 37.23 & 41.3 & 45.59 & 46.17 & 51.77 & 59.33 & 62.75 & 65.77 & 31.31 \\
\hline
\end{tabular}

(--) Data not available.

(\#) Total Alkalinity as C03--. 
TABLE A.13. contd

\begin{tabular}{|c|c|c|}
\hline 42 & 14 & 45 \\
\hline 171 & 207 & 237 \\
\hline 8.19 & 8.81 & $B .67$ \\
\hline 348 & 306 & 242 \\
\hline 0.106 & 0.059 & $<0.03$ \\
\hline 0.013 & 0.013 & 0.016 \\
\hline 6.82 & 6.55 & 10.5 \\
\hline 80.004 & $<0.004$ & $<0.004$ \\
\hline 10.02 & $i 0.02$ & $<0.02$ \\
\hline$<0.004$ & $<0.004$ & $<0.004$ \\
\hline 0.027 & $<0.005$ & $<0.005$ \\
\hline 9 & 9.2 & 8.1 \\
\hline 1.63 & 2.01 & 3.31 \\
\hline 00.002 & $<0.002$ & $<0.002$ \\
\hline 64.8 & 66.4 & 56.1 \\
\hline 00.1 & $\langle 0\}$. & $<0.1$ \\
\hline$\{0.3$ & $<0.3$ & $<0.3$ \\
\hline 0.06 & $<0.06$ & $<0.06$ \\
\hline 7.51 & 8.19 & 8.39 \\
\hline 0.153 & 0.156 & 0.224 \\
\hline 10.02 & 80.02 & $<0.02$ \\
\hline 0.024 & $<0.008$ & 60.008 \\
\hline 6.3 & 6.6 & 6 \\
\hline 23 & 23 & 22.6 \\
\hline 4.3 & 6.3 & <.3 \\
\hline 1.1 & 1.4 & 60.5 \\
\hline 74 & 82 & 78.3 \\
\hline 62.95 & 61.73 & $.50 .5 !$ \\
\hline 10.32 & 11.04 & 11.04 \\
\hline 0.00 & 0.00 & 0.00 \\
\hline 0.138 & 0.171 & 0.169 \\
\hline$\hat{6} .79$ & 0.98 & 0.97 \\
\hline 0.82 & 1.06 & \\
\hline 10.4 & $<0.4$ & $\$ 0.4$ \\
\hline 3.53 & 3.62 & 3.45 \\
\hline 3.92 & 4.11 & 3.79 \\
\hline 41.28 & 41.4 & 35.88 \\
\hline 14.32 & 11.37 & \\
\hline 77.55 & 93.28 & 112.87 \\
\hline
\end{tabular}

(---) Data not available.

(\#) Total Alkalinity as CO3--. 
TABLE A.14. Flow Through Leach Test on whole Grout with Groundwater (COLLT1)

\begin{tabular}{|c|c|c|c|c|c|c|c|c|c|c|c|}
\hline SAMPLE & -..-- & $\begin{array}{l}\text { WELL } \\
\text { HATER }\end{array}$ & 1 & 2 & 3 & 4 & 5 & 6 & 7 & 8 & 9 \\
\hline TIME & days & $-\cdots$ & 3 & 10 & 14 & 17 & 21 & 24 & 28 & 31 & 35 \\
\hline $\mathrm{ph}$ & & 8.1 & 12.90 & 12.70 & 12.81 & 12.65 & 12.33 & 12.17 & 11.43 & 12.27 & 11.03 \\
\hline Eh & av & 295 & 130 & 172 & 123 & 114 & 238 & 163 & 241 & 197 & 193 \\
\hline Al & $\lg / 1$ & $<0.1$ & 21.00 & 18.00 & 33.00 & 18.10 & 12.50 & 10.30 & 4.14 & 4.75 & 7.06 \\
\hline$B z$ & $g / 1$ & 0.06 & $<0.002$ & 0.005 & $<0.002$ & $<0.002$ & $<0.002$ & $<0.002$ & $<0.002$ & $<0.002$ & $<0.002$ \\
\hline $\mathrm{Ca}$ & $g / 1$ & 56 & 3.3 & 4.5 & 4.3 & 1.2 & 1.2 & 1.8 & 0.9 & 1.7 & 0.6 \\
\hline Cd & $g / !$ & $<0.005$ & $<0.004$ & $<0.004$ & $<0.004$ & $<0.004$ & $<0.004$ & $<0.004$ & $<0.004$ & 60.004 & 60.004 \\
\hline $6 r$ & $g / 1$ & $<0.015$ & $<0.02$ & 60.02 & $<0.02$ & $\leqslant 0.02$ & $<0.02$ & $<0.02$ & $<0.02$ & 10.02 & $\langle 0.02$ \\
\hline Cu & 11 & 00.005 & 0.012 & 0.005 & 0.024 & 0.024 & 0.009 & 0.006 & $<0.004$ & 10.004 & $(0.004$ \\
\hline $\mathrm{Fe}$ & $/ 1$ & $\{0.01$ & 0.114 & 0.020 & 0.067 & 0.037 & 0.033 & 1.620 & $<0.005$ & 0.013 & 0.005 \\
\hline$k$ & $\operatorname{sog} / 1$ & 7.8 & 45.0 & 49.0 & 83.0 & 49.0 & 34.0 & 29.0 & 16.0 & 31.0 & 23.0 \\
\hline $\mathrm{Hg}$ & $g / 1$ & 14.4 & 40.06 & 10.06 & $<0.06$ & $<0.06$ & $<0.06$ & 0.09 & $<0.06$ & $\{0.06$ & $<0.06$ \\
\hline $\mathrm{in}$ & gil & $<0.01$ & $<0.002$ & $<0.002$ & $<0.002$ & $<0.002$ & $<0.002$ & 10.002 & $<0.002$ & 60.002 & 60.002 \\
\hline $\mathrm{Ha}$ & agil & 25 & 1280 & 1210 & 2000 & 1100 & 720 & 643 & 262 & 630 & 410 \\
\hline$P$ & $a q / 1$ & $\{0.1$ & 0.1. & 00.1 & 0.20 & 0.14 & $<0.1$ & $<0.1$ & 0.1 & 10.1 & 0.1 \\
\hline dE FBA & agil & $\{0.31$ & $<0.3$ & 60.3 & 0.61 & 0.43 & $<0.3$ & 60.3 & $\{0.3$ & 60.3 & $<0.3$ \\
\hline $\mathrm{Fb}$ & in $/ 1$ & 0.05 & 10.06 & 10.06 & 0.06 & $<0.06$ & $<0.06$ & $<0.06$ & $<0.06$ & $<0.06$ & 80.06 \\
\hline $5 i$ & $g / 1$ & IS & B.5 & 13.0 & 15.1 & 11.6 & 11.3 & 11.1 & 10.8 & 10.4 & 11.2 \\
\hline $5 r$ & & 0.25 & 0.046 & 0.090 & 0.094 & 0.089 & 0.061 & 0.070 & 0.055 & 0.069 & 0.053 \\
\hline in & $g / 1$ & $\leqslant 0.01$ & 0.670 & 0.100 & 0.285 & 0.158 & 0.027 & 0.026 & 0.110 & 0.024 & $<0.02$ \\
\hline Ir & $\log / 1$ & 80.008 & $<0.008$ & $<0.008$ & $<0.008$ & $<0.008$ & $<0.008$ & $\$ 0.008$ & $<0.008$ & $<0.008$ & $<0.00 B$ \\
\hline F- & $g / 1$ & -80.5 & 11.0 & 11.0 & 18.0 & 11.0 & 9.0 & 8.0 & 5.8 & 8.6 & 6.3 \\
\hline $\mathrm{Cl}-$ & & 22 & 12 & 22 & 22 & 20 & 23 & 20 & .21 & $2 i$ & 23 \\
\hline 102- & & 0.03 & $<3.0$ & $<0.1$ & 10.1 & $<0.1$ & 00.1 & 0.1 & 0.1 & $<0.1$ & 60.1 \\
\hline $\mathrm{HO3-}$ & $g / 1$ & 0.1 & 19.0 & 13.0 & 9.0 & 15.0 & 30.0 & 6.0 & 2.9 & a.5 & 5.1 \\
\hline $504--$ & n! & 86 & 150 & 180 & 270 & 160 & 130 & 120 & 80 & 120 & 100 \\
\hline $\mathrm{HCOS}-$ & & $17 i$ & 0.0 & 0.0 & 0.0 & 0.0 & 0.0 & 0.0 & 0.0 & 0.0 & 0.0 \\
\hline $\mathrm{CO} 3-$ & & 2.1 & 6276.9 & 3074.4 & 5315.9 & 3062.2 & 1747.2 & 1259.0 & 438.0 & 246 & $\$ 10$ \\
\hline $\mathrm{OH}-$ & $g / 1$ & 0 & 0.0 & 0.0 & 0.0 & 0.0 & 0.0 & 0.0 & 0.0 & 250.5 & 52.7 \\
\hline 8 & $69 / 1$ & 0.06 & 1.500 & 1.200 & 2.210 & 2.200 & 1.150 & 0.511 & 1.300 & 0.587 & 0.368 \\
\hline $25 \mathrm{H3803}$ & $g / 1$ & 4. 34 & 8.57 & 6.90 & 12.64 & 12.60 & 0.60 & 3.92 & 7.47 & 3.35 & 2.10 \\
\hline$T B C$ & gil & 1 & 17.1 & 7.4 & 20.1 & 11.4 & 6.9 & $\cdots$ & -..- & $\cdots$ & $-\ldots$ \\
\hline $\mathrm{P}[14-\ldots$ & $g / 1$ & $\langle 1.0$ & \$5.0 & $\$ 5.0$ & $\$ 5.0$ & 85.0 & $\langle\mathbf{s . 0}$ & 85.0 & $\langle 5.0$ & $\langle 5.0$ & 15.0 \\
\hline CATIONS & eqil & 5.20 & 56.97 & 54.69 & 89.30 & 47.90 & 32.24 & 28.80 & 11.85 & 28.28 & 18.45 \\
\hline AHIOES & seq/1 & 5.54 & 119.00 & 61.03 & 102.00 & 50.68 & 38.38 & 26.80 & 13.45 & 26.62 & 19.91 \\
\hline TOT.ALK., E & $q / 1$ & $\$ 2.16$ & 3433 & 1669 & 2835 & 1674 & 1032 & 697 & 325 & 388 & 303 \\
\hline IC & dị $/ 1$ & 34.1 & 71.5 & 27.2 & 62.7 & 28.4 & 56.3 & $-\cdots$ & --- & --- & $\cdots$ \\
\hline YORE VOL. & & & 0.31 & 0.51 & 1.37 & 1.98 & 3.16 & 4.12 & 4.33 & 5.67 & 6.37 \\
\hline
\end{tabular}

(-) Data not available.

(\#) Total Alkalinity as CO3-.. 
TABLE A.14.

contd

\begin{tabular}{|c|c|c|c|c|c|c|c|c|c|c|c|}
\hline SAMPLE I & -....- & 10 & 11 & 12 & 13 & 14 & 15 & 16 & 17 & 18 & 19 \\
\hline TIME & days & 45 & 52 & 56 & 59 & 63 & 66 & 70 & 74 & 78 & 31 \\
\hline pH & & 11.92 & 11.63 & 11.59 & 10.73 & 10.51 & 10.95 & 10.33 & 10.19 & 10.61 & 11.37 \\
\hline Eh & ar & 122 & 180 & 243 & 207 & 241 & 219 & 256 & 310 & 194 & 573 \\
\hline AI & $09 / 1$ & 3.47 & 3.17 & 1.66 & 1.04 & 0.11 & 0.12 & $<0.03$ & 60.03 & 00.03 & $0.3 B$ \\
\hline $8 \mathrm{a}$ & $g / 1$ & 0.019 & $<0.002$ & 0.003 & 0.004 & 0.004 & 0.005 & 0.008 & 0.006 & 0.004 & 0.002 \\
\hline$C_{d}$ & $a g / 1$ & 1.2 & 0.9 & 1.2 & 0.7 & 1.3 & 1.4 & 3.9 & 2.6 & 1.4 & 1.2 \\
\hline Cd & $g / 1$ & $<0.004$ & $<0.004$ & $<0.004$ & $<0.004$ & $<0.004$ & $<0.0014$ & 60.004 & $<0.004$ & $<0.004$ & $\therefore 0.004$ \\
\hline$[r$ & $. g / 1$ & 10.02 & $<0.02$ & $<0.02$ & $<0.02$ & 60.02 & 10.02 & $<0.02$ & $<0.02$ & 60.02 & $\$ 0.02$ \\
\hline $\mathrm{Cu}$ & $9 / 1$ & $<0.004$ & $<0.004$ & $<0.004$ & $<0.004$ & $<0.004$ & $<0.004$ & $<0.004$ & $<0 . v 014$ & 0.0014 & 60.004 \\
\hline Fe & $2 g / 1$ & 0.036 & 0.006 & $<0.005$ & $<0.005$ & $<0.005$ & $<0.005$ & 60.005 & $<0.005$ & 60.005 & 60.1005 \\
\hline$k$ & $g / 1$ & 18.4 & 17.0 & 14.0 & 12.0 & 11.0 & 9.4 & 9.1 & 8.4 & 8.6 & 10.1 \\
\hline $\mathrm{ng}$ & $\operatorname{mg} / 1$ & $<0.06$ & $<0.06$ & $<0.06$ & 10.06 & 10.06 & 0.19 & 2.50 & 1.06 & 0.14 & 30.016 \\
\hline In & $\Delta / 1$ & $<0.002$ & $<0.002$ & $<0.002$ & $<0.002$ & $<0.002$ & $<0.002$ & $\langle 0.002$ & 10.602 & 60.002 & 00.002 \\
\hline $\mathrm{Ha}$ & $g / 1$ & 366 & 305 & 204 & 162 & 125 & 110 & 99 & 95 & 114 & 159 \\
\hline$f$ & $\lg /$ & $<0.1$ & $<0.1$ & 60.1 & $<0.1$ & 0.1 & 0.1 & 0.1 & $\langle 0.1$ & 0.1 & 0.1 \\
\hline as $\mathrm{PO4}$ & $2 g / 1$ & 10.3 & $<0.3$ & $\{0.3$ & $<0.3$ & $<0.3$ & $<0.3$ & 60.3 & $<0.3$ & 60.3 & 40.3 \\
\hline $\mathrm{PG}$ & $19 / 1$ & $<0.06$ & 80.06 & $<0.06$ & $<0.06$ & $<0.06$ & 10.06 & 0.06 & 60.06 & 80.06 & 0.06 \\
\hline $\mathrm{Sj}$ & $49 / 1$ & 13.6 & 13.2 & 11.4 & 10.0 & 9.6 & 9.3 & 9.0 & 9.9 & 11.9 & 14.7 \\
\hline $5 r$ & $2 g / 1$ & 0.065 & 0.051 & 0.063 & 0.064 & 0.063 & 0.058 & 0.092 & 0.081 & 0.055 & 0.042 \\
\hline in & $9 / 1$ & 0.023 & $<0.02$ & 10.02 & $<0.02$ & 0.02 & 0.049 & 0.02 & 80.02 & 0.101 & 60.02 \\
\hline Ir & $\lg / \mathrm{l}$ & $<0.008$ & $<0.008$ & 10.008 & $<0.008$ & $<0.008$ & 0.1 & $<0.008$ & 0.1 & 0.008 & 60.600 \\
\hline F- & ig/l & 4.8 & 5.6 & 6.6 & 5.7 & 5.8 & 5.6 & 5.1 & 6.0 & 5.9 & 7.4 \\
\hline $\mathrm{Cl}-$ & ag/l & 23 & 22 & 23 & 23 & 23 & 25 & 24 & 24 & 23 & 23 \\
\hline H02- & $a g / 1$ & 0.1 & $\{0.1$ & $<0.1$ & 0.1 & $<0.1$ & 0.1 & \{0.1 & $0 . j$ & 0.1 & 0.1 \\
\hline $\mathrm{NOS-}$ & $g / 1$ & 4.9 & 3.6 & 3.6 & 2.0 & 1.3 & 1.2 & 0.7 & 1.1 & 1.4 & 2.5 \\
\hline 504-- & agil & 96 & 88 & 96 & 83 & 79 & 85 & 8.5 & 76 & 70 & 8ิ4 \\
\hline $\mathrm{HCOJ}^{-}$ & $\operatorname{og} / 1$ & 0.0 & 0.0 & 0.0 & 0.0 & 0.0 & 0.0 & 2.0 & 20.5 & $i_{0}, j$ & 173.0 \\
\hline co3-- & gil & 172 & 488.0 & 345.7 & $i 24$ & 110 & 78 & 76 & $6 \bar{i}$ & 52 & 52 \\
\hline OHH- & $g / 1$ & 146.5 & 0.0 & 0.0 & 13.0 & 3.4 & 13.0 & 0.0 & 0.0 & 20.4 & $\hat{\mathbf{t}} \hat{0}$ \\
\hline $\bar{B}$ & $9 g / 1$ & 0.310 & 0.257 & 0.181 & 0.156 & 0.129 & 0.142 & i. 119 & 0.159 & 6.234 & 0.301 \\
\hline as $4350 j$ & $\lg / 1$ & 1.77 & 1.47 & 1.03 & 0.89 & 0.74 & 0.81 & 0.60 & 0.93 & 1.30 & 1.49 \\
\hline Toc & ail & $\ldots$ & $\ldots$ & $\cdots$ & $-\cdots$ & --- & --- & --- & 1.4 & i.5 & 2.2 \\
\hline FO4--- & gil & $\langle 5.0$ & $\{5.0$ & 65.0 & $<5.0$ & 6.0 & $\langle 5.0$ & 8.0 & $(5)$. & 15.6 & $(5.0$ \\
\hline CATIOHS & seq/l & 16.45 & 13.75 & 9.29 & 7.39 & 5.79 & 5.11 & 4.54 & 4.57 & $5.20^{\circ}$ & 7.25 \\
\hline AHIONS & aeqil & 17.45 & 14.18 & 10.29 & 7.61 & 6.49 & 6.16 & 5.26 & 4.99 & 5.50 & 7.37 \\
\hline TOT.ALK. & $\operatorname{sg} / 1$ & 434 & 341 & 217 & 147 & 116 & 101 & 77 & 72 & 83 & 137 \\
\hline IE & $a g / 1$ & -- & $\cdots$ & $\cdots$ & $\cdots$ & $\cdots$ & --- & --- & 22.5 & 27.5 & 38.8 \\
\hline FUAE VOL. & & 6.13 & 9.76 & 11.7 & 13.01 & 15.91 & 18.13 & 20.62 & 33.69 & 34.96 & $3.2 j$ \\
\hline
\end{tabular}

\footnotetext{
(---) Data not available.

(\#) Total Alkalinity as C03--.
} 
TABLE A.15. Flow Through Leach Test on Crushed Grout with Groundwater (BCGT)

HELL

\begin{tabular}{|c|c|c|c|c|c|c|c|c|c|c|c|}
\hline SAMPLE & $\cdots$ & HATER & 1 & 2 & 3 & 4 & 5 & 6 & 7 & $B$ & 7 \\
\hline TIME & days & & 3 & 7 & 10 & 14 & 17 & 21 & 24 & 27 & $3 !$ \\
\hline $\mathrm{pH}$ & & 8.10 & 11.89 & 12.06 & 11.95 & 10.95 & 10.53 & 10.02 & 9.55 & 8.52 & \\
\hline Eh & $\mathbf{s y}$ & 295 & 360 & 285 & 354 & 225 & 294 & $24 !$ & 370 & 258 & \\
\hline कीl & $9 / 1$ & $<0.1$ & 11 & 17.3 & 12.6 & 5.76 & 1.35 & 0.17 & 0.13 & 0.07 & 0.12 \\
\hline Ba & $\lg / 1$ & 0.060 & 0.004 & 0.003 & 0.002 & 0.002 & 0.003 & 0.011 & 0.01 & 0.014 & $0.0:$ \\
\hline$\tilde{C a}_{\mathrm{a}}$ & $90 / 1$ & 56.0 & 1.44 & 1.62 & 1.76 & 1.28 & 1.71 & 5.84 & 5.83 & 8.51 & 6.7 \\
\hline 61 & igfi & $\therefore 0.005$ & $<0.004$ & 80.004 & 10.004 & 60.004 & $<0.004$ & 60.004 & $\{0.004$ & 00.004 & $(0,60$ \\
\hline ir & $\operatorname{ag} / 1$ & 60.015 & 0.027 & 0.034 & 0.025 & $<0.02$ & 60.02 & $<0.02$ & 0.02 & $<0.02$ & $<0.02$ \\
\hline Cu & $9 / i$ & $\therefore 0.005$ & 0.11 & 0.005 & 0.005 & $<0.004$ & 10.004 & $<0.004$ & 10.004 & 10.004 & 16.00 \\
\hline$\overline{F_{p}}$ & als $/ 1$ & 60.601 & 0.025 & 0.017 & 0.007 & $\{0.005$ & $<0.005$ & 10.005 & 10.005 & 0.005 & $\{0.00\}$ \\
\hline$k$ & $g / 1$ & 7.8 & 22 & 20 & 15 & 11 & 8.7 & 8.2 & 8.4 & 7.9 & \\
\hline is & $\Delta g / 3$ & 14.40 & $<0.06$ & 10.06 & 0.06 & $<0.06$ & 0.421 & 5.07 & 5.86 & 6.67 & 5.55 \\
\hline Mn & $69 / 1$ & $(0.01$ & $<0.002$ & 60.002 & $<0.002$ & 30.002 & $(0.0012$ & $<0.002$ & $<0.002$ & $(0.002$ & $(0.002$ \\
\hline $\mathrm{Ha}$ & $a g / !$ & 25 & 681 & 639 & 433 & 262 & 158 & 91.9 & 80 & 65 & 66 \\
\hline$P$ & $\lg i$ & $\langle 0.1$ & 0.1 & 0.09 & 0.1 & $<0.1$ & $<0.1$ & 60.1 & $<0.1$ & 10.1 & 0.1 \\
\hline is $F 04$ & $y / 1$ & 40.3 & $<0.3$ & 0.27 & $<0.3$ & $\{0.3$ & $\{0.3$ & 10.3 & 50.3 & $<0.3$ & 0.3 \\
\hline $\mathrm{Fb}$ & 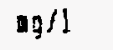 & $(0.65$ & 0.05 & 10.06 & 00.06 & $<0.06$ & 00.06 & 60.06 & $: 0.06$ & 10.00 & 60.06 \\
\hline$\overline{j i}$ & i! & 15.0 & 16.5 & 25.6 & 24.3 & 17.4 & 12.1 & 10.3 & 9.92 & 9.57 & 7.62 \\
\hline $5 r$ & $/ 1$ & 0.250 & $0.02 B$ & 0.022 & 0.019 & 0.022 & 0.036 & 0.073 & 0.077 & 0.095 & 0.088 \\
\hline in & $i 1$ & 60.01 & 0.037 & 0.027 & $<0.02$ & $<0.02$ & $<0.02$ & $<0.02$ & $\{0.02$ & $<0.02$ & 00.02 \\
\hline It & & 60.008 & $<0.008$ & 0.018 & 0.018 & 0.019 & 0.041 & 0.081 & 0.036 & 0.009 & 0.0018 \\
\hline F- & & 80.5 & to & 51 & 35 & 21 & 13 & 9 & 10 & 8 & 10 \\
\hline $\mathrm{CI}-$ & i1 & 22 & 27 & 27 & 25 & 25 & 24 & 27 & 27 & 27 & 23 \\
\hline $\mathrm{HO}_{2}^{2-}$ & il & 00.03 & 10.3 & $(0.3$ & 60.3 & $<0.3$ & 60.3 & 60.3 & 10.3 & 80.3 & 0.3 \\
\hline MOS- & 11 & 0.1 & 40 & 17 & 8.5 & 3.8 & 0.9 & $<0.05$ & 0.05 & 80.05 & 60.05 \\
\hline SOS:- & aty $/ 1$ & 86 & 470 & 250 & 150 & 110 & 84 & 79 & 77 & 77 & 75 \\
\hline $\mathrm{HCG3-}$ & $9 / 1$ & 171.0 & 0.00 & 0.00 & 0.00 & 0.00 & 0.00 & 44.90 & 61.73 & 84.18 & 50.51 \\
\hline c003-- & $10 / 1$ & ? & 541.80 & 441.60 & 358.80 & 440.00 & 132.46 & 55.20 & 22.08 & 5.52 & 11.04 \\
\hline $\mathrm{OH}^{-}$ & $\operatorname{ag} / 1$ & 0.0 & 35.03 & 117.30 & 36.02 & 31.73 & 0.00 & 0.00 & 0.00 & 0.00 & 0.00 \\
\hline 5 & $\lg i$ & 9.1360 & 2.13 & 2.64 & 1.75 & 0.954 & 0.487 & 0.27 & 0.22 & 0.164 & $\dot{0} .208$ \\
\hline H3803 & agil & 0.34 & 12.18 & 15.1 & 10.09 & 5.41 & 2.78 & 1.54 & 1.26 & 1.05 & 1.87 \\
\hline TEE & 1011 & 1.0 & ---- & --- & ---- & --- & -..- & --- & --- & $-\cdots$ & $--\cdot$ \\
\hline POS- & ạ/l & 60.1 & 60.4 & 10.4 & $<0.4$ & 0.4 & 10.4 & $\{0.7$ & $\langle i, 4$ & 60.4 & 60.4 \\
\hline CATIOHS & seq/! & 5.28 & 30.25 & 26.36 & 19.30 & 11.74 & 7.21 & 4.92 & 4.47 & 4.02 & 3.98 \\
\hline AHIGKS & Вевй $l]$ & 5.54 & 36.97 & 30.55 & 22.83 & 20.70 & 7.54 & 5.46 & 4.64 & 4.35 & 3.83 \\
\hline GT.ALK.: & ogil & 92 & 709.5 & 648.6 & 510.6 & 496 & 132.48 & 77.28 & 52.44 & 46.32 & 35.86 \\
\hline ic & an/l & 34.1 & $-\cdots$ & ---- & ---- & -- & --- & $\cdots$ & ---- & -..- & --- \\
\hline OAE VOL. & & & 0.37 & 1.14 & 1.66 & 2.55 & $3.3 !$ & 4.53 & 5.67 & $5.8 i$ & 6.15 \\
\hline
\end{tabular}

(--) Data not available.

(\#) Total Alkalinity as c03--. 
TABLE A.15. contd

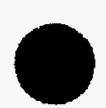

\begin{tabular}{|c|c|c|c|c|c|c|c|c|}
\hline SAMPLE & -.- & 10 & 11 & 12 & 13 & 14 & 15 & 16 \\
\hline TIME & days & 38 & 45 & 49 & 52 & 56 & 61 & 64 \\
\hline $\mathrm{pH}$ & & 8.65 & 8.31 & 8.13 & 8.28 & 8.17 & 7.84 & 7.94 \\
\hline Eh & ay & 284 & 341 & 396 & 397 & 385 & 370 & 413 \\
\hline Al & $8 g / 1$ & 0.11 & 0.034 & 0.032 & 0.039 & $<0.03$ & $<0.03$ & $<0.05$ \\
\hline $8 a$ & $9 / 1$ & 0.012 & 0.018 & 0.017 & 0.02 & 0.018 & 0.014 & 0.012 \\
\hline$C_{2}$ & $g / 1$ & 9.52 & 20.3 & 24.4 & 29.7 & 28.8 & $2 \overline{8} .0$ & 29.2 \\
\hline Cod & $g / 1$ & $<0.004$ & $<0.004$ & $<0.004$ & 60.004 & $<0.004$ & $<0.004$ & 10.004 \\
\hline Cr & ig $/ 1$ & $<0.02$ & $<0.02$ & $<0.02$ & $<0.02$ & 10.02 & 30.02 & 10.02 \\
\hline $\mathrm{Cu}$ & $9 / 1$ & $<0.004$ & $<0.004$ & $<0.004$ & $<0.004$ & $<0.004$ & $<0.004$ & $<0.004$ \\
\hline Fe & $g / 1$ & $<0.005$ & $<0.005$ & $<0.005$ & $<0.005$ & 0.006 & $<0.005$ & 60.005 \\
\hline$k$ & $0 / 1$ & 9.4 & 8.8 & 8.2 & 8.3 & 7.7 & 7.0 & 7.0 \\
\hline$y_{9}$ & $\Delta q / 1$ & 5.92 & 9.37 & 9.85 & 11.3 & 10.80 & 9.95 & 9.97 \\
\hline $\mathrm{kn}$ & $\ln / 1$ & $<0.002$ & $<0.002$ & $<0.002$ & 50.002 & $<0.002$ & $<0.002$ & 80.002 \\
\hline $\mathrm{Na}$ & $a g / 1$ & 61 & 38 & 29.9 & 27.6 & 29 & .29 & 28 \\
\hline$F$ & $g / 1$ & $<0.1$ & $<0.1$ & $<0.1$ & $<0.1$ & 00.1 & $<0.1$ & $<0.1$ \\
\hline as PỌ & $g / 1$ & 60.3 & 60.3 & 60.3 & 10.3 & 10.3 & 00.3 & $0 . j$ \\
\hline $\mathrm{Pb}$ & $\lg / 1$ & 10.06 & $<0.06$ & 10.08 & $<0.06$ & $<0.06$ & 60.06 & 10.06 \\
\hline$S i$ & ag/l & 8.93 & 8.1 & 8.97 & 10.3 & 9.8 & 8.7 & 8.9 \\
\hline sr & $g / 1$ & 0.107 & 0.16 & 0.177 & 0.201 & 0.203 & 0.203 & 0.208 \\
\hline In & $g / 1$ & 10.02 & $<0.02$ & $<0.02$ & $\{0.02$ & 10.02 & $<0.02$ & 10.02 \\
\hline ir & $9 / 1$ & $<0.008$ & $<0.008$ & 60.008 & $<0.008$ & $<0.008$ & 10.008 & 0.1 \\
\hline F- & $2 g / 1$ & 10 & 7.3 & 7.6 & 7.4 & 8.8 & 9.1 & 9.8 \\
\hline Cl- & $g / 1$ & 24 & 22 & 21 & 21 & 23 & 21 & 23 \\
\hline $\mathrm{HO}_{2}-$ & ogil & 80.3 & $<0.3$ & $<0.3$ & 60.3 & $<0.3$ & $\langle 0.3$ & \{́́.j \\
\hline HOJ- & $\Delta g / 1$ & $<0.05$ & 60.05 & 10.05 & 0.05 & 0.05 & 60.05 & 60.05 \\
\hline 504-- & $g / 1$ & 75 & 71 & 69 & 66 & 77 & 73 & 78 \\
\hline $\mathrm{HCCOB-}$ & $n / 1$ & 67.34 & 50.51 & 56.12 & 89.79 & 56.12 & 22.45 & Si. 51 \\
\hline CDJ-- & $8 / 1$ & 5.52 & $5.5 \hat{2}$ & 0.00 & 0.00 & 5.52 & 0.00 & 0.00 \\
\hline OH- & $g / 1$ & 0.00 & 0.00 & 0.00 & 0.00 & 0.00 & 0.00 & 0.00 \\
\hline B & $9 / 1$ & 0.2 & 0.135 & 0.126 & 0.125 & $0.14 j$ & 0.152 & 6.150 \\
\hline $25 \mathrm{H3BOJ}$ & $\operatorname{mog} / 1$ & 1.14 & 0.77 & .0 .72 & 0.71 & 0.82 & 0.87 & 0.86 \\
\hline Ioc & $g / 1$ & $-\infty$ & -- & $-\infty$ & $\cdots$ & $\cdots$ & --- & $-\cdots$ \\
\hline PO4--- & $\log / 1$ & $\langle 0.5$ & $<0.4$ & 60.4 & $<0.4$ & 80.4 & 60.4 & $<0.4$ \\
\hline Critions & meqil & 3.86 & 3.67 & 3.54 & 3.63 & 3.70 & 2.37 & 3.69 \\
\hline ANIOAS & neq/1 & 4.05 & 3.50 & 3.35 & 3.67 & 3.82 & 2.97 & 3.62 \\
\hline TOT. ALK. * & $\Delta g i$ & 38.64 & 30.36 & 27.6 & 44.16 & 33 & li & 25 \\
\hline IC & $99 i$ & -- & --- & -- & ---- & --- & $\cdots$ & -... \\
\hline GRE YOL. & & 7.58 & 9.04 & 10.21 & 21.16 & 11.67 & 22.43 & 12.35 \\
\hline
\end{tabular}

(--) Data not available.

(\#) Total Alkalinity as CO3--. 


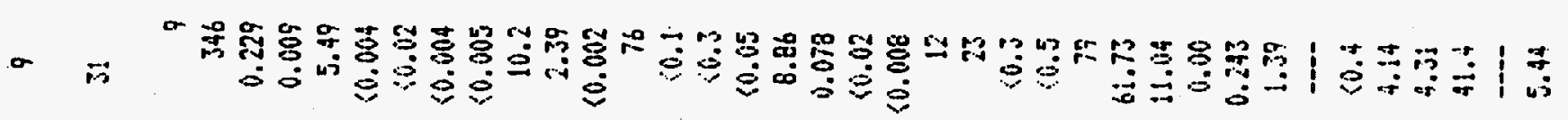

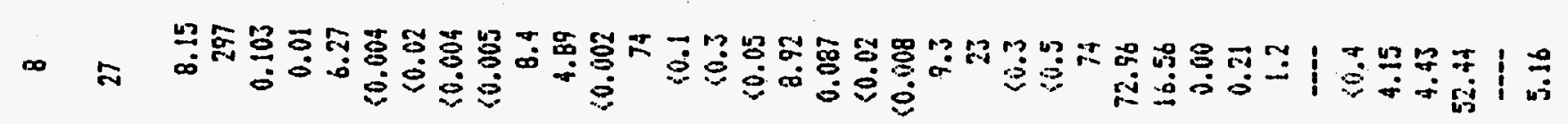

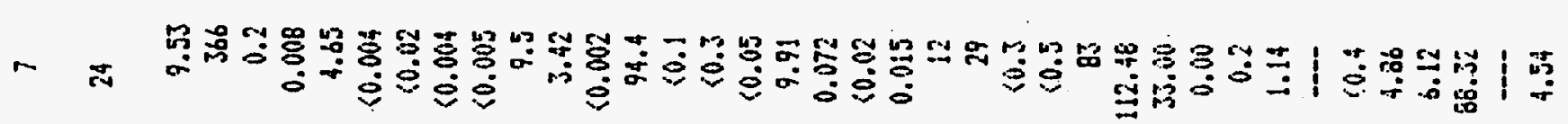

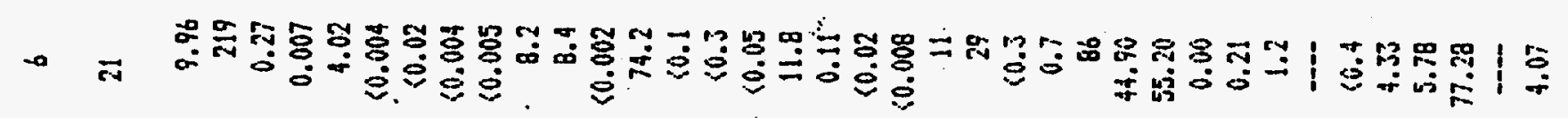

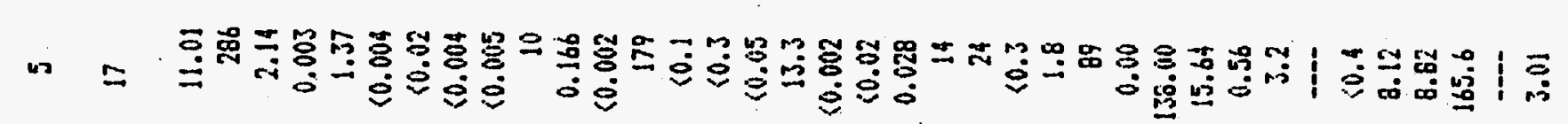

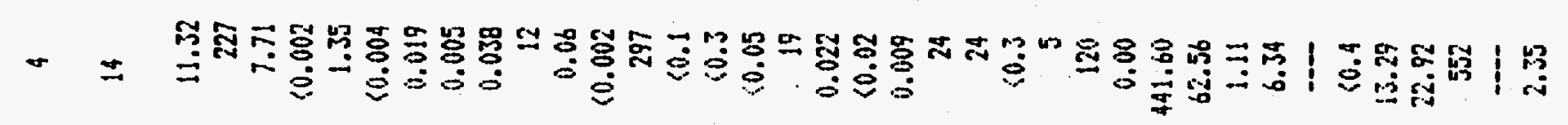

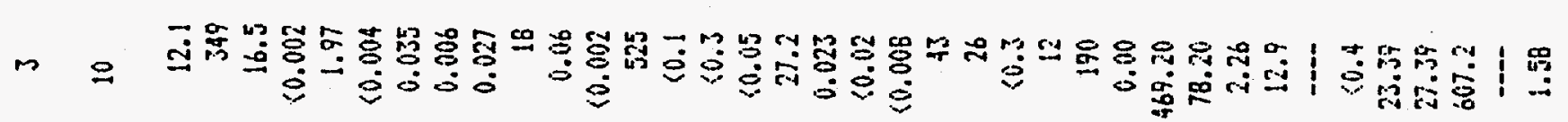

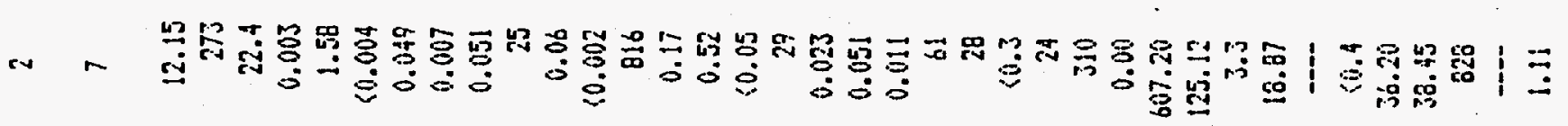

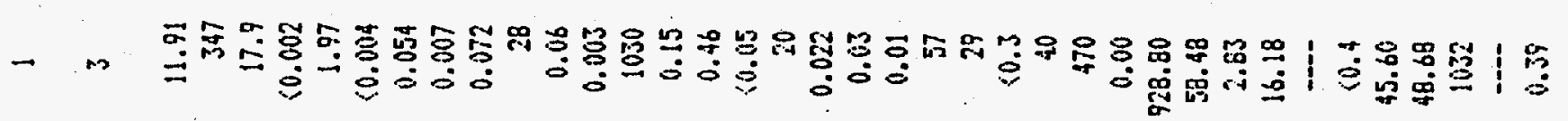

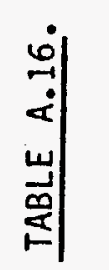

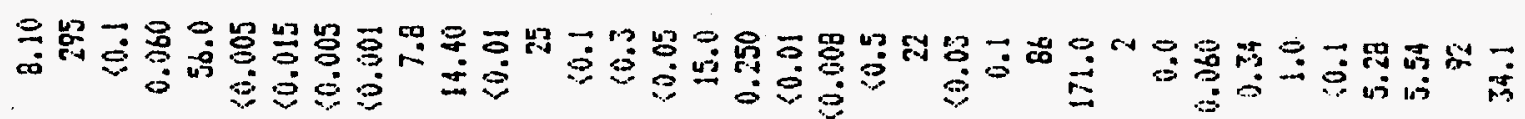

声㿥

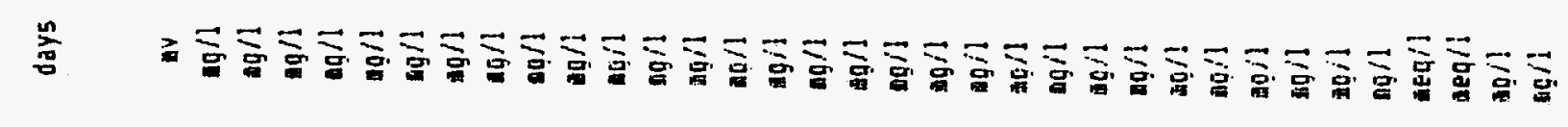

i 营

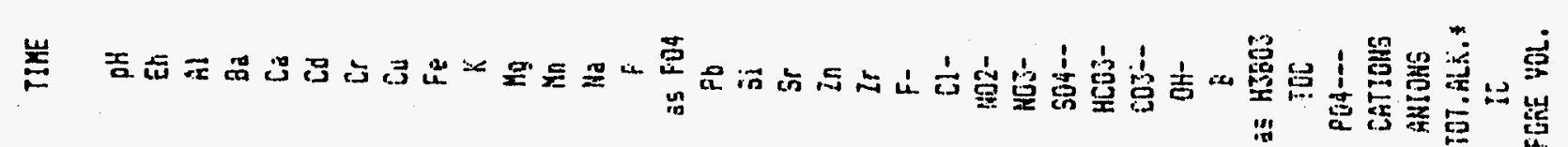




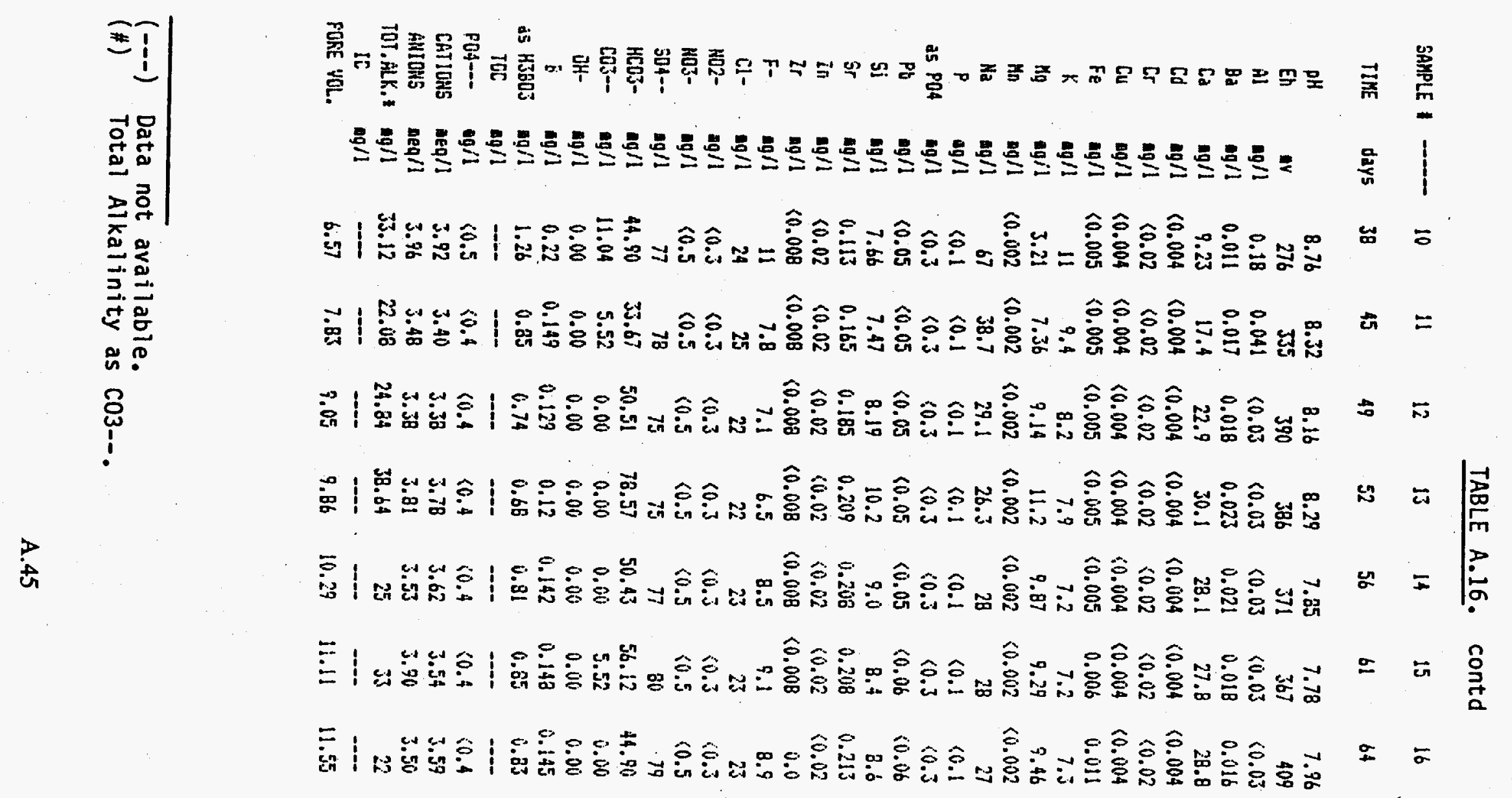


TABLE A.17. Flow Through Leach Test on Crushed Grout with Groundwater (BCGC)

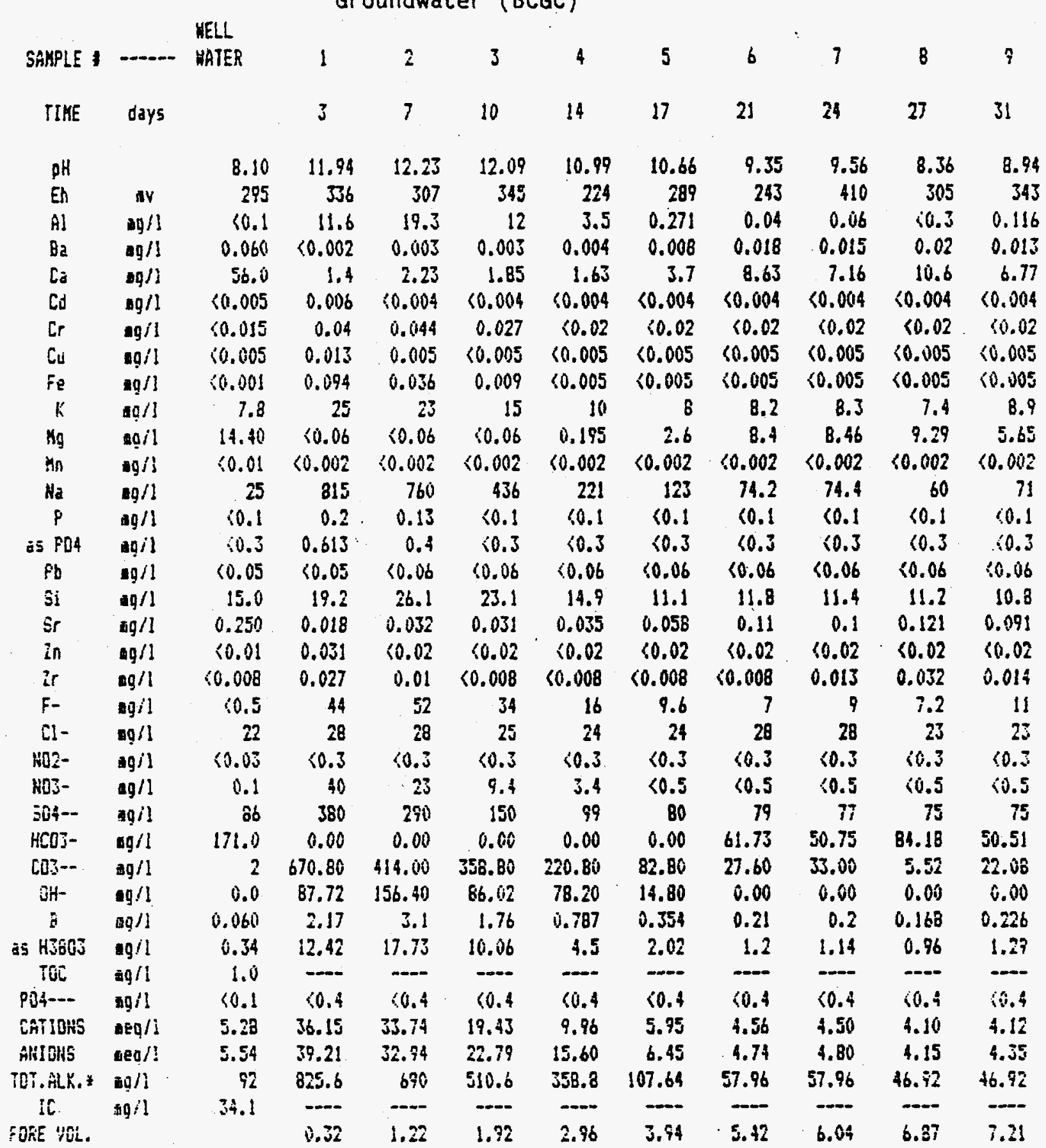

(--) Data not available.

(\#) Total Alkalinity as C03--. 


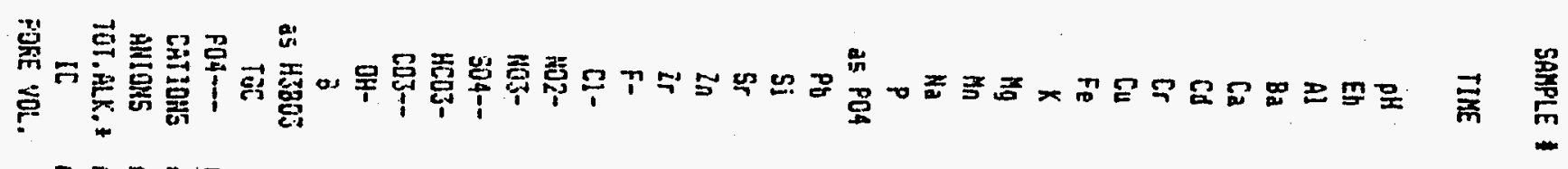

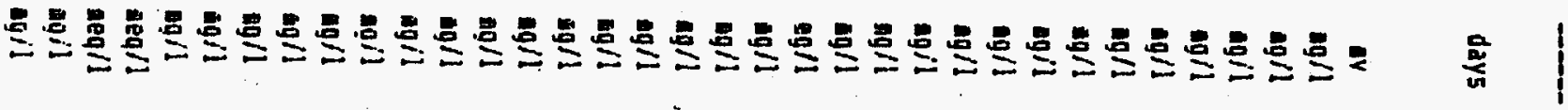

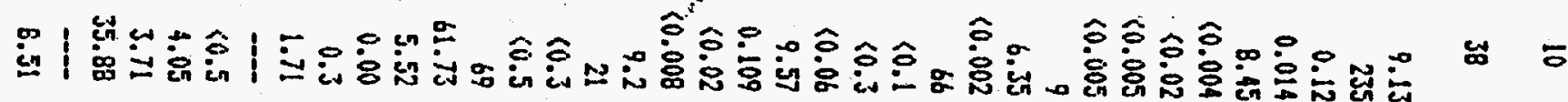
苟|

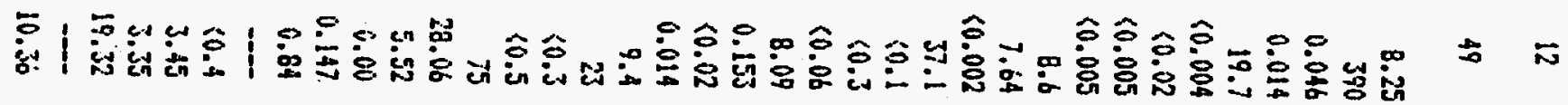

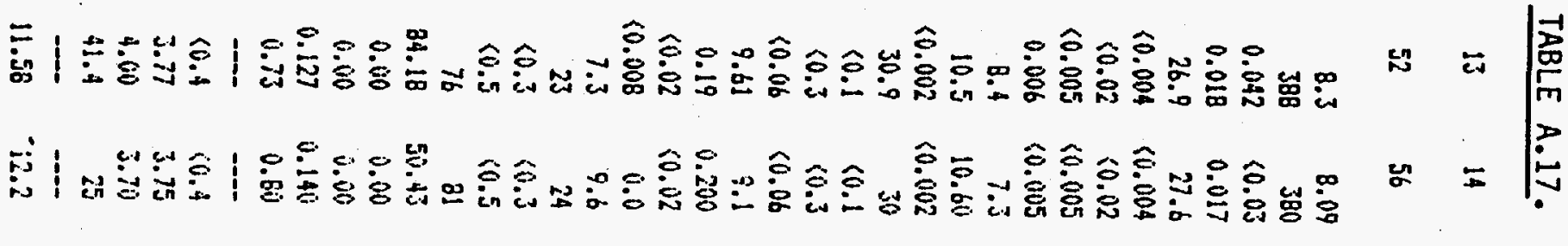

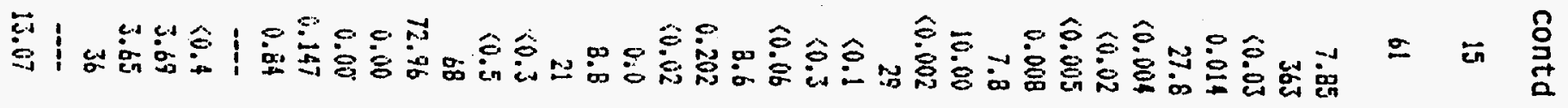

整| 
TABLE A.18. Column Adsorption Test on Crushed Grout in Sediment (CGT)

\begin{tabular}{|c|c|c|c|c|c|c|c|c|c|c|c|}
\hline SAMPLE I & -...- & $\begin{array}{l}\text { WELL } \\
\text { WATER }\end{array}$ & $!$ & 2 & 3 & 4 & 5 & 6 & 7 & 8 & 9 \\
\hline TIME & days & & 3 & 7 & 11 & 15 & 18 & 22 & 25 & 29 & 32 \\
\hline ph & & 8.10 & 12.29 & 12.5 & 11.78 & 11.91 & 11.78 & 11.07 & 11.41 & 11.19 & 11.05 \\
\hline Eh & av & 295 & 257 & 349 & 357 & 285 & 327 & 233 & 274 & 237 & 345 \\
\hline A! & $\log / 1$ & $<0.1$ & 6.23 & 12.3 & 9.97 & 9.34 & 5.42 & 4.88 & 4.73 & 4.46 & 4.07 \\
\hline$B a$ & $19 / !$ & 0.060 & 0.008 & 0.011 & 0.009 & 0.005 & 0.006 & 0.004 & 0.007 & 0.003 & 0.005 \\
\hline Cà & $0 / 1$ & 56.0 & 30.3 & 4.01 & 4.16 & 3.14 & 4.02 & 3.07 & 4.37 & 2.97 & 4.19 \\
\hline Cd & $q g / 1$ & $: 0.005$ & 0.004 & $<0.004$ & $<0.004$ & 10.004 & $<0.004$ & 10.004 & $<0.004$. & $<0.004$ & $<0.004$ \\
\hline Cr & $9 / 1$ & 60.015 & 0.073 & 0.022 & $<0.02$ & $<0.02$ & $<0.02$ & $<0.02$ & $<0.02$ & $<0.02$ & 60.02 \\
\hline Cu & $g / 1$ & $\therefore 0.005$ & 0.159 & 0.081 & 0.064 & 0.024 & $0.01 B$ & 0.012 & 0.012 & 0.005 & $<0.004$ \\
\hline Fe & $m / l$ & $(0.001$ & 0.028 & 0.097 & 0.159 & 0.071 & 0.136 & 0.048 & 0.166 & 0.052 & 0.13 \\
\hline$\kappa$ & $\Delta g / 1$ & 7.8 & 7.7 & 2.7 & 1.5 & 1 & 0.6 & 0.8 & 0.7 & 0.8 & 0.7 \\
\hline $\mathrm{Mg}$ & $\lg / 1$ & 14.40 & 0.322 & 0.166 & 0.163 & 0.062 & $<0.06$ & 10.06 & 20.06 & 0.07 & 0.15 \\
\hline fin & $\lg / 1$ & 10.01 & $<0.002$ & 0.605 & 0.006 & $\langle 0.002$ & 0.006 & $<0.002$ & 0.006 & $<0.002$ & 0.006 \\
\hline $\mathrm{Ha}$ & $\operatorname{ag} / 1$ & 25 & 1260 & 774 & 549 & 452 & 361 & 310 & 272 & 233 & 220 \\
\hline$P$ & $8 g / 1$ & 0.1 & 0.36 & 0.63 & 0.61 & 0.56 & 0.62 & 0.64 & 0.61 & 0.6 & 0.5 \\
\hline as $\mathrm{FOA}_{4}$ & $4 \mathrm{~g} / 1$ & 60.3 & 1.1 & 1.93 & 1.87 & 1.72 & 1.9 & 1.96 & 1.27 & 1.84 & 1.53 \\
\hline Pb & $g / 1$ & 10.05 & $<0.05$ & $<0.05$ & 20.05 & $<0.06$ & $<0.06$ & $<0.06$ & 40.06 & $\{0.08$ & 80.06 \\
\hline$\Xi i$ & $g / 1$ & 15.0 & 126 & 132 & 119 & 110 & 91.3 & 72.5 & 63.2 & 47.1 & 4ó. 2 \\
\hline Sr & ogil & 0.250 & 0.13 & 0.013 & 0.01 & 0.007 & 0.007 & 0.005 & 0.007 & 0.007 & 0.007 \\
\hline in & $g / 1$ & $<0.01$ & $<0.02$ & 10.62 & 10.02 & 0.054 & 0.026 & 0.041 & 0.035 & $<0.02$ & 00.02 \\
\hline ir & $40 / 1$ & 10.008 & $<0.008$ & $<0.008$ & $\angle 0.008$ & $\angle 0.00 B$ & $<0.008$ & $<0.008$ & 10.008 & 10.008 & $<0.006$ \\
\hline$F-$ & ig/1 & $<0.5$ & 35 & 30 & 19 & 12 & 9.3 & 7 & 6.5 & 6.1 & 0.3 \\
\hline CI- & $\operatorname{ma} / 1$ & 22 & 40 & 24 & 23 & 20 & 21 & 20 & 20 & 23 & 24 \\
\hline $142-$ & 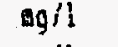 & $<0.03$ & 0.3 & 0.3 & 0.3 & $<0.3$ & $<0.3$ & 60.3 & 10.3 & 80.3 & $<0.3$ \\
\hline & $\| q / 1$ & 0.1 & 58 & 6 & 1.9 & 0.6 & 0.6 & 60.5 & $<0.5$ & $<0.5$ & $<0.5$ \\
\hline $564--$ & $0 y / !$ & 86 & 540 & 130 & 86 & 69 & 73 & 74 & 74 & 76 & $3 E$ \\
\hline HCD3- & $9 / 1$ & 171.0 & 0.00 & 0.00 & 0.00 & 0.00 & 0.00 & 0.00 & 0.00 & 0.00 & 0.00 \\
\hline $\mathrm{Cus} 3-$ & 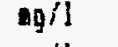 & 2 & 542.80 & 516.00 & 412.80 & 303.60 & 249.40 & 552.00 & 248.40 & 253.92 & 187.80 \\
\hline OH- & $6 g / 1$ & 0.0 & 291.03 & 270.47 & 146.20 & 132.94 & 101.66 & 62.56 & 46.72 & 6.26 & 23.40 \\
\hline B & \&il & 0.060 & 9.26 & 2.47 & 1.4 & 0.731 & 0.535 & 0.383 & 0.355 & $0 . j$ & 0.3 \\
\hline d5 43303 & $\operatorname{sg} / 1$ & 0.34 & 28.39 & 7.63 & 4.29 & 4.18 & 3.06 & 2.24 & 2.03 & 1.71 & 1.71 \\
\hline TAC & 90 & 1.0 & --- & -- & $\cdots$ & --- & $\cdots$ & $\because-$ & $\cdots$ & $\cdots$ & $\cdots$ \\
\hline Pot--- & $8 q ! 1$ & (0).1 & 60.5 & $\langle 0.4$ & 60.4 & 1.2 & 1.4 & 1.4 & i.j & 1.2 & $! .2$ \\
\hline CATIOHS & neq $/ 1$ & 5.28 & 56.52 & 33.94 & 24.13 & 17.84 & 15.91 & 13.65 & 12.06 & 10.31 & 9.31 \\
\hline ANroHS & nequil & 5.54 & 50.44 & 36.19 & 25.85 & 20.82 & 16.92 & 24.60 & 13.53 & 11.42 & 10.31 \\
\hline TOT. HLK.; & nọil & 92 & 1057.8 & 993.3 & 670.8 & 538.2 & 427.8 & 662.4 & 351.2 & 264.76 & 209.1 \\
\hline if & g: 1 & 34.1 & - & $\cdots$ & --- & --- & --- & $\cdots$ & --- & --- & $-\cdots$ \\
\hline FGRE VDL. & & & 2.86 & 4.8 & 5.76 & 8.29 & 9.18 & 11.13 & 12.36 & 14.53 & 15.79 \\
\hline
\end{tabular}

(---) Data not available.

(\#) Total Alkalinity as CO3--. 
TABLE A.18. contd

\begin{tabular}{|c|c|c|c|c|c|c|c|c|c|c|}
\hline SAMPLE & $\ldots$ & 10 & 11 & 12 & 13 & 14 & 15 & 16 & 17 & 18 \\
\hline TIME & days & 35 & 39 & 46 & 53 & 57 & 60 & 64 & 69 & 72 \\
\hline pH & & 10.19 & 10.47 & 10.47 & 10.56 & 10.53 & 10.30 & 10.23 & 9.87 & 10.58 \\
\hline Eh & or & 241 & 330 & 258 & 309 & 354 & 348 & 367 & 317 & 368 \\
\hline Al & $6 g / 1$ & 3.63 & 3.58 & 2.99 & 2.08 & 1.93 & 1.64 & 1.41 & 1.05 & 0.84 \\
\hline $\mathrm{Ba}$ & $\therefore 9 / 1$ & $<0.002$ & 0.005 & 0.003 & $<0.002$ & 10.002 & $<0.002$ & $\$ 0.002$ & $<0.002$ & 80.002 \\
\hline$\tau_{3}$ & $a g / 1$ & 2.87 & 4.04 & 3.13 & 1.7 & 1.7 & 1.8 & 2.0 & 2.5 & 5.1 \\
\hline Co & ag $/ 1$ & $<0.004$ & $<0.004$ & $<0.004$ & $<0.004$ & $<0.004$ & $<0.004$ & $\$ 0.004$ & 60.004 & 60.004 \\
\hline $\mathrm{Cr}$ & $2 g / 1$ & $<0.02$ & 10.02 & $<0.02$ & $<0.02$ & $<0.02$ & $<0.02$ & $\{0.02$ & 60.02 & 10.02 \\
\hline $\mathrm{Cu}$ & $9 g / 1$ & $<0.004$ & 0.008 & $<0.004$ & $<0.004$ & $<0.004$ & 0.006 & $<0.004$ & $<0.004$ & 00.004 \\
\hline $\mathrm{Fe}$ & $g / 1$ & 0.045 & 0.176 & 0.119 & 0.027 & 0.027 & 0.027 & 0.023 & 0.016 & 0.071 \\
\hline$k$ & $9 g / 1$ & 0.5 & 0.8 & 0.6 & 1.2 & 0.7 & 0.8 & 60.3 & 2.1 & 3.6 \\
\hline $\mathrm{Mg}$ & $g / 1$ & 0.064 & 0.152 & 0.09 & 0.089 & $<0.06$ & 60.06 & $<0.06$ & 60.06 & $\therefore 0.06$ \\
\hline$M$ & $g / 1$ & $<0.002$ & 0.006 & 0.004 & $<0.002$ & $<0.002$ & 10.002 & $\$ 0.002$ & 10.002 & 0.002 \\
\hline $\mathrm{Ha}$ & og/l & 174 & 202 & 186 & 163 & 144 & 130 & 121 & 109 & 55 \\
\hline 8 & $\log / 1$ & 0.45 & 0.46 & 0.4 & 0.33 & 0.25 & 0.18 & 0.18 & 0.13 & 30.1 \\
\hline as f04 & $\log / 1$ & 1.38 & 1.41 & 1.23 & 1.01 & 0.77 & 0.55 & 0.55 & 0.40 & 10.3 \\
\hline$F b$ & $g / 1$ & $<0.06$ & $<0.06$ & 10.06 & 60.06 & 60.06 & 0.06 & 60.06 & 00.06 & 10.06 \\
\hline $\mathrm{Si}$ & ig/l & 37.2 & 47.8 & 43.4 & 33.1 & 26.0 & 22.7 & 21.0 & 19.5 & 18.1 \\
\hline Sr & $g / 1$ & 0.005 & 0.006 & 0.005 & 0.004 & 0.004 & 0.003 & 0.003 & 0.004 & 0.010 \\
\hline $2 n$ & $\lg / 1$ & $<0.02$ & 80.02 & $<0.02$ & $<0.02$ & $<0.02$ & $<0.02$ & $<0.02$ & 10.02 & 60.62 \\
\hline $2 r$ & $\log / 1$ & $<0.008$ & $<0.008$ & $\$ 0.008$ & 0.01 & $<0.000$ & $<0.008$ & 10.1208 & $<0.008$ & <i. 008 \\
\hline F- & ag $/ 1$ & 5.7 & 6.4 & 6.6 & 6.4 & 6.8 & 6.5 & 6.5 & 6.5 & 7.3 \\
\hline $\mathrm{Cl}-$ & $g / 1$ & 22 & 23 & 24 & 23 & 22 & 22 & 22 & 22 & 24 \\
\hline $\mathrm{NO}^{2}-$ & $g / 1$ & $<0.3$ & $<0.3$ & 60.3 & $<0.3$ & $i 0 . j$ & $<0.3$ & 10.3 & 60.3 & 60.3 \\
\hline $103-$ & $g / 1$ & $<0.5$ & $<0.5$ & $i 0.5$ & 60.5 & 10.5 & 60.5 & $<0.5$ & 0.5 & 60.5 \\
\hline $504--$ & $\operatorname{sg} / 1$ & .73 & 79 & 77 & 77 & 77 & 76 & 75 & 75 & 82 \\
\hline $\mathrm{H}[\mathrm{CO} 3-$ & $\lg / 1$ & 22.45 & 0.00 & 81.73 & 33.67 & 0.00 & 22.45 & 16.75 & 11.22 & 16.04 \\
\hline CO?-- & $m g / 1$ & 176.64 & 204.20 & 149.04 & 121.44 & 99.36 & 88.32 & 71.60 & 60.72 & 55.20 \\
\hline OH- & $a g / 1$ & 0.00 & 0.00 & 0.00 & 0.00 & 9.30 & 0.00 & 0.00 & $\dot{0} .00$ & 0.00 \\
\hline B & $g / 1$ & 0.259 & 0.329 & 0.3 & 0.258 & 0.228 & 0.214 & 0.220 & 0.214 & 0.216 \\
\hline as 43803 & $g / 1$ & 1.49 & 1.67 & 1.71 & 1.47 & 1.30 & 1.22 & 1.30 & 1.22 & 1.14 \\
\hline TOC & $a g / 1$ & $-\cdots$ & -... & $\cdots$ & $\cdots$ & $\cdots$ & $\ldots$ & $\cdots$ & - & $-\cdots$ \\
\hline PO4--- & $\log / 1$ & $i$ & 0.9 & 0.9 & 60.4 & 60.4 & $\therefore 0.4$ & $60 . t$ & 60.4 & 60.4 \\
\hline CATIOHS & aeq/1 & 8.60 & 9.02 & 8.27 & 7.21 & 6.37 & 5.77 & 5.36 & 4.72 & 9.47 \\
\hline AMIONS & eqqil & 8.73 & 9.47 & 8.64 & 7.19 & 6.45 & 5.86 & 5.17 & 4.73 & 4.86 \\
\hline TOT.ÂLK.* & $-i g / 1$ & 187.68 & 204.24 & 173.4 & 138 & 116 & 99 & 80 & 86 & $\dot{0 j}$ \\
\hline IC & ogil & ---- & ---- & $-\cdots$ & $-\cdots$ & --- & ---. & --- & ---- & .... \\
\hline FORE VOL. & & 17.68 & 18.66 & 20.67 & 23.15 & 25.16 & 26.74 & 20.42 & 30.36 & $3 j$ \\
\hline
\end{tabular}

(--) Data not available.

(\#) Total Alkalinity as c03-.. 


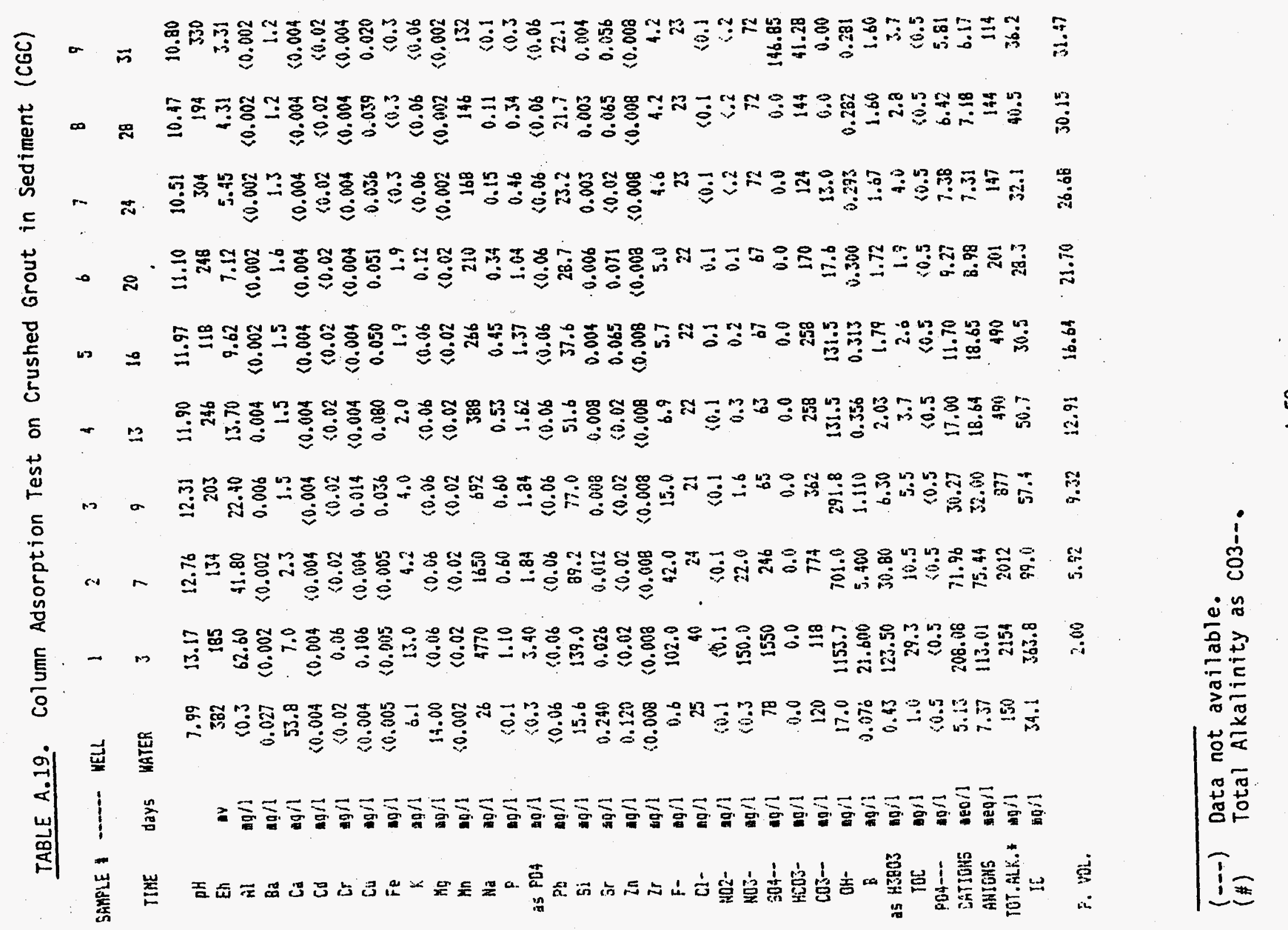


TABLE A.19. contd

\begin{tabular}{|c|c|c|c|c|c|c|c|c|c|c|c|c|}
\hline SAFPLE I & -..- & 11 & 13 & 15 & 17 & 19 & 21 & 24 & 26 & 28 & 30 & 32 \\
\hline TIME & days & 38 & 44 & $5 !$ & 60 & 66 & 73 & 67 & 94 & 104 & 113 & 122 \\
\hline $\mathrm{pH}$ & & 9.91 & 10.54 & 10.44 & 10.81 & 10.27 & 10.37 & 10.87 & 10.84 & 10.10 & 10.16 & 8.71 \\
\hline Eh & av & 339 & 303 & 290 & 295 & 278 & 321 & 335 & 254 & 344 & 349 & 366.60 \\
\hline Al & $\log / 1$ & 1.90 & 1.30 & 1.01 & 1.32 & 2.18 & 2.14 & 2.78 & 3.69 & 4.43 & 3.61 & 5.63 \\
\hline $\mathrm{Ba}$ & $9 g / 1$ & $<0.002$ & 0.008 & 0.009 & 0.010 & 0.012 & 0.010 & 0.011 & 0.011 & 0.008 & 0.0017 & 0.004 \\
\hline $\mathrm{Ca}$ & $g / 1$ & 2.2 & 18.0 & 28.7 & 39.6 & 50.9 & 36.5 & 49.0 & 49.1 & 33.4 & 52.5 & 40.30 \\
\hline Cd & $90 / 1$ & $<0.004$ & $<0.004$ & $<0.004$ & $<0.004$ & $<0.004$ & 20.004 & 80.004 & $\angle 0.004$ & $<0.004$ & $<0.004$ & 60.004 \\
\hline Gr & $g / 1$ & $<0.02$ & $<0.02$ & $<0.02$ & 60.02 & 60.02 & 60.02 & 60.02 & 10.02 & $<0.02$ & $\langle 0.02$ & 60.02 \\
\hline $\mathrm{Cu}$ & $g / 1$ & $<0.004$ & $<0.004$ & $<0.004$ & $<0.004$ & $<0.004$ & 60.004 & 30.004 & $<0.004$ & 60.604 & 60.064 & $\$ 0.604$ \\
\hline$F_{e}$ & $9 / 1$ & 0.016 & $<0.005$ & $<0.005$ & 0.014 & 60.005 & 0.007 & 0.008 & 0.006 & 0.006 & 0.005 & 0.01 \\
\hline$k$ & $\ln / 1$ & 3.9 & 28.0 & 27.0 & 21.0 & 16.0 & 14.0 & 13.0 & 11.0 & 9.7 & 3.4 & 8.00 \\
\hline $\mathrm{Mg}$ & agil & $<0.06$ & 50.06 & $<0.06$ & $<0.06$ & 60.06 & $\$ 0.06$ & $>0.06$ & 60.06 & $<0.00^{\prime}$ & 60.016 & $\langle\hat{v} . \dot{v} b$ \\
\hline $\mathrm{Mn}$ & oil & $<0.002$ & $<0.002$ & 60.002 & 10.002 & 10.002 & 10.002 & 0.002 & $<0.002$ & $<0.002$ & 60.002 & 60.002 \\
\hline $\mathrm{Ha}$ & $9 / 1$ & 96 & 45 & 26 & 26 & 25 & 25 & 25 & 26 & 25 & 26 & 25.70 \\
\hline$p$ & $g / 1$ & $<0.1$ & $<0.1$ & $<0.1$ & $<0.1$ & $<0.1$ & $<0.1$ & 0.1 & $<0.1$ & 00.1 & $<0.1$ & (0.) \\
\hline is PO4 & $\operatorname{ag} i 1$ & 60.3 & $<0.3$ & $<0.3$ & $<0.3$ & $<0.3$ & $\{0.3$ & 10.3 & 60.3 & 00.3 & 60.3 & 60.3 \\
\hline Po & $\lg / 1$ & $<0.06$ & 80.06 & $<0.06$ & 10.06 & 10.06 & 0.06 & $<0.06$ & 10.06 & 10.06 & 0.06 & 0.06 \\
\hline $5 i$ & $2 g / 1$ & 15.3 & 12.7 & 11.0 & 11.6 & 10.7 & 9.7 & 6.8 & 7.6 & 6.2 & 4.8 & $2.7 !$ \\
\hline $5 r$ & $g / 1$ & 0.010 & 0.117 & 0.201 & 0.289 & 0.409 & 0.452 & 0.613 & 0.698 & 0.716 & 0.762 & 0.67 \\
\hline in & og/l & 0.060 & 0.110 & $<0.2$ & 0.054 & $<0.2$ & 0.029 & 10.02 & 60.02 & 60.02 & $\therefore 0.02$ & 60.02 \\
\hline Ir & $9 / 1^{\circ}$ & $<0.008$ & $<0,008$ & $<0.008$ & $<0.008$ & $(0.008$ & $<0.008$ & $(0.008$ & (0.008 & 0.008 & 60.606 & 10.600 \\
\hline F- & $0 / 1$ & 7.5 & 7.6 & 4.6 & 5.2 & 5.2 & 5.9 & 7.2 & 7.8 & 8.0 & 8.5 & B. 70 \\
\hline $\mathrm{Cl}-$ & $g / 1$ & 25 & 24 & 24 & 24 & 20 & 21 & 22 & 22 & 22 & 22 & 23.00 \\
\hline H02- & $g / 1$ & $<0.1$ & 0.1 & $<0.3$ & $<0.3$ & $\langle 0.3$ & $\{0.3$ & $\{0.3$ & 60.3 & $\langle 0.3$ & 0.3 & iu.j \\
\hline 103- & $10 / 1$ & 3.2 & <.2 & $<0.5$ & $<0.5$ & 60.5 & $<0.5$ & 40.5 & $<0.5$ & 60.5 & (0.5 & ห̂́.ร \\
\hline $504--$ & agil & 82 & 80 & 71 & 73 & 69 & 69 & 73 & 73 & 72 & 73 & 71.00 \\
\hline $\mathrm{HCOS-}$ & agil & 10.49 & 57.71 & 83.94 & 41.97 & 57.95 & 0.60 & 26.23 & 0.00 & 15.74 & 57.71 & $3 i .3 i$ \\
\hline COS-- & agil & 61.92 & 5.16 & 15.48 & 10.32 & 41.20 & 5.16 & 41.28 & 41.92 & 30.96 & 46.44 & 31.00 \\
\hline ㅇt- & $\lg / 1$ & 0.00 & 0.00 & 0.00 & 0.00 & 0.00 & 0.00 & 0.00 & 2.56 & 0.00 & 0.011 & 0.017 \\
\hline a & $\operatorname{mg} / 1$ & 0.270 & 0.250 & 0.214 & 0.224 & 0.204 & 0.203 & 0.202 & 0.196 & i. 150 & 0.175 & 0.18 \\
\hline as H360J & 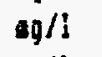 & 1.54 & 1.43 & 1.22 & 1.28 & 1.67 & 1.16 & 1.15 & 1.12 & 1.06 & 2.10 & 1.03 \\
\hline TDC & agil & 1.5 & 1.5 & 2.1 & 2.2 & 1.9 & 3.6 & 0.3 & 3.5 & 10.4 & 2.3 & 0.62 \\
\hline POA--. & $\mathrm{gg} / 1$ & 00.5 & $<0.5$ & $<0.1$ & $<0.4$ & $<0.4$ & 60.4 & 60.4 & 60.4 & (iv. 4 & 10.4 & $\therefore 0.4$ \\
\hline CATIONS & aeq/1 & 4.39 & 3.58 & 3.27 & 3.64 & 4.07 & 3.30 & 3.89 & 3.97 & $3.3 j$ & 4.00 & 3.36 \\
\hline AHIORS & aeq/I & 5.06 & 3.88 & 4.32 & 3.53 & 4.61 & 2.51 & $4: 36$ & 4.13 & 3.85 & 5.12 & 4.17 \\
\hline IOT. AHLK.: & $\lg / 1$ & 67 & 34 & 57 & 31 & 70 & $s$ & 54 & 46 & 39 & 75 & 46.40 \\
\hline IC & $\lg / 1$ & 11.7 & 11.7 & 4.7 & 4.5 & $i 6.7$ & 5.4 & 4.3 & 3.4 & 4.2 & 5.0 & 7.54 \\
\hline F. VOL. & & 38.91 & 49.85 & 56.08 & 62.83 & 70.23 & 76.30 & 87.70 & 94.30 & 101.80 & 160.72 & 122.67 \\
\hline
\end{tabular}

(\#) Data not available.
(\#) 
TABLE A.19. contd

\begin{tabular}{|c|c|}
\hline 34 & 35 \\
\hline 158 & 188 \\
\hline 8.32 & 9.56 \\
\hline 343.00 & 290.00 \\
\hline 3.57 & 3.74 \\
\hline 0.01 & 0.00 \\
\hline 39.50 & 42.70 \\
\hline$<0.004$ & $<0.004$ \\
\hline 00.02 & 10.02 \\
\hline$<0.004$ & $\{0.004$ \\
\hline$<0.005$ & 60.005 \\
\hline 8.20 & 6.20 \\
\hline 0.69 & $<0.06$ \\
\hline 10.002 & $<0.002$ \\
\hline 26.10 & 26.40 \\
\hline$<0.1$ & $<0.1$ \\
\hline$<0.3$ & 60.3 \\
\hline$<0.06$ & $<0.06$ \\
\hline 3.54 & 3.27 \\
\hline 0.53 & 0.52 \\
\hline 60.02 & $<0.02$ \\
\hline$\therefore 0.00 B$ & 20.008 \\
\hline 8.70 & 11.00 \\
\hline 23.60 & 22.10 \\
\hline$<0.3$ & 40.3 \\
\hline 60.5 & 60.5 \\
\hline 73.60 & 74.60 \\
\hline 22.45 & 37.28 \\
\hline 38.64 & 16.56 \\
\hline 0.60 & 0.00 \\
\hline 0.15 & 0.15 \\
\hline 0.65 & 0.88 \\
\hline ---- & $-\cdots$ \\
\hline 60.7 & 60.4 \\
\hline 3.39 & 3.45 \\
\hline 4.28 & 3.85 \\
\hline 49.68 & 35.38 \\
\hline
\end{tabular}

$160.36 \quad 195.60$

(--) Data not available.

(\#) Total Alkalinity as C03-.. 
TABLE A.20. Column Adsorption Test on Crushed Grout in Sediment (SCGI)

\begin{tabular}{|c|c|c|c|c|c|c|c|c|c|c|c|}
\hline SAMPLE I & -...- & MELL & 1 & 2 & 3 & 4 & 5 & 6 & $7^{\circ}$ & 8 & 9 \\
\hline TIKE & days & WATER & 3 & 7 & 9 & 13 & 16 & 20 & 24 & $2 B$ & 31 \\
\hline pH & & 7.99 & 11.95 & 12.48 & 12.72 & 12.03 & 11.87 & 11.86 & 11.27 & 11.02 & 11.49 \\
\hline Eh & av. & 382 & 256 & 133 & 179 & 244 & 166 & 273 & 323 & 198 & 345 \\
\hline Al & ogil & 60.1 & 0.17 & 25.60 & 20.80 & 19.30 & 17.40 & 14.60 & 11.70 & 8.59 & 7.54 \\
\hline $\mathrm{Ba}$ & $9 / 1$ & 0.060 & 0.018 & $<0.002$ & $<0.002$ & $<0.002$ & 0.002 & $<0.002$ & $<0.002$ & 00.002 & 0.002 \\
\hline$C_{a}$ & $2 g / 1$ & 56.0 & 45.2 & 2.7 & 1.9 & 1.6 & 1.4 & 1.2 & 1.2 & 1.3 & 1.1 \\
\hline Cot & $9 / 1$ & 0.005 & $<0.004$ & 60.004 & 60.004 & $<0.004$ & $<0.004$ & $<0.004$ & 60.004 & $\therefore 0.004$ & 0.0014 \\
\hline Cr & $29 / 1$ & $\langle 0.015$ & 0.05 & $<0.02$ & $<0.02$ & 0.02 & $<0.02$ & $<0.02$ & 00.02 & 00.02 & 60.02 \\
\hline $\mathrm{Cu}$ & $\log / 1$ & $<0.005$ & 0.036 & 0.042 & 0.012 & 0.008 & 60.004 & $<0.004$ & $<0.004$ & 80.004 & 60.0014 \\
\hline $\mathrm{Fe}$ & qil & $<0.01$ & $<0.005$ & 0.014 & 0.032 & 0.088 & 0.087 & 0.049 & 0.073 & 0.0174 & 0.037 \\
\hline$k$ & $29 / 1$ & 7.8 & 8.3 & 4.3 & 3.0 & 3.0 & 2.2 & 2.1 & 60.3 & 60.3 & 60.3 \\
\hline $\mathrm{Mg}$ & $g / 1$ & 14.40 & $\{0.06$ & $<0.06$ & 80.06 & 0.06 & 60.06 & 00.06 & 60.66 & 60.06 & 30.06 \\
\hline Hin & $90 / 1$ & $<0.01$ & $<0.02$ & 10.02 & 0.02 & 0.02 & 00.02 & 0.02 & 10.002 & 60.002 & 0.002 \\
\hline $\mathrm{Ha}$ & $\lg / 1$ & 25 & 1030 & 1920 & 990 & 585 & 468 & 345 & 272 & 213 & 191 \\
\hline$P$ & $\operatorname{mog} / 1$ & 60.1 & $<0.1$ & 0.20 & $<0.1$ & 0.21 & 0.21 & 0.25 & $0.2 !$ & 0.18 & 0.14 \\
\hline as P04 & $\log / 1$ & $<0 . j$ & $<0.3$ & 0.60 & 6.3 & 0.64 & 0.64 & $\dot{0} .77$ & 0.64 & 0.55 & 0.43 \\
\hline$P b$ & $\lg / 1$ & $<0.05$ & $<0.06$ & $<0.06$ & 80.06 & $<0.06$ & 20.06 & 80.06 & 0.06 & 6.06 & 80.06 \\
\hline $\mathrm{ji}$ & $g / 1$ & 15.0 & 72.0 & 169.0 & 107.0 & 70.5 & 57.6 & 43.4 & 33.6 & 29.8 & 27.3 \\
\hline $5 r$ & a & 0.250 & 0.418 & 0.020 & 0.014 & 0.010 & 0.008 & 0.007 & 0.005 & 0.005 & 0.005 \\
\hline in & $9 g / 1$ & $<0.01$ & 0.056 & 0.066 & 60.02 & 60.02 & 0.108 & 0.036 & 0.215 & 60.02 & 0.024 \\
\hline $2 r$ & $g / !$ & $<0.008$ & $<0.008$ & 50.008 & $(0.008$ & $<0.008$ & $\langle 0.008$ & $(0,008$ & 60.008 & $40.00 \overline{8}$ & 60.000 \\
\hline F- & $g / 1$ & <0.5 & 32.0 & 34.0 & 19.0 & 13.0 & 10.0 & 7.3 & 5.8 & 5.0 & 4.2 \\
\hline Cl- & $\log / 1$ & 22 & 47 & 27 & 21 & 21 & 20 & 21 & 22 & 24 & 23 \\
\hline $\mathbb{N O} \hat{L}^{-}$ & ig/1 & $00.0 j$ & 60.1 & 0.1 & 0.1 & 60.1 & 0.1 & 60.1 & 00.1 & 60.1 & 80.1 \\
\hline NQZ3- & $\operatorname{sg} / 1$ & 0.1 & 160.0 & 56.0 & 6.4 & 0.8 & 0.2 & 0.1 & 30.2 & 0.2 & 60.2 \\
\hline $504--$ & $9 / 1$ & ab. & 1440 & 560 & {$[8]$} & 106 & 79 & 68 & 68 & bo & 66 \\
\hline HCOJ- & $\log / 1$ & 151.5 & 0.0 & 0.0 & 0.0 & 0.0 & 0.0 & 0.0 & 0.0 & 3.0 & 304.27 \\
\hline COS-- & $g / 1$ & 15 & 362 & 566 & 414 & 376 & 258 & 362 & 158 & 176 & 30.96 \\
\hline BH- & $g / l$ & 0.0 & 57.8 & $86 \dot{j} .0$ & 438.0 & 117.3 & 204.6 & 14.2 & 82.7 & 29.5 & 0.00 \\
\hline B & $\lg / 1$ & 0.060 & 9.720 & 5.340 & 2.240 & 1.420 & 1.000 & 0.300 & 0.467 & 1.290 & 1.090 \\
\hline as 43603 & $\operatorname{ag} / 1$ & 0.34 & 55.59 & 33.74 & 12.80 & 3.12 & 5.72 & 4.60 & 2.68 & 7.30 & 6.23 \\
\hline TOC & agil & 1.0 & -- & -- & $\cdots$ & --- & $\cdots$ & -.- & $\ldots$ & $\cdots$ & $\cdots$ \\
\hline PO4--- & $a g / 1$ & $\{1.0$ & $(4.0$ & $\langle 4.0$ & $\langle 4.0$ & 34.0 & 24.0 & $: 4.0$ & 4.00 & 34.0 & 64,6 \\
\hline CÁTIONS & Ieqil & $5.2 B$ & 47.06 & 83.73 & 43.22 & 25.60 & 20.48 & 15.12 & 11.89 & 7.33 & 8.j6 \\
\hline AHIOHS & aea/1 & 5.54 & 51.60 & 84.76 & 45.04 & 39.60 & 23.38 & 15.30 & 12.41 & $10.6 i$ & 0.26 \\
\hline 70T.ÂLK.: & $\lg / l$ & 90 & 464 & 2067 & 1187 & 1083 & 617 & 387 & 302 & 248 & 181 \\
\hline It & $\log / 1$ & 34.1 & $\cdots$ & $\cdots$ & $\cdots$ & -- & $\cdots$ & $\cdots$ & $\cdots$ & $-\cdots$ & $\cdots$ \\
\hline FOKE YOL. & & & 1.64 & 5.72 & 9.06 & $11.6 ?$ & 15.97 & 20.61 & 24.4 & 27.94 & 30.02 \\
\hline
\end{tabular}

(---) Data not available.

(\#) Total Alkalinity as CO3-.. 


\section{Appendix B}

Cumulative Fraction Leached and Effective Diffusion Coefficient Data 


\section{Appendix B}

\section{List of Tables}

Tables B.1 - ANS 16.1 Leach Test of Whole Grout with Groundwater (DIFF2-4)

Tables B.2 - ANS 16.1 Leach Test of Whole Grout with Groundwater (DIFF3-4)

Tables B.3 - ANS 16.1 Leach Test of Whole Grout with Groundwater (DIFF4-8)

Tables B.4 - ANS 16.1 Leach Test of Whole Grout with Groundwater (DIFF5-5)

Tables B.5 - ANS 16.1 Leach Test of Whole Grout with Groundwater (Radionuclides only)

Tables B.6 - Static Leach Test of Whole Grout with Groundwater (DIFF2-17)

Tables B.7 - Static Leach Test of Whole Grout with Groundwater (DIFF2-18)

Tables B.8 - Static Leach Test of Whole Grout with Groundwater (DIFF4-13)

Tables B.9 - Static Leach Test of Whole Grout with Groundwater (DIFF5-17)

Tables B.10 - Static Leach Test of Whole Grout with Groundwater (Radionuclides only)

Tables B.11 - Flow-Through Test--Whole Grout/Sediment/Groundwater (DIFCOLT2)

Tables B.12 - Flow-Through Test--Whole Grout with Groundwater (DFCOL3A)

Tables B.13 - Flow-Through Test--Whole Grout/Sediment/Groundwater (DIFCOLI)

Tables B.14 - Flow-Through Test--Whole Grout with Groundwater (COLT2A)

Tables B.15 - Flow-Through Test-Whole Grout/Sediment/Groundwater (DFCOL1)

Tables B.16 - Flow-Through Test-Whole Grout with Groundwater (COLT1A)

Tables B.17 - Flow-Through Test-Crushed Grout with Groundwater (DIFBCGT)

Tables B.18 - Flow-Through Test-Crushed Grout with Groundwater (DIFBCGI)

Tables B.19 - Flow-Through Test-Crushed Grout with Groundwater (DIFBCGC) 
Tables B.20 - Flow-Through Test-Crushed Grout/Sediment/Groundwater (DIFCGT)

Tables B.21 - Flow-Through Test-Crushed Grout/Sediment/Groundwater (DIFCGC)

Tables B.22 - Flow-Through Test-Crushed Grout/Sediment/Groundwater (SCGI)

Tables B.23 - Flow-Through Test-Crushed Grout/Sediment/Groundwater (CGI) (Radionuclides only) 
TABLE B.la. Potassium (K) Leach Fractions from ANS 16.1 Leach Test--Whole Grout in Groundwater (DIFF2-4)

\begin{tabular}{|c|c|c|c|c|c|}
\hline SAMPLE & $A n / A O$ & CUs. An/Ao & TIME Jays & Dinc. & S cun, \\
\hline CRN $z-4-i$ & $-7.155-03$ & $-7.15 E-03$ & $3.00 E-04$ & $4.61 E-02$ & 4.61E-12 \\
\hline CRH 2-4-2 & $3.34 E-03$ & $-3.32 E-03$ & $3.3 \mathrm{JE}-02$ & $4.19 E-10$ & $5.47 E-10$ \\
\hline Cni 2-4-3 & $4.77 E-04$ & $-3.34 E-03$ & $2.92 E-01$ & $1.13 E-11$ & $1.200-10$ \\
\hline CRI 2-4-4 & $1.00 E-02$ & $3.685-03$ & $1.00 E+00$ & $1.48 E-69$ & $1.40 E-10$ \\
\hline $\operatorname{Can} 2-4-5$ & $6.68 E-03$ & 1. $34 E-62$ & $2.10 E \div 00$ & $6.92 E-10$ & $2.605-10$ \\
\hline Crit 2-4-6 & $6.68 E-03$ & $2.00 E-02$ & $3.02 E+00$ & $1.67 E-09$ & 4.16E-10 \\
\hline CRH $2-4-7$ & $5.82 E-02$ & 7.82E-02 & i.jiE+0! & $3.00 E-67$ & $1.46 E-19 t$ \\
\hline $\operatorname{con} 2-4-5$ & $2.4 B E-92$ & 1. OJE-0̂! & $2.00 E+01$ & $2.63 E-69$ & $1.66 E-99 \overline{7}$ \\
\hline CRU 2-4-? & 1.53E- 02 & $1,18 E-01$ & 7.70E 001 & $1.39 E-09$ & 1.62E-69 \\
\hline SFH 2-4-10 & $2.96 E-\hat{b} 2$ & $1.48 E-01$ & 4. $10 E \div 01$ & $1.8 d E-09$ & 1.6TE- $6 \overline{9}$ \\
\hline SRM $2-4-11$ & 1. $53 \mathrm{JE}-02$ & $1.63 E-61$ & 5. $00 E+01$ & 1. $6 \mathrm{JE}-09$ & 1.66E-19? \\
\hline CKN $2-4-12$ & $1.5 \mathrm{JE}-02$ & $1.78 E-0) !$ & b. $30 E+v 1$ & $3.71 E-10$ & 1. $58 E-69$ \\
\hline $\operatorname{CSH} 2-4-13$ & $2.48 E-02$ & $2.03 E-01$ & $3.20 E+01$ & $7.0 \mathrm{E}-10$ & 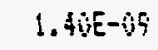 \\
\hline CFin $2-4-15$ & $1.91 E-02$ & $2.22 E-01$ & $1.22 E+12$ & $5.39 E-10$ & 1.37E-6? \\
\hline CRI $2-4-14$ & $1.76 E-02$ & $2,425-0 !$ & $1.34 E+12$ & $4.25 E-69$ & 1. 4aE-iq \\
\hline LRH 2-4-16 & 2.00E-02 & $2.62 E-01$ & $1.22 E+\hat{v} 2$ & 2. $20 E-10$ & $1.30 E-09$ \\
\hline CRH $2-4-17$ & $2.86 E-02$ & $2.30 E-01$ & $3.02 E+62 z$ & $1.70 E-10$ & ร. $: 3 E-10$ \\
\hline CFN 2-4-18 & $2.29 E-02$ & $3,13 E-01$ & $4.10 E+02$ & $1.39 E-10$ & 0.0GE+00 \\
\hline
\end{tabular}

TABLE B.1b. Sodium (Na) Leach Fractions from ANS 16.1 Leach Test--Whole Grout in Groundwater (DIFF2-4)

\begin{tabular}{|c|c|c|c|c|c|}
\hline SAMPLE & $\hat{A n} / \hat{A O O}$ & EUะ. An/Ao & TiME days & jint. & I [ [ij. \\
\hline ind $2-4-1$ & $5.81 E-03$ & 5.81 IE-03 & 3. 00E-94 & 3.05E-12 & 3. SEE-0E \\
\hline bxil $2-4-2$ & $2.33 E-02$ & $2,31 E-\hat{y} 2$ & a.j3E-iz & 2. $635-128$ & J.16E-78 \\
\hline CRet $2-4-3$ & $1.335-02$ & $4.3 \mathrm{BJE}-\hat{02}$ & $2.53=-\hat{i} j$ & i. $65 E-\hat{4}$ & $2,41 E-60$ \\
\hline 6 SH 2-4-4 & $3.43 E-02$ & $3.175-02$ & $1.60 E+00$ & ม.74E-6o & 2.6E- \\
\hline Silk $i-4-5$ & $2.7 T E-62$ & i. $09 E-0 i$ & $2.10 E \div 0 \hat{0}$ & 1.J9E-\$E & 1. \\
\hline 6 政 2-4-6 & $1.52 E-02$ & $1.255-01$ & $3.02 E+10$ & $8.71 E-67$ & abIE-vE \\
\hline CRH $2-4-7$ & $9.715-02$ & 2. $z+E-\hat{v} !$ & $1,31 E+6 i$ & B. $I 1 E-i g$ & 1.28E-15 \\
\hline in $2-4-6$ & $4.37 E-3 i$ & $2.67 E-41$ & Z. DiE & B.jet-95 & $1.35-60$ \\
\hline $25142-4-9$ & $3.27 E-0 i$ & $3.60 E-61$ & C. $70 E+61$ & $0.77 E-67$ & 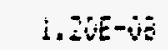 \\
\hline Cfit $2-4-16$ & $5.1 A E-6 i$ & S.SIE-0! & 4. $16 \overline{1}=3$ & $5,4 E-6 \%$ & 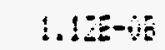 \\
\hline orin $2-4-11$ & $2.585-62$ & S. $F T E-i \mathrm{i}$ & 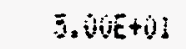 & $2.6 \bar{E}-6 \%$ & I. IIE-1E \\
\hline $6 x+2-12$ & J.1 $1 \overline{X E}-1 \mathrm{H}$ & $4, \dot{v} E E-\hat{\mathrm{d}}$ & t. $36 \bar{v}+61$ & $4.0 E E-69$ & $1.65-78$ \\
\hline$C R H=4-1 j$ & $4.05 E-13$ & $4.5 \pi E-6 !$ & $7,-00 E+0 j$ & $2,6 B E-79$ & ¿.50E-AG \\
\hline 识 $2-3-14$ & $3.96 E-12$ & $4.75 E-i j$ & $1.23=+0 z$ & 2. JE- & E. \\
\hline Ef $2-4-15$ & 3.PTE- & $5,3 j \varepsilon-\dot{u} 1$ & $i . j+E+4 i$ & i. & 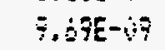 \\
\hline Fid 2-4-10 & 3. $99 E-\mathrm{ij} 2$ & $5.7 D E-9 \mathrm{i}$ & $1.32 E+12$ & S.-BIE-10 & $5.635-69$ \\
\hline CFi $z-4-! ?$ & ב. & b. $. \dot{b E}-\hat{0} !$ & $3,6 Z E+9$ & $7,9 E-10$ & S. ZUE-6\% \\
\hline$x-2-4-86$ & $4.9 B E-V Z$ & $5.36 \bar{E}-01$ & $4.10 E-12$ & $3.44 E-10$ & $\therefore, O G E+\infty 0$ \\
\hline
\end{tabular}


TABLE B.1e. Boron (as H3BO3) Leach Fractions from ANS 16.1 Leach Test--Whole Grout in Groundwater (DIFF2-4)

\begin{tabular}{|c|c|c|c|c|c|}
\hline SAKPLE & $\mathrm{An} / \mathrm{AO}_{\mathrm{O}}$ & CUn. An/AD & TIKE days & bine. & $D$ cus. \\
\hline CRY 2-4-1 & $7.54 E-04$ & $7.54 E-04$ & 3. 00 -04 & $5.12 E-04$ & $5.12 E-64$ \\
\hline CRH $2-4-2$ & $2.20 E-03$ & $2.95 E-03$ & $8.33 E-02$ & $1.82 E-10$ & $3.27 E-10$ \\
\hline CRH $2-4-3$ & $1.22 E-03$ & 4.18E-03 & $2.52 E-01$ & $7.42 \mathrm{E}-11$ & $1.87 E-10$ \\
\hline CRK $2-4-4$ & $9.11 E-04$ & $5.09 E-03$ & $1.00 E+00$ & $1.2 \mathrm{JE}-11$ & 6.10E-11 \\
\hline ERN $2-4-5$ & $5.34 E-04$ & $5.62 E-03$ & $2.10 E+00$ & $4.42 E-12$ & $4.71 E-1 !$ \\
\hline CAn 2-4-6 & $2.04 \mathrm{E}-03$ & $7.666-03$ & $3.02 E+00$ & $1.57 \mathrm{E}-10$ & $0.09 E-11$ \\
\hline Cกิ 2-4-7 & 2. $83 \mathrm{E}-04$ & $7.95 E-03$ & $1.31 E+01$ & 7. OQE- 14 & $1.51 E-11$ \\
\hline Cกีแ 2-4-8 & $2.83 E-04$ & $9.23 E-03$ & $2.60 E+01$ & $3.42 E-13$ & $.1 .06 E-11$ \\
\hline CRM 2-4-9 & $2.20 E-94$ & $8.45 E-0 j$ & $2.70 E+01$ & $2.875-13$ & $8.77 E-12$ \\
\hline CRH $2-4-10$ & $6.60 E-04$ & $9.11 E-0 J$ & 4. $i D E+01$ & $3.35 E-13$ & b. $3 J E-12$ \\
\hline CRU $2-4-11$ & $1.26 E-04$ & $9.23 E-03$ & $5.00 E+01$ & $1,\{1 E-\{3$ & $5.34 E-12$ \\
\hline CRH $2-4-12$ & $1.57 E-04$ & $9.37 E-03$ & $6.30 E+01$ & $1.03 E-13$ & $4.30 E-12$ \\
\hline$\left[\sin ^{2} 2-4-13\right.$ & $3.77 E-04$ & $9.7 T E-63$ & $9.20 E+0 \mathrm{I}$ & 1. $22 E-13$ & $3.25 E-12$ \\
\hline $\mathrm{Cng} 2-4-14$ & $2.20 E-04$ & $9.39 E-03$ & $1.22 E+02$ & $7.16 E-14$ & 2.5oE-12 \\
\hline CAM 2-4-15 & 2. $7 B E-04$ & $1.03 E-02$ & $1.34 E+02$ & $9.90 E-13$ & 2. $17 E-12$ \\
\hline ERK $2-4-16$ & $3.77 E-014$ & $1.07 E-02$ & 1.92E+102 & $7.4 \mathrm{JE}-\mathrm{i4}$ & $1.95 E-12$ \\
\hline $\operatorname{CrH} 2-4-17$ & $7.54 E-04$ & $1.14 E-02$ & $3.02 E+02$ & $1.18 E-1 \bar{J}$ & $1.35 E-12$ \\
\hline Conth $2-4-18$ & $1.88 E-04$ & $1.16 E-02$ & $4.10 E+02$ & 1.35E-i4 & 1. $03 E-12$ \\
\hline
\end{tabular}

TABLE B.1f. Fluoride (F) Leach Fractions from AMS 16.1 Leach Test--Whole Grout in Groundwater (DIFF2-4)

\begin{tabular}{|c|c|c|c|c|c|}
\hline SAMPLE & Anitho & CUA. An/ABO & TIME days & inc. & D cun. \\
\hline CFI 2-4-1 & $1.43 E-64$ & $1.43 E-04$ & $3.00 E-014$ & $1.84 E-65$ & $1.84 E-05$ \\
\hline CRI $2-4-2$ & $3.33 E-64$ & $4.76 E-\hat{G 4}$ & $8.33 E-02$ & $4.17 E-12$ & 8.50E-12 \\
\hline CSH $2-4-3$ & 3. 33E-04 & 8. $09 E-64$ & 2. $92 E-01$ & 5. $49 E-12$ & i. $12 \mathrm{E}-12$ \\
\hline CAll $:-4-4$ & $1.47 E-03$ & $2.26 E-0]$ & $1,00 E+00$ & $3.22 E-11$ & 1.6JE-1! \\
\hline Sfill $2-4-5$ & $1.43 E-03$ & $3.71 E-0] 3$ & 2. $10 E+100$ & $3.16 \mathrm{E}-11$ & $2.05 E-! 1$ \\
\hline $\operatorname{CRN} 2-4-6$ & $5.71 E-04$ & 4. $28 E-03$ & $3.02 E+00$ & $1.22 E-11$ & 1. $90 E-11$ \\
\hline CFin 2-4-7 & $1.765-13$ & $6.04 E-03$ & $1.31 E+0\}$ & $2.755-12$ & 8.7JE-12 \\
\hline CRH $2-4-8$ & ล. 1BE-014 & $6.66 E-0 j$ & 2. $00 E+01$ & $1.64 E-12$ & 5.74E-!? \\
\hline CRA $2-4-9$ & J.BIE- & 7. $04 E-03$ & $2.705+0 \mathrm{i}$ & $3.55 \mathrm{E}-1 \mathrm{~J}$ & 5. $75 E-12$ \\
\hline Chin 2-4-10 & 5.2JE-64 & $i .56 E-\hat{v} j$ & $4.10 E+61$ & $5.8 B E-13$ & $4.37 E-12$ \\
\hline ERN $2-4-11$ & $4.765-65$ & $7.61 E-63$ & 5. $.0 E+6 i j$ & $1.39 E-14$ & 3.63E-12 \\
\hline Can $2-4-12$ & $9.51 E-05$ & $7.71 E-03$ & $6.3 \hat{v E}+01$ & $3.79 E-j 4$ & $2.755-12$ \\
\hline San $2-4-13$ & $5.5 J E-65$ & $7.305-03$ & 7.200 E +01 & 1. $04 E-14$ & $2.075-12$ \\
\hline CRN $2-4-14$ & 3.56E-A4 & 3.6DE-0J & $1.22 E+62$ & 1. BGE-12 & $1.98 E-12$ \\
\hline Ona $2-4-15$ & $4.76 E-0104$ & १.13E-טज & 1. $34 E+02$ & $2.56 E-12$ & 1. $75 z-1 z$ \\
\hline CAH 2-4-16 & $9.51 E-05$ & $3.2 J E-\hat{J}$ & 1. $82 E+102$ & $4.74 E-15$ & $1.40 \overline{0}-12$ \\
\hline 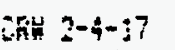 & 2.JBE-04 & $\{.475-6 j$ & 3. $2 E \pm \div 02$ & :. IIE-14 & $9.2 \div E-13$ \\
\hline CAก $2-4-16$ & $9.51 E-05$ & $3.56 E-03$ & 4.10E+102 & $3.74 E-: 5$ & b. \\
\hline
\end{tabular}


TABLE B.1g. Nitrate (NO3) Leach Fractions from ANS 16.1 Leach Test--Whole Grout in Groundwater (DIFF2-4)

\begin{tabular}{|c|c|c|c|c|c|}
\hline SAIPLE & An/Ao & Cue. $\mathrm{An} / \mathrm{AO}$ & IIME days & Dinc. & D Cun. \\
\hline CRA 2-4-1 & $1.33 E-02$ & $1.33 E-02$ & $3.00 E-04$ & $1.57 E-01$ & 1.59E-6i! \\
\hline CRU $2-4-2$ & $1.94 E-02$ & $3.27 E-02$ & B. 3JE- 62 & 1. $42 E-08$ & 4.02E-08 \\
\hline CRH 2-4-3 & 1.12E-02 & $4.37 E-02$ & $2.72 \mathrm{E}-01$ & $6.255-69$ & 2. $177 E-48$ \\
\hline CRU 2-4-4 & $1.94 E-02$ & 6. J3E- 02 & $1.00 E+60$ & $5.57 E-0 ?$ & 1.2SE- \\
\hline CAL $2-4-5$ & 1. $33 E-02$ & 7. ÓOE-02 & $2.10 E+00$ & $2.74 E-09$ & 8.75E-19 \\
\hline CRI 2-4-6 & 9.17E-03 & $8.48 E-02$ & $3.02 E+00$ & $2.51 E-09$ & $7.45 E-199$ \\
\hline CRII 2-4-7 & $6.13 E-02$ & $1.46 E-01$ & $1.31 E+01$ & $3.3 j E-109$ & 5.11E-09 \\
\hline CAH $2-4-8$ & $2.25 E-02$ & $1.69 \mathrm{E}-01$ & $2.00 E+01$ & $2.16 E-09$ & i. $45 E-09$ \\
\hline CFH 2-4-9 & $-1.23 E-02$ & $1.31 E-01$ & $2.70 E+01$ & $8.97 E-10$ & $3.79 E-199$ \\
\hline ㅅำ $2-4-10$ & 6. $955-02$ & $2.50 E-01$ & $4.10 E+01$ & $1.04 E-08$ & 5. $30 E-69$ \\
\hline CRH $z-4-11$ & $7.15 E-03$ & $2.57 E-01$ & $5.00 E+01$ & $3.59 E-10$ & $4.725-09$ \\
\hline CoN $2-4-12$ & $7.15 E-03$ & $2.65 E-\hat{01} 1$ & $6.305+01$ & $2.135-10$ & $3.75 E-09$ \\
\hline CRH $2-4-13$ & $1.94 E-02$ & $2.34 E-01$ & $7.20 E+01$ & $4.31 E-10$ & $3.02 E-19$ \\
\hline CAn 2-4-14 & $7 .: 5 E-03$ & 2.91E-01 & $1.22 E+02$ & $7.57 E-11$ & 2.17E-69 \\
\hline CEN $2-4-15$ & $8.48 E-02$ & $3.70 E-01$ & $1.34 E+62$ & $8.00 E-00$ & $3.305-39$ \\
\hline CRI $2-4-16$ & $1.53 E-02$ & $3.91 E-01$ & $1.82 E+02$ & $1.23 E-10$ & $0.00 E+00$ \\
\hline СR̆ 2-4-17 & 5. $01 E-02$ & $4.41 E-01$ & $3.02 E+02$ & $5.19 E-10$ & $7.60 E-69$ \\
\hline CAU $2-4-18$ & $2.35 E-02$ & 4.65E-01 & $4.10 E+12$ & $2.10 E-10$ & $1.65 E-09$ \\
\hline
\end{tabular}


TABLE B.1i. Carbon-14 (C-14) Leach Fractions from ANS 16.1 Leach Test--Whole Grout with Groundwater (2-4)

\begin{tabular}{|c|c|c|c|c|c|c|c|}
\hline SAKPLE & ${ }^{u C i}$ & \% LEACHED & TIME DAYS & An/Ao & Cun An/AO & $D$ inc. caz/set & D cul enzisec \\
\hline CAN $2-4-1$ & $1.93 E-04$ & $0.012 \%$ & $3.00 E-04$ & $1.21 E-04$ & $1.21 E-04$ & $1.13 E-10$ & $1.53 E-10$ \\
\hline CRt 2-4-2 & $6.87 E-04$ & 0.01547 & 8.3JE-02 & $4.21 E-04$ & $5.43 E-04$ & $7.55 E-12$ & $1.11 E-$ \\
\hline CRH $2-4-3$ & $2.43 E-04$ & $0.069 \%$ & $2.92 E-01$ & $1.52 E-04$ & $0.94 E-64$ & $1.14 E-12$ & $5.17 E-$ \\
\hline CRH $2-4-4$ & $8.42 E-04$ & 0.1212 & $1.00 E+00$ & $5.15 E-04$ & $1.21 E-03$ & $3.93 E-12$ & $4.58 E-$ \\
\hline CRH 2-4-5 & $3.20 E-04$ & 0.1417 & $2.10 E+00$ & $1.99 E-04$ & $1.41 E-03$ & $6.12 E-13$ & $2.36 \mathrm{E}-$ \\
\hline CRin 2-4-6 & $3.19 E-04$ & $0.161 \%$ & $3.02 E+00$ & $1,97 E-04$ & $1.61 E-113$ & $1.46 E-12$ & 2.67E- \\
\hline CAH 2-4-7 & 1. $60 E-03$ & $0.258 \%$ & $1.31 E+01$ & $9.77 E-04$ & $2.58 E-13$ & $8.47 E-13$ & $1.60 \mathrm{E}$ \\
\hline CRH 2-4-6 & $1.93 E-04$ & $0.270 \%$ & 2. V0E +01 & $1.21 E-04$ & 2.70E- $(1) 3$ & 6. $28 E-14$ & 1.14E- \\
\hline En $2-4-9$ & $1.93 E-04$ & $0.283 \%$ & 2. $i \hat{U E E}+\hat{H S}$ & $1.21 E-04$ & $2.33 E-103$ & 8. $77 E-14$ & $9.25 E-$ \\
\hline CNA $2-4-10$ & $1.93 E-04$ & $0.295 \%$ & 4.: $0 E+01$ & $1.21 E-04$ & $2.95 E-03$ & $3.16 E-14$ & 6.63E- \\
\hline LAM 2-4-11 & $1.93 E-04$ & $0.307 \%$ & $5.00 E+(01$ & $1.21 \mathrm{E}-04$ & $3.07 E-03$ & 1. $6 \mathrm{BE}-13$ & 5.99ะ- \\
\hline Can $2-4-12$ & $1.93 E-94$ & $0.319 \%$ & 5. $40 E+01$ & $1.21 E-04$ & $3.19 E-03$ & $5.33 E-14$ & 4.77E- \\
\hline$C F H 2-4-13$ & 1. $93 E-04$ & 0.3312 & $9.20 E+01$ & $1.21 E-04$ & $3.31 E-i j 3$ & $1.81 E-14$ & 3.73E- \\
\hline CRH $2-4-14$ & $1.93 E-04$ & $0.343 \%$ & $1.22 E+02$ & $1.21 E-04$ & $3.43 E-03$ & 2. $38 E-14$ & $3.02 E-$ \\
\hline sn $2-4-15$ & $1.93 E-04$ & $0.355 \%$ & $1.34 E+02$ & $1.21 E-04$ & $3.55 E-03$ & $1.63 E-13$ & $2.955-$ \\
\hline CRH 2-4-13 & $9.795-04$ & $0.417 \%$ & 1. $32 E+102$ & $6.13 E-04$ & $4.175-03$ & $3.21 E-13$ & $2.955-$ \\
\hline CFH 2-4-17 & $1.93 E-04$ & $0.429 \%$ & $3.02 E+02$ & $1.29 E-09$ & $4.29 E-03$ & $3.46 E-15$ & $1.71 E-$ \\
\hline CรH 2-4-i8 & 1.93E-04 & $0.442 \%$ & $4.10 E+02$ & $1.21 E-04$ & $4.42 E-03$ & $5.53 E-15$ & $1.49 E-1$ \\
\hline
\end{tabular}

TABLE B.1i. Carbon-14 (C-14) Leach Fraction from ANS 16.1 Leach Test--Whole Grout in Groundwater (2-5)

\begin{tabular}{|c|c|c|c|c|c|c|c|}
\hline SAMPLIE & $u C i$ & Z LEACHED & TIME DAYS & $A \pi / A O$ & Cun $A \cap / A O$ & $D$ inc. CAz $2 / 5 E E$ & D cun enziser \\
\hline CSN $2-5-1$ & $2.43 E-04$ & $0.012 x$ & 3.00E-04 & 1.18E-O4 & $1.18 E-64$ & 1. $69 \mathrm{E}-10$ & $1.48 E-10$ \\
\hline CAN $2-5-2$ & $1.83 E-03$ & $0.099 \%$ & 8. $33 E-02$ & 6.72E- -04 & $9.90 E-64$ & $3.27 E-1 i$ & $3.73 E-11$ \\
\hline Cfin $2-5-3$ & $6.29 E-j) 4$ & $0.129 \%$ & 2.32E-01 & $3.01 E-04$ & $1.29 E-03$ & $4.535-12$ & 1.A1E-11 \\
\hline Crill $2-5-4$ & $9.58 E-64$ & $0.175 \%$ & $1.00 E+00$ & $4.57 E-0.4$ & $1.75 E-13$ & $3.13 E-12$ & $9.67 E-12$ \\
\hline ChH $2-5-5$ & 8. $17 E-04$ & 0.2142 & $2.10 E+100$ & 3. $30 E-04$ & $2.145-03$ & $2.395-12$ & 0.99E- \\
\hline Cân $2-5-6$ & $1.75 E-04$ & 0.2232 & $3.02 E+00$ & $9.45 E-05$ & $2.235-03$ & $3.39 E-13$ & $5.23 E-12$ \\
\hline CFin $2-5-7$ & $1.56 E-03$ & $0.298 \%$ & $\lfloor .31 E+01$ & T.49E-64 & $2.985-10$ & $5.045-13$ & 2.15E-12 \\
\hline $64=2-5-8$ & $i .20 E-64$ & $0.333 \%$ & C.DOE+01 & $3.44 E-104$ & $3.33 E-13$ & $5.125-13$ & i.75E-12 \\
\hline LFH $2-5-9$ & $1.93 E-04$ & $0.342 \%$ & $2.7 \hat{V E}+01$ & $9.45 E-05$ & $3.425-03$ & $5.375-14$ & $1.37 E-12$ \\
\hline San $2-5-10$ & $1.93 E-04$ & 0.3525 & $4.20 E+01]$ & $7.45 E-05$ & $3.5 \leq 2-103$ & j. $34 E-14$ & $9.54 E-13$ \\
\hline in $2-5-11$ & $3.26 E-04$ & $0.367 \%$ & $5.00 E \div 01$ & $1.595-1.4$ & 3.67E-43 & $3.78 E-13$ & $8.535-13$ \\
\hline CSill $2-5-12$ & $5.21 E-04$ & $0.392 \%$ & $6.40 E+01$ & $2.50 \mathrm{E}-04$ & 3. $32 E-103$ & $2.275-13$ & $3.672-1$ \\
\hline 2nin $2-5-13$ & $2.70 E-104$ & 0.4052 & $7.20 E+01$ & $! .31 E-04$ & $4.65 E-03$ & 2.15E-14 & 5. CoE-1 \\
\hline GRH $2-5-14$ & $1.93 E-04$ & $0.415 \%$ & $1.22 E+62$ & $9.45 E-65$ & $4.15 E-6 !$ & 2. $34 E-: 4$ & $4.47 \mathrm{E}-\mathrm{i}$ \\
\hline inin $2-5-15$ & $1.93 E-64$ & $0.424 \%$ & $1.34 E+02$ & $8.45 E-65$ & $4.24 E-63$ & 1.0GE-13 & $4.25 E-13$ \\
\hline CRH $2-5-16$ & $1.02 E-03$ & 0.4736 & $1.82 E+62$ & $4.91 E-64$ & $4.73 E-13$ & $2 . \therefore B E-13$ & $3.76: E-1$ \\
\hline [f: $2-5-17$ & 1.73E- 74 & 6. $483 \%$ & $=02 E+02$ & $\hat{0.45 E-j 5}$ & $4.53 E-\hat{j} 3$ & $2.675-15$ & $2 \div 4 E-i$ \\
\hline
\end{tabular}


TABLE B.2a. Potassium (K) Leach Fractions from ANS 16.1 Leach Test--Whole Grout in Groundwater (DIFF3-4)

\begin{tabular}{|c|c|c|c|c|c|}
\hline SAMPLE & $A \cap / A D$ & CUS. An/AO & TIME days & Dine. & D cus. \\
\hline CRN 3-4-1 & $-1.87 E-03$ & $-1.87 E-03$ & $3.005-04$ & & \\
\hline CAN $3-4-2$ & $1.49 E-02$ & $1,31 E-02$ & $8.33 E-02$ & 8. J9E-09 & 6. 42EE-09 \\
\hline $\operatorname{CRN} 3-4-3$ & $1.03 E-02$ & 2. $33 E-02$ & $2.92 E-01$ & $5.22 E-09$ & 5. $84 E-09$ \\
\hline CAS 3-4-4 & $2.43 E-02$ & 4. $76 E-02$ & $1.00 E+00$ & $3.72 E-09$ & $7.095-199$ \\
\hline CRH $3-4-5$ & $1.96 E-02$ & $6.72 E-02$ & $2.10 E+00$ & $5.965-09$ & D. TZE-09 \\
\hline CRH 3-4-6 & $1.03 E-02$ & $7.75 E-02$ & $3.02 E+00$ & $3.96 E-09$ & 6. $22 E-09$ \\
\hline CRN 3-4-7 & $6.63 E-02$ & $1.44 E-01$ & $1.31 E+01$ & 3. 39E-09 & $.9 .95 E-09$ \\
\hline CRH 3-4-8 & $2.89 E-02$ & $1.73 E-01$ & $2.00 E+01$ & $3.58 E-09$ & $4.6 T E-09$ \\
\hline CRH 3-4-9 & $1.96 E-102$ & 1. $32 \mathrm{E}-01$ & $2.70 E+01$ & 2.29E-09 & $-4.29 E-19$ \\
\hline $\operatorname{Cn} 23-4-10$ & $3.365-02$ & $2.26 E-01$ & $4.10 E+01$ & $2.43 E-07$ & $4.41 E-09$ \\
\hline $\operatorname{can} 3-4-1 !$ & $1.76 E-02$ & $2.46 E-01$ & $5.00 E+01$ & $2.70 E-07$ & $4.345-39$ \\
\hline CRY $3-4-i z$ & $1.03 E-02$ & $2.56 E-0 !$ & 6.30E+011 & $4.40 E-10$ & $3.75 E-09$ \\
\hline CRU $3-4-13$ & $2.89 E-02$ & $2.85 E-01$ & $7.20 E+01$ & $9.58 E-10$ & $3.025-07$ \\
\hline CRU 3-4-14 & 1. 3áE-0̂2 & $3.04 E-01$ & $1.22 E+02$ & $5.39 E-10$ & $2.66 E-69$ \\
\hline CRA 3-4-15 & $1.96 E-02$ & $3.24 E-01$ & $1.345+02$ & $4.27 E-09$ & $2.80 E-099$ \\
\hline Can $3-4-16$ & $1.76 E-02$ & $3.44 E-01$ & $1.82 E+02$ & $2.01 E-10$ & $2.36 E-69$ \\
\hline C月H $3-4-17$ & 2. $05 E-02$ & $3.64 E-01$ & $3.02 E+02$ & $8.74 E-11$ & 1..22E-vi \\
\hline
\end{tabular}

TABLE B.2b. Sodium (Na) Leach Fractions from ANS 16.1 Leach Test--Whole Grout in Groundwater (DIFF3-4)

\begin{tabular}{|c|c|c|c|c|c|}
\hline SAKPLE & $A n / A ́ O$ & Cus. An/Ao & TIME days & 0 ine. & 0 cus. \\
\hline CRN 3-4-1 & $2.985-03$ & 2. 8 8EE- 03 & $3.00 E-04$ & $8.01 E-03$ & $8.01 E-03$ \\
\hline CFI $3-4-2$ & $3.44 E-02$ & $3.74 E-02$ & $8.33 E-62$ & $4.45 E-08$ & $5.25 E-08$ \\
\hline CRH $3-4-3$ & $2.25 E-02$ & $5.99 E-02$ & 2.72E-Gi & $2,50 E-68$ & $3.85 E-68$ \\
\hline $\operatorname{CRN} 3-4-4$ & 4.01E-02 & $1.00 E-01$ & $1.00 E+00$ & 2. $3 B E-48$ & $3.13 E-08$ \\
\hline 대 $3-4-5$ & $3.22 E-02$ & $1.32 E-01$ & $2.10 E+00$ & 1.6IE-0E & $2.61 E-06$ \\
\hline CRN J-4-6 & $1.65 E-02$ & $1.49 E-v i 1$ & $3.02 E+00$ & $1.03 E-68$ & $2.29 E-10$ \\
\hline CFE $3-4-i$ & 1.07E-01 & $2.55 E-01$ & 1.jIE+01 & 1. OIE-CB & $1.81 E-16$ \\
\hline $\operatorname{con} 3-4-8$ & 4.91E-02 & 3. $04 E-01$ & 2.00E+01 & $1.03 E-68$ & $1.62 E-08$ \\
\hline ERH $3-4-?$ & $3.675-02$ & $3.41 E-0 !$ & 2. $\bar{j} 0 E+0\rfloor$ & 8.11E-05 & 1.59E- 68 \\
\hline 动 3-4-10 & $5.53 E-02$ & $3.96 E-\hat{v} 1$ & 4. $30 E+01$ & $0.57 E-69$ & :.52E-06 \\
\hline Cñ $3-4-11$ & $2.74 E-02$ & $4.24 E-01$ & $5.00 E+01$ & $5.25 E-08$ & $1.46 E-08$ \\
\hline Cกิม $3-4-12$ & $3.12 E-02$ & $4.55 E-01 !$ & b. $30 E+0 i$ & $4.05 E-69$ & 1.39E-68 \\
\hline CFH 3-4-1j & $4.74 E-022$ & $5.02 E-01$ & $9.20 E+01$ & $2.5 i E-05$ & 1.17E-66. \\
\hline Can 3-4-14 & $3.70 E-02$ & $5.41 E-01$ & 1.22E+02 & $2.265-09$ & $1.66 E-48$ \\
\hline SR 3-4-15 & $-6.335-03$ & $5.35 E-01$ & $1.34 E+12$ & $4.45 E-10$ & 3.0?E-1P \\
\hline CRH j-4-16 & j. & $5.72 E-01$ & 1. $32 E+02$ & i.IIE-iv & $0.24 E-. j 8$ \\
\hline SFin $5-4-17$ & $5.20 \mathrm{E}-12$ & $6.24 E-01$ & $3.02 E+02$ & $5.61 E-10$ & 2. $22 E-04$ \\
\hline
\end{tabular}


TABLE B.2e. Boron (asH3B03) Leach Fractions from ANS 16.1 Leach Test--Whole Grout in Groundwater (DIFF3-4)

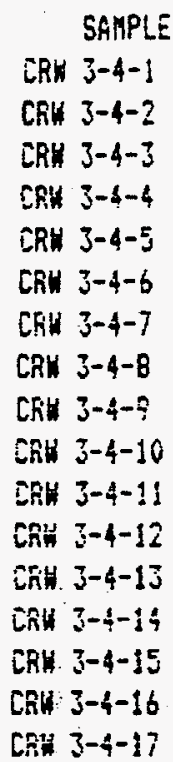

$$
\begin{aligned}
& \text { An/AO } \\
& 5.84 E-04 \\
& 3.35 E-03 \\
& 2.06 E-03 \\
& 2.77 E-03 \\
& 1.60 E-03 \\
& 0.46 E-04 \\
& 2.58 E-03 \\
& 6.76 E-04 \\
& 3.59 E-04 \\
& 7.07 E-04 \\
& 2.77 E-04 \\
& 9.22 E-015 \\
& 3.59 E-04 \\
& 9.22 E-05 \\
& 2.15 E-04 \\
& 3.36 E-04 \\
& 6.46 E-04
\end{aligned}
$$

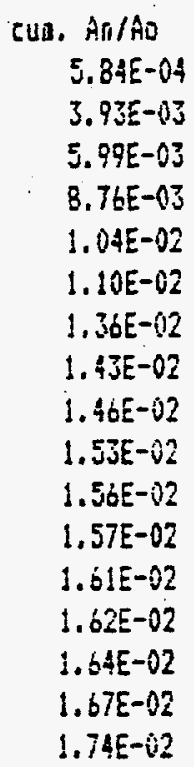

CUB. An/AD

5. $84 E-04$

$3.935-13$

$5.99 E-03$

$8.76 E-03$

$1.04 E-02$

1. $10 \mathrm{E}-02$

$1.36 \mathrm{E}-1.12$

$1.43 \mathrm{E}-02$

$1.46 E-02$

1. $53 \mathrm{E}-02$

1.56E-12

1.57E-02

$1.61 E-\hat{v} 2$

$1.62 E-02$

1. $64 E-02$

$1.67 E-02$

1. $74 E-62$

TIME JaYs
$3.00 E-04$
$8.33 E-02$
$2.92 E-01$
$1.00 E+00$
$2.10 E+00$
$3.02 E+00$
$1.31 E+01$
$2.00 E+01$
$2.70 E+01$
$4.10 E+01$
$5.00 E+01$
$6.30 E+01$
$7.20 E+01$
$1.22 E+02$
$1.34 E+02$
$1.32 E+02$
$3.12 E+02$

$D$ inc.

3.07E-04

4. $22 \mathrm{E}-10$

2. $10 E-10$

1. $13 E-16$

$3.96 E-11$

$1.56 E-11$

5. $51 E-12$

1. $965-12$

3.12E-13

1. $07 E-12$

5. STE-13

$3.55 E-14$

$1.56 E-13$

1. $26 E-14$

$5.15 E-13$

5. $98 E-14$

8. $63 E-14$
0 cus.

$3.07 E-64$

$5.82 E-10$

3. $25 E-10$

\begin{tabular}{|c|c|c|c|c|c|}
\hline SAMPLLE & An/AO & Cus. An/AO & TIME days & 8 inc. & 0 св. \\
\hline CFIN 3-4-i & $0.00 E+(10$ & $0.00 E+00$ & $3.00 E-044$ & & $0.00 E+00$ \\
\hline $\operatorname{cst} 3-4-2$ & $4.66 E-05$ & $4.66 E-05$ & a. $33 E-02$ & 8.15E-14 & 6. $14 E-14$ \\
\hline Cก́n 3-4-3 & $5.59 E-64$ & E. 65E-04 & $2.92 E-0 !$ & 1.5AE-11 & 3. $73 E-12$ \\
\hline ลกับ 3-4-4 & $4.66 E-04$ & $1.07 E-03$ & $1.00 E+00$ & $3.21 E-12$ & 3.59E-12 \\
\hline iiㄴ 3-4-5 & $1.54 E-03$ & $2.61 E-03$ & $2.10 E+00$ & $3.66 E-11$. & l. AiE-ii \\
\hline Cs: 3-4-6 & $1.49 E-03$ & $4.10 E-03$ & $3.02 E+60$ & 8.3सE-11 & $1.74 E-11$ \\
\hline Sin 3-4-7 & d. 9aE-64 & $4.30 E-6 j$ & $1.3 \mathrm{iE}+0 \mathrm{i}$ & $4.32 E-13$ & $5.50 E-12$ \\
\hline CRAN 3-4-8 & $1.96 E-03$ & $6.75 E-63$ & 2. $00 E+01$ & 1. $64 E-11$ & 7.13E-12 \\
\hline CFH 3-4-? & $1.12 E-03$ & $7.37 E-i 3$ & $2.70 E+01$ & $7.45 E-12$ & 7.16E-12 \\
\hline $\operatorname{Cnn} 3-4-10$ & $4.66 E-614$ & 8. $3 \mathrm{JE} E-\hat{\mathrm{B}} \mathrm{3}$ & 4. $1 \mathrm{DE}+01$ & $4.66=-13$ & $5.30 E-12$ \\
\hline Con $j-4-11$ & $6.05 E-04$ & $8.74 E-13$ & $5.00 E+0 !$ & $2.575-12$ & $5.00 E-12$ \\
\hline Con 3-4-12 & $4.66 E-0.55$ & $8.79 E-63$ & $6.30 E+61$ & $3.045-15$ & 4.011E-12 \\
\hline Can $3-4-13$ & $1.40 E-j) 4$ & $9.135-03$ & $9.20 E+01$ & 2.23E- & $2.835-12$ \\
\hline CSN 3-4-14 & $9.31 E-15$ & จ. .2EE-0J & ¿.22E+02 & 1. $28 E-19$ & $2.13 E-12$ \\
\hline 5 37-4-15 & $5.35 E-344$ & $9.75 E-03$ & $1.34 E+\sqrt{12} 2$ & $3.1=5-12$ & $2.22 E-12$ \\
\hline if $3-2-16$ & $5.76 E-94$ & 1. $07 \mathrm{E}-\mathrm{-02}$ & 1. $62 E+12$ & J. $\hat{0} \mathrm{OEE}-\mathrm{i} 3$ & 1. $9 B E-12$ \\
\hline $304-4-17$ & P.JIE-ט̂E & 1.08E-0? & $3.02 E+02$ & $1.60 E-15$ & โ.21E-!2 \\
\hline
\end{tabular}

$2.40 E-10$

$1.6 \hat{N E}-10$

$1.25 E-10$

4. 42E-I:

J. $18 E-1 i$

$2.48 E-11$

i. $60 \mathrm{OE}-11$

1.53E-11

1. 23E-1!

3.79E-12

$6.71 E-12$

6. $27 \mathrm{E}-12$

4.81E-12

$3.15 E-12$

TABLE B.2f.

Fluoride (F) Leach Fractions from ANS 16.1 Leach Test--Whole Grout in Groundwater (DIFF3-4) 
TABLE B.2g. Nitrate (N03) Leach Fractions from ANS 16.1 Leach Test--Whole Grout in Groundwater (DIFF3-4)

\begin{tabular}{|c|c|c|c|c|c|}
\hline SAMPLE & An/Ao & CUn. An/AO & TIME days & Dinc. & D cus. \\
\hline CFN 3-4-1 & $5.00 E-03$ & 5. $00 \mathrm{E}-03$ & $3.00 \mathrm{E}-04$ & $2.25 E-02$ & $2.25 E-02$ \\
\hline CRH 3-4-2 & $2.80 E-02$ & 3. $30 E-02$ & Q.33E-02 & 2. $55 E-06$ & $4.09 E-08$ \\
\hline CRU $3-4-3$ & 1. $60 E-02$ & 4. $90 E-02$ & $2.92 E-01$ & $1.27 E-i 8$ & $2.57 E-08$ \\
\hline CnN 3-4-4 & 2.10E-02 & $7.00 E-02$ & $1.00 E+00$ & 6. $52 E-09$ & 1. S5E-46 \\
\hline CRH 3-4-5 & $1.60 E-02$ & $8.60 E-02$ & $2.10 E+00$ & $3.97 E-09$ & $1.10 E-00$ \\
\hline $\operatorname{cnn} 3-4-6$ & $8.00 E-03$ & $9.40 E-02$ & $3.02 E+00$ & $2.40 E-09$ & $9 . ! 55-199$ \\
\hline CAH 3-4-7 & $7.40 E-02$ & $1.68 E-01$ & $1.31 E+01$ & $4.85 E-69$ & $0.75 E-08$ \\
\hline CRI 3-4-5 & $2.50 E-02$ & $1.93 E-01$ & 2. $00 E+01$ & $2.67 E-09$ & 5.83E-09 \\
\hline 대 3-4-9 & $1.605-02$ & $2.09 E-01$ & $2.70 E+01$ & $1.53 \mathrm{E}-\hat{\mathrm{N} g}$ & E. $60 E-099$ \\
\hline CRY 3-4-10 & 1. BOE-02 & $2.27 E-01$ & $4.10 E+01$ & $6.96 E-10$ & 4.41E-ij9 \\
\hline CRM 3-4-11 & $7.00 E-03$ & $2.3 A E-01$ & $5.60 E+01$ & $3.44 E-10$ & $3.61 E-09$ \\
\hline CRH 3-4-12 & $1.00 E-0.2$ & $2.44 E-01$ & $6.30 E+01$ & $4.17 E-10$ & 3. $15 E-09$ \\
\hline CRU 3-4-13 & $2.20 E-02$ & $2.66 E-01$ & $\div .20 E+01$ & 5. $53 \mathrm{~J} E-10$ & $2.30 E-09$ \\
\hline Cnin 3-4-14 & $7.00 E-03$ & $2.73 E-01$ & $1.22 E+02$ & $7.25 E-11$ & $0.00 E+00$ \\
\hline $\mathrm{CNH} 3-4-15$ & $1.10 E-02$ & $2.84 E-01$ & $1.34 E+02$ & $1.35 E-09$ & 2. $67 E-199$ \\
\hline $\operatorname{CRN} 3-4-16$ & $1.50 E-02$ & $2.99 E-01$ & $1.82 E+02$ & 1. $1 B E-10$ & $1.76 E-99$ \\
\hline CRH $3-4-17$ & $4.90 E-02$ & $3.48 E-01$ & $3.02 E+02$ & $4.97 E-10$ & $1.52 E-67$ \\
\hline
\end{tabular}

TABLE B.2g. Nitrate (NO3) Leach Fractions from ANS 16.1 Leach Test--Whole Grout in Groundwater (DIFF3-4)

\begin{tabular}{|c|c|c|c|c|c|}
\hline SAKPLE & $\mathrm{An} / \mathrm{AD}$ & LLIS. Ân/AO & TIME days & ij inc. & 5 Gut. \\
\hline $\operatorname{can} 3-4-j$ & 3. $00 E-03$ & $5.005-03$ & $3_{0} 60 \mathrm{EE}-044$ & $2.25 E-3 i$ & 2. $255-62$ \\
\hline Can $3-4-2$ & $2.80 E-02$ & $3.30 E-02$ & 8.j3E-132 & 2. $355-0.66$ & $4.67 E-6 \mathrm{jE}$ \\
\hline Exil $3-4-3$ & 1. Sôf -102 & $4.30 E-02$ & $2.52 E-631$ & $1.27 E-63$ & $2.5 \overline{1}=-60$ \\
\hline 슨 3-4-4 & 2.10E- & 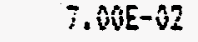 & $1.00 E \div 60$ & $6.52 E-69$ & !.55E- 55 \\
\hline Chin $3-4-5$ & 1. $60 E-102$ & $8.60 E-\hat{0} 2$ & $2.10 E+00$ & $3.37 \mathrm{E}-69$ & $1.1 \mathrm{vE}-\mathrm{i} 8$ \\
\hline ธละ 3-4-b & $0.00 E-03$ & $9.90 E-\hat{v} 2$ & 3. $62 E+60$ & $2.465-99$ & 9. $.5 E-49$ \\
\hline 鼓 $3-4-7$ & $7.46 E-62$ & 1. $60 E-01]$ & L.j1E+0! & $4.85 E-69$ & $=.75 E-69$ \\
\hline SRA $5-4-6$ & $2.50 \leq-02$ & $1.73 E-01$ & 2. 60 E +01 & $2.675-69$ & 5. $335-49$ \\
\hline SAN 3-4-9 & 1. $60 E-02$ & 2. BPE-6! & $2.70 E \div 01$ & 1. 53E- -197 & $5.605-69$ \\
\hline Chit $5-4-10$ & 1. BUE- -12 & 2. $27 \mathrm{E}-\hat{\mathrm{v}} \mathrm{i}$ & 4. DOETQGS & $6.76 E-10$ & $4,415-65$ \\
\hline $5 n$ 3-4-11 & $7.615-63$ & $2.34 E-i 11$ & $5.6 v E+61$ & $3,44 E-10$ & $5 ., 3 E-89$ \\
\hline $653-4-12$ & 1. $010 E-02$ & $2 . \$ 4 E-v 1$ & 2. $30 \hat{E}+\hat{v} !$ & 4.1TE- & 3.15E-49 \\
\hline $8+43-43$ & $2.705-02$ & $2.005-0 !$ & $7.20 \mathrm{E}+61$ & $5.53 E-10$ & S.SWE-A \\
\hline$-54-4-34$ & $7.09 E-03$ & $2.735-1) !$ & $1.22 E+0$ & T.2EE-1! & Z.LIE- \\
\hline IfH $j-4-15$ & $1.10 E-02$ & $2.34 E-31$ & $1.345+02$ & 1. 35E-64 & 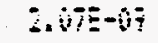 \\
\hline Enk $3-4-16$ & i. $560-02$ & 2. $39[-0 !$ & $\therefore .325+02$ & 1. $1 E E-16$ & C.TBE-TE \\
\hline $5 i-3-1 j$ & $4.70 E-12$ & $3.40 E-31$ & 3. $\quad 02 E \div 0$ & $4.97=-8 i$ & 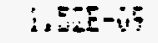 \\
\hline
\end{tabular}


TABLE B.2h. Sulfate (SO4) Leach Fractions from ANS 16.1 Leach Test--Whole Grout in Groundwater (DIFF3-4)

\begin{tabular}{|c|c|c|c|c|c|}
\hline SAMPLE & $\hat{A} \cap / A_{D}$ & Cus. An/AO & TIME days & D inc. & $D$ ius. \\
\hline CRY 3-4-1 & $5.84 E-04$ & 5. $84 E-34$ & 3. OODE-04 & 3:07E-04 & $3.07 E-\hat{v}$ \\
\hline CRH 3-4-2 & $3.35 E-03$ & $3.93 \mathrm{E}-03$ & B. $33 E-02$ & $4.22 E-10$ & $5.82 \mathrm{E}-10$ \\
\hline CRH 3-4-3 & $2.065-03$ & $5.99 E-03$ & 2. 32E-01 & $2.10 E-10$ & $3.255-10$ \\
\hline CRI 3-4-4 & $2.77 E-03$ & $8.76 E-03$ & 1.00E +00 & 1. $13 E-10$ & 2. $4 \hat{v E}-10$ \\
\hline CRH 3-4-5 & $1.60 E-03$ & $1.04 E-02$ & $2.10 E+00$ & $3.965-11$ & 1. $.00 E-10$ \\
\hline CAN 3-4-6 & $6.46 E-04$ & 1. $10 E-02$ & $3.02 E+0 j$ & $1.56 E-11$ & $1.25 E-10$ \\
\hline CRH $3-4-7$ & $2.5 B E-03$ & $1.36 E-02$ & $1.31 E+01$ & 5. $3 ! E-12$ & 4. $12 E-1 !$ \\
\hline CRH 3-4-8 & $0.76 E-04$ & $1.43 E-02$ & $2.00 E+01$ & $1.70 E-12$ & $3 .: 0 E-1 i$ \\
\hline CRH 3-4-7 & 3. OGE-04 & $1.405-02$ & $2.70 E+01$ & E.12E-1j & $2 .+65-11$ \\
\hline CNU $3-4-10$ & T.0TE-04 & $1.53 E-02$ & 4. $10 E+01$ & $1.07 E-12$ & $\therefore 30 E-1 !$ \\
\hline CNin $3-4-11$ & $2.77 E-64$ & $1.56 E-02$ & 5. $00 E+0 !$ & $5.3 \bar{E}-13$ & 1. $335-11$ \\
\hline Conil $3-4-12$ & $7.22 E-05$ & i.57E-02 & b. $30 E+01$ & $3.55 E-14$. & i. $23 E-\mathrm{i} i$ \\
\hline CRH $3-4-i j$ & 3.5รEE-04 & $1.61 E-02$ & $7.20 E+0 !$ & $1.56 \mathrm{E}-13$ & $8.7 P E-12$ \\
\hline CRN 3-4-14 & 9.22ZE-05 & $1.62 E-\hat{v} z$ & 1.22E+0.2 & $1.26 E-14$ & $6.71 E-12$ \\
\hline CNA $3-4-15$ & 2. $15 \mathrm{E}-\hat{6} 4$ & $1.64 E-02$ & $1.34 E+02$ & $5.15 E-13$ & $6.27 E-12$ \\
\hline CAH $3-4-16$ & 3. 3BE-04 & $1.67 E-02$ & $1.82 E+102$ & $5.39 E-14$ & $4.81 E-12$ \\
\hline CNA $3-4-17$ & $0.36 E-04$ & $1.74 E-02$ & 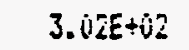 & 8. $0 \mathrm{JE}-14$ & $3.135-12$ \\
\hline
\end{tabular}

\section{TABLE B.2i. Technetium-99 (TC-99) Leach Fractions from ANS 16.1 Leach Test-- Whole Grout in Groundwater (3-4)}

\begin{tabular}{|c|c|c|c|c|c|c|c|}
\hline SAMPLE & $\mathrm{UCI}$ & ¿ LEACHED & TIME DAYS & AnjÁno & CUL Ân/ÂO & jinc. caz/sec & D cun caZisec \\
\hline Efit $3-4-1$ & $1.35 E-04$ & 0.009\% & $3.00 E-04$ & 7. $42 E-05$ & $9.425-05$ & $6.97 \mathrm{E}-11$ & $9.47 E-11$ \\
\hline Cofit $3-4-2$ & $3.9 B E-03$ & $0.278 \%$ & $8.33 E-02$ & $2.69 E-03$ & $2.70 E-03$ & 3.1SE-10 & $2.98 E-10$ \\
\hline Ext $3-4-3$ & $1.62 E-83$ & $0.388 \%$ & $2.82 E-01$ & $1.09 E-03$ & $5.86 E-013$ & 5.05E-!! & $i .655-i 0$ \\
\hline CFin 3-4-4 & $3.2 B E-63$ & $0.607 \%$ & $1.00 E+00$ & $2.21 E-03$ & $6.09 E-6.3$ & $7.94 E-i i$ & 1.1.EE-10 \\
\hline Crin 3-4-5 & $1.64 E-0.33$ & $0.720 \mathrm{~h}$ & $2.10 E+00$ & $1.11 E-03$ & $7.20 \mathrm{E}-03$ & i. Fóe- $1 i$ & $7.71 E-11$ \\
\hline Ean 3-4-6 & $5.98 E-04$ & $0.761 \%$ & $3.02 E+00$ & $4.075-04$ & $7.61 E-03$ & $6.36 E-12$ & 6.14E-i1 \\
\hline CAH $3-4-i$ & $5.175-03$ & $! .11 \hat{\theta}_{\mathrm{s}}$ & $1.31 E+01$ & $3.47 E-\hat{*} 3$ & $1.11 E-12$ & $1 . \$ 1 E-11$ & $3.02 E-11$ \\
\hline isin $3-4-3$ & $\therefore .365-93$ & $1.203 \%$ & $2.00 E+01$ & $9.21 E-0 \mathfrak{Z}$ & i. $20 E-62$ & $3.71 E^{2}: 2$ & 2.JîE-ij \\
\hline ㅈํำ 3-4-7ิ & $1.02 E-03$ & $1.272 \%$ & $2.70 E+01$ & $6.73 E-04$ & $1.27 E-02$ & $2.93 E-12$ & $1.72 E-11$ \\
\hline Sin 3-4-10 & 1.64E-0I & $1.365 \%$ & $4 .: 0 E+01$ & $1.11 E-03$ & $1.365-02$ & $2.795-12$ & $1.49 E-11$ \\
\hline $843-4-11$ & $3.96 E-64$ & 1.4104 & $5.00 E+01$ & $2.70 E-144$ & $1.91 \varepsilon-012$ & 5.23E-:3 & $1.27 E-1$ \\
\hline $\operatorname{Cosin} 3-4-12$ & $5.13 E-64$ & $1.445 \%$ & 6. $40 E+01$ & $3.49 E-04$ & $1.44 E-0.2$ & $4.53 E-13$ & $1.04 \varepsilon-: i$ \\
\hline 재 3-4-13 & 1.13E-03 & $1.521 \%$ & $7.20 E+01$ & $7.66 \bar{E}-04$ & $1.52=-12$ & $7.4 ! 5-: 3$ & 8.06E-12 \\
\hline G甘木 3-4-14 & $8.77 E-04$ & $1.581 \%$ & $1.22 E-02$ & 5. $96 E-64$ & !.58E-0.2 & $5.37 E-13$ & $6.56 E-12$ \\
\hline $6 \sin 5-4-15$ & $2.35 E-64$ & $1.590 \%$ & $1.34 E+02$ & $9.42 E-i 5$ & i. $59 E-02$ & I.O1E-ij & $0.04 E-12$ \\
\hline Cfis $3-4-16$ & $\vdots .44 E-i 3$ & $1.689 \%$ & 1. $32 E+112$ & $9.83 E-044$ & $1.6=5-02$ & $\overline{8} .4 \overline{3 E}-1 \overline{5}$ & 5. $02 \mathrm{E}-1$ \\
\hline Sil 3-4-17 & 1. $42 E-03$ & $1.785 \%$ & $3.02 E+02$ & $9.60 E-04$ & $1.78 E-12$ & i. $55 E-: 3$ & 3. $30 \mathrm{~B}-1$ \\
\hline
\end{tabular}


TABLE B.2i. Technetium-99 (TC-99) Leach Fractions from ANS 16.1 Leach Test-Whole Grout in Groundwater (3-5)

\begin{tabular}{|c|c|c|c|c|c|c|c|}
\hline SAMPLE & $U C \mathrm{Cj}$ & I LEACHED & TIKE DAYS & An/AB & $C U: A n / A D$ & $\theta$ inc. calsec & 1 cus col/5ec \\
\hline CFN 3-5-1 & 1. $35 E-04$ & $0.010 \%$ & $3.00 E-04$ & 9.51E-05 & $9.51 E-0.5$ & 6. $63 E-11$ & $9.00 E-11$ \\
\hline CRIN $3-5-2$ & $2.36 E-03$ & $0.170 \%$ & $8.33 E-02$ & $1.60 E-03$ & $0.70 E-03$ & $1.04 E-10$ & $1.03 E-10$ \\
\hline CAN 3-5-3 & $1.68 E-63$ & $0.284 \%$ & $2.72 E-01$ & $1.14 E-03$ & $2.84 E-03$ & $6.12 E-11$ & 8.24E-1! \\
\hline 대 $3-5-4$ & $1.88 E-03$ & 0.4126 & $1.00 E+00$ & 1. $28 E-03$ & $4.12 E-03$ & $2.30 E-11$ & $5.06 E-11$ \\
\hline CRH 3-5-5 & 1. $15 E-03$ & $0.490 \%$ & $2.10 E+00$ & $7.82 \mathrm{E}-04$ & $4.90 E-03$ & $9.05 E-12$ & $3.41 E-11$ \\
\hline CRA 3-5-6 & :.J5E-14 & $0.499 \%$ & $3.02 E+00$ & $9.538-05$ & $4.99 E-03$ & $3.29 E-13$ & $2.46 E-11$ \\
\hline CRM $3-5-7$ & $5.02 E-03$ & $0.842 \%$ & $1.31 E+01$ & $3.42 E-03$ & $3.42 E-03$ & $9.91 E-1 \hat{n}$ & !. $2 Z E-11$ \\
\hline CRN 3-5-6 & ¿.21E-03 & $0.925 \%$ & $2.00 E+01$ & $8.34 E-04$ & $9.25 E-03$ & $2.84 E-12$ & ¿.2BE-11 \\
\hline CRH 3-5-9 & 3.55E-6i4 & 0.9636 & $2.70 E+01$ & $5.63 E-64$ & 9. $33 E-63$ & $1.94[-j 2$ & $1.07 E-11$ \\
\hline CEL j-5-10 & $6.31 E-03$ & i. $072 \%$ & 4. $00 E+01$ & $3.91 E-04$ & $1.07 E-\hat{i} 2$ & $1.63 E-12$ & $3.37 E-12$ \\
\hline CRW 3-5-11 & $4.41 E-04$ & 1.1053 & 5. $60 E+01$ & $3.03 E-64$ & 1.10E-02 & $6.13 E-13$ & 7. $26 E-12$ \\
\hline CFin $3-5-12$ & $6.57 E-0.4$ & $1.148 \%$ & $6.40 E+01$ & $4.52 \mathrm{E}-04$ & $1.15 E-02$ & $7.07 E=13$ & $6.15 t-12$ \\
\hline BRE 3-5-13 & $3.74 E-04$ & $1.174 \%$ & $9.20 E+01$ & $2.57 E-04$ & $1.17 E-62$ & $7.77 E-14$ & 4. $37 E-12$ \\
\hline CRN $3-5-14$ & 9. $64 E-34$ & 1.2392 & $1.22 E+02$ & $6.57 E-04$ & 1.24E- -2 & $6.10 E-13$ & $3.76 E-12$ \\
\hline CEN $3-5-15$ & $1.35 E-04$ & 1.2494 & $1.34 E+02$ & $9.97 E-05$ & $1.25 \mathrm{E}-02$ & 7.51E-14 & $3.47 E-12$ \\
\hline Cfill $3-5-16$ & $1.36 E-03$ & $1.342 \pi$ & $1.82 E+02$ & $9.32 E-04$ & $1.34 E-012$ & $7.07 E-13$ & $2.95 E-12$ \\
\hline CNA $3-5-17$ & $7.15 E-04$ & $1.391 \%$ & $3.02 E+02$ & $4.69 E-04$ & 1. 39E- 02 & $4.72 E-14$ & $1.91 E-12$ \\
\hline CRY 3-5-18 & $2.72 E-03$ & $! .577 \%$ & 4.10E+112 & $1.86 E-03$ & 1.55E-0.2 & $1.25 E-12$ & โ. $81 E-12$ \\
\hline
\end{tabular}

TABLE B.3a. Potassium (K) Leach Fractions from ANS 16.1 Leach Test--Whole Grout in Groundwater (DIFF4-8)

\begin{tabular}{|c|c|c|c|c|c|}
\hline SAKPLE & An/Ao & Eua. An/AO & TIME daYs & $D$ inc. & D cus. \\
\hline CRI 4-8-1 & $-2.07 E-03$ & $-2.07 E-03$ & $3.00 E-014$ & & \\
\hline CRN $4-8-2$ & $1.66 E-012$ & $1.45 E-02$ & 8. $33 E-02$ & 1. $635-4 a$ & 7.63E-69 \\
\hline CRH $4-8-3$ & 8. $80 E-\hat{v} 3$ & $2.33 E-02$ & $2.52 E-(0)$ & $3.63 E-69$ & $5.82 E-09$ \\
\hline CRy 4-8-4 & $2.17 E-02$ & $4.50 \mathrm{E}-02$ & $1.00 E+100$ & $6.395-09$ & $6.34 E-19$ \\
\hline CFI 4-3-5 & 1. $6 \in E-02$ & $6.16 E-02$ & $2.10 E+100$ & $4.25 E-69$ & $5.652-99$ \\
\hline SRH 4-8-6 & $6.21 E-03$ & $6.78 E-02$ & $3.02 E+00$ & $1.45 E-199$ & $4.76 E-09$ \\
\hline CAN $4-8-7$. & $6.31 E-02$ & $1.3|E-0|$ & $1.31 E+01$ & 3.53E-จ9 & 4. $10 E-69$ \\
\hline CRH $4-8-8$ & $2.69 E-\hat{v} 2$ & ¿. $58 E-01$ & $2.005 \div 01$ & $3.09 E-09$ & $3.90 E-199$ \\
\hline Cx+H 4-ô- & $1.665-62$ & โ. TAE-0̇ & $2.70 E+01$ & $1.64 E-j 9$ & $3.3215-69$ \\
\hline Coll $4-8-10$ & $3.21 E-\hat{v} 2$ & $2.06 E-01$ & 4.10E+Gi & $2.21 E-39$ & 3.65E-69 \\
\hline 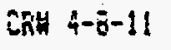 & $\$ .83 E-03$ & $2 . j 6 E-\dot{v i} i$ & $5.00 E+6 i$ & $0.78 E-10$ & $3.35 E-69$ \\
\hline Crit $4-\hat{\varepsilon}-12$ & $5.31 E-63$ & $2.26 E-01$ & $6.30 E+61$ & $3.62 E-10$ & $2.67 E-49$ \\
\hline CFH $4-8-13$ & $3.21 E-02$ & $2.58 E-6 !$ & $9.20 \mathrm{DE}+01$ & ¿.:9E-6\% & $2.57 E-69$ \\
\hline ani 4-8-14 & $\therefore .91 E-02$ & $2.77 E-61$ & $1.22 E+62$ & $5.43 E-10$ & $2.27 E-69$ \\
\hline$i n i^{4} 4-8-15$ & $2.04 E-102$ & $2.97 E-0 !$ & $1.34 E+02$ & $4.54 E-5 ?$ & $2.92 E-19$ \\
\hline $6+44-8-16$ & $3.17 E-i z$ & $3: 9 E-1)$ & i. ECE+iaz & $2,475-40$ & $2.06=-09$ \\
\hline BKH $4-8-1\}$ & $2.695-12$ & $3.465-0 !$ & $\therefore .02 E+02$ & (.5)E-10 & $\therefore .52 E-49$ \\
\hline
\end{tabular}


TABLE B.3b. Sodium (Na) Leach Fractions from ANS 16.1 Leach Test--Whole Grout in Groundwater (DIFF4-8)

\begin{tabular}{|c|c|c|c|c|c|}
\hline SAMPLE & $A n / A D$ & cus. An'îno & TIME days & D inc. & D cus. \\
\hline CRH 4-8-1 & $2.705-03$ & $2.70 E-03$ & $3.00 \mathrm{E}-04$ & $6.59 E-03$ & $0.59 E-013$ \\
\hline CFIH $4-8-2$ & $3.48 E-02$ & 3. $75 E-02$ & $8.3 \mathrm{JE}-02$ & $4.50 E-08$ & 5. $30 E-08$ \\
\hline CRN $4-3-3$ & 2.01E-02 & $5.77 E-02$ & $2.72 E-01$ & 2. $00 E-08$ & $3.575-68$ \\
\hline Cก) 4-6-4 & $3.5 \pi E-02$ & $3.34 E-02$ & $1.00 E+00$ & $1.895-08$ & $2.735-60$ \\
\hline CRU 4-8-5 & 2.61E-02 & ¿. 20E-01 & 2. $10 E+00$ & 1. $36 E-08$ & $2.13 E-18$ \\
\hline Cñu 4-B-b & 1.38E- 02 & 1.J3E-01 & $3.62 E+00$ & $7.17 E-0 \%$ & 1. $.64 E-06$ \\
\hline CAM 4-8-7 & 1.0iE-01 & $2.3+5-01$ & $1.31 E+0\}$ & $3.78 E-09$ & 1. $.38 E-38$ \\
\hline CAN $\div-8-8$ & $4.33 E-02$ & $2,77 E-01$ & $2.00 E+01$ & $8.60 E-39$ & $1.37 E-68$ \\
\hline CRI $4-8-9$ & $3.2 ! E-02$ & $3.09 E-01$ & $2.70 E+01$ & 6.17E-0? & $1.2 B E-3 B$ \\
\hline Can 4-8-10 & $5.11 E-02$ & 3.60 E- 01 & 4.10E+01 & 5. $00 E-09$ & :. $19 E-60$ \\
\hline LFit $4-8-11$ & 2.61E-62 & 3.67E-01 & $5.00 E+01$ & $4.79 E-69$ & $1.18 E-3 B$ \\
\hline CAN $4-8-12$ & $2.97 E-02$ & 4.16E-01 & b. $30 E+01$ & $3.695-69$ & $1.11 E-08$ \\
\hline CRH $4-8-1 . j$ & $4.965-02$ & $4.66 E-01$ & $9.20 E+61$ & $2.81 E-09$ & $\therefore .60 E-08$ \\
\hline$C{ }^{3} 4-6-14$ & 3. $79 E-02$ & $5.04 E-01$ & $1.22 E+02$ & 2.12E-07 & S.81E-09 \\
\hline เก⿻ 4-8-15 & $3.79 E-02$ & $5.42 E-01$ & $1.3+E+02$ & $1.59 E-08$ & $3.69 E-09$ \\
\hline $\operatorname{CAH} 4-6-16$ & $3.82 E-02$ & $5.80 E-01$ & $1.82 E+02$ & $7.62 E-10$ & 8.63E-0.9 \\
\hline CAN $4-8-17$ & $5.80 E-02$ & $6.38 E-01$ & $3.02 E+02$ & $6.965-10$ & $6.50 E-09$ \\
\hline
\end{tabular}

TABLE B.3e. Boron (as H3B03) Leach Fractions from ANS 16.1 Leach Test--Whole Grout in Groundwater (DIFF4-8)

\begin{tabular}{|c|c|c|c|c|c|}
\hline SAMPLE & $A_{n} / A_{0}$ & CUI. An/Ao & IIME days & $D$ int. & D clit. \\
\hline CRIN 4-8-1 & $5.45 E-04$ & 5. 45EE-04 & 3.0OE-04 & Z. 6 BE-04 & $2.66 E-04$ \\
\hline CNH $\div-8-2$ & $3.82 E-03$ & $4.36 E-03$ & B. $33 E-02$ & $5.475-10$ & $7.15 E-10$ \\
\hline CRy 4-8-3 & $2.155-03$ & $6.51 E-03$ & $2.925-01$ & $2.28 E-10$ & $4.54 E-10$ \\
\hline Cก̈ $4-8-4$ & $2.69 E-03$ & $9.20 E-0]$ & $1.00 E+00$ & $1.07 E-10$ & $2.655-10$ \\
\hline CNA 4-8-5 & 3. 88E-04 & 1.02E-02 & $2.10 E+00$ & $1.51 E-11$ & $1.55 E-10$ \\
\hline CSN $4-8-6$ & $3.41 E-04$ & i. $05 E-02$ & 3. $02 E+60$ & $4.36 E-12$ & $1 .: 5 E-10$ \\
\hline CRE $4-6-7$ & $1.53 \mathrm{E}-03$ & 5. 21E-02 & $1.31 E+01$ & 2.06E-12 & $3.46 E-11$ \\
\hline CRH $4-5-8$ & $3.75 E-14$ & $1.24 E-02$ & $2.00 E+01$ & $6.01 E-1 j$ & $2,42 E-11$ \\
\hline Lin $4-3-9$ & 2.39E-04 & $1.27 E-02$ & $2.70 E+01$ & $3.405-13$ & $1.86 E-11$ \\
\hline CRH $4-3-10$ & $4.43 E-04$ & $1.31 E-02$ & $4,10 E+0 \mathrm{i}$ & $4.21 E-13$ & 1.3IE-11 \\
\hline SFil $4-8-11$ & $1.02 E-04$ & $1.32 E-92$ & 5. $00 E+01$ & $7.33 E-14$ & 1. OFE-!! \\
\hline CRH $4-8-12$ & 1. $0125-04$ & $1.3 j E-v 2$ & $6.30 E+0 i$ & 4. SEE-14 & 3. $22 E-! 2$ \\
\hline Cat 4-8-13 & 4. $69 E-14$ & $1.37 \varepsilon-0.2$ & $9.20 \mathrm{ve}+0 \mathrm{i}$ & $1.7 \mathrm{TE}-1.3$ & $\therefore, 41 E-12$ \\
\hline 证 4-8-14 & $1.705-044$ & 1.39E- 62 & $1.22 E+02$ & 4.30E-14 & $4.76=-12$ \\
\hline ERN $4-8-15$ & $2.73 E-614$ & 1. $42 E-02$ & $1.34 E+02$ & $8.265-13$ & $4.8 \div E-12$ \\
\hline CRan 4-6-16 & $3.75 E-64$ & 1. $45 E-82$ & 1. $62 E+62$ & $7.355-14$ & $3.645-1: 2$ \\
\hline 2A1 $4-8-17$ & ร.79ะ-104 & $1.51 E-\hat{0} 2$ & 3. $02 E+02$ & 0.75E-14 & $2,37 E-12$ \\
\hline
\end{tabular}


TABLE B.3f. Fluoride (F) Leach Fractions from ANS 16.1 Leach Test--Whole Grout in Groundwater (DIFF4-8)

\begin{tabular}{|c|c|c|c|c|c|}
\hline SARPPLE & An/ÂO & EUn. An/ÂO & TIME days & $D$ inc. & 0 cus. \\
\hline 쥰 4-8-1 & $1.03 E-04$ & $1.03 E-04$ & $3.00 E-04$ & $9.60 E-06$ & $9.60 E-06$ \\
\hline CAM $4-8-2$ & 6.19E-04 & $7.23 E-04$ & Q.33E-02 & I. IZE-II & $1.96 E-11$ \\
\hline CRH 4-8-3 & 4.65E-04 & $1.19 E-03$ & 2. $.92 E-01$ & $1.07 E-11$ & $1.51 \mathrm{E}-11$ \\
\hline CAN $4-8-4$ & $1.50 E-\hat{0} \mathbf{J}$ & $2.68 E-03$ & 1. ONEE+00 & $3.31 E-11$ & 2.25E-1j \\
\hline Сลㄱ 4-8-5 & $1.29 E-03$ & $3.37 E-03$ & $2.10 \mathrm{E}+00$ & $2.58 E-11$ & 2.jJ5E-11 \\
\hline CRH 4-8-6 & $4.65 E-04$ & $4,44 E-03$ & $3.022 E+00$ & $8.10 E-12$ & 2.04E-11 \\
\hline CRH $4-8-7$ & 1.45E-03 & $5.38 \mathrm{E}-0 \mathrm{~J}$ & $1.31 E+01$ & $1.85 E-12$ & $8.26 E-12$ \\
\hline CAN 4-8-6 & $4.13 E-04$ & $6.30 E-03$ & $2.00 E+01$ & $7.29 E-13$ & $6.20 E-12$ \\
\hline CRH 4-8-9 & $2.06 E-04$ & $6.50 \mathrm{E}-0.3$ & $2.70 E+01$ & $2.54 E-13$ & 4. $90 E-12$ \\
\hline Cกิ 4-8-10 & $4.13 E-04$ & $6.92 E-0 J$ & 4.10E+(11) & $3.66 E-13$ & $3.65 E-12$ \\
\hline$C R H 4-B-11$ & $5.16 E-05$ & $6.97 \mathrm{E}-0 \mathrm{~J}$ & $5.00 E+01$ & 1.67E-14 & $3,64 E-12$ \\
\hline Can $4-8-12$ & $5.16 E-65$ & $7.02 E-03$ & b. $30 E+01$ & 1.:IE-I4 & $2.45 E-: 2$ \\
\hline CFil $4-6-13$ & $5.16 E-05$ & $7.07 E-0 J$ & $9.20 E+01$ & $.3 .05 E-15$ & $1.70 \mathrm{ve}-12$ \\
\hline Cละ $4-3-14$ & $8.77 E-04$ & $7.95 E-03$ & $1.22 E+0 z$ & $1.14 E-12$ & $1.62 \mathrm{E}-12$ \\
\hline CRH 4-8-15 & $8.52 E-04$ & $8.80 E-03$ & 1. $34 E+02$ & 3. 66E-12. & $1.81 E-12$ \\
\hline Crit 4-5-16 & $8.26 E-04$ & $9.63 E-03$ & $1.82 E+02$ & $3.57 E-13$ & 1.59E-12 \\
\hline CRU $4-8-17$ & $1.16 E-02$ & $2.12 E-02$ & $3.02 E+62$ & 2.79E-11 & 4. $67 E-12$ \\
\hline
\end{tabular}

TABLE B.3g. Nitrate. (NO3) Leach Fractions from ANS 16.1 Leach Test--Whole Grout in Groundwater (DIFF4-8)

\begin{tabular}{|c|c|c|c|c|c|}
\hline SARPLE & An/Ao & CUA, An/AO & TIME days & inc. & O cu. \\
\hline$C_{n} n$ 4-B-1 & $3.32 E-03$ & $3.32 \mathrm{E}-\mathrm{6} 3$ & 3. $00 \mathrm{E}-04$ & $9.96 \mathrm{E}-03$ & $9.365-03$ \\
\hline CRV 4-9-2 & $1.55 E-02$ & $1.68 E-02$ & 8.J3E-02 & $9.05 E-09$ & 1. $33 E-6 B^{\circ}$ \\
\hline CAH 4-8-3 & $6.65 E-13$ & $2.555-02$ & $2.92 E-01$ & $2.15 E-09$ & 6. $37 E-69$ \\
\hline CAN 4-8-4 & $8.67 \varepsilon-03$ & $3.44 E-02$ & 1.00E+00 & $1.16 E-09$ & $3.69 E-09$ \\
\hline CRY 4-8-5 & $1.33 E-02$ & $4.77 E-02$ & $2.10 E+00$ & $2.74 E-09$ & $3.38 E-099$ \\
\hline CAL \&-8-6 & $2.22 E-43$ & $4.99 E-\hat{v} 2$ & $3.02 E+100$ & $3.84 E-10$ & $2.56 E-09$ \\
\hline $\mathrm{CAN} 4-9-7$ & $3.10 E-02$ & $8.09 E-02$ & $1.31 E+01$ & 8. 5.5E-10 & $1.5 \overline{F E}-0 \bar{i}$ \\
\hline CFin $4-8-8$ & $7.36 E-03$ & B. $37 E-02$ & $2.00 E+0 i$ & $2.57 E-10$ & $1.2 \mathrm{BE}-6 \mathrm{q}$ \\
\hline CRH $+-8-9$ & $4.43 E-63$ & $9.31 E-62$ & $2.70 E+01$ & $1.17 E-10$ & $1.00 E-69$ \\
\hline CRH $\div-5-10$ & i. $85 E-02$ & 1.12E-01 & $4.10 E+01$ & $7.62=-10$ & $9.56 \mathrm{E}-10$ \\
\hline Cאू⿻ 4-8-1! & $5.54 E-03$ & :. I7E- 01 & $5.00 E+61$ & $2.15 E-10$ & $3.6 \div E-10$ \\
\hline टरू 4-o-12 & 1. TIE-02 & 1. J5E-01 & b. JûEtọ! & $1.31 E-39$ & $\overline{3} .06 E-16$ \\
\hline CAil $4-5-1 j$ & 1. $38 E-02$ & 3.54E-0! & $9.20 E \div 01$ & $4.66 E-10$ & $8.07 E-10$ \\
\hline Sid 4-8-14 & $5.54 E-93$ & $1.605-01$ & $1.22 E+192$ & 4.55E-i! & j. $53 E-10$ \\
\hline Sny 4-8-15 & $7.76 E-03$ & i. $67 \mathrm{E}-0 \mathrm{~S}$ & $1.345+012$ & $6.655-10$ & $6.54 E-10$ \\
\hline Coil $4-5-16$ & $9.7 \pm-03$ & $1.77 \mathrm{E}-0 \mathrm{i}$ & $1.825+i j 2$ & $5.2: E-11$ & $5.415-16$ \\
\hline Sifit $4-8-17$ & $4.32 E-02$ & $2.21 E-01$ & $3.62 E+02$ & $3.07 E-10$ & $5.55-10$ \\
\hline
\end{tabular}


TABLE B.3i. Iodine-125 (I-125) Leach Leach Fractions from ANS 16.1 Leach Test--Whole Grout in Groundwater (4-8)

\begin{tabular}{|c|c|c|c|c|c|c|c|}
\hline SAMPLE & $u C i$ & : LEACHED & TIME DAYS & An/Ao & CUn AN/AO & D inc. en2/sec & $D$ cue ca $2 / 5 e c$ \\
\hline CRH 4-8-1 & $3.39 E-02$ & $0.369 \%$ & 3.00E-04 & $3.69 E-03$ & 3. $69 E-03$ & $1.01 E-07$ & $1.37 \mathrm{E}-0 \mathrm{0})$ \\
\hline CRH 4-8-2 & $2.46 E-01$ & $3.036 \%$ & 3. $33 E-02$ & $2.67 E-02$ & $3.04 E-02$ & $2.91 E-08$ & $3.335-08$ \\
\hline CRH 4-8-3 & $1.27 \mathrm{E}-01$ & $4.420 \%$ & $2.92 E-01$ & $1.38 E-62$ & $4.42 E-02$ & $9.08 E-119$ & $2.01 \mathrm{E}-1$ \\
\hline CAN $4-8-4$ & 2.01E-01 & $6.004 \%$ & $1.00 E+00$ & $2.18 E-02$ & 6. SÚE-012 & $6.80 \mathrm{E}-09$ & $1 . j: E-$ \\
\hline CRH 4-8-5 & $1.21 E-01$ & $7.922 \%$ & $2.10 E+00$ & $1.32 E-02$ & $7.92 \mathrm{E}-0.2$ & $2.59 E-09$ & $8.99 E-6$ \\
\hline CRU $4-B-6$ & $4.55 E-02$ & 3.4162 & $3.02 E+100$ & $4.94 E-03$ & $8.92 E-02$ & $8.81 E-10$ & $7.06 E-09$ \\
\hline CAH :-3-7 & $4.37 E-01$ & 13.1702 & $1.31 E+01$ & $4.75 E-02$ & $1.32 E-61$ & $1.935-09$ & j.99E-0 \\
\hline CRI $4-8-8$ & $1.08 E-01$ & $14.342 \%$ & $3.00 E+01$ & $1,17 E-02$ & $1.43 E-0 i$ & $5.665-10$ & $3.10 E-09$ \\
\hline CNE 4-8-9 & $7.55 E-1.2$ & 15.1652 & $2.70 E+01$ & B. $23 E-03$ & $1.52 E-01$ & $3.89 E-10$ & $2.56 E-1$ \\
\hline CRH $4-8-10$ & $1.28 E-011$ & 16.5554 & 4. $10 E+01$ & $1.39 E-02$ & $1.66 E-1) 1$ & $3.99 E-10$ & 2.01E- \\
\hline CFI $+-B-11$ & $1.31 E-02$ & $16.697 \%$ & $5.00 E+01$ & $1.43 E-03$ & $1.67 E-01$ & 1.37E-11 & $1.68 E-$ \\
\hline CRH $\{-8-12$ & $1.63 E-02$ & 16.875\% & 6. $40 E+01$ & $1.78 \mathrm{E}-03$ & $1.69 E-01$ & $1.10 E-11$ & $1 . j 4 E-$ \\
\hline CRH $4-8-13$ & $7.23 E-12$ & 17.6635 & $9.20 E+01$ & $7.88 E-03$ & $1.77 E-01$ & $7.37 E-11$ & 1.02E-09 \\
\hline CSN $4-E-14$ & $4.92 E-02$ & $18.198 \%$ & $1.22 \mathrm{E}+12$ & $5.35 E-03$ & $1.82 E-001$ & $\{.07 E-11$ & 3. $17 E-10$ \\
\hline$C R+\div-8-15$ & $1.50 E-02$ & 18.3624 & $1.34 E+02$ & $1.65 E-03$ & 1. $34 E-01$ & $2.90 \mathrm{E}-11$ & $7.57 E-10$ \\
\hline CRN 4-6-16 & $6.28 E-62$ & $19.047 \%$ & $1.82 E+02$ & $6.85 E-03$ & $1.90 E-01$ & $3.85 E-11$ & a. $00 E-1$ \\
\hline
\end{tabular}

TABLE B.3i. Iodine-125 (I-125) Leach Leach Fractions from ANS 16.1 Leach Test--Whole Grout in Groundwater (4-9)

\begin{tabular}{|c|c|c|c|c|c|c|c|}
\hline SAMPLE & $\mathrm{UCi}$ & \% LEACHED & TIME DAYS & - An/Â & Cun An/Ao & $D$ inc. inliser & 3 cun co2iser \\
\hline CEN $4-9-1$ & $3.42 E-02$ & $0.364 \%$ & $3.00 E-04$ & $3.64 E-03$ & $3.64 E-103$ & $9.80 E-06$ & 1. J3E-017 \\
\hline Con $4-\{-2$ & 2.13E-01 & $2.630 \%$ & B. $33 E-02$ & $2.27 E-02$ & $2.63 \mathrm{E}-022$ & $2.10 E-08$ & 2.50E-0B \\
\hline Cof $4-9-3$ & $5.87 E-01$ & 3.877\% & $2.925-(11$ & $6.25 E-02$ & 8. $3 B E-62$ & 1. $85 E-07$ & 8. $12 E-018$ \\
\hline Gain 4-9-4 & $2.07 E-011$ & $11.061 \%$ & $1.00 E+00$ & $2.20 E-02$ & $3.11 E-6 !$ & $6.72 E-19$ & $3.70 E-08$ \\
\hline Cfin 4-?-5 & $1.50 E-01$ & $12.678 \%$ & $2.10 E+00$ & $1.60 E-02$ & $1.275-61$ & $3.81 E-09$ & $2.30 E-08$ \\
\hline $\operatorname{Con} 4-3-6$ & $7.575-0_{2}$ & $13.465 \%$ & $3.02 E+00$ & $8.075-03$ & $1.35 E-01$ & 2.35E-09 & $1.51 E-18$ \\
\hline CRH 4-9-i & J. 04E-01 & 18. BE6\% & $1.31 E+01$ & $5.37 E-02$ & $1.89 E-01$ & $2,46 E-09$ & 3.16E- 09 \\
\hline CRN $+-7-8$ & $1.51 E-011$ & $20.463 \%$ & $2.00 E+01$ & $1.61 E-02$ & $2.05 E-61$ & 1.06E-09 & 6.30E-09 \\
\hline CRU $4-i-9$ & $1.02 E-01$ & $21.550 \%$ & $2.70 E+01$ & 1.095-02 & $2.16 E-01$ & 6.7AE-10 & $5.79 E-19$ \\
\hline Crid $4-;-10$ & $\vdots .68 E-01$ & $23.340 \%$ & $4.10 E+0 i$ & $1.79 E-62$ & $2.33 E-6 !$ & $0.62 E-10$ & $4.14 E-0 ?$ \\
\hline Can 4-?-11 & $1.83 E-02$ & 23.5374 & $5.60 E \div 01$ & $4.96 E-03$ & $2.355-01$ & $2 . \dot{B D E}-11$ & $3.74 E-09$ \\
\hline CAH $\{-\{-12$ & $2.40 E-12$ & $23.794 \%$ & $6.40 E+61$ & $2.59 E-63$ & $2.38 E-01$ & $2.3 \mathrm{BE}-11$ & $3.215-09$ \\
\hline CลN 4-9-13 & $9.33 E-02$ & $24.790 \%$ & $9.20 E+01$ & $9.96 E-03$ & $3.46[-i]$ & $1.10 E-10$ & $2.23 E-4) 4$ \\
\hline [RH $4-9-14$ & $5.51 E-12$ & $25.376 \%$ & $1.23 E+02$ & $5.66 E-0 j$ & 2.54E- $51 \mathrm{i}$ & $4.93 E-11$ & $1.84 E-\sqrt{19}$ \\
\hline Cก) $4-9-15$ & 1. 18E-02 & $25.504 \%$ & $1.345 \div 02$ & $1.26 E-03$ & 2.55t-01 & $2.67 E-11$ & 1. $22 E-69$ \\
\hline Crith 4-9-10 & $4.4 ! E-6 \mathrm{j} 2$ & $25.973 \%$ & a.A2E+A2 & $4.67[-0]$ & $2.60 E-4 i$ & 1.5iE-ii & $2 .: 0 E-67$ \\
\hline 609 4-9-17 & $3.0 G E-02$ & $30.024 \%$ & S. OAZE+oi & O. $51 E-j 3$ & $2.60 E-01$ & $5.44 E-1 !$ & 1. VEE- 09 \\
\hline
\end{tabular}


TABLE B.4a. Potassium (K) Leach Fractions from ANS 16.1 Leach Test--Whole Grout in Groundwater (DIFF5-5)

\begin{tabular}{|c|c|c|c|c|c|}
\hline SAMPLE & An/AO & Cun. Anjita & TIKE days & D ins. & 9 cus. \\
\hline ERK 5-5-i & $-4.33 E-63$ & $-4.33 E-63$ & 3. ODE-64 & 1. ÓYE-02 & 1.69E-12 \\
\hline CRH $5-5-2$ & $9.615-03$ & $5.29 E-03$ & 3. $335-02$ & $3.47 E-69$ & $1.05 E-09$ \\
\hline Cinl 5-5-3 & $6.25 E-03$ & i. $15 E-02$ & $2.72 E-\hat{v} !$ & 1.9JE-09 & $1.43 E-69$ \\
\hline CRH 5-5-4 & $2.02 E-02$ & $3.17 E-02$ & $1.60 E+100$ & $6.63 E-09$ & $3.15 E-69$ \\
\hline LRH 5-5-5 & $1.54 E-02$ & $4.71 E-02$ & 2. $10 E+60$ & 3.67E-09 & $3.3 ! 5-19$ \\
\hline Cก́H $5-5-6$ & $1.06 E-12$ & $5.77 E-02$ & $3.02 E+00$ & $4.20 E-09$ & $3.45 E-199$ \\
\hline CRE $5-5-7$ & $6.835-0 ?$ & $1.20 E-01$ & $1.31 E+01$ & $4.13 E-09$ & $3.795-09$ \\
\hline CRH $5-5-6$ & 2. $98 E-92$ & $1.56 E-01$ & $2.00 E+0 !$ & $3.5(E E-6)$ & $3.205-69$ \\
\hline CRH 5-5-9 & $2.02 E-02$ & $1.76 E-01$ & $2.70 E+01$ & $2.43 E-09$ & $3.395-0 \%$ \\
\hline CAH $5-5-10$ & 3. 4óE-ij2 & 2. $\lfloor\lfloor E-0\rfloor\rfloor$ & 4.10E+111 & $2.57 \pm-09$ & 3. ESE-09 \\
\hline Can 5-5-11 & $1.06 E-0.2$ & $2.2|E-\hat{v}|$ & $5.00 E+01$ & $7.84 E-i 6$ & $3.35 E-09$ \\
\hline CRin $5-5-12$ & $1,01 E-62$ & $2.3 \mathrm{EE}-01$ & $6.305+01$ & $4.25 E-10$ & $2.375-69$ \\
\hline CRH 5-5-13 & $2.98 E-62$ & $2.61 E-01$ & $7.20 E+01$ & $1.02 E-09$ & $2.57 E-69$ \\
\hline 5.⿲丿丨乚 5-5-14 & $1.9 \angle \mathcal{E}-02$ & $2.00 E-\hat{O} 1$ & $1.22 E+02$ & $5.87 E-10$ & $2.27 E-69$ \\
\hline [KH 5-5-15 & $1.97 E-02$ & J.OAEE-OI & $1.34 E+0 \hat{2}$ & $4.32 E-69$ & $2.42 E-69$ \\
\hline Crii 5-5-16 & $2.02 E-02$ & $3.20 E-01$ & 1. $22 E+\hat{v} 2$ & $2.135-10$ & 2. $06 \mathrm{E}-09$ \\
\hline CRH 5-5-1T & $3.74 E-6 \underline{z}$ & $3.48 E-01$ & $3.62 E+02$ & 1.55E-10 & $1.52 E-69$ \\
\hline
\end{tabular}

TABLE B.4b. Sodium (Na) Leach Fractions from ANS 16.1 Leach Test--Whole Grout in Groundwater (DIFF5-5)

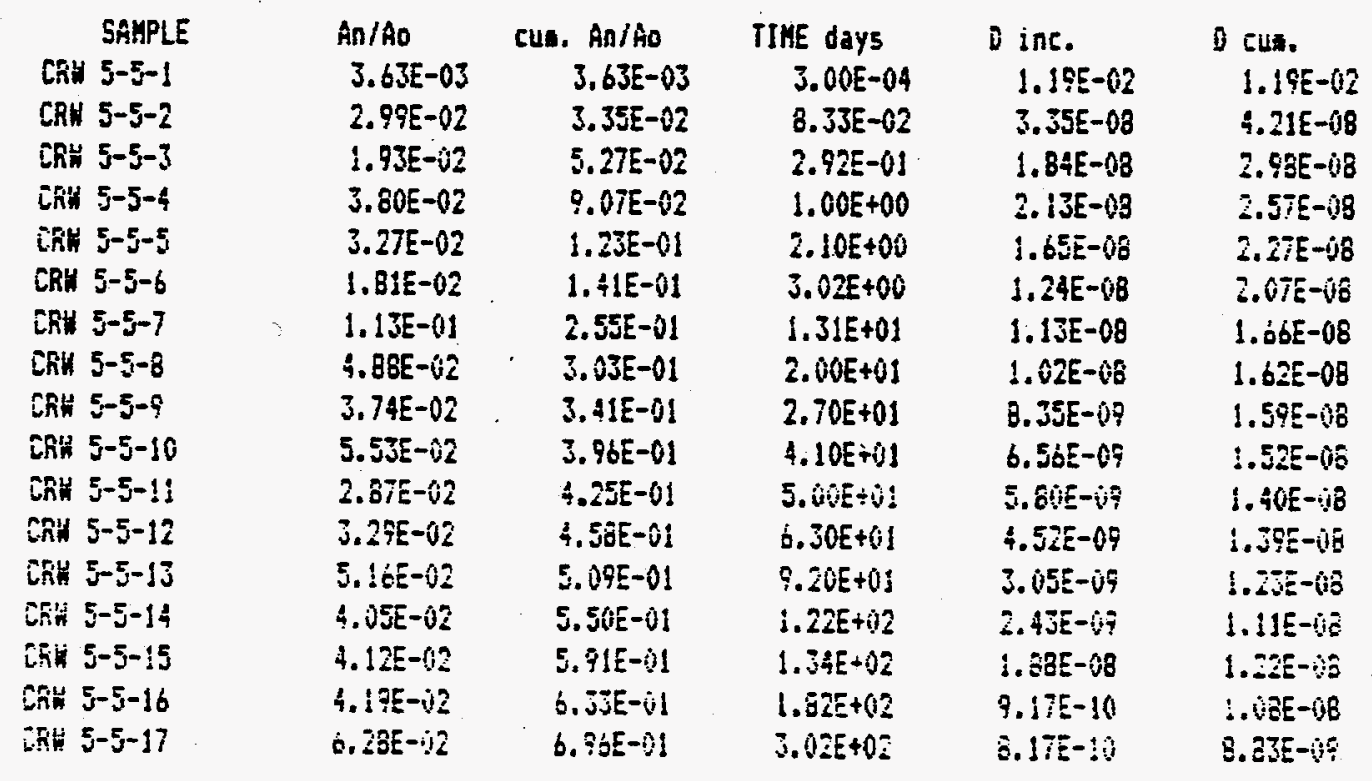


TABLE B.4e. Boron (as H3B03) Leach Fractions from ANS 16.1 Leach Test--Whole Grout in Groundwater (DIFF5-5)

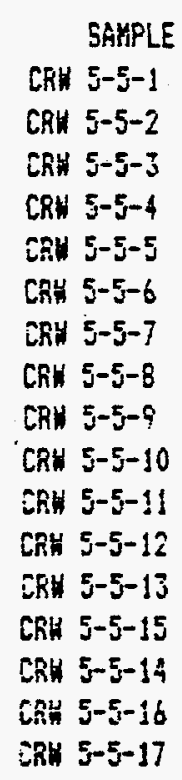

An/AO
$8.55 E-04$
$3.04 E-03$
$1.46 E-03$
$2.37 E-03$
$1.39 E-03$
$7.91 E-04$
$3.01 E-03$
$6.01 E-04$
$4.12 E-04$
$3.23 E-04$
$2.22 E-04$
$2.53 E-04$
$5.38 E-04$
$3.80 E-04$
$2.22 E-04$
$3.4 B E-04$
$7.60 E-04$

$\begin{array}{lr}\text { EU. An/AO } & \text { TIhE JaYs } \\ 8.55 E-04 & 3.00 E-04 \\ 3.89 E-03 & 8.33 E-02 \\ 5.35 E-03 & 2.72 E-01 \\ 7.72 E-03 & 1.00 E+00 \\ 9.12 E-03 & 2.10 E+00 \\ 9.91 E-03 & 3.02 E+00 \\ 1.20 E-02 & 1.31 E+01 \\ 1.35 E-02 & 2.00 E+01 \\ 1.39 E-02 & 2.70 E+01 \\ 1.48 E-02 & 4.10 E+01 \\ 1.50 E-02 & 5.00 E+01 \\ 1.52 E-02 & 6.30 E+01 \\ 1.56 E-02 & 9.20 E+01 \\ 1.61 E-02 & 1.22 E+02 \\ 1.64 E-02 & 1.34 E+02 \\ 1.67 E-02 & 1.32 E+02 \\ 1.75 E-02 & 3.02 E+02\end{array}$

$D$ inc. 6.50E-04

$3.47 \mathrm{E}-10$

$1.05 E-10$

8. $34 E-11$

3. $01 E-11$

2.35E-11

$8.02 E-12$

$1.55 E-12$

1. O1E-12

1. $46 E-12$

3. $44 E-13$

2. $63 \mathrm{E}-1 \mathrm{j}$

$3.31 E-13$

2.14E-13

5. $46 E-13$

6. JSE-14

1. $205-13$
D cus.

$6.58 E-14$

5. $70 E-10$

3.07E-10

1. $.37 E-10$

$1.24 E-1 \hat{v}$

1. $02 E-10$

$3.995-11$

2. $96 \mathrm{E}-11$

2. 25E-Ii

$1.665-11$

1. 40E-11

!. 15E-!1

6. $45 E-12$

6. 6 SE-:2 2

$6.26 E-12$

4. $80 \mathrm{E}-12$

$3.16 E-12$

TABLE B.4f:

Fluoride (F) Leach Fractions from ANS

16.1 Leach Test--Whole Grout in Groundwater (DIFF5-5)

\begin{tabular}{|c|c|c|c|c|c|}
\hline SAMPLE & $A n / A D$ & 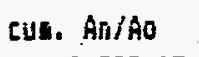 & TIME days & $D$ inc. & 8 cus. \\
\hline Cris . 5-5-1 & $9.59 E-05$ & $9.595-05$ & $3.00 E-04$ & $8.29 E-06$ & $3.29 E-16$ \\
\hline CRH $5-5-2$ & $4.80 E-04$ & 5. $75 E-\hat{A} 4$ & 8.J3E-02 & $8.64 E-12$ & $1.24 E-11$ \\
\hline CEH 5-5-3 & $4.32 E-64$ & $1.01 E-03$ & $2.92 E-0 \mathrm{i}$ & $9.22 E-12$ & 1. OFE-1! \\
\hline Cin 5-5-4 & $1.53 E-03$ & $2.54 E-03$ & $1.00 E \div 00$ & $3.48 E-i 1$ & 2. $02 E-11$ \\
\hline CAn $5-5-5$ & $1.63 E-03$ & $4.17 E-03$ & $2.10 E+00$ & $4.12 E-11$ & 2.59E-11 \\
\hline CAN 5-5-6́ & $8.15 E-04$ & $4.99 \mathrm{E}-03$ & $3.02 E+60$ & 2.50E-11 & $2.58 E-11$ \\
\hline Cñ 5-5-7 & $2.40 E-03$ & $7.38 E-0.3$ & $1.31 E+0 !$ & $5.16 E-12$ & $1.30 E-1:$ \\
\hline CFU 5-5-8 & $8.15 E-04$ & $8.20 E-03$ & $2.00 E+0 i$ & $2.84 E-1 \overline{6}$ & 1. $65 E-11$ \\
\hline Crint 5-5-9 & 4. $80 E-04$ & $8.68 E-03$ & 2.7ikE+61 & $1.37 E-12$ & $8.73 E-12$ \\
\hline CRU 5-5-10 & $7.19 E-04$ & $7.40 E-13$ & $4.10 E+61$ & 1.11E-12 & $6.74 E-12$ \\
\hline CRU $5-5-11$ & 1.32E-04 & 9.59E- 03 & 5. 00E+0ij & $2.560-3$ & 5. $76 E-15$ \\
\hline Cink $5-5-12$ & $-3.59 E-15$ & $\hat{i} .49 E-\hat{0} 3$ & 6.30E+31 & $3.84 E-14$ & $4.46-12$ \\
\hline 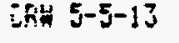 & $2.46 E-014$ & $7.73 \mathrm{E}-0 \mathrm{3}$ & $9.20 E+01$ & s.5TE-13 & $3.225-12$ \\
\hline CN14 5-5-14 & $1.25 E-63$ & $1.10 \mathrm{E}-32$ & !.22E+62 & Z. B.WE-!? & $5.69 E-12$ \\
\hline $2645-5-15$ & S. $35 E-34$ & $\therefore .17 E-12$ & $1.34 E+0$. & 5. $30 E-12$ & $3.15 E-12$ \\
\hline CRH 5-5-ió & $i .44 E-i .4$ & 1. $1 B E-42$ & $1 . \overline{8} 25+12$ & $\therefore .662-14$ & $2.40 E-12$ \\
\hline Zain 5-5-17 & $2.88 E-34$ & $1.21 E-02$ & J.0.EE+12 & 3.TIE-14 & L.5.5- 12 \\
\hline
\end{tabular}


TABLE B.4g. Nitrate (NO3) Leach Fractions from ANS 16.1 Leach Test--Whole Grout in Groundwater (DIFF5-5)

\begin{tabular}{|c|c|c|c|c|c|}
\hline $\begin{array}{l}\text { SAHPLE } \\
\text { CRU } 5-5-1\end{array}$ & $\begin{array}{l}\text { An/AO } \\
2.06 E-03\end{array}$ & $\begin{array}{l}\text { CUL. An/AO } \\
2.06 E-03\end{array}$ & $\begin{array}{l}\text { TIME day5 } \\
3.00 E-04\end{array}$ & $\begin{array}{l}\text { Dinc. } \\
3.22 E-03\end{array}$ & $\begin{array}{l}0 \text { clid. } \\
3.82 E-03\end{array}$ \\
\hline CRK $5-5-2$ & $1.03 E-02$ & $1.24 E-02$ & $8.33 E-02$ & $3.98 E-09$ & $5.74 E-09$ \\
\hline CAn 5-5-3 & $6.13 E-03$ & $1.855-62$ & 2.92E-0i & 1. $67 E-09$ & $3.69 E-69$ \\
\hline CRI 5-5-4 & $1.03 E-102$ & $2.88 E-102$ & $1.00 E+00$ & $1.57 E-09$ & 2. $60 E-79$ \\
\hline Crill 5-5-5 & $7.21 \mathrm{E}-03$ & $3.60 E-02$ & $2.10 E+00$ & 3. $06 E-10$ & $1.74 E-v 9$ \\
\hline CAL 5-5-6 & $4.12 E-03$ & $4.02 E-02$ & $3.02 E+00$ & $6.37 E-10$ & 3.67E-i9 \\
\hline CRH 5-5-7 & $2.99 E-02$ & $7.00 \mathrm{E}-02$ & $1.3 j E+0 i$ & $7.90 E-1 \hat{0}$ & $1.175-09$ \\
\hline CRN 5-5-8 & $1.24 E-02$ & $6.24 E-02$ & $2.00 E+01$ & $5.53 E-1 \hat{v}$ & 1.06E-0.9 \\
\hline CRH $5-5-9$ & 1. $13 E-02$ & $9.37 E-02$ & $2.70 E+01$ & $7.66 \mathrm{E}-10$ & $-1.02 E-69$ \\
\hline Cne 5-5-10 & $2.68 E-12$ & $1.20 E-01$ & $4.10 E+01$ & $1.54 E-39$ & 1. $11 E-69$ \\
\hline CRH 5-5-11 & $7.2 \mathrm{E}=-03$ & i. 2BE-0! & $5.00 E+01$ & $3.64 E-10$ & $1.025-69$ \\
\hline CFu 5-5-12 & $1.03 E-02$ & $1,38 E-01$ & $6.30 E+01$ & $4.42 E-10$ & $9.46 E-10$ \\
\hline ChA 5-5-13 & $3.65 E-62$ & $2.24 E-01$ & $9.20 E+01$ & 3.55E-07 & $1.82 E-09$ \\
\hline CRH 5-5-14 & $8.24 E-3 j$ & $2.33 E-01$ & $1.22 E+02$ & $1.005-10$ & $1.48 E-49$ \\
\hline Cfil 5-5-15 & $1.08 E-02$ & $2.44 E-0 !$ & $1.34 E \div 02$ & $1.30 E-09$ & 1. $4 C E-6 ?$ \\
\hline CRH $5-5-16$ & $1.34 E-02$ & $2.57 E-01$ & 1. $B 2 E+02$ & $9.36 E-1 i$ & 1.30E-09 \\
\hline CRW $5-5-17$ & $5.05 E-02$ & $3.07 E-01$ & $3.02 E+02$ & $5.2 j E-10$ & 1.15E-49 \\
\hline
\end{tabular}

\section{TABLE B.4i. Cesium-137 (CS-137) Leach Fractions from ANS 16.1 Leach Test-- Whole Grout in Groundwater (5-5)}

\begin{tabular}{|c|c|c|c|c|c|c|c|}
\hline SAMPLE & $\mathrm{u}[\mathrm{i}$ & I LEACHED & TIME DAYS & $A n / A D$ & CUE AR/AO & $D$ inc. [A2/5es & i) cus coz $/ \mathrm{sec}$ \\
\hline CRH 5-5-1 & $0.00 E+00$ & $0.000 \%$ & 3.00E-04 & B. $36 E-09$ & 8.36E-09 & 5. $38 E-19$ & 7.30E-19 \\
\hline CRU $5-5-2$ & $0.00 E+00$ & $0.000 \%$ & 8. 33E-02 & $8.365-09$ & $1.67 E-63$ & 2. กTE-2! & $1.05 E-20$ \\
\hline CRH $5-5-3$ & $0.60 E+00$ & $0.000 \%$ & $2.925-01$ & $8.36 E-09$ & $2.51 E-0.8$ & $3.45 E-21$ & $6.75 E-21$ \\
\hline CFI $5-5-4$ & $1.01 E-03$ & $0.031 \%$ & $1.00 E+00$ & $3.13 E-\hat{04}$ & 3. $13 E-04$ & $1.45 E-12$ & $3.065-13$ \\
\hline Can 5i-5i-5 & 0. 00 E +00 & $0.031 \%$ & $2.10 E+00$ & $8.36 E-09$ & $3.13 E-04$ & $1.05 E-21$ & $1.46 E-15$ \\
\hline CRn 5-5-6 & $0.00 E+60$ & $0.032 \%$ & $3.02 E+00$ & $2.53 E-06$ & 3.16E-04 & $2.40 E-16$ & $1.03 E-13$ \\
\hline CRH 5-5-7 & $0.00 E+00$ & 0.0321 & $1.31 E+01$ & $8.36 E-09$ & $3.16 E-04$ & $6.20 E-2 j$ & $2.38 E-14$ \\
\hline CAn 5-5-5 & $0.00 E+100$ & $0.032 \%$ & 2. $00 E+01$ & $5.35 E-07$ & $3.165-04$ & $1.23 \mathrm{E}-18$ & $1.56 E-14$ \\
\hline LRH 5-5-9 & $0.00 E+00$ & j.032\% & $2.70 E+01$ & $6.11 E-06$ & 3. .2E- -194 & $2.23 E-16$ & 1.20E-14 \\
\hline CRH $5-5-10$ & J. $60 E+00$ & $0.032 \%$ & $4.10 E+0 \mathrm{i}$ & $8.36 E-69$ & $3.22 E-54$ & $1.50 E-22$ & i. $52 E-15$ \\
\hline CRU 5-5-11 & $0.00 E+100$ & $0.032 \%$ & $\Sigma, 60 E+01$ & $8.36 E-69$ & $3.22 E-04$ & $4.71 E-22$ & b.50E-15 \\
\hline Ent $5-5-12$ & $0.00 E+000$ & 0.0322 & 6. $40 E+01$ & a. 3aE-A? & $3.22 E-34$ & $2.54 E-22$ & $5.08 E-15$ \\
\hline Lofin 5-5-13. & $0.00 E+00$ & $0.032 \%$ & $9.20 E \div 01$ & 6.3oE-99 & $3.225-04$ & $3.64 E-2 j$ & $3.53 E-15$ \\
\hline She 5-5-14 & $0.00 E+00$ & $0.032 \%$ & $1.22 E+112$ & $3.36 E-10$ & 3.22E-144 & $1.04 E-24$ & $2.6 \leq 5-15$ \\
\hline Con $5-5-15$ & $0.00 E+00$ & 0.0324 & $1.34 E+62$ & $1.69 E-356$ & $3.24 E-34$ & $3 .: 7 E-17$ & $2.45 E-15$ \\
\hline Con $5-5-16$ & $5.002+60$ & $0.032 \%$ & $1.22 E+0.2$ & $1.05 E-06$ & $3.255-64$ & 3. $37 E-1 ?$ & 1..22E-15 \\
\hline
\end{tabular}


TABLE B.4i. Cesium-137 (CS-137) Leach Fractions from ANS 16.1 Leach Test-Whole Grout in Groundwater (5-14)

\begin{tabular}{|c|c|c|c|c|c|c|c|}
\hline SAYYPLE & $\mathrm{uCi}$ & ¿ LEACHED & IIME DAYS & $\hat{A} \cap / A_{0}$ & EUE Ân/ÂO & inc. cazisec & 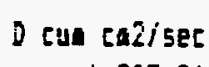 \\
\hline CRH $5-14-1$ & $0.00 E+00$ & $0.000 \%$ & $3.00 E-04$ & $3.04 E-10$ & $8.04 E-10$ & $5.09 E-21$ & $6.90 E-21$ \\
\hline CFin $5-14-2$ & $0.00 E+00$ & $0.000 \%$ & $8.33 E-02$ & $8.04 E-10$ & $1.61 E-09$ & $2.81 E-23$ & $9.95 E-23$ \\
\hline CRH $5-14-3$ & $5.18 E-05$ & $0.002 \%$ & $2.32 E-01$ & $1.51 E-05$ & $1.52 E-015$ & $1.16 E-14$ & $2.52 \mathrm{E}-$ \\
\hline CRM 5-14-4 & $0.00 E+10$ & 0.0027 & $1.00 E+00$ & $8.04 E-10$ & 1.52E-05 & $9.805-24$ & $7.35 E-$ \\
\hline DRK $5-14-5$ & $1.32 E-04$ & $0.005 \%$ & $2.10 E+00$ & $3.85 E-05$ & $5.37 E-05$ & $2.36 E-14$ & $4.40 \mathrm{E}-$ \\
\hline CRH 5-14-6 & $8.00 E-05$ & $0.608 \%$ & $3.02 E+00$ & $2.34 E-05$ & $7.71 E-65$ & $2.10 E-14$ & t.31E- \\
\hline CRU $5-14-7$ & $6.61 E-05$ & $0.010 \%$ & $1.31 E+01$ & 1. $93 E-05$ & $9.64 E-65$ & $3.395-16$ & $2.2 \mathrm{EE}-$ \\
\hline$C R^{2} N 5-14-B$ & 5. ESE-05 & $0.011 \%$ & $2.00 E+01$ & $1.71 E-05$ & $1.14 E-64$ & 1. $2 a E-15$ & $2.06 E-15$ \\
\hline CFH $5-14-?$ & $0.00 E+00$ & $0.011 \%$ & $2.70 E+01$ & $8.04 E-10$ & 1. 14E-04 & $3.75 E-24$ & $1.53 E-$ \\
\hline CRU 5-14-10 & $0.00 E+00$ & $0.011 \%$ & $4.10 E+01$ & $5,47 E-08$ & $1.14 E-04$ & $6.57 E-21$ & $1.01 E-15$ \\
\hline CAH $5-14-11$ & $0.00 E+00$ & $0.011 \%$ & $5.00 E+01$ & $3.04 E-10$ & $1.14 E-04$ & $4.64 E-24$ & 3.27E-! \\
\hline CRH $5-14-12$ & $0.00 E+00$ & $0.011 \%$ & $6.40 E+01$ & $8.04 E-10$ & $1.14 E-04$ & $2.40 E-24$ & $0.46 E-16$ \\
\hline CRH $5-14-13$ & $7.19 E-05$ & $0.014 \%$ & $9.20 E+01$ & $2.16 E-05$ & 1.35E-64 & $5.70 E-16$ & $6.36 E-16$ \\
\hline Can $5-14-14$ & $8.01 E-05$ & $0.016 \%$ & $1.22 E+02$ & $2.34 E-05$ & $1.59 E-04$ & $3.32 E-16$ & $6.60 E-16$ \\
\hline IRH $5-14-15$ & $0.00 E \div 10$ & $0.016 \%$ & $1.34 E+02$ & $8.04 E-10$ & 1.59E-04 & $7.365-24$ & $0.01 E-1$ \\
\hline $\operatorname{con} 5-14-16$ & $0.00 E+00$ & $0.016 \%$ & 1. $82 E+02$ & $8.04 E-10$ & i.53E-114 & $5.65 E-25$ & $4.435-16$ \\
\hline
\end{tabular}

TABLE B.4j. Strontium-85 (SR-85) Leach Fraction from ANS 16.1 Leach Test-Whole Grout in Groundwater (5-5)

\begin{tabular}{|c|c|c|c|c|c|c|c|}
\hline SAMFLE & $\mathrm{uCi}$ & ¿ LEACHED & TIME DAYS & An/ÂO & CUn An/ÂO & $D$ ine. & o cua. \\
\hline CRY $5-5-1$ & $0.00 E+00$ & 0.02324 & $3.00 E-04$ & $2.22 E-\hat{0} 4$ & $2.22 E-04$ & $3.82 E+00$ & $5.12 E-10$ \\
\hline CRH $5-5-2$ & $0.005+00$ & $0.0311 \%$ & $8.33 E-03$ & 3. 99E-05 & 3.1 iE-114 & $4.62 E-12$ & $3.64 E-11$ \\
\hline CKH $5-5-3$ & $7.555-04$ & $0.2316 \%$ & $2.92 E-01$ & $2.00 E-03$ & $2.32 E-03$ & 6. $23 E-11$ & $5.75 E-11$ \\
\hline CFH $5-5-4$ & $0.00 E+00$ & $0.2479 \%$ & $1.00 \equiv+100$ & $1.635-044$ & $2.48 E-03$ & $3.95 E-13$ & $1.92 E-11$ \\
\hline 매 $5-5-5$ & $0.00 E+100$ & 0.26617 & $2.10 E+10$ & $1.82 E-04$ & 2. $66 E-03$ & 5.11E-13 & 1.66E-11 \\
\hline con 5-5-5 & $0.00 E+00$ & $0.2757 \%$ & $3.02 E+00$ & $7.62 E-65$ & $2.76 E-03$ & $3.46 E-13$ & $7.88 E-12$ \\
\hline Crin 5-5-7 & $1.29 E-63$ & $0.6899 \%$ & $1.31 E+01$ & $4.14 E-03$ & $6.70 E-03$ & 1.52E-11 & $1.14 E-11$ \\
\hline CRU 5-5-8 & $3.75 E-04$ & $1.0577 \%$ & $2.00 E+01$ & $3.68 E-0 \mathrm{j}$ & :.06E-02 & $5.795-11$ & 1. $75 E-11$ \\
\hline Lor $5-5,9$ & $1.28 E-63$ & $1.6143 \%$ & $2.7 \hat{N E}+01$ & $5.57 E-03$ & 1. DiE- 02 & ¿. $655-10$ & $3.02 E-11$ \\
\hline CNH $5-5-10$ & $0.00 E+00$ & $1.6143 !$ & $4.10 E+01$ & $1.50 E-07$ & $1.61 E-62$ & $4.84 E-20$ & $1.97 E-11$ \\
\hline CWN $5-5-11$ & $0.00 E+00$ & 1.6156\% & $5.00 E+01$ & 1.27E-05 & $1.62 E-92$ & $1.14 E-15$ & $1.53 E-! 1$ \\
\hline 动 $5-5-12$ & $0.00 E+00$ & $1.6454 \%$ & 6. $4 \hat{4} E+01$ & 2. 9PE-04 & $1.655-02$ & $3.25 E-13$ & :.32E-!1 \\
\hline DF $5-5-13$ & $9.22 \mathrm{E}-04$ & $2.06: 6 \%$ & $9.20 E+01$ & $4.16 E-0.3$ & $2.66 E-62$ & 2.IAE-11 & $1.45 E-11$ \\
\hline Ehit $5-5-14$ & $0.60 E+100$ & 2.06655 & $1.22 E+i 2$ & $6.76 E-055$ & $2.075-02$ & 5.76E-15 & 1.jOE-11 \\
\hline SRiv $5-5-15$ & $1.84 E-04$ & 2.20217 & $1.34 E+12$ & 1. $34 E-\dot{v} \overrightarrow{3}$ & $2.26-62$ & 2. & 1.:3E- 11 \\
\hline CFN $5-5-15$ & $1.25 E-64$ & $2.3655 \%$ & $1.825+02$ & $1.63 E-03$ & $2.37 E-02$ & 2. 2ลE-12 & $5.62 \mathrm{E}-12$ \\
\hline
\end{tabular}


TABLE B.4j. Strontium-85 (SR-85) Leach Fraction from ANS 16.1 Leach Test-Whole Grout in Groundwater (5-14)

\begin{tabular}{|c|c|c|c|c|c|c|c|}
\hline SAHFLE & UCj & ; LEACHED & TIME DAYS & $A n / A D$ & Cun An/Ao & $D$ ine. & 0 cua. \\
\hline CRA $5-14-1$ & $0.00 E+00$ & 0.00002 & 3. $00 E-04$ & $1.835-07$ & 1.83E-07 & $2.67 \mathrm{E}-06$ & 3.5BE-16 \\
\hline CRM 5-14-2 & $2.32 E-04$ & $0.1264 \%$ & B. 33E-03 & $1.26 E-03$ & $1.26 E-03$ & $9.35 E-10$ & 6.14E-10 \\
\hline CRI $5-14-3$ & $0.00 E+00$ & 0.14942 & $2.92 \mathrm{E}-01$ & $2.30 \mathrm{E}-04$ & $1.49 E-03$ & B. $3 B E-\{3$ & $2.45 E-11$ \\
\hline CRII 5-14-4 & $0.00 E+00$ & 0.15962 & $1.60 E+00$ & $1.03 E-04$ & 1. $60 E-03$ & i.59E-13 & $0.16 E-12$ \\
\hline CRN-5-14-5 & $1.79 E-04$ & $0.2817 \%$ & $2.10 E+00$ & $1.22 E-03$ & $2.82 \mathrm{E}-03$ & 2.J7E-11 & $1.21 \mathrm{E}-11$ \\
\hline CRH-5-14-6 & $0.00 E+00$ & 0.28172 & $3.02 E+00$ & 1.80E-07 & 2.82E-03 & $1.24 E-18$ & $8.42 E-12$ \\
\hline CAH $5-14-7$ & $1.88 E-04$ & 0.38376 & $1.31 E+01$ & i. $02 E-03$ & $3.84 E-03$ & $9.43 E-13$ & $3.60 E-12$ \\
\hline CRN $5-14-8$ & $0.00 E+00$ & $0.3837 \%$ & $2.00 E+01$ & $1.80 E-07$ & $3.84 E-03$ & $1.41 E-19$ & $2.36 E-12$ \\
\hline CFH 5 $5-14-9$ & $0.00 E+00$ & $0.3890 \%$ & $2.70 E+01$ & $5.35 E-05$ & J.89E-03 & $1.75 E-14$ & $1.80 \mathrm{VE}-12$ \\
\hline CANI 5-14-10 & 1. $43 E-04$ & 0.47062 & $4.10 E+01$ & $8.16 E-04$ & $4.71 E-003$ & $1.4 \Delta E-12$ & 1. $73 E-12$ \\
\hline CRE $5-14-11$ & $0.00 E+00$ & $0.4740 \%$ & $5.00 E+01$ & $3.43 E-05$ & $4.74 E-03$ & $8.45 E-15$ & $1.44 E-12$ \\
\hline CAN $5-1 i-12$ & $0.00 E+00$ & $0.4780 \%$ & $6.40 E+01$ & $3.98 E-05$ & 4. $3 B E-63$ & $5.56 E-15$ & $1.16 E-12$ \\
\hline CRN $5-14-13$ & $7.51 E-05$ & $0.5196 \%$ & $9.20 E+01$ & $4.16 E-04$ & $5.20 E-03$ & $2.19 E-13$ & $9.40 E-13$ \\
\hline CRE $5-14-14$ & $6.69 E-15$ & $0.5567 \%$ & $1.22 \mathrm{E}+02$ & $3.71 E-04$ & $5.57 E-03$ & $2.09 E-13$ & $8.14 E-13$ \\
\hline CRH 5-14-15 & $0.00 E+00$ & $0.5604 \%$ & $1.34 E+02$ & $3.67 E-05$ & $5.60 E-03$ & $1.53 E-14$ & $7.5 ! E-13$ \\
\hline CRN 5-14-16 & $0.00 E+100$ & 0.56042 & $1.82 E+02$ & $3.47 E-07$ & $5.60 E-63$ & 1.05E-17 & $5.53 E-13$ \\
\hline
\end{tabular}

TABLE B.5i. Americium-241 (AM-241) Leach Fractions from ANS 16.1 Leach Test-Whole Grout in Groundwater (1-8)

\begin{tabular}{|c|c|c|c|c|c|c|c|}
\hline SAMFLE & $\mathrm{UCi}$ & ¿ LEACHED & TIKE DAYS & Anis/Ao & CuL An/AO & D inc. car $2 / 5 e c$ & D cun caźisec \\
\hline CAN $1-8-1$ & $1.03 E-04$ & 0.0016 & $3.00 E-04$ & $1.11 E-05$ & $1.11 E-05$ & $9.29 E-\{3$ & $1.26 E-12$ \\
\hline CRIN $1-8-2$ & $1.58 E-04$ & 0.0027 & 8.33E-02 & $1.24 E-05$ & $2.36 \mathrm{E}-0 \mathrm{v5}$ & $6.43 E-15$ & $2.04 E-14$ \\
\hline CRN $1-3-3$ & $8.21 E-05$ & $0.003 \%$ & $2.92 E-01$ & $6.31 E-06$ & $2.99 E-05$ & $1.92 E-15$. & $9.34 E-15$ \\
\hline Cfill $1-8-4$ & i. $56 E-04$ & 0.0042 & $1.00 E+00$ & i. $38 E-05$ & $4.37 E-05$ & $2.75 E-15$ & $5.825-15$ \\
\hline CRH $1-8-5$ & $1.43 E-04$ & $0.005 \%$ & $2.10 E+00$ & $1.11 E-05$ & $5.47 E-05$ & $1.85 E-15$ & $4.36 E-15$ \\
\hline CAn 1-8-6 & $1.58 E-04$ & 0.0072 & $3.02 E+00$ & $1.24 E-x 5$ & $6.72 E-65$ & $5.66 E-15$ & $4.56 E-15$ \\
\hline CरH $1-8-7$ & $1.84 E-04$ & 0.0032 & $i .31 E+03$ & $1.41 E-05$ & 3. $13 E-05$ & $2.73 E-16$ & $1.54 E-15$ \\
\hline CRN $1-8-8$ & i. $17 E-144$ & 0.0094 & $2.00 E+01$ & $8.89 E-06$ & $9.02 E-05$ & $3.30 E-16$ & $1.24 E-15$ \\
\hline CAM $1-8-9$ & $3.64 E-0.5$ & 0.0102 & $2.70 E+01$ & $7.56 E-06$ & $9.77 E-(15)$ & $3.33 E-16$ & $1.08 E-15$ \\
\hline 5 5. $1-8-10$ & $9.03 E-65$ & $0.010 \%$ & 4.10E+01 & 7.01E-06 & $1.055-04$ & 1.03E-16 & 8.:DEE-16 \\
\hline ERH i-3-11 & $2.665-04$ & $0.613 \%$ & 5. 00 ENE+01 & $2.03 E-05$ & $1.35 E-04$ & $2.82 E-15$ & $3.55 E-16$ \\
\hline LAN $1-8-12$ & $B .21 E-605$ & 0.0152 & $0.70 E+01$ & $6.501 E-06$ & $1,32 E-34$ & $1.50 \mathrm{E}-13$ & 5. $26 E-16$ \\
\hline $\operatorname{Con}:-8-1 j$ & ร. $642-05$ & $0.019 \%$ & $7.20 E+01$ & $7 .+1 E-i b$ & $1.39 E-34$ & $6.62 E-17$ & $6.41[-16$ \\
\hline CR:W :-8-14 & $3.76 E-04$ & $0.017:$ & $1.22 E+02$ & $3.13 E-05$ & $1.70 E-64$ & $1 .+1 E-15$ & $7.25 E-16$ \\
\hline CRE :-6-15 & $1.52 E-74$ & $0.010 \%$ & $1.345+42$ & $1.18 E-95$ & $1.32 E-\mathrm{in}_{4}$ & $1.51 E-15$ & $7.55 E-16$ \\
\hline Cati 1-6-16 & $4.2 ! E-14$ & $0.021 \%$ & 1. $82 E+02$ & $3.21 E-05$ & $2 . ! 4 E-14$ & $8.57 E-16$ & i.6PE-16 \\
\hline CFid: $:-8-17$ & $4.35 E-014$ & ט. $025 n$ & 3. $62 E+02$ & $3.31 E-\mathrm{i}^{5} \mathrm{~J}$ & $2.48 E-04$ & $2.21 E-j j$ & $0.20 E-16$ \\
\hline
\end{tabular}


TABLE B.5i. Americium-241 (AM-241) Leach Fractions from ANS 16.2 Leach Test-Whole Grout in Groundwater (1-13)

\begin{tabular}{|c|c|c|c|c|c|c|c|}
\hline SAMPLE & $u[j$ & ¿ LEACHED & TIKE DAYS & $A_{n} / A_{0}$ & CUA An/AO & $D$ inc. cal/ses & D Eus en $2 / \sec$ \\
\hline CRN $1-13-1$ & $1.37 E-04$ & $0.001 \%$ & 3. COE-04 & $1.29 E-05$ & $1.29 E-05$ & $1.29 E-12$ & $1.75 E-12$ \\
\hline CRE $1-13-2$ & 3.35E-04 & 0.0042 & B. $33 E-02$ & $2.65 E-05$ & $3.94 E-05$ & $3.03 E-14$ & $5.90 E-1$ \\
\hline CRH $1-13-3$ & $1.37 E-04$ & 0.0052 & $2.92 E-01$ & 1.09E-05 & $5.03 E-05$ & 5. $88 E-15$ & $2.74 E-1$ \\
\hline CRH 1-13-4 & 8. $17 E-05$ & $0.006 \%$ & $1.00 E+00$ & $6.62 \mathrm{E}-06$ & $5.69 E-65$ & $6.57 E-16$ & :.02E:- \\
\hline CAH $1-13-5$ & 8. $17 E-0.5$ & $0.006 \%$ & $2.10 E+00$ & $6.57 E-06$ & $6.35 E-65$ & $6.775-16$ & b. $07 \mathrm{E}-$ \\
\hline CRM 1-13-6 & 8. 17E-05 & $0.007 \%$ & $3.02 E+00$ & $6.64 E-06$ & 7.01E-0.5 & $1.67 E-15$ & $5.155-$ \\
\hline CRU $1-13-7$ & $1.17 E-04$ & $0.008 \%$ & $1.31 E+01$ & $9.36 E-66$ & $7.95 E-05$ & $7.86 E-17$ & 1. $.5 \mathrm{JE}-$ \\
\hline CRA 1-13-8 & $1.84 E-04$ & 0.0094 & $2.00 E+01$ & 1. $50 E-05$ & $9.45 E-05$ & $9,72 \mathrm{E}-\mathrm{ib}$ & 1.41E- \\
\hline CRH :-13-9 & $2.13 E-014$ & 0.0112 & $2.70 E+01$ & $1.73 E-05$ & $1.12 E-04$ & $1.81 E-15$ & $1.46 E-$ \\
\hline CRH $1-13-10$ & $1.31 E-04$ & $0.612 \%$ & $4.10 E+01$ & $1.09 E-05$ & $1.23 E-04$ & $2.56 E-16$ & $\therefore .16 E-15$ \\
\hline CKH $1-13-11$ & $1.52 E-04$ & $0.013 \%$ & $5.00 E+01$ & $1.21 E-05$ & $1.355-04$ & 1.05E-15 & $1.15 E-15$ \\
\hline CAN $1-13-12$ & $2.94 E-04$ & $0.016 \%$ & $6: 40 E+01$ & $2.31 E-05$ & 1.5BE-0.4 & $1.955-15$ & 1.23E-15 \\
\hline ำ $1-13-13$ & $8.17 E-05$ & 0.0162 & $\$ .20 E+01$ & $6.71 E-06$ & $1.65 E-04$ & $5.62 E-17$ & $3.31 E-1$ \\
\hline CRH $1-13-14$ & i.11E-04 & 0.0172 & $1.22 E+02$ & $8.80 E-06$ & 1. $73 E-04$ & $1.16 E-16$ & $7.79 E-1$ \\
\hline CfN : $-13-15$ & $2.39 E-04$ & $0.019 \%$ & $1.34 E+02$ & $1.89 E-05$ & 1. $32 E-014$ & $4.035-15$ & $8.73 E-1$ \\
\hline $\operatorname{CAN} i-1 j-16$ & $2.16 E-04$ & $0.021 \%$ & $1.82 E+02$ & $1.72 E-05$ & $2.10 E-04$ & $2.56 E-16$ & 7.6SE-1 \\
\hline CRN $1-13-17$ & $3.82 E-04$ & 0.0247 & $3.02 E+012$ & $2.99 E-05$ & $2.395-(14$ & $1.87 E-16$ & 6.00E-16 \\
\hline
\end{tabular}

TABLE B.5j. Plutonium-238 (PU-238) Leach Fractions from ANS 16.1 Leach Test-Whole Grout in Groundwater (1-8)

\begin{tabular}{|c|c|c|c|c|c|c|c|}
\hline SAHPLE & $\mathrm{VCI}$ & Z LEACHED & TIME DAYS & An'ínO & Eu Anitho & Dint. chzísei & 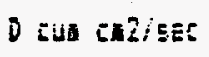 \\
\hline san $1-3-1$ & a. $035-05$ & 0. 6012\% & 3.00E-04 & $5.7 \dot{b} \bar{c}-6 \hat{c}$ & J.jée-vó & $2,495-13$ & S.JEE-:3 \\
\hline $28 H$ i-3-2 & O. 0 JE- 15 & $6.001 \%$ & $8.33 E-\hat{2} 2$ & $5.76 E-66$ & $1.15 E-i 5$ & 1. $30 E-5$ & $4.8 T-15$ \\
\hline Lnin $:-5-3$ & $8.03 E-05$ & 0.0027 & $2.92-\mathrm{ij}$ & 5. $76 E-\hat{v} b$ & $1, \overline{73} \bar{E}-05$ & 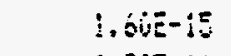 & $\therefore .15 E-15$ \\
\hline อกี่ $1-50$ & $3.03 E-05$ & $0.002 \%$ & $\therefore .00 E+0 \hat{0}$ & $5.76 \varepsilon-66$ & 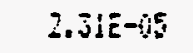 & $4 . \overline{O J E}-i c$ & i. $6.5-15$ \\
\hline En 1-ริ-5 & ล.. $23 E-05$ & $0.003 \%$ & $2.10 E+60$ & $5.76 E-106$ & 2. $32 \mathrm{EE}-\mathrm{-S}$ & 5. X广E-:2 & $1.21 E-1 E$ \\
\hline EnN 1-5-b & $1.20 \mathrm{E}-0.4$ & 0.6045 & $.3 .32 E+00$ & Sิ. SSE- $\hat{x}$ & J. $345-\hat{1} 5$ & S. $\quad 3 \overline{B E}-\mathrm{i} E$ & $1,415-15$ \\
\hline $\sin 1-8-7$ & $3.0 \mathrm{JE}-6 \mathrm{~J}$ & 6.6045 & $1.31 E+6 !$ & 5.76E-6í & $4.3: 5-15$ & $2 . \bar{E}=-17$ & $4.35-16$ \\
\hline 6hi i-6-2 & E. OJE-65 & $0.005 \%$ & $2.00 \mathrm{E}+0 !$ & $5.75 E-1) 6$ & $4.375-05$ & 1. SEE-16 & $3.65 \bar{E}-16$ \\
\hline E月 I-E-7 & E. $.35-65$ & 0. $0 \mathrm{Es}$ & $2.70 E \div 0 \mathrm{i}$ & 5. : & $5.47 E-45$ & $1.34 E-16$ & $3.36 E-16$ \\
\hline Sin $1-90-10$ & $3.63 E-65$ & $0.006 \%$ & ?.10E+10! & $5.76 E-06$ & 6. $04 \mathrm{E}-\mathrm{i}, 5$ & 6. $77:-17$ & 3. $T 2 E-16$ \\
\hline inis $\mid-0-11$ & 3. $6 \mathrm{BE}-\mathrm{v} 5$ & $0.007 \%$ & $5.60 E+01$ & $5.76 E-66$ & $\dot{0} .6 \angle E-05$ & $2.25 \bar{E}-1 \mathrm{~s}$ & 2. $260 E-16$ \\
\hline Gin $-9-12$ & $3.63 E-65$ & $0.017 \%$ & $6.40 E+01$ & $5.76 E-06$ & 7. $20 \mathrm{E}-6 \mathrm{0}$ & $1.13 E-15$ & $2.47 E-10$ \\
\hline$-741-\bar{s}-13$ & ล., $03 E-015$ & $0.008 \%$ & $9.20 E+01$ & $5.70 E-j e ́$ & $7.77 E-05$ & $\therefore . \dot{0} \mathrm{~L} E-17$ & $2.015-15$ \\
\hline 6his $1-8-14$ & 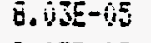 & 10.600\% & $1.22 E+02$ & 5. $75 E-65$ & 3. $35 E-15$ & i. $305-1 i$ & 2.75E-1E \\
\hline [nt: $:-8$ & $8.035-35$ & $0.009 \%$ & $1.34 E+i i z$ & E.jóse-ví & E.72E-OE & S. & !.EE-! \\
\hline Shitio & 8.63E-65 & 4.66i\% & i. $22 E+42$ & S. It & 7.50E-35 & $2.7 \mathrm{EE-15}$ & $4.52 E-18$ \\
\hline $5+14-3-13$ & $0.135-65$ & $3.60 \%$ & $3.00 \mathrm{E}+6 \bar{z}$ & $5.76 E-36$ & $1,01 E-34$ & 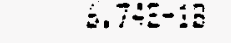 & $\therefore A E-16$ \\
\hline
\end{tabular}


TABLE B.5j. Plutonium-238 (PU-238) Leach Fractions from ANS 16.1 Leach Test-Whole Grout in Groundwater (1-13)

\begin{tabular}{|c|c|c|c|c|c|c|c|}
\hline SAMPLE & $\mathrm{uCi}$ & 7 LEACHED & TIME OAYS & An/Ao & CUn in /AO & 0 inc. caz/sec & $D$ cun cuz/5er \\
\hline CRU $1-13-1$ & $8.03 E-05$ & 0.0012 & $3.00 E-04$ & $6.01 E-06$ & $6.01 E-06$ & $2.80 E-13$ & 3. $305-13$ \\
\hline CFIV $1-13-2$ & $1.18 E-04$ & 0.0012 & 8.33E-02 & $8.74 E-06$ & $1.47 E-0 S$ & $3.2 a E-15$ & $0.26 E-15$ \\
\hline CAL 1-13-3 & $8.03 E-05$ & $0.002 \%$ & $2.92 E-01$ & $6.01 E-06$ & $2.07 E-05$ & $1.80 E-15$ & $4.67 E-15$ \\
\hline $\operatorname{CRN} 1-13-4$ & $8.03 E-05$ & 0.0037 & $1.00 E+00$ & $6.01 E-06$ & $2.68 E-05$ & $5.40 E-i b$ & $3.27 E-15$ \\
\hline CRH 1-13-5 & $8.03 E-05$ & $0.003 \%$ & 2. $10 E+00$ & 6.01E-06 & $3.28 E-05$ & $5.66 E-16$ & $1.62 E-15$ \\
\hline CRn 1-13-6 & B.03E-05 & 0.0047 & $3.02 E+00$ & $6.01 E-06$ & $3.88 E-05$ & $1.37 E-15$ & $1.57 E-15$ \\
\hline CRY $1-13-7$ & $3.03 E-05$ & $0.004 \%$ & $1.31 E+01$ & $6.01 E-06$ & $4.48 E-05$ & $3.235-17$ & $4.85 E-16$ \\
\hline CRN $1-13-8$ & $8.03 E-05$ & $0.005 \%$ & $2.00 E+01$ & $6.015-06$ & $5.08 E-05$ & $1.56 E-16$ & $4.66 E-16$ \\
\hline $\operatorname{CR}=1-13-9$ & Q.03E-05 & 0.0062 & $2.70 E+0 i$ & $6.01 E-06$ & $5.68 E-05$ & $2.16 E-16$ & $3.785-16$ \\
\hline CAU $1-13-10$ & $8.03 E-05$ & $0.006 \%$ & $4.10 E+01$ & $6.01 E-\hat{0} 6$ & $6.28 E-65$ & $7.64 E-17$ & $3,04 E-16$ \\
\hline CRH $1-1 j-11$ & $8.03 E-05$ & $0.007 \%$ & $5.00 E+01$ & $6.01 E-106$ & $6.88 E-05$ & $2.56 E-16$ & $3.00 E-16$ \\
\hline Câl : $-13-12$ & B. $03 \mathrm{JE}-05$ & 0.0072 & $6.40 E+001$ & $6.01 E-06$ & $7.48 E-05$ & $1.32 E-16$ & $2.77 E-16$ \\
\hline Cân $1-13-13$ & B.03E-05 & $0.608 \%$ & $9.20 E+01$ & $6.01 E-06$ & $8.08 E-05$ & $4.51 E-17$ & $2.25 E-16$ \\
\hline CAn $1-13-14$ & 8. $\hat{0} 3 E-05$ & $0.009 \%$ & $1.22 E+02$ & $6.01 E-06$ & $8.68 E-05$ & $5.40 E-17$ & $1.955-16$ \\
\hline CRA $1-13-15$ & $8.03 E-05$ & 0.0092 & $1.34 E+02$ & $6.01 E-06$ & $9.28 E-05$ & $4.66 E-16$ & $2.03 E-16$ \\
\hline CRI: $:-13-16$ & 8.0JE-65 & $0.010 \%$ & $1.62 E+02$ & $6.15 E-06$ & $9.90 E-05$ & $3.26 E-17$ & $1.70 E-16$ \\
\hline CRM $1-13-17$ & $9.64 E-05$ & $0.011 \%$ & $3.02 E+02$ & $7.17 E-06$ & $1.06 E-04$ & 1.06E-17 & $1.18 E-16$ \\
\hline
\end{tabular}

TABLE B.6a. Potassium $(K)$ Leach Fractions from Static Leach Test--Whole Grout in Groundwater (DIF2-17)

\begin{tabular}{|c|c|c|c|}
\hline $\begin{array}{r}\text { SAMPLE } \\
\text { CRI: } 2-17-1\end{array}$ & $\begin{array}{l}\text { Cus. An/Ao } \\
1.21 E-01\end{array}$ & $\begin{array}{l}\text { TIME days } \\
7.00 \mathrm{E} \div 00\end{array}$ & $\begin{array}{l}\text { D cus. } \\
\cdot \quad 6.59 E-09\end{array}$ \\
\hline CRH $2-17-2$ & 1.4FE-01 & 1. $30 E+01$ & $5.42 E-69$ \\
\hline CRI $2-17-3$ & 1.79E-01! & $2.00 E+01$ & $5.08 E-09$ \\
\hline 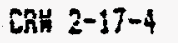 & $1.97 \varepsilon-01$ & $2.7 \hat{v E}+01$ & $4.55 E-09$ \\
\hline CR $2-17-5$ & $3.26 E-02$ & $3.40 E+01$ & $9.9 \mathrm{iE-}: 1 \mathrm{i}$ \\
\hline CRY 2-17-i & $2.20 E-01$ & $4.10 E+61$ & $4.09 E-69$ \\
\hline CRY $2-17-7$ & $3.28 E-01$ & $4.80 E+001$ & $3.76 E-09$ \\
\hline CRY $2-1 T-8$ & 2.33E-01 & $5.50 E+0 \hat{i}$ & $3.2 B E-09$ \\
\hline CRU $2-17-9$ & $2.46 E-51$ & $6.20 E+01$ & $3.50 E-09$ \\
\hline sin $2-17-10$ & $2.59 E-0 !$ & 7. OHE +01 & $3.37 E-07$ \\
\hline Crat $2-17-11$ & $2.68 E-01$ & $7.60 E+01$ & $3.395-69$ \\
\hline CRA $2-17-12$ & $2.20 E-01$ & i. $64 E+0 \hat{2}$ & $2.67 E-195$ \\
\hline CFI $2-15-13$ & $3.62 E-01$ & $1.34 E+02$ & 2. $\{2 E-39$ \\
\hline ChH $2-i \bar{i}-i q$ & $3.34 E-61$ & $1.70 E+02$ & $2.12 E-49$ \\
\hline 5) $2-j i-15$ & $3.49 E-03$ & $3.15 E+02$ & $1.95 E-69$ \\
\hline
\end{tabular}


TABLE B.6b. Sodium (Na) Leach Fractions from Static Leach Test--Whole Grout in Groundwater (DIF2-17)

\begin{tabular}{|c|c|c|c|}
\hline SAMPLE & EUn. $A n / A_{0}$ & TIME days & D cun. \\
\hline CRY $2-17-1$ & $1.91 E-01$ & 7. $00 E+00$ & $1.65 E-018$ \\
\hline CRN $2-17-2$ & $2.34 E-01$ & $1.30 E+01$ & 1.39E-08 \\
\hline CRH $2-17-3$ & $2.76 E-01$ & $2.00 E+01$ & 1.39E-08 \\
\hline CRE 2-17-4 & $3.10 E-01$ & $2.70 E+01$ & 1.26E-08 \\
\hline EFH $2-17-5$ & $3.42 E-01$ & $3.40 E+01$ & $1.26 E-08$ \\
\hline 대 $2-17-6$ & $3.51 E-01$ & $4.10 E+01$ & $1.12 E-06$ \\
\hline C5H $2-17-7$ & $3.77 E-01$ & $4.80 E+01$ & $1.16 E-08$ \\
\hline กค月 2-17-8 & $3.39 E-61$ & 5. $56 E+01$ & 1.07E-OB \\
\hline 댁 2-17-9 & $4.07 E-01$ & $6.20 E+01$ & $1.07 \mathrm{E}-\mathrm{VB}$ \\
\hline CRH $2-17-10$ & $4.360 E-01$ & 7.00E+01 & 1. $12 E-08$ \\
\hline CRH $2-17-11$ & 4. $4 P E-01$ & $7.60 E+01$ & 1.09E-0B \\
\hline CAH $2-17-12$ & $4.75 E-01$ & $1.04 E+02$ & $8.84 E-09$ \\
\hline CRH $2-17-13$ & $5.08 E-01$ & $1.34 E+02$ & $8.41 E-69$ \\
\hline CRA 2-17-14 & $5.55 E-01$ & 1. $30 E+02$ & $7.15 E-69$ \\
\hline CRN $2-17-15$ & 6. 06E-01 & $3.15 E+02$ & $5.73 E-69$ \\
\hline CRA $2-18-16$ & $6.225-01$ & $4.24 E+02$ & $4.43 E-09$ \\
\hline
\end{tabular}

TABLE B.6C. Aluminum. (A1) Leach Fractions from Static Leach Test--Whole Grout in Groundwater (DIF2-17)

\begin{tabular}{|c|c|c|c|}
\hline SAMFLE & CUS. An/ÂO & TIKE days & O cul. \\
\hline ลกำ $2-17-1$ & $3.72 E-03$ & $7.00 E+00$ & b. $24 E-12$ \\
\hline CR: 2-17-2 & $4.23 E-03$ & $1.30 E+01$ & $4.36 E-12$ \\
\hline CRN 2-17-3 & $4.84 E-63$ & $2.00 E+01$ & $3.70 E-12$ \\
\hline CRH $2-17-4$ & $5.33 E-03$ & 2.70EE+01 & $3.33 E-12$ \\
\hline SRH $2-1 \bar{j}-5$ & $5.76 E-63$ & $3.40 E+01$ & 3. $09 E-12$ \\
\hline Can 2-17-6 & $5.63 E-03$ & 4. $10 E+01$ & $2.625-12$ \\
\hline CKN 2-17-7 & $6.265-03$ & $4.60 E+01$ & $2.58 E-12$ \\
\hline $\operatorname{Con} 2-17-8$ & $6.40 E-013$ & $5.50 E+01$ & $2.35 E-12$ \\
\hline Con 2-17-9 & $6.66 E-03$ & $6.20 E+01$ & $2.26 E-12$ \\
\hline $\operatorname{con} 2-17-10$ & $7.05 E-03$ & $7.00 E \div 01$ & $2.25 E-12$ \\
\hline$C$ Ca $2-17-1 i$ & $7.26 E-03$ & $7.60 E+0 !$ & $2.20 E-12$ \\
\hline CRIN $2-17-12$ & $7.91 E-03$ & $1.04 E+02$ & $1.90 E-12$ \\
\hline [FW $2-17-13$ & $8.74 E-63$ & $1.34 E+02$ & $1.60 E-12$ \\
\hline Cont $2-17-14$ & $1.0 \mathrm{BE}-02$ & $1.90 E+02$ & $1.715-12$ \\
\hline CKH $2-17-15$ & $1.17 E-02$ & $3.15 E+02$ & 1.36E - -12 \\
\hline
\end{tabular}


TABLE B.6e. Boron (as H3BO3) Leach Fractions from Static Leach Test--Whole Grout in Groundwater (DIF2-17)

\begin{tabular}{|c|c|c|c|}
\hline SAKPLE & Cun. An/AO & TIHE days & $D$ evi. \\
\hline CRI 2-17-1 & $7.88 E-02$ & $7.00 E+00$ & $2.3 \mathrm{iE}-69$ \\
\hline CRN 2-17-2 & $9.17 E-02$ & $1.30 E+01$ & 2.05E-09 \\
\hline CRW 2-17-3 & $1.02 E-01$ & 2.00E+01 & $1.64 E-09$ \\
\hline С月H $2-17-4$ & $1.17 E-01$ & 2. $70 E+0 !$ & 1.59E-09 \\
\hline CAN $2-17-6$ & 1.37E-01 & $4.10 E+01$ & $1.45 E-09$ \\
\hline Cay 2-17-7 & L.18E-01 & $4.80 E+01$ & $9.17 E-10$ \\
\hline $\operatorname{CRH} 2-17-8$ & $1.35 E-01$ & $5.50 E+01$ & $1.05 E-09$ \\
\hline CRH 2-17-9 & $1.43 E-01$ & $6.20 E+01$ & $1.03 E-09$ \\
\hline CKit $2-17-10$ & $1.53 E-01$ & $7.60 E+01$ & 1. $05 E-69$ \\
\hline $\operatorname{CRM} 2-17-11$ & $1.60 \mathrm{E}-01$ & $7.60 \mathrm{E}+01$ & 1.07E-199 \\
\hline CRN $2-17-12$ & 1.6BE-01 & $1.04 E+02$ & $8.6 J E-10$ \\
\hline CAN $2-17-13$ & 1. $68 \mathrm{E}-01$ & $1.34 E+02$ & B.36E-10 \\
\hline CRH $2-17-14$ & 2.14E-01 & 1. $30 E+02$ & $7.05 E-10$ \\
\hline CRบ $2-17-15$ & $2.42 E-01$ & $3.15 E+02$ & $5.39 E-10$ \\
\hline
\end{tabular}

TABLE B.6f. Fluoride (F) Leach Fractions from Static Leach Test--Whole Grout in Groundwater (DIF2-17)

\begin{tabular}{|c|c|c|c|}
\hline SAMPLE & Cus. ĥn'Ao & TIKE days & D Eแ. \\
\hline CRH 2-17-i & $3.95 E-03$ & $7.00 E+00$ & $7.06 E-12$ \\
\hline Can 2-17-2 & $4.42 E-03$ & 1. $30 E+01$ & $4.76 E-12$ \\
\hline CKN $2-17-3$ & $5.04 E-03$ & $2.00 E+O 1$ & 4.01E-12 \\
\hline CRA 2-17-4 & $5.40 E-03$ & $2.70 E+01$ & 3. $42 E-12$ \\
\hline CAN $2-17-5$ & $5.99 E-03$ & $3.40 E+01$ & 3.34E-12 \\
\hline CRN 2-17-6 & 6. 22E- 013 & $4.10 E+01$ & $2.99 E-12$ \\
\hline CEN $2-17-7$ & $6.50 E-03$ & $4.80 E+01$ & $2.79 E-12$ \\
\hline CRN 2-17-3 & $6.91 E-03$ & $5.50 E+01$ & $2.75 E-12$ \\
\hline $\mathrm{CRH} 2-17-9$ & $7.23 E-63$ & 3. $20 E+01$ & 2.67E- 12 \\
\hline CAH $2-17-10$ & $7.40 E-03$ & $7.00 E+01$ & $2.48 E-12$ \\
\hline Cfil: $2-17-11$ & $7.70 E-03$ & $7.60 E+01$ & $2,47 E-12$ \\
\hline $\cos 22-17-12$ & $6.7 \mathrm{aE}-03$ & $1.04 E+02$ & $2.34 E-12$ \\
\hline IF⿻ $2-17-13$ & $3.64 E-03$ & $1.34 E+02$ & $2.19 E-12$ \\
\hline CAH $2-17-14$ & $1.05 E-02$ & $1.90 E+02$ & 1.85E-12 \\
\hline CFU $2-i 7-15$ & 1.19E-A2 & 3. $15 E+02$ & $1,43 E-12$ \\
\hline CAN 2-16-16 & 1.11E-62 & $4.24 E \div 02$ & $3.22 E-13$ \\
\hline
\end{tabular}


TABLE B.6g. Nitrate (NO3) Leach Fractions from Static Leach Test--Whole Grout in Groundwater (DIF2-17)

\begin{tabular}{|c|c|c|c|}
\hline SAMPLE & EUA. An/AD & TIME days & 0 ius. \\
\hline CRY 2-17-1 & 5.03E-02 & $7.00 E+00$ & $1.15 E-109$ \\
\hline CRH $2-17-2$ & $6.05 E-02$ & $1.30 E+01$ & 3. $90 E-10$ \\
\hline CRH $2-17-3$ & $7.30 E-02$ & $2.00 E+01$ & 8. $\$ 4 E-10$ \\
\hline CRIN 2-17-4 & $8.13 E-02$ & $2.70 E+01$ & $7.76 E-10$ \\
\hline CRY 2-17-5 & $1.22 E-01$ & $3.40 E+01$ & $1.3 B E-09$ \\
\hline CAN 2-17-6 & $1.57 E-01$ & $4.30 E+01$ & $1.30 E-09$ \\
\hline CRK 2-17-7 & $1.97 E-01$ & $4.80 E+01$ & $2.55 E-09$ \\
\hline CAM $2-17-8$ & 2.00E-0! & $5.50 E+01$ & $2.30 E-09$ \\
\hline CFI 2-17-9 & 2. $02 E-01$ & $6.20 E+01$ & $2.09 E-69$ \\
\hline Crin $2-17-10$ & $2.33 E-61$ & $7.00 E \div 01$ & $2.58 E-69$ \\
\hline CRH 2-17-11 & $2.27 E-01$ & $7.60 E+01$ & $2.38 E-69$ \\
\hline $\operatorname{con} 2-17-12$ & 2.58E-01 & 1. $04 E+02$ & $2.27 E-69$ \\
\hline $\cos 2-17-13$ & $1.88 E-01$ & $1.34 E+02$ & a.36E-10 \\
\hline CRH 2-17-14 & $2.87 E-0.1$ & 1. $3 G E+02$ & $1.565-69$ \\
\hline CRY $2-1 \bar{i}-15$ & 3.50E-01 & $3.15 E+02$ & $1.45 E-69$ \\
\hline
\end{tabular}

TABLE B.6h. Sulfate (SO4) Leach Fractions from Static Leach Test--Whole Grout in Groundwater (DIF2-17)

\begin{tabular}{|c|c|c|c|}
\hline SAKPLE & CUS. Ân/AO & TIKE days & $D$ cus. \\
\hline CRH $2-17-1$ & $2.16 E-01$ & $7.00 E+00$ & 2.39E-08 \\
\hline CRH 2-17-2 & $2.26 \mathrm{E}-01$ & $1.30 E+01$ & $1.37 E-08$ \\
\hline CRH $2-17-3$ & $2.72 E-01$ & $2.00 E+01$ & i. $29 E-16$ \\
\hline CAH 2-17-4 & $2.97 \mathrm{E}-01$ & $2.70 E \div 01$ & $1.20 E-68$ \\
\hline CNA 2-17-5 & $2.86 E-01$ & $3.40 E \pm 01$ & $8.325-69$ \\
\hline CRy 2-17-6 & $2.54 E-01$ & 4. $10 E+01$ & $5.36 E-69$ \\
\hline CEN 2-17-7 & $3.365-01$ & $4.80 E+01$ & 3. $955-10 ?$ \\
\hline CRH 2-17-6 & $3.375-01$ & $5.50 E+01$ & $7.61 E-09$ \\
\hline Cกㄴ 2-17-9 & 3. $58 E-01$ & $6.20 E+01$ & 7. FốE- -69 \\
\hline CAN $2-17-10$ & $3.73 E-01$ & 7. $\mathrm{VOE}+\mathrm{vi}$ & $7.445-09$ \\
\hline CRH $2-17-11$ & $3.64 E-0\}$ & $7.605+0 !$ & $6.45 E-69$ \\
\hline $\cos 2-17-12$ & $3.60 E-01$ & 1. $04 E \div 62$ & $5.35 E-69$ \\
\hline CRA $2-17-13$ & $4.67 E-0 !$ & $1.34 E+62$ & D.3̈É-09 \\
\hline CAK $2-17-14$ & 4. 6âE-01 & 1.96E-62 & 4. $84 E-69$ \\
\hline ind $i-1 \bar{j}-15$ & 6. $13 E-01$ & $3.155+82$ & $5.50-39$ \\
\hline
\end{tabular}


TABLE B.6i. Carbon-14 (C-14) Leach Fractions from Static Leach Test--Whole Grout with Groundwater (2-17)

\begin{tabular}{|c|c|c|c|c|}
\hline 5AMPLE & UCi & TIME DAYS & An/Ao & $D$ LOW CN2/5EE \\
\hline CRH $2-17-1$ & $8.90 E-03$ & $7.00 E+00$ & $5.23 E-03$ & $1.25 E-11$ \\
\hline $\operatorname{conh} 2-17-2$ & $9.52 E-03$ & $1.30 \mathrm{E}+01$ & 5.59E-03 & $7.71 E-12$ \\
\hline $\operatorname{CnH} 2-17-3$ & $1.02 E-02$ & 2. $\hat{0 O E}+01$ & $5.97 E-03$ & $5.70 E-12$ \\
\hline $\operatorname{csn} 2-17-4$ & $1.02 E-12$ & $2.70 E+01$ & $5.99 E-03$ & $4.26 E-12$ \\
\hline CRH 2-17-5 & $9.42 E-03$ & $3.40 E+01$ & $5.54 E-03$ & $2.295-12$ \\
\hline CRN $\hat{2}-17-6$ & $9.01 E-03$ & 4. $10 E+01$ & $5.29 E-03$ & 2. 19E-12 \\
\hline Can 2-17-7 & $7.32 E-03$ & $4.80 E+01$ & $4.30 E-03$ & $1.235-12$ \\
\hline CRU 2-17-8 & $6.94 E-03$ & $5.50 E+01$ & $4,08 E-03$ & $9,38 \mathrm{E}-13$ \\
\hline CRN 2-17-9 & $7.39 E-03$ & $6.20 E+01$ & $4.34 E-03$ & $9.74 E-13$ \\
\hline CNA $2-17-10$ & $6.71 E-03$ & $7.00 E+0 \mathrm{~S}$ & $3.95 E-0 j$ & $7 . i 2 E-13$ \\
\hline Crin :-17-11 & $5.79 E-03$ & $7.60 \mathrm{E}+01$ & $3.40 E-13$ & $4.38 E-13$ \\
\hline CRK $2-17-12$ & $7.37 E-13$ & 1. $045+02$ & $4.33 E-03$ & $5.77 \mathrm{E}-13$ \\
\hline $\operatorname{Cax} 2-17-13$ & $8.31 E-03$ & $1.34 E+02$ & $4.36 E-03$ & 5. $30 E-13$ \\
\hline 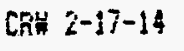 & $1.18 E-02$ & $1.90 E+02$ & 6. $92 E-1) 3$ & $8.0 B E-13$ \\
\hline CRA $2-17-15$ & $5.25 E-03$ & $3.15 E+02$ & 3. $09 E-03$ & $3.65 E-14$ \\
\hline
\end{tabular}

TABLE B.6i. Carbon-14 (C-14) Leach Fraction from Static Leach Test--Whole Grout in Groundwater (2-18)

\begin{tabular}{|c|c|c|c|c|}
\hline SAMPLE & $\mathrm{UCI}$ & TIKE DAYS & An/As̃ & i cula chiijes \\
\hline CRIN $2-18-1$ & $1.24 E-02$ & $7.00 E+00$ & $7.49 E-03$ & 2.56E-11 \\
\hline CRH $2-18-2$ & $9.02 E-03$ & $1.30 E+01$ & $5.44 E-03$ & $7.30 E-12$ \\
\hline CAH 2-18-3 & $9.65 E-03$ & $2.00 E+01$ & $5.82 E-03$ & $5.125-12$ \\
\hline CNA $2-18-4$ & $9.095-03$ & $2.70 E+01$ & $5.48 E-03$ & $3.56=-12$ \\
\hline 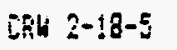 & $6.835-03$ & $3.40 z+01$ & $4.125-03$ & 1. $.3 V E-12$ \\
\hline CR: $2-18-6$ & $6.02 E-03$ & $4.10 E+\hat{V} !$ & $3.63 E-0.3$ & :. \\
\hline $\mathrm{CRH}:-18-7$ & $7.86 E-03$ & 4. Sंve+0I & $4.74 E-v 3$ & $1.56 E-12$ \\
\hline CAH $2-18-8$ & $7,47 E-63$ & 5. SUE+QI & $4.565-103$ & 1.:aE-15 \\
\hline int $2-18-7$ & $6.675-03$ & $0.2 \mathrm{VE}+01$ & $4.035-05$ & $2.4 ! E-53$ \\
\hline Sati $2-18-10$ & $1.20 E-1]$ & $7 . G O E+C i$ & $7.27 E-74$ & $2.4 J E-14$ \\
\hline ink z-:o-!1 & $5.58 E-63$ & 7.60E $\div 01$ & $3.37 E-13$ & $4.75 \mathrm{E}-13$ \\
\hline $37 \mathrm{Z}-1 \overline{\mathrm{e}}-12$ & 7. $30 E-63$ & $1.04 E+0 z$ & $4.05=-43$ & S. SOEt-i \\
\hline CAR $2-12-13$ & $7.96 E-13$ & $1.34 E+02$ & $4.30 E-63$ & $5.51 E-13$ \\
\hline $\operatorname{Con} 2-16-14$ & 1. $1 E E-12$ & $\therefore, 705+32$ & $7 .: 2 E-63$ & $5,5=-15$ \\
\hline inin $2-15-15$ & $5: 1 E-4 i$ & 3. $15 E+\hat{2} Z$ & $3,3 Z E-\lambda J$ & $3,35-: \div$ \\
\hline
\end{tabular}


TABLE B.7a. Potassium ( $K$ ) Leach Fractions from Static Leach Test--Whole Grout in Groundwater (DIF3-18)

\begin{tabular}{|c|c|c|c|}
\hline SAMPLE & EUA. Ân/ÂO & THME days & 0 cus. \\
\hline CRH 3-18-1 & $1.31 E-01$ & $7.00 E+00$ & $7.77 E-09$ \\
\hline CNAH 3-18-2 & $1.52 \mathrm{E}-0 \mathrm{1}$ & 1. $30 E+01$ & $5.60 E-69$ \\
\hline CRH $3-18-3$ & $1.92 E-01$ & 2. $.00 E+01$ & $5.35 E-69$ \\
\hline CRy 3-18-4 & $2.36 E-61$ & $2.70 E+\hat{v i}$ & $6.235-08$ \\
\hline Can 3-18-5 & $2.36 E-01$ & $3.40 E+01$ & 5. 86EE-69 \\
\hline SaH $3-18-6$ & $2.41 E-01$ & $4,10 E+01$ & $4.86 E-69$ \\
\hline CRN $3-10-7$ & $2.625-01$ & $4.80 E+01$ & $4.94 E-09$ \\
\hline CAH 3-16-3 & $2.67 E-01$ & $5.5 \hat{V E}+\hat{v} 1$ & $4.70 E-09$ \\
\hline CRA 3-18-? & $2.36 E-01$ & $6.20 E+\hat{0} !$ & $4.265-09$ \\
\hline CRH $3-18-10$ & $2.96 \varepsilon-01$ & T. OOE $+\hat{v 1}$ & $4.605-0 \%$ \\
\hline 触 $3-i 0-11$ & S. vile-0it & 7. $60 E+01$ & $4.29 E-69$ \\
\hline Сล⿻ 3-16-12 & $3.23 E-01$ & $3,04 E+02$ & $3.62 E-6\}$ \\
\hline [ñ 3-18-13 & $3.33 E-61$ & 1.34E $\div 02$ & $3.02 E-09$ \\
\hline CRA $3-16-14$ & $3.66 E-61$ & $1.90 \varepsilon+02$ & 2.75E-09 \\
\hline $\cosh 5-13-15$ & $3.76 E-01$ & $3.15 E+02$ & $1.7 \bar{E}-\mathrm{i} A$ \\
\hline
\end{tabular}

TABLE B.7b. Sodium (Na) Leach Fractions from Static Leach Test--Whole Grout in Groundwater (DIF3-18)

\begin{tabular}{|c|c|c|c|}
\hline SAMPLE & Cua. An/AO & TIME days & D Eun. \\
\hline CFH 3-18-1 & $2.10 E-01$ & $7.00 E+60$ & 2.17E- 08 \\
\hline CRU $3-18-2$ & $2.28 E-01$ & $1.30 E+01$ & $1.39 E-08$ \\
\hline CAN 3-18-3 & $2.89 E-01$ & $2.00 E+01$ & $1.515-08$ \\
\hline CRN 3-18-4 & $3.20 E-61$ & $2.70 E+01$ & $1.40 E-08$ \\
\hline CFin 3-:a-5 & $3.53 E-01$ & $3.40 E+01$ & $1.35 E-16$ \\
\hline CAN 3-18-b & $3.655-61$ & 4.10E+01 & $1.20 E-08$ \\
\hline CAN 3-18-7 & $3.78 E-01$ & $4.80 E+01$ & $1.31 E-06$ \\
\hline CAN $3-j B-6$ & 4.10E-v̂l & $5.50 E+01$ & $1.21 E-0 \varepsilon$ \\
\hline CRE 3-18-9 & $4.30 E-01$ & 6. $20 E+0 !$ & $1.265-68$ \\
\hline CÁH 3-16-10 & $4.58 E-01$ & $7.00 E+01$ & $1.255-05$ \\
\hline 5n 3-10-11 & $4.72 E-61$ & $7.600 \div 01$ & $1.21 E-00$ \\
\hline $\operatorname{con} 3-16-12$ & $4.99 E-01$ & 1. $04 E+02$ & $1.04 E-06$ \\
\hline $\operatorname{con} 3-18-13$ & $5.34 E-6 i$ & $\therefore .34 E+02$ & $9.295-69$ \\
\hline CAH 3-15-14 & $5.76 E-01$ & $1.96 E+02$ & B.30E- 69 \\
\hline 语等 & 6. $17 E-01$ & $3.15 E+02$ & 5. FBE-00? \\
\hline
\end{tabular}


TABLE B.7C. Aluminum (A1) Leach Fractions from Static Leach Test--Whole Grout in Groundwater (DIF3-18)

\begin{tabular}{|c|c|c|c|}
\hline SAMPLE & Cun. An/Ao & TIME days & 0 гua. \\
\hline CxN 3-18-1 & 5. $05 E-03$ & $7.00 E+00$ & $1.15 E-11$ \\
\hline CRN $3-18-2$ & $4.71 E-03$ & $1.30 E+O 1$ & $5.39 E-12$ \\
\hline CRH 3-18-3 & $0.23 E-03$ & 2. $00 E+01$ & D. $13 E-12$ \\
\hline CRY $3-18-4$ & $6.79 E-03$ & $2.70 E+01$ & $5.40 E-12$ \\
\hline CRH $3-18-5$ & $7.38 E-13$ & $3.40 E+01$ & 5. $07 E-12$ \\
\hline CRN $3-18-6$ & $7.51 E-03$ & $4.10 E+01$ & 4.35E-12 \\
\hline CRU $3-18-7$ & 3. 28E-03 & $4.60 E+01$ & $4.52 E-12$ \\
\hline CAH 3-18-8 & $8.35 E-63$ & 5. $50 E+01$ & $4.01 E-12$ \\
\hline CRN 3-18-9 & 3. $82 E-03$ & $6.20 E+01$ & $3.97 E-12$ \\
\hline CRA 5 - $10-10$ & $9.06 \mathrm{E}-03$ & $7.00 E+01$ & $3.735-12$ \\
\hline CRA 3-18-11 & $9.58 E-03$ & $7.60 E+01$ & $3.82 E-12$ \\
\hline CAN $3-18-12$ & $1.06 E-02$ & $1.04 E+02$ & $3.41 E-12$ \\
\hline CFI $3-18-13$ & 1. $15 E-02$ & $1.34 E+02$ & 3. JOE-12 \\
\hline CRH $3-18-14$ & $1.26 E-02$ & $1.90 E+02$ & $2.63 E-12$ \\
\hline CRH $3-18-15$ & $1.44 E-02$ & $3.15 E+\hat{0} 2$ & 2. $07 E-12$ \\
\hline
\end{tabular}

TABLE B.7e. Boron (as H3B03) Leach Fractions from Static Leach Test--Whole Grout in Groundwater (DIF3-18)

\begin{tabular}{|c|c|c|c|}
\hline SAKPLE & CUA. Ân/AD & TIKE days & D cur. \\
\hline CRN 3-18-1 & $1.49 E-02$ & $7.00 E+00$ & $1.01 E-10$ \\
\hline CRN $3-18-2$ & $1.38 E-02$ & $1.30 E+01$ & $4.62 E-11$ \\
\hline CRN $3-18-3$ & $1.76 E-02$ & $2.00 E \div 01$ & $4.87 E-1 j$ \\
\hline CAn 3-18-4 & $1.90 E-02$ & $2.70 E+01$ & $4.22 E-11$ \\
\hline CRN 3-18-5 & 2.17E-02 & $3.40 E+01$ & $4.39 E-11$ \\
\hline CAn 3-18-b & $2.32 E-02$ & $4.10 E+01$ & $4.15 E-11$ \\
\hline CRU $3-18-7$ & $2.395-02$ & $4.80 E+01$ & $3.76 \mathrm{E}-11$ \\
\hline CRN $3-18-8$ & $2.47 E-02$ & $5.50 E+\hat{v I}$ & $3.525-11$ \\
\hline CNAH 3-18-9 & $2.53 E-02$ & $6.20 E+0 !$ & 3. JFE-!1 \\
\hline $\operatorname{CSN} 5-18-10$ & $2.775-62$ & $7.00 E+01$ & $3.46 E-1 i$ \\
\hline LRH 3-18-11 & $2.83 E-12$ & $7.60 E+01$ & $3.335-11$ \\
\hline $\operatorname{con} 3-18-12$ & 3. $065-62$ & 1. $04 E+02$ & 2. $00 E-11$ \\
\hline Cก) $3-18-13$ & $3.455-62$ & $1.34 E+\sqrt{2}$ & $2.82 E-11$ \\
\hline CAM $3-10-14$ & $3.72 E-02$ & $\therefore, 70 E+02$ & $2,30 \mathrm{~V}-11$ \\
\hline $\operatorname{con} 3-10-15$ & $4.07 E-02$ & $3.15 E+02$ & $1.66=-11$ \\
\hline
\end{tabular}


TABLE B.7f. Fluoride (F) Leach Fractions from Static Leach Test--Whole Grout in Groundwater (DIF3-18)

\begin{tabular}{|c|c|c|c|}
\hline SAMPLE & Cus. Antho & TIKE days & D cish. \\
\hline CRH 3-18-1 & $3.80 E-03$ & $7.00 E+00$ & 6.52E-12 \\
\hline CRN 3-18-2 & $3.7 B E-03$ & $1.30 E+0 !$ & $3.47 E-12$ \\
\hline CRU $3-18-j$ & 4.88E-03 & 2.00E+0! & $3.77 E-12$ \\
\hline Can $3-18-4$ & $5.53 E-03$ & $2.70 E+01$ & $3.506-12$ \\
\hline CAN 3-i8-5 & $6.15 E-03$ & $3.40 E+01$ & $3.52 E-12$ \\
\hline Can 3-18-6 & $6.39 E-03$ & $4.10 E+01$ & $3.15 E-12$ \\
\hline CFH $3-18-7$ & $6.83 E-03$ & $4.80 E+01$ & $3.07 E-12$ \\
\hline $\operatorname{Con} 3-18-8$ & 7.07E-6J & $5.50 E+01$ & 2. $58 E-12$ \\
\hline CRy 3-18-9 & $7.95 E-03$ & $6.2 \mathrm{VE}+01$ & $2.83 E-12$ \\
\hline $\operatorname{CR} 3-18-10$ & $8.35 E-03$ & 7. $00 E+01$ & $3.15 E-12$ \\
\hline CRY 3-18-11 & $8.15 E-03$ & $7.60 \varepsilon+01$ & $2.365-12$ \\
\hline $\cos 3-18-12$ & $9.14 E-03$ & $1.04 E+02$ & $2.54 E-12$ \\
\hline CRH $3-18-13$ & $3.08 E-03$ & $1.34 E+02$ & 1.35E-12 \\
\hline CRN 3-18-14 & $1.09 E-02$ & $1.90 E+02$ & $1.98 E-12$ \\
\hline CRN $3-j 8-15$ & $1.28 E-02$ & $3.155+02$ & L.SOOE-IZ \\
\hline
\end{tabular}

TABLE B.7g. Nitrate (N03) Leach Fractions from Static Leach Test--Whole Grout in Groundwater. (DIF3-18)

\begin{tabular}{|c|c|c|c|}
\hline SAMPLE & CUE. An/AO & TIME Jays & D снь. \\
\hline CRH 3-18-1 & 6. $30 E-02$ & $7.00 E+00$ & $1.75 E-09$ \\
\hline CAH 3-18-2 & $6.72 E-02$ & $1.30 E+01$ & $1.10 E-09$ \\
\hline CRE 3-j8-3 & 6. 53E- 02 & $2.00 E+01$ & $1.16 E-09$ \\
\hline CRI 3-18-4 & $9.51 E-02$ & $2.70 E+01$ & $1.06 E-09$ \\
\hline CRN 3-j8-5 & $1.45 E-61$ & $3.40 E+01$ & $1.74 E-09$ \\
\hline CRM. 3-18-6 & $1.87 E-01$ & $4.10 E+01$ & $2.71 E-49$ \\
\hline ERH $3-18-7$ & $2.32 E-01$ & $4.80 E+01$ & $3.78 E-69$ \\
\hline $\operatorname{con} 3-18-8$ & $2.375-01$ & $5.50 E+01$ & $3.625-39$ \\
\hline CRH 3-18-4 & $1.84 E-01$ & $6.20 E+01$ & $1.73 E-09$ \\
\hline CAN 3-16-10 & $2.71 E-01$ & $7.00 E+\hat{0} !$ & $3.69 E-69$ \\
\hline CFI 3-18-11 & $2.76 E-01$ & $7.60 E+01$ & $3.60 E-195$ \\
\hline CAM 3-18-12 & $3.01 E-01$ & $1.04 E+02$ & 3.13E-69 \\
\hline CRA 3-16-13 & $3.06 E-01$ & $1.34 E+02$ & $2.59 E-49$ \\
\hline CRI $3-18-14$ & 3. $4: E-01$ & $1.90 E+02$ & $2.27 E-i \overline{9}$ \\
\hline 站勧 $3-18-15$ & 4. ISE-01 & 3. $15 E+122$ & $2.11 E-09$ \\
\hline
\end{tabular}


TABLE B.7h. Sulfate (SO4) Leach Fractions from Static Leach Test--Whole Grout in Groundwater (DIF3-18)

\begin{tabular}{|c|c|c|c|}
\hline SAMPLE & Cus. An/AO & IIME days & D cus. \\
\hline $\mathrm{CSH} 3-18-1$ & $2.96 E-01$ & $7.00 E+00$ & 4.66E-08 \\
\hline $\operatorname{CRU} 3-18-2$ & $2.36 E-01$ & $1.30 E+01$ & $1.53 E-08$ \\
\hline CRN $3-18-3$ & $3.23 E-01$ & 2. $00 E+01$ & 1. $88 E-08$ \\
\hline CRA $3-18-4$ & $3.53 E-01$ & $2.70 E+01$ & $8.82 E-08$ \\
\hline CFH 3-18-5 & $3.52 E-01$ & $3.40 E+01$ & $1.35 E-08$ \\
\hline CRM 3-18-6 & $3.25 E-01$ & $4.10 E+01$ & $9.865-09$ \\
\hline $\mathrm{CRH} 3-18-7$ & $4.24 E-01$ & $4.80 E+01$ & $1.47 E-0 B$ \\
\hline CAN 3-18-8 & $4.15 E-01$ & $5.50 E+01$ & $1.28 E-0 B$ \\
\hline $\operatorname{CrN} 3-18-9$ & $4.40 E-01$ & $6.20 E+01$ & $1.27 E-08$ \\
\hline CRY $3-16-10$ & $4.77 E-01$ & $7.00 E+01$ & $1.39 E-08$ \\
\hline CRH 3-18-11 & $4.855-01$ & $7.60 E+01$ & 1.28E-0B \\
\hline CRM $3-18-12$ & $4.57 E-01$ & $1.04 E+02$ & $7.56 E-109$ \\
\hline CRH $3-18-13$ & $4.84 E-01$ & $1.34 E+02$ & $7.24 E-69$ \\
\hline CAN $3-18-14$ & $5.96 E-01$ & $1.90 E+02$ & $9.11 E-69$ \\
\hline CRH 3-18-15 & $3.61 E-01$ & $3.15 E+02$ & $1.09 E-08$ \\
\hline
\end{tabular}

TABLE B.7i. Technetium-99 (TC-99) Leach Fractions from Static Leach Test-Whole Grout with Groundwater (3-18)

\begin{tabular}{|c|c|c|c|c|}
\hline SAMPLE & $\mathrm{uCi}$ & TIHE DAYS & $A n / A O$ & D CHA Caziset \\
\hline CRH 3-18-1 & $3.71 E-03$ & 7.00E+00 & $2.55 E-03$ & 3.03E-!2 \\
\hline CRN $3-18-2$ & $5.35 E-0 J$ & $1.30 E+01$ & $3.73 E-03$ & $3.40 E-12$ \\
\hline CAN 3-18-3 & $1.14 E-02$ & $2.00 E+01$ & $7.97 E-03$ & $1.01 E-11$ \\
\hline CRN 3-18-4 & $1.57 E-02$ & $2.70 E+01$ & $1.09 E-02$ & 1. 4GE-11 \\
\hline CRI 3-18-5 & $1.89 E-02$ & $3.40 E+01$ & $1.32 E-02$ & $1.62 E-11$ \\
\hline Cris 3-18-6 & $2.12 E-02$ & $4.10 E+01$ & $1.48 E-02$ & $1.68 E-11$ \\
\hline CRN 3-!8-7 & $2.28 E-02$ & 4. $80 E+01$ & $1.59 E-02$ & 1. 66E-11 \\
\hline Cond 3-16-8 & $2.31 E-02$ & $5.50 E+01$ & $1.61 E-02$ & $1.49 E-11$ \\
\hline $\cos 3-18-4$ & $2.54 E-02$ & $6.20 E+0.1$ & $1.77 E-\hat{v} 2$ & $1.60 E-11$ \\
\hline CRN 3-18-10 & $2.61 E-02$ & $7.00 E+01$ & $1.82 E-1.2$ & $1.49 E-11$ \\
\hline CRบ 3-18-11 & $2.64 E-02$ & $7.6 \mathrm{VE}+01$ & $1.84 E-02$ & $1.41 E-1$ \\
\hline $\mathrm{CRH} 3-18-12$ & D. $37 E-02$ & 1. $\hat{0} 4 E+02$ & 2. 36E- -12 & 1.70t-1 \\
\hline CRM 3-18-13 & 4.09E-12 & $1.34 E+02$ & $2.85 E-\mathrm{i}_{2}$ & 1. 32E-ii \\
\hline CRH $3-18-14$ & $4.795-02$ & $1.90 E+02$ & $j, 48 E-\hat{6} 2$ & 2. $.02 E-j i$ \\
\hline CRW $5-18-15$ & $3.34 E-12$ & $3.15 E+02$ & $2.3 \mathrm{JE}-\mathrm{j2}$ & 5. $44 E-12$ \\
\hline CAN 5-16-16 & $6.91 E-6 i 2$ & $4.24 E+02$ & $4.32 E-62$ & 1. TJE-1 \\
\hline
\end{tabular}



Technetium-99 (TC-99) Leach Fractions from Static Leach Test--
Whole Grout with Groundwater (3-19)

\begin{tabular}{|c|c|c|c|c|}
\hline SAKPLE & $\mathrm{UCi}$ & TIME DAYS & An $/ A_{0}$ & $D$ cUA caz/set \\
\hline $\cos 5-19-1$ & $5.20 E-03$ & $7.00 E+00$ & $2.81 E-03$ & $3.62 E-12$ \\
\hline CRN $3-19-2$ & $9.37 E-03$ & $1.30 E+01$ & $5.07 E-03$ & $6.3 J E-12$ \\
\hline CRN 3-19-3 & $1.53 E-02$ & $2.00 E+01$ & $8.29 E-03$ & $1.10 E-11$ \\
\hline CRH 3-19-4 & $2.04 E-02$ & $2.7 \hat{O E}+01$ & 1. $10 E-62$ & I. HAE-II \\
\hline CRN 3-19-5 & $2.325-02$ & $3.40 E+01$ & $1.26 \mathrm{E}-02$ & $1.48 E-i 1$ \\
\hline CRN $3-19-6$ & $2.70 E-02$ & $4.10 E+01$ & $1.96 E-02$ & $1.66 E-11$ \\
\hline CAL $3-19-7$ & $2.80 E-02$ & $4.30 E+01$ & $1.51 E-02$ & $1.53 E-11$ \\
\hline CRI 3-19-8 & $3.05 E-02$ & $5.50 E+01$ & $1.55 E-02$ & $1.58 E-11$ \\
\hline CRY 3-19-9 & $3.12 E-02$ & $6.20 E+01$ & $1.69 E-02$ & $1.47 E-11$ \\
\hline CRU $5-19-10$ & $3.30 \mathrm{E}-02$ & 7. DOE+OI & $1.7 a \varepsilon-02$ & $1.46 E-11$ \\
\hline CRH 3-19-11 & $3.33 E-02$ & $7.60 E+01$ & $1.30 E-02$ & $1.37 E-11$ \\
\hline CRH $3-i 9-12$ & $3.96 E-02$ & $1.04 E+02$ & $2 .: 15-02$ & $1.31 E-i i$ \\
\hline CRH 3-i9-13 & $4.73 E-02$ & $1.34 E+02$ & $2.56 E-0.2$ & $1.50 E-11$ \\
\hline CRU 3-19-14 & $5.925-02$ & $1.90 E+02$ & $3.20 E-62$ & 1.7SE-i! \\
\hline
\end{tabular}

TABLE B.8a.

Potassium (K) Leach Fractions from Static Leach Test--Whole Grout
in Groundwater (DIF4-13)

\begin{tabular}{|c|c|c|c|}
\hline SAMPLE & [UR. An/ÂO & THE days & 5 cus. \\
\hline CFH $4-13-1$ & $1.16 E-6 j\}$ & 7. $00 E+60$ & อ. $11 E-j Q$ \\
\hline $\operatorname{con} 4-13-2$ & 1. $345-01$ & $1.30 E+01$ & $4.755-09$ \\
\hline CRI $4-13-3$ & 1. $.63 E-01$ & $2.00 E+01$ & $4.26 E-09$ \\
\hline САH 4-1j-4 & $1.93 E-01$ & $2.70 E+01$ & 4.44E-09 \\
\hline CRH 4-13-5 & $2.695-01$ & $3.40 E+01$ & $4.12 E-69$ \\
\hline CRY $\{-13-6$ & $2.11 E-01$ & 4. $10 E+01$ & $3.70 E-09$ \\
\hline $\cos +15-7$ & $2.30 E-01$ & 4. OOE+OI & $3.78 E-29$ \\
\hline CNA $4-15-3$ & $2,4 j E-01$ & $5.50 E+01$ & $3.76 E-09$ \\
\hline $67 \mathrm{~A}+13-4$ & $2.61 E-01$ & $6.20 E+01$ & $3.32 E-49$ \\
\hline CNA $4-13-10$ & $2.74 E-0 !$ & $3.60 \mathrm{E}+0 \mathrm{i}$ & $3.595-09$ \\
\hline CEN $\{-15-11$ & $2.83 E-61$ & 7. SuEtol & $3.655-96$ \\
\hline Cof $4-1 j-12$ & $2.96 E-01$ & 1. $64 E+02$ & $3.13 E-69$ \\
\hline $6+44-13-13$ & $3,00 E-01$ & j. $34 E+02$ & $\therefore+4 E-G$ \\
\hline Cnit 4-13-14 & $3.31 E-01$ & $\therefore>05+02$ & $2.13 E-69$ \\
\hline $2 K^{2} \quad 4-1 j-15$ & $3.56 E-61$ & $3.15 E+7 i$ & $1.565-69$ \\
\hline
\end{tabular}


TABLE B.8b. Sodium (Na) Leach Fractions from Static Leach Test--Whole Grout in Groundwater (DIF4-13)

\begin{tabular}{|c|c|c|c|}
\hline $\begin{array}{r}\text { SAMPLE } \\
\text { CRU } 4-1 j-1\end{array}$ & $\begin{array}{l}\text { Cuu. An/Ao } \\
1.92 E-01\end{array}$ & $\begin{array}{l}\text { TIME days } \\
7.00 \mathrm{E}+00\end{array}$ & $\begin{array}{l}0 \text { cus. } \\
1.70 E-188\end{array}$ \\
\hline CRN $4-13-2$ & $2.12 E-01$ & $1.30 E+01$ & $1.17 E-08$ \\
\hline CRM $4-13-3$ & $2.61 E-01$ & $2.00 E+01$ & $1.19 E-08$ \\
\hline CRN $4-13-4$ & $3.01 E-01$ & $2.70 E+01$ & $1.21 E-08$ \\
\hline $\operatorname{con}+-13-5$ & $3.30 E-01$ & $3.40 E+01$ & $1.19 E-08$ \\
\hline CRK $4-13-6$ & $3.37 E-01$ & $4.10 E+01$ & $1.05 E-0 \mathrm{~B}$ \\
\hline $\mathrm{CAH} 4-13-7$ & $3.72 E-01$ & $4.80 E+01$ & $1,69 E-60$ \\
\hline CRH $4-13-8$ & $3.81 E-01$ & $5.50 E+01$ & 1.01E-08 \\
\hline $\cos 4-13-9$ & 4. $60 E-01$ & $6.20 E \div 01$ & $1.01 E-08$ \\
\hline CAH $4-1 j-10$ & $4.16 E-01$ & $7.00 E+01$ & $1.01 E-08$ \\
\hline$C K^{\prime} 4-1 j-11$ & $4.42 E-01$ & $7.60 \mathrm{E}+01$ & 1. $\$ 4 E-08$ \\
\hline CRA $4-13-12$ & $4.72 E-01$ & $1.04 E+02$ & $8.38 E-09$ \\
\hline CRn $4-13-13$ & $5.015-01$ & $1.34 E+02$ & $8.615 E-69$ \\
\hline CAN $4-13-14$ & క. $15 E-01$ & $1.90 E+02$ & $6.24 E-19$ \\
\hline CRill 4-13-15 & $5.87 E-001$ & $3.15 E+02$ & $5.23 E-09$ \\
\hline
\end{tabular}

TABLE B.8c. Aluminum (A1) Leach Fractions from Static Leach Test--Whole Grout in Groundwater (DIF4-13)

\begin{tabular}{|c|c|c|c|}
\hline SAHPLE & Sun. An/Ao & TIME days & D. eut. \\
\hline CRK $4-13-1$ & $4.22 E-03$ & $7.00 \mathrm{E}+00$ & a.14E-12 \\
\hline $\cos 4-13-2$ & $4.01 E-013$ & $1.30 E+01$ & $3.37 E-12$ \\
\hline CRN $4-13-\overline{3}$ & $4.84 E-03$ & $2.00 E+01$ & $3.35 E-12$ \\
\hline CRA $4-13-4$ & $2.665-05$ & $2.70 E+01$ & $8.4 J E-13$ \\
\hline CKI $4-13-5$ & $3.34 E-03$ & $3.40 E+01$ & $1.65 E-12$ \\
\hline Con 4-13-6 & $3.87 E-03$ & 4.10E+ii & 1. $17 E-12$ \\
\hline $\mathrm{C}$ - $34-13-7$ & $5.77 E-0 \mathrm{~J}$ & $4.80 E+01$ & $2.22 E-12$ \\
\hline CFH $4-13-8$ & $5.75 E-6 j$ & 5. SWE + & Z. GóE-12 \\
\hline $\operatorname{CN} 44-13-9$ & $6.25 E-63$ & $0.20 E+01$ & $2.02 E-12$ \\
\hline $\operatorname{CAH} 4-13-10$ & S. 56E-03 & T. $06 E+01$ & 1.775E-!2 \\
\hline C凋 $4-13-11$ & D. $\bar{i} 4 E-0\}$ & 7. 6 OE+O! & $1.72 E-12$ \\
\hline CAN $4-15-12$ & $7.62 E-03$ & $1.04 E+102$ & $1.79 E-12$ \\
\hline $67 x:-1 j-13$ & $8.052-03$ & 1. $345 \div 02$ & 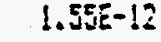 \\
\hline Cn: $4-15-14$ & $8.74 E-63$ & $1.90 E+02$ & 1.35E-:2 \\
\hline Efe' 4-15-15 & 1. $: E E-02$ & $3.15 E \div 02$ & i. $060-12$ \\
\hline
\end{tabular}


TABLE B.8e. Boron (as H3BO3) Leach Fractions from Static Leach Test--Whole Grout in Groundwater (DIF4-13)

\begin{tabular}{|c|c|c|c|}
\hline SAKPLE & CUn. An/AO & TiMe days & 0 cus. \\
\hline CRY $4-13-1$ & $1.12 E-02$ & $7.00 E+00$ & $5.75 E-11$ \\
\hline $\operatorname{CRN} 4-13-2$ & 1. $12 E-02$ & $1.30 E+01$ & 3.11E-11 \\
\hline Cก้̂ $4-13-3$ & 1.30E-02 & $2.00 E+01$ & $2.32 E-11$ \\
\hline CRH $4-13-4$ & $1.47 E-02$ & $2.70 E+01$ & $2.56 E-11$ \\
\hline ERH $4-13-5$ & $1.58 E-02$ & $3.40 E+01$ & $2.36 E-11$ \\
\hline $\operatorname{CAN} 4-13-6$ & $1.60 E-02$ & 4.:DEE+01 & $2.01 E-11$ \\
\hline CAN $4-15-7$ & $1.70 E-02$ & $4.80 E \div 01$ & $1.94 E-11$ \\
\hline $\operatorname{CAN} 4-13-8$ & $1.75 E-62$ & $5.50 E+01$ & $1.79 E-11$ \\
\hline $\operatorname{CAN} 4-13-9$ & 1. $655-02$ & $6.20 E+01$ & $1.77 E-11$ \\
\hline $\operatorname{Cs} 4-13-10$ & $1.99 E-02$ & $7.00 E+01$ & $1.81 E-11$ \\
\hline CRH $4-13-11$ & $2.01 E-02$ & $7.60 E+01$ & $1.71 E-11$ \\
\hline CAN $4-13-12$ & $2.17 E-02$ & $1.04 E+02$ & $1.48 E-11$ \\
\hline CRA $4-13-13$ & $2.38 E-62$ & $3.34 E+02$ & $1.365-11$ \\
\hline CAN $4-13-14$ & $2.46 E-02$ & $1.90 E+02$ & $1.02 E-11$ \\
\hline CRA $4-13-15$ & $3.32 E-02$ & $3.15 E \div \hat{1} 2$ & $1.12 E-11$ \\
\hline
\end{tabular}

TABLE B.8f. Fluoride (F) Leach Fractions from Static Leach Test--Whole Grout in Groundwater (DIF4-13)

\begin{tabular}{|c|c|c|c|}
\hline SAMPLE & CuA. An/AD : & TIME days & $D$ ius. \\
\hline CRN $9-13-1$ & $4.25 E-03$ & $7.00 E+00$ & $8.29 E-12$ \\
\hline CRN $4-13-2$ & $4.15 E-03$ & $1.30 E+01$ & 4. $25 \mathrm{NE}-12$ \\
\hline Cay $4-13-3$ & $5.01 E-03$ & 2. DoEtoi & $4.03 E-12$ \\
\hline $\operatorname{CaH} 4-13-4$ & $5.29 E-03$ & $2.70 E+0 !$ & 3.32E-12 \\
\hline CRA $4-13-5$ & $5.91 E-03$ & $3.40 E+01$ & 3.30E-12 \\
\hline CRM $4-1 j-6$ & $6.19 E-03$ & $4.10 E+01$ & 3.00E-12 \\
\hline Cxy $4-13-7$ & $5.91 E-03$ & $4.80 E+0 \mathrm{i}$ & $2.34 E-12$ \\
\hline CAN $4-13-8$ & $0.7 B E-63$ & $5.50 E+01$ & $2.6 B E-12$ \\
\hline CRH 4-13-9 & $6.95 E-63$ & $6.20 \mathrm{E}+01$ & $2.5 \hat{V E}-12$ \\
\hline CRL $:-13-10$ & $7.71 E-03$ & $7.00 E+01$ & $2.72 E-12$ \\
\hline CFI $4-13-11$ & $7.67 E-03$ & $7.605+01$ & $2.46 E-12$ \\
\hline CRI $4-13-12$ & $8.70 E-03$ & $1.04 E+02$ & $2.33 E-12$ \\
\hline [5H $4-15-13$ & $9.56=-63$ & 1.34E-62 & $2.1 \$-1 \overline{2}$ \\
\hline $\cos 4-1 \overline{3}-14$ & $1.06 E-02$ & 1. $90 E+02$ & $1.69 E-12$ \\
\hline $\operatorname{CH} 4-13-15$ & $1.13 E-\hat{0} \hat{2}$ & 3.:35E+ 22 & $1.315-12$ \\
\hline
\end{tabular}


TABLE B.8g. Nitrate (NO3) Leach Fractions from Static Leach Test--Whole Grout in Groundwater (DIF4-13)

\begin{tabular}{|c|c|c|c|}
\hline SAMPLE & Cus. An/tho & TIME days & 0 cul. \\
\hline CRH 4-13-1 & 5. $40 E-02$ & $7.00 E+00$ & $1.34 E-09$ \\
\hline CRA $4-13-2$ & $6.44 E-02$ & $1.30 E+01$ & $1.02 E-09$ \\
\hline CRH $4-13-3$ & $6.74 E-02$ & $2.00 E+01$ & 7.29E-10 \\
\hline CRH $4-13-4$ & $7.42 E-02$ & $2.70 E+01$ & $6.54 E-10$ \\
\hline CRN $4-13-5$ & 1. 13E-01 & $3.40 E+01$ & $1.20 E-09$ \\
\hline CRH 4-13-6 & $1.46 E-01$ & 4.10E+0i & $1.67 E-09$ \\
\hline CAY $4-13-7$ & $1.64 E-01$ & $4.80 E+01$ & $1.80 E-09$ \\
\hline CRI $4-13-8$ & $1.95 E-01$ & $5.50 E \div 01$ & $1.99 E-09$ \\
\hline CRII $4-13-9$ & 1. $86 E-01$ & $0.20 E+01$ & $1.79 E-019$ \\
\hline CRU $4-15-10$ & $7.66 E-01$ & $7.00 E+01$ & $5.16 E-08$ \\
\hline CRH $4-13-11$ & $2.18 E-03$ & $7.60 E+61$ & $2.21 E-03$ \\
\hline CRU $4-13-12$ & 2.B2E-01 & $1.04 E+02$ & $2.6 B E-09$ \\
\hline CRH $4-13-13$ & $2.11 E-01$ & 1. $34 E+02$ & 1. $06 E-69$ \\
\hline CAN $4-13-14$ & $2.99 E-01$ & $1.90 E+02$ & $1.72 E-09$ \\
\hline CAll $4-13-15$ & $3.61 E-01$ & $3.15 E+02$ & $1.56 E-699$ \\
\hline
\end{tabular}

TABLE B.8h. Sulfate (SO4) Leach Fractions from Static Leach Test--Whole Grout in Groundwater (DIF4-13)

\begin{tabular}{|c|c|c|c|}
\hline SAKPLE & CUA. AnIAO & TIME daYs & D cus. \\
\hline CRH 4-13-1 & 2.41E-01 & $7.00 E+00$ & $2.84 E-08$ \\
\hline CRN $4-13-2$ & 2.01E-01 & 1.30E+01 & $9.96 E-09$ \\
\hline CRI $4-13-3$ & $2.36 E-01$ & $2.00 E+01$ & $7.96 E-09$ \\
\hline CR: 4-13-4 & $2.67 E-01$ & $2.70 E+01$ & 7.57E-199 \\
\hline CAN $4-13-5$ & $2.51 E-01$ & $3.40 E+0 !$ & $6.41 E-09$ \\
\hline CRN $4-13-6$ & $2.16 E-01$ & 4. $10 E+01$ & $4.10 E-09$ \\
\hline $\operatorname{Csin} 4-13-7$ & $2.64 E-01$ & $9.60 E \div 01$ & $4.94 E-09$ \\
\hline Con $4-13-8$ & $2.95 E-\hat{v} 1$ & S. S0E+ôl & $5.47 E-09$ \\
\hline CFil $+-13-7$ & 3.01E-0i & $6.20 E+0 i$ & $5.26 E-\hat{v i n}$ \\
\hline CRH $4-13-10$ & 3.6BE-61 & $7.00 E+01$ & 8.47E-69 \\
\hline aㅔ $4-13-11$ & $3.25 E-01$ & $\bar{i} .50 \mathrm{OE}+0 \mathrm{~S}$ & $5.32 E-69$ \\
\hline CAY $4-13-12$ & $3.30 E-61$ & 1. $\hat{v} 4 E+\hat{v} 2$ & $3.09 E-09$ \\
\hline CAH $4-13-13$ & $3.72 \mathrm{E}-0 \mathrm{i}$ & $1.34 E+02$ & 3. $30 E-19$ \\
\hline LAH $4-13-14$ & $3.96 E-611$ & $1.90 E+02$ & $3.30 E-099$ \\
\hline CRA $4-13-15$ & $5.26 E-0 !$ & $3.15 E+02$ & $3.75 E-97$ \\
\hline
\end{tabular}


TABLE B.8i. Iodine-125 (I-125) Leach Fractions from Static Leach Test--Whole Grout with Groundwater (4-19)

\begin{tabular}{|c|c|c|c|c|}
\hline SAMPLE & UCI LEACH & TIME DAYS & $\mathrm{An} / \mathrm{Ao}$ & i) Cun $\mathrm{Ca} 2 / \mathrm{sec}$ \\
\hline CRY 4-19-1 & $1.07 E+00$ & $7.00 E+00$ & 1. $|3 E-0| 0\}$ & 5.53E- 39 \\
\hline CRH $4-19-2$ & $1.11 E+00$ & $1.30 E+01$ & 1. $|J E-D|$ & $3.20 E-$ \\
\hline CRH $4-19-3$ & $1.36 E+00$ & 2. $\hat{0}(E E+01$ & $1.43 E-61$ & $3.12 E-$ \\
\hline CAN $4-19-4$ & $1.5 A E+00$ & $2.70 E+01$ & 1. OOF-01 & S.i4E \\
\hline $\operatorname{con} 4-17-5$ & $1.69 E+00$ & $3.40 E+01$ & $1.78 E-011$ & $2.86 E$ \\
\hline Can $4-19-6$ & $1.82 E+00$ & $4.10 E+01$ & $1.92 E-01$ & $2.74 E-6$ \\
\hline CRH $:-j 9-\bar{i}$ & $1.91 E+00$ & $4.80 \mathrm{E}+0 \mathrm{I}$ & $2.625-6 !$ & $2.58 E-1$ \\
\hline CAN $4-19-8$ & 1.96E+00 & $5.50 E+01$ & $2.06 E-0 !$ & 2.B̈́E-1 \\
\hline CRN 4-19-9? & $2.06 E+00$ & $6.20 E+v i 1$ & $2.16 E-01$ & $2.33 E-1$ \\
\hline CFK +-19-10 & $2.15 E+00$ & $7.60 \mathrm{E}+01$ & $2.26 E-011$ & $2.23 E-$ \\
\hline CRH $4-19-11$ & $2.22 E \div 00$ & 7.60 tois & $2.3+5-61$ & $2.30-i$ \\
\hline CRA 4-19-12 & $2.59 E+60$ & $1.04 E+i 2$ & $2.73 E-1 i d$ & $2,15 E-i$ \\
\hline CAM $4-19-13$ & $2.86 E+00$ & $1.34 E+02$ & $3.32 E-151$ & $2.67 E-69$ \\
\hline CNu 4-19-14 & $3.23 E+60$ & $1.70 E+\hat{q}$ & $3.40 E-01$ & 1. $3 \mathrm{BE}-0$ \\
\hline
\end{tabular}

TABLE B.8i. Iodine-125 (I-125) Leach Fractions from Static Leach. Test--Whole Grout with Groundwater (4-13)

\begin{tabular}{|c|c|c|c|c|}
\hline SAMPLE & UCI LEACH & TIKE DAYS & $A_{n} / A_{O}$ & D cu $\mathrm{cs} 2 / 5 \mathrm{~s}$ \\
\hline CRN $4-13-1$ & 8. $55 E-01$ & $7.00 E+00$ & $3.23 \mathrm{E}-02$ & 3.:0E-09 \\
\hline CAH 4-13-2 & 8.35E-01 & $1.30 E+01$ & 8.02E- 62 & $1.59 E-09$ \\
\hline CRN $4-13-3$ & $9.28 E-01$ & $2.00 E+01$ & $8.93 E-\hat{\gamma 2}$ & 1. $28 E-09$ \\
\hline CRA 4-13-4 & $7.18 E-01$ & $2.76 E+0 \mathrm{~L}$ & D. $71 E-02$ & 5. $6 a E-10$ \\
\hline CRU $4-13-5$ & $1.03 E+00$ & $3.40 E+01$ & $9.87 E-102$ & $9.19 E-10$ \\
\hline CAH 4-13-6 & :. $03 E+110$ & $4.10 E+01$ & $9.92 E-02$ & $7.68 \mathrm{E}-10$ \\
\hline CAN $4-13-7$ & $1.01 E+00$ & $4.80 E+01$ & $9.73 E-12$ & $6.32 E-10$ \\
\hline CRN 4-13-8 & $9.76 E-01$ & $5.50 E+0 i$ & $9.58 E-\hat{v 2}$ & 5. $34 E-10$ \\
\hline CSA 4-13-9 & $9.5 B E-01$ & $6.20 E+01$ & $9.21 E-02$ & $4.36 E-10$ \\
\hline CAH $\{-1]-10$ & $9.46 E-\{1 !$ & $7.00 E+01$ & $9.09 E-02$ & $3.38 E-10$ \\
\hline CAH $4-13-11$ & $9.46 E-i 11$ & $7.60 E+01$ & 9.03E-02 & $3.4 E E-10$ \\
\hline С月а 4-13-12 & $9.33 E-01$ & $1.04 E+02$ & $3.97 E-\hat{2} 2$ & $2.46 E-10$ \\
\hline CEN $4-13-13$ & $1.22 E+00$ & $1.34 E+02$ & 1. $18 E-01$ & $3.31 E-10$ \\
\hline LFin $4-13-14$ & i. $36 E+60$ & 1. $8010+02$ & 1.j.j:E-0! & $2.90 E-10$ \\
\hline $\cos 4-13-15$ & $1.34 E+00$ & $3.25 E+02$ & $1.48 E-31$ & $2.22 E-10$ \\
\hline $15-16$ & $4.61 E+10$ & $4.24 E+02$ & 4.6ZE-01 & $1.52 E-69$ \\
\hline
\end{tabular}


TABLE B.9a. Potassium (K) Leach Fractions from Static Leach Test--Whole Grout in Groundwater (DIF5-17)

\begin{tabular}{|c|c|c|c|}
\hline SAKPLE & Cun. An/Ao & TIME days & $D$ cull. \\
\hline CRH $5-17-1$ & $3.76 E-02$ & $7.00 E+100$ & $3.52 E-09$ \\
\hline CRH $5-17-2$ & $1.27 \mathrm{E}-01$ & 1. $30 E+0 !$ & 3. $97 E-09$ \\
\hline CRin $5-17-3$ & $1.60 E-01$ & $2.00 E+01$ & $4.09 E-09$ \\
\hline CAH $5-17-4$ & 1.BIE-01 & $2,70 E+01$ & $3.88 E-09$ \\
\hline CRH 5-17-5 & $2.00 E-01$ & $3.40 E+01$ & $3.78 \mathrm{E}-19$ \\
\hline CRY $5-17-6$ & $2.07 E-01$ & $4.10 E+01$ & $3.70 E-09$ \\
\hline CRA 5-17-7 & $2.29 E-01$ & $4.80 E+01$ & $3.78 E-19$ \\
\hline $\cos 5-17-8$ & $2.47 E-01$ & $5.50 E+01$ & $3.96 E-09$ \\
\hline CRH $5-17-9$ & $2.66 E-01$ & $6.20 E+01$ & $4.17 E-09$ \\
\hline CAN $5-17-10$ & $2.71 E-01$ & $7.00 E+01$ & $3.69 E-09$ \\
\hline CRH $5-17-11$ & $2.805-01$ & $7.60 \mathrm{E}+01$ & $3.66 E-199$ \\
\hline CRI $5-17-13$ & $2.93 E-01$ & $1.34 E+02$ & $2.25 E-109$ \\
\hline $\mathrm{CFH} 5-17-14$ & $3.24 E-01$ & $1.90 E+02$ & $1.36 E-09$ \\
\hline CAH $5-17-15$ & $3.43 E-01$ & $3.15 E+02$ & $1.37 E-09$ \\
\hline
\end{tabular}

TABLE B.9b. Sodium (Na) Leach Fractions from Static Leach Test--Whole Grout in Groundwater (DIF5-17)

\begin{tabular}{|c|c|c|c|}
\hline SAMPLE & CUn. Ân/ÂO & TIME days. & 0 cus. \\
\hline CRH $5-17-1$ & 1.32E-01 & $7.00 E+00$ & $7.98 E-09$ \\
\hline CRU 5-17-2 & $2.24 E-01$ & $1.30 E+0 i$ & $1.24 E-\hat{0} 8$ \\
\hline CRY $5-17-3$ & $2.82 E-01$ & 2. $0 \mathrm{BE}+01$ & 1.39E-08 \\
\hline CRU $5-17-4$ & 3. $08 E-01$ & $2.70 E+01$ & $1.30 E-08$ \\
\hline Cกn 5-17-5 & $3.35 E-61$ & $3.40 E+01$ & 1. $27 E-08$ \\
\hline CRN 5-17-6 & $3.41 E-01$ & $4.10 E+01$ & $1.05 E-06$ \\
\hline CRH $5-17-7$ & $3.90 E-01$ & $4.80 E+01$ & $1.23 E-68$ \\
\hline CRU $5-17-9$ & $3.37 E-01$ & $5.50 E+01$ & ¿.14E-NG \\
\hline CRN $5-17-9$ & $4.29 E-01$ & $6.20 E+01$ & $1.20 E-0 B$ \\
\hline CFH $5-17-10$ & 4. 3AEE-01 & $7.000 E+01$ & $1.06 E-08$ \\
\hline CRU $5-13-11$ & $4.51 E-01$ & $i .60 E+01$ & 1. $.99 E-30$ \\
\hline CRU $5-17-13$ & 5.JiE-(1) & $1.34 E+02$ & $7.29 E-09$ \\
\hline Can $5-17-14$ & $5.41 E-01$ & $1.90 E+02$ & 6. B6E-69 \\
\hline Can $5-17-15$ & $5.93 E-01$ & J.15E+iz & $5.235-69$ \\
\hline
\end{tabular}


TABLE B.9C. Aluminum (A1) Leach Fractions from Static Leach Test--Whole Grout in Groundwater (DIF5-17)

\begin{tabular}{|c|c|c|c|}
\hline SAMPLE & cun. An/Ao & TIHE days & D cus. \\
\hline CRI 5-17-1 & $1.795-03$ & $7.00 E+00$ & $1.47 \mathrm{E}-12$ \\
\hline CRH 5-17-2 & $4.17 E-03$ & $1.30 E+01$ & $4.30 E-12$ \\
\hline CRA 5-17-3 & $5.51 E-03$ & $2.00 E \div 0 !$ & $4.86 E-12$ \\
\hline CRN 5-17-4 & $5.96 E-03$ & $2.70 E+01$ & $4.2 \geq 2-12$ \\
\hline $\cos 5-17-5$ & $1.81 E-03$ & $3.40 E+01$ & $3.10 E-13$ \\
\hline CRH 5-17-6 & $6.695-03$ & $4.10 E+01$ & $3.50 E-12$ \\
\hline $\operatorname{cosin} 5-17-7$ & $7.32 E-03$ & $4.80 E+01$ & $3.56 E-12$ \\
\hline CRI $5-17-8$ & $7.47 E-03$ & $5.50 E+01$ & $3.25 E-12$ \\
\hline CRH 5-17-9 & $0.14 E-03$ & $6.20 E+01$ & $3.43 E-12$ \\
\hline CRA $5-17-10$ & B. $42 E-03$ & $7.60 E+01$ & $3.25 E-12$ \\
\hline Cot 5-17-11 & $8.58 E-03$ & $7.606 E+01$ & $3.11 E-12$ \\
\hline CRH $5-17-13$ & $1.01 E-02$ & $\because .34 E+02$ & $2.43 E-12$ \\
\hline CRit 5-17-14 & $1.12 \leq-02$ & $1.5 \mathrm{VE}+02$ & 2. $13 E-12$ \\
\hline CRH 5-i7-:5 & $1.2 B E-012$ & $3.15 E+02$ & I. $6 T E-12$ \\
\hline
\end{tabular}

TABLE B.9e. Boron (as H3B03) Leach Fractions from Static Leach Test--Whole Grout in Groundwater (DIF5-17)

\begin{tabular}{|c|c|c|c|}
\hline SAMPLE & CUn. An/Ao & TIRE dày & D cus. \\
\hline CRU $5-17-1$ & 6. $105-03$ & $7.00 E+00$ & $1.7 i E-11$ \\
\hline Cont $5-17-2$ & $1.22 \mathrm{E}-02$ & $\mathrm{i} .30 \mathrm{E}+\mathrm{O} \mathrm{i}$ & $3.67 E-11$ \\
\hline CRN $5-17-3$ & $1.47 E-02$ & $2.00 E+01$ & $3.47 E-11$ \\
\hline CRN 5-17-4 & $1.61 E-02$ & $2.70 E+01$ & 3.07E-11 \\
\hline CRU $5-17-5$ & $1.60 E-02$ & $3.40 E+01$ & 3. $05 E-[1]$ \\
\hline CRH 5-17-6. & $1.88 E-02$ & $4.10 E+01$ & $2.77 \mathrm{E}-111$ \\
\hline CHW 5-17-7 & 2.0BE-02 & $4.80 E+01$ & $2.92 \mathrm{E}-11$ \\
\hline CRN $5-17-8$ & 2.14E-02 & $5.50 E+01$ & $2.68 E-11$ \\
\hline CRN 5-17-9 & $2.32 E-02$ & $6.20 E+01$ & $2.78 E-11$ \\
\hline CRU $5-17-10$ & $2.39 E-62$ & 7.00E+0! & $2.615-11$ \\
\hline CRU $5-17-11$ & $2.42 E-02$ & $7.60 E+01$ & $2.47 E-! 1$ \\
\hline CAM $5-17-13$ & $2.61 E-02$ & $1.34 E+02$ & 1.63E-1! \\
\hline CRW 5-17-14 & 3. $11 E-\hat{v} 2$ & 1. $\hat{v} E E+\hat{v} \hat{z}$ & $1.655-1 !$ \\
\hline CAY $5-17-15$ & $3.45 E-12$ & 3.15E:02 & 1. $21 E-!:$ \\
\hline
\end{tabular}


TABLE B.9f. Fluoride (F) Leach Fractions from Static Leach Test--Whole Grout in Groundwater (DIF5-17)

\begin{tabular}{|c|c|c|c|}
\hline SAMPLE & Cus. An/Ao & TIKE days & 0 cus. \\
\hline CRH $5-17-1$ & 4. $05 E-03$ & 7. $00 E+00$ & $7.53 E-12$ \\
\hline CRN $5-17-2$ & $3.80 E-03$ & $1.30 E+01$ & $3.56 E-12$ \\
\hline CRH $5-17-3$ & $4.95 E-0 J$ & $2.00 E+01$ & $3.93 E-12$ \\
\hline CAN 5-17-4 & $5.31 E-03$ & $2.70 E+01$ & $3.35 E-12$ \\
\hline CRH 5-17-5 & $5.30 E-03$ & $3.405+01$ & $3.28 E-12$ \\
\hline CR $5-17-6$ & $6.13 E-63$ & $4.10 E+01$ & $2.94 E-12$ \\
\hline CRH 5-17-7 & $6.82 E-03$ & $4.80 \mathrm{E} \div 01$ & $3.10 E-12$ \\
\hline CRI 5-17-8 & 7. $30 E-03$ & $5.50 E+01$ & $3.11 E-12$ \\
\hline CRU 5-17-9 & $3.705-03$ & $6.20 E+01$ & 3.06E-12 \\
\hline CRM $5-17-10$ & $8.19 E-63$ & $7.00 \mathrm{E}+01$ & 3.07E-12 \\
\hline CRH 5-17-11 & B. $73 E-03$ & $7.60 \mathrm{E}+01$ & $3.21 E-12$ \\
\hline 새 $5-17-13$ & $1.03 E-02$ & $1,34 E+02$ & $2.53 E-12$ \\
\hline CSW $5-17-14$ & $1.35 E-02$ & $1.90 E+02$ & $3.09 E-12$ \\
\hline CAN 5-17-15 & $1.29 E-02$ & $3.15 E+02$ & 1.69E-12 \\
\hline
\end{tabular}

TABLE B.9g. Nitrate (NO3) Leach Fractions from Static Leach Test--Whole Grout in Groundwater (DIF5-17)

\begin{tabular}{|c|c|c|c|}
\hline $\begin{array}{l}\text { SAKPLE } \\
\text { CFY 5-:7-1 }\end{array}$ & Cus. An $/ A O$ & TIME days & $D$ cus. \\
\hline $\begin{array}{l}\text { CRU } 5-17-1 \\
\cos 5-17-2\end{array}$ & $\begin{array}{l}3.985-V 6 \\
5.93 E-02\end{array}$ & $1.30 E+01$ & $\begin{array}{l}1.51 E-17 \\
8.68 E-10\end{array}$ \\
\hline CRN $5-17-3$ & $7.56 E-02$ & 2.00E+01 & $9.17 E-10$ \\
\hline CAN 5-17-4 & $8.41 E-62$ & $2.70 \varepsilon+01$ & $8.40 E-10$ \\
\hline CRN 5-1i-5 & $1.2 B E-01$ & $3.40 E+01$ & $1.55 E-09$ \\
\hline CRN S-17-6 & 1.6bE- $i j$ & 9.10E+01 & $2.16 E-09$ \\
\hline 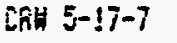 & $1.97 E-01$ & $4.80 E+01$ & $2.59 E-69$ \\
\hline CRH $5-17-9$ & $3.10 E-01$ & $5.50 E+0 !$ & $2.57 E-09$ \\
\hline CKN $5-17-9$ & $2.13 E-01$ & $6.20 E+01$ & $2.35 E-09$ \\
\hline Con 5-17-10 & $2.35 E-01$ & 7. OAE +01 & $2.34 E-09$ \\
\hline CFW $5-17-i 1$ & $2.46 E-01$ & $7.60 E+01$ & $2.87 E-6 \%$ \\
\hline CAN $5-17-13$ & $2.81 E-01$ & $1.34 E+02$ & $2.08 E-49$ \\
\hline 제 $5-17-14$ & $2.95 E-01$ & $1.90 \mathrm{E}+02$ & 1.72E-09 \\
\hline CFH $5-17-15$ & $3.59 E-1 \mathrm{il}$ & 3.1 SEE +02 & $1.56 E-99$ \\
\hline
\end{tabular}


TABLE B.9h. Sulfate (SO4) Leach Fractions from Static Leach Test--Whole Grout in Groundwater (DIF5-17)

\begin{tabular}{|c|c|c|c|}
\hline SAMPLE & Cun. Ân/AO & TIME days & $\theta$ cun. \\
\hline CRU $5-17-1$ & 2.40E-01 & $7.00 E+00$ & $2.84 E-08$ \\
\hline CRH $5-17-2$ & $2.00 E-01$ & $1.30 E+01$ & $9.88 E-109$ \\
\hline CRM $5-17-3$ & $2.35 E-01$ & $2.00 E+01$ & $9.96 E-09$ \\
\hline CAN $5-17-4$ & $2.66 E-01$ & $2.70 E+01$ & $9.57 E-09$ \\
\hline Con 5-17-5 & $2.72 E-01$ & $3.40 E+01$ & $7.60 E-09$ \\
\hline CAM 5-17-6 & 2. $.61 E-01$ & $4.10 E+01$ & $5.78 E-09$ \\
\hline CFA $5-17-7$ & $3.54 \varepsilon-01$ & $4.60 E+01$ & $9.56 E-69$ \\
\hline ลคบ $5-17-8$ & $3.58 E-01$ & $5.50 E+01$ & $8.94 E-09$ \\
\hline CFIN $5-17-9$ & $3.77 E-01$ & $6.20 E+01$ & $9.00 E-09$ \\
\hline CRI $5-17-10$ & $3.94 E-01$ & 7.00E+01 & $8.47 E-69$ \\
\hline CRA $5-17-11$ & $9.10 E-01$ & 7. $60 \mathrm{E} \div 01$ & 8.76E- 09 \\
\hline $\cos 5-17-13$ & $4.12 E-01$ & $1.34 E+02$ & $4.97 E-09$ \\
\hline CRA $5-17-14$ & $9.76 E-011$ & $1.90 E+02$ & 5. $13 E-09$ \\
\hline $\operatorname{sen} 5-17-15$ & 6. $47 E-01$ & $3.15 E+02$ & b. $85 E-199$ \\
\hline
\end{tabular}

TABLE B.9i. Cesium-137 (CS-137) Leach Fractions from Static Leach Test--Whole Grout with Groundwater (5-17)

\begin{tabular}{|c|c|c|c|c|}
\hline SAYPLE & $\mathrm{uCi}$ & TIKE DAYS & Án/ÁO & D fla cazisec \\
\hline CRN-5-17-1 & $0.00 E+00$ & $7.00 E+00$ & 8, 06E-69 & $2.96 E-23$ \\
\hline CRH $-5-17-2$ & $0.00 E+00$ & $1.30 E+0 !$ & Q. 66 E- 99 & $1.605-23$ \\
\hline$[\pi N-5-1 i-3$ & $0.00 E+00$ & $2.00 E+01$ & $8.06 E-69$ & $1.04 E-23$ \\
\hline [RI $-5-1]-4$ & $3.165-63$ & $2.70 E+01$ & $9.25 E-04$ & $1.02 E-13$ \\
\hline CRH-5-17-5 & $7.23 E-04$ & $3.40 E+01$ & $2.14 E-04$ & $4.30 E-15$ \\
\hline CRH-5-17-6 & $2.99 E-03$ & 4.10E+01 & $8.75 E-04$ & $6.62 E-34$ \\
\hline CRH-5-17-7 & $6.74 E-14$ & $4,60 E+01$ & 1. . \$BE-04 & $2,61 E-15$ \\
\hline$C W N-5-17-8$ & $1.43 E-03$ & $5.50 E+61$ & 4. $19 E-04$ & $1.02 E-14$ \\
\hline CKH-5-17-? & $2.01 E-03$ & $6.20 E+\hat{01}$ & $5.90 E-(19$ & i. $30 E-14$ \\
\hline $\cos 1-5-17-10$ & $1.46 E-03$ & $7.00 E+01$ & $4.27 E-64$ & $8.335-15$ \\
\hline CRII-5-17-11 & $2.31 E-43$ & $7.60 E+01$ & $6.7 j E-04$ & $1,93 E-14$ \\
\hline $6 \operatorname{con}-5-17-12$ & $2.265-03$ & $1.04 E+02$ & $6.635-64$ & $1.35 E-14$ \\
\hline CAU $-5-! 7$ & $1.28 E-63$ & $1.34 E \div 02$ & $3.75 E-64$ & $3.37 E-: 5$ \\
\hline
\end{tabular}


TABLE B.9i. Cesium-137 (CS-137) Leach Fractions from Static Leach Test--Whole Grout with Groundwater (5-19)

\begin{tabular}{|c|c|c|c|c|}
\hline GAMPLE & uCj & TIME DAYS & An/AO & if cue csisec \\
\hline CRH $-5-19-1$ & $6.92 E-04$ & $7.00 E+00$ & $2.04 E-04$ & $1.915-14$ \\
\hline CRK-5-19-2 & $1.54 E-03$ & $1.30 E+01$ & $4.54 E-04$ & $5.09 E-14$ \\
\hline CRH $-5-19-3$ & $3.39 E-03$ & $2.00 E+01$ & $9.995-04$ & 1. $60 \bar{E}-1 j$ \\
\hline CRI $-5-19-4$ & $2.03 E-113$ & $2.70 E+01$ & $5.97 E-014$ & $4.23 E-14$ \\
\hline SNV-5-19-5 & $1.50 E-03$ & $3.40 E+01$ & 4. 43E-09 & 1.35E-14 \\
\hline CRR-5-19-6 & $3.48 E-03$ & $4.10 E+01$ & $1.03 E-03$ & B. $25 E-14$ \\
\hline cfin-5-19-7 & $2.245-03$ & $4.80 E+01$ & $6.61 E-04$ & 2. $72 E-14$ \\
\hline$C R H-5-19-8$ & $2.4 B E-0]$ & $5.50 E+01$ & $7.3 ! E-04$ & $3.11 E-14$ \\
\hline CRN-5-;9-9 & $0.00 E+00$ & $6.20 E+01$ & 5.11E-69 & $54,40 E-24$ \\
\hline CRU $5-19-10$ & $1.15 E-03$ & 7. $00 E+01$ & $3.39 E-04$ & $5.25 E-15$ \\
\hline CRN-5-19-i1 & $0,00 E+00$ & $7.60 E+01$ & 5.11E-09 & $2.77 E-24$ \\
\hline CKit $-5-19-12$ & $1.17\{-0\}$ & $1.04 E+02$ & $3.47 E-64$ & $3.70 E-15$ \\
\hline CRA $-5-19-13$ & $1.63 E-03$. & 1.3HE+ +02 & $4.81 E-64$ & $5.52 \varepsilon-15$ \\
\hline ERH-5-19-14 & $1.66 E-03$ & $1.90 E+02$ & 4.70E-69 & $4.65=-15$ \\
\hline
\end{tabular}

TABLE B.9j. Strontium-85 (SR-85) Leach Fraction from Static Leach Test-Whole Grout in Groundwater (5-17)

\begin{tabular}{|c|c|c|c|c|}
\hline SAMPLE & TIME DAYS & $A n / A D$ & D. cus. & UË: LEACHED \\
\hline $\operatorname{CRH} 5-17-1$ & $7.00 E+00$ & $4.67 E-08$ & 3. $38 E-32$ & 6. $00 E+00$ \\
\hline CRN $5-17-2$ & $1.30 E+01$ & $3.47 E-03$ & $2.96 E-12$ & $2.18 E-04$ \\
\hline CRH 5-17-3 & $2.00 E+01$ & $2.89 E-04$ & $1.34 E-14$ & $0.00 E+00$ \\
\hline CRY 5-i7-4 & $2.70 E+01$ & $2.89 E-04$ & $9.90 E-15$ & V. $.00 E+00$ \\
\hline CRH 5-i7-5 & J.40E+01 & $3.22 E-03$ & $9.74 E-13$ & $1.84 E-64$ \\
\hline Can $5-17-6$ & $4.10 E+01$ & $5.02 E-03$ & $1.97 E-12$ & 2. $83 E-04$ \\
\hline Cละ 5-17-7 & $4.80 E+01$ & $4.19 E-03$ & $1.17 E-12$ & $2.07 E-94$ \\
\hline CAN $5-17-8$ & $5.50 E+01$ & $5.70 E-03$ & $1.87 E-12$ & $2.85 E-04$ \\
\hline CAH $5-17-9$ & $6.20 E+01$ & $6.20 E-03$ & $1.99 E-12$ & $2.92 E-1.4$ \\
\hline CRU 5-17-i0 & $7.00 E+01$ & $7.94 E-03$ & $2.89 E-12$ & $3.78 E-94$ \\
\hline CRU $5-17-11$ & $7.6 \hat{E E}+01$ & 2. $94 E-03$ & $2.52 E-13$ & $0.00 E+00$ \\
\hline CRH $5-17-12$ & $1.04 E+102$ & $2.74 E-03$ & $1.84 E-13$ & $0.96 E+00$ \\
\hline CEM $5-17-13$ & $1.34 E+32$ & $4.81 E-02$ & 7.ลิระด-:ล & S. SBEE-OS \\
\hline CAL $5-17-14$ & $\therefore .70 E+\sqrt[1]{2}$ & Q.11E-63 & 6.40E-12 & $3.35 E-04$ \\
\hline CSH $5-17-15$ & J.15E+02 & $2.5 B E-02$ & $6.75 E-12$ & $\vdots .30 E-60$ \\
\hline SRI $5-17-16$ & $4.24 E+02$ & $1.765-01$ & $2.34 E-10$ & 2.07E-02 \\
\hline
\end{tabular}


TABLE B.9j. Strontium-85 (SR-85) Leach Fraction from Static Leach Test-Whole Grout in Groundwater (5-19)

\begin{tabular}{|c|c|c|c|c|c|}
\hline SAMPLE & TIME. DAYS & An/AD & D cun. & D inc. & UCi LEACHED \\
\hline CRH-5-19-1 & 7. $00 E+00$ & $6.01 E-03$ & $1.655-11$ & $3.31 E-11$ & $3.77 E-04$ \\
\hline CRH-5-19-2 & $1.30 E+01$ & $3.50 E-03$ & $3.01 E-12$ & 4. 35E-11 & 1. $88 E-04$ \\
\hline$C R M-5-19-3$ & $2,00 E+01$ & $7.51 E-04$ & $9.03 E-14$ & $2.43 E-12$ & $0.00 E+00$ \\
\hline CAH-5-19-4 & $2.70 E+01$ & $7.51 E-04$ & $6.69 E-14$ & $3.46 E-12$ & $0.00 E+00$ \\
\hline$C R H-5-19-5$ & $3.40 E+01$ & $6.28 E-03$ & $3.72 E-12$ & 3.15E-10 & $3.46 E-04$ \\
\hline CRU $1-5-19-6$ & $4.10 E+01$ & $1.21 E-03$ & $1.15 E-13$ & $1.44 E-11$ & $0.06 E+00$ \\
\hline$C R^{4}-5-19-7$ & 1.8OE+01 & 1. $14 E-02$ & $8.68 E-12$ & $1.51 E-09$ & દ. $38 E-04$ \\
\hline$C R H-5-19-8$ & $5.50 E+01$ & $1.23 E-02$ & $8.82 E-12$ & $2.04 E-09$ & $6.41 E-04$ \\
\hline CAH-5-19-9 & $6.20 E+01$ & $1.20 E-02$ & 7.49E-12 & $2.22 E-09$ & $5.71 E-64$ \\
\hline CRH-5-19-10 & 7.00E+01 & $3.68 E-03$ & 6. $18 E-13$ & $1.795-10$ & $0.00 E+00$ \\
\hline $\begin{array}{l}C F H-5-19-11 \\
\text { CRH } \\
5-19-12\end{array}$ & $\begin{array}{l}7.60 E+01 \\
1.04 E+02\end{array}$ & $\begin{array}{l}3.68 E-03 \\
3.68 E-03\end{array}$ & $\begin{array}{l}5.69 E-13 \\
4.16 E-13\end{array}$ & $\begin{array}{l}3.51 E-10 \\
1.99 E-11\end{array}$ & $\begin{array}{l}0.00 E+00 \\
i .00 E+00\end{array}$ \\
\hline CAK-5-19-13 & $1.34 E+02$ & 3.76E-03 & $2.2 B E-12$ & $1.61 E-10$ & $3.81 E-04$ \\
\hline CRU-5-17-14 & 1. $30 E+12$ & $1.14 E-02$ & $2.21 E-12$ & 3.66E-11 & $4.55 E-04$ \\
\hline
\end{tabular}

TABLE B.10i. Americium-241 (AM-241) Leach Fractions from Static Leach Test-Whole Grout in Groundwater (1-17)

\begin{tabular}{|c|c|c|c|c|}
\hline SAMPLE & UCi & TIKE DAYS & An $/ A O$ & $0 \mathrm{cus} \mathrm{cs} / \mathrm{set}$ \\
\hline CSA $1-17-1$ & $1.78 E-04$ & $7.00 E+00$ & $1.32 E-05$ & $\because 7.39 E-17$ \\
\hline CRN $1-17-2$ & $8.17 E-05$ & $1.30 E+01$ & $6.14 E-06$ & B. $66 E-18$ \\
\hline CAH $:-17-3$ & $2.05 E-04$ & $2.00 E+01$ & $1.51 E-05$ & $3.40 E-17$ \\
\hline $\operatorname{Con} 1-17-4$ & $8.17 E-05$ & $2.70 E+01$ & b. $14 E-06$ & $4.17 E-18$ \\
\hline CRE $1-17-5$ & $1.23 E-03$ & $3.40 E+01$ & $8.94 E-05$ & $7.02 E-16$ \\
\hline Cail 1-17-6 & $1.58 E-04$ & $4.10 E+01$ & 1.17E-65 & $9.92 E-16$ \\
\hline CRU $1-17-j$ & $8.1 T E-05$ & $4.80 E+01$ & $6.14 E-06$ & $2.35 E-18$ \\
\hline CRA $1-17-8$ & $1.64 E-04$ & $5.50 E+01$ & $1.21 E-05$ & $7.97 E-18$ \\
\hline CRA :-17-9 & $1.11 E-04$ & $6.20 E+01$ & $8.25 E-06$ & $3.285-18$ \\
\hline CRH $1-17-10$ & $1.52 E-04$ & $7.00 E+01$ & $1.13 \mathbf{E}-0 \mathrm{E}$ & $5.40 E-16$ \\
\hline CRU $1-17-11$ & $2.66 E-04$ & $7.605+01$ & $1.96 E-05$ & $1.50 E-17$ \\
\hline CRY $1-17-12$ & $1.58 E-04$ & 1. $04 E+0 z$ & $1.17 E-05$ & 3..71E-18 \\
\hline [aU $:-17-13$ & i. $58 E-04$ & $1.3+E+02$ & $1.17 E-65$ & $3.04 E-10$ \\
\hline ERM $\{-1 T-14$ & $2.45 E-04$ & $1.90 E+02$ & 1. $60 E-65$ & $5.11 E-18$ \\
\hline CRH $1-17-15$ & $4.70 E-04$ & $3.15 E+02$ & $3.44 E-65$ & 1. $12 E-17$ \\
\hline
\end{tabular}


TABLE B.10i. Americium-241 (AM-241) Leach Fractions from Static Leach Test-Whole Grout in Groundwater $(1-18)$

\begin{tabular}{|c|c|c|c|c|}
\hline SAYPLE & $u[j$ & TIKE OAYS & An/AO & 0 sue con $2 / \mathrm{sec}$ \\
\hline$C A N+-1 B-1$ & $1.04 E-03$ & $7.00 E+00$ & $7.56 E-015$ & 2.55E-15 \\
\hline $\operatorname{can} 1-18-2$ & 8.17E-04 & $1.30 E+01$ & $5.97 E-0.05$ & 3.57E-16 \\
\hline$C R=1-18-3$ & $8.02 E-04$ & $2.00 E+01$ & $5.86 E-05$ & 5. $38 E-16$ \\
\hline CRN $1-18-4$ & $8.72 E-04$ & $2.70 E+01$ & $6.37 E-05$ & $4.70 \mathrm{E}-16$ \\
\hline CRN $1-18-5$ & $7.21 E-104$ & $3.40 E+01$ & $5.27 E-05$ & $2.55 E-16$ \\
\hline CAK $1-18-6$ & 8.J1E-04 & 4. $10 E+01$ & $6.07 E-05$ & $2.81 E-16$ \\
\hline CRH :-10-7 & $7.62 E-04$ & $4.30 E+01$ & $5.5 \pi E-05$ & $2.02 E-10$ \\
\hline CRU $1-18-8$ & $7.62 \mathrm{E}-04$ & $5.56 E+01$ & $5.57 E-05$ & 1.7DE-16 \\
\hline CRN 1-18-9 & $7.62 E-04$ & $6.20 \mathrm{EE}+01$ & J.57E- 0.5 & 1. SBEE-16 \\
\hline CAN $:-16-i 0$ & $7.625-34$ & 7. $00 E+01$ & $5.57 E-0.5$ & $1.305-16$ \\
\hline $\operatorname{con}:-18-11$ & $9.33 E-04$ & $7.60 E+0 !$ & S.81E-05 & 1. $.71 E-36$ \\
\hline CAN $1-18-12$ & $7.96 E-0.94$ & 1. $04 E+02$ & $5.82 E-i 15$ & 1. $12 \mathrm{E}-16$ \\
\hline CRH $1-10-13$ & $3.96 E-04$ & $1.34 E \div 02$ & $5.62 E-15$ & $7.30 E-17$ \\
\hline CFit :-18-14 & $7.76 E-694$ & $1.90 E+62$ & $5.32 E-65$ & $5.57 E-17$ \\
\hline CFH $1-18-15$ & $4.35 E-64$ & $3.15 E+02$ & $3.19 E-05$ & 1.01E-17 \\
\hline
\end{tabular}

TABLE B.10j. Plutonium-238 (PU-238) Leach Fractions from Static Leach Test-Whole Grout in Groundwater (1-17)

\begin{tabular}{|c|c|c|c|c|}
\hline SAMPLE & vij $\mathrm{i}$ & TIME DAYS & An/AO & 0 cun ch $2 / \sec$ \\
\hline chin $1-17-1$ & $1.16 E-03$ & 7. $00 \mathrm{O} \div 00$ & $7.90 E-05$ & $2.66 E-15$ \\
\hline CAN $1-17-2$ & $1.46 E-03$ & $1.30 E+01$ & $9.75 E-65$ & $2.27 E-15$ \\
\hline CRA $1-17-3$ & $1.2 i E-03$ & 2. $\mathrm{OOE} \div 01$ & $8.62 E-05$ & $1.11 E-15$ \\
\hline CRU $1-17-4$ & i. $39 E-63$ & $2.70 E+01$ & $9.46 E-05$ & $9.89 E-16$ \\
\hline CRW $1-17-5$ & $1.20 E-03$ & $3.40 E+01$ & $3.18 E-35$ & 5. $.8 E E-16$ \\
\hline CRN :-17-b́ & i. $44 E-63$ & $4.10 E+01$ & $5.79 E-65$ & $0.97 E-16$ \\
\hline CRH : $-17-7$ & ¿.19E-63 & 4. $60 E \div 01$ & $3.07 E-05$ & 4. $055-16$ \\
\hline Can $1-17-8$ & $1.20 E-03$ & $5.50 E+0 !$ & S. $13 E-05$ & 3. $65 \mathrm{E}-16$ \\
\hline CNAll 1-17-9 & 1. $15 E-13$ & $0.20 E+01$ & $i .35=-45$ & $2.97 E-16$ \\
\hline CRH $1-17-10$ & $1.22 E-03$ & T. OOE+UI & 3. $29 E-05$ & 2. $33 E-16$ \\
\hline ChA $1-17-11$ & $1.29 E-03$ & $7.30 E+01$ & 3. $30 E-0.5$ & $3.04 E-36$ \\
\hline Cก" : $-\{i-12$ & $i .20 E-13$ & 1. $04 E+32$ & $8.15 E-i j \bar{z}$ & 1. BiE-16 \\
\hline Crial $:-1 j-13$ & $1.16 E-13$ & $1.34 E+02$ & T. $70 E-i 5$ & 1.37E-16 \\
\hline
\end{tabular}


TABLE B.10j. Americium-241 (Am-241) Leach Fractions from Static Leach Test-Whole Grout in Groundwater (1-18)

\begin{tabular}{|c|c|c|c|c|}
\hline SAMPLE & UCi & TIME DAYS & An/AOO & $D$ cun cažisec \\
\hline CRA $1-18-1$ & $1.09 E-03$ & 7. $.00 E+00$ & $8.015-05$ & $2.86 E-15$ \\
\hline CRN $1-18-2$ & $1.29 E-03$ & $1.30 E+01$ & $9.44 E-05$ & $2.15 E-15$ \\
\hline CRW $1-18-3$ & $1.13 E-03$ & $2.00 E+01$ & $8.29 E-05$ & $1.07 E-15$ \\
\hline CRN $1-10-4$ & $1.33 E-03$ & $2.70 E+01$ & $9.74 E-05$ & 1.1UE-15 \\
\hline Cnl i-18-5 & $1.08 E-133$ & $3.40 E+01$ & $7.97 E-05$ & $5.85 E-16$ \\
\hline CRA 1-18-6 & $1.09 E-03$ & 4.J0E+01 & $8.015-05$ & 4. $295-16$ \\
\hline CRH $:-18-7$ & $1.19 E-03$ & $4.30 E+01$ & $8.71 E-05$ & $4.955-15$ \\
\hline CR: :-18-8 & $1.23 E-03$ & $5.50 E+01$ & $9.07 E-05$ & $4.68 E-16$ \\
\hline CRH 1-18-9 & $1.16 E-03$ & 6. $20 E+01$ & 6. $52 E-05$ & $3.675-16$ \\
\hline CRU $1-18-10$ & $1.20 E-63$ & $7.00 E+01$ & 8. $83 \mathrm{E}-05$ & $3.4 a E-16$ \\
\hline CRH :-18-11 & $1.19 E-03$ & $7.60 E+01$ & $8.76 E-05$ & 3. $10 E-10$ ó \\
\hline CEN $1-18-12$ & 1.25E-03 & $1.04 E+02$ & $9.15 E-1.5$ & $2.52 E-16$ \\
\hline CAH $:-18-13$ & $1.21 E-03$ & 1. $3 A E+62$ & 3.86E-05 & $1.84 E-16$ \\
\hline
\end{tabular}


TABLE B.11a. Potassium (K) Leach Fractions from Flow Through Test Grout/Sediment with Groundwater (DIFCOLT2)

\begin{tabular}{|c|c|c|c|c|c|}
\hline SAHPLE & $\mathrm{An} / \mathrm{Ao}$ & CuL. An/Âo & DÁYS & D inc. & 0 cus. \\
\hline EFFLUENT 1 & $0.00 E+00$ & $0.00 E+00$ & $3.0 \hat{O E}+00$ & $0.00 E+00$ & $0.00 E+00$ \\
\hline EFFLUEKT 2 & $0.00 E+00$ & $0.00 E+00$ & $6.00 E+00$ & $0.60 E+00$ & $0.00 E+00$ \\
\hline EFFLUEKT 3 & $0.00 E+00$ & $0.00 E+00$ & $9.00 E+00$ & $0.00 E+00$ & $0.00 E+00$ \\
\hline EFFLUEKT 4 & $0.00 E+00$ & $0.00 E+00$ & $1.30 E+01$ & $0.00 E+00$ & $0.00 E+00$ \\
\hline EFFLUERT 5 & $0.60 E+00$ & O. OUE +OO & $1.70 E+01$ & $0.60 E+00$ & $0.00 E+0 \hat{O}$ \\
\hline EFFLUEHT $b$ & $0.00 E+00$ & $0 . \hat{v} \cup E+00$ & $2.60 E+01$ & $0.00 E+00$ & $0.06 E \div 00$ \\
\hline EFFLUEHT 7 & $0.60 E+00$ & $0.00 E+C 0$ & 2. $30 E+01$ & $0.00 E+00$ & $0.60 E+00$ \\
\hline EFFLUIERT 3 & $0.00 E+60$ & $0.00 E+00$ & $2.70 E+01$ & $0.60 E+00$ & $0.00 E+00$ \\
\hline EFFLUENT $\hat{3}$ & $0.00 E+00$ & $0.00 E+00$ & J. $60 E+01$ & $0.000 E+00$ & 0. $00 E \div 0$ ? \\
\hline EFFLIENT 10 & $0.00 E+00$ & $0.00 E+00$ & $3.40 E+01$ & $0.00 E+06$ & $0.00 E \div 100^{\circ}$ \\
\hline EFFLUEHT II & $0.00 E+60$ & $0.00 E \div 00$ & $3.70 E+01$ & $0.00 E+60$ & $0.06 E+60$ \\
\hline EFFLUENT 12 & $0.00 E+00$ & $0.00 E+60$ & $\$ .10 E+01$ & $0.00 E \div 60$ & $0.06 E+00$ \\
\hline EFFLUENT 13 & $0.00 E+10$ & $0.00 \mathrm{E}+00$ & $\$ .40 E+0:$ & O. OUE+00 & 0. $06 E+10$ \\
\hline COLLHAN T2 & & & & & \\
\hline EFFLUENT 16 & 6. $60 E+00$ & $0.00 E+00$ & $5.80 E+01$ & $0.00 E+00$ & $0.00 E+00$ \\
\hline EFFLUENT 17 & $0.00 E+00$ & $0.00 E+00$ & $6.205+01$ & $0.00 E+00$ & $0.00 E+00$ \\
\hline EFFLUEHT IB & $0.00 E+00$ & $0.60 E+00$ & 3. $50 E+01$ & $0.0 \mathrm{OE}+00$ & U. $Q \hat{O O E}+00$ \\
\hline EFFLUENT 19 & $0.00 E+00$ & $0.00 E+00$ & $6.90 E+01$ & $0.00 E+00$ & $0.00 E+00$ \\
\hline EFFLUENT 20 & $0.00 E+00$ & $0.00 E+00$ & $7.20 E+01$ & $0.00 E+00$ & 0.00E+00 \\
\hline EFFLUENT 21 & $0.00 E+00$ & $0.00 E+00$ & $7.60 E+01$ & $0.00 E+\hat{V O O}$ & $0.00 E \div 60$ \\
\hline EFFLUEHT 22 & $0.00 E+00$ & $0.00 E+00$ & $8.00 E+01$ & $0.00 E+00$ & $0.00 E+00$ \\
\hline EFFLUEHT 23 & $0.00 E+00$ & $0.00 E+00$ & $8.40 E+01$ & $0.00 E+00$ & $0.00 E+00$ \\
\hline EFFLUE:IT 24 & $0.00 E+00$ & $0.00 E \div 00$ & $0.70 E+01$ & $0.60 E+00$ & D. $00 E+00$ \\
\hline EFFIUENT 26 & $0.00 \varepsilon+00$ & $0.00 E+00$ & $9.40 E+01$ & $0.00 E+00$ & 1. $60 \mathrm{E}+00$ \\
\hline EFFLUENT 23 & $0.00 E+00$ & $0.00 E+60$ & 1. $60 E+62$ & $0.00 E+00$ & $0.00 E+00$ \\
\hline EFFLUENT 30 & $1.70 \mathrm{E}-0 \mathrm{~J}$ & $1.90 E-0 J$ & $1.07 E+02$ & $7.73 E-11$ & $03.78 E-! 4$ \\
\hline EFFLUJEHT 32 & $1.24 E-02$ & $1.43 E-02$ & 1. $15 E+6 z$ & $2.76 \bar{c}-\hat{0} ?$ & 4.60E-!2 \\
\hline EFFLLENT 34 & $1.07 E-62$ & 2.50E-02 & $1.21 E+02$ & 3. PUE -09 & $1.34 E-11$ \\
\hline EFELUENT 36 & $2.21 E-02$ & $4.71 E-02$ & $1.23 E+02$ & $1.30 E-68$ & $4.51 E-11$ \\
\hline EFFLUEUT З? & $5.61 E-0.2$ & $1.03 E-01$ & 1. $42 E+02$ & $2.26 E-68$ & $1.955-10$ \\
\hline EFFLUERT \&! & $3.14 E-\hat{\theta} 2$ & i. J5E-01 & 1. $49 E+02$ & $3.05 E-08$ & 3.10E-10 \\
\hline EFFLUERT 43 & $3.99 E-02$ & $1.75 E-01$ & $1.60 E+02$ & 2. $: 2 E-68$ & $4.75 E-i 0$ \\
\hline EFFLUENT 45 & $4.11 E-02$ & $2.16 E-01$ & 1. TZE+02 & $2.05 E-65$ & $\overline{1 . D E-10}$ \\
\hline EFFLUERT 47 & $3.905-32$ & 2.36E-01 & $1 . \equiv 1 E+02$ & $8.98 E-198$ & $7.965-10$ \\
\hline EFFLUEHT 49 & $8.43 E-02$ & $3.20 \mathrm{E}-01$ & $2.2: 5+02$ & $9.26 E-65$ & $1.20 E-09$ \\
\hline EFFLUERT 50 & $6.44 E-02$ & 3.84E-0! & 2.51E+02 & i.13E-ibo & $1.53 E-65$ \\
\hline
\end{tabular}


TABLE B.Ilb. Sodium (Na) Leach Fractions from Flow Through Test Grout/Sediment with Groundwater (DIFCOLT2)

\begin{tabular}{|c|c|c|c|c|c|}
\hline SAHPLE & An $/$ AO & CUL. An/AD & DAYS & Dinc. & D cuts. \\
\hline EFFLUEHT 1 & $2.52 E-02$ & $2.52 E-02$ & $3.00 E+00$ & 5. 53E-10 & 5. $53 E-10$ \\
\hline EFFLUERT 2 & 3.04E-02 & $5.56 E-02$ & $6.00 E+00$ & $4.66 E-09$ & $1.3+E-09$ \\
\hline EFFLUENT 3 & $2.83 E-02$ & $8.40 E-02$ & $9.00 E+60$ & $6.90 E-09$ & $2.04 E-09$ \\
\hline EFFLUENT \& & $3.65 E-02$ & $1.20 E-01$ & $1.30 E+01$ & $9.43 E-09$ & $2.90 \mathrm{E}-0 \mathrm{3}$ \\
\hline EFFLUENT 5 & $2.37 E-92$ & $1.44 E-\hat{v} !$ & $1.30 E+01$ & $5.96 E-69$ & 3. $18 E-09$ \\
\hline EFFLUENHT 6 & $1.39 E-02$ & $1.58 E-01$ & $2.00 E+01$ & $4.13 E-09$ & $3.25 E-69$ \\
\hline EFFLUENT 7 & $2.27 E-02$ & $1.61 E-01$ & $2.30 E+01$ & $1.26 E-08$ & $3.69 E-697$ \\
\hline EFFLUENT $\hat{a}$ & $3.10 E-102$ & $2.12 E-0 !$ & $2.70 E+01$ & $1.535-08$ & 5. $45 E-09$ \\
\hline EFFLUERT $\rightarrow$ & $2.34 E-02$ & $2.35 E-0 !$ & $3.00 E+01$ & $1.30 \mathrm{E}-60$ & $6.79 E-09$ \\
\hline EFFLUEHT 10 & $2.27 E-62$ & $2.58 E-01$ & $3.40 E+01$ & $1.67 E-06$ & $3.81 E-09$ \\
\hline EFFLUENT 11 & 1.60E-02 & $2.74 E-01$ & $3.705+01$ & $1.05 E-0 B$ & b. $3 i 5-i q ?$ \\
\hline EFFLUENT 12 & $2.00 E-62$ & $2.94 E-\hat{E l}$ & 4. $10 E+i j$ & $1.02 E-\hat{0} B$ & 7.205-09 \\
\hline EFFLUENT 13 & $1.41 E-02$ & 3. (BEE-0) & $4.40 E+011$ & $9.70 E-09$ & $7.75 E-69$ \\
\hline CDLLIMN T2 & & & & & \\
\hline EFFLUEHT !6 & $2.30 E-102$ & $3.36 E-01$ & $5.80 E+01$ & $2.11 E-09$ & $7.23 E-19$ \\
\hline EFFLUENT: $: 7$ & $2.21 E-02$ & $3.58 E-01$ & $6.20 E+01$ & $1.90 E-08$ & $7.71 E-09$ \\
\hline EFFLUENT 18 & $7.92 E-03$ & $3.66 E-01$ & $6.50 E+01$ & $4.61 E-09$ & $7.82 E-69$ \\
\hline EFFLUENT 19 & $1.61 E-02$ & $3.32 E-01$ & $6.90 E+0 i$ & $1.13 E-08$ & 7. BSE-05 \\
\hline EFFLUENT 20 & $8.41 E-03$ & $3.30 E-01$ & $7.20 E+01$ & $5.77 E-07$ & 7. PTE-Ti \\
\hline EFFIUENT 21 & 7.62E-0J & J. $96 E-01$ & $7.60 E+01$ & $2.75 E-09$ & a.01E-1? \\
\hline EFFLUEHT 22 & $1.52 E-02$ & $4.33 E-01$ & $8.00 E+01$ & $1 . j 7 E-0 B$ & $3.64 E-19$ \\
\hline EFFLUENT 23 & $8.735-03$ & $4.225-01$ & $8.40 E+01$ & $4.07 E-09$ & 5. $\{1 E-3\}$ \\
\hline EFFLUENT 24 & $5.74 E-03$ & $4.2 B E-0 !$ & $0.70 E+0 !$ & $3.26 E-09$ & $8.10 E-09$ \\
\hline EFFLUENT 26 & $1.42 E-02$ & $4.42 E-01$ & $9.4 \hat{v} E+01$ & $3.88 E-09$ & B. OBE-09 \\
\hline EFFLUEMT 28 & $1.78 E-02$ & $4.60 E-01$ & 1. $00 E+02$ & $8.84 E-09$ & D. 43E-D? \\
\hline EFFLUENT 30 & D.89E-0́3 & $4.67 E-01$ & $1.07 E+62$ & $1.04 E-00$ & E. $3 \mathbf{3 E}-09$ \\
\hline EFFLUERT 32 & B. $\{B E-03$ & $4.75 E-\hat{i} \mid$ & $1.15 E \div 12$ & $3.30 E-64$ & 3.12E-07 \\
\hline EFFLUENT 34 & $4.3 B E-63$ & $4.80 E-01$ & $1.21 E+02$ & D. SAE-10 & $i . T 2 E-0 ;$ \\
\hline EFFLUENT 3̇ & $8.65 E-03$ & $4.80 E-01$ & $1.28 E+02$ & i. $38 E-\hat{09}$ & $7.68 E-i q$ \\
\hline EFFLUENT 37 & $1.61 E-62$ & 5. $04 E-01$ & 1. $42 \mathrm{ZF}+\mathrm{O2}$ & $1.86 E-09$ & $\bar{i} .2 \mathrm{TE}-\mathrm{i} \mathrm{H}$ \\
\hline EFFLUENT \&1 & $6.29 E-03$ & $5.11 E-6 !$ & $1.47 E+02$ & $1.22 E-67$ & $i .26 E-99$ \\
\hline EFFLUEKT 43 & $6.40 E-i 13$ & $5,17 E-11$ & $1.3 \theta E+02$ & $5.45 E-16$ & $7.12=-09$ \\
\hline EFFLUENT & $5.806-01\}$ & E. 23E-01 & $1.72 E+02$ & $4.1 \mathrm{XE}-10$ & D.62E-09 \\
\hline EFF:UERT 47 & $4.57 E-03$ & $5.2 \pi \equiv-1 i$ & !. $61 E+02$ & $4.75 E-10$ & $0.6: E-69$ \\
\hline EFFLUENT 49. & 1. PВE- 12 & $5.475-31$ & $2.21 E+02$ & $5.135-10$ & 5. $955-09$ \\
\hline
\end{tabular}


TABLE B.11C. Aluminum (Al) Leach Fractions from Flow Through Test Grout/Sediment with Groundwater (DIFCOLT2)

\begin{tabular}{|c|c|c|c|c|c|}
\hline SAKPLE & $A n / A 0$ & CUR. An/ÂU. & BAYS & Dinc. & i) cus. \\
\hline EFFLUENT I & $6.05 E-05$ & 6.05E-05 & $3.00 E+00$ & 3. $: 8 E-15$ & $3.18 E-15$ \\
\hline EFFLUENT 2 & $1.5 \pi-04$ & $2.17 E-04$ & $6.00 E+00$ & $1.24 E-13$ & $2.05 E-14$ \\
\hline EFFLUENT 3 & $2.06 E-04$ & 4.24E-04 & 9. $00 E+00$ & $3.65 E-13$ & 5. 19E-14 \\
\hline EFFLUENT 4 & $4.06 E-04$ & $8.29 E-04$ & $1.30 E+01$ & $1.17 E-12$ & 1. $3 B E-13$ \\
\hline EFF:LIENT 5 & $3.32 E-04$ & $1.16 E-03$ & $1.70 E+01$ & $1.03 E-12$ & $2.065-13$ \\
\hline EFFLUEHT 6 & $1.91 E-04$ & $1.35 E-03$ & $2.00 E+01$ & $7.32 E-13$ & $2.36 E-13$ \\
\hline EFFLUENT 7 & $3.51 E-04$ & $1.70 E-03$ & $2.30 E+01$ & $3.06 E-12$ & 3. $2 \mathrm{aE}-13$ \\
\hline EFFLUEKT \& & 5. $8 B E-64$ & 2.29E-03 & $2.70 E+01$ & 5. $62 E-12$ & 5. $06 E-12$ \\
\hline EFFLUEHT 9 & 4.5:E-04 & $2.74 E-0.3$ & $3.00 E+01$ & $6.69 E-12$ & $6.52=-13$ \\
\hline EFFLUENT:0 & 5.366 - 014 & $3.28 E-03$ & $3.40 E+01$ & $5.97 E-12$ & $8.2 \mathrm{AE}-1 \mathrm{~J}$ \\
\hline EFFLUENT $! 1$ & $4.19 E-04$ & $3.70 E-03$ & $3.70 E+01$ & $7.21 \mathrm{E}-12$ & Z.ti:E-\{3 \\
\hline EFFLUENT 12 & $5.30 E-04$ & $4.23 E-03$ & $4.10 E+01$ & $7.11 E-12$ & 1. $13 E-12$ \\
\hline EFFLLEHT 13 & $3.61 E-04$ & $4.59 E-03$ & $4.40 E+01$ & $6.42 E-12$ & $1.24 E-12$ \\
\hline COLUMA T2 & & & & & \\
\hline EFFLUENT i6 & 1. $12 E-03$ & 5. $70 E-03$ & $5.80 E+01$ & $3.355-12$ & $1.46 \bar{z}-12$ \\
\hline EFFLUEKT 17 & $9.60 E-04$ & $6.605-03$ & $6.20 E+01$ & 3. 606-11 & $1.36=-12$ \\
\hline EFFLUEHT :A & $3.415-34$ & $7.01 E-63$ & $6.50 E+01$ & $8.56 E-12$ & $1.96=-12$ \\
\hline EFFLUENT 19 & $6.83 E-04$ & $7.69 E-03$ & $6.90 E+01$ & $2.03 E-11$ & $2.23 E-12$ \\
\hline EFFLUEHT 20 & $3.68 E-04$ & $8.06 E-03$ & $7.20 E \div 01$ & $1.10 E-11$ & $2.35 E-12$ \\
\hline EFFLUENT 21 & 3. $00 \mathrm{E}-04$ & $8.36 E-03$ & $1.60 E+01$ & $4.32 \mathrm{E}-12$ & $2.39 E-12$ \\
\hline EFFLUENT 22 & 5.77E-04 & 6. $94 E-0 J$ & $8.00 E+01$ & $1.705-11$ & $2.60=-12$ \\
\hline EFFLUENT 23 & $3.07 E-04$ & $3.24 E-03$ & B.40E+01 & $5.02 E-12$ & $2.55 E-12$ \\
\hline EFFLUENT 24 & $1.83 E-6 t$ & $9.4 J E-03$ & $8.70 E+01$ & $3.31 E-12$ & $2.66 E-12$ \\
\hline EFFLUENT 26 & $4.53 E-04$ & $9.86 E-0 J$ & $9.40 E \pm 001$ & $3,94 E-12$ & Z. 10 EE-12 \\
\hline EFFLUEKT 28 & 5. $30 E-04$ & $1.04 E-\hat{v} 2$ & 1. $00 E+02$ & $6.16 E-j 2$ & 2. 2XE-12 \\
\hline EFFLUEHT 30 & $1.44 E-04$ & $1.06 E-02$ & 1.07E +02 & $4.58 E-13$ & $2.71 E-12$ \\
\hline EFFLUERT 32 & $1.595-194$ & $1.07 E-02$ & 1. $15 E+12$ & $4.59 E-13$ & $3.60 \mathrm{E}-12$ \\
\hline EFFLUEHT 34 & $7.47 E-05$ & $1.08 E-02$ & $1.21 E+02$ & $1.91 E-13$ & $2.515-12$ \\
\hline EFF1UEHT 36 & 1.52E-04 & 1. $69 E-02$ & $1.26 E \div 02$ & $0.12 E-13$ & $2.44 E-12$ \\
\hline EFFLUESTT & $3.92 E-04$ & 1.13E-62 & $1.42 E+02$ & $1.105-12$ & $2.36 E-15$ \\
\hline EFFLUENT 41 & 1.29E-64 & 1.15E-02 & $1.49 E+0 \bar{i}$ & 5. $30 \bar{E}-13$ & $2.30 E-12$ \\
\hline EFFLUENT 43 & $1.01 E-34$ & 1.16E-02 & $1.60=+02$ & $1.36 E-1 j$ & $\therefore$ ¿IEE-1Z $i$ \\
\hline EFFLUENT 45 & $7.04 E-15$ & 1. $16 E-32$ & 1.72E+ +2 & $5.94 E-\{4$ & $2,05 E-12$ \\
\hline EFIUEHT 47 & $5.18 E-055$ & $3.17 E-62$ & $1.815+02$ & 2. 3NE-14 & 1. $76=12$ \\
\hline EFFLUEHT 49 & $1.36 E-3.44$ & 1. 18E-02 & $2.215+02$ & $2.41 E-14$ & : . $5 \div \bar{\varepsilon}-1 \bar{z}$ \\
\hline EFFLUJEHT SO & $3.85 E-05$ & $1.10 E-02$ & $2.5 I E \div 02$ & $4.04 E-: 5$ & $1.45 E-12$ \\
\hline
\end{tabular}


TABLE B.11e. Boron (as H3B03) Leach Fractions from Flow Through Test Grout/Sediment with Groundwater (DIFCOLT2)

\begin{tabular}{|c|c|c|c|c|c|}
\hline SAMPLE & $A \cap / A O$ & cun. An/AO & DAYS & $D$ ine, & D cus. \\
\hline EFFLUENT 1 & $6.90 E-03$ & $6.90 E-03$ & 3.00E+00 & 4.13E-11 & $4.13 E-11$ \\
\hline EFFLUENT 2 & $2.26 E-03$ & $9.17 E-03$ & $6.00 E+00$ & $2.59 E-11$ & $3.64 E-11$ \\
\hline EFFLUENT 3 & $2.06 E-03$ & 1.12E-02 & $9.00 E+00$ & $3.64 E-11$ & J.डAE-i! \\
\hline EFFLUENT 4 & $1.77 E-03$ & $1.30 E-02$ & $1.30 E+01$ & $2.23 E-1 !$ & 3.JEE-11 \\
\hline EFFLUEHT 5 & $9.63 E-04$ & $1.40 E-02$ & $1.70 E+01$ & $9.01 E-12$ & $2.9 B E-11$ \\
\hline EFFLUENT 6 & $7.16 E-04$ & $1.17 E-02$ & 2. $00 E+01$ & $1.10 E-11$ & 2.80E-11 \\
\hline EFFLUEMT 7 & $8.96 E-04$ & ¿.56E-02 & $2.30 E+01$ & 1. $79 E-11$ & $2.74 E-1:$ \\
\hline EFFLUENT 8 & $9.99 E-04$ & $1.66 E-02$ & $2.70 E+01$ & $1.62 \mathrm{E}-\mathrm{ii}$ & $2.65 E-11$ \\
\hline EFFIUENT 9 & 6. $92 \mathrm{E}-04$ & $1.73 \mathrm{E}-02$ & $3.60 E \div 01$ & S. 58E- $\$ 1$ & $2.58 E-1 j$ \\
\hline EFFLLENT IO & $6.75 E-04$ & 1. $80 \mathrm{E}-\mathbf{V 2}$ & $3.40 E+01$ & $1,60 E-11$ & $2.97 E-i:$ \\
\hline EFFLUENT 11 & $4.6 B E-04$ & $1.84 E-02$ & $3.70 E+01$ & $3.99 E-12$ & 2. JYE-ij \\
\hline EFFLUEHT 12 & $5.775-04$ & $1.90 E-02$ & $4.10 E+0 i$ & อ. $135-12$ & $2.29 E-i i$ \\
\hline EFFIUENT 13 & 3. $77 E-64$ & 1. $94 E-02$ & $4.40 E+01$ & $7.60 E-12$ & $2.325-1 !$ \\
\hline CธLปลัก 12 & & & & & 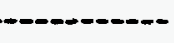 \\
\hline EFFLUENT I6 & $1.52 E-03$ & 2. *9E-02 & $5.80 E+01$ & 6. $20 \mathrm{E}-12$ & 1. $36 E-11$ \\
\hline EFFLUENT :T & $8.46 E-\hat{v i}$ & $2.17 E-02$ & $6.20 E+0 s$ & $2.79 E-i i$ & $1.98 E-11$ \\
\hline EFFLUEHT IB & $3.29 E-04$ & 2. $21 E-\hat{0} 2$ & 6. $50 E+01$ & $1.11 E-11$ & 1. FEE-11 \\
\hline EFFLUEKT I? & $9.36 E-04$ & 2.31E-02 & 6. $90 E+01$ & $3.82 E-11$ & $2.01 E-11$ \\
\hline EFFLLUENT 20 & 4.97E-014 & $2.36 E-02$ & $7.20 E+01$ & $2.01 E-11$ & 2.01E-11 \\
\hline EFFLUENT 21 & $5.65 E-04$ & $2.41 E-02$ & 7.60E+01 & $1.54 E-11$ & !. \$9E-11 \\
\hline EFFLUENT 22 & 8. $75 E-04$ & $2.50 \mathrm{E}-02$ & 8.00E+01 & $3.88 E-11$ & $2.03 E-11$ \\
\hline EFFLUENT 23 & $5.27 E-04$ & $2.55 E-02$ & $8.40 E+0 !$ & 1. AEE-1! & 2. Vิ2E-ij \\
\hline EFFLUEHT 24 & $4.50 E-04$ & $2.60 E-02$ & $8.70 E+01$ & $2.00 E-1 !$ & $2.0 \geq 5-1 !$ \\
\hline EFF!UERT 26 & $9.23 E-04$ & $2.69 \mathrm{E}-02$ & $9.40 E \div 01$ & $1.64 E-11$ & $2.00 E-1 j$ \\
\hline EFFLUEHT 2B & 1.09E-0. & $2.30 E-02$ & $1.00 E+02$ & J.J5E-1! & I. $04 E-11$ \\
\hline EFFLUENT 30 & $6.15 E-\hat{v} 4$ & $2.86 E-02$ & 1.0TE+02 & $6.32 E-12$ & i.99E-:i \\
\hline EFF:LUENT 32 & Q. $14 E-04$ & $2,34 E-52$ & $1.15 E \div 02$ & $1.15 E-11$ & $1.965-11$ \\
\hline EFFLUENT 34 & $4.72 E-0.4$ & 2. $99 E-02$ & $1.2 \mathrm{JE}+\overrightarrow{\mathrm{V} Z}$ & $7.55 E-12$ & $1.925-11$ \\
\hline EFFLUEHT 36 & 1.02E-03 & $3.09 E-02$ & $1.28 E+02$ & $2.75 E-11$ & $1.945-11$ \\
\hline EFFLUENT 3 ? & $1.45 E-63$ & $3.24 E-62$ & 1. $4 Z E+02$ & 1.5IE-11 & $i$, 汭E-ij \\
\hline EFFLUEMT \&1 & $6.55 E-64$ & $3.305-02$ & $1.49 E+02$ & 1.JJE-!! & $1.70 E-11$ \\
\hline EFFLUENT 43 & 6.72E-64 & $3.37 E-\hat{v} 2$ & $1.60 E+02$ & S. BaE-12 & i. $35 E-1:$ \\
\hline EFFLUENT 45 & 9. DóE-04 & $3.47 E-02$ & 1.TaE+iz & i. IIE-II & $1 . \overline{X E}-1 !$ \\
\hline EFFLUENT $4 \bar{i}$ & $7.34 E-04$ & $3.55 E-02$ & $1.5: E+02$ & $1.4 U E-11$ & $1.81 E-11$ \\
\hline EFFLUENT 49 & 2.96E-03 & 3.BAE- 32 & 2.BiEtitz & 5. $14 E-11$ & 1. $i 4 E-1 !$ \\
\hline EFFLUEHT SO & $2.90 E-63$ & $4,33 E-02$ & $2.515+02$ & $2.29 E-: i$ & :.TFE-: \\
\hline
\end{tabular}


TABLE B.11f. Fluoride (F) Leach Fractions from Flow Through Test Grout/Sediment with Groundwater (DFCOLT2A)

\begin{tabular}{|c|c|c|c|c|c|}
\hline SAMPLE & An/Ao & CUn. $A n / A_{0}$ & DAYS & D inc. & B cus. \\
\hline EFFLUENT 1 & $7.05 E-04$ & $7.05 E-04$ & $3.00 E+00$ & $5.23 E-13$ & $5.235-13$ \\
\hline EFFLUENT 2 & $4.61 E-04$ & $1.17 \mathrm{E}-0 \mathrm{~J}$ & $6.00 E+00$ & 1. $30 E-12$ & $7.15 E-13$ \\
\hline EFFLUEHT 3 & 4.36E-04 & $1.66 \mathrm{E}-03$ & $9.00 E+00$ & $2.56 E-12$ & $7.675-13$ \\
\hline EFFLUENT 4 & $1.07 E-03$ & $2.73 E-03$ & $1.30 E+01$ & $9.76 E-12$ & 1. $3 ! E-12$ \\
\hline EFFLUEHT 5 & $3.6 B E-04$ & $3.10 \mathrm{E}-03$ & $1.70 E+01$ & $1.60 E-12$ & $1.78 E-12$ \\
\hline EFFLUENT 6 & $2.55 E-04$ & $3.35 \mathrm{E}-03$ & $2.00 E+01$ & $1.68 E-12$ & 1. $77 E-12$ \\
\hline EFFL'UENT $i$ & $3.21 E-04$ & $3.67 E-05$ & 2.J0E+01 & $3.1 ! E-12$ & 1.85E- 12 \\
\hline EFFLLEENT 8 & $4.57 E-04$ & $4.13 E-03$ & $2.70 E+01$ & $4.11 E-12$ & $1.98 E-12$ \\
\hline EFFLUENT ? & $2.68 E-04$ & $4.40 E-03$ & $3.00 E+01$ & $2.86 E-12$ & $2.03 E-12$ \\
\hline EFFLLENT 10 & $3.51 E-04$ & $4 . i 5 E-03$ & $3.40 E+01$ & $3.10 E-12$ & $2.09 E-12$ \\
\hline EFFLUENT 11 & $2.44 E-04$ & $4.99 E-03$ & $3.70 E+01$ & $2.97 E-12$ & $2.12 E-12$ \\
\hline EFFLUENT 12 & $3.095-04$ & $5.30 E-03$ & $4.10 E+01$ & $2.73 E-12$ & $2.16 E-12$ \\
\hline EFFLUEHT 13 & $3.84 E-04$ & $5.69 E-03$ & 4. 40E $\div 01$ & 8. $i d E-12$ & 2. $32 E-12$ \\
\hline \multicolumn{6}{|l|}{ COLUAN T2 } \\
\hline EFFLUEHT ió & 6. $56 E-04$ & $6.34 E-03$ & $5.80 E+01$ & $1.41 E-12$ & $2.17 E-12$ \\
\hline EFFLLIENT 17 & $5.44 E-04$ & $6.69 E-03$ & $6.20 E+01$ & 1.40E-11 & $2.41 E-12$ \\
\hline EFFLUENT 18 & $2.75 E-04$ & $7.165-03$ & $6.50 E+01$ & $6.74 E-12$ & $2.97 E-12$ \\
\hline EFFLUENT I9 & $6.35 E-04$ & 7. BOE-03 & $6.90 E+01$ & $2.13 E-11$ & $2.78 E-12$ \\
\hline EFFLUENT 20 & $3.47 E-04$ & $8.14 E-03$ & $7.205+01$ & $1.19 E-11$ & $2.70 E-12$ \\
\hline EFFLUENT 21 & $3.29 E-04$ & $8.47 E-03$ & $7.60 E+01$ & $6.31 E-12$ & 2. $76 E-12$ \\
\hline EFFLUENT 22 & $5.87 E-04$ & $9.065-03$ & 3.00E+0! & $2.125-11$ & $3.23 \mathrm{E}-1.2$ \\
\hline EFFLUEHT 23 & $3.93 E-04$ & $9.45 E-03$ & $3.40 E+01$ & $7.9 B E-12$ & $3.35 E-12$ \\
\hline EFTLUUEHT 24 & $3.05 E-04$ & $9.76 E-03$ & $8.70 E+01$ & 1.11E-11 & $3.45 E-12$ \\
\hline EFFLUEHT 26 & B. 43E-04 & $1.06 E-02$ & $7.40 E+01$ & $1.66 E-11$ & j. $77 \varepsilon-12$ \\
\hline EFFLUENT 28 & $1.23 E-03$ & 1. $18 E-02$ & 1.00E+02 & $5.17 E-11$ & 4. $42 E-12$ \\
\hline EFFLUEHT 30 & $4.90 E-04$ & $1.23 E-02$ & 1.67E+102 & $6.395-12$ & $4.46 E-12$ \\
\hline EFFIUENT 32 & $5.23 E-04$ & 1.28E-02 & $1.15 E+02$ & J. $385-12$ & $4.525-12$ \\
\hline EFFUEKT 34 & $2.95 E-04$ & 1.3:E-02 & $1.21 E+02$ & $3.61 E-12$ & $4.5 \dot{v} E-12$ \\
\hline EFFLLENT 36 & 6. 2bE-04 & $1.38 E-02$ & $1.2 E E+122$ & $1.25 E-11$ & 4.6JE-12 \\
\hline EFFLUENT 37 & $1.47 E-03$ & $1.52 E-02$ & $1.42 E \div 62$ & $1.67 E-11$ & 5. $15 E-12$ \\
\hline EFFLUENT Ai & $7.72 E-04$ & $1.605-02$ & $1.535+12$ & $9.16 E-12$ & $5.20 E-12$ \\
\hline EFFLUENT 43 & 8.12E-Ni & $1.68 E-02$ & $1.6015+02$ & $2.66 E-11$ & $5.5 B E-12$ \\
\hline EFFLUERT 45 & P. $32 E-04$ & 1. J IOE-02 & $1.72 E+002$ & $1.35 E-11$ & 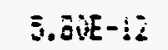 \\
\hline EFFLUENT 47 & $5.57 E-04$ & $1.83 E-02$ & i.EIE+ & อ.รIE-12 & $5.865-12$ \\
\hline EFFLUENT 49 & $2.3] E-63$ & 2.12E-6z & $2.21 E+02$ & 1.27E-11 & $\dot{Q}, 40 E-12$ \\
\hline EFFLLENT SO & $1.705-03$ & $2.31 E-\hat{02}$ & $2.51 E+02$ & i.25E-i1 & $6.7 \mathrm{TE}-12$ \\
\hline
\end{tabular}


TABLE B.11g. Nitrate (NO3) Leach Fractions from Flow Through Test Grout/Sediment with Groundwater (DFCOLT2A)

\begin{tabular}{|c|c|c|c|c|c|}
\hline SAMPLE & $A n / A O$ & [UR. Ân/AO & DAYS & $D$ inc. & D cua. \\
\hline EFFLUEHT 1 & B. $4 J E-02$ & $3.47 \mathrm{E}-02$ & $3.00 E+00$ & 7.53E-09 & $7.53 E-69$ \\
\hline EFFLUEKT 2 & $1.60 E-02$ & $1.01 E-01$ & $6.00 E+00$ & 1.56E-09 & $5.32 E-109$ \\
\hline EFFLUEHT 3 & 1.59E-02 & 2.16E-01 & $9.00 E+00$ & $2.025-69$ & $4.75 E-69$ \\
\hline EFFLUENT 4 & i. J9E-02 & 1. 30́E-01 & $1.3 \hat{V E}+01$ & $1.605-09$ & $4.12 E-69$ \\
\hline ETFLLEET 5 & $1.66 E-02$ & $1.47 E-01$ & $1.70 E+01$ & $3.23 E-09$ & 4. $01 E-O Q$ \\
\hline EFFLUERT 6 & $7.87 E-03$ & 1.55E-0! & $2.00 E+01$ & $1.60 E-09$ & $3.78 E-09$ \\
\hline EFFLUEHT ? & 1.5ล2E-02 & $1.70 E-01$ & $2.30 E+01$ & $6.94 E-09$ & $3.96 E-69$ \\
\hline EFFLUENT 9 & 1.07E-02 & $1.81 E-01$ & $2.705+01$ & $2.25 E-i) 9$ & $3.8 ! 5-67$ \\
\hline EFFLUERT ? & $8.04 E-03$ & 1.B9E-01 & 3.00E+01 & $2.50 \mathrm{E}-6 \mathrm{6}$ & 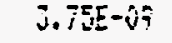 \\
\hline EFFLUEHT IO & $9.04 E-13$ & $1.98 E-01$ & $3.40 E+01$ & 2. $06 E-09$ & $5.63 E-67$ \\
\hline EFFLUENT 11 & 6. $12 E-63$ & $2.04 E-01$ & $3.70 E+01$ & 1. $26 E-7)$ & 3.5AE- \\
\hline EFFLUEMT :2 & $7.76 E-03$ & 2.12E-01 & $4.10 E+01$ & $1.36 E-19$ & 3.59E-69 \\
\hline EFFLUEHT 13 & $7.715-03$ & $2.20 E-01$ & $4.4 \hat{v E}+6 !$ & $3.73 E-09$ & 3. $68 E-69$ \\
\hline COLUAH T2 & & & & & -......... \\
\hline EFFLUEHT io & $1.22 E-02$ & $2.325-01$ & $5.80 E+01$ & $4.24 E-10$ & 3. $06 E-19$ \\
\hline EFFLUENT 17 & $6.03 E-03$ & 2. 38E-01 & $6.20 E+011$ & $1.72 E-69$ & $3.26 E-19$ \\
\hline EFFLUENT I8 & $3.26 E-03$ & $2.4 I E-01$ & $6.50 E+01$ & $9.59 E-10$ & $3.13 E-67$ \\
\hline EFFLUJENT :9 & $7.70 \mathrm{E}-0 \mathrm{3}$ & $2=49 E-01$ & 6. FิVE+01 & $3 .: 3 E-09$ & $3.06 E-19$ \\
\hline EFFLUENT 20 & $4.47 E-03$ & $2.53 \mathrm{~J}-01$ & $7.20 E+01$ & $1.97 E-09$ & 2. $35 E-39$ \\
\hline EFFLUEKT 2i & $3.64 E-03$ & $2.57 \mathrm{E}-01$ & $i .60 E+6 i$ & $5.58 E-10$ & $3.05 E-09$ \\
\hline EFFLUENT 22 & $6.91 E-03$ & $2.64 E-01$ & $8.00 E+01$ & $2.73 \bar{E}-09$ & $2.395-39$ \\
\hline EFLLUENT 23 & $5.11=-03$ & $2.69 E-61$ & $8.40 E+01$ & 1. COE 69 & $3.00 E-09$ \\
\hline EFFLUENT 24 & $4.65 E-03$ & $2.74 E-01$ & $8.70 E+01$ & $2.57 E-199$ & $2.30 E-69$ \\
\hline EFFLUEHT 26 & $1.29 E-62$ & $2.87 E-01$ & $9.40 E+01$ & 3. $35 E-99$ & $3.14 E-09$ \\
\hline EFFIUEAT 2B & 1. $38 E-02$ & 3. $05 E-01$ & 1. $00 E \div \hat{2} 2$ & 1. $20 E-05$ & $3.4:=-05$ \\
\hline EFFLUEKT SQ & 1.5SE-.32 & $\dot{j}_{.} \mathbf{z i} E-\hat{j} !$ & $1.07 E+02$ & b. $T 7 E-j S$ & $3.42 E-09$ \\
\hline EFFLUEKT 32 & $1.70 E-62$ & $5.35 E-61$ & 2. $1 E E \div 02$ & $6.31 E-y 9$ & 3. ESE-09 \\
\hline EFFLLIERT 39 & $1.41 E-02$ & J. 5JE-ט́l & $1.21 E+02$ & E. $24 E-63$ & $3.70 E-67$ \\
\hline EFFLIEETT JE & 2. $90 E-12$ & $3.22 E-\hat{y} !$ & $1.28 E+62$ & 2.5วE-18 & $4.22 E-09$ \\
\hline EFF:UEHT 33 & $0.01 E-02$ & $4.92 E-01$ & $1.42 E+02$ & $3.14 E-0 B$ & $5.35-66$ \\
\hline EFFLIEHT 41 & $3.65 E-0.22$ & 4.7FE-01 & $1.53 E \div 62$ & $2.05 E-66$ & - $16 E-\hat{D}$ \\
\hline EFF!UENT 43 & $3.53 E-02$ & $5.17 E-0 i$ & $1.605+02$ & 5. S7E-Ấ & $7,12 E-67$ \\
\hline EFIUENI \&5 & $3.64 E-02$ & $5.53 E-01$ & $1.72 E \div 02$ & 1.75E-15 & T.64E- \\
\hline EFFLUENT 47 & $6 .: 05-6 z$ & b. iAE-oi & 1. BSE+62 & 1. & \$.: $: 7 E-69$ \\
\hline EFTLUEKT 49 & $7.55 E-45$ & $6.225-01$ & $2.21 E+12$ & $7.74 E-1 !$ & E.:iE- $\$ 9$ \\
\hline EFF:IENT 50 & 5. $11 E-\hat{j} 2$ & T. BDE-OA & $2.51 E+\hat{0} 2$ & 2.:IEE-iE & 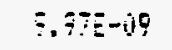 \\
\hline
\end{tabular}


TABLE B.11h. Sulfate (SO4) Leach Fractions from Flow Through Test Grout/Sediment with Groundwater (DFCOLT2A)

\begin{tabular}{|c|c|c|c|c|c|}
\hline SAMPLE & An/Ao & cun. An/Á & DAYS & D inc. & $D$ cus. \\
\hline EFFLUENT ! & $1.61 E-01$ & $1.61 E-01$ & $3.00 E+00$ & $2.72 E-0 B$ & $2.72 E-08$ \\
\hline EFFLUEHT 2 & $1.595-02$ & $1.77 \mathrm{E}-01$ & $6.00 E+00$ & 1.55E-09 & $1.64 E-08$ \\
\hline EFFLUENT 3 & 2. $10 E-02$ & $1.98 E-01$ & $9.00 E \div 00$ & 1.60E-09 & $1.37 \mathrm{E}-08$ \\
\hline EFFLUJENT 4 & 1.28E-0.2 & $2.11 E-01$ & 1. $30 E+01$ & $1.42 E-\hat{07}$ & 1. $13 E-08$ \\
\hline EFFLUENT 5 & $6.22 E-03$ & $2.17 E-0 !$ & $1.70 E+01$ & $4.565-10$ & $9.52 E-69$ \\
\hline EFFLUENT 6 & $9.02 E-03$ & $2.26 E-01$ & 2. $00 E+01$ & 2. $11 E-09$ & $8.6 B E-69$ \\
\hline EFFLLUENT 7 & ว. 87E-0) & 2. $36 E-01$ & 2. $30 E+01$ & $2.93 E-69$ & $8.36 E-199$ \\
\hline EFFLUERT a & $6.92 E-1) 3$ & $2.45 E-01$ & $2.70 E+01$ & $1.57 E-69$ & $7.545-09$ \\
\hline EFFLUENT 9 & $1.15 E-0.2$ & $2.50 E-01$ & 3. OQEE+(i) & $5.305-07$ & $3.72 E-09$ \\
\hline EFFLLENT 10 & b.23E-03 & 2. $62 E-01$ & $3.40 E+01$ & 9. $38 E-10$ & 6.81E-09 \\
\hline EFFLUENT 11 & $1.62 E-03$ & $2.63 E-\hat{01}$ & 3.TOEE+UI & $5.20 E-11$ & $6.26 E-04$ \\
\hline EFFLUENT 12 & $1.34 E-63$ & $2.65 E-01$ & 4.10E+01 & $5.50 E-11$ & $5.65 E-07$ \\
\hline EFTLLENT IJ & $6.61 E-03$ & $2.71 E-01$ & $4.40 E+01$ & $2.60 E-09$ & $5.74 E-09$ \\
\hline COLUMH T2 & & & & & - \\
\hline EFFLUENT 16 & 1.37E-03 & $2.73 E-01$ & $5.8 \mathrm{AE}+0 \mathrm{i}$ & $1.15 E-11$ & $4.35 E-09$ \\
\hline EFFLUENT 17 & $0.00 E+100$ & $2.73 E-01$ & $6.20 E+01$ & $0.00 E+00$ & $4.075-09$ \\
\hline EFFLUEKT 1B & $0.00 E+00$ & $2.73 E-01$ & $6.50 E+01$ & $0.00 E+00$ & $3.98 E-69$ \\
\hline EFFLUENT 19 & $1.23 E-02$ & $2.85 E-01$ & $6.70 E+01$ & $7.965-09$ & $4.28 E-09$ \\
\hline EFFLUEHT 20 & $3.11 E-63$ & $2.89 E-01$ & $7.20 E+01$ & $9.54 E-10$ & $4.10 E-09$ \\
\hline EFFLUEHT 21 & $1.24 E-02$ & $3.01 E-01$ & $3.60 E+01$ & $8.96 E-07$ & 4.16E-69 \\
\hline EFFLUENT 22 & $5.34 E-03$ & $3.07 E-0 !$ & 6. 60)E+ô! & $2.17 E-0 ?$ & $4.2 \times E-09$ \\
\hline EFFLUENT 23 & $3.74 E-63$ & $3.11 E-01$ & $8.40 E+01$ & $9.035-10$ & $4.685-69$ \\
\hline EFFLUENT 24 & $7.05 \mathrm{E}-63$ & $3.18 E-01$ & $8.70 E+01$ & $5.75 E-09$ & $4.2 ! E-0 ?$ \\
\hline EFFLUEHT 26 & $1.95 E-02$ & $3.37 E-01$ & $9.400 E+01$ & $8.85 E-09$ & $4.46 E-09$ \\
\hline EFFLUEHT $2 B$ & $2.85 E-02$ & $3.66 E-01$ & $1.00 E+02$ & $2.77 E-08$ & $5.08 E-19$ \\
\hline EFFLUEHT 30 & $5.91 E-604$ & $3.67 E-01$ & $1.07 E+0 z$ & $2.62 E-11$ & $4.75 E-97$ \\
\hline EFFLUENT 32 & $0.00 E+00$ & 3. $\quad \bar{O} T E-9 \mathrm{I}$ & $1.15 E \div 02$ & $0.00 E+00$ & $4.42 E-69$ \\
\hline EFF:JEHT 34 & $6.5 B E-6 j 3$ & $3.73 E-0 !$ & $1.2 ! E+02$ & 1. $79 E-09$ & $4.20 E-39$ \\
\hline
\end{tabular}


TABLE B.11i. Technetium-99 (TC-99) Leach Fractions from Flow Through Test Grout/Sediment with Groundwater (TI)

\begin{tabular}{|c|c|c|c|c|c|c|c|}
\hline SAMPLE & PORE VOLLUKE & $\mathrm{UCI}$ & TIKE DAYS & $A n / A O$ & CUn Ánifá & DIHC CK2JSEC & DCLYY CK2/SEE \\
\hline CRN I1-1 & $1.44 E+00$ & $1.55 E-02$ & $3.00 E+00$ & 8. $46 E-03$ & $8.46 E-03$ & 6. $67 E-11$ & 6. $69 E-11$ \\
\hline CRW TI-2 & $2.91 E+00$ & $3.66 E-03$ & $6.00 E+00$ & $2.01 E-03$ & 1. $05 E-62$ & $4.40 E-i i$ & $5.125-11$ \\
\hline CRH $11-3$ & $4.49 E+00$ & $4.50 E-03$ & $9.00 E+00$ & $2.45 E-03$ & $1.29 E-12$ & $6.56 E-1 !$ & $5.20 E-11$ \\
\hline CKH II-4 & $6.006 E+00$ & $3.97 E-03$ & 1. $30 E+01$ & $2.17 E-0 j$ & i.5IE-0. & $3.42 E-11$ & $4.3 \mathrm{iE}-\mathrm{i} 1$ \\
\hline SFH T1-5 & $8.4 J E+00$ & $2.67 E-03$ & $1.70 E+01$ & $1.46 E-03$ & $1.05 E-12$ & $1.74 E-11$ & $4.515-11$ \\
\hline Cny & $9.65 E+100$ & $2.045-03$ & $2.00 E+0 i$ & 1. $11 E-0 \bar{Z}$ & $1.735-02$ & $1.53 E-i 1$ & $4.37 E-11$ \\
\hline CEN $\mathrm{i}:-7$ & $1.15 E+01$ & $2.21 E-03$ & $2.30 E+01$ & $1.20 E-03$ & $1.29 E-02$ & 1.5EE-11 & $4.34 E-11$ \\
\hline CAH T1-B & 1. $41 E+01$ & $2.49 E-03$ & $2.70 E+01$ & $1.36 E-6]$ & $2.02 E-02$ & !.35E-1! & $4.25 E-11$ \\
\hline 哧 II-9 & $1.60 E+01$ & $1.78 E-03$ & J.0OE+OI & $3.70 E-04$ & $2.12 E-12$ & $2.165-11$ & $4.20 E-11$ \\
\hline CRH $T j-i 0$ & $1.86 E+01$ & 1. $97 E-03$ & $3.40 E+01$ & 1.08E-60j & $2.23 E-62$ & $8.43 E-12$ & $4.09 E-1 !$ \\
\hline 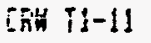 & $2,06 E+01$ & $2.91 E-03$ & $3.70 E+01$ & $1.59 E-6 j$ & $2.39 E-12$ & $3.00 E-11$ & $4.315-1 !$ \\
\hline Son $71-12$ & $2.3+E+01$ & $6.63 E-03$ & $4.10 E+01$ & $3.6 z E-\hat{v} 3$ & $2.75 E-02$ & $9.53 E-11$ & 5.16E-11 \\
\hline CR: T:-13 & $2.53+0 j$ & $4.305-03$ & $4.40 E+01$ & $2.34 E-03$ & 2. $38 E-12$ & 3.7aE- 11 & $5.56 E-11$ \\
\hline
\end{tabular}




\section{TABLE B.11i. Technetium-99 (TC-99) Leach Fractions from Flow Through Test Grout/Sediment (T2)}

\begin{tabular}{|c|c|c|c|c|c|c|c|}
\hline $\begin{array}{l}\text { SAHPLE } \\
\text { CRN T2-1 }\end{array}$ & $\begin{array}{l}\text { PORE VOLUAE } \\
8.97 \mathrm{E}-01\end{array}$ & $\begin{array}{l}\text { UC: } \\
\text { T.6IE-02 }\end{array}$ & $\begin{array}{l}\text { TIME DAYS . } \\
3.00 E+00\end{array}$ & $\begin{array}{l}\text { An/Ao } \\
4.20 E-02 \\
1.35 E-02\end{array}$ & $\begin{array}{l}\text { CUH An/AO } \\
4.20 E-12 \\
5.55 E-02\end{array}$ & $\begin{array}{r}\text { SINC CKLISEC } \\
1.535-69 \\
1.84 E-09\end{array}$ & $\begin{array}{r}\text { DCUM CK2/SEC } \\
1.5 \mathrm{JE}-09 \\
1.34 E-09\end{array}$ \\
\hline CRE T2-2 & $1.65 E+00$ & $2.46 E-02$ & 6. $00 E+00$ & & $\begin{array}{l}5.55 E-02 \\
7.70 E-02\end{array}$ & $\begin{array}{l}1.84 E-69 \\
2.72 E-69\end{array}$ & \\
\hline $\begin{array}{l}\text { Cfin } 12-3 \\
\text { Cak } 12-4\end{array}$ & $\begin{array}{l}3.35 E+00 \\
5.68 E+00\end{array}$ & $\begin{array}{l}3.94 E-02 \\
3.07 E-02\end{array}$ & $\begin{array}{l}\text { 9. } 00 E+00 \\
\text { i. } 30 E+01\end{array}$ & $\begin{array}{l}2.16 E-02 \\
1.56 E-02\end{array}$ & $\begin{array}{l}7.70 E-02 \\
9.39 E-02\end{array}$ & $\begin{array}{l}9.125-67 \\
i .92 E-07\end{array}$ & 1. $71 E-19$ \\
\hline โКํㅠ T2-5 & $6.37 E \div 00$ & $6.74 E-03$ & $1.70 E+01$ & $3.69 E-63$ & $9.75 E-0 i$ & $1.03 E-10$ & $1.465-39$ \\
\hline CRAl $72-6$ & $7.36 E+10$ & $1.15 E-02$ & $2.00 E+01$ & $6.26 E-03$ & 1. OAE-ũ1 & $4.52 E-10$ & $1.415-09$ \\
\hline CNA $72-\bar{\imath}$ & $8.88 E+10$ & $1.60 E-02$ & $2.30 E+01$ & $8.74 E-03$ & 1. $13 E-01$ & $7.74 E-10$ & $1.44 E-09$ \\
\hline CRAN T2-8 & $1.09 E+01$ & $1.28 \mathrm{E}-02$ & $2.70 E+01$ & $6.99 E-03$ & $1.20 E-01$ & $3.31 E-10$ & 1.3EE-69 \\
\hline Chin $72-9$ & $1.23 E+01$ & 1.33E-01 & $3.00 E+01$ & $7.2 b E-\hat{v} J$ & $1.27 E-01$ & $6.05 E-60$ & $6.96 E-69$ \\
\hline CRU T2-10 & $1.42 E+01$ & $1.3 B E-02$ & $3.40 E+01$ & $7.55 E-03$ & 1. $34 E-01$ & 3. 6ิธE-10 & $1.30 E-09$ \\
\hline CFH T2-11 & $1.57 E+01$ & 1.15E-03 & $3.70 E+01$ & $0.27 E-04$ & $1.35 E-61$ & 4.54E-12 & $1.28 E-69$ \\
\hline CRH $7 i-12$ & $1.76 E+01$ & $1.20 \mathrm{E}-\mathrm{v} 3$ & $4.10 E+01$ & $0.59 E-04$ & $1.365-01$ & $2.94 E-12$ & $1.17 E-09$ \\
\hline En $72-13$ & $1.32 E+01$ & $2.935-03$ & 4. $40 E+001$ & $1.60 E-03$ & 1. $37 E-41$ & 2. $85 E-11$ & $1.12 E-\hat{v}\}$ \\
\hline CRK T2-14 & $3.1 B E+011$ & $3.85 E-03$ & $4.80 E+01$ & $2.115-0 J$ & 1.37E-01 & $2.22 E-11$ & 1.03E-09 \\
\hline EF⿱ $12-15$ & $2.37 E \div 01$ & $1.97 \mathrm{E}-03$ & $5.20 E+01$ & 1. $68 E-0 J$ & $1.40 E-31$ & 1. 47E-1! & $7.69 E-10$ \\
\hline CAU T2-16́ & $2.73 E+01$ & $3.83 E-03$ & $5.30 E+01$ & 2. $10 E-03$ & $1.4 \mathbf{J E}-\mathbf{0 1}$ & $1.72 \mathrm{E}-\mathrm{i} \mathrm{i}$ & $3.13 E-10$ \\
\hline CFin T2-17 & $3.14 E+01$ & $4.79 E-03$ & 6.20E+01 & 2. $62 E-03$ & $1.95 E-01$ & b.24E-:! & $8.365-10$ \\
\hline CAN T2-18 & $3.33 E+01$ & $9.8 B E-04$ & $6.50 E+01$ & $5.41 E-04$ & $5.46 E-01$ & $3.36 E-12$ & $3.52 E-10$ \\
\hline CRH i2-19 & $3.71 E+01$ & $2.36 E-03$ & $6.90 E+01$ & $1.29 E-03$ & $1.47 E-61$ & i.13E-1! & 8. $17 \mathrm{E}-10$ \\
\hline Can $12-20$ & $3.91 E+01$ & $1.43 E-03$ & $7.20 E \div 01$ & $7.81 E-04$ & $1,4 B E-01$ & $7.015-12$ & $7.81 E-10$ \\
\hline CSH T2-21 & 4. 1BE+0! & 2.50E-03 & $3.60 E+001$ & $1.37 E-0 j$ & 1.45E-(1! & $1.27 E-11$ & $7.635-10$ \\
\hline CRH T2-22 & $4.63 E+01$ & $5.05 E-03$ & $8.10 E \div 01$ & $2.76 E-0 j$ & $1.52 E-01$ & $4.25 E-11$ & $7.43 E-10$ \\
\hline CR日 $12-23$ & $4.92 E+01$ & $1.46 E-03$ & $8.40 E+01$ & B.01E-04 & 1.5JE-01 & 2. $17 E-12$ & $7.245-10$ \\
\hline CRH $\{2-24$ & $5.10 E+01$ & $1.20 E-03$ & $8.70 E+03$ & $6.60 E-04$ & $1.53 E-01$ & $4.4 ! E-12$ & $7.05 E-10$ \\
\hline CKH $12-25$ & 5. 35E+01 & $1.52 E-03$ & $9.10 E+01$ & $8.34 E-04$ & $1.54 E-01$ & $4.72 E-12$ & $6.81 E-16$ \\
\hline Crill $72-26$ & $5.63 E+\hat{v} 1$ & $\therefore .06 E-03$ & $9.40 E+0 !$ & $5.81 E-04$ & 1.55E-01 & $3.87 E-12$ & $6.655-10 \hat{0}$ \\
\hline 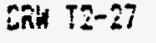 & $0.02 E+01$ & $1.21 E-0 \mathrm{~J}$ & $5.705+01$ & $6.64 E-04$ & !.55E-ij] & $4.47 E-12$ & 6. SNE -10 \\
\hline CRI $12-28$ & $6.41 E+01$ & 6. $88 E-04$ & 1. $00 E+62$ & 3.TIE-04 & $1.56 E=01$ & $1.44 E-12$ & b.jJE-iv \\
\hline $\sin 72-29$ & b.5SE+01 & $5.49 E-04$ & 1. $04 E+02$ & $3.01 E-04$ & $1.56 E-01$ & c. $: 2 E-: J$ & S. $1: 5=-10$ \\
\hline CRH T2-30 & $6.7 B E+01$ & $1.35 E-03$ & $1.07 E+02$ & 7. $11 E-04$ & $1.57 E-01$ & $6.29 E-12$ & $6.60 E-! \hat{v}$ \\
\hline 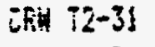 & $7.08 E+01$ & 6. 63E-94 & 1. $11 E+02$ & $4.7 Z E-64$ & !.5jE-0! & $1.515-12$ & $5.32 \geq-10$ \\
\hline CEH $72-32$ & $7.23 E+01$ & $7.76 E-04$ & 1.15E+02 & $4.25 E-34$ & $1.58 E-0 i$ & $1, i \pi t-i z$ & $5.04 E-10$ \\
\hline$\left[\pi T^{2} T 2-3\right]$ & $i .41 E+01$ & $8.44 E-04$ & $1.18 E+02$ & $4.62 E-\hat{8} 4$ & $1.58 E-7 !$ & $2.45 E-12$ & $5.5 .5 E-10$ \\
\hline -7ñ $72-34$ & 7.4óetoi & $6.47 E-04$ & $1.21 E \div 02$ & J.54E-04 & 1.59E-0! & 1. $27 \mathrm{E}-12$ & $5.42 E-10$ \\
\hline [nt in & T.TOE+CA & $2,53 E-63$ & $1.25 E+02$ & $3.30 E-34$ & i.5SE- $\mathrm{ij}$ & $4.73 E-\{1$ & $5.30-10$ \\
\hline 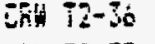 & 7. $92 E+(11$ & 8. $4 ? E-64$ & $1.23 E+02$ & $4.65 E-04$ & $1.605-6 i$ & $2.45 E-12$ & $5.21 E-10$ \\
\hline ind $72-3 i$ & $3.235+01$ & $7,45 E-64$ & $1.32 E=02$ & 4. VEE-64 & S. OVE- & $1.13=-22$ & $5, \mathrm{VEE}-1 \mathrm{0}$ \\
\hline $3 \pi^{2}+2-36$ & 8. $80 E+01$ & 1. $61 E-63$ & $1.37 E+02$ & 8. oิiE-64 & ¿.sie-6i & $2.72 E-15$ & $4.5 \pm-10$ \\
\hline $572-3$ & $9.02 E+0 !$ & $5.595-64$ & 1. $12 E+02$ & 3. DEE-64 & $\therefore, b:[-1)$ & $\therefore 4: 5-12$ & $4.7 \div-10$ \\
\hline BN $T 2-40$ & $\overline{3}, 29 E+0 i$ & $2.34 E-\hat{v} 4$ & $1,46 E+02$ & $1.6 \mathrm{IE}-\hat{\mathrm{y} q}$ & $\therefore .62 E-v !$ & $1.725-15$ & $9.67 E-10$ \\
\hline init $72-4 i$ & $3.55 E+6 !$ & $4.3 .3 E-64$ & $1.475 \div 02$ & $2.65 E-14$ & $1.32=-9 !$ & $0.64=-15$ & $9.5:-10$ \\
\hline Sin $12-42$ & 9. 泟E+ii & $5.30 E-64$ & i. $.57 E+02$ & $2.35-64$ & $1.62 E-01$ & $2.17 E-18$ & $4.37 E-10$ \\
\hline 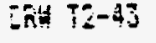 & 1. $.32 E+122$ & $4.22 E-64$ & a.suEt+vi & $2.31 E-14$ & $1.62 \mathrm{E}-\mathrm{ij}$ & $2.8=-10$ & $4.36-10$ \\
\hline Shy $92-44$ & 1. $04 E+42$ & $4.56 E-i 44$ & $1.63 E+i \hat{j} \hat{i}$ & $2.47 E-64$ & C.SBE- 11 & $0.15 E-10$ & $4.23 E-10$ \\
\hline Ifid in & 1. $A E E+12$ & i. $215-2 j$ & 1. $72 \pm+12$ & $\therefore .6 E E-64$ & i. $.63-1 \mathrm{i}$ & $1.01 E-12$ & $4.6 \div E-80$ \\
\hline Lnit iz-40 & $1.06 E+10=$ & 7.: : $3 E-05$ & $1.735+02$ & $5.62 E-65$ & 1. $60.35-19$ & i. $50-14$ & J.7iE- 0 \\
\hline ESH TE-4i & 1.11E-12 & $50.67-94$ & $1.2 \mathrm{i} E+\mathrm{u}_{2}$ & $4,425-74$ & $\{.6+E-i j$ & C.TE- :- & $3.57 E-10$ \\
\hline 0 - & $1,1+2+12$ & $5.20 E-34$ & $\therefore .55=+12$ & $2.355-64$ & $1.0+E-1]$ & $5.47 E-15$ & $3.30-10$ \\
\hline [nit T-4 & 6.j4E+6i & $3.355-6$ & 1. QSEQ⿻日木 & 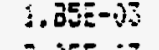 & 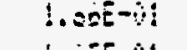 & \begin{tabular}{l}
$2.0: E-: !$ \\
\hdashline
\end{tabular} & $7.50-10$ \\
\hline $\sin 12-5$ & $1 . E=E+Q_{i}$ & $3.76 E=-6.5$ & $2 . Z E+i z$ & Z. $02 E-4 j$ & $1.30 E-01$ & $\therefore$ (1) & $3.20=-1 j$ \\
\hline
\end{tabular}


TABLE B.12a. Potassium (K) Leach Fractions from Flow Through Test whole Grout with Groundwater (DFCOL $3 A C$ )

\begin{tabular}{|c|c|c|c|c|c|}
\hline SAMPLE & $A \cap / A B$ & EUn. An/AO & DAYS & D inc. & D cur. \\
\hline EFFLUEHT I & $1.94 E-02$ & $1.34 E-02$ & $3.00 E+00$ & $4.18 E-10$ & $4.18 E-10$ \\
\hline EFFLUENT 2 & $2.05 E-62$ & $3.99 E-02$ & $9.00 E+00$ & 8. $72 \mathrm{E}-10$ & $5.705-10$ \\
\hline EFFLUEKT $j$ & $4.72 E-02$ & $8.90 E-j 32$ & $1.60 E+01$ & $8.07 E-69$ & $1.655-69$ \\
\hline EFFLUENT 4 & i. $13 E-0 i$ & $2.02 E-01$ & $\therefore .90 E \div 01$ & $3.31 E-v i 7$ & $7.18 E-69$ \\
\hline EFFLUENT 5 & $1.14 E-01$ & 3. 1OEE-01 & $2.50 E+01$ & 1. $055-07$ & 1. $97 E-68$ \\
\hline EFFLUENT 6 & $2.35 E-02$ & $3.39 E-01$ & $2.70 E+01$ & $1.24 E-08$ & $1.45 E-05$ \\
\hline EFFLUENT 7 & $3.17 E-03$ & $3.47 E-01$ & $3.20 E+01$ & 3.02E-09 & 1. $4 \hat{v E}-\overline{\mathrm{i}} \hat{\mathrm{o}}$ \\
\hline EFFLLIEHT 9 & $2.24 E-02$ & $3.70 \mathrm{E}-01$ & $3.90 E+01$ & $4.85 E-19$ & i.JOE- $i j \bar{j}$ \\
\hline EFFLUENT II & $3.73 E-02$ & $4.07 E-01$ & $4.50 E+01$ & $2.16 E-D B$ & $1.43 E-33$ \\
\hline EFFLUENT 13 & $3.10 E-02$ & $4.38 E-01$ & $5.20 E \div 01$ & $1.27 E-08$ & $1.46 E-05$ \\
\hline EFFLUEAT 15 & $3.07 E-02$ & $5.69 E-01$ & $6.00 E+01$ & ¿. $10 E-60$ & 1. $43 E-38$ \\
\hline EFFLUENT 17 & $4,01 E-13$ & $4.73 E-01$ & $6.70 E+01$ & 2.7AE-ji & 1. 3JE- 15 \\
\hline EFFLUEHT 19 & $4.08 E-63$ & $4.77 E-01$ & i. $30 E+01$ & $4.33 E-10$ & $1.28 E-10$ \\
\hline EFFLUERT 22 & $1.51 E-02$ & $4.92 E-01$ & $8.70 E+01$ & $1.24 E-09$ & $1.13 E-05$ \\
\hline EFFLUENT 24 & $4.27 E-133$ & $4.96 E-01$ & $9.40 E+01$ & $4.50 \mathrm{E}-10$ & 1. $10 E-08$ \\
\hline EFFLUENT 26 & $1.50 E-02$ & $5.11 E-01$ & 1.01E+12 & $6.015-09$ & $1.07 E-60$ \\
\hline EFFLUENT 28 & $1.35 E-62$ & $5.25 E-01$ & 1. $13 E+02$ & $1.31 E-07$ & $1.01 E-08$ \\
\hline EFFLUENT 30 & 3.10E-\$3 & $5.28 E-01$ & $1.22 E+102$ & $1.86 E-10$ & $9.60 E-09$ \\
\hline EFFLUEHT 32 & $5.70 E-03$ & $5.34 E-\hat{01}$ & $1.5 B E \div 02$ & $5.60 E-1 !$ & $7.57 E-6 \overline{9}$ \\
\hline EFFLUENT 33 & $0.00 E+00$ & $5.34 E-01$ & $1.83 E+02$ & $0.00 E+00$ & $6.36 E-67$ \\
\hline
\end{tabular}

TABLE B.12b. Sodium (Na) Leach Fractions from Flow Through Test Whole Grout with Groundwater (DFCOL3AC)

\begin{tabular}{|c|c|c|c|c|c|}
\hline SAMPLE & AniAo & EUh. An/ÂO & DAYS & inc. & D cus. \\
\hline EFFLUEHT ! & $2.99 E-02$ & $2.99 E-02$ & $3.00 E+00$ & $8.94 E-10$ & $9.74 E-10$ \\
\hline EFFLUEHT 2 & $3.03 E-02$ & $6.02 E-02$ & $7.00 E+00$ & $1.91 E-08$ & $1.345-09$ \\
\hline EFFLUEHT 3 & $7.28 E-02$ & 1.33E-01 & $1.60 E+i j\}$ & $1.775-6 B$ & $3.675-09$ \\
\hline EFFLUENT 4 & $1.67 E-01$ & $3.00 E-01$ & $1.90 E+01$ & $7.265-6 i$ & $1.67 E-\mathrm{iE}$ \\
\hline EFFILUENT 5 & $1.65 E-01$ & $4.675-01$ & $2.5(0 E+01$ & $2.3 ! E-\hat{v} 7$ & $3.575-16$ \\
\hline EFFLUENT 6 & $3.79 E-02$ & 5. U6E-01 & $2.90 E+01$ & $3.15 E-6.6$ & $3.735-80$ \\
\hline EFFLUEHT 7 & $1.38 E-02$ & $5.20 E-01$ & $3.20 E+[i\}$ & $\dot{8} . \dot{\partial u E}-\hat{v} \%$ & $3.565-68$ \\
\hline EFFLUEHT 9 & $3.80 E-1) 2$ & $5.58 E-01$ & $3.76 E \div 01$ & ¿. $.39 E-8 E$ & 3.5GE-DS \\
\hline EFFLUEHT II & c. $22 E-62$ & $6.205-61$ & 4. $5 \dot{E} E+01$ & 6. $.2 E-88$ & 3. 酒E-6E \\
\hline EFFLUENT IJ & $2.07 E-62$ & $6 .+1 E-0 i$ & E.20E+a! & $5,08 E-65$ & $3.75 E-48$ \\
\hline EFFLUENT I5 & $1.84 E-02$ & 6.5GE- & D. $60 E-61$ & 5. 汿E-19 & $3.54 E-3 \overline{3}$ \\
\hline EFELUENT ! 7 & 1. $36 E-02$ & $6.7 \pi E-31$ & อ. ZuE+ui & 3.27E-35 & $3.36-46$ \\
\hline EFFUENT I? & $1.27 E-12$ & S. $325-7 !$ & $\bar{i} .30 \mathrm{i}+0 \mathrm{i} \mathrm{i}$ & $4.15-69$ & $3.27 E-60$ \\
\hline EFFLUEKT 22 & $2 .: 2 E-1) 2$ & I. & B.70E+6i & $2,40 E-14$ & $3,60 E-35$ \\
\hline EFFLUENT 24 & b. GEE-03 & $7.14 E-01$ & $7.4 \dot{W} E+i j\}$ & $1.10 \mathrm{E}-\mathrm{9}$ & $2.7 \mathrm{EE}-\mathrm{BE}$ \\
\hline EFFLLENT 26 & 2. $04 E-62$ & $7.34 E-i !$ & i. $011 E+i 2$ & 1. & $\therefore .3 \mathrm{VE}-\mathrm{ijE}$ \\
\hline EFIIJEWT 28 & $1.53 E-42$ & F. STE-B! & 1.13E+1\% & $3.8 \div-8$ & $2.735-160$ \\
\hline EFFLUENT Ji & $\therefore, 6 B E-42$ & T. Q́4E-iai & $\therefore .2 Z E+V i$ & $\therefore 2 \mathrm{BE}-9 ?$ & $2.6 \times-6$ \\
\hline EFFLUENT Ja & $1.26 E-62$ & T. IaE- & !.5EE+12 & $2.96 E-10$ & $\therefore 21 E-i B$ \\
\hline EFFLUENT & $7.72[-1]$ & I. & !. & $1.5 \mathrm{SE}-10$ & !. \\
\hline
\end{tabular}


TABLE B.12e. Boron (as H3B03) Leach Fractions from Flow Through Test whole Grout with Groundwater (DFCOL3AA)

\begin{tabular}{|c|c|c|c|c|c|}
\hline 5AMPLE & $A n / A o$ & cun. An/Ao & DAYS & $D$ inc. & D cun. \\
\hline EFFLUENT 1 & $3.34 E-03$ & $3.34 E-03$ & $3.00 E+00$ & $1.24 E-11$ & $1.24 E-1$ \\
\hline EFFLUEHT 2 & $1.70 E-03$ & $5.04 E-03$ & $9.00 E+00$ & $5.98 E-12$ & $9.41 E-1$ \\
\hline EFFLLEHT 3 & $4.62 E-03$ & $9.66 E-03$ & $1.60 E+01$ & $7.13 E-11$ & $1.95 E-\mathrm{i}$ \\
\hline SFFLUENT 4 & $1.06 E-02$ & $2.03 E-02$ & i. $90 E+0 \mathrm{i}$ & 2. $33 E-6 ?$ & $7.23 E-1$ \\
\hline EFFLUEYT 5 & $1.19 E-02$ & $3.22 E-02$ & $2.50 E+01$ & $1.15 E-09$ & $1.3 B E-11$ \\
\hline EFFLUEHT 6 & $2.11 E-03$ & $3.43 E-02$ & $2.90 E+01$ & $1.00 E-10$ & $1.35 E-10$ \\
\hline EFFLUEKT 7 & $8.63 E-14$ & $3.52 E-02$ & $3.20 E+01$ & $3.375-11$ & $1.29 E-10$ \\
\hline EFFLUELT 9 & 1.92E-03 & $3.71 \mathrm{E}-02$ & $3.90 E+01$ & 3. $54 E-11$ & $1.18 E-10$ \\
\hline EFFLUENT 11 & $3.44 E-03$ & $4.055-02$ & $4.50 E+01$ & 1. $35 \mathrm{E}-10$ & $1.22 E-16$ \\
\hline EFFLUEKT IJ & $9.23 E-i)^{4}$ & 4. $14 E-02$ & $5.20 E+01$ & :. $13 E-11$ & $1.10 E-10$ \\
\hline EFFLUENT IS & $9.7 \mathrm{BE}-04$ & $4.24 E-02$ & 6.00E +01 & $1.12 E-1 !$ & $1.00 E-1 \hat{0}$ \\
\hline EFFLUENT 17 & 1. $02 \mathrm{E}-0 \mathrm{~J}$ & $4.34 E-02$ & 6. $70 E+01$ & $1.30 E-11$ & $9.40 E-11$ \\
\hline EFFLUENT IO & $6.45 E-04$ & 4.41E-02 & $7.30 E+01$ & $\pm .08 E-11$ & 8. $89 E-11$ \\
\hline EFFLUEKT 22 & $! .24 E-03$ & $4.53 E-02$ & B. $70 E+01$. & 8. $32 E-12$ & $7.58 E-11$ \\
\hline EFFLUEHT 24 & $3.41 E-64$ & $4.57 E-02$ & $9.40 E+01$ & $2.87 E-12$ & $7.41 E-! 1$ \\
\hline EFFLUENT 26 & $9,20 E-04$ & $4.66 E-02$ & $1.01 E+02$ & $2.25 E-11$ & $7.17 E-11$ \\
\hline EFFLUEMTT 28 & 1.00E-03 & $4.76 E-02$ & 1.13E+02 & $8.95 E-12$ & $6.69 E-11$ \\
\hline EFFLUEKT 30 & $\therefore .63 E-03$ & $4.92 E-02$ & 1.22E+02 & $5.17 E-11$ & $6.635-11$ \\
\hline EFFLUENT 32 & $2.14 E-03$ & $5.14 E-02$ & 1. $58 E+02$ & 6. $35 \mathrm{E}-12$ & 5.57E-11 \\
\hline EFFLUENT 33 & $1.70 E-03$ & $5.31 E-02$ & $1.88 E+02$ & $7.44 E-12$ & $5.005-11$ \\
\hline
\end{tabular}

TABLE B.12f. Fluoride (F) Leach Fractions from Flow Through Test whole Grout with Groundwater (DFCOL 3AA)

\begin{tabular}{|c|c|c|c|c|c|}
\hline SAMPLE & An/Ao & cus. An/Ao & DAYS & jinc. & D cus. \\
\hline EFFLUENT 1 & $7.81 E-04$ & T.81E-04 & 3. OOE +10 & 6.79E-1J & $6.79 E-13$ \\
\hline EFFLUEHT 2 & $7.68 E-04$ & $1.575-03$ & $9.00 E+00$ & $1.29 E-12$ & $7.13 E-13$ \\
\hline EFFLUEHT 3 & $1.57 E-03$ & $3.14 E-03$ & $1,60 E+01$ & $3.22 E-12$ & 2. $05 E-12$ \\
\hline EFFLLUENT 4 & $3.61 E-03$ & $6.75 E-03$ & $1.90 E+01$ & $3.37 E-10$ & 5. $00 \mathrm{E}-12$ \\
\hline EFFLUENT 5 & $2.925-03$ & $9.63 E-03$ & $2.5 \hat{0} E+\hat{0}]$ & b. 35E-11 & 1.25E-i: \\
\hline EFFIUEHT 6 & $7.965-04$ & $1.05 E-02$ & $2.90 E+01$ & $1.43 \mathrm{JE}-11$ & 1.26E-11 \\
\hline EFFLUEHT 7 & $5.40 E-04$ & $1.10 E-02$ & $3.20 E+01$ & 1.3E-1! & $1.25 E-15$ \\
\hline EFFLLIENT 9 & $1.54 E-03$ & $1.25 E-62$ & 3. ToE+Â! & $2.29 E-1:$ & 1. $35 \bar{E}=-i j$ \\
\hline EFFLUEST II & $2.32 E-v 3$ & 1. $43 E-62$ & $4.50 E+01$ & $3.37 E-11$ & $1.64 E-11$ \\
\hline EFFLUENT :3 & $1.50 E-63$ & 1. 0 AE -02 & S.ZOE+61 & $=965-11$ & 1. $\mathrm{i} 2 \mathrm{E}-\mathrm{i} i$ \\
\hline EFFLUERT IE & 1. 53E-13 & $1.74 E-62$ & D. BuE 001 & $2.79 E-11$ & !.79E-1! \\
\hline EFF!UENT I7 & i. $00 E-6 j$ & :.89E-ij2 & 5. $705+41$ & !. $\quad 3 \mathrm{BE}-! !$ & $1.79 E-11$ \\
\hline EFFLUENT I? & $7.77=-04$ & 1. 汭E- 62 & $7.30 E+91$ & $2.4 E E-1 !$ & 1. BIE-1! \\
\hline EFFLUENT 22 & $1.4: E-03$ & 2.: $: 3 E-02$ & 3. $70 E+01$ & $1.08 E-11$ & $1.74 E-1 !$ \\
\hline FLUUEKT 24 & $4.75 E-64$ & $2 .: \Delta E-\hat{2} z$ & $9.40 E \div 01$ & $5.57 E-12$ & !.SFE-11 \\
\hline EFFLLIENT 20 & 1. $04 E-15$ & $2.34 E-02$ & $1.61 E \div 02$ & $7.1 \mathrm{EE}-1 !$ & L.E:E-!: \\
\hline EFFLUENT 20 & $2.21 E-13$ & $2.5 b E-02$ & 1.13E+102 & $4.35 E-1 !$ & 1.: $: 4 E-! 1$ \\
\hline EFFLUEKT 30 & $2.6 J E-\hat{U} J$ & 2. $3 \overrightarrow{i E}-92$ & :. $22 E+0^{n}$ & $1.345-16$ & Z.19E-!1 \\
\hline EFFLUEHT 32 & $3.455-13$ & S.1TE-b2 & i. $58 E+02$ & $1.69 E-1:$ & 2. $: 2 E-! !$ \\
\hline EFFLLENT 30 & $\{.32 E-93$ & $3.35 E-0_{i}$ & $i .60 E+0 \bar{Z}$ & $3.40 E-12$ & :.395-i: \\
\hline
\end{tabular}


TABLE B.12g. Nitrate (NO3) Leach Fractions from Flow Through Test whole Grout with Groundwater (DFCOL3AA)

SARPLE
EFFLUEHT 1
EFFLUENT 2
EFFLUENT 3
EFFLUENT 4
EFFLUENT 5
EFFLUENT 6
EFFLUENT 7
EFFLUENT 9
EFFLUENT 11
EFFLUENT 13
EFFLUENT 15
EFFLUENT 17
EFFLUENT 19
EFFLUENT 22
EFFLUENT 24
EFFLUENT 26
EFFLUENT 23
EFFLUENT 30
EFFLUENT 32
EFFLUENT 33

An/AD
$1.77 E-02$
$1.81 E-02$
$4.41 E-02$
$1.01 E-01$
$1.04 E-01$
$1.80 E-02$
$7.73 E-03$
$1.8 B E-02$
$3.53 E-02$
$1.07 E-02$
$9.90 E-03$
$1.02 E-02$
$8.31 E-03$
$1.78 E-02$
$5.46 E-03$
$1.67 E-02$
$1.28 E-02$
$1.22 E-02$
$2.74 E-02$
$7.54 E-03$

cus. An/Âũ

$1.77 E-02$

$3.58 E-02$

7. $99 E-02$

$1.81 E-01$

$2.85 E-01$

3.03E-01

$3.11 E-01$

$3.29 E-01$

3. $65 E-01$

$3.75 E-01$

3. 85E-01

3. 35E-01

4. $04 E-01$

$4.23 E-01$

4.27E-01

4. 44E-0!

4.57E-01

4. $69 \mathrm{E}-01$

$4.36 E-01$

5. $04 E-01$
1 inc.

3. $50 \mathrm{NE}-10$

6. $78 E-10$

6. 49E-09

$2.665-07$

8. $71 E-08$

$7.31 E-0 \%$

2.70E-09

J. 42E-09

1. $74 E-6 B$

1. $52 E-69$

1. 14E-0?

$1.735-09$

1.79E-37

$1.72 E-09$

7. $34 \mathrm{E}-10$

$7.57 E-09$

1. $63 \mathrm{JE}-\hat{6} 9$

2. $87 \mathrm{E}-69$

1. $68 E-38$

$1.46 E-10$ tิ cus.

3. $50 \mathrm{E}-10$

4.76E-10

1. $33 E-09$

5. $77 E-99$

1. $09 E-0 B$

1.10E-VE

1. VTE-

1. $01 E-\mathrm{j} B$

5.06E- 68

1. $14 E-00$

3. $56 E-09$

$9.08 E-09$

8. 3TE-69

7. 2 JE- 69

7. $50 \mathrm{E}-0 \overline{9}$

7. 52E- 69

7. $40 E-39$

7.J1E-09

b. $53 E-69$

5. 49E-03 
TABLE B.12i. Technetium-99 (TC-99) Leach Fractions from Flow Through Test Grout with Groundwater (3A)

\begin{tabular}{|c|c|c|c|c|c|c|c|}
\hline SAMFLE & PORE YOLUHE & $\mathrm{UCI}$ & TIKE OAYS & $A \cap / A D$ & CUX ÂnZAOD & DINC CKLJSEC & DCIN CK2/SEC \\
\hline LIJA-1 & $1.06 E+00$ & $5.28 E-63$ & 3. $00 E+00$ & $2.95 E-03$ & $2.75 E-103$ & $3.72 E-12$ & 9.72E -12 \\
\hline LT3A-2 & $1.70 E+00$ & $1.06 E-03$ & $9.00 E+10$ & $5.93 E-04$ & $3.55 E-0 \hat{3}$ & $1.76 \mathrm{E}-12$ & $4.67 E-12$ \\
\hline LTJA-3 & $3.16 E+00$ & $0.90 E-03$ & $1.60 E+01$ & $4.98 E-03$ & $8.53 E-03$ & $1.30 E-10$ & $1.525-11$ \\
\hline LT3A-4 & $6.60 E+000$ & $2.35 E-02$ & $2.00 E+01$ & $1.32 E-\hat{v} 2$ & $2.1 F E-62$ & 2. $: J E-1) 7$ & $\bar{x} .36 \bar{E}-11$ \\
\hline LT3A-5 & $9.94 E+00$ & $4.31 E-02$ & $2.50 E+01$ & $2.41 E-02$ & $4.58 E-02$ & $4.14 E-69$ & $2.60 \mathrm{E}-10$ \\
\hline$\angle T 3 A-6$ & $1.24 E+01$ & $3.10 E-03$ & $2.90 E+01$ & $1.73 E-03$ & $4.75 E-62$ & $3.22 E-11$ & $2.60 E-10$ \\
\hline LT3A-? & $1.39 E+01$ & $1.69 E-03$ & $3.20 E+01$ & $9.47 E-04$ & $4.85 E-02$ & 1.32E-!! & $2.45 E-10$ \\
\hline LITA & 1.65E+01 & $4.17 E-03$ & $3.60 E+01$ & $2.33 E-03$ & $5.08 E-02$ & $4.73 E-1 \mathrm{i}$ & $2.40 E-10$ \\
\hline $613 A^{2}-9$ & 1.39E+01 & $3.03 E-03$ & $3.30 E+01$ & $1.72 E-63$ & $5.25 E-02$ & 4.36E-1! & $2.50 E-10$ \\
\hline $\operatorname{LT} 3 \hat{A}-10$ & $2.24 E+01$ & $3.12 E-03$ & $4.20 E+01$ & $1.75 E-0 j$ & $5.43 E-6 i \bar{i}$ & $3.90 \mathrm{E}-11$ & $2.34 E-10$ \\
\hline$[33+1]$ & $2.49 E+01$ & $1.79 E-0 . j$ & $4.50 E+01$ & $1.00 E-03$ & $5.53 E-0 \bar{Z}$ & $1.30 E-11$ & $2.27 e-10$ \\
\hline $173 A-12$ & $2.83 E+01$ & 1.30E-03 & $4.70 E+01$ & $7.28 E-04$ & J. $6 \mathrm{ve}-02$ & $4.60 E-12$ & $2.14 E-10$ \\
\hline $133 A-13$ & 3.04E+01 & $1.62 E-03$ & $5.20 E+01$ & $3.09 E-04$ & $5.69 E-02$ & $1.21 \mathrm{E}-1 !$ & $2.065-10$ \\
\hline LTSÂ-14 & $3.45 E+01$ & $3.38 E-03$ & $5.60 E+01$ & $1.695-03$ & $5.88 E-02$ & $3.105-11$ & $2.06 E-10$ \\
\hline LT3A-15 & $3.575+01$ & $7.925-04$ & $6.00 E+01$ & $4.43 E-04$ & $5.93 E-02$ & $1.91 \leq-12$ & $1.96 E-10$ \\
\hline LT3A-16 & $3.75 E+01$ & $1.26 E-03$ & $6.30 E+01$ & $7.04 E-04$ & $0.00 E-6,2$ & $7.28 E-12$ & $! .91 E-10$ \\
\hline$[T 3 A-!]$ & $3.89 E+01$ & $1.46 E-03$ & $6.60 E \div \hat{0 I}$ & B.iJE-04 & $6.68 E-62$ & $8.67 E-12$ & $! .87 E-10$ \\
\hline LIJA-18 & $4.10 E+01$ & $2,71 E-03$ & 7.0OE+01 & $1.52 E-03$ & $6.23 E-02$ & 2. $60 E-11$ & $1.355-10$ \\
\hline LI3A-19 & $4.23 E+101$ & 6. 32E-04 & $7.305+01$ & 3.54E-04 & $0.27 E-1 j z$ & $1.64 E-12$ & $1.80 E-10$ \\
\hline $473-70$ & $4.32 E+01$ & $1.57 E-03$ & $7,70 E+61$ & $8.79 E-04$ & $6.35 E-62$ & b.71E-12 & $1.75 E-10$ \\
\hline$[13 \hat{A}-21$ & $4.59 E \div 01$ & $3.15 E-03$ & $8.40 E+01$ & $1.76 E-1) 3$ & $6.53 E-0.2$ & 1. $39 E-11$ & $1.70 E-10$ \\
\hline $4 T 34-22$ & $4.73 E+01$ & $1.4 J E-03$ & B. $70 E+01$ & $7.97 E-04$ & $6.61 E-02$ & I. $23 E-11$ & $1.605-10$ \\
\hline LTSA-23 & $4,83 E \div 01$ & $7.47 E-04$ & $9.10 E+01$ & $4.18 E-04$ & $6.65 E-02$ & 1.52E-12 & $1.625-10$ \\
\hline LT3ิ & $4.86 E+01$ & $4.70 E-04$ & $9.40 E+01$ & 2. $j 4 E-04$ & $6.6 B E-62$ & 1.11E-12 & $1.50 E-10$ \\
\hline $173 \hat{A}-25$ & $5.21 E+01$ & $3,21 E-03$ & S. $.00 E+01$ & $1.77 E-03$ & $6.66 E-i \mathrm{i} 2$ & $2.77 E-11$ & $1.60 \bar{E}-10$ \\
\hline Liș-26 & $5.46 E+01$ & $1.10 E-03$ & $1.01 E+02$ & $3.13 E-014$ & $6.92 E-62$ & $5.55 E-! 2$ & $1.58 E-10$ \\
\hline LI3A-27 & $5.71 E+01$ & $1.01 E-03$ & $1.04 E+12$ & $5.665-64$ & $0.95 E-02$ & 4. $16 E-i 2$ & 1. $5 E E-10$ \\
\hline $1734-26$ & $6.48 E+0 i$ & $1.07 E-02$ & 1.13E+02 & $5.97 E-93$ & $7.57 E-0.2$ & $1.05 E-i 0$ & 4.70E-! 0 \\
\hline Lishy & $7.04 E+0 i$ & $1.64 E-03$ & 1. $19 E+02$ & $9.17 E-39$ & $7.66 E-62$ & $6.535-12$ & $1.655-10$ \\
\hline$(T 3 A-30$ & T.JJE+ôt & $9.18 E-04$ & $1.22 \mathrm{E}+02$ & $5.135-04$ & $7.72 E-\hat{12}$ & $4.70 \mathrm{UE}-12$ & 1.SJE-10 \\
\hline [13:-3! & $7.69 E+6 !$ & $3.28 \mathrm{E}-03$ & $1.26 E+002$ & $\bar{i} .15 E-04$ & $7.75 E-02$ & $4.44 E-12$ & $2.615-10$ \\
\hline$[33-32$ & $3.96 E+01$ & $2.315-03$ & 1. $58 \mathrm{BE}+02$ & $1.29 E-03$. & $7.92 E-1.2$ & 1. OPE- 12 & [. .33E- 10 \\
\hline$[15 \overline{-153}$ & 1. $34 E+i \mathrm{i} 2$ & S. ôAE-0.j & $1.68 E+62$ & $3.83 E-9 j$ & 3.30E-6z & L.7FE- & i. $2 A E-i j$ \\
\hline
\end{tabular}


TABLE B.12i. Technetium-99 (TC-99) Leach Fractions from Flow Through Test, Grout with Groundwater (3B)

\begin{tabular}{|c|c|c|c|c|c|c|c|}
\hline SAMPLE & PORE VOLUME & $\mathrm{uCi}$ & TIME DAYS & $\mathrm{An} / \mathrm{AO}$ & CUM AnIAO & DIHC CH2/SEC & DCUH CHa'SECC \\
\hline LT3B-1 & $1.39 E+00$ & $1.315-03$ & $3.00 E+00$ & $7.01 E-04$ & $7.01 E-04$ & 5. $21 E-13$ & $5.21 E-13$ \\
\hline LTBB-2 & $3.66 E+00$ & $2.11 E-03$ & 6. $.00 E+00$ & $1.13 E-03$ & $1.83 E-(13$ & $1.57 E-11$ & $1.77 E-12$ \\
\hline LT38-3 & $4.62 E+100$ & $1.62 E-03$ & $9.00 E+00$ & $8.695-04$ & $2.70 E-03$ & $9.32 E-12$ & $2.57 E-12$ \\
\hline LTBB -4 & $7.10 E+00$ & $4.77 E-03$ & $1.30 E+01$ & $2.56 E-03$ & $5.26 E-03$ & $5.4 ! E-11$ & $6.75 E-12$ \\
\hline $1338-5$ & $9.11 E+00$ & $2.82 \mathrm{E}-03$ & $1.60 E+01$ & 1.5IE-03 & $6.77 E-03$ & $3.19 E-11$ & 9.0 SE- 12 \\
\hline $173 B-6$ & 1.1FE+01 & $3.30 E-03$ & $2.00 E+01$ & $1.77 E-03$ & $8.535-03$ & $2.57 E-11$ & $1.16 E-11$ \\
\hline $4738-7$. & $1.51 E+01$ & $3.77 \mathrm{E}-03$ & $2.50 E+01$ & $2.01 E-03$ & $1.05 E-012$ & $2.75 E-11$ & $1.91 E-11$ \\
\hline LT38-8 & $1.75 E+01$ & $2.96 E-03$ & $2.90 E+01$ & $1.58 E-03$ & $1.21 E-02$ & $2.54 E-11$ & 1.61E-11 \\
\hline LT3B-9 & $1.86 E+01$ & $2.08 E-03$ & $3.20 E+01$ & $1.11 E-03$ & $1.32 E-02$ & I.7JE-1! & $1.74 E-11$ \\
\hline LT3B-10 & $2.12 E+01$ & $3.32 E-03$ & $3.60 E+(1) 1$ & $1.77 E-03$ & $1.50 E-0.2$ & $2.60 E-1 i$ & 1.99E-11 \\
\hline LT3E-11 & $2.36 E+01$ & $2.59 E-03$ & $3.90 E+01$ & $1.38 E-63$ & $1.64 E-02$ & $2.66 E-11$ & $2.19 E-11$ \\
\hline $1738-12$ & $2.70 E+0 i$ & $2.59 E-03$ & $4.20 E \div 01$ & $1.39 E-03$ & 1.78E-02 & $2.3 B E-1 !$ & 2.3FE-11 \\
\hline LT3B-13 & $2.94 E+01$ & $1.26 E-03$ & $4.50 E+01$ & $6.75 E-04$ & $1.85 E-02$ & $5.61 E-12$ & $2.40 E-1 !$ \\
\hline LI38-14 & $3.28 E+01$ & $2.93 E-03$ & $4.90 E+01$ & $1.57 E-6 j$ & $2.005-02$ & 2. $0 \mathrm{BE}-1 j$ & $2.60 E-11$ \\
\hline $1735-15$ & $3.50 E+01$ & $2.01 E-0 j$ & $5.20 E+0 !$ & $1.07 E-03$ & $2.11 E-02$ & $1.61 E-11$ & $2.72 E-11$ \\
\hline $1538-16$ & $3.89 E+01$ & $2.38 E-03$ & $5.60 E+01$ & $1.54 E-0 j$ & $2.26 E=02$ & $1.96 E-11$ & $2.71 E-11$ \\
\hline $4 T 3 B-17$ & $4.04 E+01$ & $1.15 E-03$ & $6.00 E+01$ & 6. $14 E-04$ & 2. $33 \mathrm{E}-012$ & $3.49 E-12$ & $2.865-11$ \\
\hline $4 T 38-18$ & 4.19E+01 & $1.86 E-03$ & 6.JUE+1iI & $9.94 E-04$ & 2. $42 E-02$ & $1.385-11$ & $2.96 E-11$ \\
\hline LT3B-19 & 4.J3E+01 & $2.04 E-0 \bar{J}$ & $6.60 E+01$ & $1.095-03$ & $2.53 E-02$ & $1.47 E-11$ & 3.09E-1i \\
\hline $213 B-20$ & $4.55 E+01$ & $1.73 E-03$ & $7.00 E+01$ & $9.27 E-04$ & $2.63 E-02$ & $7.10 E-12$ & J.13E-11 \\
\hline LT3B-21 & 4. $64 E+01$ & 8.97E-04 & $7.30 E+01$ & $4.80 E-04$ & $2.67 \mathrm{E}-\mathrm{V2}$ & $3.215-12$ & $3.11 E-11$ \\
\hline $473 B-22$ & $4.73 E+01$ & १.98E-04 & $7.70 E+01$ & $5.34 E-\hat{y} q$ & $2.73 E-62$ & $2.35 E-12$ & J. DTE-i $i$ \\
\hline $6738-23$ & $5.12 E+01$ & $3.91 E-03$ & $8.20 E+01$ & $2.09 E-03$ & $2.74 E-1.2$ & $2.965-11$ & $3 . j 4 E-11$ \\
\hline $1738-24$ & $5.39 E+01$ & $1.52 E-03$ & $8.50 E+01$ & 8. $10 E-04$ & $3.02 E-62$ & $1.02 E-11$ & $3.40 E-i i$ \\
\hline$: 13 B-25$ & $5.65 E+01$ & $1.61 E-03$ & $8.30 E \div 01$ & $8.62 E-044$ & $3.10 E-192$ & 6. $14 E-12$ & $3.44 E-11$ \\
\hline LIJB-26 & $5.80 E+01$ & $1.29 E-63$ & $7.20 E+01$ & $6.90 E-04$ & 3. IIE-02 & $3.65 E-12$ & $3.48 E-11$ \\
\hline$\lfloor 135-27$ & $5.02 E+0 !$ & $1.44 E-05$ & $9.60 E \div 01$ & $7.705-04$ & $3.25 E-32$ & $4.89 E-12$ & $3.49 E-1 !$ \\
\hline LTडE-28 & D.:IE+ô & $3.12 E-0 j$ & $9.70 E+01$ & $1.67 E-03$ & $3.42 E-02$ & J. 39E-11 & $3.755-13$ \\
\hline L130-29 & b. 19E+01 & $2.17 E-03$ & $1.02 E+02$ & $1.16 E-03$ & $3.53 E-1) 2$ & i.66E-!! & 3.89E-1! \\
\hline LTSE-30 & $6.51 E+01$ & $3.24 E-03$ & $1.11 E+02$ & $1.73 E-013$ & $3.71 E-02$ & 8. $3 B E-12$ & $3.93 E-1:$ \\
\hline L135-31 & a. $78 E+01$ & $1.62 E-03$ & $1.17 E \div 02$ & $3.65 E-104$ & $3.7, F E-02$ & 5.515-12 & $3.90 E-11$ \\
\hline $1756-32$ & อ. $37 E+01$ & 1.72E-03 & $1.20 E+102$ & $1.03 E-03$ & $3.90 E-612$ & $1.76 E-1 i$ & 4. \\
\hline LI38-33 & 7. $04 E+01$ & $2.05 E-63$ & $1.24 E+02$ & $1.10 \mathrm{E}-03$ & $4.01 E-02$ & $9.53 E-12$ & $\{.11 E-11$ \\
\hline ITSB-34 & $5.32 E+0 i$ & $5.17 E-O 4$ & 1. $52 E+02$ & $2.76 E-\dot{v} \alpha$ & $4.03 E-02$ & $5.57 E-i 4$ & J.4ie-ii \\
\hline$(13 E-35$ & $1.46 E+02$ & $6.35 E-14$ & $1.32 E+02$ & $3.40 E-04$ & $4.07 E-02$ & $1.36 \mathrm{E}-13$ & Z.6FE-11 \\
\hline
\end{tabular}


TABLE B.13a. Potassium (K) Leach Fractions from Flow Through Test Grout/Sediment with Groundwater (DIFCOLI)

\begin{tabular}{|c|c|c|c|c|c|}
\hline SAKPLE & An/AO & Cun. $A_{n} / A_{0}$ & DAYS & D.inc. & D cus. \\
\hline EFFLUENT 1 & $0.00 E+00$ & $0.00 E+00$ & $3.00 E+00$ & $0.60 E+00$ & $0.00 E+00$ \\
\hline EFFLUENT 2 & $0.00 E+00$ & $0.00 E+00$ & $6.00 E+00$ & $0.00 E+00$ & $0.00 E+00$ \\
\hline EFFLUENT 3 & $0.00 E+00$ & $0.00 E+00$ & $9.00 E+00$ & $0.00 E+00$ & $0.60 E+100$ \\
\hline EFFLUIENT \& & $0.00 E+00$ & $0.00 E+00$ & $1.30 E+01$ & $0.00 E+00$ & $0.00 E+\hat{w} \hat{v}$ \\
\hline EFFLUERT 5 & $0.00 E+00$ & $0.00 E+00$ & $1.70 E+03$ & $0.00 E+00$ & $0.60 E+00$ \\
\hline EFFLUIENT 6 & $0.00 E+00$ & $0.00 E+00$ & $2.00 E+01$ & $0.00 E+00$ & $0.00 E+00$ \\
\hline EFFLUENT 7 & $0.00 E+00$ & $0.00 E+00$ & $2.30 E+0 i$ & $0.00 E+00$ & $0.00 E+60$ \\
\hline EFFLUENT 8 & $0.00 E+00$ & $0.00 E+00$ & $2.70 E+0 !$ & $0.0015+00$ & $0.60 E+60$ \\
\hline EFFLUENT 7 & $0.60 E+00$ & $0.00 E+00$ & $3.00 E+01$ & $0.00 E+00$ & $0.00 \mathrm{E}+00$ \\
\hline EFFLUENT 10 & $0.00 E+00$ & $0.00 E+00$ & $3.40 E+0:$ & $0.00 E+00$ & $0.60 E+00$ \\
\hline EFFLUENT 11 & $0.00 E+00$ & $0.00 E \div 00$ & $3.70 E+01$ & $0.00 E+00$ & $0.00 E+00$ \\
\hline EFFLUENT i2 & $0.60 E+00$ & $0.00 E+00$ & $4.10 E+01$ & 0. VUE +00 & $0.00 E+00$ \\
\hline EFFLUENT 13 & $0.00 E+0 \hat{V}$ & $0.00 E+00$ & $4.40 E+01$ & $0.00 E+00$ & $0.00 E+00$ \\
\hline EFFLUENT I4 & $0.00 E+00$ & $0.00 E+00$ & $4.90 E+01$ & $0.00 E+00$ & $0.06 E+00$ \\
\hline EFFLUERT IE & $0.00 E+10$ & $0.00 E+00$ & $5.20 E+01$ & $0.00 E+00$ & $0.00 E+60$ \\
\hline EFFLUENT 16 & $0.00 E+60$ & $0.00 E+00$ & $5.80 E+01$ & $0.00 \mathrm{DE}+00$ & $0.00 E+60$ \\
\hline EFFLUENT 17 & $0.60 E+00$ & $0.00 E+00$ & $6.20 E+01$ & $0.00 E+00$ & $0.60 E+60$ \\
\hline EFFLUENT 18 & $0.00 E+00$ & $0.00 E+00$ & $6.50 E+01$ & $0.00 E+00$ & $0.00 E \div 00$ \\
\hline EFFLUEHT 19 & $0.00 E+00$ & $0.00 E+00$ & $6.9 \hat{v} E+0 i$ & $0.00 E+00$ & $0.00 E+00$ \\
\hline EFF!UENT 20 & $0.00 E+00$ & $0.00 E+00$ & $7.20 E+01$ & $0.00 E+00$ & $0.00 E+00$ \\
\hline EFFLUEHT 22 & $0.00 E+00$ & $0.00 E+00$ & $8.00 E+01$ & $0.00 E+00$ & 0. $00 E+00$ \\
\hline EFFLUENT 23 & $0.00 E+00$ & $0.00 E+00$ & $8.40 E+01$ & $0.00 E+00$ & $0.00 E \div 00$ \\
\hline EFFLUEHT 24 & $0.00 E+00$ & $0.00 E+00$ & $8.70 E+01$ & $0.00 E+00$ & $0.00 E \div 00$ \\
\hline EFFLUENT 26 & $0.00 E+00$ & $0.00 E+00$ & $9.40 E+01$ & $0.00 E+00$ & $0.00 E+00$ \\
\hline EFFLUERT 28 & $0.00 E \div 00$ & $0.00 E \div 00$ & $1.00 E+02$ & $0.00 E+00$ & $0.00 E+00$ \\
\hline EFFLUENT 30 & $0.00 E+00$ & $0.00 E+00$ & $1.07 E+02$ & $0.00 E+00$ & $0.00 E+60$ \\
\hline EFFLUERT 32 & $0.00 E+00$ & $0.00 E+00$ & 1. $15 E+62$ & $0.00 E+10$ & $0.00 E+10$ \\
\hline EFFLUENT 34 & $0.00 E+00$ & $0.00 E+00$ & $1.21 E+02$ & $0.00 E+00$ & $0.00 E+00$ \\
\hline EFFLUEKT 36 & 1.8BE-03 & $1.38 E-03$ & $1.28 E+02$ & $1.05 E-10$ & 8. $0 B E-14$ \\
\hline EFFLUENT 39 & $6.97 E-02$ & $7.16 E-02$ & $1.42 E \div 02$ & $3.92 E-0.8$ & $1.06 E-10$ \\
\hline EFFLUEHT 41 & 4. $5 \mathbf{J E}-02$ & $1.17 E-01$ & $1.49 E+02$ & $7.21 E-08$ & $2.70 \mathrm{E}-10$ \\
\hline EFFLUEKT 43 & $7.33 E-02$ & I. SIE-ต̂l & $1.56 E+62$ & 1.79E- $\$ 7$ & ธ. 3EE-10 \\
\hline EFFLUEMT 45 & $7.36 E-02$ & $2.55 E-0 !$ & $1.58 E+02$ & $7.15 E-i \hat{\varepsilon}$ & 1.2E-69 \\
\hline EFFLIEHT 47 & 6. $8 B E-12$ & 3.33E-21 & i. $77 E+\hat{v a}$ & $1.16 E-4 ? ?$ & $1.34 E-09$ \\
\hline EFFLUERT 49 & $5.80 E-02$ & $3.92 E-01$ & $2.13 E \div 02$ & $6.14 E-09$ & 2. $12 E-6 ?$ \\
\hline EFFLUENT $\$ 0$ & $3.65 E-82$ & 4. ZPE-6: & $2.435+02$ & $4.03 E-49$ & $2.22 E-19$ \\
\hline
\end{tabular}


Sodium Leach Fractions from Flow Through Test Grout/Sediment with Groundwater (DIFCOLI)

\begin{tabular}{|c|c|c|c|c|c|}
\hline SAMPLE & An/Ao & CUA. An/AOO & DAYS & Dinc. & D cua. \\
\hline EFFLUERT 1 & $3.72 E-02$ & $3.72 E-62$ & $3.00 E+00$ & $1.35 E-09$ & $1.35 E-69$ \\
\hline EFFLUENT 2 & $3.71 E-02$ & $7.43 E-02$ & $6.00 E+00$ & $7.63 \mathrm{E}-09$ & $2.70 E-19$ \\
\hline EFFLUENT 3 & $3.14 E-62$ & $1.06 E-01$ & $9.00 E+00$ & $9.52 E-09$ & $3 . \leq 4 E-0 ?$ \\
\hline EFFLUENT 4 & 3.51E-02 & 1. HiE- $\hat{i} i$ & 1.jôE+vi & $? .04 E-0\}$ & $4,47 E-6 \hat{q}$ \\
\hline EFFLUEHT 5 & $1.32 E-02$ & $1.5 A E-01$ & i.76E+0i1 & $1.91 E-09$ & $4,0,5 E-69$ \\
\hline EFFLUENT $b$ & $1.90 E-02$ & $1.73 E-01$ & $2.00 E \div 01$ & $6.65 E-09$ & 4.JEE-09 \\
\hline EFFLUENT 7 & $2.58 E-02$ & $1.99 E-01$ & $2.30 E+0 i$ & $1.36 E-v 8$ & 5. $03 E-099$ \\
\hline EFFIUENT a & 3. $25 E-02$ & $2.31 E-01$ & $2.70 E \div 01$ & $1.94 E-08$ & $4.76 E-69$ \\
\hline EFFLUENT 9 & $2.58 \mathrm{E}-02$ & $2.53 E-01$ & $3.00 E+01$ & $2.47 E-63$ & $5.625-69$ \\
\hline EFFLUENT IO & $2.08 E-02$ & $2.79 E-01$ & $3.40 E \div 01$ & $1.01 E-08$ & 5.BJE- \\
\hline EFFLUENT 11 & $1.49 E-02$ & $2.335-01$ & $3.70 E+01$ & 3.63E-6B & 5. $i S E-i \hat{~}$ \\
\hline EFFLUENT 12 & $1.75 E-02$ & $3.12 E-9 i$ & $4.10 E+01$ & $1.09 E-08$ & $6.03 E-09$ \\
\hline EFFLUENT 13 & 1. $32 E-02$ & $3.25 E-01$ & $4.40 E+0 \mathrm{I}$ & $9.59 E-v 9$ & $5.10 E-69$ \\
\hline EFFLUENT 14 & $1.995-02$ & $3.45 E-0 !$ & $4.50 \mathrm{NE}+01$ & 3.6 $3 E-19 ?$ & $0.00 E-i \xi$ \\
\hline EFFLUEMT 15 & $7.635-03$ & $3.5 \mathrm{BE}-0 \mathrm{i}$ & $5.205 \div 01$ & 3. BJE-0? & S.joE-jo \\
\hline EFFLUENT I6 & $2.54 E-02$ & $3.7 \mathrm{AE}-01$ & $5.80 E+01$ & 1.16E-va & 6. $85 E-0 \%$ \\
\hline EFFLUENT 17 & $1.925-02$ & $3.77 E-01$ & $6.20 E \div 01$ & $1.625-i 8$ & 7. RvE-Gi \\
\hline EFFLUEHT 18 & 1. $18 E-02$ & $4.095-01$ & 6. $5 \hat{v E E}+01$ & $1.15 E-06$ & $7.30 E-69$ \\
\hline EFF!UENT 19 & 1.61E-02 & $4.255-01$ & $6.90 E+01$ & $1.27 E-08$ & 7. $\angle A E-69$ \\
\hline EFFLUEHT $2 \hat{V}$ & $3.71 E-03$ & $4.29 E-\hat{v} !$ & $7.20 E+01$ & $1.27 E-09$ & $3.37 E-69$ \\
\hline EFFLUEHT 22 & $1.67 E-02$ & $4.46 E-01$ & $8.00 E+01$ & 3. E6E-69 & $7.40 E-09$ \\
\hline EFFLUENT 23 & $1.07 E-02$ & $4.56 E-01$ & $6.40 E+01$ & 6. $90 E-07$ & $7.43 E-69$ \\
\hline EFFLUENT 24 & 6. $22 E-03$ & $4.63 E-013$ & $8.70 E+01$ & $4.8 E E-09$ & 7.1 SE-09 \\
\hline EFFLUENT 26 & $1.855-62$ & $4.82 E-01$ & $9.40 E+01$ & $7.4 \mathrm{ZE}-09$ & $7.355-0 \%$ \\
\hline EEFLUJEKT 28 & $2.12 E-12$ & $5.03 E-91$ & 1.00E+02 & 1. $42 E-08$ & $7.0 \& E-00^{\circ}$ \\
\hline EFFLUENT 30 & 7.J3E-03 & $5 . i 2 E-61$ & $1.07 E+02$ & 2. $16 E-\hat{1} 9$ & $7.53 E-69$ \\
\hline EFF!UEHT 32 & $8.79 E-03$ & $5.21 E-01$ & $1.15 E+02$ & $1.57 E-69$ & $7.34 E-39$ \\
\hline EFFLUENT 34 & 1. $19 E-02$ & 5. उJE- & $1.21 E+02$ & $5.47 E-\hat{~} 9$ & $i .32 E-09$ \\
\hline EFFLUENT 36 & $3.2 \mathrm{JE}-\mathrm{A3}$ & $5.42 E-01$ & $1.26 E+02$ & $2.53 E-09$ & $\bar{i} .25 E-j \rho$ \\
\hline EFFLUENT 39 & :. B1E- 02 & 5. $60 E-01$ & $1.42 E+i 22$ & $2.665-69$ & $7.255-69$ \\
\hline EFF!UENT 41 & $0.14 E-03$ & 5. SóE-Ji & $1.49 E+122$ & $1.3 ! E-67$ & $7.195-69$ \\
\hline EFFLUEHT 43 & T.SIE-0J & $5.74 E-\hat{v i}$ & $1.56 E+02$ & $2.28 E-07$ & $0.575-48$ \\
\hline EFFLUEHT 45 & $9.235-03$ & $5.53 E-01$ & $1.58 E \div 02$ & 1. BE-Vi & D. $6 E E-1 \%$ \\
\hline EFFLUEKT 47 & $7.54 E-\hat{03}$ & ร. $\{i E-01$ & 1. FiE+ia & $3.42 E-0 !$ & E. EXE-:i \\
\hline EFF!UENT $\$ 9$ & 2. $68 E-1 / 2$ & $0.12 E-01$ & $2.13 E+02$ & $\bar{C} .57 E-1 \mathrm{~A}$ & $\therefore$ ¿. \\
\hline EFFLUENT 50 & $1.42 E-12$ & $0.26 E-6 !$ & 2. $43 E+02$ & c. QUE- & 5. $60 \mathrm{E}-19 \%$ \\
\hline
\end{tabular}


TABLE B.13c. Aluminum (A1) Leach Fractions from Flow Through Test Grout/Sediment with Groundwater (DIFCOLI)

\begin{tabular}{|c|c|c|c|c|c|}
\hline SAMPLE & An/AO & SUn. An/Ao & DAYS & 0 inc. & O cull. \\
\hline EFFLUENT I & $6.20 E-05$ & $6.20 E-05$ & $3.00 E+00$ & $3.76 E-15$ & $3.76 E-15$ \\
\hline EFFLUEKT 2 & 3. $38 E-04$ & 4.00E-04 & $6.00 E+00$ & $6.49 E-13$ & T.81E-14 \\
\hline EFFLUENT 3 & $2.21 E-04$ & $6.21 E-04$ & $9.00 E+00$ & $4.74 E-1 J$ & $1.26 E-13$ \\
\hline EFFLLIEHT \& & $4.24 E-04$ & $1.04 E-03$ & 1. $30 E+01$ & $1.44 E-12$ & $2.40 E-13$ \\
\hline EFFLLUEHT 5 & $3.13 E-04$ & $1.36 E-03$ & $1 . T 0 E+01$ & 1.0 $0 \mathrm{iE}-12$ & $3.18 E-13$ \\
\hline EFFLUENT 6 & $3.16 E-04$ & $1.67 \mathrm{E}-03$ & 2.00E+01 & $2.40 E-12$ & $4.11 E-13$ \\
\hline EFFLUENT 7 & $5.35 E-04$ & $2.21 E-0 J$ & 2. $30 E+01$ & $3.01 E-12$ & $6.22 E-13$ \\
\hline EFFLUEHT 8 & 7. 40E-14 & $2.95 E-03$ & $2.70 E+01$ & $1.00 \mathrm{E}-11$ & $9.94 E-13$ \\
\hline EFFLUENT 7 & $6.04 E-04$ & $3.55 E-03$ & 3.0OE+0i & $1.35 E-11$ & 1. $23 E-12$ \\
\hline EFFLUENT 10 & $7.03 E-014$ & $4.26 \mathrm{E}-03$ & $3.40 E+0 i$ & $1.16 E-11$ & 1. $56 E-12$ \\
\hline EFFLUERT II & $5.455-04$ & $4.80 E-03$ & $3.70 E+01$ & $\{.37 E-11$ & 4.8งE-12 \\
\hline EFFLUENT 12 & b. $87 E-04$ & $5.49 E-13$ & $4.10 E+01$ & $1.35 E-11$ & $2.15 E-12$ \\
\hline EFFLUENT 13 & 4. OBE-04 & 5. 35E-0J & $4.40 E+01$ & $1.21 E-11$ & $2.36 E-12$ \\
\hline EFFLUERT I\$ & $7.27 E-04$ & $6.68 E-03$ & $4.90 E+01$ & $1.15 E-11$ & $2.67 E-12$ \\
\hline EFFLUEHT IS & $2.93 E-014$ & $6.98 E-0 j$ & $5.20 E+01$ & $5.66 \mathrm{E}-12$ & 2.74E-12 \\
\hline EFFLUENT 16 & 8.79E-04 & $7.85 E-03$ & $5.80 E+01$ & $1.38 E-11$ & $3.12 E-! 2$ \\
\hline EFFLUEKT 17 & $7.64 E-04$ & B.625-10] & $0.20 E+01$ & $2.57 E-11$ & $3.51 E-12$ \\
\hline EFFLUERT I8 & $4.76 E-04$ & $9.095-03$ & $6.50 E+01$ & $1.87 E-1 !$ & 3. $7 \mathrm{JE}-12$ \\
\hline EFFLUEKT 19 & $5.13 E-04$ & $9.61 E-03$ & 6. $30 E+0 !$ & $1.29 E-1 !$ & $3.72 E-12$ \\
\hline EFFLUENT 20 & $1.51 E-04$ & $9.76 \mathrm{E}-03$ & $7.20 E+01$ & $2.11 E-12$ & $3.38 E-12$ \\
\hline EFFLUENT 22 & $5.43 E-04$ & $1.03 E-02$ & 2. $00 E+01$ & 4.10E-12 & $3.875-12$ \\
\hline EFFLUEMT 23 & $4.18 E-04$ & $1.07 E-02$ & $8.40 E+01$ & $1.05 E-11$ & $4.01 E-12$ \\
\hline EFFLUERT 24 & $2.44 E-04$ & $1.10 E-02$ & $8.70 E+01$ & $6.64 E-12$ & $4.055-12$ \\
\hline EFFILENT 26 & $7.91 E-04$ & $1.18 E-02$ & $9.40 E+01$ & $1.36 \varepsilon-11$ & 4. $31 E-12$ \\
\hline EFFLUENT 28 & 8. $56 E-04$ & $1.26 E-02$ & $1.00 E+02$ & $3.315-11$ & $4.665-15$ \\
\hline EFFIJENT 30 & $2.73 E-04$ & 1.29E-02 & $1.07 E+02$ & $1.84 E-12$ & $4.55 E-12$ \\
\hline EFFLUENT 32 & $2.18 E-04$ & 1.31E-02 & $1 . ! 5 E+02$ & $9.65 E-13$ & $4.3 B E-12$ \\
\hline EFELUENT 34 & 3.10E-04 & $1.34 E-02$ & $1.21 E+02$ & $3.69 E-12$ & $4.365-12$ \\
\hline EFFLUENT 36 & $1.84 E-04$ & 1.36E-02 & $1.25 E+02$ & 1.01E-12 & $4.235-12$ \\
\hline EFFLUENT 39 & $3.18 E-04$ & $1.39 E-02$ & $1.425+02$ & $8.14 E-13$ & $4.305-12$ \\
\hline EFFLUENT A1 & ¿.01E-04 & $1.40 E-\hat{0} 2$ & $1.49 E \div 02$ & $3.565-13$ & 3. $27 E-12$ \\
\hline EFFLUEHT 43 & $1.09 E-014$ & $1.41 E-02$ & $1.56 E+02$ & $4.33 E-13$ & $3.75 E-12$ \\
\hline EFFLUENHT 45 & $3,41 E-05$ & $1.42 E-0\}$ & $1.58 E-02$ & 9.3AE-:4 & $5.5=-12$ \\
\hline EFFLUENT 47 & $3.63 E-05$ & $1.4 \overline{Z E}-02$ & $1.77 E+62$ & 3.6TE-14 & $3.365-1 \bar{z}$ \\
\hline EFFLUEKT 49 & $3.56 E-3 ?$ & $1.42 E-62$ & $2.13 E+02$ & $\therefore 295-18$ & $2.79 \mathrm{E}-12$ \\
\hline EFFLUENT 50 & $4.70 E-35$ & $1.4 J E-6 Z$ & $2.43 E+62$ & $0.55 t-15$ & $2.47 E-12$ \\
\hline
\end{tabular}


TABLE B.13e. Boron (as H3B03) Leach Fractions from Flow Through Test Grout/Sediment with Groundwater (DIFCOLIA)

\begin{tabular}{|c|c|c|c|c|c|}
\hline SAMPLE & $\mathrm{An} / \mathrm{AO}$ & CUA. An/AO & DAYS & $D$ inc. & $D$ cus. \\
\hline EFFLUEHT I & $1.13 E-02$ & $1.13 E-02$ & $3.00 E+000$ & $1.25 E-10$ & $1.25 E-10$ \\
\hline EFFLUENT $\hat{2}$ & $5.11 E-03$ & $1.64 E-02$ & $6.00 E+00$ & $1.49 E-10$ & $1.32 E-10$ \\
\hline EFFLUENT 3 & $3.15 E-1\} 3$ & $1.96 E-02$ & $9.00 E+00$ & $9.61 E-11$ & $1.25 E-10$ \\
\hline EFFLUENT 4 & $3.26 E-03$ & 2. $2 \mathrm{gE}-62$ & 1. $3 \hat{v E E}+\hat{U} \mathfrak{1}$ & 3.5:5-1! & 1. LaE-10 \\
\hline EFFLUERT 5 & $2.27 E-03$ & 2.51E-02 & $1.70 E+01$ & $5.66 E-11$ & 1.09E-10 \\
\hline EFFLUENT : & $1.41 E-03$ & $2.65 E-02$ & $2.005+01$ & 4. $80 E-11$ & 1.0XE-10 \\
\hline EFFLUENT 7 & 2.17E-0J & $2.37 E-02$ & 2. $30 E+01$ & 1.3IE-10 & 1. $.5 E-10$ \\
\hline EFFLUENT 8 . & $2.78 E-03$ & $3.15 E-02$ & 2. $70 E+01$ & $1.42 E-10$ & i. $03 E-10$ \\
\hline EFFLUEHT $q$ & $1.37 E-03$ & $3.2 B E-02$ & $3.00 E+0 !$ & $6.98 E-11$ & $1.05 E-10$ \\
\hline EFFLUEHT :O & :.JIE- 63 & 3. $42 E-02$ & $3,40 E+01$ & $4.63 \mathrm{E}-11$ & 1. $.1 E-10$ \\
\hline EFFLUENT $\|$ & 8. $86 E-04$ & $3.50 \mathrm{0E}-02$ & 3. $70 E+01$ & $3.63 E-11$ & 9. $73 E-! 1$ \\
\hline EFFLGENT :2 & i. $01 E-03$ & $3,6 \mid E-02$ & 4.10E+01 & 2. T3E-11 & $\$ .30 E-1 \mathrm{~S}$ \\
\hline EFFLUENT 13 & $\therefore .79 E-04$ & $3.67 E-02$ & $4.40 E+01$ & $2.55 E-11$ & 3. $39 E-1:$ \\
\hline EFFLUEKT 14 & $1,14 E-103$ & $3.79 E-02$ & 4. $30 E+0 !$ & $2.83 E-11$ & ב.5EE-11 \\
\hline EFF!UEKT IJ & $4.27 E-04$ & $3.33 E-02$ & $5.20 E+0 \mathrm{i}$ & $1.20 E-i:$ & 3.27E-1! \\
\hline EFFLUENT 16 & $1.41 E-03$ & $3.97 \mathrm{E}-02$ & $5.80 E+\hat{v} 1$ & $3.57 E-11$ & $7.97 E-11$ \\
\hline EFF!LLENT 17 & $7.23 E-04$ & 4. $04 E-02$ & $6.20 \mathrm{VE}+01$ & $2.30 \mathrm{E}-11$ & $7.75 E-11$ \\
\hline EFFLUEHT 18 & b. $27 E-04$ & $4.11 E-02$ & $6.56 E+0 i$ & $3.25 E-11$ & $7.61 E-11$ \\
\hline EFFLUERT IS & $1.36 E-03$ & $4.24 E-02$ & $6.90 E \div 01$ & $9.09 E-i 1$ & $7.55 E-11$ \\
\hline FLUENT 20 & $4.18 E-04$ & $4.2 \mathrm{BE}-02$ & $7.20 E+01$ & $1.6 \mathrm{iE}-1 \mathrm{i}$ & $7,47 E-11$ \\
\hline FLUENT 22 & $1.165-03$ & $4.40 E-02$ & 3. $00 E+01$ & 1.6TE-11 & T. $1 \bar{v} \bar{c}-1 !$ \\
\hline FLUERT 23 & $5.34 E-64$ & $4.45 E-02$ & $0.40 E+01$ & $\mathrm{i} .72 E-\mathrm{ii}$ & S. $9 E=-11$ \\
\hline FLLUEHT 24 & $3.94 E-34$ & $4.49 E-02$ & $8.70 E+0 !$ & 1.TSE-11 & S. BOEE-I: \\
\hline FFLUEKT 26 & 0.71E-04 & $4.58 E-02$ & $9.40 E+01$ & $1.04 E-11$ & อ.54E-11 \\
\hline FFLUENT 28 & 1. $00 E-03$ & $4.685-02$ & 1.00E+02 & $3.18 E-! 1$ & 0. $4 \angle E-1 !$ \\
\hline FFLUENT 30 & $6.21 E-64$ & $4.74 E-02$ & $2.07 \mathrm{E}+02$ & 4. $55 E-12$ & b. $16 \bar{c}-11$ \\
\hline FFLUEHT 32 & $6.2 \mathrm{IE}-014$ & 4. $30 \mathrm{je}-\mathrm{v2}$ & $1.15 E+02$ & $i .84 E-12$ & $5.39 E-11$ \\
\hline FFLUENT is & $7.71 E-04$ & $4.38 E-\hat{v 2}$ & $1.21 E+0 \bar{z}$ & 2. SPE-il & $3.77 E-1 j$ \\
\hline FFLUENT 36 & $7.56 E-04$ & $4.96 E-02$ & $1.28 E+62$ & $1.70 \mathrm{E}-11$ & $5.63 E-1 !$ \\
\hline FFLULNT 39 & $1.50 E-03$ & $5.11 E-02$ & $1.42 E+0 z$ & !.6ZE-:! & 5. उfE-i: \\
\hline FFLUENT 41 & b. $40 E-04$ & 5. $17 E-02$ & 1.49E-12 & 1. $+3 \bar{E}-\mathbf{i l}$ & E. \\
\hline FFLLEKTT 43 & $0.475-14$ & 5. $20 E-62$ & $i .5 b E+v 2$ & $2.8 \mathrm{JE}-\mathrm{il}$ & J.:19E-1: \\
\hline$F F\lfloor$ LEHT 45 & 1.13E-63 & $5.37 E-02$ & $1.58 E+012$ & I. $A \mid E-: I$ & $\Xi . \therefore 4 E-: I$ \\
\hline FFLUEHT 47 & $1.055-03$ & 5. $4 \mathrm{EE}-02$ & I.TTE $40 i$ & $2.77 E-1 !$ & 4.97:-:! \\
\hline FFluent 49 & 3.2FE-03 & $5.31 E-02$ & $2.13 E+12$ & t.71E-:I & $\lceil .09 E-1: 1$ \\
\hline FFLLIENT 50 & $2.4 \div E-03$ & b. $16 \bar{E}=-42$ & $2.43 E+12$ & 1. aิ5-:: & $4.2 B-1 j$ \\
\hline
\end{tabular}


TABLE B.13f. Fluoride (F) Leach Fractions from Flow Through Test Grout/Sediment with Groundwater (DIFCOLIA)

\begin{tabular}{|c|c|c|c|c|c|}
\hline SAKPLE & An/Ao & CUA. An/ÂO & DAYS & Dine. & $D$ cun. \\
\hline EFFLUEEHT 1 & $1.24 E-03$ & $1.24 E-03$ & $3.00 E+00$ & $1.51 E-12$ & ¿.5JE-12 \\
\hline EFFLUENT 2 & $9.36 E-04$ & $2.18 E-03$ & $6.00 E+00$ & $4.99 E-12 \hat{2}$ & 2. $32 E-12$ \\
\hline EFELUENT 3 & $7.17 E-04$ & $2.90 E-03$ & 9. 60 EOOO & $4.97 E-12$ & $2.73 E-12$ \\
\hline EFFLUENT 4 & $7.96 E-0.4$ & $3.695-03$ & $1.30 E+01$ & $5.07 E-: 2$ & $3.675-12$ \\
\hline EFFLUEHT 5 & $4.72 E-04$ & $4.16 E-03$ & 1. $T 0 E+01$ & $2.44 E-12$ & $2.95 E-12$ \\
\hline EFFLUENT & $4.04 E-04$ & $4.57 E-03$ & $2.00 E+01$ & $3.33 E-12$ & $3.06 E-12$ \\
\hline EFFLLUENT 7 & $5.40 E-14$ & $5,11 E-03$ & $2.30 E+01$ & $3.15 E-12$ & $3.33 E-12$ \\
\hline EFFLUEHT & $7.42 E-04$ & $5.85 E-03$ & $2.70 E+01$ & $1.01 E-11$ & $3.72 E-12$ \\
\hline EFfluERT 9 & $5.17 E-114$ & $6.375-03$ & $3.00 E+013$ & $9.93 E-12$ & $3: 96 E-12$ \\
\hline EFFLUENT 10 & 5. 05E-04 & $6.67 E-\hat{0} 3$ & $3.40 E+61$ & $5.98 E-12$ & $4.07 E-12$ \\
\hline EFFLUENT 11 & $3.67 E-04$ & $7.24 E-0 j$ & j.70E 01 & $6.245-12$ & 4. $15 E-12$ \\
\hline EFFLUENT 12 & 4.75E-04 & $7.71 E-03$ & 4. 10E+01 & $6.44 E-12$ & $4.265-12$ \\
\hline EFFLUENT 13 & $3.43 E-04$ & $3.065-03$ & $4.40 E+01$ & 6.51E-12 & $4.33 E-12$ \\
\hline EFFLUENT 14 & $5.07 E-04$ & $9.56 E-03$ & $4.90 E+01$ & $5.61 E-12$ & $4.39 E-12$ \\
\hline EFEUENT 15 & $2.26 E-014$ & o.,TIE-0j & $5.20 E+01$ & $3.36 E-12$ & $4.36 E-12$ \\
\hline EFFLUENT it & $6.06 E-04$ & $9.40 E-03$ & 5.8OE+01 & $6.59 E-12$ & $4.46=-12$ \\
\hline EFELUENT 17 & $4.32 E-04$ & $9.33 E-63$ & $6.20 E+01$ & $3.20 E-12$ & $4.57 E-12$ \\
\hline EFFLUENT IB & $3.96 E-04$ & $1.02 E-32$ & $6.50 E+01$ & $1.36 E-11$ & $4.72 E-12$ \\
\hline EEFLUENT 19 & $7.80 \mathrm{E}-04$ & 1.10E-02 & $6.70 E+01$ & $2.995-11$ & $5.15 E-12$ \\
\hline EFFLUENT 20 & $2.32 E-04$ & $1.12 E-02$ & $7.20 E+01$ & $4.32 E-12$ & $5.14 E-12$ \\
\hline EFFLUENT 22 & $5.21 E-04$ & 1. $16 E-02$ & $8.00 E+0 !$ & 3.TAE-12 & $5.07 E-12$ \\
\hline EFFLUENT 23 & $3.08 E-04$ & $1.21 E-02$ & $8.40 E+01$ & 5.72E-12 & 5.06E-12 \\
\hline EFFLUENT 24 & $2.31 E-04$ & $1.23 E-12$ & 8.70E+0! & $5.96 E-12$ & 5. $10 E-12$ \\
\hline EFFLLIEKT 26 & $5.65 E-04$ & $1.29 E-02$ & $9.40 E+01$ & $6.92 E-12$ & 5. $16 E-12$ \\
\hline EFFLUENT 28 & $7.75=-04$ & $1.36 E-02$ & $1.00 E+0 z$ & $1.30 E-11$ & $5.455-12$ \\
\hline EFFLIENT 30 & $4.32 E-64$ & $1.41 E-02$ & 1. $07 E+02$ & $4.62 E-12$ & $5.435-12$ \\
\hline EFFLUEHT 32 & $3.24 E-04$ & $1.44 E-62$ & $1.15 E+02$ & $2.13 E-12$ & $5.265-12$ \\
\hline EFFLUENT 34 & $7.27 E-014$ & 1.5:E-i2 & $1.21 E+02$ & $2.035-11$ & 5. $34 \mathrm{E}-12$ \\
\hline EFFLUENT 36 & $5.23 E-64$ & $1.5 i \mathrm{E}-02$ & $1.28 E+02$ & $1.01 E-11$ & $5.65 t-12$ \\
\hline EFFLUENT 39 & $1.37 E-03$ & $1 . j I E-\hat{v} 2$ & i. $42 E+0 \hat{2}$ & 1.5IE-11 & $0.02 E-12$ \\
\hline EFFLLENT A1 & $6.15 E-14$ & $1.77 E-62$ & $1.49 E+\bar{v} 2$ & $1.3 z E-11$ & b. $10 E-1 \bar{c}$ \\
\hline EFFLLERT 43 & $i .65 E-04$ & 1.85E-62 & $1.56 E+62$ & 2.13E-i! & $0.40 E-12$ \\
\hline EF:UUEसT 45 & $1.015-03$ & $1.75 E-12$ & $1.68 E+6 i$ & $1.3+E-1$ & 6. biE-iz \\
\hline EFF:UEHT 47 & $1.73 E-03$ & $\hat{i} \cdot 125-\hat{v i}$ & $1 . j \bar{j} E+0 \bar{Z}$ & $7.93 E-11$ & $3,43 E-12$ \\
\hline EFPLUENT 49 & $2.83 E-03$ & $2 .+1 E-\dot{a} 2$ & 2. $13 E+62$ & 1.4IE-!! & $7.70 E-12$ \\
\hline EFIUEHT 56 & $2.15 E-03$ & $2.62 E-62$ & 2. $+3 E+02$ & 1. $30 \mathrm{E}-\mathrm{i}$ ! & 3.30E-12 \\
\hline
\end{tabular}


TABLE B.13g. Nitrate (NO3) Leach Fractions from Flow Through Test Grout/Sediment with Groundwater (DIFCOLIA)

\begin{tabular}{|c|c|c|c|c|c|}
\hline SAHPLE & $\mathrm{An} / \mathrm{Ao}$ & CUA. ÂR/Â & DAYS & $D$ ine. & 3 Eus. \\
\hline EFFLUENT 1 & 1.19E-01 & 1.19E-01 & $3.00 E+00$ & 3. $3 \mathrm{AE}-68$ & 1. $3 B E-08$ \\
\hline EFFLUEHT 2 & $2.81 E-02$ & 1. $47 E-O 1$ & $6.00 E+00$ & $4.51 E-09$ & $1.065-08$ \\
\hline EFFLUENT 3 & $1.70 E-02$ & $1.64 E-01$ & $9.00 E+00$ & $2.79 E-09$ & $8.76 E-09$ \\
\hline EFFLUENT 4 & $1.74 E-02$ & $1.81 E-01$ & $1.30 E+01$ & $2.43 E-09$ & $7.42 E-69$ \\
\hline EFFLUENT 5 & $1.03 E-02$ & $1.72 E-01$ & $1.70 E \div 01$ & 1.17E-09 & $6.34 E-67$ \\
\hline EFFLUENT 6 & $8.68 E-\hat{v} 3$ & $2.00 E-01$ & $2.600+01$ & $1.61 E-\hat{v} 9$ & 5. $89 E-69$ \\
\hline EFFLUEHT 7 & 4.:17E-03 & 2. OSE-DI & $2.30 E+01$ & $4.87 E-10$ & $4.705-69$ \\
\hline EFFLUENT В & $1.06 E-6 j 2$ & 2. $15 E-01$ & $2.70 E+01$ & $2.06 E-09$ & $4.44 E-019$ \\
\hline EFFLUENT 9 & $7.91 E-03$ & 2. $23 E-01$ & 3. $00 E+01$ & $2.32 E-69$ & $3075 E-67$ \\
\hline EFFLUENT IO & $7.42 \mathrm{E}-03$ & 2. $31 E-01$ & $3.40 E+6 i$ & $1.29 E-09$ & $3.30 E-\hat{A} 9$ \\
\hline EFFLUENT II & $5.16 E-013$ & $2.36 E-0 !$ & $3.70 E+01$ & $1.23 E-199$ & 3. $33 E-35$ \\
\hline EFFLUEHT 12 & $0.595-03$ & $2.425-61$ & 4.10E $\div 01$ & $\therefore .24 E-1,9$ & $3.46 E-68$ \\
\hline EFFLUENT IJ & $4.59 E-02$ & 2. $88 E-0 !$ & A. $400 E+01$ & 1. $17 E-07$ & 4. $37 E-69$ \\
\hline GFFLUENT 14 & $3.035-02$ & $3.17 E-01$ & $4.905+01$ & 2. OIE-08 & $5.485-109$ \\
\hline EFFLUENT I5 & $3.53 E-63$ & $3.2 \geq E-01$ & $5.20 E+01$ & $3.21 E-10$ & 5. $i \in E-j \%$ \\
\hline EFFLUEKT 16 & $1.04 E-02$ & $3.32 E-01$ & $5.80 E+\hat{0} 1$ & 1. $34 E-09$ & $4,76 E-199$ \\
\hline EFFLUEHT 17 & 4. $30 E-i j 3$ & 3.37E-OI & $6.30 E+01$ & $3.15 E-10$ & $4.75 E-69$ \\
\hline EFFLUEHT IO & $4.13 E-03$ & $3.41 E-01$ & $6.50 E+0 \mathrm{i}$ & $1.41 E-09$ & $4.735-69$ \\
\hline EFFLUENT 19 & $1.41 E-62$ & $3.555-01$ & $6.90 E+01$ & Q. JAE & 5. $375-17$ \\
\hline EFFLUVHT & $6.10 E-03$ & $3.6 \mathrm{iE}-6 \mathrm{i}$ & $7.20 E+01$ & $3.42 E-09$ & $4,36 E-49$ \\
\hline EFFLUENT 22 & $9.29 E-63$ & 3. วิ0E-BI & 8. जOE +01 & $1.20 E-99$ & $4.655-19$ \\
\hline EFFIUENT 23 & $4.14 E-63$ & $3.75 E-01$ & 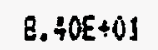 & $1.035-69$ & $4.73 E-09$ \\
\hline EFFLUENT 24 & J. $45 E-03$ & $3.78 E-01$ & $8.70 E+01$ & 1.J3E-09 & $4.575-09$ \\
\hline EFFLUEHT is & $5.65 E-\mathrm{b}^{3} 3$ & $3.84 E-01$ & $9.40 E+61$ & $7.40 E-10$ & $4.23 E-67$ \\
\hline EFFLUENT 20 & $\bar{i} .68 E-0 \overline{3}$ & 3. SIE-0! & 1. $000+02$ & $1.36 E-69$ & $4.22 E-69$ \\
\hline CFFLUENT 30 & $5.30 E-03$ & $3.97 E-0 i$ & $1.07 E+02$ & $6.75 E-10$ & $4.17 E-i=?$ \\
\hline EFF!UENT 32 & $4.30 E-63$ & 4.01E-0! & 1. $15 E+02$ & $3.7 T E-10$ & 3. $36 E-39$ \\
\hline EFFLUENT 34 & $6.13 E-0 J$ & 4. $07 E-O 1$ & $1.21 E+02$ & 1. $446-69$ & 3. YOEE-0Q \\
\hline EFFLUENT 36 & 3. $945-03$ & 4. JEE-0I & 1. $2 \mathrm{BE}+i \mathrm{z}$ & $3.385-0.5$ & $3.92 E-69$ \\
\hline EFFLUELT 34 & $3.73 E-62$ & $4.53 E-01$ & $1,42 E+1 \mathrm{i}$ & 1.: 3 E-6. & 4.:-E-09 \\
\hline EFIUENT al & 2. $54 \mathrm{~A}-\hat{\mathrm{v} z} \mathrm{z}$ & 4.7ดE-31 & $1.19 E+12 \hat{2}$ & $2.24 E-i a$ & 4. SOE- 69 \\
\hline EFFLUEHT 43 & 3.73E-122 & $5, i \dot{0} E-\hat{0} \mid$ & $1.56 E+62$ & $3.08 E-\hat{v a}$ & $5.41 E-6 ?$ \\
\hline EFFLUEHT 45 & 3.5EE-62 & $5,525-0 !$ & 1, SEE-12 & ¿.SEE-\$5 & $5-7 B E-7 \bar{T}$ \\
\hline EFFLUENT 47 & $5.60 E-1.23$ & B. $\dot{v} B[-\hat{v}]$ & $1.7 T E+63$ & $7.8+E-\hat{D E}$ & $7,25 E-i f$ \\
\hline EFFLUENT 49 & $4.86 \overline{6}-03$ & $0.135-01$ & $2.13 E+12$ & $4 .: 75-1 !$ & 6. 6 GE- -99 \\
\hline EFFLUEAT 50 & 1. $i \div 5-0 !$ & $\bar{i} .3 \vec{i} \mathrm{E}-\mathrm{v} !$ & $2.43 E+12$ & P. $0 \leq E-38$ & $\therefore .1 B E-3 E$ \\
\hline
\end{tabular}


TABLE B.13i.

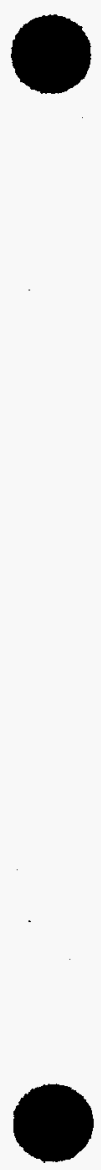

Iodine-125 (I-125) Leach Fractions from Flow Through Test, Grout/Sediment with Groundwater (I1)

\begin{tabular}{|c|c|}
\hline SAMPLE & PORE VOLUME \\
\hline CRH 11-1 & $1.33 E+00$ \\
\hline CAN $11-2$ & $2.72 E+00$ \\
\hline CRH $11-3$ & $4.15 E+00$ \\
\hline CRI II-4 & $5.89 E+00$ \\
\hline CRH 11-5 & $6.92 E+100$ \\
\hline CRI $11-6$ & $8.01 E+00$ \\
\hline CRH $11-7$ & $9.67 E+100$ \\
\hline CAN II-8 & $1.19 E+01$ \\
\hline CAH 11-9 & $1.37 E+01$ \\
\hline CRH II-10 & $1.58 E+01$ \\
\hline CRU I1-11 & $1.74 E+01$ \\
\hline CKN $11-12$ & $1.97 E+01$ \\
\hline [ลี $[1-1]$ & 2.14E+01 \\
\hline CRU $11-14$ & $2 .\{2 E+01$ \\
\hline CRH $11-15$ & $2.54 E+01$ \\
\hline CRH 11-16 & 2. $.88 E+01$ \\
\hline [R: $11-17$ & $3.24 E+01$ \\
\hline CRN $11-18$ & $3.50 E+01$ \\
\hline ERI 11-19 & $3.86 E+011$ \\
\hline CRH $11-20$ & $3.96 E+0.11$ \\
\hline CFU $11-22$ & $4.255+01$ \\
\hline CFH 11-23 & $4.47 E+0\}$ \\
\hline CAH 11-24 & $4.62 E+011$ \\
\hline CRH $11-25$ & $4.83 E+01$ \\
\hline $\mathrm{C} \times 4{ }^{\prime} 11-26$ & $5.10 E+01$ \\
\hline CAN $[i-7]$ & $5.50 E+01$ \\
\hline CRU II-28 & 5. $78 E+01$ \\
\hline BFH I;-2S & D. AAE+AI \\
\hline SEA $11-30$ & $6.14 E+01$ \\
\hline CRU $\mid 1-3]$ & 3. 40E+ô 1 \\
\hline CAH II-32 & $0.64 E+0 i$ \\
\hline CRH $11-33$ & $6.98 E+01$ \\
\hline Can $11-34$ & $7.24 E+01$ \\
\hline CRiN I1-35 & $7,40 E \div 01$ \\
\hline Crin 11:-36 & $7.72 E+01$ \\
\hline She Ii $-5 i$ & $0.04 E+j i$ \\
\hline - - 재 11-38 & $8.57 E+01$ \\
\hline - Eh : I1-37 & $0.92 E+61$ \\
\hline CRE i1-40 & $9.22 E+01$ \\
\hline Chit $11-41$ & $9.4 \pi E+01$ \\
\hline Ffis $11-42$ & $9.53 E+01$ \\
\hline Sne 11-45 & $1.03 E+62$ \\
\hline 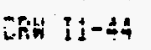 & $1.05 E+02$ \\
\hline Sh⿻ 1:-45 & :. $1 Z E+0 Z$ \\
\hline Can $11-46$ & $1.15 \div \div 02$ \\
\hline Crith II -47 & $1.23 E+012$ \\
\hline Cru :1-48 & $1.27 E+02$ \\
\hline Chin $11-49$ & $1,+9 E+62$ \\
\hline fin $11-50$ & $1.595+02$ \\
\hline
\end{tabular}

An/Ao

COR. UCI

2.57E-02

$1.35 E-02$

1. $.22 E-02$

$1.16 E-02$

$1.14 E-02$

1.47E-02

$1.37 E-02$

1. $03 E-02$

$1.03 E-02$

$6.70 E-03$

8. $31 E-03$

$6.43 E-03$

8. $03 \mathrm{E}-03$

5. $325-03$

1. $53 E-12$

1. $11 E-02$

6. 42E-03

2.15E-02

5. $50 \mathrm{E}-63$

1. $43 E-02$

1. $28 E-02$

$4.88 E-03$

$6.53 E-03$

1. I2E- 02

1. 30E-02

1. 4 SE-02

1. $27 \mathrm{E}-02$

5. $40 E-0 \bar{J}$

1. 34E-02

1. 25E-02

i. $77 E-02$

1. T3E-0?

$1.61 E-02$

1. $69 \bar{E}-02$

$2.36 E-102$

$3.066-02$

2. $34 \mathrm{E}-0 \mathrm{i}$

2. $37 E-62$

2. $13 E-02$

4. (0)E-02

$3.31 E-02$

2. $6 \mathrm{iE}-02$

$6.53 E-i) 2$

T. $57 E-02$

$4.49 E-02$

4.54E-12

$3.70 E-01$

$4.25 E-01$
TIME DAYS

$3.00 E+00$

6. $00 E+00$

$9.00 E+00$

$1.30 E+01$

$1.70 E+01$

$2.00 E+01$

$2.30 E+01$

$2.70 E+01$

3. OOE+O!

3. 40E+01

$3.70 E+01$

4.10E+01

4. $40 E+01$

4. $90 E+01$

5. $20 E+01$

5. $80 E+01$

$6.20 E+01$

$6.50 E+01$

$6.90 E+01$

$7.20 E+01$

8. $00 E+01$

6. $40 E+01$

B. $70 E+01$

7.10E+01

$9.40 E+01$

S. 70E+01

$1.00 E+02$

$1.04 E+02$

1. $07 E+02$

i. $: 1 E+02$

1. $15 E+02$

1. $18 E+02$

$1.21 E+02$

$1.25 E+\hat{2}$

1. $2 \mathrm{eE}+02$

$1.32 E+1) 2$

1. उ?E $\div 02$

1. $42 E+02$

$1.46 E+02$

$1.49 E+02$

$1.53 E+02$

$1 . \overline{6 E}+\hat{12}$

i. $59 E+02$

1. $.06 E+42$

1. $74 E+02$

1. $77 E+02$

1. $81 E+02$

$2 . ! 3 E+02$

$2.43 E+02$
1.17E-01

2. $64 E-02$

2.01E-02

1.15E-02

1.19E-02

1.17E-02

1.51E-02

1. 40E-02

1. $06 E-02$

$1.06 E-02$

6. 89E-03

$9.16 E-03$

$6.62 E-03$

$8.26 E-03$

$5.47 \mathrm{E}-03$

:. 57 - 02

1. $.14 E-\hat{0} 2$

6.61E-03

$2.21 \mathrm{E}-02$

5. $66 \mathrm{E}-03$

1. $47 \mathrm{E}-02$

1. $32 E-02$

5. $02 E-03$

$6.72 E-03$

1. 35E-02

1. $85 E-02$

$1.39 E-02$

1. 30E-02

5. $56 E-03$

1. $37 E-02$

1. $2 E E-02$

$1.83 E-02$

$1.785-62$

1. 6 EE -02

1. $35 E-02$

2. 42E-02

3. $775-02$

2. $37 \mathrm{xE}-02$

$2.44 E-62$

$2.20 \mathrm{~V}-\mathrm{v}) 2$

4. $12 E-02$

$3.41 E-62$

2. 6 BE-02

3. $78 E-62$

i. 7 i $\mathrm{E}-62$

$4.625-02$

4. $67 E-i 2$

3. $81 E-01$

4. 37E-ii!
CU. An/AO

1.17E-01

$1,43 E-01$

1.63E-01

$1.75 E-01$

1. $37 E-01$

1. $38 E-01$

2.13E-01

2. $28 E-01$

2.38E-(i)

2. 49E-i)!

2.56E-0 $0 \mathrm{~s}$

2. $65 E-01$

2.71E-01

2. $80 E-01$

2. 85E-01

$3.01 E-01$

$3.125-01$

3.19E-01

$3.41 E-01$

3.47E-01

$3.61 E-01$

$3.74 E-01$

3.79E-01

3. $86 E-01$

3. 36E-01

4. 16E-0i

$4.31 E-\mathrm{i} i \mathrm{i}$

4. $\{4 \varepsilon-01$

4. $50 E-80$

4. $65 \mathrm{JE}-01$

4. $i 6 E-01$

4. . $55 E-01$

5. $12 E-01$

5.29E- -11

5. $46 E-00 !$

5.73E-01]

6. $22 E-0 i$

6. 4 EE- i!

b. Sb́E-il!

b. $36 E-01$

$7.20 E-01]$

7.6JE-6!

7. $30 \mathrm{E}-0 \mathrm{ii}$

B. $58 \mathrm{E}-\hat{\mathrm{p} !}$

5, J JE- ij

3. อ2E-

1. $15=-10$

$1 .+15+60$

$1.355+00$
D IHC CM2/SEC$$
1.33 E-08
$$

6.14E-09

3. $55 E-09$

8. 04E-10

$9.41 E-10$

i. $34 E-09$

2. U1E-09

1.19E-09

1. $09 E-09$

6. $73 E-10$

$4.62 E-10$

$5.06 E-10$

4. $27 \mathrm{E}-10$

3. OBE -10

$3 .: 7 \mathrm{E}-10$

8. 89E- 10

$9.91 E-10$

4. $25 E-10$

2. $94 E-07$

$3.12 \mathrm{E}-10$

5. $38 E-10$

1. 49E-09

2. $95 E-10$

2. $72 \mathrm{E}-10$

1. $2 \mathrm{FE}-09$

3. $02 E-09$

$1.97 \mathrm{E}-10 \%$

$1.0 .5 E-69$

$3.01 E-10$

1. $14 E-09$

$1.06 E-19$

$3.25 E-09$

2.76E-09

1. $\mathrm{BJE}-0 \mathrm{~S}$

B. TUE-0?

3. $54 E-09$

ร. $01 E-09$

1. $045-08$

$3.5 a E-6)$

$4.70 \mathrm{E}-6 \mathrm{6}$

1. 02E-68

i. $: 3 \mathrm{E}-60$

D. 34E- 19

$5.96 E-99$

$5 . \quad 1=E-16$

2. $43 E-18$

1. $:$ !E-

?.7E- 3

: . TIE-

D CUN CH2/SEC

1. $33 E-68$

1. $00 E-08$

8.69E-09

6. $90 E-09$

6. $12 E-09$

5. $81 E-09$

5. 05E-19

5. $14 E-09$

5. $08 E-09$

4.91E-09

$4.90 E-69$

4. 82E-69?

$4.50 E-019$

4. उ5E-09

4. $43 E-09$

$4.305-69$

4. $29 E-09$

$4.44 E-69$

4.79E- 09

4. $39 E-09$

4. $71 E-69$

$4.76 E-09$

4. $91 E-09$

4. $79 E-199$

5.11E-199

5.57E-09

5. $71 E-09$ 
TABLE B.13i. Iodine-125 (I-125) Leach Fractions from Flow Through Test Grout/Sediment with Groundwater (12)

\begin{tabular}{|c|c|c|c|c|c|c|c|}
\hline $\begin{array}{l}\text { SAYPLE } \\
\text { CRM 12-1 }\end{array}$ & $\begin{array}{l}\text { PORE YOL UKES } \\
1.66 E+00\end{array}$ & $\begin{array}{l}\text { COR } 4 C i \\
6.30 E-02\end{array}$ & $\begin{array}{l}\text { TIKE DAYS } \\
3.00 E+00\end{array}$ & $\begin{array}{l}\text { An/Ao } \\
6.26 E-02\end{array}$ & $\begin{array}{l}\text { CUM An/Ao } \\
6.26 E-02\end{array}$ & $\begin{array}{r}\text { DINC CX2/SEC } \\
4.13 E-09\end{array}$ & $\begin{array}{r}\text { DCUH CM2/SEC } \\
4.13 E-09\end{array}$ \\
\hline CRN $12-2$ & $3.21 E+100$ & $1.51 E-02$ & $6.00 E+00$ & i. 50 E-02 & $7.76 E-02$ & $2.76 E-09$ & $3.17 E-09$ \\
\hline CRI 12-3 & $6.66 E+00$ & $3.49 E-02$ & $9.00 E+00$ & $3.47 E-02$ & 1.12E-01 & $5.715-09$ & $4.43 E-09$ \\
\hline CRH 12-4 & $8.98 E+00$ & $1.50 E-02$ & $1.30 E+01$ & $1.43 E-02$ & $1.27 E-01$ & $6.77 E-10$ & $3.94 E-09$ \\
\hline CRH 12-5 & $1.10 E+01$ & $1.12 E-02$ & $1.70 E \div 01$ & $1.11 E-02$ & $1.38 E-01$ & $2.85 E-10$ & $3.56 E-09$ \\
\hline CRN 12-6 & $1.24 E+01$ & $1.03 \mathrm{E}-01$ & 2. $00 E+01$ & $1.03 E-01$ & $2.41 E-01$ & 2.12E-08 & $3.62 E-09$ \\
\hline CKH 12-7 & $1.45 E+01$ & 1.10E-02 & $2.30 E+01$ & $1.09 E-02$ & $2.52 E-01$ & 2.04E-10 & $7.26 E-09$ \\
\hline CAN 12-8 & $1.74 E+01$ & $1.2 \mathrm{BE}-02$ & $2.70 E+01$ & $1.27 E-02$ & $2.65 E-01$ & $2.30 E-10$ & $7.33 E-09$ \\
\hline CEH 12-9 & $1.95 E+01$ & $1.45 E-02$ & 3. OOEE+01 & $1.44 E-02$ & 2. TSE-OI! & $2.71 E-10$ & $7.115-03$ \\
\hline CRU $12-10$ & $2.24 E+01$ & 1. $58 \mathrm{~B}-\mathrm{i} 2 \mathrm{2}$ & $3.40 E+01$ & $1.56 \varepsilon-\hat{v} 2$ & $2.95 E-01$ & $2.75 E-10$ & $\bar{i} .34 E-69$ \\
\hline CRN 12-11 & $2.48 E+01$ & 1. $75 E-02$ & $3.70 E+01$ & $1.73 E-02$ & $3.12 E-01$ & $3.14 E-10$ & 7.19E-19 \\
\hline CRN 12-12 & $2.77 E+01$ & $1.75 E-02$ & $4.10 E+01$ & $1.74 E-02$ & $3.30 E-01$ & $2.61 E-10$ & $7.55 E-69$ \\
\hline CRE :2-13 & $3.01 E+01$ & $1.23 E-02$ & $4.40 E+01$ & $1.82 E-02$ & $3.48 E-61$ & $2.87 E-10$ & 3. OOE-0S \\
\hline
\end{tabular}


TABLE B.14a. Potassium (K) Leach Fractions from Flow Through Test Whole Grout with Groundwater (COLI2A-C)

\begin{tabular}{|c|c|c|c|c|c|}
\hline SAMPLE & $\mathrm{An} / \mathrm{AO}_{0}$ & CUn. An/Ao & DAYS & D inc. & O Cun. \\
\hline EFFLUERT ! & $5.06 E-02$ & $5.06 E-02$ & $3.00 E+60$ & $2.53 E-69$ & $2.53 E-69$ \\
\hline EFFLUENT 2 & $2.92 E-02$ & $7.98 E-02$ & $1.00 E+01$ & $1.23 E-09$ & $1.89 E-09$ \\
\hline EFFLLENT 3 & $2.10 E-02$ & $1.01 E-01$ & $1.50 E+01$ & $3.88 E-09$ & $2.15 E-09$ \\
\hline EFFLUENT 4 & $8.20 E-03$ & $i .09 E-01$ & $1.70 E+01$ & $1.37 E-09$ & $2.07 E-09$ \\
\hline EFFLUENT 5 & $2.63 E-02$ & $1.35 E-01$ & $2.10 E+01$ & $9.70 E-09$ & $2.53 E-69$ \\
\hline EFFLUENT 6 . & $1.26 E-02$ & 1. 48E-01 & $2.40 E+01$ & $4.67 E-09$ & $2.70 E-0.9$ \\
\hline EFFLUEHT $?$ & $0.25 E-03$ & $1.54 E-01$ & $2.80 E+01$ & $7.53 E-10$ & $2.51 E-699$ \\
\hline EFFLUIENT a & $1.04 E-02$ & $1.64 E-01$ & $3.10 E+01$ & 4. $18 E-09$ & $2.58 E-09$ \\
\hline EFFLUEHT $?$ & 1. $14 E-02$ & 1.TDE-OOI & $3.50 E+01$ & 3.55E-09 & $2.62 E-69$ \\
\hline COLLHAN 28 & & & & & \\
\hline EFFLUENT 10 & $1.83 E-02$ & $1.94 E-01$ & $4.50 E+01$ & $1.58 \mathrm{E}-\hat{69}$ & $2.48 E-09$ \\
\hline EFFLUENT II & $1.49 E-02$ & $2.09 E-01$ & $5.20 E+01$ & $2.595-09$ & $2.83 E-09$ \\
\hline EFFLUENT 12 & $1.15 E-02$ & $2.20 E-01$ & $5.60 E+01$ & $5.23 E-09$ & $2.39 E-69$ \\
\hline SFFLUENT 13 & $2.96 E-0.3$ & $2.23 \mathrm{E}-01$ & 5. $90 E+01$ & $6.655-10$ & $2.74 E-09$ \\
\hline EFFLUENT 14 & $3.495-03$ & $2.27 E-01$ & $6.30 E+01$ & $5.515-10$ & 2. $22 E-09$ \\
\hline EFFLUERT 15 & $7.76 E-04$ & $2.28 \mathrm{E}-01$ & $6.60 E+01$ & $5.11 E-11$ & $2.69 E-09$ \\
\hline EFFLLENT $16^{\circ}$ & 1.17E-02 & $2.39 E-01$ & $7.00 E+01$ & $6.91 E-09$ & $2.71 E-\hat{v 9}$ \\
\hline EFFLUENT 17 & $3.99 E-03$ & $2.43 E-01$ & $7.40 E+0: 1$ & 8.5IE-10 & $2.755-09$ \\
\hline EFFLUENT 19 & $3.71 E-03$ & $2.43 E-01$ & $7.80 E+01$ & $7.775-10$ & $2.72 E-73$ \\
\hline EFFLUENT 19 & $3.11 E-03$ & $2.50 E-01$ & $8.10 E+01$ & $1.01 E-99$ & $2.22 E-39$ \\
\hline EFFLUENT 21 & $7.43 E-03$ & $2.58 E-01$ & $8.80 \sum+01$ & $1.14 E-67$ & $2.8 J E-69$ \\
\hline EFFLUENT 23 & $5.60 E-03$ & $2.63 E-011$ & $7.40 E+01$ & $9.38 E-10$ & $2.46 E-09$ \\
\hline EFFLUENT 25 & $1.23 E-03$ & $2.64 E-01$ & $1.015+02$ & $3.58 E-11$ & 2.29E-is \\
\hline EFFLUENT 27 & $1.55 E-03$ & $2.66 E-01$ & 1.09E+02 & $4.70 \mathrm{E}-11$ & $2.32 E-09$ \\
\hline EFFLUEHT 29 & $1.56 E-03$ & 2. $6 B E-01$ & $1.15 E+62$ & $9.02 E-11$ & $2.19 E-69$ \\
\hline EFFLUENT $3 !$ & $2.61 E-03$ & $2.70 E-01$ & $1.22 E+42$ & $1.96 E-10$ & Z.0TE-09 \\
\hline EFFLUEHT 34 & $6.24 E-013$ & $2.76 E-01$ & $1.36 E+02$ & 3.6SE-10 & $2.00 \mathrm{E}-09$ \\
\hline EFFLUENT 36 & $2.19 E-03$ & 2. $T P E-\hat{O A I}$ & $1.43 E+02$ & $1.62 \mathrm{E}-10$ & $1.91 E-67$ \\
\hline EFFLLENT 38 & $1.465-03$ & $2.80 E-01$ & $1.50 E+62$ & $7.57 E-11$ & $1.325-49$ \\
\hline EFFLUENT 40 & 4.70E-0J & 2. $25 E-0 i$ & $1.62 E+12$ & 2.83E-10 & $1.685-09$ \\
\hline EFFLUENT $\$ 2$ & $4.54 E-03$ & $2.85 E-01$ & $1.71 E+02$ & $5 . \dot{V I E-I U}$ & $1.73 E-09$ \\
\hline EFFLUENT 44 & $1.33 E-013$ & $2.91 E-61$ & $2.06 E+02$ & $3.23 E-12$ & $1,4 J E-69$ \\
\hline
\end{tabular}


IABLE B.14b. Sodium ( $\mathrm{Na}$ ) Leach Fractions from Flow Through Test whole Grout with Groundwater (COLT2A-C)

\begin{tabular}{|c|c|c|c|c|c|}
\hline ' SAMPLE & An $/ A O$ & Eun. An/AO & DAYS & D inc. & D cus. \\
\hline EFFLUENT ! & 8. $3 B E-02$ & 8. $3 B E-02$ & $3.00 E+00$ & $6.94 E-09$ & $6.94 E-09$ \\
\hline EFFLUENT 2 & $4.61 E-02$ & $1.30 E-01$ & $1.00 E+01$ & $3.08 E-09$ & $5.00 E-09$ \\
\hline EFFLUENT 3 & $3.15 E-02$ & $1.61 E-01$ & $1.40 E+01$ & $8.77 E-09$ & $5.575-09$ \\
\hline EFFLUEENT 4 & $1.34 E-02$ & $1.75 E-01$ & $1.70 E+01$ & $3.63 E-09$ & 5.33E-67 \\
\hline EFFLUEHT 5 & 4. $12 E-02$ & $2.16 E-01$ & $2.10 E+01$ & 2.JBE- $6 B$ & 7.71E- \\
\hline EFFLULNT 6 & $2.07 E-02$ & $2.37 E-01$ & $2.40 E+01$ & $1.27 E-08$ & $8.49 E-0.99$ \\
\hline EFFLUENT 7 & $9.01 E-03$ & $2,46 E-0 !$ & $2.80 E+01$ & ¿.5 $5 E-09$ & $7.5 B E-v_{i}$ \\
\hline EFFLUENT $B$ & $1.57 E-02$ & $2.61 E-01$ & $3.10 E+01$ & $9.57 E-09$ & i. 47E- \\
\hline EFFLUENT 9 & $1.71 E-02$ & 2.79E-01 & $3.50 E+0 !$ & 7.16E-09 & $7.78 E-j 99$ \\
\hline & & & & & \\
\hline $\begin{array}{l}\text { EFFLUEHT } 10 \\
\text { EFFLUENT }\end{array}$ & $\begin{array}{l}3.09 E-02 \\
2.39 E-02\end{array}$ & $\begin{array}{l}3.04 \mathrm{E}-01 \\
3.3 \mathrm{JJE}-\hat{\mathrm{v} 1}\end{array}$ & $\begin{array}{l}4.50 E+01 \\
5.20 E+01\end{array}$ & $\begin{array}{l}4.51 E-69 \\
6.71 E-69\end{array}$ & $\begin{array}{l}1.38 E-49 \\
7.55 E-09\end{array}$ \\
\hline EFFLUEKT 12 & $2.02 E-02$ & $3.54 E-01$ & $5.60 E+01$ & $1.635-08$ & 7. $35 E-09$ \\
\hline EFFLUENT IJ & $6.02 E-03$ & $3.60 E-01$ & $5.70 E+6 i$ & $2.75 E-69$ & 5. $10 E-09$ \\
\hline EFFLUENT I4 & 3. $87 E-0 J$ & $3.665-01$ & $0.30 E+01$ & $2.13 E-99$ & B. $07 E-09$ \\
\hline EFFLUENT 15 & i. $5 J E-03$ & 3.68E-01 & $6.60 E+01$ & $1.96 E-10$ & $7.70 E-69$ \\
\hline EFFLUEMT 16 & 2.1:E-02 & 3.89E-01 & $7.00 E+01$ & $2.23 E-08$ & 8.19E-ig \\
\hline EFFLUENT 17 & $1.16 E-02$ & 4.0IE-0i & $7.40 E+01$ & $7.17 E-09$ & $3.22 E-09$ \\
\hline EFFLUENT 18 & $7.39 E-03$ & 4. 08E-01 & $7.80 E+01$ & $3.08 E-69$ & $8.25 E-69$ \\
\hline EFFLUENT 19 & $6.77 E-03$ & $4.15 E-\hat{v} !$ & $8.10 E+01$ & $4.80 E-09$ & $7.94 E-09$ \\
\hline EFFLUEMT $2 !$ & $1.36 E-02$ & $4.28 E-01$ & $8.80 E+0 !$ & 3. BIE-09 & $8.07 E-09$ \\
\hline EFFLUENT 23 & $1.26 E-02$ & $4.41 E-01$ & $9.40 E+01$ & $4.77 E-69$ & E. ABE- $\hat{Q} 9$ \\
\hline EFFLUENT 25 & $9.02 E-03$ & $4.50 E-0\}$ & $1.01 E+02$ & $1.92 E-09$ & $7.95 E-99$ \\
\hline EFFLUENT $2 \bar{T}$ & $9.32 E-03$ & 4.57E-01 & 1.09E+02 & $1.69 E-09$ & $7.74 E-69$ \\
\hline EFFLUENT 29 & $8.17 E-43$ & $4.68 E-01$ & $1.15 E+02$ & $2.46 E-05$ & $7.75 E-69$ \\
\hline EFFLUENT 31 & $9.73 E-03$ & $4.77 E-01$ & $1.22 E+02$ & $2.72 E-69$ & $7.05 E-69$ \\
\hline EFFLUEMT 34 & $1.77 E-02$ & $4.75 E-01$ & $1.36 E+02$ & $2.43 E-69$ & $7.23 E-619$ \\
\hline EFFLUEKT 36 & $6.8 P E-6 \bar{J}$ & $5.02 E-0 \mathrm{i}$ & $1.4 \overline{3} E \div 02$ & $1.60 \varepsilon-08$ & $7.22 E-09$ \\
\hline EFFLUENT 36 & $6.665-013$ & 3.69E-01 & $1.50 E+02$ & $1.57 E-69$ & $\overline{7} .21 E-i q$ \\
\hline EFIUUENT to & 1.53E- 32 & $5.22 E-01$ & $1.62 \mathrm{E}+02$ & $2.26 E-69$ & 7.01E- \\
\hline EFFLUENT 42 & $8.73 E-i 3$ & $5.31 E-01$ & $1.71 E+02$ & $1.86 E-69$ & c. $79 E-19$ \\
\hline EFFLUEHT 44 & $2.29 E-63$ & $5.33 E-01$ & 2. $065 \div 02$ & $9.515-12$ & $5.31 E-49$ \\
\hline
\end{tabular}


TABLE B.14C. Aluminum (A1) Leach Fractions from Flow Through Test whole Grout with Groundwater (COLT2A-C)

\begin{tabular}{|c|c|c|c|c|c|}
\hline SAMPLE & $\mathrm{An} / \mathrm{AO}_{0}$ & cus. An/Ao & DAYS & $D$ inc. & cus. \\
\hline EFFLLENT 1 & $1.47 E-03$ & $1.47 E-0 j$ & $3.00 E+00$ & $2.12 E-12$ & $2.12 \mathrm{E}-12$ \\
\hline EFFLUEHT 2 & $8.61 E-04$ & $2.33 E-03$ & $1.00 E+01$ & $1.07 E-12$ & 1.60E-12 \\
\hline EFFLUEHT 3 & $5.96 E-104$ & $2.92 E-03$ & $1.40 E+01$ & 3.: $4 E-12$ & 1. $81 E-12$ \\
\hline EFFLUENT \& & 2. BIE-04 & $3.20 E-03$ & $1.70 E+01$ & $1.60 E-12$ & $1.79 E-12$ \\
\hline EFFLUENT 5 & $8.58 E-014$ & $4.06 E-03$ & $2.10 E+01$ & $1.03 E-11$ & 2. $33 E-12$ \\
\hline EFFLUEKT 6 & $3.86 E-04$ & $4.45 \mathrm{E}-03$ & $2.40 E+01$ & $4.42 \mathrm{E}-12$ & $2.44 E-12$ \\
\hline EFFLUEKT 7 & 1. BBE-04 & $4.64 E-03$ & $2.80 E+01$ & $6.775-13$ & $2.27 E-16$ \\
\hline EFFLUEHT O & 3.25E-04 & $4.96 E-03$ & $3.10 E+01$ & 4.095-12 & $2.35 E-12$ \\
\hline EFFLUEHT 9 & $2.85 E-014$ & $5.24 E-03$ & $3.50 E+01$ & $1.99 E-12$ & $2 \sigma 33 E-12$ \\
\hline COLUKH $2 B$ & & & & & \\
\hline EFFLUENT 10 & 5. $77 E-04$ & $5.84 E-0 j$ & $4.50 E \div 01$ & $1.66 E-12$ & $2.25 E-12$ \\
\hline EFFLUENT 11 & $4.98 E-04$ & $6.34 E-\hat{0} 3$ & $5.20 E+01$ & $2.91 E-12$ & $2.29 E-12$ \\
\hline EFFLUENT 12 & $3.675-04$ & $6.71 E-03$ & $5.60 E+01$ & $5.45 E-12$ & $2.38 E-12$ \\
\hline EFFLIERT II & $1.74 E-0.5$ & $6.73 E-003$ & $5.70 E+01$ & 2.31E-14 & $2.27 E-12$ \\
\hline EFFLUEHT 14 & 7. $85 E-05$ & $6.83 E-03$ & 6.30E+01 & $4.38 E-13$ & $2.19 E-12$ \\
\hline EFFL'UENT I5 & 2.19E-05 & $6.85 E-03$ & $6.60 E+01$ & $4.07 E-14$ & $2.11 E-12$ \\
\hline EEFLUEMT 16 & $2.86 E-04$ & $7.135-03$ & 7.00E+01 & $4.11 E-12$ & 2.15E-12 \\
\hline EFFLUENT 17 & $0.00 E+00$ & $7.13 E-03$ & $7.40 E+01$ & $0.00 E+00$ & $2.04 E-12$ \\
\hline EFFLUEHT IB & $7.00 \mathrm{E}-06$ & $7.14 E-0 \bar{J}$ & $7.80 E+01$ & $2.76 \mathrm{E}-15$ & $1.74 E-12$ \\
\hline EFFLUENT IS & $4.10 E-05$ & $7.18 E-03$ & Q.:OOE+01 & $1.36 E-13$ & 1.89E-12 \\
\hline EFFLUENT 21 & $8.34 E-06$ & $7.19 E-03$ & $8.80 E+01$ & $1.42 \mathrm{E}-1 \mathrm{E}$ & $1.74 E-12$ \\
\hline EFFLUJENT 23 & $0.00 E+00$ & 7. 19E-03 & $9.40 E+01$ & $0.00 E+00$ & 1. $63 E-12$ \\
\hline EFF!UENT 25 & $0.00 E+00$ & 7.19E-03 & $1.01 E+02$ & $0.00 E+001$ & $1.52 \mathrm{E}-12$ \\
\hline EFFLUENT 27 & $0.00 E+00$ & $7.19 E-03$ & $1.09 E+02$ & $0.00 E+00$ & $1.41 E-12$ \\
\hline EFFLUEHT 29 & $5.90 E-016$ & $7.19 E-03$ & $1.15 E+02$ & $1.28 E-15$ & 1. J3E-12 \\
\hline EFFLUEMT 31 & $1.31 E-05$ & $7.21 E-03$ & $1.22 E+62$ & $4.94 E-15$ & $1.26 E-12$ \\
\hline EFFLUEHT 34 & $0.00 E+00$ & $i .21 E-03$ & $1.36 E+02$ & $0.00 E+00$ & 1.13E-12 \\
\hline EFFLUENT 36 & $0.00 E+00$ & $7.21 E-03$ & $1.43 E+02$ & $0.00 E+000$ & 1. $0 B E-12$ \\
\hline EFFLUENT 38 & $0.00 E+00$ & $7.21 \mathrm{E}-\mathrm{vj}$ & 1.50E+02 & $0.00 E+00$ & $1.03 E-12$ \\
\hline EFPLUEHT 40 & $1.57 E-005$ & $7.22 \mathrm{E}-03$ & $1.62 E+02$ & $3.15 E-15$ & $9.55 E-15$ \\
\hline EFFLUENT 42 & $1.71 E-016$ & $7.23 \mathrm{E}-0 \mathrm{~J}$ & $1.71 E+02$ & $7.12 \mathrm{E}-17$ & $9: 05 E-13$ \\
\hline EFFLUEKT $\$ 4$ & $0.00 E+00$ & $7.23 E-03$ & $2.06 E+02$ & $0.00 E+0.5$ & $7.51 E-13$ \\
\hline
\end{tabular}


TABLE B.14e.

Boron (as $\mathrm{H} 3 \mathrm{~B} 03$ ) Leach Fractions from Flow Through Test whole Grout with Groundwater (COLT2A-C)

\begin{tabular}{|c|c|c|c|c|c|}
\hline SAMPLE & An/Ao & Cus. An/AO & DAYS & D. inc. & $D$ cus. \\
\hline EFFLUEसT 1 & $2.88 E-03$ & $2.88 E-03$ & $3.00 E+00$ & 8.21E-12 & $8.21 E-12$ \\
\hline EFFLUENT 2 & $2.90 E-03$ & $5.78 E-03$ & $1.00 E+01$ & $1.22 \mathrm{E}-11$ & $9.91 E-12$ \\
\hline EFFLUENT 3 & $1.94 E-03$ & $7.73 E-03$ & $1.40 E+01$ & 3.JJE-11 & $1.26 \mathrm{E}-11$ \\
\hline EEFLUENT 4 & $8.49 E-04$ & $8.58 E-03$ & $1.70 E+01$ & $1.47 E-11$ & $1.28 E-11$ \\
\hline EFFLUENT 5 & $2.10 E-03$ & $1.07 E-02$ & $2.10 E+01$ & $6.19 E-11$ & $1.61 E-11$ \\
\hline EFFLUEHT 6 & $8.02 E-04$ & $1.15 E-02$ & $2.40 E+01$ & $1.90 E-11$ & $1.63 E-11$ \\
\hline EFFLUEET 7 & $4.64 E-04$ & $1.19 E-02$ & $2.80 E+01$ & 4.15E-12 & 1.51E-11 \\
\hline EFFLUENT 8 & $7.81 E-04$ & $1.27 E-02$ & $3.10 E+011$ & $2.37 \mathrm{E}-11$ & 1.55E-11 \\
\hline EFF!UEHT ? & 6. 13E-04 & $1.33 \mathrm{JE}-02$ & $3.50 E+01$ & $9.17 E-12$ & $1.51 E-11$ \\
\hline COELUKK 28 & & & & & \\
\hline EFFLUEHT 10 & $1.70 E-03$ & $1.50 E-i 2$ & 4.50E+01 & $1.36 E-11$ & 1. A5E-19 \\
\hline EFFLUENT 11 & $1.23 E-03$ & $1.63 E-02$ & $5.20 E+01$ & $1.7 B E-\mathrm{i} I$ & !.SIE- 11 \\
\hline EFFLUENTI 12 & $1.09 E-03$ & $1.74 E-\hat{v} 2$ & $5.60 E+01$ & $4.73 E-11$ & $1.57 E-11$ \\
\hline EFFLUENT IJ & $2.32 E-04$ & $1.76 \mathrm{E}-02$ & $5.90 E+01$ & $4.07 E-12$ & $1.55 E-11$ \\
\hline EFFLUEETT I4 & $4.38 E-(14$ & $1.60 E-02$ & $6.30 E+01$ & 8. $65 E-12$ & 1.5JE-11 \\
\hline EFFLUENT 15 & $9.97 E-05$ & $1.81 E-02$ & $6.60 E+01$ & B.45E- 13 & 1.48E-1! \\
\hline EFFLUENT 16 & $1.30 E-03$ & 1. $94 E-62$ & $7.60 E+01$ & $8.47 E-11$ & $1.60 E-11$ \\
\hline EFFLUENT IT & $0.78 E-04$ & $2.01 E-02$ & $7.40 E \div 01$ & $2.46 E-11$ & $1.62 E-11$ \\
\hline EFFLUEHT 16 & $5.15 E-i 4$ & $2.06 E-02$ & $7.80 E+01$ & $1.50 \mathrm{E}-11$ & $1.61 E-11$ \\
\hline EFFLUENT I\$ & 5.12E-04 & $2.11 E-02$ & $6.10 E+01$ & $2.74 E-11$ & $1.6 \overline{E E}-11$ \\
\hline EFFLUENT 21 & $8.38 E-044$ & $2.20 E-02$ & $8.80 E+01$ & $1.43 E-11$ & $1.62 E-11$ \\
\hline EFFLUENT 23 & $1.11 E-6 J$ & $2.31 E-02$ & 9. $10 E+01$ & $3.66 E-11$ & 1.6BE-11 \\
\hline EFFLUENT 25 & $3.93 E-04$ & $2.40 E-02$ & $1.01 E+02$ & $1.88 E-11$ & 1.6FE-11 \\
\hline EFFLUENT 27 & $8.36 E-014$ & $2.48 E-02$ & $1.09 E+02$ & $1.36 E-11$ & $1.67 \mathrm{E}-11$ \\
\hline EFFLUENT 29 & $8.34 E-04$ & $2.565-02$ & $1.15 E+02$ & $2.57 E-11$ & $1.67 E-11$ \\
\hline EFFLUENT 31 & 8.60E-0. & $2.65 E-02$ & i. $22 E+62$ & 2.12E-11 & 1.TIE-i: \\
\hline EFFLUEHT 34 & $1.29 E-03$ & $2.78 E-02$ & 1. $36 E+02$ & $1.30 E-11$ & $1.68 E-11$ \\
\hline EFFLUENT $3 b$ & $0.49 E-04$ & $2.84 E-02$ & $\therefore 43 E+02$ & $1.42 E-11$ & $1.6 B E-11$ \\
\hline EFFLUEHT 38 & $7.70 E-04$ & 2. ร2E-02 & 1. $50 E+02$ & $2.10 E-11$ & I. G9E-i! \\
\hline EFFLUENT 40 & i.50E-03 & $3.07 E-\hat{0} 2$ & 1.22E+62 & $2.90 \mathrm{E}-\mathrm{ii}$ & 1. $72 E-11$ \\
\hline EFFL UENT 12 & $1.12 E-03$ & $3.185-02$ & $1.71 E+12$ & $3.06 E-1 !$ & 1.76E-1! \\
\hline EFFLUENT 44 & $4.0 \mathrm{iE}-04$ & $3.22 E-62$ & $2.06 E+02$ & $2.73 E-\mathrm{i} 3$ & $1.45 E-! !$ \\
\hline
\end{tabular}


TABLE B.14f. Fluoride (F) Leach Fractions from Flow Through Test whole Grout with Groundwater (COLT2A-A)

\begin{tabular}{|c|c|c|c|c|c|}
\hline SAMPLE & An/AO & CUn. An/Ao & DAYS & D inc. & icus. \\
\hline EFFLUENT 1 & $1.105-03$ & ¿. $10 E-03$ & $3.00 \mathrm{E}+60$ & $1.19 E-12$ & $1.19 E-12$ \\
\hline EFFLUENT 2 & $7.08 E-04$ & $1.80 E-03$ & $1.00 E+01$ & $7.26 \mathrm{E}-13$ & $9.64 E-13$ \\
\hline EFFLUENT 3 & $5.20 E-04$ & $2.32 E-0 j$ & $1.40 E+01$ & $2.39 E-12$ & 1.14E-12 \\
\hline EFFLUENT 4 & $2.65 E-04$ & $2.59 E-03$ & $1.70 E+01$ & $1.43 E-12$ & 1.17E-12 \\
\hline EFFLUEHT 5 & 8. 44E-04 & $3.43 E-03$ & $2.10 E+01$ & $1.00 E-11$ & 1.56E-12 \\
\hline EFFLUENT 6 & 5.22E-04 & 3.95E-03 & $2.40 E+01$ & $8.06 E-12$ & $1.93 E-12$ \\
\hline EFFLUEHT 7 & $3.24 E-104$ & $4.28 E-03$ & $2.80 E+01$ & $2.02 E-12$ & $1.94 E-12$ \\
\hline EFFLULENT 8 & $4.90 E-04$ & $4.77 E-03$ & $3.10 E+01$ & $9.31 E-12$ & $2.17 E-12$ \\
\hline EFFLUEHT $?$ & $7.39 E-04$ & $5.51 E-0 j$ & $3.50 E+01$ & $1.345-11$ & $2.57 E-12$ \\
\hline COLUNA 2B & & & & & \\
\hline EFFLUENT 10 & $6.15 E-04$ & $6.12 E-63$ & 4. SEE+01 & 1.79E-12 & $2.47 E-12$ \\
\hline EFFLUENT i1 & $7.53 E-044$ & $6.88 E-03$ & $5.20 E+01$ & 3. $64 E-12$ & $2.69 E-12$ \\
\hline EFFLLENT 12 & 3.59E-04 & T.73E-03 & 5. $00 E+01$ & $2.95 E-11$ & $3.17 E-12$ \\
\hline EFFLUENT 13 & $4.71 E-04$ & $8.21 E-6 j$ & $5.90 E+01$ & $1.68 E-11$ & $3.38 E-12$ \\
\hline EFFLLERT 14 & $2.58 E-14$ & $8.46 E-0 j$ & 6. $30 E+01$ & $3.02 E-12$ & 3.3iE-!2 \\
\hline EFFLUERT 15 & $5.74 E-05$ & $8.52 E-03$ & $6.60 E+01$ & $2.80 E-i J$ & $3.26 E-12$ \\
\hline EFFLUENT 16 & $8.76 E-04$ & $7.40 E-03$ & 7.00E $\div 01$ & $3.87 E-11$ & j. $74 E-12$ \\
\hline EFFLUERT IT & $9.02 E-04$ & 1. $03 \mathrm{E}-02$ & $7.40 E+01$ & $4.34 E-11$ & $4.25 E-12$ \\
\hline EFFLUIEHT 18 & b. $355-04$ & $1.095-02$ & $7.80 E+0 \mathrm{~s}$ & $2.27 E-11$ & $4.54 E-12$ \\
\hline EFFLUERT IO & $5.25 E-04$ & 1. $15 E-02$ & 2. $10 E+01$ & $2.89 E-11$ & $4.81 E-12$ \\
\hline EFFLUEHT 21 & $1.30 E-03$ & $1.28 E-02$ & $8.80 E+01$ & $3.94 E-11$ & 5. 4BE-12 \\
\hline EFFLUENT 23 & 1.61E-03 & $1.44 E-02$ & $9.40 E+01$ & $7.75 E-11$ & t.51E-12 \\
\hline EFFLUEUT 25 & $1.23 E-03$ & 1.56E-02 & 1.01E+02 & $3.57 E-11$ & $7.14 E-12$ \\
\hline EFFLUENT 27 & $1.16 E-03$ & $1.68 E-02$ & $1.07 E+02$ & $2.6 \mathrm{JE}-11$ & $7.64 \mathrm{E}-12$ \\
\hline EFFLUEAT 29 & B. $74 E-04$ & $1.76 E-\hat{v} 2$ & $1.15 E+02$ & $2.82 E-11$ & $8.01 E-12$ \\
\hline EFFLUENT 31 & $1.025-03$ & $1.87 E-02$ & $1.22 E+02$ & 2. $99 \mathrm{E}-\$ 1$ & $6.95 E-12$ \\
\hline EFFLUENT I4 & $2.5 B E-03$ & $2.12 E-02$ & i. $36 E+02$ & S.JEE-11 & $9.82 E-12$ \\
\hline EFFLUENT 36 & $1.31 E-03$ & $2.25 E-02$ & $1.43 E+02$ & 5.80E-11 & 1. 65E-i1 \\
\hline EFFIUUENT 38 & $1.15 E-03$ & $2.37 E-02$ & $1.50 E+02$ & $4.67 E-1 i$ & I.IIE-ii \\
\hline EFFLUERT 40 & $2.11 E-03$ & $2.58 E-62$ & $1.62 E+02$ & $5.705-11$ & $1.22 E-: i$ \\
\hline SFFLUENT $\$ 2$ & $2.19 E-03$ & $2.30 \mathrm{E}-02$ & $1.71 E+02$ & $1.16 E-10$ & $1.36 E-11$ \\
\hline EFFLUENT 44 & $5.795-03$ & 3.jBE-v2 & 2. $66 E+62$ & $6.10 E-11$ & $1.64 E-11$ \\
\hline EFFLUENT 45 & $6.5: E-4]$ & $4.6 \mathrm{BE}-\mathrm{V} 2$ & $2.36 E+02$ & $1.2 \mathrm{EE}-10$ & $2.04 E-13$ \\
\hline
\end{tabular}


TABLE B.14g. Nitrate (NO3) Leach Fractions from Flow Through Test whole Grout with Groundwater (COLT2A-A)

\begin{tabular}{|c|c|c|c|c|c|}
\hline SAMPLE & $\mathrm{An} / \mathrm{AO}$ & Cun. An/Ao & DAYS & Dinc. & 0 cus. \\
\hline EFFLUEHT 1 & $4.51 E-02$ & $4.51 \mathrm{E}-02$ & $3.00 E+00$ & $2.01 E-09$ & 2.01E-39 \\
\hline EFFLUEKT 2 & $2.59 E-02$ & $7.10 E-02$ & $1.00 E+01$ & $9.73 E-10$ & $1.50 E-09$ \\
\hline EFFLUENT 3 & $1.69 E-02$ & B. $80 \mathrm{E}-02$ & $1.40 E+01$ & $2.53 E-09$ & $1.64 E-69$ \\
\hline EFFLUENT 4 & $1.20 E-02$ & $1.00 E-01$ & $1.70 E+01$ & $2.93 E-\hat{j} 9$ & $1.74 E-19$ \\
\hline EFFLUEHT 5 & $1.92 E-02$ & $1.19 E-01$ & $2.10 E+01$ & $5.19 E-09$ & 2.01 $E-0 ?$ \\
\hline EFFLUENT 6 & $7.95 E-03$ & 1.27E-01 & $2.40 E+01$ & $1.87 E-09$ & 2. $00 E-69$ \\
\hline EFFLUENT 7 & $4.20 E-\hat{Q} 3$ & 1.3IE-01 & 2. $00 E+01$ & $3.40 E-10$ & 1. $33 E-09$ \\
\hline EFFLUENT 8 & $3.73 E-03$ & $1.39 E-01$ & 3. $10 E+01$ & 2. $44 E-09$ & $1.865-09$ \\
\hline EFFLUENT ? & $7.77 \mathrm{E}-03$ & $1.47 E-01$ & $3.50 E+01$ & $1.47 E-09$ & $1.23 E-09$ \\
\hline COLUAN $2 B$ & & & & & \\
\hline EFFLUENT 10 & $1.58 E-02$ & $1.63 \mathrm{E}-01$ & $4.50 E+0 \hat{10}$ & $1.18 E-09$ & $3.27 E-69$ \\
\hline EFFLUENT 11 & 1. $3 \mathbf{3} E-02$ & $1.76 E-01$ & $5.20 E+01$ & $2.06 E-09$ & $3.41 E-09$ \\
\hline EFFLUENT 12 & $1.09 E-02$ & $1.87 E-01$ & 5. $60 E+01$ & 4.78E-09 & $3.64 E-09$ \\
\hline EFFLUENT I3 & $2.7 B E-03$ & $1.90 E-01$ & $5.90 E+01$ & $5.84 E-10$ & $3.45 E-09$ \\
\hline EFFLUENT If & $4.34 E-03$ & $1.34 E-01$ & $6.30 E+01$ & B. $525-10$ & $3.33 E-09$ \\
\hline EFFLUENT 15 & $9.65 E-(14$ & $1.55 E-01$ & 6. $60 E+01$ & $7.91 E-11$ & $3.22 E-09$ \\
\hline EFFLUE!IT 16 & $1.46 E-02$ & $2.10 E-01$ & 7. $\hat{V G E}+G 1$ & 1.0EE-ve & 3. $01 E-0 h$ \\
\hline EFFLLENT 17 & $5.85 E-03$ & 2. $\{t E-0 \mid$ & $7.40 E+01$ & 1. $835-09$ & j. $68 E-69$ \\
\hline EFFLUENT 18 & $4.26 \mathrm{E}-\mathrm{bi}$ & 2. $20 \mathrm{E}-\hat{\mathrm{O} i}$ & 7. 80ETOO1 & $1.02 E-09$ & $3.49 E-i j ?$ \\
\hline EFFLUEHT 19 & $4.44 E-03$ & $2.24 E-01$ & $8.10 E+01$ & $2.06 E-07$ & $3.64 E-0 \hat{Y}$ \\
\hline EFFLUENT 21 & Q. $.99 E-\hat{v} 3$ & 2.33E-01 & B. $80 E+01$ & $1.61 E-09$ & $3.61 E-09$ \\
\hline EFFLUENT 23 & $7.75 E-03$ & $2.41 E-01$ & $7.40 E+01$ & $1.30 E-09$ & $3.63 E-09$ \\
\hline EFFLUEHT 25 & $5.81 E-113$ & $2 .\{7 E-01$ & $1.01 E+02$ & $7.965-10$ & 3. $63 E-67$ \\
\hline EFFLUENT 27 & $6.66 E-03$ & $2.5 j E-01$ & $1.05 E+02$ & $8.62 E-10$ & $3.60 E-39$ \\
\hline EFFLUEKT 29 & $6.37 E-03$ & $2.600 E-01$ & $1.15 E+02$ & $1.49 E-07$ & 3. $41 E-19$ \\
\hline EFFLUENT 31 & $7.00 E-03$ & $2.67 E-01$ & $1.22 E+02$ & $1.40 E-0 E$ & $3.44 E-09$ \\
\hline EFFLUERT 34 & 2. $00 E-02$ & $2.87 E-01$ & 1. $36 E+02$ & $3.13 E-09$ & 1.7FE-09 \\
\hline EFFLUENT j6 & $6.57 E-03$ & $2.33 E-01$ & $1.43 E+02$ & $1.40 E-29$ & $1.76 E-09$ \\
\hline EFFLUEKT 3 B & $6.26 E-03$ & $3.005-01$ & 1. $50 E+02$ & 1.3PE-09 & $1.77 E-099$ \\
\hline EFFLUENT 40 & i. $65 E-02$ & $3.16 E-01$ & $1.62 E+02$ & $3.5 \mathrm{IE}-09$ & $1.83 E-019$ \\
\hline EFFLUENT 42 & $8.10 E-0\}$ & $3.2 A E-0 !$ & $1.71 E+02$ & $1.60 E-09$ & 1.82E-69 \\
\hline EFFLUENT 44 & $2.65 E-012$ & $3.51 E-01$ & $2.065+\hat{0} 2$ & $1.28 E-69$ & $1.77 E-09$ \\
\hline EFFLIENT 45 & 1. $02 E-62$ & $3.61 E-01$ & 2. $3 \dot{E E}+0 \mathrm{C}$ & $3.00 E-10$ & 1.64E-09 \\
\hline
\end{tabular}


TABLE B.14i. Iodine-125 (I-125) Leach Fractions from Flow Through Test, Grout with Groundwater (2A)

\begin{tabular}{|c|c|c|c|c|c|c|c|}
\hline SAMPLE & PORE VOLUME & $\operatorname{COR} \mathrm{UCI}$ & TIME DAYS & An/Ad & CUn An/AO & DINC CN $/$ SEC & DCUY CK2/SEC \\
\hline CRN LTI2A-1 & $1.02 E+00$ & $4.57 E-02$ & $3.00 E+00$ & $4.90 E-02$ & $4.90 E-02$ & $2.46 E-09$ & $2.46 E-09$ \\
\hline CRH LT2A-2 & $1.83 E+00$ & $3.34 E-02$ & $1.00 E+01$ & $3.58 E-02$ & $8.47 E-02$ & $4.82 E-09$ & $7.37 E-09$ \\
\hline CRH LTI2A-3 & $2.65 E+00$ & $1.99 E-02$ & $1.40 E+01$ & $2.13 E-02$ & $1.06 E-01$ & $5.25 E-09$ & $1.16 E-08$ \\
\hline CRH LT2A-4 & $3.05 E+00$ & $8.4 J E-0 j$ & $1.70 E+01$ & $9.035-03$ & 1.15E-01 & $1.11 E-09$ & 1.36E-08 \\
\hline CRH L12A-5 & $4.69 E+100$ & 1. $19 E-02$ & $2.10 E+01$ & $1.23 E-02$ & $1.28 E-01$ & $1.30 E-09$ & $1.66 E-08$ \\
\hline CRH LT2A-6 & $5.895+00$ & $3.53 E-03$ & $2.40 E+01$ & $1.02 E-02$ & $1.36 E-01$ & $1.41 E-09$ & $1.76 E-08$ \\
\hline CRH LT2RA-7 & $6.66 E+00$ & $5.22 E-03$ & $2.80 E+01$ & $5.595-03$ & $1.44 E-011$ & $2.51 E-10$ & 2. $12 E-08$ \\
\hline CRH $L T 2 A-8$ & $7.83 E+00$ & $1.91 E-02$ & $3.10 E+0 i$ & $2.05 E-02$ & $1.64 E-01$ & 5. $68 E-09$ & $2.77 E-08$ \\
\hline CRH LT2Â-9 & $9.32 E+00$ & $1.75 E-02$ & $3.50 E+01$ & $1.88 E-02$ & $1.33 E-01$ & $2.82 E-09$ & $3.44 E-60$ \\
\hline
\end{tabular}


Iodine-125 (I-125) Leach Fraction from Flow Through Test, Grout with Groundwater (2B)

\begin{tabular}{|c|c|c|c|c|c|c|}
\hline SAMPLE & PORE VOLUAE & CORR. UCj & TIME DAYS & $\hat{n} \mathrm{n} / \mathrm{AO}$ & CUL An/AO & DIKC CK2/5EC \\
\hline CRH LT2B-1 & $7.20 E-01$ & 1.21E-02 & $3.00 E+00$ & $1.23 E-02$ & $1.26 E-62$ & $1.67 E-10$ \\
\hline CRM LT2B-2 & $1.38 E+00$ & $1.50 E-02$ & $1.00 E+01$ & $1.59 E-02$ & $2.87 E-02$ & $9.45 E-10$ \\
\hline CRH LT2D-3 & $2.10 E+60$ & $1.10 \mathrm{E}-02$ & $1.90 E+01$ & $1.16 E-02$ & $4.04 E-0.2$ & $1.55 E-09$ \\
\hline CRN LT28-4 & $2.70 E+100$ & $1.25 E-02$ & $1.70 E+01$ & $1.33 E-02$ & $5.36 E-62$ & 2. JáE- $\hat{v} 9$ \\
\hline CRI LT28-5 & $4.45 E+00$ & $2.39 E-02$ & $2.10 E+01$ & $2.58 E-02$ & $7.70 E-02$ & $5.11 E-09$ \\
\hline CRN LT2B-6 & $5.87 E+00$ & $2.20 E-02$ & $2.40 E+01$ & $2.34 E-02$ & $1.02 E-01$ & $7.34 E-09$ \\
\hline CRN $1528-7$ & $6.90 E+00$ & $4.90 E-03$ & $2.80 E+01$ & $5.20 \mathrm{E}-03$ & $1.08 E-01$ & $2.15 E-10$ \\
\hline CRN LT2B-B & $7.52 E+00$ & $8.48 E-03$ & $3.10 E+01$ & $9.01 E-03$ & $1.17 E-01$ & $1.09 E-09$ \\
\hline CRN $1 T 2 B-9$ & $8.43 E+00$ & 1. DSE-02 & $3.50 E+01$ & $1.16 E-02$ & $1.28 \mathrm{E}-0 \mathrm{I}^{\circ}$ & $1.075-09$ \\
\hline CKH LIZE-10 & $1.02 E+01$ & $2.27 \mathrm{E}-02$ & $4.50 E+01$ & $2.41 E-02$ & $1.525-61$ & $1.48 E-67$ \\
\hline CRH LT2E-II & $1.21 E+01$ & $1.66 E-02$ & $5.20 E+01$ & $1.76 E-02$ & $1.705-01$ & 1. $24 E-09$ \\
\hline CAN LTZB-12 & $1.47 E+01$ & $1.43 E-02$ & $5.60 E+01$ & $3.52 E-02$ & $1.85 E-01$ & $2.65 E-09$ \\
\hline CRH 172B-13 & $1.63 E+01$ & $8.32 E-03$ & $5.90 E \div 01$ & $9.36 E-03$ & $1.74 E-01$ & 1.16E-19 \\
\hline CRN LT28-14 & $1.72 E+01$ & $6.26 E-03$ & $6.30 E+01$ & $6.54 E-63$ & $2.01 E-01$ & $3.39 E-10$ \\
\hline CA & $1.74 E+01$ & $4.555-04$ & $6.60 E \div 01$ & $4.83 E-04$ & 2.6!E-01 & 3.15E-12 \\
\hline CRH LT2B-16 & $2.01 E+01$ & $2.04 E-02$ & $7.00 E+01$ & $2.17 E-02$ & $2.23 E-01$ & $3.73 E-09$ \\
\hline CRY LT2S-!17 & $2.355+01$ & $8.32 E-03$ & $7.405+0 !$ & 3.69E-03 & $2.32 E-6 i$ & 7. $14 E-10$ \\
\hline CRH LT2B-iB & $2.57 E+01$ & $5.87 \mathrm{E}-03$ & $3.80 E+01$ & 6.23E-03 & 2. $38 E-011$ & $3.45 E-10$ \\
\hline CAH LT28-19 & $2.65 E+01$ & $5.655-03$ & $8.10 E+01$ & $6.00 E-03$ & $2.74 E-01$ & $4.35 E-10$ \\
\hline CRH LT2B-20 & $2.87 E+01$ & $9.17 E-03$ & $8.50 E+01$ & $9.74 E-03$ & $2.54 E-01$ & $7.52 E-10$ \\
\hline CRH LT2B-21 & $3.18 E+01$ & $6.275-03$ & $8.20 E+01$ & $6.66 E-0 J$ & $2.61 E-01$ & 5. $95 E-10$ \\
\hline CRH LT2B-22 & $3.475+01$ & $6.61 E-03$ & $9.10 E+01$ & $7.02 E-03$ & $2.6 E E-01$ & $5.34 E-10$ \\
\hline CAH $172 B-23$ & $3.72 E+01$ & $2.62 E-03$ & $9.40 E+01$ & $2.79 E-0.3$ & $2.70 E-01$ & $9.20 E-11$ \\
\hline CFH 1728-24 & $4.01 E+01$ & $6.24 E-03$ & $9.80 E+01$ & $6.63 E-03$ & $2.77 E-01$ & $3.48 E-10$ \\
\hline CAN $1723-25$ & $4.135+01$ & $3.83 E-03$ & $1.01 E+02$ & $4.075-03$ & $2.8 ! E-j !$ & $2.22 E-10$ \\
\hline CRH LT28-26. & $4.45 E+01$ & $8.81 E-03$ & $1.65 E+02$ & $9.35 E-03$ & $2.91 E-01$ & $5.93 E-10$ \\
\hline CBN LTAD-27 & $4.56 E+01$ & $2.03 \bar{E}-03$ & $1.09 E+02$ & $2.79 E-03$ & $2.952-01$ & b. $31 E-11$ \\
\hline CKH LT28-26 & $4.69 E+01$ & $6.63 \mathrm{~J}-03$ & $1.125 \div 02$ & $7.03 E-03$ & $3.00 E-i j 1$ & $0.64 E-10$ \\
\hline CAH LT23-29 & $4.82 E+01$ & $5.77 E-63$ & $1.15 E+02$ & $6 . \mathbf{j} \mathbf{J E}-\mathbf{0} \mathbf{J}$ & $3 . \dot{v} B E-0 i$ & $4 . \neq 5 E-10$ \\
\hline CRH LT28-30 & $5.02 E+01$ & $6.16 E-03$ & $1.19 E+0.2$ & $0.54 E-03$ & J. $I J E-6 i$ & $3.40 E-10$ \\
\hline 재 $1725-31$ & $5.18 E+01$ & $5.41 E-0 j$ & $1.22 E+02$ & $5.74 E-63$ & $3.17 E-j j$ & $4.43 E-10$ \\
\hline CRN LTII-32 & $5.34 E+01$ & $4.45 E-03$ & $1.26 E \div 02$ & $4.73 E-13$ & $3 . Z 3 E-01$ & 1.77E-10 \\
\hline CEN $1728-33$ & $56 B E+01$ & 1. 0.5E- 02 & 1. J3E +02 & $2.22 E-122$ & $\therefore .35 E-j i$ & $5.23 E-10$ \\
\hline LEK LT:E-34 & $\Xi .91 E+01$ & $3.93 E-03$ & $1.36 E \div 02$ & $4.17 E-\hat{1} 3$ & $3.395-0 !$ & $3.075-10$ \\
\hline CFH LT2B-35 & $6.14 E+01$ & $5.015-03$ & $1.40 E+02$ & $5.325-03$ & $j .4+E-0 i$ & $2.24 E-10$ \\
\hline ERH LT2B-jb & $6.27 E+01$ & $3.325-0 j$ & $1.43 E+102$ & 4. OSE-6) & $3.48 E-6 i$ & $2.2 \mathrm{iE}-\mathrm{i} \hat{\mathrm{j}}$ \\
\hline Cny $1725-3 i$ & $6 .+65-013$ & $3.70 E-03$ & $1.77 E+02$ & $4.145-6 j$ & $3.52 E-6 i$ & $1.365-10$ \\
\hline CKH LT2E-38 & b. $523 E+01$ & $6.70 E-03$ & $1.50 E+102$ & $7 .: 2 E-03$ & $3.595-61$ & $6.30 \mathrm{0}-10$ \\
\hline CKH LT2E-3Y & S. $63 E+01$ & 7. 60์ -0̂J & $1.53 E+02$ & $8.67 E-13$ & 3. SEE-8.1 & $7.7: I E-j 0$ \\
\hline ORH LT2E-40 & 7. ¿3E+100 & 5. จุE-0\} & 1.3AE+0Z & $6.29 E-03$ & J.TAE-01 & $2.0 E E-10$ \\
\hline CRH LT28-41 & $7.55 E+01\}$ & $3.18 E-03$ & $1.6 B E+06$ & $3.075-63$ & $3.77 E-4 j$ & $8.05 E-11$ \\
\hline CAN LT2E-42 & $7.75 E+01$ & $3.93 E-0 J$ & 1.71E+02 & 4.: : :E-1:3 & 3. $31 E-\hat{u}:$ & $2.04 E-j 0$ \\
\hline CFN LTZ3-43 & $\bar{i} .38 E+10 !$ & $4.15 E-03$ & $1.75 E+02$ & $4.44 E-63$ & S. $36 E-\nabla !$ & $\therefore 575-10$ \\
\hline CFin 1T23-44 & $7.3 \mathrm{JE}+61$ & $5.995-63$ & $2.06 E+02$ & S. $3 \in E-63$ & 3.72E-6j & $2.50 \mathrm{E}-11$ \\
\hline CFH $1 T 2 B-45$ & $1.13 E+02$ & $9.24 E-6 j$ & $2.36 E+02$ & $\overline{5} \bar{B} \mathrm{IE}-0 \mathrm{i}$ & $4.02 E-01$ & $1.16 E-10$ \\
\hline
\end{tabular}


TABLE B.15b. Sodium (Na) Leach Fractions from Flow Through Test Grout/Sediment with Groundwater (DFCOLC1)

\begin{tabular}{|c|c|c|c|c|c|}
\hline SARPLE & An/Ao & Cus. An/AO & DAYS & $D$ inc. & $D$ cul. \\
\hline EFFLUEKT 1 & $4.63 E-02$ & 1. $63 E-02$ & $3.00 E+00$ & $2.26 \mathrm{E}-09$ & $2.26 E-19$ \\
\hline EFFLUEKT 2 & $4.71 E-02$ & $9.33 \mathrm{E}-02$ & $6.00 E+00$ & 1.36E-0B & $4.59 E-09$ \\
\hline EFFLUENT 3 & $5.58 E-02$ & $1.49 E-01$ & $9.00 E+00$ & 3. 25E-08 & $7.82 E-09$ \\
\hline EFFLUENT 4 & $4.565-02$ & $1.95 E-01$ & $1.30 E+01$ & $1.79 E-08$ & $9.23 E-199$ \\
\hline EFFLUENT 5 & $1.17 E-02$ & $2.06 E-01$ & $1.70 E+01$ & $1.61 E-09$ & $8.655-09$ \\
\hline EFFLLUENT $b$ & $1.48 E-02$ & $2.21 E-01$ & $2.00 E+01$ & 5. $66 E-09$. & $8.09 E-09$ \\
\hline EFFLUENT 7 & 1. $83 \mathrm{E}-02$ & $2.39 E-01$ & $2.30 E+01$ & $1.02 E-08$ & Q. 86E-0.9 \\
\hline EFFLUENT В & 2.32E-02 & $2.63 E-01$ & $2.70 E+01$ & 1.06E-0B & $8.57 E-09$ \\
\hline EFFLUENT 9 & $1.74 E-02$ & 2. 50E-01 & $3.00 E+01$ & $1.22 E-08$ & $9.08 E-10 \%$ \\
\hline EFFLGEHT 10 & $1.78 E-62$ & $3.005-01$ & $3.40 E+01$ & $9.93 E-0 ?$ & \{.33E-199 \\
\hline EFFLUENT II & $1.46 E-62$ & $3.15 E-01$ & $3.70 E+01$ & 1.07E-68 & $9.22 E-19$ \\
\hline EFFLUEHT 12 & $1.62 E-02$ & $3.31 E-01$ & 4.10E+01 & $6.605-09$ & $7.575-08$ \\
\hline EFFLUENT IJ & $1.21 E-62$ & $3.43 E-01$ & $4.40 E+01$ & $3.78 E-09$ & $9.53 E-6 ?$ \\
\hline EFFLUENT I4 & $1.51 E-02$ & $3.58 E-01$ & $4.96 E+01$ & $5.37 E-09$ & $9.75 E-09$ \\
\hline EFFLUENT 15 & $1.24 E-62$ & $3.70 E-01$ & $5.20 E+01$ & $1.09 E-08$ & $9.77 E-09$ \\
\hline EFFLUEHT 16 & $2.00 E-02$ & $3.908-01$ & $5.60 E+01$ & 7.70E-69 & $9.89[E-09$ \\
\hline EFFLUENT 17 & $2.03 E-02$ & 4. $11 \mathrm{IE}-01$ & $6.20 E \div 01$ & 1. 95E-39 & $1.04 E-1 \mathrm{j} 3$ \\
\hline EFFLUENT 18 & $1.04 E-02$ & $4.21 E-01$ & $6.50 E+01$ & $7.665-09$ & $1.05 E-68$ \\
\hline EFFLUENT 19 & $1.96 E-02$ & $4.36 E-01$ & 6. 30E+01 & $1.13 E-08$ & $1.10 E-18$ \\
\hline EFFLLEERT 20 & 7. $13 E-03$ & $4.43 E-01$ & $7.20 E+01$ & $5.04 E-09$ & $1.06 E-\hat{v} B$ \\
\hline EFFLUENT 21 & $2.50 E-03$ & 4.45E-01 & $7.60 E+01$ & $3.06 E-10$ & $1.05 E-08$ \\
\hline EFFLUEHT 22 & 3.09:E-03 & 4. 48E-01 & B.00E+01 & $5.68 E-10$ & 1.00E-08 \\
\hline EFFLUEHT 23 & $6.14 E-03$ & 4. $54 E-01$ & $8.40 E+0 !$ & $2.44 E-09$ & $9.53 E-69$ \\
\hline EFFLUENT 24 & $9.31 E-03$ & $4.64 E-01$ & a. $70 E \div 01$ & $1.04 E-08$ & $9.695-i 7$ \\
\hline
\end{tabular}


TABLE B.15c. Aluminum (A)) Leach Fractions from Flow Through Test Grout/Sediment with Groundwater (DFCOLCl)

\begin{tabular}{|c|c|c|c|c|c|}
\hline SAMPLE & $A n / A O$ & Cus. An/ÂO & DAYS & Dinc. & $D$ cul. \\
\hline EFFLUEHT 1 & $2.53 E-04$ & $2.53 E-04$ & $3.00 E+00$ & $6.77 E-14$ & $6.775-14$ \\
\hline EFFLUEKT 2 & $3.81 E-04$ & $6.34 E-04$ & $6.00 E+00$ & $8.91 E-13$ & 2.12E-13 \\
\hline EFFLLEKT 3 & b. $57 E-04$ & $1.29 E-03$ & $9.00 E+00$ & $4.51 E-12$ & 5. $86 E-13$ \\
\hline EFFLUENT 4 & $7.79 E-04$ & $2.07 E-03$ & $1.30 E+01$ & $5.23 E-12$ & 1.045-12 \\
\hline EFFLUENT 5 & 1.72E-04 & $2.26 E-03$ & $1.70 E+01$ & $4.37 E-13$ & $9.52 E-13$ \\
\hline EFFLUENT 6 & $2.83 E-04$ & $2.54 E-03$ & 2.00E+01 & $2.08 E-12$ & $1.02 E-12$ \\
\hline EFF!UENT 7 & $4.17 E-04$ & $2.765-03$ & $2.30 E+01$ & $5.25 \mathrm{E}-12$ & $1.21 E-12$ \\
\hline EFFLUENT 9 & $6.57 E-04$ & $3.62 E-03$ & $2.7 \mathrm{DE}+01$ & 8. $52 E-12$ & 1.53E-12 \\
\hline EFFLUENT 9 & $4.565-014$ & $4.67 \mathrm{E}-0 \mathrm{~J}$ & $3.00 E+01$ & 8.3AE-12 & 1.75E-12 \\
\hline EFFLUENT 10 & $6.94 E-0.4$ & $4.17 E-03$ & $3.40 E+01$ & $1.22 E-11$ & $2.12 E-12$ \\
\hline EFFLUENT 11 & $5.43 E-04$ & $5.31 E-0 j$ & $3.70 E+01$ & $1.47 E-11$ & $2.41 E-12$ \\
\hline EFFLUENT 12 & $6.28 E-04$ & $5.94 E-03$ & 4. $10 E+0 \mathrm{j}$ & $1.22 E-11$ & $2.72 E-12$ \\
\hline EFFLUENT I3 & $4.35 E-04$ & $6.38 E-03$ & $4.40 E+01$ & $1.13 E-11$ & $2.92 \mathrm{E}-12$ \\
\hline EFFLUENT 14 & 5.1GE-04 & $6.88 E-03$ & $4.70 E+01$ & $6.11 E-12$ & $3.06 E-12$ \\
\hline EFFLUENT 15 & $4.36 E-64$ & $7.32 E-\hat{U} J$ & $5.20 E+01$ & $1.365-1 !$ & $3.26 \mathrm{E}-12$ \\
\hline EFFLUEKT 16 & $6.20 E-04$ & $7.74 E-03$ & $5.80 E+01$ & $7.4 J E-12$ & $3.44 E-12$ \\
\hline SFFLUEHT 17 & 7.14E-04 & $8.66 \mathrm{E}-03$ & $0.20 E+01$ & $2.42 E-11$ & $3.82 E-12$ \\
\hline EFFLUEMT :B & $3.60 E-04$ & $9.02 E-03$ & $6.50 E+01$ & 1.16E-11 & $3.965 E-12$ \\
\hline EFFLUENT 19 & $4.625-04$ & $9.48 E-0 j$ & $6.90 E+01$ & $1.13 E-11$ & $4.12 E-12$ \\
\hline EFFLUENT 20 & $2.25 E-04$ & $9.70 E-03$ & $7.20 E+01$ & $5.01 E-12$ & $4.14 E-12$ \\
\hline EFFLUEKT 21 & $6.72 E-05$ & $9.77 E-03$ & $7.60 E \div 01$ & $2.64 E-13$ & $3.975-12$ \\
\hline EFFLUEKT 22 & $6.04 E-05$ & $8.83 E-03$ & 8.00E+0i & $2.25 E-13$ & $3.82 E-12$ \\
\hline EFFLUEMT 23 & 1. $0 B E-04$ & $9.94 E-63$ & B. 40E+01! & $7.57 E-13$ & $3.72 E-12$ \\
\hline EFFLLERT 24 & $1.91 E-04$ & $1.01 E-02$ & 3. $70 E+01$ & $4.39 E-12$ & 3.73E -12 \\
\hline
\end{tabular}


TABLE B.15d. Silica (Si) Leach Fractions from Flow Through Test Grout/Sediment with Groundwater (DFCOLC1)

\begin{tabular}{|c|c|c|c|c|c|}
\hline SAKPLE & An/ $\mathrm{fo}$ & CUA. An/Áo & DAYS & Dinc. & $D$ cun. \\
\hline EFFLUENT I & $3.155-03$ & $3.15 E-03$ & $3.00 E+100$ & 1.04E-11 & $1.04 E-11$ \\
\hline EFFLUENT 2 & $3.88 E-03$ & $7.03 E-03$ & $6.00 E+00$ & $9.28 E-11$ & 2.61E-11 \\
\hline EFFLUEMT 3 & J.8IE-03 & $1.08 \mathrm{E}-02$ & $9.00 E+00$ & $1.52 E-10$ & 4. $13 E-11$ \\
\hline EFFLUEKT \$ & $2.72 E-03$ & $1.36 E-02$ & $1.30 E+01$ & $6.37 E-11$ & $4.46 E-11$ \\
\hline EFFLUENT 5 & $7.84 E-04$ & $1.43 \mathrm{E}-02$ & $1.70 E+01$ & $7.25 E-12$ & 3. $03 E-11$ \\
\hline EFFLUEHT 'ं & 1.0BE-03 & 1.54E-ט2 & 2. $00 E+01$ & $3.02 E-11$ & $3.76 E-11$ \\
\hline EFFLUEKT 7 & $1.17 E-03$ & 1. G6E-02 & $2.30 \mathrm{E}+01$ & 9.14E-11 & $3.79 E-1:$ \\
\hline EFFLLEKT 8 & 1. $55 E-03$ & $1.81 E-02$ & $2.705+01$ & $4.74 E-11$ & $3.66 E-11$ \\
\hline EFFLUENT 7 & $9.36 E-04$ & $1.91 E-02$ & 3. OUE +OOI & $3.8 ; E-11$ & j.8́cE-1i \\
\hline EFFLUENT 10 & $\therefore .210 E-03$ & $2.04 E-02$ & $3.40 E+01$ & $4 . \hat{v Z E}-11$ & J.DTE-11 \\
\hline EEFLUENT 11 & 8. T9E-04 & $2.13 E-02$ & 3. $70 E+01$ & $3.36 \bar{E}-11$ & $3.87 E-11$ \\
\hline EFFLUENT 12 & $9.80 E-04$ & $2.22 E-02$ & $4.10 E+01$ & $2.965-1 !$ & $3.32 E-11$ \\
\hline EFFLUENT 13 & $6.57 E-04$ & $2.29 E-02$ & $4.40 E+01$ & $2.58 E-\$ 1$ & $3.77 \mathrm{E}-11$ \\
\hline EFFLUENT 14 & $8.20 E-04$ & $2.37 E-02$ & $4.90 E+01$ & $1.5 B E-\$ 1$ & $3.63 \mathrm{E}-! 1$ \\
\hline EFFLUENT IJ & $5.995-04$ & $2.43 E-02$ & $5.20 E \div 01$ & $2.55 E-11$ & 3. OOE-11 \\
\hline EFFLUEHT 16 & $1.02 E-0 \hat{J}$ & $2.5 \bar{J} E-\hat{v} 2$ & $5.80 E+01$ & 2. OUE-!! & 3.50E-!: \\
\hline EFFLUEHT 17 & $4.71 E-64$ & $2.5 \mathrm{AE}-02$ & $6.20 E+01$ & i. $655-11$ & J.4GE-ij \\
\hline EFFLUENT 18 & 1.17E-14 & $2.59 E-02$ & $6.50 E+01$ & $1.2 \mathrm{JE}-12$ & $3.27 E-11$ \\
\hline EFFLUEHT IQ & 2. $79 E-\mathrm{i} 14$ & $2.62 E-\hat{2}$ & $6.90 E+01$ & $4.115-12$ & J.15E-1! \\
\hline EFFLUENT 20 & $1.275-04$ & $2.63 E-\hat{0} 2$ & $7.205+01$ & $1.59 E-12$ & 3. $65 E-11$ \\
\hline EFLUENT 21 & $5.79 E-05$ & $2.64 E-02$ & $7.60 E+01$ & $1.96 \mathrm{E}-1 \mathrm{j}$ & 2.70E-1! \\
\hline EFFLUEHT 22 & ¿.10E-04 & $2.65 E-02$ & 8. $00 E \div 01$ & $3.49 E-13$ & $2.79 E-1 j$ \\
\hline EFFLUEHT 23 & $3.42 E-04$ & $2.608 E-02$ & E.40E+01 & $7.575-12$ & $2.71 \mathrm{E}-1 \mathrm{j}$ \\
\hline EFFLUERT 24 & $5.28 E-04$ & $2.74 E-02$ & $8.7 C E+01$ & 3. J5E-11 & $2.73 E-11$ \\
\hline
\end{tabular}


TABLE B.15e. Boron (as H3B03) Leach Fractions from Flow Through Test Grout/Sediment with Groundwater (DFCOLC1)

\begin{tabular}{|c|c|c|c|c|c|}
\hline SAAMPLE & $\mathrm{An} / \mathrm{AO}$ & CUA. An/AO & DAYS & 0 ine. & I cus. \\
\hline EFFLUENT 1 & $4.39 E-03$ & 4. $39 E-03$ & $3.00 E+00$ & $2.03 E-11$ & 2.03E-11 \\
\hline EFFLUENT 2 & $3.47 E-03$ & $7.86 E-03$ & $6.00 E+00$ & $7 .\{1 E-11$ & $3.26 E-11$ \\
\hline EFFLUEKT 3 & 2.7JE-03 & $1.06 E-02$ & $9.00 E+00$ & $7.7 b E-11$ & $3.94 \varepsilon-11$ \\
\hline EFFLUIENT & $1.67 E-03$ & $1.23 E-02$ & $1.30 E+01$ & $2.41 E-1 !$ & $3.65 E-: 1:$ \\
\hline EFFLUENT 5 & $6.30 E-04$ & $1.29 E-02$ & $1.70 E+01$ & $4.69 E-12$ & $3.09 E-11$ \\
\hline EFFLUEHT $b$ & $9.36 E-04$ & 1. 3BE-02 & $2.00 E+03$ & $2.28 E-11$ & $3.025-11$ \\
\hline EFFLUEHT 7 & 9.17E-04 & $1.47 E-02$ & 2.30E+ +01 & $2.54 E-11$ & $2.99 E-11$ \\
\hline EFFLUENT $B$ & 1.01E-Ǵ3 & $1.57 E-02$ & $2.70 E+01$ & $2.02 E-11$ & $2.91 E-11$ \\
\hline EFFLUEEHT ? & $6.76 E-04$ & $1.64 E-02$ & $3.00 E+01$ & $1.83 \mathrm{E}-11$ & $2.35 E-11$ \\
\hline EFFLUENT 10 & a. 47E-04 & 1.7JE-02 & $3.40 E+0 i$ & 1. OLE-11 & 2.7SE-1i \\
\hline EFFLUENT II & $6.22 E-04$ & $1.77 E-02$ & $3.70 E+01$ & $1.93 E-11$ & $2.74 E-1 !$ \\
\hline EFFLUENT 12 & $7.63 E-04$ & $1.87 E-02$ & $4.10 E+01$ & $1.795-11$ & $2.69 E-11$ \\
\hline EFFLUEHT 13 & 5. $90 E-04$ & $1.92 E-02$ & $4.40 E+0 i$ & 2. $66 E-1 !$ & $2.665-11$ \\
\hline EFFLUEHT 14 . & $7.20 E-04$ & $2.00 E-02$ & 4. $90 E+01$ & $1.22 E-11$ & $2.57 \mathrm{E}-1 \mathrm{i}$ \\
\hline EFFLUEHT IS & $5.58 E-04$ & $2.05 E-02$ & $5.20 E+01$ & $2.21 E-11$ & $2.56 E-11$ \\
\hline EFFLUENT 16 & 9. $6 B E-04$ & $2.15 E-02$ & $5.50 E+01$ & $1.81 E-1 !$ & 2.52E-!! \\
\hline EFFLUEHT 17 & $5.02 E-04$ & $2.20 E-02$ & $6.20 E+01$ & $1.13 E-11$ & $2.47 E-11$ \\
\hline EFFLUEHT 18 & 3. $6 I E-04$ & $2.24 E-02$ & $6.50 E+01$ & $1.18 E-11$ & $2.43 E-11$ \\
\hline EFFLUENT 19 & $7.23 E-04$ & $2.31 E-02$ & $6.30 E+01$ & $2.77 E-11$ & $2.44 E-11$ \\
\hline EFFIUENT 20 & $4.04 E-04$ & 2. 3SE-02 & $7.20 E+01$ & $1.62 E-1 !$ & $2.42 E-11$ \\
\hline EFFLUEHT 21 & $1.87 E-0.14$ & $2.37 E-02$ & $7.60 E+01$ & $2.04 E-12$ & $2.33 E-11$ \\
\hline EFFLUERT 22 & 3.20E-04 & $2.40 E-02$ & $8.005+01$ & $6.32 E-12$ & $2.23 E-11$ \\
\hline EFFLUENT 23 & $6.84 E-04$ & $2.47 E-02$ & $8.40 E+01$ & $3.04 E-11$ & $2.25 E-11$ \\
\hline EFFLUENT 24 & $6.53 E-04$ & $2.54 E-02$ & $8.70 E+01$ & 5. $T E E-i j$ & $2.34 E-11$ \\
\hline
\end{tabular}


TABLE B.15f. Fluoride (F) Leach Fractions from Flow Through Test Grout/Sediment with Groundwater (DFCOLC1A)

\begin{tabular}{|c|c|c|c|c|c|}
\hline SANPLE & An/Ao & Cun. An/Ao & DAYS & 0 inc. & 0 cus. \\
\hline EFFLUEHT 1 & 1. 65E-03 & 1. 05E-03 & $3.00 E+00$ & $1.17 E-12$ & $1.17 E-12$ \\
\hline EFFLUENT 2 & $8.04 E-04$ & $1.865-03$ & $6.00 E+00$ & $3.98 E-12$ & $1.82 E-12$ \\
\hline EFFLUENT 3 & $8.07 E-04$ & $2.66 E-03$ & $9.00 E+00$ & $6.80 E-12$ & 2.49E-12 \\
\hline EFFLUENT 4 & $6.69 E-04$ & $3.33 E-03$ & $1.30 E+01$ & $3.365-12$ & 2.70E-12 \\
\hline EFFLUENT 5 & $2.19 E-04$ & $3.55 E-03$ & $1.70 E+01$ & $5.65 E-13$ & 2. 3EE -12 \\
\hline EFFLUERT 6 & $3.20 E-04$ & $3.87 E-03$ & 2.00E+01 & 2. $66 E-12$ & $2.37 E-12$ \\
\hline EFF!UENT 7 & $3.47 E-04$ & $4.22 E-03$ & $2.30 E+01$ & $3.64 E-12$ & $2.45 E-12$ \\
\hline EFFLUEKT \& & $4.93 E-04$ & 4.71E-03 & $2.70 E+01$ & $4.80 E-12$ & $2.60 E-12$ \\
\hline EFFLUIENT 9 & $3.54 E-04$ & $5.07 E-03$ & 3. ONEE+01 & 5. $025-12$ & 2:31E-12 \\
\hline EFFIUEHT 10 & $4.34 E-04$ & $5.50 E-03$ & 3. 40E+01 & $4.77 E-12$ & $2.625-12$ \\
\hline EFFLUENT is & $3.505-04$ & $5.85\{-03$ & $3.70 E+01$ & 6. $11 E-12$ & $2.935-12$ \\
\hline EFFLUENT 12 & $4.30 E-044$ & $6.28 E-03$ & $4.10 E+01$ & $5.69 E-12$ & $3.04 E-12$ \\
\hline EFFLUENT 13 & $3.26 E-04$ & $6.6: E-03$ & $4.40 E+01$ & $6.34 E-12$ & $3.14 E-12$ \\
\hline EFFLLEHT 14 & $4.345-04$ & $1.04 E-03$ & $4.90 E+01$ & $4.44 E-12$ & $3.20 \mathrm{E}-12$ \\
\hline EFFLUEHT 15 & $3.45 E-014$ & $7.385-03$ & $5.20 E+01$ & $8.44 E-12$ & $3.32 \mathrm{E}-12$ \\
\hline EFFLUERT 16 & $5.70 E-04$ & $7.95 E-03$ & $5.80 E+01$ & $6.27 E-12$ & $3.45 E-12$ \\
\hline EFFLUERT 17 & $4.42 E-014$ & $3.40 E-0]$ & $6.20 E+01$ & $9.27 E-1 E$ & $3.60 E-12$ \\
\hline EFFLUEHT IB & $4.50 E-04$ & 8. $85 E-03$ & $6.50 E+01$ & $1.8 i E-11$ & $3.21 E-12$ \\
\hline EFFIUENT 19 & $7.07 E-04$ & $9.55 E-03$ & b. $90 \mathrm{E}+01$. & $2.65 E-11$ & $4.19 E-12$ \\
\hline EFFLUEHT 20 & $4,18 E-04$ & $9.97 E-03$ & $7.20 E+01$ & i.73E-11 & $4.37 E-12$ \\
\hline EFFLUENT Z1 & $1.70 E-04$ & $1.01 E-02$ & $T_{.00} 00+01$ & $1.70 E-12$ & 4. $28 E-12$ \\
\hline EFFLUENT 22 & $2.35 E-04$ & 1.04E-02 & $8.00 E+01$ & $3.40 E-12$ & $4.26 E-12$ \\
\hline EFFLUEHT 23 & $3.34 E-04$ & $\$ .08 E-02$ & $B .40 E+01$ & $1.01 E-11$ & $4.375-12$ \\
\hline EFFLUEKT 24 & $4.14 E-04$ & $1.12 E-02$ & $8.70 E+01$ & 2. $06 E-11$ & $4.55 E-12$ \\
\hline
\end{tabular}


TABLE B.15g. Nitrate (NO3) Leach Fractions from Flow Through Test Grout/Sediment with Groundwater (DFCOLC1A)

\begin{tabular}{|c|c|c|c|c|c|}
\hline SAKPLE & An/AD & Cun. An/ĥo & BAYS & $2 \mathrm{inc}$ & D clis. \\
\hline EFFLUEHT ! & $6.53 E-02$ & 6. $53 \mathrm{E}-02$ & $3.00 E+00$ & 4. $50 E-09$ & $4.50 E-077$ \\
\hline EFFLUEEHT 2 & $2.83 E-02$ & $9.36 E-02$ & b. $00 E+00$ & $4.32 E-199$ & $4.62 E-0.9$ \\
\hline EFFLUENT 3 & $2.23 E-02$ & 1.:BEE-01 & $9.00 E+00$ & 5. $18 E-09$ & $4.7 Z E-09$ \\
\hline EFFLLUENT 4 & $1.41 E-02$ & 1. उOE-01 & $1.30 E+01$ & :.71E-09 & $4.11 E-09$ \\
\hline EFFLUEKT 5 & $6.32 E-03$ & $1.3 \mathrm{bE}-\hat{\mathrm{v}} \mathrm{l}$ & $1.70 E+01$ & $4.72 E-10$ & $3.46 E-09$ \\
\hline EFFLUENT 6 & $3.355-03$ & $1.45 E-6 !$ & 2. $00 E+01$ & 1.81E-69 & j.JIE-0Q \\
\hline EFELUENT 7 & $1.21 E-02$ & I.57E-0! & 2. $30 E+01$ & $4,40 E-69$ & 3.3EE-iG \\
\hline EFFLUENT a & $7.51 E-03$ & $1.64 E-01$ & $2.70 E+01$ & 1.11E-09 & $3.16 E-09$ \\
\hline EFFLUEHT ? & a. $16 E-0]$ & $1.72 E-01$ & $3.60 E+01$ & $2.61 E-69$ & $3.13 E-67$ \\
\hline EFFLLEHT 10 & $7.59 E-03$ & 1. 80E-0i & $3.4 \hat{v E}+0$ & $1.46 E-195$ & S. GiE- \\
\hline EFFLUENT 11 & $5.90 E-03$ & $1.36 \mathrm{E}-\hat{\mathrm{v}} \mid$ & $3.76 E+61$ & $1.74 E-69$ & $2.75 E-97$ \\
\hline EFFLUENT 12 & $6.81 E-03$ & 1. $33 E-01$ & $4 . i 0 E+0 i$ & $1 .\{3 E-09$ & $2.56 E-69$ \\
\hline EFFLUENT 13 & 6. B3E-03 & 1.79E-0! & 4. $30 E+0 !$ & $2.79 E-69$ & $2.86 E-69$ \\
\hline EFFLUEHT I4 & $7.81 E-03$ & 2. $07 E-01$ & $4.705+01$ & $1.43 E-09$ & $3.00 E-019$ \\
\hline EFFLUENT IS & $5.025-03$ & $2.13 E-61$ & $5.20 E+0:$ & $2.40 E-3 i$ & $2.83 E-97$ \\
\hline EFFLUEHT 16 & 8.33E- 03 & $2.2 \mathrm{EE}-\mathrm{D} !$ & $5.80 E+01$ & 1. 34E-0ิ9? & $2.79 E-19$ \\
\hline EFFLUEhT i7 & 4. $1 B E-03$ & $2.26 E-01$ & $0.20 E+01$ & $3.27 E-10$ & Z. $. \bar{B} \mathrm{BE}-69$ \\
\hline EFFLUENT I8 & $3.76 E-03$ & $2.29 E-61$ & 6.J0E+01. & ¿.26E-09 & $2.73 E-69$ \\
\hline EFFLUEHT IS & b. $45 E-03$ & $2.36 E-01$ & $6.90 E+01$ & $2.21 E-77$ & $2.55 E-09$ \\
\hline EFFLUENT 20 & $3.76 E-03$ & $2.40 E-01$ & $7.20 E+0 \mathrm{i}$ & 1. $40 E-69$ & $2.935-\hat{5} 5$ \\
\hline EFFLUENT 21 & $2.34 E-03$ & $2.42 E-01$ & $7.60 E+01$ & J. $2 ! E-! 0$ & $2.68 E-59$ \\
\hline EFFLUERT 22 & $3.50 E-03$ & $2.45 E-\hat{01}$ & 8. $00 E+01$ & $7.55 E-10$ & 2.65E-0? \\
\hline EFFLUEKT 23 & $5.00 E-03$ & $2.51 E-01$ & $8.40 E+0]$ & $2.03 E-09$ & $2.53 E-6 ?$ \\
\hline EFF!UEKT 24 & $7.695-63$ & $2.575-01$ & $0.70 E+01$ & $\bar{T} . \mathrm{iONE}-\hat{\mathrm{v}} \mathrm{q}$ & $2.06 E-1) 9$ \\
\hline
\end{tabular}


TABLE B.15h.

SANPLE
EFFLUENT !
EFFLUENT $?$
EFFLUENT 3
EFFLUENT 4
EFFLUENT 5
EFFLUENT 6
EFFLUENT 7
EFFLUENT 8
EFFLUENT 9
EFFLUENT 10
EFFLUENT 11
EFFLUENT 12
EFFLUENT 13
EFFLUENT 14
EFFLUENT 15
EFFLUENT 16
EFFLUENT 17
EFFUUENT 16
EFFLUENT 19
EFFLUENT 20
EFFLUENT 21
EFFLUENT 22
EFFLUENT 23
EFFLUENT 24

Sulfate (SO4) Leach Fractions from Flow Through Test Grout/Sediment with Groundwater (DFCOLC1A)

\begin{tabular}{|c|c|c|c|c|}
\hline$A \cap / A O$ & Cun. An/AO & DAYS & $D$ ine. & $D$ cua. \\
\hline $1.345-01$ & $1.34 E-01$ & $3.00 E+00$ & 1.39E-08 & 1. $89 E-08$ \\
\hline $3.00 \varepsilon-02$ & $1.64 E-01$ & $6.00 E+00$ & $5.52 E-09$ & $1.42 E-08$ \\
\hline $2.57 E-02$ & $1.90 E-01$ & $9.00 E+00$ & $6.87 E-\hat{v} 9$ & $1.26 E-08$ \\
\hline 1.18E-02 & $2.01 E-01$ & $1.30 E+01$ & $1.19 E-09$ & $9.87 E-09$ \\
\hline $5.99 E-03$ & 2.07E-01 & $1.70 E+01$ & $4.24 E-10$ & 8. $655-09$ \\
\hline 1. $24 E-02$ & $2.20 E-01$ & $2.00 E+01$ & $3.97 \mathrm{E}-09$ & $8.09 E-09$ \\
\hline 9.11E-03 & $2.29 E-01$ & $2.30 E+01$ & $2.51 E-09$ & $7.7 \pm E-11 ?$ \\
\hline $9.57 E-03$ & $2.38 E-01$ & $2.70 E+01$ & $1.31 E-\hat{v} 9$ & $7.54 E-09$ \\
\hline $1.37 E-02$ & 2.52E-01 & $3.00 E+01$ & $7.56 E-09$ & $-7.1 B E E-69$ \\
\hline $4.44 E-03$ & $2.57 E-01$ & $3.40 E+01$ & $4.3 B E-16$ & $6.81 E-97$ \\
\hline $2.62 \mathrm{E}-0 \mathrm{~J}$ & $2.59 E-01$ & $3.70 E+01$ & $3 .+1 E-10$ & $6.25 E-69$ \\
\hline $1.28 E-03$ & $2.60 E-01$ & $4 . ; 0 E \div 01$ & $5.09 E-11$ & $5.65 E-39$ \\
\hline 3. BDE-03 & $2.64 E-01$ & $4.40 E+01$ & $3.65 E-10$ & $5.26 E-69$ \\
\hline 6. $97 E-03$ & $2.71 E-01$ & $4.90 E+01$ & 1.14E-09 & 5.15E-199 \\
\hline $2.58 E-03$ & $2.74 E-01$ & $5.20 E+(01$ & $4.71 E-1 a$ & $4.85 E-69$ \\
\hline $9.13 E-03$ & $2.83 E-01$ & $5.30 E+01\}$ & $1.61 E-09$ & $4.70 \mathrm{E}-\hat{\mathrm{v}} \mathrm{7}$ \\
\hline $0.00 E+100$ & $2.65 E-0 i$ & $6.30 E+01$ & $0.60 E+00$ & $4.39 E-197$ \\
\hline $0.00 E+60$ & 2. $.83 E-0 !$ & $6.50 E+01$ & $0.00 E+00$ & $4.195-09$ \\
\hline $0.00 E+00$ & $2.33 E-01$ & $6.90 E \div 01$ & $0.00 E+00$ & $3.955-69$ \\
\hline $5.49 E-33$ & 2. $58 E-\hat{0} 1$ & $3.20 E+01$ & 2. $99 E-09$ & $4.10 E-09$ \\
\hline $1.07 E-03$ & $2.87 E-01$ & $7.60 E+01$ & 6. $70 E-1 !$ & $3.88 E-09$ \\
\hline 6. $89 E-03$ & $2.96 E-01$ & $8.00 E+01$ & $2.85 E-09$ & $3.77 E-69$ \\
\hline $1.86 E-02$ & $3.15 E-01$ & B. $40 E+01$ & $2.255-68$ & $4.36 E-19$ \\
\hline $6.99 E-03$ & $3.22 E-01$ & $8.70 E+01$ & $5.67 E-69$ & 4.2JE-199 \\
\hline
\end{tabular}


TABLE B.15i. Cesium-137 (CS-137) Leach Fractions from Flow Through Test, Grout/Sediment with Groundwater (C1)

\begin{tabular}{|c|c|c|c|c|c|c|c|}
\hline SAMPLE & PDRE VOLUME & $u C i$ & TIME DAYS & An/ÂO & CUM An/ÂO & DINC CK2/SEC & DCUM CK2/SEE \\
\hline CRW C1-1 & $1.78 E+00$ & $4.35 E-11$ & $3.00 E+100$ & $1.06 E-11$ & 1. 66 E-11 & 1.18E-20 & 1.18E-28 \\
\hline CRM C1-2 & $3.74 E+00$ & $2.57 \mathrm{E}-10$ & $6.00 E+00$ & $6.27 E-11$ & $7.32 E-11$ & $4.80 E-26$ & 2. $32 E-27$ \\
\hline CRM CI-3 & b. $52 \mathrm{E}+00$ & $5.61 E-11$ & $9.00 E+00$ & $1.37 \mathrm{E}-11$ & $8.69 E-11$ & $2.23 E-27$ & 2. $34 E-27$ \\
\hline CAN CI-4 & $9.80 E+00$ & $5.85 E-11$ & 1. JGE+Ui & $3.42 E-11$ & 1.01E-10 & $1.66 E-27$ & $2.46 E-27$ \\
\hline CRE C1-5 & $1.08 E+01$ & $2.05 E-11$ & $1.70 E+01$ & $5.01 E-12$ & $1.06 E-10$ & $2.30 E-2 \hat{0}$ & $2.09 E-27$ \\
\hline CRH C1-6 & $1.20 E+01$ & $1.43 E-10$ & $2.00 E+01$ & $3.49 E-11$ & $1.41 E-10$ & $1.69 E-26$ & 5.13E-27 \\
\hline CRN C1-7 & $1.35 E+01$ & $3.49 E-11$ & $2.30 E+01$ & $8.51 E-12$ & $1.50 E-10$ & $8.86 E-28$ & $3.06 E-27$ \\
\hline CRN C1-8 & $1.57 E+01$ & $2.61 E-10$ & $2.70 E+01$ & 6. $36 E-11$ & $2.13 E-10$ & $3,3 i E-26$ & $5.30 \mathrm{E}-2 \mathrm{~J}$ \\
\hline CRU C1-9 & $1.73 E+01$ & $3.89 E-11$ & $3.00 E+03$ & $9.42 E-12$ & $2.23 E-10$ & $1.25 E-27$ & $5.20 E-27$ \\
\hline CFI C1-10 & $1.98 E+01$ & $5.07 E-11$ & $3.40 E+01$ & $1.24 E-11$ & $2.355 E-10$ & $1.25 E-27$ & $5.12 \mathrm{E}-2$ \\
\hline CRA CI-11 & $2.1 B E+01$ & $4.22 E-11$ & $3.70 E+01$ & $1.03 E-11$ & $2.45 E-10$ & 1.46E-27 & 5. $12 \mathrm{E}-27$ \\
\hline CRA C1-12 & $2.43 E+01$ & $5.18 E-11$ & $4,10 E+01$ & $1.26 E-11$ & $2.58 E-10$ & 1. $30 E-27$ & 5. 11 E-27 \\
\hline CRA C1-13 & $2.62 E+01$ & $6.94 E-11$ & $4.40 E+01$ & 1.69E-1! & $2.75 E-10$ & $3.97 E-27$ & $5.41 E-27$ \\
\hline CRIN C1-14 & $2.87 E+01$ & $7.74 E-11$ & 4.9GE+OC1 & 1. $89 E-11$ & $2.74 E-10$ & $2 . j 5 E-27$ & $5.54 E-27$ \\
\hline CRㅂ $61-15$ & $3.07 E+01$ & $3.55 E-11$ & $5.20 E+01$ & $8.65 E-12$ & $3.02 E-10$ & $1.15 E-27$ & $5.54 E-27$ \\
\hline Chy $C 1-16$ & $3.37 E+01$ & $1.16 E-10$ & $5.80 E+01$ & $2.82 \mathrm{E}-11$ & $3.315-10$ & $3.76 E-27$ & $5,93 E-27$ \\
\hline CKA $51-17$ & $3.80 E+01$ & i.05E-10 & $6.20 E+01$. & $2.56 E-11$ & $3.56 E-10$ & $7.21 E-27$ & $6.45 E-27$ \\
\hline CAH C1-18 & 4.11E+01 & $6.20 E-11$ & $6.50 E+01$ & $1.51 E-11$ & $3.71 E-j 0$ & $3.17 E-2 i$ & $6.68 E-27$ \\
\hline CRH Cl-19 & $4.54 E+01$ & $9.61 E-11$ & $6.90 E+01$ & $2.34 E-11$ & $3.95 E-10$ & $4.495-27$ & $7.11 E-27$ \\
\hline$C F H C I-20$ & $4.78 E+01$ & 4. $42 E-11$ & $7.20 E+0 \mathrm{i}$ & $1.08 E-j 1$ & $4.06 E-10$ & $1.61 E-27$ & $7.19 E-27$ \\
\hline ER $[1-2]$ & $4.26 E 5+01$ & $6,29 E-12$ & $7.605+01$ & $1.5 \mathrm{JE}-12$ & $4.07 E-10$ & $1.93 E-29$ & $6.87 E-27$ \\
\hline Chin C1-22 & $4.96 E+001$ & 2.61E-11 & 2.16E+01 & $6.35 E-12$ & $4.13 E-10$ & $2.71 E-28$ & $6.65 E-27$ \\
\hline CRM $[1-23$ & 5. $10 E+01$ & $2.88 E-11$ & $8.40 E+01$ & $7.02 E-12$ & $4.20 E-10$ & $7.58 E-23$ & $6.63 E-27$ \\
\hline CEA CI-24 & 5.30E+01 & $1.06 E-11$ & $8.70 E+01$ & 2.5BE-12 & 4. $235-10$ & $3.14 E-29$ & $6.46 E-27$ \\
\hline ERK $[1-25$ & J.56E+01 & $2.15 E-11$ & $9.10 E+01$ & $5.23 E-12$ & $4.29 E-10$ & $2.24 E-2 E$ & $6.35 E-27$ \\
\hline
\end{tabular}


TABLE B.15i. Cesium-1379 (CS-137) Leach Fractions from Flow Through Test, Grout/Sediment with Groundwater (C2)

\begin{tabular}{|c|c|c|c|c|c|c|c|}
\hline SAMPLE & PORE YDLUME & $\mathbf{u L i}$ & TIME DAYS & $\mathrm{An} / \mathrm{AO}$ & CUH An/AO & DINC CN2/SEC & DCUA CK2/SEE \\
\hline CRM $[2-1$ & $1.64 E+00$ & 1. $84 E-10$ & $3.00 E+00$ & $4.38 E-11$ & 4.3BE-11 & $2.04 E-27$ & $3.04 E-27$ \\
\hline CRN C2-2 & 3. $3 \theta E+00$ & $3.26 \mathrm{E}-11$ & 6.00E+00 & $7.77 E-12$ & $5.16 E-11$ & $7.46 E-28$ & i.41E-27 \\
\hline CRM C2-3 & $5.03 E+00$ & $2.10 E-11$ & $9.00 E+00$ & $5.00 E-12$ & $5.66 E-11$ & $3.08 E-28$ & $1.13 E-27$ \\
\hline$C R N C_{2}-4$ & $9.30 E+00$ & $4.98 E-11$ & $1.30 E+01$ & $1.19 E-11$ & $6.85 E-11$ & $1.16 E-27$ & $1.15 E-27$ \\
\hline CRU C2-5 & $1.06 E+01$ & $2.64 E-11$ & $1.70 E+01$ & 6.30E-12 & $7.48 E-11$ & $3.68 E-28$ & $1.05 E-27$ \\
\hline CRN C2-6 & $1.22 E+01$ & $3.37 E-11$ & $2.00 E+0 !$ & $6.03 E-12$ & B.2BE-11 & $9.01 E-2 B$ & $1.09 E-27$ \\
\hline CRA $02-7$ & $1.41 E+01$ & 5. OSE-11 & $2.30 E+01$ & $1.21 E-11$ & $9.49 E-11$ & $1.82 E-27$ & $1.24 E-27$ \\
\hline CRH C2-5 & $1.79 E+01$ & $3.82 \mathrm{E}-11$ & $2.70 E+01$ & $9.12 E-12$ & $1.04 E-10$ & $6.86 E-28$ & $1.27 E-27$ \\
\hline CAH C2-9 & $2.03 E+0 i$ & $1.28 E-11$ & $3.00 E+01$ & 3. $045-12$ & $1.07 E-10$ & $1.29 E-28$ & $1.215-27$ \\
\hline$C F N=2-10$ & $2.36 E+j 1$ & $1.79 E-11$ & $3.40 E+01$ & $4.27 E-12$ & L.ije-io & 3. 50 E- 20 & $\{.16 E-2 i$ \\
\hline $\operatorname{CN}[2-11$ & $2.62 E+01$ & 3.20E-11 & $3.70 E+01$ & $1.97 E-11$ & $1.31 E-10$ & $5.44 E-27$ & $1 .+8 E-27$ \\
\hline CRH C2̂-12 & $2.94 E+101$ & $2.79 E-10$ & $4.10 E+01$ & $6.65 E-11$ & 1.98E-10 & $3.65 E-26$ & $3.03 E-27$ \\
\hline Sa $[2-13$ & $3.19 E+01$ & $4.59 E-11$ & $4,40 E+01$ & $1.09 E-11$ & 2. $09 E-10$ & $1.67 E-27$ & $3.14 E-27$ \\
\hline
\end{tabular}

TABLE B.15j. Strontium-85 (SR-85) Leach Fractions from Flow Through Test, Grout/Sediment with Groundwater (C2)

\begin{tabular}{|c|c|c|c|c|c|c|c|}
\hline SAMPLE & PORE VOLUME & $\mathrm{uCi}$ & TIKE OAYS & An/AO & CUH An $/ A D$ & DINC CX2/SEC & DCUN CH2/SEE \\
\hline $\begin{array}{l}\text { ind } C_{2}-1 \\
\text { CRA } C 2-2\end{array}$ & $\begin{array}{l}1.64 E+00 \\
3.36 E+00\end{array}$ & $\begin{array}{l}4.14 E-11 \\
1.33 E-10\end{array}$ & $\begin{array}{l}3.00 E+00 \\
3.00 E+00\end{array}$ & $\begin{array}{l}5.21 E-10 \\
1.54 E-09\end{array}$ & $\begin{array}{l}5.21 E-10 \\
2.07 E-69\end{array}$ & $\begin{array}{l}5.74 E-25 \\
2.94 E-23\end{array}$ & $\begin{array}{l}3.67 E-25 \\
2.26 E-24\end{array}$ \\
\hline CFU C2-3 & $5.03 E \div 00$ & $2.26 \mathrm{E}-11$ & $3.00 E+00$ & 2. $35 E-10$ & $2.35 E-69$ & 1. $50 E-24$ & $1.95 E-24$ \\
\hline CAH C2-4 & $9.305+00$ & $5.89 E-11$ & $1.30 E+01$ & $7.41 E-10$ & $3.09 E-09$ & $4.54 E-24$ & $2.34 E-24$ \\
\hline CAn C2-5 & $1.06 E+01$ & $6.37 E-11$ & $1.70 E+01$ & $8.02 E-10$ & $3.39 E-09$ & $5.755-24$ & $2.83 E-24$ \\
\hline CRit C2-6 & $1.22 E+01$ & $7.80 E-11$ & $2.00 E+01$ & $9.83 E-10$ & $4.68 E-99$ & $1.35 E-23$ & $3.78 E-24$ \\
\hline $\mathrm{CAH}[2-7$ & $1.41 E+01$ & $2.84 E-11$ & 2. $30 E+01$ & $3.57 E-10$ & $5.23 E-09$ & $1.5 \mathrm{JDE}-24$ & $3.7 E E-24$ \\
\hline CAK C2-8 & $1.79 E+01$ & $2.36 E-11$ & $2.70 E+01$ & $3.73 E-10$ & $5.61 E-09$ & 1.15E-24 & $3.705-24$ \\
\hline Cris $C_{2}-9$ & $2.03 E+01$ & $3.26 E-11$ & $3.00 E+01$ & $4.11 E-10$ & c. $02 E-09$ & $2.36 E-24$ & $3.83 E-24$ \\
\hline CRH C $2-1 \hat{v}$ & $2.36 E+11$ & $2.23 E-11$ & $3.40 E+01$ & $2.30 E-10$ & $6.30 E-09$ & $6.50 E-25$ & 3. 70E-24 \\
\hline CKN C2-11 & 2.:2E+01 & $8.54 E-11$ & J.70E+01 & $1.08 E-09$ & $7.37 \mathrm{E}-09$ & 1. $62 E-23$ & $4.67 E-24$ \\
\hline CRH $\mathrm{C}_{2}-12$ & 2. $94 E+011$ & 2. $48 E-10$ & $4.10 E+01$ & $3.12 E-69$ & $1.05 E-100$ & $8.05 E-23$ & $8.53 E-24$ \\
\hline CAN $C 2-13$ & $3.19 E+\hat{0} 1$ & 7.23E-11 & $4.40 E+01$ & $9.16 E-10$ & $1.14 E-018$ & $3.17 E-23$ & $9,405-24$ \\
\hline
\end{tabular}


TABLE B.16a. Potassium (K) Leach Fractions from flow Through Test whole Grout with Groundwater (COLTIA-C)

\begin{tabular}{|c|c|c|c|c|c|}
\hline SARPLE & An/AO & Cus. An/Ao & DAYS & Dinc. & I cus. \\
\hline EFFIUEHT 1 & $9.82 E-03$ & 7. $.82 E-03$ & $3.00 E+00$ & $9.53 E-11$ & $9.53 E-1 !$ \\
\hline EFFLUENT 2 & $6.90 E-03$ & $1.67 E-02$ & $1.00 E+01$ & $6.89 E-11$ & 3.29E-11 \\
\hline EFFLUENT 3 & $5.42 E-02$ & $7.10 \mathrm{E}-02$ & $1.40 E \div 01$ & $2.60 E-\hat{v} 6$ & 1.07E-69 \\
\hline EFFLUEKT \& & $0.00 E+00$ & $7.10 E-02$ & $1.70 E+01$ & $0.00 E+00$ & $3.76 E-10$ \\
\hline EFFLUEHT 5 & $2.60 E-02$ & $9.69 E-02$ & $2.10 E+01$ & $9.48 E-09$ & $1.33 E-69$ \\
\hline EFFLUENT 6 & $1.72 E-02$ & $1.14 E-01$ & $2.40 E+01$ & $8.75 E-09$ & $3.61 E-69$ \\
\hline EFFLLUEHT 7 & $4.86 E-03$ & 1.19E-01 & $2.80 E+111$ & $4.54 E-10$ & 1. $50 E-69$ \\
\hline EFFLUENT 8 & $1.64 E-02$ & $1.35 E-01$ & $3.10 E+01$ & 1. $65 E-68$ & $1.75 E-09$ \\
\hline EFFLUENT ? & $9.01 E-03$ & $1.4 A E-01$ & $3.50 E+01$ & 1. $78 E-09$ & $1.77 \mathrm{E}-09$ \\
\hline COLUYH IS & & & & & \\
\hline EFFLUENT 10 & $1.57 E-02$ & $1.60 E-01$ & $4.50 E+61$ & $1.16 E-109$ & 1.69E- \\
\hline EFFLUERT II & $1.26 E-02$ & i. TJE-01 & $5.20 E+01$ & 1. E5E-09 & $1.70 E-69$ \\
\hline EFFLUENT 12 & 1.01E-02. & $1.83 E-01$ & $5.60 \hat{E}+0 !$ & $4.11 E-0 ?$ & 1.7TE-05 \\
\hline EFFLUEHT 13 & $6.765-03$ & $1.90 E-01$ & $5.90 E+01$ & $3.36 E-\hat{0} 9$ & 1.6iE-69 \\
\hline EFFLUENT 14 & $6.18 E-03$ & $1.96 E-01$ & $6.30 E+011$ & $1.73 E-09$ & 1. ลCE-69 \\
\hline EFFLUENT 15 & $2.99 E-03$ & $1.99 E-01$ & $6.60 E+01$ & $7.58 E-10$ & $1.77 E-08$ \\
\hline EFFLUENT 16 & $2.73 E-03$ & $2.01 E-01$ & $7.00 E+01$ & $3.75 E-10$ & $1.72 E-09$ \\
\hline EFFLUENT 17 & $1.24 E-63$ & 2. $\hat{3} \mathrm{JE}-01$ & $7.40 E+01$ & 6. $96 E-11$ & 1. $54 E-09$ \\
\hline EFFLLEHT 18 & $9.99 E-04$ & $2.04 E-01$ & $7.30 E+01$ & $5.62 E-11$ & $1.56 E-09$ \\
\hline EFFLUEKT I9 & $1.93 E-0 \mathrm{~J}$ & $2.06 \mathrm{~b}-01$ & $8.10 E+01$ & $3.865-10$ & !.EEE-09 \\
\hline
\end{tabular}

TABLE B.16b. Sodium (Na) Leach Fractions from Flow Through Test Whole Grout with Groundwater (COLTIA-C)

\begin{tabular}{|c|c|c|c|c|c|}
\hline SHMFLE & AnIAB & Cun. An/Áno & OÂYS & 5 inc. & 0 cul: \\
\hline EFFLUENT 1 & 1. PQE-62 & ¿. $92 E-02$ & S. OAEE+ Ô & $3.50 E-10$ & S. Dof-10 \\
\hline EFFLIENT $?$ & $1.15 E-012$ & $3.08 E-02$ & 1. $60 E+01 !$ & $1.92 \mathrm{E}-1.6$ & $2.20 E-10$ \\
\hline EFFLLENT 3 & 0.2TE-02 & $1.13 \mathrm{E}-01$ & $1.40 E+01$ & $6.64 E-10$ & $2.73 E-69$ \\
\hline EFFLLIENT 4 & $2.54 E-0 j$ & 1.1GE-6i & $1.70 E+01$ & $1.32 E-10$ & $2.35-69$ \\
\hline EFFLUEHT 5 & $4.30 E-62$ & ¿.56E-01 & $3.105+01$ & 2. BE- & $3.46[-69$ \\
\hline EFFLUENT B & 2. $31 \mathrm{i} E-\hat{0} \hat{i}$ & $1.85 E-61$ & $2.40 E+0 !$ & $2.515-63$ & $4.23 E-6 i$ \\
\hline EEELUEHT $?$ & $8.155-03$ & $1.73 E-41$ & $2.80 E+i j$ & $1.25 E-19$ & $3.95=-6 \%$ \\
\hline EFFLIENT 3 & $3.47 E-02$ & $2.1 B E-6 i$ & $3.96+61$ & $2.46=-60$ & $3.29-19$ \\
\hline EFF!LEKT 9 & $1.3 \leq E-\hat{v} 2$ & $2.3|E-\hat{0}|$ & $3.50 E \div 01$ & $4.30 E-j 5$ & $3.67 E-07$ \\
\hline Exith Is & & & & & \\
\hline EFFLUEHT i0 & $2.73 E-02$ & $3.61 E-v 1$ & 4. Bove0! & $\begin{array}{l}40 \text { UE } \\
=\end{array}$ & 5. $545-\therefore 7$ \\
\hline EFFüEHT li & $2.22 E-32$ & $2.33 \mathrm{E}-\mathrm{vi1}$ & J. ẤE+bi & $5.77 E-i ; j$ & $5.24 z-19$ \\
\hline FF!LEUT !2 & 1.70E- $6 z$ & $30 \mathrm{E}-\mathrm{n}$ & $5,50+61$ & $3,: 5=-6$ & E.07E-6i \\
\hline EFFLUENT :3 & $1.2 E E-02$ & ¿. IJE-OI & $5 . ; 0\left[\begin{array}{c}0.1] \\
0\end{array}\right.$ & 1. $245-60$ & $5.735-93$ \\
\hline EFFLUENT 14 & $1.12 E-j 2$ & $3.24 E-9 !$ & $6.3 \mathrm{ivE}+\mathrm{iij}$ & $5.60=-69$ & E. $32=-99$ \\
\hline EF! vent 15 & $9.22 E-13$ & S. $23 E-3 i$ & b. $64=+6 !$ & $7.225-67$ & $5,5=-49$ \\
\hline EFilenth Is & $9.02 E-03$ & B. $42 E-7 i$ & $7.60 E-91$ & C. & J.9YE-9i \\
\hline EFFEUEHT 17 & $7 .-5 E-13$ & 3.5AE-01 & $7,90 E+6 i$ & $3.20 E-69$ & ב. \\
\hline EFFLUEHT IB & $0.965-93$ & $3.5 \mathrm{E}-\mathrm{ii}$ & 7. EUE+U! & $2,3=5$ & $=.5 Z-19$ \\
\hline EFLLENT IF & $0.51 E-03$ & 3. & a. $: 0 E+0 i$ & $4.445-69$ & $5.70-09$ \\
\hline
\end{tabular}


TABLE B.16C. Aluminum (A1) Leach Fractions from flow Through Test whole Grout with Groundwater (COLTIA-C)

\begin{tabular}{|c|c|c|c|c|c|}
\hline & & & & & \\
\hline SAHPLE & An/Ao & EUS. An/AD & DAYS & Dine. & $D$ cun. \\
\hline EFFLUENT I & 4. $16 E-04$ & 4. $16 E-04$ & $3.60 E+0.0$ & $1.71 E-13$ & $1.7 ! 5-13$ \\
\hline EFFLUEKT 2 & $2.26 E-04$ & $6.42 E-04$ & $1.005+01$ & $7.39 E-19$ & $1.22 E-13$ \\
\hline EFFLUEKT 3 & $1.79 E-03$ & $2.43 E-03$ & $1.40 E+01$ & $2.82 E-11$ & $1.25 E-12$ \\
\hline EFFLUERT : & 6. $99 E-04$ & $3.33 E-03$ & $1.70 E+01$ & $9.755-12$ & $1.71 E-12$ \\
\hline EFFLUERT 5 & $9.27 E-04$ & $4.06 E-03$ & $2.105+01$ & $1.21 E-11$ & $2.32 E-12$ \\
\hline EFFLUENT $b$ & $6.24 E-04$ & 4.6 BE-03 & $2.40 E+01$ & $1.15 E-11$ & $2.70 E-12$ \\
\hline EFFLUENT 7 & $1.80 E-04$ & $4.86 E-03$ & $2.80 E+01$ & 6. $26 E-13$ & $2.50 E-12$ \\
\hline EFFLUENT 8 & $2.48 E-04$ & $5.11 E-03$ & J.!OE+0! & $2.3 P E-12$ & $2.49 E-12$ \\
\hline EFFLUEHT ? & $3.11 E-04$ & $5.42 E-03$ & $3.50 E+01$ & 2.36E-12 & 2. $49 E-12$ \\
\hline COLUMA IB & & & & & 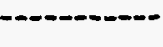 \\
\hline EFFLUENT 10 & $3.76 E-04$ & $5.80 E-03$ & 4. $50 E+01\}$ & $6.68 E-13$ & $2.21 E-12$ \\
\hline EFFLUENT 11 & $3.16 E-04$ & $6.11 E-03$ & $5.20 E+01$ & $1.1 / E-12$ & $2.1 \mathrm{EE}-12$ \\
\hline EFFLUERT 12 & $1.92 \pm-04$ & $6.30 E-05$ & $5.60 E+01$ & $1.46 E-12$ & $2.10 E-12$ \\
\hline EFFLLENT II & $1.14 E-04$ & $6.42 E-03$ & $5.90 E+0 i$ & $9.35 E-13$ & $2.07 E-12$ \\
\hline EFFLUENT 14 & $1.46 E-06$ & $0.42 E-03$ & ¿. $30 E+101$ & $9.58 E-17$ & 1. 34ะ-12 \\
\hline EFFLLIERT 15 & $2.82 E-06$ & $6.42 E-03$ & $6.60 E+01$ & 6. $73 E-16$ & $1.355-12$ \\
\hline EFFLUENT I6 & $0.00 E+00$ & $6 .\{2 E-03$ & $7.00 E+01$ & $0.00 E+00$ & $6.755-12$ \\
\hline EFFLUEHT 17 & $0.00 E+00$ & $6.42 E-03$ & $7.40 E+01$ & $6.00 E+00$ & $1.65 E-12$ \\
\hline EFFLUEHT IO & $0.00 E+00$ & b. $42 E-03$ & $7.80 E \div 01$ & $0.005+00$ & 1. $57 E-12$ \\
\hline EFFLUENT 19 & 1.7TE-05 & $6.44 E-03$ & $8.10 E+01$ & $3.27 E-14$ & $1.325-12$ \\
\hline
\end{tabular}

TABLE B.16e. Boron (as H3BO3) Leach Fractions from Flow Through Test Whole Grout with Groundwater (COLTIA-C)

\begin{tabular}{|c|c|c|c|c|c|}
\hline SAKPLE & Ân/ÂO & CU日. Ân/Ao & gaYs & D inc. & 8 cur. \\
\hline EFFLUENT I & $1,435-03$ & $1.43 E-03$ & $3.00 E \div 00$ & 2.02E-12 & $2.025-12$ \\
\hline EFFLUENT 2 & $7.23 E-04$ & $2.15 E-03$ & $1.00 E+01$ & $\bar{i} .595-1.3$ & 1.3EE-12 \\
\hline EFFLUENT 3 & 5. $84 E-03$ & B. $00 E-03$ & $1.90 E+01$ & $3.01 E-10$ & 1. 35E- 19 \\
\hline EFFLUEKT 4 & $4,16 E-03$ & $1.22 E-02$ & $1.70 E+91$ & $3.52 E-10$. & $2.5 B E-11$ \\
\hline EFFLUEMT $\Xi$ & $4.69 E-03$ & $\therefore, 62 E-02$ & 2. $16 E+01$ & $2.355-10$ & $3.72 \pm-11$ \\
\hline EFFLUERT $b$ & $1.385-03$ & $1.76 E-02$ & $2.30 E+01$ & $5.63 E-1 i$ & $3.345-11$ \\
\hline EFFLUENT $7{ }^{\circ}$ & $2.78 E-63$ & $2.04 E-12$ & 2. $20.0+0 !$ & 1. 49E-10 & $4.4 ! E-! !$ \\
\hline EFFLUENT 8 & $1.40 E-03$ & $2.18 E-02$ & $3.10[+\hat{i}]$ & $7.66 E-1 !$ & $4.55=-1:$ \\
\hline EFLLUEST 9 & ć. $37 E-64$ & $2.25 E-02$ & $3.50 E+01$ & $1.155-11$ & $4,256-11$ \\
\hline OHLIN is & & & & & \\
\hline EFFLUEHT 10 & i. $35=-63$ & $2,37 E-3 i$ & A. Sivt +01 & $3.17=-12$ & Solst-D \\
\hline EFFLUEHT II & $1.02 E-03$ & $2.99 E-02$ & $5.26 E+01$ & ¿.ZiE-\$i & $3.54 \bar{E}-11$ \\
\hline EFFLUEKT :2 & $i .43 E-04$ & 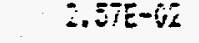 & $566 E-61$ & $2.31 E-11$ & J.46E-1: \\
\hline EFFLUENT :3 & S. $.35-1.49$ & $2.52 E-02$ & $5 .=0 E+i i$ & 2.38E-11 & $\therefore 460-i j$ \\
\hline EFFLUEHT IA & 5. $619 E-64$ & $2.00-82$ & 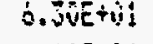 & ¿.:BZE-:i & $3.36 E-11$ \\
\hline EFFLUEKT 15 & 5. $73 E-i d 4$ & $2.73 E-62$ & $8.06+20$ & $2.34 E-11$ & $3.35=-1:$ \\
\hline EFFLIEAT IS & $4.76 E-14$ & $\therefore .78 E-62$ & $7.606 \div 61$ & i. IIE-1i & $3.275-11$ \\
\hline EFFLUEMT IT & $\bar{i} .15 E-04$ & $2.85 E-62$ & $7.40 E+0 i$ & $2.73 \mathrm{E}-1$. & $3,25-\vdots 1$ \\
\hline EFFLLENT IO & $8.15 E-04$ & $2,75-12$ & 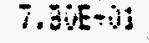 & SOAE-I! & 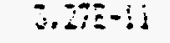 \\
\hline EFFUUEHT : & $6.34 E-04$ & $3.00-32$ & 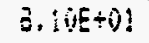 & $\div .21 E-11$ & $3.30-i 1$ \\
\hline
\end{tabular}


TABLE B.16f. Fluoride (F) Leach Fractions from Flow Through Test whole Grout with Groundwater (COLTIA-A)

\begin{tabular}{|c|c|c|c|c|c|}
\hline SAMPLE & An/AO & Cus. An/AO & DAYS & Dinc. & D cus. \\
\hline EFFLUENT 1 & $1.16 E-03$ & $1.16 E-03$ & $3.00 E+00$ & 1. $34 \mathrm{E}-12$ & $1.34 E-12$ \\
\hline EFFLUERT 2 & B. $05 E-04$ & $1.97 \mathrm{E}-03$ & $1.00 E+01$ & $9.39 E-13$ & $1.15 E-12$ \\
\hline EFFLUEET 3 & $5.90 E-03$ & $7.87 E-03$ & $1.40 E+01$ & $3.07 E-10$ & $1.31 E-11$ \\
\hline EFFLUENT 4 & $0.00 E+00$ & $7.87 E-03$ & $1.70 E+01$ & $0.00 E+00$ & $1.08 E-11$ \\
\hline EFFLUENT 5 & $3.29 E-03$ & $1.12 \mathrm{E}-02$ & $2.10 E+01$ & $3.52 E-10$ & $1.76 E-11$ \\
\hline EFFLUENT 6 & 2.29E-03 & $1.35 E-02$ & $2.40 E+01$ & $1.565-10$ & $2.23 \mathrm{JE}-11$ \\
\hline EFFLUEHT 7 & $9.10 E-04$ & $1.44 E-02$ & $2.80 E+01$ & $1.59 \mathrm{E}-11$ & $2.18 E-11$ \\
\hline EFFLUEKT $B$ & $2.14 E-0 J$ & $1.65 E-02$ & $3.10 E+01$ & $1.78 E-16$ & $2.60 E-11$ \\
\hline EFFLUENT & $1.32 E-03$ & $1.78 E-02$ & $3.50 E+01$ & $4.26 E-1 !$ & $2.69 E-11$ \\
\hline \multicolumn{6}{|l|}{ COLLUH IB } \\
\hline EFFLUEKT 10 & $2.635-03$ & $2.05 E-02$ & $4.50 E+01$ & $3.26 E-11$ & $2.76 E-1 !$ \\
\hline EFFLUERT II & $2.23 E-03$ & $2.27 \mathrm{E}-02$ & $5.20 E+0 \mathrm{i}$ & $5.84 E-11$ & $2.73 E-i \mathrm{i}$ \\
\hline EFFLUENT 12 & $2.195-03$ & $2.49 E-02$ & $5.605+01$ & $1.92 E-10$ & j.27E-11 \\
\hline EFFLUENT IJ & $1.84 E-03$ & $2.675-02$ & 5. $50 E+01$ & $2.55 E-10$ & 3. 38E-11 \\
\hline EFFLUENT 14 & $2.01 E-03$ & $2.87 E-02$ & $6.30 E+01$ & $1.835-10$ & j. $8 E E-! !$ \\
\hline EFFLUERT 15 & $1.65 E-63$ & $3.04 E-02$ & 6. $60 E+101$ & $2.315-10$ & $4.14 E-1 !$ \\
\hline EFFLUENT 16 & $1.79 E-03$ & $3.22 E-02$ & $7.00 E+01$ & $1.61 E-10$ & $4.38 E-11$ \\
\hline EFFLUENT 17 & $1.49 \varepsilon-03$ & $3.36 E-02$ & $7.40 E+01$ & $1,19 E-10$ & $4.53 E-11$ \\
\hline EFFLUERT 18 & $1.00 E-03$ & $3.47 E-02$ & $7.80 E+01$ & 5.6EE-11 & $4.56 E-11$ \\
\hline EFFLUENT 19 & $7.97 E-04$ & $3.54 E-02$ & $8.10 E+01$ & $0.64 E-11$ & $4.60 E-11$ \\
\hline
\end{tabular}

TABLE B.16g. Nitrate (NO3) Leach Fractions from Flow Throuth Test Whole Grout with Groundwater (COLTIA-A)

\begin{tabular}{|c|c|c|c|c|c|}
\hline SAKPLE & AnI $/$ AO & Cun. An/Ao & DAYS & D inc. & o Eus. \\
\hline EFFLUEMT I & $1.07 \mathrm{E}-02$ & 1.07FE-02 & $3.00 E+00$ & $1.13 E-10$ & $1.13 E-10$ \\
\hline EFFLUEMT ? & $6.42 E-03$ & $1.715-02$ & $1.00 E+01$ & $5.97 E-11$ & Q.36E-ij \\
\hline EFFLUEHT 3 & 1.3BE- 02 & $3.09 E-02$ & $1,40 E+01$ & 1. $67 E-09$ & 2. $.22 \mathrm{E}-10$ \\
\hline EFFLUEHT 4 & $1.64 E-02$ & $4.73 E-02$ & $1.70 E+01$ & $5.51 E-199$ & $3.90 \mathrm{E}-10$ \\
\hline EFFLUEHT 5 & $6.35 E-02$ & $1.11 E-01$ & $2.10 E+01$ & 5. 6́6E-ง8 & 1.73E-697 \\
\hline EFFLUUENT 6 & 1.0JE-102 & $1.21 E-01$ & $2,46 E+01$ & $3.11 E-69$ & 1.81E-iी \\
\hline EFFLUEKT 7 & 3. $55 E-03$ & 1. 25E-01 & $2.80 E+01$ & $2.43 E-10$ & $1.04 E-19$ \\
\hline EFFLUEMT a & $1.27 E-62$ & ¿. उTE-AI & $3.10 E+01$ & ล. $31 E-1 j \overline{7}$ & 1. $806-09$ \\
\hline EFFLUENT $\rightarrow$ & $6.35 E-63$ & $1.44 E-01$ & $3.50 E+0 !$ & $9.84 E-10$ & $1.75 E-8 \overline{9}$ \\
\hline \multicolumn{6}{|l|}{ coluth iB } \\
\hline EFFLUENT IO & $1.525-02$ & $1.59 E-01$ & $4.50 E+01$ & 1.:1VE-iV & 1. $66 E-27$ \\
\hline EFFLUEHT !I & 1. $0 \pm E-v 2$ & $1.69 E-01$ & $3.20 E+0 !$ & 1.27E-69 & :.65-69 \\
\hline EFFLUENT I2 & $1.23 E-02$ & $1.61 E-01$ & $5.60 E+63$ & D. OZE-vิ & 1. $74 E-i 7$ \\
\hline Effluent is & $0.55 E-63$ & i. $36 E-\hat{v i}$ & $5.70 E+0 !$ & $3.25 E-i j 7$ & 1.7EE-09 \\
\hline EFFLUEHT I4 & 4. $77 E-03$ & 1. $7 J E-01$ & $6.305+4 !$ & a. IIE-0Y & 1. TFE-6i \\
\hline EFFLUERT :S & $4.40 E-03$ & i. $975-01$ & $0.00 E+6 i$ & $3.65 E-09$ & $1.75 E-49$ \\
\hline EFELLENT 16 & $3.30 E-j]$ & $2.015-11$ & $7.00 E \div 01$ & c.52E-10 & $1.7 ! E-09$ \\
\hline EFLLUENT 17 & 4. $v E E-0 \overline{3}$ & 2. USEE-V̂l & $7.70 E+0 !$ & $3.67 E-10$ & 1. FFE- \\
\hline EFFIIENT 13 & $3.465-133$ & $2.095-51$ & $7.30 E \div 01$ & อ. & !. ร9E-ग9 \\
\hline EFFLIENT :9 & $3.75 E-0 j$ & $2.125-\hat{0} 1$ & 3.:논 & 1. $25 E-69$ & 1.8:E-69 \\
\hline
\end{tabular}


TABLE B.16i. Cesium-137 (CS-137) Leach Fractions from Flow Through Test, Grout with Groundwater (1B)

\begin{tabular}{|c|c|c|c|c|c|c|c|}
\hline SAHPLE & PORE YOLUYE & $u C i$ & TIKE DAYS & An/AO & CUn An/AOO & DINC CK2/SEC & DCUH CH2/SEC \\
\hline CRH IB-1 & $9.30 E-01$ & $1.09 E-10$ & $3.00 E+00$ & $2.64 E-11$ & $2.64 E-11$ & $7.51 E-28$ & $7.51 E-28$ \\
\hline CRN $18-2$ & $2.42 E+00$ & $2.32 E-10$ & $1.00 E+01$ & $5.62 E-11$ & $8.26 E-11$ & $1.25 E-23$ & $2.21 E-27$ \\
\hline CRH 1B-3 & $3.60 E+00$ & $5.80 E-11$ & $1.40 E+01$ & $1.40 E-11$ & $9.66 \mathrm{E}-11$ & $2.39 E-27$ & $2.16 E-27$ \\
\hline CAN 18-4 & $4.42 E+00$ & $1.09 E-10$ & $1.70 E+01$ & $2.63 E-11$ & $1.23 E-10$ & $9.86 E-27$ & $2.88 E-27$ \\
\hline$C$ CRU IB-5 & $6.8 \hat{2 E}+00$ & $2.87 E-10$ & $2.10 E+01$ & $6.95 E-11$ & $1.92 \mathrm{E}-10$ & $4.06 E-26$ & $5.71 E-27$ \\
\hline CRY 18-6 & $8.52 E+10$ & 1. $32 E-10$ & $2.40 E+01$ & $3.18 E-11$ & $2.24 E-10$ & $1.44 E-26$ & $6.78 E-27$ \\
\hline CRE $1 B-7$ & $9.47 E+00$ & $6.38 E-11$ & $2.80 E+01$ & $1.54 E-11$ & $2.40 E-10$ & $2.0 \mathrm{iE}-2 \bar{i}$ & 6. $64 E-27$ \\
\hline CRU $18-6$ & $1.09 E+01$ & $6.50 E-11$ & $3.10 E+01$ & $1.57 E-11$ & $2.55 E-10$ & $3.52 E-27$ & $6.81 E-27$ \\
\hline CRH 18-9 & $1.32 E+01$ & $1.62 E-10$ & $3.50 E+01$ & $3.91 E-11$ & $2.955-10$ & $1.29 E-26$ & $8.02 E-27$ \\
\hline CRN $16-10$ & $1.56 E+01$ & $1.41 E-10$ & $4.50 E+01$ & $3.42 E-1 !$ & $3.29 E-10$ & $3.17 E-27$ & $7.77 E-27$ \\
\hline$C F H \mid B-11$ & $1.78 E+01$ & $1.02 E-10$ & $5.20 E+01$ & $2.46 E-11$ & $3.53 E-10$ & $3.81 E-27$ & $7.77 \mathrm{E}-27$ \\
\hline ENK $16-12$ & $2.04 E+01$ & $1.78 E-10$ & $5.60 E+01$ & $4.30 E-11$ & $3.96 E-10$ & $2.24 E-26$ & $9.06 E-27$ \\
\hline CRH 18-13 & $2.30 E+011$ & $8.72 E-11$ & $5.30 E+01$ & $2 . \| 1 E-11$ & $4.18 E-10$ & $6.35 E-27$ & $9.56 E-27$ \\
\hline CRR $18-14$ & $2.61 E+01$ & $2.39 E-10$ & $6.30 E+01$ & $5.7: E-11$ & $4.75 E-10$ & $2.82 E-26$ & $1.16 E-26$ \\
\hline CRH 16-15 & $2.91 E+01$ & $3.71 E-10$ & $0.60 E+01$ & B. $99 E-11$ & $5.65 E-10$ & $1.15 E-25$ & $1.57 E-26$ \\
\hline CRH 10-16 & $3.25 E+01$ & $2.58 E-10$ & 7.00E+01 & $6.24 E-11$ & $6.29 E-10$ & $3.27 E-26$ & $1.82 E-26$ \\
\hline [RH 18-1] & $3.55 \mathrm{JE}+01$ & $2.17 E-10$ & $7.40 E+01$ & $5.25 E-11$ & $6.60 E-10$ & $\therefore 60 E-206$ & $2.32 E-26$ \\
\hline CRH 1B-18 & $3.75 E+01$ & $2.19 E-10$ & $7.80 E+01$ & $5.3 \mid E-11$ & $7.33 E-10$ & $2.65 E-26$ & $2.23 E-26$ \\
\hline ENK $1 B-19$ & $3.89 E+01$ & $1.55 E-10$ & $8.10 E+01$ & $3.75 E-11$ & $7.715-10$ & $2.01 E-26$ & $2.37 E-26$ \\
\hline CAN $18-20$ & 4.11E+01 & $1.12 E-10$ & $8.50 E+01$ & $2.70 E-11$ & $7.98 E-10$ & 6. $1 J E-27$ & $2.42 E-26$ \\
\hline
\end{tabular}

TABLE B.16j. Strontium-85 (SR-85) Leach Fractions from Flow Trhough Test Grout with Groundwater (1A)

\begin{tabular}{|c|c|c|c|c|c|c|c|}
\hline SAMFLE & FOAE VOLUKE & UEi & TIME DAYS & AnjÂO & CUB An $/ A D$ & JINC CX2/SEC & BCUH CK2/SEE \\
\hline CNH $1 A-1$ & 3.10E-01 & $4.02 E-11$ & $3.00 E \div 00$ & $5.49 E-10$ & $5.49 E-10$ & $3.67 E-25$ & $3.65 E-25$ \\
\hline CEN $15-2$ & 5.jOE-6! & $2.01 E-11$ & $1.00 E+01$ & $2.75 E-10$ & $8.24 E-10$ & $2.14 E-25$ & $2.49 E-25$ \\
\hline CEH iñ-3 & $1.37 E+60$ & $1.76 E-10$ & $1.40 E+01$ & 2.40E-09 & $3.23 E-09$ & $1.54 E-23$ & $3.735-24$ \\
\hline$C$ CN & $1.98 E \div 00$ & $6.21 E-11$ & $1.70 E+01$ & B. $49 E-10$ & $4.07 E-09$ & $1.68 E-24$ & 3.58E-24 \\
\hline Es: $1 \hat{n}-5$ & $3.16 E \div 00$ & 1. $78 E-10$ & $2.40 E+01$ & $2.43 E-09$ & b. $51 E-09$ & $1.11 E-23$ & $0.47 E-24$ \\
\hline CBN $19-6$ & $4.12 E+00$ & $1.47 E-30$ & $3.10 E+01$ & $2.01 E-09$ & $8.51 E-09$ & $4.13 E-23$ & B. $565-24$ \\
\hline $5 R^{2} 14-0$ & $0.24 E+00$ & 1.04 $10-10$ & $3.50 E+01$ & $1.42 E-79$ & 5. $34 E-69$ & $2.79 E-23$ & ¿. $v 4 E-23$ \\
\hline CEM $1 \hat{A}-9$ & $7.13 E+00$ & $6.21 \mathrm{E}-11$ & $4.50 E+0 \mathrm{i}$ & $8.43 E-10$ & 1. 68 EE-6B & $2.2 \mathrm{EE}-24$ & $9.49 E-24$ \\
\hline
\end{tabular}


TABLE B.16j. Strontium-85 (SR-85) Leach Fractions from Flow Through Test, Grout with Groundwater (1B)

\begin{tabular}{|c|c|c|c|c|c|c|c|}
\hline $\begin{array}{l}\text { SAMPLE } \\
\text { CRAB !B-1 }\end{array}$ & $\begin{array}{l}\text { PORE VOLLME } \\
9.30 E-01\end{array}$ & $\begin{array}{l}u[\mathrm{i} \\
4.75 E-11\end{array}$ & $\begin{array}{l}\text { TIKE DAYS } \\
3.00 E+100\end{array}$ & $\begin{array}{l}A n / A D \\
6.4 B E-10\end{array}$ & $\begin{array}{l}\text { CUK An/AO } \\
6.48 E-10\end{array}$ & $\begin{array}{r}\text { DINC CK2/SEC } \\
4.53 E-25\end{array}$ & $\begin{array}{c}\text { DCUK CK2/SEE } \\
4.53 E-25\end{array}$ \\
\hline CRN $18-2$ & $2.42 E+00$ & $1.60 E-10$ & $1.00 E+01$ & $2.18 E-09$ & $2.83 E-09$ & $1.88 E-23$ & $2.59 \mathrm{E}-24$ \\
\hline CRH $18-3$ & $3.60 E+00$ & $3.98 E-11$ & $1.40 E+01$ & 1.23E-09 & $4.05 E-19$ & $1.82 E-23$ & $3.80 E-24$ \\
\hline CRN 1B-4 & $4.42 E+00$ & $1.01 E-10$ & $1.70 E+01$ & 1.3BE-09 & 5. 43E-09 & $2.69 E-23$ & $5.61 E-24$ \\
\hline Cfit 1B-5 & $6.82 E+00$ & $4.34 E-11$ & $2.10 E+01$ & $5.93 E-10$ & $6.02 E-09$ & $2.95 E-24$ & $5.58 E-24$ \\
\hline CRY 1B-6 & $8.52 E+00$ & $3.05 E-11$ & $2,40 E+001$ & $4.16 E-10$ & $6.44 E-199$ & $2.46 E-24$ & $5.58 E-24$ \\
\hline CRH 18-7 & $9.47 E+00$ & $1.39 E-10$ & $2.80 E+01$ & $1.90 E-09$ & Q.35E- 09 & $3.03 E-23$ & $8.025-24$ \\
\hline CRN 1B-B & $1.09 E+01$ & $1.06 E-10$ & $3.10 E+01$ & 1. $44 E-09$ & $9.78 E-09$ & $2.97 E-2 j$ & 7. $9 B E-24$ \\
\hline CRE 1B-9 & $1.32 E+01$ & $2.39 E-10$ & $3.50 E+01$ & $3.26 E-09$ & 1. $30 E-08$ & $8.92 E-23$ & $! .57 \mathrm{E}-23$ \\
\hline CRN $18-10$ & 1.56E+01 & 1. $31 E-10$ & $4.50 E+01$ & 1. TPE-09 & $1,48 E-18$ & $8.72 E-24$ & $1.58 E-2 j$ \\
\hline ISH $1 B-11$ & $1.78 E+01$ & $1.01 E-10$ & $5.20 E+01$ & $1.38 E-09$ & $1.62 E-08$ & $1.20 E-23$ & 1.6JE-23 \\
\hline CRM $15-12$ & $2.04 E+01$ & $1.51 E-10$ & $5.60 E+01$ & 2. $06 E-05$ & $1.83 E-18$ & $5.13 E-23$ & $1.97 E-23$ \\
\hline CAM $13-13$ & $2.30 E+01$ & $2.44 E-10$ & $5.90 E+01$ & $3.30 E-09$ & $2.16 E-108$ & ¿.58E-22 & $2.56 E-23$ \\
\hline CRN 18-14 & $2.61 E+01$ & $1.41 E-10$ & $0.30 E+01$ & i. $93 \mathrm{JE}-09$ & 2.35E-68 & $3.13 E-23$ & 2. $84 E-23$ \\
\hline CRM $1 B-15$ & $2.91 E+01$ & $1.33 E-10$ & $6.60 E+01$ & $1.81 E-09$ & $2,53 E-08$ & $4.6 \mathrm{EE}-23$ & $3.15 E-23$ \\
\hline CRM 18-16 & $3.25 E+01$ & $1.32 E-10$ & $7.00 E+01$ & $1.80 E-09$ & $2.71 E-i 8$ & $2.72 E-23$ & $3.40 E-23$ \\
\hline CEY $1 B-17$ & 3.55E+01 & 1.39E- 10 & $7.40 E+01$ & 1. B6E-09 & $2.90 E-08$ & $3.33 E-23$ & $3.68 E-23$ \\
\hline CRM 1B-1B & $3.73 E+01$ & $7.09 E-11$ & $7.80 E+01$ & $9.67 E-10$ & $3.00 E-08$ & $8.82 E-24$ & $3.73 E-23$ \\
\hline CKH $18-19$ & $3.89 E+01$ & b. $16 E-11$ & $8.10 E+01$ & $3.40 E-10$ & $3.08 E-08$ & $1.01 E-23$ & $3.79 E-23$ \\
\hline CRN $18-20$ & $4.11 E+01$ & $1.015-10$ & $8.50 E+01$ & $1.38 E-09$ & $3.22 E-6 B$ & $1.60 \mathrm{E}-2 \mathrm{~J}$ & $3.95 E-23$ \\
\hline
\end{tabular}

TABLE B.17a. Potassium (K) Leach Fractions from Flow Through Test Crushed Grout with Groundwater (DIFBCGTC)

\begin{tabular}{|c|c|c|c|c|c|}
\hline SAMPLE & An/AQ & CLU. An/AO & DAYS & ine. & is eus. \\
\hline EFIUUEHT 1 & $5.56 E-02$ & $5.53 \mathrm{E}-12$ & 3. $A 0 E+W 0$ & $5.86 E-21$ & 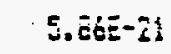 \\
\hline EFFLUEH ? & $9.83 E-02$ & $1.54 E-01$ & 7. OUE +60 & 6. $545-25$ & $\therefore .325-20$ \\
\hline EFFLUEHT 3 & $3.76 E-102$ & i. $54 E-v 1$ & 1. $64 E+61$ & 3. 3.3E- 30 & $2.12 E-2$ \\
\hline EFFLUENT 4 & $2.79 E-02$ & $2.24 E-\hat{v} 1$ & i. $40 E+0 i 1$ & $1.5 i E-i i$ & 2. $.55=-i 5$ \\
\hline EFfluent 5 & T. $66 E-33$ & $2.3 \mid E-6\}$ & $1.706+6 !$ & $1.775-\ddot{z}$ & $2.5, E-60$ \\
\hline EFPLUENT 6 & 5.i1E-0J & $2.30-\hat{B} !$ & 2.. $: \dot{B E}+0 \dot{0}$ & $5.965-22$ & $2.12 E-98$ \\
\hline EFELUENT 7 & $3.425-63$ & $i, 3 ; E-\hat{v i}$ & $2.465+3 i$ & $\therefore$ S.QVE- 2 & $1.5 \times 5-60$ \\
\hline EFFLUERT a & $7.66 z-64$ & $2,40 E-01$ & $2.70 E+01$ & $3,5 E-23$ & $1.655-60$ \\
\hline EFFLUENT ? & $4.13 E-0 j$ & $2,\{4 E-0 !$ & $3.16 E-i 1$ & $3.96-22$ & $\therefore 44 E-36$ \\
\hline EFFLUEHT IO & $2.4 j E-62$ & $2.6 \partial E-\hat{v} 1$ & $3.30 E+63$ & B. $.5 E-2 !$ & :.52E- $: 5$ \\
\hline EFEUENT ! & $1.53 E-132$ & $2.34 E-3 i$ & $4.562+01$ & a. & 1. . $3 E E-1 \mathrm{~B}$ \\
\hline EFFLUENT IA & $4.75 E-43$ & $2.35 E-b 1$ & 4. जEE $\div 4\}$ & $\therefore .83 E-32$ & $1.36=-6$ \\
\hline EFELUEHT IJ & $4.76[-13$ & $2.74 E-61$ & $5.26 E+61$ & $510-2 !$ & !.juE- \\
\hline EFT1:EWT I4 & $0.0 \mathrm{e}+00$ & 2. ? $94 E-3 !$ & S.E:E+1! & W. We+wi & :.Z1E-\%E \\
\hline EF?WEHi is & $\therefore, 60+60$ & COYE-OU! & $0.1 \mathrm{D}-1.1$ & $6 . ; 6 E+\%$ & $2 . \mathrm{AEE}-\mathrm{C}$ \\
\hline EFFGUEHT is & $40005+60$ & C. F十E-6? & $\therefore 46+6 i$ & D. WuEto & i. 650 \\
\hline
\end{tabular}


TABLE B.17b. Sodium (Na) Leach Fractions from Flow Through Test Crushed with Groundwater (DIFBCGTC)

\begin{tabular}{|c|c|c|c|c|c|}
\hline SAKPLE & Ân/ÂO & CUL. An/AD . & DAYS & D inc. & D cua. \\
\hline EFFLUEHT ! & $1.50 E-01$ & 1.50E-0! & $3.005+00$ & $4.21 E-20$ & $4.21 E-20$ \\
\hline EFFLUENT 2 & $2.87 E-01$ & $4.36 E-01$ & $7.00 E+00$ & $5.56 E-19$ & $2.545-07$ \\
\hline EFFLUENT 3 & $1.30 E-01$ & $5.66 E-01$ & $1.00 E+01$ & $3.58 E-19$ & $3.43 E-0.7$ \\
\hline EFFLUERT \& & $1.28 E-01$ & $6.94 E-01$ & $1.40 E+01$ & $2.75 E-19$ & 2. 19E-07 \\
\hline EFF!UEKT 5 & b. $10 E-02$ & $7.55 E-01$ & $1.70 E+0 !$ & $1.44 E-19$ & $4.50 E-07$ \\
\hline EFFLUENT 6 & $4.89 E-02$ & 8. $64 E-01 !$ & $2.10 E+01$ & $6.39 E-20$ & $4.56 E-07$ \\
\hline EFFLUEHT 7 & $1.79 E-02$ & 8.22E-01 & $2.40 E+01$ & $1.80 E-20$ & 4.j3E-0 $\bar{i}$ \\
\hline EFFLIJENT 8 & 1.7BE- 02 & B. $40 E-01$ & $2.70 E+01$ & 2.0ิ3E-20 & $4.24 E-0 j$ \\
\hline EFFLUENT १ & $8.39 E-03$ & $8.48 E-01$ & 3.10E+01 & $2.36 \mathrm{E}-21$ & $3.39 E-6 j$ \\
\hline EFFLUENT 10 & $3.06 E-02$ & 8.79E-01 & 3. $80 E+01$ & $1.48 E-20$ & $3.7 \mathrm{gE}-0 \mathrm{0}$ \\
\hline EFFLUERT II & $1.07 E-02$ & $8.70 E-01$ & 4. $50 E+61$ & $2.10 E-21$ & $3.31 E-177$ \\
\hline EFFLLENT I2 & $2.60 E-63$ & $3.92 E-01$ & $4.70 E+\square !$ & $5.215-23$ & $3.04 E-67$ \\
\hline EFFLUENT 13 & $9.22 E-04$ & 3. $93 E-0) !$ & $5.20 E+01$ & $1.00 E-22$ & $2.86 E-67$ \\
\hline EFFLUEHT I4 & $9.42 E-019$ & B. $94 \mathrm{E}-\hat{\mathrm{v}} 1$ & $5.60 E+0 \mathrm{i}$ & $6.75 E-23$ & $2.66 E-017$ \\
\hline EFFLUENT - 15 & $1.37 E-0.3$ & $8.96 E-01$ & C. $1 \hat{V E}+01$ & $1.02 E-22$ & $2.56 \mathrm{E}-67$ \\
\hline EFFLUENT I6 & $5.97 E-04$ & $8.965-01$ & 6. $4 \hat{E E}+01$ & $5.59 E-2 j$ & $2.46 E-67$ \\
\hline
\end{tabular}

TABLE B.17e. Boron (as H3BO3) Leach Fractions from Flow Through Test Crushed with Groundwater (DIFBCGTA)

\begin{tabular}{|c|c|c|c|c|c|}
\hline SAMFLE & An/AD & CLS. Ân/ÂO & DAYS & inc. & 0 E \\
\hline EEFLUENT 1 & $3.04 E-02$ & $3.04 E-02$ & $3.00 E+00$ & 1.TAE-21 & $74 E-21$ \\
\hline EFFLUEMT 2 & $7.79 E-02$ & $1.06 E-01$ & $7.00 E+00$ & $4 . j 0 E-20$ & $5 E-21$ \\
\hline EFFLUENT 3 & $3.50 E-02$ & $1.43 E-01$ & $1.00 E+01$ & $2.55 E-20$ & $16 E-30$ \\
\hline EFFLUEHT 4 & $3.06 E-02$ & 1. $74 E-\hat{v} !$ & $1.40 E+01$ & $1.58 E-\hat{2} \dot{j}$ & $! .225-20$ \\
\hline EFFIUEHT 5 & $1.23 E-022$ & $1.065-01$ & $1.70 E+01$ & $5.69 E-21$ & $\vdots .15 t$ \\
\hline EFFLUEKT 6 & $9.34[-6]$ & $1.965-01$ & $2.10 E+01$ & $2.35 E-21$ & i.0.03E \\
\hline EFF!LERT 7 & $3.12 E-03$ & $1.979-01$ & $2.40 E+0 i$ & $5.47 E-32$ & $5.20 E$ \\
\hline EFFLUEKT \& & $3.21 E-63$ & $2.02 E-01$ & $2.70 E+01$ & 6.5AE-22 & 8. 52E-2! \\
\hline EFFLUEKT? & $3.31 E-03$ & $2.055-9 !$ & $3.10 E+01$ & $4.4 i E-2 i$ & T. .JE-2! \\
\hline EFFLUENT io & $7.03 E-03$ & $8.80 E-89$ & $3.80 E+01$ & $7.845-22$ & 6.69E-2! \\
\hline EFFLUENT I! & $3.435-43$ & 3.23E-09 & 4. जUEE+6! & $2,25 E-22$ & 5..3EE-2! \\
\hline EFF!LENT IZ & $2.57 E-03$ & $7.565-67$ & $4.90 E+0 \mathrm{i}$ & $\bar{B}, \bar{B}: \bar{E}-Z Z$ & 5. $35-2:$ \\
\hline EFFLLEKT IJ & $1.835-63$ & T.12E-69 & $5.20 \mathrm{VE}+\mathrm{uj}$ & $4.24 E-2 z$ & $5.25 E-2$ \\
\hline EFFLUENT It & {$[.355-03$} & S.6:E-6? & $3.6 \mathrm{vE}+\dot{01}$ & i.47E-2i & $4.3 \mathrm{BE}-2$ \\
\hline EFFLLENT 15 & $2.31 E-63$ & $6.07 E-i 9$ & 3. $105+61$ & 2. $315-22$ & $4.8:=-2$ \\
\hline EFFLUEMT 16 & 1.27E-03 & $\therefore .35 E-67$ & S. $+0 E+01$ & $2.51 E-22$ & \\
\hline
\end{tabular}


TABLE B.17f. Fluoride (F) Leach Fractions from Flow Through Test Crushed with Groundwater (DIFBCGTA)

SAKPLE
EFFLUEMT I
EFFLUEHT 2
EFFLUENT 3
EFFLUEHT 4
EFFLUENT 5
EFFLUEHT 6
EFFLUEHT 7
EFFLUENT
EFFLUEHT
EFFLUENT 10
EFFLUEHT 11
EFFLUENT 12
EFFLUENT 13
EFFLLENT 14
EFFLUENT 15
EFFLUEHT 16

\begin{tabular}{|c|c|c|}
\hline An/AO & Cua. An/AO & DAYS \\
\hline 1. SEE-62 & $1.555-02$ & 3. $60 E \div 00$ \\
\hline $4.05 E-02$ & $5.60 \mathrm{E}-02$ & $7.00 E+00$ \\
\hline $1.89 E-0.2$ & $7.49 E-02$ & 1. $O B E \div(1)$ \\
\hline $1.30 E-02$ & $9.39 E-02$ & $1.40 E+01$ \\
\hline $9.85 E-03$ & $1.04 E-\hat{0} 1$ & $1.70 E+01$ \\
\hline $1.07 E-02$ & $1.14 E-01$ & $2.10 E+01$ \\
\hline $5.35 E-03$ & $1.20 E-01$ & $2.40 E+01$ \\
\hline $5.80 E-03$ & $1.26 E-01$ & 2.70E +OI \\
\hline $3.23 E-03$ & 1.29E-0! & $3.10 E+01$ \\
\hline $1.91 E-02$ & $1.43 E-0 i$ & $3.80 E+01$ \\
\hline [. $02 E-12$ & 1.5JE-01 & $4.50 E+01$ \\
\hline $8.65 E-93$ & $1.62 E-01$ & $4.90 E+0$ \\
\hline $6.73 E-03$ & 1.67E-01 & $5.20 E+0$. \\
\hline $4.472 E-6]$ & $1.735-01$ & 5. áve+ô \\
\hline $6.75 x-13$ & 1. $80 \mathrm{E}-\hat{\mathrm{v}} 1$ & $6.10 E+0$ \\
\hline & ¿. $84 E-01$ & 6.40 \\
\hline
\end{tabular}

0 inc.

$4.49 E-22$

1.11E-20

T. SEEE-21

B. $07 E-2 !$

3. $76 E-21$

$3.06 E-21$

i.ólE- $2 i$

2. $15 E-21$

$4.25 E-22$

3. $15 E-7 !$

2. $00 \mathrm{E}-21$

$4.96 \mathrm{E}-2 \mathrm{i}$

5. $74 E-2 !$

1. $47 E-\hat{z}:$

2. $31 E-21$

$2,64 E-21$
D cus.

4. 4 TE-22

$2.53 E-21$

3. $.6 \mathrm{E}-2 !$

J. J5E-21

3.5TE-2!

$3.52 E-21$

$3.37 \mathrm{~F}-21$

3. 3UE -21

3.0.

3. טगJE- 21

2. $942-2 !$

$3.61 E-21$

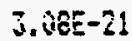

3. 01E-2i

2. $\$ 9 E-29$

$2.96 E-2 !$

TABLE B.17g. Nitrate (NO3) Leach Fractions from Flow Through Test Crushed with Groundwater (DIFBCGTA)

\begin{tabular}{|c|c|c|}
\hline SAMPLE & An/ÂO & CUR. AnIAOO \\
\hline EFFLUEHT ! & $3.34 E-01$ & 3.34E-0 \\
\hline EFFLUEKT 2 & 2. $88 E-01$ & $6.23 \mathrm{E}-0$. \\
\hline EFFIUEKT 3 & 3.6TE-102 & $\bar{i}, 19 E-0$ \\
\hline EFFLUENT \& & $7.00 E-02$ & $7.695-0$ \\
\hline EFFLUELT 5 & $1.02 E-02$ & $8.00 \mathrm{E}-0$ \\
\hline EFFLUEM : & $5.47 E-03$ & $8.05 E-\hat{v}$ \\
\hline EFFLUEST 7 & $2.44 E-03$ & $6.07 E-0$ \\
\hline EFFLUENT B & $3.37 E-63$ & $3.11 E-i$ \\
\hline EFELUENT ? & $1.47 E-03$ & $0.12 E-c$ \\
\hline EFFLUEHT IO & 6. $44 E-i 3$ & E.15E- \\
\hline EFFLUENT i! & $6.57 E-\mathrm{iJ}$ & $6.25 \mathrm{E}-$ \\
\hline EFFLUENT :2 & $\bar{J} . J \mathbf{J} E-\hat{H} J$ & $3.315-$ \\
\hline EFFUUET 15 & $4.25 E-03$ & 6. $35 E-$ \\
\hline EFELUENT If & $2.32 E-103$ & $3.37 E-i$ \\
\hline EFHUNT IE & $3.41 E-63$ & $3.41 E-1$ \\
\hline EFLUEMT 16 & $\{.72 E-133$ & $3.42 E-1$ \\
\hline
\end{tabular}

BÂYS

$3.00 E+00$

i. $00 E+00$

1. 00 OOSOI

1.. tôe+ol

$1.70 E+0 !$

2.: $0 E+01$

$2.40 \mathrm{v}+\hat{0} 1$

$2.7 \hat{v E}+\hat{O} \mathrm{~S}$

J. $.10 E \div 0 !$

J. JôE+6i

$4.30 \mathrm{~V}=01$

$4.90 \mathrm{Q}+\hat{\mathrm{i} i}$

5. $2 \mathrm{jE}-\mathrm{Q} 1 \mathrm{1}$

S. $6 \hat{i} E+\hat{i} \mathrm{i}$

S. jNE

S. 46E+i!
0 inc.

2. $30 \mathrm{E}-19$

5.625-19

1. $9 E$ EE-19?

B. $24 E-30$

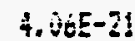

3.0 $015-32$

3. $3 \mathrm{BEE}-22$

$7.25 E-32$

8. $38 E-23$

6. 5 .5E-32

6. $24 E-12$

1. ETE-Z:

2. ZFE-3

$4.6=-22$

อ. $4 E-3$

5.75
(1) cuis.

3. $025-67$

6. $152-07$

6.72E-3i

6. 5xE-ij]

5. $6 \mathrm{jE}-\mathrm{i} 7 \mathrm{7}$

4.

4. 12E-Bi

3. $60 E-61$

$3.15 E-4 \%$

2. TSE-Si

2. $31 E-6 i$

2. $2 \mathrm{ZE}-\mathrm{GT}$

$2.3 \mathrm{E}-\mathrm{i} i$

$2.04 \bar{E}-6 \overline{7}$

L.

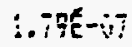


TABLE B.17h. Sulfate (SO4) Leach Fractions from Flow Through Test Crushed with Groundwater (DIFBCGTA)

SAKPLE
EFFLUENT 1
EFFLUENT 2
EFFLLENT 3
EFFLUENT 4
EFFLUENT 5
EFFLUENT 6
EFFLUENT 7
EFFLUENT 8
EFFLUENT 7
EFFLUENT 10
EFFLUENT 11
EFFLUENT 12
EFFLUENT 13
EFFLUENT 14
EFFLUEHT 15
EFFLUENT 16

$\begin{array}{lr}\text { An/AO } & \text { CUn. An/ADO } \\ 9.65 E-01 & 9.65 E-01 \\ 8.68 E-01 & 1.83 E+00 \\ 2.48 E-01 & 2.08 E+00 \\ 1.87 E-01 & 2.27 E+00 \\ 2.89 E-01 & 2.30 E+00 \\ 8.00 E-03 & 2.31 E+00 \\ 0.00 E+00 & 2.31 E+00 \\ 0.00 E+00 & 2.31 E+00 \\ 0.00 E+00 & 2.31 E+00 \\ 0.00 E+00 & 2.31 E+00 \\ 0.00 E+00 & 2.31 E+00 \\ 0.00 E+00 & 2.31 E+00 \\ 0.00 E+00 & 2.31 E+00 \\ 0.00 E+00 & 2.31 E+00 \\ 0.00 E+00 & 2.31 E+00 \\ 0.00 E+00 & 2.31 E+00\end{array}$

DAYS

$3.00 E+00$

$7.00 E+00$

$1.00 E+01$

$1.40 E+01$

$1.70 E+01$

2.10E+01

$2.40 E+01$

$2.70 E+01$

$3.10 E+01$

3. BOE +01

4. $50 E+01$

4. $7 \hat{0 E}+\hat{0} \mathrm{i}$

5. $20 \mathrm{E}+01$

$5.60 E+01$

6. IOE+U1

6. $40 E+0 !$
Dinc.

1. $75 E-18$

$6.07 E-19$

$3.48 E-20$

$1.415-20$

2. $37 E-22$

$1.72 E-23$

$0.00 E+00$

$0.00 E+100$

$0.00 E+0 O$

$0.00 E+00$

D. OOE +00

O. (D)E +00

$0.00 E+00$

$0.00 E+00$

$0.00 E+60$

0. waEtó
1 cus.

3. $91 E-65$

5. 16E-त6

$3.61 E-i 6$

2.58E-06

2.13E-96

$1.72 E-06$

$1.51 E-06$

$1.34 E-115$

1. 1 iE $-i b$

$9.51 E-67$

6. $635-07$

T. 3ate-07

6. $95[5-01]$

$6.452-67$

5. $722-07$

5. $655-07$

TABLE B.17i. Technetium-99 (TC-99) Leach Fractions from Flow Through Test, Crushed with Groundwater (BCGT)

\begin{tabular}{|c|c|c|c|c|c|c|c|}
\hline SAMPLE & PORE VDLUME & $\mathrm{uCi}$ & TIME DAYS & An/ÂO & CUY An/ÂO & DINE CH2 $/ 5 E C$ & DCUK CK2/SEC \\
\hline BCET-1 & $3.705-01$ & $5.84 E-01$ & $3.00 E+00$ & $2.20 E+00$ & $2.20 E+00$ & $1.20 E-05$ & $1.20 E-05$ \\
\hline BCET -2 & $1.14 E+\infty 0$ & $4.07 E-02$ & $7.00 E+00$ & $1.53 E-01$ & $2.36 E+00$ & $3.46 E-19$ & $5.16 E-06$ \\
\hline BCET -3 & $1.66 E+00$ & $7.09 E-03$ & $1.10 E+01$ & $2.6 \mathrm{BE}-02$ & $2.38 E \div 00$ & $1.18 E-20$ & 3.2BE-06 \\
\hline BCET -4 & $2.55 E+00$ & $1.24 E-02$ & $\therefore .50 E+01$ & 4. $67 E-02$ & $2.43 E+90$ & $3.59 E-20$ & $2.41 E-06$ \\
\hline 3EST -5 & $3.31 E+00$ & $2.66 E-03$ & $1.80 E+01$ & $1.01 E-02$ & $2.44 E+00$ & $2.51 E-21$ & 2. $61 E-06$ \\
\hline BCET-6 & $4.53 E+00$ & $2.45 E-03$ & $2.20 E+01$ & $3.25 E-13$ & $2.45 E \div 00$ & $1.26 E-21$ & $1.64 E-016$ \\
\hline BCET-7 & 5. OTE+00 & $1.50 E-03$ & $2.50 E+01$ & $5.66 E-03$ & $2.46 E+70$ & $7.97 E-22$ & $1.34 E-16$ \\
\hline ECGT-8 & $5.82 E+00$ & $1.32 E-03$ & $2.90 E+01$ & $4.97 E-03$ & $2.46 E+00$ & $3.63 E-22$ & $1.25 E-06$ \\
\hline BCET-9 & $6.15 E+00$ & $6.70 E-04$ & $3.20 E+01$ & $2.60 E-03$ & $2.46 E+i \hat{v}$ & $1.66 E-22$ & $1.13 E-06$ \\
\hline BCET-10 & $7.58 E+00$ & $1.54 E-0 J$ & $3.50 E+01$ & 5. $80 E-03$ & $2.47 E+00$ & $7.35 E-22$ & 1.03E-06 \\
\hline BCET-11 & $9.04 E+00$ & $1.11 E-03$ & $j .90 E+01$ & $4 .: 77 E-03$ & $2 . \div T E+90$ & $2.50=-22$ & $7.2 b E-07$ \\
\hline EC6T-12 & $1.02 E+01$ & $2.24 E-03$ & $4.60 E+0 \mathrm{i}$ & $8.46 E-1 j j$ & $2.48 E+00$ & $5.44 E-22$ & $7.25 E-1 j 7$ \\
\hline $\operatorname{scsi}-13$ & $1.12 E+01$ & $\therefore .4 i E-0 j$ & 5. $30 E+0 i$ & $5.35 E-103$ & $2.49 E+00$ & $2.00 E-22$ & $3, \quad 2 Z E-07$ \\
\hline SEET-14 & $1.17 E+01$ & $7.45 E-04$ & $5.70 E+01$ & 2. BOE-03 & $2.49 E \div 60$ & $1.60 E-22$ & $5.34 E-67$ \\
\hline $300 T-15$ & $1.24 E+01$ & $6.045-124$ & 6. $00 E \div W 1$ & $2.51 E-03$ & $2.47 E+40$ & $1.56 E=2 ?$ & $6.02 E-17$ \\
\hline 8coi-16 & $1.2 B E+01$ & $8.3 j[E-\hat{0} 4$ & t. $40 E+i)$ & $3.16 \mathrm{E}-\mathrm{ij}$ & $2.50 E+00$ & $\therefore 47 E-27$ & $5.64 E-6 j$ \\
\hline
\end{tabular}


TABLE B.17i. Technetium-99 (TC-99) Leach Fractions from Flow Through Test, Crushed with Groundwater (BCGT)

\begin{tabular}{|c|c|c|c|c|c|c|c|}
\hline SAMPLE & PORE VOLUAE & $\mathrm{uCI}$ & IIKE DAYS & $\mathrm{An} / \mathrm{AnO}_{0}$ & CUH An/AO & DINC CM2/5EC & UEUA EK2/SEL \\
\hline BCET-1 & $3.70 E-01$ & 5. 84E-01 & $3.00 E+100$ & $2.20 E+00$ & $2.20 E+00$ & $1.20 E-05$ & $1.20 E-0$ \\
\hline BCET-2 & $1.14 E+00$ & $4.07 E-02$ & $7.00 E+00$ & $1.53 E-01$ & $2.365+10$ & $3.46 E-19$ & 5. $16 E-06$ \\
\hline BCET-3 & $1.66 E+00$ & $7.09 E-03$ & $1.10 E+01$ & $2.68 E-02$ & $2.38 E+00$ & $1.18 E-20$ & 3. $28 E-$ \\
\hline BCóT-4 & $2.55 \mathrm{E}+100^{\circ}$ & $1.24 E-02$ & $1.50 E+01$ & $4.67 E-102$ & $2.43 E+100$ & $3.59 E-20$ & $2.41 E$ \\
\hline BCST-5 & $3.31 E+\infty 0$ & $2.66 E-03$ & $1.80 E+01$ & 1.01E-02 & $2.44 E+00$ & $2.51 E-21$ & 2.01E- \\
\hline BCGT-6 & $4.53 E+60$ & $2.45 E-03$ & $2.20 E+01$ & $9.25 E-03$ & $2.45 E+00$ & 1. $26 E-21$ & D. SUE- \\
\hline БСБ̄T-7 & $5.07 E+00$ & $1.50 \mathrm{E}-0 \mathrm{3}$ & $2.50 E+01$ & $5.60 E-63$ & $2.46 E+00$ & $7.97 E-22$ & $1.945-$ \\
\hline ВCET-8 & $5.82 E+00$ & $1.32 E-03$ & $2.90 E+01$ & $4.97 E-63$ & $2.46 E+100$ & $3.635-22$ & $1.25 \mathrm{E}-$ \\
\hline SCOT-G & $6.15 E+00$ & $6.90 E-64$ & $3.20 E+01$ & $2.60 E-03$ & $3.46 E+100$ & $1, \Delta B E-22$ & 1. $1135-$ \\
\hline SCET-10 & $7.50 E+100$ & $1.54 \mathrm{E}-0 \mathrm{3}$ & $3.50 E+01$ & $5.20 E-13$ & $2.4 T E+00$ & T.35E-22 & $1.635-$ \\
\hline BCGT-1i & $7.64 E+00$ & 1. $11 E-13$ & $3.70 E+01$ & $4.17 E-03$ & $2.47 E+i 10$ & $2.56 E-22$ & $3.2 E E-6$ \\
\hline $\operatorname{BCET-12}$ & $1.02 E+(01$ & $2.24 E-03$ & 4. $60 E+0 \mathrm{i}$ & $B .46 E-03$ & 2. $46 E+00$ & $5.44 E-22$ & $7.35 E-1$ \\
\hline BCAT-1J & $1.12 E+01$ & $1.41 E-03$ & $5.30 E+i j 1$ & $5.33 E-63$ & $2.49 E+60)$ & $2.60 E-22$ & $0.52 E-i$ \\
\hline BCGT-14 & $1.17 E+01$ & $7.43 E-\hat{04}$ & $5.70 E+01$ & 2. BGE-0J & 2. $49 E+(6)$ & $\therefore .66 E-22$ & $6.34 E-i$ \\
\hline BCET -15 & $1.24 E \div 01$ & b. $64 E-104$ & $6.005+01$ & $2.51 E-6 j$ & $2.475 \div 60$ & 1.50E -22 & $0.02 E-0$ \\
\hline$B C E T-16$ & $1.28 E+01$ & $3.37 E-04$ & $6.40 E+0 i$ & $3 . i b E-0 j$ & $2.50 E+60$ & $1.47 E-32$ & $5.67 E-$ \\
\hline
\end{tabular}

TABLE B.18a. Potassium (K) Leach Fractions from Flow Through Test Crushed with Groundwater (DIFBCGIC)

\begin{tabular}{|c|c|c|c|c|c|}
\hline SAMPLE & An/AOO & cua, Ân/Áto & DAYS & 0 inc. & 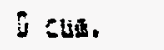 \\
\hline EFFLUENT I & $8.34 E-02$ & $8.34 E-02$ & $3.00 E+00$ & $1.31 E-20$ & $1.31 E-20$ \\
\hline EFFLUERT 2 & $1.30 E-01$ & $2.14 E-01$ & i.OGE+100 & $1.155-19$ & $4.73 E-j E$ \\
\hline EFFLUENT 3 & $5.015-02$ & $2.64 E-10 !$ & $1.00 E+01$ & 5. $32 E-20$ & $5.35-98$ \\
\hline EFFLUEKT 4 & $3.33 E-02$ & $2.38 E-00 !$ & 1. \{ $\{E E+0\}$ & i. $53 E-20$ & $5.23 E-40$ \\
\hline EFFLUEHT 5 & $1.54 E-\sqrt{2}$ & 3. IJE-01 & $1.70 E \div 01$ & $9.14 E-21$ & $4.6-i E-48$ \\
\hline EFFLUEKT 6 & $4.48 E-03$ & $3.17 E-0 !$ & $2.10 E+01$ & $5.37 \mathrm{E}-\hat{2} 2$ & $4.05-08$ \\
\hline EFF:UEHT 7 & $3.355-03$ & $3.26 E-01$ & $2.40 E+01$ & $3.73 E-21$ & J. \\
\hline EFFIUENT 3 & $3.89 E-63$ & $3.30 E-01$ & $2.70 E+\hat{v} !$ & $9.6 \mathrm{BE}-2 \pi$ & J.joE- $\mathrm{ij} \bar{j}$ \\
\hline EFFLÜEST 9 & $3.08 E-03$ & $3.37 E-01$ & $3.10 \overline{0}+01$ & $2.05 E-21$ & $3.15 E-13$ \\
\hline EFFLUENT 10 & $3.21 E-02$ & $3.75 E-\hat{v} 1$ & S.duE+u! & $2.3 \mathrm{WE}-20$ & S.:DE -36 \\
\hline EFFUUENT I! & $2.12 E-12$ & $3.76 E-01$ & $4.56 \bar{c}+\overline{v i j}$ & B. बuE-2i & उ. \\
\hline EFFLUEWT 12 & 5.:1E-03 & 4.01E-0i & 4.50E+0i & !.T3E-2! & $2.895-68$ \\
\hline EFFLUEHT 13 & $3.55 E-1,49$ & $4.32 E-b i$ & $5.20 E+\hat{v i}$ & $\$ .205-23$ & $2.15=-10$ \\
\hline EFF:UEHT IA & B. Wót +10 & 4. $62 E-61$ & $5.0 \hat{E E}+i \mathrm{i}$ & ¿. 6oetwi & E.JUE- \\
\hline EFLUENT :5 & D. $.6 E+Q 0$ & 4. $32 E-3 !$ & b. $\{\hat{v E+i i\}}$ & W. WhE+Bu & E \\
\hline EFFLUEKT 16 & 6. $60 E+00$ & $4.02 E-01$ & 6. $40 E+0 i$ & A. But-in & $\therefore 21 \bar{E}-i$ \\
\hline
\end{tabular}


TABLE B.18b. Sodium (Na) Leach Fractions from Flow Through Test Crushed with Groundwater (DIFBCGIC)

\begin{tabular}{|c|c|c|c|c|c|}
\hline SAMFLE & An/Ao & CUS. Ân/AO & DAYS : & Dinc. & D cus. \\
\hline EFFLUEMT I & $2.41 E-01$ & $2.41 E-01$ & $3.00 E+00$ & $1.095-19$ & $1.495-07$ \\
\hline EFFLUENT ? & $3.47 E-01$ & 5. $88 E-01$ & $7.00 E+00$ & B. $15 E-19$ & $5.38 E-67$ \\
\hline EFFLUEHT 3 & $1.42 E-01$ & $7.305-01$ & $1.00 E+01$ & $4.27 E-19$ & $7.02 E-07$ \\
\hline EFFLUENT 4 & $1.27 E-01$ & $3.57 E-01$ & $1.40 E+01$ & $2.70 E-19$ & $9.06 E-67$ \\
\hline EFFLUENT $\bar{J}$ & $6.20 E-02$ & $9.19 E-01$ & $1.70 E+01$ & 1.4IE-19 & $1.05 E-66$ \\
\hline EEF!UENT 6 & $3.14 E-02$ & $9.50 E-01$ & $2.10 E \div 01$ & $2.63 E-20$ & 1. GEE-6io \\
\hline EFFLUENT 7 & $1.955-02$ & $9.70 E-01$ & $2.40 E+01$ & $2.15 E-20$ & $1.11 E-06$ \\
\hline EFFLULENT 8 & $1.81 E-02$ & $9.89 E-01$ & $2.70 E+01$ & $2.09 E-20$ & $1.34 E-06$ \\
\hline EFF:LEHT ? & 8.56E-03 & $9.96 E-01$ & $3.10 \mathrm{E}+01$ & 2.99E-2! & 1.liE-î́ó \\
\hline EFFLUEHT IO & $2.83 E-02$ & $1.02 E+00$ & $3.80 E+01$ & $i .2 J E-200$ & Q.5JE-67 \\
\hline EFFLLEHT $\|$ & $9.78 E-03$ & $1.03 E+00$ & $4.50 E \div 01$ & $1.85 E-21$ & $3.03 E-47$ \\
\hline EFFLUENT 12 & $2.30 E-03$ & $1.04 E+00$ & 4. $90 E+\hat{O} 1$ & $3.5 ! 5-22$ & $7.38 E-07$ \\
\hline EFFLUENT 13 & $1.49 E-04$ & $1.04 E+00$ & $5.20 \mathrm{VE}+0 \mathrm{O}$ & $2.81 E-24$ & $6.955-07$ \\
\hline EFFLUENT 14 & $6.33 E-04$ & $1.04 E+00$ & $5.60 E+01$ & $3.06 E-23$ & $0.45 E-07$ \\
\hline EFFIUEHT IS & 1.00E-0̃ & 1. $\hat{B} 4 E+00$ & $6.10 E+01$ & $5.335-23$ & $3.3 Z E-67$ \\
\hline EFFLUENT I6 & $2.95 E-04$ & $1.04 E+00$ & $6.40 E+01$ & $1.36 E-23$ & $5.55 E-07$ \\
\hline
\end{tabular}

\section{TABLE B.18e.}

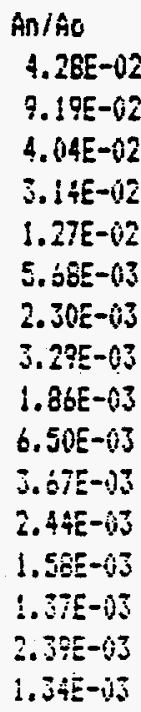

cus. ÂRiẪo

4.26E-02

$1.35 E-01$

¿.75E-01

2.06E-i1

2. $195-01$

2. $25 E-01$

$2.27 \mathrm{E}-\mathrm{OA}$

2.30E-0!

2.32E-01

$2.37 E-01$

2. 42E-1)!

$2.95 E-\hat{i j}$

$2.96 E-0)$

Z. $48 \mathrm{E}-0 \mathrm{i}$

2. 5uE-Oi!

2.5ZE-91
บิด

3. $00 E+00$

$7.00 E+00$

1. $00 E+01$

1. $4 \hat{\mathrm{V} E}+01$

$1.70 E \div 01$

2.10E+01

$2.40 E+01$

$2.70 E+01$

$3.10 E+11$

$3.60 E+0 \mathrm{i}$

$4.5 G E+01$

4. SOE +Di:

5. $2 \hat{\mathrm{vE}}+\mathrm{Ui}:$

5. $60 \mathrm{E}+\hat{0} \mathrm{i}$

b. $10 E+0 \mathrm{j}$

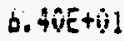

$\checkmark$ ine.

3. $45 E-21$

5. $71 E-20$

$3.45 E-20$

1. $65 E-20$

6. $27 E-21$

3. 5 JE -2

2. $98 \mathrm{BE}-22$

3. ?2E- 22

1. $42 E-2$

6. TOE- $\mathrm{Z}$

z. ETE-ב

3.

5.:SE-20

1. $42 E-3$

Jine-zis

Z.E!E-2 is cust.

$3.45 E-21$

$1.365-20$

1. $73 E-20$

2.39E-65

2. 1 EE-

$1.76 E-66$

1. $69 E-60$

!. $50 \mathrm{E}-\mathrm{v}$

!. 3iE-\$8

$\therefore$. $75-10$

c.

S. ATE-Ti

־. उEE-99

3. 71E-19

ว.97E-99

T. 
TABLE B.18g. Nitrate (NO3) Leach Fractions from Flow Through Test Crushed Grout with Groundwater (DIFBCGIA)

\begin{tabular}{|c|c|c|c|c|c|}
\hline SAMFLE & An/AO & CUn. An/Ao & DAYS & $D$ ine. & 0 eun. \\
\hline EFFLUENT I & $3.51 E-01$ & $3.51 E-01$ & $3.00 E+00$ & $2.32 E-19$ & $3.55 E-07$ \\
\hline EFFLUEHT 2 & $3.84 E-01$ & $7.35 E-01$ & 7.00E+00 & $9.98 E-19$ & 1.00E-07 \\
\hline EFFLUEET 3 & $1.23 E-01$ & $8.58 \mathrm{E}-01$ & $1.00 E+01$ & $3.21 E-19$ & 1. $27 E-66$ \\
\hline EFFLUENT \& & B. $11 E-02$ & Q. $40 E-01$ & $1.40 E+01$ & $1.11 E-19$ & $1.45 E-106$ \\
\hline EFFLUERT 5 & $2.24 E-02$ & $9.62 E-01$ & $1.70 E+61$ & $1.95 E-20$ & $1.425-60$ \\
\hline EFFLUENT $b$ & $9.60 E-03$ & $9.72 E-01$ & $2.10 E+0 i$ & 2. $46 E-21$ & $1.27 \mathrm{c}-\hat{\mathrm{v}} \mathrm{b}$ \\
\hline EFFLUEAT 7 & $2.11 E-113$ & $3.74 E-01$ & $2.40 E+01$ & $2.50 E-22$ & $1.11 E-06$ \\
\hline EFFLUEHT & $2.78 E-03$ & $9.76 E-01$ & $2.70 E+0 \mathrm{i}$ & $4.94 E-2 \hat{2}$ & $1.155-67$ \\
\hline EFFLUENT ? & $\therefore .26 \mathrm{E}-03$ & $9.7 a E-01$ & $3.1 \hat{0 E} \div 01$ & c.52E-2J & ร.7.71E- 6.60 \\
\hline EFFLUENT 10 & 5. $09 E-03$ & $9.53 E-0 !$ & $3.80 E+0\}$ & $4.12 E-22$ & T.92E-6a \\
\hline EFFLUEAT II & $5.66 E-03$ & $9.88 E-01$ & $4.50 E+01$ & b. $17 \leq-22$ & $7.40 E-07$ \\
\hline EFFLUENT 12 & $5.47 E-03$ & $9.94 E-01$ & $4.90 E \div 0 I$ & 1.97E-2! & $6.90 E-0 ? ?$ \\
\hline EFFLUEHT I3 & $3.606-03$ & $8.98 E-\hat{0} !$ & $5.20 E+01$ & $1.705-2 !$ & $6.50 E-0 !$ \\
\hline EFFLUENT 14 & $2.34 E-03$ & $1.00 E+00$ & $5.60 E+01$ & $4.16 E-22$ & E. $90 E-607$ \\
\hline EFFLLENT 15 & $3.7 \mathrm{iE}-03$ & $1.00 \mathrm{E}+00$ & 6. $10 E+01$ & $7.255-22$ & 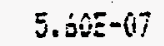 \\
\hline EFFLUENT 16 & $1.98 E-63$ & $1.01 E+00$ & 6. $30 E \div 01$ & 6.14E- $-2 E$ & 4. $70 E-05$ \\
\hline
\end{tabular}

TABLE B.18h. Sulfate (S04) Leach Fractions from Flow Through Test Crushed Grout with Groundwater (DIFBCGIA)

\begin{tabular}{|c|c|c|c|c|c|}
\hline SAMPLE & $A \cap / A B D$ & CUE. An $j$ ÂO & BAYS & Dinc. & 0 cus. \\
\hline EFFIUEHT 1 & $1.01 E+00$ & $1.01 E+00$ & $3.00 \mathrm{E}+100$ & $1.93 \mathrm{JE}-1 \mathrm{~B}$ & $1.20 E-05$ \\
\hline EFFLUEENT 2 & $1.10 E+00$ & 2. $11 E+00$ & $7.00 E+010$ & $9.74 E-19$ & $5.16 E-66$ \\
\hline EFFLUEHT 3 & $3.455-61$ & $2.46 E+06$ & 1. $0 \hat{N E}+\hat{01}$ & $6.70 \mathrm{E}-2 \mathrm{v}$ & $3 . t J E-i t$ \\
\hline EFFLUENT 4 & 2. $\mid 2 E-01$ & $2.67 E+00$ & $1.90 E+01$ & $1.815-20$ & $2.58 E-06$ \\
\hline EFFLUEEHT 5 & $4.81 E-02$ & $2.72 E+00$ & $1.70 E+01$ & $7.675-22$ & $2.12 E-06$ \\
\hline EFFLUERT 6 & $5.6 \mathrm{IE}-02$ & $2.77 E+00$ & 2. $10 E+01$ & $8.47 E-22$ & $1.72 E-06$ \\
\hline EFFLUEHT $i$ & $1.54 E-02$ & $2.79 E+00$ & $2.40 E+01$ & $5.575-23$ & 1.50E-06 \\
\hline EFFLUEKT 3 & $0.00 E+00$ & $2.79 E+00$ & $2.70 E+0 \mathrm{~S}$ & $0.00 \mathrm{Q}+60$ & 1. $34 E-6 c$ \\
\hline EFFLLLET & $1.85 E-03$ & $2.79 E+0 \mathrm{j}$ & 3.16E-N1 & $6.21 E-25$ & 1.6oE-ivo \\
\hline EFFLUEHT 10 & $0.66 E+100$ & 2. $7 F E+v 0$ & $3.80 E+01$ & $0.00 E+60$ & $3.515-v 7$ \\
\hline EFFLUEHT 11 & i. $.00=+00$ & $2.79 E \div 60$ & 4.50E & 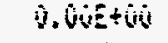 & E.65E- \\
\hline EFFIIEHT L2 & $0.06 E+100$ & $\therefore 79 E+00$ & 4. FoE+OI! & $3.00 \overline{0}+60$ & $7.37 \mathrm{E}-6 \mathrm{7}$ \\
\hline EFFLUENT 13 & $0.60 E \div 00$ & $2.795=00$ & $5.26 E+i j\}$ & $0.006+60$ & $6.75 E-67$ \\
\hline EFFLLENT IA & $0.00 E+00$ & $2.75 E \div 00$ & $5.60 E+0 i$ & Q. $90 \bar{v}+60$ & $6.95-57$ \\
\hline EFF!UEHT 15 & 1. . $8 B E-02$ & $2.80 E+00$ & B. $! O E+61$ & $\therefore .1 \subseteq E-33$ & 5. \\
\hline EFFLUENT 16 & $2.89 E-0 j$ & $2.302+60$ & b. $40 E+0) !$ & $7.365-25$ & $5.04 E$ \\
\hline
\end{tabular}


TABLE B.18i. Iodine-125 (I-125) Leach Fractions from flow Through Test, Crushed with Groundwater (BCGI)

\begin{tabular}{|c|c|c|c|c|c|c|c|}
\hline SAMPLE & PORE VOLUME & CORR UCj & TIKE DAYS & An/ÂO & CUS Ân/Aú & DINC CA2/SEC & DCHN CN2/SEC \\
\hline CRY ECEI-1 & $3.90 E-01$ & $1.37 E-02$ & $3.00 E+00$ & $7.79 E-01$ & $7.79 E-01$ & $2.91 E-06$ & $2.31 E-06$ \\
\hline CRU BCÂI-2 & $1.11 E+10$ & $2.22 E-02$ & $7.005+00$ & $1.26 E+000$ & $2.04 E+00$ & $2.35 E-17$ & $5.16 E-06$ \\
\hline CAN BCSI-3 & $1.58 E+00$ & $7.37 E-03$ & $1.00 E+01$ & 4.19E-0I & $2.46 E \div 00$ & $4.37 E-18$ & $3.615-86$ \\
\hline CRU BC6:-4 & $2.35 E+00$ & $4.68 E-03$ & $1.40 E+01$ & $2.66 \mathrm{E}-01$ & $2.73 E+00$ & $i .04 E-13$ & 2.5SE-06 \\
\hline CRH BCE]-5 & $3.01 E+00$ & $1.93 E-03$ & $1.70 E+01$ & $1.10 E-01$ & 2. $84 E+00$ & $2.995-17$ & $2.12 E-1.66$ \\
\hline CRY BCG:-6 & $4.07 E+00$ & $1.53 E-03$ & 2. $10 E+01$ & $8.722 E-02$ & $2.93 E+60$ & $1.12 E-17$ & 6.72E- 106 \\
\hline CAH BCE!-7 & $4.54 E+00$ & $2.64 E-04$ & $2.40 E+01$ & $1.50 E-02$ & $2.34 E+10$ & $5.62 E-2 !$ & 1.51E-06 \\
\hline CAN BCEI-B & $5.16 E+00$ & $2.57 E-04$ & $2.70 E+01$ & $1.46 E-02$ & $2.95 E+10$ & $4.675-21$ & ¿. JHE-106 \\
\hline CRE BCE!-9 & $5.44 E+00$ & $3.26 E-64$ & $3.10 E+01$ & $1.85 E-\hat{v} 2$ & $2.37 E \div 00$ & $.5 .04 E-21$ & $! .97 E-06$ \\
\hline CAN BCSI-10 & $6.57 E+00$ & $8.43 E-04$ & 3. $.00 E+01$ & $4.79 E-02$ & $3.02 E+00$ & $1.75 E-20$ & $9.5 \mathrm{IE}-07$ \\
\hline CAN SCDI-11 & $7.83 E+00$ & $2.34 E-04$ & $4.50 E+01$ & $1.33 E-62$ & 3. $0 \mathrm{BE}+\mathrm{WB}$ & $1.67 E-21$ & $5.015 E-0107$ \\
\hline CFH ล̊Cô!-12 & $8.65 E+(10$ & $2.36 E-09$ & $4.90 E+01$ & $1.34 E-62$ & $3.05 E+60$ & $3.81 E-21$ & $7.37 E-07$ \\
\hline CEA BCE]-13 & $7.86 E+00$ & $1.41 E-04$ & $5.2 \hat{V E}+\hat{\theta} !$ & $3.01 E-\hat{0} 3$ & $3.68 E+60$ & $1.60 E-21$ & $0.75 E-37$ \\
\hline CRV BCEI-14 & $1.03 E+01$ & $9.09 E-05$ & $5.60 E+01$ & 5. $17 E-03$ & $3.06 E+10$ & $3.935-22$ & $6.48 E-07$ \\
\hline CKH BCÂ]-15 & $1.11 E+01$ & $2.04 E-04$ & $6.105+01$ & $1,16 E-02$ & $3.672+60$ & $1.65 \mathrm{~J}-21$ & 5. $52 E-0 \bar{i}$ \\
\hline CAN BCOI-16 & $1.16 E+01$ & $9.50 E-05$ & $6.40 E+01$ & $3.40 E-\hat{8} 3$ & $3.065+00$ & $0.04 E-72$ & $5.64 \varepsilon-07$ \\
\hline
\end{tabular}

TABLE B.19a. Potassium (K) Leach Fractions from Flow Through Test Crushed with Groundwater (DIFBCGCC)

\begin{tabular}{|c|c|c|c|c|c|}
\hline SAKPLE & An/AD & Cus. Añ $/$ Ao & DAYS & y inc. & D cul. \\
\hline EFFLUENT I & $5.75 E-162$ & $\$ .75 E-122$ & $3.00 E+00$ & $6.22 E-21$ & $6.22 E-21$ \\
\hline EFFLUUEHT 2 & $1.45 E-01$ & $2.02 E-01$ & $7.005+00$ & i. $+2 E-19$ & 4. $77 E-08$ \\
\hline EFFLUEHT 3 & $5.24 E-62$ & $2.55 E-01$ & $1.00 E+01$ & $5.805-20$ & $4.8 E E-06$ \\
\hline EFFLUENT 4 & $2.42 E-02$ & 2.J $J E-01$ & $1.40 E+01$ & $9.865-21$ & 4.47E-6B \\
\hline EFFLLEHT 5 & $2.06 E-13$ & $2.61 E-01$ & $1.70 E+21$ & 1. $65 E-22$ & 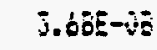 \\
\hline EFFLLIENT $b$ & $0.21 E-03$ & $2.57 E-0 i$ & $2.10 E+01$ & $1.03 E-2 !$ & $3.235-45$ \\
\hline EFFLLUEHT 7 & $3.24 E-i 3$ & $2.90 \mathrm{DE}-01$ & 2. $4(0 E+0) 1$ & $5.93 E-23$ & $2.825-08$ \\
\hline EFFLUENT 8 & $0.00 E+00$ & 2.90E-01 & $2.705+0 !$ & $0.005+00$ & 2.5IE- $\mathrm{iE}$ \\
\hline EFFLUENT : & $3.89 E-03$ & $2.5 \div E-01$ & $3 .\{0 E+1\}$ & S.13E-72 & $2.13 E-98$ \\
\hline EFFLUEKT $1 \hat{v}$ & $1.64 E-02$ & 3. IIE-V̂I & $3.8 \hat{E} E+01$ & $4.25 E-21$ & $2.67 \mathrm{c}-2 \mathrm{~b}$ \\
\hline EFFUENT II & $9.15 E-03$ & $3.20 E-\hat{v} !$ & $4.50 E+01$ & $1.60 E-2 !$ & 1. \\
\hline EFFLIEHT 12 & $5.11 E-63$ & 3. $25 E-011$ & $4.70 E+01$ & I.TAE-Z1 & i. $725-16$ \\
\hline EFFLUENT IJ & $7.67 E-13$ & $3.33 E-31$ & $5.2 \hat{v E}+01$ & $\bar{i},\{4 E-21$ & $1.34 E-166$ \\
\hline EFFLLEKT I4 & $0.00 E+00$ & $5.5 E-6 i$ & $5.60 E+01$ & $0.0 \mathrm{ve}+0.09$ & $1.61 E-9 \overline{8}$ \\
\hline EFLUEHT IS & $6.06 E+60$ & $3.35 E-01$ & $6.10 E+01$ & S. & $1,+4-98$ \\
\hline SFFLUENT 16 & $0.00 E+60$ & S. $335-i q i$ & b. $90 E+i j$ & 0. GuE + Q & {$[, 42 E-1) E$} \\
\hline
\end{tabular}


TABLE B.19b. Sodium (Na) Leach Fractions from Flow Through Test Crushed with Groundwater (DIFBCGCC)

\begin{tabular}{|c|c|c|c|c|c|}
\hline SAMPLE & An/Ao & cus. $A n / A o$ & DAYS & 0 inc. & D cun. \\
\hline EFFLUENT 1 & $1.53 E-01$ & 1.53E-01 & $3.00 E+00$ & $4,41 E-20$ & $4,41 E-20$ \\
\hline EFFLUIENT 2 & $4.06 E-01$ & $5.57 E-01$ & 7. $00 E+00$ & $1.12 E-18$ & $4.70 E-177$ \\
\hline EFFLLIENT 3 & $1.73 E-01$ & $7.32 E-01$ & $1.00 E+01$ & $6.34 E-19$ & $7.02 E-07$ \\
\hline EFFLUENT 4 & $1.25 E-01$ & $8.57 \mathrm{E}-0 \mathrm{l}$ & $1.40 E+01$ & $2.61 E-19$ & $9.05 E-07$ \\
\hline EFFLUENT 5 & $5.81 E-02$ & $9.15 E-01$ & $1.70 E+01$ & $1.315-17$ & $9.81 E-67$ \\
\hline EFFLUENT 6 & $4.35 E-02$ & $9.59 E-01$ & $2.10 E+0 \mathrm{i}$ & $5.05 E-20$ & $1.15 E-i)$ \\
\hline EFFLUEHT 7 & i. $82 E-\hat{0} 2$ & 9.77 E-01 & $2.40 E+01$ & $1.87 E-20 \mathrm{j}$ & 1. $35 E-07$ \\
\hline EFFLUEHT 3 & $1.73 E-02$ & $9.74 E-01$ & $2.70 E+01$ & $1.91 E-20$ & 1. J3E- 37 \\
\hline EFFLIIENT 9 & $9.24 E-03$ & 1. $\hat{N} \hat{E} E+00$ & $3.10 E+001$ & $3.49 E-21$ & $-1.16 E-37$ \\
\hline EFFLUEKT 10 & $3.11 \mathrm{E}-0.2$ & $1.04 E+00$ & $3.50 E+01$ & $1.60 \mathrm{E}-20$ & $9.50 E-07$ \\
\hline EFFLUENT II & $1.26 E-02$ & $1.05 E+00$ & $4.50 E+01$ & $3.14 E-21$ & ล. WUE-67 \\
\hline EFFLUENT I2 & 4. $12 E-0 j$ & $1.05 E+00$ & $4.90 E+01$ & $1.12 E-21$ & $i .40 E-07$ \\
\hline EFFLUENT 13 & $3.63 E-03$ & 1. $0 D E+00$ & $5.20 E+01$ & 1. $67 E-21$ & 3. $70 E-07$ \\
\hline EFFLUENT I4 & $1.52 \mathrm{E}-0 \mathrm{3}$ & $1.06 E \div 00$ & $5.60 E+01$ & $1.75 E-22$ & $6.50 E-67$ \\
\hline EFFLUEHT 15 & $1.59 E-63$ & $1.08 E+\infty 0$ & $6.10 E+01$ & 1. $34 E-22$ & $5.70 E-66$ \\
\hline EFFLUENT I6 & $8.79 E-04$ & $3.06 E+00$ & $6.40 E+01$ & $1.2 ! 5-22$ & $5.001 E-0 \dot{v} 6$ \\
\hline
\end{tabular}

TABLE B.19e. Boric Acid (as H3B03) Leach Fractions from Flow Through Test Crushed Grout with Groundwater (DIFBCGCA)

\begin{tabular}{|c|c|c|c|c|c|}
\hline SAMPLE & $\mathrm{An} / \mathrm{AD}$ & cus. An/AD & DAYS & sinc. & i ธus. \\
\hline EFFUEENT I & $2.64 E-02$ & $2.64 E-02$ & 3. $.0 E+00$ & $1.31 E-21$ & $1.31 E-21$ \\
\hline EFFLUENT $?$ & 1. $09 E-01$ & $1.35 \mathrm{E}-01$ & $7.00 E+00$ & $7.78 E-20$ & $1.47 E-20$ \\
\hline EFFLUEHT 3 & $4.61 E-02$ & $1.61 E-01$ & 1. $30 E+01$ & $4.50 E-30$ & $1.85 E-20$ \\
\hline EFFLUENT 4 & $2.95 E-62$ & 2. $11 E-01$ & $1.40 E \div 0 i$ & $1.46 E-20$ & $2.37 E-05$ \\
\hline EFFLUEERT 5 & $1.08 E-02$ & $2.2 \leq E-01$ & 1.TOE+01 & $4.535-2\}$ & $2.13 E-0 B$ \\
\hline EFFLUENT 6 & $j .87 \mathrm{E}-\hat{0} \mathrm{~J}$ & 2.29E-î! & 2.11E $E+01$ & 1.66E-2: & $\therefore .74 E-18$ \\
\hline EFF!UEHT 7 & $3.03 E-03$ & $2.32 E-\hat{v i}$ & $2.40 E \div 01$ & 5.1 AE-22 & $1.695-65$ \\
\hline EFFLIJENT 8 & $3.05 E-03$ & 2. 35E-01 & $2.70 E+01$ & $5.70 \mathrm{E}-2 Z$ & $1.5: E-06$ \\
\hline EFF!UEMT ? & $2.00 E-13$ & $2.37 E-01$ & $-10 E+0 i$ & 1. DAE $-Z$ & 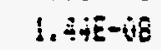 \\
\hline EFFLUENT 10 & :.15E-102 & $2 . \$ 9 E-0 \mathrm{i}$ & $3.30 E+i j$ & 2. $: \Delta E-Z 1$ & $i .28 E-0 \%$ \\
\hline EFFLUENT II & $3.53 E-03$ & $2.5 Z E-01$ & $4.50 E+0 !$ & 2. JAE -22 & 1. JEE-AE \\
\hline EFFLUENT 12 & $1.725-1 j$ & $2.54 E-41$ & $4.7 \mathrm{vE}+61$ & a. $575-29$ & $9,75 E-69$ \\
\hline EFFLUEKT 13 & $2.52 E-03$ & $2,5 j E-01$ & 5. $20 E+6 i$ & B. & 1. $\mathrm{BEE}-\mathrm{YG}$ \\
\hline EFFLUENT 14 & $1.53 E-0 j$ & $\bar{Z} .59 E-\hat{y} 1$ & 5. SUE+01 & 1. $95-22$ & $9.55-69$ \\
\hline EFF!UENT 15 & $2.4 \pi E-0 j 3$ & 2.bIE-DI & b. LVE+61 & $3.2 \mathrm{E}-22$ & E.74:-35 \\
\hline EFFLUENT: :ó & $2.54 E-62$ & $2.89 E-6 i$ & $0.40 E+01$ & $\therefore-\overline{s e c}-1 \bar{F}$ & 1. $. E-05$ \\
\hline
\end{tabular}


TABLE B.19f. Fluoride (F) Leach Fractions from Flow Through Test Crushed Grout with Groundwater (DIFBCGCA)

\begin{tabular}{|c|c|c|c|c|c|}
\hline SAKPLE & $A n / A O$ & CUn. An/AO & DAYS & 0 inc. & D cus. \\
\hline EFFLUERT ! & $1.45 E-02$ & 1. $15 E-02$ & $3.00 E+00$ & $3.94 E-22$ & $3.94 E-22$ \\
\hline EFFLUERT ? & $4.89 E-02$ & $6.335-02$ & $7.00 E+00$ & $1.62 E-20$ & $3.24 E-21$ \\
\hline EFFLUIENT 3 & $2.42 E-02$ & 6. 76E-02 & 1. OOE +01 & $1.24 E-20$ & 4. J3E-21 \\
\hline EFFLUENT 4 & $1.69 E-02$ & $1.04 E-01$ & $1.40 E+01$ & $4.81 E-21$ & $4.40 E-21$ \\
\hline EFFLUEHT S & $9.26 E-133$ & $1.14 E-01$ & $1.70 E+01$ & 3.J.JE-2! & $4,30 E-21$ \\
\hline EFFLUENT 6 & $9.91 E-03$ & $1.24 E-01$ & 2. IOE+01 & $2.6 \mathrm{JE}-2 \mathrm{I}$ & $4.11 E-21$ \\
\hline EFFLUERT 7 & $5.44 E-0 J$ & d. $29 E-01$ & $2.40 E+01$ & $1.67 E-21$ & $3.92 E-21$ \\
\hline EFFLUERT 8 & $5.76 \mathrm{E}-03$ & $1.35 E-01$ & $2.70 E+01$ & $2.12 E-21$ & $3.60 E-2 i$ \\
\hline EFFLUENT 9 & $3.67 E-03$ & $1.39 E-01$ & $3.10 E+01$ & 5.5.5 $1 E-22$ & $-3.49 E-23$ \\
\hline EFFLIIENT 10 & $1.17 E-02$ & 1.50E-01 & J. $.00 E+01$ & $2.18 E-21$ & $3.35 E-2 i$ \\
\hline EFFLUENT 11 & $9.39 E-03$ & $1.605-01$ & $4.50 E+01$ & $1.68 E-2 !$ & $3.20 E-2 !$ \\
\hline EFFLUENT 12 & $5.61 E-03$ & $1.65 E-01$ & 4. $90 E+01$ & 2. $196 E-2 !$ & $3.15 E-21$ \\
\hline EFFLUEKT 13 & 8. $54 E-03$ & $1.74 E-01$ & $5.20 E+01$ & $9.24 E-21$ & $3.28 E-2 !$ \\
\hline EFFLUENT 14 & 5. B7E-0J & $1.80 E-01$ & $5.60 E+01$ & $2.62 E-21$ & $3.25 E-2 !$ \\
\hline EFFLUEHT IS & $7.48 E-03$ & 1. $.87 E-01$ & $6.10 E+01$ & $2.95 E-21$ & $3.24 E-21$ \\
\hline EFFLUEHT 16 & $4.69 E-03$ & $1.92 E-01$ & $6.90 E+01$ & $3.45 E-21$ & $3.25 E-21$ \\
\hline
\end{tabular}

TABLE B.19g. Nitrate (NO3) Leach Fractions from Flow Through Test Crushed Grout with Groundwater (DIFBCGCA)

\begin{tabular}{|c|c|c|c|c|c|}
\hline SAMPLE & $A_{n} / A_{0}$ & CUI. An'Á & DAYS & $D$ inc. & i cus. \\
\hline EFFLUEMT I & $2.84 E-01$ & 2. $34 E-01$ & $3.00 E+00$ & $1.52 E-17$ & 2.:GE-07 \\
\hline EFFLUERT 2 & $4.64 E-01$ & $7.48 E-01$ & $7.00 E+00$ & $1.45 E-18$ & $1.10 E-06$ \\
\hline EFFLUENT 3 & 1. $42 \mathrm{E}-01$ & $8.89 E-01$ & $1.00 E+01$ & $4.25 E-1 ?$ & 1. $5 \hat{60 E}-96$ \\
\hline EFFLUENT 4 & $7.31 E-02$ & $9.63 E-01$ & $1.40 E+0 i$ & B. $75 E-2 \hat{v}$ & $1.72 E-106$ \\
\hline EFFLUENT 5 & $4.425-03$ & $9.67 E-91$ & $1.70 E+08$ & $7.585-22$ & $1.005-36$ \\
\hline EFFLUENT '́ & $6.65 E-103$ & $7.74 E-01$ & $2.10 E+0 !$ & 1. $B B E-21$ & $1.27 E-06$ \\
\hline EFFLUERT 7 & $2.78 E-03$ & $9.76 E-01$ & $2.40 E+(11)$ & $4.35 E-22$ & !.3E- \\
\hline EFFLUENT $B$ & $3.75 E-03$ & $9.80 E-01$ & $2.70 E+01$ & E. $58 E-22$ & i. $12 E-66$ \\
\hline EFFLUEHT ? & $1.52\{-0]$ & $9.82 E-0 !$ & $3.10 E \div 01$ & 7.39E-23 & $8.70 E-07$ \\
\hline EFFLUENT 10 & $5.85 E-0\}$ & Q. $6 B E-\hat{\imath} !$ & $3.60 E+9 i$ & $5.43 E-22$ & $8.50 E-07$ \\
\hline EFFIUEHT II & $5.60 E-03$ & Q. 抒E-0! & 2. $50 E+60 !$ & $5.99 E-22$ & $3.06=-67$ \\
\hline EFFLUENT 12 & $2.74 E-\hat{v} 3$ & $5.96 E-61$ & $4.90 E+01$ & $4.97 E-22$ & $7.4 \mathrm{ijE}-0 \mathrm{ij}$ \\
\hline EFFLUENT 13 & $5.47 E-63$ & 1. $.00 E+00$ & $5.20 E+\{01$ & 3. iפE-21 & $=.765-17$ \\
\hline EFFLJENT I4 & 2. $30 E-\hat{B} 3$ & i. $00 \mathrm{E}+00$ & 5. ذטิ $\div 01$ & 5.TIE- & $6.50-97$ \\
\hline EFFLLENT IS & $3.52=-03$ & $\therefore . N 1 E+00$ & $0.10 E+01$ & S.10E-:Z & $5.70 E-i j \bar{j}$ \\
\hline EFLUEHT IS & $2.32 E-1 \bar{J}$ & 1. $81 E+00$ & c. $40 E+3 !$ & $3.41 E-i 2$ & 5. 60E-j? \\
\hline
\end{tabular}


TABLE B.19i. Cesium-137 (CS-137) Leach Fractions from Flow Through Test Crushed with Groundwater (BCGC).

\begin{tabular}{|c|c|c|c|c|c|c|c|}
\hline SAAMPLE & PORE VOLUME & $\mathrm{UCi}$ & TIKE DAYS & AnIÁo & CUK An:AAO & DIRC CK2/SEC & ถับบ CH2/SEC \\
\hline CRU SECEL-1 & $3.76 E-01$ & $1.09 E-10$ & $3.00 E+00$ & $1.62 E-j 0$ & $1.82 E-10$ & 8. $935-39$ & 8.73E-37 \\
\hline CAN BCEC-2 & $1.44 E+00$ & $9.31 E-10$ & $7.00 E+00$ & $1.56 E-09$ & $1.745-69$ & $5.09 E-36$ & $3.49 E-37$ \\
\hline CRH SCEC-3 & $2.25 E+00$ & 1.39E-10 & $1.00 E+01$ & $3.165-10$ & $2.06 E-39$ & $3.55 E-37$ & $3.41 E-3\}$ \\
\hline CRI BCEC-4 & $3.49 E+00$ & $1.86 E-10$ & $1.40 E+01$ & $3.12 E-10$ & 2.37E-199 & $2.035-37$ & $3.23 E-57$ \\
\hline CRA BCEC-5 & $4.64 E+00$ & $2.655-10$ & $1.70 E+01$ & $4.44 E-10$ & $2.51 E-09$ & $6.99 E-37$ & $3.75 E-37$ \\
\hline CRIN BCEC-6 & $6.38 E+00$ & $3.99 E-10$ & $2.10 E+01$ & $6.69 E-10$ & $3.48 E-09$ & $3.35 E-37$ & $4.65 E-37$ \\
\hline CFH SCAC-7 & $7.11 E+00$ & $1.67 E-10$ & $2.40 E+0\}$ & $2.79 E-10$ & $3.76 E-09$ & $2.765-37$ & $4.75 E-37$ \\
\hline CAN BCGC-8 & $8.0 B E+00$ & $2.25 E-10$ & $2.70 E+01$ & $3.77 E-10$ & $4.14 E-69$ & $4.43 E-5 \bar{i}$ & $5 .: j E-j i$ \\
\hline CAN SCEC-9 & $0.48 E+60$ & $9.09 E-11$ & $3.105+01$ & :.52E-10 & $4.295-69$ & $4.85 E-38$ & $9.7 B E-3 i$ \\
\hline ERy SCEC-iO & $1.00 E+01$ & $1.63 E-10$ & $3.60 E+01$ & $2.31 E-10$ & $4.5 i=-19$ & $0.54 E-38$ & $4.43 E-3 i$ \\
\hline CNA SC6C-11 & $1.15 E \div 01$ & $3.36 E-10$ & $4.50 E+01$ & $5.635-10$ & 5. $3 \mathrm{JE}-69$ & $4.245-3 i$ & $4.715-3 \bar{i}$ \\
\hline SAH BCEC -12 & $1.22 E+01$ & $1.05 E-10$ & $4.90 E+0 !$ & $1.8 \mathrm{BE}-10$ & $5.32 E-i j \overline{7}$ & $3.015-37$ & $4.64 E-5 j$ \\
\hline CNin 5C6C-13 & 1. $36 E+01$ & $3.205-10$ & $5.205+01$ & $5.50 E-10$ & $5.87 E-95$ & $1.07 E-30$ & $5.35-3]$ \\
\hline CKH BCEC-14 & $1.43 E+01$ & 1. $63 E-10$ & $5.60 E+01$ & $2.6 \mathrm{iE}-10$ & $6.15 E-69$ & $1.66 E-37$ & $5.43 E-37$ \\
\hline CRA ScGC-15 & $1.54 E+01$ & $2,35 E-10$ & 6. IUEE+0: & $3.94 E-10$ & $6.54 E-49$ & $2.6 \mathrm{E}=-37$ & $5.65 E-37$ \\
\hline CNAN BCEC-16 & $1.6 \mathrm{vE}+01$ & $1.58 E-10$ & b. $40 E+01$ & $2.64 E-10$ & $3.81 E-09$ & $2.74 E-37$ & $5.33 E-37$ \\
\hline
\end{tabular}

TABLE B.19j. Strontium-85 (SR-85) Leach Fractions from Flow Through Test, Crushed with Groundwater (BCGC)

\begin{tabular}{|c|c|c|c|c|c|c|c|c|}
\hline SAMPLE & PORE YOLUKE & $U C i$ & TIKE DAYS & $A n / A D$ & CUH An/Ao & DINC EX2/SEC & JCu: & CX2/SEC \\
\hline CRU BCAC-1 & $3.20 E-01$ & $2.76 E-11$ & $3.00 E+00$ & $1.72 E-68$ & 1.72E- 08 & $5.60 E-34$ & & $5.60 E-34$ \\
\hline CRA BCGC-2 & $1.22 E+60$ & $2.42 E-10$ & $7.00 E+00$ & $1.51 E-07$ & $1.68 E-07$ & $3.365-31$ & & $2.29 E-32$ \\
\hline CRL BCEC-3 & $1.92 E+00$ & $5.92 E-11$ & $1.00 E+01$ & $3.74 E-08$ & $2.065-07$ & $3.4 B E-32$ & & $2.39 E-32$ \\
\hline CAN" BCGC-4 & $2.96 E+00$ & $1.76 E-10$ & $1.40 E+01$ & $1.10 E-07$ & $3.16 E-07$ & $1.79 E-31$ & & 4.0.3E- \\
\hline CRY BCEC -5 & $3.94 E+00$ & $1.65 E-10$ & $1.70 E+01$ & $1.035-67$ & $4.19 E-07$ & $2.66 E-31$ & & $5.85 E-$ \\
\hline CRU aCOĆ- & $5.42 E+\hat{0}$ & $1.88 E-10$ & $2.10 E+01$ & $1.17 E-07$ & 5.37E-07] & $2.02 E-3:$ & & $7.75 E-$ \\
\hline CRA BCAC-7 & $6.04 E+00$ & $5.82 E-11$ & $2.40 E+01$ & $3.64 E-08$ & 5.73E-07 & $3.27 E-32$ & & $7.73 E-$ \\
\hline CAะ $8 C B C-3$ & b. $37 E+00$ & $7.19 E-11$ & $2.70 E+01$ & $4.50 E-08$ & $6 .: 2 E-\{17$ & $4.44 E-32$ & & $7.995-1$ \\
\hline CAN BCEC-9 & $7.21 E+00$ & $3.14 E-11$ & $3.10 E+01$ & $1.965-08$ & $6.38 E-57$ & $5.675-33$ & & $7 .+1 E-$ \\
\hline CAM SCOE- 10 & $8.51 E+90$ & i. $23 E-10$ & $3.30 E+01$ & $7.66 \mathrm{E}-08$ & $7.14 E-07$ & $4.46 E-32$ & & $7.595-$ \\
\hline IAH ECoc-11 & $3.75 E+00$ & 1.50E-10 & $4.50 E+01$ & 9.3. $3 E-08$ & 9.0BE-07 & B. $23 E-32$ & & 8. $19 E-32$ \\
\hline CFI SCEC-1? & $1.64 E+01$ & $5.78 E-11$ & $9.90 E+0\}$ & $3.6 \mathrm{EE}-08$ & $3.445-07$ & $2.76 E-32$ & & $3.21 E-32$ \\
\hline CAN $\operatorname{RCBC}-13$ & $1.16 E+01$ & $1.67 E-10$ & $5.20 E+01$ & $1.03 E-07$ & $\overline{3}, 46 E-07$ & $2.03 E-31$ & & $3.77 E-32$ \\
\hline CRH SOECL-14 & $1.22 E+01$ & $7.49 E-11$ & $5.60 E+01$ & $4.68 E-98$ & ใ.ระEE-017 & Z.2EE-32 & & $8.99 E-32$ \\
\hline LFN ECAC-15 & $1.31 E+01$ & 7. $795-11$ & b. IOE+0! & $4.875-36$ & $1.34 E-\hat{y} b$ & $2.60 E-3 \hat{2}$ & & $1 . S 1 E-5$ \\
\hline ifN $3 \operatorname{Coc}-i b$ & $\{.36 E+01\}$ & 4.6IE-11 & $6.40 E+01$ & $2.80 \mathrm{E}-0.0$ & $1.0 j E-06$ & $2.275-52$ & & $i .02 E-3 i$ \\
\hline
\end{tabular}


Confrourti:
TABLE B.20b. Sodium (Na) Leach Fractions from Flow Through Test Crushed
Grout/Sediment with Groundwater (DIFCGTC)

SAMPLE
EEFLUUEHT 1
EFFLUENT 2
EFFLUENT 3
EFFLUENT 4
EFFLUENT 5
EFFLUENT 6
EFFLUENT 7
EFFLUENT 8
EFFLUENT 9
EFFLUENT 10
EFFLUENT 11
EFFLUENT 12
EFFLUENT 13
EFFLUENT 14
EFFLUEHT 15
EFFLUEHT 16
EFFLUENT 17
EFFLUENT 18

An/AO
$1.84 E-01$
$8.98 E-02$
$2.81 E-02$
$5.6 B E-02$
$1.67 E-02$
$3.11 E-02$
$1.69 E-02$
$2.99 E-02$
$1.16 E-02$
$1.59 E-02$
$9.64 E-03$
$1.80 E-02$
$1.91 E-02$
$1.34 E-02$
$3.11 E-03$
$8.99 E-03$
$1.14 E-02$
$5.49 E-03$.

CU1. An/AO -

DAYS
$3.00 E+00$
$7.00 E+00$
$1.10 E+01$
$1.50 E+01$
$1.30 E+01$
$2.20 E+01$
$3.50 E+01$
$2.90 E+01$
$3.20 E+01$
$3.50 E+01$
$3.90 E+01$
$4.60 E+01$
$5.30 E+01$
$5.70 E+01$
$6.60 E+01$
$5.40 E+01$
$5.30 E+01$
$7.20 E+01$

$\checkmark$ inc.

$2.36 E-20$

iิ Eua.

\subsection{E-01}

2.74E-01

$2.01 E-20$

$2.36 E-20$

3. $02 E-0 !$

3. 59E-01

$3.76 E-01$

$4.07 E-01$

4.23E-01

4.54E-01

$4.65 E-01$

4. $81 \mathrm{E}-01$

$4.91 E-01$

5. $09 E-01$

5. $23 E-01$

5. 4IE-0i

$5.50 E-01$

5. 59E-01

$5.71 E-01$

$3.655-21$

$4.92 E-\mathrm{j} \overline{8}$

$3.95 E-08$

$2.17 \mathrm{E}-20$

4. 40E- $-\mathrm{i} E$

$4.23 E-21$

$3.31 E-\hat{v B}$

$1.00 E-20$

$4.07 E-00$

6. $20 \mathrm{E}-2 !$

J. $21 E-60$

1. $25 \mathrm{E}-20$

3. $355-68$

$3.77 \mathrm{E}-21$

3. $27 E-48$

7. $23 E-2 !$

3. $i 4 E-1 B$

1. IิEE-21

3. 52E-

2. BSE-2!

3.20 - 68

3. V⿺E-2!

3. IGE-

$5.13 E-21$

3. $6 G E-98$

$4,49 E-21$

3. $\dot{W} T E-T B$

2. 6) UE-Z

3. 02E-18

2. 8BE-2!

2.

5. 36 E-01

1. $36 \mathrm{E}-21$

2. $54 E-13$

TABLE B.20c.

Aluminum (A1) Leach Fractions from Flow Through Test Crushed Grout/Sediment with Groundwater (DIFCGTC)

\begin{tabular}{|c|c|c|c|c|c|}
\hline SAMPLE & An/AD & CUs. AniAO & DÂYS & $D$ inc. & $\exists$ cLiB. \\
\hline EFFLUERT I & $1.15 E-03$ & $1.155-03$ & $3.065+60$ & $9.175-25$ & S.17E-25 \\
\hline EFFLUEMT 2 & $2.30 E-6 J$ & $3.95 E-03$ & $7.00 E+00$ & 1. SGE-ZJ & $4.23 E-24$ \\
\hline EFFIUENT 3 & $5.75 E-04$ & 4. $. \dot{J} E-0 J$ & $1.165+01$ & $2.115-24$ & 4. $055-24$ \\
\hline EFFLUEKT \& & i. $57 \mathrm{TE}-0 \mathrm{~J}$ & $6.195-\hat{v} 3$ & 1.50̂E+01 & i. $65 \mathrm{E}-23$ & $5.35 E-24$ \\
\hline EFFIUENT 5 & $3.31 E-04$ & $6.53 E-03$ & $1.30 E+i 0$ & ¿. $5 T E-24$ & $4.725-24$ \\
\hline EFFLUENT 'G & $6.51 E-0.4$ & $7.1 B E-03$ & $2.20 E+01$ & 4. $46 E-24$ & $4.37 E-34$ \\
\hline EFFLUENT 7 & 3. $955-34$ & $7.57 \mathrm{E}-0 \mathrm{~J}$ & $2.50 E \div+11$ & $3.39 E-24$ & บ. $.77 E-Z ;$ \\
\hline EFFLUENT 8 & 5. 80 E- 194 & $8.35 E-03$ & $2.76 E+01$ & $E .52 E-24$ & $5.00 E-24$ \\
\hline EFIUEHT ? & $2.92 E-\hat{04}$ & 3.SAE-0j & $3.20 E+4 !$ & $2.415-34$ & 4. 3 gE- -34 \\
\hline EFLUEHT 16 & $4.69 E-14$ & $9.05 E-03$ & $3.505+i v 1$ & 5.1EE-24 & 4.97:-37 \\
\hline EEFEUEHT 11 & $\overline{2} .33 E-04$ & $9.25 E-03$ & $3.905+61$ & 1. BEE-24 & L. E) \\
\hline EFF!UEMT 12 & $3.335-34$ & $3.68[-0]$ & 4.56 E +iil & 1.1:E-24 & i. $24=-24$ \\
\hline EFFLUENT IJ & $3.225-04$ & J. $605-02$ & S. Jive-vi & $0.71 f-25$ & J. $=2 E-34$ \\
\hline EFluEht it & $2.40 E-44$ & $1,02 E-02$ & 5. $: \hat{G E+\hat{n} !}$ & $2.65 E-24$ & S. 3JE-Zi \\
\hline EFFUENT 15 & $1.5 E=-34$ & 1. $\mathrm{j}$ AE-i2 & Q. WBE & $1.20 \bar{E}-24$ & $\therefore$ 口. $4 E-3$ \\
\hline EFrouENi it & i.joE- 04 & $1.05 E-32$ & S. $40 E+41$ & 5. $39 E-25$ & S. BSE- -4 \\
\hline EFFLUEHT Li & 1. $345-14$ & i. & $0.905 \div 64$ & 3. IDE -25 & 3. $13 E=-2$ \\
\hline EFFLIENT IO & 5.b5E-195 & $1.07 E-\hat{v} 2$ & $\therefore \quad \angle 0 E+6 i$ & $265 E-25$ & Z.JE-34 \\
\hline
\end{tabular}


TABLE B.20d. Silica (Si) Leach Fractions from Flow Through Test Crushed Grout/Sediment with Groundwater (DIFCGTC)

\begin{tabular}{|c|c|c|c|c|c|}
\hline SAMPLE & $A n / A O$ & CUn. An/Âo & DAYS & $\bar{D}$ inc. & $D$ EUh, \\
\hline EFFLUENT 1 & $8.59 E-03$ & 8.575- 03 & 3. $\hat{U} 0 E+\hat{O} 0$ & $5.11 E-23$ & 5.11E-23 \\
\hline EFFLUEKT 2 & $7.27 E-03$ & $1.59 E-02$ & $7.60 E+00$ & $1.32 E-22$ & $7.48 E-23$ \\
\hline EFFLUENT 3 & 2. $50 E-0 J$ & $1.88 E-02$ & $1.10 E+01$ & 3. $77 E-23$ & $6.65 E-23$ \\
\hline EFFLUERI 4 & $6.56 E-i 33$ & $2.53 \mathrm{JE}-\mathrm{v} 2$ & $1.50 E+01$ & $2.69 E-22$ & 5. $895-23$ \\
\hline EFFLUEHT 5 & $1.96 E-03$ & 2.73E-02 & 1. $80 E+0 !$ & $5.36 E-23$ & $3.60 E-2 J$ \\
\hline EFFLLENT 6 & $3.25 E-03$ & $3.05 E-02$ & $2.20 E+01$ & $1.09 E-22$ & อ. ว1E-23 \\
\hline EFFLUEENI 7 & $1.70 E-03$ & 3. $22 E-02$ & $2.50 E+01$ & $6.30 E-23$ & ô.645-2j \\
\hline EFFLUENT 3 & 2.jJE- 03 & $3.46 E-02$ & 2. $4(10 E+01$ & $T .87 E-2 \mathrm{~J}$ & 3. $58 E-23$ \\
\hline EFFLUEHT 9 & P.52E-64 & 3. 5oc-02 & $3.20 E \div 01$ & $2.55 E-23$ & $8.21 E-23$ \\
\hline EFFLUEHT $1 \hat{V}$ & $1.06 E-03$ & $3.66 E-02$ & $3.505+01$ & 3. $50 E-23$ & $7.77 \mathrm{E}-23$ \\
\hline EFF!UENT i1 & $7.18 E-64$ & $3.755-02$ & $3.90 E+01$ & $1.62 E-3 j$ & $7.51 E-33$ \\
\hline EFFLUEHT 12 & $1.63 E-63$ & 3. $92 E-62$ & $4.60 E+01$ & 1. : :E-23 & $6.93 E-13$ \\
\hline EFELUENT 13 & $1.27 E-03$ & $4,04 E-62$ & $5.302+01$ & S. J5E-Z3 & $0.42 E-23$ \\
\hline EFFLUEMT IA & $0.15 E-64$ & $4.10 E-02$ & 5. $7 \hat{\mathrm{v} E}+01$ & j.vaE-2.j & b. $155-23$ \\
\hline EFFLUENT 15 & $3.24 E-04$ & $4 . j 4 E-62$ & $6.00 E+0 \mathrm{I}$ & $5.68 E-24$ & 5. $93 E-23$ \\
\hline EFFLUENT Ió & $2.96 E-694$ & $4.17 E-02$ & b. $40 E+0 i$ & $2.32 E-24$ & $5.64 E-23$ \\
\hline EFF:UEHT 17 & 2. $77 E-04$ & $4.19 E-02$ & $6.90 E+61$ & $1.72 E-24$ & $5.305-23$ \\
\hline EFFLUEHT I8 & $i, 04 E-64$ & $4.21 E-\hat{v} z$ & $7.20 E+01$ & $\vec{j} .01 E-25$ & 5.11E-Z3 \\
\hline
\end{tabular}

TABLE B.20e. Boron (as H3B03) Leach Fractions from Flow Through Test Crushed Grout/Sediment with Groundwater (DIFCGTA)

\begin{tabular}{|c|c|c|c|c|c|}
\hline SAMPLE & $\mathrm{An} / \mathrm{AD}^{\circ}$ & Cun. Ânitho & DAYS & ì inc. & ì sun. \\
\hline EFIUENT I & $4.74 E-02$ & $4.74 E-02$ & $3.00 E+00$ & $1.56 E-21$ & 1.56e-21 \\
\hline EFFLUENT 2 & $9.8 \hat{v} E-\hat{U} 3$ & $5.72 E-02$ & $7.605+60$ & $2.39 E-22$ & $9.71 E-22$ \\
\hline EFFLLENT 3 & $2.355-a 3$ & $5.95 E-02$ & $1.10 \varepsilon \div 01$ & i. $5 \dot{0} E-2 j$ & $0.76 E-22$ \\
\hline EFFLUENT \& & $5.6 B E-63$ & $0.52 E-12$ & $i .50 E \div \hat{H}$ & $2.16 E-22$ & 5. $39 E-\not Z$ \\
\hline EFFLUEHT 5 & $1.49 E-03$ & $6.67 E-62$ & 1. $80 E+01$ & 3. 3อE-23 & $5.14 E-22$ \\
\hline EFFLUEKT $S$ & $2.25 E-6 j$ & $0.89 E-02$ & $2.20 E+01$ & $5.25 E-23$ & $4.47 E-2 Z$ \\
\hline EFFLUENT 7 & $1.255-03$ & $7.02 E-62$ & $2.50 E+i i j$ & $5.37 E-23$ & 4. \\
\hline EFFLUEHT 8 & 2.:0E-63 & $\overline{1} .23 E-02$ & $2.70 E+01$ & 6.iBE-2J. & $3.75 E-2$ \\
\hline EFFLUENT ? & ล. ธอร- 14 & $7.31 E-02$ & $3.20 E+0 !$ & $2 .: 2 E-33$ & S.4. \\
\hline EFFLWEHT 10 & 1. $14 E-03$ & $7.43 E-6 z$ & 3. $50 E+\hat{0}:$ & $4.60 E-23$ & $3.20 E-3$ \\
\hline EFFLUEHT is & $\overline{1} .70 E-04$ & 7.51E-62 & $3.70 E \div 01$ & X.:AE-ZJ & 3. \\
\hline EFFLUEHT IE & 1.6SE-6] & $\bar{i} .67 E-12$ & $4.606 \div 0:$ & $1.92 E-33$ & 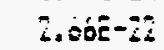 \\
\hline EFFIUEHT 13 & 2. & $i=8 i E-02$ & $5.305+01$ & ?.44E-3J & $2.95=-8$ \\
\hline EFELIIENT I4 & $\{.34 E-i 3$ & อ. $015-\hat{v i}$ & $5.70 E+0 j$ & $5,13 E-25$ & $\therefore 3+2$ \\
\hline EF?LENT 15 & $7.65 E-04$ & 8. $6 \mathrm{BE}^{-192}$ & 2. GaE+T! & $3,5 \mathrm{SE}-3 \mathrm{~S}$ & $\therefore 2 E-2$ \\
\hline EFEVERT IS & $7.34 E-34$ & E. $\hat{i S E-\hat{v} \mathbf{z}}$ & $0.4(0[-1)$ & Z.E:E-2i & $2.17=-23$ \\
\hline EFELYENT $: 7$ & 1.23E-33 & 3. 3ive- & อ. $36 E+6 !$ & $3 . X j E-53$ & $\therefore$ - \\
\hline EFfuent lo & S.ETE-YA & 3.3iE-6i & T.20E+i!! & $3,0 I E-25$ & $Z, X E-Z$ \\
\hline
\end{tabular}




\section{TABLE B.20f.}

Fluoride (F) Leach Fractions from Flow Through Test Crushed Grout/Sediment with Groundwater (DIFCGTA)

\begin{tabular}{|c|c|c|c|c|c|}
\hline SÂMPLE & An/AO & cua. An/AO & DAYS & $D$ inc. & 0 ELis. \\
\hline EFFLUENT I & $3.33 E-113$ & B. $33 \mathrm{JE}-0 \mathrm{j}$ & $3.00 E+00$ & $5.90 E-23$ & S. $4 \hat{v E}-Z \bar{J}$ \\
\hline EFFLUENT 2 & $6.06 E-03$ & $1.49 E-02$ & 7. UOE+100 & $9.15 E-23$ & $6.595-23$ \\
\hline EFFLUEHT 3 & $1.70 E-03$ & $1.66 E-102$ & $1.10 E+01$ & $3.34 E-23$ & $5.20 E-23$ \\
\hline EFFLLLENT 4 & $2.61 \mathrm{E}-0 \mathrm{Z}$ & $1.72 E-02$ & $1.50 E+01$ & $4.59 E-23$ & $5.11 E-23$ \\
\hline EFFLUEHT 5 & $i .\{4 E-64$ & $1.79 \mathrm{E}-\mathrm{C2}$ & 1. OOE+OUI & $6.4 J E-24$ & $4.60 \mathrm{VE}-23$ \\
\hline EFFLUENT: & 1. $20 E-\hat{v} 3$ & 2. iZE-02 & $2.20 E+01$ & 1. SUE-ZJ & $4.2 \pi c-23$ \\
\hline EFFLUENT 7 & $6.77 E-64$ & 2. IEE-02 & $2.50 E+619$ & 1. DEE- 23 & $3.75-3$ \\
\hline EFFLUEIT \& & $1.36 E-10 j$ & 2. $32 E-\hat{v} 2$ & $2.90 E+001$ & Z. ÓLE-ZS & $3.30 E-23$ \\
\hline EFFLUEHT 9 & $5.355-04$ & $2.36 E-12$ & $3.20 \mathrm{E}+\mathrm{ii}$ & $9.85 E-24$ & $\operatorname{sic} \theta-23$ \\
\hline EFFuJEIT io & $3.295-64$ & $2.46 \mathrm{E}-32$ & $3.5 j \bar{j}+i j i$ & 2. IJE-2J & 3 SEE- 3 \\
\hline EFFLUEHT II & 5. T6E-64 & $2.52 E-62$ & $3.70 \mathrm{E}+0 \mathrm{j}$ & $5.75 E-24$ & $3.36 E-23$ \\
\hline EFFILEHT IZ & 1. $16 E-\hat{i}]$ & $2 . \Delta \mathrm{BE}-\hat{\mathrm{v} z}$ & A. BVE $\div \hat{V} 1$ & $7.675-24$ & $3.13 E-23$ \\
\hline EFFLUENT 13 & $1.37 E-13]$ & $2.77 \bar{E}-12$ & $5.30 E+01$ & $1.62 E-2 j$ & $3.025-23$ \\
\hline EFFLUENT I4 & $1.21 \mathrm{I}-\hat{\mathrm{v}} \mathrm{Z}$ & $2.39 E-02$ & $5.70 E+01$ & $4.16 E-2 j$ & $3.05 E-23$ \\
\hline EFFLLERT 15 & $B .88 E-\hat{v} 4$ & $2.36 E-02$ & $6.00 E+011$ & $4.27 E-23$ & $3.6 B E-23$ \\
\hline EFFLUENT $i b$ & $9.59 E-114$ & $3.00 \mathrm{E}-02$ & $6.40 E+01$ & $2.96 E-23$ & $3.36 E-23$ \\
\hline EFFLLEHT 17 & $1.39 E-03$ & 3. $22 E-\hat{\mathrm{v} 2}$ & $6.905 \div 01$ & $4.25-23$ & $3.12 E-25$ \\
\hline EFFLUENT I8 & $9.17 E-04$ & $3.31 E-02$ & $i .20 E+01$ & $5.4 \partial E-2 j$ & J. $16 E-23$ \\
\hline
\end{tabular}

Nitrate (NO3) Leach Fractions from Flow Through Test Crushed Grout/Sediment with Groundwater (DIFCGTA)

\begin{abstract}
SAHPLE
EFFLUENT I

EFFLUEHT 2

EFFLUEHT 3

EFFLUENT \&

EFFLUEHT 5

EFFLUEHT 6

EFFLUEHT $;$

EFFLUEKT a

EFFLUENT ?

EFivent it

EFFIUENT II

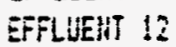

EFFLLENT 13

EFFLUENT :4

EFFLUENT 15

EFflueir 16

EFulueri 17

EFLLEHT IS
\end{abstract}

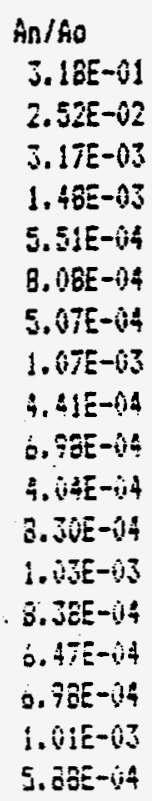

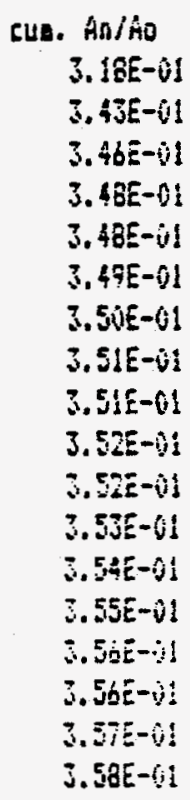

CUE. AR/AO

3. ISE- ถิ

3. 43E- 41

$3.46 \mathrm{E}-\mathrm{bl}$

$3.46 E-0 \mathrm{i}$

3.4BE- $\mathrm{il}$

3. 49E-01

3.SUE-0!

3.51E-

3. 5|E-0!

3.52E-1i

$3.52-01$

$3.53 E-01$

$3.54[-01$

¿. $55 E-v \hat{~} 1$

B. $5 \mathrm{j}[-31$

3. $56[-i)$

$3.55-6 !$

3. 5 SE- -61

DAYYS

3. $00 E+100$

$7.60 E+60$

1. $10 E+01$

1.50E+01

1. BuEt+11

2. 20E+(1)

$2.5 \mathrm{kE}+\hat{N i}$

$2.70 E+01$

$3.20 E+01$

$3.50 E+01$

3. TET

4. OUE +0 i

S. juE TiI

5. $7 \hat{0 E}+\mathrm{Q}$

S. WE-T!

b. $4 \dot{x}=+i a !$

5. 5 ineibl

3. $2 \mathrm{EE}+0$ ! jinc.

I.).ปtE- 2

1. STE-a!

4.

i. $47 E-23$

4.

6. $78 E-34$

S. SEE -2

1. $59 \mathrm{E}-\mathrm{S}$

5. $46 E-24$

1. SIE-

S. $14 E-2$

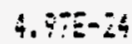

ล. $3 \mathrm{BE}-29$

$2.61 E-23$

ב.

i. $57 \mathrm{E}-\mathrm{ij}$

Z.A.

- $25 \mathrm{E}-3$ is cat.

1. 6 6E- -07

3. $32 E-615$

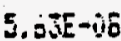

4. $13 E-60$

उ. $44 \mathrm{E}-\mathrm{-45}$

2. $.25-18$

2. $465-98$

3.1 $1.5-j \mathrm{j}$

1. $34 \mathrm{E}-\mathrm{AS}$

1. .77E-SE

1.59E- is

¿.JSE-15

1. $177^{3}-86$

1. $165-68$

$1.10 E-6 \bar{S}$

1. $6 \mathrm{BE}-\mathrm{it}$

T. $375-69$

จ. 
TABLE B.20i. Technetium-99 (TC-99) Leach Fractions from Flow Through Test, Crushed/Sediment with Groundwater (CGT)

\begin{tabular}{|c|c|c|c|c|c|c|c|}
\hline SAMPLE & PORE VOLUAE & $\mathrm{UCI}$ & IIME DAYS & An/AO & CUH An/AO & DIHC CK2/SEC & DCUN EM2/SEL \\
\hline [BT-] & $2.66 E+00$ & $5.84 E-01$ & $3.00 E+60$ & $4.325-01$ & 4. $32 E-01$ & $3.34 E-97$ & $3.34[-6]$ \\
\hline CET-2 & $4.80 E+00$ & $4.07 E-02$ & 7. $00 E+00$ & $3.01 E-02$ & $4.62 E-01$ & $4.90 \mathrm{E}-21$ & $1.77 E-01$ \\
\hline CoT-3 & $3.76 E+00$ & $7.09 E-03$ & $1.10 E+01$ & $5.25 E-03$ & $4.6 B E-01$ & $1.67 E-22$ & $1.13 E-07$ \\
\hline CET-4 & $8.29 E+60$ & $1.24 E-02$ & $1.50 E+01$ & $9.16 E-03$ & $4.77 E-01$ & $5.06 E-22$ & $8.72 E-38$ \\
\hline CET-5 & $9.18 E+00$ & $2.66 E-03$ & $1.30 E+01$ & $1.97 E-03$ & $4.79 E-(1)$ & $3.56 E-23$ & $7.27 E-08$ \\
\hline$C E T-6$ & $1.1 \mid E+\hat{0} 1$ & $2.45 E-03$ & $2.20 E+01$ & $1.82 E-0 J$ & $4.81 E-01$ & 1.7AE-2J & $5.75 E-68$ \\
\hline CET-7 & $1.24 E+01$ & $1.50 E-03$ & $2.50 E+01$ & $1.11 E-03$ & $4.82 E-01$ & 1.1JE-2J & $5.23 E-08$ \\
\hline CST -8 & $1.49 E+01$ & 1. $32 E-03$ & $2.90 E+01$ & $9.76 E-04$ & 4.83E-01 & 5. $15 E-24$ & $4.51 E-198$ \\
\hline CET-9 & $1.60 E+01$ & $6.90 \mathrm{E}-04$ & $3.20 E+01$ & $5.10 E-04$ & $4.63 E-01$ & $2.385-24$ & 4.5 VE- 68 \\
\hline $\operatorname{CET}-10$ & $1.77 E+11$ & $1.54 E-03$ & $3.50 E+0 \mathrm{i}$ & $1.14 E-0]$ & $4.34 E-01$ & $1.05 E-2 \bar{J}$ & J. $74 E-\hat{v o}$ \\
\hline CET-1I & $1.27 E+01$ & $1.11 E-03$ & $3.90 E+01$ & S.19E-04 & $4.35[-01]$ & $3.63 E-24$ & $3.52 E-68$ \\
\hline $\operatorname{CET}-12$ & $2.07 E+01$ & $2.24 E-03$ & $4.60 E+01$ & $1.63 E-03$ & $4.8 \pi E-01$ & $7.70 E-24$ & 2. $79 E-018$ \\
\hline CET-13 & $2 . j 1 E+01$ & i. $41 E-0 j$ & $5.30 E+01$ & 1.0SE-0j & $4.68 E-01$ & $3.79 E-24$ & Z. $54 E-j E$ \\
\hline COT -14 & 2.52E+01 & $7.435-\hat{v} 4$ & $5.70 E+01$ & $5.50 E-04$ & $4.88 E-01$ & $2.35 E-24$ & $2.41 E-68$ \\
\hline COT-15 & $2.57 E+01 !$ & $6.64 E-04$ & b. DOE+01 & $4.32 E-64$ & $4.89 E-01$ & $2.21 E-24$ & 2. $29 E-06$ \\
\hline CET-16 & $2.84 E+01$ & $8.37 E-04$ & $6.40 E+01$ & $6.2 \mathrm{GE}-04$ & $4.50 E-01$ & $2.08 E-24$ & $2.15 E-08$ \\
\hline$[6]-1]$ & $3.09 E+01$ & $1.42 \mathrm{E}-03$ & $6.90 E \div 0:$ & ¿.05E-03 & $4.315-01$ & $4.735-24$ & i. $39 E-60$ \\
\hline COT-iB & J.JOE +01 & $6.76 E-04$ & $7.20 E+01$ & 5. 15E-04 & $4.91 E-01$ & $2.69 E-24$ & 1. $91=-08$ \\
\hline
\end{tabular}


TABLE B.21a. Potassium (K) Leach Fractions from Flow Through Test Crushed Grout/Sediment with Groundwater (DIFCGCC)

\begin{tabular}{|c|c|c|c|c|c|}
\hline SAMPLE & An $/ A O$ & CUL. AnjÁ & DAYS & Dinc. & D Eus. \\
\hline EFFLUEHT 1 & $4.73 E-03$ & $4.73 \mathrm{E}-03$ & $3.00 E+60$ & $1.55 E-23$ & $1.55 E-23$ \\
\hline EFFLUENT 2 & $0.00 E+00$ & $4.73 E-03$ & $7.00 E+00$ & $0.00 E+00$ & $0.64 E-24$ \\
\hline EFFLUENT 3 & $0.00 E+00$ & $4.73 E-03$ & $9.00 E+00$ & $0.00 E+00$ & $5.16 E-24$ \\
\hline EFFLUEHT 4 & $0.00 E+60$ & $4.735-03$ & 1.30E+01 & $0.00 E+00$ & $3.57 E-24$ \\
\hline EFFLUEHT 5 & $0.00 E+100$ & $4.73 E-03$ & $1.60 E+01$ & $0.00 E+00$ & $2.70 E-24$ \\
\hline EFFLLUEHT $b$ & $0.00 E+00$ & $4.73 E-0 J$ & $2.00 E+01$ & $0.00 E \pm 00$ & $2.32=-24$ \\
\hline EFFLUENT 7 & $0.0 G E \div 00$ & $4.73 E-03$ & $2.40 E+0 !$ & $0.00 E+0 i j$ & $1.745-24$ \\
\hline EFFLUEKT 8 & $0.60 E+60$ & $4.73 \mathrm{~J}-0 \mathrm{~J}$ & $2.60 E+011$ & $0.00 E+00$ & !.bóE-24 \\
\hline EFFLUENT 9 & $0.00 E+00$ & $4.73 E-03$ & $3,10 E+01$ & $0.00 E+20$ & 1. Sive- 39 \\
\hline EFFLUEHT 11 & $0.00 E+60$ & $4.73 E-03$ & $3.80 E+0 \mathrm{i}$ & $0.04 E \div 00$ & 1.2ZE-24 \\
\hline EFF!UENT 13 & $1.00 E-01$ & 1.05E-0.1 & $4.4 \hat{v} E+6 i$ & $9.555-26$ & $5.23 E-22$ \\
\hline EFFLUEMT :5 & $5.43 E-62$ & 1. $60 E-01$ & $.5 .10 E+01$ & $2.30 E-20$ & 1. $14 E-2 i$ \\
\hline EFFLUENT I7 & $4.05 E-02$ & 2. $\hat{0 A E}-\hat{\mathrm{O}} \mathrm{i}$ & $0.0 \hat{E}+01$ & $9.34 E-21$ & $1.395-3$ \\
\hline EFFLUENT 19 & $2.42 \mathrm{E}-02$ & $2.24 E-i \mathrm{i}$ & $0.60 E+6 i$ & 0.5IE-2! & 3. 36E-70? \\
\hline EFFLLEHT $2 !$ & $1.31 E-02$ & $2.4|E-0|$ & $7.30 E+01$ & $3.46 E-21$ & J. otE- $0 \%$ \\
\hline EFFLUENT 24 & $1.80 E-12$ & $2.58 E-01$ & $0.70 E+01$ & $1 . i 0 E-i j$ & $3.67 E-09$ \\
\hline EFFLUENT 26 & $7.60 E-63$ & 2.59E-31 & 9. $90 E+0 !$ & $1.72 E-21$ & 5.5oE-7i \\
\hline EFFLUENT Z3 & $0.4 B E-03$ & 2. $35 E-0 i$ & $1.01 E+02$ & 6. ร́o $E-22$ & $3.41 E-6 \%$ \\
\hline EFFLUEKT 30 & $0.46 E-03$ & $2.22 E-01$ & $1.13 E+02$ & $2.5 x E-22$ & 3. उ०E-0? \\
\hline EFFLUENT 32 & $1.09 E-003$ & $2.83 E-01$ & $1.22 E+02$ & $1.43 E-23$ & S. $15 E-19$ \\
\hline EFLLUEHT 34 & $6.3 j E-03$ & $2.90 \mathrm{E}-01$ & $1.58 E+02$ & $4.17 E-2 J$ & 2.56E- 59 \\
\hline EFFLUEHT 35 & $0.00 E+00$ & 2. $90 E-01$ & $1.865+02$ & $0.60 E+00$ & $2.16 E-96$ \\
\hline
\end{tabular}


TABLE B.21b. Sodium (Na) Leach Fractions from Flow Through Test Crushed Grout/Sediment with Groundwater (DIFCGCC)

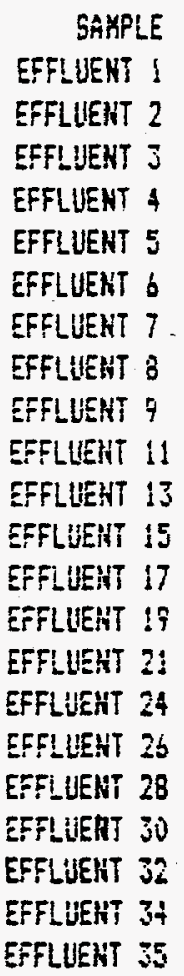

An/AO
$2.50 E-01$
$1.6 B E-01$
$5.97 E-02$
$3.42 E-02$
$2.37 E-02$
$2.46 E-02$
$1.67 E-02$
$1.10 E-02$
$3.69 E-0 J$
$1.72 E-02$
$5.49 E-03$
$0.00 E+00$
$0.66 E+00$
$0.00 E+00$
$0.00 E+00$
$0.00 E+00$
$0.06 E+00$
$0.00 E+00$
$0.00 E+00$
$0.00 E+00$
$9.71 E-05$
$2.61 E-04$

CUI. AN/AOO DAYS

An ho

4. $19 E-01$

4. $7 \mathrm{BE}-01$

$5.12 E-01$

ร. उB́E-01

5. $61 E-01$

$5.79 E-01$

5. $90 E-01$

$5.94 E-01$

6. $11 E-01$

6. $: i E-01$

6. $17 E-\hat{v} !$

O. $.17 \mathrm{E}-\hat{\mathrm{u}} !$

$6.17 E-01$

b. $17 E-01$

6. $17 E-01$

6. $17 E-01$

b. $17 E-01$

6. $17 E-0$ !

6. $17 E-01$

D. IIE-VI

b. $17 E-01$
$3.00 E+10$

7.00E+00

$3.00 E+00$

$\therefore .30 E+01$

$1.60 E+01$

2.00E+01

2. $40 E-01$

2. BOE+OI

3. $10 E \div 0 \mathrm{i}$

3. BNE+01

4. $40 E+O i$

5. JOE $+0 \mathrm{I}$

S. QOE +Oid

b. GOE $O$ OI

T. JiE +Ui

3. 70 E +01

9.46E+W1

1. OIE +0.2

1. $13 E+02$

$1.22 E+02$

1. $58 E+02$

1. 8BE+02
0 inc.

$4.35 E-20$

$7.05 E-20$

$5.30 E-20$

6. $65 E-2 !$

7. $48 E-21$

5. $62 E-21$

3. $98 E-21$

i. $6.3 E-21$

$3.72 E-22$

4. $73 E-21$

$2.55 E-2 z$

Q. $00 \mathrm{OQ}+00$

0 . WUE?

D. OUETOO

$0.00 E+60$

$0.06 E \div 00$

D. VUE+QO

O. NOE+OOO

C. DGE 0 OO

0. $60 E+00$

3. $79 \pm-2 i$

1. $67 E-25$
0 cus.

7. $74 E-18$

!. 36E- 37

¿.45E-67

1. $\{\mathrm{JE}-\hat{0}\}$

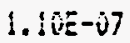

$4.57 E-08$

3. E2E-vi

$7.92 E-01 a$

7. TEE-ie

6. $16 E-16$

S. TEE-TE

4. $765-98$

$4.225-16$

3. $34 E-08$

$3.47 E-i 60$

$2.51 E-i j a$

2. $07 E-68$

2.5IE-

$2.24 E-j \mathrm{~s}$

$2.695-68$

I. 30E-

4. JEE- 
TABLE B.21C. Aluminum (A1) Leach Fractions from Flow Through Test Crushed Grout/Sediment with Groundwater (DIFCGCC)

\begin{tabular}{|c|c|c|c|c|c|}
\hline SAMPLE & An $/$ AO & Cul. An/Ao . & DAYS & Dinc. & $D$ cus. \\
\hline EFFLUEHT 1 & $4.27 E-03$ & $4.27 E-03$ & 3. $10 E+60$ & $1.26 E-23$ & $1.26 E-23$ \\
\hline EFFLUENT $?$ & $5.58 E-03$ & $9.85 E-03$ & $3.00 E+10$ & $7.75 \mathrm{E}-2 \mathrm{~J}$ & 2. $38 E-2 J$ \\
\hline EFFLUEERT 3 & $2.57 E-03$ & $1.24 E-02$ & $9.60 E+00$ & $1.10 E-22$ & $3.56 E-23$ \\
\hline EFFLUERT 4 & $1.65 \mathrm{E}-03$ & $1.41 E-02$ & 1. $30 E+01$ & $1.54 E-23$ & $3.165-23$ \\
\hline EFFLUENT $\Xi$ & 1.1PE-03 & 1.5JE-02 & $1.60 E+01$ & $1.70 E-2 J$ & $3.035-23$ \\
\hline EFFIUEHT ' & !. J6E- 43 & $1.64 E-02$ & $2.50 E+01$ & $1.30 E-2 j$ & $2.5: 5-23$ \\
\hline EFFLUENT 7 & a. $79 E-04$ & $1.73 E-02$ & $2.40 E+01$ & 8.5IE-24 & $2.60 E-23$ \\
\hline EFFLUENT & $4.77 E-64$ & $1.78 E-02$ & 2. $30 E+0 !$ & $3.07 E-24$ & 2.J5E-2J \\
\hline EFfliteht 9 & $1.36 E-04$ & $1.79 E-02$ & $3.105+01$ & $5.05 E-25$ & $2.16 E-23$ \\
\hline EFFLUENT II & $5.11 E-04$ & $1.84 E-02$ & $3.80 E+01$ & $1.52 E-24$ & $1.36=-23$ \\
\hline EFFLUENT 13 & $4.50 E-04$ & 1. B3E-02 & $4.40 E+01$ & 1.91E-27 & 1. $.9 \mathrm{E}-23$ \\
\hline EFFLLUENT 15 & $1.51 E-64$ & $1.90 E-62$ & $5.10 E+01$ & i. $65 E-25$ & $\therefore, 46 E-23$ \\
\hline EFFLUUHT IT & $2.365-04$ & $1.93 E-02$ & $0.00 E+01$ & $3.17 \pm-25$ & $3.29 E-23$ \\
\hline ESFLUEHT I9 & $4.13 E-04$ & 1. $77 E-02$ & S. $60 E+01$ & $2.54 E-24$ & $1.225-23$ \\
\hline EFFLUERT 21 & $3.83 E-04$ & $2.01 E-02$ & $7.30 E+01$ & 1.73E-24 & .. $.5 E-23$ \\
\hline EFFLUENT 24 & $6.48 E-64$ & $2.07 E-02$ & $8.70 E+01$ & 1. $42 E-24$ & 1. $03 E-23$ \\
\hline EFFLUERT 26 & $7.67 E-04$ & 2.: $5 E-02$ & $9.40 E \div 01$ & $9.03 E-24$ & $1,025-23$ \\
\hline EFFLUEHT 28 & $1.06 E-03$ & $2.26 E-02$ & $1.01 E+02$ & $1.87 E-23$ & 1. $.65 E-23$ \\
\hline EFFLUENT 30 & $1.01 E-03$ & 2. $36 E-02$ & $1.13 E+02$ & $6.30 E-24$ & 1. . VZE- 23 \\
\hline EFFLUENT 32 & $1.78 E-03$ & $2.53 E-02$ & $1.22 E+102$ & $3.81 E-23$ & 1. . V9E- -33 \\
\hline EFFLUENT 34 & $4.21 E-03$ & $2.96 E-3.2$ & $1.58 E+02$ & 1.56E-23 & $1.15-23$ \\
\hline EFFLUENT 35 & $3.085-03$ & $3.26 E-02$ & 1. $88 E+02$ & $1.5725-23$ & $1.165-23$ \\
\hline
\end{tabular}


TABLE B.21e. Boron (as H3BO3) Leach Fractions from Flow Through Test Crushed Grout/Sediment with Groundwater (DIFCGCA)

\begin{tabular}{|c|c|c|c|c|c|}
\hline SAHPLE & $\mathrm{An} / \mathrm{AO}_{\mathrm{O}}$ & CUA. An/AO & DAYS & $D$ inc. & $y$ clil. \\
\hline EFFLUEHT 1 & $7.37 E-02$ & $7.37 \mathrm{E}-02$ & $3.00 E+00$ & $3.76 E-2 !$ & $3.76 E-21$ \\
\hline EFFLUENT $i$ & $3.57 E-02$ & $1.09 E-01$ & 7.00E+00 & $3 . \mid 7 E-21$ & $3.55 E-2 \mathrm{i}$ \\
\hline EFFLUEHT 3 & $5.97 E-03$ & $1.15 E-01$ & $9.00 E+60$ & $5.90 E-22$ & 3.07E-21 \\
\hline EFFLUENT 4 & $1.72 E-03$ & 1.17E-0i & $1.30 E+01$ & $1.67 E-23$ & 2. $\mid$ ISE-21 \\
\hline EFFLUERT 5 & $1.52 E-03$ & 1.19E-01 & $1.60 E \div 01$ & $3.09 E-23$ & $1.83 E-31$ \\
\hline EFFLUEET 6 & $1.95 E-03$ & $1.21 E-01$ & $2.00 E+01$ & $3.56 E-2 j$ & 1.51E-21 \\
\hline EFFLUENT 7 & $1.85 E-03$ & $1.22 E-01$ & $2.40 E+01$ & 3. $90 E-23$ & 1. Jủe-21 \\
\hline EFFLUENT 8 & $1.22 E-03$ & $1.24 E-01$ & $2.80 E+01$ & $2.00 E-23$ & $1.13 E-2 j$ \\
\hline EFFLUEKT Y & 4. $63 E-04$ & 1.24E-01 & $3.10 E+0 !$ & $5.33 E-24$ & i. VIE-21 \\
\hline EFFLUENT 11 & $3.10 E-03$ & $1.27 E-01$ & $3.80 E+01$ & $5.00 E-2 j$ & $0.35-22$ \\
\hline EFFLUENT 13 & $3.265-03$ & 1.30E-01 & 4.40E+01 & 1.025-22 & 8. $045-22$ \\
\hline EFFLUENT 15 & $1.97 E-193$ & $1.32 E-0 !$ & 5.10E+01 & $1.75 E-23$ & $7,03 E-22$ \\
\hline EFF!UENT 17 & $1.72 E-03$ & 1.3AE-01 & D. OOE $\div 01$ & $1.68 E-23$ & S. 19E- 22 \\
\hline EFFLUENT 19 & $2.41 E-03$ & $1.36 E-01$ & $6.50 E+011$ & $8.44 E-2 J$ & $5.33 E-22$ \\
\hline EFFLUENT 21 & $1.335-03$ & $1.37 E-01$ & $7.30 E+01$ & $2.08 E-23$ & $5.37 E-22$ \\
\hline EFFLLEEHT 24 & $1.64 E-03$ & 1.39E-01 & $8.70 E+01$ & $9.15 E-24$ & $4.62 \mathrm{E}-22$ \\
\hline EFF: UERT 26 & 1. JóE-03 & $1.40 E-01$ & 9. $40 E+011$ & $2.86 E-23$ & $4.36 E-22$ \\
\hline EFFLUENT $2 B$ & $1.42 E-03$ & $1.42 E-01$ & $1.01 E+02$ & $3.32 E-2 j$ & $4.14 E-22$ \\
\hline EFFLUENT 30 & $1.52 E-03$ & $1.43 \mathrm{E}-01$ & $1.13 E+02$ & $1.43 E-23$ & $3.78 E-22$ \\
\hline EFFLUSENT 32 & $2.15 E-03$ & $1.45 E-01$ & $1.22 E+02$ & $5.59 E-23$ & $3.615-22$ \\
\hline EFFLUEMT 34 & $4.72 E-03$ & $1.50 \mathrm{E}-01$ & $1.58 E+02$ & $2.00 \mathrm{E}-2 \mathrm{~J}$ & 2. $37 E-23$ \\
\hline EFFLUENT 35 & $3.33 E-03$ & 1.5JE-0! & I. $B B E+02$ & $1.77 \mathrm{E}-2 \mathrm{~S}$ & $2 . \operatorname{dig}-22$ \\
\hline
\end{tabular}


TABLE B.21f. Fluoride (F) Leach Fractions from Flow Through Test Crushed Grout/Sediment with Groundwater (DIFCGCA)

\begin{tabular}{|c|c|c|c|c|c|}
\hline SAMPLE & An/Ao & Cun. Aniŝo & DAYS & jinc. & D cus. \\
\hline EFFLUEHT 1 & $9.19 E-03$ & $9.195-03$ & $3.00 E+00$ & $5.86 E-23$ & ร. $26 E-23$ \\
\hline EFFLUENT 2 & 7.37E-03 & $1.66 E-02$ & 7. $00 E+00$ & $1.35 E-22$ & 3.15E-23 \\
\hline EFFLUENT 3 & $2.22 E-03$ & 1.8BE-02 & $9.00 E+00$ & 8. i5E-2J & 6. $155-23$ \\
\hline EFFLUEHT 4 & $1.02 E-03$ & $1.98 E-02$ & $1.30 E+01$ & $5.95 E-24$ & $6.275-23$ \\
\hline EFFLUEHT 5 & $8.64 E-114$ & $2.07 E-02$ & $1.60 E+01$ & $9.97 E-24$ & $5.55 E-23$ \\
\hline EFFLUENT 6 & 1.01E-03 & $2.17 \mathrm{E}-02$ & $2.00 E+01$ & $9.49 E-24$ & $4.69 E-23$ \\
\hline EFFLUEMT 7 & $9.035-04$ & $2.26 \mathrm{E}-02$ & 2. $4 \hat{U} E+01$ & S.3!E-24 & $4.425-23$ \\
\hline EFFLUENT B & $5.6 T E-04$ & $2.31 E-02$ & $2.80 E+01$ & 4. J3E-24 & $3.985-23$ \\
\hline EFFLUENT 9 & 2. $166-104$ & $2.34 E-02$ & $3.10 E+\dot{V} 1$ & $1.27 E-24$ & 3. \\
\hline EFFLUERT Ii & $2.92 E-63$ & $2.03 E-102$ & S.BUE+0I & $4.77 E-23$ & $3.7 B E-2 J$ \\
\hline EFFLUENT 13 & $3.47 E-03$ & $2.98 E-62$ & $4.405 \div 01$ & $1 .: 4 E-22$ & $4.1 B E-23$ \\
\hline EFFLUENT 15 & $1.13 E-03$ & $3.69 E-62$ & $5 .: 0 E+01$ & $1.03 E-2 J$ & J.37E-23 \\
\hline EFFLUENT 17 & $1.41 E-03$ & $3.23 E-02$ & 6. $00 E+01$ & $1.1 \mathrm{JE}-2 \mathrm{~J}$ & $3.6 ! 5-23$ \\
\hline EFFLGEHT If & $1.35 E-03$ & $3.36 E-02$ & D. $60 E+01$ & $2.67 \mathrm{E}-23$ & $3.57 E-23$ \\
\hline EFFLLEETT 21 & $1.46 E-03$ & 3.51E-02 & $7.30 E+01$ & $2.51 E-23$ & $3.51 E-23$ \\
\hline EFFLUEKT 24 & $2.28 E-03$ & $3.74 E-\hat{v} 2$ & $8.70 E+01$ & $1.76 E-23$ & $3.34 E-23$ \\
\hline EFFLUENT 26 & 2. : $6 E-03$ & $3.95 E-02$ & 9.40E+01 & $7.14 E-23$ & $3.46 E-23$ \\
\hline EFFLUENT 28 & $2.52 E-03$ & $4.21 E-02$ & $1.01 E+012$ & $1.05 E-22$ & $3.64 E-23$ \\
\hline EFFLUEHT 30 & $3.19 E-03$ & $4.52 E-02$ & 1. $13 E+02$ & $6.28 E-2 j$ & $3.77 E-23$ \\
\hline EFFLLENT 32 & $4.51 E-03$ & 4. $96 E-02$ & $1.22 E+02$ & $2.45 E-32$ & $4.22 E-23$ \\
\hline EFFLUENT 34 & $1.3 \mathrm{BE}-02$ & S. उaE-02 & $1.58 E+02$ & 1.70E-22 & $5.32 E-23$ \\
\hline EFFLUENT 35 & 1.17E-02 & $7.52 E-02$ & 1. $88 E+02$ & $2.17 E-22$ & $6.26 E-23$ \\
\hline
\end{tabular}


TABLE B.21g. Nitrate (NO3) Leach Fractions from Flow Through Test Crushed Grout/Sediment with Groundwater (DIFCGCA)

\begin{tabular}{|c|c|c|c|c|c|}
\hline SAMPLE & An/Ao & Cun. An/Ao & DAYS & $D$ inc. & 0 Eus. \\
\hline EFFLUE:IT ! & $2.71 E-61$ & 2. 91E-01 & $3.00 E+00$ & $5.89 E-2 \mathrm{j}$ & $1.36 E-07$ \\
\hline EFFIUUENT 2 & d. $29 E-02$ & $3.74 E-01$ & $7.00 E+00$ & $1.71 E-20$ & $1.01 E-07$ \\
\hline EFFLUIENT 3 & $4.30 E-03$ & $3.79 E-01$ & $9.00 E+00$ & $3.06 E-22$ & B. $.31 E-G B$ \\
\hline EFFLUEHT 4 & $0.00 E+00$ & $3.79 E-01$ & $1.30 E+01$ & O. $60 E+00$ & 5. $75 E-06$ \\
\hline EFFLIIENT 5 & $0.60 E+00$ & $3.79 E-01$ & $1.60 E+01$ & $0.00 E+00$ & 4.6IE-08 \\
\hline EFFLUENT & $0.00 E+06$ & $3.79 E-01$ & $2.00 E+0 i$ & $0.60 E+60$ & $3.74 E-08$ \\
\hline EFFILUENT 7 & $0.00 E+00$ & 3.79E-01 & $2.40 E+011$ & $0.00 E+00$ & $3.12 E-66$ \\
\hline EFFLUENT a & $0.00 E+100$ & 3. TFE-Oi & $2.30 E+61$ & $0.605+00$ & $2.67 E-00$ \\
\hline EFFLUEHT ? & $0.00 E+D O$ & $3.79 E-01$ & $3.10 E+01$ & $0.00 E+00$ & $2.41 E-i B S$ \\
\hline EFFLUEHT 11 & 6.00E+ +00 & $3.79 E-01$ & $3.80 E+01$ & $0.60 E+00$ & 1. $77 E-08$ \\
\hline EFFLUENT 13 & $0.00 E+00$ & $3.79 E-01$ & $4.40 E+0 i$ & $0.00 E+06$ & 1.70E-56 \\
\hline EFFLUENT IS & $1.21 E-03$ & $3.80 E-01$ & $5.10 E+01$ & 1.16E-2S & $1.475-68$ \\
\hline EFFLUENT 17 & $1.32 E-03$ & उ. $01 E-0 !$ & $6.00 E+0 !$ & $9.64 E-24$ & $1.25 E-16$ \\
\hline EFFLUENT 17 & $1.26 E-03$ & $3.82 \mathrm{E}-01$ & $6.60 E+01$ & $2.32 E-2 J$ & $1.13 E-00$ \\
\hline EFFLUEHT Z1 & $1.1 a E-1,3$ & $3.84 E-01$ & $3.30 E+01$ & $1.655-23$ & 1. $02 E-16$ \\
\hline EFFIUENT 24 & $1.48 E-43$ & $3.85 E-01$ & $9.70 E+01$ & 7. $46 E-24$ & 9.IOE-OS \\
\hline EFFLUENT 26 & $1.29 E-03$ & $3.865-01$ & $3.40 E+01$ & $2.54 E-23$ & E. $12 \bar{E}-0\}$ \\
\hline EFFLUENT 28 & $1.96 E-003$ & 3. $68 E-01$ & 1. $(1 \mathrm{1} E+1) 2$ & $3.545-23$ & $7.8 \div E-69$ \\
\hline EFFLUENTT 30 & $1.73 E-03$ & $3.90 \mathrm{E}-01$ & $1.13 E+62$ & $1.365-23$ & $7.01 E-49$ \\
\hline EFFLUENT 32 & 2. 3JE-60 & $3.92 E-61$ & $1.22 E+02$ & $6.56 E-25$ & $0.49 E-69$ \\
\hline EFFLUENT 34 & $7.315-63$ & $3.99 E-\hat{V I}$ & $1.58 E+02$ & $4.79 E-25$ & $5,355-39$ \\
\hline EFFLUENT 35 & $4.82 E-63$ & 4. $04 E-01$ & $1.36 E+002$ & $3.705-23$ & 2.42E-19 \\
\hline
\end{tabular}


TABLE B.21i. Cesium-137 (Cs-137) Leach Fractions from Flow Through Test Crushed/Sediment with Groundwater (CGC)

\begin{tabular}{|c|c|c|c|c|c|c|c|}
\hline SAMPLE & PORE VOLUKE & $\mathrm{UCi}$ & IIME DAYS & An/AO & CUN An/AOO & DIKC CH2/SEC & DCLับ CX2/SEC \\
\hline CAN CBC-1 & $2.00 E+00$ & $7.75 E-11$ & . $\quad 3.00 E+00$ & $2.54 E-11$ & $2.54 E-11$ & $4.49 E-40$ & 4. $49 E-40$ \\
\hline CRI CEC-2 & $5.92 E+00$ & $1.22 E-10$ & $7.00 E+00$ & 4.00E-11 & $6.54 E-11$ & $3.63 E-39$ & $1.27 E-38$ \\
\hline CRH CEC-3 & $9.32 \mathbb{E}+00$ & $6.58 E-11$ & $9.00 E+00$ & $2 . j 6 E-11$ & $3.70 E-1 !$ & $7.76 E-39$ & $1.75 E-39$ \\
\hline CRI CEC-4 & $1.29 E+01$ & $6.52 E-11$ & $1.30 E+01$ & $2.14 E-11$ & 1. $0 B E-10$ & $2.14 E-39$ & $1.28 E-39$ \\
\hline CRA CER-5 & $1.66 E+01$ & $6.56 E-11$ & $1.6 \hat{0 E}+01$ & $2.16 E-11$ & $1.30 E-10$ & $4.25 E-39$ & 2.19E-39 \\
\hline CAn Coc-6 & $2 . ! J E+01$ & $9.35 E-11$ & $2.00 E+01$ & $3.07 E-11$ & $1.61 E-10$ & $5.09 E-37$ & $2.68 E-j 9$ \\
\hline CRU CEC-T & $2.67 E+01$ & $9.50 E-11$ & $2.40 E+01$ & $3.12 E-11$ & $1.92 E-10$ & 5.7บE-39 & 3.19E-j9 \\
\hline CSH CGC-ó & $3.01 E+01$ & $8.84 E-11$ & $2.80 E+01$ & $2.80 E-11$ & $2.21 E-10$ & $5.11 E-39$ & $3.62 E-39$ \\
\hline CRY Cố-9 & $3.15 E+01$ & $3.36 E-11$ & j. $10 E+01$ & 1. $: U E-11$ & $2.32 E-10$ & $1: 12 E-38$ & 3.61E-39 \\
\hline $\cos \operatorname{coc}-10$ & $3.465+01$ & $7.78 E-11$ & $3.50 E+01$ & $2.32 E-11$ & 2. $53 E-10$ & $3.72 E-35$ & $3.96 E-79$ \\
\hline CriH CGC-11 & $3.89 E+61$ & $7.14 E-1 !$ & $3.80 E+01$ & $2.35 E-11$ & $2.32 E-10$ & $5.03 E-39$ & $4.345-39$ \\
\hline $\mathrm{CAn}^{4} \mathrm{Ca}-12$ & $4.54 E+01$ & 1.00E-10 & 4.10E+01 & $3.49 E-1 !$ & $3.17 E-10$ & $9.86 E-39$ & 5. $68 E-39$ \\
\hline ERH SAC-1] & $4.99 E+01$ & $1.44 E-10$ & $4.40 E+01$ & $4.71 E-11$ & $3.64 E-10$ & $1.79 E-30$ & $6.25 E-39$ \\
\hline CAY CEC-14 & $5.40 E+01$ & $1.07 E-10$ & $4.80 E+01$ & $3.51 E-11$ & $3.97 E-10$ & $6.665-37$ & S. EAE-3\} \\
\hline CKH CEC- 15 & $5.61 E+01$ & $3.365-11$ & $5.16 E+0 !$ & $1.10 E-11$ & $4.10 E-10$ & 1.11E-3? & 3. $85 E-39$ \\
\hline CRN CGC-16 & 6. OGE +01 & 6. $\$ 1 E-11$ & $5.60 E+01$ & $2.10 \mathrm{E}-11$ & 4.JiE-10 & 1.76́E-39 & $0.89 E-37$ \\
\hline 데 $\mathrm{Coc}-1]$ & 6. $2 B E+01$ & $4.735-11$ & $6.00 E+01$ & $1.55 E-11$ & $4.46 E-10$ & 1.6IE-37 & $0.30 E-39$ \\
\hline CFH CAC-18 & $6.70 E+01$ & $5.84 E-11$ & b. $30 E+01$ & $1.82 E-11$ & $9.66 E-10$ & $3.375-37$ & $7.155-39$ \\
\hline CRH CAC-19 & $T .02 E+0 \mathrm{ii}$ & 1. $\hat{0} A E-\mathrm{j} 0$ & $6.60 E \div 01$ & 3. $43 E-11$ & $5.00 E-10$ & $9.51 E-37$ & 7. $2 J E-3 \varphi$ \\
\hline CAN CAC-20 & $7.35 E+\hat{v} 1$ & $5.80 E-11$ & 7. .OE +01 & 1.3iE-il & $5.19 E-10$ & 1. $365-37$ & 3. $00 E-35$ \\
\hline Can Csc-21 & $7.63 E+0:$ & 5. $065-11$ & 7:JAE+01 & $1.665-11$ & $5.36 E-10$ & $2.5 j E-39$ & $6.17 E-39$ \\
\hline GFin Cac-22 & $3.01 E+01$ & $9.36 E-11$ & $7.30 E+01$ & J.ט7E-11 & $5.66 E-10$ & 5. $11 E-39$ & 3. $66 E-39$ \\
\hline Crill CaC-23 & $8.44 E+01$ & 8. $06 E-11$ & 8. $40 E+01$ & $2.65 E-11$ & $5.935-10$ & $1.86 E-39$ & $9.70 E-39$ \\
\hline CAN Cof -24 & $8.77 E+01$ & $7.10 \mathrm{E}-1 \mathrm{i}$ & $8.70 E+01$ & 2.J3E-11 & b. 10́E-10 & 6.53E-37 & $9.07 E-39$ \\
\hline CRA CEC-25 & 7.13E+01 & $7.75 E-11$ & $9.10 E+01$ & 2.5EE-11 & $6.42 E-10$ & $3.51 E-39$ & $5.40 E-3 T$ \\
\hline $\operatorname{css} \operatorname{coc} c-26$ & $7.43 E+01$ & $7.73 E-11$ & $9.40 E+01$ & 2.5AE-11 & $6.67 E-10$ & $5.50 E-39$ & $9.84 E-37$ \\
\hline $\operatorname{con} \operatorname{coc}-27$ & $3.26 E+01$ & $1.99 E-10$ & $9.60 E+0 i$ & $6.53 E-11$ & $7.32 E-10$ & $2.30 E-36$ & $1.14 E-3 E$ \\
\hline $\operatorname{CNA} \cos C-28$ & 1. $02 E+02$ & 3.62E-11 & $1.01 E+02$ & $2.35 E-11$ & $7.6 J E-10$ & $7.43 E-37$ & i.195-36 \\
\hline GH CDC-29 & $1.65 E+012$ & 3.87E-11 & j. $04 E \div 02$ & 2.92E-11 & $7.90 \mathrm{E}-10$ & $6.86 E-39$ & $1.25 E-30$ \\
\hline Chu Cat-30 & 1.1!E+12 & $9.9 ! E-! 1$ & 1.1.jE+02 & $3.25 E-11$ & $0.22 \sum-10$ & $1.945-37$ & $1.24 E-36$ \\
\hline EAH câ-3i & 1. $1 S E+02$ & $5.32 E-10$ & i.19E+62 & $1.75 E-10$ & $3.975-10$ & $1.47 E-3 i$ & $1.74 E-58$ \\
\hline EFIN 6ES-32 & $1.23 E+12$ & $2.11 E-: 0$ & $1.22 E+02$ & S. $91 E-11$ & $1.07 E-69$ & 5. $30 E-30$ & $\vdots .94 E-38$ \\
\hline [AN CBt-3] & $\therefore .28 E+112$ & $3.04 E-10$ & $1.26 E+02$ & 7. 99E-\$1 & ¿ITE-M? & $5.3 F E-3 \bar{C}$ & $\therefore .24 E-36$ \\
\hline CFH CISC-54 & i. $6 \hat{v E}+\hat{0} 2$ & 2. $02 E-09$ & 1. $56 E+02$ & $6.55 E-10$ & $1.03 E-67$ & $\therefore 30 E-3 i$ & $4.4: 5-33$ \\
\hline Cxin & $1.65 E+62$ & $1.53 E-09$ & $1.88 E+62$ & 5. $04 E-10$ & $2.335-69$ & 2. WE-Si & $\therefore, 05-30$ \\
\hline
\end{tabular}


TABLE B.21j. Strontium-85 (Sr-85) Leach Fractions from Flow Through Test, Crushed/Sediment with Groundwater (CGC)

\begin{tabular}{|c|c|c|c|c|c|c|c|}
\hline SAMPLE & PORE VOLURE & UCj & TIME DAYS & $\mathrm{An} / \mathrm{AO}_{\mathrm{O}}$ & CUK An/Ao & DIRC CK2/SEC & DCUK CMZ/SEC \\
\hline CRH CEC-1 & $2.00 E+00$ & $3.96 E-11$ & $3.00 E+00$ & $1.25 E-09$ & $1.25 E-09$ & 1.08E-36 & $1.08 E-36$ \\
\hline CAU CEC -2 & $5.92 E+00$ & $7.77 E-11$ & $7.00 E+00$ & $2.45 E-09$ & $3.71 E-09$ & $3.26 E-35$ & $4.08 E-36$ \\
\hline CRN CAC-3 & $9.32 E+00$ & 8.59E-11 & $9.00 E+00$ & $2.71 E-09$ & $6.42 E-09$ & $1.22 E-34$ & $9.52 E-36$ \\
\hline CRM CEC -4 & $1.29 E+01$ & $8.81 E-11$ & $1.30 E+01$ & $2.78 \mathrm{BE}-09$ & $7.20 E-19$ & $3.62 E-35$ & i. $35 E-35$ \\
\hline CRH CEC-5 & $1.66 E+01$ & $8.05 E-11$ & $1.60 E+01$ & $2.54 E-09$ & $1.17 E-08$ & $5.93 E-35$ & 1.79E-35 \\
\hline CAH CEC-Ó & $2.17 E+01$ & $1.10 E-10$ & 2.00E+01 & 3. $49 E-09$ & $1.52 E-08$ & $6.58 E-35$ & $2.41 E-35$ \\
\hline CEN CEC -7 & $2.6 \pi+01$ & $1.18 E-10$ & $2.40 E+01$ & $3.72 E-09$ & $1.90 E-08$ & $8.39 E-35$ & 3.i.iE -35 \\
\hline CAM CEC-8 & 3. $\hat{0} 1 E+01$ & $1.01 E-10$ & $2.80 E+01$ & $3.19 E-09$ & $2.2 ! E-06$ & $6.15 E-35$ & $3.64 E-35$ \\
\hline CFH CSC-9 & $3.15 E+01$ & $3.20 E-11$ & 3.10E+01 & 1.01E-09 & $2.32 E-18$ & $9.375-36$ & $3.60 E-35$ \\
\hline CAN CEC- 10 & $3.46 E+01$ & $7.62 E-11$ & $3.50 E+(11$ & $2.41 E-07$ & 2.55E-68 & $3.135-35$ & $3.885-35$ \\
\hline CRA CEC-11 & $3.99 E+01$ & $9.82 E-! 1$ & $3.30 E+01$ & $3.10 E-09$ & $2.87 E-68$ & $8.80 E-35$ & $4.50 E-35$ \\
\hline CFiN $[G C-12$ & $4.54 E+01$ & $1.40 E-10$ & 4.:0E+01 & $4.42 E-09$ & $3.31 E-616$ & $1.58 E-34$ & $5.55 E-35$ \\
\hline ERN $[5 i-13$ & $4.97 E+01$ & $1.06 E-10$ & $4.40 E+01$ & $3.35 E-09$ & $3.64 E-O B$ & $9.09 E-35$ & $6.27 E-35$ \\
\hline Cnat CEC-14 & $5.40 E+01$ & $9.16 \mathrm{E}-11$ & $4.80 E+01$. & $2.89 E-09$ & $3.93 E-08$ & 4. $53 E-35$ & $6.70 E-35$ \\
\hline CFW CEL-iE & $5.61 E+01$ & $4.46 E-11$ & 5. $1 \hat{V E}+01$ & $1.71 E-09$ & $4.07 E-08$ & $\lfloor .615-35$ & $6.77 E-35$ \\
\hline CAN CGC-16 & $6.00 E+01$ & $9.17 E-11$ & $5.60 E+01$ & $2.90 E-09$ & $4.36 E-08$ & $3.34 E-35$ & $7.07 E-35$ \\
\hline CRH $C A C-17$ & $0.2 B E+01$ & $6.76 E-11$ & $6.00 E+01$ & $2.14 E-09$ & $4.58=-68$ & $3.05 E-35$ & $7.26 E-35$ \\
\hline CRA COĆ-13 & $6.70 E+01$ & $1.02 E-10$ & $6.30 E+01$ & $3.23 E-09$ & $4.90 E-08$ & $9.5 \mathrm{JE}-35$ & $3.93 E-35$ \\
\hline CRU COC- 19 & J.02E+01 & 3. $58 E-11$ & $6.60 E+01$ & 2.TIE-09 & $5.17 E-68$ & $5.94 E-35$ & $8.43 E-35$ \\
\hline CRA COCO-20 & $7.35 E+01$ & $6.96 E-11$ & $7.00 E+01$ & $2.20 E-09$ & $5.35 E-08$ & $2.61 E-35$ & $5.635-35$ \\
\hline CFU CEC-2I & $7.63 E+01$ & $6.07 E-11$ & $7.30 E+01$ & $1.92 E-09$ & $5.58 E-08$ & $3.36 E-35$ & 8. $68 E-35$ \\
\hline CAH CGC-22 & $8.01 E+01$ & $9.09 E-11$ & $7.7 \hat{E E}+0 \mathrm{I}$ & $2.87 E-09$ & $5.87 E-08$ & $4.46 \bar{E}-j \bar{J}$ & $9.31 E-35$ \\
\hline CRH CEC-23 & $8.44 E+01$ & $1.00 E-10$ & $8.40 E+01$ & $3.17 E-09$ & b. 19E-68 & $2.80 E-35$ & 9. $4 B E-35$ \\
\hline CAn CGC-24 & $8.77 E+01$ & $7.5 A E-11$ & $8.70 E+01$ & $2.39 E-09$ & $6.43 E-68$ & $6.68 E-35$ & $9.37 E-35$ \\
\hline ERH C6C-25 & ?.13E+01 & 6. 55E-11 & 9.10E+01 & $2.76 E-69$ & $6.70 E-68$ & $3.95 E-35$ & $1.02 E-34$ \\
\hline CAN CEC-20́ & $9.43 E+01$ & $7.81 E-11$ & $9.40 E+01$ & $2.47 E-09$ & $6.94 E-68$ & $5.57 E-35$ & i. $07 E-34$ \\
\hline $\operatorname{CAH} \cos -27$ & $3.86 E+01$ & $1.27 E-10$ & $9.80 E+01$ & $4.03 E-07$ & $7.35 E-05$ & $3.76 E-35$ & $1.15 E-34$ \\
\hline CRH COC-26 & $1.02 E+02$ & B. $62 E-11$ & $1.01 E+02$ & $2.53 E-v 9$ & $7.60 E-68$ & $5.87 E-35$ & $1.19 E-34$ \\
\hline cin cac- 29 & $1.05 E+02$ & $8.96 \mathrm{E}-11$ & $1.04 E+02$ & $2.835-09$ & $7.88 E-68$ & $6.48 E-35$ & $1.245-34$ \\
\hline CRH CEE-3O & $1.11 E+62$ & $1.32 E-10$ & $1.13 E+02$ & 4. $17 \mathrm{E}-09$ & 3. $30 E-08$ & $3.23 E-35$ & ¿.27E-34 \\
\hline CRH CGC-31 & 1.19E+02 & 2.00E-10 & 1.19E+02 & $6.32 E-09$ & $5.73 E-08$ & $1.93 E-34$ & 1.39E-34 \\
\hline CAN COL -32 & $1.23 E+02$ & $7.91 E-11$ & $1.22 E+02$ & $2.50 E-65$ & $7.13 E-06$ & $6.53 E-35$ & $1.44 E-34$ \\
\hline CHn $[60-53$ & $1.28 E+02$ & $1.14 E-10$ & $1.26 E+02$ & $3.615-09$ & $9.54 E-0.8$ & $7.05=-35$ & $1.50 E-34$ \\
\hline CFin CGC -34 & 1. $6 \hat{v} E+1.2$ & $7.61 E-10$ & $! .58 E+02$ & $2.46 E-08$ & $1.19 E-67$ & $2.35 E-34$ & i. $88 E-34$ \\
\hline LAN CEC-3J & $1.85 E+12$ & $5.76 E-10$ & $1.88 E+0 \bar{Z}$ & $\therefore .32 E-69$ & $1.38 E-37$ & $2.75 E-34$ & $\therefore .10 E-34$ \\
\hline
\end{tabular}


TABLE B.22b.

SAMPLE
EFFLUEHT ।
EFFLUEHT ?
EFFLUEHT 3
EFFLUENT 4
EFFLUEHT 5
EFFLUENT 6
EFFLUENT 7
EFFLUENT 8
EFFLUEHT 9

TABLE B.22C.

SAMPLE
EFFLUENT !
EFFLUENT ?
EFFLUENT 3
EFFLUENT 4
EFFLUENT 5
EFFLUENT
EFFLUENT ?
EFFLUENT 8
EFFLUEHT ?

TABLE B.22d.
Sodium ( $\mathrm{Na}$ ) Leach Fractions from Flow Through Test Crushed Grout/Sediment with Groundwater (SCGIC)

\begin{tabular}{|c|c|c|c|c|}
\hline $\mathrm{An} / \mathrm{Ao}$ & Cus. An/Go & DAYS & inc. & D CUE. \\
\hline $4.30 E-02$ & $4.30 E-02$ & 3. $00 E+00$ & $1.66 E-2 !$ & !. étE-2! \\
\hline !.94E-01 & $2.43 E-01$ & $7.00 E+00$ & $7.40 E-20$ & $1.76 E-20$ \\
\hline $8.54 E-02$ & $3.29 E-01$ & $9.00 E+00$ & $1.21 E-19$ & 2.50E-20 \\
\hline $3.90 E-02$ & $3.6 \partial E-01$ & $1.30 E+01$ & $0.61 E-21$ & $2.26 E-20$ \\
\hline $4.45 E-02$ & 4.12E-01 & $1.6 \mathrm{VE}+01$ & $2.64 E-20$ & $2.21 E-20$ \\
\hline 4. $32 E-02$ & $4.55 E-01$ & $2.00 E+01$ & $1.74 E-20$ & $3.16 E-20$ \\
\hline $3.33 E-02$ & $4.89 E-01$ & $2.40 E+(i t$ & $1.27 E-20$ & $2.07 E-20$ \\
\hline $1.75 E-02$ & 5. $06 E-01$ & $2.80 E+61$ & $4.14 E-21$ & $1.90 E-20$ \\
\hline 1. $35 E-02$ & $5.20 \mathrm{E}-0 \mathrm{i}$ & $3.10 E+01$ & $4.75 E-21$ & $1.81 E-20$ \\
\hline
\end{tabular}

Aluminum (A1) Leach Fractions from Flow Through Test Crushed Grout/Sediment with Groundwater (SCGIC)

\begin{tabular}{|c|c|c|c|c|}
\hline N/AO & [Uh. Â/AOD & OAYS & 0 inc. & IS EUA. \\
\hline $0.00 E+00$ & $0.00 E+00$ & $3.00 E+00$ & $0.00 E+\infty 0$ & 0. $60 E+400$ \\
\hline $3.57 E-03$ & $3.37 E-03$ & $7.00 E+00$ & $2.83 E-23$ & $\begin{array}{l}3.3 i E-24 \\
-\therefore\end{array}$ \\
\hline $2.365-03$ & $5.73 E-03$ & $9.00 E+00$ & $9.22 E-23$ & i.5BE-24 \\
\hline $72 E-63$ & $7.45 E-03$ & $1.30 E+01$ & $1.68 E-23$ & 8. $87 E-24$ \\
\hline $23 E-03$ & $7.685-03$ & $1.60 E+01$ & $6.665-23$ & $1.22 \mathrm{E}-23$ \\
\hline $15-03$ & $1.22 E-02$ & $2.00 E+01$ & $5.90 E-23$ & $1.55 E-23$ \\
\hline OOE -03 & $1.32 E-02$ & $2.405+01$ & $4.58 E-23$ & $1.75 E-23$ \\
\hline $11 E-03$ & $1.52 \mathrm{E}-02$ & $2.80 E+61$ & $1.37 E-23$ & 1. $.72 \mathrm{E}-3 \mathrm{~s}$ \\
\hline $7 E-04$ & $1.60 E-02$ & $3.10 E+01$ & 1.60E-23 & 1.71E-23 \\
\hline
\end{tabular}

Silica (Si) Leach Fractions from Flow Through Test Crushed Grout/Sediment with Groundwater (SCGIC)

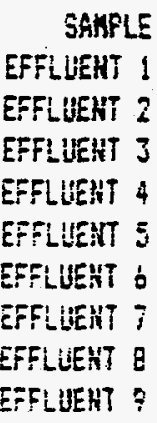

cu.

$$
\begin{aligned}
& \text { An/Ao } \\
& 1.43 E-03 \\
& 9.63 E-03 \\
& 1.36 E-02 \\
& \vdots .56 E-02 \\
& 1.60 \mathrm{E}-62 \\
& 2.00 E-02 \\
& 2.13 E-\hat{0} 2 \\
& 2.20 \mathrm{i}-02 \\
& 2.25 E-\hat{2} 2
\end{aligned}
$$

$D$ inc. 1. 4ZE-24 1. $675-22$ 2. :5E -22 2. $25 E-23$ 6. $47 E-25$ 3.59E-23 $\therefore .345-23$ t. $365-24$ 6. IEE-
0 Eis.

1. 4ZE-24

$2.7 \mathrm{EE}-2 \mathrm{~S}$

$4,43 E-23$

4. $012-83$

4. $23 E-3$

¿. $16 E-25$

.

ร.SEE-IS

3. $3 \mathrm{E}-\mathrm{Z}$ 
TABLE B.22e. Boron (as HCBO3) Leach Fractions from Flow Through Crushed Grout/Sediment with Groundwater (SCGIA)

\begin{tabular}{|c|c|c|c|c|c|}
\hline SAMPLE & An/Ao & CUL. An/ÂO & BAYS & D inc. & 0 cus. \\
\hline EFFLUEHT 1 & $3.05 E-02$ & $3.05 E-02$ & $3.00 E+00$ & $6.46 E-22$ & $3.46 E-22$ \\
\hline SFFLUENT 2 & $3.88 E-02$ & 6. P3E-02 & $7.00 E+000$ & ב.74E-2! & $1.43 E-21$ \\
\hline EFFLUEHT 3 & $1.24 E-02$ & $\hat{0} .17 E-\hat{0} 2$ & $9.00 E+00$ & $2.56 E-21$ & $1.54 E-21$ \\
\hline EFFLUENT 4 & b. $0 B E-03$ & $8.7 \mathrm{BE}-02$ & $1.30 E+01$ & $2.10 E-22$ & $1.37 E-2 !$ \\
\hline EFFLUEHT J & $6.04 E-03$ & $9.38 E-02$ & $1.60 E+01$ & $4.87 E-22$ & 1. $24 E-21$ \\
\hline EFFLUEHT 6 & $6.41 E-03$ & $1.00 E-01$ & $2.00 E+01$ & $3.63 E-22$ & $1.04 E-21$ \\
\hline EFFLUENT 7 & $3.46 E-03$ & $1.04 E-01$ & $2.40 E+01$ & $1.36 E-22$ & $9.3 G E-2 Z$ \\
\hline EFFLUENT B & $7.38 E-0 j$ & $1.11 E-01$ & $2.80 E+01$ & $7.35 E-22$ & $9.16 E-22$ \\
\hline EFFLUEHT 9 & $5.37 E-63$ & $1.16 E-01$ & $3.10 E+01$ & $7.60 \mathrm{E}-22$ & $9.10 E-72$ \\
\hline
\end{tabular}

TABLE B.22f. Fluoride (F) Leach Fractions from Flow Through Crushed Grout/Sediment with Groundwater (SCGIA)

\begin{tabular}{|c|c|c|c|c|c|}
\hline SAHPLE & $\mathrm{Ani} / \mathrm{AO}$ & Cun. An/tho & Dinls & Int. & 0 ius. \\
\hline EFFLUENT I & $2.63 E-03$ & $2.63 E-03$ & $3.00 E+00$ & 4. COE-24 & $4.305-24$ \\
\hline EFFLUEHT 2 & 5. $89 E-03$ & 8. $52 E-03$ & $7.00 \mathrm{E}+00$ & $8.63 E-23$ & 2. $16 E-2]$ \\
\hline EFFLUEMT 3 & 2. $80 E-63$ & $1.13 E-02$ & $9.00 E+00$ & j.30E-22 & 2. $85 E-23$ \\
\hline EFFLLUEHT 4 & $1.49 E-63$ & $1.28 E-02$ & $1.30 E+01$ & $1.25 E-2 J$ & $2.62 E-2$ \\
\hline EFFLUEHT S & $1.02 E-03$ & $1.44 E-02$ & $1.60 E+61$ & $3.53 E-23$ & $2.71 E-23$ \\
\hline EFFLUEKT O & $1.56 E-0.3$ & $1.60 E-12$ & $2.00 E+01$ & $2.27 E-23$ & $3.60 E-23$ \\
\hline EFFLUEHT 7 & $1.2 ! E-03$ & $1.72 E-02$ & $2.40 E+01$ & $1.57 E-2 j$ & $2.5 c=-23$ \\
\hline EFFLUENH 8 & 7. $68 E-04$ & $1.79 E-02$ & $2.80 E+0 i$ & 6.76E-29 & $2.38 E-23$ \\
\hline EFFLUENT ? & $5.05 E-04$ & $1.84 E-02$ & $3.10 E+01$ & 6. FEe-24 & $2.275-23$ \\
\hline
\end{tabular}

TABLE B.22g. Nitrate (NO3) Leach Fractions from Flow Through Crushed Grout/Sediment with Groundwater (SCGIA)

\begin{tabular}{|c|c|c|c|c|c|}
\hline SAMPLE & Añî́o & 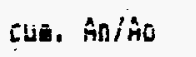 & DิẤs & Dinc. & S Сux. \\
\hline EFEUERT 1 & $2.8 T E-01$ & 2. BTE-ôt & $3.60 E+10$ & E.7JE-30 & E. ŻiE-0́s \\
\hline EFFIUEHT 2 & 2. IIE- & $4.9 E E-01$ & 3. OAE+on & I. $:: E-: ?$ & a. $27 E-6 z$ \\
\hline EFFUUEHT 3 & ¿. $.79-19$ & $5 . i C E-\hat{0} !$ & $7.00 E+96$ & S. S9E-II & 1. VEE-vi \\
\hline EFFLUENT 4 & $1.29 E-33$ & $5 . j 7 E-v i$ & $1.30 E+\dot{v} 1$ & F IEE-24 & $7.45 E-0 \overline{0}$ \\
\hline fFiuturt s & D. $00 E+00$ & S.: :FE-31 & i.jôE+U1 & $\dot{\mathrm{v}}, \mathrm{be} \div \mathrm{b}$ & S. \\
\hline EFFLUER b & 6. $60 \mathrm{DE}+00$ & $5.17 E-\hat{v i}$ & $2.60 E+61$ & D. DOETW & $4.5 \overline{z E}-4 \overline{\mathrm{c}}$ \\
\hline EFFLUEMT $i$ & $0,60 E+60$ & $5.175-3 i$ & $2.40 E+6 !$ & A. WBE-QB & 4. \\
\hline EFFIUENTT & $0,00 E+00$ & $5.15 E-01$ & 2. SôE+O1 & $0.0,0+w i j$ & $3.48 E-i t \sigma$ \\
\hline EFFiUEMT 7 & $6.60 E \div 60$ & $5.1: \vdots-31$ & 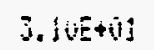 & 0, Bif + 60 & 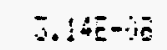 \\
\hline
\end{tabular}


TABLE B.22h. Sulfate (S04) Leach Fractions from Flow Through Crushed Grout/Sediment with Groundwater (SCGIA)

\begin{tabular}{|c|c|c|c|c|c|}
\hline SAMPLE & $A n / A O$ & CUL, An/AO & DAYS & $D$ inc. & j cus. \\
\hline EFF!UENT I & 7.17E-01 & $7.17 \mathrm{E}-01$ & $3.00 E+00$ & $3.56 E-19$ & $3.70 \mathrm{e}-11$ \\
\hline EFFLUENT 2 & $5.33 E-01$ & $1.25 E+00$ & 7. $00 E+60$ & $7.08 E-19$ & $3.06 E-12$ \\
\hline EFFLUEKT 3 & 7. $34 E-02$ & $1.35 E+60$ & $7.00 E+00$ & $1.61 E-19$ & 1. $34 E-12$ \\
\hline EFFLUERT 4 & $2.10 E-02$ & $1.37 E+00$ & $1,30 E+01$ & 2.5IE-21 & :.34E-i2 \\
\hline EFFLUENT 5 & $1.085-03$ & $1.37 E+60$ & $1.6 \mathrm{0E}+6 \mathrm{ij}$ & $1.57 E-2 j$ & 1.01E-12 \\
\hline EFFLUIEHT 6 & $0.00 E+00$ & $1.37 E+00$ & $2.00 E+01$ & $0.00 E+00$ & $3.165-13$ \\
\hline EFFLUENT 7 & $0.00 E+00$ & $1.37 E+00$ & $2.40 E+01$ & $0.00 E+00$ & $6.67 \equiv-13$ \\
\hline EFFLUENT 8 & $0.00 E+00$ & $1.37 E+00$ & $2.80 E+01$ & $0.00 E+00$ & $5.64 E-15$ \\
\hline EFFLUENT 7 & $0.00 E+00$ & $1.37 E+000$ & J. $10 E+01$ & $0.00 E+00$ & $4.975-13$ \\
\hline
\end{tabular}

TABLE B.22i. Technetium-99 (TC-99) Leach Fractions from Flow Through Test, Crushed/Sediment with Groundwater (SCGT)

\begin{tabular}{|c|c|c|c|c|c|c|c|}
\hline SAMPLE & FDRE VOLUHE & $\mathbf{i t i}$ & TIME DAYS & Añitiò & CUA $A \bar{n} / A_{\bar{O}}$ & DINC CH2/SEL & DCUA CH2/SEC \\
\hline SEET-1 & $3.36 E+0 \hat{v}$ & 5. $.95-01$ & $3.00 E+00$ & $4.365-01$ & 4. $36 E-61$ & $1.315-36$ & i. $.31 E-0 b$ \\
\hline SETT-2 & $6.44 E+00$ & $0.54 E-02$ & $7.60 E+10$ & $6.32 E-02$ & $4.97 E-01$ & $8.37 E-21$ & $5.7 \pm E-06$ \\
\hline SEST-3 & $7.955+60$ & $1.25 E-02$ & $1.10 E+01$ & $9.28 E-43$ & $5.09 E-01$ & $2.62 E-22$ & $9.535-06$ \\
\hline $360 T-4$ & $1.15 E+01$ & $9.09 E-03$ & 1.5JE+ & $0.73 E-03$ & 5. $15 E-01$ & $1.0 \mathrm{c} E-22$ & $\lfloor .36 E-015$ \\
\hline $565 T-5$ & $1.29 E+01$ & $2.08 E-03$ & $1.80 E+01$ & $1.54 E-03$ & $5.17 E-01$ & 8. $405-24$ & $1.535-65$ \\
\hline SCÊT-6 & $1.58 E+01$ & $2.78 E-03$ & $2.20 E+01$ & $2.06 E-03$ & $5.19 E-01$ & $5.24 E-24$ & $2.005-05$ \\
\hline SEST-7 & 1.76E+01 & $1.14 E-03$ & $2.50 E+01$ & 8. $47 E-04$ & $5.20 E-111$ & $2.54 E-24$ & $2.27 E-65$ \\
\hline $5[E]-8$ & $2.20 E+01$ & $1.37 \mathrm{E}-0 \mathrm{3}$ & $2.90 E+01$ & $1.01 E-03$ & $5.21 E-01$ & $2.155-24$ & $2635 E-60 \bar{J}$ \\
\hline SEET-9 & 2. $3 \mathrm{BE}+0 \mathrm{i}$ & $5.07 E-04$ & $3.20 \mathrm{E}+0 \mathrm{i}$ & $3.765-04$ & $5.21 E-0 !$ & $4.995-35$ & $2.90 E-65$ \\
\hline SCET-10 & $2.66 E+01$ & $7.16 E-04$ & $3.50 E \div$ vi1 & $5.30 E-04$ & $5.22 E-6 !$ & $5.775-25$ & 3. $16 E-j 5$ \\
\hline SC67-il & $2.83 E+0 !$ & $5.82 E-\hat{4} 4$ & $3.90 E+0 !$ & $4.31 E-04$ & $5.22 E-0 !$ & $3.865-25$ & 3. J4E- \\
\hline SCET -12 & $3.26 E+01$ & $1.52 E-03$ & $4.60 E+04$ & 1. $42 E-0 J$ & $5.24 E-61$ & 2. $19 E-24$ & $4 .: 7 E-05$ \\
\hline SCST-!3 & $3.76 E+01$ & 8. $315-64$ & 5. $30 E+01$ & $6.595-64$ & $5.24 E-0 i$ & $5.53 E-25$ & $4 . \bar{j} 1 E-\hat{v} \bar{s}$ \\
\hline 3COT-14 & $4.05 E \div 01$ & $4.15 E-04$ & $5.70 E+01$ & 3.07E-0. & 5. $55 E-011$ & $2.345-\overline{i j}$ & $5.43 E-65$ \\
\hline SCET-15 & $4.26 E+401$ & $3.37 E-69$ & 6. $00 E+01$ & $2.50 E-\hat{v} 4$ & 5.25E-01 & $2.21 \equiv-25$ & $5.72 E-65$ \\
\hline SСET-16 & $4.50 E+01$ & 5. 17 E- & $6.40 E+01$ & J. $33 E-044$ & S. 25E-0i & $3.07 E-25$ & $6.10 E-45$ \\
\hline SCลT $T-1 \bar{i}$ & $4.61 E+0 i$ & $6.64 E-64$ & $0.30 E+01$ & $5.0 \leq E-014$ & $5.765-01$ & $4.40 E-75$ & $6.575-i j 5$ \\
\hline $360 \bar{i}-i \hat{\varepsilon}$ & $4.995+01$ & 4.05EE-ia4 & $7.20 E \div 6 \mathrm{i}$ & 3 B. 00 - 64 & 5.23E-6! & $3.5=-25$ & b. $\equiv t=-05$ \\
\hline
\end{tabular}

B.111 
TABLE B.22j. Iodine-125 (I-125) Leach Fractions from Flow Through Test Crushed/Sediment with Groundwater (SCGI)

\begin{tabular}{|c|c|c|c|c|c|c|}
\hline SAKPLE & $v i j$ & TIME ôAYS & An/ÁD & EUa Ans/âo & DINC CK2/SEC & DEUM SKA/SEL \\
\hline CSW SCEI-1 & \{. 5BE- Uิ & $3.00 E+00$ & $4.25 E-01$ & $4.29 E-01$ & $1.965-97$ & 1. $.865-67$ \\
\hline CRA SC6I-2 & $9.3 \hat{E}-02$ & 7. $00 E+00$ & $2.65 E-01$ & $6.95 E-0 !$ & $1.47 E-19$ & $2.935-67$ \\
\hline CEV SCE!-J & $8.90 \mathrm{0E}-03$ & P. $Q A E+0 O$ & $2.600-92$ & T.21E- 11 & $4.35 E-2 i$ & 2. $46 E-\hat{0} 7$ \\
\hline CRY SCEI-4 & $4.12 E-03$ & $1.30 E+01$ & $1.26 E-102$ & $7.33 E-\hat{y} !$ & $2.80 \varepsilon-22$ & 1. $.95-97$ \\
\hline CRN SCE:-5 & 乏..77E-04 & $1.600+01$ & $3.07 E-03$ & $7.36 E-D 1$ & J. 3BE-23 & i. $5 E-6 i$ \\
\hline CAN SCEI-b & $6.76 E-64$ & 2.00E+01 & $2.31 E-03$ & $7.39 E-61$ & $3.115-23$ & 3. $22 E-0 i$ \\
\hline CRH SC6I-7 & $3.16 E-194$ & $2.40 E+01$ & $1.10 E-63$ & $7.40 E-\hat{O} !$ & $2.86 E-24$ & ¿.UEE-OT \\
\hline 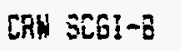 & $3.50 E-014$ & $2,80 E+01$ & $1.275-03$ & $7.41 E-01$ & 3. BuE-2i & $3.67 E-60$ \\
\hline [EH SCEI-9 & 1. $01 E-014$ & $3.10 E+j !$ & $6.585-04$ & $\bar{i}, 42 E-\hat{i} !$ & 1.53E-24 & 7. BiE- \\
\hline 政 sco:-10 & $2.74 E-04$ & $3.50 E+0 i$ & $1.08 E-03$ & $7.45 E-i i$ & $2.4+5-24$ & $0 .=5 E-68$ \\
\hline
\end{tabular}




\section{TABLE B.22k. Strontium-85 (Sr-85) Leach Fractions from Flow Through Test,}

Crushed/Sediment with Groundwater (SCGC)

\begin{tabular}{|c|c|c|c|c|c|c|c|}
\hline SAMPLE & PORE VOLUME & Uזi & TIME DAYS & $A \cap / A_{0}$ & CUn An/ÂO & DIKC CH2/SEC & DCUK CK2/SEC \\
\hline CRY SCEC-1 & $9.80 E-01$ & $3.91 E-11$ & $3.00 E+00$ & $1.24 E-09$ & $1.24 E-09$ & $4.09 E-37$ & 4.09E-37 \\
\hline CRY SCAC -2 & $2.47 E+00$ & $4.10 E-11$ & $7.00 E+00$ & $1.29 E-09$ & $2.53 E-09$ & $3.51 E-36$ & $3.36 E-37$ \\
\hline CRN SCGC-J & $4.45 E+00$ & $1.24 E-10$ & $9.00 E+00$ & $3.91 E-09$ & $6.44 E-09$ & $9.63 E-35$ & $3.70 E-36$ \\
\hline CRN SCEC-A & $7.75 E+00$ & $9.27 E-11$ & $1.30 E+01$ & $2.93 E-09$ & $9.37 E-09$ & $1.55 E-35$ & $5.43 E-36$ \\
\hline CRN SCEC-5 & $9.93 E+00$ & $5.89 E-11$ & $1.60 E+01$ & $1.86 E-09$ & $1.12 \mathrm{E}-08$ & i. 22E-35 & $6.34 E-36$ \\
\hline CRH SCEC-d & 1.19E+01 & $5: 78 E-11$ & $2.00 E+01$ & 1.83E-09 & $1.31 E-v B$ & $6.97 E-36$ & $6.85 E-36$ \\
\hline CRH SCAC-7 & $1.33 E+01$ & $5.48 E-11$ & $2.40 E+01$ & $1.73 E-69$ & $1.46 E-03$ & $7.02 E-36$ & $7.33 E-3 b$ \\
\hline CFY 5 CEC-8 & 1. $3 B E+01$ & $7.46 E-12$ & $2.80 E+01$ & $2.36 E-10$ & $1.50 E-6 B$ & $1.30 E-37$ & 5. $48 E-36$ \\
\hline CRH SCAC-9 & $1.56 E+(11$ & $5.97 E-11$ & $3.10 E+01$ & 1.89E-09 & 1.69E-08 & $1.26 E-35$ & $7.42 E-36$ \\
\hline ERA SCGC-10 & $1.90 E+01$ & $9.67 E-11$ & $3.50 E+01$ & $3.05 E-09$ & $2.00 E-0 B$ & $1.95 E-35$ & $9.16 E-36$ \\
\hline CRH SCGC-11 & $2.19 E+01$ & $9.42 E-11$ & $3.80 E+01$ & $2.98 E-69$ & $2.29 E-68$ & $3.14 E-35$ & $1.11 E-35$ \\
\hline CRH SCBC-12 & $2.80 E+0 i$ & $1.78 E-10$ & $4.10 E+01$ & $5.63 E-09$ & $2.86 E-68$ & $9.91 E-35$ & $1.60 E-35$ \\
\hline Can SCE[-1] & $3.20 E+01$ & $1.43 \mathrm{E}-10$ & $4.40 E+C 1$ & $4.51 E-09$ & $3.31 E-08$ & $6.37 E-35$ & $2.00 E-35$ \\
\hline IRE SCEC-14 & $3.57 E+01$ & $9.82 E-11$ & $4.80 E+01$ & $3.105-09$ & $5.62 E-08$ & $2.01 E-35$ & $2.19 E-35$ \\
\hline CAM SCSC-15 & $3.75 E+01$ & $6 . \$ 0 E-11$ & $5.10 E+01$ & $2.02 E-07$ & $3.32 E-08$ & $1.455-35$ & $2.30 E-35$ \\
\hline CAH SCBC-16 & 4.09E+01. & $8.79 E-11$ & $5.60 E+01$ & $2.78 E-09$ & $4.10 E-08$ & $1.15 E-35$ & $2.41 E-35$ \\
\hline Can scac-17 & $4.35 E+01$ & $8.72 E-11$ & $6.00 E+01$ & $2.82 E-09$ & 4.JBE- 68 & $2.055-35$ & $2.5 i E-j 5$ \\
\hline CEH SCEC-18 & $4.7 \mathrm{iE}+01$ & $9.39 E-11$ & c. $30 E+01$ & $2.97 E-09$ & $4.68 E-05$ & $3.11 E-35$ & $2.79 E-35$ \\
\hline CAN SCOC-19 & $5.30 E+01$ & 3.01E-11 & $0.60 E+01$ & $2.53 E-09$ & $4.935-06$ & 2. $00 \mathrm{E}-35$ & $2.96 E-35$ \\
\hline CEH SCEC-20 & $5.29 E+01$ & $7.23 E-11$ & $7.00 E+01$ & $2.30 E-09$ & $5.16 E-08$ & 1.1IE-35 & $3.06 E-35$ \\
\hline CAH SCOC-21 & $5.54 E+01$ & $7.31 E-11$ & $7.30 E+01$ & $2.31 E-69$ & $5.39 E-08$ & 1.89E-35 & $3.20 E-35$ \\
\hline CFin scoc-22 & 5.91E+01 & $5.12 E-10$ & $7.70 E+01$ & $3.5 \mathrm{SE}-09$ & $5.74 E-08$ & $2.61 E-35$ & $3.45 E-35$ \\
\hline Cât SCaC-23 & b. 32E+01 & i. $08 E-10$ & 8.40EE+01 & $3.40 E-09$ & 6. $0 A E-G B$ & $1.25 E-35$ & $3.55 E-j 5$ \\
\hline CRA SCEC-24 & $6.63 E+01$ & $8.94 E-11$ & $8.70 E+01$ & $2.83 E-09$ & $6.37 E-0 \mathrm{E}$ & $3.71 E-35$ & 3.75E-35 \\
\hline CAN SCEC-25 & $6.95 E+0 \mathrm{i}$ & 8.02E-11 & $9.10 E+01$ & $2.53 \mathrm{E}-09$ & $6.62 E-18$ & $1.345-35$ & $3.88 E-35$ \\
\hline CAN Scac-26 & $7.23 E+01$ & $7.375-11$ & $9.40 E+01$ & $2.335-09$ & 6. 65E-08 & 1. $92 E-35$ & $4.02 E-35$ \\
\hline GR SCGC -27 & 7.6IE+V́1 & $3.71 E-11$ & $9.80 E+01$ & $3.07 E-09$ & $7.16 E-08$ & $1.97 E-35$ & $4.21 E-35$ \\
\hline CRH SCEC-2B & $7.72 E+01$ & $7.26 \mathrm{E}-11$ & $1.01 E+02$ & $2.29 E-69$ & $7.39 E-08$ & $1.36 E-35$ & $4.35 E-35$ \\
\hline $\cos \sec -29$ & $8.23 E+01$ & $8.88 E-11$ & $1.04 E+02$ & $2.81 E-09$ & 7.STE-0B & $2.46 E-35$ & $4.55 E-35$ \\
\hline CRU SEGC-30 & $8.83 E+01$ & $1.80 E-10$ & $1.13 E+02$ & $5.70 E-09$ & $8.24 E-08$ & $2.315-35$ & 4.63E-35 \\
\hline CAN SCEC-31 & $9.64 E+01$ & $2.00 E-10$ & $1.19 E+02$ & b. J3E-v9 & $8.87 E-68$ & $7.49 E-35$ & $5.32 E-35$ \\
\hline CAH SCAC-j2 & $3.53 E+61$ & $7.12 E-11$ & $1.22 E+02$ & $2.25 E-09$ & $9.10 E-68$ & $2.17 E-35$ & $5.46 E-35$ \\
\hline SRy Scoc-33 & $1,63 E+02$ & 1.37E-10 & $1.26 E+02$ & 4. उBE- 09 & $9.54 E-16$ & $4.61 E-35$ & $5.8 ! 5-35$ \\
\hline 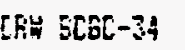 & $1.31 E+02$ & 6.87E-10 & $1.58 E+62$ & 2.17E-0a & $1.17 E-07$ & $7.40 E-35$ & $6.985-35$ \\
\hline NAN $3 \operatorname{coc}-35$ & $1.51 E+\hat{v 2}$ & $5.07 E-10$ & $1.68 E+02$ & 1. $60 \mathrm{NE}-0 \bar{E}$ & $1.33 E-07$ & $3.25 E-55$ & $7.535-35$ \\
\hline
\end{tabular}


TABLE B.221. Cesium-137 (Cs-137) Leach Fractions from Flow Through Test, Grout/Sediment with Groundwater (SCGC)

\begin{tabular}{|c|c|c|c|c|c|c|c|}
\hline SAMPLE & PORE VOLUKE & UCi & TIME DAYS & An/Ao & $\mathrm{CUM}$ An/Ao & DINL CN2/SEC & DCUS CN2/SEC \\
\hline CRN SCEC-1 & $9.80 E-01$ & $2.27 E-11$ & $3.00 E+00$ & $7.45 E-12$ & $7.45 E-12$ & 1. $\{9 E-41$ & [. $49 E-4]$ \\
\hline CRH SCEC-2 & $2.47 E+00$ & $1.65 E-10$ & $7.00 E+00$ & $5.4 J E-11$ & 6.17E-11 & $6.165-39$ & $4.36 E-40$ \\
\hline CRH SCEE-3 & $4.45 E+00$ & $4.06 E-11$ & $9.00 E+00$ & $1.33 E-11$ & $7.50 E-11$ & $1.14 E-39$ & $5.035-40$ \\
\hline CRN SCEC-4 & $7.75 E+100$ & $5.52 E-1 !$ & $1.30 E+01$ & 1.81E-1! & 7.31E-11 & $5.34 E-40$ & $5.37 E-40$ \\
\hline CRN SCGC-5 & $9.93 E+00$ & 4. 30E-11 & $1.60 E+01$ & $1.41 E-11$ & $1.07 E-10$ & $7.07 E-40$ & 5. $79 E-40$ \\
\hline CAH SCEC-6 & $1.19 E+01$ & $2.20 E-10$ & $2.00 E+01$ & $7.24 E-11$ & $1.80 E-10$ & $1.10 E-36$ & $1.30 E-39$ \\
\hline CRN SCEC-7 & $1.33 E+01$ & $3.63 E-11$ & $2.40 E+01$ & 1.1SE-11 & $1.72 E-1 \hat{V}$ & $3.33 E-40$ & i. $.23 E-39$ \\
\hline CKN SCAC-8 & $1.38 E+01$ & $2.20 E-11$ & $2.80 E+01$ & $7.24 E-12$ & $1.99 E-10$ & $1.23 \mathrm{JE}-40$ & $1.14 E-39$ \\
\hline CRN SCEC-9 & $1.56 E+01$ & $4.19 E-11$ & $3.10 E+01$ & $1.38 E-11$ & $2.13 E-10$ & $6.725-40$ & $1.17 E-39$ \\
\hline CRN SCEE-10 & $1.90 E+01$ & $6.46 E-11$ & $3.50 E+01$ & $2.12 E-11$ & 2. $34 E-10$ & $9.42 E-40$ & $1.26 E-39$ \\
\hline CFH SCBC-1i & $2.19 E+01$ & $5.62 E-11$ & $3.80 E+01$ & $1.655-11$ & $2.52=-10$ & $1.21 E-39$ & $1.35 E-37$ \\
\hline CRH SCGC-12 & $2.80 E+01$ & $1.18 E-10$ & 4. $10 E+01$ & $3.68 E-11$ & 2. $71 E-10$ & $4.715-39$ & $1.66 E-37$ \\
\hline CRH SCEC-13 & $3.20 E+01$ & $1.40 E-10$ & $4.40 E+01$ & $4.60 E-11$ & $3.37 E-10$ & $6.605-39$ & 2.018E-39 \\
\hline $\operatorname{con} \operatorname{scos} c-14$ & $3.57 E+01$ & $1.09 E-10$ & $4.80 E+01$ & $3.59 E-11$ & $3.73 E-10$ & $2.70 E-39$ & 2.J3E-39 \\
\hline CAN SCSC-15 & $3.75 E+01$ & $2.95 E-11$ & S.10E+01 & $9.695-12$ & $3.83 E-10$ & $3.32 E-40$ & $2.31 E-39$ \\
\hline CRH SCEL-i6 & $4.09 E+01$ & $1.25 E-10$ & $5.60 E+01$ & $4.12 E-11$ & $4.24 E-10$ & $2.62 E-39$ & $2.56 E-39$ \\
\hline CRN SCEC-17 & 4.35E+0: & $5.66 E-11$ & $6.00 E+01$ & $1.93 E-11$ & $4.43 E-10$ & $3.56 E-40$ & $2.63 E-37$ \\
\hline CR: SCGC-18 & $4.71 E+01$ & $6.70 \mathrm{E}-11$ & $6.30 E+01$ & 2.20E-11 & $4.655-10$ & $1.72 E-39$ & $2.76 E-39$ \\
\hline CRH SCEC-19 & S.00E+01 & $6 .|1 E-1|$ & $6.60 E+61$ & 2. $01 E-11$ & 4.85E-10 & $1.26 \mathrm{E}-39$ & $2.87 E-39$ \\
\hline CAH SCsC-20 & $5.29 E+01$ & $5.05 E-11$ & 7.00E+01 & $1.66 E-11$ & $5.02 E-10$ & $5.76 E-40$ & $2.89 E-39$ \\
\hline СRН SCE[-2] & $5.54 E+\hat{O} \mathrm{I}$ & $1.75 E-11$ & $7.30 E+01$ & 5. $74 E-12$ & $5.07 E-10$ & $1.17 E-40$ & $2.84 E-39$ \\
\hline CAn Scac-22 & $5.91 E+01$ & $9.99 E-11$ & $7.70 E+01$ & $3.28 E-11$ & $5.40 E-10$ & $2.25 E-3 ?$ & $3.05 E-33$ \\
\hline CRH SCEC-23 & $6.32 E+01$ & $7.38 E-11$ & $8.40 E+01$ & $2.42 E-11$ & $5.65 E-10$ & $6.36 E-40$ & $3.05 E-39$ \\
\hline CFH SCGC-24 & $6.53 E+01$ & $1.58 E-11$ & $0.70 E+01$ & $5.18 E-12$ & $5.705-10$ & 1. $25 E-40$ & $3.60 E-39$ \\
\hline CAH SCEC-25 & $6.95 E+01$ & $1.65 E-11$ & $9.10 E+01$ & $5.40 E-12$ & $5.75 E-10$ & c. :I!E-41 & $2.52 E-39$ \\
\hline CAL SCSC-2b & $7.23 E+01$ & $1,\{i E-11$ & $3.40 E+01$ & $4.63 E-12$ & $5.80 E-10$ & $7.60 \mathrm{E}-91$ & $2.88 E-39$ \\
\hline [SH SCSC-2] & $7.63 E+01$ & $2.05 E-11$ & $9.80 E+01$ & $6.73 E-12$ & $5.36 E-1 \hat{0}$ & $? .47 E-41$ & $2.62 E-39$ \\
\hline CAU SCAE-28 & $7.52 E+01$ & $5,91 E-11$ & $1.01 E+02$ & $1.94 E-11$ & $0.06 E-10$ & 1.3JE-39 & 2. $92 E-39$ \\
\hline$\therefore$ SRE 5 CEC-29 & $8.23 E+01$ & $1.41 E-10$ & $1.04 E+02$ & 4. $03 E-11$ & $6.53 E-10$ & $0.71 E-39$ & $3.37 E-39$ \\
\hline CAN SCEC-30 & B. $83 E+01$ & 1.78E-10 & $1.13 E+02$ & ร. $65 E-11$ & 7.11E-10 & $2.43 E-3 e$ & $3.60 E-39$ \\
\hline CA: 5EeC-31 & $7.64 E+01$ & $1.62 E-10$ & $1.17 E+\sqrt{2} 2$ & $5.33 E-1 !$ & $7.64 E-16$ & $5.315-39$ & $3.85 E-39$ \\
\hline CNH SCEL-32 & 9. SIE+401 & 5. $77 E-1 i$ & $1.22 E+02$ & i. $65 E-11$ & T. aJE-10 & $1.545-57$ & $4.04 \varepsilon-33$ \\
\hline CR: $5 C 5 C-33$ & $1.035+62$ & $8.29 E-11$ & $1.26 E+02$ & $2.72 E-11$ & B.10E-10 & $1.355-37$ & $4.1 F E-3 P$ \\
\hline CAN SCGL-34 & $1.31 E+62$ & $5.50 E-10$ & $1.58 E+02$ & $1.8 \mathrm{iE}-\mathrm{i} 0$ & $9.915-10$ & $5 .: 25-39$ & $5.00 E-39$ \\
\hline CRU STEC $C-35$ & $1.51 E+02$ & $4.065-10$ & $1.38 E+02$ & $1.33 E-10$ & 1.15-67 & $5.71 E-3 \hat{3}$ & $5.41 E-39$ \\
\hline
\end{tabular}


TABLE B.23i. Iodine-125 (I-125) Leach Fraction from Flow Through Test, Crushed/Sediment with Groundwater (CGI)

\begin{tabular}{|c|c|c|c|c|c|c|c|}
\hline SAMPLE & PDRE VOLUKE & CORR UCi & IIME DAYS & $A n / A D$ & Cus An/Âo & DINC CS2/SEC & DCUM CHב/SEC \\
\hline CAH $[B]-1$ & 1. $1 \mathrm{EE}+00$ & 8. $99 \mathrm{E}-02$ & $3.00 E+00$ & $2.37 E-01$ & $2.37 E-01$ & B. $3 J E-6 B$ & $8.71 E-96$ \\
\hline CAn CE!-2 & $5.02 E+00$ & $9.30 E-02$ & $7.00 E+00$ & $2.45 E-61$ & $4.81 E-01$ & $3.24 E-19$ & i. $37 \mathrm{E}-1 \mathrm{iT}$ \\
\hline Crí CôI-3 & $8.44 E+00$ & $1,41 E-02$ & $9.00 E+00$ & $3.71 E-0.2$ & $5.18 E-011$ & $2.27 E-206$ & $1.77 E-07$ \\
\hline CRH Cal-4 & $1.25 E+01$ & $5.15 E-63$ & {$[.30 E+01$} & $1.36 E-02$ & $5.32 E-111$ & 8.60E-?2 & $\therefore .298-07$ \\
\hline [FH $C E]-5$ & $1.6 J E+01$ & $8.20 E-04$ & $1.60 E+01$ & $2.16 E-63$ & $5.34 E-61$ & $4.26 E-23$ & 1. $65 E-67$ \\
\hline CAH CGI-6. & $3.14 E+01$ & $7.74 E-04$ & $2.00 E+01$ & $2.04 E-03$ & $5.36 E-113$ & $2.24 E-2 E$ & $3.79 E-08$ \\
\hline CAn Cal-7 & $2.56 E+01$ & 5. $04 E-64$ & $2.40 E+01$ & $1.33 E-03$ & $5.375-91$ & 1. 0eE-23 & i. $.35 E-0 R$ \\
\hline CRH C61-6 & $3.02 E+01$ & $4.47 E-04$ & $2.80 E+01$ & $1.13 E-03$ & $5.39 E-61$ & 8. 4. (1)E-24 & $6.28 E-08$ \\
\hline Cfat cal-? & $3.18 \varepsilon+01$ & $2.01 E-04$ & 3. $10 E+01$ & $5.23 E-04$ & 5. J9E-OI & $2.36=-24$ & $5.67 E-06$ \\
\hline Can Coi-10 & $3.56 E+01$ & $4.38 E-04$ & $3.50 E+01$ & 1.:5E-03 & $5.30 E-01$ & i. $175-24$ & $5.03 E-68$ \\
\hline
\end{tabular}


Appendix C

Mass Balance of Leach Solutions 


\section{Appendix C}

\section{List of Tables}

Table C.1 - Mass Balance for ANS 16.1 Leach Test of Whole Grout with Groundwater (MB2-4.WK1)

Table C.2 - Mass Balance for ANS 16.1 Leach Test of Whole Grout with Groundwater (MB3-4)

Table C.3 - Mass Balance for ANS 16.1 Leach Test of Whole Grout with Groundwater (MB4-8)

Table C.4 - Mass Balance for ANS 16.1 Leach Test of Whole Grout with Groundwater (MB5-5)

Table C.5 - Mass Balance for Static Leach Test of Whole Grout in Groundwater (MB2-17)

Table C.6 - Mass Balance for Static Leach Test of Whole Grout in Groundwater (MB3-18)

Table C.7 - Mass Balance for Static Leach Test of Whole Grout in Groundwater (MB4-13)

Table C.8 - Mass Balance for Static Leach Test of Whole Grout in Groundwater (MB5-17)

Table C.9 - Mass Balance for Flow-Through Leach Test on Whole Grout/Sediment with Groundwater (MBCOLT1)

Table C.10 - Mass Balance for Flow-Through Leach Test on Whole Grout/Sediment with Groundwater (MBCOLT1)

Table C.11 - Mass Balance for Flow-Through Leach Test on Whole Grout/Sediment with Groundwater (MBCOLC1)

Table C.12 - Mass Balance for Flow-Through Leach Test on Whole Grout with Groundwater (MBCOLLT3)

Table C.13 - Mass Balance for Flow-Through Leach Test on Whole Grout with Groundwater (MBCOLLT2)

Table C.14 - Mass Balance for Flow-Through Leach Test on Whole Grout with Groundwater (MBCOLLT1)

Table C. 15 - Mass Balance for Flow-Through Leach Test on Crushed Grout with Groundwater (MBBCGT)

Table C.16 - Mass Balance for Flow-Through Leach Test on Crushed Grout with Groundwater (MBBCGI) 
Table C.17 - Mass Balance for Flow-Through Leach Test on Crushed Grout with Groundwater (MBBCGC)

Table C.18 - Mass Balance for Flow-Through Leach Test on Crushed Grout/Sediment with Groundwater (MBCGT)

Table C.19 - Mass Balance for Flow-Through Leach Test on Crushed Grout/Sediment with Groundwater (MBCGC)

Table C.20 - Mass Balance for Flow-Through Leach Test on Crushed Grout/Sediment with Groundwater (MBSCGI) 
TABLE C.1. Mass Balance for ANS 16.1 Leach Test whole Grout with Groundwater (MB2-4.WK1)

$\mathrm{Ca}$

SAHPLE RO. EFFLUENT HGU CONC. ELUTED SUH ELUTED

\begin{tabular}{|c|c|c|c|c|}
\hline & pp $\mathrm{Ca}_{\mathrm{a}}$ & $\mathrm{Pp} \perp \mathrm{Cz}$ & of $\mathrm{Ca}$ & $29 \mathrm{Cz}$ \\
\hline $2-4-1$ & 49.60 & 56.00 & -3.84 & -3.84 \\
\hline $2-4-2$ & 23.50 & 56.00 & -19.50 & -23.34 \\
\hline $2-4-3$ & 9.90 & 56.00 & -27.66 & -51.00 \\
\hline $2-4-4$ & 9.30 & 56.00 & -28.02 & -79.02 \\
\hline $2-4-5$ & 1.10 & 56.00 & -32.94 & -111.96 \\
\hline $2-4-6$ & 13.20 & 56.00 & -25.68 & -137.64 \\
\hline $2-4-7$ & 1.60 & 56.00 & -32.64 & -170.28 \\
\hline $2-4-8$ & 2.40 & 56.00 & -32.16 & -202.44 \\
\hline $2-4-9$ & 3.00 & 56.00 & -31.80 & -234.24 \\
\hline $2-4-10$ & 1.40 & 56.00 & -32.76 & -267.00 \\
\hline $2-4-11$ & 2.70 & 56.00 & -31.98 & -298.98 \\
\hline $2-4-12$ & 1.60 & 56.00 & -32.64 & -331.62 \\
\hline $2-4-13$ & 1.10 & 56.00 & -32.94 & -364.56 \\
\hline $2-4-14$ & 1.00 & 56.00 & -33.00 & -397.56 \\
\hline $2-4-15$ & 1.00 & 56.00 & -33.00 & -430.56 \\
\hline $2-4-10$ & 1.00 & 56.00 & -33.00 & -463.56 \\
\hline $2-4-77$ & 0.80 & 56.00 & -33.12 & -496.68 \\
\hline $2-4-18$ & 0.90 & 56.00 & -33.06 & -529.74 \\
\hline
\end{tabular}

K

SAKPLE NO. EFFLUENT HGH COKC. ELUTED SUK ELUTED

\begin{tabular}{|c|c|c|c|c|}
\hline & $p p=K$ & ppe $K$ & Ig $K$ & $\leqslant k$ \\
\hline $2-4-1$ & 6.30 & 7.80 & -0.90 & -0.90 \\
\hline $2-4-2$ & 8.50 & 7.80 & 0.42 & -0.48 \\
\hline $2-4-3$ & 7.90 & 7.80 & 0.06 & -0.12 \\
\hline $2-4-4$ & 9.80 & 7.80 & 1.26 & 0.84 \\
\hline $2-4-5$ & 9.20 & 7.80 & 0.84 & 1.68 \\
\hline $2-4-6$ & 9.20 & 7.80 & 0.84 & 2.52 \\
\hline $2-4-7$ & 20.00 & 7.80 & 7.32 & 9.84 \\
\hline $2-4-8$ & 13.00 & 7.80 & 3.12 & 12.96 \\
\hline $2-4-\overline{7}$ & 11.60 & 7.60 & 1.92 & 14.86 \\
\hline $2-4-10$ & 14.00 & 7.80 & 3.72 & 18.60 \\
\hline $7-4-11$ & 11.00 & 7.30 & 1.82 & $20.5 i$ \\
\hline $2-4-12$ & 11.00 & 7.20 & 1.92 & 22.94 \\
\hline$i-4-i j$ & 13.60 & 7.80 & 3.12 & 25.56 \\
\hline $2-4-14$ & 11.80 & 7.80 & 2.40 & 27.96 \\
\hline $2-4-15$ & 11.90 & 7.30 & 2.46 & 30.42 \\
\hline $2-4-: 0$ & 12.00 & 7.80 & 2.52 & 32.94 \\
\hline $2-4-17$ & 13.30 & 7.80 & 3.60 & 36.54 \\
\hline $2-4-18$ & 12.60 & 7.80 & 2.35 & 39.42 \\
\hline
\end{tabular}

$\mathrm{Ha}$

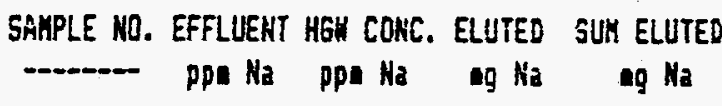

\begin{tabular}{|c|c|c|c|c|}
\hline & PpE $\mathrm{Na}$ & PpD Ha & ${ }_{9} \mathrm{~g}$ & $\operatorname{ag} \mathrm{Hz}$ \\
\hline $2-4-1$ & 46.00 & 25.00 & 12.60 & 12.60 \\
\hline $2-\{-2$ & 109.00 & 25.00 & 50.90 & 63.00 \\
\hline $2-4-3$ & 99.00 & 25.010 & 39.60 & 102.60 \\
\hline $2-4-4$ & 149.00 & 25.00 & 74.40 & 177.00 \\
\hline $2-4-5$ & 125.00 & 25.00 & 60.00 & 237.00 \\
\hline $2-4-6$ & 30.00 & 25.60 & 33.00 & 270.00 \\
\hline $2-4-7$ & 383.00 & 25.60 & 214.80 & 484.80 \\
\hline $2-4-8$ & 183.00 & 25.60 & 94.80 & 579.60 \\
\hline $2-4-9$ & 143.00 & 25.00 & 70.50 & 650.40 \\
\hline $2-4-10$ & 210.00 & 25.00 & 111.00 & 761.40 \\
\hline $2-4-11$ & 118.00 & 25.00 & 55.80 & 817.20 \\
\hline $2-4-12$ & 138.00 & 25.00 & 67.80 & 885.00 \\
\hline $2-4-13$ & 200.00 & 25.00 & 105.00 & $79 \hat{0} .00$ \\
\hline $2-4-14$ & 168.00 & 25.00 & 85.80 & 1075.80 \\
\hline $2-4-15$ & 168.50 & 25.00 & 86.10 & 1161.90 \\
\hline $2-4-16$ & 169.00 & 25.00 & 86.40 & 1248.30 \\
\hline $2-4-17$ & 241.00 & 25.00 & 129.60 & 1377.90 \\
\hline $2-4-18$ & 205.00 & 25.00 & 108.00 & 1485.90 \\
\hline
\end{tabular}

A1

SAMPLE NG. EFFLUENT HGH COHC. ELUTED SUY ELUTED

\begin{tabular}{|c|c|c|c|c|}
\hline -....... & Pp: Al & [p: A] & $49 \mathrm{Al}$ & a $\mathrm{Al}$ \\
\hline $2-4-1$ & 0.11 & 0.10 & 0.01 & 0.01 \\
\hline $2-4-2$ & 0.05 & 0.10 & -0.03 & $-i) .02$ \\
\hline $2-4-3$ & 0.03 & 0.10 & -0.14 & $-0.0 i$ \\
\hline $2-4-4$ & 0.04 & 0.10 & -0.04 & -0.10 \\
\hline $2-4-6$ & 0.03 & 0.10 & -0.04 & -0.14 \\
\hline $2-4-7$ & 0.65 & 0.10 & -0.03 & $-i 1.17$ \\
\hline $2-4-5$ & 1.08 & 0.10 & 6.59 & (1.41 \\
\hline $2-4-9$ & 0.03 & 0.10 & -0.04 & 6.37 \\
\hline $2-4-10$ & 0.03 & 0.10 & -0.64 & 0.33 \\
\hline $2-4-i i$ & 0.03 & 0.10 & -0.04 & 0.29 \\
\hline $2-4-12$ & 0.63 & i. 10 & -0.04 & 0.25 \\
\hline $2-4-13$ & 0.03 & 0.10 & -0.04 & 0.20 \\
\hline $2-4-14$ & 0.08 & 0.10 & -0.64 & J.ió \\
\hline $2-4-i 5$ & 0.03 & 0.10 & -0.14 & 0.12 \\
\hline $2-4-16$ & 0.63 & 0.10 & -0.04 & 0.88 \\
\hline $2-i-17$ & 0.03 & 0.00 & $-i, \hat{y}$ & 0.04 \\
\hline $2-4-18$ & 0.03 & 3.10 & -6.64 & -1.6 \\
\hline
\end{tabular}


TABLE C.1. contd

Si

SAKFLE NB. EFFLUENT HGH CONC. ELUTED SUM ELUTED

$\begin{array}{lrlll} & & & & \\ 2-4-1 & 13.50 & 15.00 & -0.90 & -0.90 \\ 2-4-2 & 11.70 & 15.00 & -1.98 & -2.88 \\ 2-4-3 & 11.40 & 15.00 & -2.16 & -5.04 \\ 2-4-4 & 10.60 & 15.00 & -2.64 & -7.68 \\ 2-4-5 & 8.60 & 15.00 & -3.84 & -11.52 \\ 2-4-6 & 12.40 & 15.00 & -1.56 & -13.08 \\ 2-4-7 & 10.30 & 15.00 & -2.82 & -15.90 \\ 2-4-8 & 8.50 & 15.00 & -3.66 & -19.56 \\ 2-4-9 & 8.60 & 15.00 & -3.64 & -23.40 \\ 2-4-10 & 7.60 & 15.00 & -4.44 & -27.84 \\ 2-4-11 & 3.60 & 15.00 & -3.84 & -31.68 \\ 2-4-12 & 3.20 & 15.00 & -5.28 & -36.96 \\ 2-4-13 & 5.10 & 15.00 & -5.94 & -42.90 \\ 2-4-14 & 4.30 & 15.00 & -6.42 & -49.32 \\ 2-4-15 & 4.55 & 15.00 & -6.27 & -55.59 \\ 2-4-16 & 4.80 & 15.00 & -6.12 & -61.71 \\ 2-4-17 & 5.20 & 15.00 & -5.88 & -67.59 \\ 2-4-18 & 3.40 & 15.00 & -6.96 & -74.55\end{array}$

$F$

GAKPLE HO. EFFLUENT HGY CONC. ELUTED SUH ELUTED

\begin{tabular}{|c|c|c|c|c|}
\hline $2-4-1$ & 0.80 & 0.50 & 0.18 & 0.18 \\
\hline $2-4-2$ & 1.20 & 0.50 & 0.42 & 0.60 \\
\hline $2-4-3$ & 1.20 & 0.50 & 0.42 & 1.02 \\
\hline $2-4-4$ & 3.60 & 0.50 & 1.86 & 2.86 \\
\hline $2-4-5$ & 3.50 & 0.50 & 1.80 & 4.68 \\
\hline $2-4-6$ & 3.70 & 0.50 & 0.72 & 5.40 \\
\hline $2-4-7$ & 4.20 & 0.50 & 2.22 & 7.62 \\
\hline $2-4-6$ & 1.80 & 0.50 & 0.78 & 0.40 \\
\hline$i-4-9$ & 1.30 & 0.50 & 0.46 & 8. 38 \\
\hline $2-4-10$ & i. 60 & 0.50 & 0.66 & 7.54 \\
\hline $2-4-11$ & 0.60 & 0.50 & 0.06 & 9.60 \\
\hline $2-4-12$ & 0.70 & v. 50 & 0.12 & 9.72 \\
\hline $2-4-13$ & 0.70 & 0.50 & 0.12 & 9.84 \\
\hline $2-4-14$ & 2.30 & 6.50 & 1.06 & 10.92 \\
\hline $2-4-15$ & 1.50 & 0.50 & 0.50 & 11.52 \\
\hline $2-4-16$ & 0.70 & 0.50 & 0.12 & 11.64 \\
\hline $2-4-17$ & 1.00 & 0.50 & 0.30 & 11.34 \\
\hline $2-4-15$ & 0.70 & 0.50 & 6.12 & 12.06 \\
\hline
\end{tabular}

$\mathrm{Kg}$

SAMPLE NO. EFFLUERT HGH CONC. ELUTED SIIK ELUTED

\begin{tabular}{|c|c|c|c|c|}
\hline & D & ppe $\mathrm{Ng}$ & o $\mathrm{Ng}$ & Ig $\mathrm{Mg}$ \\
\hline $2-4-1$ & 13.10 & 14.40 & -0.78 & $-0,78$ \\
\hline $2-4-2$ & 5.94 & 14.40 & -5.08 & -5.86 \\
\hline $2-4-3$ & 6.63 & 14.40 & -4.66 & -10.52 \\
\hline $3-4-4$ & 1.00 & 14.40 & -8.04 & -18.56 \\
\hline $2-4-5$ & 0.32 & 14.40 & -8.45 & -27.01 \\
\hline $2-4-6$ & 9.70 & 14.40 & -2.82 & -29.83 \\
\hline $2-4-7$ & 0.06 & 14.70 & -8.60 & -36.43 \\
\hline $2-4-8$ & 0.75 & 14.40 & -8.19 & -96.62 \\
\hline $2-4-9$ & 2.80 & 14.40 & -6.90 & -55.50 \\
\hline $2-4-10$ & 0.46 & 14.40 & -8.36 & -61.74 \\
\hline $2-4-11$ & 5.53 & 14.40 & $-5,32$ & -67.27 \\
\hline $2-4-12$ & 2.57 & 14.40 & -7.10 & $-i 4.36$ \\
\hline $2-4-13$ & 0.13 & 14.40 & -8.56 & -82.93 \\
\hline $2-4-14$ & 0.31 & 14.40 & -8.45 & -91.38 \\
\hline $2-4-15$ & 0.30 & 14.40 & -8.46 & -87.84 \\
\hline $2-4-16$ & 0.28 & 14.40 & -8.47 & -108.32 \\
\hline $4-17$ & 0.06 & 14.40 & -8.60 & -116.92 \\
\hline $2-4-18$ & 0.15 & 14,40 & -8.55 & -125.47 \\
\hline
\end{tabular}

NOJ

SAMFLE NO. EFFLUENT HEN CONC. ELUTED SUK ELUTED

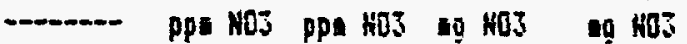

\begin{tabular}{|c|c|c|c|c|}
\hline$i-4-1$ & 1.40 & 0.10 & 0.78 & $0.7 B$ \\
\hline $2-4-2$ & 2.00 & 0.10 & 1.14 & 1.92 \\
\hline $2-4-3$ & 1.20 & 0.10 & 0.66 & 2.56 \\
\hline $2-4-4$ & 2.00 & 0.10 & 1.14 & 3.72 \\
\hline $2-4-5$ & 1.40 & 0.10 & 0.78 & 4.50 \\
\hline $2-4-6$ & 0.90 & 6.10 & 0.48 & 4.75 \\
\hline $2-4-7$ & 6.10 & 0.16 & 3.600 & 8.53 \\
\hline $2-4-6$ & 2.30 & 6.00 & 1.32 & 9.90 \\
\hline $2-4-9$ & 1.30 & 0.10 & 0.72 & 10.02 \\
\hline $2-4-10$ & 6.70 & $0 .: 0$ & 4.08 & 14.70 \\
\hline $2-4-11$ & v. 00 & 0.10 & 0.42 & 15.12 \\
\hline $2-4-12$ & 0.39 & 0.10 & 6.42 & 15.54 \\
\hline $2-4-13$ & 2.00 & 0.10 & 1.14 & 35.68 \\
\hline $2-4-14$ & 0.80 & $0 . \hat{\imath} \hat{\jmath}$ & i. 42 & 17.60 \\
\hline $2-4-15$ & 2.20 & 0.10 & 0.66 & 17.76 \\
\hline $2-4-16$ & 1.60 & 4.10 & $3, \div 0$ & 18.86 \\
\hline $2-4-17$ & 5.00 & 9.10 & 2.34 & 21.60 \\
\hline $2-4-18$ & $2 . \div 0$ & vi.io & 1.38 & 22.78 \\
\hline
\end{tabular}


SAMPLE ROEFFLUEHT HGN CONC. ELUUTED SLH ELUTE PpE $B$ ppe $B$ ug $B$ a $B$

$\begin{array}{lllll}2-4-1 & 0.56 & 0.34 & 0.14 & 0.14 \\ 2-4-2 & 1.04 & 0.34 & 0.42 & 0.56 \\ 2-4-3 & 0.73 & 0.34 & 0.23 & 0.80 \\ 2-4-4 & 0.63 & 0.34 & 0.17 & 0.37 \\ 2-4-5 & 0.51 & 0.34 & 0.10 & 1.07 \\ 2-4-6 & 0.39 & 0.34 & 0.39 & 1.46 \\ 2-4-7 & 0.43 & 0.34 & 0.05 & 1.52 \\ 2-4-8 & 0.43 & 0.34 & 0.05 & 1.57 \\ 2-4-9 & 0.41 & 0.34 & 0.04 & 1.61 \\ 2-4-10 & 0.55 & 0.34 & 0.13 & 1.74 \\ 2-4-11 & 0.38 & 0.34 & 0.02 & 1.76 \\ 2-4-12 & 0.39 & 0.34 & 0.03 & 1.79 \\ 2-4-13 & 0.46 & 0.34 & 0.07 & 1.87 \\ 2-4-14 & 0.41 & 0.34 & 0.04 & 1.91 \\ 2-4-15 & 0.43 & 0.34 & 0.05 & 1.96 \\ 2-4-16 & 0.46 & 0.34 & 0.07 & 2.03 \\ 2-4-17 & 0.58 & 0.34 & 0.14 & 2.18 \\ 2-4-18 & 0.40 & 0.34 & 0.04 & 2.21\end{array}$

IC

SAHPLE HOEFFLUEMT HER COHC. ELUTEO SUH ELUTE

\begin{tabular}{|c|c|c|c|c|}
\hline & pte ic & Dpa if & $\lg$ & 0 Ii \\
\hline $2-4-1$ & 23.98 & 34.10 & -6.67 & -6.07 \\
\hline $2-4-2$ & 25.62 & 34.10 & -5.45 & -11.52 \\
\hline $2-4-3$ & 17.33 & 34.10 & -10.06 & -21.50 \\
\hline$z-4-4$ & 20.51 & 34.10 & $-\hat{5} .15$ & -29.7 \\
\hline $2-4-5$ & 90.23 & 34.10 & -3.30 & -50.63 \\
\hline$z-a-1-j$ & $21, \bar{i}$ & 34.16 & $-\bar{i} .30$ & -45.31 \\
\hline$i-4-i$ & $24 .: 8$ & 34.90 & -5.35 & D.5. \\
\hline$i-\dot{a}-\bar{c}$ & İ. & 34.10 & -8.55 & $-02,21$ \\
\hline$z-9-5$ & 17.90 & 34.10 & -10.20 & 72,4 \\
\hline $7-4-10$ & $i=7 i$ & 34.10 & -8.03 & -81.8 \\
\hline$=-4-11$ & 19.93 & 34.10 & -2.98 & -30.92 \\
\hline$=-4-12$ & $47.1 i$ & 54.10 & Fivi & -81.3 \\
\hline$-4-15$ & Zidio & 34.10 & -3.30 & -87.38 \\
\hline$-4-14$ & $i 7.7 j$ & 34.10 & $-6.7 \div$ & 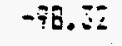 \\
\hline 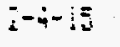 & 5.50 & 39.10 & $\dot{v} .0 \dot{0}$ & $-7 \overline{17}$ \\
\hline $4-i 5$ & $2: 30$ & 34,10 & $-\bar{T} . \overline{1}$ & $-105,20$ \\
\hline $4-i$ & 25.60 & 34.10 & $-i, 3 i$ & -105.75 \\
\hline $4-\vdots 5$ & 80 & 34.10 & -3.5 & -105.Z3 \\
\hline$\therefore-37$ & 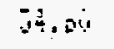 & 37.010 & 3.3 & -10.50 \\
\hline $4-20$ & $\therefore,=0$ & 5,10 & $-3, i$ & $-111 . \overline{7}$ \\
\hline
\end{tabular}

SAMPLE HCEFFLUENT HGW COHC. ELUTED SUM ELUTE

\begin{tabular}{|c|c|c|c|c|}
\hline & $p p=504$ & pp: SDA & 504 & EDQ 504 \\
\hline $2-4-1$ & 81.00 & 86.00 & -3.00 & -3.00 \\
\hline $2-4-2$ & 93.00 & 86.60 & 4.20 & 1.20 \\
\hline $2-4-3$ & 84.00 & 26.00 & -1.20 & \\
\hline $2-4-4$ & 29.60 & 86.00 & 1.30 & i.j \\
\hline $2-4-5$ & 84.00 & 86.00 & -1.20 & 0.6 \\
\hline $2-4-6$ & 75.00 & 86.00 & -6.60 & -6.6 \\
\hline $2-4-7$ & 107.00 & 36.60 & 12. ait & \\
\hline $2-4-8$ & 82.60 & 36.00 & -2.40 & \\
\hline $2-4-9$ & 82.00 & 86.00 & -2.46 & $1 . \bar{c}$ \\
\hline $2-4-10$ & 72.00 & 86.60 & -3.40 & -6.60 \\
\hline $2-4-11$ & 86.60 & 86.00 & 6.60 & $-6.5 \hat{0}$ \\
\hline $2-4-1 \hat{2}$ & 81.00 & $8 t .60$ & -3.00 & -9.60 \\
\hline $2-4-13$ & 83.00 & 86.60 & -1.80 & -11.90 \\
\hline $2-4-34$ & 60.60 & $B 6.00$ & -15.60 & $-2 \pi .66$ \\
\hline $2-4-15$ & 60.00 & 66.00 & -15.66 & -42.60 \\
\hline & 71.00 & 86.00 & $-9.6 \hat{v}$ & -51.60 \\
\hline & 75.00 & 86.00 & -6.60 & -56.20 \\
\hline $2-4-18$ & 70.00 & 66.00 & -9.60 & $-6 \bar{i} .80$ \\
\hline
\end{tabular}

Tf

SAMPLE NOEFFLUENT HGH CONC. ELUTED SUH ELUTE

\begin{tabular}{|c|c|c|c|c|}
\hline-1 & ppe $7 \mathrm{~T}$ & ppe TC & 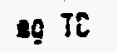 & $\lg$ TS \\
\hline $2-4-1$ & 22.70 & 33.10 & -6.24 & -6.24 \\
\hline $2-4-2$ & 23.47 & 33.10 & -5.78 & -12.02 \\
\hline $2-4-3$ & 16.36 & 33.10 & -10.04 & -22.06 \\
\hline $2-4-4$ & 19.11 & 33.10 & -8.39 & -30.46 \\
\hline $2-4-5$ & 16.78 & 33.10 & -8.59 & -39.65 \\
\hline $2-4-6$ & 20.68 & 33.10 & -7.45 & -46.50 \\
\hline $2-4-7$ & 18.70 & 35.10 & -6.64 & -55.14 \\
\hline $2-4-6$ & 18.60 & 33.10 & -8.70 & -63.84 \\
\hline $7-4-7$ & 15.70 & 33.10 & -10.44 & -74.20 \\
\hline $2-4-10$ & $: 8.34$ & 35.10 & -3.86 & $-8 . .14$ \\
\hline $2-4-11$ & 17.77 & 30.10 & -7.20 & -72.33 \\
\hline $2-4-i \bar{i}$ & $46 . \overline{35}$ & 5.10 & 7.75 & -84.38 \\
\hline $2-4-13$ & 18.61 & 33.100 & $-3.5 i$ & -52.96 \\
\hline $2-4-14$ & i5. 40 & 33.10 & -8.82 & -101.70 \\
\hline $2-4-15$ & $3 \bar{s}^{3} .10$ & 33.10 & 1.20 & -100.56 \\
\hline $2-4-i 3$ & 21.20 & $3 \overline{3} .10$ & -7.14 & -107.72 \\
\hline $2-4-17$ & 33.60 & 33.10 & 6.30 & -107.42 \\
\hline $2-4-18$ & 33,90 & $33 .: 9$ & 0.48 & $-10 \leq .74$ \\
\hline
\end{tabular}


TABLE C.2. Mass Balance for ANS 16.1 Leach Test whole Grout with Groundwater (MB3-4)

$C$

SAMPLE NO. EFFLUENT HGN CONC. ppe Ca ppe Ca

$\begin{array}{ll}3-4-1 & 51.90 \\ 3-4-2 & 13.90 \\ 3-4-3 & 7.80 \\ 3-4-4 & 7.50 \\ 3-4-5 & 12.80 \\ 3-4-6 & 13.50 \\ 3-4-7 & 14.20 \\ 3-4-8 & 2.00 \\ 3-4-9 & 2.60 \\ 3-4-10 & 1.20 \\ 3-4-11 & 1.90 \\ 3-4-12 & 1.30 \\ 3-4-13 & 1.00 \\ 3-4-14 & 1.20 \\ 3-4-15 & 0.65 \\ 3-4-16 & 0.09 \\ 3-4-17 & 0.80\end{array}$

ELUTED SUH ELUTED $19 \mathrm{Ca} \quad \mathrm{Ca}$

$\begin{array}{lrr}56.00 & -2.46 & -2.46 \\ 56.00 & -25.26 & -27.72 \\ 56.00 & -28.92 & -56.64 \\ 56.00 & -29.10 & -65.74 \\ 56.00 & -25.92 & -111.66 \\ 56.00 & -25.50 & -137.16 \\ 56.00 & -25.08 & -162.24 \\ 56.00 & -32.40 & -194.64 \\ 56.00 & -32.04 & -226.68 \\ 56.00 & -32.38 & -259.56 \\ 56.00 & -32.46 & -292.02 \\ 56.00 & -32.82 & -324.84 \\ 56.00 & -33.00 & -357.84 \\ 56.00 & -32.88 & -390.72 \\ 56.00 & -33.21 & -423.93 \\ 56.00 & -33.55 & -457.48 \\ 56.00 & -33.12 & -490.60\end{array}$

k

SAMFLE HO. EFFLUERT HEN CONC. ppa $K$

ppe $K$

7.80
7.80
7.80
7.80
7.80
7.80
7.80
7.80
7.80
7.60
7.80
7.80
7.80
7.80
7.80
7.80
7.30

ELUTED SUH ELUTED ag $K$ og

$\begin{array}{rr}-0.24 & -0.24 \\ 1.92 & 1.68 \\ 1.32 & 3.00 \\ 3.12 & 6.12 \\ 2.52 & 8.64 \\ 1.32 & 9.96 \\ 8.52 & 16.46 \\ 3.72 & 22.20 \\ 2.52 & 24.72 \\ 4.32 & 28.64 \\ 2.52 & 31.56 \\ 1.32 & 32.89 \\ 3.72 & 36.00 \\ 2.52 & 39.12 \\ 2.52 & 41.64 \\ 2.52 & 44.16 \\ 2.64 & 46.30\end{array}$

GAMPLE NO. EFFLUENT HGW CONC. ppe $\mathrm{Na}$ ppe $\mathrm{Ka}$

$3-4-1$
$3-4-2$
$3-4-3$
$3-4-4$
$3-4-5$
$3-4-6$
$3-4-7$
$3-4-8$
$3-4-7$
$3-4-10$
$3-4-11$
$3-4-12$
$3-4-13$
$3-4-14$
$3-4-15$
$3-4-16$
$3-4-17$

36.00
152.00
108.00
173.00
144.00
86.00
418.00
206.00
161.00
229.00
126.00
140.00
200.00
169.00
165.00
161.00
217.00

$\mathrm{Na}$

25.00

25.00

25.00

25.100

$25.00^{\circ}$

25.00

25.00

25.60

25.00

25.00

25.60

25.00

25.00

25.00

25,00

25.00

25.00
ELUTED SUH ELUTED

$\begin{array}{rr}6.60 & 6.60 \\ 76.20 & 82.80 \\ 49.80 & 132.60 \\ 88.30 & 221.40 \\ 71.40 & 292.80 \\ 36.60 & 329.40 \\ 235.80 & 565.20 \\ 108.60 & 673.80 \\ 61.60 & 755.40 \\ 122.40 & 877.80 \\ 60.60 & 938.40 \\ 69.00 & 1067.40 \\ 105.60 & 1112.40 \\ 86.40 & 1198.30 \\ 84.00 & 1282.80 \\ 81.60 & 1364.40 \\ 115.20 & 1479.60\end{array}$

Al

SAMPLE NO. EFFLUET HGU CONC. PpE Al PPE Al

0.13
0.68
0.05
0.63
0.10
0.09
1.71
0.04
0.04
0.05
0.64
0.63
0.03
0.03
0.03
0.03
0.03

0.10
0.10
0.10
0.30
0.10
0.10
0.10
0.10
0.10
0.10
0.10
0.10
0.10
0.10
0.10
0.10
0.10

ELUTED SUH ELUTED ho $\mathrm{fil} \quad \mathrm{g} \mathrm{hl}$

$3-4-1$
$j-4-2$
$3-4-3$
$j-4-4$
$3-4-5$
$3-4-6$
$3-4-7$
$j-4-8$
$j-4-9$
$j-4-10$
$j-4-11$
$3-4-12$
$3-4-13$
$3-4-14$
$j-4-15$
$3-4-16$
$3-4-17$

0.02
-0.01
-0.03
0.32
0.00
-0.01
0.97
-6.04
-3.04
-0.03
-0.64
-0.04
-0.04
-0.04
-0.04
-0.04
-0.04

0.02 0.01 $-6.02$ 0.29 6. 29 0.29 3.25 1.22 i. 18 1.15 $1 . ! 2$ 1.07 1.03 0.79 0.95 0.91 0.36 
SAMPLE NO. EFFLUENT HGW CONC. ELUTED SUA ELUTED ppa $\mathrm{Si}$ ppa $\mathrm{Si}$ ig $\mathrm{Si} \quad \mathrm{Si}$

$\begin{array}{lllll}3-4-1 & 13.80 & 15.00 & -0.72 & -0.72 \\ 3-4-2 & 11.30 & 15.00 & -2.22 & -2.34 \\ 3-4-3 & 11.00 & 15.00 & -2.40 & -5.34 \\ 3-4-4 & 10.00 & 15.00 & -3.00 & -8.34 \\ 3-4-5 & 10.50 & 15.00 & -2.70 & -11.04 \\ 3-4-6 & 11.90 & 15.00 & -1.86 & -12.90 \\ 3-4-7 & 10.30 & 15.00 & -2.82 & -15.72 \\ 3-4-8 & 9.30 & 15.00 & -3.42 & -19.14 \\ 3-4-9 & 8.30 & 15.00 & -4.02 & -23.16 \\ 3-4-10 & 7.30 & 15.00 & -4.62 & -27.78 \\ 3-4-11 & 7.60 & 15.00 & -4.44 & -32.22 \\ 3-4-12 & 11.50 & 15.00 & -2.10 & -34.32 \\ 3-4-13 & 4.30 & 15.00 & -6.42 & -40.74 \\ 3-4-14 & 4.60 & 15.00 & -6.24 & -46.38 \\ 3-4-15 & 4.75 & 15.00 & -6.15 & -53.13 \\ 3-4-16 & 4.90 & 15.00 & -6.06 & -59.19 \\ 3-4-17 & 4.70 & 15.00 & -6.18 & -65.37\end{array}$

F

SAMFLE MO. EFFLUENT HGU CONC. ELUTED SUN ELUTED pps $F$ ppa $F$ ig $F$

$\begin{array}{lllll}3-4-j & 0.60 & 0.50 & 0.06 & 0.06 \\ 3-4-2 & 1.70 & 0.50 & 0.72 & 0.78 \\ j-4-3 & 1.50 & 0.50 & 0.60 & 1.38 \\ j-4-4 & 3.30 & 0.50 & 1.98 & 3.36 \\ j-4-5 & 3.70 & 0.50 & 1.92 & 5.23 \\ j-4-6 & 2.00 & 0.50 & 0.70 & 6.18 \\ j-4-7 & 4.70 & 0.50 & 2.52 & 8.70 \\ j-4-3 & 2.70 & 0.50 & 1.44 & 10.14 \\ j-4-3 & 1.50 & 0.50 & 6.60 & 10.74 \\ j-4-10 & 1.80 & 0.50 & 0.78 & 11.52 \\ j-4-11 & 0.90 & 0.50 & 0.24 & 11.76 \\ 3-4-12 & 0.80 & 0.50 & 0.18 & 11.54 \\ 3-4-13 & 0.70 & 0.50 & 0.12 & 12.06 \\ 3-4-14 & 2.50 & 0.50 & 1.26 & 13.32 \\ j-4-15 & 1.55 & 0.50 & 0.59 & 14.01 \\ 3-4-16 & 0.70 & 0.50 & 0.12 & 14.13 \\ j-4-17 & 0.90 & 0.50 & 0.24 & 14.37\end{array}$

SAKFLE NO. EFFLUEHT HGW CONC.

3
$3-4-1$
$3-4-2$
$j-4-3$
$3-4-4$
$3-4-5$
$3-4-6$
$3-4-7$
$3-4-8$
$j-4-9$
$3-4-10$
$j-4-11$
$j-4-12$
$3-4-13$
$j-4-14$
$j-4-15$
$3-4-16$
$3-4-17$
Pan

$\begin{array}{ll}13.20 & 14.40 \\ 2.46 & 14.40 \\ 4.20 & 14.40 \\ 0.29 & 14.40 \\ 1.69 & 14.40 \\ 8.50 & 14.40 \\ 0.66 & 14.40 \\ 0.20 & 14.40 \\ 1.60 & 14.40 \\ 0.16 & 14.40 \\ 4.67 & 14.40 \\ 1.31 & 14.40 \\ 0.14 & 14.40 \\ 0.34 & 14.40 \\ 0.34 & 14.40 \\ 0.34 & 14.40 \\ 0.06 & 14.40\end{array}$

$-0.72$

$-7.15$

$-5.82$

$-8.47$

$-7.63$

$-3.54$

$-6.60$

$-3.52$

$-7.58$

$-3.54$

$-0.20$

$-7.49$

$-6.56$

$-3.44$

$-8.44$

$-6.44$

$-8.60$

SUM ELUTEO

ig $\mathrm{kg}$

$$
-0.72
$$

$-13.69$

$-22.16$

$-29.78$

$-33.32$

$-41.73$

$-50.45$

$-58.13$

$-66.67$

$-72.87$

$-30.36$

$-88.72$

$-97.32$

$-165.79$

$-114.25$

$-\$ 22.03$

103

SAHFL :NO. EFFLUENT HGH COHE.

ELUTED SIS ELUTES PPO $\mathrm{ND3}$ PPE NGS og $\mathrm{Hez}$ aุ 103

$3-4-1$
$3-4-2$
$3-4-3$
$3-4-4$
$3-4-5$
$3-4-5$
$3-4-7$
$j-4-5$
$3-4-5$
$j-4-10$
$j-4-11$
$3-4-12$
$j-4-13$
$3-4-14$
$3-4-15$
$j-4-16$
$3-4-97$

0.60
2.90
1.70
2.20
1.70
0.90
7.50
2.30
1.30
1.70
0.60
1.10
2.30
0.30
1.20
1.60
5.60

0.10

0.30

0.30

0.10

1.68

1.98

0.10

0.96

2.34

$1.26, \quad 4.20$

$0.96 \quad 5.16$

0.10

0. 48

5.64

4.44

10.08

0.10

1. 50

11.58

6.10

3.76

12.54

6. 10

1.68

13. 62

i. 10

i. 52

6.60

19. 04

0.10

t..10

1.32

0.42

14.64

15. 56

6.10

0.10

0.50

16.38

17.84

i. 10

0.70

17.94

2.74

20.39 
TABLE C.2. contd

H3BO3

SAKFLE ND. EFFLUENT HGN CONC. ELUTED SUM ELUTED

$\begin{array}{lll} & \text { ppE B } & \text { ppe B } \\ 3-4-1 & 0.53 & 0.34 \\ 3-4-2 & 1.43 & 0.34 \\ 3-4-3 & 1.01 & 0.34 \\ 3-4-4 & 1.24 & 0.34 \\ 3-4-5 & 0.36 & 0.34 \\ 3-4-6 & 0.35 & 0.34 \\ 3-4-7 & 1.18 & 0.34 \\ 3-4-8 & 0.56 & 0.34 \\ 3-4-9 & 0.46 & 0.34 \\ 3-4-10 & 0.37 & 0.34 \\ 3-4-11 & 0.33 & 0.34 \\ 3-4-12 & 0.37 & 0.34 \\ 3-4-13 & 0.46 & 0.34 \\ 3-4-14 & 0.37 & 0.34 \\ 3-4-15 & 0.41 & 0.34 \\ 3-4-16 & 0.45 & 0.34 \\ 3-4-17 & 5.49 & 0.34\end{array}$

I $B$ B $B$

0.11

0.65

0.40

0.54

0.31

0.13

0.50

0.13

0.07

0.14

0.05

0.02

0.07

0.02

0.04

0.07

3.09
0.11

0.77

1.17

1.71

2.02

2.15

2.65

2.78

2.86

2.99

3.05

3.07

3.14

3.16

3.20

3.26

6.35
504

SAMPLE NO. EFFLUENT HGH CONC. ELUTEO SUM ELUTED ppa 504

PPE 504

19584

og SO4

$3-4-1$
$3-4-2$
$3-4-3$
$3-4-4$
$3-4-5$
$3-4-6$
$3-4-7$
$3-4-8$
$3-4-9$
$3-4-10$
$3-4-11$
$3-4-12$
$3-4-13$
$3-4-14$
$3-4-15$
$3-4-16$
$3-4-17$

79.00

102.00

91.00

95.00

66.00

82.00

107.00

84.00

82.00

75.00

37.60

78.00

82.00

60.00

64.00

68.10

71.00
86.00
86.00
86.00
86.00
86.00
86.00
86.00
86.00
86.00
86.00
86.00
86.00
86.00
86.00
86.00
66.00
86.00

-4.20
9.60
3.00
5.40
0.60
-2.40
12.60
-1.20
-2.40
-6.60
0.60
-4.60
-2.40
-15.60
-13.20
-10.20
-9.00

$-4.20$

5.40

8.40

13.30

13.80

11.40

24.60

22.30

20.40

13.80

14.40

5.60

7.20

$-8.40$

$-21.50$

$-32.40$

$-41.40$ 
TABLE C.3. Mass Balance for ANS 16.1 Leach Test whole Grout with Groundwater (MB4-8)

Ca

SAHPLE NO. EFFLUEHT HGW COKC, ELUTED SUR ELUTED

\begin{tabular}{|c|c|c|c|c|}
\hline 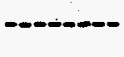 & $\mathrm{pos} \mathrm{Ca}$ & ppa Ca & $\mathrm{g} \mathrm{Ca}$ & $9 \mathrm{Ca}$ \\
\hline $4-8-1$ & 50.60 & 56.00 & -3.24 & -3.24 \\
\hline $4-8-2$ & 19.20 & 56.00 & -22.08 & -25.32 \\
\hline $4-8-3$ & 9.10 & 56.00 & -28.14 & -53.46 \\
\hline $4-8-4$ & 9.90 & 56.00 & -27.66 & -81.12 \\
\hline $4-6-5$ & 12.40 & $56.00^{\circ}$ & -26.16 & -107.28 \\
\hline $4-8-6$ & 14.50 & 56.00 & -24.90 & -132.18 \\
\hline $4-8-7$ & 1.70 & 56.00 & -32.58 & -164.76 \\
\hline $4-3-8$ & 2.70 & 56.00 & -31.98 & -196.74 \\
\hline $4-8-9$ & 2.50 & 56.00 & -32.10 & -228.84 \\
\hline $4-8-10$ & 1.40 & 56.00 & -32.76 & -261.60 \\
\hline $4-8-11$ & 2.30 & $56 . \hat{00}$ & -32.22 & -293.82 \\
\hline $4-3-12$ & 1.10 & 56.00 & -32.94 & -326.76 \\
\hline $4-5-13$ & 1.10 & 56.00 & -32.94 & -359.70 \\
\hline $4-8-14$ & 0.30 & 56.00 & -33.06 & -392.76 \\
\hline $4-8-15$ & 0.90 & 56.00 & -33.06 & -425.82 \\
\hline $4-8-16$ & 0.90 & 56.00 & -33.06 & -458.88 \\
\hline $4-8-17$ & 0.90 & 56.00 & -33.06 & -491.94 \\
\hline
\end{tabular}

K

SAMFLE NO. EFFLUENT hGN COMC. ELUTED SUM ELUTED pp: $K$ ppI $k$ sg $k$ in $K$

\begin{tabular}{|c|c|c|c|c|}
\hline $4-8-1$ & 7.40 & 7.80 & -0.24 & -0.24 \\
\hline $4-8-2$ & 11.00 & 7.80 & 1.92 & 1.68 \\
\hline $4-\hat{b}-3$ & 9.50 & 7.80 & 1.02 & 2.70 \\
\hline $4-8-4$ & 12.06 & 7.80 & 2.52 & 5.22 \\
\hline $4-8-5$ & 11.00 & 7.80 & 1.32 & 7.14 \\
\hline $4-8-6$ & 9.00 & 7.80 & 0.72 & 7.36 \\
\hline $4-8-7$ & 20.60 & 7.80 & 7.32 & 15. 18 \\
\hline $4-8-6$ & 13.00 & 7.80 & 3.12 & 16.30 \\
\hline $4-5-7$ & $1: .00$ & $7.8 \hat{v}$ & 1, 92 & 20.22 \\
\hline $4-6-10$ & 14.00 & 7.80 & 3.72 & 23.94 \\
\hline $4-8-11$ & 7.70 & 7.30 & 1.14 & 25.08 \\
\hline $4-8-12$ & 9.60 & 7.30 & 1.06 & 26.16 \\
\hline $4-2-\{3$ & 14.00 & 7.80 & 3.72 & 27.68 \\
\hline $4-8-14$ & 13.50 & 7.60 & 2.22 & 72.10 \\
\hline $4-3-15$ & $11.7 \mathrm{E}$ & 7.50 & 2.37 & 34.97 \\
\hline $4-\varepsilon-1=$ & 12.60 & 7.80 & 2.52 & 36.99 \\
\hline $2-E-17$ & $13.6 \mathrm{ft}$ & 7.80 & $3 . \vdots 2$ & 40.11 \\
\hline
\end{tabular}

His

SAMPLE HO. EFFLUENT hâ̆ COHC. ELUTED SUH ELUTED

\begin{tabular}{|c|c|c|c|c|}
\hline & $p p=\mathrm{Ha}$ & pp: Ha & $q \mathrm{Na}$ & $\lg \mathrm{Ma}$ \\
\hline $4-8-1$ & 34.00 & 25.110 & 5.40 & 5.40 \\
\hline $4-8-2$ & 141.00 & 25.00 & 69.60 & 75.60 \\
\hline $4-8-3$ & 92.00 & 25.00 & 40.20 & $\$ 15.20$ \\
\hline $4-8-4$ & 144.00 & 25.60 & 71.40 & 166.60 \\
\hline $4-8-5$ & 112.00 & 25.00 & 52.20 & 238.80 \\
\hline $4-6-6$ & 71.00 & 25.00 & 27.60 & 266.40 \\
\hline $4-3-7$ & 3600.00 & 25.00 & 201.60 & 407.40 \\
\hline $4-8-8$ & 169.00 & 25.00 & 86.40 & 553.60 \\
\hline $4-8-9$ & 132.00 & 25.00 & 64.20 & 618.00 \\
\hline $4-8-10$ & 195.00 & 25.60 & 102.00 & 720.00 \\
\hline $4-8-11$ & 112.00 & 25.00 & 52.20 & 772.20 \\
\hline $4-8-12$ & 124.00 & 25.00 & 57.40 & 831.60 \\
\hline $4-8-13$ & 190.00 & 25.00 & 99.00 & 930.50 \\
\hline $4-8-14$ & 151.00 & 25.00 & 75.60 & 1006.20 \\
\hline $4-8-15$ & 151.50 & 25.00 & 75.70 & 1002.10 \\
\hline $4-8-16$ & 152.00 & 25.00 & 76.20 & 1158.30 \\
\hline $4-3-17$ & 218.00 & 25.00 & 115.80 & 1274.10 \\
\hline
\end{tabular}

Al

SAMFLE NO. EFFLLENT hGW COAC. ELUTED SUH ELLTED

\begin{tabular}{|c|c|c|c|c|}
\hline & ppe Al & $p p h \quad \hat{l}$ & $m \mathrm{HI}$ & $\hat{n}$ \\
\hline $4-8-1$ & 0.12 & 0.10 & 0.01 & \\
\hline $4-8-2$ & 0.05 & 0.10 & -0.03 & \\
\hline $4-5-3$ & 0.12 & 0.10 & 0.01 & \\
\hline $4-8-4$ & 0.29 & 0.10 & 0.11 & \\
\hline $4-6-5$ & 0.09 & 0.10 & -0.01 & \\
\hline $4-3-5$ & 0.08 & 0.10 & $-6.0 !$ & \\
\hline $4-8-7$ & 0.67 & 0.10 & 0.34 & $\dot{0}$. \\
\hline $4-6-8$ & 0.03 & 0.20 & -6.64 & 0.2 \\
\hline $4-6-9$ & 0.03 & 1.: $: 0$ & -3.64 & 0. \\
\hline $4-8-10$ & 0.03 & 0.10 & -0.04 & a. \\
\hline $4-3-1 !$ & 0.03 & i. 10 & -3.04 & 0. \\
\hline $4-6-12$ & 0.03 & 0.16 & -4.04 & 0.2 \\
\hline $4-3-13$ & 0.03 & 0.10 & -0.34 & $\hat{i}$. \\
\hline $4-8-14$ & 0.63 & $0 . ! 6$ & -0.04 & \\
\hline $4-8-15$ & 0.03 & $0.1 \hat{0}$ & $-\hat{v} .04$ & 0. \\
\hline $4-8-16$ & 0.03 & 0.10 & -0.04 & 0. \\
\hline $4-2-17$ & 0.03 & 0.10 & -0.04 & u. \\
\hline
\end{tabular}


TABLE C.3. contd

Si

SAMPLE NO. EFFLUENT HGK CONC. ELUTED SUA ELUTED

$\begin{array}{lllll} & \text { pp Si } & \text { ppe Si } & \text { S Si } & \text { S Si } \\ 4-8-1 & 13.50 & 15.00 & -0.90 & -0.90 \\ 4-8-2 & 11.20 & 15.00 & -2.28 & -3.18 \\ 4-8-3 & 11.80 & 15.00 & -1.92 & -5.10 \\ 4-8-4 & 10.20 & 15.00 & -2.68 & -7.98 \\ 4-8-5 & 10.40 & 15.00 & -2.76 & -10.74 \\ 4-8-6 & 12.50 & 15.00 & -1.50 & -12.24 \\ 4-8-7 & 9.90 & 15.00 & -3.06 & -15.30 \\ 4-3-8 & 9.70 & 15.00 & -3.16 & -18.48 \\ 4-6-7 & 8.80 & 15.00 & -3.72 & -22.20 \\ 4-8-10 & 7.30 & 15.00 & -4.62 & -26.82 \\ 4-6-11 & 9.60 & 15.00 & -3.24 & -30.06 \\ 4-8-12 & 6.40 & 15.00 & -5.16 & -35.22 \\ 4-8-13 & 5.20 & 15.00 & -5.88 & -41.10 \\ 4-8-14 & 4.40 & 15.00 & -6.36 & -47.46 \\ 4-6-15 & 4.70 & 15.00 & -6.18 & -53.64 \\ 4-8-16 & 5.00 & 15.00 & -6.00 & -59.64 \\ 4-8-17 & 5.00 & 15.00 & -6.00 & -65.64\end{array}$

$\mathbf{F}$

SAMFLE ND. EFFLUENT HGW COKC. ELUTED SUH ELUTED

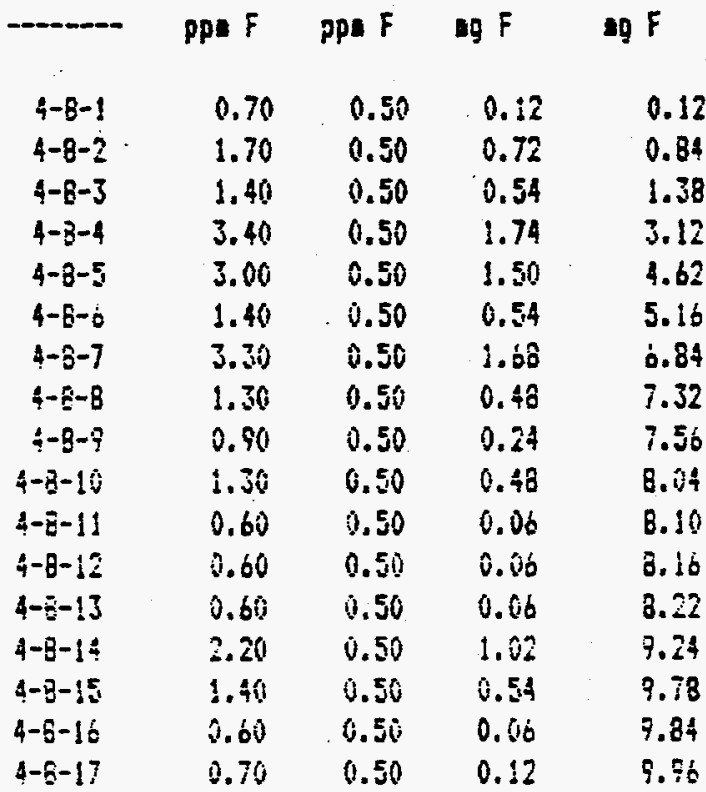

ig

SAMPLE RO. EFFLUENT HGW CONC. ELUTED SUH ELUTED

\begin{tabular}{|c|c|c|c|c|}
\hline 五 & pps $\mathrm{kg}$ & ppe ilg & ag $\mathrm{kg}$ & ig \\
\hline $4-3-1$ & 13.10 & 12.90 & 0.12 & \\
\hline $4-8-2$ & 5.94 & 2.70 & -4.18 & -4 \\
\hline $4-8-3$ & 6.63 & 6.50 & -3.76 & -7 \\
\hline $4-8-4$ & 1.00 & 1.20 & -7.14 & -14.9 \\
\hline $4-8-5$ & 0.32 & 4.20 & -7.55 & -22.5 \\
\hline $4-8-6$ & 9.70 & 10.20 & -1.72 & -24.43 \\
\hline $4-9-7$ & 0.06 & 0.06 & -7.70 & -32.1 \\
\hline $4-6-8$ & 0.75 & 2.40 & -7.29 & -39.42 \\
\hline $4-8-9$ & 2.00 & 4.22 & -6.06 & -45.4 \\
\hline $4-8-10$ & 0.46 & 1.10 & -7.46 & -52.31 \\
\hline $4-8-11$ & 5.53 & 7.80 & -4.42 & -57.37 \\
\hline $4-8-12$ & 2.57 & 3.67 & -6.20 & -63.56 \\
\hline $4-8-13$ & 0.13 & 0.24 & -7.66 & -71.25 \\
\hline $4-8-14$ & 0.31 & 0.82 & -7.55 & -78.78 \\
\hline $4-6-15$ & 0.51 & 0.32 & -7.43 & -86.28 \\
\hline $4-8-16$ & 0.71 & 14.40 & -7.31 & -93.5 \\
\hline $4-8-17$. & 0.06 & 14.40 & -7.70 & -101.23 \\
\hline
\end{tabular}

NO3

SAMPLE NO. EFFLUEHT HGU CONC. ELUTED SUM ELUTED

\begin{tabular}{|c|c|c|c|c|}
\hline 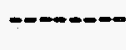 & ppe kDJ & pps $\mathrm{HOZ}^{2}$ & I9 & if $\mathrm{MLJ}$ \\
\hline $4-8-1$ & 0.46 & 0.10 & 0.18 & 0.1 \\
\hline $4-8-2$ & 1.50 & 0.20 & 0.84 & 1.0 \\
\hline $4-6-3$ & 0.70 & 0.10 & 0.36 & 1. 38 \\
\hline $4-6-4$ & 0.90 & 0.10 & 0.46 & 1.8́ \\
\hline $4-8-5$ & 1.30 & 0.10 & 0.72 & 2.58 \\
\hline $4-6-6$ & 0.36 & 0.20 & 0.12 & 2.70 \\
\hline $4-8-7$ & 2.90 & 0.20 & 1.63 & 9.30 \\
\hline $4-6-3$ & 0.80 & (i. 10 & 0.42 & 4.36 \\
\hline $4-3-9$ & 0.50 & 0.10 & 0.24 & 5.04 \\
\hline $4-8-10$ & 1.30 & 0.10 & 1.02 & 0.66 \\
\hline $4-3-1 !$ & 4.60 & 6.10 & 0.30 & 6.36 \\
\hline $4-8-12$ & 1.70 & 0.10 & 0.96 & 7.38 \\
\hline $4-\overline{5}-13$ & 1.30 & 3.10 & 1.02 & 3.34 \\
\hline $4-\hat{b}-14$ & 0.50 & (j. 20 & 0.30 & 8.64 \\
\hline $4-8-15$ & 0.30 & 6.10 & 0.42 & 7.6 \\
\hline $4-5-16$ & 1.00 & 0.10 & 0.54 & 5.30 \\
\hline $4-6-17$ & 4.60 & i. $: 0$ & 2.34 & $i 1=$ \\
\hline
\end{tabular}




\section{TABLE C.3. contd}

H3BO3

SAMPLE NOEFFLUENT HGH CONC. ELUTED SUH ELUTE

$\begin{array}{lllll} & \text { pp B } & \text { pp B } & 0.8 & \text { B B } \\ 4-8-1 & 0.50 & 0.34 & 0.10 & 0.10 \\ 4-8-2 & 1.46 & 0.34 & 0.67 & 0.77 \\ 4-8-3 & 0.97 & 0.34 & 0.38 & 1.15 \\ 4-8-4 & 1.13 & 0.34 & 0.47 & 1.62 \\ 4-8-5 & 0.63 & 0.34 & 0.17 & 1.79 \\ 4-8-6 & 0.44 & 0.34 & 0.06 & 1.35 \\ 4-8-7 & 0.73 & 0.34 & 0.27 & 2.12 \\ 4-8-8 & 0.45 & 0.34 & 0.07 & 2.19 \\ 4-8-9 & 0.41 & 0.34 & 0.04 & 2.23 \\ 4-8-10 & 0.47 & 0.34 & 0.08 & 2.31 \\ 4-8-11 & 0.37 & 0.34 & 0.02 & 2.33 \\ 4-8-52 & 0.37 & 0.34 & 0.02 & 2.35 \\ 4-8-13 & 0.46 & 0.34 & 0.07 & 2.42 \\ 4-8-14 & 0.39 & 0.34 & 0.03 & 2.45 \\ 4-8-15 & 0.42 & 0.34 & 0.05 & 2.50 \\ 4-8-16 & 0.45 & 0.34 & 0.07 & 2.56 \\ 4-8-17 & 0.51 & 0.34 & 0.10 & 2.66\end{array}$

504

SAMPLE NO. EFFLUENT HGW CONC. ELUTED - SUH ELUTE

\begin{tabular}{|c|c|c|c|c|}
\hline & ppa SO4 & PPS 504 & 29504 & 49504 \\
\hline $4-8-1$ & 80.00 & 86.00 & -3.60 & -5.60 \\
\hline $4-8-2$ & 106.00 & 86.00 & 12.00 & 8.40 \\
\hline $4-3-3$ & 111.00 & 86.00 & 15.00 & 23.40 \\
\hline $4-8-4$ & 90.00 & 86.00 & 2.40 & 25.80 \\
\hline $4-8-5$ & 65.00 & 86.00 & -0.60 & 25.20 \\
\hline $4-8-6$ & 104.00 & 86.00 & 10.80 & 36.00 \\
\hline $4-8-7$ & 97.00 & 36.00 & 6.60 & 42.60 \\
\hline $4-8-8$ & 109.00 & 86.00 & 13.80 & 56.40 \\
\hline $4-8-9$ & 33.00 & 86.00 & -1.80 & 54.60 \\
\hline $4-8-10$ & 73.00 & 86.00 & -7.80 & 46.80 \\
\hline $4-8-11$ & 81.60 & 86.00 & -3.00 & 43.80 \\
\hline $4-8-12$ & 85.00 & 86.00 & -0.60 & 43.20 \\
\hline $4-8-13$ & 84.00 & 86.00 & -1.20 & 42.00 \\
\hline $4-8-14$ & 58.00 & 86.00 & -16.80 & 25.20 \\
\hline $4-8-15$ & 63.50 & 86.00 & -13.50 & 11.70 \\
\hline $4-8-16$ & 69.00 & 86.00 & -10.20 & 1.50 \\
\hline $4-8-17$ & 76.00 & 86.00 & -5.00 & -4.50 \\
\hline
\end{tabular}


TABLE C.4. Mass Balance for ANS 16.1 Leach Test whole Grout with Groundwater (MB5-5)

Ca

SAMPLE NO. EFFLUENT HEU CONC. ELUTED SUM ELUTED ppa $\mathrm{Ca}$ ppe $\mathrm{Ca}$ ag $\mathrm{Ca}$ ag

$\begin{array}{rrrrr}5-5-1 & 50.60 & 56.00 & -3.24 & -3.24 \\ 5-5-2 & 1.60 & 56.00 & -32.64 & -35.88 \\ 5-5-3 & 7.80 & 56.00 & -28.92 & -64.80 \\ 5-5-4 & 3.00 & 56.00 & -28.80 & -93.60 \\ 5-5-5 & 12.60 & 56.00 & -26.04 & -119.64 \\ 5-5-6 & 13.10 & 56.00 & -25.74 & -145.38 \\ 5-5-7 & 1.50 & 56.00 & -32.70 & -178.08 \\ 5-5-8 & 2.30 & 56.00 & -32.22 & -210.30 \\ 5-5-9 & 2.70 & 56.00 & -31.98 & -242.28 \\ 5-5-10 & 1.40 & 56.00 & -32.76 & -275.04 \\ 5-5-11 & 2.60 & 56.00 & -32.04 & -307.08 \\ 5-5-12 & 1.30 & 56.00 & -32.52 & -339.60 \\ 5-5-13 & 1.10 & 56.00 & -32.94 & -372.54 \\ 5-5-11 & 1.20 & 56.00 & -32.88 & -405.42 \\ 5-5-15 & 1.05 & 56.00 & -32.37 & -438.39 \\ 5-5-16 & 0.70 & 56.00 & -33.06 & -471.45 \\ 5-5-17 & 0.80 & 56.00 & -33.12 & -504.57\end{array}$

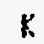

SAMPLE ND. EEFLUENT HGN CONC. ELUTED SUM ELUTED

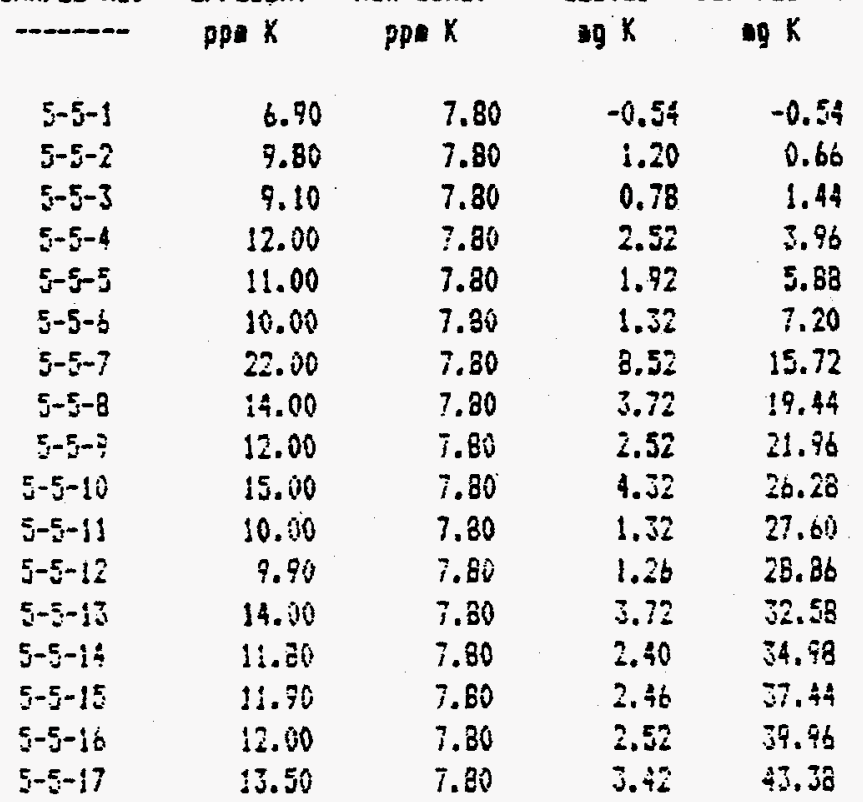

$\mathrm{Na}$

SARPLE RO. EFFLUEHT HGU CONC. ELUTED SUK ELUTED

\begin{tabular}{rr} 
& \multicolumn{1}{c}{ ppr } \\
$5-5-1$ & 38.00 \\
$5-5-2$ & 132.00 \\
$5-5-3$ & 94.00 \\
$5-5-4$ & 161.00 \\
$5-5-5$ & 142.00 \\
$5-5-6$ & 90.00 \\
$5-5-7$ & 430.00 \\
$5-5-8$ & 200.00 \\
$5-5-9$ & 159.00 \\
$5-5-10$ & 223.00 \\
$5-5-11$ & 128.00 \\
$5-5-12$ & 143.00 \\
$5-5-13$ & 210.00 \\
$5-5-14$ & 170.00 \\
$5-5-15$ & 172.50 \\
$5-5-16$ & 175.00 \\
$5-5-17$ & 250.00
\end{tabular}

Pp: $\mathrm{Na}$

ig $\mathrm{Ha}$

ig $\mathrm{Ka}$

25.00
25.00
25.00
25.00
25.00
25.00
25.00
25.00
25.00
25.00
25.00
25.00
25.00
25.00
25.00
25.60
25.00$$
3.30
$$

7.80

64.20

41.40

B1.60

70.20

39.00

243.60

105.00

30.40

i18. 80

61.30

70.80

111.00

57.00

88.50

30.60

135.00
72.00

113.40

175.00

265.20

304.20

547.20

652.20

732.60

851.40

913.20

984.00

1095.00

1182.00

1270.50

1360.50

1495.50
Al

SAMPLE ND. EFFLUENT Hă CONC. ppe $\mathrm{nl}$

pos Al

$$
\begin{aligned}
& 0.09 \\
& 0.03 \\
& 0.03 \\
& 0.19 \\
& 0.10 \\
& 0.08 \\
& 2.11 \\
& 0.09 \\
& 0.64 \\
& 0.06 \\
& 0.07 \\
& 0.03 \\
& 0.04 \\
& 0.05 \\
& 0.07 \\
& 0.03 \\
& 0.03
\end{aligned}
$$

0.10
0.10
0.10
0.10
0.10
0.10
0.10
0.10
0.10
0.10
0.10
0.10
0.10
0.10
0.10
0.10
0.10
ELUTED SUH ELUTED $9 \mathrm{Al}$ ol $\mathrm{Al}$

$\begin{array}{rr}-0.01 & -0.01 \\ -0.04 & -0.05 \\ -0.04 & -0.09 \\ 0.05 & -0.04 \\ 0.00 & -3.04 \\ -0.01 & -0.05 \\ 1.31 & 1.06 \\ -0.01 & 1.15 \\ -0.04 & 1.12 \\ -0.02 & 1.09 \\ -0.01 & 1.09 \\ -3.04 & 1.04 \\ -3.04 & 1.01 \\ -0.04 & 0.07 \\ -0.04 & 0.72 \\ -0.04 & 0.06 \\ -3.04 & 0.04\end{array}$


TABLE C.4. contd

$5 i$

SAMPLE NO. EFFLUEKT HGN CONC, ELUTED SUK ELUTED ppe $\mathrm{si}$ pps $5 \mathrm{i}$ i $\mathrm{Si} \quad \mathrm{Si}$

$\begin{array}{lllll}5-5-1 & 15.50 & 15.00 & -0.90 & -0.90 \\ 5-5-2 & 10.20 & 15.00 & -2.88 & -3.78 \\ 5-5-3 & 10.80 & 15.00 & -2.52 & -6.30 \\ 5-5-4 & 10.10 & 15.00 & -2.94 & -9.24 \\ 5-5-5 & 10.70 & 15.00 & -2.58 & -11.82 \\ 5-5-6 & 12.30 & 15.00 & -1.62 & -13.14 \\ 5-5-7 & 11.10 & 15.00 & -2.34 & -15.78 \\ 5-5-8 & 8.70 & 15.00 & -3.78 & -19.56 \\ 5-5-9 & 8.00 & 15.00 & -4.20 & -23.76 \\ 5-5-10 & 7.40 & 15.00 & -4.56 & -28.32 \\ 5-5-11 & 8.00 & 15.00 & -4.20 & -32.52 \\ 5-5-12 & 6.90 & 15.00 & -4.86 & -37.38 \\ 5-5-13 & 4.90 & 15.00 & -6.06 & -43.44 \\ 5-5-14 & 4.20 & 15.00 & -6.12 & -49.56 \\ 5-5-15 & 4.85 & 15.00 & -6.09 & -55.65 \\ 5-5-16 & 4.90 & 15.00 & -6.06 & -61.71 \\ 5-5-17 & 5.40 & 15.00 & -5.76 & -67.47\end{array}$

F

SAMPLE NO. EFFLUEKT HEW CONC. ELUTED SUN ELUTED

$\begin{array}{lll}- & p p=F & p p=F \\ 5-5-1 & 0.70 & 0.50 \\ 5-5-2 & 1.50 & 0.50 \\ 5-5-3 & 1.40 & 0.50 \\ 5-5-4 & 3.70 & 0.50 \\ 5-5-5 & 5.50 & 0.50 \\ 5-5-6 & 2.20 & 0.50 \\ 5-5-7 & 5.50 & 0.50 \\ 5-5-8 & 2.20 & 0.50 \\ 5-5-9 & 1.50 & 0.50 \\ 5-5-10 & 2.30 & 0.50 \\ 5-5-11 & 0.90 & 0.50 \\ 5-5-12 & 0.30 & 0.50 \\ 5-5-13 & 1.00 & 0.50 \\ 5-5-14 & 5.10 & 0.50 \\ 5-5-15 & 1.95 & 0.50 \\ 5-5-10 & 0.80 & 0.50 \\ 5-5-17 & 1.10 & 0.50\end{array}$

\& $\mathrm{F} \quad$ \& $\mathrm{F}$

$\begin{array}{rr}0.12 & 0.12 \\ 0.60 & 0.72 \\ 0.54 & 1.26 \\ 1.72 & 3.18 \\ 2.04 & 5.22 \\ 1.02 & 6.24 \\ 3.60 & 9.24 \\ 1.02 & 10.26 \\ 0.60 & 10.86 \\ 0.90 & 11.76 \\ 0.26 & 12.00 \\ -0.12 & 11.88 \\ 0.30 & 12.18 \\ 1.56 & 13.74 \\ 0.87 & 14.61 \\ 0.19 & 14.79 \\ 0.36 & 15.15\end{array}$

SAMPLE HO.

$5-5-1$
$5-5-2$
$5-5-3$
$5-5-4$
$5-5-5$
$5-5-6$
$5-5-7$
$5-5-8$
$5-5-9$
$5-5-10$
$5-5-11$
$5-5-12$
$5-5-13$
$5-5-14$
$5-5-15$
$5-5-16$
$5-5-17$

Hg EFFLUENT HON CONC.
ppe hg pDE Hg

ELUTED SUH ELUTED og $\mathrm{hg}$

$19 \mathrm{Hg}$

$\begin{array}{lllr}13.00 & 14.40 & -0.84 & -10.84 \\ 2.00 & 14.40 & -7.44 & -8.20 \\ 5.09 & 14.40 & -5.59 & -13.87 \\ 0.25 & 14.40 & -9.49 & -22.36 \\ 2.59 & 14.40 & -7.09 & -29.44 \\ 8.70 & 14.40 & -3.42 & -32.36 \\ 0.06 & 14.40 & -8.60 & -41.47 \\ 0.30 & 14.40 & -9.46 & -49.73 \\ 1.50 & 14.40 & -7.74 & -57.67 \\ 0.32 & 14.40 & -8.45 & -66.11 \\ 3.38 & 14.40 & -6.31 & -72.43 \\ 1.36 & 14.40 & -7.52 & -79.95 \\ 0.14 & 14.40 & -8.56 & -86.51 \\ 0.20 & 14.40 & -8.52 & -77.03 \\ 0.18 & 14.40 & -8.54 & -105.56 \\ 0.15 & 14.40 & -8.55 & -114.11 \\ 0.06 & 14.40 & -8.60 & -122.72\end{array}$

N03

SAMPLE NO.

EFFLUEHT ops No3

HEH CONC
op) HOJ

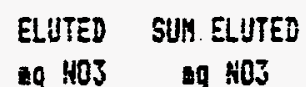

$5-5-1$
$5-5-2$
$5-5-3$
$5-5-4$
$5-5-5$
$5-5-6$
$5-5-7$
$5-5-5$
$5-5-9$
$5-5-10$
$5-5-11$
$5-5-12$
$5-5-13$
$5-5-14$
$5-5-15$
$5-5-16$
$5-5-17$

0.10

0.10

0.10

0.10

0.10

0.10

i. 10

6. 10

0. 10

0.10

0.10

0.10

1.. 10

0.10

i.10

0.16

1. 10

$\begin{array}{rr}0.12 & 0.12 \\ 0.60 & 0.72 \\ 0.36 & 1.08 \\ 0.60 & 1.68 \\ 0.42 & 2.10 \\ 0.24 & 2.34 \\ 1.74 & 4.08 \\ 0.72 & 4.80 \\ 0.50 & 5.46 \\ 1.56 & 7.02 \\ 0.42 & 7.44 \\ 0.00 & 6.04 \\ 5.04 & 13.08 \\ 0.48 & 13.56 \\ 10.74 & 24.36 \\ 21.00 & 45.30 \\ 12.66 & 57.36\end{array}$


TABLE C.4. contd

43803

SARPLE NO. EFFLUENT hGY CONC. ELUTED SUM ELUTED

$$
\text { ppe } 8 \text { ppe } 8
$$

0.61

1.30

0.80

1.09

0.78

0.59

1.29

0.53

0.47

0.60

0.41

0.42

0.51

0.41

0.43

0.45

0.58 ig $B$

0.16

0.58

0.28

0.45

0.26

0.15

0.57

0.11

0.08

0.16

0.04

0.05

0.10

0.04

0.05

0.07

0.14 $\lg B$

$\begin{array}{ll}0.16 & 5-5-1 \\ 0.74 & 5-5-2 \\ 1.01 & 5-5-3 \\ 1.46 & 5-5-4 \\ 1.73 & 5-5-5 \\ 1.88 & 5-5-6 \\ 2.45 & 5-5-7 \\ 2.56 & 5-5-6 \\ 2.64 & 5-5-9 \\ 2.80 & 5-5-10 \\ 2.84 & 5-5-11 \\ 2.89 & 5-5-12 \\ 2.39 & 5-5-13 \\ 3.03 & 5-5-14 \\ 3.08 & 5-5-15 \\ 3.15 & 5-5-16 \\ 3.29 & 5-5-17\end{array}$

504

SAMPLE HO. EFFLUEHT HGH COHC.

ELUTED pp. S04 PPE SO4 I9504

SUK ELUTED

Ig 504

80.00
102.00
70.00
94.00
90.00
83.00
106.00
85.00
85.00
87.00
84.00
83.00
84.00
57.00
61.50
66.00
79.00

86.00

86.00

86.00

86.00

86.00

86.00

86.00

86.00

60.10

86.00

86.00

86.00

8ó.00

36.00

36.00

86.60

86.00
$-3.60$

9.60

2.40

4.60

2.40

$-1.80$

12.00

$-0.60$

$-0.60$

1.50

$-1.20$

$-1.80$

$-1.20$

$-17.40$

$-14.70$

$-12.60$

$-4.20$

\section{$-3.60$}

6.00

8.40

13.20

15.60

13.80

75.60

25.20

24.60

26.40

25.20

23.40

22.20

4.80

$-9.50$

$-21.90$

$-26.10$ 


\section{TABLE C.5. Mass Balance for Static Leach Test whole Grout in Groundwater (MB2-17)}

$\mathrm{Ca}$

SAMPLE HO, EFFLUENT HGK CONC. ELUTED TOTAL CHANGE ppe Ca ppe Ca

$A B-2-17-1$

AB- $2-17-2$

$\mathrm{BB}-2-17-3$

$M B-\hat{\imath}-17-4$

$76-\hat{\imath}-17-5$

$M B-2-17-6$

$M B-z-17-7$

$M B-2-17-6$

$\mathrm{KB}-2-17-9$

$\mathrm{rB}-2-17-10$

$\mathrm{AB}-2-17-11$

$\mathrm{KE}-2-17-12$

AB $-2-17-13$

BB-2-17-14

IB $-2-17-15$

$M E-2-18-16$

$\begin{array}{llll}3.30 & 56.00 & -31.62 & -31.62 \\ 3.10 & 56.00 & -2.76 & -34.38 \\ 4.20 & 56.00 & -1.99 & -36.36 \\ 4.60 & 56.00 & -2.35 & -38.71 \\ 2.00 & 56.00 & -4.13 & -42.34 \\ 0.30 & 56.00 & -5.15 & -47.99 \\ 4.40 & 56.00 & -0.33 & -48.32 \\ 4.00 & 56.00 & -2.82 & -51.14 \\ 4.10 & 56.00 & -2.54 & -53.68 \\ 4.10 & 56.00 & -2.60 & -56.27 \\ 4.00 & 56.00 & -2.66 & -58.93 \\ 4.00 & 56.00 & -2.60 & -61.53 \\ 4.20 & 56.00 & -2.48 & -64.01 \\ 4.70 & 56.00 & -2.29 & -66.30 \\ 3.60 & 56.00 & -3.23 & -69.52 \\ 1.60 & 56.00 & -3.82 & -73.34\end{array}$

1.60
$-3.82$
Ha

SAMPLE NO. EFFLUENT HGU CONC.

ppe $\mathrm{Na}$ pp: $\mathrm{Na}$

25.00
25.00
25.00
25.00
25.601
25.00
25.00
25.00
25.00
25.00
25.00
25.00
25.00
25.00
25.00
25.00

$48-2-17-1 \quad 740.00$

NE-2-17-2

$K B-2-17-3$

MB-2-17-4

$X B-2-17-5$

$M B-2-17-6$

$78-2-17-7$

กร- $2-17-8$

$38-2-17-9$

$62-2-17-10$

75-2-17-11

NB- $2-17-i \hat{2}$

HB- $2-17-13$

KR-2-17-14

पI $5-2-17-15$

$18-2-16-16$

\subsection{0 \\ 930.00 \\ $\$ 80.00$ \\ 1017.00 \\ 1055.00 \\ 982.00 \\ 950.00 \\ 939.00 \\ 970.00 \\ 940.00 \\ 960.00 \\ 1008.60 \\ 1100.00 \\ 1200.00 \\ 1163.00}

ELUTED TOTAL CHAHGE a $\mathrm{Ha} \quad \mathrm{ga} \mathrm{Na}$

$\begin{array}{rr}429.00 & 429.00 \\ 95.75 & 524.75 \\ 94.75 & 617.50 \\ 75.25 & 694.75 \\ 69.75 & 764.70 \\ 72.75 & 837.45 \\ 7.70 & 865.15 \\ 28.65 & 293.80 \\ 39.65 & 933.45 \\ 64.30 & 997.75 \\ 29.25 & 1027.00 \\ 57.75 & 1684.75 \\ 75.55 & 1160.30 \\ 164.35 & 1264.65 \\ 113.75 & 1376.40 \\ 36.55 & 1414.75\end{array}$

$x$

SAIPLE HO. EFFLUENT HGH COHC. ppa $K$ ppa K

\begin{tabular}{|c|c|c|c|c|}
\hline$M B-2-1 \bar{i}-1$ & 34.00 & $\bar{i} .80$ & 15.72 & 15.72 \\
\hline HE $[-2-17-2$ & 38.00 & 7.80 & $3.3 !$ & 19.45 \\
\hline $4 B-2-17-3$ & 42.60 & 7.80 & $3.9 !$ & 23.34 \\
\hline $75-2-17-4$ & 43.00 & 7.801 & 2.31 & 25.65 \\
\hline $48-i-17-5$ & $44 . \mathrm{ju}$ & $\mathrm{I} . \mathrm{BN}$ & 2.30 & 23.01 \\
\hline$M 8-i-17-6$ & 45.00 & 7.80 & 2.76 & 30.97 \\
\hline$X 5-2-17-7$ & 49.00 & $7.8 \hat{0}$ & 2.36 & 32.3 \\
\hline$X I=-2-i \bar{i}-\overline{0}$ & 42.80 & $\bar{i} .80$ & i.si & 72.84 \\
\hline$\because z-j-1 \bar{j}-\bar{z}$ & $=i, 00$ & 7.00 & i.ii & $34, E E$ \\
\hline $58-2-17-90$ & 28.00 & 1.20 & $\vdots .7 !$ & 30.26 \\
\hline $4-2-7:-1:$ & 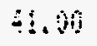 & $\bar{i} .0$ & $\{.: 1$ & 3.7 \\
\hline $5-j-i \div-12$ & $4 ., 06$ & 7.30 & i.só & $39.6 \mathrm{G}$ \\
\hline $4=-17-12$ & 23.60 & $\bar{i} .30$ & 28 & 4.67 \\
\hline$Y \vec{I}-z-1 \bar{T}-\mathbf{Z}:$ & 27.60 & 7.30 & 4.16 & 40.65 \\
\hline $4 \vdots-Z-: ?-! \vdots$ & 97.90 & 7.80 & 1.76 & $\div 8,65$ \\
\hline$M E-Z-Z E-\vdots 2$ & క. & $\therefore 50$ & 2.50 & 5.57 \\
\hline
\end{tabular}

ELUTED TBTAL CHANGE ag $x$ $\lg K$

SAMELE HO

$$
\text { mon hi }
$$

हigh hi

HEH CONE. yps $A$

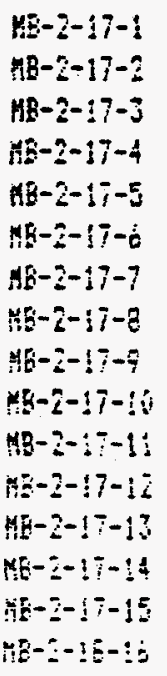

10.50
11.40
12.20
12.65
12.80
12.60
12.10
11.50
11.30
11.50
13.20
12.50
12.50
16.40
15.40
31.60

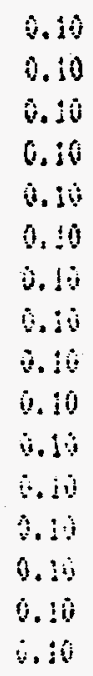

ELITE sg TATAL CHAHEE
so $\mathrm{Al}$ $\therefore$ 0.65 6.42 7.31 1.69 0.64 i. $: 4$ 1). 6.10 6. 24 เ. 45 0. 06 अ. 37 1. 1.44 2. $\therefore 61$ i.
ช. 3 ต

$\div .20$

ร. 55

4i. 51

10.92

11.16

11.6!

$\$ 2.29$

ićs

$: 27$

15.

5.62

26.34

2210 
Si

SAMPLE NO. EFFLUENT HGW CONC. PPE $\mathrm{Si}$ PPE $\mathrm{Si}$

\begin{tabular}{|c|c|c|c|c|}
\hline$M B-2-17-1$ & 10.30 & 15.00 & -2.52 & -2.52 \\
\hline $18-2-17-2$ & 11.00 & 15.00 & -0.09 & -2.61 \\
\hline$M B-2-17-3$ & 11.30 & 15.00 & -0.02 & -2.63 \\
\hline$N E-2-17-4$ & 11.60 & 15.00 & -0.01 & -2.64 \\
\hline$B B-2-17-5$ & 12.20 & 15.00 & 0.19 & $-2,45$ \\
\hline $1 B-2-17-6$ & 12.80 & 15.00 & 0.55 & -1.90 \\
\hline$M B-2-17-7$ & 12.40 & 15.00 & -0.35 & -2.25 \\
\hline $18-2-17-8$ & 12.30 & 15.00 & -0.19 & -2.44 \\
\hline $1 B-2-17-9$ & 12.50 & 15.00 & -0.02 & -2.45 \\
\hline $4 B-2-17-10$ & 14.60 & 15.00 & 1.13 & -1.32 \\
\hline$A B-2-17-11$ & 13.70 & 15.00 & -0.56 & -1.88 \\
\hline$M E-2-17-12$ & 13.50 & 15.00 & -0.19 & -2.06 \\
\hline $48-2-17-13$ & 14.30 & 15.00 & 0.40 & -1.66 \\
\hline $4 B-2-17-14$ & 14,80 & 15.00 & 0.26 & -1.39 \\
\hline $48-2-17-15$ & 16.90 & 15.00 & 1.25 & -0.14 \\
\hline $78-2-10-16$ & 17.40 & 15.00 & 0.39 & 0.25 \\
\hline
\end{tabular}

F

SAMPLE NDE.

EFFLUENT ppe $F$

HSH CONC. ppe $F$

\subsection{0}

9.40

\subsection{0}

औह- $2-17-2$

औ5-2-17-3

$n \mathrm{~B}-\hat{z}-17-4$

$x \mathrm{x}-2-17-5$

$76-2-1 j-6$

$55-\bar{i}-1\}-\bar{j}$

NE-2-17-8

ME $-2-17-9$

IIE-2-17-16

15- $;-17-11$

$4 \mathrm{~B}-2-17-12$

HE- $2-17-13$

ติ- $-17-14$

*⿻一𠃋十 $7-17-15$

iิ $5-2-18-16$
10.00

10.00

iิ. 50

11.00

7.90

16.00

3. 70

9.50

?. 40

11.00

12.00

13. 60

15.00

12.00
ELUTED TOTAL CHAKGE

Ig $\mathrm{Si}$

$19 \mathrm{Si}$

\begin{tabular}{|c|c|}
\hline-2.53 & $X B-2-17-1$ \\
\hline-2.61 & $1 B-2-17-2$ \\
\hline 2.63 & $18-2-17-3$ \\
\hline .64 & $1 B-2-17-4$ \\
\hline 45 & $48-2-17-5$ \\
\hline 90 & $x B-2-17-6$ \\
\hline 25 & KB $-2-17-7$ \\
\hline & n8-2-17-9 \\
\hline & $M B-2-17-9$ \\
\hline & $48-2-17-10$ \\
\hline & $485-2-17-11$ \\
\hline & $M B-2-17-12$ \\
\hline & $48-2-17-13$ \\
\hline & $18-2-17-14$ \\
\hline & $18-2-17-15$ \\
\hline 沙 & $48-2-18-16$ \\
\hline
\end{tabular}

SAMPLE NO. EFFLUENT HGN COHC.

$$
\text { ppe Mg }
$$

0.06
0.06
0.06
0.09
0.08
0.06
0.07
0.06
0.05
0.06
0.06
0.06
0.06
0.12
0.06
0.06

pps ing

14.40
14.40
14.40
14.40
14.40
14.40
14.40
14.40
14.40
14.40
14.40
14.40
14.40
14.40
14.40
14.40

ELITED sg $\mathrm{Hg}$

-3.60
-0.72
-0.72
-0.70
-0.72
-0.73
-0.71
-0.72
-0.72
-0.71
-0.72
-0.72
-0.72
-0.68
-0.75
-0.72

TOTAL CHANGE

eg

$$
\begin{array}{r}
-8.60 \\
-9.32 \\
-10.04 \\
-10.74 \\
-11.46 \\
-12.20 \\
-12.91 \\
-13.63 \\
-14.35 \\
-15.06 \\
-15.78 \\
-16.50 \\
-17.21 \\
-17.50 \\
-18.65 \\
-19.36
\end{array}
$$

\begin{tabular}{|c|c|c|}
\hline 5.16 & 5.16 & $M E-2-17-1$ \\
\hline 0.61 & 5.77 & $A B-2-17-2$ \\
\hline 0.80 & 6.57 & $4 E-2-17-3$ \\
\hline 0.97 & 7.05 & $78-2-17-4$ \\
\hline 0.77 & 7.82 & $i N B-2-17-5$ \\
\hline 1.07 & 8.90 & $k E-2-17-6$ \\
\hline-0.14 & 8.76 & $A B-2-17-7$ \\
\hline$\hat{6} .53$ & 5.29 & $K B-2-17-6$ \\
\hline 0.91 & 7.71 & $M B-2-17-9$ \\
\hline $0.2 j$ & 9.74 & $M E-2-1 j-10$ \\
\hline 0.39 & 10.35 & $45-2-17-11$ \\
\hline 1.40 & 11.35 & $m b-2-17-12$ \\
\hline 1.12 & 12.80 & $M E-2-\{;-1 j$ \\
\hline 1.17 & 14.05 & $M E-i-i j-14$ \\
\hline 1.32 & $15 . \overline{6} 6$ & $m i-2-17-15$ \\
\hline-1.08 & $i 4.78$ & $48-i-15-i 6$ \\
\hline
\end{tabular}

\begin{tabular}{|c|c|}
\hline 3.92 & 3.42 \\
\hline 0.76 & 4.19 \\
\hline$\hat{0} .51$ & 4.57 \\
\hline 4.00 & 9.29 \\
\hline-1.44 & 7.35 \\
\hline 1.87 & 9.75 \\
\hline 0.19 & 3.74 \\
\hline 0.14 & 10.09 \\
\hline 1.67 & 11.58 \\
\hline$-i, 41$ & 11.58 \\
\hline$\therefore .87$ & $\{3.47$ \\
\hline 5. & 9.23 \\
\hline$-i .0 i$ & 7.56 \\
\hline 7.50 & 15.45 \\
\hline 3.84 & 17.29 \\
\hline 1.07 & 30.37 \\
\hline
\end{tabular}

NG3

ELUTED TOTAL CHAHGE SAMPLE HO. Ig $F \quad g F$

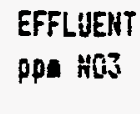

$$
\begin{array}{r}
5.80 \\
6.60 \\
6.90 \\
14.00 \\
10.45 \\
16.00 \\
15.00 \\
14.00 \\
16.00 \\
14.00 \\
16.00 \\
7.50 \\
4.20 \\
17.00 \\
22.00 \\
22.00
\end{array}
$$

H6y conte. pre NCS

0.10
0.10
0.10
0.10
0.10
0.10
0.10
4.20
0.10
0.10
0.10
0.16
0.10
0.10
0.10
0.10

ELUTEO to $\mathrm{NOJ}$

TOTAL CHANGE so $\mathrm{Ha}$ 
TABLE C.5. contd

HJBOJ

504

SAMPLE NO. EFFLUENT HGW CONC. ELUTED TOTAL CHAHGE SAMPLE NO. EFFLUENT HGW CONC. ELUTED TOTAL CHAHGE ppa 8 PDI 8 a 8

$\begin{array}{lllll}M B-2-17-1 & 3.72 & 0.34 & 2.03 & 2.03 \\ M B-2-17-2 & 3.99 & 0.34 & 0.33 & 2.36 \\ M B-2-17-3 & 4.12 & 0.34 & 0.26 & 2.62 \\ M B-2-17-4 & 4.44 & 0.34 & 0.38 & 3.00 \\ M B-2-17-5 & 4.07 & 0.34 & -0.02 & 2.98 \\ M B-2-17-6 & 4.97 & 0.34 & 0.52 & 3.51 \\ M B-2-17-7 & 4.07 & 0.34 & -0.31 & 3.20 \\ M B-2-17-8 & 4.23 & 0.34 & 0.28 & 3.48 \\ M B-2-17-9 & 4.18 & 0.34 & 0.16 & 3.64 \\ M B-2-17-10 & 4.33 & 0.34 & 0.2 B & 3.93 \\ M B-2-17-11 & 4.33 & 0.34 & 0.20 & 4.13 \\ M B-2-17-12 & 4.34 & 0.34 & 0.21 & 4.33 \\ M B-2-17-13 & 4.85 & 0.34 & 0.51 & 4.34 \\ M B-2-17-14 & 5.60 & 0.34 & 0.68 & 5.51 \\ M B-2-17-15 & 6.35 & 0.34 & 0.71 & 6.23 \\ M B-2-16-16 & 7.09 & 0.34 & 0.74 & 6.97\end{array}$

\begin{tabular}{|c|c|}
\hline $76-2-17-1$ & 161.00 \\
\hline$A B-2-17-2$ & 158.00 \\
\hline$B E-2-17-3$ & 168.00 \\
\hline$M B-2-17-4$ & 170.00 \\
\hline$x 8-2-17-5$ & 159.00 \\
\hline $48-2-17-6$ & 148.00 \\
\hline nE-2-17-7 & 165.00 \\
\hline $4 B-2-j 7-8$ & 159.00 \\
\hline$K B-2-17-9$ & 160.00 \\
\hline$K B-2-17-10$ & 159.00 \\
\hline$H E-2-17-11$ & 150.00 \\
\hline$n-2-17-12$ & 150.00 \\
\hline $18-2-17-13$ & 175.00 \\
\hline M8-2-17-14 & 168.00 \\
\hline$M E-2-17-15$ & 213.00 \\
\hline
\end{tabular}

pps 304

19504

99504

IC

TC

GAYTLE NO. EFFLUENT HON CONC. ppe IC ppl IC ag IC ag iC

\begin{tabular}{|c|c|c|c|c|}
\hline$n B-2-17-i$ & 20.50 & 34.10 & -8.16 & -8.16 \\
\hline $18-2-17-2$ & 20.00 & 34.10 & -0.99 & -7.14 \\
\hline $4 E-2-17-3$ & 19.70 & 34.10 & -1.49 & -10.63 \\
\hline$M B-2-i 7-4$ & $\$ 8.10$ & 34.10 & -1.13 & -11.76 \\
\hline $48-2-17-5$ & 18.70 & 34.10 & -0.44 & -12.20 \\
\hline$M E-2-1 j-6$ & 19.30 & 34.10 & -0.08 & -12.28 \\
\hline $15-2-17-i$ & 18.30 & 34.10 & -1.34 & -13.62 \\
\hline $9-2-17-8$ & 22.10 & $33.1 \hat{v}$ & 1.49 & -12.13 \\
\hline $48-2-17-9$ & 17.10 & 34.10 & -3.60 & -15.73 \\
\hline $45-2-17-10$ & 41.50 & 34.10 & 13.79 & -1.34 \\
\hline $72-17-11$ & 30.30 & 34.10 & -6.35 & -3.29 \\
\hline $47-2-17-12$ & 14.30 & 34.10 & $-9.4 j$ & -17.72 \\
\hline$n-\hat{z}-17-1 j$ & 31.50 & 34.10 & 9.60 & -3.72 \\
\hline$P E-2-17-14$ & 23.60 & 34.10 & -4.87 & -13.59 \\
\hline $15-2-17-15$ & 33.40 & $34 .: 0$ & 5.35 & -8.23 \\
\hline $6-2-13-16$ & 28.30 & 34.10 & -3.10 & -11.33 \\
\hline
\end{tabular}

SARYPLE NO. EFFLUENT HGM CONC.

ELUTED TOTAL CHAMGE ppa iC ppi IC

af TC

sg TC

\begin{tabular}{|c|c|c|c|c|}
\hline$K 8-2-17-1$ & 3.10 & 33.10 & -18.00 & -18.00 \\
\hline$E-2-17-2$ & 2.30 & 53.10 & -1.68 & -19.68 \\
\hline $48-2-17-3$ & 2.90 & 33.10 & -1.96 & -21.14 \\
\hline$n g-2-17-4$ & 3.00 & 33.10 & -3.45 & -22.59 \\
\hline$k E-2-17-5$ & 2.50 & 30.10 & -1.81 & -24.39 \\
\hline $48-2-17-6$ & 2.00 & 33.10 & $-2.1 i$ & -26.50 \\
\hline B $-2-1 ;-7$ & 2.40 & 33.60 & -1.32 & $-27 . \hat{1}$ \\
\hline $48-2-17-5$ & 2.30 & 33.10 & -1.50 & $-29.4 j$ \\
\hline $45-2-17-9$ & 2.40 & 53.10 & -1.48 & -30.89 \\
\hline$K E-2-j 7-j 0$ & 5.30 & 53.10 & 0.00 & -30.60 \\
\hline $16-\bar{z}-17-11$ & 4.20 & 3.10 & -1.38 & -32.62 \\
\hline$M B-2-17-i 2$ & 2.20 & $3 .: 8$ & -9.96 & -35.38 \\
\hline $48-2-\{7-13$ & 2.76 & 3.10 & -1.13 & -36.50 \\
\hline$M B-2-1 i-14$ & 3.76 & 33.10 & -1.63 & -37.53 \\
\hline $45--17-15$ & 4.30 & 50.10 & $-\hat{4}, 79$ & -30.52 \\
\hline$m B-7-15-i b$ & 3.20 & 3.16 & $-2.2 \mathrm{i}$ & -40.73 \\
\hline
\end{tabular}


TABLE C.6. Mass Balance for Static Leach Test Whole Grout with Groundwater (MB3-18)

$i \dot{a}$

SAMPLE HQ. EFFLUERT HGH CONC. ELUTED TOTAL CHANGE

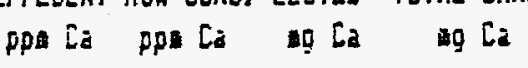

\begin{tabular}{|c|c|c|c|c|}
\hline$x B-3-18-1$ & 3.30 & 50.00 & -31.62 & -31.62 \\
\hline $45-3-18-2$ & 3.60 & 56.60 & -2.46 & -34.07 \\
\hline $58-3-1 E-3$ & 1.40 & 56.00 & -3.94 & -38.01 \\
\hline$M E-3-3 \theta-4$ & 4.10 & 56.10 & -1.11 & -39.12 \\
\hline$h E-3-18-5$ & 2.40 & 56.00 & -3.62 & -42.74 \\
\hline$N E-3-13-6$ & 0.70 & 56.60 & -4.64 & -43.76 \\
\hline ก $6-3-16-7$ & 4.50 & 56.60 & -0.49 & -44.24 \\
\hline$y_{2}-3-13-3$ & 3.50 & 50.60 & -2.94 & -47.16 \\
\hline ก็D-3-1E-? & 4.60 & 56.00 & -2.55 & -49.72 \\
\hline $15-5-10-10$ & 4.20 & 50.00 & -2.48 & -52.21 \\
\hline $4 B-3-6=-11$ & 4.10 & 56.60 & -2.65 & -54.86 \\
\hline $18 B-3-18-12$ & 4.00 & 56.60 & -2.66 & -57.51 \\
\hline$i E-j-i c-i j$ & $4.20^{\circ}$ & 56.60 & -2.48 & -59.89 \\
\hline$M B-3-16-14$ & 5.47 & 56.60 & -1.83 & -61.82 \\
\hline $58-3-18-15$ & 3.50 & 36.00 & -3.71 & -65.53 \\
\hline
\end{tabular}

Mg

SAYFLE HO. EFFLUEHT HEV COHE. ELUTED TOTAL CHAMEE

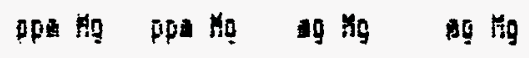

\begin{tabular}{|c|c|c|c|c|}
\hline $46-3-15-2$ & 0.06 & 14.40 & -8.60 & -5.60 \\
\hline$y=-\bar{i}-1 \subseteq-\bar{i}$ & 0.06 & 14.40 & -6.72 & -9.32 \\
\hline $55-3-15-3$ & (1.06 & 13.40 & -0.72 & -10.04 \\
\hline $35-5-15-4$ & 4,6 & 14,40 & -6.72 & -10.76 \\
\hline $4 E-3-1 E-5$ & 8.06 & 14.40 & -0.72 & $-11.4 j$ \\
\hline ME $-3-10-3$ & 0.06 & 14.40 & -0.72 & $-\$ 1.4 j$ \\
\hline$M-j-i b-i$ & 0.6 & 14.40 & -0.72 & -12.19 \\
\hline $56-3-16-8$ & 3.66 & 54.40 & -4.72 & $-12 . \overline{1}$ \\
\hline 돌- & 8.66 & 14.40 & $-\dot{4} .72$ & $-\{3.62$ \\
\hline $4 E-j-i \varepsilon-10$ & is is & 14.40 & -1.72 & -14.34 \\
\hline $45-j-15-1 !$ & 8.60 & 14.40 & $-6,72$ & -15.06 \\
\hline$m E-5-i c-: 2$ & in & $i 4.40$ & -3.72 & $-\mid \vdots, \pi$ \\
\hline $55-j-19-13$ & a. & 24.40 & -0.72 & -16.49 \\
\hline $3 E-3-1 E-14$ & 6. ite & $1+40$ & -0.72 & -17.21 \\
\hline $45-3-15-15$ & i. ie & $\{4,40$ & -6.72 & - \\
\hline
\end{tabular}

k

SAYYPLE HO. EFFLUEHT Hơ CONC. ELLTED TOTÂL CHAMGE ppa $K$ ppi $K$ so $k$ ug

\begin{tabular}{|c|c|c|c|c|}
\hline$M E-3-18-1$ & 35.00 & 7.80 & 16.32 & 16.32 \\
\hline$x 6-3-16-2$ & 37.60 & 7.30 & 2.56 & 13.86 \\
\hline$x E-3-16-3$ & 43.00 & 7.80 & 5.66 & 23.94 \\
\hline $36-3-10-4$ & 45.00 & 7.30 & 2.96 & 26.90 \\
\hline $5 E-3-18-5$ & 46.60 & 7.80 & 2.46 & 29.36 \\
\hline $18-j-i 6-b$ & $47.6 \hat{0}$ & 7.60 & j.06 & 29.96 \\
\hline$M E-j-18-7$ & 45.60 & 7.80 & 0.76 & $30.7 i$ \\
\hline $46-3-18-8$ & 43.00 & 7.80 & 0.66 & $3 ! .33$ \\
\hline $68-3-18-9$ & 44.60 & 7.60 & 2.36 & 3.74 \\
\hline $68-3-18-10$ & 43.00 & 7.30 & 1.21 & 34.95 \\
\hline $4 \theta-3-16-11$ & 31.60 & 7.80 & 0.56 & 35.51 \\
\hline $48-3-18-12$ & 43.60 & 7.00 & 2.36 & 38.57 \\
\hline $48-3-18-13$ & 42.00 & 7.80 & 1.16 & 37.53 \\
\hline $16-3-18-14$ & 46.60 & 7.50 & 4.11 & 43.64 \\
\hline $4 \bar{B}-3-! 0-15$ & 45.00 & 7.80 & 1.31 & 44.95 \\
\hline
\end{tabular}

Hig

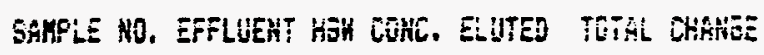

\begin{tabular}{|c|c|c|c|c|}
\hline 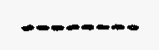 & iph Ha & DFF & $\Delta 9$ & Ş \\
\hline$M[-3-1 \hat{a}-\mathbf{2}$ & $7 i c .00$ & 25.00 & 450.60 & 450.30 \\
\hline$m e-j-18-z$ & 773. & 25. & 3.55 & $405.1=$ \\
\hline$x_{E}-3-1=-3$ & 932.00 & 25.60 & $\$ 1.5$ & 65.30 \\
\hline $45-3-15-4$ & Fo\% & 25.0 & $b \bar{j} .35$ & $=00,85$ \\
\hline$y \bar{z}-j-1 \bar{j}-5$ & 1005.34 & 25.60 & 31.20 & 758,65 \\
\hline $1 B-3-18-6$ & 1049.60 & 55.60 & $\$ 8.20$ & $7=2.15$ \\
\hline $6 \bar{b}-3-16-7$ & 1000.60 & $25.6 i$ & $3.8 \%$ & 863.55 \\
\hline$(E-j-1 j-j$ & 962.60 & 25.60 & 25.95 & 829.30 \\
\hline $16-5-16-9$ & 沘. & E. & 92.05 & 97.45 \\
\hline $16-j-15-10$ & 930.60 & 25.60 & 8.55 & 53.55 \\
\hline $4 B-j-1 \bar{b}-11$ & 50.60 & 25.30 & 27.75 & 95.90 \\
\hline $46-j-: 3-12$ & 570.00 & 5,4 & 泣.25 & 10!.85 \\
\hline $48-5-15-13$ & $0: 5.60$ & 25.8 & 74.28 & 105.00 \\
\hline$-5-18-14$ & 1630.60 & 96 & 92.30 & 1190.70 \\
\hline $4=-3-12-15$ & 1146,00 & 25.6 & 5.3 & 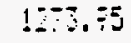 \\
\hline
\end{tabular}


TABLE C.6. contd

504

SAMYPLE NOD. EFFLUENT HGU COHC. ELUTED TOTAL CHANGEE PpD SO4 ppe SO4 SOA SO4 SO4

\begin{tabular}{|c|c|c|c|c|}
\hline$A B-3-18-1$ & 184.00 & 86.00 & 58.80 & 58.80 \\
\hline $45-3-18-i$ & 156.00 & 86.00 & $-\$ 1.90$ & 46.70 \\
\hline $58-j-18-3$ & 179.00 & 86.00 & 17.30 & 64.20 \\
\hline $68-3-18-4$ & 182.00 & 86.00 & 6.45 & 70.65 \\
\hline$M B-3-18-5$ & 173.00 & 86.00 & -0.60 & $70 . \hat{1}$ \\
\hline r6-3-18-b & 164.00 & 86.00 & -6.00 & 64.65 \\
\hline $4 B-3-18-7$ & 103.00 & 36.00 & 15.30 & 79.95 \\
\hline $35-3-18-8$ & $172: 00$ & 86.00 & -1.75 & 78.20 \\
\hline$x E-3-18-5$ & 173.00 & 86.00 & 4.70 & 83.10 \\
\hline $48-3-10-10$ & 178.00 & 86.00 & 7.35 & 90.45 \\
\hline $38-3-18-11$ & 173.00 & 86.00 & 1.60 & 92.015 \\
\hline $46-3-18-12$ & 150.00 & 86.00 & -9.45 & 82.60 \\
\hline $1 \mathrm{E}-3-18-13$ & 113.60 & 86.00 & -18.64 & 63.76 \\
\hline $48-3-18-14$ & 191.010 & 86.00 & 47.82 & 111.78 \\
\hline $4 B-j-16-15$ & 237.60 & 86.00 & 32.25 & 144.63 \\
\hline
\end{tabular}

MOJ

SAMPLE NO. EFFLUENT HaW CONC. ELUTED TOTHL CHANGE

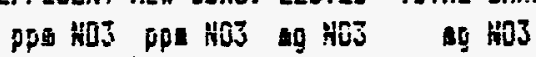

\begin{tabular}{|c|c|c|c|c|}
\hline $58-3-16-1$ & 6.20 & 0.10 & 3.36 & 3.66 \\
\hline $4-3-18-2$ & 0.10 & 0.10 & 0.24 & 3.70 \\
\hline $49-3-13-3$ & 7,40 & 0.16 & i. & 4.78 \\
\hline $72-3-18-4$ & 7.70 & 0.10 & 0.54 & 5.53 \\
\hline $45-3-16-5$ & 12.15 & 0.10 & 3.60 & 3.59 \\
\hline $15 i-j-18-5$ & 16.00 & 0.10 & 5.36 & i0.89 \\
\hline $45-3-18-i$ & 18.60 & 0.10 & 1.99 & 12.58 \\
\hline $46-3-60-6$ & 17.60 & 0.10 & $0.2 \%$ & 13.18 \\
\hline $45-3-16-\overline{7}$ & 10.50 & 0.50 & -3.06 & 10.92 \\
\hline$\pi \bar{b}-3-i b-10$ & 13.00 & 0.10 & 5.02 & 15.14 \\
\hline $75-j-16-11$ & 17.60 & 3.10 & 0.29 & 13.44 \\
\hline $48-3-15-12$ & 13.60 & 0.10 & $\vdots .44$ & 16.88 \\
\hline$k E-3-12-13$ & 19.50 & 8.10 & 1.39 & 13.28 \\
\hline $45-3-15-14$ & 19.30 & 0.50 & 6.67 & 17.35 \\
\hline $8-3-15-15$ & 24.06 & 0.10 & $3 .: 4$ & 23.23 \\
\hline
\end{tabular}

Si

SAMPLE HO. EFFLUENT HEN COHC. ELUTED TOTAL CHANGE -....- ppa Si ppis Si ng Si

\begin{tabular}{|c|c|c|c|c|}
\hline$x B-3-16-1$ & S..$\hat{v}$ & 15.00 & -3.12 & -3.12 \\
\hline$n B-j-18-2$ & 9.10 & 15.00 & -0.68 & $-j . j \dot{v}$ \\
\hline $68-3-18-3$ & 9.70 & 15.00 & 0.06 & -3.74 \\
\hline $16-3-18-4$ & 10.00 & 15.00 & -0.09 & -3.92 \\
\hline$K B-j-18-5$ & 10.60 & 15.00 & 0.11 & -3.71 \\
\hline$K B-3-18-6$ & 11.20 & 15.00 & .0 .47 & -3.35 \\
\hline $\mathrm{HE}-3-18-7$ & 11.20 & 15.60 & -0.17 & -3.54 \\
\hline $66-j-18-8$ & 11.30 & 15.00 & -1.15 & -3.67 \\
\hline $48-3-18-9$ & 10.10 & 15.00 & -0.31 & -4.58 \\
\hline $48-3-18-10$ & 12.30 & 15.00 & $1.3 i$ & -3.26 \\
\hline$K E-3-18-11$ & 12.70 & 15.60 & -0.12 & $-3 . j 6$ \\
\hline $48-3-18-12$ & 12.80 & 15.00 & -0.06 & -3.43 \\
\hline$x 8-3-18-33$ & 13.60 & 15.60 & 0.37 & -3.06 \\
\hline$-3-18-54$ & 13.50 & 15.60 & -6.13 & -3.19 \\
\hline $18-3-18-15$ & 16.50 & 15.00 & 1.72 & -1.45 \\
\hline
\end{tabular}

Al

SAHPLE H. EFFLLENT HGH CONC. ELITEG TOTAL CHGHEE -..-- ppa Al gpe $A !$ ag Al a

\begin{tabular}{|c|c|c|c|c|}
\hline $45-3-16-1$ & 14.00 & 0.10 & 8.37 & 8.34 \\
\hline$M B-3-1 \hat{C}-2$ & 11.70 & 0.10 & -0.57 & 7.77 \\
\hline $4 B-j-1 B-3$ & 15.10 & 0.10 & 2.51 & 10.28 \\
\hline $48-3-18-4$ & 15.40 & 0.10 & 0.35 & $i 1.21$ \\
\hline $45-j-18-5$ & 15.75 & 0.30 & 0.97 & 12.19 \\
\hline $46-3-13-5$ & 96.20 & 0.10 & $\therefore .18$ & 12.40 \\
\hline $48-3-18-7$ & 5.60 & $\hat{0} .10 \hat{0}$ & 0.50 & 12.70 \\
\hline$n B-3-1 E-8$ & 14.50 & 0.10 & Q.i! & 15.01 \\
\hline กิ5-j-j8-7 & 14.60 & 0.10 & 0.70 & 3.73 \\
\hline $66-j-18-16$ & 14.70 & 1.10 & 3.90 & 14.70 \\
\hline$n 5-3-i 6-i !$ & 14.30 & 0.10 & 0.36 & 15.68 \\
\hline$m B-3-1 E-12$ & 15.70 & $\dot{y} . j 0$ & is & $1 . .75$ \\
\hline$M 5-j-15-1 j$ & 13.06 & 0.10 & 1.45 & 1.2 .0 \\
\hline $5 \bar{B}-5-16-14$ & 10,00 & 6.10 & \$. & 3.60 \\
\hline $45-3-10-15$ & $2 ., 80$ & $\dot{x}, j \hat{j}$ & $\therefore 70$ & 3.97 \\
\hline
\end{tabular}


TABLE C.6. contd

F

SAMFLE NO. EFFLUEMT HEU COHC. ELUTED TOTAL CHAKGE

ppe $F$ ppo $F$ ig $F$ ig $F$

\begin{tabular}{|c|c|c|c|c|}
\hline$M B-j-18-1$ & B.40 & 0.50 & 4.74 & 4.74 \\
\hline $4 \theta-3-18-2$ & 7.70 & 0.50 & -0.03 & 4.72 \\
\hline$H i-j-18-3$ & $9.4 \hat{0}$ & 0.50 & 1.38 & 0.10 \\
\hline $48-j-18-4$ & 10.00 & 0.50 & 0.80 & 6.90 \\
\hline $18-3-18-5$ & 10.50 & 0.50 & 0.77 & 7.67 \\
\hline$x B-3-15-6$ & 11.00 & 0.50 & 1.07 & 7.97 \\
\hline $45-3-10-7$ & $: 0.20$ & 0.50 & 0.04 & 8.02 \\
\hline $45-3-16-8$ & 7.30 & 0.50 & 6.30 & 3.32 \\
\hline$x B-3-1 B-7$ & 9.50 & 0.50 & 0.47 & 8.79 \\
\hline $19-3-18-10$ & $\ddot{I I .000}$ & 0.50 & $: .13$ & 9.92 \\
\hline $4 E-3-10-11$ & 7.70 & 0.50 & $-i 1.26$ & 9.57 \\
\hline $65-3-16-12$ & 11.00 & 0.50 & 2.24 & 10.91 \\
\hline $40-3-10-13$ & 23.50 & 0.50 & 3.62 & 18.93 \\
\hline $1 E-3-15-i 24$ & $\mathrm{j} \dot{3} .00$ & 0.50 & -5.15 & 13.78 \\
\hline$A B-3-18-15$ & 16.100 & 0.50 & 2.42 & 16.21 \\
\hline
\end{tabular}

$43 B a 3$

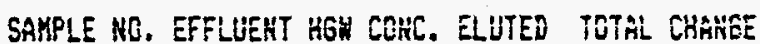
pps $B$ ppI $B$ a B D $B$

\begin{tabular}{|c|c|c|c|}
\hline$K B-3-18-1$ & 5.04 & 0.34 & 2.82 \\
\hline $18-3-18-2$ & 4.29 & 0.34 & -0.22 \\
\hline$k 8-j-18-3$ & 5.15 & 0.34 & $0.7 i$ \\
\hline $46-3-18-4$ & 5.20 & 0.34 & 0.27 \\
\hline $36-3-10-5$ & $5.60^{\circ}$ & 0.34 & 0.52 \\
\hline$n 8-3-18-6$ & a.! & 6.34 & $0.7 ?$ \\
\hline$x B-3-18-7$ & 5.43 & 0.34 & -0.19 \\
\hline na $8-3-18-3$ & 5.26 & 6.34 & 0.16 \\
\hline $19-3-10-9$ & 5.13 & v. 34 & 0.20 \\
\hline $68-3-10-10$ & 5.37 & 6.34 & 0.30 \\
\hline $4 B-3-18-11$ & 5.14 & 0.34 & 0.1! \\
\hline $146-j-18-12$ & 5.49 & 0.34 & 0,45 \\
\hline $48-3-18-13$ & 6.39 & 6.34 & 0.74 \\
\hline $68-3-16-14$ & 6.63 & v.j4 & 0.50 \\
\hline $46-3-16-15$ & 7.30 & 0.34 & 0.66 \\
\hline
\end{tabular}




\section{TABLE C.7. Mass Balance for Static Leach Test Whole Grout}

with Groundwater (MB4-13)

$\mathrm{Ca}$

SAMFLE HO. EFFLUENT HGW CONC. ppe Ca ppa ca

$48-4-13-1$

$35-4-13-2$

$M B-4-13-3$

$M E-4-13-4$

$M E-4-1 j-5$

$x B-4-13-6$

ME-4-13-7

$M E-4-13-3$

$78-4-13-9$

$M 5-4-13-10$

$35-4-13-11$

ME $5-4-13-12$

$3:-4-13-13$

$5:-4-1 j-14$

$n \bar{k}-4-1 j-15$

$\begin{array}{llll}4.00 & 56.00 & -31.20 & -31.20 \\ 0.50 & 56.00 & -4.70 & -35.90 \\ 1.50 & 56.00 & -2.18 & -38.07 \\ 4.40 & 56.00 & -0.39 & -39.06 \\ 3.75 & 56.00 & -2.77 & -42.03 \\ 3.10 & 56.00 & -3.36 & -42.42 \\ 4.70 & 56.00 & -1.57 & -43.78 \\ 4.60 & 56.00 & -2.74 & -46.72 \\ 4.60 & 56.00 & -2.57 & -49.29 \\ 4.60 & 56.00 & -2.57 & -51.36 \\ 4.10 & 56.00 & -2.87 & -54.73 \\ 4.60 & 56.00 & -2.30 & -57.02 \\ 5.18 & 56.00 & -2.22 & -59.25 \\ 4.54 & 56.00 & -2.75 & -61.59 \\ 5.70 & 56.00 & -2.04 & -64.03\end{array}$

5.70
ELUTED TOTAL CHANGE $a \mathrm{Ca} \quad \mathrm{Ca}$
$\mathrm{Ha}$

SAMPLE NO. EFFLUENT HaV CONE. ppa ina pon ha

25.00
25.00
25.00
25.60
25.60
25.60
25.00
25.00
25.00
25.00
25.00
25.00
25.00
25.60
25.00

748.06
762.00
883.00
764.00
762.00
1020.00
989.00
740.00
936.00
960.600
920.00
945.00
990.00
975.60
170.00

ELUTEO

两 $\mathrm{Ha}$

\begin{tabular}{|c|c|}
\hline 90.80 & 433.80 \\
\hline 44.55 & 478.35 \\
\hline 167.45 & 527.60 \\
\hline 91.56 & $679.3 i j$ \\
\hline 57.85 & ij7.05 \\
\hline 80.55 & 757.85 \\
\hline 31.15 & 731.00 \\
\hline 13.30 & 809.80 \\
\hline 44.55 & 854.35 \\
\hline 53.85 & 913.20 \\
\hline 22,75 & 335.75 \\
\hline 59.75 & 995.70 \\
\hline 57.00 & 1062.70 \\
\hline 58.75 & 1119.45 \\
\hline 53.50 & 1272.95 \\
\hline
\end{tabular}

$k$

SAHFLE WO. EFFLUENT HGW CONC. Ppe $K$ ppl $K$

\begin{tabular}{|c|c|c|}
\hline $57-4-13-1$ & 32.00 & 7.80 \\
\hline$n i-4-13-2$ & $35.6 \hat{v i v}$ & $i .30$ \\
\hline $12-4-13-3$ & 37.00 & 7.80 \\
\hline $36-4-15-4$ & 43.00 & 7.60 \\
\hline$y-4-1]-5$ & 43.50 & 7.00 \\
\hline$n E-4-13-6$ & 44.60 & 7.80 \\
\hline$m 5-4-95-7$ & 42.00 & 7.80 \\
\hline$x-4-13-3$ & 43.00 & 7.80 \\
\hline $35-4-13-5$ & 43.20 & 7.80 \\
\hline $15-4-13-10$ & 43.001 & 7.60 \\
\hline $53-4-13-1 !$ & 42,00 & 7.00 \\
\hline$x \hat{0}-4-1, j-12$ & 42.00 & $? .30$ \\
\hline$n \hat{E}-4-1 \mathbf{j}-1 \mathbf{j}$ & 40.60 & 7.30 \\
\hline $12-4-13-14$ & 49.06 & 7.60 \\
\hline $46-4-13-15$ & 47.60 & 7.80 \\
\hline
\end{tabular}

ELUTED TOTAL CHARGE ig $\mathrm{K}$

19 $\mathrm{K}$

$\begin{array}{ll}15.12 & 15.12 \\ 2.46 & 17.50 \\ 3.76 & 21.34 \\ 3.36 & 25.30 \\ 2.06 & 27.36 \\ 2.36 & 27.66 \\ 6.61 & 20.27 \\ 2.31 & 30.58 \\ 1.76 & 32.34 \\ 1.36 & 34.16 \\ 3.36 & 35.26 \\ 1.71 & 36.97 \\ 0.51 & 37.40 \\ 4.61 & 41.49 \\ 5.61 & 45.10\end{array}$

A.

SARPLE Hio. EFFLUENT hä COHC.

gpon hal

jps Al

\begin{abstract}
His $-4-13-1$
$M 6-4-13-2$

$m 5-4-13-3$

$M E-4-13-4$

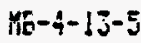

$48-4-15-6$

$A B-4-15-7$

$58-4-13-5$

k5-4-15-9

KE-4-15-10

$135-4-13-11$

in-4-13-12

AD $-4-15-13$

$45-4-13-14$

$x=4-13-15$
\end{abstract}

12.30
10.70
12.20
4.30
6.45
6.60
12.30
11.80
11.70
11.70
11.20
12.30
13.06
14.50
17.60

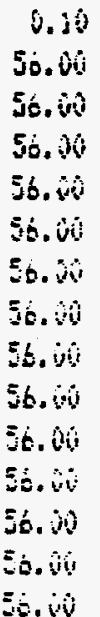

Elited Tith CHAitas ing is a $\mathrm{Al}$

\begin{tabular}{|c|c|}
\hline 3.32 & 1.32 \\
\hline$-\dot{v}, 35$ & 6.9 \\
\hline $1.4 j$ & 8.4 \\
\hline-3.78 & 4.6 \\
\hline i.ji & \\
\hline 2.10 & 0.7 \\
\hline 2.57 & $i .70$ \\
\hline 6.31 & $\lfloor\dot{v}, 0 \mid$ \\
\hline 0.52 & 10.5 \\
\hline 3.59 & 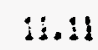 \\
\hline 0.35 & $: 3.3$ \\
\hline$\therefore=$ & {$[2.71$} \\
\hline 0.75 & 13.50 \\
\hline i. $\equiv ;$ & 15.2 \\
\hline 2.25 & 17.4 \\
\hline
\end{tabular}


TABLE C.7. contd

Si

SAMFLE HO. EFFLUENT HGW CONC. ppI $s i$ ppe $5 i$.

\begin{tabular}{|c|c|}
\hline$M B-4-13-1$ & 10.00 \\
\hline$m B-4-13-2$ & 9.70 \\
\hline $76-4-13-3$ & 10.20 \\
\hline $118-4-13-4$ & 6.10 \\
\hline$x B-4-13-5$ & 5.50 \\
\hline $48-4-13-6$ & $5 . \hat{v}$ \\
\hline$M B-4-13-7$ & 11.80 \\
\hline$x 6-4-13-8$ & 12.00 \\
\hline $45-4-13-7$ & 12.40 \\
\hline$m-4-13-10$ & 13.30 \\
\hline $46-4-13-11$ & 13.40 \\
\hline $12-4-13-12$ & 13.60 \\
\hline $58-4-15-13$ & 13.40 \\
\hline $15-4-13-14$ & $14 . \hat{s 0}$ \\
\hline $48-4-13-15$ & 15.70 \\
\hline
\end{tabular}

$F$

GAHFLE WD.

EFFLUENT ppin $;$

Conc.

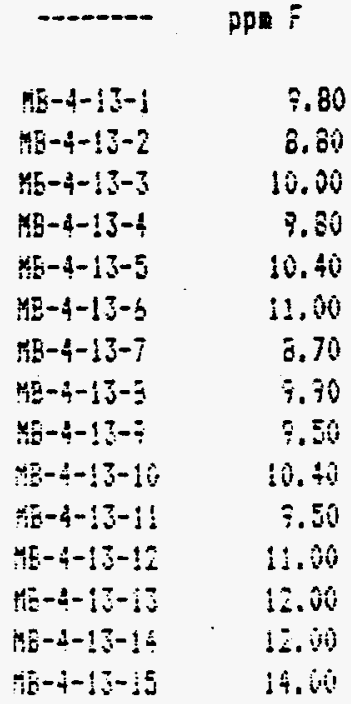
15.00
15.00
15.00
15.00
15.00
15.00
15.001
15.061
15.00
15. 00
15.00
15.00
15. 10
15.00

0.50
0.50
0.50
0.50
0.50
0.50
0.50
0.50
0.50
0.50
0.50
0.50
0.50
0.50
0.50

ELUTEO TOTAL CHANGE ig $5 \mathrm{i}$

$\begin{array}{rr}-3.00 & -3.00 \\ -0.43 & -3.43 \\ 0.03 & -3.40 \\ -2.70 & -6.10 \\ -0.81 & -6.90 \\ -1.11 & -7.20 \\ 3.56 & -3.62 \\ -0.64 & -3.66 \\ 0.09 & -3.57 \\ 0.41 & -3.16 \\ -0.03 & -3.19 \\ 0.04 & -3.15 \\ -0.19 & -3.34 \\ 0.76 & -2.58 \\ 0.53 & -2.05\end{array}$

SAMPLE HO.

\begin{tabular}{|c|}
\hline $48-4-13-1$ \\
\hline $4 B-4-13-2$ \\
\hline $46-4-13-3$ \\
\hline $48-4-13-4$ \\
\hline$K 5-4-13-5$ \\
\hline $73-4-i j-6$ \\
\hline $48-4-13-7$ \\
\hline $48-4-13-8$ \\
\hline $13-4-13-\hat{7}$ \\
\hline $36-4-13-10$ \\
\hline$A B-4-13-11$ \\
\hline $15-4-15-12$ \\
\hline $18-4-13-1 j$ \\
\hline$n^{n}-4-15-14$ \\
\hline $4-13-1$ \\
\hline
\end{tabular}

ng

EFFLUEHT HGH COHC. ELUTED TRTAL CHANGE

ppl Hg ppe hg ug hg to th

$\begin{array}{llll}0.06 & 14.40 & -6.60 & -8.60 \\ 0.06 & 14.40 & -0.72 & -3.32 \\ 0.06 & 14.40 & -6.72 & -10.04 \\ 0.06 & 14.40 & -0.72 & -10.76 \\ 0.06 & 14.40 & -0.72 & -13.47 \\ 0.06 & 14.40 . & -0.72 & -11.47 \\ 0.06 & 14.40 & -0.72 & -12.19 \\ 0.06 & 14.40 & -6.72 & -12.71 \\ 0.06 & 14.46 & -0.72 & -13.82 \\ 0.07 & 14.40 & -0.71 & -14.35 \\ 0.06 & 14.40 & -0.72 & -15.06 \\ 0.06 & 14.40 & -0.72 & -15.77 \\ 0.06 & 14.40 & -6.72 & -16.45 \\ 0.66 & 14.40 & -0.72 & -17.21 \\ 0.06 & 14.40 & -0.72 & -17.72\end{array}$

Has

ELUTED TOTAL CHAHEE $\log$

$\log$

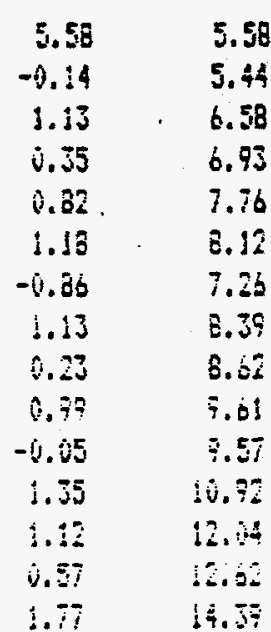

SAKPLE NO.

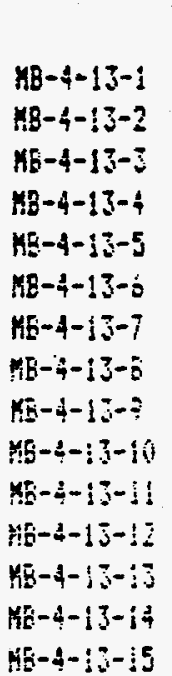

Dp Haj

5.60
5.50
6.00
6.20
7.60
13.60
13.00
14.60
15.50
71.60
5.20
15.600
5.50
15.60
20.30

Haม Citic. joe $\mathrm{HOJ}$

$$
\begin{aligned}
& 0.10 \\
& 4.16 \\
& \text { o.jo } \\
& \text { i. } 10 \\
& \text { v. } 10
\end{aligned}
$$

\begin{tabular}{|c|c|}
\hline 3.50 & 3.30 \\
\hline 6.57 & 3.37 \\
\hline 6.65 & 4.6i \\
\hline$\dot{0} .41$ & 9.49 \\
\hline 2.34 & $6 . \overline{3}$ \\
\hline$\therefore .38$ & 5.87 \\
\hline i. 54 & $\overline{3} .52$ \\
\hline 1.24 & 10.76 \\
\hline V. & io. \\
\hline 35.5 & $46.3 \mathrm{i}$ \\
\hline-83.34 & 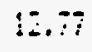 \\
\hline $\bar{x}$ & 10.76 \\
\hline$-4.3 c$ & 2.5 \\
\hline 5,4 & 17.77 \\
\hline$\therefore \%$ & 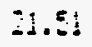 \\
\hline
\end{tabular}

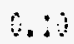

$$
\begin{aligned}
& \dot{v}_{0} \dot{j} \\
& \text { ใ. } 10 \\
& \text { b. } 13 \\
& \text { 4. } 10 \\
& \therefore \\
& \text { s. } 20 \\
& \therefore \text { ii } \\
& \text { i. ii } \\
& \text { i. } 10
\end{aligned}
$$

Elite: ag Noj
TOTAL CHANaE

ํㅗำ 
TABLE C.7. contd

HJBO3

504

\begin{tabular}{|c|c|c|c|c|c|c|c|c|c|}
\hline SAKPLE NO. & $\begin{array}{l}\text { EFFLLUENT } \\
\text { ppa B }\end{array}$ & $\begin{array}{c}\text { HGU COKC. } \\
\text { ppa B }\end{array}$ & $\begin{array}{l}\text { ELUTED } \\
A B\end{array}$ & $\begin{array}{c}\text { TOTAL CHANGE } \\
\text { Ig } B\end{array}$ & SAKPLE NO. & $\begin{array}{l}\text { EFFLUEMT } \\
\text { PPQ SO }\end{array}$ & $\begin{array}{l}\text { HEW COKC. } \\
\text { pps } 504\end{array}$ & $\begin{array}{l}\text { ELUTEA } \\
\text { SQ SUA }\end{array}$ & $\begin{array}{c}\text { TOTAL CHAHEE } \\
\text { SOB }\end{array}$ \\
\hline $88-4-13-1$ & 4.05 & 0.34 & 2.23 & 2.23 & $M B-4-13-1$ & 170.00 & 36.00 & 50.40 & 50.40 \\
\hline $118-4-13-2$ & 3.75 & 0.34 & 0.01 & 2.23 & $\mathrm{AB}-4-13-2$ & 149.00 & 86.00 & -8.40 & 42.00 \\
\hline $48-4-13-3$ & 4.86 & 0.34 & 0.36 & 2.57 & $48-4-13-3$ & 150.00 & 26.00 & $\bar{i} .35$ & 49.35 \\
\hline $36-4-13-4$ & 4.30 & 0.34 & 0.33 & 2.92 & $3 B-4-13-4$ & 161.00 & 66.00 & 6.50 & 55.85 \\
\hline $45-4-13-5$ & 4.35 & 0.34 & 0.23 & 3.15 & $4 B-4-13-5$ & 149.00 & 96.00 & -3.45 & 52.40 \\
\hline $18-4-! 3-6$ & 4.41 & 0.34 & 0.26 & 3.18 & $48-4-13-6$ & 137.00 & 56.60 & -10.05 & 45.20 \\
\hline$M B-4-13-7$ & 4.68 & 0.34 & 0.01 & 3.19 & $48-4-13-7$ & 144.00 & 66.00 & 3.75 & 51.95 \\
\hline $3 E-4-13-8$ & 3.73 & 0.34 & 0.10 & 3.28 & $M E-4-13-6$ & 150.00 & 56.00 & 0.50 & 58.45 \\
\hline$K 8-4-13-7$ & 3.95 & 0.34 & 0.19 & 3.48 & $K B-4-13-7$ & 147.00 & 36.00 & $\vdots .45$ & 59.55 \\
\hline $13 E-4-13-i \hat{v}$ & 4.10 & 0.34 & 0.27 & 3.75 & $58-4-13-10$ & 172.00 & 80.00 & 18.05 & 79.90 \\
\hline $66-4-13-11$ & 3.87 & 0.34 & 0.05 & 3.80 & $136-4-13-11$ & 143.00 & 86.00 & -13.10 & 64.80 \\
\hline $48-4-15-12$ & 4.17 & 0.34 & $\hat{0.30}$ & 4.15 & $58-4-13-12$ & 140.00 & 36.90 & i. 05 & 65.35 \\
\hline $15-4-13-13$ & 4.49 & 0.34 & 0.38 & 4.54 & $78-4-13-13$ & 150.00 & $\ddot{a b . ~ \hat{v v}}$ & 8.70 & 74.55 \\
\hline $15-4-13-14$ & 4.70 & 0.34 & 0.15 & 4.69 & $36-4-13-14$ & $15 j .00 \mathrm{i}$ & 80.60 & 5.60 & 79.55 \\
\hline $48-4-13-15$ & 5.43 & 0.34 & 0.82 & 5.51 & $M B-4-13-15$ & 193.00 & 86.00 & 27.35 & i06.70 \\
\hline
\end{tabular}


$C_{d}$

Mass Balance for Static Leach Test Whole Grout
in Groundwater (MB5-17)

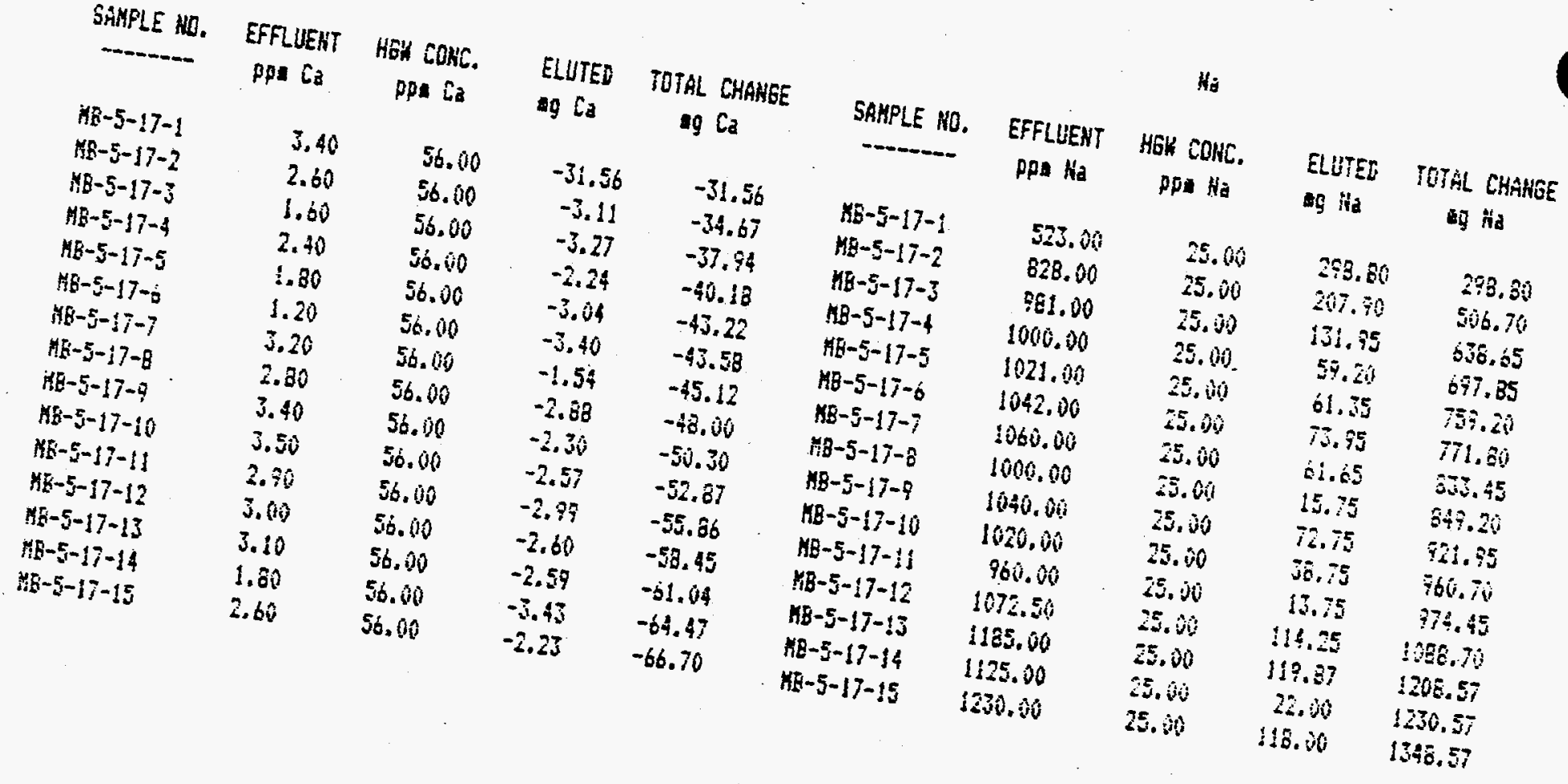

\begin{tabular}{|c|c|}
\hline - & $\begin{array}{l}\text { EFFLUEHT } \\
\text { DOE } K\end{array}$ \\
\hline$A \bar{B}-5-1 / \bar{T}-1$ & 27.8 \\
\hline $\begin{array}{l}75-5-17-2 \\
45-5-17-3\end{array}$ & 34.60 \\
\hline $8-5-17-3$ & $3 \hat{3}, \hat{v i}$ \\
\hline $8-5-17-5$ & 41.00 \\
\hline$-5-17-6$ & 42.50 \\
\hline$-5-17-7$ & 49.00 \\
\hline$-5-17-8$ & 93.80 \\
\hline $5-\{7-j$ & 44.00 \\
\hline $5-17-96$ & 45.00 \\
\hline $5-17-1 !$ & 45,04 \\
\hline$-1 ;-12$ & 42.00 \\
\hline$-1 ;-13$ & 42.60 \\
\hline $17-44$ & +2.00 \\
\hline $17-15$ & 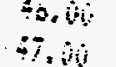 \\
\hline
\end{tabular}

H6H Cons.

7.80
7.60
7.80
7.60
7.80
7.30
7.30
7.80
7.80
7.80
7.80
7.80
.50
.80
.00

ELUTED

s $k$

11.52
5.36
4.31
2.76
2.56
3.46
2.31
2.36
2.41
3.05
1.35
$3.7 !$
.31
.11
51

TOTAL CHAMGE

on $x$

$$
11.52
$$

16.68

20.99

23.75

26. 31

27.21

ล.). 42

30.76

33.19

20.35

30.4

3.72

78.43

42.54

45.65

ii)

SAKPLE NO. EESLUENT HEW CONC.

\begin{tabular}{|c|c|}
\hline $\begin{array}{l}68-5-17-5 \\
6 E-5-17-2 \\
35-5-17-3 \\
48-5-17-5 \\
48-5-17-5 \\
45-5-17-5 \\
18-5-17-5\end{array}$ & $\begin{array}{l}5.30 \\
11.80 \\
14.70 \\
14.30 \\
15.25 \\
15.70\end{array}$ \\
\hline
\end{tabular}

7) $-5-17-3$

$76-5-17-9$

$75-5-17-16$

药- $5-17-11$

$45-5-i 7-12$

$78-5-17-15$

$k 5-5-17-14$

$18-5-17-15$
4.19

0.10

0.10

i. .00

a.

i.

v. 30

6.10

B. 10

$\therefore i c$

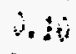

$\therefore .6$

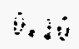

i.

$\therefore i$
EUTES TOTAL CAANGE

ป⿻ำ

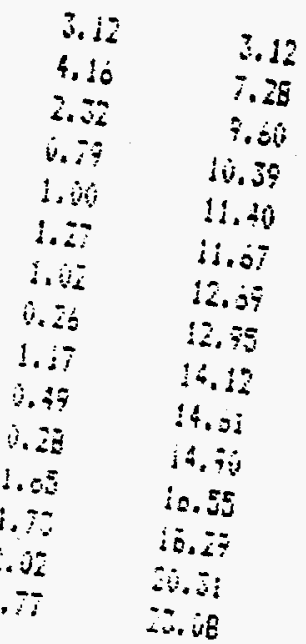


TABLE C.8. contd

HJBO3

504

\begin{tabular}{|c|c|c|c|c|c|c|c|c|c|}
\hline SAMPLE MD. & $\begin{array}{c}\text { EFFLUENT } \\
\text { pp: B" }\end{array}$ & $\begin{array}{c}\text { HEH CONC. } \\
\text { PPE } \overline{8}\end{array}$ & $\begin{array}{l}\text { EIUTED } \\
\text { Ig B }\end{array}$ & $\begin{array}{c}\text { TOTAL CHANGE } \\
\text { G }\end{array}$ & SAMPLE NO. & $\begin{array}{l}\text { EFFLUENT } \\
\text { ppE SO4 }\end{array}$ & $\begin{array}{c}\text { HEN COHC. } \\
\text { PP: SBA }\end{array}$ & $\begin{array}{l}\text { ELUTEO } \\
\text { Ig SOA }\end{array}$ & $\begin{array}{c}\text { TOTAL CHANGE } \\
\text { IO SOA }\end{array}$ \\
\hline$-5-17-1$ & 2.37 & 0.34 & 1.22 & 1.22 & $x B-5-17-1$ & 170.00 & 86.00 & 50.40 & 50.40 \\
\hline$A B-5-17-2$ & 4.23 & 0.34 & 1.22 & 2.44 & $K E-5-17-2$ & 149.00 & 86.60 & -8.40 & 42.20 \\
\hline$H E-5-17-3$ & 4.74 & 0.34 & 0.50 & 2.94 & $A B-5-17-3$ & 156.00 & 86.60 & 7.35 & 49.35 \\
\hline $58-5-17-4$ & 4.83 & 0.34 & 0.27 & 3.21 & $38-5-17-4$ & 161.60 & $B 6.00 j$ & 0.50 & 55.35 \\
\hline$x 5-5-17-5$ & 5.00 & 0.34 & 0.33 & 3.54 & $48-5-17-5$ & 157.00 & B́. 00 & 3.35 & $5 \pi .20$ \\
\hline$x E-5-17-6$ & 5.36 & 0.34 & 0.54 & 3.75 & $A B-5-17-6$ & 153.00 & 56.60 & -1.05 & 54.86 \\
\hline $\mathrm{X}[-5-1]-7$ & 5.24 & 0.34 & 0.18 & 3.93 & $M E-5-17-7$ & 174.60 & 36.60 & 15.85 & 70.75 \\
\hline $48-5-17-8$ & 5.01 & 0.34 & 0.11 & 4.04 & $156-5-17-8$ & 108.60 & 66.00 & $0.3 \mathrm{ji}$ & 71.55 \\
\hline KE-5-17-9 & 5.20 & 0.34 & 0.35 & 4.39 & $M B-5-17-?$ & 168.00 & b. & 4.30 & $75,0.5$ \\
\hline $8-5-17-10$ & 5.03 & 0.34 & 0.14 & 4.53 & $K E-5-17-10$ & 167.00 & 35.60 & 3.50 & 79.15 \\
\hline $40-5-17-11$ & 4.74 & 0.34 & 0.06 & 4.59 & $4 B-5-17-11$ & 166.00 & BE.0i & 3.45 & 82.60 \\
\hline $16-5-17-12$ & 4.74. & 0.34 & 0.22 & 4.81 & $n B-5-17-12$ & 163.00 & $86,0)$ & 2.30 & 84.50 \\
\hline$-5-17-15$ & 4.74 & 0.34 & 0.22 & 5.03 & $48-5-17-13$ & 160.00 & 86.60 & 2.05 & 36.55 \\
\hline$-17-14$ & 6.29 & 0.34 & 1.15 & 6.18 & $n a-5-17-14$ & 170,30 & 56.40 & 13.30 & 500.15 \\
\hline $3-5-17-15$ & 6.32 & v. 34 & 0.68 & 6.85 & $48-5-17-15$ & 229.60 & 86.00 & 36.30 & 136.45 \\
\hline
\end{tabular}


TABLE C.9. Mass Balance for Flow Through Leach Test on whole Grout/Sediment with Groundwater (MBCOLTI)

SHFLLE HO, EFFLUENT FOAE VOL. EZUTẼ̃ SUK ELUTED

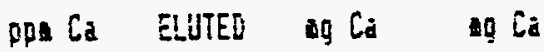

\begin{tabular}{|c|c|c|c|c|}
\hline 1 & 19.1 & 1.44 & -3.33 & -3.33 \\
\hline 2 & 3.6 & 2.91 & -4.82 & -9.15 \\
\hline 3 & 3.3 & 4.47 & -5.21 & -13.36 \\
\hline 4 & 2.9 & 6.66 & -7.21 & -20.57 \\
\hline 5 & 2.2 & 3.47 & -6.09 & -26.67 \\
\hline$b$ & 2.0 & 9.65 & -3.99 & -30.65 \\
\hline 7 & 2.2 & 11.47 & -6.13 & -36.79 \\
\hline 3 & $2 . i$ & 14.06 & -8.75 & -45.53 \\
\hline 9 & 1.3 & 16.00 & -6.58 & -52.11 \\
\hline 10 & 1.6 & 18.56 & -3.72 & -60.83 \\
\hline 11 & 1.6 & 20.64 & $-i .06$ & $-6 \bar{i} .31$ \\
\hline $1 \bar{i}$ & 1.4 & 23.36 & -9.29 & -57.21 \\
\hline 13 & 1.4 & 25.27 & -6.52 & -33.73 \\
\hline 16 & 0.8 & 27.25 & -6.85 & -90.57 \\
\hline 17 & 0.8 & 31.44 & -14.48 & -105.66 \\
\hline 18 & 0.8 & 33.26 & -6.29 & -111.35 \\
\hline 19 & 0.7 & 37.15 & $-13,46$ & -129.81 \\
\hline 20 & $0 . \bar{i}$ & 39.13 & -6.85 & -131.66 \\
\hline 21 & $\hat{0} . \hat{z}$ & 41.76 & -9.08 & $-i 46.34$ \\
\hline 22 & $0 . \hat{0}$ & $46.20^{\circ}$ & -15.54 & -156.28 \\
\hline 23 & $0 . \overline{8}$ & 47.11 & -7.84 & -160.12 \\
\hline 24 & 0.7 & 51.02 & -6.55 & $-172: 71$ \\
\hline 20 ó & 0.97 & 56.32 & -18.25 & -190.37 \\
\hline $2 \hat{0}$ & $1.1 \hat{y}$ & 64.06 & $-\hat{i} \dot{E} .60$ & -217.56 \\
\hline 30 & 1.67 & 67.83 & -12.82 & -230.38 \\
\hline 32 & $\$ .5 ?$ & 72.30 & -15.23 & -245.61 \\
\hline 34 & 4.74 & 74.58 & -7.32 & -252.73 \\
\hline $3 \dot{b}$ & 1.30 & 79.24 & -15.78 & -268.71 \\
\hline 39 & j.83 & 90.19 & -37.69 & -305.80 \\
\hline 4 & 2.42 & 95,45 & -17.64 & -323.44 \\
\hline 43 & 3.22 & i0i.70 & -20.65 & -344.08 \\
\hline 45 & 4.90 & 107.75 & -14.35 & -363.43 \\
\hline 47 & 5.07 & 111.33 & $-14,41$ & -374.85 \\
\hline 45 & 6.65 & 133.14 & -67.37 & -442.21 \\
\hline $5 i t$ & 14.10 & 151.35 & -49.67 & -491.28 \\
\hline
\end{tabular}

SAKPLE NO. EFFLUERT POFE VOL. ELUTED SLK ELLITED ppa $\mathrm{Ha}$ ELUTED $\mathrm{lg} \mathrm{Ha}$ sq $\mathrm{Ha}$

\begin{tabular}{|c|c|c|c|c|}
\hline 1 & 794 & 1.44 & 69.31 & 69.31 \\
\hline 2 & 730 & 2.71 & 83.27 & ¿52.58 \\
\hline 3 & 810 & 4.49 & 77.63 & $230.2 !$ \\
\hline 4 & 760 & 6.66 & 39.53 & 50.63 \\
\hline 5 & 600 & 8.47 & 65.14 & 395.18 \\
\hline 6 & 540 & 9.65 & 30.64 & 433.21 \\
\hline 7 & 570 & 11.47 & 62.08 & 445.29 \\
\hline 8 & 550 & 14.06 & 85.:1: & 586.40 \\
\hline 9 & 550 & 16.00 & 63.75 & 244.15 \\
\hline 10 & 414 & 18.56 & 62.33 & 706.40 \\
\hline 11 & $36 j$ & 20.64 & 44.60 & 750.48 \\
\hline 12 & 348 & 23.36 & 54.97 & 805.47 \\
\hline 13 & 346 & 25.27 & 38.37 & 643.85 \\
\hline 16 & 347 & 27.25 & 39.90 & 823.75 \\
\hline 17 & 241 & 31.44 & 56.65 & 940.40 \\
\hline 18 & 203 & 33.26 & 20.28 & $900.6 \bar{i}$ \\
\hline 17 & 135 & 37.15 & 41.39 & 1002.06 \\
\hline 20 & 171 & 39.13 & 15.09 & 1020.16 \\
\hline 21 & 168 & 41.76 & 23.54 & 1045.70 \\
\hline 22 & 163 & 46.26 & 30.87 & 1082.57 \\
\hline 23 & is! & 47.11 & 22.48 & 1105.04 \\
\hline 24 & 148 & 51.02 & 14.70 & 1115.75 \\
\hline 26 & 135.00 & 56.32 & 36.49 & 1156.24 \\
\hline 28 & 119.00 & 64.010 & 45.54 & $: 201.77$ \\
\hline 30 & 100.00 & $6 \hat{i} .8 \overline{3}$ & 17.70 & 1219.47 \\
\hline 32 & 103.00 & 72.30 & 21.22 & $: 241.27$ \\
\hline 34 & 104.00 & 74.58 & 11.27 & 1252.50 \\
\hline 36 & 101.00 & 79.24 & 2.17 & 1274.73 \\
\hline 39 & 85.40 & 90.19 & 41.40 & :3io.jJ \\
\hline $4 i$ & 74.00 & $\mathbf{7 5 . 4 5}$ & 16.15 & 1352.26 \\
\hline $4 j$ & 67.00 & 101.70 & 16.43 & $1340.6 \%$ \\
\hline 45 & 54.60 & 167.75 & $15.0 \bar{i}$ & 13E?.76 \\
\hline 47 & 77.20 & $11 ! .35$ & $: 1.70$ & 138540 \\
\hline 43 & 62.30 & :33.14 & 50.72 & 1426.30 \\
\hline $5 i$ & 5.70 & 153.55 & 3.44 & 1450.82 \\
\hline
\end{tabular}


contd

SAAFFLE ND. EFFLUEAT PORE VOL. ELUTED SUK ELUTED

\begin{tabular}{|c|c|c|c|c|}
\hline & fon K & ELUTED & o K & $\lg k^{\prime}$ \\
\hline 1 & 4.2 & 1.44 & -0.32 & -0.32 \\
\hline 2 & 0.3 & 2.91 & -0.69 & -1.01 \\
\hline 3 & 0.3 & 4.49 & -0.74 & -1.76 \\
\hline 4 & 0.3 & 6.66 & -1.02 & -2.77 \\
\hline 5 & 2.3 & 8.47 & -0.62 & -3.40 \\
\hline 6. & 0.3 & 9.65 & -0.55 & -3.95 \\
\hline 7 & 0.3 & 11.47 & -0.85 & -4.81 \\
\hline 8 & 0.3 & 14.06 & -1.22 & -6.02 \\
\hline 9 & 2.0 & 16.00 & -0.70 & -5.73 \\
\hline 10 & 2.2 & 10.56 & -0.30 & -7.62 \\
\hline 11 & 1.9 & 20.64 & -0.77 & -8.39 \\
\hline 12 & 2.4 & 23.36 & -0.92 & -9.31 \\
\hline 13 & 1.8 & 25.27 & -0.32 & -10.03 \\
\hline 16 & 2.8 & 27.25 & -6.62 & -10.65 \\
\hline 17 & 2.0 & 31.44 & -1.52 & -12.17 \\
\hline is & 3.4 & 33.26 & -6.50 & -12.67 \\
\hline 19 & 3.2 & 37.15 & -1.12 & -13.79 \\
\hline 20 & 2.1 & 39.13 & $-0.7 i$ & -14.50 \\
\hline 21 & 2.1 & 41.76 & -0.94 & -15.43 \\
\hline 22 & 1.7 & 46.26 & -1.72 & $=-17.15$ \\
\hline 23 & 2.2 & 49.11 & -1.00 & -16.15 \\
\hline 24 & 1.7 & 51.02 & -0.73 & -18.36 \\
\hline 26 & 4.60 & 56.32 & -1.26 & -20.14 \\
\hline 23 & 6.20 & 64.06 & -0.78 & -20.72 \\
\hline 36 & 9.00 & 67.63 & 0.23 & -20.03 \\
\hline 32 & 14.40 & 72.30 & 1.85 & -18.79 \\
\hline 34 & 19.00 & 74.58 & 1.60 & -17.19 \\
\hline 36 & 17.10 & 79.24 & 3.30 & -13.59 \\
\hline 39 & 20.60 & 90.19 & 8.36 & -5.53 \\
\hline $4 !$ & 22.00 & 95.45 & 4.67 & $-\hat{0} .36$ \\
\hline $4 j$ & 23.00 & 161.70 & 5.75 & 5.09 \\
\hline 45 & 29.60 & 107.75 & 6.13 & 11.22 \\
\hline 47 & $2: .00$ & 111.33 & 2.96 & 14.88 \\
\hline 97 & 37.00 & 133.14 & 12.50 & 20.74 \\
\hline 5 & 16.00 & 151.85 & 9.60 & 36.34 \\
\hline
\end{tabular}

SAKPLE RO. EFFLUENT FORE VOL. ELUTEO SOLH ELUTED Pp: Al ELUTED AQ Al ị All

\begin{tabular}{|c|c|c|c|c|}
\hline 1 & 1.52 & 1.44 & 0.13 & 0.13 \\
\hline 2 & 3.70 & 2.91 & 0.33 & 0.46 \\
\hline 3 & 4.50 & $\therefore .49$ & 0.44 & 0.89 \\
\hline 4 & 6.40 & 6.06 & 0.56 & 1.75 \\
\hline 5 & 6.30 & a. 47 & 0.70 & 2.45 \\
\hline á & 5.56 & 9.65 & 6.40 & 2.36 \\
\hline 7 & 0.60 & 11.47 & 0.74 & 3.60 \\
\hline$B$ & 7.77 & 14.00 & 1.24 & $4.3 \%$ \\
\hline$?$ & 7.90 & 16.69 & 0.55 & 5.79 \\
\hline 10 & 7.17 & 10.50 & 1.13 & 6.92 \\
\hline 11 & 6.31 & 20.64 & 1.39 & 7.01 \\
\hline 12 & 6.68 & 23.36 & 1.12 & 6.93 \\
\hline 13 & 6.46 & 25.27 & 6.76 & 3.69 \\
\hline 16 & 10.00 & $27.2 \bar{\Sigma}$ & 1.25 & 10.71 \\
\hline 17 & 7.34 & 31.94 & 1.90 & $12.8 \mathrm{i}$ \\
\hline ia & 0.01 & 35.20 & 0.67 & $13,+9$ \\
\hline 19 & 5.65 & 37.15 & 1.35 & 14.84 \\
\hline 20 & 5.02 & 39.13 & 0.56 & 15.45 \\
\hline 21 & 4.32 & 41.76 & 6.53 & 20.14 \\
\hline 22 & 4.15 & $4 c ́ .2 o ́$ & i. 14 & 17.28 \\
\hline 23 & $3.5 !$ & $49.1 !$ & 0.01 & 57.39 \\
\hline 24 & 3.12 & $5 i .02$ & 0.56 & 18.25 \\
\hline 26 & 2.80 & 56.32 & 0.50 & 17.15 \\
\hline 28 & 2.30 & 64.66 & 1.07 & $20.2 !$ \\
\hline 30 & 1.31 & 67.33 & 0.37 & 20.50 \\
\hline 32 & 1.25 & 72,30 & 0.35 & 20.51 \\
\hline 34 & 1.14 & $74,50$. & 0.15 & 20.70 \\
\hline 36 & 1.13 & 73.24 & $\dot{0} .30$ & 21.26 \\
\hline 39 & $\therefore 23$ & 80.19 & 0.77 & 32.04 \\
\hline $4 i$ & $\hat{0} .87$ & 95.45 & 0.25 & 22.29 \\
\hline 43 & B. & 161.70 & 0.20 & 22.49 \\
\hline 45 & 0.47 & $\operatorname{lng} 5$ & 0.14 & 22.63 \\
\hline 47 & 0.30 & 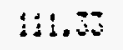 & 3.66 & 22.69 \\
\hline 49 & 8.30 & [S3.14 & 0.27 & 22.36 \\
\hline 50 & $\dot{0} .17$ & :EL.ड̃ & 3.60 & 23.64 \\
\hline
\end{tabular}


TABLE C.9. contd

WHAT ELEMEN EFFLUEHT PORE VOL. ELUTED SUM ELUTED

\begin{tabular}{|c|c|c|c|c|}
\hline & $p p \wedge S i$ & ELUTED & $19 \mathrm{Sj}$ & $99 \mathrm{Si}$ \\
\hline 1 & 116.0 & b. 44 & 9.10 & 9.10 \\
\hline 2 & 167.0 & 2.91 & 13.99 & 23.09 \\
\hline 3 & 152.0 & 4.49 & 13.55 & 36.64 \\
\hline 4 & 121.0 & 6.66 & 14.40 & 51.03 \\
\hline 5 & 93.0 & 8.47 & 8.84 & 57.37 \\
\hline$b$ & 37.4 & 9.65 & 6.09 & 65.96 \\
\hline 7 & 96.3 & 11.47 & 9.26 & 75.22 \\
\hline 8 & 90.9 & 14.06 & 12.30 & 87.52 \\
\hline 9 & 81.6 & 16.00 & 8.09 & 95.61 \\
\hline 10 & 67.6 & 18.56 & $8.4 j$ & 104.04 \\
\hline 11 & 50.4 & 20.64 & 5.65 & 109.69 \\
\hline 12 & 55.0 & 23.36 & 6.81 & 116.50 \\
\hline 13 & 52.9 & 25.27 & 4.53 & 121.03 \\
\hline 16 & 41.0 & 27.25 & 3.22 & 124.25 \\
\hline 17 & 24.9 & $3 i .44$ & 2.60 & 126.84 \\
\hline 18 & 21.1 & 33.26 & 0.67 & 127.54 \\
\hline 10 & 22.0 & 37.15 & 1.70 & 129.24 \\
\hline 20 & 19.8 & 39.13 & 0.59 & 129.84 \\
\hline 21 & 20.2 & 41.76 & 0.86 & 130.67 \\
\hline 22 & 88.7 & 46.26 & 1.04 & 131.74 \\
\hline 23 & 15.9 & 49.11 & 0.14 & 131.88 \\
\hline 24 & 16.9 & 51.02 & 0.23 & 132.11 \\
\hline 26 & 15.80 & 56.32 & 0.27 & 132.37 \\
\hline 28 & 11.10 & 64.06 & -1.89 & 130.48 \\
\hline 30 & 8.34 & 67.83 & -1.57 & 128.91 \\
\hline 32 & 10.90 & 72.30 & -1.15 & 127.76 \\
\hline 34 & 11.50 & 74.58 & -0.44 & 127.32 \\
\hline 36 & 13.00 & 73.24 & -3.58 & 120.74 \\
\hline 37 & 9.11 & 90.19 & -4.04 & 122.70 \\
\hline $4 i$ & 6.50 & 75.45 & -2.80 & ii9.50 \\
\hline 43 & 6.29 & 101.70 & -3.41 & 116.50 \\
\hline 45 & b. ioto & 107.75 & $-3.1 i$ & 113.38 \\
\hline 43 & 8.43 & 111.33 & $-1.4 \bar{i}$ & 191.71 \\
\hline 47 & 5.00 & 133.14 & -11.47 & 100.44 \\
\hline 50 & 7.68 & 151.85 & -9.27 & $9 ! .17$ \\
\hline
\end{tabular}

SAKPLE ND. EFFLUENT FORE VOL. ELUTED SUM ELUTED Pp Mg ELUTED ag $\mathrm{Mg}$ aq No

\begin{tabular}{|c|c|c|c|c|}
\hline 1 & 0.06 & 1.44 & -1.26 & -1.26 \\
\hline 2 & 0.06 & 2.91 & -1.28 & -2.54 \\
\hline 3 & 0.06 & 4.49 & -1.38 & -3.92 \\
\hline 4 & 0.06 & 6.66 & -1.39 & -5.81 \\
\hline 5 & 0.06 & 3.47 & -1.58 & -7.39 \\
\hline$b$ & 0.16 & 9.65 & -1.03 & -8.42 \\
\hline 7 & 0.06 & 11.97 & -1.59 & $-10.6 \mathrm{i}$ \\
\hline 8 & 0.06 & 14.06 & -2.26 & -12.27 \\
\hline 9 & 0.06 & 16.60 & -1.67 & -13.760 \\
\hline 10 & 0.06 & 18.56 & -2.23 & -ió.l? \\
\hline 11 & 0.06 & 20.64 & -1.81 & -IE. B! \\
\hline 12 & 0.05 & 23.36 & -2.37 & -20.38 \\
\hline 13 & 0.06 & 25.27 & -1.07 & -22.65 \\
\hline 16 & 0.6́ & 27.25 & -1.73 & -23.78 \\
\hline 17 & 0.06 & 31.44 & 6́ & -27.43 \\
\hline 18 & 0.66 & 33.26 & -1.57 & -29.02 \\
\hline 19 & 3.06 & 37.15 & -3.37 & -32.41 \\
\hline 20 & 0.06 & 53.13 & -1.73 & -34.14 \\
\hline 21 & 1..06 & 41.16 & -2.37 & -36.44 \\
\hline 22 & 0.06 & 40.25 & -3.83 & -40.36 \\
\hline 23 & 0.06 & 49.11 & -2.49 & -42.85 \\
\hline 24 & $\dot{0} .06$ & 51.02 & $-1.6 i$ & -44.52 \\
\hline 26 & 0.06 & 56.32 & -4.62 & -49.14 \\
\hline 28 & 0.06 & 64.06 & $-5 . j 5$ & -55.39 \\
\hline 30 & i. 06 & 67.83 & -3.27 & -59.12 \\
\hline 32 & 0.06 & 72.30 & -3.50 & -53.05 \\
\hline 34 & 0.06 & 74.58 & $-1,79$ & -65.67 \\
\hline 36 & 0.06 & 79.24 & -4.07 & -67.14 \\
\hline 39 & 0.06 & 70.19 & -9.58 & -78.59 \\
\hline 41 & 0.06 & 75.45 & -4.39 & -83.28 \\
\hline 43 & 4.16 & 101.70 & -5.45 & $-8 \bar{E} . j 3$ \\
\hline 45 & 0.05 & 107.75 & $-5 .: B$ & $-7 \leq .01$ \\
\hline 47 & 0.60 & 111.35 & -5.12 & -97.14 \\
\hline 49 & 0.06 & 133.14 & -19.05 & -115.17 \\
\hline 50 & 0.32 & 151.85 & -15.79 & $-13 i .5$ \\
\hline
\end{tabular}


TABLE C.9. contd

SAMPLE HO. EFFLUENT PDRE YOL. ELUTED SUK ELUTED

\begin{tabular}{|c|c|c|c|c|}
\hline & $P D \subseteq F$ & ELUTED & of $F$ & of \\
\hline 1 & 13.0 & 1.44 & 1.13 & 1.13 \\
\hline 2 & 8.5 & 2.91 & 0.74 & 1.86 \\
\hline 3 & 8.5 & 4.49 & 0.79 & 2.65 \\
\hline 4 & 13.0 & 6.66 & 1.70 & 4.35 \\
\hline 5 & 5.7 & 0.47 & 0.59 & 4.74 \\
\hline$\dot{d}$ & 6.0 & 9.65 & 0.41 & 5.35 \\
\hline 7 & 5.0 & 11.47 & 0.51 & 5.86 \\
\hline 8 & 5.0 & 14.06 & 0.73 & 6.59 \\
\hline 7 & 4.0 & 16.60 & 0.42 & $7.0 !$ \\
\hline 10 & 4.0 & 18.56 & 0.56 & 7.57 \\
\hline 11 & 3.5 & 20.64 & 0.37 & 7.87 \\
\hline 12 & 3.4 & 23.36 & 0.47 & 8.46 \\
\hline 13 & 5.6 & 25.27 & 0.61 & 9.07 \\
\hline 16 & 4.7 & 27.25 & 0.55 & 8.61 \\
\hline 17 & 3.6 & 31.44 & 0.81 & 10.43 \\
\hline 18 & 4.1 & 33.26 & 0.11 & iv. 84 \\
\hline 19 & 4.4 & 37.15 & 0.95 & 11.79 \\
\hline 20 & 4.0 & 39.13 & 0.43 & 12.22 \\
\hline 21 & 4.0 & 41.76 & 0.58 & 12.80 \\
\hline 22 & 3.6 & 46.26 & 0.87 & 13.67 \\
\hline 23 & 3.8 & 49.11 & $\hat{v .57}$ & 14.26 \\
\hline 24 & 4.3 & 51.02 & 0.45 & 14.71 \\
\hline 26 & 3.10 & 56.32 & 0.86 & 15.55 \\
\hline 28 & 3.00 & 64.06 & $1.2 i$ & 16.79 \\
\hline 30 & 3.60 & 67.33 & 0.73 & 17.52 \\
\hline 32 & 3.30 & 72.30 & 6.75 & 12.30 \\
\hline 34 & 3.60 & 74.50 & 0.44 & 18.74 \\
\hline 36 & 3.70 & 79.24 & 0.73 & 19.68 \\
\hline 39 & 3.70 & 90.19 & 2.19 & 21.67 \\
\hline 41 & $\$ .00$ & 95.45 & 1.15 & 23.12 \\
\hline $4 j$ & 3.60 & 101.70 & ¿.21 & 24.23 \\
\hline 45 & 4.30 & 107.75 & 1.44 & 25.67 \\
\hline $4 i$ & 9.20 & 111.33 & $\dot{4} .35$ & 26.50 \\
\hline 49 & 3.00 & 133.14 & 4.23 & 30.73 \\
\hline 30 & 502 & 151.35 & 2.75 & 30.67 \\
\hline
\end{tabular}

SAKPLE NO. EFFLUENT FORE YGL. ELUTEO SUM ELUTED

\begin{tabular}{|c|c|c|c|c|}
\hline & pDE $\mathrm{NOZ}$ & ELUTED & בסות פִי & Jo \\
\hline 1 & 70.0 & 1.44 & 6.30 & 0.30 \\
\hline 2 & 13.0 & 2.91 & 1.19 & 7.49 \\
\hline 3 & 12.0 & 4.49 & 1.18 & a. \\
\hline 4 & 7.7 & 6.66 & 1.03 & 3.70 \\
\hline 5 & 11.0 & 8.47 & 1.23 & 10.93 \\
\hline 6 & 3.0 & 9.65 & $0.5 \overline{0}$ & $1.5 !$ \\
\hline 7 & $10 . \hat{v}$ & 11.47 & 3.13 & 22.04 \\
\hline 8 & 5.0 & 19.06 & $-0.7 \%$ & 13.44 \\
\hline 9 & 5.0 & 16.000 & 1.59 & $: 4.03$ \\
\hline 10 & 4.3 & 12.50 & $0.6 \bar{j}$ & 14.70 \\
\hline 11 & 3.6 & 20.64 & 0.46 & 15.16 \\
\hline 12 & 3.5 & 23.36 & 0.53 & 15.74 \\
\hline 13 & 5.0 & 25.27 & 0.55 & 15.32 \\
\hline 16 & $3.9-$ & 27.25 & 0.47 & 10.80 \\
\hline 17 & 1.7 & 31.44 & 0.42 & 17.22 \\
\hline 18 & $2 . j$ & 33.26 & 0.23 & 87.44 \\
\hline 19 & 2.3 & 37.15 & 0.54 & $1 \bar{i} .76$ \\
\hline 20 & 2.2 & 39.13 & 0.20 & $10 \hat{0} .24$ \\
\hline 21 & 2.0 & 41.76 & $\dot{v} . j !$ & 18.55 \\
\hline 22 & 3.8 & 46.26 & 0.48 & 19.03 \\
\hline 25 & 2.1 & 99.11 & 0.36 & $17.3 \%$ \\
\hline 24 & 2.8 & 51.02 & 0.32 & 19.11 \\
\hline 26 & 2.40 & 56.32 & 0.76 & 20.47 \\
\hline 28 & 2.00 & 64.06 & $0.9 i$ & 21.37 \\
\hline 30 & 4.30 & 67.83 & 1.11 & 22.30 \\
\hline 32 & 4.50 & 72.30 & 1.23 & 33.73 \\
\hline 34 & 7.00 & 74.53 & 6. & 24.72 \\
\hline 36 & 7.00 & 79.24 & $2.6 !$ & 26.73 \\
\hline 39 & 3.20 & 90.17 & 4.13 & 36.93 \\
\hline 41 & 7.80 & 95.45 & 2.54 & 3.45 \\
\hline 43 & 6.90 & 161.70 & 2.06 & 36.11 \\
\hline 45 & 6.80 & 107.75 & 2.54 & 36.64 \\
\hline 47 & 19.00 & $11 ! .3$ & 2.33 & 42.0 \\
\hline 49 & 0.50 & 130.14 & 0.55 & 43.42 \\
\hline $5 i)$ & 4.72 & 15i.E5 & 5 & 48.97 \\
\hline
\end{tabular}


TABLE C.9. contd

SAFFLE NO. EFFLUERT POGE VOL. ELLTED SUM ELUTED

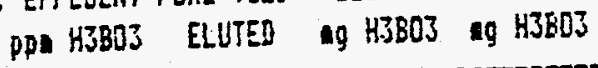

\begin{tabular}{|c|c|c|c|c|}
\hline 1 & 18.87 & 1.94 & 1.67 & 1.67 \\
\hline 2 & 6.29 & 2.91 & 0.55 & 2.22 \\
\hline 3 & 5.37 & 4.49 & 0.50 & 2.71 \\
\hline 4 & 3.49 & 6.66 & 0.43 & 3.14 \\
\hline 5 & 2.40 & 8.47 & 0.23 & 3.38 \\
\hline 6 & 2.68 & 9.65 & 0.17 & 3.55 \\
\hline 7 & 2.24 & 11.47 & 0.22 & 3.77 \\
\hline 8 & 1.83 & 14.06 & 0.24 & 4.01 \\
\hline 9 & 1.71 & 16.00 & 0.17 & 4.17 \\
\hline 10 & 1.37 & 18.56 & 0.17 & 4.34 \\
\hline 11 & 1.21 & 20.64 & 0.11 & 4.45 \\
\hline 12 & 1.16 & 23.36 & 0.14 & 4.59 \\
\hline 13 & 1.10 & 25.27 & 0.09 & 4.69 \\
\hline 16 & 1.38 & 27.25 & 0.19 & 4.98 \\
\hline 17 & 1.07 & 31.44 & 0.19 & 5.07 \\
\hline 18 & 1.11 & 33.26 & 0.09 & 5.16 \\
\hline 19 & 1.21 & 37.15 & 0.21 & 5.37 \\
\hline 20 & 1.10 & 39.13 & 0.09 & 5.46 \\
\hline 21 & 3.25 & 41.76 & 0.15 & 5.61 \\
\hline 22 & 1.04 & 46.26 & 0.20 & 5.81 \\
\hline 23 & 1.01 & 49.11 & 0.12 & 5.73 \\
\hline 24 & 1.18 & 51.02 & 0.10 & 6.03 \\
\hline 26 & 0.97 & 56.32 & 0.21 & 6.24 \\
\hline 23 & 0.85 & 64.06 & 0.25 & 6.48 \\
\hline $3 i$ & 0.73 & 83.83 & 0.14 & 6.62 \\
\hline 32 & 1.00 & 72.30 & 0.12 & 6.81 \\
\hline 34 & 1.09 & 74.58 & 0.11 & 6.92 \\
\hline 36 & 1.13 & 79.24 & 0.23 & 7.15 \\
\hline 39 & i). 82 & 90.19 & 0.33 & 7.48 \\
\hline 41 & 0.79 & 95.45 & 0.15 & 3.62 \\
\hline 43 & 0.74 & 101.70 & 0.16 & 7.78 \\
\hline 45 & 0.93 & 107.75 & 0.22 & 8.00 \\
\hline $4 i$ & 1.13 & 111.33 & 0.18 & a.:3 \\
\hline 49 & 0.35 & 133.14 & 0.67 & 3.05 \\
\hline 50 & $3.2 \hat{v}$ & IEl.E5 & 6.54 & 9.39 \\
\hline
\end{tabular}

SAMPLE NO. EFFLUENT PORE VOL. ELUTED SUM ELLTEES PDE SO4 ELUTED SO SO4 SO4

\begin{tabular}{|c|c|c|c|c|}
\hline 1 & 540 & 1.14 & 40.92 & 40.22 \\
\hline 2 & 130 & 2.91 & 4.65 & 44.97 \\
\hline 3 & 140 & 4.49 & 5.34 & 50.31 \\
\hline 4 & 110 & 0.00 & 3.26 & 53.57 \\
\hline 5 & 100 & 8.47 & 1.58 & 55.15 \\
\hline 6 & 117 & 9.65 & 3.25 & 57.44 \\
\hline 7 & 108 & 11.97 & $2.5 !$ & 5.75 \\
\hline 8 & 360 & 14.00 & 3.25 & 8.96 \\
\hline$?$ & $1 \pm 0$ & 36.60 & 2.93 & 65.:3 \\
\hline 10 & 96 & 18,56 & 1.80 & 0.73 \\
\hline 11 & 85 & 20.64 & 0.23 & 8.60 \\
\hline 12 & 38 & 23.36 & 0.34 & 67.34 \\
\hline 13 & 100 & 25.73 & $1,6 i$ & $67.0 \mathrm{~s}$ \\
\hline I6 & 26 & 27.25 & 6.25 & $=5.26$ \\
\hline 17 & 86 & $31 .+49$ & 8.60 & $\dot{0}$ \\
\hline 18 & $B 5$ & 33.26 & $-\hat{3} .1 !$ & 6i. \\
\hline 19 & 38 & $3 i .15$ & 2.32 & $F_{0}, 06$ \\
\hline 20 & 31 & 39.13 & 0.02 & 72.88 \\
\hline 21 & 107 & 41.76 & 3.76 & 70.14 \\
\hline 22 & $3 !$ & $40.2 b$ & $i, 4 i$ & 77.53 \\
\hline 23 & $9 i$ & 49.11 & 0.37 & 70.34 \\
\hline 24 & 160 & 51.02 & $1.8 j$ & 601.12 \\
\hline 26 & 94.50 & 56.32 & 1.82 & 31.54 \\
\hline 38 & 83.06 & 64.06 & -1.45 & 80.49 \\
\hline 30 & 87.00 & 67.33 & 0.27 & 80.72 \\
\hline 32 & 73.60 & 72.30 & -1.90 & $7 \bar{a} . j 0$ \\
\hline 34 & 97.00 & 74.56 & $1 . \bar{i} \bar{i}$ & 86.33 \\
\hline 36 & 31.00 & 79.24 & b & 73.88 \\
\hline 39 & 22.00 & 50.17 & -2.74 & 7.13 \\
\hline 41 & 81.10 & 3045 & -1.35 & 74.45 \\
\hline 43 & 78.00 & $10 \div .70$ & -3.5 & 7.36 \\
\hline 45 & $32.6 j$ & 10.75 & $-\{.51$ & $=\overline{7} .8 \div$ \\
\hline 47 & 34.190 & 191.5 & $-1,95$ & 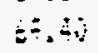 \\
\hline 49 & 30,6 & 4 & - & 50 \\
\hline 50 & 70.30 & $: 3.35$ & $-\vdots .5$ & 3 \\
\hline
\end{tabular}


TABLE C.10. Mass Balance for Flow Through Leach Test on whole Grout/Sediment with Groundwater (MBCOLT1)

SÁMPLE NO. EFFLUENT FORE VOL. ELUTED SUM ELUTED ppi la Eluted qu la ug la

\begin{tabular}{|c|c|c|c|c|}
\hline 1 & 19.1 & 1.33 & -3.32 & $-j . j 2$ \\
\hline 2 & 3.8 & 2.72 & -4.72 & -8.24 \\
\hline 3 & 3.6 & 4.15 & -5.08 & -13.32 \\
\hline 4 & 3.0 & 5.89 & -6.25 & -19.56 \\
\hline 5 & 2.5 & 6.82 & -3.73 & -23.30 \\
\hline 6 & 2.4 & 8.01 & -3.96 & -27.25 \\
\hline $\bar{i}$ & 1.8 & 9.67 & -6.09 & -33.34 \\
\hline 8 & 1.9 & 11.84 & -8.32 & -41.66 \\
\hline 7 & 1.7 & 13.74 & -6.62 & -48.29 \\
\hline 10 & 1.6 & 15.78 & -7.52 & -55.81 \\
\hline 11 & 1.6 & 17.41 & -6.01 & -61.82 \\
\hline 12 & 1.2 & 19.68 & -8.42 & $-j 0.24$ \\
\hline$\vdots 3$ & 1.1 & 21.39 & -5.36 & -76.59 \\
\hline 14 & 1.0 & 24.16 & -10.31 & -86.91 \\
\hline$\$ 5$ & 1.1 & 25.35 & -4.42 & -91.33 \\
\hline 16 & 1.0 & 28.83 & -12.97 & -104.31 \\
\hline 17 & 1.0 & 32.37 & -13.20 & -117.50 \\
\hline 18 & 1.0 & 34.97 & -9.67 & -127.19 \\
\hline 17 & 0.9 & 38.56 & -13.37 & -140.59 \\
\hline 20 & 1.0 & 39.63 & -3.99 & -144.57 \\
\hline 22 & 0.8 & 42.29 & -9.94 & -154.52 \\
\hline 23 & 0.9 & 44.70 & -8.99 & -163.51 \\
\hline 24 & 0.9 & 46.18 & -5.52 & -169.03 \\
\hline 26 & 0.85 & 50.59 & -17.97 & -187.00 \\
\hline 28 & 1.10 & 57.84 & -25.47 & -212.48 \\
\hline 30 & 1.37 & $6 \mathrm{i} .38$ & -13.10 & -225.58 \\
\hline 32 & 1.11 & 66.40 & -16.67 & -244.24 \\
\hline 34 & 1.18 & 72.36 & -22.13 & -206.37 \\
\hline 36 & 1.31 & 77.15 & -17.58 & -283.96 \\
\hline 39 & 2.69 & 65.25 & -43.70 & -327.65 \\
\hline 41 & 3.58 & 94.66 & -19.20 & -346.93 \\
\hline 43 & 5.10 & 162.67 & $-2 i .55$ & -374.48 \\
\hline 45 & $0.4 \varepsilon$ & 111.34 & -31.10 & -405.58 \\
\hline 47 & 1500 & 123.04 & -32.33 & -437.71 \\
\hline 45 & 12.50 & 149.07 & $-i 6.70$ & $-514,61$ \\
\hline 50 & 14.00 & 160.17 & -56.37 & -570.98 \\
\hline
\end{tabular}

SAMPLE ND. EFFLUENT PORE VOL. ELUTED SUM ELUTED pP $\mathrm{Na}$ ELUTED $\mathrm{Na} \mathrm{Na}$. $\mathrm{Ha}$

\begin{tabular}{|c|c|c|c|c|}
\hline 1 & 1130 & 1.33 & 99.55 & 99.55 \\
\hline 2 & 1080 & 2.72 & 97.34 & 196.89 \\
\hline 3 & 690 & 4.15 & 83.79 & 282.68 \\
\hline 4 & 820 & 5.67 & 9.3 .70 & 376.37 \\
\hline 5 & 530 & 6.92 & 35.23 & 411.62 \\
\hline 6 & 710 & 8.01 & 56.50 & 962.20 \\
\hline 7 & 640 & 9.67 & 69.16 & 531.36 \\
\hline 8 & 590 & 11.74 & 86.65 & 610.24 \\
\hline 9 & 590 & 13.74 & 68.59 & 607.83 \\
\hline 10 & 420 & 15.78 & 55.27 & 742.52 \\
\hline !! & 387 & 27.41 & 37.57 & 782.79 \\
\hline 12 & 364 & 19.58 & 52.13 & 834.92 \\
\hline 13 & 330 & 21.39 & 35.33 & 270.25 \\
\hline 14 & 308 & 24.16 & 53.10 & 923.35 \\
\hline 15 & 280 & 25.35 & 20.56 & 943.90 \\
\hline 16 & 313 & 22.63 & 67.89 & $1011: 80$ \\
\hline 17 & 237 & 32.37 & 51.32 & 1063.31 \\
\hline 13 & 204 & 34.97 & 31.53 & 1054.64 \\
\hline 19 & $2 \hat{v 2}$ & 38.56 & 43.64 & 1137.60 \\
\hline 20 & $17 !$ & 39.03 & 10.58 & 1148.27 \\
\hline 22 & 250 & 92.29 & 40.54 & 1188.61 \\
\hline 23 & 216 & 44.70 & $3 i .18$ & 1219.99 \\
\hline 24 & 202 & 46.10 & 17.75 & 1237.73 \\
\hline 20 & 177.00 & 50.99 & 49.53 & 1287.26 \\
\hline 28 & 147.00 & 57.84 & 50.61 & $\{343.67$ \\
\hline 30 & 123.00 & $6 j .38$ & 24.94 & 1368.81 \\
\hline 32 & 123.60 & 66.40 & 33.33 & 1402.14 \\
\hline 34 & 164.00 & 72.36 & 31.89 & 1434.03 \\
\hline 36 & 161.60 & 77.15 & 24.60 & 1458.65 \\
\hline 39 & 34.20 & $89 . .5$ & 48.36 & isoi.3: \\
\hline 41 & 67.60 & 94.60 & ib.41 & 253.62 \\
\hline 43 & 34.30 & 102.67 & $2 ! .16$ & $1544.7 \mathrm{e}$ \\
\hline 45 & 64.30 & $11: 94$ & 24.68 & 1563.46 \\
\hline 47 & 51.80 & 123.04 & 20.45 & 1509.01 \\
\hline 49 & 56.50 & 147.07 & 55.54 & !6:5.:5 \\
\hline 50 & 52.90 & 169.37 & 5.94 & 1603.14 \\
\hline
\end{tabular}


TABLE C.10. contd

SAKPLE NO. EFFLUENT POFE YOL. ELUTED SUM ELUTED PPE $K$ ELUTED IQ $K$ Ig $K$

\begin{tabular}{|c|c|c|c|c|}
\hline 1 & 6.3 & 1.33 & -0.14 & -0.14 \\
\hline 2 & 1.9 & 2.72 & -0.56 & -0.69 \\
\hline 3 & 1.9 & 4.15 & -0.57 & -1.26 \\
\hline 4 & 0.3 & 5.89 & -0.92 & -2.18 \\
\hline 5 & 1.6 & 6.92 & -0.43 & -2.61 \\
\hline 6 & 2.8 & 8.01 & -0.37 & -2.98 \\
\hline 7 & 1.4 & 9.67 & -0.72 & -3.70 \\
\hline 8 & 1.7 & 11.94 & -0.91 & -4.61 \\
\hline 9 & 1.8 & 13.74 & -0.73 & -5.34 \\
\hline 10 & 1.3 & 15.76 & -0.90 & -6.24 \\
\hline 11 & 1.3 & 17.41 & -0.72 & -6.96 \\
\hline 12 & 1.8 & 19.68 & -0.92 & -7.58 \\
\hline 13 & 1.4 & 21.39 & -0.74 & -8.62 \\
\hline 14 & 1.3 & 24.16 & -1.22 & -9.84 \\
\hline 15 & 0.7 & 25.35 & -0.57 & -10.41 \\
\hline 16 & 3.0 & 28.83 & -1.13 & -11.55 \\
\hline 17 & 2.5 & 32.37 & -1.25 & -12.79 \\
\hline 18 & 2.1 & 34.97 & -1.00 & -13.80 \\
\hline 19 & 1.9 & 38.56 & -1.43 & -15.23 \\
\hline 20 & 1.8 & 39.63 & -0.43 & $-\{5.67$ \\
\hline 22 & 1.2 & 42.29 & -1.19 & -16.86 \\
\hline 23 & 1.3 & 44.70 & -1.06 & -17.92 \\
\hline 24 & 0.9 & 46.18 & -0.69 & -18.61 \\
\hline 26 & 2.60 & 50.99 & -1.69 & -20.30 \\
\hline 23 & 2.60 & 57.84 & -2.41 & -22.72 \\
\hline 30 & 2.51 & 61.38 & -1.27 & -23.99 \\
\hline 32 & $3 . \hat{v} \hat{v}$ & 6ó. 40 & -1.03 & -25.62 \\
\hline 34 & 4.70 & 72.36 & -1.17 & -26.79 \\
\hline 36 & 8.70 & 77.15 & 0.29 & -26.50 \\
\hline 37 & $2 ! .00$ & 89.25 & 10.82 & -15.68 \\
\hline 41 & 27.60 & 94.68 & 7.06 & -8.82 \\
\hline 43 & 29.00 & 102.67 & 11.47 & 2.36 \\
\hline 45 & 26.60 & ili.94 & 11.43 & 14.29 \\
\hline 45 & 22.00 & 123,69 & 10.68 & 24.96 \\
\hline 47 & 13.00 & 149.07 & 9.13 & 34.13 \\
\hline 3i) & $12,0 \mathrm{v}$ & 163.17 & 5.72 & 39.65 \\
\hline
\end{tabular}

SAMPLE NO. EFFLLENT FORE VOL. ELUTED SUM ELUTED PPE Al ELUTED Al Al $\mathrm{Al}$

\begin{tabular}{|c|c|c|c|c|}
\hline 1 & 1.52 & 1.33 & 0.13 & 0.13 \\
\hline 2 & 7.50 & 2.72 & 0.70 & 0.82 \\
\hline 3 & 4.80 & 4.15 & 0.46 & 1.28 \\
\hline 4 & 7.50 & 5.69 & 0.87 & 2.15 \\
\hline 5 & 9.30 & 6.92 & 0.89 & 2.79 \\
\hline 6 & 3.90 & B. 01 & 0.65 & 3.44 \\
\hline 7 & 9.94 & 9.67 & $! .1 !$ & 4.55 \\
\hline 8 & 10.00 & 11.94 & 1.52 & 6.07 \\
\hline 9 & 10.30 & 13.74 & 1.24 & 7.32 \\
\hline 10 & 10.60 & 15.76 & 1.45 & 6.77 \\
\hline 11 & 10.30 & 17.41 & 1.13 & .8 .89 \\
\hline 12 & 9.29 & 19.66 & $\mathrm{i} .4 \mathrm{i}$ & 11.31 \\
\hline 13 & 6.41 & 21.39 & 0.96 & 12.27 \\
\hline 14 & 8.07 & 24.16 & 1.50 & 13.71 \\
\hline 15 & 7.85 & 25.35 & 0.01 & 14.37 \\
\hline 16 & 7.77 & 20.83 & $1.3 i$ & 16.18 \\
\hline 17 & 6.66 . & 32.37 & 1.57 & 17.75 \\
\hline 18 & 5.67 & 34.97 & 0.78 & 16.74 \\
\hline 19 & 4.45 & 38.56 & 1.86 & 18.74 \\
\hline 20 & 4.69 & 39.63 & 0.33 & 20.13 \\
\hline 22 & 5.75 & 42.29 & 1.62 & 21.14 \\
\hline 23 & 5.64 & 44.70 & 0.74 & 23.08 \\
\hline 24 & 5.13 & 46.18 & 0.50 & 22.59 \\
\hline 26 & 5.10 & 50.99 & $\therefore .53$ & 24.21 \\
\hline 28 & 3.50 & 57.84 & 1.76 & 25.99 \\
\hline 30 & 2.44 & 61.36. & $\dot{0} .56$ & 26.54 \\
\hline 32 & 1.97 & 66.40 & 0.64 & 27.18 \\
\hline 34 & 1.65 & 72.36 & 0.64 & 27.81 \\
\hline 36 & i.27 & 77.15 & 0.36 & 28.19 \\
\hline 39 & 0.50 & 89.25 & 0.65 & 28.35 \\
\hline 41 & 0.67 & $94.60 \hat{~}$ & 0.21 & 29.05 \\
\hline 43 & 0.52 & $162.8 ?$ & 0.22 & 29.28 \\
\hline 45 & 0.30 & 111.74 & 1.:17 & 29.45 \\
\hline $4 i$ & $\dot{u} .21$ & 123.04 & $0.0 \bar{E}$ & 27.53 \\
\hline 49 & 0.10 & $149.0 \bar{i}$ & 0.00 & 53.53 \\
\hline 50 & 0.17 & $165.17 i$ & $\dot{a}, i j$ & 29.05 \\
\hline
\end{tabular}


TABLE C.10. contd

SAMPLE MO. EFFLUERT PORE VOL. ELUTED SUM ELUTED ppe Si ELUTED $\mathrm{Si}$ ag $\mathrm{Si}$

\begin{tabular}{|c|c|c|c|c|}
\hline 1 & 103.0 & 1.33 & 7.83 & 7.93 \\
\hline 2 & 150.0 & 2.72 & 12.71 & 20.64 \\
\hline 3 & 159.0 & 4.15 & 13.95 & 34.59 \\
\hline 4 & 115.0 & 5.89 & 11.79 & 46.38 \\
\hline 5 & 103.0 & 6.92 & 6.14 & 52.52 \\
\hline 6 & 108.0 & 8.01 & 6.87 & 59.38 \\
\hline$i$ & 89.4 & 9.67 & 8.37 & 67.75 \\
\hline 8 & 65.7 & 11.94 & 10.80 & 78.65 \\
\hline 9 & 76.8 & 13.74 & 7.54 & 36.19 \\
\hline 10 & 65.5 & 15.78 & 6.98 & 93.16 \\
\hline 11 & 56.9 & 17.41 & 4.63 & 97.79 \\
\hline 12 & 51.1 & 19.68 & 5.55 & 103.34 \\
\hline 13 & 45.4 & 21.39 & 3.52 & 106.86 \\
\hline 14 & 41.3 & 24.16 & 4.93 & 111.80 \\
\hline 15 & 35.3 & 25.35 & 1.64 & 113.43 \\
\hline 16 & 41.9 & $2 \mathrm{~B} .63$ & 6.34 & 119.78 \\
\hline 17 & 28.0 & 32.37 & 3.12 & i22.89 \\
\hline 18 & 24.4 & 34.97 & 1.66 & 124.55 \\
\hline 19 & 29.7 & 38.56 & 3.57 & 128.12 \\
\hline 20 & 33.0 & 39.63 & 1.30 & 129.43 \\
\hline 22 & 38.0 & 42.29 & 4.32 & 133.75 \\
\hline 23 & 30.3 & 44.70 & 2.50 & 136.25 \\
\hline 24 & 28.0 & 46.18 & 1.30 & 137.55 \\
\hline 26 & 21.50 & 50.99 & 2.12 & 139.67 \\
\hline 28 & 14.30 & 57.84 & -0.32 & 139.35 \\
\hline 30 & 11.90 & 61.38 & -0.74 & 138.60 \\
\hline 32 & 13.40 & 06,40 & -0.54 & 138.06 \\
\hline 34 & 11.10 & 72.36 & -1.57 & 136.49 \\
\hline 36 & 9.95 & 77.15 & -1.64 & 134.85 \\
\hline 39 & 8.65 & 89.25 & -5.20 & 229.64 \\
\hline 41 & 3.19 & 94.68 & -2.87 & 126.77 \\
\hline 43 & 7.31 & 102.67 & -4.16 & 122.61 \\
\hline 45 & 8.05 & 111.94 & -4.36 & 113.24 \\
\hline 47 & 8.55 & $: 23.04$ & -4.56 & 113.30 \\
\hline 49 & 3.50 & 147.07 & -11.46 & 101.52 \\
\hline 50 & 7.06 & 169.17 & -10.78 & 91.13 \\
\hline
\end{tabular}

SAKFLE NO. EFFLUENT PORE VOL. ELUTED SUM ELUTED PPO Hg ELUTED ag Hg ag Mg

\begin{tabular}{|c|c|c|c|c|}
\hline 1 & 0.06 & 1.33 & -1.26 & -1.26 \\
\hline 2 & 0.06 & 2.72 & -1.31 & -2.57 \\
\hline 3 & 0.06 & 4.15 & -1.35 & -3.92 \\
\hline 4 & 0.06 & 5.89 & -3.64 & -5.50 \\
\hline 5 & 0.06 & 6.92 & $-\hat{0} .97$ & $-6.5 j$ \\
\hline$b$ & 0.06 & 3.01 & -1.63 & -7.56 \\
\hline 7 & 0.06 & 9.67 & -1.57 & -9.13 \\
\hline 8 & 0.06 & 11.94 & -2.14 & -11.27 \\
\hline 9 & 0.06 & 13.74 & -1.70 & -12.37 \\
\hline :0 & 0.06 & 15.73 & -1.73 & -14.70 \\
\hline 11 & 0.06 & 17.41 & -1.54 & -16.44 \\
\hline 12 & 0.06 & 19.68 & -2.14 & -16.58 \\
\hline 13 & 0.06 & 21.37 & -1.31 & -20.20 \\
\hline$\dddot{14}$ & 1.06 & 24.16 & -2.62 & $-22.8 \mathrm{i}$ \\
\hline 15 & 0.06 & 25.35 & -1.12 & -23.94 \\
\hline 16 & 0.06 & 28.83 & -3.23 & -27.22 \\
\hline 17 & 0.06 & 32.37 & -3.39 & -30.57 \\
\hline 18 & 0.06 & 34.97 & -2.40 & -33.02 \\
\hline 19 & 0.06 & 30.56 & -3.39 & -52.41 \\
\hline 20 & 0.06 & 38.63 & -1.01 & $-3 j .42$ \\
\hline 22 & 0.06 & 42.29 & -2.51 & -39.93 \\
\hline 23 & 0.06 & $\$ 4.70$ & -2.26 & -42.21 \\
\hline 24 & 0.06 & 46.18 & -1.40 & -43.61 \\
\hline 26 & 0.06 & 50.97 & -4.54 & -48.15 \\
\hline 28 & 0.06 & 57.84 & -0.47 & -54.02 \\
\hline 30 & 0.06 & 61.38 & -3.34 & -57.90 \\
\hline 32 & $\dot{v} . \dot{v} 6$ & 56.40 & -4.74 & -62.70 \\
\hline 34 & 0.06 & 72.36 & -5.63 & -66.33 \\
\hline 36 & 0.06 & 77.15 & -4.52 & $-72 . B_{E}^{E}$ \\
\hline 39 & 0.06 & 85.25 & $-11.4 j$ & $-54.2 E$ \\
\hline 41 & $0.0 \dot{0}$ & 74.83 & -5.13 & -59.41 \\
\hline 43 & $4 .: 5$ & $102.6 !$ & -7.50 & -76.90 \\
\hline 45 & $0.3 \bar{j}$ & fis. 74 & $-\hat{s} .50$ & -105. if \\
\hline $4 \bar{i}$ & $1 . \dot{c o b}$ & 123.04 & -2.80 & $-1: 4.14$ \\
\hline 45 & 2.18 & 189.07 & -20.00 & $-175,2=$ \\
\hline 53 & 3.45 & $: 5: 17$ & -14.50 & - \\
\hline
\end{tabular}


TABLE C.10. contd

SAMPLE HO. EFFLUENT PORE VOL. ELUTED SUM ELUTED

\begin{tabular}{|c|c|c|c|c|}
\hline & ppe F & ELUTED & $\Leftrightarrow F$ & $9 \mathrm{~F}$ \\
\hline 1 & $22.0 \hat{0}$ & 1.33 & 1.94 & 1.94 \\
\hline 2 & 16.0 & 2.72 & 1.46 & 3.40 \\
\hline 3 & 12.0 & 4.15 & 1.11 & 4.51 \\
\hline 4 & 11.0 & 5.69 & 1.24 & 5.75 \\
\hline 5 & 11.0 & 6.92 & 0.73 & 6.48 \\
\hline 6 & 9.0 & 8.01 & 0.63 & 7.11 \\
\hline 7 & 8.0 & 9.67 & 0.84 & 7.95 \\
\hline 3 & B. 0 & 11.94 & 1.15 & 9.10 \\
\hline p & 7.0 & 13.74 & 0.79 & $\$ .90$ \\
\hline 10 & 0.2 & 15.78 & 0.79 & 10.69 \\
\hline 11 & 5.7 & 17.41 & 0.57 & 11.26 \\
\hline 12 & 5.3 & 19.68 & 0.74 & 12.00 \\
\hline 13 & 5.1 & 21.39 & 0.53 & 12.53 \\
\hline 14 & 4.7 & 34.16 & 0.79 & 13.32 \\
\hline 15 & 4.9 & 25.35 & 0.35 & 13.67 \\
\hline 16 & 4.5 & 28.83 & 0.94 & 14.62 \\
\hline i7 & 3.3 & 32.37 & 0.67 & 15.29 \\
\hline iB & 4.0 & 34.97 & 0.62 & 15.90 \\
\hline 19 & 5.5 & 38.56 & 1.22 & 17.12 \\
\hline 20 & 5.8 & 39.63 & 0.38 & 17.50 \\
\hline 22 & 4.6 & 42.29 & 0.74 & 18.24 \\
\hline 23 & 3.7 & 44.70 & 0.52 & 18.77 \\
\hline 24 & 4.1 & 46.18 & 0.36 & 19.13 \\
\hline 26 & 3.20 & 50.79 & 0.88 & 20.01 \\
\hline 28 & 3.30 & 57.84 & 1.21 & 21.21 \\
\hline 30 & 3.30 & 61.38 & 0.67 & $21.8 B$ \\
\hline 32 & 3.10 & 66.40 & 0.88 & 22.77 \\
\hline 34 & 2.60 & 72.36 & 0.85 & 23.62 \\
\hline 36 & 3.30 & 37.15 & 0.91 & 24.52 \\
\hline 37 & 3.10 & 89.25 & 2.13 & 26.66 \\
\hline 41 & 3.10 & 94.68 & 0.96 & 27.61 \\
\hline 43 & 2.70 & 302.07 & 1.19 & 38.80 \\
\hline 45 & 3.00 & 111.94 & 1.57 & 30.37 \\
\hline 47 & 4.20 & 123.04 & $2.7 E$ & 33.15 \\
\hline $4 ?$ & 3.60 & 149.07 & 4.41 & 37.50 \\
\hline 50 & 2.75 & 169.17 & 3.34 & 40.90 \\
\hline
\end{tabular}

SAKPLE NO. EFFLUENT PORE VOL. ELUTED SUK ELUTEU PPI NOJ ELUTED ag NOS A MOJ

\begin{tabular}{|c|c|c|c|c|}
\hline 1 & 36.0 & 1.33 & 2.64 & 3.64 \\
\hline 2 & 22.0 & 2.72 & 2.06 & 10.70 \\
\hline 3 & 13.0 & 4.15 & 1.25 & 11.95 \\
\hline 4 & 11.0 & 5.89 & 1.28 & 13.34 \\
\hline 5 & 11.0 & 0.92 & 0.76 & 14.00 \\
\hline 6 & 8.8 & 8.01 & 0.64 & 14.64 \\
\hline 7 & 3.0 & 9.67 & 0.33 & 14.97 \\
\hline 3 & 5.3 & 11.74 & 0.80 & 15.77 \\
\hline$p$ & 5.0 & 13.74 & 0.60 & 16.36 \\
\hline 10 & $4 . \hat{2}$ & $15.7 \mathrm{~B}$ & 0.57 & 16.93 \\
\hline 11 & 3.7 & 17.41 & 0.40 & 57.33 \\
\hline 12 & 3.4 & 19.68 & 0.51 & 17.93 \\
\hline 13 & 29.0 & 21.37 & 3.35 & 21.18 \\
\hline 14 & 12.0 & 24.16 & 2.23 & 23.41 \\
\hline 15 & 3.5 & 25.35 & 0.27 & 23.69 \\
\hline 16 & 3.5 & 28.53 & 0.30 & 24.49 \\
\hline 17. & 1.6 & 32.37 & 0.36 & 24.85 \\
\hline 18 & 2.0 & 34.97 & 0.33 & 25.18 \\
\hline 19 & 4.5 & 38.56 & 1.07 & 25.25 \\
\hline 20 & 6.8 & 39.63 & 0.45 & 26.74 \\
\hline 22 & 3.7 & 12.29 & 0.65 & 27.35 \\
\hline 23 & 2.3 & 44.70 & 0.36 & 27.75 \\
\hline 24 & 2.8 & 4o..18 & $\dot{0} .27$ & 23.02 \\
\hline 26 & 1.60 & 50.59 & 0.49 & 28.51 \\
\hline $2 B$ & 1.50 & 57.84 & 0.65 & 29.16 \\
\hline 30 & 1.30 & 61.38 & 0.93 & 29.59 \\
\hline 32 & 1.60 & 60.46 & $0.5 i$ & 30.10 \\
\hline 34 & 1.40 & 72.36 & 0.52 & 30.02 \\
\hline 30 & 2.30 & 77.15 & 0.71 & 31.34 \\
\hline 39 & 3.60 & 39.25 & 2.37 & 34.21 \\
\hline 41 & 5.30 & 94.58 & !. & 30.12 \\
\hline 4 & $5.3 \hat{0}$ & 102.67 & 2.81 & $3 E .53$ \\
\hline 45 & 4.40 & 111.84 & 2.70 & 41.63 \\
\hline$\$ i$ & 5.70 & 123.14 & 4.21 & 45.34 \\
\hline 49 & 0.50 & 195.07 & 31.71 & 96.55 \\
\hline 50 & 7.59 & 165.17. & 12.74 & 58.46 \\
\hline
\end{tabular}


TABLE C.10. contd

SAMPLE NO. EFFLUENT PORE YOL. ELUTED SUM ELUTED PPE HJEOJ ELUTED

$\begin{array}{rrrrr}1 & 29.97 & 1.33 & 2.67 & 2.67 \\ 2 & 13.16 & 2.72 & 1.21 & 3.88 \\ 3 & 8.00 & 4.15 & 0.74 & 4.62 \\ 4 & 6.86 & 5.89 & 0.77 & 5.39 \\ 5 & 3.00 & 6.32 & 0.53 & 5.92 \\ 6 & 4.84 & 8.01 & 0.33 & 6.25 \\ 7 & 4.90 & 3.67 & 0.51 & 6.77 \\ 8 & 4.60 & 11.94 & 0.66 & 7.42 \\ 9 & 2.99 & 13.74 & 0.32 & 7.74 \\ 10 & 2.58 & 15.78 & 0.31 & 8.05 \\ 11 & 2.24 & 17.41 & 0.21 & 3.26 \\ 12 & 1.89 & 19.66 & 0.24 & 8.50 \\ 13 & 1.72 & 21.39 & 0.16 & 3.66 \\ 14 & 1.77 & 24.16 & 0.27 & 8.93 \\ 15 & 1.60 & 25.35 & 0.10 & 9.03 \\ 16 & 1.75 & 28.33 & 0.33 & 9.36 \\ 17 & 1.05 & 32.37 & 0.17 & 9.53 \\ 18 & 1.18 & 34.97 & 0.15 & 9.68 \\ 19 & 1.66 & 38.56 & 0.32 & 10.00 \\ 20 & 1.79 & 39.63 & 0.11 & 10.11 \\ 22 & 1.72 & 42.29 & 0.25 & 10.36 \\ 23 & 1.18 & 44.70 & 0.14 & 10.49 \\ 24 & 1.27 & 46.18 & 0.09 & 10.59 \\ 26 & 0.97 & 50.99 & 0.21 & 10.79 \\ 23 & 0.85 & 57.84 & 0.24 & 11.03 \\ 30 & 0.96 & 61.38 & 0.15 & 11.18 \\ 32 & 0.36 & 66.40 & 0.21 & 11.39 \\ 34 & 0.79 & 72.36 & 0.18 & 11.57 \\ 36 & 0.89 & 77.15 & 0.18 & 11.75 \\ 39 & 0.77 & 89.25 & 0.35 & 12.10 \\ 41 & 0.75 & 94.36 & 0.15 & 12.25 \\ 43 & 0.71 & 102.67 & 0.20 & 12.45 \\ 45 & 0.78 & 111.74 & 0.28 & 12.75 \\ 47 & 0.67 & 123.04 & 0.25 & 12.53 \\ 45 & 0.78 & 149.07 & 0.78 & 13.75 \\ 50 & 6.77 & 167.17 & 0.58 & 14.34\end{array}$

SAMPLE NO. EFFLUENT PORE VDL. ELUTED SOUH ELUTED PPE SO4 ELUTED IQ SOA Ug SOA

\begin{tabular}{|c|c|c|c|c|}
\hline 1 & 880 & 1.33 & 71.53 & 71.53 \\
\hline 2 & 230 & 2.72 & 13.56 & 65.09 \\
\hline 3 & 175 & 4.15 & 8.62 & 93.71 \\
\hline 4 & 160 & 5.89 & 8.72 & 102.44 \\
\hline 5 & 150 & 6.92 & 4.47 & 106.90 \\
\hline 6 & 144 & 8.01 & 4.28 & 111.19 \\
\hline 7 & 137 & 6.67 & -5.73 & 116.92 \\
\hline 8 & 116 & 11.34 & $4.6 !$ & 121.53 \\
\hline ? & 110 & 13.74 & 2.33 & 124.96 \\
\hline 10 & 102 & 15.76 & 2.21 & $126 . c 7$ \\
\hline $1 !$ & 97 & 17.41 & 1.21 & 127.36 \\
\hline 12 & 93 & 19.68 & 1.08 & 128.96 \\
\hline 13 & 97 & 21.37 & 1.27 & 130.24 \\
\hline 14 & 96 & 24.16 & !.83 & $! 32.1 !$ \\
\hline 15 & 96 & 25.35 & 0.01 & 132.92 \\
\hline 16 & 98 & 28.63 & 2.83 & 135.75 \\
\hline 17 & 38 & 32.37 & 0.38 & 136.23 \\
\hline 18 & 32 & 34.97 & -0.70 & $135.5 \overline{2}$ \\
\hline 19 & 78 & 38.56 & 2.92 & i3̄. 44 \\
\hline 20 & 110 & 39.63 & 1.74 & $: 40.18$ \\
\hline 22 & 106 & 42.29 & 3.60 & 143.70 \\
\hline 23 & 91 & 44.70 & 0.82 & 144.60 \\
\hline 24 & 57 & 46.18 & 1.20 & 195.70 \\
\hline 26 & 86.00 & 50.59 & 0.60 & 145.70 \\
\hline 28 & 38.00 & 57.84 & 0.73 & 140.63 \\
\hline 30 & 80.00 & 61.36 & $0.0 \hat{v}$ & 140.63 \\
\hline 32 & $7 \% . \$ 0$ & 66.40 & -2.36 & 144.25 \\
\hline 34 & 72.00 & 72.36 & -5.65 & ijo.jo \\
\hline 36 & 61.00 & 77.15 & -1.62 & 136.78 \\
\hline 39 & 79.60 & 59.25 & -5.74 & 131.2: \\
\hline 41 & $7 \pi .00$ & 94.38 & $-3.3 i$ & 127.93 \\
\hline 43 & 76.00 & 102.67 & -5.41 & 122.52 \\
\hline 45 & 30.60 & iilas4 & -3.77 & 113.75 \\
\hline 47 & 72.00 & 120.04 & -16.53 & 168.22 \\
\hline 47 & 30.60 & $145.6 ?$ & $-: 0153$ & 37.04 \\
\hline 50 & 30.00 & 169.17 & $-\overline{8} .80$ & Bi. \\
\hline
\end{tabular}


TABLE C.11. Mass Balance for Flow Through Leach Test on Whole Grout/Sediment with Groundwater (MBCOLC1)

SAKPLE NO. EFFLUENT PORE VOL. ELUTED SUH ELUTED ppa Ca ELUTED a $\mathrm{Ca}$ ag Ca

$\begin{array}{ccccc}1 & 10.2 & 1.76 & -5.26 & -5.26 \\ 2 & 3.0 & 3.94 & -7.39 & -12.66 \\ 3 & 3.1 & 6.52 & -8.81 & -21.47 \\ 4 & 2.4 & 9.80 & -11.35 & -32.82 \\ 5 & 2.0 & 10.78 & -3.42 & -36.24 \\ 6 & 1.9 & 11.99 & -4.23 & -40.47 \\ 7 & 1.7 & 13.53 & -5.40 & -45.87 \\ 8 & 1.8 & 15.73 & -7.70 & -53.57 \\ 7 & 1.6 & 17.31 & -5.55 & -59.11 \\ 10 & 1.4 & 19.79 & -8.75 & -67.86 \\ 11 & 1.3 & 21.84 & -7.24 & -75.10 \\ 12 & 1.2 & 24.34 & -8.84 & -83.95 \\ 13 & 1.1 & 26.20 & -6.60 & -90.55 \\ 14 & 1.0 & 28.68 & -8.32 & -99.36 \\ 15 & 1.3 & 30.69 & -7.10 & -106.46 \\ 16 & 1.2 & 33.67 & -10.55 & -117.01 \\ 17 & 1.0 & 38.00 & -15.38 & -132.39 \\ 18 & 0.9 & 41.07 & -10.93 & -143.32 \\ 17 & 0.8 & 45.41 & -15.46 & -158.77 \\ 20 & 0.9 & 47.79 & -8.46 & -167.23 \\ 21 & 1.1 & 48.63 & -2.98 & -170.21 \\ 22 & 1.0 & 49.54 & -3.23 & -173.44 \\ 23 & 1.1 & 51.04 & -5.31 & -178.76 \\ 24 & 1.5 & 53.00 & -5.90 & -185.65\end{array}$

SAMPLE NO. EFFLUENT PDRE VOL. ELUTED SUN ELITED PPE $\mathrm{Kz}$ ELUTED $q \mathrm{Nz}$ ig $\mathrm{Na}$

\begin{tabular}{|c|c|c|c|c|}
\hline 1 & 1120 & 1.78 & 125.85 & 125.65 \\
\hline 2 & 940 & 3.94 & 127.62 & 253.47 \\
\hline 3 & 940 & 6.52 & 152.43 & 405.90 \\
\hline 4 & 610 & 9.80 & 123.90 & 529.80 \\
\hline 5 & 530 & 10.78 & 31.96 & 561.75 \\
\hline 6 & 540 & 11.99 & 40.24 & 601.79 \\
\hline 7 & 524 & 13.53 & -49.62 & $651.6 \mathrm{i}$ \\
\hline 8 & 470 & 15.73 & $63.2 !$ & 714.82 \\
\hline 9 & 490 & 17.31 & 47.44 & 762.26 \\
\hline 10 & 362 & 19.79 & 53.97 & 815.23 \\
\hline 11 & 327 & 21.84 & 39.95 & 356.20 \\
\hline 12 & 297 & 24.34 & 43.91 & 900.11 \\
\hline 13 & 300 & 26.20 & 33.03 & 933.14 \\
\hline 14 & 282 & 28.65 & 41.15 & 974.29 \\
\hline 15 & 284 & $30.5 ?$ & :3.61 & 1007.81 \\
\hline 16 & $30 B$ & 33.67 & 54.45 & 1062.36 \\
\hline$j 7$ & 222 & 38.00 & 55.08 & 1117.44 \\
\hline 18 & 168 & 41.07 & 28.35 & 1145.79 \\
\hline iq & 167 & 45.41 & 39.79 & 1125.58 \\
\hline 20 & 151 & 47.79 & 19.36 & 1204.54 \\
\hline $2 i$ & 151 & 48.63 & 6.83 & 1211.70 \\
\hline 22 & 165 & 49.54 & 8.23 & 1220.00 \\
\hline 23 & 199 & 51.04 & 16.85 & 1236.86 \\
\hline 24 & 226 & 53.00 & 25.44 & $i 262.29$ \\
\hline
\end{tabular}


TABLE C.11. contd

SAMPLE NO. EFFLUENT PORE VOL. ELUTED SUR ELUTED

\begin{tabular}{ccccc} 
& DPE K & ELUTED & $19 K$ & $19 K$ \\
\hline 1 & 2.4 & 1.78 & -0.62 & -0.62 \\
2 & 0.3 & 3.94 & -1.05 & -1.67 \\
3 & 0.3 & 6.52 & -1.25 & -2.92 \\
4 & 0.3 & 9.80 & -1.59 & -4.50 \\
5 & 0.3 & 10.78 & -0.47 & -4.98 \\
6 & 2.0 & 11.99 & -0.45 & -5.43 \\
7 & 2.6 & 13.53 & -0.52 & -5.95 \\
8 & 1.8 & 15.73 & -0.85 & -6.80 \\
9 & 1.3 & 17.31 & -0.66 & -7.46 \\
10 & 1.8 & 19.79 & -0.96 & -8.43 \\
11 & 1.9 & 21.84 & -0.78 & -9.21 \\
12 & 1.7 & 24.34 & -0.98 & -10.19 \\
13 & 1.0 & 26.20 & -0.32 & -11.01 \\
14 & 0.9 & 28.68 & -1.11 & -12.12 \\
15 & 10.3 & 30.69 & -1.01 & -13.13 \\
16 & 2.5 & 33.67 & -1.02 & -14.15 \\
17 & 2.2 & 38.00 & -1.57 & -15.72 \\
18 & 1.3 & 41.07 & -1.29 & -17.01 \\
19 & 2.4 & 45.41 & -1.51 & -18.52 \\
20 & 1.7 & 47.79 & -0.34 & -19.46 \\
21 & 1.6 & 48.63 & -0.34 & -19.79 \\
22 & 0.9 & 49.54 & -0.41 & -20.20 \\
23 & 2.2 & 51.04 & -0.54 & -20.74 \\
24 & 3.7 & 53.00 & -0.52 & -21.26 \\
& & & &
\end{tabular}

SAHPLE NO. EFFLUENT FORE VOL. ELUTED SUH ELUTED

\begin{tabular}{|c|c|c|c|c|}
\hline & PPE Âl & ELUTED & $\Delta \mathrm{All}$ & $\| \stackrel{A l}{A l}$ \\
\hline 1 & 4.72 & 1.76 & 0.53 & 0.53 \\
\hline 2 & 5.60 & 3.94 & 0.79 & 1.33 \\
\hline 3 & 3.40 & 6.52 & 1.38 & 2.71 \\
\hline 4 & 7.80 & 7.80 & 1.63 & 4.34 \\
\hline 5 & 6.50 & 10.78 & 0.40 & 4.74 \\
\hline$\dot{c}$ & 7.70 & 11.99 & 0.55 & 5.34 \\
\hline 7 & 8.84 & 13.53 & 0.37 & 0.21 \\
\hline$B$ & 9.80 & $: 5.73$ & i.j8 & 7.59 \\
\hline 9 & 9.48 & 17.31 & 0.96 & 3.54 \\
\hline 10 & 9.19 & 17.79 & 1.40 & 10.00 \\
\hline 11 & 8.73 & 21.34 & 1.14 & 11.14 \\
\hline 12 & 8.23 & 24.34 & 1.31 & 12.45 \\
\hline 13 & 7.70 & 26.20 & 0.71 & 13.37 \\
\hline 14 & 6.76 & 26.66 & 1.07 & 14.43 \\
\hline 15 & 7.16 & 30.69 & 0.92 & 15.35 \\
\hline 16 & $6.8 i$ & 33.67 & 1.30 & 10.65 \\
\hline 17 & 5.45 & 38.000 & 1.50 & $18 .: 5$ \\
\hline 18 & 3.91 & 41.07 & 6.76 & i6.9! \\
\hline 19 & 3.56 & 45.41 & 0.37 & 19.37 \\
\hline 20 & 3.16 & 47,79 & 0.47 & 20.34 \\
\hline 21 & 2.71 & 48.63 & 0.14 & 20.99 \\
\hline 22 & 2.21 & 49.54 & 0.12 & 20.61 \\
\hline 23 & 2.46 & 51.04 & 0.23 & 20.84 \\
\hline 24 & 3.28 & 53.60 & 0.40 & 21.24 \\
\hline
\end{tabular}


TABLE C.11. contd

SAMPLE NO. EFFLUENT PORE VDL. ELUTED SUK ELUTED

$$
\text { PDE Si ELUTED } \quad \mathrm{Si} \quad \mathrm{Si}
$$

$\begin{array}{rrrrr}1 & 158.0 & 1.78 & 16.44 & 16.44 \\ 2 & 160.0 & 3.94 & 20.22 & 36.66 \\ 3 & 135.0 & 6.52 & 19.99 & 56.65 \\ 4 & 52.0 & 9.80 & 14.19 & 70.84 \\ 5 & 80.0 & 10.78 & 4.11 & 74.95 \\ 6 & 87.2 & 11.99 & 5.64 & 80.59 \\ 7 & 76.2 & 13.53 & 6.09 & 86.68 \\ 8 & 72.0 & 15.73 & 8.10 & 94.78 \\ 9 & 65.5 & 17.31 & 5.15 & 99.93 \\ 10 & 56.2 & 19.79 & 6.60 & 106.53 \\ 11 & 49.8 & 21.34 & 4.61 & 111.13 \\ 12 & 46.6 & 24.34 & 5.10 & 116.23 \\ 13 & 43.6 & 26.20 & 3.43 & 119.67 \\ 14 & 41.8 & 28.68 & 4.29 & 123.96 \\ 15 & 39.1 & 30.69 & 3.13 & 127.09 \\ 16 & 42.7 & 33.67 & 5.33 & 132.42 \\ 17 & 23.8 & 38.00 & 2.46 & 134.86 \\ 13 & 18.1 & 41.07 & 0.61 & 135.49 \\ 19 & 30.2 & 45.41 & 1.46 & 136.95 \\ 20 & 19.3 & 47.79 & 0.66 & 137.61 \\ 21 & 20.6 & 48.63 & 0.30 & 137.71 \\ 22 & 24.6 & 49.54 & 0.56 & 138.48 \\ 23 & 33.6 & 51.04 & 1.80 & 140.28 \\ 24 & 36.9 & 53.00 & 2.77 & 143.05\end{array}$

SAMPLE ND. EFFLUEHT PORE YOL. ELUTED SUM ELUTED

$$
\text { PPS MI ELUTED }
$$

ig $\mathrm{Kg}$

\begin{tabular}{|c|c|c|c|c|}
\hline 1 & 0.06 & 1.76 & -1.60 & -1.60 \\
\hline 2 & 0.06 & 3.94 & -1.94 & -3.55 \\
\hline 3 & 0.06 & 6.52 & -2.32 & $-5 . \overline{8} \overline{7}$ \\
\hline 4 & 0,06 & 9.80 & -2.95 & -8.82 \\
\hline 5 & 0.06 & 10.78 & -0.38 & -9.70 \\
\hline 6 & 0.06 & 11.99 & -1.69 & -10.79 \\
\hline 7 & 0.06 & 13.53 & -1.39 & $-12 .: 8$ \\
\hline 8 & 0.06 & 15.73 & -1.98 & -14.16 \\
\hline 9 & 0.06 & 17.31 & -1.42 & -15.50 \\
\hline 10 & 0.06 & 17.79 & -2.23 & -17.01 \\
\hline 11 & 0.06 & 21.34 & -1.85 & -19.66 \\
\hline 12 & 0.06 & 24.34 & -2.25 & -21.31 \\
\hline 13 & 0.06 & 26.20 & $-1.8 \bar{j}$ & -23.58 \\
\hline 14 & 0.06 & 28.66 & -2.25 & -25.22 \\
\hline 15 & 0.66 & 30.69 & -1.81 & -37.62 \\
\hline ib & 0.06 & 33.67 & -2.68 & -30.31 \\
\hline 17 & 0.06 & 38.60 & -3.90 & -34.20 \\
\hline 18 & 0.06 & 41.67 & -2.76 & -36.17 \\
\hline 19 & 0.06 & 45.41 & $-3.9 !$ & -40.87 \\
\hline 20 & 0.06 & 47.39 & -2.14 & -43.02 \\
\hline 21 & 0.06 & 48.63 & -6.76 & -43.77 \\
\hline 22 & 0.06 & 49.54 & -0.82 & -44.57 \\
\hline 23 & 0.06 & 51.04 & -1.35 & -45.74 \\
\hline 24 & 0.06 & 53.00 & -1.76 & $-4 i .71$ \\
\hline
\end{tabular}

og No 
TABLE C.11. contd

SAMPLE NG. EFFLUERT PORE VOL. ELLTED SUM ELUTED

\begin{tabular}{|c|c|c|c|c|}
\hline & pas & ELUTED & $90 \bar{r}$ & $f$ \\
\hline 1 & 15.0 & 1.79 & 1.67 & 1.67 \\
\hline 2 & 9.0 & 3.34 & 1.27 & 2.34 \\
\hline 3 & $a .2$ & 6.52 & 1.26 & 4.22 \\
\hline 4 & 5.5 & 9.80 & 1.06 & 5.28 \\
\hline 5 & 6.0 & 16.78 & 0.35 & 5.63 \\
\hline b & 7.0 & 11.99 & $0.5 !$ & 6.13 \\
\hline 7 & 0.0 & 13.53 & 0.55 & 6.68 \\
\hline 8 & 6.0 & 15.73 & 0.78 & 7.46 \\
\hline$\xi$ & 6.0 & 17.31 & 0.56 & 8.02 \\
\hline 90 & 4.5 & 19.79 & 0.69 & 8.71 \\
\hline 11 & 4.7 & 21.34 & $0.5 \dot{6}$ & 9.27 \\
\hline 12 & 4.7 & 24.34 & 0.68 & 9.55 \\
\hline 13 & 4.8 & 26.20 & 0.52 & 10.46 \\
\hline 14 & 4.8 & 28.68 & 0.69 & 11.15 \\
\hline 15 & 4.7 & 30.69 & 0.55 & 11.70 \\
\hline 16 & 5.2 & 33.67 & 0.70 & 12.00 \\
\hline 17 & 3.0 & 38.00 & 0.70 & 13.30 \\
\hline 16 & 4.1 & 41.07 & 0.71 & 14.01 \\
\hline 19 & 4.5 & 45.41 & 1.12 & 15.13 \\
\hline 20 & 4.8 & 47.79 & 0.66 & 15.79 \\
\hline 21 & 5.5 & 46.63 & 0.27 & 16.07 \\
\hline 22 & 6.7 & 49.54 & 0.36 & 16.43 \\
\hline 23 & 7.0 & 51.04 & 6.63 & 17. vo \\
\hline 24 & 3. 7 & 53.00 & 0.06 & 17.32 \\
\hline
\end{tabular}

SAMPLE NO. EFFLUEAT POKE YDL. ELUTED SUK ELUTED PPS NOS ELUTED GO NOJ NOS

\begin{tabular}{|c|c|c|c|c|}
\hline 1 & 42.0 & 1.78 & 4.22 & 4.82 \\
\hline 2 & 15.0 & 3.94 & 2.08 & 6.89 \\
\hline 3 & 10.0 & 6.52 & 1.65 & 8.54 \\
\hline 4 & 5.0 & 9.60 & 1.04 & 5.58 \\
\hline 5 & 7.5 & 10.78 & 0.47 & 10.05 \\
\hline$\dot{0}$ & 8.0 & 11.79 & 0.62 & 10.67 \\
\hline 7 & 9.0 & 13.53 & 0.88 & 21.55 \\
\hline 8 & 4.0 & 15.73 & 0.55 & $12 . ! !$ \\
\hline 9 & 6.0 & 13.31 & y.óv & 12.71 \\
\hline 10 & 3.6 & 19.79 & 0.56 & 13.27 \\
\hline 11 & 3.4 & 21.84 & 0.44 & 13.70 \\
\hline 12 & 3.2 & 24.34 & 0.50 & 14.21 \\
\hline 13 & 4.3 & 25.20 & 3.50 & 14.71 \\
\hline 14 & 3.7 & 28.88 & 0.53 & 15.29 \\
\hline 15 & 3.4 & 30.67 & 0.43 & $15.7 \mathrm{i}$ \\
\hline 16 & 3.3 & 33.67 & 0.62 & 16.33 \\
\hline 17 & 1.2 & 38.00 & 0.31 & 16.64 \\
\hline 18 & 1.5 & 41.07 & 0.26 & 16.92 \\
\hline 19 & 1.3 & 45.41 & 0.48 & 17.37 \\
\hline 20 & 1.9 & 47.79 & 0.23 & $i \bar{i} .6 \bar{T}$ \\
\hline 21 & 3.3 & 48.63 & 0.17 & 17.84 \\
\hline 22 & 4.4 & 49.54 & 0.25 & 18.09 \\
\hline 23 & 4.4 & 51.64 & $\hat{1}, 4 \hat{2}$ & $16.5 \mathrm{i}$ \\
\hline 24 & 4.6 & 53.00 & 0.57 & $19.60^{\circ}$ \\
\hline
\end{tabular}




\section{TABLE C.11. contd}

SAHFLE ND. EFFLUERT PORE VOL. EZUTED SUM ELUTED

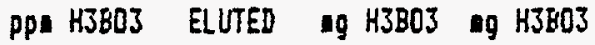

$\begin{array}{lllll}1 & 9.49 & 1.78 & 1.05 & 1.05 \\ 2 & 6.29 & 3.94 & 0.83 & 1.88 \\ 3 & 4.28 & 6.52 & 0.66 & 2.54 \\ 4 & 2.23 & 9.80 & 0.40 & 2.94 \\ 5 & 2.74 & 10.78 & 0.15 & 3.09 \\ 6 & 3.22 & 11.99 & 0.23 & 3.32 \\ 7 & 2.54 & 13.53 & 0.22 & 3.53 \\ 6 & 2.05 & 15.73 & 0.24 & 3.78 \\ 9 & 1.33 & 17.31 & 0.16 & 3.34 \\ 10 & 1.61 & 19.79 & 0.20 & 4.14 \\ 11 & 1.47 & 21.84 & 0.15 & 4.29 \\ 12 & 1.61 & 24.34 & 0.21 & 4.50 \\ 13 & 1.52 & 26.20 & 0.14 & 4.64 \\ 14 & 1.42 & 28.68 & 0.17 & 4.81 \\ 15 & 1.37 & 30.69 & 0.13 & 4.95 \\ 16 & 1.55 & 33.67 & 0.23 & 5.18 \\ 17 & 0.77 & 38.00 & 0.12 & 5.30 \\ 18 & 0.78 & 41.07 & 0.09 & 5.37 \\ 19 & 0.96 & 45.41 & 0.17 & 5.56 \\ 26 & 0.97 & 47.79 & 0.10 & 5.66 \\ 21 & 1.17 & 48.63 & 0.05 & 5.70 \\ 22 & 1.62 & 49.54 & 0.08 & 5.78 \\ 23 & 2.05 & 51.04 & 0.17 & 5.94 \\ 24 & 1.66 & 53.00 & 0.17 & 6.11\end{array}$

SAKPLE NO. EFFLUENT PORE VOL. ELUTED SUK ELUTED ppe SO4 ELUTED IO SO4 SO4

$\begin{array}{rrrrr}1 & 380 & 1.78 & 33.79 & 33.79 \\ 2 & 140 & 3.94 & 7.53 & 41.32 \\ 3 & 125 & 6.52 & 6.50 & 47.62 \\ 4 & 100 & 7.80 & 2.97 & 50.78 \\ 5 & 110 & 10.78 & 1.52 & 52.30 \\ 6 & 126 & 11.59 & 3.13 & 55.43 \\ 7 & 109 & 13.53 & 2.29 & 57.32 \\ 8 & 103 & 15.73 & 2.41 & 60.13 \\ 9 & 120 & 17.31 & 3.37 & 63.60 \\ 10 & 93 & 19.79 & 1.12 & 64.72 \\ 11 & 91 & 21.84 & 0.66 & 65.33 \\ 12 & 88 & 24.34 & 0.32 & 65.70 \\ 13 & 94 & 26.20 & 0.76 & 66.67 \\ 14 & 97 & 28.86 & 1.76 & 63.43 \\ 15 & 91 & 30.69 & 0.65 & 69.08 \\ 16 & 98 & 33.67 & 3.31 & 71.33 \\ 17 & 63 & 38.60 & -6.43 & 64.75 \\ 18 & 78 & 41.67 & -1.59 & 63.37 \\ 19 & 81 & 45.41 & -1.40 & 61.97 \\ 20 & 95 & 47.79 & 1.38 & 63.35 \\ 21 & 91 & 46.63 & 0.27 & 63.52 \\ 22 & 115 & 49.54 & 1.70 & 65.33 \\ 23 & 135 & 51.04 & 4.75 & 70.07 \\ 24 & 100 & 55.60 & 1.77 & 71.84\end{array}$


TABLE C.11. contd

SAMPLE NO. EFFLUERT PORE YOL. ELUTED SUH ELUTED PPE IC ELUTED $A$ IC 9 IC

$\begin{array}{rrrrr}1 & 43.4 & 1.78 & 1.07 & 1.07 \\ 2 & 45.3 & 3.94 & 1.56 & 2.63 \\ 3 & 40.7 & 1.52 & 1.10 & 3.73 \\ 4 & 27.1 & 9.80 & -1.49 & 2.24 \\ 5 & 54.1 & 10.78 & 1.26 & 3.50 \\ 6 & 23.0 & 11.99 & -0.87 & 2.64 \\ 7 & 23.1 & 13.53 & -1.09 & 1.54 \\ 3 & 31.4 & 15.73 & -0.38 & 1.16 \\ 7 & 28.3 & 17.31 & -0.60 & 0.57 \\ 10 & 33.6 & 19.79 & -0.07 & 0.49 \\ 11 & 26.4 & 21.84 & -1.02 & -0.53 \\ 12 & 25.6 & 24.34 & -1.37 & -1.90 \\ 13 & 31.7 & 26.20 & -0.29 & -2.19 \\ 14 & 27.0 & 29.68 & -1.14 & -3.32 \\ 15 & 27.7 & 30.69 & -0.83 & -4.15 \\ 16 & 35.1 & 33.67 & 0.19 & -3.96 \\ 17 & 26.1 & 38.60 & -2.23 & -6.19 \\ 18 & 45.4 & 41.07 & 2.24 & -3.95 \\ 19 & 21.5 & 45.41 & -3.53 & -7.46 \\ 20 & 21.7 & 47.79 & -1.91 & -9.39 \\ 21 & 33.9 & 48.63 & -0.01 & -9.41 \\ 22 & 28.2 & 49.54 & -0.35 & -9.75 \\ 23 & 44.5 & 51.04 & 1.01 & -8.75 \\ 24 & 62.2 & 53.06 & 3.56 & -5.19\end{array}$

SAMPLE ND. EFFLUEMT PORE VOL. ELUTED SUM ELUTED PP TOC ELUTED I TOC IIOC

\begin{tabular}{|c|c|c|c|c|}
\hline 1 & 15.8 & 1.78 & 1.70 & 1.70 \\
\hline 2 & 9.4 & 3.94 & 1.18 & 2.88 \\
\hline 3 & 6.3 & 6.52 & 0.96 & 3.34 \\
\hline 4 & 3.7 & 9.80 & 0.57 & 4.41 \\
\hline 5 & 6.5 & 10.78 & 0.35 & 4.76 \\
\hline 6 & 4.3 & 11.99 & 0.26 & 5.01 \\
\hline 7 & 3.0 & 13.53 & 0.20 & 5.21 \\
\hline 3 & 2.5 & 15.73 & 0.21 & 5.42 \\
\hline 9 & 3.0 & $\{7.31$ & 3.20 & 3.62 \\
\hline 10 & 2.4 & :9.7? & 0.23 & 5.85 \\
\hline 11 & 2.3 & 21.84 & 0.17 & 0.02 \\
\hline 12 & 2.5 & 24.34 & 0.24 & 0.26 \\
\hline 13 & 3.0 & 26.20 & 0.24 & 6.50 \\
\hline 14 & 2.4 & 23.65 & 0.22 & 6.71 \\
\hline 15 & 2.5 & 30.69 & 4.19 & 6.90 \\
\hline 16 & 2.4 & 33.67 & 0.27 & 7.17 \\
\hline 17 & 1.3 & 38.10 & 0.07 & 7.26 \\
\hline 18 & 1.2 & 41.07 & 0.05 & 7.31 \\
\hline 19 & 1.3 & 45.91 & 0.68 & 7.39 \\
\hline 20 & 1.4 & 47.79 & 3.66 & 7.45 \\
\hline 21 & 2.5 & 48.63 & 0.08 & 7.53 \\
\hline 22 & 3.4 & 49.54 & 0.14 & 7.67 \\
\hline 23 & 3.3 & 51.04 & 0.23 & 7.70 \\
\hline 24 & 2.9 & 53.00 & 0.24 & 5.14 \\
\hline
\end{tabular}


TABLE C.12. Mass Balance for Flow Through Leach Test on Whole Grout with Groundwater (MBCOLLT3)

SAMPLE NO. EFFLUEHT PORE VOL. ELUTED SUA ELUTED

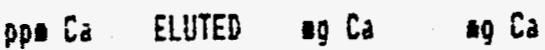

$\begin{array}{rrrrr}1 & 1.6 & 1.06 & -5.42 & -5.42 \\ 1 & 1.3 & 1.70 & -3.29 & -8.72 \\ 3 & 3.05 & 3.18 & -7.37 & -16.08 \\ 4 & 3.92 & 6.60 & -16.75 & -32.83 \\ 5 & 4.8 & 9.94 & -16.07 & -48.90 \\ 6 & 1.3 & 12.36 & -12.45 & -61.35 \\ 7 & 1.4 & 13.93 & -8.06 & -69.41 \\ 9 & 1.4 & 16.95 & -25.78 & -95.19 \\ 11 & 2.0 & 24.89 & -30.16 & -125.36 \\ 13 & 1.1 & 30.12 & -28.54 & -153.90 \\ 15 & 1.8 & 35.89 & -27.36 & -181.77 \\ 17 & 1.1 & 38.91 & -15.59 & -197.36 \\ 19 & 0.7 & 42.29 & -17.51 & -214.87 \\ 22 & 1.7 & 47.29 & -25.54 & -240.41 \\ 24 & 0.8 & 48.65 & -7.06 & -247.46 \\ 26 & 1.7 & 54.58 & -30.26 & -277.72 \\ 26 & 4.7 & 64.77 & -49.17 & -326.89 \\ 30 & 6.6 & 73.33 & -39.73 & -366.62 \\ 32 & 17.4 & 39.62 & -59.12 & -425.73 \\ 33 & 26.40 & 104.20 & -40.58 & -466.31\end{array}$

SAMPLE KE. EFFLLENT FORE YOL. ELUTED SUK ELUTED pp: $k$ EIUTED in $\mathrm{X}$ $\lg k$

\begin{tabular}{|c|c|c|c|c|}
\hline 1 & 38.0 & 1.06 & 3.01 & 3.01 \\
\hline 2 & 61.0 & 1.70 & 3.20 & 6.21 \\
\hline 3 & 62.50 & 3.18 & 7.61 & 13.82 \\
\hline 4 & 63.25 & 6.60 & 17.85 & 31.65 \\
\hline 5 & 64.0 & 9.54 & 17.65 & 49.30 \\
\hline 6 & 24.0 & 12.36 & 3.65 & $5 \% .97$ \\
\hline$?$ & 16.4 & 13.93 & 1.27 & 54.26 \\
\hline 9 & 15.2 & 18.75 & 3.49 & 57.75 \\
\hline 11 & 16.2 & 24.39 & 5.81 & 63.56 \\
\hline 13 & 9.9 & 30.42 & 1.69 & 64.65 \\
\hline 15 & 9.9 & 35.69 & 1.08 & 65.73 \\
\hline 17 & 10.0 & 33.91 & 0.62 & 66.35 \\
\hline 19 & 9.8 & 42,27 & 0.64 & 66.39 \\
\hline 22 & 14.8 & 47.29 & 2.91 & 59.70 \\
\hline 24 & 13.6 & 48.65 & 0.50 & 70.57 \\
\hline 26 & $(2,0$ & 54,59 & 2.34 & 72.71 \\
\hline 28 & $10 . \dot{0}$ & 64.77 & 2.11 & 75.02 \\
\hline 30 & 3.4 & 73.303 & 0.46 & 75.50 \\
\hline 32 & 3.4 & 89.62 & 0.72 & 70.42 \\
\hline 33 & 7.60 & 164.20 & -1.10 & 75.32 \\
\hline
\end{tabular}

SAKFLE HO. EFFLUENT PORE VOL. ELUTED SLM ELUTED

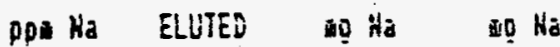

\begin{tabular}{|c|c|c|c|c|}
\hline 1 & 827 & 1.06 & 79.53 & 79.33 \\
\hline 2 & 1380 & 1.70 & 31.53 & 161.46 \\
\hline 3 & 1420 & 3.18 & $194.1 i$ & 355.50 \\
\hline 4 & 1440 & 6.60 & $454.7 ?$ & 810.57 \\
\hline 5 & 1460 & 9.94 & 450.63 & 1201.20 \\
\hline$b$ & 467 & 12.36 & 101.02 & 1362.22 \\
\hline$j$ & 275 & 13.85 & ت0. 80 & 1379.12 \\
\hline 9 & $24 !$ & 18.95 & 201.75 & 1501.07? \\
\hline if & 324 & 24.69 & 166.89 & 16000.45 \\
\hline 13 & 132 & 30.42 & $5 \overline{3} .63$ & 1725.59 \\
\hline 35 & 121 & 35.89 & $47.3 \hat{i}$ & 9773.06 \\
\hline 17 & 155 & 38.51 & $36.7 j$ & $i 209.9 \bar{i}$ \\
\hline 17 & 133 & 42.29 & 34.60 & 1843.97 \\
\hline 22 & 175 & 47.29 & 70.52 & $\$ 914.49$ \\
\hline 24 & 165 & $\$ 6.65$ & 17.90 & 1932.39 \\
\hline 26 & 123 & 54.38 & 54.54 & 1927.03 \\
\hline 28 & 79 & 64.77 & 51.35 & 2030.38 \\
\hline 30 & 61 & 73.33 & 20.3 & 2057.52 \\
\hline 32 & 46 & 89.82 & 32.6 & 2699.08 \\
\hline 33 & 40.10 & 164.20 & 20.70 & 2120.38 \\
\hline
\end{tabular}

SAMPLE NO. EFFLUET POAE VAL. ELUTES SIM ELUTED PPE Al ELUTED AQ Ai AQ Al

\begin{tabular}{|c|c|c|c|c|}
\hline 1 & 12.30 & 1.06 & 1.26 & 1.20 \\
\hline 2 & 22.00 & 1.70 & 1.32 & 2.57 \\
\hline 3 & 12.17 & 3.18 & 1.68 & 4.25 \\
\hline 4 & 7.25 & 0.00 & 2.30 & 6.55 \\
\hline 5 & 2.34 & 9.94 & 9.70 & 7.26 \\
\hline 6 & 6.05 & 12.36 & 1.36 & 3.02 \\
\hline 7 & 3.38 & 13.53 & $0.4 \overline{0}$ & 9.10 \\
\hline ? & 2.88 & 16.95 & !.3! & $10.4 !$ \\
\hline 11 & 4.23 & 24.94 & 2.31 & 12.72 \\
\hline 13 & 0.32 & 36,72 & b. 11 & 12.03 \\
\hline ij & 0.06 & 35.59 & -4.62 & 22.81 \\
\hline 17 & 1.01 & 38.71 & 0.28 & $i 3 . \hat{v} \bar{j}$ \\
\hline 19 & 0.56 & 42.27 & 0.24 & 13.31 \\
\hline 22 & 1.35 & 9.29 & i. 5 & 13.719 \\
\hline 24 & $\{.22$ & 42.85 & i. : 4 & 14.84 \\
\hline 26 & i. 52 & 54.56 & 6.23 & 14.28 \\
\hline 28 & 6.63 & 64.77 & $-10.3 j$ & 14.21 \\
\hline 30 & 0.03 & 73.33 & $-2,6$ & 14.15 \\
\hline 32 & $0.13 j$ & 39.22 & -1.11 & 14.65 \\
\hline $3 j$ & 0.03 & 104.20 & -9.86 & 13,5 \\
\hline
\end{tabular}


TABLE C.12. contd

SAKPLE NO. EFFLUENT PORE VOL. ELUTED SUK ELUTED pp $\mathrm{Si}$ ELUTED $\mathrm{Si}$ $\mathrm{Si}$

$\begin{array}{rrrrr}1 & 13.2 & 1.06 & -0.18 & -0.18 \\ 2 & 16.7 & 1.70 & 0.10 & -0.08 \\ 3 & 16.35 & 3.18 & 0.19 & 0.11 \\ 4 & 16.17 & 6.60 & 0.38 & 0.49 \\ 5 & 16.0 & 9.94 & 0.31 & 0.80 \\ 6 & 10.4 & 12.36 & -1.05 & -0.25 \\ 7 & 12.2 & 13.93 & -0.41 & -0.66 \\ 9 & 11.3 & 18.95 & -1.75 & -2.41 \\ 11 & 12.9 & 24.89 & -1.17 & -3.58 \\ 13 & 9.7 & 30.42 & -2.76 & -6.34 \\ 15 & 9.6 & 35.89 & -2.77 & -9.11 \\ 17 & 12.4 & 38.91 & -0.74 & -9.85 \\ 19 & 10.0 & 42.29 & -1.59 & -11.44 \\ 22 & 12.6 & 47.29 & -1.13 & -12.57 \\ 24 & 11.4 & 48.65 & -0.46 & -13.03 \\ 26 & 9.3 & 54.59 & -3.17 & -16.19 \\ 28 & 8.5 & 64.77 & -6.24 & -22.43 \\ 30 & 9.3 & 73.33 & -4.57 & -27.02 \\ 32 & 13.4 & 89.62 & -2.45 & -29.47 \\ 33 & 14.90 & 104.20 & -0.14 & -29.61\end{array}$

SAMFIE HO. EFFLUENT PORE VOL. ELUTED SUS ELUTED

\begin{tabular}{|c|c|c|c|c|}
\hline & pDEF F & ELUTED & $\lg \mathrm{F}$ & $9 \mathrm{~F}$ \\
\hline I & 12.7 & 1.06 & 1.22 & 1.22 \\
\hline 2 & 21.0 & 1.70 & i.23 & 2.45 \\
\hline$j$ & 13.00 & 3.18 & 2.44 & 4.88 \\
\hline 4 & 16.50 & 6.60 & 5.14 & 10.03 \\
\hline 5 & 15.0 & 9.94 & 4.55 & .14 .50 \\
\hline 6 & 0.0 & 12.36 & 1.25 & $: 5.83$ \\
\hline 7 & 0.2 & 13.33 & 0.34 & 16.68 \\
\hline 9 & 5.6 & 15.75 & 2.11 & 19.06 \\
\hline 11 & $i .0$ & 24.89 & 3.63 & $22, \pi 1$ \\
\hline 13 & 5.0 & 30,42 & 2.34 & 25.05 \\
\hline$\vdots 5$ & 5.2 & 35.89 & 2.32 & 27.47 \\
\hline $1 i$ & 5.0 & 36.31 & 1.56 & 29.03 \\
\hline 19. & 5.3 & 42.29 & 1.53 & 30.50 \\
\hline 22 & 6.3 & 47.29 & 2.73 & 33.26 \\
\hline 24 & 6.3 & 43.65 & 0.74 & 34.02 \\
\hline 36 & 5.1 & 54.58 & 2.56 & 36.59 \\
\hline 28 & $5 . j$ & 54.77 & 4.50 & 41.19 \\
\hline 30 & 5.6 & 73.33 & 4.10 & 45.29 \\
\hline 32 & 4.0 & 09.02 & 5.36 & 50.65 \\
\hline 33 & 2.57 & 108.80 & 2.34 & 53.49 \\
\hline
\end{tabular}

SAKPLE NO. EFFLUEHT PORE VOL. ELUTED SUM ELUTED DPE Hg ELUTED a Mo to Mg

$\begin{array}{rrrrr}1 & 0.06 & 1.06 & -1.39 & -1.39 \\ 2 & 0.06 & 1.70 & -0.84 & -2.23 \\ 3 & 0.06 & 3.19 & -1.94 & -4.17 \\ 4 & 0.06 & 6.60 & -4.48 & -6.65 \\ 5 & 0.06 & 9.94 & -4.36 & -13.03 \\ 6 & 0.06 & 12.36 & -3.17 & -16.20 \\ 7 & 0.06 & 13.93 & -2.06 & -16.26 \\ 9 & 0.06 & 18.35 & -6.56 & -24.84 \\ 11 & 0.06 & 24.89 & -7.79 & -32.62 \\ 13 & 0.06 & 30.42 & -7.25 & -39.87 \\ 15 & 0.06 & 35.89 & -7.17 & -47.04 \\ 17 & 0.06 & 38.91 & -3.96 & -51.00 \\ 19 & 0.06 & 42.29 & -4.43 & -55.43 \\ 22 & 0.06 & 47.29 & -6.55 & -61.96 \\ 24 & 0.06 & 48.65 & -1.78 & -63.76 \\ 26 & 0.06 & 54.58 & -7.77 & -71.53 \\ 28 & 0.06 & 54.77 & -13.36 & -84.89 \\ 30 & 3.45 & 73.33 & -8.49 & -93.38 \\ 32 & 10.70 & 89.62 & -5.05 & -98.44 \\ 33 & 13.40 & 104.20 & -0.82 & -99.26\end{array}$

SAFFLE HO. EFFLUENT PORE VBL. ELUTED SUM ELUTED PPE HOJ ELUTED US NOJ NOS

\begin{tabular}{|c|c|c|c|c|}
\hline$f$ & 13.0 & 1.06 & 1.29 & 1.29 \\
\hline 2 & $22 . \hat{v}$ & .1 .70 & 1.32 & 2.00 \\
\hline 3 & 23.60 & 3.18 & 3.19 & 5.78 \\
\hline 4 & 23.50 & b. 60 & 7.52 & 13.31 \\
\hline 5 & 24.0 & 5.74 & $7.5:$ & 20.22 \\
\hline 6 & 5.7 & 12.36 & 1.32 & 22.14 \\
\hline 7 & 3.7 & 15.73 & 6.56 & 22.70 \\
\hline 9 & 3.0 & 18.95 & $1.3 i$ & 24.077 \\
\hline 11 & 4.7 & 24.69 & 2.57 & $2 \varepsilon .64$ \\
\hline 13 & 1.5 & 36.42 & i. $7 \mathrm{~F}$ & 27.42 \\
\hline 15 & i.5 & 35.87 & 0.72 & 20.14 \\
\hline 37 & 2.3 & 30.71 & 0.74 & 25.28 \\
\hline 19 & 2.0 & 42.29 & 0.60 & 23.48 \\
\hline 32 & 3.5 & 47.27 & 1.60 & $3 i .00$ \\
\hline 24 & 3.2 & tE. & 6.40 & 31.47 \\
\hline 26 & 2.3 & 54.58 & 1.35 & $=2.70$ \\
\hline 25 & 1.4 & 64.77 & 1.25 & 33.95 \\
\hline$j i$ & 1.2 & 73.33 & i. 29 & 34.35 \\
\hline 32 & 1.4 & 37.62 & 1.79 & 56.92 \\
\hline 33 & $0.5 \mathrm{i}$ & :iat.76 & 0.55 & 37.37 \\
\hline
\end{tabular}


contd

SAKPLE NO. EFFLUENT PORE VOL. ELUTED SUM ELUTED

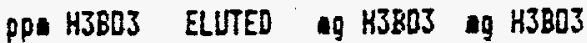

$\begin{array}{rrrrr}1 & 0.24 & 1.06 & 0.79 & 0.79 \\ 2 & 7.03 & 1.70 & 0.40 & 1.19 \\ 3 & 8.09 & 3.18 & 1.08 & 2.27 \\ 4 & 0.62 & 6.60 & 2.66 & 4.73 \\ 5 & 9.15 & 9.74 & 2.77 & 7.70 \\ 6 & 2.53 & 12.36 & 0.50 & 8.20 \\ 7 & 1.72 & 13.93 & 0.20 & 8.40 \\ 9 & 1.30 & 19.95 & 0.45 & 8.85 \\ 11 & 1.80 & 24.39 & 0.82 & 9.67 \\ 13 & 0.76 & 30.42 & 0.22 & 9.89 \\ 15 & 0.79 & 35.89 & 0.23 & 10.12 \\ 17 & 1.19 & 38.91 & 0.24 & 10.36 \\ 17 & 0.82 & 42.29 & 0.15 & 10.51 \\ 22 & 1.11 & 47.29 & 0.36 & 10.87 \\ 24 & 0.97 & 48.65 & 0.08 & 10.95 \\ 26 & 0.73 & 54.58 & 0.22 & 11.17 \\ 28 & 0.67 & 64.77 & 0.32 & 11.49 \\ 30 & 0.62 & 73.33 & 0.39 & 11.37 \\ 32 & 0.67 & 89.62 & 0.51 & 12.38 \\ 33 & 0.63 & 104.20 & 0.40 & 12.76\end{array}$

SAAPLE NO. EFFLUENT PDRE VOL. ELUTED SUR ELUTED PPI IC ELUTED II IC ag IC

\begin{tabular}{|c|c|c|c|c|}
\hline 1 & 43.4 & 1.06 & 0.93 & 0.93 \\
\hline 2 & 45.3 & 1.70 & 0.67 & 1.60 \\
\hline 3 & 40.7 & 3.18 & 0.72 & 2.52 \\
\hline 4 & 27.1 & 6.60 & -2.26 & 0.26 \\
\hline 5 & 54.1 & 9.34 & 6.28 & 6.53 \\
\hline 6 & 23.0 & 12.36 & -2.52 & 4.01 \\
\hline 7 & 23.1 & 13.93 & -1.63 & 2.39 \\
\hline 9 & $3 i .4$ & 18.95 & -1.27 & 1.12 \\
\hline 11 & 28.3 & 24.89 & -3.26 & -2.14 \\
\hline 13 & 33.6 & 30.42 & -0.24 & -2.38 \\
\hline 15 & 26.4 & 35.69 & -3.97 & -6.35 \\
\hline $1 i$ & 25.6 & 38.91 & -2.40 & -8.70 \\
\hline 19 & 31.7 & 42.29 & -0.77 & -7.52 \\
\hline 22 & 23.0 & 47.29 & -3.34 & -12.86 \\
\hline 24 & 27.7 & 46.65 & -0.81 & $-13.6 i$ \\
\hline 26 & $35 . !$ & 54.38 & 0.56 & -13.12 \\
\hline 25 & $26 . j$ & $64.7 ?$ & -7.65 & -20.77 \\
\hline 30 & 45.4 & 13.33 & 9.06 & $-11.6 \%$ \\
\hline 32 & 21.5 & 89.62 & - \pm 9.27 & -30.76 \\
\hline 33 & 21.7 & 164.20 & -17.07 & -46.63 \\
\hline
\end{tabular}

SARPLE NO. EFFLUENT PORE YOL. ELUTED SIMT ELUTED PDE SO4 ELUTED SOS SO4

\begin{tabular}{|c|c|c|c|c|}
\hline 1 & 102 & 1.06 & 1.59 & 1.59 \\
\hline 2 & 215 & 1.70 & 7.76. & 9.36 \\
\hline 3 & 212 & 3.18 & 17.53 & 26.89 \\
\hline 4 & 211 & 6.60 & 40.19 & 67.08 \\
\hline 5 & 210 & 9.34 & 38.94 & 106.02 \\
\hline 6 & 109 & 12.36 & 5.23 & 111.26 \\
\hline 7 & 99 & 13.93 & 1.72 & 113.17 \\
\hline 9 & 88 & 18.75 & $=0.84$ & 114.12 \\
\hline 11 & 99 & 24.89 & 7.26 & 121.38 \\
\hline 13 & 77 & 30.42 & -4.68 & 116.70 \\
\hline 15 & 82 & 35.89 & -2.66 & 114.64 \\
\hline 17 & 83 & 38.71 & $-\hat{v}, 85$ & 113.79 \\
\hline 19 & 81 & 42.29 & -1.59 & 112.20 \\
\hline 22 & 87 & 47.29. & 1.11 & $11 j^{3} .6 i$ \\
\hline 24 & 87 & 42.05 & 0.13 & 113.74 \\
\hline 26 & 81 & 54.58 & -2.79 & 110.95 \\
\hline 28 & 76 & 64.77 & -9.58 & 101.57 \\
\hline 30 & 74 & 73.33 & -9.60 & 91.71 \\
\hline 32 & 70 & 89.62 & -12.25 & 79.46 \\
\hline 33 & 74.60 & 104.20 & -15.63 & 63.83 \\
\hline
\end{tabular}

SAMFLE NO. EFFLUEHT PORE VOL. ELUTED SWH ELUTED PPE TOC ELUTES SO TOC OQ TOC

\begin{tabular}{|c|c|c|c|c|}
\hline 1 & 15.8 & 1.06 & 1.47 & 1.47 \\
\hline 2 & 8.4 & 1.70 & 0.51 & 1.78 \\
\hline 3 & 6.8 & 3.18 & 0.50 & 2.79 \\
\hline 4 & 3.1 & 6.60 & 0.86. & 3.05 \\
\hline 5 & 6.5 & 9.34 & 1.73 & 5.36 \\
\hline 6 & 4.3 & 12.36 & 0.74 & 6.12 \\
\hline 7 & 3.0 & 13.93 & v. 3j & 6.92 \\
\hline 3 & 2.5 & 18.75 & 0.69 & 7.11 \\
\hline$! 1$ & 3.0 & 24.83 & 1.10 & 8.21 \\
\hline ij & 2.4 & $30,4 \hat{z}$ & 0.73 & 8.94 \\
\hline 15 & 2.3 & 35.39 & $\dot{0}, \dot{0} \bar{z}$ & 7.61 \\
\hline 17 & 2.5 & BE.?1 & 0.42 & 10.03 \\
\hline 19 & 3.0 & 42.29 & 0.54 & 10.07 \\
\hline 22 & 2.4 & 47.27 & 6.53 & 11.30 \\
\hline 24 & 2.5 & 48. 25 & 0.19 & 11.49 \\
\hline 23 & 2.4 & 54.53 & i. 78 & 12.27 \\
\hline 20 & 1.3 & 84.77 & $0.3 i$ & 12.5 \\
\hline 30 & 1.2 & 75.33 & $\hat{0.19}$ & $12.7 i$ \\
\hline 32 & 1.3 & 69.22 & 9.86 & 13.28 \\
\hline 3 & 1.4 & 104.26 & j.ร: & 2.33 \\
\hline
\end{tabular}


TABLE C.13. Mass Balance for Flow Through Leach Test on whole Grout with Groundwater (MBCOLLT2)

SAMPLE NO. EFFLUENT PORE VOL. ELUTED SUM ELUTED pDE Ca ELUTED eq Ca $\mathrm{lg} \mathrm{Ca}$

\begin{tabular}{rrrrr}
\hline 1 & 3.2 & 1.02 & -5.34 & -5.34 \\
2 & 3.0 & 1.88 & -4.52 & -9.86 \\
3 & 3.0 & 2.65 & -4.05 & -13.92 \\
4 & 3.0 & 3.05 & -2.10 & -16.02 \\
5 & 1.6 & 4.69 & -8.85 & -24.87 \\
6 & 2.3 & 5.89 & -6.39 & -31.26 \\
7 & 1.6 & 6.66 & -4.15 & -35.41 \\
8 & 1.7 & 7.63 & -6.30 & -41.72 \\
9 & 1.3 & 9.82 & -10.31 & -52.52 \\
10 & 1.7 & 10.24 & -2.26 & -54.79 \\
11 & 0.8 & 12.09 & -10.14 & -64.92 \\
12 & 1.2 & 14.72 & -14.37 & -79.22 \\
13 & 1.2 & 16.25 & -8.32 & -87.54 \\
14 & 0.7 & 17.18 & -5.10 & -92.64 \\
15 & 1.10 & 17.38 & -1.09 & -93.73 \\
16 & 1.5 & 20.06 & -14.50 & -108.23 \\
17 & 1.4 & 23.52 & -18.73 & -126.76 \\
18 & 1.2 & 25.71 & -11.71 & -138.87 \\
19 & 1.0 & 26.54 & -4.53 & -143.40 \\
21 & 1.9 & 31.79 & -28.19 & -171.59 \\
23 & 2.4 & 37.23 & -28.55 & -200.54 \\
25 & 2.3 & 41.30 & -21.67 & -222.21 \\
27 & 2.1 & 45.59 & -22.74 & -245.15 \\
29 & 1.5 & 46.17 & -13.94 & -259.68 \\
31 & 1.36 & 51.77 & -19.51 & -278.60 \\
34 & 3.35 & 59.13 & -38.44 & -317.04 \\
36 & 4.17 & 62.75 & -18.61 & -335.65 \\
38 & 3.17 & 65.37 & -15.83 & -351.48 \\
40 & 3.74 & 71.31 & -28.72 & -380.20 \\
42 & 6.82 & 77.55 & -30.44 & -410.64 \\
44 & 6.55 & 93.28 & -77.16 & -487.80 \\
45 & 10.50 & 112.87 & -66.42 & -576.22 \\
& & & &
\end{tabular}

SAMPLE NO. EFFLUENT PORE VOL. ELUTED SUM ELUTED

\begin{tabular}{|c|c|c|c|c|}
\hline & Pp: $\mathrm{Na}$ & ELUTED - & a $\mathrm{Na}$ & ag $\mathrm{Ha}$ \\
\hline 1 & 1800 & 1.02 & 179.60 & 179.60 \\
\hline 2 & 1200 & 1.68 & 100.24 & 279.84 \\
\hline 3 & 910 & 2.65 & $67.6 \hat{0}$ & 347.44 \\
\hline 4 & 760 & 3.05 & 29.16 & 376.61 \\
\hline 5 & 570 & 4.69 & 88.66 & $465,2 \bar{i}$ \\
\hline$b$ & 400 & 5.89 & 44.64 & 509.81 \\
\hline 7 & 278 & 6.66 & 19.33 & 529.24 \\
\hline B & 317 & 7.63 & 33.69 & 563.13 \\
\hline 9 & 212 & 9.82 & 36.72 & 600.64 \\
\hline 10 & 509 & 10.24 & 20.17 & 620.21 \\
\hline 11 & 391 & 12.09 & 67.17 & 667.38 \\
\hline 12 & 243 & 14.72 & 56.36 & 744.25 \\
\hline 15 & 137 & 16.25 & 17.60 & 761.25 \\
\hline 14 & 235 & 17.18 & 19.37 & 780.63 \\
\hline 15 & $24 i$ & 17.30 & 4.28 & 泣ะ.ง! \\
\hline 16 & 248 & 20.06 & 59.29 & 844.20 \\
\hline 17 & 120 & 23.52 & $32.6 !$ & 076.80 \\
\hline 18 & $12 !$ & 25.71 & 20.60 & 877.66 \\
\hline 19 & 160 & 26.54 & 11.12 & 308.78 \\
\hline 21 & 110 & 31.79 & $44.2 \bar{i}$ & 655.04 \\
\hline 23 & 31 & 37.23 & 35.62 & 998.60 \\
\hline 25 & 88 & 41.30 & 25.60 & 1014.26 \\
\hline 27 & 87 & 45.59 & 26.43 & 1040.67 \\
\hline 29 & 115 & 48.17 & 23.63 & 1663.72 \\
\hline 31 & 102 & 51.77 & 27.50 & 1071.22 \\
\hline 34 & 93 & 59.13 & $49 . \overline{\mathrm{o}} \mathrm{T}$ & 1141.08 \\
\hline 36 & 79 & 62.75 & 29.46 & libc.5s \\
\hline 38 & 68 & $6 \bar{s} .7 i$ & $1 \overline{6} .6 \mathrm{i}$ & 1179.30 \\
\hline 40 & 8 & 71.31 & 37.43 & 1216.79 \\
\hline $4 \hat{2}$ & 65 & 77.55 & 24.54 & $124 i .+2$ \\
\hline 44 & 86 & $=3.23$ & 64.60 & 1306.62 \\
\hline 45 & 56 & $112.3 i$ & 6,44 & 136tat \\
\hline
\end{tabular}


TABLE C.13. contd

SAMPLE NO. EFFLUENT FORE VOL. ELUTED SUH ELUTED PPE $K$ ELUTED IQ $K$ Ig $K$

$\begin{array}{rrrrr}1 & 70.0 & 1.02 & 6.29 & 6.29 \\ 2 & 51.0 & 1.38 & 3.69 & 9.98 \\ 3 & 42.0 & 2.65 & 2.61 & 12.59 \\ 4 & 34.0 & 3.05 & 1.04 & 13.63 \\ 5 & 28.0 & 4.69 & 3.29 & 16.92 \\ 6 & 21.0 & 5.89 & 1.57 & 18.49 \\ 7 & 18.0 & 6.66 & 0.78 & 19.27 \\ 8 & 19.0 & 7.83 & 1.30 & 20.57 \\ 9 & 15.0 & 9.82 & 1.42 & 21.89 \\ 10 & 24.4 & 10.24 & 0.69 & 22.68 \\ 11 & 21.0 & 12.09 & 2.42 & 25.10 \\ 12 & 15.0 & 14.72 & 1.88 & 26.98 \\ 13 & 11.0 & 16.25 & 0.49 & 37.47 \\ 14 & 14.0 & 17.18 & 0.57 & 28.04 \\ 15 & 14.50 & 17.38 & 0.13 & 28.17 \\ 16 & 15.0 & 20.06 & 1.91 & 30.09 \\ 17 & 9.7 & 23.52 & 0.65 & 30.74 \\ 13 & 10.6 & 25.71 & 0.61 & 31.35 \\ 19 & 11.4 & 26.54 & 0.30 & 31.64 \\ 21 & 10.5 & 31.79 & 1.41 & 33.05 \\ 23 & 9.5 & 37.23 & 0.92 & 33.97 \\ 25 & 8.3 & 41.30 & 0.20 & 34.17 \\ 27 & 8.4 & 45.59 & 0.26 & 34.42 \\ 29 & 8.3 & 48.17 & 0.26 & 34.68 \\ 31 & 9.00 & 51.77 & 0.43 & 35.11 \\ 34 & 9.20 & 59.13 & 1.02 & 36.13 \\ 30 & 3.80 & 62.75 & 0.36 & 36.49 \\ 38 & 8.60 & 05.77 & 0.24 & 36.73 \\ 40 & 9.20 & 71.31 & 0.77 & 37.50 \\ 42 & 9.00 & 77.55 & 0.74 & 38.24 \\ 44 & 9.20 & 93.28 & 2.18 & 40.43 \\ 45 & 9.10 & 112.37 & 0.58 & 41.01\end{array}$

SAMPLE ND. EFFLUENT FORE YOL. ELUTED SUM ELLITED PPI Al ELUTED $\mathrm{GI}$ AD

\begin{tabular}{|c|c|c|c|c|}
\hline 1 & 17.00 & 1.02 & $1.7 !$ & 1.71 \\
\hline 2 & 13.00 & 1.88 & 1.10 & 2.81 \\
\hline 3 & 12.00 & 2.65 & 0.81 & 3.72 \\
\hline 4 & 8.85 & 3.05 & 0.35 & 4.07 \\
\hline 5 & 5.49 & 4.69 & 0.38 & 4.34 \\
\hline 6 & 4.16 & 5.89 & 0.48 & 5.43 \\
\hline 7 & 4.75 & 6.66 & $0 . J \dot{0}$ & 5.78 \\
\hline 8 & 2.50 & 7.83 & 0.28 & b. 66 \\
\hline 9 & 7.27 & 9.82 & 1.42 & $i .46$ \\
\hline 10 & 5.97 & 10.24 & 0.24 & .72 \\
\hline 11 & 3.17 & 12.09 & 0.56 & 8.29 \\
\hline 12 & 0.35 & 14.72 & 0.06 & 8.35 \\
\hline 13 & 2.42 & 16.25 & 0.35 & 8.71 \\
\hline 14 & 2.42 & 17.18 & $0.2 \mathrm{i}$ & 0.72 \\
\hline 15 & 2.43 & 17.38 & $0.0 \mathrm{~s}$ & 0.97 \\
\hline 1é & 0.06 & 20.06 & $-0.0 \mathrm{i}$ & 8.95 \\
\hline 17. & 0.17 & 23.52 & 0.02 & 8.96 \\
\hline 18 & 0.73 & 25.71 & 0.14 & 9.11 \\
\hline 19 & 0.14 & 26.54 & 0.00 & 9.12 \\
\hline $2 !$ & 0.03 & 31.79 & -0.64 & 5.06 \\
\hline 23 & 0.03 & 37.23 & -0.04 & 9.04 \\
\hline 25 & 0.03 & 41.30 & $-0 . \hat{v} 3$ & 9.52 \\
\hline 27 & 0.15 & 45.59 & 0.02 & 9.04 \\
\hline 29 & 0.18 & 48.17 & 0.02 & 9.06 \\
\hline 31 & 0.07 & 51.77 & $-0.0 i$ & 9.05 \\
\hline 34 & 0.13 & 59.13 & $-\dot{v} .05$ & 3. 59 \\
\hline 36 & 0.07 & 02.75 & -0.01 & 8.90 \\
\hline 38 & 0.16 & 65.77 & 0.02 & 9.00 \\
\hline 40 & 0.11 & 71.31 & 0.60 & 9.00 \\
\hline 42 & 0.06 & 77.55 & -0.63 & B. 88 \\
\hline 44 & 0.03 & 73.28 & -0.11 & 3.67 \\
\hline 45 & 0.03 & 112.87 & -0.14 & 8.73 \\
\hline
\end{tabular}


TABLE C.13. contd

SAMPLE NO. EFFLUENT PORE VOL. ELLUTED SUM ELUTED poe $5 \mathrm{i}$ ELUTED $9 \mathrm{Si}$. $\mathrm{Si}$

\begin{tabular}{|c|c|c|c|c|}
\hline 1 & 14.5 & 1.02 & -0.05 & -0.05 \\
\hline 2 & 12.0 & 1.88 & -0.26 & -0.31 \\
\hline$j$ & 10.8 & 2.65 & -0.32 & -0.63 \\
\hline 4 & 10.0 & 3.05 & -0.20 & -0.83 \\
\hline 5 & 10.5 & 4.69 & -0.73 & -1.56 \\
\hline 6 & 9.7 & 5.39 & -0.63 & -2.19 \\
\hline 7 & 10.3 & 6.66 & -0.36 & -2.55 \\
\hline 8 & 10.4 & 7.83 & -0.53 & -3.08 \\
\hline 9 & 10.2 & 9.82 & -0.85 & -4.03 \\
\hline 10 & 11.4 & 10.24 & -0.15 & -4.18 \\
\hline 11 & 11.0 & 12.03 & -0.73 & -4.91 \\
\hline 12 & 11.0 & 14.72 & -1.04 & -5.96 \\
\hline 13 & 9.5 & 16.25 & -0.83 & -6.79 \\
\hline 14 & 12.8 & 17.18 & -0.20 & -6.99 \\
\hline 15 & 12.30 & 17.38 & -0.05 & -7.04 \\
\hline 16 & 31.7 & 20.06 & -0.82 & -7.87 \\
\hline 17 & 9.9 & 23.52 & -1.76 & -9.63 \\
\hline 18 & 10.6 & 25.71 & -0.96 & -10.57 \\
\hline 19 & 13.5 & 26.54 & -0.12 & -10.71 \\
\hline 21 & 9.8 & 31.79 & -2.69 & -13.40 \\
\hline 23 & 8.? & 37.25 & -3.31 & -16.71 \\
\hline 25 & 7.5 & 41.30 & -2.23 & -18.94 \\
\hline 27 & 9.1 & 45.59 & -2.51 & -21.45 \\
\hline 29 & 12.1 & 48.17 & -0.74 & -22.19 \\
\hline 31 & 10.50 & 51.77 & -1.61 & -23.80 \\
\hline 34 & 9.53 & 55.13 & -3.99 & -27.79 \\
\hline 36 & B. & 62.75 & -2.29 & -30.68 \\
\hline 36 & 10.50 & 65.77 & -1.35 & -31.43 \\
\hline 40 & 10.40 & 71.31 & -2.53 & -33.96 \\
\hline 42 & 7.51 & 77.55 & -4.64 & -38.60 \\
\hline 44 & B.:37 & 93.28 & -10.63 & -49.22 \\
\hline 45 & ¿.34 & 112.57 & -12.34 & -82.16 \\
\hline
\end{tabular}

SAMPLE ND. EFFLUENT PORE VOL. ELUTEd SUH ELUTED

\begin{tabular}{|c|c|c|c|c|}
\hline & ppe kq & ELUTED & g $\mathrm{Mg}$ & gو \\
\hline 1 & 0.06 & 1,02 & -1.41 & -1.41 \\
\hline 2 & 0.06 & 1.86 & -1.17 & -2.60 \\
\hline 3 & 0.08 & 2.65 & -1.06 & -3.86 \\
\hline 4 & 0.06 & 3.05 & -0.55 & -4.22 \\
\hline 5 & 0.06 & 4.69 & -2.27 & -ó.48 \\
\hline 6 & 0.06 & 5.39 & -1.66 & -8.14 \\
\hline 7 & 0.06 & 6.66 & -1.06 & -7.21 \\
\hline 3 & 0.06 & 7.85 & -1.22 & -10.83 \\
\hline 9 & 0.06 & 8.82 & -2.75 & -13.58 \\
\hline 10 & 0.06 & 10.24 & -0.58 & -14.10 \\
\hline 11 & 0.06 & 12.09 & -2.56 & -16.72 \\
\hline 12 & 0.08 & 14.72 & -3.64 & -20.35 \\
\hline 13 & 0.06 & 16.25 & -2.12 & -22.47 \\
\hline 14 & 0.06 & 17.18 & -1.29 & $-23,76$ \\
\hline 15 & 0.06 & 17.38 & -0.28 & -24.03 \\
\hline 16 & 0.06 & 20.60 & -3.71 & -27.74 \\
\hline 17 & 0.28 & 23.52 & -4.71 & $-32,45$ \\
\hline 18 & 0.17 & 25.71 & -3.00 & -35.45 \\
\hline 19 & p. Á & 26.54 & -1.15 & -36.60 \\
\hline 21 & 0.41 & 33.79 & -7.60 & -43.68 \\
\hline 23 & 0.70 & 37.23 & -7.18 & -50.86 \\
\hline 25 & 0.54 & 41.30 & -5.43 & -56.29 \\
\hline 27 & 0.39 & 45.57 & -5.79 & $-\dot{2} 2.08$ \\
\hline 29 & 0.66 & 48.17 & -3.57 & -65.65 \\
\hline 31 & 0.22 & $5 i .77$ & -4.92 & -70.57 \\
\hline 34 & 0.65 & 59.13 & -9.75 & -80.32 \\
\hline 36 & 1.22 & 22.75 & -4.50 & -84.91 \\
\hline 38 & 0.88 & 65.77 & -3.93 & -38.34 \\
\hline 40 & 1.24 & 71.31 & -7.01 & -95.25 \\
\hline 42 & 1.63 & 77.55 & -7.66 & -103.51 \\
\hline 44 & 2.01 & 93.29 & -18.71 & -122.22 \\
\hline 45 & 3.31 & 112.67 & -20.77 & -142.99 \\
\hline
\end{tabular}


TABLE C.13.

contd

SAKPLE NO. EFFLUENT PORE VOL. ELUTEO SUK ELUTED PPS $F$ ELUTED $Q F$ I $F$

$\begin{array}{rrrrr}1 & 14.0 & 1.02 & 1.37 & 1.37 \\ 2 & 11.0 & 1.83 & 0.30 & 2.26 \\ 3 & 9.0 & 2.65 & 0.65 & 2.91 \\ 4 & 9.0 & 3.05 & 0.34 & 3.25 \\ 5 & 7.0 & 4.69 & 1.06 & 4.31 \\ 6 & 6.0 & 5.89 & 0.65 & 1.96 \\ 7 & 5.8 & 6.66 & 0.40 & 5.37 \\ 8 & 5.8 & 7.83 & 0.62 & 5.98 \\ 9 & 5.2 & 9.82 & 0.93 & 6.91 \\ 10 & 6.1 & 10.24 & 0.23 & 7.14 \\ 11 & 7.2 & 12.09 & 1.23 & 8.37 \\ 12 & 5.9 & 14.72 & 1.41 & 9.78 \\ 13 & 5.6 & 16.25 & 0.77 & 10.55 \\ 14 & 5.1 & 17.18 & 0.42 & 10.98 \\ 15 & 5.50 & 17.33 & 0.10 & 11.08 \\ 16 & 5.9 & 20.06 & 1.44 & 12.51 \\ 17 & 4.8 & 23.52 & 1.48 & 13.99 \\ 18 & 5.3 & 25.71 & 1.04 & 15.03 \\ 19 & 6.6 & 26.54 & 0.50 & 15.53 \\ 21 & 5.2 & 31.79 & 2.45 & 17.98 \\ 23 & 5.4 & 37.23 & 2.64 & 20.63 \\ 25 & 5.5 & 41.30 & 2.02 & 22.64 \\ 27 & 5.0 & 45.59 & 1.92 & 24.56 \\ 29 & 6.1 & 48.17 & 1.43 & 25.99 \\ 31 & 5.20 & 51.77 & 1.68 & 27.67 \\ 34 & 6.30 & 59.13 & 4.23 & 31.91 \\ 36 & 6.50 & 62.75 & 2.15 & 34.06 \\ 38 & 6.00 & 65.77 & 1.39 & 35.95 \\ 40 & 6.80 & 71.31 & 3.46 & 39.41 \\ 42 & 6.30 & 77.55 & 3.59 & 43.00 \\ 44 & 6.60 & 33.28 & 9.52 & 52.52 \\ 45 & 6.00 & 112.97 & 10.69 & 63.21\end{array}$

SAGPLE ND. EFFLUENT PORE VOL. ELUTED SUM ELUTED DPE HOJ ELUTED I NOJ

\begin{tabular}{|c|c|c|c|c|}
\hline 1 & 26.0 & 1.02 & $2.82 z$ & 2.62 \\
\hline 2 & 18.0 & 1.88 & 1.53 & 4.15 \\
\hline 3 & 13.0 & 2.85 & 0.79 & 5.13 \\
\hline 4 & 18.0 & 3.05 & $0 . \pi 1$ & 5.84 \\
\hline 5 & 7.0 & 4.69 & 1.12 & 6.97 \\
\hline 6 & 4.0 & 5.67 & 0.16 & 7.43 \\
\hline 7 & 3.3 & 6.66 & 0.24 & 7.67 \\
\hline$B$ & 4.1 & 7.83 & 0.46 & 3.14 \\
\hline 9 & 2.4 & 9.82 & 0.45 & 8.59 \\
\hline 10 & 6.8 & 10.24 & 0.23 & 8.87 \\
\hline 11 & 5.6 & 12.09 & 1.01 & 7.88 \\
\hline 12 & 3.3 & 14.72 & 0.83 & 10.72 \\
\hline 13 & 1.5 & 16.25 & 0.21 & 10.93 \\
\hline 14 & 3.7 & 17.18 & 0.33 & 11.26 \\
\hline 15 & 4.00 & 17.38 & 0.08 & ii.j4 \\
\hline 16 & 4.3 & 20.06 & 1.12 & 32.45 \\
\hline 17 & 1.4 & 23.52 & 0.45 & 12.90 \\
\hline 18 & 1.6 & 25.71 & 0.33 & 13.23 \\
\hline 19 & 2.5 & 26.54 & 0.20 & 13.42 \\
\hline 21 & 1.6 & 31.79 & 0.78 & 14.21 \\
\hline 23 & 1.2 & 37.23 & 0.59 & 14.60 \\
\hline 25 & 1.2 & 41.30 & 0.44 & 15.24 \\
\hline 27 & 1.3 & 45.59 & $0.5 i$ & 15.75 \\
\hline 29 & 2.0 & 48.17 & 0.48 & 16.24 \\
\hline 31 & 1.60 & $51.7 \%$ & 6.54 & 16.73 \\
\hline 34 & 2.20 & 58.13 & 1.53 & 10.31 \\
\hline 36 & 1.50 & 02.75 & 0.50 & 18.8! \\
\hline 38 & 1.70 & 6.5 .77 & 0.45 & 16.29 \\
\hline 40 & 2.40 & 71.33 & 1.20 & 20.56 \\
\hline 42 & 1.10 & 77.55 & 0.62 & 21.17 \\
\hline 44 & 1.40 & 53.28 & 2.03 & 25.20 \\
\hline 45 & 0.50 & 112.07 & 0.78 & 23.70 \\
\hline
\end{tabular}


TABLE C.13. contd

SAMPLE NO. EFFLUENT FORE VOL. ELUTED SUH ELUTED DPE H3BDZ ELUTED I HJBDOZ

$\begin{array}{lllll}1 & 5.72 & 1.02 & 0.54 & 0.54 \\ 2 & 6.86 & 1.88 & 0.56 & 1.10 \\ 3 & 5.15 & 2.65 & 0.37 & 1.47 \\ 4 & 4.46 & 3.05 & 0.16 & 1.63 \\ 5 & 2.79 & 4.69 & 0.40 & 2.03 \\ 6 & 1.62 & 5.89 & 0.15 & 2.18 \\ 7 & 1.49 & 6.66 & 0.09 & 2.27 \\ 8 & 1.62 & 7.23 & 0.15 & 2.42 \\ 9 & 0.93 & 9.82 & 0.12 & 2.54 \\ 10 & 2.68 & 10.24 & 0.10 & 2.63 \\ 11 & 2.00 & 12.09 & 0.30 & 2.94 \\ 12 & 1.38 & 14.72 & 0.27 & 3.21 \\ 13 & 0.72 & 16.25 & 0.06 & 3.27 \\ 14 & 1.52 & 17.28 & 0.11 & 3.38 \\ 15 & 1.53 & 17.38 & 0.02 & 3.40 \\ 16 & 1.55 & 20.06 & 0.32 & 3.72 \\ 17 & 0.83 & 23.52 & 0.17 & 3.89 \\ 18 & 0.93 & 25.71 & 0.13 & 4.02 \\ 19 & 1.24 & 26.54 & 0.07 & 4.09 \\ 21 & 0.20 & 31.79 & 0.24 & 4.33 \\ 23 & 0.85 & 37.23 & 0.28 & 4.61 \\ 25 & 0.89 & 41.30 & 0.22 & 4.03 \\ 27 & 0.83 & 45.59 & 0.21 & 5.04 \\ 29 & 1.15 & 48.17 & 0.21 & 5.24 \\ 31 & 0.94 & 51.77 & 0.21 & 5.46 \\ 34 & 0.78 & 59.13 & 0.32 & 5.76 \\ 36 & 0.79 & 62.75 & 0.16 & 5.54 \\ 36 & 0.98 & 65.77 & 0.19 & 6.13 \\ 40 & 1.02 & 71.31 & 0.37 & 6.51 \\ 42 & 0.79 & 77.55 & 0.28 & 6.78 \\ 44 & 0.98 & 93.28 & 1.00 & 7.78 \\ 45 & 0.37 & 112.67 & 1.22 & 9.01\end{array}$

SAMPLE NO. EFFLUENT PORE VOL. ELUTED SUK ELUTED PDN SO4 ELUTED I SO4 SO4

\begin{tabular}{|c|c|c|c|c|}
\hline 1 & 240 & 1.02 & 15.58 & 15.50 \\
\hline 2 & 180 & 1.88 & 8.62 & 23.60 \\
\hline 3 & 144 & 2.65 & 4.43 & 26.03 \\
\hline 4 & 120 & 3.05 & 1.35 & 27.38 \\
\hline 5 & 110 & 4.69 & 3.90 & 33.39 \\
\hline$b$ & 100 & 5.87 & 1.67 & 34.95 \\
\hline 7 & 120 & 0.66 & 2.60 & 37.55 \\
\hline 8 & 90 & 7.83 & -0.46 & 38.01 \\
\hline 9 & 30 & 9.22 & -1.18 & 36.83 \\
\hline 10 & 114 & 10.24 & $i . i j$ & 33.61 \\
\hline 11 & 119 & 12.39 & 6.06 & 44.65 \\
\hline 12 & 92 & 14.72 & 1.57 & 45.62 \\
\hline 13 & 30 & 16.25 & -0.91 & 44.71 \\
\hline 14 & 78 & 17.15 & $1.1 j$ & 45.81 \\
\hline 15 & 105 & 17.38 & 0.38 & 46.19 \\
\hline 16 & 112 & 20.06 & $6.7 i$ & 53.10 \\
\hline 17 & 76 & 23.52 & -3.43 & 49.67 \\
\hline 18 & 20 & 25.71 & -1.30 & 40.37 \\
\hline 19 & 34 & 26.54 & -0.16 & $\$ 8.20$ \\
\hline 21 & 84 & 31.79 & -1.04 & 47.16 \\
\hline 23 & 82 & 37.23 & -2.106 & 45.60 \\
\hline 25 & 78 & 41.30 & -3.23 & 41.77 \\
\hline 27 & 74 & 45.59 & -5.11 & 36.67 \\
\hline 29 & 75 & 48.17 & -2.82 & 33.85 \\
\hline 31 & 73 & 51.77 & -4.64 & 29.21 \\
\hline 34 & 71 & 59.13 & -10.95 & 18.25 \\
\hline jó & 73 & b2. 75 & -2.27 & 15.38 \\
\hline 36 & 79 & 65.77 & $-2.1 \hat{v}$ & 13.25 \\
\hline 40 & 30 & 71.31 & -3.30 & 3.79 \\
\hline 42 & 74 & 77.55 & -7.93 & 2.56 \\
\hline 44 & 32 & 9.25 & -0.24 & -3.60 \\
\hline 45 & 79 & 112.87 & -13.99 & -17.67 \\
\hline
\end{tabular}


TABLE C.13. contd

SARPLE NO. EFFLUENT PORE VOL. ELUTED SUM ELUTED pPE IC ELUTED IO IC IO IC

$\begin{array}{rrrrr}1 & 41.1 & 1.0 & 0.71 & 0.71 \\ 2 & 49.9 & 1.9 & 1.35 & 2.06 \\ 3 & 28.7 & 2.7 & -0.41 & 1.65 \\ 4 & 19.3 & 3.1 & -0.59 & 1.06 \\ 5 & 30.1 & 4.7 & -0.64 & 0.42 \\ 6 & 20.9 & 5.9 & -1.57 & -1.16 \\ 7 & 24.3 & 6.7 & -0.75 & -1.90 \\ 8 & 24.3 & 7.3 & -1.14 & -3.04 \\ 9 & 34.9 & 9.8 & 0.16 & -2.89 \\ 10 & 49.2 & 10.2 & 0.63 & -2.26 \\ 11 & 63.5 & 12.1 & 5.40 & 3.14 \\ 12 & 16.3 & 14.7 & -4.65 & -1.51 \\ 13 & 17.0 & 16.3 & -2.59 & -4.10 \\ 14 & 12.6 & 17.2 & 0.78 & -3.32 \\ 15 & 17.3 & 17.4 & -0.33 & -3.65 \\ 16 & 16.2 & 20.1 & -4.77 & -8.42 \\ 17 & 15.6 & 23.5 & -6.36 & -14.78 \\ 18 & 15.3 & 25.7 & -4.08 & -18.87 \\ 19 & 15.2 & 26.5 & -1.56 & -20.42 \\ 21 & 15.1 & 31.8 & -9.90 & -30.32 \\ 23 & 15.0 & 37.2 & -10.33 & -40.65 \\ 25 & 18.8 & 41.3 & -6.16 & -46.81 \\ 27 & 12.0 & 45.6 & -9.40 & -56.21 \\ 29 & 38.5 & 48.2 & 1.12 & -55.09 \\ 31 & 21.90 & 51.3 & -4.36 & -59.45 \\ 34 & 24.74 & 59.1 & -6.83 & -66.28 \\ 36 & 17.47 & 62.8 & -5.97 & -72.26 \\ 36 & 20.90 & 65.8 & -3.95 & -76.21 \\ 46 & 21.26 & 71.3 & -7.06 & -83.27 \\ 42 & 14.32 & 77.6 & -12.21 & -95.51 \\ 44 & 11.37 & 93.3 & -35.47 & -136.35 \\ 45 & 12.89 & 112.9 & -41.32 & -172.29\end{array}$

SAMPLE NO. EFFLUENT FORE YOL. ELUTED SUM ELUTED PDS TOC ELUTED IV TOC TOC

\begin{tabular}{|c|c|c|c|c|}
\hline 1 & 13.6 & 1.02 & 1.28 & 1.28 \\
\hline 2 & 9.5 & 1.88 & 0.73 & 2.00 \\
\hline 3 & 8.4 & 2.65 & 0.57 & 2.57 \\
\hline 4 & 6.9 & 3.05 & 0.23 & 2.81 \\
\hline 5 & 4.3 & 4.69 & 0.54 & 3.34 \\
\hline 6 & 3.5 & 5.39 & 0.30 & 3.64 \\
\hline 7 & 3.7 & 0.60 & 0.20 & 3.84 \\
\hline 8 & 3.5 & 7.83 & 0.29 & 4.13 \\
\hline 9 & 2.9 & 9.32 & 0.37 & 4.51 \\
\hline 10 & 3.7 & 10.24 & 0.11 & 4.62 \\
\hline 11 & 4.5 & 12.09 & 0.54 & 5.26 \\
\hline 12 & 19.1 & 14.72 & 4.72 & 7.98 \\
\hline 13 & 19.9 & 16.25 & 2.86 & 12.85 \\
\hline 14 & 46.7 & 17.16 & 4.22 & 17.06 \\
\hline 15 & 20.9 & 17.38 & 0.39 & 17.46 \\
\hline 16 & 18.0 & 30.06 & 4.52 & 21.53 \\
\hline 17 & 16.0 & 23.52 & 5.15 & 27.12 \\
\hline 18 & 14.0 & 25.71 & 2.82 & 29.75 \\
\hline 19 & 12.0 & $2 b .54$ & 0.51 & 30.85 \\
\hline $2 i$ & $10: 0$ & 31.79 & 4.69 & 35. 54 \\
\hline $2 j$ & 2.3 & 37.23 & 0.70 & 36.24 \\
\hline 25 & 2.6 & 41.30 & 0.63 & 36.37 \\
\hline 27 & 2.2 & 45.59 & 0.52 & 37.37 \\
\hline 29 & 2.0 & 46.17 & 0.25 & 57.64 \\
\hline 31 & 3.52 & 53.73 & 0.70 & 38.54 \\
\hline 34 & 5.65 & 59.13 & 3.42 & 41. S \\
\hline 36 & $4.52^{\circ}$ & 62.75 & 1.26 & 43.22 \\
\hline 38 & 3.55 & 65.77 & 0.76 & 43.99 \\
\hline 40 & 3.08 & 71.31 & 1.14 & 45.13 \\
\hline 42 & 0.32 & 77.55 & $-0 . i:$ & 45.02 \\
\hline 44 & 1.06 & 75.25 & 0.09 & 45.11 \\
\hline 45 & 1.06 & 112.87 & 6.00 & 45.11 \\
\hline
\end{tabular}


TABLE C.14. Mass Balance for Flow Through Leach Test on whole Grout with Groundwater (MBCOLLTI)

SAMFLE HO EFFLUEHT FORE VOL. ELUTED SUH ELUTED pp: Ca ELUTED Q $\mathrm{Ca} \quad \mathrm{Ca}$

$\begin{array}{lllll}1 & 3.3 & 0.31 & -1.70 & -1.70 \\ 2 & 4.5 & 0.51 & -1.07 & -2.77 \\ 3 & 4.3 & 1.37 & -4.62 & -7.39 \\ 4 & 1.2 & 1.98 & -3.47 & -10.86 \\ 5 & 1.2 & 3.16 & -0.72 & -17.58 \\ 6 & 1.8 & 4.12 & -5.40 & -22.98 \\ 7 & 0.9 & 4.83 & -4.07 & -27.05 \\ 8 & 1.7 & 5.67 & -4.74 & -31.79 \\ 9 & 0.6 & 6.37 & -4.03 & -35.82 \\ 10 & 1.2 & 8.13 & -10.02 & -45.34 \\ 11 & 0.3 & 9.76 & -9.34 & -55.17 \\ 12 & 1.2 & 11.70 & -11.04 & -66.22 \\ 13 & 0.7 & 13.61 & -10.97 & -77.19 \\ 14 & 1.3 & 15.91 & -13.06 & -90.25 \\ 15 & 1.4 & 16.13 & -12.60 & -102.85 \\ 16 & 3.9 & 20.62 & -13.49 & -116.34 \\ 17 & 2.6 & 33.09 & -69.22 & -185.56 \\ 13 & 1.4 & 34.96 & -10.60 & -196.16 \\ 19 & 1.2 & 36.21 & -7.12 & -203.23\end{array}$

SAMPLE NO. EFFLUENT PORE VOL. ELUTEÑ SUM ELUTEA DPE $K$ ELUTED ag $k$ ig $k$

\begin{tabular}{|c|c|c|c|c|}
\hline $\mathbf{i}$ & 45.0 & 0.31 & 1.20 & 1.20 \\
\hline 2 & 49.0 & 0.51 & 0.86 & 2.05 \\
\hline 3 & 83.0 & 1.37 & 6.72 & 8.77 \\
\hline 4 & 47.0 & 1.76 & 2.61 & 11.30 \\
\hline 5 & 34.0 & 3.16 & 3.21 & 14.600 \\
\hline 6 & 29.0 & 4.12 & 2.11 & i6.71 \\
\hline 7 & 10.0 & 4.83 & 0.60 & 17.31 \\
\hline$\overline{\mathrm{g}}$ & 31.0 & 5.57 & 2.02 & 19.34 \\
\hline 9 & $23 . \hat{0}$ & 6.37 & i.11 & 20.44 \\
\hline 10 & 13.4 & 8.13 & $i .34$ & 22.38 \\
\hline 11 & 17.0 & 9.76 & 1.50 & 23.74 \\
\hline 12 & $(4,0)$ & $: 1.70$ & 1.25 & 25.17 \\
\hline 13 & 12.0 & 13.61 & 0.35 & 26.02 \\
\hline 14 & 11.0 & $\mid 5.71$ & 0.76 & 30.79 \\
\hline :5 & 5.4 & ig.:13 & $0.3 i$ & 27.16 \\
\hline 10 & 7.1 & 20.52 & 0.34 & 37.49 \\
\hline $1 \bar{i}$ & Q. 4 & 33.09 & 0.73 & 23.27 \\
\hline 18 & 0.6 & 34.96 & 0.15 & 20.43 \\
\hline :? & $10 .:$ & J6́. & 0.30 & 2.33 \\
\hline
\end{tabular}

SAMPLE NO. EFFLUENT FORE VDL. ELUTED SLR ELUTED Pp: $\mathrm{Na}$ ELUTED I $\mathrm{Na}$ a $\mathrm{Ha}$

\begin{tabular}{|c|c|c|c|c|}
\hline 1 & 1280 & 0.31 & 40.42 & 40.42 \\
\hline 2 & 1210 & $0.5 !$ & 24.52 & 65.04 \\
\hline 3 & 2000 & 1.37 & 176.46 & 241.50 \\
\hline 4 & 1100 & 1.98 & 68.13 & 307.62 \\
\hline 5 & 720 & 3.16 & 85.20 & 374.82 \\
\hline 6 & 643 & 4.12 & 61.64 & 450.46 \\
\hline 7 & 262 & 4.83 & 17.48 & 473.94 \\
\hline 3 & 230 & $5.0 j$ & 52.80 & 526.74 \\
\hline 7 & 410 & 6.37 & 29.00 & 554.74 \\
\hline 10 & job & 6.13 & 62.35 & 517.09 \\
\hline 11 & 305 & 9.76 & 47.42 & 004.50 \\
\hline 12 & 204 & 11.70 & 36.06 & 700.58 \\
\hline i3 & 162 & 13.61 & 27.13 & 727.76 \\
\hline 14 & 125 & 15.91 & 33.89 & 751.66 \\
\hline 15 & 110 & 16.13 & 19.50 & 771.26 \\
\hline ló & 97 & 20.62 & 19.:1: & 790.41 \\
\hline 17 & 95 & 33.09 & 96.52 & 881.22 \\
\hline 13 & 114 & 34.76 & 17.27 & 898.51 \\
\hline 19 & 159 & 36.21 & 13.90 & 915,31 \\
\hline
\end{tabular}

GAMPLE NO. EFFLUENT FORE YDL. ELUTED SUY ELUTEE

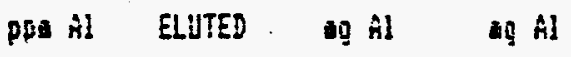

\begin{tabular}{|c|c|c|c|c|}
\hline 1 & 21.00 & 0.31 & 0.67 & 0.67 \\
\hline 2 & 18.0ิ & U. & 0.37 & 1.05 \\
\hline 3 & 33.00 & 1.37 & 2.74 & 3.98 \\
\hline 4 & 18.10 & 1,83 & i.14 & 5.13 \\
\hline 5 & $\$ 2.50$ & 3.16 & 1.52 & 0.05 \\
\hline 6 & 10.30 & 4.12 & 1.02 & $7.6 c^{\prime}$ \\
\hline$j$ & 4.14 & 4.93 & 0.30 & 9.76 \\
\hline 8 & 4.75 & $\overline{3.67}$ & $r_{1}=1$ & $\overline{0.37}$ \\
\hline 9 & 9.60 & 0.37 & 3.51 & a. \\
\hline 10 & 3.47 & 6.13 & 6.02 & 9.49 \\
\hline 11 & 3.17 & 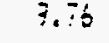 & 8.5 & 0.01 \\
\hline 12 & 1.60 & 14.70 & 0.21 & 10.3 \\
\hline$: 3$ & 1.34 & $13.6:$ & i. 19 & 10.5: \\
\hline 14 & 3.:1: & 15.31 & 6.60 & 20.51 \\
\hline 15 & 0.12 & i6. & 6.60 & $\therefore 0.5 \%$ \\
\hline lót & 0.13 & 30.62 & -0.02 & 30.50 \\
\hline$i \bar{i}$ & 0.43 & 33.09 & -4.69 & iv. $4 !$ \\
\hline is & 3.03 & 24.56 & $-\dot{0} . \Delta I$ & 16.5 \\
\hline 17 & 300 & 30.21 & 6.64 & $! \cdot 0.43$ \\
\hline
\end{tabular}


contd

SARPLE NO. EFFLUENT PORE VOL. ELUTED SUK ELUTED ppl $5 i$ ELUTED $\$ Q \mathrm{Si}$ ag $\mathrm{Si}$

$\begin{array}{rrrrr}1 & 0.5 & 0.31 & -0.21 & -0.21 \\ 2 & 13.0 & 0.51 & -0.04 & -0.25 \\ 3 & 15.1 & 1.37 & 0.01 & -0.24 \\ 4 & 11.6 & 1.98 & -0.22 & -0.46 \\ 5 & 11.3 & 3.16 & -0.45 & -0.91 \\ 6 & 11.1 & 4.12 & -0.39 & -1.30 \\ 7 & 10.8 & 4.63 & -0.31 & -1.61 \\ 9 & 10.4 & 5.67 & -0.40 & -2.01 \\ 9 & 11.2 & 0.37 & -0.28 & -2.29 \\ 10 & 13.6 & 0.13 & -0.26 & -2.54 \\ 11 & 13.2 & 9.76 & -0.30 & -2.85 \\ 12 & 11.4 & 11.70 & -0.73 & -3.57 \\ 13 & 10.0 & 13.61 & -0.99 & -4.57 \\ 14 & 9.6 & 15.91 & -1.30 & -5.86 \\ 15 & 9.3 & 18.13 & -1.32 & -7.19 \\ 16 & 9.0 & 20.62 & -1.56 & -6.74 \\ 17 & 9.9 & 33.09 & -6.65 & -15.39 \\ 19 & 11.9 & 34.96 & -0.60 & -15.99 \\ 19 & 14.9 & 36.21 & -0.01 & -16.01\end{array}$

SAMPLE NO. EFFLUENT PORE VOL. ELUTED SUIT ELUTED ppe $\mathrm{Hg}$ ELUTED Ig Kg $\mathrm{Kg}$

$\begin{array}{lllll}1 & 0.06 & 0.31 & -0.45 & -0.45 \\ 2 & 0.06 & 0.51 & -0.29 & -0.74 \\ 3 & 0.06 & 1.37 & -1.25 & -1.98 \\ 4 & 0.06 & 1.38 & -0.38 & -2.37 \\ 5 & 0.06 & 3.16 & -1.71 & -4.58 \\ 6 & 0.09 & 4.12 & -1.37 & -5.96 \\ 7 & 0.06 & 4.83 & -1.03 & -6.90 \\ 8 & 0.06 & 5.67 & -1.22 & -3.21 \\ 9 & 0.06 & 6.37 & -1.01 & -9.22 \\ 10 & 0.06 & 8.13 & -2.55 & -11.77 \\ 11 & 0.06 & 9.76 & -2.36 & -14.13 \\ 12 & 0.06 & 11.70 & -2.81 & -16.94 \\ 13 & 0.06 & 13.61 & -2.77 & -19.71 \\ 14 & 0.06 & 15.91 & -3.33 & -23.04 \\ 15 & 0.19 & 18.13 & -3.19 & -25.22 \\ 16 & 2.50 & 20.62 & -2.77 & -29.20 \\ 17 & 1.06 & 33.09 & -16.76 & -45.96 \\ 18 & 0.14 & 34.96 & -2.69 & -48.36 \\ 19 & 0.06 & 36.21 & -1.61 & -50.47\end{array}$

SAKPLE NO. EFFLUENT PORE VOL. ELUTED SUK ELUTED

\begin{tabular}{|c|c|c|c|c|}
\hline & $p p=F$ & ELUTED & of $F$ & $g$ \\
\hline$i$ & 11.0 & 0.31 & 0.34 & 0.31 \\
\hline 2 & 11.0 & 0.51 & 0.22 & 0.56 \\
\hline$j$ & 18.0 & 1.37 & 1.56 & 2.12 \\
\hline 4 & 11.0 & 1.98 & 0.67 & 2.79 \\
\hline 5 & 9.0 & 3.16 & 1.04 & 3.83 \\
\hline 6 & 8.0 & 4.12 & 0.75 & 4.58 \\
\hline 7 & 5.6 & 4.63 & 0.37 & 4.97 \\
\hline 3 & 8.6 & 5.67 & 0.71 & 5.67 \\
\hline 9 & 6.3 & 6.37 & 0.42 & 6.09 \\
\hline 10 & 4.8 & 3.13 & 0.77 & 6.88 \\
\hline 11 & 5.6 & 9.76 & 0.86 & 7.74 \\
\hline 12 & 6.6 & 11.70 & 1.23 & 3.77 \\
\hline 13 & 5.7 & 13.61 & 1.03 & 10.01 \\
\hline 14 & 5.8 & 15.91 & i. 27 & 11.27 \\
\hline 15 & 5.6 & 18.13 & 1.18 & 12.45 \\
\hline 16 & $5 .:$ & 20.62 & 1.19 & $: 3.64$ \\
\hline $1 \bar{i}$ & 6.0 & 33.09 & 7.13 & 20.76 \\
\hline 18 & 5.9 & 34.76 & 1.05 & 21.81 \\
\hline \$? & 7.4 & 36.21 & 0.90 & 22.71 \\
\hline
\end{tabular}

SARPLE NO. EFFLUENT PORE VOL. ELUTED SUY ELUTED

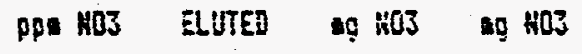

\begin{tabular}{|c|c|c|c|c|}
\hline 1 & 39.0 & $0.3 i$ & จ. ถ่ & $0 . \dot{1}$ \\
\hline 2 & 13.0 & $0.5 i$ & 0.37 & 0.98 \\
\hline 3 & 3.0 & 1.37 & 0.80 & 1.78 \\
\hline 4 & 15.0 & 1.78 & 0.74 & 2.72 \\
\hline 5 & 30.0 & 3.16 & 3.67 & 6.39 \\
\hline$b$ & 6.0 & 4.12 & 0.59 & 6.57 \\
\hline$i$ & 2.7 & 4.83 & $0.2 !$ & 7.18 \\
\hline b & 8.5 & 5.67 & 9.73 & 7.31 \\
\hline 7 & $5 .:$ & 6.37 & 4.36 & 8.28 \\
\hline 10 & $4 . ?$ & 8.12 & $\dot{B}$ & 9.15 \\
\hline 11 & 3.6 & 9.76 & 6.59 & 9.75 \\
\hline$: 2$ & 3.6 & 11,70 & 0.71 & 10.45 \\
\hline 13 & 2.0 & $13.5 !$ & 4.38 & 10.83 \\
\hline 14 & $\vdots .3$ & $15.5 i$ & 0.23 & 12.:2 \\
\hline 15 & 1.2 & 90.13 & 0.25 & 11.37 \\
\hline 16 & j. $\overline{4}$. & 20.02 & 0.21 & i1.57 \\
\hline 17 & 3.1 & 32,07 & 1.30 & 12.38 \\
\hline 13 & 1.4 & 34.36 & 8.35 & 13.45 \\
\hline 19 & 2.3 & 30.3 & 0.37 & 1.42 \\
\hline
\end{tabular}


contd

SAMPLE ND. EFFLUERT PORE VOL. ELUTED SUH ELUTED PPE H3BO3 ELUTED Ig $\mathrm{KJBOJ}$ of $\mathrm{H} 3 \mathrm{BO}$

$\begin{array}{rrrrr}1 & 8.57 & 0.31 & 0.27 & 0.27 \\ 2 & 6.90 & 0.51 & 0.14 & 0.40 \\ 3 & 12.64 & 1.37 & 1.10 & 1.50 \\ 4 & 12.60 & 1.98 & 0.78 & 2.28 \\ 5 & 6.60 & 3.16 & 0.77 & 3.64 \\ 6 & 2.92 & 4.12 & 0.26 & 3.30 \\ 7 & 7.47 & 4.83 & 0.53 & 3.83 \\ 8 & 3.35 & 5.67 & 0.26 & 4.09 \\ 9 & 2.10 & 6.37 & 0.13 & 4.22 \\ 10 & 1.77 & 6.13 & 0.26 & 4.48 \\ 11 & 1.47 & 9.76 & 0.19 & 4.67 \\ 12 & 1.63 & 11.70 & 0.14 & 4.81 \\ 13 & 0.89 & 13.61 & 0.11 & 4.92 \\ 14 & 0.74 & 15.31 & 0.10 & 5.02 \\ 15 & 0.81 & 18.13 & 0.11 & 5.12 \\ 16 & 0.68 & 20.62 & 0.09 & 5.21 \\ 17 & 0.91 & 33.09 & 0.74 & 5.95 \\ 18 & 1.33 & 34.96 & 0.19 & 6.14 \\ 19 & 1.49 & 36.21 & 0.15 & 6.27\end{array}$

SAMPLE MO. EFFLUERT PORE YOL. ELUTED SUM ELUTED

\begin{tabular}{|c|c|c|c|c|}
\hline & ppa IC & ELUTED & tI & $\log$ If \\
\hline 1 & 33.4 & 0.31 & 0.30 & 0.30 \\
\hline 2 & 45.3 & $0.5 !$ & 0.23 & 0.53 \\
\hline 3 & 40.7 & 1.37 & 6.59 & 1.12 \\
\hline 4 & 27.1 & 1.98 & -0.45 & 0.60 \\
\hline 5 & $54 . i$ & 3.16 & 2.15 & 3.13 \\
\hline 6 & 23.0 & 4.12 & -1.11 & 2.02 \\
\hline 7 & $23 . !$ & 4.83 & $-\hat{b} . \varepsilon 1$ & i.2i \\
\hline 3 & 31.4 & 5.67 & -0.23 & 0.96 \\
\hline 3 & 28.3 & 0.37 & $-i .42$ & 4.55 \\
\hline 10 & 33.6 & 3.13 & -4.68 & 0.47 \\
\hline 11 & 26.4 & 9.76 & $-1, j j$ & -0.24 \\
\hline 12 & 25.6 & $1: .70$ & $-1.7 !$ & -2.5 \\
\hline 43 & $3 i .7$ & ij.ti & $-\hat{0}, 4 \hat{0}$ & -3.63 \\
\hline 19 & 37.0 & 15.31 & -1.70 & -9.72 \\
\hline 15 & 27.7 & 13.13 & -1.47 & -5.19 \\
\hline ib & $35 .:$ & 20.62 & 0.26 & -5.93 \\
\hline 17 & 26.1 & 30.99 & -16.35 & $-1 \dot{0} .26$ \\
\hline$: 8$ & 45.7 & 34.95 & 2.19 & -14.98 \\
\hline 19 & $2: .5$ & 30.21 & -1.63 & -15.72 \\
\hline
\end{tabular}

SAMPLE NO. EFFLUEMT FOFE VOL. ELUTED SUN ELUTED DPE SDS ELUTED \$p 504 g. 504

\begin{tabular}{|c|c|c|c|c|}
\hline 1 & 150 & 0.31 & 2.06 & 2.06 \\
\hline 2 & 180 & 0.51 & 1.95 & 4.01 \\
\hline 3 & 270 & 1.37 & 16.44 & 20.95 \\
\hline 4 & 160 & 1.98 & 4.68 & 25.14 \\
\hline 5 & 130 & 3.16 & 5.37 & 30.54 \\
\hline 6 & 120 & 4.12 & 3.37 & 33.73 \\
\hline$i$ & 30 & 4.83 & -0.44 & 33.49 \\
\hline g & 120 & 5.67 & 2.97 & 36.45 \\
\hline ? & 100 & 6.37 & 1.02 & 37.47 \\
\hline 10 & 96 & 8.13 & 1.83 & 59.38 \\
\hline 11 & 88 & 9.76 & 0.34 & 39.24 \\
\hline 12 & 96 & 11.70 & 2.02 & 41.65 \\
\hline 13 & 83 & 13.61 & $-\dot{0} . \dot{b} \dot{u}$ & 41. ivo \\
\hline 14 & 79 & 15.9: & $-1.8 i$ & 37.39 \\
\hline 15 & 85 & 18.13 & -0.23 & 37.16 \\
\hline 16 & 83 & 20.32 & -6.32 & 36.38 \\
\hline 17 & 76 & 33.09 & -12.96 & 25.42 \\
\hline 18 & $7 \dot{b}$ & 34.96 & -1.94 & 23.46 \\
\hline 19 & 84 & 36.21 & -4.20 & 23.22 \\
\hline
\end{tabular}

SAMPLE NO. EFFLUENT PORE VOL. ELUTED SUN ELUTED PDS TOC ELUTES DO TOC 19 TOC

\begin{tabular}{|c|c|c|c|c|}
\hline 1 & 15.8 & 0.31 & 0.48 & 0.18 \\
\hline 2 & 9.9 & 0.51 & 0.18 & 6.65 \\
\hline 3 & 6.8 & 1.37 & $0.5 i$ & 1.17 \\
\hline 4 & 3.7 & i. is & 0.17 & 1.54 \\
\hline 5 & 6.5 & 3.16 & 0.68 & 2.01 \\
\hline 6 & 4.3 & 4.12 & 0.33 & 2.34 \\
\hline $\bar{i}$ & 3.0 & 4.63 & 0.15 & 2.49 \\
\hline 8 & 2.5 & $5.6 i$ & 1.13 & 2.52 \\
\hline 9 & 3.0 & 6.37 & 0.14 & 2.78 \\
\hline 10 & 2.4 & 3.15 & 0.26 & j. 32 \\
\hline 11 & 2.3 & 3.70 & 4.2 & 3.24 \\
\hline 12 & 2.5 & $1 i . i 0$ & 0.30 & 3.53 \\
\hline 13 & $\mathbf{j . \hat { v }}$ & 1.3 .63 & 0.40 & 3.13 \\
\hline is & 2.4 & 15.71 & $\dot{0} . \overline{2} 2$ & 4.25 \\
\hline 15 & 2.5 & 18.13 & 3.34 & 4.57 \\
\hline 16 & $2 . \div$ & 26.62 & 8.36 & 4.35 \\
\hline 17 & $\mathbf{1 . 3}$ & 3.67 & $0.4:$ & 5.37 \\
\hline 18 & 1.2 & 34,95 & 0.65 & 5.32 \\
\hline 3 & 1.3 & 50.2 & 0.04 & 5.45 \\
\hline
\end{tabular}


TABLE C.15. Mass Balance for Flow Through Leach Test on Crushed Grout with Groundwater (MBBCGT)

SAMPLE NO. EFFLLENT PORE YOL. ELUTED SUH ELUTED

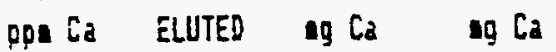

$\begin{array}{rrrrr}1 & 1.44 & 0.37 & -4.32 & -4.32 \\ 2 & 1.62 & 1.14 & -5.95 & -13.27 \\ 3 & 1.76 & 1.06 & -6.03 & -19.30 \\ 4 & 1.28 & 2.55 & -10.41 & -29.71 \\ 5 & 1.71 & 3.31 & -8.82 & -38.53 \\ 6 & 5.84 & 4.53 & -13.06 & -51.61 \\ 7 & 5.83 & 5.07 & -5.79 & -57.41 \\ 8 & 8.51 & 5.82 & -7.61 & -65.02 \\ 9 & 6.71 & 0.15 & -3.48 & -68.50 \\ 10 & 9.52 & 7.58 & -14.21 & -82.71 \\ 11 & 20.30 & 9.04 & -11.14 & -93.85 \\ 12 & 24.40 & 10.21 & -7.80 & -101.75 \\ 13 & 29.70 & 11.16 & -5.34 & -107.10 \\ 14 & 28.8 & 11.67 & -2.97 & -110.06 \\ 15 & 28.0 & 12.43 & -4.55 & -114.61 \\ 16 & 29.2 & 12.85 & -2.41 & -117.02\end{array}$

SAMPLE NO. EFFLUENT PORE YOL. ELUTED SUK ELUTED

\begin{tabular}{|c|c|c|c|c|}
\hline & PPE K & ELUTED & $\log$ & $0 K$ \\
\hline 1 & 22.00 & 0.37 & 1.12 & 1.12 \\
\hline 2 & 20.00 & 1.19 & 2.01 & 3.13 \\
\hline 3 & 15.60 & 1.66 & 0.80 & 3.93 \\
\hline 4 & 11.00 & 2.55 & 0.61 & 4.54 \\
\hline$\xi$ & 8.70 & 3.31 & 0.15 & 4.69 \\
\hline 6 & 8.20 & 4.53 & 0.10 & 4.79 \\
\hline$i$ & 8.40 & 5.07 & 0.07 & 4.86 \\
\hline 8 & 7.90 & 5.32 & 0.02 & 4.38 \\
\hline 9 & 9.00 & b. 15 & 0.08 & 4.96 \\
\hline 10 & 9.40 & $7.5 B$ & 0.49 & 5.45 \\
\hline 11 & 8.80 & 9.04 & 0.31 & 5.76 \\
\hline 12 & 3.20 & 10.21 & 0.10 & 5.36 \\
\hline 13 & 8.30 & 11.16 & 6.20 & 5. ร6 \\
\hline 14 & 7.7 & {$[1.67$} & -0.01 & 5.95 \\
\hline$: 5$ & 7.0 & $22.4 j$ & -0.13 & 5.32 \\
\hline 16 & 7.0 & 12.55 & $-\dot{y} \cdot \hat{0} \bar{i}$ & 5.75 \\
\hline
\end{tabular}

SAYPLE NO. EFFLUENT PGRE VOL, ELUTED SUM ELUTED pps $\mathrm{Ha}$ ELUTED $\mathrm{Ma}$ Ma

\begin{tabular}{|c|c|c|c|c|}
\hline 1 & 681.00 & 0.37 & 51.89 & 51.89 \\
\hline 2 & 633.00 & 1.19 & 101.06 & $: 52.97$ \\
\hline$j$ & 433.60 & 1.66 & 45.36 & 196.32 \\
\hline 4 & 262.00 & 2.55 & 45.67 & 245.42 \\
\hline 5 & 158.00 & 3.31 & 21.61 & 265.03 \\
\hline 6 & 91.90 & 4.53 & 17.45 & $2 \mathrm{a}_{2} \cdot 48$ \\
\hline 7 & $B \hat{v} .00$ & 5.07 & 6.35 & 283.83 \\
\hline 8 & 65.00 & 5.82 & 6.41 & 295.24 \\
\hline 9 & 68.60 & 6.15 & 3.03 & 298.20 \\
\hline 10 & 61.00 & 7.56 & 11.01 & $36 \% .20$ \\
\hline 11 & 36.00 & 9.04 & 4.06 & 313.34 \\
\hline 12 & 29.90 & 10.21 & 1.23 & 314.56 \\
\hline 13 & 27.60 & 11.16 & 0.53 & 315.09 \\
\hline 14 & 29 & 11.67 & 0.41 & 315.51 \\
\hline 15 & 29 & 12.53 & 0.00 & 310.11 \\
\hline 16 & $2 B$ & 12.85 & $0.3 \hat{0}$ & 316.40 \\
\hline
\end{tabular}

SAMFLE NO. EFFLUENT FORE VOL. ELUTED SUM ELUTED ppa Al ELUTED ag Al ag

\begin{tabular}{|c|c|c|c|c|}
\hline 1 & 11.00 & 0.37 & $0,8 b$ & $0.80^{\circ}$ \\
\hline 2 & 17.30 & 1.14 & 2.83 & 3.69 \\
\hline 3 & 12.60 & $1.66^{\circ}$ & 1.39 & 5.08 \\
\hline 4 & 5.76 & 2.55 & 1.48 & 6.16 \\
\hline 5 & 1.35 & $3.3:$ & 0.20 & 6.30 \\
\hline$\dot{b}$ & 0.17 & 4.55 & 0.02 & 2.36 \\
\hline 7 & 4.13 & 5.67 & $\hat{\mathrm{B}} \cdot \hat{\mathrm{B}} \hat{\mathrm{v}}$ & 0.5 \\
\hline 8 & $0.3 \bar{i}$ & 5.32 & $-\dot{v}, \dot{j 0}$ & 5.38 \\
\hline 9 & 0.12 & 6.15 & 0.00 & $\leq .30$ \\
\hline 10 & 0.11 & $\bar{i} .5 \bar{E}$ & 0.60 & 6.30 \\
\hline$: 1$ & 3.03 & 5.04 & $-3,4$ & 6. \\
\hline 12 & $\hat{b}, 0 \overline{3}$ & $i \hat{4} .23$ & -3.02 & 2.35 \\
\hline 13 & 0.04 & 11.16 & $-i j .0 i$ & 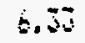 \\
\hline 14 & 0.03 & 11.67 & $-0, i 1$ & $0.5 i$ \\
\hline$: 5$ & 60.15 & 12.43 & $-4,42$ & 0.31 \\
\hline 10 & 0.03 & $: 2.85$ & $-\hat{i}, \hat{i} \hat{j}$ & $0.3 i$ \\
\hline
\end{tabular}


TABLE C.15. contd

SAHFLE NO. EFFLUENT PORE VOL. ELLUTED SUK ELUTED pp $\mathrm{Si}$ ELUTED $29 \mathrm{Si}$ $2 \mathrm{5i}$

$\begin{array}{rrrrr}1 & 16.50 & 0.37 & 0.12 & 0.12 \\ 2 & 25.60 & 1.14 & 1.74 & 1.86 \\ 3 & 24.30 & 1.66 & 1.03 & 2.90 \\ 4 & 17.40 & 2.55 & 0.46 & 3.35 \\ 5 & 12.10 & 3.31 & -0.47 & 2.88 \\ 6 & 10.30 & 4.53 & -1.23 & 1.66 \\ 7 & 9.32 & 5.07 & -0.59 & 1.67 \\ 6 & 9.57 & 5.82 & -0.87 & 0.20 \\ 9 & 9.62 & 6.15 & -0.38 & -0.18 \\ 10 & 8.93 & 7.58 & -1.86 & -2.04 \\ 11 & 9.10 & 7.04 & -1.84 & -3.86 \\ 12 & 3.37 & 10.21 & -1.51 & -5.39 \\ 13 & 10.30 & 11.16 & -0.35 & -6.34 \\ 11 & 9.8 & 11.67 & -0.57 & -6.91 \\ 15 & 8.9 & 12.43 & -0.39 & -7.90 \\ 16 & 3.7 & 12.85 & -0.55 & -0.44\end{array}$

SARFLe ho. EFFLUENT PORE VOL. ElUted SUL ELUTED

\begin{tabular}{|c|c|c|c|c|}
\hline & $p p=F$ & ELUTED & $\lg F$ & $\operatorname{~g} F$ \\
\hline 1 & 40.00 & 0.37 & 3.12 & 3.12 \\
\hline 2 & 51.00 & 1.14 & $8.3 !$ & 11.44 \\
\hline 3 & 35.00 & 1.66 & 3.84 & 15.27 \\
\hline 4 & 21.00 & 2.55 & 3.90 & 18.17 \\
\hline 5 & 13.00 & 3.31 & 2.03 & 21.20 \\
\hline b & 9.60 & $4.5 j$ & 2.22 & 23.42 \\
\hline 7 & 10.00 & 5.07 & 1.10 & 24.52 \\
\hline 8 & 8. (6) & 5.82 & 1.20 & 25.72 \\
\hline 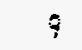 & 10.00 & 6.15 & $0.6 i$ & 26.39 \\
\hline 10 & 10.00 & $\bar{i} .50$ & 2.30 & 29.30 \\
\hline ii & 7.30 & 9.64 & 2.12 & 31.42 \\
\hline 12 & 7.00 & 10.21 & 1.78 & 33.19 \\
\hline 15 & 7.40 & 11.16 & 1.40 & 34.60 \\
\hline 14 & 8.3 & 11.67 & 0.90 & 55.50 \\
\hline 5 & 9.1 & 12.43 & 1.40 & 30.30 \\
\hline ió & $\{.8$ & 32.35 & 0.84 & 37.73 \\
\hline
\end{tabular}

SAMFLE NO. EFFLUENT PQRE VOL. ELUTEU SUM ELUTEJ PPE MQ ELUTED Hg Hg

$\begin{array}{lllll}1 & 0.06 & 0.37 & -1.10 & -1.10 \\ 2 & 0.06 & 1.14 & -2.29 & -3.40 \\ 3 & 0.06 & 1.66 & -1.55 & -4.75 \\ 4 & 0.06 & 2.55 & -2.05 & -7.00 \\ 5 & 0.42 & 3.31 & -2.21 & -9.81 \\ 6 & 5.07 & 4.53 & -2.33 & -12.14 \\ 7 & 5.26 & 5.07 & -0.94 & -13.07 \\ 8 & 6.87 & 5.82 & -1.14 & -14.22 \\ 9 & 5.55 & 6.15 & -0.60 & -14.01 \\ 10 & 5.92 & 7.56 & -2.47 & -17.23 \\ 11 & 9.37 & 9.04 & -1.45 & -18.73 \\ 12 & 9.85 & 10.21 & -1.04 & -19.77 \\ 13 & 11.30 & 11.16 & -0.55 & -20.32 \\ 14 & 10.80 & 11.67 & -0.35 & -20.66 \\ 15 & 9.75 & 12.43 & -0.66 & -21.32 \\ 16 & 9.97 & 12.85 & -0.36 & -21.68\end{array}$

SAYPLE NO. EFFLUEHT PORE VOL. ELUTES SUK ELUTED

\begin{tabular}{|c|c|c|c|c|}
\hline & $p p=N 03$ & ELUTED & ig $\mathrm{HO3}$ & NQS \\
\hline 1 & 40.00 & 0.37 & 3.is & 3.16 \\
\hline 2 & 17.00 & 1.14 & 2.70 & 5.74 \\
\hline 3 & 8.50 & 1.00 & 0.93 & 6.87 \\
\hline 4 & 3.30 & 2.55 & $0.7 \hat{v}$ & 7.58 \\
\hline 5 & 0.90 & 3.31 & ค.:โ3 & $7 . i 1$ \\
\hline 6 & 0.05 & 4.53 & $-(i, j)$ & 7.65 \\
\hline 7 & 0.05 & 5.07 & -3.01 & $7.0 \overline{9}$ \\
\hline 8 & 0.05 & 5.82 & $-\dot{\mathrm{j}} .01$ & $\overline{1.60}$ \\
\hline 9 & 4.65 & 6.15 & $-3,00$ & $i .45$ \\
\hline 10 & 0.05 & 7.55 & -0.02 & 7.56 \\
\hline 11 & 0.05 & 5.04 & $-0.0 \%$ & 7.64 \\
\hline 12 & 0.05 & 10.21 & -0.61 & 7.65 \\
\hline $\mathbf{i j}$ & 0.05 & $11 .: 15$ & $-j_{1}, i j$ & $\bar{i} .62$ \\
\hline 14 & 4.05 & di.5T & $-\dot{j} \cdot 31$ & $\bar{i} .8 i$ \\
\hline 15 & 6. 05 & 12,43 & $-\dot{0}, 01$ & 7.31. \\
\hline 16 & 0.05 & 12.35 & -1.60 & $\bar{i} .6 \dot{\mathrm{v}}$ \\
\hline
\end{tabular}


TABLE C.15. contd

SAMPLE NO. EFFLUENT PORE VOL. ELUTED SUH ELUTED pDE HJBOZ ELUTED

$\begin{array}{lllll}1 & 12.18 & 0.37 & 0.94 & 0.94 \\ 2 & 15.10 & 1.14 & 2.43 & 3.37 \\ 3 & 10.09 & 1.66 & 1.08 & 4.45 \\ 4 & 5.41 & 2.55 & 0.96 & 5.41 \\ 5 & 2.78 & 3.31 & 0.40 & 5.81 \\ 6 & 1.54 & 4.53 & 0.31 & 6.12 \\ 7 & 1.26 & 5.07 & 0.11 & 6.23 \\ 8 & 1.05 & 5.32 & 0.11 & 6.34 \\ 9 & 1.39 & 0.15 & 0.11 & 6.45 \\ 10 & 1.14 & 7.58 & 0.24 & 6.70 \\ 11 & 0.77 & 9.04 & 0.13 & 6.83 \\ 12 & 0.72 & 10.31 & 0.10 & 6.93 \\ 13 & 0.71 & 11.16 & 0.08 & 7.00 \\ 14 & 0.32 & 11.67 & 0.05 & 7.06 \\ 15 & 0.97 & 12.43 & 0.09 & 7.14 \\ 16 & 0.96 & 12.85 & 0.05 & 7.19\end{array}$

SAMPLE NO. EFFLUENT PORE VOL. PPE SO4 ELUTED

$\begin{array}{rrrrr}1 & 470.00 & 0.37 & 30.38 & 30.38 \\ 2 & 250.00 & 1.14 & 27.00 & 57.37 \\ 3 & 150.00 & 1.66 & 7.11 & 64.37 \\ 4 & 110.00 & 2.55 & 4.57 & 69.05 \\ 5 & 34.00 & 3.31 & -0.32 & 68.73 \\ 6 & 79.00 & 4.53 & -1.83 & 66.90 \\ 7 & 77.00 & 5.07 & -1.04 & 65.86 \\ 8 & 77.00 & 5.82 & -1.44 & 64.42 \\ 9 & 75.00 & 3.15 & -0.70 & 63.65 \\ 10 & 75.00 & 7.58 & -3.36 & 60.23 \\ 11 & 71.00 & 9.04 & -4.68 & 55.60 \\ 12 & 67.00 & 10.21 & -4.25 & 51.35 \\ 13 & 68.00 & 11.16 & -3.66 & 47.67 \\ 14 & 77 & 11.67 & -0.94 & 46.75 \\ 15 & 73 & 12.43 & -2.10 & 44.66 \\ 16 & 78 & 12.85 & -1.72 & 43.94\end{array}$


TABLE C.16. Mass Balance for Flow Through Leach Test on Crushed Grout with Groundwater (MBBCGI)

SAMPLE NO. EFFLUEHT PORE VOL. ELUTEE SUM ELUTED ppe Ca ELUTED a Ca

$\begin{array}{rrrrr}1 & 1.97 & 0.39 & -4.50 & -4.50 \\ 2 & 1.53 & 1.11 & -8.38 & -12.88 \\ 3 & 1.37 & 1.58 & -5.43 & -18.31 \\ 4 & 1.35 & 2.35 & -9.00 & -27.31 \\ 5 & 1.37 & 3.01 & -7.71 & -35.02 \\ 6 & 4.02 & 4.07 & -11.78 & -46.80 \\ 7 & 4.65 & 4.54 & -5.16 & -51.95 \\ 8 & 0.27 & 5.10 & -6.57 & -58.55 \\ 7 & 5.49 & 5.44 & -3.02 & -61.57 \\ 10 & 3.23 & 8.57 & -11.30 & -72.37 \\ 11 & 17.40 & 7.83 & -10.40 & -83.27 \\ 12 & 22.90 & 9.05 & -8.63 & -91.90 \\ 13 & 30.10 & 9.86 & -4.49 & -76.39 \\ 14 & 28.1 & 10.29 & -2.56 & -98.95 \\ 15 & 27.3 & 11.11 & -4.54 & -103.89 \\ 16 & 28.8 & 11.55 & -2.56 & -106.45\end{array}$

SAMFLE HO. EFFLLEHT PORE VOL. ELUTED SUH ELUTED

\begin{tabular}{|c|c|c|c|c|}
\hline & $p p=k$ & ELUTED & $\lg k$ & $\log K$ \\
\hline 1 & 28.10 & 0.39 & 1.68 & 1.58 \\
\hline 2 & 25.00 & $1.1 !$ & 2.65 & 4.33 \\
\hline 3 & 18.60 & 1.58 & 1.02 & 5.36 \\
\hline i & 12.00 & 2.35 & 0.69 & 6.05 \\
\hline 5 & 10,60 & 3.01 & 0.31 & 6.36 \\
\hline 6 & 6.20 & 4.07 & 0.09 & 6.45 \\
\hline 7 & 7.50 & 4.54 & 0.17 & 6.62 \\
\hline 0 & 5.40 & 5.16 & 0.08 & 6.70 \\
\hline$\overline{7}$ & 30.20 & 5.44 & 0.14 & 6.84 \\
\hline 10 & 11.00 & 6.57 & 0.77 & 3.58 \\
\hline 11 & 5.40 & 7.33 & 9.43 & 8.05 \\
\hline 12 & 3.20 & 9.65 & 0.10 & B.: : \\
\hline 13 & 7.50 & $\exists 36$ & 0.02 & 5.23 \\
\hline 14 & 7.2 & 10.29 & $-\hat{t}$ & B.11 \\
\hline 15 & 7.2 & 12.31 & $-3.1 !$ & $3.6 !$ \\
\hline io & 7.3 & 1.55 & -6.65 & $7 . \overline{70}$ \\
\hline
\end{tabular}

SAMPLE RO. EFFLUERT POFE VOL. ELUTED SLM ELUTED ppa Ha ELUTẼ du $\mathrm{Ha}$ ag Ha

$\begin{array}{rrrrr}1 & 1030.00 & 0.39 & 63.79 & 83.79 \\ 2 & 816.00 & 1.11 & 121.76 & 215.55 \\ 3 & 525.00 & 1.50 & 56.24 & 255.79 \\ 4 & 297.00 & 2.35 & 44.73 & 306.57 \\ 5 & 179.00 & 3.01 & 21.73 & 322.30 \\ 6 & 74.20 & 4.07 & 11.15 & 335.45 \\ 7 & 94.40 & 4.54 & 0.97 & 340.42 \\ 8 & 74.00 & 5.16 & 6.49 & 346.32 \\ 9 & 76.00 & 5.44 & 3.65 & 349.37 \\ 10 & 67.00 & 6.57 & 10.15 & 360.12 \\ 11 & 38.70 & 7.83 & 3.69 & 363.61 \\ 12 & 29.10 & 9.05 & 1.07 & 364.86 \\ 13 & 26.30 & 9.86 & 0.23 & 355.30 \\ 14 & 23 & 10.29 & 0.27 & 365.37 \\ 15 & 20 & 11.11 & 0.46 & 365.82 \\ 16 & 27 & 11.55 & 0.20 & 366.32\end{array}$

SAMPLE HO. EFFLUEHT FGRE YOL. ELUTES SUM ELUITED

\begin{tabular}{|c|c|c|c|c|}
\hline & po: ñi & ELUTES & $\$ \mathrm{Al}$ & 294 \\
\hline $\mathrm{i}$ & 17.90 & 0.39 & 1.45 & 1.98 \\
\hline 2 & 22.40 & :.:1 & 3.43 & \$. 泣 \\
\hline 3 & 16.50 & 1.58 & 1.55 & $6.5 t$ \\
\hline 4 & $7 . i 1$ & 2.35 & $\therefore .25$ & $i .3 \hat{~}$ \\
\hline$\Xi$ & $2 .: 4$ & $j_{2} \mathrm{ij}$ & 0.28 & 5.11 \\
\hline a & 6.37 & 4.67 & B. & $3 .: 4$ \\
\hline$i$ & 0.20 & 4. 34 & 0.01 & E.15 \\
\hline$\vdots$ & 3.16 & S.:S & 0.06 & B. $i E$ \\
\hline$\vdots$ & 8.23 & 5.44 & B. 34 & 8.15 \\
\hline 20 & 6.16 & $2 . \bar{j}$ & 0.02 & $\vdots .0 \mathrm{E}$ \\
\hline $1 !$ & 0.74 & 7.53 & $-\therefore$ & 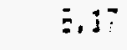 \\
\hline$i z$ & 0.30 & $\overline{105}$ & -8.63 & ล. \\
\hline 13 & 0.6 & i.js & $-1, \because$ & $\Xi: Z$ \\
\hline i4 & 6.63 & 10.29 & $-i, 8$ & 3.11 \\
\hline$\vdots$ & 0.0 & .1.1! & 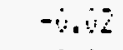 & B. \\
\hline$i=$ & 18 & :2.85 & $-i, \therefore$ & $\vdots 6$ \\
\hline
\end{tabular}


TABLE C.16.

contd

SAMPLE NO. EFFLUENT PQRE VOL. ELUTED SUM ELUTED pp』 $\mathrm{Si}$ ELUTED $\mathrm{Si} \quad \mathrm{Si}$

$\begin{array}{rrrrr}1 & 20.00 & 0.39 & 0.42 & 0.42 \\ 2 & 29.00 & 1.11 & 2.16 & 2.57 \\ 3 & 21.20 & 1.58 & 1.23 & 3.80 \\ 4 & 19.00 & 2.35 & 0.66 & 4.46 \\ 5 & 13.30 & 3.01 & -0.24 & 4.22 \\ 6 & 11.80 & 4.07 & -0.73 & 3.49 \\ 7 & 9.91 & 4.54 & -0.5 ! & 2.96 \\ 8 & 8.52 & 5.16 & -0.81 & 2.17 \\ 9 & 3.86 & 5.44 & -0.37 & 1.81 \\ 10 & 7.66 & 0.57 & -1.77 & 0.03 \\ 11 & 7.47 & 7.83 & -2.03 & -2.00 \\ 12 & 3.19 & 9.05 & -1.78 & -3.77 \\ 13 & 10.20 & 9.36 & -0.83 & -4.00 \\ 14 & 8.6 & 10.29 & -1.55 & -5.15 \\ 15 & 3.4 & 11.11 & -1.16 & -6.31 \\ 16 & 8.6 & 11.55 & -0.61 & -5.92\end{array}$

SAMPLE ND. EFFLUENT PORE VOL. ELUTED SUK ELUTED

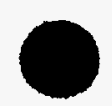

\begin{tabular}{|c|c|c|c|c|}
\hline & $p p \in F$ & ELUTED & g $F$ & 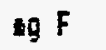 \\
\hline 1 & 57.00 & 0.39 & 4.71 & 4.71 \\
\hline 2 & 63.00 & d.i! & 9.31 & 14.02 \\
\hline$j$ & 43.00 & 1.58 & 4.27 & 18.29 \\
\hline 4 & 24.00 & 2.35 & 3.87 & 22.16 \\
\hline 5 & 14.00 & 3.01 & 1.70 & 24.07 \\
\hline 6 & 11.00 & 4.07 & 2.38 & 26.45 \\
\hline $\bar{i}$ & 12.00 & 4.54 & 1.jó & 27.60 \\
\hline$B$ & 9.30 & 5.16 & 1.17 & 28.77 \\
\hline 9 & 12.00 & 5.44 & 0.69 & 29.46 \\
\hline 10 & 11.00 & 6.57 & 2.54 & 31.59 \\
\hline 11 & 7.80 & 7.83 & 1.97 & 33.56 \\
\hline 12 & 7.10 & 9.05 & 1.72 & 35.68 \\
\hline 13 & $6.5 i$ & 9.86 & 1.04 & 36.72 \\
\hline i4 & 8.5 & $: 0.27$ & 0.74 & $37 \div$ \\
\hline$i 5$ & 3.1 & $11,1 !$ & $1.5 !$ & 78.96 \\
\hline : & 5.7 & 11.55 & 0.79 & 39.75 \\
\hline
\end{tabular}

5AMPLE NO. EFFLUENT POAE YOL. ELUTED SIMA ELUTES

\begin{tabular}{|c|c|c|c|c|}
\hline & ppe Hq & ELUTED & Ig & ing \\
\hline 1 & 0.06 & 0.39 & -1.16 & -1.16 \\
\hline 2 & 0.06 & 1.11 & -2.15 & -3.31 \\
\hline 3 & 0.166 & 1.58 & -1.90 & $-4 i 31$ \\
\hline 4 & 0.06 & 2.35 & -2.27 & $-i .00$ \\
\hline 5 & 0.17 & 3.01 & -1.95 & -3.90 \\
\hline 6 & 3.40 & 4.07 & -1.27 & -10.22 \\
\hline 7 & 3.42 & 4.54 & -1.06 & -11.29 \\
\hline 8 & 4.89 & 5.26 &.$-1.2 !$ & -12.50 \\
\hline 9 & 2.39 & 5.44 & -0.69 & -13.19 \\
\hline 10 & 3.21 & 6.57 & $-2.6 \mathrm{i}$ & -15.00 \\
\hline 11 & 7.36 & 7.63 & -1.79 & -11.57 \\
\hline 12 & 3.14 & 9.05 & $-1.2 \hat{i}$ & -18.55 \\
\hline 13 & 11.20 & 9.86 & -0.48 & -15.34 \\
\hline 14 & 9.97 & 10.29 & -0.35 & -19.72 \\
\hline 15 & 9.29 & 11.11 & -0.83 & -20.54 \\
\hline 16 & 9.46 & 11.55 & -3.93 & -20.57 \\
\hline
\end{tabular}

SAMPLE NO. EFFLUENT FORE VOL. ELUTES SUH ELUTES ppa NOJ ELUTED eg 303 og 105

\begin{tabular}{|c|c|c|c|c|}
\hline 1 & 40.00 & 0.39 & 3.33 & 3.33 \\
\hline 2 & 24,00 & 1.1! & 3.68 & 7.61 \\
\hline 3 & 32.00 & 1.58 & 1.20 & 8.20 \\
\hline 4 & 5.00 & 2.35 & 0.81 & 9.01 \\
\hline 5 & 1.80 & 3.01 & 0.24 & 9.25 \\
\hline 6 & 3.70 & 4.07 & $0 . i 4$ & 9.38 \\
\hline 7 & i0.5 & 4.54 & $-\hat{b}, 0 i$ & 3.37 \\
\hline 8 & 10.5 & 5.16 & $-0.0 !$ & 9.36 \\
\hline 9 & 6.5 & 5.94 & $-6, i j$ & 3.25 \\
\hline 10 & 0.5 & 6.57 & -6.62 & 9.33 \\
\hline 11 & 0.5 & 7.85 & -3.63 & 9.30 \\
\hline 12 & 6.5 & $9, \mathrm{is}$ & -1.03 & 9.20 \\
\hline 13 & 0.5 & 7.00 & $-3, i_{2} 2$ & 9.26 \\
\hline 14 & 60.5 & 16.29 & $-6.6 !$ & 7.25 \\
\hline : & iv.j & 11.11 & $-i 1.62$ & 9.23 \\
\hline is & 6.5 & AB.E & $-i \Delta . i j$ & 9.2 \\
\hline
\end{tabular}


TABLE C.16. contd

SARRPLE ND. EFFLUERT FORE VOL. ELUTED SUM ELUTED

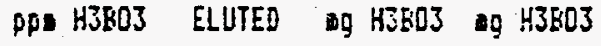

$\begin{array}{ccccc}1 & 16.18 & 0.39 & 1.32 & 1.32 \\ 2 & 18.87 & 1.11 & 2.85 & 4.17 \\ 3 & 12.90 & 1.58 & 1.26 & 5.44 \\ 4 & 6.34 & 2.35 & 0.99 & 6.42 \\ 5 & 3.20 & 3.01 & 0.40 & 6.23 \\ 6 & 1.20 & 4.07 & 0.19 & 7.02 \\ 7 & 1.14 & 4.54 & 0.08 & 7.10 \\ 8 & 1.20 & 5.16 & 0.11 & 7.22 \\ 9 & 1.39 & 5.44 & 0.06 & 7.28 \\ 10 & 1.26 & 6.57 & 0.22 & 7.50 \\ 11 & 0.85 & 7.83 & 0.14 & 7.64 \\ 12 & 0.74 & 9.05 & 0.10 & 7.74 \\ 13 & 0.68 & 9.86 & 0.06 & 7.80 \\ 14 & 0.81 & 10.29 & 0.04 & 7.64 \\ 15 & 0.65 & 11.15 & 0.09 & 7.93 \\ 16 & 0.83 & 11.55 & 0.05 & 7.98\end{array}$

SAMPLE NO. EFFLUENT PORE VOL. ELUTED SUK ELUTED PDE 504 ELUTED Ig SO4 SOS

\begin{tabular}{|c|c|c|c|c|}
\hline 1 & 470.00 & 0.39 & 32.02 & 32.02 \\
\hline 2 & 310.00 & 1.11 & 34.48 & 60.50 \\
\hline 3 & 190.00 & 1.58 & 10.45 & 76.95 \\
\hline 4 & 120.00 & 2.35 & $5.6 \hat{v}$ & 32.54 \\
\hline 5 & 39.60 & 3.01 & $\dot{4} .42$ & 82.97 \\
\hline 6 & 86.60 & 4.07 & 0.00 & 52.97 \\
\hline 7 & 33.00 & 4.54 & -0.30 & 82.67 \\
\hline 6 & $74.0 \hat{v}$ & 5.16 & -1.55 & 81.63 \\
\hline 9 & 79.00 & 3.44 & -6.42 & 6ै. \\
\hline $1 \hat{v}$ & 77.00 & 6.57 & $-\bar{i} .1 \bar{j}$ & 78.46 \\
\hline 11 & 78.00 & 7.03 & -2.16 & 70.33 \\
\hline 12 & 75.00 & $\$ .05$ & -2.37 & 73.46 \\
\hline 13 & 75.00 & $? .86$ & -1.70 & 71.55 \\
\hline 14 & 77 & 10.29 & -0.78 & 70.76 \\
\hline 15 & $8 \dot{0}$ & 11.11 & -1.12 & 69.64 \\
\hline 16 & $7 i$ & 11.55 & -1 - só & 65.78 \\
\hline
\end{tabular}


TABLE C.17. Mass Balance for Flow Through Leach Test on Crushed Grout with Groundwater (MBBCGC)

SAKPLE NG. EFFLUENT PORE VOL. ELUTED SLA ELUTED ppa $\mathrm{C}_{3}$ ELUTED $\mathrm{Ca}$ \& $\mathrm{Ca}$

$\begin{array}{rrrrr}1 & 1.40 & 0.32 & -3.74 & -3.74 \\ 2 & 2.23 & 1.22 & -10.35 & -14.08 \\ 3 & 1.85 & 1.92 & -8.10 & -23.18 \\ 4 & 1.63 & 2.96 & -12.09 & -34.27 \\ 5 & 3.70 & 3.94 & -10.96 & -45.23 \\ 6 & 8.63 & 5.92 & -14.99 & -60.22 \\ 7 & 7.16 & 6.04 & -6.47 & -66.69 \\ 3 & 10.60 & 6.87 & -8.06 & -74.75 \\ 9 & 6.77 & 7.21 & -3.58 & -78.33 \\ 10 & 8.45 & 6.51 & -13.22 & -91.54 \\ 11 & 17.50 & 9.75 & -10.21 & -101.75 \\ 12 & 19.70 & 10.36 & -4.73 & -106.48 \\ 13 & 26.90 & 11.58 & -7.59 & -114.07 \\ 14 & 27.6 & 12.20 & -3.76 & -117.04 \\ 15 & 27.8 & 13.07 & -5.25 & -123.08 \\ 16 & 29.2 & 13.59 & -2.98 & -126.06\end{array}$

SARPLE NO. EFFLUENT PORE VOL. ELUTED SUM ELUTED

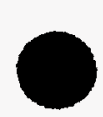
pp: $K$ ELUTED I $K$

ig $x$

\begin{tabular}{|c|c|c|c|c|}
\hline 1 & 25.00 & 0.32 & 1.18 & 1.18 \\
\hline 2 & 23.00 & 1.22 & 2.72 & 4.10 \\
\hline 3 & 15.00 & 1.92 & 1.08 & 5.18 \\
\hline 4 & 10.00 & 2.96 & 0.49 & 5.67 \\
\hline 5 & 3.00 & 3.94 & 0.04 & 5.71 \\
\hline b́ & 8.20 & 5.42 & 0.13 & 5.84 \\
\hline 7 & 8.30 & 0.04 & 0.07 & 5.30 \\
\hline ầ & 7.40 & 6.87 & -0.07 & $5.8 j$ \\
\hline 3 & 8.90 & $i .21$ & 0.08 & 5.91 \\
\hline 10 & 9.00 & 3.51 & 0.33 & 6.25 \\
\hline 11 & 8.50 & 9.75 & 0.19 & 6.43 \\
\hline 12 & 3.60 & 10.36 & 0.10 & 6.59 \\
\hline 13 & 8.46 & 11.50 & 0.16 & 6.69 \\
\hline 14 & 7.30 & 12.20 & -6.07 & 6.63 \\
\hline 15 & 3.30 & 13.37 & 0.00 & $6.6 j$ \\
\hline ic & 7.30 & 13.59 & -0.06 & 6.57 \\
\hline
\end{tabular}

SAMPLE NO. EFFLUENT PORE VOL. ELUTED SUH ELUTED PDI $\mathrm{Na}$ ELUTED

ig Na

ig $\mathrm{Ha}$

$\begin{array}{rrrrr}1 & 815 & 0.32 & 54.05 & 54.05 \\ 2 & 760 & 1.22 & 141.42 & 195.47 \\ 3 & 436 & 1.92 & 61.51 & 256.98 \\ 4 & 221 & 2.96 & 43.58 & 300.55 \\ 5 & 123 & 3.74 & 20.53 & 321.08 \\ 6 & 74 & 5.42 & 15.57 & 336.65 \\ 7 & 74 & 6.64 & 6.55 & 343.20 \\ 8 & 60 & 6.67 & 6.21 & 349.41 \\ 9 & 71 & 7.21 & 3.34 & 352.76 \\ 10 & 66 & 8.51 & 11.40 & 364.15 \\ 11 & 43 & 9.75 & 4.75 & 368.90 \\ 12 & 37 & 10.36 & 1.58 & 370.47 \\ 13 & 31 & 11.58 & 1.54 & 372.01 \\ 14 & 30 & 12.20 & 0.66 & 372.68 \\ 15 & 29 & 13.07 & 0.78 & 373.46 \\ 16 & 29 & 13.59 & 0.42 & 373.88\end{array}$

SAKPLE KD. EFFLUENT PORE VOL. ELUTED SUK ELUTED PPA Al ELUTED A Al SQ $\mathrm{Al}$

\begin{tabular}{|c|c|c|c|c|}
\hline 1 & 11.60 & 0.32 & $0.7 \%$ & 0.79 \\
\hline 2 & 19.30 & $i .22$ & 3.69 & 4.48 \\
\hline 3 & 12.00 & 1.92 & 1.78 & 0.26 \\
\hline 4 & 3.50 & 2.95 & 0.76 & 7.02 \\
\hline 5 & 0.27 & 3.94 & 0.04 & 7.05 \\
\hline 6 & 0.04 & 5.42 & -6.02 & 7.03 \\
\hline$i$ & 0.06 & 6.04 & -0.01 & $i .03$ \\
\hline$B$ & 40.3 & $6.3 \bar{T}$ & -0.02 & 7.01 \\
\hline 9 & 0.12 & 7.21 & 0.00 & 7.01 \\
\hline 10 & 0.12 & $3.5 i$ & $\dot{0} .01$ & 7.62 \\
\hline 11 & 0.04 & 9.75 & $-0,02$ & 7.00 \\
\hline 12 & 0.05 & 10.36 & -0.01 & 6.39 \\
\hline 13 & 0.04 & 11.58 & -0.02 & 6.78 \\
\hline 14 & 0.63 & $: 2.20$ & $-6.0 j$ & 6.97 \\
\hline 15 & 0.03 & $i \vec{j}, \vec{i}$ & $-3,0 \mathrm{OL}$ & 6.96 \\
\hline 16 & $0.0 j$ & $13.5 \%$ & $-0.0 \mathrm{~b}$ & 6.95 \\
\hline
\end{tabular}


TABLE C.17. contd

SAMFLE NO. EFFLUENT PORE VOL. ELUTED SUM ELUTED PPE $\mathrm{Si}$ ELUTED a $\mathrm{Si}$ a $\mathrm{Si}$

$\begin{array}{rrrrr}1 & 19.20 & 0.32 & 0.29 & 0.29 \\ 2 & 26.10 & 1.22 & 2.14 & 2.42 \\ 3 & 23.10 & 1.39 & 1.21 & 3.64 \\ 4 & 14.90 & 2.96 & -0.02 & 3.61 \\ 5 & 11.10 & 3.94 & -0.82 & 2.80 \\ 6 & 11.30 & 5.42 & -1.01 & 1.78 \\ 7 & 11.40 & 6.04 & -0.48 & 1.31 \\ 8 & 11.20 & 6.87 & -0.67 & 0.63 \\ 9 & 10.60 & 7.21 & -0.31 & 0.33 \\ 10 & 9.57 & 8.51 & -1.51 & -1.18 \\ 11 & 9.25 & 9.75 & -1.52 & -2.71 \\ 12 & 8.09 & 10.36 & -0.90 & -3.61 \\ 13 & 9.61 & 11.58 & -1.41 & -5.01 \\ 14 & 9.1 & 12.20 & -0.78 & -5.79 \\ 15 & 8.6 & 13.07 & -1.10 & -6.37 \\ 16 & 0.9 & 13.59 & -0.69 & -7.66\end{array}$

SAMPLE NO. EFFLUENT PORE VOL. ELUTED SUM ELUTED

\begin{tabular}{|c|c|c|c|c|}
\hline & ppo $F$ & ELUTED & $g F$ & $g F$ \\
\hline 1 & 44.00 & 0.32 & 2.98 & 2.98 \\
\hline 2 & 52.00 & 1.22 & 9.91 & 12.89 \\
\hline$j$ & 34.00 & 1.52 & 5.01 & 17.90 \\
\hline 4 & 16.00 & 2.96 & 3.95 & 21.34 \\
\hline 5 & 9.60 & 3.94 & 1.91 & 23.25 \\
\hline 6 & 7.00 & 5,42 & 2.06 & 25.31 \\
\hline 7 & 9.00 & 6.09 & 1.13 & 26.43 \\
\hline 8 & 7.20 & 6.87 & $1 .: 9$ & 27.62 \\
\hline 9 & 11.00 & 7.21 & 0.76 & 23.39 \\
\hline$t i$ & 9.20 & 8.51 & 2.42 & 30.80 \\
\hline 11 & $i .80$ & 9.75 & 1.94 & 32.79 \\
\hline 12 & 3.40 & 10.36 & 1.16 & 33.70 \\
\hline 13 & 7.30 & 11.58 & $1.7 i$ & 55.67 \\
\hline 14 & 9.6 & $: 2.20$ & 1.21 & 36.30 \\
\hline 15 & 8.8 & 13.07 & i. 54 & 38.42 \\
\hline$\dot{i}$ & $\overline{9.3}$ & 13.57 & 6.70 & $3 ? .40$ \\
\hline
\end{tabular}

SAMPLE NO. EFFLUENT PORE VOL. ELUTED SUN ELUTED

\begin{tabular}{|c|c|c|c|c|}
\hline & $p p=$ ho & ELUTED & $19 \mathrm{Mg}$ & Q \\
\hline 1 & 0.06 & 0.32 & -0.95 & -0.95 \\
\hline 2 & 0.06 & 1.22 & -2.69 & -3.64 \\
\hline 3 & 0.06 & 1.92 & -2.09 & -5.72 \\
\hline 4 & 0.20 & 2.76 & -3.07 & -3.79 \\
\hline 5 & 2.60 & 3.94 & -2.39 & -11.18 \\
\hline 6 & 5.40 & 5.42 & -1.77 & -12.75 \\
\hline 7 & 8.46 & 6.04 & -0.73 & -13.69 \\
\hline 8 & 9.29 & 6.87 & -0.64 & -14.52 \\
\hline 9 & 5.65 & 7.21 & -0.61 & -15.13 \\
\hline 10 & 6.35 & 8.51 & -2.13 & $-1 j .26$ \\
\hline 11 & 9.08 & 3.75 & -1.30 & $-: 8.56$ \\
\hline 12 & 7.64 & 10.36 & -0.83 & -11.39 \\
\hline 13 & 10.50 & 11.53 & -0.51 & -20.30 \\
\hline 14 & 10.60 & 12.20 & $-\hat{0} .45$ & $-2 \dot{\mathrm{j}} .75$ \\
\hline 15 & 10.06 & 13.07 & -0.74 & -21.50 \\
\hline 16 & 30.20 & ij.59 & $-3,42$ & -21.72 \\
\hline
\end{tabular}

SAKFLE NO. EFFLUEHT PORE YOL. ELUTED GUM ELUTES

\begin{tabular}{|c|c|c|c|c|}
\hline & ppg NDZ & ELUTED & 403 & $4 \mathrm{NOS}$ \\
\hline 1 & 40.00 & 0.32 & 2.73 & 2.73 \\
\hline 2 & 23.00 & 1.22 & 4.41 & 7.14 \\
\hline 3 & 9.40 & 1.72 & 1.39 & 8.53 \\
\hline 4 & 3.40 & 2.96 & 0.73 & 9.26 \\
\hline 5 & 0.50 & 3.74 & 0.60 & 7.35 \\
\hline 6 & 0.50 & 5.42 & 0.13 & 9.47 \\
\hline 7 & 0.50 & 6.09 & 0.35 & 5.52 \\
\hline$B$ & 0.50 & 6.67 & i. & 9.60 \\
\hline 9 & 3.50 & $7.2 !$ & 0.03 & 9.22 \\
\hline 10 & 0.50 & B.ji & a.1! & 7.74 \\
\hline 11 & 0.50 & 9.75 & 0.11 & 9.34 \\
\hline$: 2$ & 0.50 & 10.36 & 6.65 & 9.39 \\
\hline $1 j$ & 0.50 & 11.55 & 0.10 & 10.00 \\
\hline 14 & 0.50 & 12.20 & $\dot{0} .05$ & 10,05 \\
\hline 15 & $\hat{0} .5 \hat{v}$ & $: 3.57$ & 4.67 & $16 .: 3$ \\
\hline 16 & $0.5 i$ & S.3. & 3.14 & $i 6.17$ \\
\hline
\end{tabular}


TABLE C.17. contd

SAMPLE NO. EFFLUENT PORE VOL. ELUTED SUA ELUTED ppD 43803 ELUTED og H3603 in H3BO3

$\begin{array}{lllll}1 & 12.42 & 0.32 & 0.83 & 0.83 \\ 2 & 17.73 & 1.22 & 3.35 & 4.17 \\ 3 & 10.06 & 1.92 & 1.45 & 5.63 \\ 4 & 4.50 & 2.96 & 0.92 & 6.55 \\ 5 & 2.02 & 3.94 & 0.35 & 6.90 \\ 6 & 1.20 & 5.42 & 0.27 & 7.18 \\ 7 & 1.14 & 6.04 & 0.11 & 7.28 \\ 8 & 0.96 & 6.87 & 0.11 & 7.39 \\ 9 & 1.29 & 7.21 & 0.07 & 7.46 \\ 10 & 1.71 & 8.51 & 0.38 & 7.84 \\ 11 & 0.84 & 9.75 & 0.13 & 7.97 \\ 12 & 0.84 & 10.36 & 0.07 & 8.04 \\ 13 & 0.73 & 11.58 & 0.10 & 9.14 \\ 14 & 0.80 & 12.20 & 0.06 & 8.20 \\ 15 & 0.84 & 13.07 & 0.09 & 8.30 \\ 10 & 0.34 & 13.59 & 0.06 & 3.35\end{array}$

SAMPLE NO. EFFLUERT PORE VOL. ELUTED SUK ELUTED PPE SO4 ELUTED 504 SOS

\begin{tabular}{|c|c|c|c|c|}
\hline 1 & 380.00 & 0.32 & $20.1 !$ & 20.11 \\
\hline 2 & 290.00 & 1.22 & 37.25 & 59.37 \\
\hline 3 & 150.00 & 1.92 & 9.58 & 68.94 \\
\hline 4 & 99.00 & 2.96 & 2.89 & $7 ! .83$ \\
\hline$\Sigma$ & 80.60 & 3.34 & -1.26 & 70.58 \\
\hline 6 & 79.60 & 5.42 & -2.21 & 68.30 \\
\hline$i$ & 77.00 & 6.04 & -1.19 & 67.17 \\
\hline 8 & 75.00 & 6.37 & -1.75 & 65.22 \\
\hline$?$ & 75.00 & 7.21 & -0.80 & 64.42 \\
\hline 10 & 69.00 & 8.51 & -4.72 & 59.69 \\
\hline 11 & 76.00 & 9.75 & -2.65 & 57.04 \\
\hline 12 & 75.00 & 10.36 & -1.43 & 55.61 \\
\hline 13 & 76.00 & 11.58 & $-2.6 \mathrm{j}$ & 53.10 \\
\hline 14 & B! & 12.20 & -1.65 & 52.35 \\
\hline 15 & b8 & $\dot{1} \mathbf{j} . \dot{\mathrm{i} i}$ & -3.35 & 99.00 \\
\hline 16 & $7 !$ & 13.59 & -1.67 & 47.33 \\
\hline
\end{tabular}


TABLE C.18. Mass Balance for Flow Through Leach Test on Crushed Grout/Sediment with Groundwater (MBCGT)

SAKPLE NO. EFFLUENT PORE VOL. ELUTED SUK ELUTED

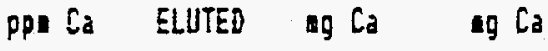

$\begin{array}{rrrrr}1 & 30.30 & 2.66 & -7.70 & -7.70 \\ 2 & 4.01 & 4.60 & -12.53 & -20.23 \\ 3 & 4.16 & 5.76 & -5.61 & -25.84 \\ 4 & 3.14 & 8.29 & -15.06 & -40.70 \\ 5 & 4.02 & 9.18 & -5.21 & -46.11 \\ 6 & 3.07 & 11.13 & -11.63 & -57.74 \\ 7 & 4.37 & 12.36 & -7.15 & -64.89 \\ 8 & 2.97 & 14.93 & -15.35 & -80.24 \\ 9 & 4.19 & 15.99 & -6.19 & -86.43 \\ 10 & 2.87 & 17.66 & -10.11 & -96.34 \\ 11 & 4.04 & 18.66 & -5.74 & -102.28 \\ 12 & 3.13 & 20.67 & -11.97 & -114.25 \\ 13 & 1.70 & 23.15 & -15.17 & -127.42 \\ 14 & 1.7 & 25.18 & -12.42 & -141.63 \\ 15 & 1.5 & 26.74 & -7.51 & -151.35 \\ 16 & 2.0 & 28.42 & -10.23 & -161.57 \\ 17 & 2.5 & 30.38 & -14.85 & -176.40 \\ 13 & 5.1 & 33.00 & -12.16 & -108.56\end{array}$

SAMPLE ND. EFFLUENT PORE YOL. ELUTED SUN ELUTED

\begin{tabular}{|c|c|c|c|c|}
\hline & ppe K & ELUTE』 & ag $K$ & $\lg K$ \\
\hline 1 & 7.70 & 2.66 & -0.03 & -6.03 \\
\hline 2 & 2.70 & $\therefore .60$ & -1.23 & -1.26 \\
\hline 3 & 1.50 & 5.76 & -0.60 & -1.94 \\
\hline 4 & 1.00 & 8.29 & -1.54 & -3.88 \\
\hline 5 & $\hat{\mathrm{v}} .60$ & 9.18 & -0.72 & -4.60 \\
\hline ó & 0.80 & 11.13 & -1.54 & -6.14 \\
\hline 7 & 0.70 & 12.36 & -0.98 & -7.12 \\
\hline ถิ & 0.00 & 14.33 & -2.03 & -9.15 \\
\hline 9 & 0.70 & 15.99 & -0.85 & $-i 0.00$ \\
\hline 10 & 0.50 & 17.68 & -1.39 & -11.37 \\
\hline 11 & 0.86 & 18.56 & -0.77 & -12.16 \\
\hline 12 & 0.60 & 20.67 & -1.63 & -13.79 \\
\hline 13 & 1.26 & 23.15 & -1.84 & $-i 5.6 j$ \\
\hline 14 & 0.5 & 25.18 & -1.58 & $-17.2 !$ \\
\hline j5 & 0.6 & 26.74 & -1.23 & -13.44 \\
\hline$: \dot{0}$ & 60.3 & 28.42 & -1.40 & $-: 5.82$ \\
\hline 17 & $2 . j$ & Jú. & -1.50 & -21.49 \\
\hline$\hat{i a ̂}$ & 3.6 & 33.60 & -1.60 & -25.50 \\
\hline
\end{tabular}

SAMPLE HO. EFFLUERT PORE VOL. ELUTED SUM ELUTED ppe $\mathrm{Ha}$ ELUTED ig $\mathrm{Ha}$ G $\mathrm{Ha}$

\begin{tabular}{|c|c|c|c|c|}
\hline 1 & 1260.00 & 2.66 & 370.03 & 370.03 \\
\hline 2 & 774.00 & 4.80 & 180.55 & 550.56 \\
\hline 3 & 549.00 & 5.76 & 56.66 & 607.24 \\
\hline 4 & 452.00 & 6.29 & 121.69 & 728.93 \\
\hline 5 & $36 j .00$ & 9.18 & 33.60 & 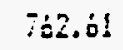 \\
\hline 6. & 310.00 & 11.13 & 62.60 & 225. 21 \\
\hline$i$ & 272.00 & 12.36 & .34 .22 & 359.43 \\
\hline 8 & 233.60 & 14.93 & 60.21 & 519.45 \\
\hline 9 & 220.00 & 15.99 & 25.23 & 942.73 \\
\hline 10 & 194.00 & 17.68 & 32.17 & 975.20 \\
\hline 11 & 202.60 & 18. ó & 18.54 & 994.64 \\
\hline 12 & 186.00 & 20.67 & 36.45 & $\$ 031.09$ \\
\hline 13 & 163.00 & 23.15 & 30.55 & 2065.04 \\
\hline 14 & 144 & 25.18 & $2 i .2 !$ & liv?b.35 \\
\hline $1 \Xi$ & 130 & 26.74 & 18.45 & \$115. 30 \\
\hline ib & 121 & 28.42 & $18 .: 7$ & 1130.47 \\
\hline 17 & 109 & 30.68 & 25.28 & 1156.74 \\
\hline 18 & 95 & 33.60 & 10.68 & 1933.43 \\
\hline
\end{tabular}

SAMFLE NO. EFFLUENT PQRE YOL. ELUTE SUM ELUTEU

\begin{tabular}{|c|c|c|c|c|}
\hline & PPA Al & ELUTED & A Al & $\Delta \mathrm{g}$ \\
\hline 1 & 6.23 & 2.86 & 1.84 & 1. 84 \\
\hline 2 & 18.30 & 4.60 & 4.37 & 6.22 \\
\hline$j$ & 9.77 & 5.76 & 1.07 & 3.27 \\
\hline 4 & 8.34 & 8.29 & 2.53 & $\$ .92$ \\
\hline 5 & 5.42 & 9.18 & 4.5 & 16.46 \\
\hline 6 & 4.38 & 11.13 & 1.65 & $: 4.51$ \\
\hline 7 & 4.73 & 12.30 & 0.85 & $12 .: 5$ \\
\hline$\vec{b}$ & 4.46 & 14.55 & $1.3=$ & 13.34 \\
\hline 5 & 4.67 & 15.57 & 0.47 & 13.37 \\
\hline 10 & 3.63 & 17.88 & $\dot{b} . \dot{b i}$ & 24.50 \\
\hline 11 & 3.58 & le. bt & 4.32 & 14.74 \\
\hline 12 & 2.97 & 20.57 & 0.55 & $: 5.00$ \\
\hline 25 & 2.83 & $\therefore . \mathrm{i}$ & 4. & 10.5 \\
\hline 14 & $\therefore .93$ & 25.18 & 0.42 & is.5 \\
\hline ij & 5.64 & 26.74 & 0.77 & $1=.34$ \\
\hline :t́ & ¿.4! & 3.42 & 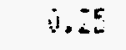 & 27.67 \\
\hline 17 & 1.05 & 30.30 & i. .6 & 17.35 \\
\hline 15 & 0.84 & 30 & 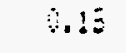 & 27.5 \\
\hline
\end{tabular}


contd

SAMPLE ND. EFFLUERT PORE VOL. ELUTED SUM ELUTED PPE Si ELUTED $\mathrm{Si}$ I $\mathrm{Si}$

$\begin{array}{rrrrr}1 & 126.00 & 2.66 & 33.26 & 33.26 \\ 2 & 132.00 & 4.80 & 28.20 & 61.46 \\ 3 & 119.00 & 5.76 & 11.25 & 72.71 \\ 4 & 110.00 & 8.29 & 27.07 & 99.78 \\ 5 & 91.30 & 9.18 & 7.65 & 107.43 \\ 6 & 72.50 & 11.13 & 12.63 & 120.06 \\ 7 & 63.20 & 12.36 & 6.63 & 126.74 \\ 8 & 47.10 & 14.33 & 9.29 & 136.03 \\ 9 & 46.20 & 15.59 & 3.73 & 139.75 \\ 10 & 37.20 & 17.68 & 4.23 & 143.98 \\ 11 & 47.80 & 13.66 & 3.62 & 147.60 \\ 12 & 43.40 & 20.67 & 6.43 & 154.63 \\ 13 & 33.10 & 23.15 & 5.06 & 157.09 \\ 14 & 26.0 & 25.18 & 2.52 & 161.60 \\ 15 & 22.7 & 26.74 & 1.35 & 162.96 \\ 16 & 21.6 & 28.42 & 1.25 & 164.20 \\ 17 & 19.5 & 30.88 & 1.25 & 165.45 \\ 18 & 16.1 & 33.04 & 0.74 & 166.19\end{array}$

SAMPLE HO. EFFLUENT PORE :OL. ELUTED SUY ELUTED ppa $F$ ELUTED U $F$ Ig $F$

\begin{tabular}{|c|c|c|c|c|}
\hline Mrir Le no. & ppa $F$ & ELUTED & ELG & of $F$ \\
\hline $\mathfrak{i}$ & 35.00 & 2. ós & 10.31 & 10.34 \\
\hline 2 & 30.00 & 4.50 & 3.11 & 17.45 \\
\hline 3 & 15.00 & 5.76 & 2.00 & 18.45 \\
\hline 4 & 12.60 & 8.29 & 3.28 & 22.73 \\
\hline 5 & 9.30 & 9.18 & 0.88 & 23.61 \\
\hline 6 & 7.00 & 11.13 & 1.43 & 25.04 \\
\hline$i$ & 6.50 & 12.30 & 0.83 & 25.87 \\
\hline 8 & 0.50 & 14.93 & 1.62 & 27.49 \\
\hline 9 & 0.30 & 15.39 & 0.69 & 28.13 \\
\hline 10 & 5.70 & 17.68 & 0.95 & 29.17 \\
\hline 11 & 6.40 & 18.66 & 0.65 & 27.22 \\
\hline 12 & b.jo & 20.57 & i.30 & 31.20 \\
\hline 13 & $b .40$ & 23.15 & 1.35 & 32.25 \\
\hline 14 & 6.8 & 25.18 & 1.44 & 34.29 \\
\hline 15 & 6.5 & 26.74 & 1.05 & 35.35 \\
\hline$: \dot{b}$ & 2.5 & 26.42 & 1.34 & 30.48 \\
\hline$\vdots j$ & 0.5 & 36.86 & 2.56 & JE.14 \\
\hline 12 & 7.3 & 33.00 & $1 . .22$ & 39.77 \\
\hline
\end{tabular}

SANPLE HO. EFFLUENT FQRE VOL. ELLUTED SUK ELUTED pp ho ELUTED ig Hg Ho

$\begin{array}{lllll}1 & 0.32 & 2.66 & -4.10 & -4.10 \\ 2 & 0.17 & 4.80 & -3.33 & -7.43 \\ 3 & 0.16 & 5.76 & -1.50 & -6.93 \\ 4 & 0.06 & 8.27 & -3.97 & -12.90 \\ 5 & 0.06 & 9.18 & -1.40 & -14.30 \\ 6 & 0.06 & 11.13 & -3.06 & -17.36 \\ 7 & 0.06 & 12.36 & -1.93 & -19.27 \\ 8 & 0.07 & 14.93 & -4.03 & -23.32 \\ 9 & 0.15 & 15.99 & -1.65 & -24.98 \\ 10 & 0.06 & 17.68 & -2.05 & -27.53 \\ 11 & 0.15 & 18.66 & -1.53 & -29.15 \\ 12 & 0.09 & 20.67 & -3.15 & -32.31 \\ 13 & 0.09 & 23.15 & -3.37 & -36.19 \\ 14 & 0.06 & 25.18 & -3.19 & -39.36 \\ 15 & 0.06 & 26.74 & -2.45 & -41.63 \\ 16 & 0.06 & 23.42 & -2.64 & -44.47 \\ 17 & 0.06 & 30.88 & -3.86 & -48.33 \\ 18 & 0.06 & 33.00 & -3.33 & -51.60\end{array}$

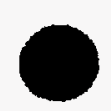

\begin{tabular}{|c|c|c|c|c|}
\hline SAMPLE NO. & $\begin{array}{l}\text { EFFLUERT } \\
\text { pPE NOJ }\end{array}$ & $\begin{array}{l}\text { FOFE VEL. } \\
\text { ELUTED. }\end{array}$ & $\begin{array}{l}\text { ELUTEI } \\
\text { AQ } \mathrm{KOJ}\end{array}$ & $\begin{array}{l}\text { SUM ELUTES } \\
\text { s) HIJ }\end{array}$ \\
\hline 1 & 58.60 & 2.56 & 17.35 & 17.35 \\
\hline 2 & 6.60 & 4.80 & 1,42 & 8.77 \\
\hline 3 & 1.70 & $5.7 \%$ & 0.19 & 18.76 \\
\hline 4 & 0.60 & a. 27 & 0.14 & 19.11 \\
\hline 5 & 0.60 & $\$ .18$ & 0.05 & 17.16 \\
\hline b & 0.50 & 11.15 & 0.09 & 19.25 \\
\hline 7 & 0.50 & 12.36 & 0.06 & 19.30 \\
\hline 8 & $\hat{0} .5 \hat{0}$ & 14.93 & 0.12 & 19.42 \\
\hline 9 & 0.50 & 15.99 & 0.65 & 19.76 \\
\hline 10 & 0.50 & 17.68 & 6.08 & $1 \div .54$ \\
\hline $1 i$ & 0.50 & 16.86 & 0.04 & 19.3 \\
\hline 12 & 0.50 & 20.67 & 0.09 & $: 936$ \\
\hline$! 3$ & 0.50 & 23.15 & v.: & 89.79 \\
\hline 14 & 0.50 & $25 .: 2$ & 6.09 & $1: .36$ \\
\hline 15 & 0.50 & 23.74 & i. 07 & 99.95 \\
\hline 36 & ป. $5 \dot{0}$ & $2 \mathrm{c} .42$ & 4.08 & $\overline{3}, 0 i$ \\
\hline 17 & $\dot{0} .50$ & 30.38 & 0.11 & 20.14 \\
\hline 16 & 0.50 & 33.60 & 4.10 & 26.3 \\
\hline
\end{tabular}




\section{TABLE C.18. contd}

SAKPLE NO. EFFLUENT PORE VOL. ELUTED SUH ELUTED ELLITED 9 H3BCZ $9 \mathrm{HJBOJ}$

$\begin{array}{rrrr}1 & 2.66 & 161.69 & 161.69 \\ 2 & 4.80 & 31.25 & 192.95 \\ 3 & 5.76 & 9.26 & 202.21 \\ 4 & 8.29 & 19.57 & 221.78 \\ 5 & 9.18 & 7.28 & 229.06 \\ 6 & 11.13 & 16.16 & 245.24 \\ 7 & 12.36 & 10.21 & 255.45 \\ 6 & 14.73 & 21.90 & 277.35 \\ 9 & 15.39 & 7.27 & 286.62 \\ 10 & 17.68 & 13.83 & 300.45 \\ 11 & 18.66 & 8.68 & 309.14 \\ 12 & 20.67 & 17.36 & 326.49 \\ 13 & 23.15 & 21.41 & 347.91 \\ 14 & 25.13 & 17.53 & 365.44 \\ 15 & 26.74 & 13.29 & 378.73 \\ 16 & 28.42 & 14.05 & 392.78 \\ 17 & 30.88 & 20.56 & 413.36 \\ 18 & 33.00 & 17.50 & 432.86\end{array}$

SAKPLE NO. EFFLUENT PORE VOL. E!UTES SUK ELUTED PPA SOA ELUTED ig 504 ing 504

\begin{tabular}{|c|c|c|c|c|}
\hline 1 & 380 & 2.66 & 83.69 & 88.09 \\
\hline 2 & 140 & 4.80 & 13.02 & $101 . i 1$ \\
\hline 3 & $1 \geq 5$ & 5.76 & 4.22 & 105.32 \\
\hline 4 & 100 & 8.29 & 3.99 & 109.31 \\
\hline 5 & 110 & 9.18 & 2.41 & 111.72 \\
\hline$\dot{0}$ & 126 & 11.13 & B. 79 & 120.50 \\
\hline 7 & 109 & 12.36 & 3.19 & 123.69 \\
\hline 8 & 103 & 14.83 & 4.92 & 128.61 \\
\hline 9 & 120 & 15.99 & 4.06 & $\{32.67$ \\
\hline 10 & 93 & 17.68 & 1.3 .3 & 134.00 \\
\hline 11 & $3 !$ & 18.66 & 0.55 & 134.56 \\
\hline 12 & 88 & 20.67 & 0.45 & 135.01 \\
\hline 13 & 34 & 23.15 & 2.23 & 137.24 \\
\hline 14 & 97 & 25.18 & 3.52 & 139.76 \\
\hline 15 & $9 !$ & 26.74 & 0.88 & 140.64 \\
\hline 16 & 78 & 25.42 & $2.2 \bar{i}$ & 192.91 \\
\hline 17 & 63 & 30.88 & -6.37 & 136.54 \\
\hline 18 & 78 & 33.60 & -1.51 & 154.03 \\
\hline
\end{tabular}


TABLE C.19. Mass Balance for Flow Through Leach Test on Crushed Grout/Sediment with Groundwater (MBCGC)

SAMPLE NO. EFFLUENT PORE VOL. ELUTED SUH ELUTED pp: Ca ElUTED $g \mathrm{Ca} \quad \mathrm{Ca}$

\begin{tabular}{|c|c|c|c|c|}
\hline 1 & 7.0 & 2.00 & -5.19 & -5.19 \\
\hline 2 & 2.3 & 5.92 & -11.16 & -16.35 \\
\hline$j$ & 1.5 & 9.32 & -9.82 & -20.17 \\
\hline 4 & 1.5 & 12.91 & -10.37 & -36.54 \\
\hline 5 & 1.5 & 16.64 & -10.77 & -47.30 \\
\hline$b$ & 1.0 & 21.70 & -14.59 & -61.90 \\
\hline 7 & 1.3 & 26.68 & -14.43 & -76.32 \\
\hline 8 & 1.2 & 30.15 & -10.06 & $-96 . \pm 0$ \\
\hline 9 & 1.2 & $3 i .47$ & -3.64 & -70.24 \\
\hline 11 & 2.2 & 38.71 & $-2 i .2 i$ & -111.45 \\
\hline 33 & 18.0 & 49.35 & -22.03 & -153.49 \\
\hline 15 & 28.7 & 56.08 & -9.01 & -142.50 \\
\hline 17 & 39.6 & 62.83 & -5.87 & -148.37 \\
\hline 19 & 50.9 & 70.23 & -2.00 & -150.37 \\
\hline 21 & 36.5 & 76.30 & -6.27 & -156.64 \\
\hline 24 & 49.0 & 87.70 & -4.23 & -160.87 \\
\hline 26 & 99.1 & 74.30 & -2.41 & -163.28 \\
\hline 23 & 39.4 & 101.60 & -6.60 & -169.28 \\
\hline 30 & 52.5 & 110.72 & -1.65 & -171.54 \\
\hline 32 & 40.30 & 122.69 & -9.76 & -181.50 \\
\hline 34 & 39.50 & 160.26 & -32.85 & -214.35 \\
\hline 35 & 42.70 & 185.00 & -17.44 & -231.77 \\
\hline
\end{tabular}

SAMPLE HO. EFFLUEHT PORE VOL. ELUITEN SUM ELLITED

\begin{tabular}{|c|c|c|c|c|}
\hline & Pp: $\mathrm{Ha}$ & E!UTED & og $\mathrm{Ha}$ & og \\
\hline 1 & $\$ 770$ & 2.00 & 502.97 & 502.97 \\
\hline 2 & 1650 & 5. 72 & 337.61 & 840.58 \\
\hline 3 & 692 & 9.32 & 120.19 & 960.77 \\
\hline 4 & 388 & 12.91 & 67.67 & 1029.34 \\
\hline 5 & 266 & 16.64 & 47.64 & 1077.48 \\
\hline 6 & 210 & 21.70 & 49.31 & 1127.10 \\
\hline 7 & 168 & 26.68 & -37.74 & $1 ! 64.84$ \\
\hline 8 & 146 & 30.15 & 22.25 & $1: 37.09$ \\
\hline 9 & 132 & $3 ! .47$ & $7.4 \overline{7}$ & $1: 94.58$ \\
\hline 11 & 96 & 38. 31 & 28.60 & 1222.56 \\
\hline 13 & 45 & 49.65 & 11.60 & 1234.17 \\
\hline 15 & 26 & 56.06 & i.,30 & 1234.54 \\
\hline 17 & 26 & 62.83 & 6.18 & 1234.72 \\
\hline 19 & 25. & 70.23 & 0.16 & 1234.87 \\
\hline 21 & 25 & .76 .30 & 0.13 & 1235.00 \\
\hline 24 & 25 & 87.70 & 0.06 & 1235.06 \\
\hline 26 & $2 \dot{D}$ & 94.30 & 0.24 & 1255.31 \\
\hline 28 & 25 & 101.30 & 0.04 & 1235.35 \\
\hline 30 & 26 & 110.72 & 0.28 & 1235.63 \\
\hline 32 & 25.70 & 122.67 & 0.44 & $i 235.67$ \\
\hline 34 & 26.10 & 160.26 & 2.13 & 1238.26 \\
\hline 35 & 26.40 & 105.00 & 1.84 & 1240.10 \\
\hline
\end{tabular}


TABLE C.19. contd

SAMPLE NO. EFFLUENT PORE YOL. ELUTED SUM ELUTED PPE $K$ ELUTED Mg $K$ ig $K$

$\begin{array}{rrrrr}1 & 13.0 & 2.00 & 0.55 & 0.55 \\ 2 & 4.2 & 5.92 & -0.75 & -0.20 \\ 3 & 4.0 & 9.32 & -0.68 & -0.88 \\ 4 & 2.0 & 12.91 & -1.10 & -1.99 \\ 5 & 1.9 & 16.64 & -1.17 & -3.15 \\ 6 & 1.8 & 21.70 & -1.58 & -4.73 \\ 7 & 0.3 & 26.68 & -1.98 & -6.71 \\ 8 & 0.3 & 30.15 & -1.38 & -8.09 \\ 9 & 0.3 & 31.47 & -1.52 & -8.62 \\ 11 & 3.9 & 38.91 & -1.54 & -10.16 \\ 13 & 26.0 & 49.85 & 11.71 & 1.56 \\ 15 & 27.0 & 56.08 & 6.34 & 7.30 \\ 17 & 21.0 & 32.83 & 4.72 & 12.62 \\ 19 & 16.0 & 30.23 & 3.22 & 15.84 \\ 21 & 14.0 & 76.30 & 1.99 & 17.83 \\ 24 & 13.0 & 87.30 & 3.14 & 20.97 \\ 36 & 11.0 & 94.30 & 1.12 & 22.09 \\ 28 & 9.7 & 101.80 & 0.76 & 22.85 \\ 30 & 9.4 & 110.72 & 0.76 & 23.60 \\ 32 & 8.00 & 122.69 & 0.13 & 23.73 \\ 34 & 8.20 & 160.36 & 0.80 & 24.53 \\ 35 & 6.20 & 185.00 & -2.10 & 22.43\end{array}$

SAKPLE NO, EFFLUERT POGE VGL. ELUTED SUM ELUTED POA Al ELUTED $\triangle \mathrm{Al}$ og $\mathrm{A}]$

\begin{tabular}{|c|c|c|c|c|}
\hline 1 & 62.60 & 2.00 & 0.63 & 6.6 .3 \\
\hline 2 & 41.80 & 5.92 & 8.66 & 15,29 \\
\hline$j$ & 22.40 & 9.32 & 4.02 & 19.31 \\
\hline 4 & 13.70 & 12.91 & 2.59 & 23.89 \\
\hline 5 & 9.62 & 16.69 & 1.88 & 23.70 \\
\hline 6 & 7.12 & 21.70 & 1.80 & 25.66 \\
\hline$i$ & 5.45 & 26.08 & 1.41 & 27.07 \\
\hline 8. & 4.31 & 30.15 & $0.7 i$ & 27.85 \\
\hline$\dddot{7}$ & 3.31 & 31.43 & 0.22 & 26.07 \\
\hline $1 i$ & 1.90 & 38.91 & 0.71 & 26.73 \\
\hline 13 & 1.30 & 49.85 & 0.70 & 27.88 \\
\hline 15 & 1.01 & 56.08 & 0.30 & 29.76 \\
\hline 17 & 1.32 & 22.83 & 0.44 & 30.21 \\
\hline 19 & 2.13 & 70.23 & $0 . \hat{6} \dot{z}$ & 31.63 \\
\hline 21 & 2.14 & 76.30 & $0 .=6$ & 31.60 \\
\hline 24 & $2.7 B$ & $5 i .70$ & 1.62 & 33.30 \\
\hline 26 & 3.59 & 94.30 & 1.26 & 34.56 \\
\hline 28 & $4.4 j$ & 101.90 & $! .72$ & j5:2B \\
\hline 30 & 3.61 & 110.72 & i.bó & 37.54 \\
\hline 32 & 4.63 & 122.69 & 2.37 & 40.81 \\
\hline 34 & 3.57 & 1600.26 & 6.71 & 47.72 \\
\hline 35 & 3.94 & $: 65.00$ & 5.04 & 52.76 \\
\hline
\end{tabular}


TABLE C.19. contd

SAMPLE ND. EFFLUENT FORE VOL. ELUTED SUH ELUTED ig $5 j$

$139.0 \quad 2.00$

$89.2 \quad 5.92$

$77.0 \quad 9.32$

$51.6 \quad 12.91$

$37.6 \quad 16.64$

$28.7 \quad 21.70$

23.226 .68

21.7

22.1

$\$ 5.3$

12.7

11.0

11.6

10.7

9.7

8.8

7.6

6.2

4.8

2.71

3.54

3.27

\subsection{5}

31.47

30.71

49.85

56.08

62.83

70.23

76.30

87.70

94.30

101.30

110.72

122.67

160.26

135.00

13.14

15.42

11.17

6.96

4.47

3.67

2.16

1.25

0.50

0.12

$-1.33$

$-1.32$

$-1.22$

$-1.69$

$-1.71$

$-3.77$

$-2.59$

$-3.51$

$-4.83$

$-7.67$

$-22.82$

$-15.38$
4 $\mathrm{Si}$

13.14

28.56

39.73

46.70

51.16

54.84

57.00

58.23

58.73

56.85

57.52

56.20

54.98

53.29

51.57

47.82

45.23

41.71

36.88

29.21

เ. 39

$-8.79$
SAMPLE NO. EFFLUENT PORE VOL. ELUTEO SLM ELUTED DPE HQ ELUTED is Ho ag

\begin{tabular}{|c|c|c|c|c|}
\hline 1 & 0.06 & 2.00 & -1.48 & -1.48 \\
\hline 2 & 0.06 & 5.93 & -2.90 & -4.37 \\
\hline 3 & 0.06 & 9.32 & $-2,51$ & -5.68 \\
\hline 4 & 0.06 & $\{2.31$ & -2.05 & -3.54 \\
\hline 5 & 0.06 & 16.64 & $-2.7 \dot{s}$ & -12.28 \\
\hline 6 & 0.06 & 21.70 & -3.74 & -16.03 \\
\hline 7 & 0.06 & 26.68 & - క. cồ & $-13.7 !$ \\
\hline 9 & 0.06 & 30.15 & -2.5 & -22.20 \\
\hline 3 & 0.06 & $3 i .47$ & $-\hat{u} .78$ & -23.25 \\
\hline 11 & 0.06 & 33.91 & -5.50 & -23.75 \\
\hline 13 & 0.06 & 49.85 & -3.62 & -36.83 \\
\hline 15 & $\hat{0} . \hat{0} t$ & 56.08 & -4.60 & $-41.4 j$ \\
\hline$i T$ & 0.06 & 02.63 & -4.79 & -46.42 \\
\hline 17 & 0.06 & 70.23 & -5.47 & -51.87 \\
\hline $2 !$ & 0.06 & 76.30 & -4.40 & $-56.3 i$ \\
\hline 24 & 0.06 & 87.76 & -6.42 & -64.79 \\
\hline 26 & 0.06 & 34.30 & -4 . EE & -69.67 \\
\hline 22 & 0.66 & 161.60 & -5.54 & -75.21 \\
\hline 30 & 0.06 & 110.72 & -6.59 & -31.60 \\
\hline 32 & 0.06 & 122.67 & -8.34 & -90.05 \\
\hline 34 & 0.06 & 160.26 & $-27.7 b$ & -118.40 \\
\hline 35 & 0.06 & 195.00 & $-18 . i 3$ & -136. \\
\hline
\end{tabular}


TABLE C.19. contd

SAMPLE NO. EFFLUENT PORE VOL. ELUTED SUM ELUTED

\begin{tabular}{rrrrr} 
& PPA $F$ & ELUTED & IF & $19 F$ \\
\hline 1 & 102.0 & 2.00 & 10.76 & 10.76 \\
2 & 42.0 & 5.92 & 8.62 & 19.38 \\
3 & 15.0 & 9.32 & 2.61 & 21.39 \\
4 & 6.7 & 12.91 & 1.22 & 23.21 \\
5 & 5.7 & 16.64 & 1.03 & 24.24 \\
6 & 5.0 & 21.70 & 1.21 & 25.45 \\
7 & 4.6 & 26.68 & 1.03 & 26.53 \\
8 & 4.2 & 30.15 & 0.68 & 27.21 \\
9 & 4.2 & 31.47 & 0.26 & 27.47 \\
11 & 7.5 & 38.31 & 2.76 & 30.23 \\
13 & 7.6 & 49.85 & 4.12 & 34.34 \\
15 & 4.6 & 56.08 & 1.35 & 35.70 \\
17 & 5.2 & 62.83 & 1.68 & 37.38 \\
19 & 5.2 & 70.23 & 1.34 & 39.22 \\
21 & 5.9 & 76.30 & 1.74 & 40.96 \\
24 & 7.2 & 87.70 & 4.05 & 45.01 \\
26 & 7.8 & 94.30 & 2.55 & 47.56 \\
28 & 6.0 & 101.20 & 2.78 & 50.54 \\
30 & 8.5 & 110.72 & 3.78 & 54.33 \\
32 & 8.90 & 122.69 & 5.33 & 59.65 \\
34 & 6.70 & 160.26 & 16.33 & 75.38 \\
35 & 11.00 & 185.00 & 13.77 & 89.75
\end{tabular}

SAMPLE NO. EFFLUENT PORE YOL. ELUTED SUM ELUTED

\begin{tabular}{|c|c|c|c|c|}
\hline & $P p=\mathrm{H}_{03}$ & ELUTED & ig & 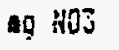 \\
\hline 1 & 150.0 & 2.00 & 15.89 & 15. .69 \\
\hline 2 & 22.0 & 5.72 & 4.55 & 20.44 \\
\hline 3 & 2.6 & 9.32 & 0.27 & $20 . i i$ \\
\hline 4 & 0.3 & 12.91 & 0.04 & 20.75 \\
\hline 5 & 0.2 & 16.64 & 0.01 & 20.76 \\
\hline 6 & 0.1 & 21.70 & 0.00 & 20.76 \\
\hline 7 & 0.2 & 26.68 & 0.03 & 20.79 \\
\hline 3 & 0.2 & 30.15 & 0.02 & 20.81 \\
\hline 9 & 0.2 & 31.47 & 0.01 & 20.61 \\
\hline i! & 0.2 & 38.71 & 6.04 & 20.85 \\
\hline 13 & 0.2 & 47.35 & 0.06 & 20.71 \\
\hline 15 & 0.5 & 56.08 & 0.13 & 21.04 \\
\hline 17 & 6.5 & 52.33 & 0.14 & 21.19 \\
\hline 19 & 0.5 & 70.23 & 0.16 & 21.34 \\
\hline 21 & 0.5 & 76.30 & 0.13 & 21.47 \\
\hline 24 & 0.5 & $37 . i v$ & 0.24 & 21.71 \\
\hline $2 b$ & 0.5 & 94.30 & 0.14 & 21.35 \\
\hline 28 & 0.5 & 101.80 & 0.16 & 20.01 \\
\hline 30 & 0.5 & 110.72 & 0.13 & 22.20 \\
\hline 32 & 0.5 & 122.69 & 0.25 & 22.45 \\
\hline 34 & 0.5 & 160.26 & $\hat{v} .8 \hat{v}$ & 23.25 \\
\hline 35 & 0.5 & 185.00 & 4.52 & 23.73 \\
\hline
\end{tabular}


TABLE C.19. contd

SAMPLE NO, EFFLUENT PORE VOL. ELUTED SUM ELUTED PPE HJEOJ ELUTED

ig $\mathrm{HJBOJ}$ a $\mathrm{HJBOJ}$

$\begin{array}{rrrrr}1 & 123.50 & 2.00 & 13.05 & 13.05 \\ 2 & 30.80 & 5.92 & 6.33 & 19.38 \\ 3 & 6.30 & 9.32 & 1.07 & 20.46 \\ 4 & 2.63 & 12.91 & 0.32 & 20.78 \\ 5 & 1.79 & 16.64 & 0.29 & 21.07 \\ 6 & 1.72 & 21.70 & 0.37 & 21.44 \\ 7 & 1.67 & 26.68 & 0.35 & 21.79 \\ 8 & 1.60 & 30.15 & 0.23 & 22.02 \\ 9 & 1.60 & 31.47 & 0.09 & 22.31 \\ 11 & 1.54 & 38.91 & 0.47 & 22.58 \\ 13 & 1.43 & 49.85 & 0.63 & 23.21 \\ 15 & 1.22 & 56.06 & 0.29 & 23.50 \\ 17 & 1.28 & 62.33 & 0.34 & 23.84 \\ 19 & 1.67 & 70.23 & 0.52 & 24.36 \\ 21 & 1.16 & 76.30 & 0.26 & 24.62 \\ 24 & 1.15 & 87.70 & 0.49 & 25.11 \\ 26 & 1.12 & 94.30 & 0.27 & 25.39 \\ 23 & 1.06 & 101.30 & 0.29 & 25.67 \\ 30 & 1.00 & 110.72 & 0.31 & 25.88 \\ 32 & 1.03 & 122.69 & 0.44 & 26.42 \\ 34 & 0.85 & 160.26 & 1.02 & 27.44 \\ 35 & 0.38 & 165.00 & 0.71 & 28.15\end{array}$

SAMPLE ND. EFFLUENT PORE VOL. ELUTED SUK ELUTED

\begin{tabular}{|c|c|c|c|c|}
\hline & ppe 304 & ELUTED & 504 & 9ด 504 \\
\hline$!$ & 1550 & 2.00 & 155.18 & 155.18 \\
\hline 2 & 246 & 5.92 & 33.24 & 188.43 \\
\hline 3 & 35 & 9.32 & -3.78 & 184.64 \\
\hline 4 & 63 & 12.71 & -4.38 & 180.27 \\
\hline 5 & $b 7$ & 16.04 & -3.76 & $176.5 !$ \\
\hline 6 & $6 j$ & 21.70 & -5.10 & 171.41 \\
\hline 7 & 72 & 26.68 & -3.70 & 167.72 \\
\hline 8 & 72 & 30.15 & $-2.5 i$ & 105.14 \\
\hline 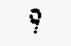 & 72 & 31.47 & -10.96 & 164.16 \\
\hline 11 & 82 & 36.71 & -1.58 & 162.58 \\
\hline 13 & $B 0$ & 49.85 & -3.46 & i5s.ii \\
\hline 15 & 71 & 56.08 & -4.95 & 154.16 \\
\hline 17 & 73 & 62.83 & -4.65 & 149.50 \\
\hline 19 & 69 & 70.23 & -6.67 & 142.84 \\
\hline 21 & 69 & 76.30 & -5.47 & 137.37 \\
\hline 24 & 73 & 37.70 & $-i .85$ & 129.51 \\
\hline 26 & 73 & 94.30 & -4.55 & 124.37 \\
\hline 28 & 72 & $10 i .30$ & -5.57 & 117.40 \\
\hline 30 & 73 & 110.72 & -6.15 & 113.26 \\
\hline 32 & $71.6 \hat{v}$ & 122.68 & -9.52 & 103.74 \\
\hline 34 & 73.00 & 160.26 & -25.89 & 77.85 \\
\hline 35 & 74.60 & iš. $0 \hat{0}$ & -14.95 & $62 . \overline{71}$ \\
\hline
\end{tabular}


TABLE C.20. Mass Balance for Flow Through Leach Test on Crushed Grout/Sediment with Groundwater (MBSCGI)

SAMPLE NO. EFFLUENT FORE VOL. ELUTED SUM ELUTED ppe ca eluted a ca a ca

$\begin{array}{rrrrr}1 & 45.2 & 1.84 & -1.06 & -1.06 \\ 2 & 2.7 & 5.72 & -11.01 & -12.07 \\ 3 & 1.9 & 9.06 & -9.62 & -21.68 \\ 4 & 1.6 & 11.69 & -7.61 & -29.29 \\ 5 & 1.4 & 15.49 & -11.03 & -40.32 \\ 6 & 1.2 & 20.61 & -14.91 & -55.23 \\ 7 & 1.2 & 24.40 & -11.05 & -66.28 \\ 8 & 1.3 & 27.94 & -10.30 & -76.38 \\ 9 & 1.1 & 31.02 & -9.00 & -85.57\end{array}$

SAMPLE NO. EFFLLUENT PORE YOL. ELUTED SUM ELUTED

\begin{tabular}{|c|c|c|c|c|}
\hline & $P P=K$ & ELUTED & $9 k$ & $\operatorname{tg} K$ \\
\hline 1 & 8.3 & 1.64 & 0.05 & 0.05 \\
\hline 2 & 4.3 & 5.72 & -0.72 & $-0.6 i$ \\
\hline 3 & 3.0 & 9.06 & -0.85 & -1.53 \\
\hline 4 & 3.0 & 11.69 & -0.67 & -2.20 \\
\hline 5 & 2.2 & 15.49 & -1.13 & -3.33 \\
\hline$\dot{b}$ & 2.1 & 20.61 & -1.55 & -4.88 \\
\hline 7 & 0.3 & 24.40 & -1.51 & -0.39 \\
\hline B & 0.3 & 27.94 & -1.41 & -7.81 \\
\hline 9 & 0.3 & 31.02 & -1.23 & $-9.0 j$ \\
\hline
\end{tabular}

SAHPLE NG. EFFLUEHT FOFE YOL. ELUTED SUM ELUTED

\begin{tabular}{|c|c|c|c|c|}
\hline & pps 5 j & SLUTED & ig $\mathrm{Sj}$ & 4 $5 i$ \\
\hline 1 & 72.0 & 1.64 & 5.58 & 5.58 \\
\hline 2 & 169.0 & 5.72 & 31.78 & 63.37 \\
\hline 3 & 107.0 & 3.06 & 16.34 & 53.70 \\
\hline 4 & 70.5 & 11.67 & i.7ó & 61.47 \\
\hline 5 & 57.6 & 15.47 & B.j1 & 70.08 \\
\hline$b$ & 43.4 & 20.61 & 7.73 & 77.81 \\
\hline $\bar{i}$ & 3.6 & 24.40 & 3.75 & B!.5' \\
\hline B & 29.7 & 25.94 & 2. है! & 84.37 \\
\hline 9 & 23.3 & 51.62 & 2.02 & 30.30 \\
\hline
\end{tabular}

SAMPLE NO. EFFLUENT PORE VOL. ELUTEE SUM ELUITED ọ $\mathrm{Ka}$

$\begin{array}{rrrrr}1 & 1030 & 1.64 & 98.36 & 98.36 \\ 2 & 1920 & 5.72 & 391.60 & 669.44 \\ 3 & 990 & 9.06 & 171.44 & 260.68 \\ 4 & 585 & 11.69 & 78.34 & 739.22 \\ 5 & 468 & 15.49 & 69.54 & 325.76 \\ 6 & 345 & 20.61 & 87.15 & 915.81 \\ 7 & 272 & 24.40 & 49.79 & 965.70 \\ 8 & 213 & 27.94 & 35.40 & 1001.10 \\ 9 & 191 & 31.02 & 27.19 & 102.39\end{array}$

SAMPLE NO. EFFLUERT FORE VGL. ELUTE⿹̃ SUM ELUTES Ppa Al ELUTED UQ Al GQ Al

$\begin{array}{rrrrr}1 & 0.17 & 1.54 & 0.01 & 0.0 ! \\ 2 & 25.60 & 5.72 & 5.26 & 5.27 \\ 3 & 20.80 & \$ .06 & 3.58 & 8.35 \\ 4 & 19.30 & 11.69 & 2.69 & 11.53 \\ 5 & 17.40 & 15.49 & 3.50 & 15.13 \\ 6 & 14.60 & 20.61 & 3.95 & 19.03 \\ 7 & 11.70 & 24.40 & 3.34 & 21.42 \\ 8 & 8.59 & 27.94 & 1.60 & 23.02 \\ 7 & 7.54 & 31.02 & 1.22 & 24.33\end{array}$

SAMPLE NO. EEFLUENT FOSE VOL. ELUTED SIMY ELUTED Dou Ha ELUTED ag hl ig

\begin{tabular}{|c|c|c|c|c|}
\hline 1 & 0.06 & i. $\hat{a}^{4}$ & -1.30 & 6́ \\
\hline 2 & 0. itió & 5.72 & -3.80 & -4.24 \\
\hline 3 & 0.68 & 3.06 & -2.46 & $-6 . i z$ \\
\hline 4 & 3.06 & :1.:9 & $-1, \$ 5$ & $-\overline{3} .67$ \\
\hline 5 & $0 . \dot{0}$ & ij. 45 & $-2.3 i$ & $-1: .49$ \\
\hline 8 & 0.00 & 20.co: & $-3.3 i$ & $-15.8 E$ \\
\hline$i$ & 3.66 & 24.40 & $-3.8 j$ & $-1 \bar{j}, \bar{j}=$ \\
\hline 6 & 0.60 & 27.74 & $-\dot{\partial}$ & -20.72 \\
\hline$;$ & 0.62 & 31.02 & $-\dot{2} . \dot{\mathrm{i}}$ & $-20,00$ \\
\hline
\end{tabular}


TABLE C.20. contd

SAMPLE NO, EFFLUENT PORE VOL, ELUTED SUH ELUTED

\begin{tabular}{crrrr} 
& PPE F & ELUTED & MQ F & B $F$ \\
\hline 1 & 32.0 & 1.84 & 3.08 & 3.08 \\
2 & 34.0 & 5.72 & 6.91 & 10.00 \\
3 & 19.0 & 9.06 & 3.29 & 13.28 \\
4 & 13.0 & 11.69 & 1.75 & 15.03 \\
5 & 10.0 & 15.49 & 1.92 & 16.95 \\
6 & 7.3 & 20.61 & 1.85 & 16.80 \\
7 & 5.8 & 24.40 & 1.07 & 19.87 \\
8 & 5.0 & 27.34 & 0.65 & 20.72 \\
9 & 4.2 & 31.02 & 0.61 & 21.33
\end{tabular}

5AMPLE HO. EFFLUENT PORE VOL. ELUTED SUM ELUTED PDE HJBOZ ELUTED

$\begin{array}{rrrrr}1 & 55.59 & 1.64 & 5.41 & 5.41 \\ 2 & 33.74 & 5.72 & 6.99 & 12.30 \\ 3 & 12.30 & 9.66 & 2.21 & 14.51 \\ 4 & 8.12 & 11.69 & 1.09 & 15.60 \\ 5 & 5.72 & 15.49 & 1.09 & 16.69 \\ 6 & 4.60 & 20.61 & 1.16 & 17.85 \\ 7 & 2.68 & 24.40 & 0.47 & 18.32 \\ 3 & 7.33 & 27.94 & 1.33 & 19.65 \\ 7 & 6.23 & 31.02 & 0.76 & 20.61\end{array}$

SAKPLE ND. EFFLUENT PORE VOL. ELUTED SUM ELUTED PPE KOJ ELUTED MO

$\begin{array}{rrrrr}1 & 160.0 & 1.24 & 15.05 & 15.65 \\ 2 & 56.0 & 5.72 & 11.54 & 27.69 \\ 3 & 6.4 & 9.06 & 1.12 & 28.31 \\ 4 & 0.8 & 11.67 & 0.10 & 20.40 \\ 5 & 0.2 & 15.49 & 1.02 & 23.42 \\ 6 & 0.1 & 20.61 & -0.01 & 22.41 \\ 7 & 0.2 & 24.40 & 0.02 & 28.43 \\ 8 & 0.2 & 27.74 & -10.02 & 28.45 \\ 7 & 0.2 & 31.62 & 0.02 & 28.47\end{array}$

SAKPLE HO. EFFLUEMT FORE YOL. ELUTEE SUM ELJTED

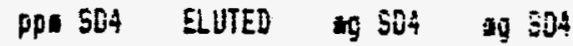

\begin{tabular}{|c|c|c|c|c|}
\hline 1 & 1440 & 1.34 & 132.32 & 132.52 \\
\hline 2 & 560 & 5.72 & 97.22 & 250.34 \\
\hline 3 & 181 & 9.06 & 36.88 & 247.22 \\
\hline 4 & 106 & 11.69 & 2.50 & 250.61 \\
\hline 5 & 79 & 15.49 & -1.41 & 248.60 \\
\hline 6 & 68 & 20.51 & -4.70 & 243.70 \\
\hline 7 & 68 & 24.40 & -3.63 & $240.07 j$ \\
\hline 8 & 68 & 27.94 & -3.39 & 23.60 \\
\hline 8 & 66 & 31.02 & -3.28 & 233.40 \\
\hline
\end{tabular}




\section{Distribution}

No. of

Copies

\section{OFFSITE}

12 DOE/Office of Scientific and Technical Information

J. A. Coleman, NE-24

DOE Office of Remedial Action and Waste Technology 19901 Germantown Road

Germantown, MD 20545

3 Westinghouse Savannah River Company

Savannah River Site Aiken, SC 29808-001

Attn:

C. A. Langton

R. M. Wallace

E. Wilhite

2 Brookhaven National Laboratory Upton, NY 19973

Attn: M. Fuhrmann

T. M. Sullivan

3 Nuclear Regulatory Commission Office of Nuclear Regulatory

Research

Washington, DC 20555

Attn: J. W. Bradbury

E. O'Donnell

P. R. Reed

$4 \quad$ Oak Ridge National Laboratory

P. O. Box X

Oak Ridge, TN 37831

Attn.: T. M. Gilliam

H. W. Godbee

E. McDaniel

T. L. Sams
No. of

Copies

2 EG\&G

P. O. Box 1625

Idaho Falls, ID 83415

Attn.: J. McConnell

R. Rogers

T. L. Jones

New Mexico State University

Dept. of Agronomy

Agriculture Building

Room 254

Los Cruces, NM 88003-003

R. O. Lokken

618 N. Georgia

Kennewick, WA 99336

FOREIGN

A. Atkinson

Atomic Energy Research

Establishment

Harwell

Didcot

Oxfordshire OX 11 ORA

UNITED KINGDOM

L. P. Buckley

Atomic Energy of Canada Ltd. Chalk River, Ontario KOJ 1J0

CANADA

R. Dayal

Ontario Hydro

800 Kipling Ave.

Toronto, Ontario M8Z 5S4

CANADA

Distr.1 
No. of

Copies

ONSITE

4 DOE Richland Operations Office

E. A. Bracken

A5-19

R. D. Freeberg

R. F. Guercia

D. E. Trader

10 Westinghouse Hanford Company

K. C. Burgard

G6-13

D. W. Hendrickson

F. N. Hodges

A. A. Kruger

F. M. Mann

J. W. Shade

J. C. Sonnichsen

M. I. Wood

J. A. Voogd

G. F. Williamson
N3-13

G6-13

G6-13
No. of

Copies

35 Pacific Northwest Laboratory

L. M. Bagaasen

P7-43

M. J. Fayer .

K9-33

G. W. Gee

K9-33

G. R. Holdren

K6-81

D. I. Kaplan

C. T. Kincaid (2)

K6-81

K9-33

K. M. Krupka

K6-81

G. V. Last

K9-48

V. L. Legore

P8-37

C. W. Lindenmeier

K6-81

P.F.C. Martin

P8-37

W. J. Martin

K1-19

S. V. Mattigod

R. J. Serne (10)

J. H. Westsik, Jr. (4)

G. A. Whyatt

Publishing Coordination

Technical Report Files (5)
K6-81

K6-81

K9-80

P7-19 